\title{
Data Recovery Excavations at the Snakeskin Bluff Site (41GU177), Guadalupe County, Texas
}

Christina Nielsen

Ashley Eyeington

Ken Lawrence

Chris Shelton

Mercedes C. Cody

See next page for additional authors

Follow this and additional works at: https://scholarworks.sfasu.edu/ita

Part of the American Material Culture Commons, Archaeological Anthropology Commons, Environmental Studies Commons, Other American Studies Commons, Other Arts and Humanities Commons, Other History of Art, Architecture, and Archaeology Commons, and the United States History Commons

Tell us how this article helped you.

This Article is brought to you for free and open access by the Center for Regional Heritage Research at SFA ScholarWorks. It has been accepted for inclusion in Index of Texas Archaeology: Open Access Gray Literature from the Lone Star State by an authorized editor of SFA ScholarWorks. For more information, please contact cdsscholarworks@sfasu.edu. 


\section{Data Recovery Excavations at the Snakeskin Bluff Site (41GU177), Guadalupe County, Texas}

Authors

Christina Nielsen, Ashley Eyeington, Ken Lawrence, Chris Shelton, Mercedes C. Cody, and Brandon S. Young

Creative Commons License

(c) (7) \&

This work is licensed under a Creative Commons Attribution-NonCommercial 4.0 International License 


\section{Data Recovery Excavations at the Snakeskin Bluff Site (41GU177), Guadalupe County, Texas}

TEXAS ANTIQUITIES PERMIT NO. 8231

OCTOBER 2019

PREPARED FOR

Central Texas Regional Water Supply Corporation and

VRRSP Consultants, LLC

PREPARED BY

SWCA Environmental Consultants 



\title{
DATA RECOVERY EXCAVATIONS AT THE SNAKESKIN BLUFF SITE (41GU177), GUADALUPE COUNTY, TEXAS
}

\author{
Prepared for \\ Central Texas Regional Water Supply Corporation \\ 3711 S. Mo-Pac Expressway, Building One, Suite 300 \\ Austin, Texas 79746 \\ and \\ VRRSP Consultants, LLC \\ 2000 NW Loop 410 \\ San Antonio, Texas 78213
}

Prepared by

Christina Nielsen, M.A., Ashley Eyeington, B.A., Ken Lawrence, M.A., Chris Shelton, M.A., Mercedes C. Cody, B.A., and Brandon S. Young, M.A., RPA

Principal Investigator

Christina Nielsen, M.A.
SWCA Environmental Consultants
4407 Monterey Oaks Boulevard
Building 1, Suite 110
Austin, Texas 78749
www.swca.com

SWCA Project No. 31410.00

Texas Antiquities Permit No. 8231

SWCA Cultural Resources Report No. 19-584

October 2019 



\section{ABSTRACT}

On behalf of Central Texas Regional Water Supply Corporation (CTRWSC) and VRRSP Consultants, LLC, SWCA Environmental Consultants (SWCA) conducted archaeological data recovery excavations at multicomponent site $41 \mathrm{GU} 177$ (the Snakeskin Bluff Site) within the proposed alignment of the Vista Ridge Regional Water Supply Project (Vista Ridge) in Guadalupe County, Texas. Investigations were conducted in compliance with Section 106 of the National Historic Preservation Act (NHPA) (54 United States Code [USC] 306108) and its implementing regulations (36 Code of Federal Regulations [CFR] 800), in anticipation of a Nationwide Permit 12 from the U.S. Army Corps of Engineers (USACE) in accordance with Section 404 of the Clean Water Act. In addition, the work is subject to compliance with the Antiquities Code of Texas (ACT), as the Vista Ridge Project will be ultimately owned by CTRWSC, a political subdivision of the State of Texas.

The Vista Ridge project will involve construction of an approximately 140-mile-long, 60 -inch-diameter water pipeline from north-central San Antonio, Bexar County, to Deanville, Burleson County, Texas. The of area of potential effects (APE) for the Phase I survey efforts included the proposed centerline alignment and a 100-foot-wide corridor (50 feet on either side of centerline), as well as temporary and permanent construction easements, and aboveground facilities, such as pump stations and the northern and southern termini sites. Between June 2015 and March 2018 the cultural resources inventory identified 78 cultural resources (i.e., 64 archaeological sites and 14 isolated finds). Included in that inventory was site 41GU177, originally discovered on August 31, 2015 on the west bank of the Guadalupe River.

Site 41 GU177 is a stratified prehistoric site on the western high bank of the Guadalupe River southeast of New Braunfels, Texas near the community of McQueeney. The site contains components deposited intermittently from approximately the Late Archaic to Transitional Archaic periods through Late Prehistoric times. The primary components investigated in the excavations span the final Late Archaic period and into the Austin phase of the Late Prehistoric, a timeframe from approximately 2,600 to 900 years ago. The field investigations, conducted between October 2016 and December 2017, included intensive shovel testing, geomorphological study with mechanical excavations, and subsequent hand excavations. This report presents the results of the data recovery investigations conducted from November-December 2017. The survey results and testing results have been previously reported on (Acuña et al. 2016; Rodriguez et al. 2017); the testing results are also presented in Appendix A.

SWCA's work at $41 \mathrm{GU} 177$ was conducted under the ACT. The state regulations mandate the evaluation of the site's eligibility for designation as a State Antiquities Landmark (SAL) or for listing on the National Register of Historic Places (NRHP). The survey and testing investigations were conducted under ACT Permit No. 7295, and the subsequent data recovery was completed under Permit No. 8231. Brandon S. Young served as initial Principal Investigator (PI) on both permits and Christina Nielsen took over as PI on the permits for the final reporting stages of the project.

Overall, $34.1 \mathrm{~m}^{3}$ of sediment was excavated from the site during the data recovery through both traditional and feature-focused unit excavations. During these excavations, approximately $22 \mathrm{~m}^{2}$ of Late Prehistoric components were exposed, consisting of rock-lined hearths and 6,496 artifacts. The Late Prehistoric artifacts included Edwards points, Perdiz points, a Fresno point, ceramics, bifaces, an end scraper, a shell bead, various informal lithic tools, ground stone, choppers, debitage, and faunal remains. The Late Prehistoric component was approximately $40-80 \mathrm{~cm}$ thick. No cultural features were identified within the underlying Archaic components of the site; however, 3,421 artifacts were recovered including a Zephyr point, bifaces, ground stone, various informal lithic tools, choppers, debitage, and faunal remains. The Transitional Archaic component was approximately $35-60 \mathrm{~cm}$ thick; however, the underlying Archaic (and possible older) components were not defined. 
The excavations were limited to the right-of-way, and consequently the exposure afforded only a partial glimpse of the overall site. Based on the assemblage, the site is interpreted as a logistical base camp as indicated by both formal and informal tool forms and site furniture. Small groups exploited the abundance of lithic raw material and riparian zone resources, making forays into the landscape to hunt and forage. A total of four radiocarbon dates from the Late Prehistoric components reveal several short-term encampments over the course of several centuries from approximately 600 to 1200 B.P. (A.D. 750 to 1350). The Toyah Phase component has some noted disturbances (especially towards the ground surface), but good integrity from where the radiocarbon sample was collected. The Austin Phase component is vertically and horizontally discrete, contains a substantial amount of archaeological materials, and the site structure and radiocarbon dates suggests multiple, discrete occupations.

The data recovery investigations at the Snakeskin Bluff site sought to address environmental, technological, chronological, and adaptive changes during the transition from Archaic to Late Prehistoric. As mentioned, the overall artifact and feature recovery at the site was low and disturbances and mixing of components was noted, especially within the upper deposits of the site. Disturbances within the Late Prehistoric Toyah Phase were most prevalent in the eastern portions of the site. The Late Prehistoric Austin Phase component contained intact, well-preserved archaeological deposits containing preserved flora and faunal material, cooking features, and diagnostic implements. The Transitional Archaic (and older) occupations were difficult to characterize, due to limited quantities of temporal diagnostic artifacts, lack of cultural features, and low artifact recovery. Despite these limitations, the data recovered from the cultural components show diachronic shifts between the technological and foraging strategies of the Archaic and Late Prehistoric.

In concurrence with the 2016-2017 testing recommendations, the Snakeskin Bluff site is considered eligible for designation as an SAL and for the NRHP. Although not all cultural components of the site were stratigraphically discrete, the Late Prehistoric Austin Phase component revealed intact, wellpreserved archaeological deposits that significantly contributed to our understanding of Late Prehistoric patterns. Given the sensitive nature of the cultural deposits at $41 \mathrm{GU} 177$, the main concern following the completion of data recovery excavations was the prevention of significant surface and subsurface impacts to the site during clearing and pipeline construction. As such, SWCA developed a site monitoring protocol; methods and results of the monitoring efforts are provided in Appendix H. Contributing components beyond the impact area will not be affected and will be preserved by avoidance; however, it is important to note that these investigations mitigated the project-specific effects, not the entire site. Any future project that could impact the site's deeper deposits, or those beyond the current right-of-way, warrant further consideration to assess the possibilities for additional contributing components. With these considerations, no further work is recommended. 


\section{CONTENTS}

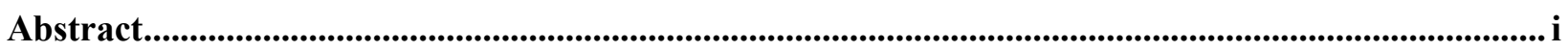

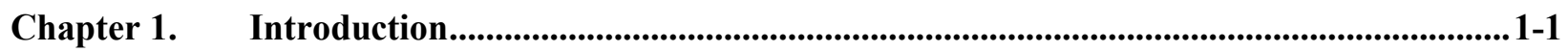

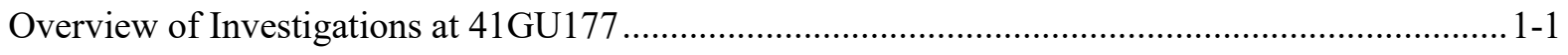

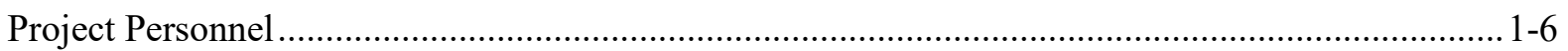

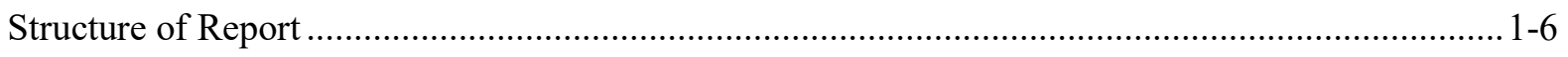

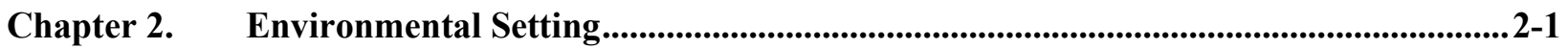

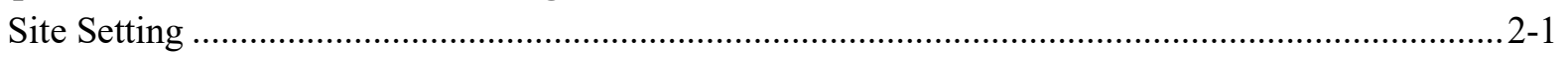

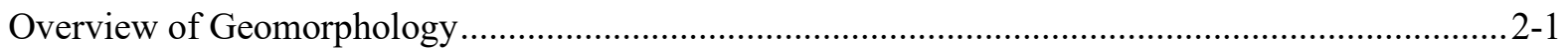

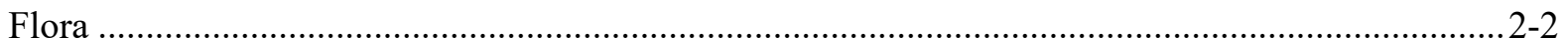

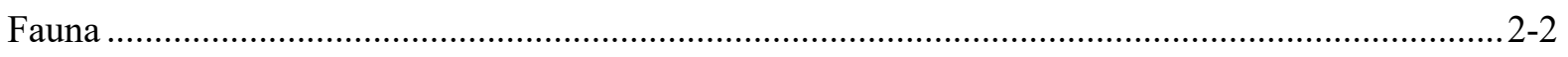

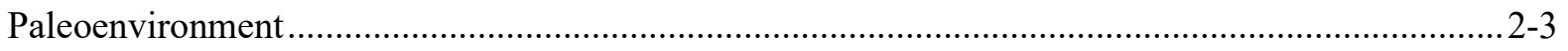

Chapter 3. Cultural Setting........................................................................................................................ 3-1

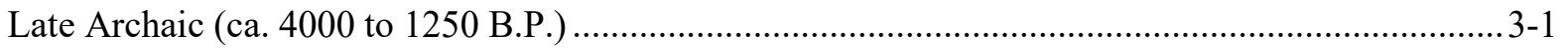

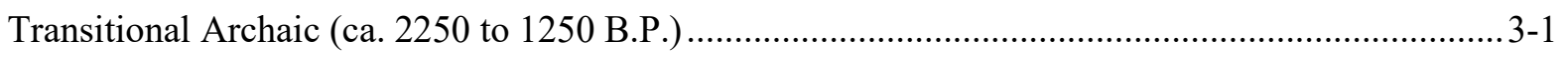

Late Prehistoric (ca. 1250 to 350 B.P.) …............................................................................... 3-2

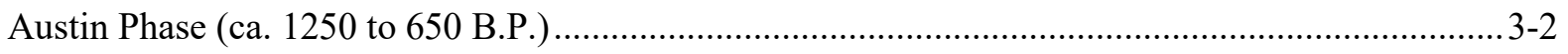

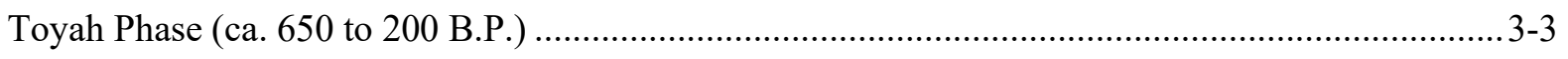

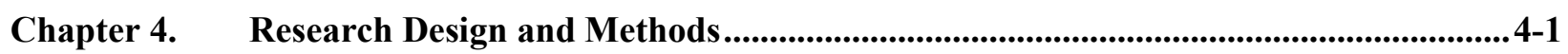

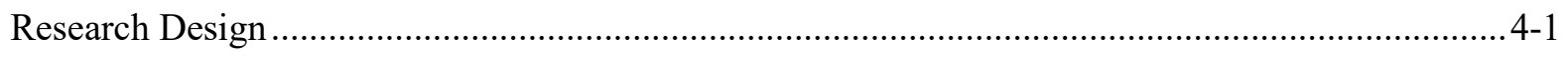

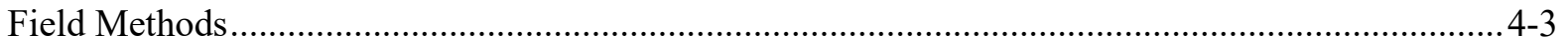

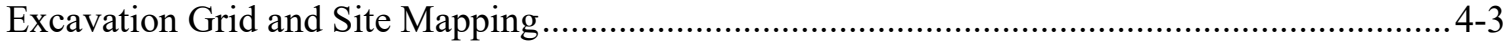

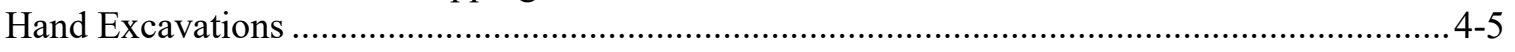

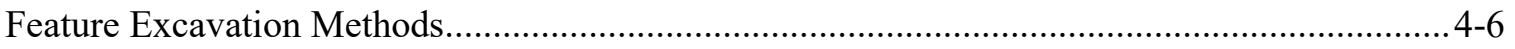

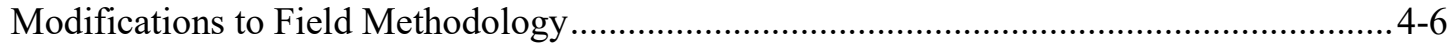

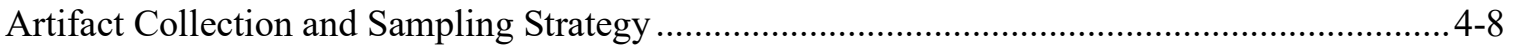

Laboratory Processing and Analysis Methods ........................................................................ $4-8$

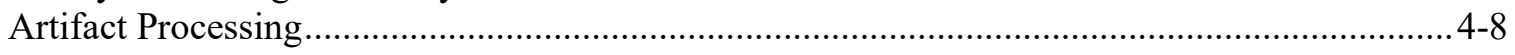

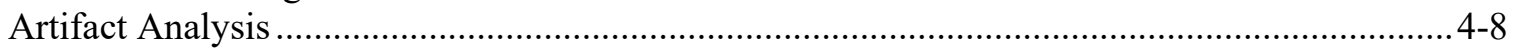

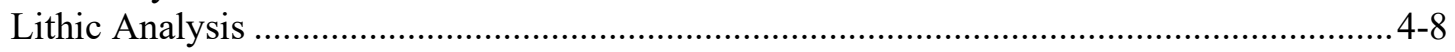

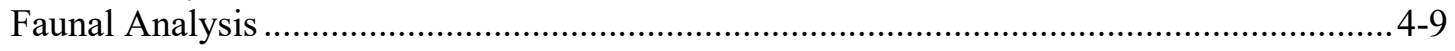

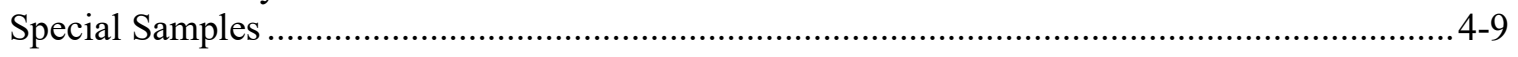

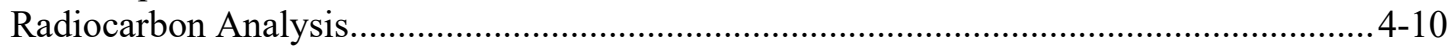

Flotation and Macrobotanical Analysis ...........................................................................

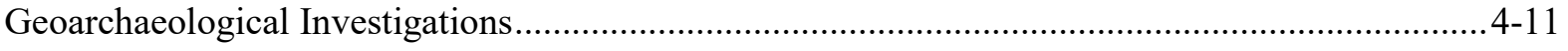

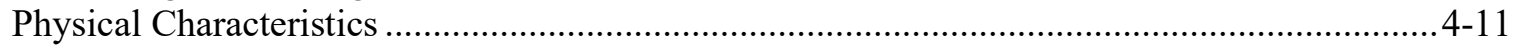

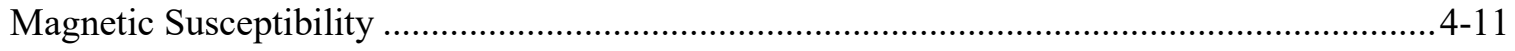

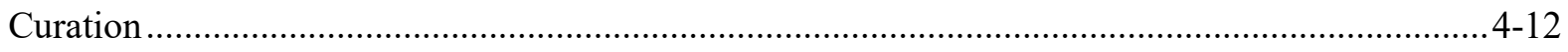

Chapter 5. Overview of Data Recovery Investigations .......................................................................5-1

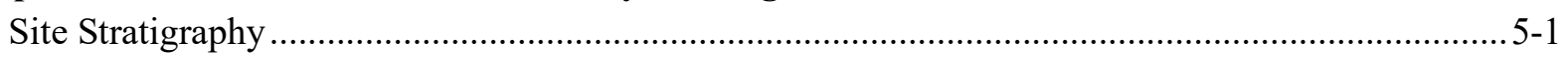

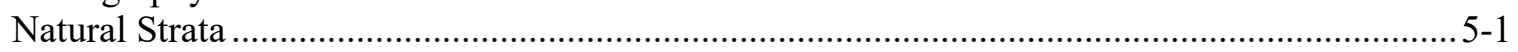

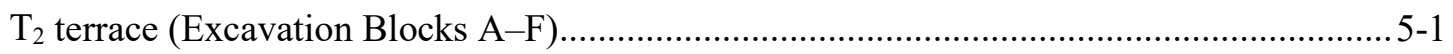

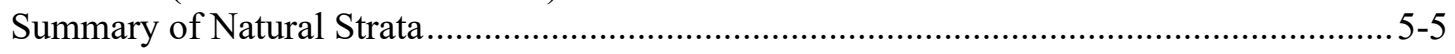

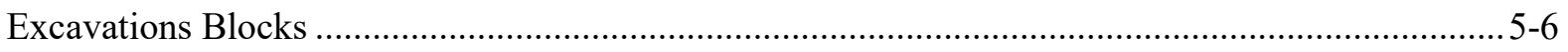




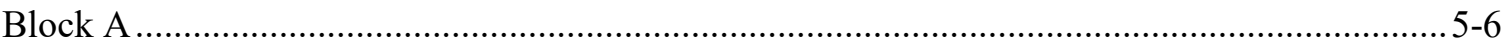

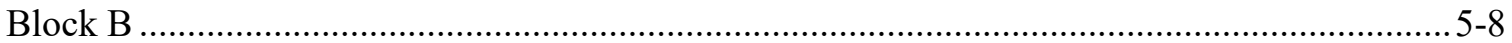

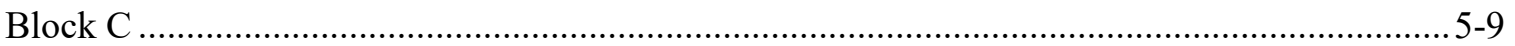

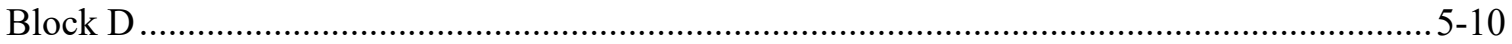

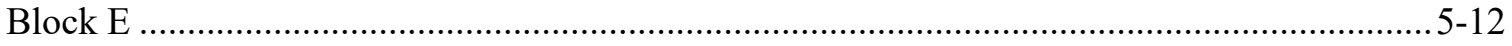

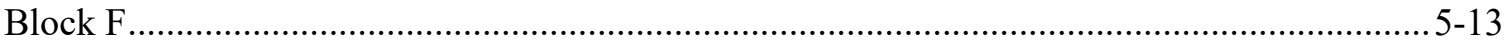

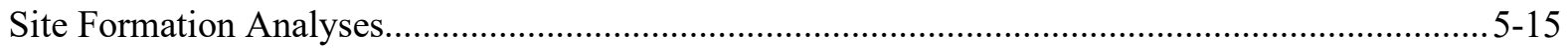

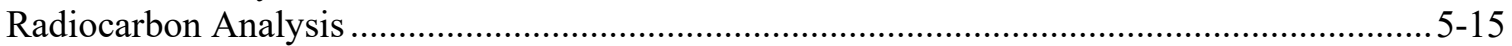

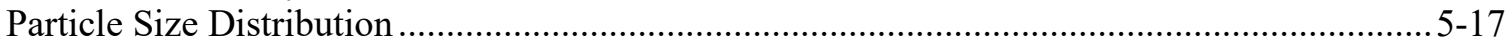

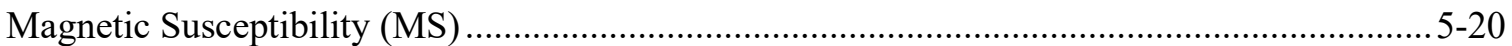

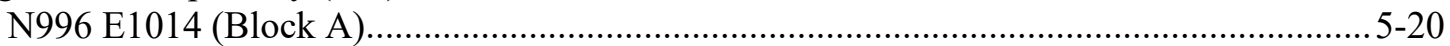

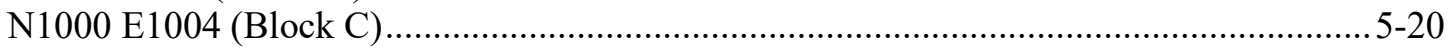

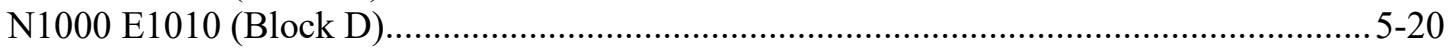

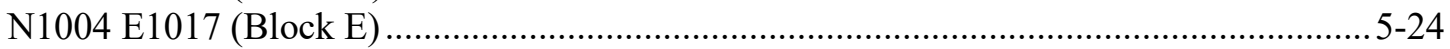

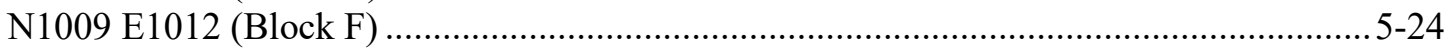

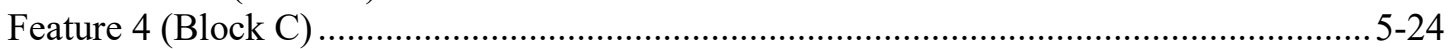

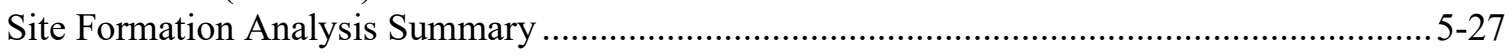

Chapter 6. Artifacts, Ecofacts, and Features....................................................................................... 6-1

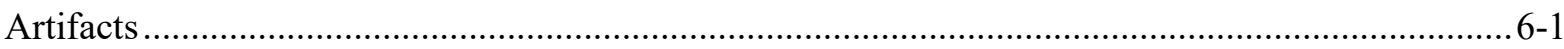

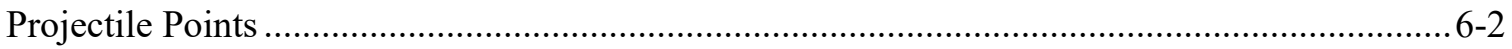

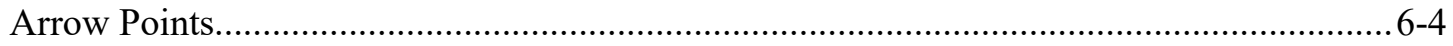

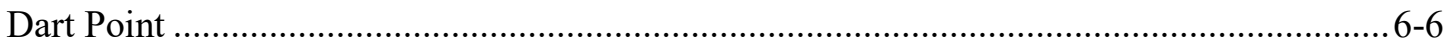

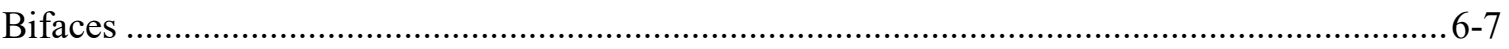

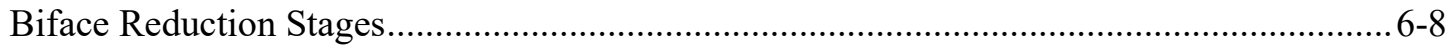

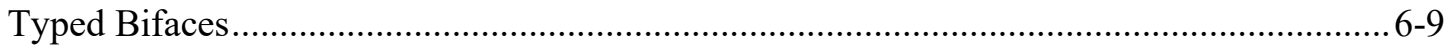

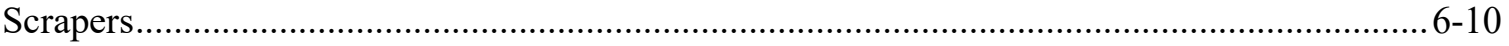

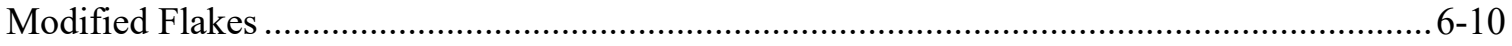

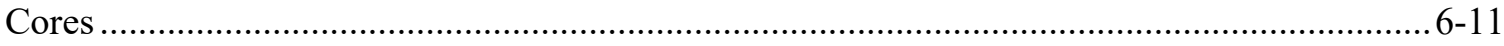

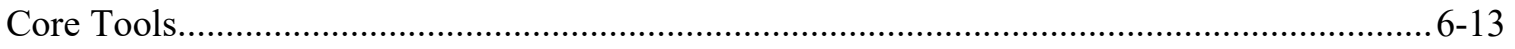

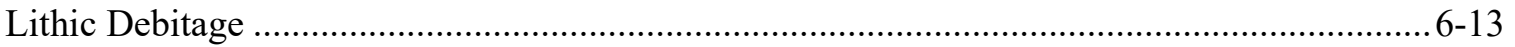

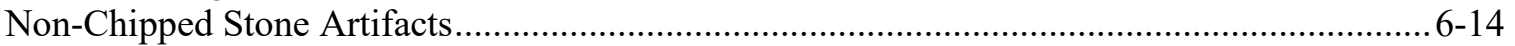

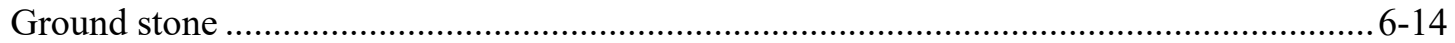

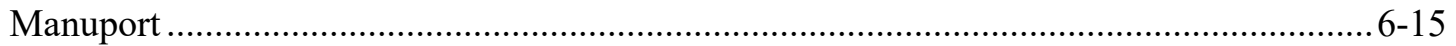

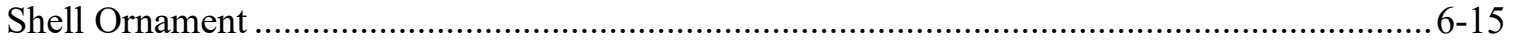

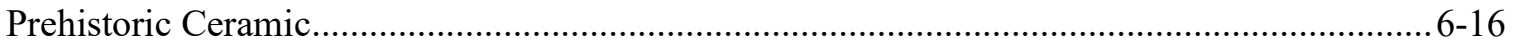

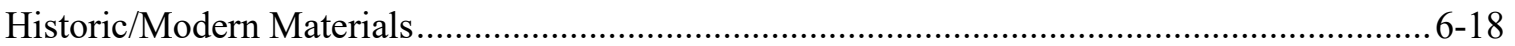

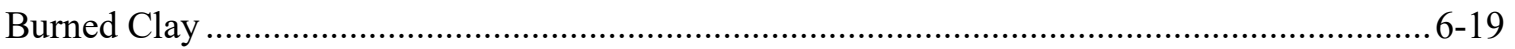

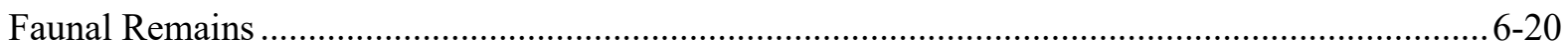

Bone

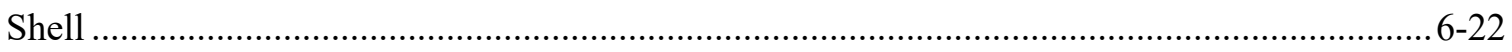

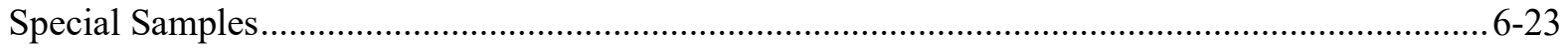

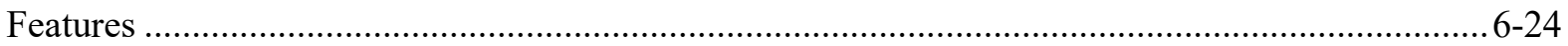

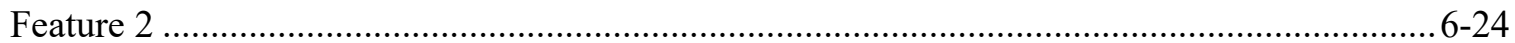

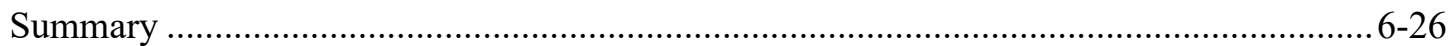

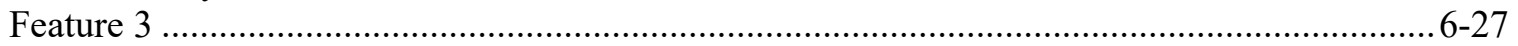

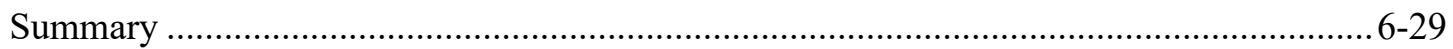

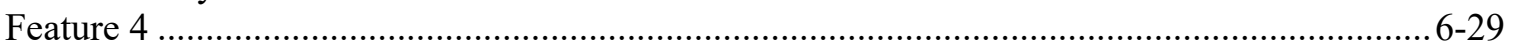

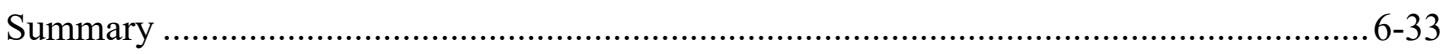

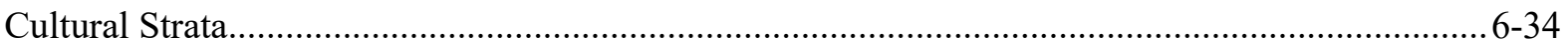


Chapter 7. Synthesis and Recommendations...................................................................................

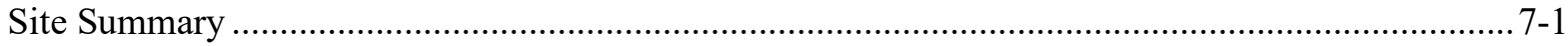

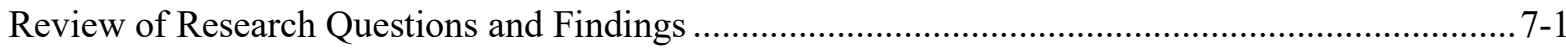

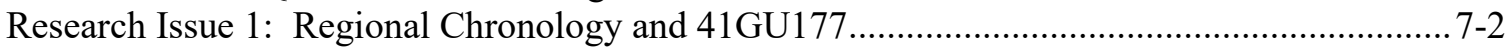

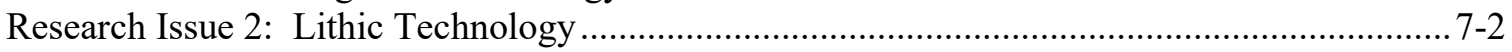

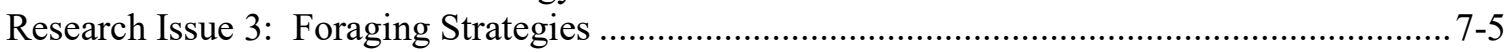

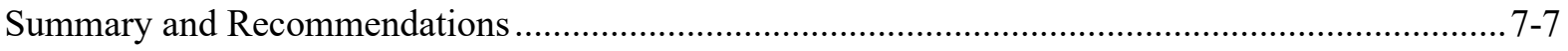

Chapter 8. Literature Cited .......................................................................................................................8 8-1

\section{Appendices}

Appendix A. 41GU177 Testing Interim Report

Appendix B. 41 GU177 Data Recovery Specimen Inventory and Burned Rock Tables

Appendix C. Artifact Analysis Tables

Appendix D. Faunal Analysis

Appendix E. Radiocarbon Assays

Appendix F. Macrobotanical Analysis

Appendix G. Geoarchaeological Analysis Raw Data

Appendix H. Construction Monitoring Memo

\section{Figures}

Figure 1.1. Location Map for VRRSP Project and Site 41GU177 ...................................................... 1-2

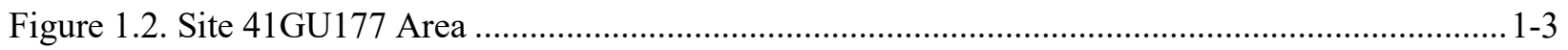

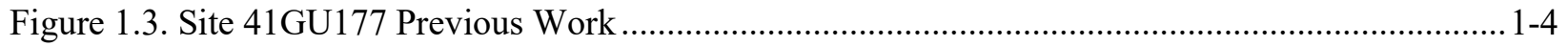

Figure 4.1. Site 41GU177 Data Recovery excavations plan map........................................................4-4

Figure 4.2. Overview of Unit Layout for Feature 4 Excavations......................................................... $4-7$

Figure 5.1. 41GU177 Cross-section Plan Map. ............................................................................. $5-2$

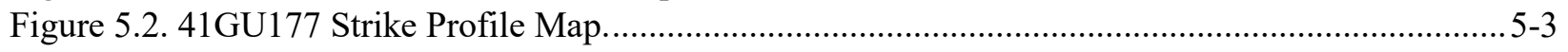

Figure 5.3. 41GU177 Dip Profile Map.............................................................................................

Figure 5.4. Overview of 41GU177 data recovery excavation blocks, facing south, upslope, away

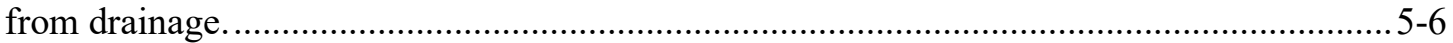

Figure 5.5. Overview of Block A after all units at termination level and geoarchaeological samples

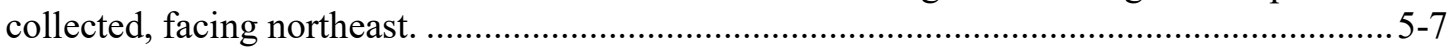

Figure 5.6. Overview of N999 E1014 in Block B at termination level $(98.10 \mathrm{~m})$, facing northeast. ........5-8

Figure 5.7. Overview of Block $\mathrm{C}$ after all units at termination level and geoarchaeological samples collected, facing southeast.

Figure 5.8. Overview of Block D after all units at termination level and geoarchaeological samples collected, facing east.

Figure 5.9. Large mammal bone recovered from N1001 E1009, Level 5 at $99.15 \mathrm{~m}$ and submitted for radiocarbon dating (Beta 492587).

Figure 5.10. Overview of Block E after all units at termination level and geoarchaeological samples collected, facing north.

Figure 5.11. Overview of N1009 E1012 in Block F at termination level $(97.70 \mathrm{~m})$, facing north.........5-14 


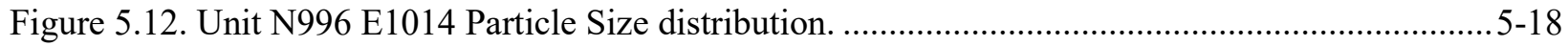

Figure 5.13. Unit N1004 E1017 Particle Size distribution. .........................................................5-19

Figure 5.14. Unit N996 E1014 profile annotation with magnetic susceptibility results.......................5-21

Figure 5.15. Unit N1000 E1004 profile annotation with magnetic susceptibility results......................5-22

Figure 5.16. Unit N1000 E1010 profile annotation with magnetic susceptibility results......................5-23

Figure 5.17. Unit N1004 E1017 profile annotation with magnetic susceptibility results.....................5-25

Figure 5.18. Unit N1009 E1012 profile annotation with magnetic susceptibility results.....................5-26

Figure 19. Feature 4 magnetic susceptibility sample cruciform. ......................................................5-27

Figure 5.20. Feature $4 \chi$ lf magnetic susceptibility results from west to east sample column.................5-28

Figure 5.21. Feature $4 \chi$ lf magnetic susceptibility results from south to north sample column..............5-29

Figure 6.1. Edwards arrow points: a) Lot 552-8, b) Lot 668-6; Perdiz arrow points: c) Lot 559-7, d)

Lot 579-10; Fresno arrow point: e) Lot 607-6; Indeterminate arrow point: f) Lot 550-

69; Zephyr dart point: g) Lot 621-7............................................................................ 6-4

Figure 6.2. Representative sample of biface assemblage. Stage 5: a) Lot 403-9, b) Lot 620-1, c) Lot 661-7; Stage 1: d) Lot 341-1; Stage 2: e) Lot 360-1; Stage 3: f) Lot 404-1......................... 6-8

Figure 6.3. Side-notched biface: a) Lot 316-7 and Gahagan biface: b) Lot 552-9. .............................. 6-9

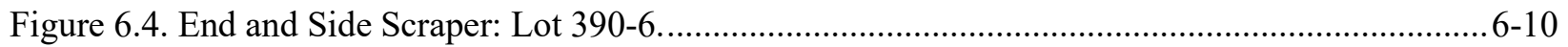

Figure 6.5. Representative sample of edge-modified and utilized flake assemblage. Edge modified flakes: a) Lot 519-10, b) Lot 590-7, c) Lot 638-5; Utilized flakes: d) Lot 320-1, e) Lot

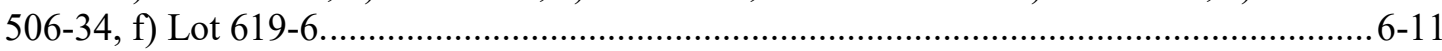

Figure 6.6. Representative sample of core assemblage. Unifacial: a) Lot 650-5; Bifacial: b) Lot

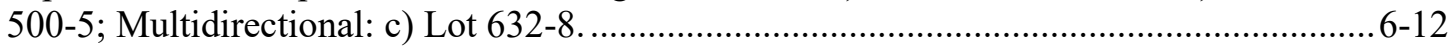

Figure 6.7. Representative sample of core tool-chopper assemblage: a) Lot 427-1, b) Lot 624-1.........6-13

Figure 6.8. Representative sample of non-chipped tool assemblage. Mano: a) Lot 492-6; Grooved Stone: b) Lot 416-1; Manuport: c) Lot 461-1.................................................................. $6-15$

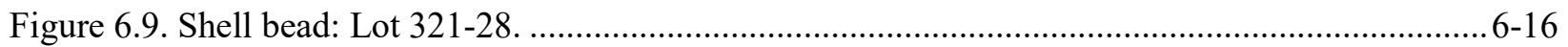

Figure 6.10. Prehistoric ceramics: a-f) Lot 462-22, g) Lot 463-4, and h) Lot 464-5.......................... 6-17

Figure 6.11. Microscopic image of prehistoric ceramic sherd temper with thermally altered angular bone fragment from Unit N1000 E1003, Level 3 (Lot 462-22)....................................... 6-17

Figure 6.12. Historic Assemblage: Non-diagnostic whiteware sherds: a) Lot 367-5, b) 488-4, c) 497-3, and d) 557-2; Colorless glass: e) Lot 673-5; Mayer China: f) Lot 578-6; Bullet casing: g) Lot 687-2; and Miscellaneous metal fragments: h) Lot 471-13, and i) Lot

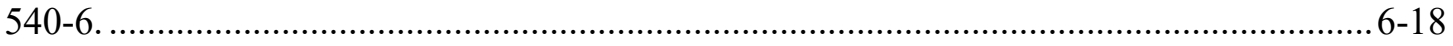

Figure 6.13. Representative sample of pieces of burned clay. Lot 405-9 ......................................... 6-19

Figure 6.14. Piece of burned clay with coronal heat alteration. Lot 538-6 ........................................ 6-20

Figure 6.15. Bone exhibiting butchering and processing taphonomy; Percussion notch: a) Lot 323-

1; cutmarks: b) Lot 539-1; percussion notch and spiral fracture: c) Lot 576-1.................. 6-21

Figure 6.16. Representative sample of mussel shell: a) Lot 589-1 (two on left) and b) Lot 605-6

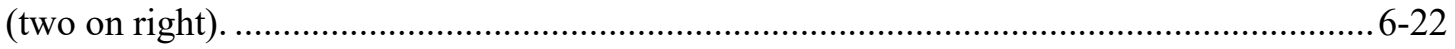

Figure 6.17. Modern Guadalupe orb shell from Palmetto State Park in Gonzales County, Texas.

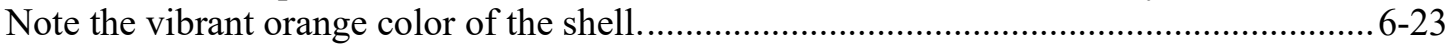

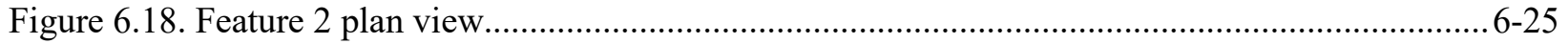

Figure 6.19. Feature 2, northwest profile showing overlapping burned rock, facing south...................6-26

Figure 6.20. Feature 3 plan view showing location of Clusters 1 and 2........................................... 6-28

Figure 6.21. Feature 4 Layer 1 plan view, note possible discard area to west...................................... 6-30

Figure 6.22. Feature 4 Layer 1 Plan map...................................................................................... $6-31$

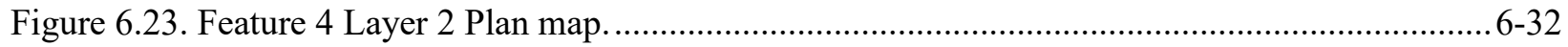

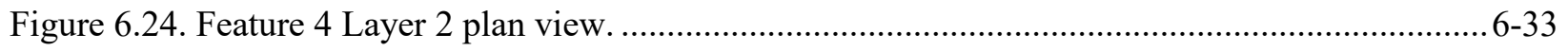




\section{Tables}

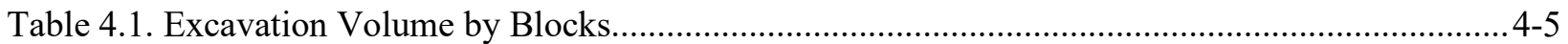

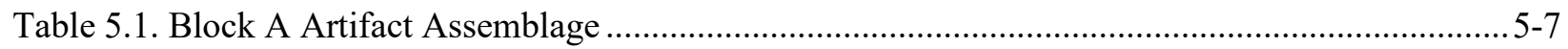

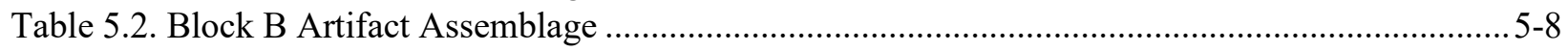

Table 5.3. Block C Artifact Assemblage …...................................................................................... 5-10

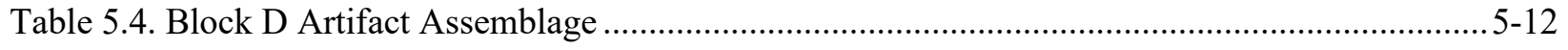

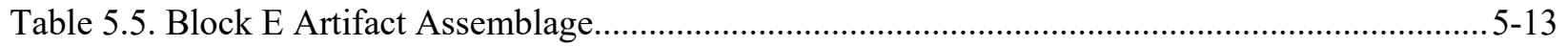

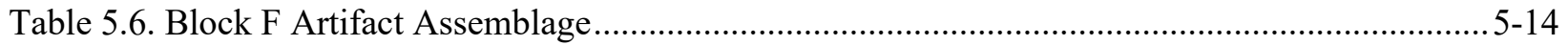

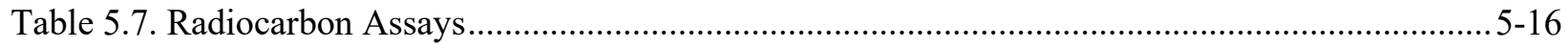

Table 6.1. 41GU177 Data Recovery Artifact Assemblage ...................................................................... 6-2

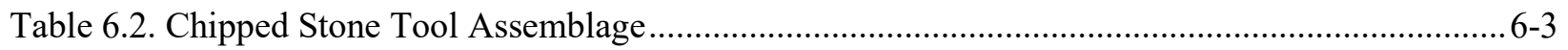

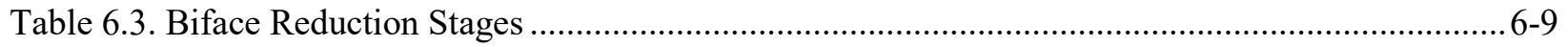

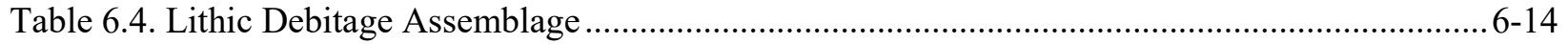

Table 6.5. Non-Chipped Stone Artifact Assemblage......................................................................... 6-14

Table 6.6. Mussel Shell Species Identified within the 41GU177 Assemblage..................................... 6-22

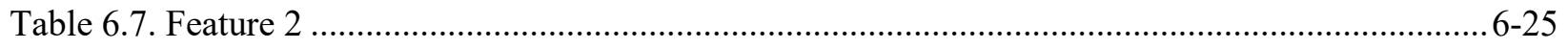

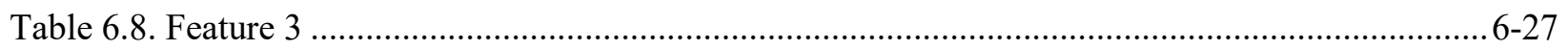

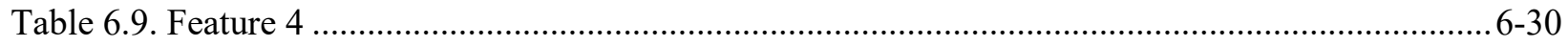

Table 7.1. Summary of Lithic Assemblage from Snakeskin Bluff Components .................................... 7-3

Table 7.2. Biface to Core Ratio among Snakeskin Bluff Components.................................................. 7-4

Table 7.3. Comparative Biface: Core Ratio Data from Parry and Kelly (1987).................................... 7-4

Table 7.4. Ratio of Debitage to Tools by Component on the Snakeskin Bluff Site ................................. 7-5 
This page intentionally left blank. 


\section{CHAPTER 1. INTRODUCTION}

On behalf of the Central Texas Regional Water Supply Corporation (CTRWSC) and VRRSP Consultants, LLC (VRRSP), SWCA Environmental Consultants (SWCA) has prepared this report presenting the results of data recovery excavations at prehistoric site 41GU177 (Snakeskin Bluff site) in Guadalupe County, Texas. The site was recorded by SWCA during Phase I survey investigations that occurred between June 2015 and March 2018 for the proposed Vista Ridge Regional Water Supply (Vista Ridge) Project (Figure 1.1). Investigations were conducted in compliance with Section 106 of the National Historic Preservation Act (NHPA) (54 United States Code [USC] 306108) and its implementing regulations (36 Code of Federal Regulations [CFR] 800), in anticipation of a Nationwide Permit 12 from the U.S. Army Corps of Engineers (USACE) in accordance with Section 404 of the Clean Water Act. In addition, the work is subject to compliance with the Antiquities Code of Texas (ACT), as the Vista Ridge Project will be ultimately owned by CTRWSC, a political subdivision of the State of Texas. The survey and testing investigations were conducted under ACT Permit No. 7295, and the subsequent data recovery was under Permit No. 8231.

The Vista Ridge project will involve installation of a 140-mile-long, 60-inch-diameter water pipeline from north-central San Antonio, Bexar County, to Deanville, Burleson County, Texas (see Figure 1.1). The area of potential effects (APE) for the Phase I survey efforts included the proposed centerline alignment and a 100-foot-wide corridor (50 feet on either side of centerline), as well as temporary and permanent construction easements, and aboveground facilities, such as pump stations and the northern and southern termini sites. Between June 2015 and March 2018, the cultural resources survey identified 78 cultural resources (i.e., 64 archaeological sites and 14 isolated finds). Included in that inventory was site $41 \mathrm{GU} 177$, originally discovered on August 31, 2015, on the west bank of the Guadalupe River.

Site $41 \mathrm{GU} 177$ is a stratified prehistoric site on the western high bank of the Guadalupe River southeast of New Braunfels, Texas, near the community of McQueeney (Figure 1.2). The site contains components deposited intermittently from approximately the Late Archaic to Transitional Archaic periods through to Late Prehistoric times. The primary components investigated in the excavations span the final Late Archaic period and into the Austin phase of the Late Prehistoric, a timeframe from approximately 2,600 to 900 years ago. The field investigations, conducted between October 2016 and December 2017, included intensive shovel testing, geomorphological study with mechanical excavations, and subsequent hand excavations. This report presents the results of the data recovery investigations conducted from November to December 2017. The survey results and testing results have been previously reported (Acuña et al. 2016; Rodriguez et al. 2017); the testing results are also presented in Appendix A. Below is a chronology of investigations at $41 \mathrm{GU} 177$ to contextualize the data recovery excavations presented in this report.

\section{OVERVIEW OF INVESTIGATIONS AT 41GU177}

Based upon the nature and depth of the soils identified during Phase I survey efforts at 41GU177 in August 2015, and the assemblage of artifacts noted during shovel testing, a second phase of survey-level investigations consisting of systematic backhoe trenching occurred at the site on October 16, 2015 (Figure 1.3). Trench excavations near the northeastern end of the site contained a partially intact burned rock feature and a moderate density of chipped-stone debitage and faunal remains. Ultimately, the 2015 investigations revealed intact prehistoric subsurface cultural materials and a buried cultural feature in the northeastern/eastern portion of the site. Given the apparent integrity of the cultural deposits and the depth of observed artifacts, SWCA recommended that a phase of test excavations was warranted to better explore the cultural deposits through hand excavations and to determine if the cultural deposits retained sufficient integrity to warrant designation as a State Antiquities Landmark (SAL). 


\section{Restricted Information}

\section{Not for Public Disclosure}

Figure 1.1. Location Map for VRRSP Project and Site 41GU177 


\title{
Restricted Information
}

\author{
Not for Public Disclosure
}

Figure 1.2. Site 41GU177 Area 


\title{
Restricted Information
}

\author{
Not for Public Disclosure
}

Figure 1.3. Site 41GU177 Previous Work 
Test excavations occurred October 12-14, 2016, until work was halted at the request of the landowner due to the recovery of a possible human bone from one of the excavation units on October 13; at that time, excavations were also halted to contact the Guadalupe County Sheriff's office. Ultimately, the bone was determined to not be human; however, the landowner halted all archaeological activity on the site. Access to continue work at the site was eventually obtained in June 2017. SWCA returned to site 41GU177 in June 2017 to complete the excavation units originally established in 2016, as well as excavate two additional backhoe trenches at the location of a proposed bore pit for a planned horizontal directional drill [HDD] that would impact parts of the site. During the excavation of the two additional backhoe trenches, in situ cultural materials were observed in the trench profiles and SWCA excavated a fifth hand excavation unit at the intersection of the two new trenches to explore the artifact and burned rock feature. Following completion of the limited excavations on July 6, 2017, there was sufficient evidence to recommend that portions of the site potentially warranted SAL designation and a phase of data recovery excavations.

In September and October 2017, SWCA conducted a program of close-interval shovel testing followed by additional backhoe trenching at the site to better determine the nature, depth, and extent of its cultural deposits (i.e., investigate if more than one temporal component was represented) and to ultimately refine the proposed data recovery areas. Following completion of the close-interval shovel testing, SWCA then proceeded to complete additional mechanical trenching on the site in October 2017. The locations of the backhoe trenches were determined by the results of the close-interval shovel testing (i.e., areas where shovel testing revealed potentially deeply buried artifacts and possible cultural features). SWCA excavated seven additional trenches across the site, which revealed similar stratigraphy and artifact densities to those observed during the close-interval shovel testing; however, several artifacts were noted at significantly deeper depths (i.e., close to 200 centimeters below surface [cmbs]) than those in the shovel tests.

Complete avoidance of the site was not possible, due to the necessary boring of the Guadalupe River (adjacent to the site) that required an approximately $50 \times 50$-foot bore pit area, a $300 \times 8 \times 10$-foot construction trench, a $65 \times 200$-foot temporary workspace, a 20 - to 25 -foot-wide haul road for construction activities, vegetation/tree clearing, and the HDD within portions of site $41 \mathrm{GU} 177$ considered to have a high density of cultural materials. Based on the results of the phased investigations at the site, SWCA refined the location and extent of potential data recovery efforts, focusing on the more significant and intact parts of the site identified during the various investigations that would be impacted by construction-related activities.

Rather than opt for mitigation of the entire site, the VRRSP team wanted to preserve and protect parts of $41 \mathrm{GU} 177$ in place in conjunction with limited data recovery excavations within the $50 \times 50$-foot bore pit area. Coordination with VRRSP, SWCA, and the Texas Historical Commission (THC) resulted in the determination that preservation in place was a viable option. SWCA subsequently prepared a preservation plan, establishing a process for protecting parts of the site in place and allowing construction to proceed following the completion of limited data recovery within the bore pit location. SWCA consulted with Dr. Christopher Mathewson from Texas A\&M University (an acknowledged expert in archaeological site capping for in place preservations) to develop the plan.

Data recovery excavations at $41 \mathrm{GU} 1277$ began the last week of November 2017 and extended through December 28, 2017, authorized by the THC through issuance of ACT Permit No. 8231 to Principal Investigator (PI), Brandon S. Young. Preservation in place involved a multistep process following the limited data recovery to ensure protection of the buried cultural materials, the process included carefully prescribed methods for vegetation clearing (with an archaeological monitor present at all times), the covering of sensitive areas outside the bore pit locale with geotechnical fabric, 18 inches of fill, and 6 
inches of rock/gravel to allow construction activities to proceed in the $300 \times 8 \times 10$-foot construction trench, the $65 \times 200$-foot temporary workspace, and the 20 - to 25 -foot-wide haul road.

\section{PROJECT PERSONNEL}

Brandon S. Young, M.A., RPA, served as the initial PI and project manager (PM) for the $41 \mathrm{GU} 177$ field investigations, overseeing overall logistics and organization, managing reporting, and agency consultation. Christina Nielsen, M.A., served as the Project Archaeologist (PA) in the field and took over as PM and PI for the final reporting stages of the project. The data recovery was completed by Brandon Young; Christina Nielsen; and SWCA archaeologists Jessica Ulmer, Ashley Eyeington, Daniel Rodriguez, Mary Rodriguez, Ben Morton, Michael Golden, Sophia Salgado, Stefan Barker, Brooke Bonorden, Zach Overfield, Cody Roush, and Jonathan Welch, between November 27 and December 28, 2017, under Permit No. 8231. Archaeologists Virginia Moore, Jake Sullivan, and Megan Veltri from Pape-Dawson Engineers, Inc. (Pape Dawson) also assisted with the excavations during the last week of December 2017. Christina Nielsen, Ashley Eyeington, Ken Lawrence, Chris Shelton, Mercedes C. Cody, and Brandon S. Young undertook the reporting effort, while Carole Carpenter and Jason Kainer expertly produced all field and report maps for the project, and Lauri Logan provided technical editing and document preparation.

\section{STRUCTURE OF REPORT}

The following report presents the environmental setting of site 41GU177 (Chapter 2) followed by a cultural history of the site and region (Chapter 3). Chapter 4 provides a discussion of methods utilized to accomplish the data recovery. Chapter 5 offers a general overview of the data recovery as it relates to excavation units, chronology, and general level of effort. Chapter 6 presents a detailed examination of excavated cultural features, recovered artifacts, faunal remains, and special samples obtained during excavations. The report ends with Chapter 7, which provides a synthesis of the site and special analyses and samples, as well as addressing and answering the research questions posited for the data recovery and offering recommendations for site protection and preservation. Supporting data are presented in seven appendices to this report. The majority of the appendices are special study results and the various analytical data. 


\section{CHAPTER 2. ENVIRONMENTAL SETTING}

Site 41GU177 in Guadalupe County is located in central Texas near a junction of varied geology and diverse vegetation regions. Specifically, the site is situated in the Blackland Prairies physiographic region, which parallels the base of the Balcones Escarpment of the Edwards Plateau (Wermund 2018). Accordingly, the site is positioned within two intermingled floral communities, namely the Post Oak Savannah and the Blackland Prairies regions (Correll and Johnston 1979:3-7).

This chapter provides an overview of the present-day environmental setting of $41 \mathrm{GU} 177$ and a review of the regional paleoenvironmental record. The discussion is based on the results of field investigations performed by SWCA archaeologists and a review of relevant literature.

\section{SITE SETTING}

The site is located approximately 2.9 miles north of McQueeney in Guadalupe County, Texas. Site $41 \mathrm{GU} 177$ is on the western (right) high bank of the Guadalupe River, with a 9- to 12-meter (m) vertical drop to the river bottom to the east and gently sloping (2-5 percent slope) terrain to the west. SWCA's investigations were confined to the portion of the site within the project area; however, SWCA suspects the site continues beyond the limits of the project area to the east and west.

Site 41GU177 is located within forested rangeland with mixed hardwood trees, large live oak trees, and moderately dense shrub undergrowth. Ground surface visibility ranges from 0 to 20 percent, with limestone gravels and cobbles exposed on the ground surface. Vegetation clearing and grading, fence line construction, and natural erosion have impacted site integrity to varying degrees. One property fence and private cemetery (Hoffman Cemetery) borders the northwestern site boundary (see Figure 1.2), and natural erosion from the high bank of the Guadalupe River has impacted the northeastern site boundary.

\section{OVERVIEW OF GEOMORPHOLOGY}

SWCA reviewed the local geology and soils as mapped by the Bureau of Economic Geology; the U.S. Department of Agriculture's Soil Conservation Service; and local U.S. Geological Survey (USGS) quadrangle maps, aerial photographs, and excavation and site profiles, which provided the basis for the general geomorphology of site $41 \mathrm{GU} 177$.

The surface geology for this area indicates that the project alignment crosses Pleistocene-age terrace deposits (Barnes 1983). These deposits are characterized as gravel, sand, silt, and clay largely derived from the Edwards Plateau situated a few miles upstream (Barnes 1983). These deposits in local areas have calcium carbonate-cemented quartz sand, silt, clay, and gravel intermixed and interbedded in terraces along streams. Low terraces of major rivers are capped by 2 to $4 \mathrm{~m}$ of clayey sand and silt. Sandy gravel on higher terraces varies somewhat in composition from river to river.

The soils at 41GU177 are mapped as Sunev loam (1 to 5 percent slopes) (Natural Resources Conservation Service [NRCS] 2018; Ramsey and Bade 1977). Briefly, the Sunev loams are occasionally identified as Venus loams (Sunev spelled backward) in older soil surveys (Ramsey and Bade 1977; Taylor et al. 1991). These are characterized as occurring on terraces or alluvial fans of rivers and large streams and composed of calcareous brown to dark brown loams and clay loams (Ramsey and Bade 1977; Taylor et al. 1991).

At 41GU177, the Guadalupe River is a competent drainage with an approximately 43-m-wide (142-foot) base and a slow southeast-flowing channel. The Guadalupe River, as it winds past the site, has a high sinuosity ratio of 1.4, indicating that it is a sinuous waterway, which is apparent from the New Braunfels 
East and McQueeney, Texas, 7.5-minute USGS quadrangle maps (Charlton 2008:138-139). This sinuosity suggests a partially dynamic depositional history where the channel has moved laterally. In this type of setting, the lateral movement can significantly erode the deposits of outside meander bends and, in contrast, deposit significant amounts of sediment upon interior meanders (Charlton 2008). Site 41GU177 is situated on an exterior bend of a meander. Not surprisingly, the right bank of the river, below $41 \mathrm{GU} 177$, does not contain broad alluvial landforms.

At this location, the Guadalupe River valley is an unpaired, stair-stepped terrace system consisting of a series of alluvial terraces that bracket the drainage created by various episodes of overbank deposition (Waters 1992:149-151). The left bank (east side) of the Guadalupe River exhibits three terrace landforms that from youngest (closest to the channel) to oldest consist of the $T_{0}, T_{1}$, and $T_{2}$ terraces. In contrast, the right bank, containing 41GU177, only has one apparent alluvial terrace landform $\left(\mathrm{T}_{2}\right)$.

On the right bank, the $T_{2}$ landform abruptly rises about 9.5 to $11 \mathrm{~m}$ (31 to 36 feet) above the Guadalupe River. The $\mathrm{T}_{2}$ landform on both banks is broad (approximately 1 kilometer) and gradually grades into the valley margins aligning the drainage valley. Another landform occurs upon the $\mathrm{T}_{2}$ terrace near site $41 \mathrm{GU} 177$, which could be part of the $\mathrm{T}_{2}$ landform or remnants of an older alluvial landform (i.e., $\mathrm{T}_{3}$ ). This landform parallels the Guadalupe River and is situated at approximately 580 to 585 feet above mean sea level (amsl) and about $1.5 \mathrm{~m}$ ( 5 feet) above the $\mathrm{T}_{2}$ terrace that surrounds it. Site $41 \mathrm{GU} 177$ is primarily situated on the $\mathrm{T}_{2}$ terrace between 575 to 580 feet amsl.

\section{FLORA}

The Blackland Prairie has rolling topography that supports a diverse assemblage including southern hackberry (Celtis laevigata), cedar elm (Ulmus crassifolia), bur oak (Quercus macrocarpa), post oak (Quercus stellata), blackjack oak (Quercus marilandica) with an understory of bunch grasses, shrubs, laurel greenbriar (Smilax laurifolia), yaupon holly (Ilex vomitoria), American beautyberry (Callicarpa Americana), and coralbean (Erythrina herbacea) (Kutac and Caran 1994; Petrides 1988; Simpson 1988). Originally, the Blackland Prairie region supported a tall grass prairie (Gould 1969).

The Post Oak Savannah region is characterized as a "Post Oak Woods, Forest and Grassland Mosaic" which supports several varieties of oaks, such as blackjack oak (Quercus marilandica), live oak (Quercus virginiana), and sandjack oak (Quercus incana) (Mahan et al. 1984:19). The Post Oak Savannah is also described as primarily containing grassy plains with confined stands or groves of trees (Kutac and Caran 1994:13). Other trees present in the area include mesquite (Acacia sp.), black hickory (Carya texana), and southern hackberry. Grassland flora includes little bluestem (Schizachyrium scoparium), silver bluestem (Bothriochloa saccharoides), and sand lovegrass (Eragrostis trichodes) (Gould 2002). Historic land use patterns have resulted in extensive clearing of large areas once dominated by the oak woodlands (Gould 1969). In addition, many of the prairie areas no longer support the native medium and tall grasses as they have been used for pasture and/or cultivated for various crops.

\section{FAUNA}

The intermingled floral communities of the Post Oak Savannah and the Blackland Prairies vegetation regions that surround the site area corresponds to the convergence of the broader Texan, Tamaulipan, and Balconian biotic provinces of Texas defined by Blair (1950). The site itself, however, is situated within the Texan biotic province.

The Texan biotic region is a region that is transitional between forested regions to the east and the grasslands to the west (Blair 1950). As such, the Texan biotic province contains a wide variety of native mammal species. Some mammals found within this biotic province include: opossum (Didelphis 
virginiana), eastern mole (Scalopus aquaticus), Brazilian free-tailed bat (Tadarida brasiliensis), armadillo (Dasypus novemcinctus), swamp rabbit (Sylvilagus aquaticus), eastern cottontail (Sylvilagus floridanus), black-tailed jackrabbit (Lepus californicus), Attwater's pocket gopher (Geomys attwateri), fulvous harvest mouse (Reithrodontomys fulvescens), northern pygmy mouse (Baiomys taylori), gray fox (Urocyon cinereoargenteus), raccoon (Procyon lotor), and striped skunk (Mephitis mephitis) occur in suitable habitats throughout area (Davis and Schmidly 1994; Schmidly 1983). Large mammal species that occur or have the potential to occur within the project area include white-tailed deer (Odocoileus virginianus), coyote (Canis latrans), bobcat (Lynx rufus), and javelina (Tayassu tajacu) while red wolf, bison, and black bear are reported to have historically ranged into or near the project area (Anderson 1981; Burt and Grossenheider 1976; Schmidly 1983).

Birds of the Texan biotic region in the wooded areas include black vultures (Coragyps atratus), turkey vultures (Cathartes aura), northern bobwhite (Colinus virginianus), mourning dove (Zanaida macroura), chuck-will's-widow (Caprimulgus carolinensis), red-bellied woodpecker (Melanerpes carolinus), downy woodpecker (Picoides pubescens), scissor-tailed flycatcher (Tyrannus forficatus), blue jay (Cyanocitta cristata), tufted titmouse (Parus bicolor), red tailed hawk (Buteo jamaicensis), American robin (Turdus migratorius), and many sparrows (Bull and Farrand 1977; Kutac and Caran 1994; Short 1981).

In addition to the mammals and birds, various species of amphibians and reptiles can be found in the Texan biotic province. The reptilian assemblage includes the Great Plains rat snake (Elaphe guttata emoryi), eastern yellowbelly racer (Coluber constrictor), Mississippi mud turtle (Kinosternon subrubrum hippocrepis), bullfrog (Rana catesbiana), southern leopard frog (Rana utricularia), and the Gulf Coast toad (Bufo valliceps) (Blair 1950; Conant and Collins 1998; Kutac and Caran 1994).

\section{PALEOENVIRONMENT}

Over the past 12,000 years, the environmental and climatic conditions of the Central Texas region have varied considerably. There is some debate about the paleoenvironmental conditions ca. $12,000-10,000$ years before present (B.P.), and two models are presently posited for the paleoenvironment of this early period. Bryant and Holloway (1985) have proposed that the Central Texas region was predominantly a deciduous forest $65,000-10,000$ years ago, with a diverse assortment of tree and plant species. In contrast, more recent research by Hall and Valastro (1995) and Toomey et al. (1993) suggests that Central Texas was dominated by grassland and mesic climatic conditions during the late Pleistocene. This interpretation fits well with the regional faunal record since megafaunal species from this period, such as mastodon, mammoth, giant bison, camel, and horse, would have required substantial areas of grassland for grazing (Dillehay 1974; Graham 1987; Graham and Lundelius 1984; Toomey et al. 1993).

About 10,000 years ago, changes in palaeoclimatic conditions led to mass extinctions of megafauna across the region (Graham 1987; Graham and Lundelius 1984). Faunal records from the Edward's Plateau indicate the average seasonal temperature warmed, and climatic conditions became progressively drier (but still retained higher effective moisture than present day) throughout the early Holocene (Bement 1994; Toomey et al. 1993). As a result, vegetation cover diminished, and a degradation of the soil mantle ensued (Toomey et al. 1993:316). Johnson and Goode (1994:20) argue that the early Holocene warming and drying trend experienced on the Edwards Plateau did not impact the Post Oak Belt. Due to the wind currents and temperature patterns of the nearby Gulf of Mexico, palaeoclimatic conditions experienced east of the Edwards Plateau may have been relatively warm and moist during this time (Johnson and Goode 1994:20).

Toomey et al. (1993:309) propose that the early Holocene warming trend continued into the middle and late Holocene and peaked ca. 5000-2500 B.P. "when climatic conditions were drier than any time during the last 20,000 years." Johnson and Goode (1994:5) also recognize this extreme xeric period and describe 
it as the "Dry Edward's Interval." Following this extensive dry period, the wetter and warmer conditions returned to the region until ca. 1500-1000 B.P. (Bryant and Holloway 1985; Johnson and Goode 1994; Toomey et al. 1993). From this time forward, present-day climatic and environmental conditions were firmly established for south-central Texas. 


\section{CHAPTER 3. CULTURAL SETTING}

The following prehistoric cultural history derives its information from several central Texas regional chronologies: Black (1989), Collins (1995, 2004), and Johnson and Goode (1994), which build upon the seminal efforts of Suhm (1960) and Prewitt (1981, 1985). Furthermore, significant archaeological sites within the Central Texas archaeological region and the Edwards Plateau have contributed important information to understanding prehistory. The following prehistoric cultural sequence is divided into two periods: Archaic and Late Prehistoric. The Archaic period is subdivided into two subperiods; Late and Transitional; while the Late Prehistoric discussion is divided into the Austin and Toyah phases, respectively.

\section{LATE ARCHAIC (CA. 4000 TO 1250 B.P.)}

During the succeeding Late Archaic period (4000 B.P. to 1300-1200 B.P.), populations continued to increase (Prewitt 1985:217). As evidenced by stratified Archaic sites such as Loeve-Fox, Cibolo Crossing, and Panther Springs Creek, the Late Archaic components contain the densest concentrations of cultural materials of all the Archaic periods. Establishment of large cemeteries along drainages also suggests certain groups had strong territorial ties (Story 1985:40).

Middle Archaic subsistence technology, including the use of rock and earth ovens, continues into the Late Archaic period. Collins (2004:121) states that, at the beginning of the Late Archaic period, the use of rock ovens and the resultant formation of burned rock middens reached its zenith and that the use of rock and earth ovens declined during the latter half of the Late Archaic. There is, however, mounting chronological data that midden formation culminated much later and that this high level of rock and earth oven use continued into the early Late Prehistoric period (Black et al. 1997:270-284; Kleinbach et al. 1995:795). A picture of prevalent burned rock midden development in the eastern part of the Central Texas archaeological region after 2000 B.P. is gradually becoming clear. This scenario parallels the widely recognized occurrence of post-2000 B.P. middens in the western reaches of the Edwards Plateau (Goode 1991).

The use of rock and earth ovens (and the formation of burned rock middens) for processing and cooking plant foods suggests that this technology was part of a generalized foraging strategy. Considering the amount of energy involved in collecting plants, constructing hot rock cooking appliances, and gathering fuel, the caloric return of most plant foods is relatively low (Dering 1999). This suggests that plant foods were part of a broad-based diet (Kibler and Scott 2000:134) or part of a generalized foraging strategy, an idea Prewitt (1981) put forth earlier. At times during the Late Archaic, this generalized foraging strategy appears to have been marked by shifts to a specialized economy focused on bison hunting (Kibler and Scott 2000:125-137). Castroville, Montell, and Marcos dart points are elements of tool kits often associated with bison hunting (Collins 1968). Archaeological evidence of this association is seen at Bonfire Shelter in Val Verde County (Dibble and Lorrain 1968), Jonas Terrace in Medina County (Johnson 1995), Oblate Rockshelter in Comal County (Johnson et al. 1962:116), John Ischy in Williamson County (Sorrow 1969), and Panther Springs Creek in Bexar County (Black and McGraw 1985).

\section{TRANSITIONAL ARCHAIC (CA. 2250 TO 1250 B.P.)}

As Collins (2004:122-123) notes, diverse and comparatively complex archaeological manifestations toward the end of the Late Archaic attest to the emergence of kinds of human conduct without precedent in the area. This period (2250-1250 B.P.), referred to as the Transitional Archaic (Turner and Hester 1999) or Terminal Archaic (Black 1989), is not recognized by all researchers. Other chronologies 
terminate the Late Archaic at around 1200-1250 B.P. (Collins 2004; Johnson and Goode 1994) to encompass this later subperiod. Johnson et al. (1962) originally designated the Transitional Archaic as a subperiod of the Archaic because of the similarities between the latest dart point types and the earliest arrow point types. Since then, however, the designation has failed to be universally accepted by researchers. In two recent chronologies for central Texas, Collins (2004) does not include the Transitional as a subperiod of the Archaic, and Johnson and Goode (1994) separate the Late Archaic into two subperiods designated Late Archaic I and Late Archaic II. The Transitional Archaic, as it is used here, closely corresponds to Johnson and Goode's (1994) Late Archaic II, but begins after the appearance of Marcos points, not with it. In this scheme, the Transitional Archaic coincides with the last two style intervals recognized by Collins (2004) for the Late Archaic subperiod.

During the Transitional Archaic, smaller dart point forms, such as Darl, Ensor, Fairland, and Frio, were developed (Turner and Hester 1999). These points were probably ancestral to the first Late Prehistoric arrow point types and may have overlapped temporally with them (Carpenter et al. 2006; Hester 1995; Houk and Lohse 1993). Several researchers believe that the increased interaction between groups at the end of the Late Archaic was an important catalyst for cultural change (Collins 2004; Johnson and Goode 1994). This change may have included increased regional stress and conflict between groups as interaction became more frequent (Houk et al. 1997). In Bexar County, for instance, researchers noted a distinct shift in settlement patterns during this period (Houk et al. 1997). Groups began to use hilltops as camps rather than just lithic procurement locations. These elevated locations would have provided points from which to observe game and other groups of humans as they moved through the surrounding creek valleys and upland prairies (Houk et al. 1997).

Overall, the Archaic period represents a hunting and gathering way of life that was successful and remained virtually unchanged for more than 7,500 years. This notion is based in part on consistent artifact and tool assemblages through time and place and on resource patches that were used continually for several millennia, as the formation of burned rock middens show. This pattern of generalized foraging, though marked by brief shifts to a heavy reliance on bison, continued almost unchanged into the succeeding Late Prehistoric period.

\section{LATE PREHISTORIC (CA. 1250 TO 350 B.P.)}

Introduction of the bow and arrow and, later, ceramics into the Central Texas archaeological region marks the Late Prehistoric period (1250-350 B.P.). Population densities dropped considerably from their Late Archaic peak (Prewitt 1985:217). Subsistence strategies did not differ greatly from the preceding period, although bison again became an important economic resource during the latter part of the Late Prehistoric period (Prewitt 1981:74). Rock and earth ovens were utilized for plant food processing (Black et al. 1997; Kleinbach et al. 1995:795). Horticulture came into play very late in the region but was of seemingly minor importance to overall subsistence strategies (Collins 1995:385). Artifact assemblages include Scallorn, Perdiz, and Edwards projectile points, worked stone, thermally altered stone, hematite, bone, and shell. The points are associated with the use of the bow and arrow in the region, probably introduced sometime around 1350-1150 B.P.

\section{AUSTIN PHASE (CA. 1250 TO 650 B.P.)}

The earlier Austin phase (identified by Scallorn and Edwards points) and the later Toyah phase (defined through Perdiz points) divide the Late Prehistoric period throughout central Texas (Black 1989; Story 1990). These divisions were originally recognized by Suhm (1960) and Jelks (1962), and remain an accepted separation of the period. Although a distinct change in the material culture between the two phases can be seen in the archaeological record, there is some debate over the cultural underpinnings that 
prompted the change. The different arrow point styles (and other associated artifacts in the assemblage) may represent distinct cultural groups (Johnson 1994), but others challenge this view (e.g., Black and Creel 1997), and attribute the change to a spread of new technological ideas in response to the increase of a different economic resource in bison populations (Ricklis 1992). Nevertheless, prehistoric communities traced through cultural remains assigned to the Austin phase (1250-650 B.P.), like many of the Archaic period cultures before them, relied on a hunting and gathering subsistence with more of an emphasis on gathering (Prewitt 1981:83). Communities attributed to the Toyah phase (650-200 B.P.) relied more on bison procurement (Prewitt 1981:84).

\section{TOYAH PHASE (CA. 650 TO 200 B.P.)}

Around 1000-750 B.P., slightly more-xeric or drought-prone climatic conditions returned to the region, and bison came back in large numbers (Huebner 1991; Toomey 1993). Using this vast resource, Toyah peoples were equipped with Perdiz point-tipped arrows, end scrapers, four-beveled-edge knives, and plain bone tempered ceramics. Toyah technology and subsistence strategies represent a completely different tradition from the preceding Austin phase. Collins (1995:388) states that formation of burned rock middens ceased as bison hunting and group mobility obtained a level of importance not witnessed since Folsom times. Although the importance of bison hunting and high group mobility hardly can be disputed, the argument that burned rock midden development ceased during the Toyah phase is tenuous. An examination of Toyah-age radiocarbon assays and assemblages by Black et al. (1997) suggest that their association with burned rock middens represents more than a "thin veneer" capping Archaic-age features. Black et al. (1997) claim that burned rock midden formations, although not as prevalent as in earlier periods, was part of the adaptive strategies of Toyah peoples. 


\section{CHAPTER 4. RESEARCH DESIGN AND METHODS}

The 41GU177 investigations entailed multiple phases, from survey, to testing, and finally to data recovery. At each phase, the fundamental objectives changed, and accordingly the means evolved to attain the changing ends. Techniques and methods were first adapted to define the limits and significance of site deposits, and then subsequently modified to gather solid, non-redundant data that would contribute to an understanding of the broad patterns of regional prehistory. This chapter presents a synopsis of the research objectives followed by a detailed review of the methods and techniques that SWCA used to investigate the site.

Due to some remaining uncertainties regarding the site's contextual integrity, SWCA proposed a flexible approach, whereby excavations could be scaled down if early results revealed poor preservation or other issues with the site. Overall, the investigations involved vegetation clearing, mechanical excavations, hand excavations, and special studies. The goal of SWCA's excavations was to perform data recovery excavations on the portions of the site that would be directly impacted by the undertaking. Specifically, the bore pit measured approximately $50 \times 50$ feet $(15.24 \times 15.24 \mathrm{~m})$ in extent, which represented $232 \mathrm{~m}^{2}$. SWCA assumed a maximum depth of cultural deposits as 1.5 to $2.0 \mathrm{~m}$ depending upon recoveries, which equated to approximately $348 \mathrm{~m}^{3}$ of potential cultural deposits at the bore pit. SWCA's prior investigations within the portion of the site that would be directly impacted resulted in the partial excavation of a single, $1 \times 1-\mathrm{m}^{2}$ unit and a total of $12 \mathrm{~m}^{2}$ of mechanical trenches (or $20 \mathrm{~m}^{3}$ ). That left approximately $328 \mathrm{~m}^{3}$ to be sampled systematically. As part of the data recovery effort, SWCA proposed to excavate approximately 10.0 percent of this remaining volume, which would represent approximately thirty-two $1-\mathrm{m}^{3}$ units. This allowed SWCA archaeologists to fully assess the integrity, data potential, and depositional setting of the site's cultural components and determine whether the relevant research issues could be addressed.

Work was performed by a crew of up to 10 archaeologists under the direction of the PI Brandon S. Young and Christina Nielsen. The PI provided project management, agency coordination, and overall quality control, while the PA supervised and directed all field investigations and all subsequent lab analyses and reporting. All work was conducted in compliance with applicable standards as defined or referenced in the ACT (13 Texas Administrative Code [TAC] 26.20) and elsewhere as appropriate.

\section{RESEARCH DESIGN}

The test investigations determined that the stratigraphic setting of site $41 \mathrm{GU} 177$ suggested multiple Holocene colluvial blankets of the toe slope from the older, upslope landform of possible Pleistocene age. Both landforms are capped by a thin veneer of recent deposition. Except for localized disturbances limited to, or near, the ground surface from bioturbation and landform modifications, the preservation and stratigraphic separation of the prehistoric occupations in this setting is most likely a direct result of the nature and timing of the sedimentation at the site. The cultural deposits observed at 41GU177 suggest at least two (and possibly up to four) cultural zones. Given the deep subsurface site component, dense artifact assemblage, buried hearth feature, and evidence for multiple cultural zones, SWCA recommended mitigation of the portion of the site within the APE that cannot be avoided by construction impacts, and therefore developed the following data recovery plan.

SWCA's focus during the data recovery was to address the environmental, technological, chronological, and adaptive changes during the transition from Archaic to Late Prehistoric patterns. The primary overarching goal was to discern the nature and timing of the transition from the end of the Archaic period to the beginning of the Late Prehistoric period on the eastern margins of the Balcones Escarpment. In addressing this issue, evidence of good faunal preservation and a diverse artifact assemblage derived from 
the phased previous investigations on 41 GU177 suggested SWCA would be able to explore the issues of site chronology, lithic technology (including hot rock cooking), subsistence strategies, and intrasite activity area patterns, and then compare these data with the known regional transition from the Late Archaic to the early Late Prehistoric periods. The following research topics were proposed for the data recovery investigations:

1) Regional Chronology and 41GU177: The data have the potential to address multiple Late Prehistoric occupations at the site, as well as the shift from the Transitional Archaic to the Late Prehistoric period. Such data could possibly refine or revise the regional chronology by clarifying when certain projectile point styles appeared on the eastern edge of the Edwards Plateau. Additionally, radiocarbon dates obtained from faunal and floral remains would augment these temporal definitions.

2) Lithic Technology: The data have the potential to elucidate what stage along the trajectory of lithic reduction and tool production the recovered artifacts represent and how this compares to other Transitional Archaic and Late Prehistoric sites in the region. For example, does the assemblage of debitage represent early reduction stages or later stages of reduction and tool production? What do the artifacts suggest regarding lithic raw material sources and transport? Given the presence of multiple occupations, can we identify changes in the lithic technology trajectories through time? Is there evidence of the transition from use of spears to those of the bow and arrow? The lithic analysis methodology was focused on gathering data to determine whether the lithic reduction strategies at 41GU177 are consistent with the findings from other Transitional Archaic to Late Prehistoric sites in the region.

3) Foraging Strategies: Through the analysis of feature technology (i.e., hot rock cooking), lithic materials, flora and faunal samples, and regional paleoenvironmental data, the investigations could identify variations in foraging strategies from the Transitional Archaic to the Late Prehistoric, as well as if there are any discernible changes amongst the Late Prehistoric occupations. Based on other studies of Transitional to early Late Prehistoric sites along the Edwards Plateau margin, foraging patterns of the times have been characterized as narrow diet breadth focused on seasonal (late fall to early winter) intensive exploitation of deer, a pattern indicated by archaeological assemblages with formal burned rock technology. However, these sites lack evidence of middens, bison, common geophytes, artiodactyl remains with evidence of intensive processing (i.e., marrow extraction and grease rendering of even low marrow utility elements), and relatively high biface-to-core ratios. Analysis focused on gathering data to determine whether the $41 \mathrm{GU} 177$ assemblage is consistent with these expectations or shows a different foraging strategy.

Based on the limited testing investigations conducted on the site, there was potential for better preservation of at least the later end of the occupational span, the Austin phase, although additional work was needed to clarify such potential. The recovery of several arrow points within the test units on the site indicated stratigraphically distinct Late Prehistoric occupations, as well as hints of an earlier Transitional Archaic occupation. The data recovery investigations were able to build upon this dataset through the correlation of stratigraphic profiles, identification of burned rock features, and the application of established and relevant research initiatives.

Although the initial focus was on the Late Prehistoric occupations, deeper units were excavated down to assess the possible Transitional Archaic occupation, as well as to provide views of the context and preservation of this earlier occupation. SWCA proposed to open broad horizontal exposures, where possible, to study spatial patterning across the site and recover a viable sample of the assemblage. This would allow excavators to expose the entirety of features or other anomalies to further address topics 
relating to the subsistence economy, use of the landscape, and relative group size during the respective periods. Although the testing investigations revealed initial possibilities, the nature of the current level of understanding of the site necessitated a flexible approach to prioritize objectives as the information was acquired.

In summary, although the initial testing investigations on $41 \mathrm{GU} 177$ revealed substantial data on the Late Prehistoric occupations, the hints of the Transitional Archaic occupation were difficult to characterize, due to limited quantities of temporal diagnostics associated with that period. Given the preponderance of projectile points associated with the Late Prehistoric Austin phase, the early part of the Late Prehistoric was the initial focus of investigations, but the objectives would adapt to what the site could potentially offer. Specific data used to make the determinations included geoarchaeological information, preserved flora and faunal material, cooking features, and diagnostic implements that serve as indicators for the presence of intact occupations. Additional research issues included:

- Technological data regarding burned rock hearth features and possible data on what may have been processed in the features (i.e., fuel wood identification and possible subsistence data derived from macrobotanical samples recovered from feature fill, which could also provide insight into season[s] of site use).

- Examination of faunal resources exploited by site occupants based on the study of well-preserved faunal materials recovered from site, including bone and freshwater mussel shells that could also shed light on the issues of seasonality.

- Determination of seasonality of site use during the Late Archaic and Late Prehistoric periods through evidence derived from macrobotanical remains and analysis of faunal material (i.e., mussel shell and bone).

- Examination of freshwater mussel exploitation and possible shell working activities based on the common recovery of freshwater mussels in the cultural deposits, including a shell fragment recovered during the testing phase that appears to have been intentionally drilled for use as a bead.

Ultimately, data recovery efforts sought to provide information that contributed to the analytical model for the site, as well as address issues pertinent to the understanding of the prehistory of Texas. Although the research goals were straightforward and addressed issues pertaining to the presence or absence of specific cultural phenomena, more intensive research avenues (i.e., foraging theory and burned rock feature technology) were also pursued.

\section{FIELD METHODS}

\section{Excavation Grid and Site Mapping}

Prior to starting the data recovery excavations, a formal grid was established with grid north correlating with the bore area boundary. The primary site datum, a 24-inch-long, 0.5 -inch diameter piece of rebar, was established at the North (N) 1000 East (E) 1000 grid point, located $1.5 \mathrm{~m}$ west and $0.5 \mathrm{~m}$ south of the southwestern bore pit area corner. A 100-m-long tape was pulled along the E1000 line, and secondary datums, also using rebar, were set every 5 m (at N1000 E1005, N1000 E1010, N1000 E1015, etc.).

Excavation units were established initially along the E1000 line, then subsequently on the E995, E1005, and E1010 lines (Figure 4.1). Each $1 \times 1-\mathrm{m}$ excavation unit was designated by the coordinate of its southwestern corner. Vertical control was maintained relative to the primary site datum, a poured concrete casing around rebar located at N1000 E1000. The datum was assigned an arbitrary elevation of $100.00 \mathrm{~m}$, 


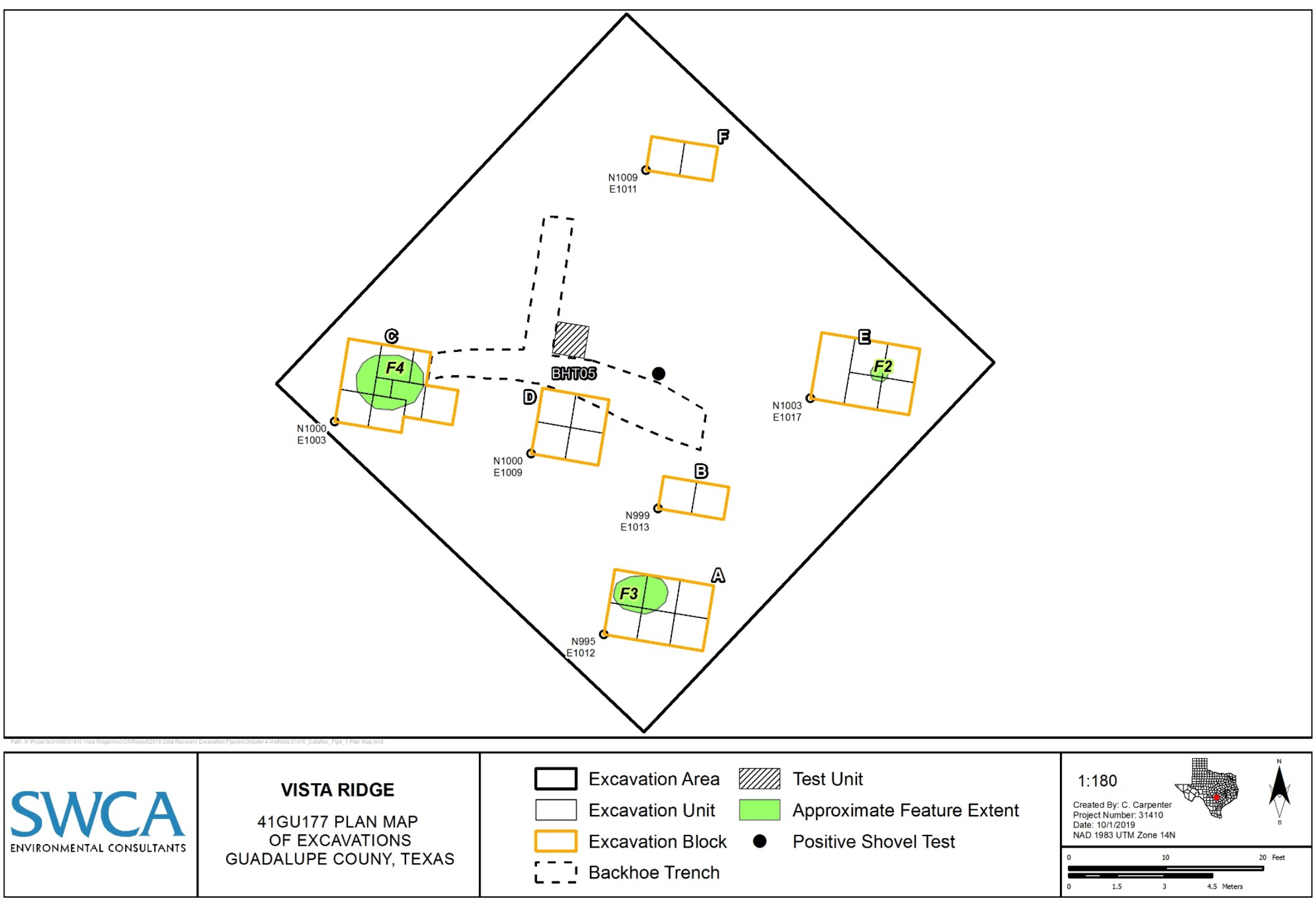

Figure 4.1. Site 41GU177 Data Recovery excavations plan map. 
which correlates with an absolute elevation of about $173.74 \mathrm{~m}$ amsl. String line datums were established in 5-m intervals along the E1000 baseline and at similar intervals for the N995, N1005, and N1010 baselines. Elevations of artifacts and levels were maintained by these datums.

\section{Hand Excavations}

Hand excavation locations were selected at the discretion of the PI and PA to expose broad horizontal exposures and investigate cultural features. A total of 22 traditional $1 \times 1-\mathrm{m}$ units were initially excavated during the data recovery within six excavations blocks (Blocks A-F) (see Figure 4.1). In addition, six feature-focused units were excavated within Block $\mathrm{C}$ to explore the horizontal extents of Feature 4 . When combined, the traditional and feature-focused units resulted in the excavation of approximately $34.1 \mathrm{~m}^{3}$. The distribution of excavation volume by block and unit is shown in Table 4.1.

Table 4.1. Excavation Volume by Blocks

\begin{tabular}{|c|c|c|c|c|c|c|c|c|}
\hline $\begin{array}{l}\text { Excavation } \\
\text { Block }\end{array}$ & $\begin{array}{c}\text { Excavation Unit } \\
\text { Type }\end{array}$ & $\begin{array}{l}\text { Excavation } \\
\text { Unit Size }\end{array}$ & Northing & Easting & $\begin{array}{l}\text { Top Elev. } \\
\text { (m) }\end{array}$ & $\begin{array}{l}\text { Bottom } \\
\text { Elev. (m) }\end{array}$ & $\begin{array}{l}\text { No. of } \\
\text { Levels }\end{array}$ & $\begin{array}{c}\text { Total } \mathrm{m}^{3} \\
\text { Excavated }\end{array}$ \\
\hline \multirow{6}{*}{$A$} & Traditional & $1 \times 1 \mathrm{~m}$ & 995 & 1012 & 99.70 & 98.20 & 15 & 1.50 \\
\hline & Traditional & $1 \times 1 \mathrm{~m}$ & 995 & 1013 & 99.65 & 98.20 & 15 & 1.45 \\
\hline & Traditional & $1 \times 1 \mathrm{~m}$ & 995 & 1014 & 99.59 & 98.20 & 14 & 1.39 \\
\hline & Traditional & $1 \times 1 \mathrm{~m}$ & 996 & 1012 & 99.70 & 98.20 & 15 & 1.50 \\
\hline & Traditional & $1 \times 1 \mathrm{~m}$ & 996 & 1013 & 99.59 & 98.20 & 14 & 1.39 \\
\hline & Traditional & $1 \times 1 m$ & 996 & 1014 & 99.60 & 97.60 & 20 & 2.00 \\
\hline \multirow{2}{*}{ B } & Traditional & $1 \times 1 \mathrm{~m}$ & 999 & 1013 & 99.58 & 98.10 & 15 & 1.48 \\
\hline & Traditional & $1 \times 1 m$ & 999 & 1014 & 99.55 & 98.10 & 15 & 1.45 \\
\hline \multirow{8}{*}{$\mathrm{C}$} & Traditional & $1 \times 1 \mathrm{~m}$ & 1000 & 1003 & 99.83 & 98.90 & 9 & 0.93 \\
\hline & Traditional & $1 \times 1 \mathrm{~m}$ & 1000 & 1004 & 99.77 & 98.30 & 15 & 1.47 \\
\hline & ${ }^{*}$ Feature-Focused & $1 \times 1 \mathrm{~m}$ & 1000.5 & 1005.5 & 99.60 & 99.30 & 3 & 0.30 \\
\hline & ${ }^{*}$ Feature-Focused & $1 \times 1.5 \mathrm{~m}$ & 1001 & 1003 & 99.60 & 99.30 & 3 & 0.45 \\
\hline & Feature-Focused & $50 \mathrm{~cm} \times 50 \mathrm{~cm}$ & 1001 & 1004 & 99.75 & 99.30 & 5 & 0.11 \\
\hline & Feature-Focused & $3 / 4$ unit & 1001 & 1004.5 & 99.77 & 99.30 & 5 & 0.35 \\
\hline & ${ }^{\star}$ Feature-Focused & $1 \times 1 \mathrm{~m}$ & 1001.5 & 1004 & 99.60 & 99.30 & 3 & 0.30 \\
\hline & ${ }^{*}$ Feature-Focused & $1 \times .5 m$ & 1001.5 & 1005 & 99.50 & 99.30 & 2 & 0.10 \\
\hline \multirow{4}{*}{$\mathrm{D}$} & Traditional & $1 \times 1 \mathrm{~m}$ & 1000 & 1009 & 99.67 & 98.59 & 11 & 1.08 \\
\hline & Traditional & $1 \times 1 \mathrm{~m}$ & 1000 & 1010 & 99.62 & 97.59 & 21 & 2.03 \\
\hline & Traditional & $1 \times 1 \mathrm{~m}$ & 1001 & 1009 & 99.60 & 98.59 & 10 & 1.01 \\
\hline & Traditional & $1 \times 1 m$ & 1001 & 1010 & 99.59 & 97.59 & 20 & 2.00 \\
\hline \multirow{6}{*}{$E$} & Traditional & $1 \times 1 \mathrm{~m}$ & 1003 & 1017 & 99.28 & 97.80 & 15 & 1.48 \\
\hline & Traditional & $1 \times 1 \mathrm{~m}$ & 1003 & 1018 & 99.23 & 97.80 & 15 & 1.43 \\
\hline & Traditional & $1 \times 1 \mathrm{~m}$ & 1003 & 1019 & 99.22 & 97.80 & 15 & 1.42 \\
\hline & Traditional & $1 \times 1 \mathrm{~m}$ & 1004 & 1017 & 99.25 & 97.70 & 16 & 1.55 \\
\hline & Traditional & $1 \times 1 m$ & 1004 & 1018 & 99.22 & 97.80 & 15 & 1.42 \\
\hline & Traditional & $1 \times 1 m$ & 1004 & 1019 & 99.22 & 97.80 & 15 & 1.42 \\
\hline \multirow{2}{*}{$F$} & Traditional & $1 \times 1 m$ & 1009 & 1011 & 99.26 & 97.70 & 16 & 1.56 \\
\hline & Traditional & $1 \times 1 m$ & 1009 & 1012 & 99.21 & 97.70 & 15 & 1.51 \\
\hline Total & & & & & & & & 34.09 \\
\hline
\end{tabular}

${ }^{*}$ Level 1 of these units stripped off and not screened due to disturbance; level 1 not counted in total number of levels or $\mathrm{m}^{3}$ excavated 
The hand excavation units were usually $1 \times 1-\mathrm{m}$ in size and excavated up to $2 \mathrm{~m}$ deep in select units or to sterile deposits, whichever was encountered first (see Chapter 5). Using standard archaeological methods, the excavation units were systematically excavated in arbitrary $10-\mathrm{cm}$ levels and documented using standardized field forms and photographs. All soils were screened through $1 / 4$-inch hardware mesh. All artifacts and pertinent faunal or floral remains were collected for analysis and bagged separately to examine site stratigraphy and occupation levels. Features encountered during the investigations were carefully exposed, documented, and sampled.

\section{Feature Excavation Methods}

As an important data set, features were focal points of the archaeological investigations. Each of the three features discovered during hand excavations were numbered, with the PA maintaining the list of consecutive numbers. Each feature was exposed in plan, drawn, and photographed. Each feature was described and documented on a special feature form. Features were cross-sectioned, and samples for magnetic susceptibility (MS) and charcoal samples for radiocarbon dating were collected as determined by the PA. Bulk matrix samples were taken from various contexts, and the coarse matrix (burned rock) was size-sorted, counted, weighed, and discarded in the field. Approximately 2 gallons of matrix from each feature was reserved for flotation or pollen/phytolith analyses, and the remaining fine matrix was screen through $1 / 4$-inch mesh for full artifact recovery.

\section{Modifications to Field Methodology}

During the course of fieldwork, it became necessary to modify certain elements of the feature excavation methodology. Specifically, the significant modifications were related to the excavation and documentation of Feature 4, a large earth oven feature spanning numerous units in Block C.

\section{FEATURE 4 METHODS}

Feature 4 was first noted as a cluster of burned rocks in the northeast quadrant of unit N1000 E1004, Level 5 in Excavation Block C (Figure 4.2). The concentration appeared to extend to the north and east, outside of the original $1 \times 1-\mathrm{m}$ unit. Due to time constraints, typical methodology of expanding the excavation with traditional units (i.e., $1 \times 1-\mathrm{m}$ units) to the north and east was abandoned for a nontraditional, feature-focused approach consisting of excavation of a 3/4-sized unit (N1001 E1004.5) around the burned rock cluster (see Figure 4.2). Excavations in this feature-focused unit identified additional burned rock extending into the west wall, so SWCA opened another featured-focused unit. this unit (N1001 E1004) measured $50 \times 50 \mathrm{~cm}$.

Once excavations into the $50 \times 50$-cm unit reached the feature, it became apparent that the burned rock feature extended further outside the three units to the north, east, and west. As such, four addition featurefocused units of various sizes (N1001 E1003, N1001.5 E1004, N1001.5 E1005, and N1000.5 E1005.5) were opened to further investigate the extent, thickness, and composition of the feature and to identify potential drop zones and/or discard areas (see Figure 4.2). Due to modern disturbances and to expedite the feature investigations, Level 1 of each of these four units was stripped off without screening and excavations began just above Feature 4 at either $99.6 \mathrm{~m}$ (30 centimeters below datum [cmbd]) or $99.5 \mathrm{~m}$ (40 cmbd); this approach was approved by Jeff Durst, with the THC, who was on site that day. None of the feature-focused units were excavated further after Feature 4 was fully documented and removed. 


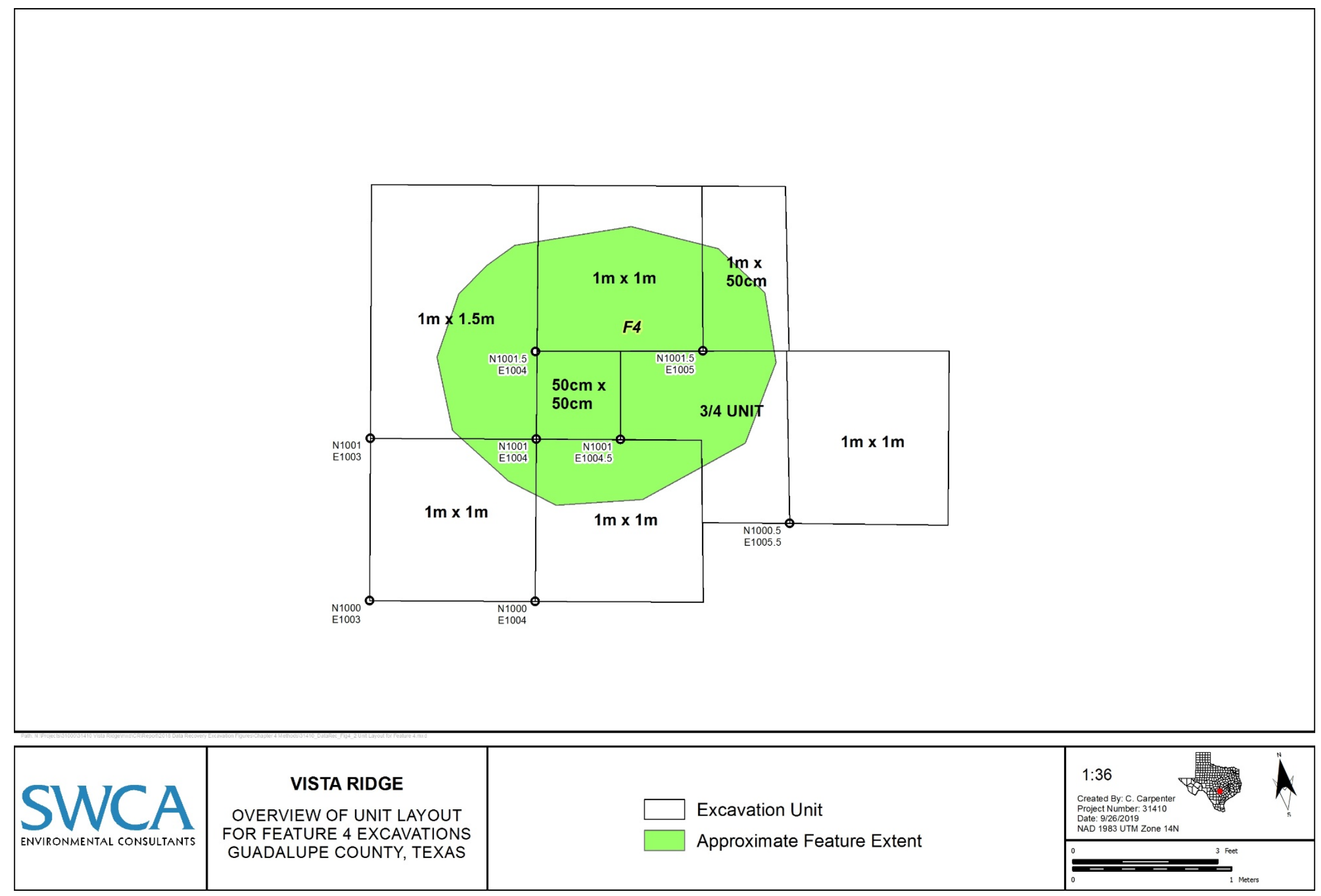

Figure 4.2. Overview of Unit Layout for Feature 4 Excavations 


\section{Artifact Collection and Sampling Strategy}

All artifacts and samples recovered from each provenience unit and level were collected, bagged, and labeled accordingly, and were assigned a general bag number in the field inventory. Point provenience artifacts and samples were assigned an individual bag number in the field inventory. Additionally, most artifacts of special interest (e.g., projectile points, formal bifaces, formal scrapers, ground stone artifacts, and antler and bone tools, etc.) were assigned a unique item (UI) number in addition to the general or individual bag number. Burned rock was size-sorted, weighed, and counted for each unit by level and/or feature but not collected. The presence of snails was also noted on unit level forms but not collected.

In conjunction with the excavations, special samples were collected from appropriate contexts across the site. Special samples included materials for radiocarbon dating (from feature contexts), matrix samples for flotation, and geomorphic samples.

\section{LABORATORY PROCESSING AND ANALYSIS METHODS}

An important component of the data recovery investigations at $41 \mathrm{GU} 177$ was the laboratory processing and analyses conducted subsequent to the fieldwork. These disparate analyses performed separately were designed to provide a more thorough picture from which to interpret the significance of the site.

\section{Artifact Processing}

The artifact processing and cataloging system was done in compliance with the Center for Archaeological Research at The University of Texas-San Antonio (CAR-UTSA) curation standards and guidelines. Artifact processing included washing, sorting, and tabulating the recovered materials with the resulting data compiled into the specimen inventory cataloging system. The field inventory was applied to the specimen inventory, which included assigning lot numbers to the bag and UI numbers previously designated in the field and assigning specimen numbers to facilitate artifact analyses. The specimen inventory for the data recovery excavations are presented in Appendix B. All artifact processing was accomplished at SWCA's laboratory.

\section{Artifact Analysis}

Once the fieldwork was complete, SWCA analyzed all recovered artifacts from stratified deposits. This included washing all artifacts and sorting into analytical categories, including projectile points, bifaces, edge-modified flakes, cores, debitage, and ground stone. Analysis of lithic artifacts focused on recording basic attributes, examining function, and exploring raw material origins. SWCA measured the tools and ground stone, and recorded relevant attributes for each category; all analysis tables are presented in Appendix C. Faunal analysis focused on determining species, number of individuals, elements, seasonality, butchering practices, and effects of post-depositional processes (Appendix D). Data from the lithic and faunal analyses were then compared to information recovered from regional contexts. Site analysis included examining artifact distributions, running special samples, and comparing overall results with other relevant data.

\section{Lithic Analysis}

\section{TAXONOMY}

From field collection through final report production, the analytical process was inherently tied to the taxonomic classification of each artifact. Stone artifacts were classified essentially following Andrefsky's 
general morphological model typology (Andrefsky 1998:74:Figure 4.7). At the broadest level, the stone artifact assemblage is divided into three primary types: chipped stone tools, non-chipped stone tools, and debitage. These three primary types are further defined and broken down by various functional types, when applicable, and characteristics reflecting nominal and metrical attributes specific to each class of artifacts. Based on this taxonomy, SWCA distinguished six categories of chipped stone tools: projectile points, bifaces, scrapers, modified flake, cores, and core tools, and two categories of non-chipped stone tools: ground stone and manuports (see Chapter 6).

\section{NOMINAL AND METRIC ATTRIBUTES}

The goal of SWCA's lithic analysis is to provide data consistent and comparable to existing data of the region; therefore, a variety of nominal and metric attributes were collected, pulling from techniques employed by Andrefsky (1998), Callahan (1979), Collins (1974), Fields and Gadus (2012), Perttula (2008), and Perttula and Nelson (2006). Attributes recorded were dependent on the established research questions and artifact types. Common recorded nominal attributes included information such as cortex, raw material type, color, patination, evidence of heat treatment, breakage, beveling, and reworking. Types of metrical attributes recorded for each specimen include maximum length, maximum width, maximum thickness, weight, as well as artifact type specific metrics such as, haft length, retouch height, angle of retouch, maximum flake scar length, and number of flake scars. The width-to-thickness ratios, based on Callahan (1979), were calculated for bifaces, as a contributing attribute for determining the reduction stage. The average edge angle of each biface was also measured using a contact goniometer.

As closely as practicable, artifact length was measured from the proximal to distal ends, width was measured perpendicular to this from the lateral margins, and thickness was measured from ventral to dorsal surfaces. In the case of lithic debitage, an analysis approach was taken focused on counts and weight of the lithic debitage with a simple sort into complete, proximal, and broken flakes types.

\section{Faunal Analysis}

The 41GU177 faunal analysis was conducted in SWCA's Austin office. The assemblage was sorted, analyzed and interpreted by Chris Shelton and reviewed by Kevin A. Miller. Each bone was individually examined and analyzed by hand. When possible, faunal identifications were made using comparative osteology manuals, such as Klein and Cruz-Uribe's The Analysis of Animal Bones from Archeological Sites (1984), Lyman's Vertebrate Taphonomy (1994), Olsen's Mammal Remains from Archaeological Sites (1973) and Reitz and Wing's Zooarchaeology (2008), as well as comparative faunal collections housed in the SWCA Austin lab.

The faunal assemblage was computer catalogued using the Microsoft Excel program. The database consisted of 15 distinct criteria, including unique lot, bag, and specimen numbers; taxon identification; common name of animal; weight; body size; presence; cultural modification presence and type; bone element identification; location of bone; presence of growth plate fusion; fragment type; and margin angle. Human taphonomy processes, such as presence or absence of butchery in the form of cut marks, chop marks and percussion impact breakage were cataloged to examine foraging techniques. The combination of these criteria can be analyzed individually or as groups in order to determine important economic, preference, and social information in regard to the site.

\section{Special Samples}

As mentioned briefly above, special samples were systematically collected from appropriate contexts across the site. Special samples included materials for radiocarbon dating (from features, geomorphic units, and other contexts), matrix samples for flotation and/or fine screening (from features), and sediment 
samples for geoarchaeological analysis (e.g., MS and phosphorus analysis). These types of samples, often critical in interpreting a site, were taken from both cultural and natural contexts. Special samples were immediately prepared and submitted to the appropriate laboratories for processing. Quick submittal of the samples ensured a fast turn-around time so that results were integrated into the report.

\section{Radiocarbon Analysis}

During the investigations at site $41 \mathrm{GU} 177$, several samples $(\mathrm{n}=6)$ of charred material, faunal remains, and plant material were collected for radiocarbon analysis. Select charcoal, bone, and flora assays were subsequently sent to Beta Analytic Inc. who conducted the analyses. Notably, radiocarbon years $\left({ }^{14} \mathrm{C}\right)$ are not calendar years, but rather a measurement of remaining ${ }^{14} \mathrm{C}$ isotopes (Bartlein et al. 1995; Blockley et al. 2007; McCormac and Baillie 1993; Mock and Bartlein 1995; Ramsey 2008, 2009:337; Stuiver and Suess 1966). Consequently, the ${ }^{14} \mathrm{C}$ years must be converted to a calendrical format (i.e., calibrated) for general interpretation and comparison (Mook and Waterbolk 1985:20). This study employs the nomenclature proposed by Nordt (1992) and used by the journal American Antiquity for distinguishing between uncalibrated radiocarbon and calibrated radiocarbon results. Specifically, the results of these analyses are reported in both conventional radiocarbon age $\left({ }^{14} \mathbf{C}\right.$ yr B.P.) and the calendar calibrated (cal yr B.P.) results. The Gregorian calendar year A.D. 1950 is recognized as the beginning point of before present (B.P.) (Hua 2009; Mook and Waterbolk 1985; Stuiver and Polach 1977; Taylor 1997:67-68, 2009).

SWCA selected an initial batch of six radiocarbon samples from a variety of archaeological and geomorphological contexts for analysis by Beta-Analytic, Inc. Only five of the samples were determined large enough to be tested. The results of the radiocarbon analyses were then interpreted in regard to natural stratigraphy and associated cultural deposits to construct a chronostratigraphy. The radiocarbon assays from Beta Analytic Inc. are presented in Appendix E.

\section{Flotation and Macrobotanical Analysis}

Additional attempts were made to obtain plant-related subsistence data from 41GU177. In open, welldrained sites like $41 \mathrm{GU} 177$, plant remains rarely preserve unless they were previously exposed to 250 $300^{\circ} \mathrm{C}$ temperatures with little oxygen, resulting in carbonization (Hard et al. 1996). Although resistant to biological deterioration once carbonized, they still may be destroyed by mechanical means (Miksicek 1987:20).

When possible, charred fragments visible to the naked eye ("macrofossils") were collected directly from the excavation units. Smaller remains go unnoticed during excavation and often slip through screens, so SWCA collected soil samples for processing by water separation, or flotation (Struever 1968), in order to maximize data recovery.

Five matrix soil samples were retained for this purpose from three features (i.e., F2-F4). The samples were processed in a 15-gallon metal wash pan filled about two-thirds with water. A large piece of fine window screen was clipped to the circumference of the rim, loose enough to hang well below the water surface. Individual soil samples were added to the water gradually and gently agitated by hand, allowing for the sediment matrix to break up and free any lightweight organic contents (light fraction) into the water column. The light fraction was then scooped from the water using a fine-mesh aquarium net. The procedure was repeated until the entire sample was processed, and the light fraction set to dry. The heavier materials caught in the window screen catchment (heavy fraction) were rinsed clean, dried, and bagged separately from the light fraction. Once dry, each of the light and heavy fractions were weighed (in grams) and the light fractions were packaged to be sent to Dr. Leslie Bush for macrobotanical analysis. 
In addition to the analysis of light fractions from the feature matrix samples, SWCA sent Dr. Bush 10 fragments of wood charcoal collected directly from feature contexts for identification. The macrobotanical analysis report and analysis tables are included in Appendix F.

\section{GEOARCHAEOLOGICAL INVESTIGATIONS}

The geoarchaeological investigations involved thorough examination and select sampling of multiple exposed stratigraphic profiles across the site. Profiles along both the strike and dip axes across and adjacent to the site were characterized using a modified soil profile documentation advocated by Birkeland (1999). These investigations conducted by Ken Lawrence incorporated a documentation of the physical characteristics of the horizon matrices, as well as a suite of associated analyses (i.e., organic carbon, radiocarbon, particle size, and MS). All geoarchaeological analysis tables are presented in Appendix G.

\section{Physical Characteristics}

The stratigraphic investigations involved a thorough examination and documentation of multiple exposed profiles. All Munsell colors, unless indicated otherwise, were recorded dry.

\section{Magnetic Susceptibility}

MS analyses of soils have been utilized for archaeological investigations for several decades and have largely been directed toward surveys and prospection (e.g., Crowther and Barker 1995; Dalan 1996, 2008). More recently, these investigations have focused on archaeological site formation and associated depositional processes (Dalan 2006, 2008). In particular, these analyses have assisted in identifying buried or thermally-altered soils associated with cultural activities, as well as identifying the horizontal extent of cultural features (e.g., Dalan and Banerjee 1998; Dalan and Bevan 2002; Ellwood et al. 1995; Frederick 2010; Jones and Leffler 2003; Mauldin and Figueroa 2006; Nickels 2010; Peters and Thompson 1999; Rivers et al. 2004).

The processes of environmental magnetism and particularly the application of MS have been extensively reviewed (e.g., Dearing 1999a, 1999b; Gale and Hoare 1991:201-229; Maher et al. 1999; Thompson and Oldfield 1986; Verosub and Roberts 1995). Briefly, however, magnetic minerals are prevalent in the natural environment and are sensitive to environmental changes (Gale and Hoare 1991:202). The measurement of MS $\chi$ (Chi) is a quantification of the 'magnetizability' of the material (Dearing 1999b:5; Gale and Hoare 1991:202-204). The mineralogy, the size and shape of the grains, internal stress, and other factors can initially influence the susceptibility value (Dearing 1999a, 1999b; Gale and Hoare 1991:204). However, of relevance to this discussion, factors including organic content, pedogenesis, thermal alteration, and cultural activities (e.g., ash-charcoal and refuse) can subsequently alter (usually increase) the susceptibility values. The implications of using MS for archaeological research is that when examining either vertical or horizontal areas, the susceptibility values can assist in identifying cultural activity areas that may otherwise be blurred at a macro level. Horizontally, the application of MS analysis has been used to define the limits of cultural features and living spaces in excavation blocks (e.g., Mauldin and Figueroa 2006). Vertically, the MS results have been applied to recognizing and delineating cultural horizons (e.g., Frederick 2010, 2012; Lawrence et al. 2013; Lawrence and Frederick 2012).

For the investigations at site 41GU177, MS samples were collected from select excavation profiles (i.e., N1000 E1004, N996 E1014, N1004 E1017, N1009 E1012, and N1000 E1010) and one feature (Feature 4). Vertically, the MS samples were collected up profile in systematic $2-3 \mathrm{~cm}$ intervals. Each of the samples were mapped into the respective profiles, effectively correlating them to site stratigraphy and excavation elevation. These samples were packed in plastic cubes and subsequently analyzed using a 
Bartington MS2 meter and MS2b sensor to examine both low ( $\chi \mathrm{lf})$ and high $(\chi \mathrm{hf})$ frequency (470-4700 $\mathrm{Hz}$, respectively) MS. The cube samples were measured at the high sensitivity setting (0.1) compared to the normal sensitivity setting (1.0). The high sensitivity setting was necessary since the frequency dependency of the MS samples were also calculated (Gale and Hoare 1991:223-224). The values for each cube sample were catalogued twice at both the low and high frequencies to provide an average value using the Systeme International (SI) scale. SWCA recorded the $\chi \mathrm{lf}, \chi \mathrm{hf}$, the average values, and each sample's weight in an Excel table. After every five readings, the Bartington MS2 meter was "zeroed" out to recalibrate the meter.

The cube samples from this analysis were subsequently calculated using the methods outlined by Gale and Hoare (1991:223-226). Briefly however, the primary metric for recognizing MS values of interest is the coefficient of frequency dependency $(\chi \mathrm{fd})$. This calculation is indicated as:

$$
\chi \mathbf{f d}=\mathbf{1 0 0}[(\chi \mathbf{l f}-\chi \mathbf{h f}) / \chi \mathbf{l f}]
$$

This calculation uses $\chi \mathrm{lf}$ as the 'normal' sensitivity value and contrasts it with the $\chi \mathrm{hf}$ value to reveal significant values of difference in percentages (Dearing 1999a:47, 1999b:17-18; Gale and Hoare 1991:226). Elevated values of $\chi \mathrm{fd}$ can represent concentrations of ultrafine magnetic grains (e.g., maghemite), which are commonly associated with pedogenesis (Dalan 2006:164; 2008:22). A number of factors may cause an increase in $\chi$ lf values including biological (e.g., bacteria) or inorganic (e.g., natural weathering of iron minerals) processes. In an archaeological context, an increase in $\chi \mathrm{lf}$ values may reflect cultural activities from thermal features and general refuse (Dalan 2008; Dalan and Banerjee 1998; Frederick 2012). Ideally, when applied to a vertical profile, collected in systematic intervals (e.g., 2$3 \mathrm{~cm}$ ), and contrasted with cultural data (e.g., artifact frequencies) and other analyses (e.g., Loss on Ignition), this analysis can provide valuable data to interpret site formation and cultural activities.

\section{CURATION}

Per specifications of the ACT permit issued for the data recovery, all project documents and artifacts must be curated at an approved curatorial facility. Artifacts recovered during the investigations were prepared for permanent storage (e.g., washing, labeling, and cataloguing) and curation using state archaeological standards. All curated artifacts will be housed at CAR-UTSA. 


\section{CHAPTER 5. OVERVIEW OF DATA RECOVERY INVESTIGATIONS}

This chapter summarizes the results of SWCA's data recovery excavations at the Snakeskin Bluff site (41GU177). Data recovery investigations to mitigate project effects were conducted from November 27 to December 28, 2017, within the proposed bore bit area. A discussion of the overall site stratigraphy and cultural strata are discussed followed by results from the hand unit excavations undertaken at the site. An overview of the various analyses results, particularly regarding site formation processes are also provided. More detailed information on recovered materials and cultural features encountered during these excavations are within Chapter 6. Previous work at the site, including initial survey and testing phase investigations, was summarized in Chapter 1 and were previously reported in an interim report and are presented in Appendix A (Rodriguez et al. 2017).

\section{SITE STRATIGRAPHY}

\section{Natural Strata}

As previously reported in the interim report, the excavation and documentation of 58 shovel tests, 13 backhoe trenches (i.e., BHT01-BHT13) and five test units (i.e., TU01-TU05) during the test excavations, observed a distinctly different stratigraphy across $41 \mathrm{GU} 177$ depending on topography and proximity to the Guadalupe River (Rodriguez et al. 2017). The excavations on the western end of the site (i.e., BHTs 01, 03, 09, and 13) suggest an older deposition than those trenches on the eastern portions of the site (i.e., BHTs $02,04,05-08$, and 10-12). The western trenches are situated on a landform approximately 580 to 585 feet amsl and about 5 feet above the eastern trenches. These trenches contain relatively shallow calcium carbonate $\left(\mathrm{CaCO}_{3}\right)$ filaments and nodules beginning around 30 to $40 \mathrm{cmbs}$, which appear to be Pleistocene terrace deposits. The eastern trenches coincide with the $\mathrm{T}_{2}$ terrace landform at 575 to 580 feet amsl, whereas the western trenches are situated on the adjacent landform that could be part of the $\mathrm{T}_{2}$ landform or remnants of an older alluvial landform (i.e., $T_{3}$ ). The stratigraphy in the eastern trenches have deep alluvial deposits that appear younger in age (Holocene) than the western trenches on the adjacent landform.

The data recovery excavations at $41 \mathrm{GU} 177$ all occurred on the $\mathrm{T}_{2}$ terrace landform centered around BHT05 and BHT06. Prior to the data recovery excavations, a backhoe was used to reopen BHT05 and BHT06 initial opened during the testing phase (see Figure 4.1). The stratigraphy observed in the excavation units correlated with the stratigraphy observed during the test excavations.

\section{$T_{2}$ terrace (Excavation Blocks A-F)}

With some exceptions, SWCA observed six strata in the excavations (Excavation Blocks A-F) on the $\mathrm{T}_{2}$ terrace that correlated with the earlier test excavations (i.e., BHTs 2, 4, 5-8, 10-12) at 41GU177 (Figures 5.1-5.3). The test excavations extended to $330 \mathrm{cmbs}$ while the data recovery excavations extended to no more than $200 \mathrm{cmbs}$.

Stratum I is an approximately 2-6-cm-thick horizon of very dark grayish brown (10YR 3/2) silt loam humate soil with partially decomposing organics and a clear and slightly wavy lower boundary. The second horizon (Stratum II) ranges in thickness from $14-35 \mathrm{~cm}$ and is a very dark grayish brown to dark grayish brown (10YR 3/2-4/2) silt loam with friable consistency, a crumb to subangular blocky structure, and a clear and smooth lower boundary. Inclusions observed within the stratum include white filaments that are interpreted to be mycorrhizal fungi (1 percent) and Heliodiscus singleyanus and Rabdotus mooreanus snail shell (10 percent). 


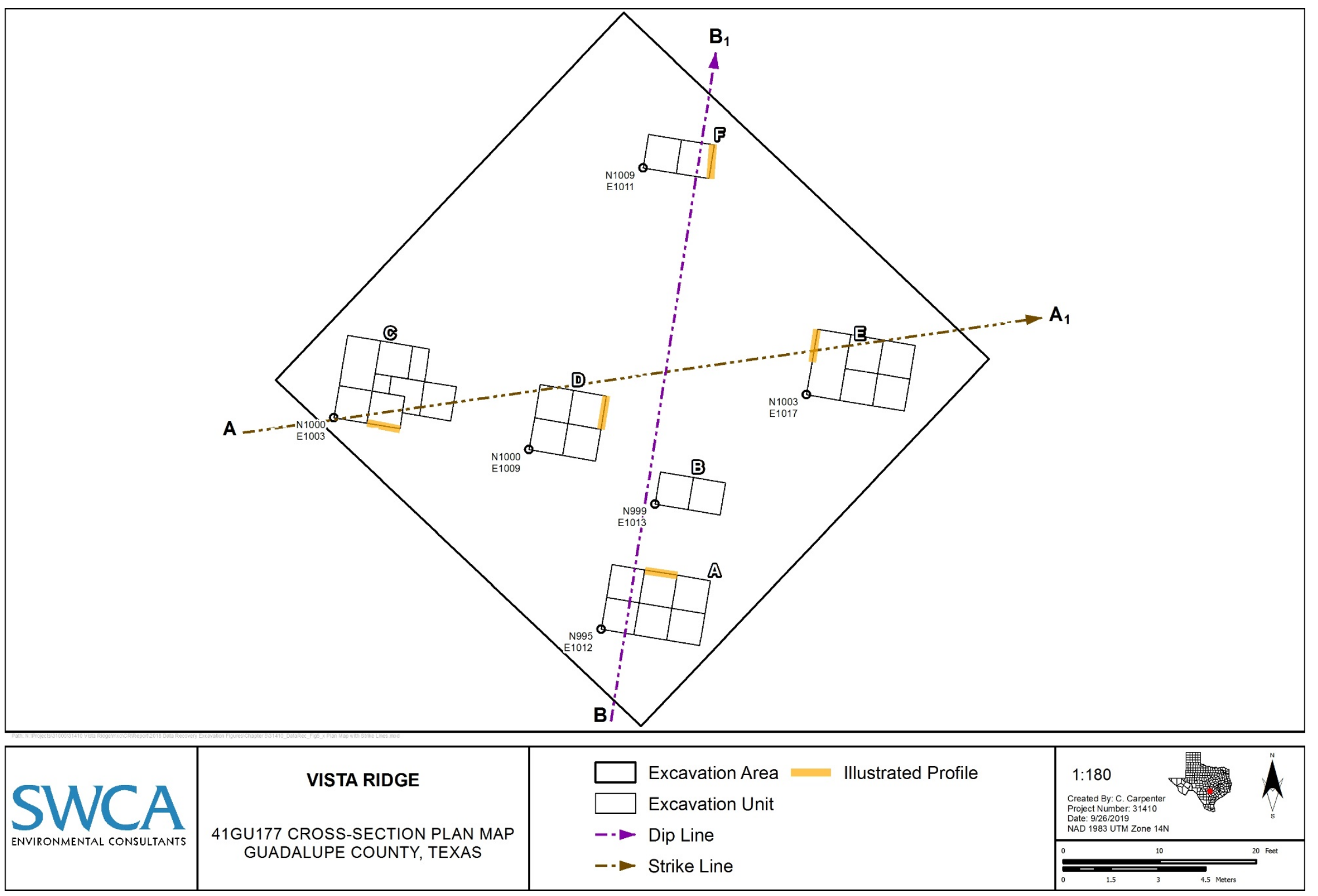

Figure 5.1. 41GU177 Cross-section Plan Map. 


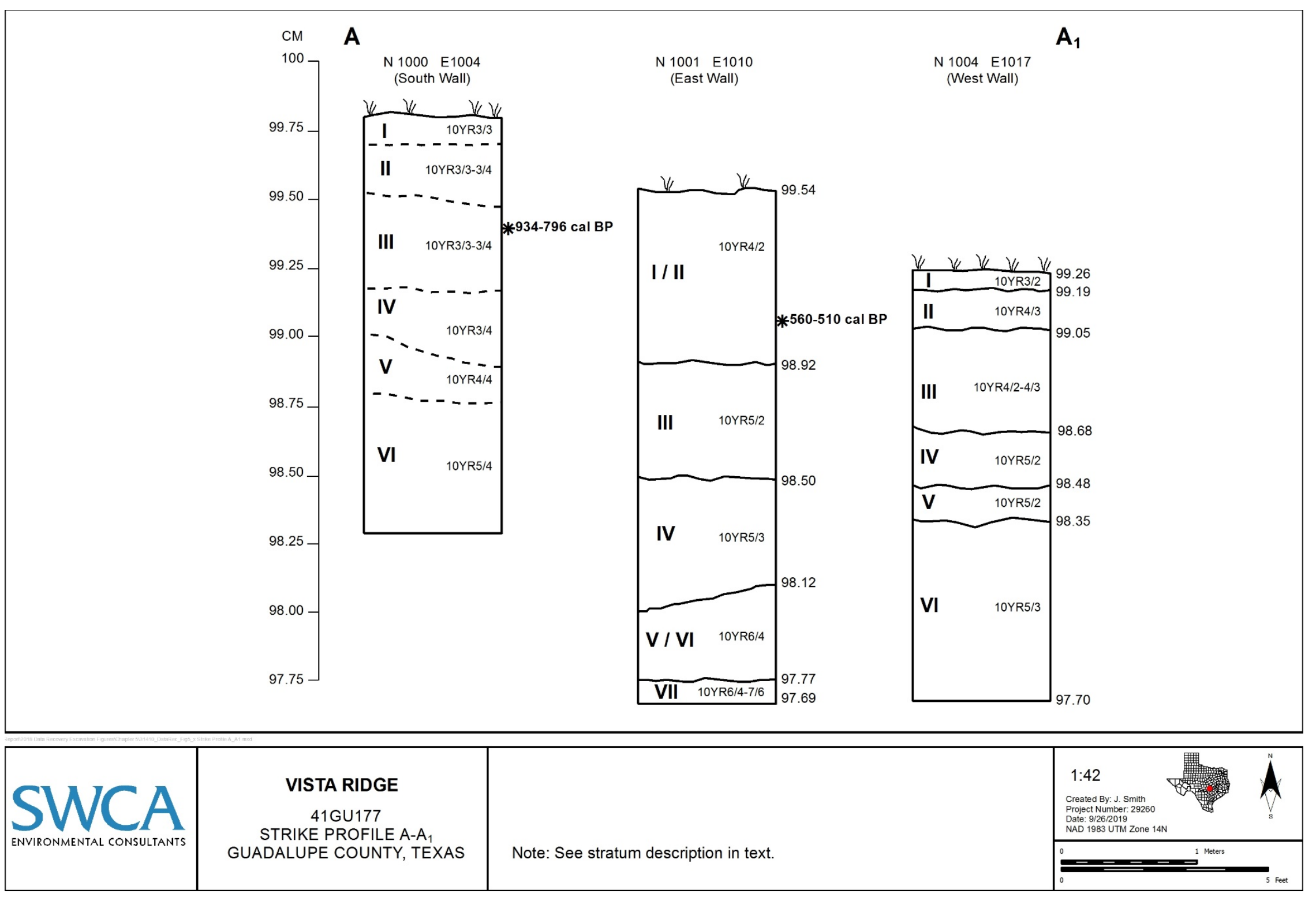

Figure 5.2. 41GU177 Strike Profile Map. 


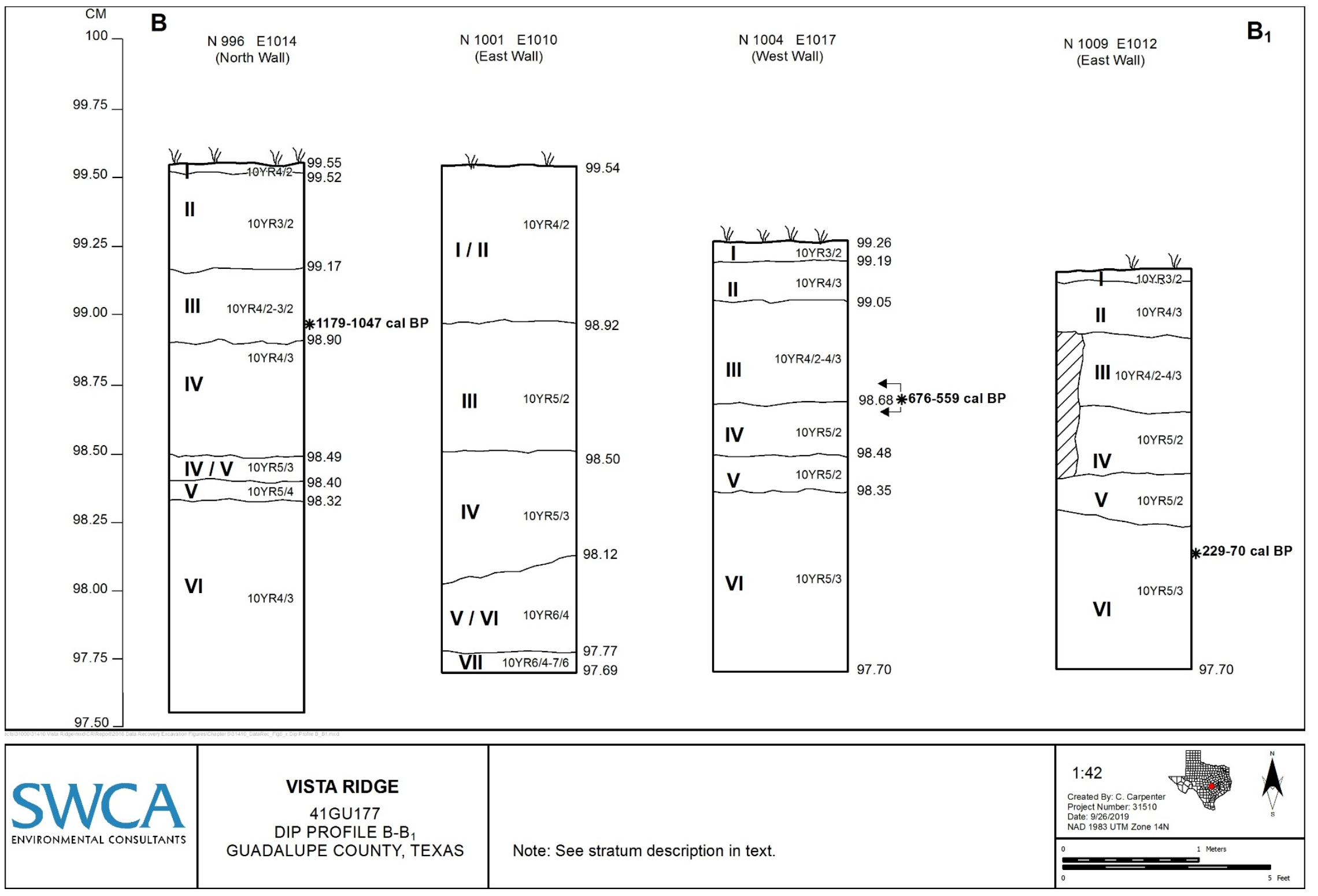

Figure 5.3. 41GU177 Dip Profile Map. 
Underlying this horizon is Stratum III, an approximately 27-42-cm-thick horizon of dark grayish brown to grayish brown (10YR 4/2-5/2) silt loam with a subangular blocky structure and friable consistency with a gradual and smooth lower boundary. Inclusions observed include common roots/rootlets (15 to 20 percent), common insect and worm burrows (10 to 15 percent), white filaments that are interpreted to be mycorrhizal fungi (<1 percent), and Heliodiscus singleyanus (1 to 5 percent) and Rabdotus mooreanus (1 to 3 percent) snail shell.

The fourth horizon (Stratum IV) was observed to range from $20-41 \mathrm{~cm}$ in thickness and consists of a grayish brown to brown (10YR 5/2-5/3) silt loam with a friable consistency, a subangular blocky structure, and exhibited a gradual and smooth lower boundary. Inclusions included roots/rootlets (5 to 15 percent), insect and worm burrows ( 5 to 10 percent), white filaments that are interpreted to be mycorrhizal fungi ( $<1$ percent), and Heliodiscus singleyanus (10 percent) and Rabdotus mooreanus ( 2 percent) snail shell.

The underlying horizon (Stratum V) was approximately $13-18 \mathrm{~cm}$ thick and consists of a grayish brown (10YR 5/2) silt loam with a friable consistency and subangular structure with a gradual and smooth to slightly sloping lower boundary. Stratum V appears to be a transitional horizon as it was not easily discernable in some excavation profiles.

Below Stratum V, the sixth horizon (Stratum VI) was observed to range in thickness from $53 \mathrm{~cm}$ to greater than $82 \mathrm{~cm}$ as the base of the stratum was not observed in most excavations. Stratum VI consists of yellowish brown to light yellowish brown (10YR 5/4-6/4) sandy loam with a friable to loose consistency, a subangular blocky parting to crumb structure, and where observed, a gradual and smooth lower boundary. Inclusions within Stratum VI included rootlets (15 percent), insect and worm burrows (5 percent), and Heliodiscus singleyanus (3 percent).

In select excavations in Block D, a seventh horizon was observed (Stratum VII). Stratum VII is a light yellowish brown to very pale brown (10YR 6/4-7/4) fine to very fine silt loam. This horizon extends beyond $330 \mathrm{cmbs}$ (measuring over $130 \mathrm{~cm}$ thick) has a friable consistency and a crumb parting to subangular blocky structure. Observed inclusions included insect burrows (10 percent), Heliodiscus singleyanus ( $<2$ percent) and Rabdotus mooreanus ( $<5$ percent) snail shell, and $\mathrm{CaCO}_{3}$ filaments ( $3-5$ percent). The lower boundary of this horizon was unobserved during data recovery excavations.

\section{Summary of Natural Strata}

In summary, the natural strata (I-VII) observed at 41GU177 suggests some disturbances localized at or near the ground surface. The disturbances are primarily associated with extensive tree root growth, possible earth movement, and erosion. The vertical extent of evident disturbance across the site varied. The excavation areas amongst extensive trees (i.e., BHT05 and Excavation Blocks C and D) had moderate, localized disturbance to approximately $40 \mathrm{cmbs}$. The underlying strata (Strata III-VI) across 41GU177 appears generally intact with some localized disturbance from tree roots and small burrows. Comparing the pedon at $41 \mathrm{GU} 177$ to that of the soil series mapped in the area, the site stratigraphy matches that attributed to the Sunev series. The pedon for the Sunev series is an Ap, A, Bk1, Bk2, and Bk3 (NRCS 2018). A discussion of the cultural stratigraphy at the site is presented in Chapter 6. 


\section{EXCAVATIONS BLOCKS}

As mentioned in Chapter 4, the data recovery excavation units were laid out in six blocks, designated as excavation blocks A-F (see Figure 4.1; Figure 5.4). The excavations included 22 traditional $1 \times 1-\mathrm{m}$ units and six feature-focused units; with a total excavated volume of approximately $34.09 \mathrm{~m}^{3}$ (see Table 4.1). The following sections summarize the results of the investigations within each of the excavation blocks.

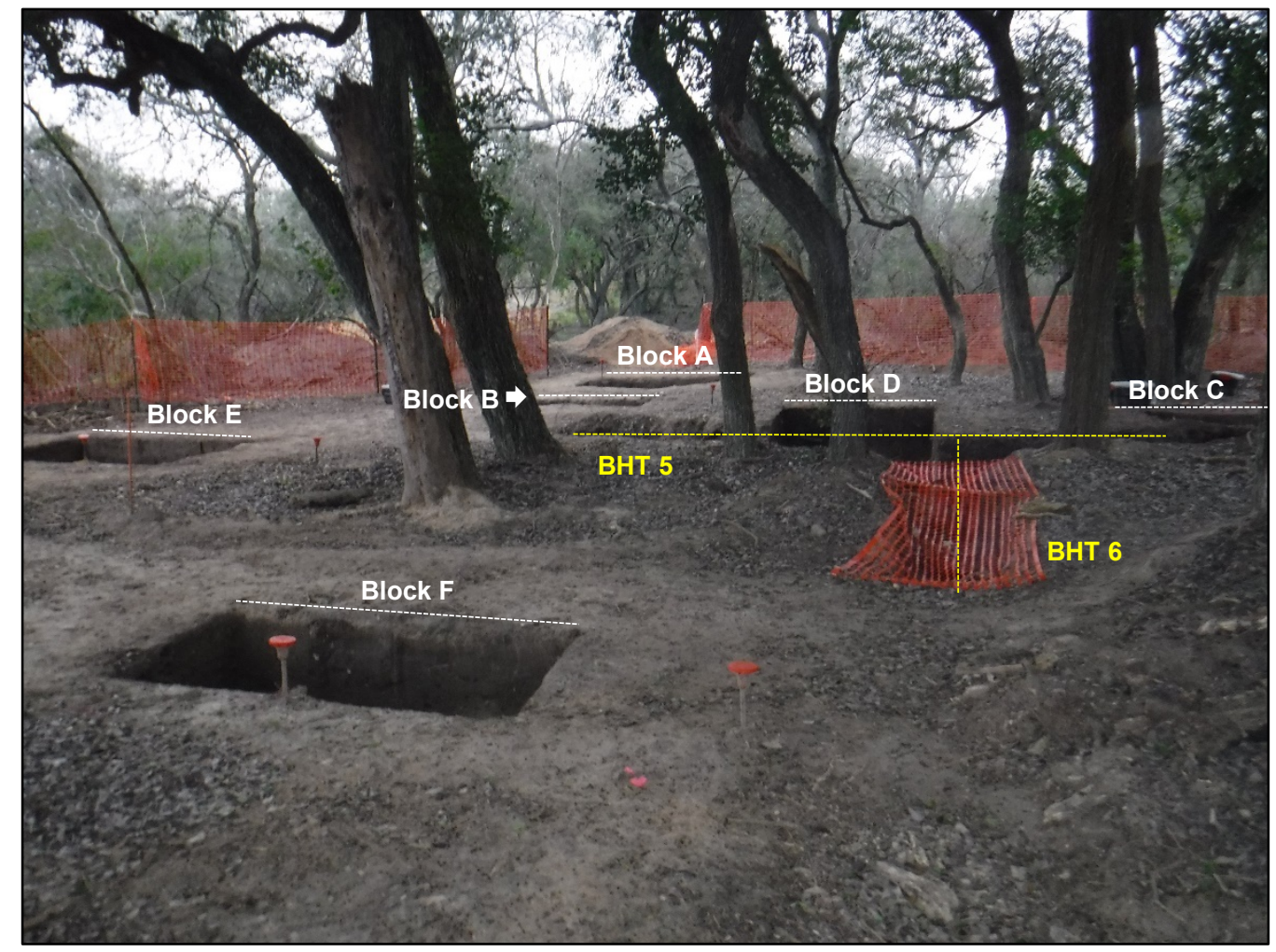

Figure 5.4. Overview of $41 \mathrm{GU} 177$ data recovery excavation blocks, facing south, upslope, away from drainage.

\section{Block A}

Based on the results of the close-interval shovel testing, the southwestern-most portion of the bore pit area was predicted to have the highest artifact density. As such, a block of units was opened up in this area and designated as Block A. Six traditional $1 \times 1-\mathrm{m}$ excavation units were opened in Block A along the N995 and N996 lines at E1012, E1013, and E1014 (see Figure 4.1). Five of the units were excavated in 14 to 15 levels, with all units terminating at $98.2 \mathrm{~m}$, while the sixth unit (N996 E1014) was excavated in 20 levels and terminated at $97.6 \mathrm{~m}$, for a total excavated volume of $9.23 \mathrm{~m}^{3}$ (Figure 5.5).

One feature (Feature 3) was recorded in this block and consisted of two discrete burned chert clusters with an associated diffuse chert scatter. The feature spanned units N995-996 E1013 (Cluster 1) and N996 E1012-1013 (Cluster 2) and is discussed in further detail in Chapter 6. One radiocarbon sample from Feature 3 (Beta 492584) was analyzed and returned a broad calibrated age of 1173-1058 cal B.P. at 68.2 percent probability (Appendix E). No temporally diagnostic artifacts were recovered in association with the feature. 


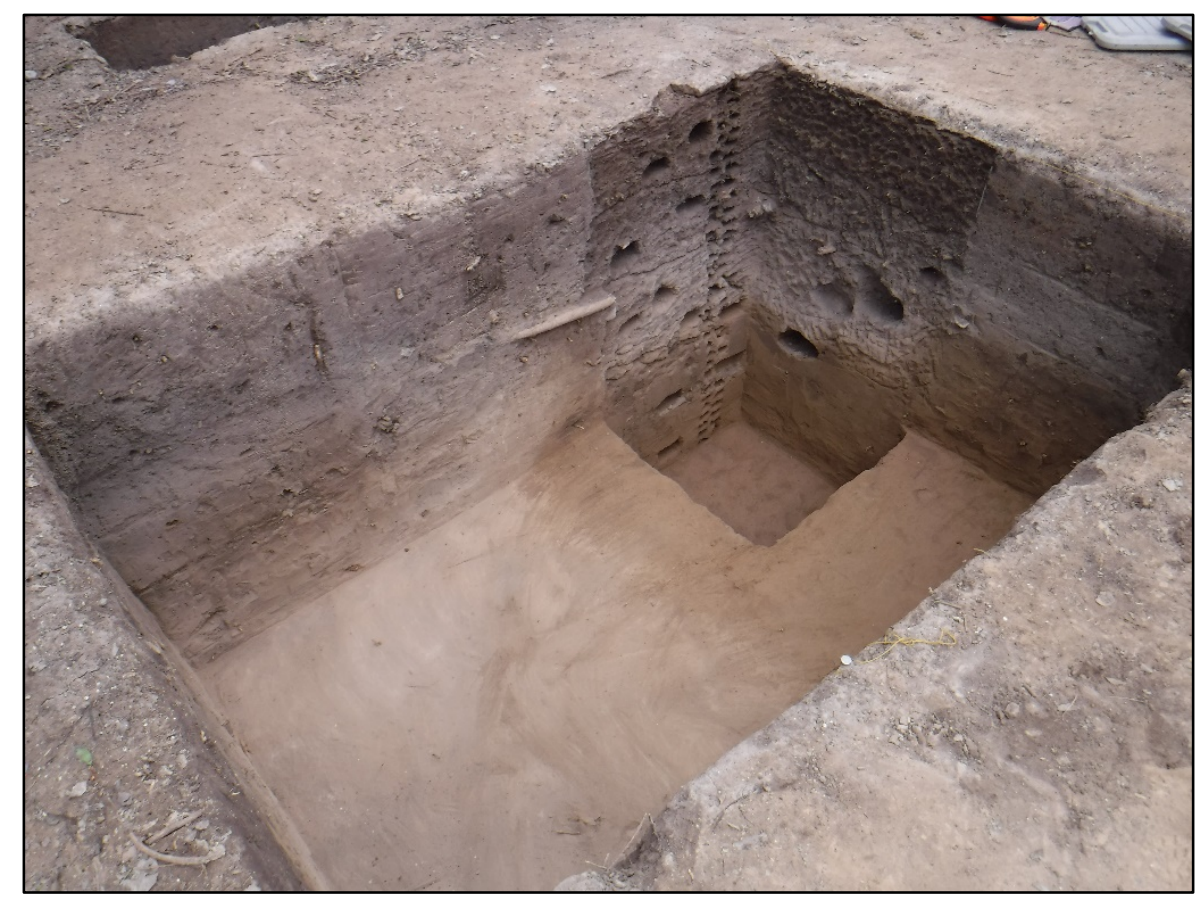

Figure 5.5. Overview of Block $A$ after all units at termination level and geoarchaeological samples collected, facing northeast.

Artifacts encountered in Block A included 13 bifaces, one scraper, eight cores, two core tools, one battered stone, one ground stone, 26 modified flakes, one historical ceramic, 38 clay artifacts (i.e., burned clay), 16 thermally altered stones, over $45 \mathrm{~kg}$ of burned rock, nearly 3,100 pieces of debitage, one shell ornament, 22 unmodified shell, and 164 unmodified animal bones (Table 5.1). In addition, three charcoal samples, two feature matrix samples, and 76 geoarchaeological samples (64 MS and 12 bulk matrix samples) were collected from Block A.

Table 5.1. Block A Artifact Assemblage

\begin{tabular}{lrr}
\hline Artifact Type & Count & Weight (g) \\
\hline Battered Stone & 1 & 183.52 \\
\hline Biface & 13 & 613.11 \\
\hline Bone & 164 & 120.11 \\
\hline Historical Ceramic & 1 & 0.82 \\
\hline Clay Artifact & 38 & 235.24 \\
\hline Core & 8 & 705.11 \\
\hline Core Tool & 2 & 848.72 \\
\hline Debitage & 3,088 & $4,775.35$ \\
\hline Ground stone & 1 & 185.48 \\
\hline Modified Flake & 26 & 730.37 \\
\hline Scraper & 1 & 20.12 \\
\hline Shell & 22 & 84.51 \\
\hline Shell Ornament & 1 & 0.06 \\
\hline Thermally Altered Stone & 16 & 725.44 \\
\hline Total & $\mathbf{3 , 3 8 2}$ & $\mathbf{9 , 2 2 7 . 9 6}$ \\
\hline
\end{tabular}

\begin{tabular}{lrr}
\hline Samples & Count & Weight (g) \\
\hline Charcoal Samples & 3 & 1.54 \\
\hline Feature Matrix & 2 & $7,300.00$ \\
\hline Geoarch MS Samples & 64 & N/A \\
\hline Geoarch Bulk Matrix & 12 & N/A \\
\hline Total & $\mathbf{8 1}$ & $\mathbf{7 , 3 0 1 . 5 4}$ \\
\hline
\end{tabular}

\begin{tabular}{lrr}
\hline Burned Rock Size $\mathbf{( c m )}$ & Count & Weight (kg) \\
\hline $0-5$ & 1549 & 13.65 \\
\hline $5-10$ & 245 & 31.20 \\
\hline $10-15$ & 4 & 1.00 \\
\hline $15+$ & 0 & 0 \\
\hline Total & $\mathbf{1 7 9 8}$ & $\mathbf{4 5 . 8 5}$ \\
\hline
\end{tabular}




\section{Block B}

Two traditional $1 \times 1-\mathrm{m}$ excavation units were opened in Block B along the N999 line at E1013 and E1014 (see Figure 4.1). Both units were excavated in 15 levels, terminating at $98.1 \mathrm{~m}$, for a total excavated volume of $2.93 \mathrm{~m}^{3}$ (Figure 5.6). No features were encountered in this block and no radiocarbon samples were submitted for this area.

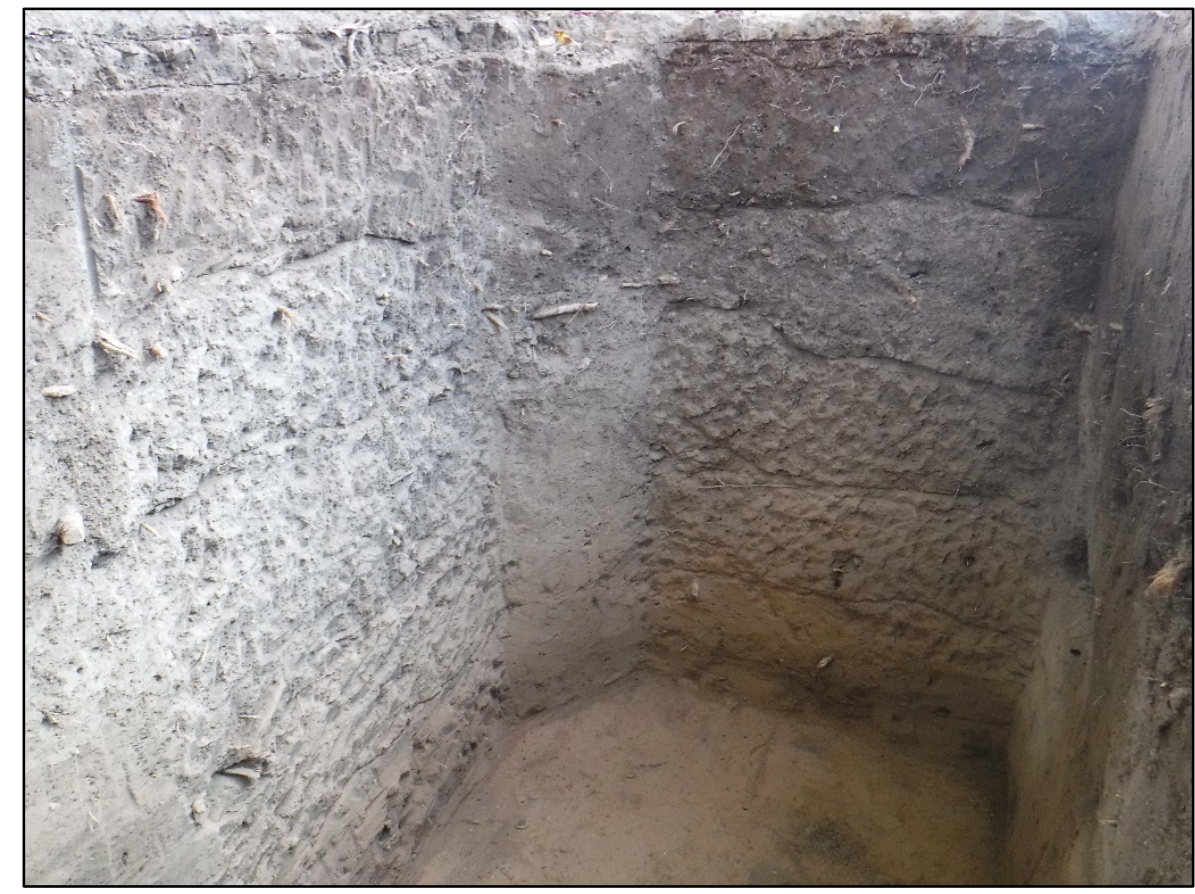

Figure 5.6. Overview of N999 E1014 in Block B at termination level (98.10 $\mathrm{m})$, facing northeast.

Artifact densities in Block B were relatively low compared to the other excavation blocks and included one biface, two modified flakes, 490 pieces of debitage, two unmodified shell, and three unmodified animal bones (Table 5.2). In addition, only $8.3 \mathrm{~kg}$ of burned rock was recovered and no charcoal samples or thermally altered stones were recovered. No geoarchaeological samples were collected from Block B.

Table 5.2. Block B Artifact Assemblage

\begin{tabular}{lrr}
\hline Artifact Type & Count & Weight (g) \\
\hline Biface & 1 & 15.46 \\
\hline Bone & 3 & 2.52 \\
\hline Debitage & 490 & 908.46 \\
\hline Modified Flake & 2 & 77.53 \\
\hline Shell & 2 & 9.86 \\
\hline Total & $\mathbf{4 9 8}$ & $\mathbf{1 , 0 1 3 . 8 3}$ \\
\hline
\end{tabular}

\begin{tabular}{lrr}
\hline Burned Rock Size (cm) & Count & Weight (kg) \\
\hline $0-5$ & 72 & 6.71 \\
\hline $5-10$ & 8 & 1.60 \\
\hline $10-15$ & 0 & 0 \\
\hline $15+$ & 0 & 0 \\
\hline Total & $\mathbf{8 0}$ & $\mathbf{8 . 3 1}$ \\
\hline
\end{tabular}




\section{Block C}

Two traditional $1 \times 1-\mathrm{m}$ excavation units were opened in Block $\mathrm{C}$ along the N1000 line at E1003 and E1004 (see Figure 4.1). One unit (N1000 E1003) was excavated in nine levels, terminating at $98.9 \mathrm{~m}$ and the other unit (N1000 E1004) was excavated in 15 levels terminating at $98.3 \mathrm{~m}$, for a total excavated volume of $4.01 \mathrm{~m}^{3}$. One feature, Feature 4, was recorded in this block. The feature spanned both units and appeared to continue into the northern walls, therefore additional feature-focused units were opened up to fully explore the extent of the Feature 4 . Over the course of the feature excavation, an additional six feature-focused units were opened, including one $50 \times 50-\mathrm{cm}$ unit (N1001 E1004), one $3 / 4$ unit (N1001 E1004.5), two $1 \times 1-\mathrm{m}$ units (N1000.5 E1005.5 and N1001.5 E1004), and two $1 \times 1.5-\mathrm{m}$ units (N1001 E1003 and N1001.5 E1005). The feature-focused units were excavated in three to five levels, and all terminated at $99.3 \mathrm{~m}$ (Figure 5.7).

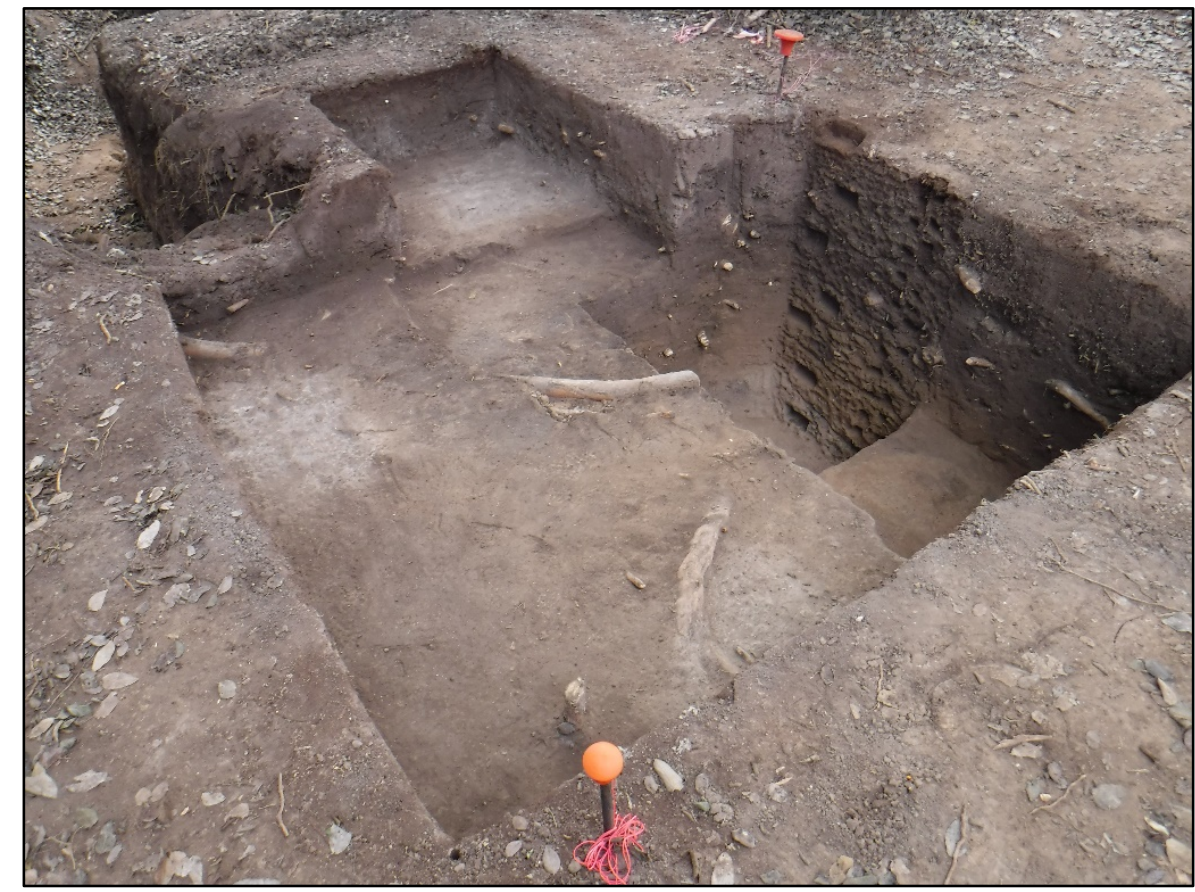

Figure 5.7. Overview of Block $C$ after all units at termination level and geoarchaeological samples collected, facing southeast.

One feature (Feature 4) was recorded in Block $\mathrm{C}$ and consisted of a burned rock earth oven. The feature spanned numerous units, including two traditional $1 \times 1-\mathrm{m}$ units and six non-traditional feature-focused units of various sizes; an explanation of the methodology used for documentation and excavation of this feature was presented in Chapter 4 (see Figure 4.2). Layer 1 of Feature 4 extended into units N1000-1001 E1003-1005 and Layer 2 spanned units N1000-1001 E1004; this feature is discussed in further detail in Chapter 6. One radiocarbon sample from Feature 4 (Beta 492583) was analyzed and returned a calibrated age of 929-802 cal B.P. at 68.2 percent probability (Appendix E). In addition, one temporally diagnostic artifact, a Late Prehistoric Toyah Phase Perdiz Point, was recovered in association with the feature.

Artifacts encountered in Block $\mathrm{C}$ included one arrow point, four bifaces, one core, five modified flakes, eight prehistoric ceramics, one historical ceramic, three historical metal fragments, three pieces of ochre, six clay artifacts (i.e., burned clay), three miscellaneous stone/minerals (i.e., manuports), 14 thermally altered stones, over $52 \mathrm{~kg}$ of burned rock, 871 pieces of debitage, 11 unmodified shell, and 303 unmodified animal bones (Table 5.3). Of note, the majority of the burned rock came from in and around 
Feature 4 and the only large burned rock (i.e., over $15 \mathrm{~cm}$ in diameter) encountered during the data recovery excavations also came from Feature 4. In addition, seven charcoal samples, two feature matrix samples, and 45 geoarchaeological samples (38 MS and seven bulk matrix samples) were collected from Block C.

Table 5.3. Block C Artifact Assemblage

\begin{tabular}{|c|c|c|c|c|c|}
\hline Artifact Type & Count & Weight (g) & Samples & Count & Weight (g) \\
\hline Arrow Point & 1 & 1.43 & Charcoal Samples & 7 & 3.79 \\
\hline Biface & 4 & 93.21 & Feature Matrix & 2 & 5,400 \\
\hline Bone & 303 & 362.59 & Geoarch MS Samples & 38 & $\mathrm{~N} / \mathrm{A}$ \\
\hline Historical Ceramic & 1 & 2.73 & Geoarch Bulk Matrix & 7 & $\mathrm{~N} / \mathrm{A}$ \\
\hline Clay Artifact & 6 & 5.36 & Total & 54 & $5,403.79$ \\
\hline Core & 1 & 188.54 & & & \\
\hline Debitage & 871 & $1,239.18$ & & & \\
\hline Historical Metal & 3 & 1.40 & & & \\
\hline Misc. Stone/ Mineral & 3 & 180.97 & & & \\
\hline Modified Flake & 5 & 105.75 & Burned Rock Size (cm) & Count & Weight (kg) \\
\hline Ochre & 3 & 1.03 & $0-5$ & 530 & 6.35 \\
\hline Prehistoric Ceramic & 8 & 12.39 & $5-10$ & 143 & 16.15 \\
\hline Shell & 11 & 43.58 & $10-15$ & 26 & 14.20 \\
\hline Thermally Altered Stone & 14 & 87.64 & $15+$ & 12 & 15.50 \\
\hline Total & 1,234 & $2,325.80$ & Total & 711 & 52.20 \\
\hline
\end{tabular}

\section{Block D}

Four traditional $1 \times 1-\mathrm{m}$ excavation units were opened in Block D along the N1000 and N1001 lines at E1009 and E1010 (see Figure 4.1). Two of the units were excavated in 10 to 11 levels terminating at $98.59 \mathrm{~m}$ and the other two units were excavated in 20 to 21 levels and terminated at $97.59 \mathrm{~m}$, for a total excavated volume of $6.12 \mathrm{~m}^{3}$ (Figure 5.8). No features were encountered in this block and the radiocarbon sample that was collected was too small to be submitted for analysis. Instead, SWCA submitted a large mammal bone (Beta 492587) from N1001 E1009 Level 5 (99.15 m) for radiocarbon dating; the bone collagen was analyzed and returned a calibrated age of 552-518 cal B.P. at 68.2 percent probability (Figure 5.9; Appendix E).

Artifacts encountered in Block D included three arrow points, seven bifaces, three cores, two ground stone, six modified flakes, three historical ceramics, $23 \mathrm{~kg}$ of burned rock, 1,782 pieces of debitage, four unmodified shell, and 168 unmodified animal bones (Table 5.4). In addition, one charcoal sample and 44 geoarchaeological samples (34 MS and 10 bulk matrix samples) were collected from Block D. 


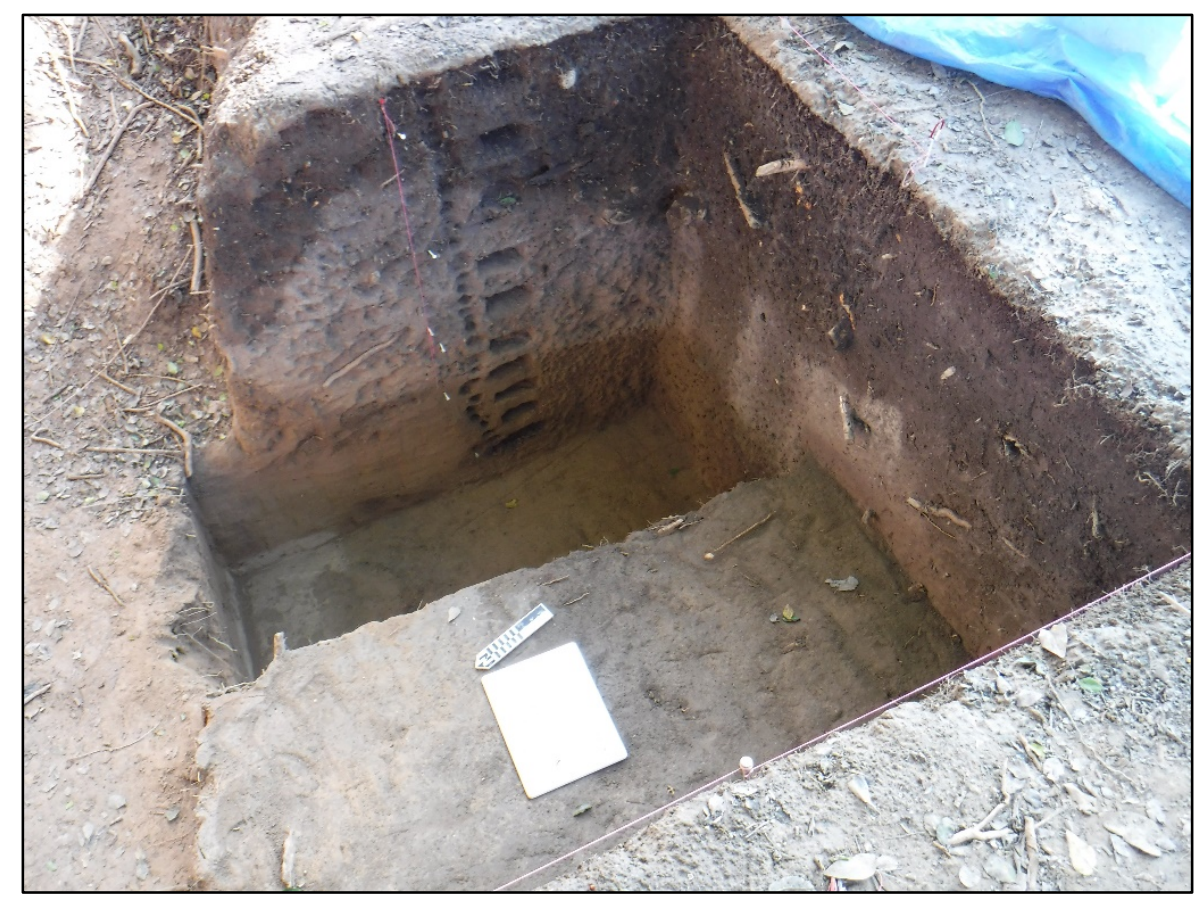

Figure 5.8. Overview of Block $D$ after all units at termination level and geoarchaeological samples collected, facing east.

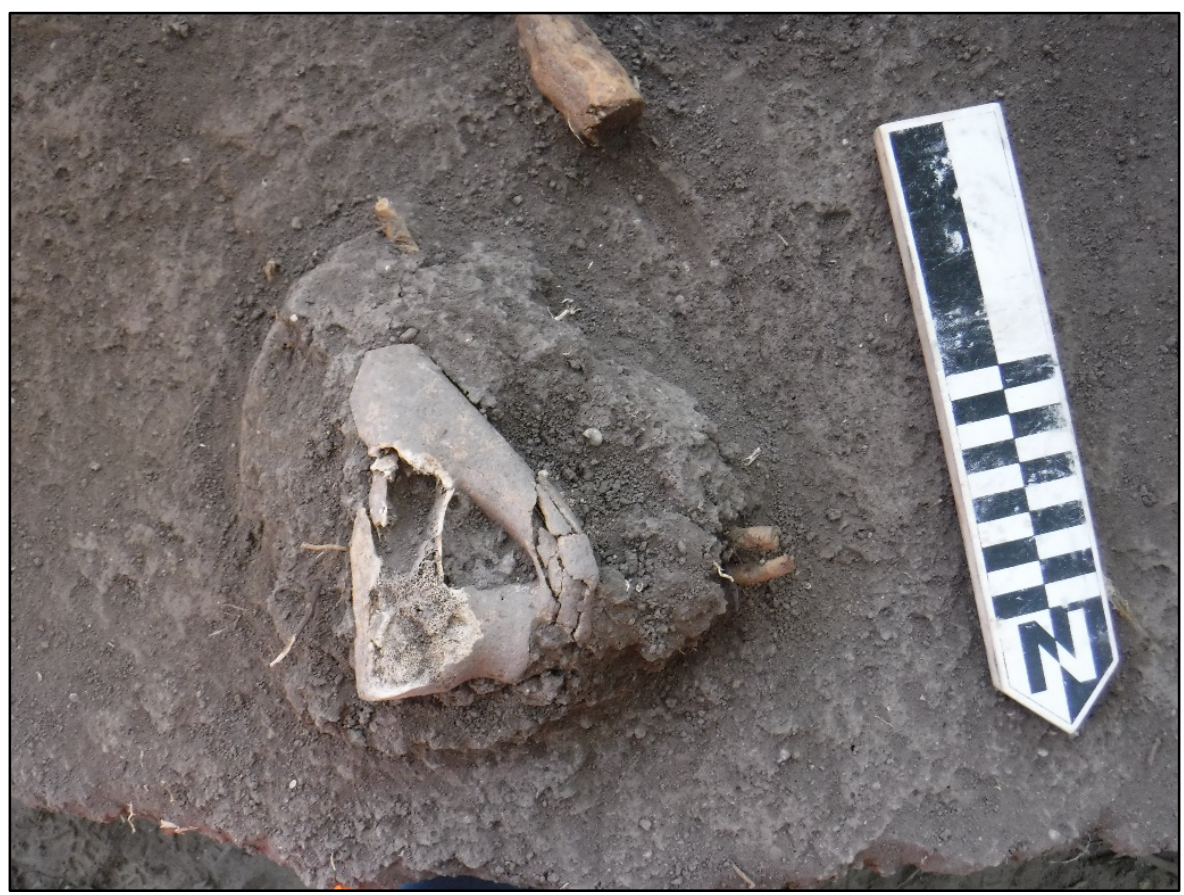

Figure 5.9. Large mammal bone recovered from N1001 E1009, Level 5 at $99.15 \mathrm{~m}$ and submitted for radiocarbon dating (Beta 492587). 
Table 5.4. Block D Artifact Assemblage

\begin{tabular}{lrr}
\hline Artifact Type & Count & Weight (g) \\
\hline Arrow Point & 3 & 2.00 \\
\hline Biface & 7 & 57.91 \\
\hline Bone & 168 & 181.86 \\
\hline Historical Ceramic & 3 & 9.26 \\
\hline Core & 3 & 501.49 \\
\hline Debitage & 1782 & $2,898.36$ \\
\hline Ground stone & 2 & 221.77 \\
\hline Modified Flake & 6 & 323.07 \\
\hline Shell & 4 & 11.67 \\
\hline Total & $\mathbf{1 9 7 8}$ & $\mathbf{4 , 2 0 7 . 3 9}$ \\
\hline
\end{tabular}

\begin{tabular}{lrr}
\hline Samples & Count & Weight (g) \\
\hline Charcoal Samples & 1 & 0.01 \\
\hline Geoarch MS Samples & 34 & N/A \\
\hline Geoarch Bulk Matrix & 10 & N/A \\
\hline Total & $\mathbf{4 5}$ & $\mathbf{0 . 0 1}$ \\
\hline & & \\
\hline Burned Rock Size (cm) & Count & Weight $\mathbf{( k g )}$ \\
\hline $0-5$ & 345 & 12.46 \\
\hline $5-10$ & 56 & 10.15 \\
\hline $10-15$ & 2 & 0.40 \\
\hline $15+$ & 0 & 0 \\
\hline Total & $\mathbf{4 0 3}$ & $\mathbf{2 3 . 0 1}$ \\
\hline
\end{tabular}

\section{Block E}

Six traditional $1 \times 1-\mathrm{m}$ excavation units were opened in Block E along the N1003 and N1004 lines at E1017, E1018, and E1019 (see Figure 4.1). Five of the six units were excavated in 15 levels, terminating at $97.8 \mathrm{~m}$ and the sixth unit (N1004 E1017) was excavated in 16 levels and terminated at $97.7 \mathrm{~m}$, for a total excavated volume of $8.72 \mathrm{~m}^{3}$ (Figure 5.10). Feature 2 was recorded in this block within units N1003 to N1004 and E1018 to E1019.

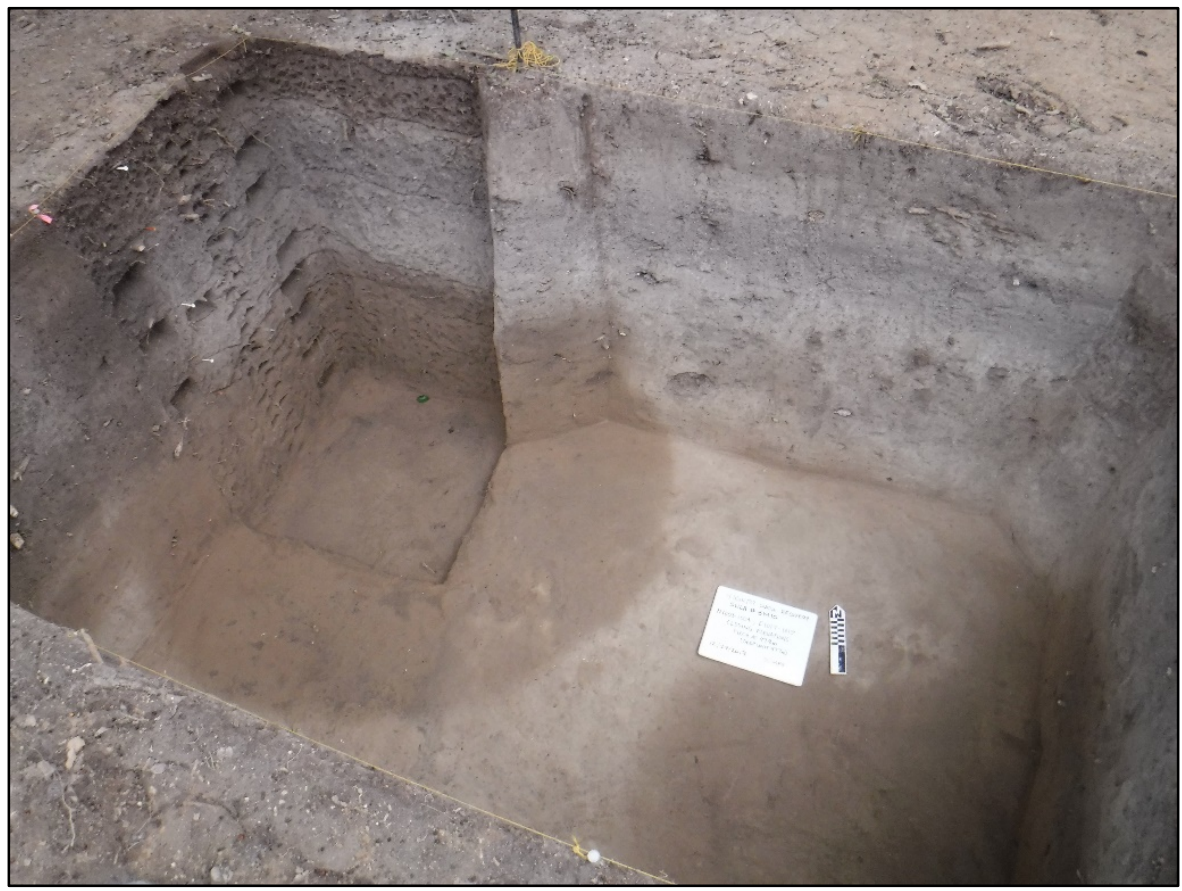

Figure 5.10. Overview of Block $E$ after all units at termination level and geoarchaeological samples collected, facing north. 
One feature (Feature 2) was recorded in Block E and consisted of a small burned rock concentration. The feature spans units N1003-1004 E1018-1019 and is centered just southwest of the N1004 and E1019 grid intersection. One radiocarbon sample obtained from the Feature 2 flotation sample (Beta 492582) was analyzed and returned a broad calibrated age of 670-566 cal B.P. at 68.2 percent probability (Appendix E). A Fresno point was recovered from the screen in Unit N1003 E1018 at the same elevation as the feature and a Zephyr dart point and an Edwards arrow point were recovered in levels below the feature.

Artifacts encountered in Block E included two arrow points, one dart point, 10 bifaces, nine cores, two core tools, 26 modified flakes, one miscellaneous stone/mineral (i.e., manuport), 28 thermally altered stones, $25.5 \mathrm{~kg}$ of burned rock, nearly 2,500 pieces of debitage, 87 unmodified shell, and 19 unmodified animal bones (Table 5.5). In addition, one feature matrix sample and 65 geoarchaeological samples (52 MS and 13 bulk matrix samples) were collected from Block E.

Table 5.5. Block E Artifact Assemblage

\begin{tabular}{lrr}
\hline Artifact Type & Count & Weight (g) \\
\hline Arrow Point & 2 & 4.01 \\
\hline Biface & 10 & 198.12 \\
\hline Bone & 19 & 16.13 \\
\hline Core & 9 & $1,263.20$ \\
\hline Core Tool & 2 & 636.46 \\
\hline Dart Point & 1 & 3.86 \\
\hline Debitage & 2,485 & $4,218.52$ \\
\hline Misc. Stone/ Mineral & 1 & 18.50 \\
\hline Modified Flake & 26 & 621.98 \\
\hline Shell & 87 & 263.39 \\
\hline Thermally Altered Stone & 28 & 8.00 \\
\hline Total & $\mathbf{2 , 6 7 0}$ & $\mathbf{7 , 2 5 2 . 1 7}$ \\
\hline
\end{tabular}

\begin{tabular}{lrr}
\hline Samples & Count & Weight (g) \\
\hline Feature Matrix & 1 & 5,100 \\
\hline Geoarch MS Samples & 52 & N/A \\
\hline Geoarch Bulk Matrix & 13 & N/A \\
\hline Total & $\mathbf{6 6}$ & $\mathbf{5 , 1 0 0}$ \\
\hline
\end{tabular}

\begin{tabular}{lrr}
\hline Burned Rock Size $\mathbf{( c m )}$ & Count & Weight $\mathbf{( k g )}$ \\
\hline $0-5$ & 447 & 11.26 \\
\hline $5-10$ & 124 & 11.97 \\
\hline $10-15$ & 17 & 2.30 \\
\hline $15+$ & 0 & 0 \\
\hline Total & $\mathbf{5 8 8}$ & $\mathbf{2 5 . 5 3}$ \\
\hline
\end{tabular}

\section{Block F}

Two traditional $1 \times 1-\mathrm{m}$ excavation units were opened in Block F along the N1009 line at E1011 and E1012 (see Figure 4.1). The units were excavated in 15 to 16 levels, with all units terminating at $97.7 \mathrm{~m}$, for a total excavated volume of $3.07 \mathrm{~m}^{3}$ (Figure 5.11). No features were encountered in this block and no radiocarbon samples were collected. Instead, SWCA submitted a deer bone (Beta 492588) recovered from N1009 E1012 Level $11(98.19 \mathrm{~m})$ for radiocarbon dating; the bone collagen was analyzed and returned a calibrated age of $282 \mathrm{cal} \mathrm{B.P.} \mathrm{to} \mathrm{the} \mathrm{present} \mathrm{at} 68.2$ percent probability (Appendix E). 


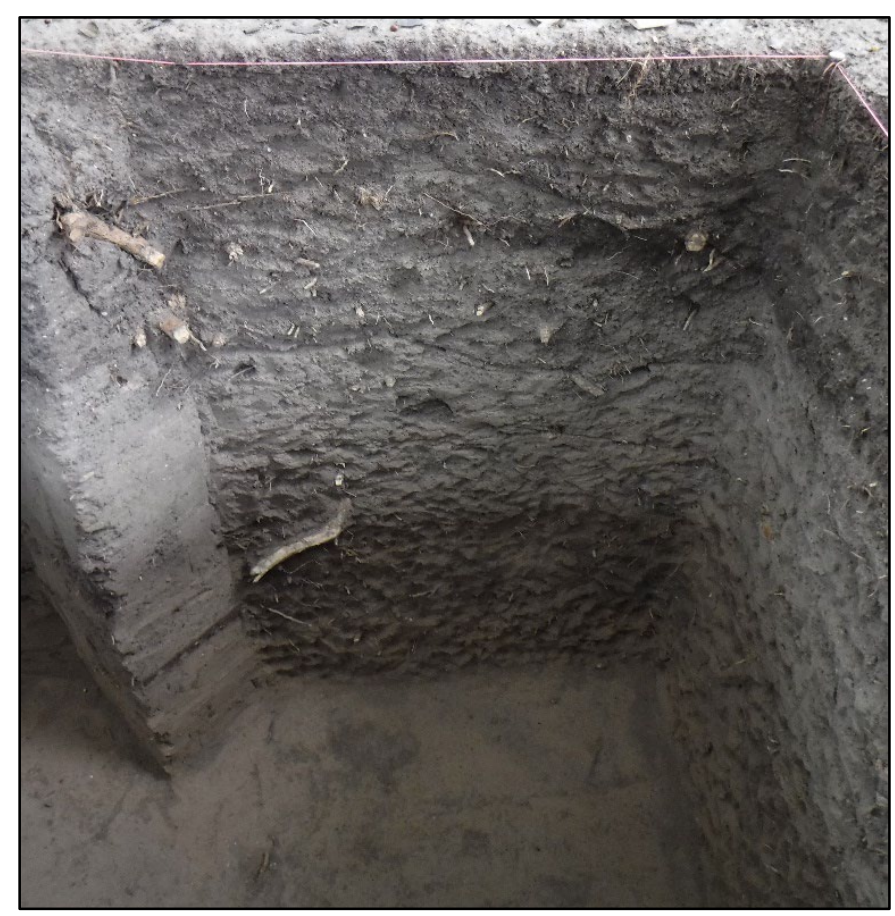

Figure 5.11. Overview of N1009 E1012 in Block F at termination level $(97.70 \mathrm{~m})$, facing north.

Artifacts densities in Block F were the lowest out of all of the excavation blocks and included one core, two modified flakes, one historical metal fragment, one historical glass fragment, only $7.3 \mathrm{~kg}$ of burned rock, 138 pieces of debitage, five unmodified shell, and eight unmodified animal bones (Table 5.6). In addition, SWCA collected 58 geoarchaeological samples from Block F, including 49 MS and nine bulk matrix samples.

Table 5.6. Block F Artifact Assemblage

\begin{tabular}{lrr}
\hline Artifact Type & Count & Weight (g) \\
\hline Bone & 8 & 21.47 \\
\hline Core & 1 & 18.02 \\
\hline Debitage & 138 & 348.56 \\
\hline Glass & 1 & 0.79 \\
\hline Metal & 1 & 0.64 \\
\hline Modified Flake & 2 & 40.71 \\
\hline Shell & 5 & 8.17 \\
\hline Total & $\mathbf{1 5 6}$ & $\mathbf{4 3 8 . 3 6}$ \\
\hline
\end{tabular}

\begin{tabular}{lrr}
\hline Samples & Count & \multicolumn{2}{r}{ Weight (g) } \\
\hline Geoarch MS Samples & 49 & N/A \\
\hline Geoarch Bulk Matrix & 9 & N/A \\
\hline Total & $\mathbf{5 8}$ & N/A \\
\hline \multicolumn{1}{l}{ Burned Rock Size (cm) } & Count & Weight $\mathbf{( k g )}$ \\
\hline $0-5$ & 91 & 5.4 \\
\hline $5-10$ & 6 & 1.9 \\
\hline $10-15$ & 0 & 0 \\
\hline $15+$ & 0 & 0 \\
\hline Total & $\mathbf{9 7}$ & $\mathbf{7 . 3}$ \\
\hline
\end{tabular}




\section{SITE FORMATION ANALYSES}

This section summarizes the results of radiocarbon, particle size distribution, and MS analyses conducted at $41 \mathrm{GU} 177$.

\section{Radiocarbon Analysis}

As stated previously, SWCA collected 11 samples of charred material during the investigations at the Snakeskin Bluff site. Of these, five samples were sent to Beta Analytic Inc. to conduct radiocarbon dating analysis (Table 5.7; Appendix E). These assays were selected based upon the combination of cultural context (e.g., feature matrix) and stratigraphic location. These analyses focused on the stratigraphy and cultural zones seemingly affiliated with the Late Prehistoric or Archaic horizons. Specifically, radiocarbon analyses were conducted on samples from Feature 2 (Beta-492582), Feature 3 (Beta-492584), Feature 4 (Beta-492583), a faunal fragment in Unit N1001 E1009 (Beta-492587), and a faunal fragment in Unit N1009 E1012 (Beta-492588) (see Figure 4.1). Each of these five assays are discussed below.

The results of the radiocarbon analyses from the charcoal sample in Feature 2 returned a broad calibrated age of 670-566 cal B.P. at 68.2 percent probability (see Table 5.7). The broad range of the radiocarbon results is due to the sample's position on the calibration curve. Specifically, the sample falls on two peaks in the curve and the bimodal range of the results cluster at 670-645 cal B.P. (38.6\% certainty) or 586-566 cal B.P. (29.6\% certainty). Chronologically, this places the Feature 2 sample collected at $98.77-98.5 \mathrm{~m}$ elevation in the Late Prehistoric. Also, the radiocarbon sample was identified as charred vetch (Vicia ludoviciana) with a $\delta^{13} \mathrm{C}$ value of -22.3 .

The results of the radiocarbon analyses from the charcoal sample in Feature 3 returned a broad calibrated age of 1173-1058 cal B.P. at 68.2 percent probability (see Table 5.7). The broad range of the radiocarbon results is due to the sample's position on the calibration curve. Specifically, the sample falls on several peaks in the curve and the multimodal range of the results cluster at $1149-1058$ cal B.P. (57.1\% certainty) or $1173-1157$ cal B.P. (11.1\% certainty). Chronologically, this places the Feature 3 sample collected at 99.0-98.94 m elevation in the Late Prehistoric. Notably, the radiocarbon sample was identified as charred bluewood condalia (Condalia hookeri) with a $\delta^{13} \mathrm{C}$ value of -25.2 .

The results of the radiocarbon analyses from the charcoal sample in Feature 4 returned a calibrated age of 929-802 cal B.P. at 68.2 percent probability (see Table 5.7). The sample results fall on several peaks in the curve and the multimodal range of the results cluster at 862-828 cal B.P. (31.4\% certainty), 929-902 cal B.P. (28.3\% certainty), or $812-802$ cal B.P. (8.4\% certainty). Chronologically, this places the Feature 4 sample collected at 99.3-99.21 m elevation in the Late Prehistoric. The radiocarbon sample was identified as charred camas bulb (Camassia sp.) with a $\delta^{13} \mathrm{C}$ value of -25.5 .

The results of the radiocarbon analyses of collagen from a faunal fragment in Unit N1001 E1009 returned a calibrated age of 552-518 cal B.P. at 68.2 percent probability (see Table 5.7). Chronologically, this places the mammal bone fragment collected at $99.15 \mathrm{~m}$ elevation in the Late Prehistoric, more specifically the Toyah phase. The radiocarbon sample had a $\delta^{13} \mathrm{C}$ value of -11.1 . 
Table 5.7. Radiocarbon Assays

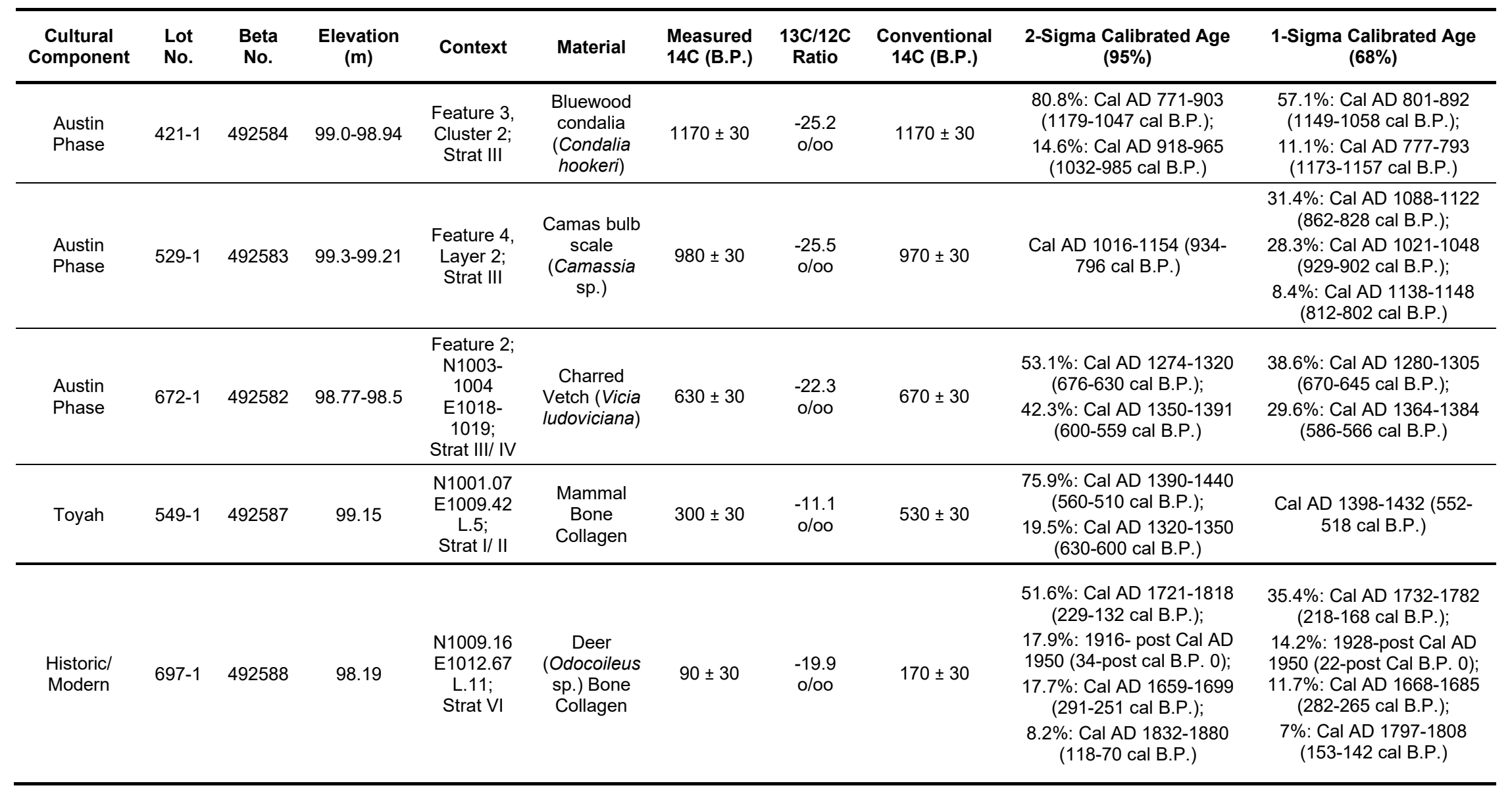


Finally, the results of the radiocarbon analyses of collagen from a faunal fragment in Unit N1009 E1012 returned a calibrated age of $282 \mathrm{cal}$ B.P. to the present at 68.2 percent probability (see Table 5.7). The broad range of the radiocarbon results for this sample is due to the sample's position on a dynamic portion of the calibration curve. Specifically, the sample falls on several peaks in the curve and the multimodal range of the results cluster at $218-168$ cal B.P. (35.4\% certainty), 22 cal B.P. to present (14.2\% certainty), 282-265 cal B.P. (11.7\% certainty), or $153-142$ cal B.P. (7\% certainty). Chronologically, this places the mammal bone fragment collected at $98.19 \mathrm{~m}$ elevation in the historic to modern era. The radiocarbon sample was identified as deer (Odocoileus sp.) with a $\delta^{13} \mathrm{C}$ value of -19.9.

Considering the overall radiocarbon results, the chronometric data indicate prehistoric occupations from the Late Prehistoric to modern times. However, comparing the chronometric results stratigraphically across the site, the results are seemingly not in an appropriate sequence. The youngest assay (282 cal B.P. to the present) came from Unit N1009 E1012 (Beta-492588) at an elevation of $98.19 \mathrm{~m}$ (see Table 5.7). Although downslope from the other radiocarbon samples, this assay is noticeably lower than the other chronometric data (see Figures 5.2 and 5.3). Additionally, the oldest assay (1173-1058 cal B.P.) recovered from the site is from Feature 3 (Beta-492584) at an elevation of 99.0-98.94 $\mathrm{m}$. The dissonance in chronostratigraphy for these results appears to suggest some translocation (i.e., vertical displacement), contamination, or a combination of factors. Therefore, the radiocarbon sample from Unit N1009 E1012 (Beta-492588) is rejected as the results do not correlate with the other radiocarbon data or with the cultural assemblage at the site.

For the site's chronostratigraphy, the remaining four radiocarbon assays are associated with Strata II and III. Stratum II only contains the faunal fragment in Unit N1001 E1009 that returned a calibrated age of 552-518 cal B.P. (see Table 5.7). Stratum III contains the samples from the three features (i.e., Features 2-4) dating at 1173-1058 cal B.P. in Feature 3 (Beta-492584), 929-802 cal B.P. in Feature 4 (Beta492583), and 670-566 cal B.P. in Feature 2 (Beta-492582). Accordingly, Stratum III appears to date to approximately 1200-600 B.P., while the overlying Stratum II dates from 600 B.P. to sometime before the present. Both of these strata are associated with the Late Prehistoric, but there appears to be a cultural distinction. Based solely on the radiocarbon data, Stratum III may be associated with the Austin Phase, whereas Stratum II appears to be associated with the Toyah Phase (Collins 2004; Gadus et al. 2006; Johnson 1994; Johnson and Goode 1994; Ricklis and Collins 1995). Accordingly, the deposits underlying Stratum III precede the Late Prehistoric Austin Phase and certainly encompass the Late Archaic.

\section{Particle Size Distribution}

Particle size and texture analysis was conducted on samples from two excavation blocks (Excavation Block A and E). These columns, near the southern and northern areas of the excavation, were selected to determine if any evident differences were present. The data generally indicate both columns are very consistent. The percentages of very coarse and coarse sand decreases with depth, the percentages of medium and fine sand increases, and fine sand remains relatively consistent (Figures 5.12 and 5.13; Appendix G1). Interestingly, the silt and clay particle size percentages steadily decrease with depth in each profile. This coarsening upward sequence in the particle size distribution is not what is commonly associated with a fluvial depositional environment. Rather, a fining upward sequence where larger clast particles increase with depth is to be expected in alluvial overbank flooding environments (Reading 1996; Reineck and Singh 1975). A generally coarsening upward sequence is typically associated with a regression or drop in water environments (e.g., sea level or coastal areas). The coarsening upward sequence at $41 \mathrm{GU} 177$ may be attributed to sediment contributions from upslope (i.e., colluvium) particularly for the upper three horizons (Strata I-III). Overall, these results do not indicate any dramatic natural or cultural disconformities. 


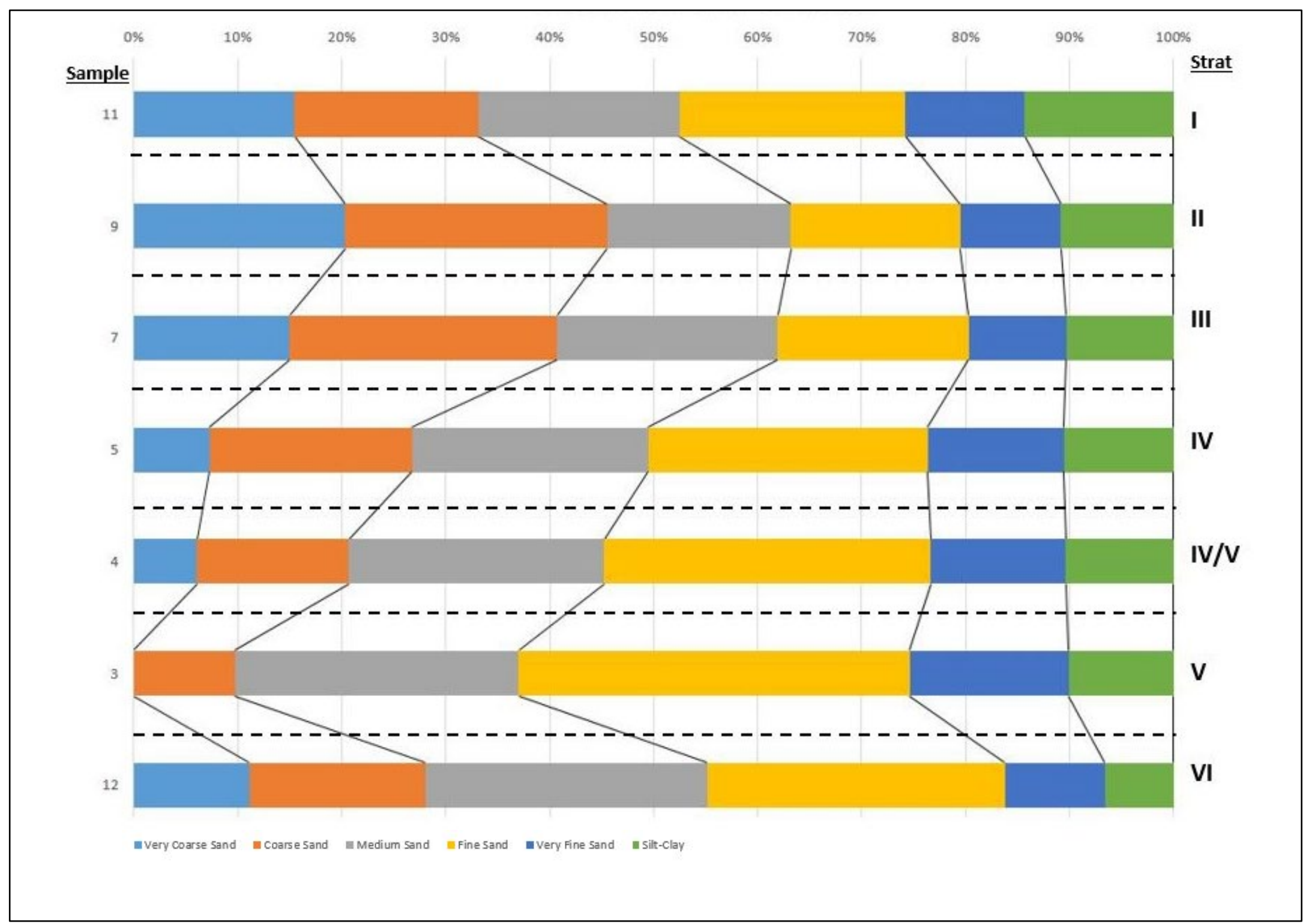

Figure 5.12. Unit N996 E1014 Particle Size distribution. 


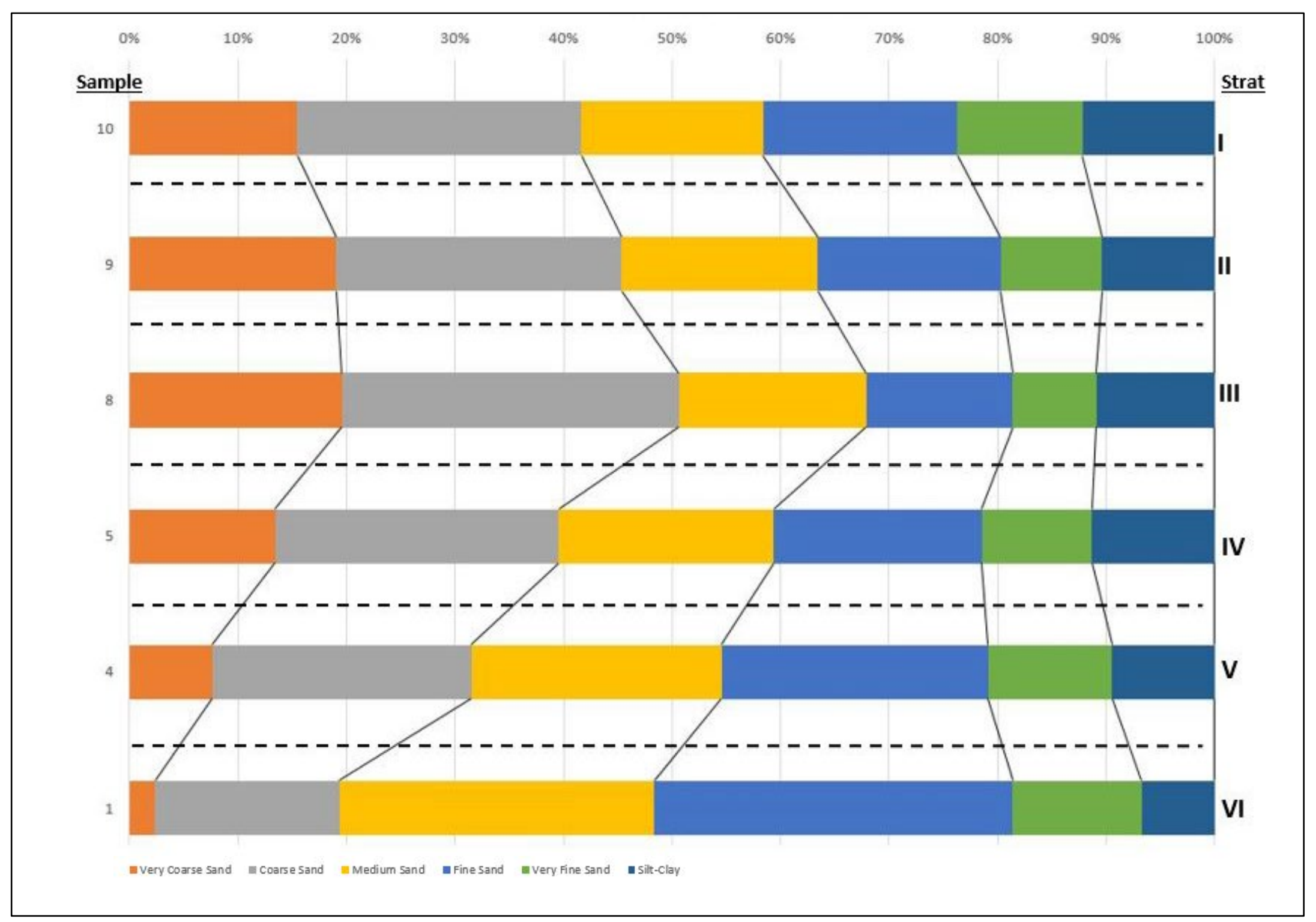

Figure 5.13. Unit N1004 E1017 Particle Size distribution. 


\section{Magnetic Susceptibility (MS)}

SWCA collected five vertical cube columns and one horizontal column for MS analysis during the investigations at 41GU177. The vertical columns included N996 E1014 (Block A), N1000 E1004 (Block C), N1000 E1010 (Block D), N1004 E1017 (Block E), and N1009 E1012 (Block F), while the horizontal column was centered on Feature 4 (Block C). As mentioned briefly in the methods section, factors including organic content, pedogenesis, thermal alteration, and cultural activities (e.g., ash-charcoal and refuse) can subsequently alter (usually increase) susceptibility values. In archaeological contexts, particularly when examining vertical values, the MS analysis can assist in identifying cultural zones or disturbance.

\section{N996 E1014 (Block A)}

The results for the vertical column in Block A exhibits elevated $\chi$ lf values in Strata II-IV with a gradual decrease in values down profile (Figure 5.14 MS N996 E1014; Appendix G2). The elevated values coincide with the observed cultural materials in this excavation block. There are several subtle spikes in value exhibited at 98.54-98.5 m elevation (Stratum IV) and at 98.38-98.31 m elevation (Stratum V), which may correlate with discrete cultural activities. Interestingly at the base of the vertical column in Stratum VI, the $\chi \mathrm{lf}$ values exhibit a gradual increase after a steady decline in values (see Figure 5.14). Extensive burrows were observed in Stratum VI that may account for the increase, but it is also possible that the increase in this horizon may be attributed to another zone of cultural activities.

\section{N1000 E1004 (Block C)}

The results for the vertical column in Block C exhibits elevated $\chi$ lf values in Strata II and IV with an overall gradual decrease in values down profile (Figure 5.15; Appendix G2). There is a noticeable spike in values at the transition from Stratum I to Stratum II, which may indicate a disconformity. This spike is likely the result of disturbance near the ground surface. There is an evident spike in value exhibited at 99.15-99.10 m elevation (Stratum IV), which may correlate with discrete cultural activities associated with Feature 4 (discussed further below). The decrease in $\chi \mathrm{lf}$ values in Strata III is notable as cultural materials were present in this horizon. This incongruent data between the low values with cultural materials may indicate that the cultural activities in this area in Stratum III were not associated with thermal activities. Overall, the results in the vertical column show minor fluctuations associated with cultural activities down profile with a gradual and steady decrease in values. With the exception of the possible disconformity at the base of Stratum I, this is what would be expected in a natural soil cycle.

\section{N1000 E1010 (Block D)}

The results for the vertical column in Block D exhibits elevated $\chi$ lf values particularly in Strata I-III, with an overall gradual decrease in values down profile (Figure 5.16; Appendix G2). The elevated values in Strata I/II is close to the surface and may be attributed to disturbance in the upper $35 \mathrm{cmbs}$. In Stratum III, there is a large increase in $\chi \mathrm{lf}$ values beginning around $98.70 \mathrm{~m}$ elevation that drops at the base of the horizon around $98.53 \mathrm{~m}$ elevation. This prominent Stratum III spike likely represents intensive cultural activities possibly associated with thermal activities. Notably, no cultural features were observed at these elevations in Block D. Overall, the results in the vertical column shows minor fluctuations associated with cultural activities down profile with a gradual and steady decrease in values that would be expected in a natural soil cycle. 


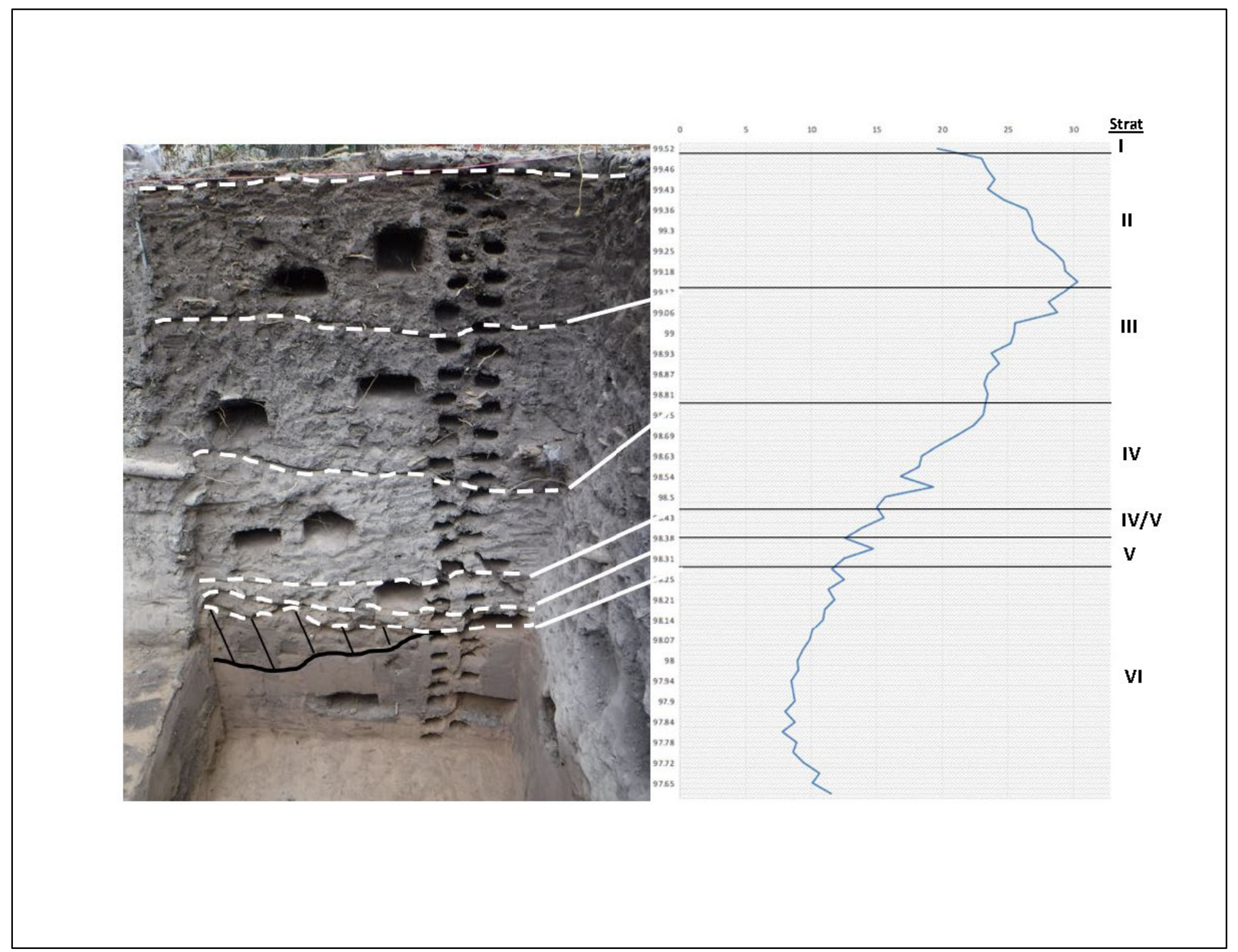

Figure 5.14. Unit N996 E1014 profile annotation with magnetic susceptibility results. 


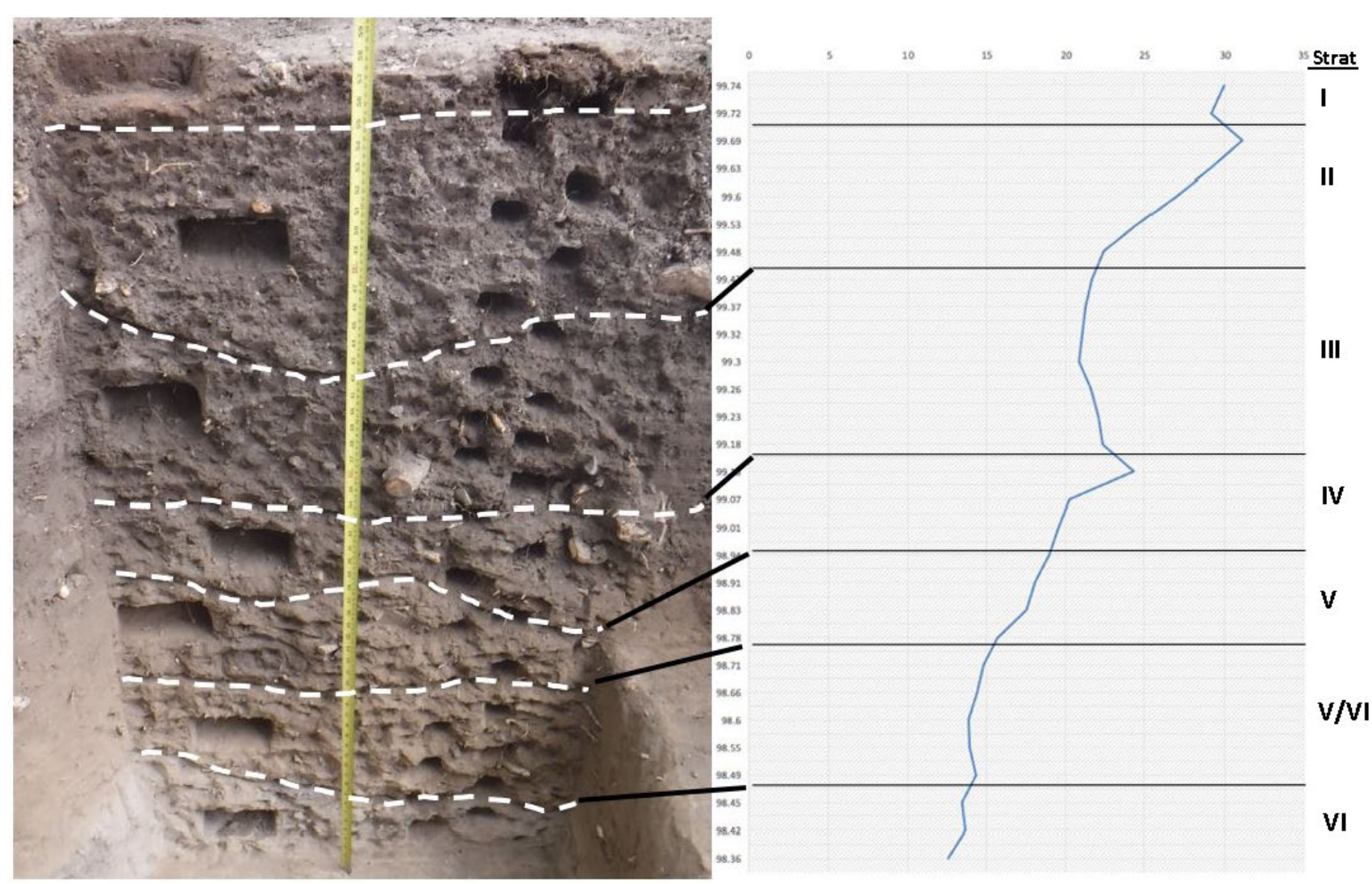

Figure 5.15. Unit N1000 E1004 profile annotation with magnetic susceptibility results. 


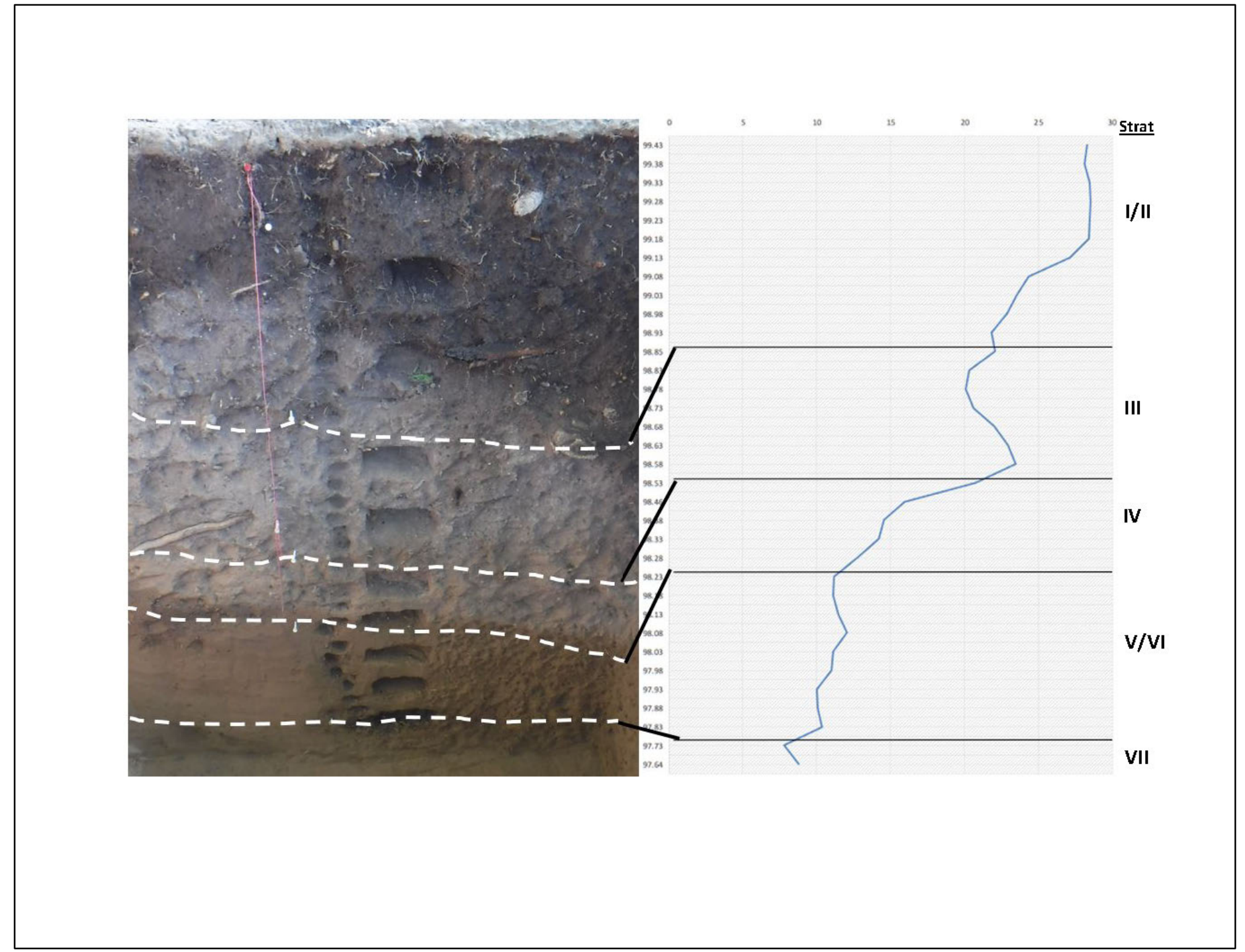

Figure 5.16. Unit N1000 E1010 profile annotation with magnetic susceptibility results. 


\section{N1004 E1017 (Block E)}

The results for the vertical column in Block E exhibits $\chi$ lf values slightly different than the other vertical columns. Specifically, there is an apparent disconformity between Stratum II and Stratum III based on the sharp increase between the two strata (Figure 5.17; Appendix G2). Block E is in an area noted during test excavations as having had some disturbance from heavy equipment (Appendix A; Rodriguez et al. 2017). Therefore, the disconformity in $\chi 1 \mathrm{ff}$ values is attributed to this disturbance. Overall, the results in the vertical column shows minor fluctuations associated with cultural activities down profile with a gradual and steady decrease in values. With the exception of the disconformity at the base of Stratum II, this is what would be expected in a natural soil cycle.

\section{N1009 E1012 (Block F)}

The results for the vertical column in Block F exhibits elevated $\chi$ lf values particularly in Strata III-IV with an overall gradual decrease in values down profile (Figure 5.18; Appendix G2). There are some noticeable fluctuations in the upper Strata I and II close to the ground surface and may be attributed to disturbance in the upper $25 \mathrm{cmbs}$. In Stratum III, there is a large increase in $\chi$ lf values beginning around $98.80 \mathrm{~m}$ elevation that drops at the base of the horizon around $98.67 \mathrm{~m}$ elevation. In Stratum IV there is another increase in $\chi$ lf values between $98.64-98.55 \mathrm{~m}$ elevations. These prominent spikes likely represent intensive cultural activities possibly associated with thermal activities. Notably, no cultural features were observed at these elevations in Block F. Overall, the results in the vertical column show minor fluctuations associated with cultural activities down profile with a gradual and steady decrease in values that would be expected in a natural soil cycle.

\section{Feature 4 (Block C)}

Feature 4 in Block $\mathrm{C}$ was investigated with a horizontal MS sampling strategy. The samples were arranged along a cruciform pattern with a row of samples collected west to east along N1001.1 beginning at E1003.1 eastward to E1006.5, while a row of samples were collected south to north along E1004.9 beginning at N1000.3 northward to N1002.7 (Figure 5.19). The sample cruciform was centered on the feature at N1001.1 E1004.9. The samples were collected horizontally every $20 \mathrm{~cm}$ with two series of sampling (i.e., A and B series). The A series collected samples were collected at the surface depth of the feature (approximately 99.34 m elevation), while the B series were collected $4 \mathrm{~cm}$ below the A series (approximately $99.3 \mathrm{~m}$ elevation). These investigations were conducted in an attempt to identify isolated areas of $\chi \mathrm{lf}$ where there was an evident increase or decrease in values. Notably, due to large burned rocks at specific locations, several samples in the series were not collected. The west to east samples were the most complete only missing three samples in the B series, while the south to north column is unfortunately missing three samples in the A series and nine samples were not collected in the B series.

Despite these gaps in the horizontal MS data, the results are informative. The west to east sample column along N1001.1 starts outside of the observed burned rock feature with elevated $\chi$ lf values followed by a sharp drop in values around E1003.5 to E1004.1 (Figure 5.20; Appendix G3). Beginning around E1004.3 at the edge of the burned rock, the $\chi$ lf values dramatically increases and remains elevated eastward through the feature to the end of the sampling column. Interestingly, the trend in $\chi$ lf values was the same for the A and B series. The drop in $\chi \mathrm{lf}$ values exhibited in the west to east sample column is interpreted to be evidence of a clean out activity of the feature.

The $\chi \mathrm{lf}$ results for the south to north sample column along E1004.9 are less informative than the west to east column. The $\chi \mathrm{lf}$ values are elevated and exhibit some variation, but only within a narrow range $(<3$ $\chi \mathrm{lf})$. Further, the values trend consistently between the A and B series (Figure 5.21; Appendix G3). These data do not indicate any isolated areas of $\chi \mathrm{lf}$ increases or decreases, but rather a consistent influence from 


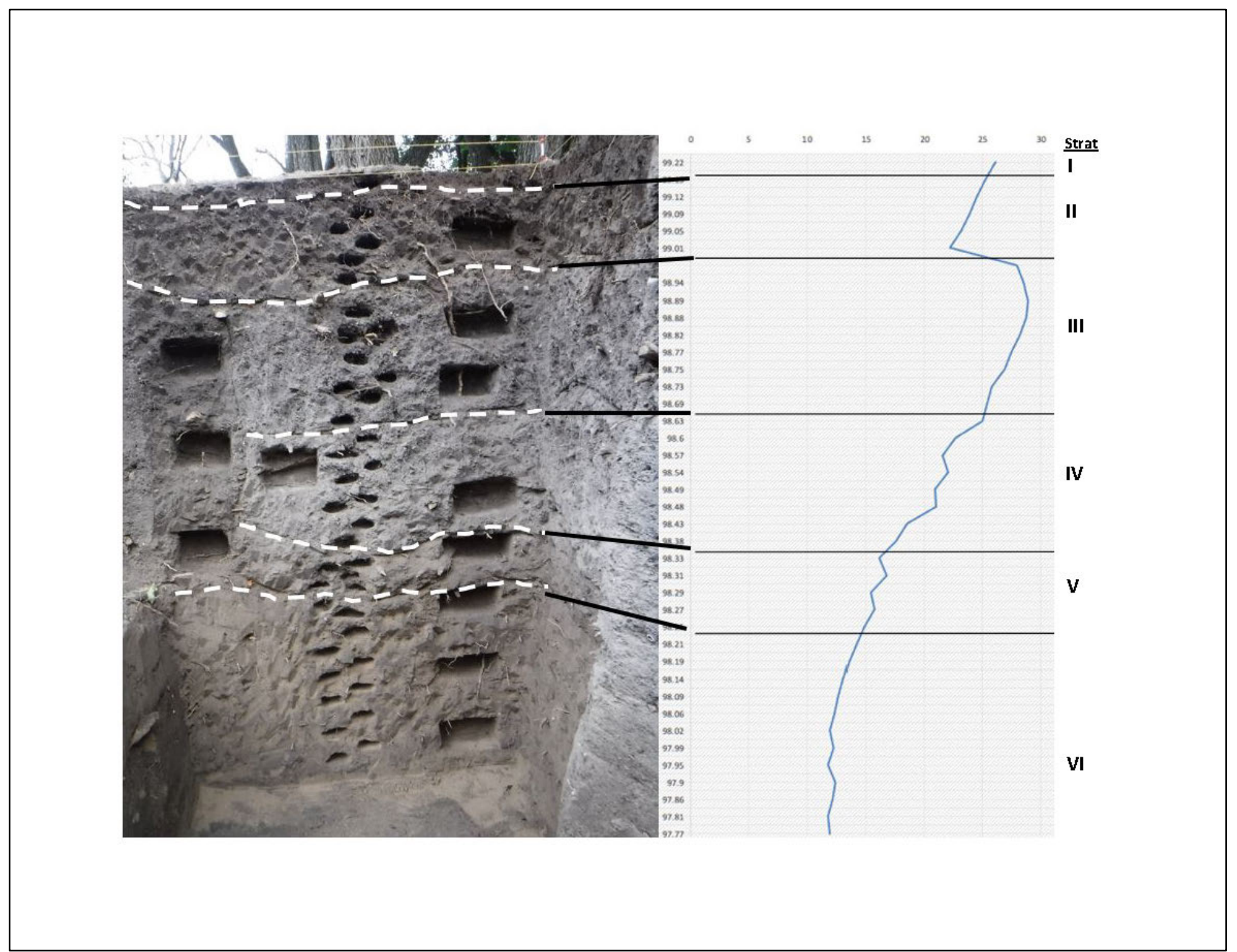

Figure 5.17. Unit N1004 E1017 profile annotation with magnetic susceptibility results. 


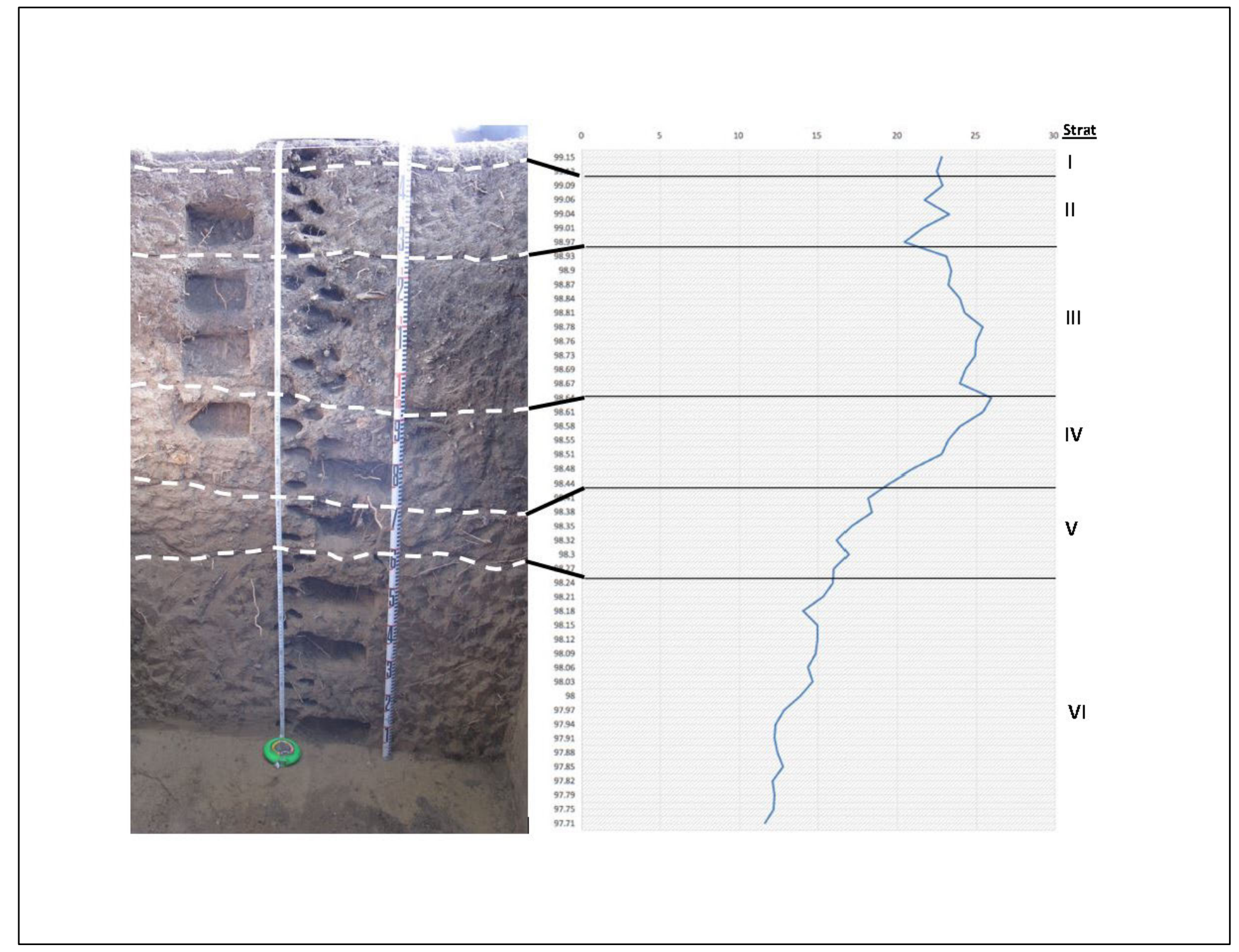

Figure 5.18. Unit N1009 E1012 profile annotation with magnetic susceptibility results. 


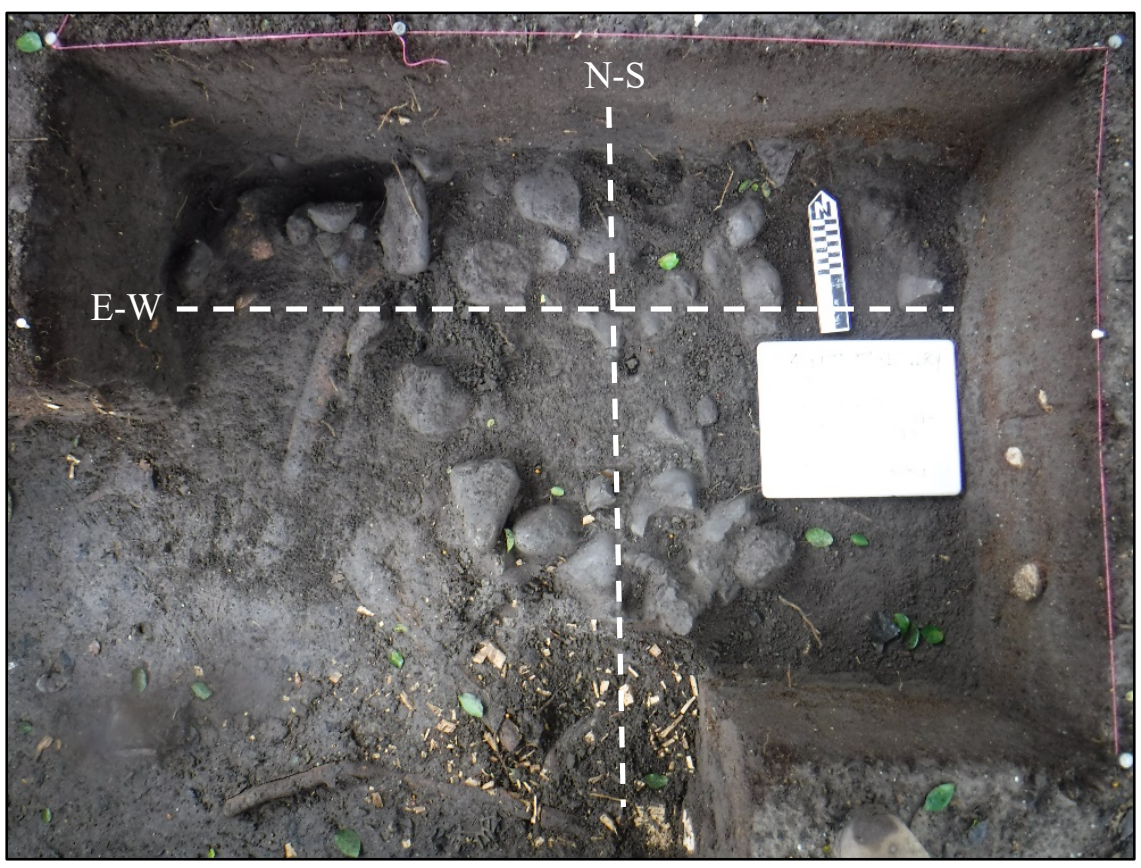

Figure 19. Feature 4 magnetic susceptibility sample cruciform.

thermal activities. The missing data points in the south to north series may have exhibited drastically different values than the available data set. Regardless, the south to north series indicates consistent elevated $\chi$ lf values that are interpreted to be influenced from thermal alteration. The widespread consistency of these values over this large area (about $2.4 \mathrm{~m}$ wide), may be inferred that the deposits were mixed from repeated use. The evidence of the feature clean out along the west to east line is consistent with this interpretation.

\section{Site Formation Analysis Summary}

Considering the combined results of radiocarbon, particle size distribution, and MS analyses at 41GU177, the data indicate generally intact alluvial deposits with some limited disturbance. The site deposits have been subjected to disturbance from bioturbation (e.g., burrows and tree roots) that have, to varying degrees, vertically displaced some deposits. Further, localized disturbance from heavy equipment or similar influence has affected the upper deposits on the eastern side of the site. Specifically, the MS results for the upper strata in the vertical columns at Excavation Blocks D, E, and F all suggested some disturbance, particularly within Excavation Block E (see Figure 5.17). The radiocarbon results also implied some slight mixing based on the dissonance in the stratigraphy and chronometric data. The youngest assay (282 cal B.P. to the present) came from Unit N1009 E1012 (Beta-492588) at an elevation of $98.19 \mathrm{~m}$, which was the deepest of the samples. This radiocarbon sample was rejected for interpretation of the chronostratigraphy at $41 \mathrm{GU} 177$. The remaining four radiocarbon samples do align stratigraphically and indicate that Stratum III appears associated with the Austin Phase, while Stratum II appears to be associated with the Toyah Phase. The deposits underlying Stratum III precede the Late Prehistoric Austin Phase and encompass the Late Archaic and possibly earlier. 


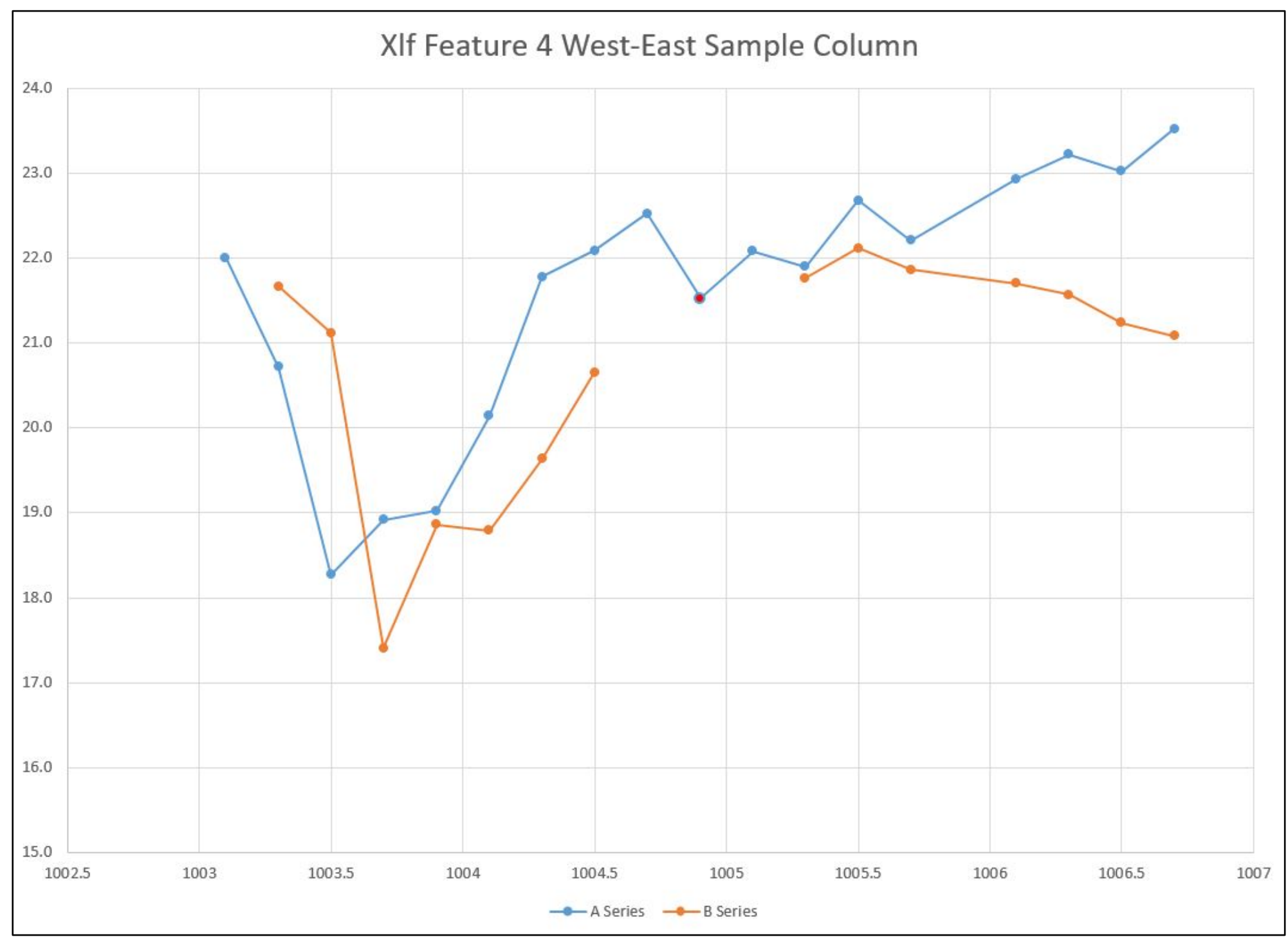

Figure 5.20. Feature $4 \chi$ lf magnetic susceptibility results from west to east sample column. 


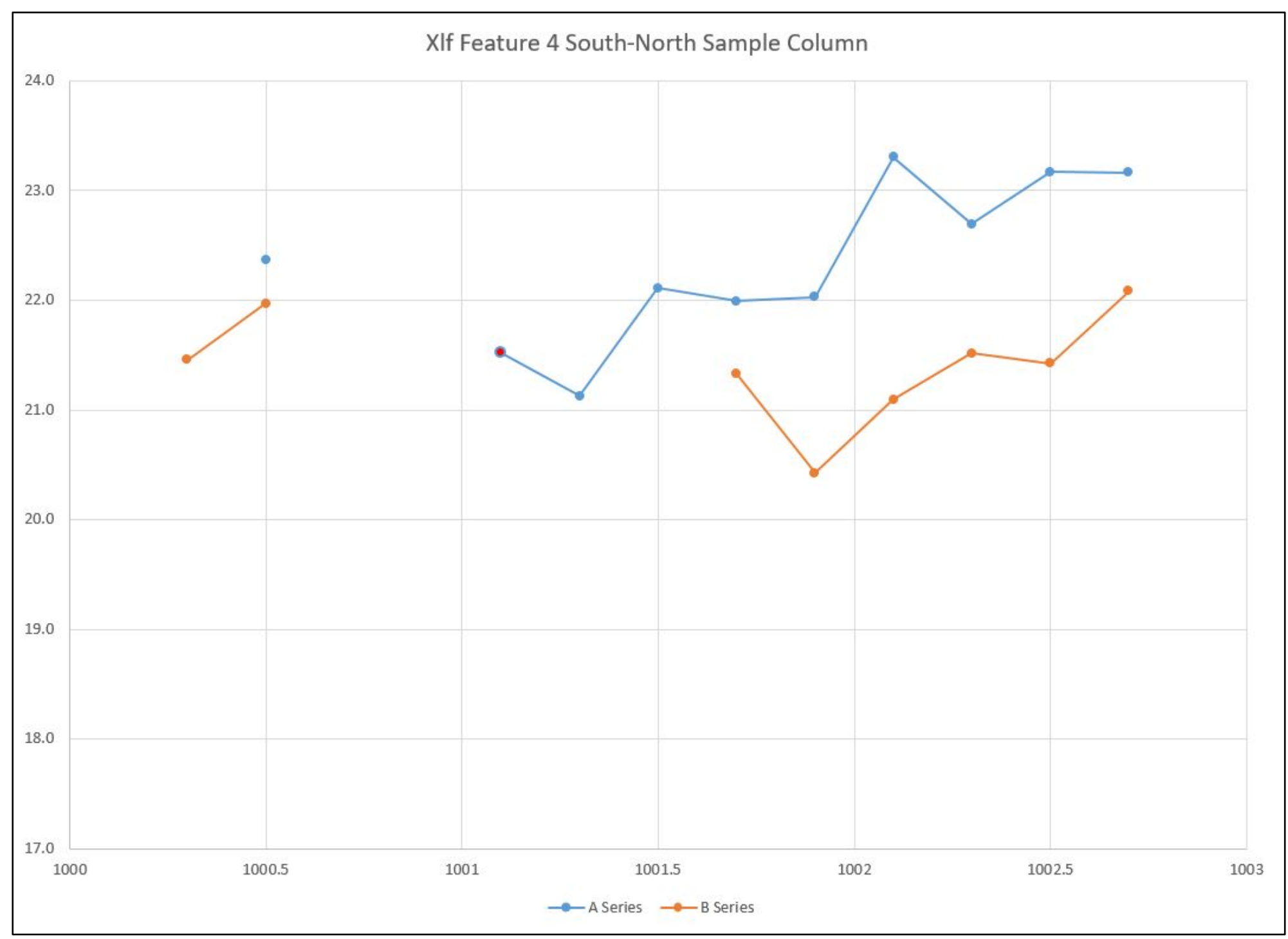

Figure 5.21. Feature $4 \chi$ lf magnetic susceptibility results from south to north sample column. 


\section{CHAPTER 6. ARTIFACTS, ECOFACTS, AND FEATURES}

Archaeology is the study of the material record to derive interpretations of past societies and their contexts. But what endures through the destructive processes of time is often only a small fraction of the overall material culture. Items manufactured from hides, wood, and other perishables disintegrated long ago, and are generally lost to the archaeological record. In addition to natural processes that bias the archaeological record, there are social filters that influence what is discarded on residential sites and what is discarded elsewhere. Accordingly, archaeological assemblages are inaccurate reflections of the total material culture of any given past society.

This chapter presents descriptive data on the features, artifacts, and ecofacts that were investigated or recovered during the excavations. The materials are not organized by component or stratum, but rather by basic descriptive categories. The intent of this chapter is to present the data as objectively as possible with only brief chronological or stratigraphic divisions. A few low-level interpretations are provided on functions of tools and features, but these are kept at a minimal level. The subsequent chapter will develop more of an interpretive context that will address the issues of moving from the material record to inferences on prehistoric society and behavior.

Cultural materials recovered during the data recovery excavations on site $41 \mathrm{GU} 177$ include features and artifact categories composed of various materials, including stone, bone, shell, ceramic, wood, and other floral or faunal remains (Appendix B). However, as a result of depositional and post-depositional (e.g., preservation) site formation processes, stone comprises the vast majority of the artifact assemblage. Inorganic remains are enormously overrepresented in the archaeological record. Burned rock, lithic tools, as well as reduction debris are quite often the only things that endure, but ethnographic studies show hunter-gatherer occupations typically leave behind as much organic remains as non-organics. As a result, the total prehistoric material assemblage often has to be inferred from lithic remains and site structure. Site 41GU177, however, yielded a relatively good collection of some perishable materials, including bone and floral remains. Some of these are artifacts, and many are ecofacts, defined as plants, animals, or rocks that were not clearly cultural modified or produced but were nevertheless culturally introduced or otherwise part of the overall site context.

Charcoal, which forms the primary basis for determining the site's chronological parameters through radiocarbon dating, was abundant and well preserved. Dates reported in this chapter are corrected, 2Sigma calibrated dates (cal B.P.). Both macro- and micro-floral remains were also well preserved.

\section{ARTIFACTS}

Over the course of the data recovery efforts, SWCA collected 9,917 artifacts (Table 6.1; Appendix B). Stone artifacts encompassed the majority of the artifact assemblage. Debitage accounted for almost 90 percent of the total assemblage. Burned rock was likewise abundant $(n=3,677)$ but was counted and discarded in the field (and is not included in the total above). Faunal remains formed the next largest category, with a total of 666 bone fragments and 131 shells; however, the majority of bone were very fragmented and likely originated from relatively few individual specimens. Modified flakes formed the next largest stone artifact category, with a total of 67 specimens recovered. This was followed in quantity by bifaces $(n=35)$ and cores $(n=22)$; recovery of other stone tool categories (e.g., projectile points, scrapers) was minimal (see Table 6.1). In addition to the stone artifact assemblage, eight prehistoric ceramics and 10 historic-age artifacts were recovered. One of the more interesting finds included a single shell ornament that may have been a decorative bead. 
Table 6.1. 41GU177 Data Recovery Artifact Assemblage

\begin{tabular}{|c|c|c|c|c|c|c|c|}
\hline Artifact Type & Count & Percent & Weight (g) & Samples & Count & Weight (g) & \\
\hline Battered Stone & 1 & 0.01 & 183.52 & Charcoal Samples & 11 & 5.48 & \\
\hline Biface & 35 & 0.35 & 977.81 & Feature Matrix & 5 & $17,800.00$ & \\
\hline Bone & 666 & 6.72 & 704.68 & Geoarch MS Samples & 237 & N/A & \\
\hline Historic Ceramic & 5 & 0.05 & 12.81 & Geoarch Bulk Matrix & 51 & N/A & \\
\hline Core & 22 & 0.22 & $2,676.36$ & Total & 305 & $17,805.48$ & \\
\hline Core Tool & 4 & 0.04 & $1,485.18$ & & & & \\
\hline Debitage & 8,854 & 89.29 & $14,388.43$ & & & & \\
\hline Historic Glass & 1 & 0.01 & 0.79 & Burned Rock Size $(\mathrm{cm})$ & Count & Percent & Weight (kg) \\
\hline Ground stone & 3 & 0.03 & 407.25 & $0-5$ & 3034 & 82.51 & 55.83 \\
\hline Historic Metal & 4 & 0.04 & 2.04 & $5-10$ & 582 & 15.83 & 72.97 \\
\hline Misc. Stone/ Mineral & 5 & 0.05 & $1,364.97$ & $10-15$ & 49 & 1.33 & 17.90 \\
\hline Modified Flake & 67 & 0.68 & $1,899.41$ & $15+$ & 12 & 0.33 & 15.50 \\
\hline Pigment & 3 & 0.03 & 1.03 & Total & 3677 & & 162.20 \\
\hline Projectile Point & 7 & 0.07 & 7.44 & & & & \\
\hline Scraper & 1 & 0.01 & 20.12 & & & & \\
\hline Shell & 131 & 1.32 & 421.18 & & & & \\
\hline Shell Ornament & 1 & 0.01 & 0.06 & & & & \\
\hline Thermally Altered Stone & 58 & 0.58 & 821.08 & & & & \\
\hline Prehistoric Ceramic & 8 & 0.08 & 12.39 & & & & \\
\hline Clay Artifact & 41 & 0.41 & 239.57 & & & & \\
\hline Grand Total & 9,917 & & $25,626.12$ & & & & \\
\hline
\end{tabular}

\section{Projectile Points}

An 11,000-year broad regional chronology has been established for Central Texas, and this broadly applicable chronology is primarily based on specific projectile point types (Collins 2004:113:Figure 3.9a). There are issues of inconsistency with this method of classification due to biases or the subjective decisions of the analyst (see Johnson 1995 and Tomka et al. 2003). Additionally, variability in form, style, blade retouch, or base treatment can be expected, making identifying a specific artifact to a specific type problematic. Nevertheless, the use of this cultural-historical approach has achieved many objectives for which it is designed, namely developing chronological schemes and culturo-temporal diagnostics throughout the state, especially the Central Texas, South Texas, and Lower Pecos regions (Hester 2004; Shafer 2005).

Chipped stone tools identified as projectile points were further classified by diagnostic types based on the original ordering provided by Suhm et al. (1954) and Turner et al. (2011). Overall technology, base and stem attributes, patterning in blade thinning and resharpening, were all taken into consideration when typological decisions were being made. Following typological designations, nominal (qualitative) and metrical (quantitative) attributes were recorded for each point specimen and entered into a database. Recorded nominal attributes included information such as cortex, raw material type, color, patination, evidence of heat treatment, breakage, beveling, and reworking. Metrical attributes recorded for each specimen were similar to those measured by Hudler (1997), including variables such as blade and stem dimensions (length, width, thickness, weight), haft length, base depth, base width, and neck width. 
Seven projectile points, including both arrow point and dart point types, were recovered from the site (Table 6.2). The projectile point assemblage consists of six arrow point types and a single dart point type (Figure 6.1). Of the seven projectile points identified, six are diagnostic types and are designated as Edwards, Perdiz, Fresno, and Zephyr. The five diagnostic arrow points are all associated with the Late Prehistoric period (ca. 1250 to 350 B.P.) and the single diagnostic dart point is associated with the Late Archaic period (ca. 4000 to 1250 B.P.). Further chronological division can be found within the arrow points with the occurrence of the two Edwards and two Perdiz points, which are both commonly found points for the Austin and Toyah phases, or horizons, of central Texas. Spatially, the seven projectile points were found predominantly in excavation Blocks D and E, with only one Perdiz point found in Block C (see Figure 4.1).

Table 6.2. Chipped Stone Tool Assemblage

\begin{tabular}{|c|c|c|c|c|c|}
\hline Category & Subcategory & Count & Percentage & Total Count & Total Percentage \\
\hline \multirow{5}{*}{ Projectile Point } & Edwards & 2 & 1 & \multirow{5}{*}{7} & \multirow{5}{*}{5} \\
\hline & Perdiz & 2 & 1 & & \\
\hline & Fresno & 1 & 1 & & \\
\hline & Zephyr & 1 & 1 & & \\
\hline & Indeterminate & 1 & 1 & & \\
\hline \multirow{8}{*}{ Biface } & Notched Biface Tool & 1 & 1 & \multirow{8}{*}{35} & \multirow{8}{*}{26} \\
\hline & Gahagan Biface & 1 & 1 & & \\
\hline & Stage 1 & 2 & 1 & & \\
\hline & Stage 2 & 2 & 1 & & \\
\hline & Stage 3 & 4 & 3 & & \\
\hline & Stage 4 & 0 & 0 & & \\
\hline & Stage 5 & 7 & 5 & & \\
\hline & Indeterminate & 18 & 13 & & \\
\hline Scrapers & End and Side-scraper & 1 & 1 & 1 & 1 \\
\hline \multirow{2}{*}{ Modified Flakes } & Edge-modified & 53 & 39 & \multirow{2}{*}{67} & \multirow{2}{*}{49} \\
\hline & Utilized & 14 & 10 & & \\
\hline \multirow{4}{*}{ Cores } & Unifacial & 1 & 1 & \multirow{4}{*}{26} & \multirow{4}{*}{19} \\
\hline & Bifacial & 5 & 4 & & \\
\hline & Multidirectional & 16 & 12 & & \\
\hline & Choppers & 4 & 3 & & \\
\hline Total & & & & 136 & \\
\hline
\end{tabular}




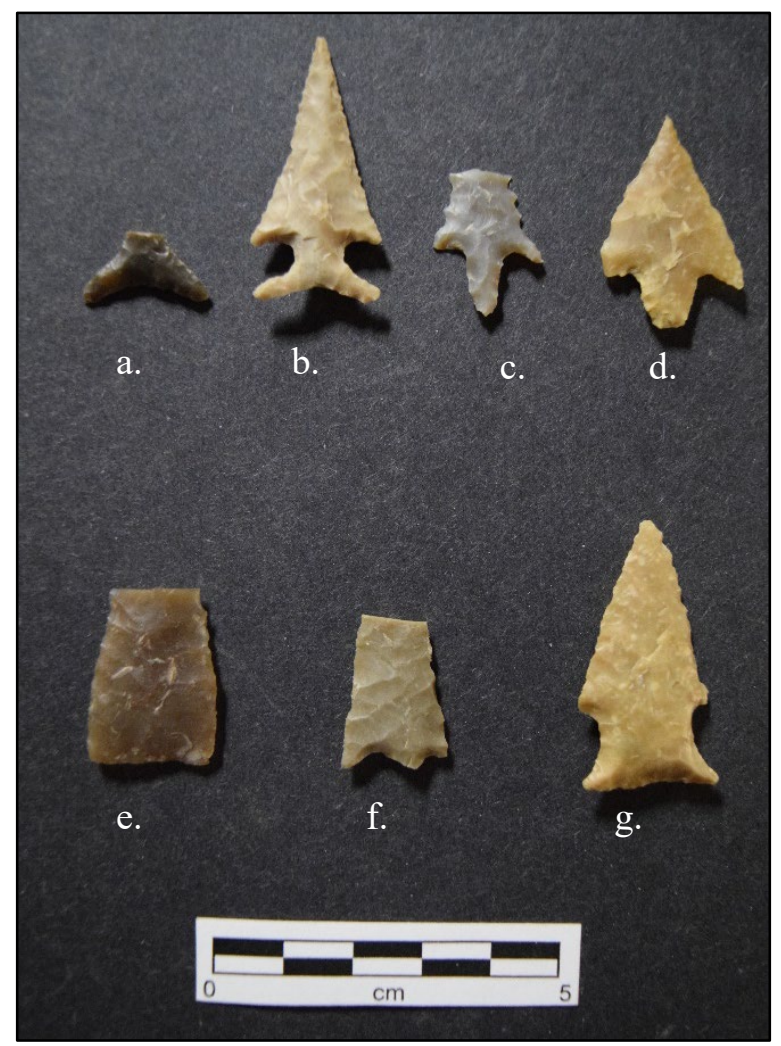

Figure 6.1. Edwards arrow points: a) Lot 552-8, b) Lot 668-6; Perdiz arrow points: c) Lot 559-7, d) Lot 579-10; Fresno arrow point: e) Lot 607-6; Indeterminate arrow point: f) Lot 550-69; Zephyr dart point: g) Lot 621-7.

\section{Arrow Points}

Six arrow points were recovered from the excavations. These six arrow points include two Edwards points, two Perdiz points, a Fresno point, and one indeterminate arrow point. All three point types are late Prehistoric in age (ca. 1250 to 350 B.P.) (Turner et al. 2011). Both Edwards and Perdiz points have strong cultural associations with two known phases or horizons in Central Texas, the Austin and the Toyah Phase, respectively (see Chapter 3).

\section{EDWARDS}

Two Edward points were encountered during the data recovery; one in unit N1001 E1009, Level 7 at an elevation of 98.99 to $98.89 \mathrm{~m}$ and one in unit N1004 E1019, Level 12 at an elevation of 98.2 to $98.1 \mathrm{~m}$ (see Figure 4.1). Edward points are common in south central Texas and portions of the south coastal plains, representing the earliest and largest arrow points in many areas (Turner et al. 2011:190). They are characterized as having straight to convex lateral edges, prominent shoulders and pointed barbs, and an expanding stem with a prominent recurved divide (Turner et al. 2011:190). Expanding stem points, such as Edwards and Scallorn, are type points associated with the Austin phase (Black 1989). They have been dated in archaeological contexts as early as the tenth century A.D., with a typical range from c.a. A.D. 900 to 1100 (ca. 1050 to 850 B.P.) (Black and McGraw 1985; Gerstle et al. 1978). This is contemporaneous with Scallorn points, but not included by Prewitt $(1981,1985)$ in the Austin phase toolkit. 
Two specimens from the site have sufficient diagnostic attributes for typological assignment as Edwards points (see Figure 6.1; see Table 6.2). Specimen Lot 552-8 is an incomplete point fragment consisting of only the stem and base. The specimen is made from very dark gray (10YR 3/1) fine-grained chert. The break appears to be a snap fracture that may have resulted from use wear. Despite the small portion of the projectile point remaining, multiple Edwards characteristics are identifiable, such as a broad expanding stem and the prominent, deep concavity along the basal margin. The stem base has a maximum width of 18.04 millimeters $(\mathrm{mm})$ and medial stem thickness of $2.76 \mathrm{~mm}$ (Appendix $\mathrm{C} 1$ ). These measurements are relative to the full Edwards point (Lot 668-6) discussed in detail below.

Specimen Lot 668-6 is a complete Edwards point made from grayish brown (10YR 5/2) fine-grained chert with slight gray (10YR 6/1) banding. Lot 668-6 has multiple identifiable Edwards characteristics, including a broad expanding stem, prominent and deep concavity along the basal margin, and fine, almost serrated, flaking along the lateral blade margins. The specimen has a maximum stem base width of $17.44 \mathrm{~mm}$ and a medial stem thickness of $3.37 \mathrm{~mm}$ (Appendix C1).

\section{PERDIZ}

Two Perdiz points were encountered during the data recovery; one in unit N1001 E1010, Level 4 at an elevation of 99.29 to $99.19 \mathrm{~m}$ and one in unit N1000.5 E1004, Level 3 at an elevation of 99.5 to $99.4 \mathrm{~m}$ (see Figure 4.1). Perdiz points are a common projectile point type found throughout most of Texas and are characterized as triangular with well-barbed shoulders and contracting stems (Turner et al. 2011:206). They may also be unifacial or bifacial modified (Suhm and Jelks 1962:283). Several subgroupings have been defined for Perdiz, including the Whitney and Morgan varieties (Jelks 1962; Suhm and Jelks 1962:24). The latter have shorter, more rounded bases. These points have long been considered diagnostic of the Toyah phase, horizon or other chronological division. They have been found in archaeological contexts that date to as early as ca. 1200 B.P. in Collins's (2004: Figure 3.9a) Central Texas chronology; however, they are more commonly dated from approximately 700/650 B.P. until 200/250 B.P. (Arnn 2012:64-65; Johnson 1994:87; Prewitt 1981:84).

Two projectile points within the assemblage have sufficient diagnostic attributes for typological assignment of Perdiz (see Figure 6.1; see Table 6.2). As commonly noted in the literature, there is quite a bit of variation in the technology and morphology of this type, and this variation is evident in the sample collected from the site (Jelks 1962; Suhm and Jelks 1962:24). Technologically, many are made on bladeflakes using reduction techniques ranging from minimal edge trimming, often unifacial, to complete bifacial working (Turner et al. 2011:206). Morphologically, the collection varies with one specimen having a classic thin and narrow body with fine flaking, while the second specimen exhibits typical Perdiz characteristics but has a broad and short body.

The narrow-bodied flake specimen (Lot 559-7) is incomplete, consisting of only the basal fragment with a portion of the blade (see Figure 6.1). Lot 559-7 is a greenish gray (10G 5/1) fine-grained chert. The break across the medial portion of the blade is a snap fracture and appears to have been caused by use wear due to break type and location. The specimen is only $14.44 \mathrm{~mm}$ at its maximum blade width. It exhibits the typical Perdiz style with a distinct contracting stem, sharp pointed barbs, is finely worked, and, in this case, has serrated blade edges (Turner et al. 2011:206). Lot 559-7 has two barbs, one long and sharply pointed and one that is short. The short barb appears to have been partially removed, as is evident from a transverse flake removed from one face of the blade, and it has been bifacially retouched. Although the long barb is twice the length of the short barb, $4 \mathrm{~mm}$ verses $2 \mathrm{~mm}$, both notch depths and widths are relatively similar in morphology (Appendix $\mathrm{C} 1$ ).

The broad-bodied specimen (Lot 579-10) is a complete projectile point with only the tip of one barb missing (see Figure 6.1). This specimen was recovered from one level above Feature 4 in the 
northeastern-most unit. Lot 579-10 is brown (10YR 5/3) fine-grained chert with mineral impurities evident in the stem. The break is small and the placement is too ambiguous to assess the cause of breakage. The maximum blade width of Lot $579-10$ is $20.62 \mathrm{~mm}$, which is significantly larger than the previously defined narrow-bodied Lot 559-7, which is only $14.44 \mathrm{~mm}$. This specimen represents a common variation from the traditional long and narrow Perdiz points, being broader in body but still retaining the typical Perdiz characteristics of a distinct contracting stem, sharp pointed barbs, and finely worked blade edges.

\section{FRESNO}

A single Fresno point was encountered in unit N1003 E1018, Level 7 at an elevation of 98.7 to $98.6 \mathrm{~m}$ (see Figure 4.1). Feature 2 corresponds to this level in the northeast portion of the unit; unfortunately, the projectile point was not found in situ, and, as such, the provenience of the point in relation to the feature cannot be determined. Fresno points are poorly defined, unstemmed triangular points found throughout central and south Texas (Turner et al. 2011: 191). Unstemmed points such as Fresno are problematic, because stemmed projectile point preforms typically go through a similar morphological phase before being completed. One argument for why unstemmed points are common in the central and south Texas regions is the limited occurrence of high-quality material, which could indicate that the unstemmed point is an adaption to the available raw material of the region (Black and Quigg 2018). Fresno points are characterized as having straight to slightly convex or concave lateral margins, triangular, and unstemmed (Turner et al. 2011:191). They are similar to Cameron projectile points, found in far south Texas, but are generally larger. Fresno points have been found in archaeological contexts relating to Late Prehistoric occupations (Mallouf et al. 1977).

The single Fresno point (Lot 607-6) encountered is an incomplete point with a snap break across its upper medial portion, resulting in only the basal margin and an undetermined portion of the blade remaining intact (see Figure 6.1; see Table 6.2; Appendix C1). It is very dark gray (10YR 3/1) fine-grained chert. The breakage has been interpreted as use wear due to the snap break and its location at the distal end of the point. The lateral margins and slightly convex and the basal margin is straight. None of the three available margins appear retouched.

\section{INDETERMINATE}

A single Indeterminate arrow point (Lot 550-69) was encountered in unit N1001 E1009, Level 5 at an elevation of 99.19 to $99.09 \mathrm{~m}$ (see Figure 4.1). The specimen consists of a blade fragment with two intact barbs. It is grayish brown (10YR 5/2) in color and made from fine-grained chert (see Figure 6.1; see Table 6.2; Appendix C1). Both breaks at the distal tip and the stem appear to be snap breaks from use wear. Lot 550-69 is thin and finely worked with oblique subparallel flaking on one blade face. Notches appear to be on the side and one barb is long and sharply pointed, while the other is short and slightly less pointed. Due to the fragmented condition of specimen Lot 550-69 it is classified as Indeterminate in type and cultural association.

\section{Dart Point}

A single dart point was recovered from the excavations. The dart point has been identified as a Zephyr projectile point. Zephyr points are typically dated from 200 to 700 A.D., which is associated with the Late Archaic period (ca. 4000 to 1250 B.P.) in Central Texas (Turner et al. 2011:174). 


\section{ZEPHYR}

A single dart point was encountered in unit N1003 E1019, Level 9 at an elevation of 98.5 to $98.4 \mathrm{~m}$ (see Figure 4.1). It has been identified as a Zephyr dart point, which are characterized as being small to medium lanceolate or triangular points (see Figure 6.1; see Table 6.2). Zephyr's typically have a gently expanding or parallel-sided stems with the edges exhibiting grinding, and concave basal margins with rounded edges (Turner et al. 2011:174). Previous Zephyr points, along with Mahomet points, were considered a subcategory of Darl types (Prewitt 1981). Zephyr points have since become their own classification based on their occurrence on numerous sites in north central Texas, including Stephen and Comanche Counties, Horn Shelter 2, and Rogers Springs (Turner at al. 2011:174).

The Zephyr dart point encountered during investigations is complete and created from brown (10YR 5/3) fine-grained chert that is speckled with slight gray (10YR 6/1) impurities (Appendix C1). The specimen exhibits many of the typical Zephyr characteristics, such as being a medium to small triangular point with gently expanding stem and slightly concave to straight basal margin. The stem is broad and the shoulders or barbs are weak and rounded. Fine flaking is noticeable along both lateral margins.

\section{Bifaces}

Simply defined, bifaces are characterized by sequential flake removal that has occurred on both surfaces of a flake or core to form a single edge. Bifacial tools include a variety of types, distinct in terms of function and/or morphology. Odell (2003:65) notes that these include "projectile points, drills, axes, adzes, and generic oval, rectangular, or triangular forms called simply bifaces". Of the total 35 bifaces encountered during the data recovery excavation, 33 are typed simply as bifaces; the remaining two include a notched biface tool and a fragmented Gahagan biface (see Table 6.2; Figure 6.2). To further define the 33 bifaces that do not have a subcategory, SWCA employed standards outlined by Andrefsky (1998) and Callahan (1979) to refine each specimen based on the level of reduction. In the case of bifaces, five stages of reduction are recognized, including: Stage 1: blank preparation, Stage 2: initial edging, Stage 3: biface thinning, Stage 4: preform, and Stage 5: finished biface. This is discussed further in the Biface Reduction Stages section below.

The 35 recovered bifaces exhibit a wide range of retouch or completion (see Figure 6.2). Only nine of the bifaces recovered are complete specimens (Appendix C2). The remainder of the specimens fall within various categories of condition with the most prominent conditions being complete $(n=9)$, distal-medial fragments $(n=9)$, and indeterminate fragments $(n=8)$. Of the 26 broken or fragmented specimens, four exhibit breakage from use wear, 19 show breakage from the manufacturing process, and the three have indeterminate breakage patterns. The material most commonly utilized consists of fine-grained chert with only one specimen made from coarse-grained chert. The most prominent chert color is gray $(10 \mathrm{YR} 5 / 1)$ or grayish brown $(10 \mathrm{YR} 5 / 2)(\mathrm{n}=11)$ with variations ranging from brown $(10 \mathrm{YR} 5 / 3)$ to very dark gray (10YR 3/1) to pale red (2.5YR 6/2). Due to the number of broken or incomplete bifaces, many of the nominal and metrical attributes could not be discerned and were recorded in the database as Indeterminate (Appendix C2). 


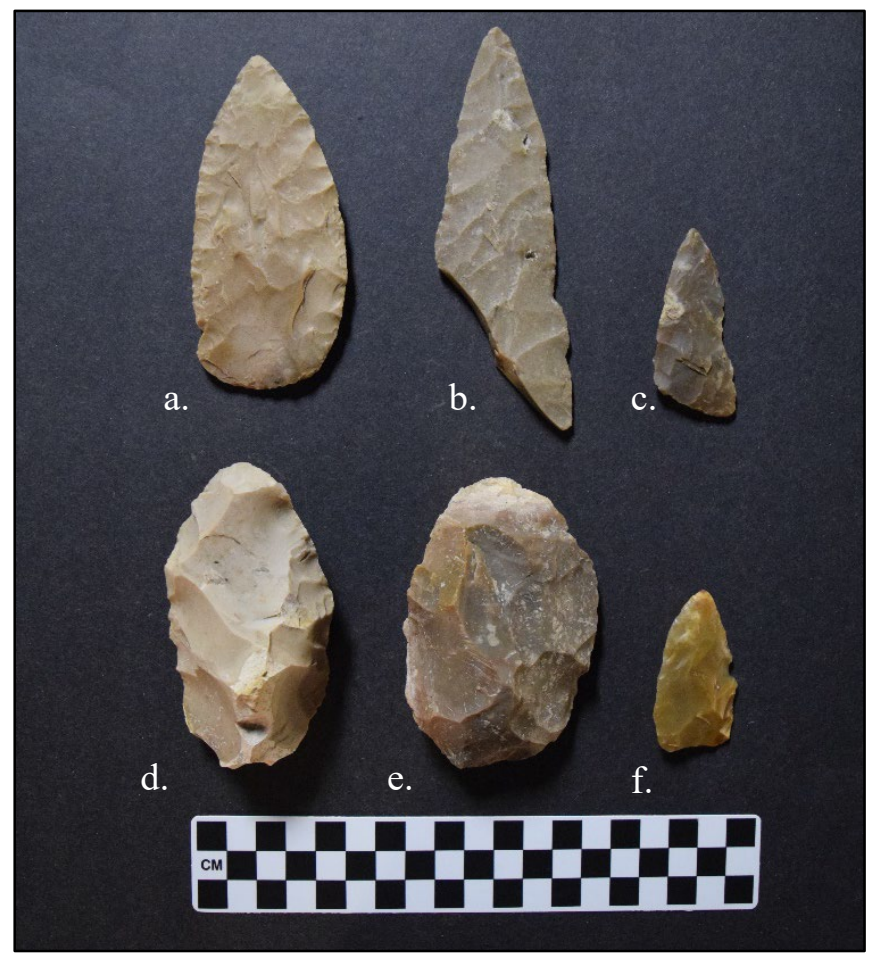

Figure 6.2. Representative sample of biface assemblage. Stage 5: a) Lot 403-9, b) Lot 620-1, c) Lot 661-7; Stage 1: d) Lot 341-1; Stage 2: e) Lot 360-1; Stage 3: f) Lot 404-1.

\section{Biface Reduction Stages}

Lithic bifacial reduction has consistently been viewed as a stage or step-like production process along a trajectory, from raw material to finished tool (Callahan 1974; Patterson 1977:60; Whittaker 1994). As a biface is reduced, it goes through several sequential stages or steps differentiated from one another by the manufacturing implement employed, the size and thickness of the biface, and its form. The sequence and nature of these stages or steps differ, depending on numerous variables, including the desired end product of the reduction process, the form and quality of the parent raw material, and the style or technique in which flint knapping is performed. Previously completed tools may be reintroduced into the production trajectory and be repaired, rejuvenated, or recycled into a different form.

Table 6.3 summarizes reduction stages based on Callahan's (1979) weight/thickness ratio index for biface reduction stage. Based on the stages defined by Callahan (1979), two bifaces are classified as Stage 1, two bifaces are Stage 2, four bifaces are Stage 3, and seven bifaces are Stage 5. The remaining 20 bifaces, including the two typed bifaces discussed below, were unable to be classified by reduction stage due to being fragmentary specimens (Appendix C2). 
Table 6.3. Biface Reduction Stages

\begin{tabular}{lll}
\hline $\begin{array}{l}\text { Biface } \\
\text { Stage }\end{array}$ & Weight/Thickness Ratio & Description \\
\hline Stage 1 & None & Blank stage: procurement of material \\
\hline Stage 2 & $2.00 \pm$ to $3.00 \pm$ & Edged biface: initial edge work along circumference \\
\hline Stage 3 & $3.00-$ to $4.00-$ & Primary thinning: most or all of cortex removed and thinning flakes to center of tool \\
\hline Stage 4 & 4.00 - to 5.00 & Secondary thinning: transverse flake removal and tool shaping \\
\hline Stage 5 & 4.00 to $6.00+$ & Shaping: final shaping and fine edge work \\
\hline
\end{tabular}

Source: Callahan (1979) and Andrefsky (1998)

\section{Typed Bifaces}

Specimen Lot 316-7 is a thin, light yellowish brown (10YR 6/4) biface tool made from fine-grained chert with a single side notch (Figure 6.3; see Table 6.2). Lot 316-7 was recovered from N995 E1012, Level 3 at an elevation of 99.5 to $99.4 \mathrm{~m}$ (see Figure 4.1). It appears to be a broken fragment of a biface that was reworked into a notched tool. There is also evidence of intentional heat treatment, due to a reddened discoloration, particularly along the margins. Lot 316-7 is only a fragment and, as such, the tool function and possible cultural affiliation cannot be determined.

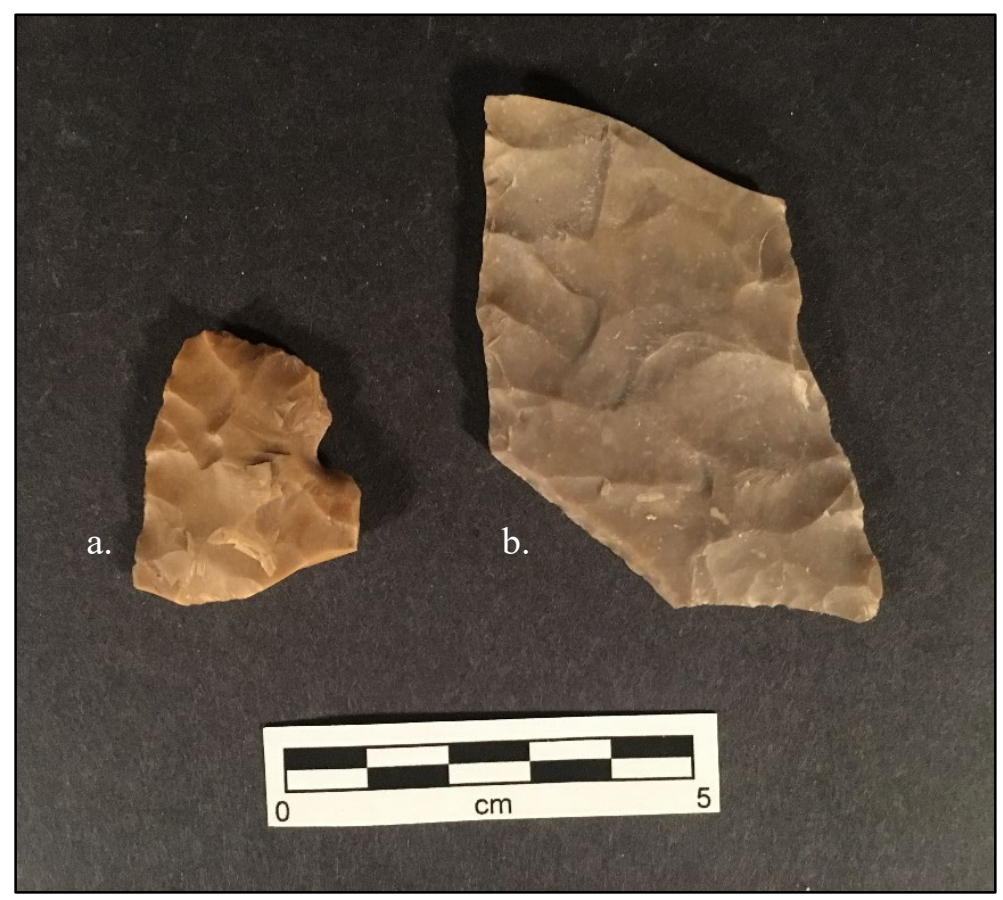

Figure 6.3. Side-notched biface: a) Lot $316-7$ and Gahagan biface: b) Lot 552-9.

Lot 552-70 is a medial-basal fragment of a gray (10YR 5/1) fine-grained chert Gahagan biface (see Figure 6.3; see Table 6.2). It was recovered from N1001 E1009, Level 7 at an elevation of 98.99 to $98.98 \mathrm{~m}$ (see Figure 4.1). Gahagan bifaces are classified as long, triangular bifaces with elongated parallel sides and straight or moderately concave bases that have been thinned (Turner et al. 2011:230). They are associated with the Late Prehistoric and are found predominately in central or eastern Texas. Although not considered type artifacts, current research is in general agreement that Gahagan bifaces are typically associated with Caddoan sites such as the George C. Davis site (Turner et al. 2011:230). 


\section{Scrapers}

Scrapers are defined as "unifacially flaked artifacts ... and unretouched flakes characterized by relatively acute working edges, often exhibiting unifacially distributed microflaking and more commonly edge rounding on either distal and/or lateral working edges" (Tomka et al. 1999:30). Scrapers are typically subdivided based on the location of retouch and/or use wear; categories include end scrapers, side scrapers, and end/side scrapers (Tomka et al. 1999:32). End scrapers are also a common component of Toyah assemblages. Johnson (1994) notes that during the Toyah period, end scrapers were heavily relied on to thin buffalo hides. They were often made from medium-sized flakes, and the tip opposite of the remnant striking platform was chipped into a scraping bit (Johnson 1994). In this case, the end scrapers, particularly those fashioned from blade flakes, are considered formal tools based on such elements as intentional tool shaping and extensive, continuous flaking along the use-areas.

A single end and side scraper (Lot 390-6) was recovered from N996 E1013, Level 6 at an elevation of 99.1 to $99.0 \mathrm{~m}$ (see Figure 4.1). The specimen was recovered one level above Feature 3, which is discussed later in this chapter. Specimen Lot 390-6 is in complete condition and derived from a reddish gray to reddish brown (5YR 5/2 to 5YR 4/3) fine grained chert (Figure 6.4; see Table 6.2). The reddish color appears to be the result of intentional heat treatment. Retouching along the distal and left lateral margin is continuously distributed and found unifacially on the dorsal face (Appendix C3). Possible breakage from post-depositional trauma was noted along the right lateral margin near the distal end. The specimen represents a typical Toyah style scraper.

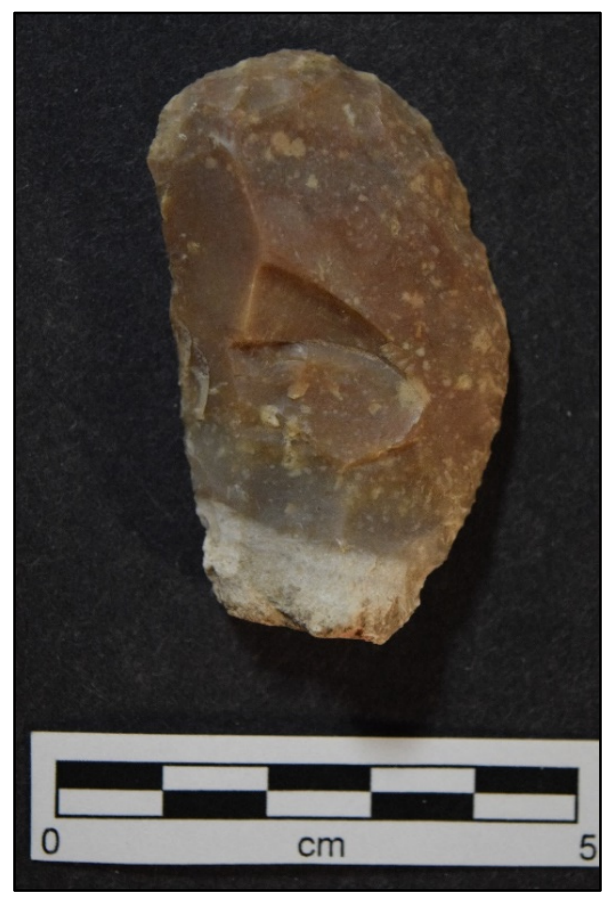

Figure 6.4. End and Side Scraper: Lot 390-6.

\section{Modified Flakes}

Modified flake tools, also commonly referred to as edge-modified flakes, are flakes with intentionally retouched edges that lack standard formal and locational characteristics (Odell 2003). Also included in this category are flakes with edges that have been modified as a result of use as tools (also called utilized flakes). Both forms are considered informal tools, having been minimally modified through use or 
minimally trimmed when manufactured. Typically, flaking scars do not extend into the interior of the flake surface and are confined to less than $10 \mathrm{~mm}$ of the lateral margins. Modification may be unifacial or bifacial, and these tools may have served multiple purposes as expedient knives, scrapers, or gravers. Utilized flakes can be the most difficult to identify accurately, since edge damage through use is created through intensity, duration, and type of use. Edge damage can also occur through post-depositional processes, such as trampling or crushing, mimicking use wear.

SWCA collected 67 modified flakes during the data recovery excavations (Figure 6.5; see Table 6.2). As the largest tool class, an attempt was made to further define the broad classification of modified flake into two sub-groups: edge-modified flakes and utilized flakes. For the purposes of this analysis the term edgemodified was used to distinguish flakes that exhibited flake removal and intentional retouching of the edges. Of the total modified flake assemblage, 53 were designated as edge-modified, and of these 53 only 18 were concluded to be culturally affiliated (Appendix C4). The remaining 35 edge-modified flakes were not complete enough or were lacking in other culturally defining traits (e.g., location and distribution of retouch, heat treatment, and breakage types). The classification of utilized flakes was used to distinguish flakes that exhibit modification from use or very fine edge modification that cannot be confirmed as culturally intentional. Of the total modified flake assemblage, only 14 were designated as utilized (Appendix C4).

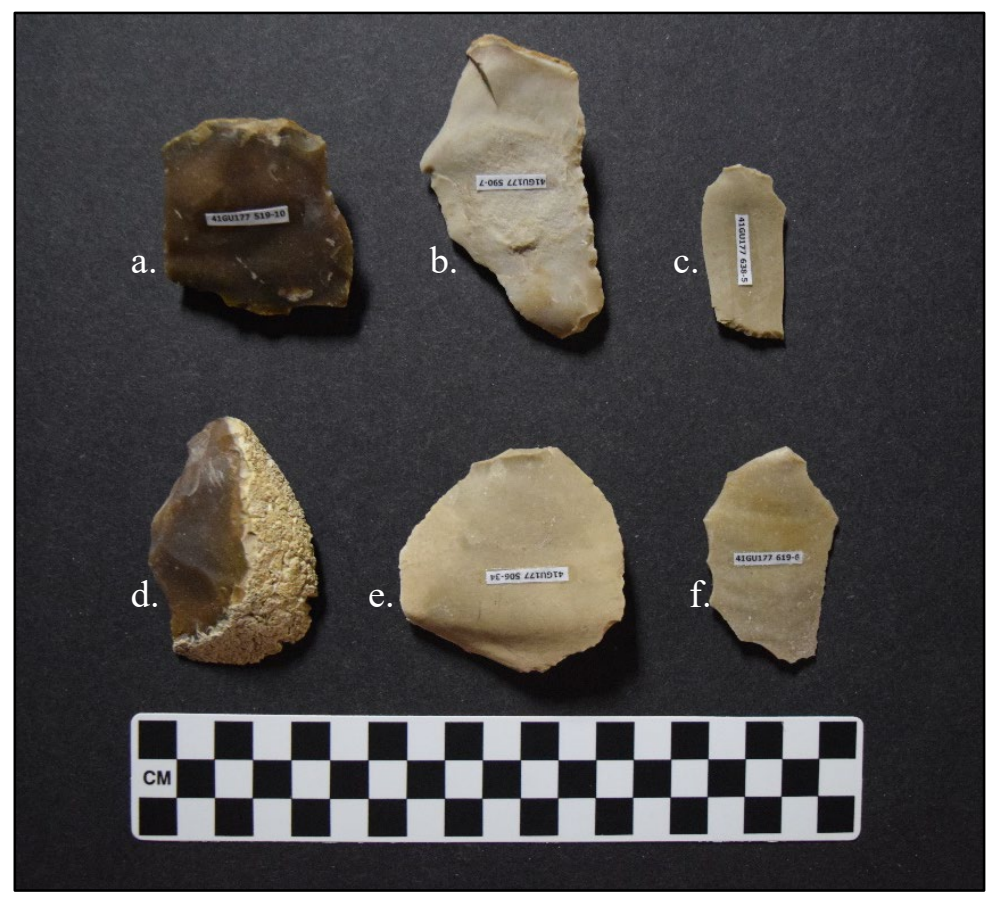

Figure 6.5. Representative sample of edge-modified and utilized flake assemblage. Edge modified flakes: a) Lot 519-10, b) Lot 590-7, c) Lot 638-5; Utilized flakes: d) Lot 320-1, e) Lot 506-34, f) Lot 619-6.

\section{Cores}

A core is a mass of lithic material (also called an "objective piece") from which another piece is detached (Andrefsky 1998). Although they can be utilized as tools, they are considered part of the lithic debitage. They exhibit negative flake scars created by fracturing, a reductive process that involves the removal of flakes from the core by striking it with a percussor, such as a billet or hammer stone. Flakes may also be 
detached through indirect percussion using a punch or through pressure. The primary purpose of cores is a source of flakes, which may be utilized or further reduced into stone tools. In some instances, a sharp margin of the core itself may be utilized as a stone tool. The butted or backed bifaces probably functioned in this role, as did the early manufacturing stage bifaces.

Similar to bifaces, cores cannot always be typed by function (e.g., expedient tools for use in cutting or chopping). To further define the core category, SWCA also used classification standards outlined by Andresky (1998) and Callhan (1979) to define each artifact based on a level of reduction. The subdivisions utilized for cores include multidirectional, bidirectional, bifacial, unifacial, bipolar, and indeterminate. Multidirectional cores have striking platforms on different axis, and flakes are removed in numerous directions. Bidirectional cores have opposing or perpendicular platform surfaces, with flakes detached in two different directions. Bifacial cores have flakes detached along both faces of an edge, with the edge serving as the platform. This category may have been used as tools more so than the other core types. Unifacial cores have flakes detached along both faces of an edge, with the edge serving as the platform. Bipolar cores are held against an anvil at the distal end as a flake is detached from the opposing ends. This can split the core longitudinally. The resulting pieces may then be used for further reduction, using the new ventral surface as a platform; in addition, small pebbles may also be split this way.

SWCA collected 22 cores from the data recovery excavations (Figure 6.6; see Table 6.2). The assemblage contained 19 complete cores, including 16 made from fine-grained chert and six from coarse-grained chert. Core sizes ranged from 18 grams to 240 grams. Of the 22 cores, 16 were categorized as multidirectional, five as bifacial, and a single core was designated as unifacial (Appendix C5).

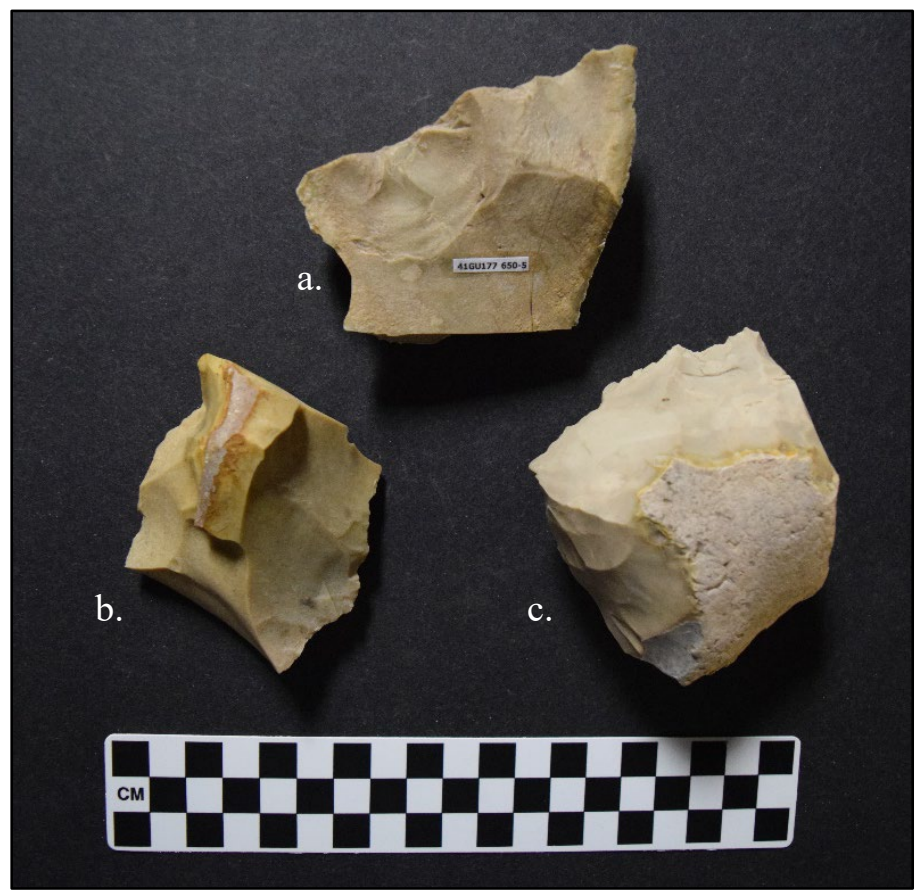

Figure 6.6. Representative sample of core assemblage. Unifacial: a) Lot 650-5; Bifacial: b) Lot 500-5;

Multidirectional: c) Lot 632-8. 


\section{Core Tools}

As briefly mentioned above, cores can at times be utilized as tools; usually, as expedient cutting or chopping tools. Cores by definition are objective pieces that have undergone flake removal (Andrefsky 1998). If the material removed constitutes the desired working tool, then the core can simply be defined as a core; however, if the flake removal produced a working edge on the core that was then either used or intended for use, then the core can now be considered a tool (Andrefsky 1998). Core tools must have intentional modification (i.e., use wear or edge retouching) but cannot be typed as a flake tool or biface tool (Andrefsky 1998).

Four such core tools were identified during the data recovery excavations (Figure 6.7; see Table 6.2). These core tools are classified as choppers, which are expedient stone tools with a transverse edge utilized for cutting or chopping. All four specimens are nodule cobbles that exhibit 50 percent or more cortex. Large flakes were removed to create a sharp edge, along which lab analysis identified evidence of crushing and striations (Appendix C6). Three of the choppers were coarse-grained chert and the fourth was fine-grained chert.

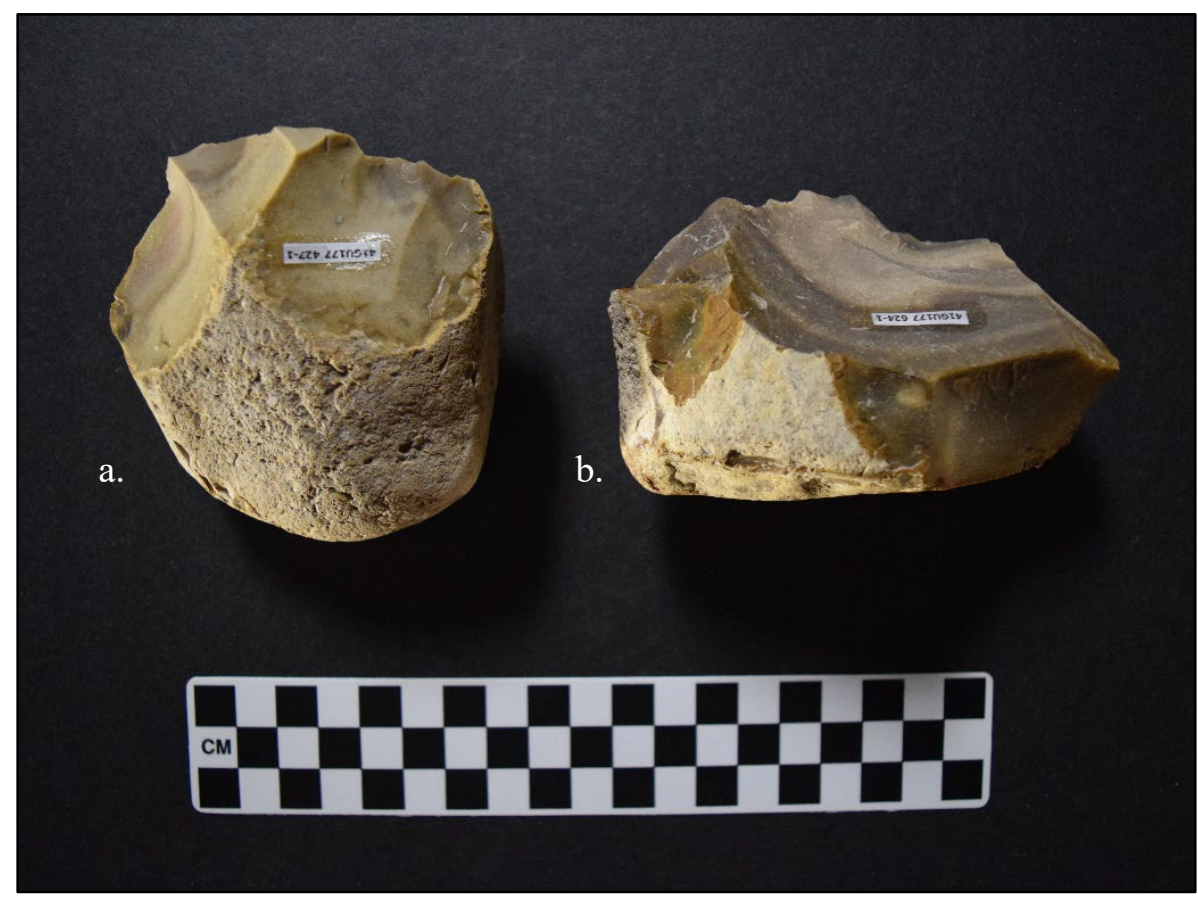

Figure 6.7. Representative sample of core tool-chopper assemblage: a) Lot $427-1$, b) Lot 624-1.

\section{Lithic Debitage}

SWCA recovered 8,716 pieces of lithic debitage from the data recovery efforts including 6,029 lithic flakes (69 percent) and 2,687 pieces of lithic shatter (31 percent) (Table 6.4; Appendix C7). The raw material consists of mostly fine-grained chert made available through local sources. The 6,029 lithic flakes collected during the data recovery were further defined by completion; 1,166 (19 percent) were complete flakes, 1,516 (25 percent) were proximal flakes, and 3,347 (56 percent) were broken or incomplete flakes. The remaining 2,687 pieces of debitage consist of lithic shatter categorized into two types: flaking shatter and thermal shatter. Only 89 pieces ( 3 percent) of the lithic shatter consisted of flaking shatter; the remaining 2,598 specimens (97 percent) were defined as thermal shatter. 
Table 6.4. Lithic Debitage Assemblage

\begin{tabular}{|c|c|c|c|c|c|}
\hline Category & Subcategory & Count & Percentage & Total Count & Total Percentage \\
\hline \multirow{3}{*}{ Lithic Flakes } & Complete & 1166 & 19 & \multirow{3}{*}{6,029} & \multirow{3}{*}{69} \\
\hline & Proximal & 1516 & 25 & & \\
\hline & Broken & 3347 & 56 & & \\
\hline \multirow{2}{*}{ Lithic Shatter } & Flaking & 89 & 3 & \multirow{2}{*}{2,687} & \multirow{2}{*}{31} \\
\hline & Thermal & 2598 & 97 & & \\
\hline Total & & & & 8,716 & \\
\hline
\end{tabular}

\section{Non-Chipped Stone Artifacts}

Non-chipped stone tools, like chipped stone tools, have been altered and formed intentionally for use (Turner et al. 2011:251). They may function in food preparation (i.e., ground stones, manos, and metates), tool sharpening or maintenance (i.e., abraders or grooved stones), or as tools themselves (i.e., hammerstones and celts). Non-chipped stone tool assemblages can consist of multiple categories, determined by inferred function, as well as morphological and material attributes. For the purpose of this study, three main categories were used to classify the assemblage: ground stone, battered stone, or manuport. The assemblage was then subcategorized into various function specific types including mano, pestle, metate, grinding stone, notched stone, grooved stone, hammerstone, and others (Appendix C8). Five non-chipped stone artifacts were recovered during the data recovery investigations (Table 6.5).

Table 6.5. Non-Chipped Stone Artifact Assemblage

\begin{tabular}{cccc}
\hline Category & Subcategory & Count & Total \\
\hline \multirow{2}{*}{ Ground stone } & Grooved Stone & 1 & \multirow{3}{*}{} \\
\cline { 2 - 4 } & Mano & 1 & $\mathbf{2}$ \\
\cline { 2 - 4 } & Indeterminate & 1 & $\mathbf{5}$ \\
\hline Manuport & Manuport & 2 & $\mathbf{5}$ \\
\hline Total & & & \\
\hline
\end{tabular}

\section{Ground stone}

Three ground stone artifacts were identified during the data recovery excavation (Figure 6.8; see Table 6.5). The three specimens were further defined as a grooved stone, a mano, and an indeterminate fragment of stone. Specimen Lot 416-1 is a grooved stone recovered from N0996 E1014, Level 16 at an elevation of $98.05 \mathrm{~m}$ (see Figure 4.1). It is an incomplete specimen with a single narrow trough along one face of the stone (Appendix C8). Due to the specimen being fragmented, it is difficult to discern the function. Grooved stones with similar morphology have been identified as abraders, which are stones utilized to sharpen, smooth, or shape bone and stone tools (Turner et al. 2011:252).

Specimen Lot 492-6 was recovered from unit N1000 E1009, Level 7 between an elevation of 99.09 to $98.99 \mathrm{~m}$ (see Figure 4.1). Lot 492-6 has been classified as a mano. Manos are small- to medium-sized oval to rounded cobbles that exhibit smoothing and pecking (Tomka et al. 1999:32). The smoothing is created as a result of grinding activities, while the pecking is used to rejuvenate use surfaces, making them rougher. The use of milling stones/manos is seen as a hallmark of an Archaic lifestyle (Collins 2004). Lot 492-6 was found to exhibit to smooth surfaces with intentional modification only occurring on 
one face (Appendix C8). Residue was noted on the modified surface that appears organic. No further analysis was completed on the residue.

The third ground stone recovered from the data recover excavations were encountered in unit N1001 E1009, Level 10 between an elevation of 98.69 to $98.59 \mathrm{~m}$ (see Figure 4.1). Lot 555-5 is a very small medial limestone fragment (see Table 6.5). The medial fragment does consist of one smooth face with evidence of possible organic residue. Lot 555-5 is very dark gray, and the breakage is sharp and irregular, possibly indicating the stone was heat-treated (Appendix C8).

\section{Manuport}

Manuports are stones that have been brought into an archaeological site from somewhere else. This may be by natural or cultural processes. Manuports are not locally sourced and therefore stand apart from other rocks and stones in a particular cultural context. Two manuports were recovered from the data recovery excavations in units N1000 E1003 and N1000.5 and E 1005.5 at elevations of $99.65 \mathrm{~m}$ and 99.5 to 99.4 $\mathrm{m}$, respectively (see Figure 4.1). Both manuports were recovered from Block $\mathrm{C}$, which is associated with Feature 4. Neither manuport was found in association with the feature. Lot 461-1 and 518-10 are both rounded limestone rocks with no evidence of cultural modification (Figure 6.8; see Table 6.5).

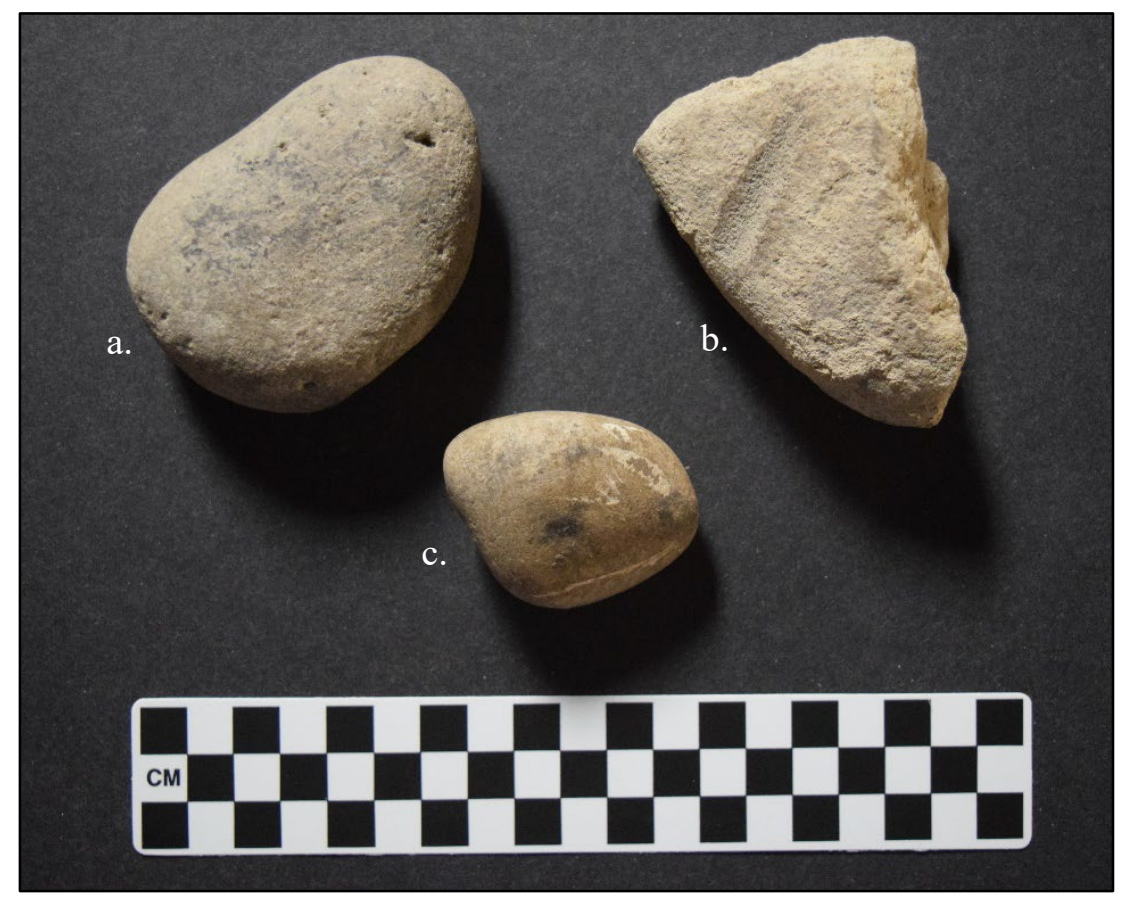

Figure 6.8. Representative sample of non-chipped tool assemblage. Mano: a) Lot 492-6; Grooved Stone: b) Lot 416-1; Manuport: c) Lot 4611.

\section{Shell Ornament}

One possible shell bead, of unknown species, was recovered from N0995 E1012, Level 7 between 99.1 and $99.0 \mathrm{~m}$ (see Figure 4.1). The shell (Lot 321-28) is round and measures approximately $9.15 \mathrm{~mm}$ in diameter and is $0.42 \mathrm{~mm}$ thick (Figure 6.9). The inner surface is a glossy, very pale brown (10YR $8 / 2$ to $10 \mathrm{YR} 8 / 3)$ while the intact periostracum is white (10YR 8/1) in color. A 2.24-mm-diameter hole is present in the center of the specimen, possibly from intentional puncture or natural causes. The shell is 
thin and drastically rounded, similar to the morphology of a rabdotus shell (Bulimulidae family), which was common across the site, but due to the small size of the specimen and lack of diagnostic features, it is problematic to confirm.

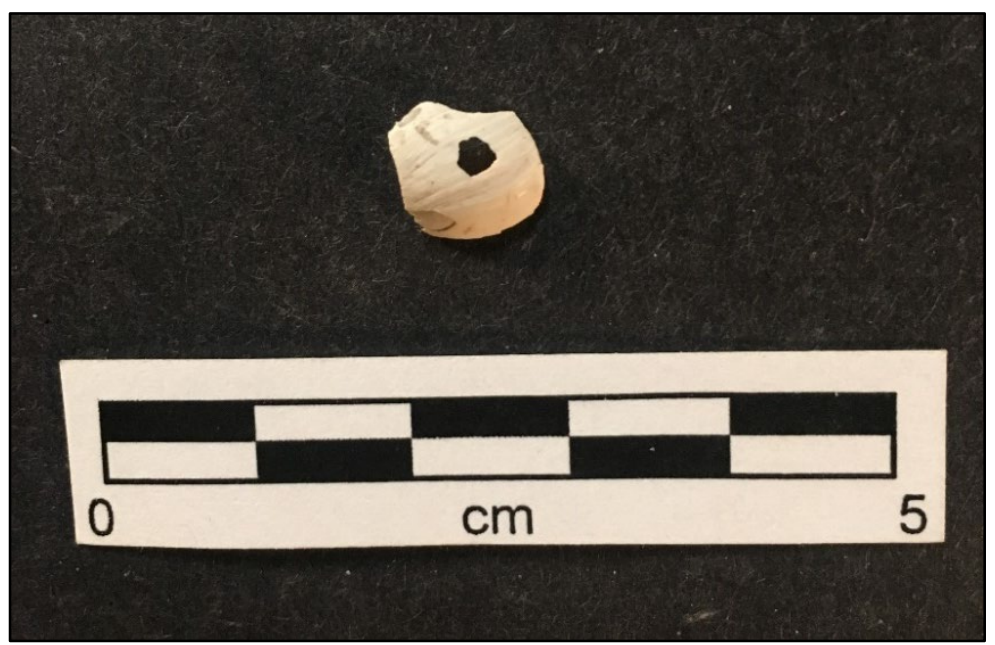

Figure 6.9. Shell bead: Lot 321-28.

\section{Prehistoric Ceramic}

Eight prehistoric ceramic sherds was recovered from site $41 \mathrm{GU} 177$ during the data recovery excavations (Figure 6.10). The eight sherds were all collected from Block C, Unit N1000 E1003; six were recovered in Level 3 between 99.6 to $99.5 \mathrm{~m}$, one within Level 4 between 99.5 to $99.4 \mathrm{~m}$, and one within Level 5 between 99.4 and $99.3 \mathrm{~m}$. Two of the sherds recovered from Level 3 also refit. Analysis of the sherds included visual inspection and microscopic examination; however, no detailed petrochemical analysis was conducted.

All of the recovered ceramics are a quarter-size $(24.3 \mathrm{~mm})$ or smaller undecorated body sherds that appear to belong to the same vessel. However, the size and function of the vessel is indeterminate. No exotic wares were identified. The thickness of the sherds ranges from 8.39 to $9.58 \mathrm{~mm}$. The sherds exhibit a reddish yellow (7.5YR 6/6) slip applied to the exterior surface and a strong brown (7.5YR 4/6) slip applied to the interior surface. The exterior surfaces appear smoothed and burnished, whereas the interior surfaces appear striated. The paste is a very dark grayish brown (10YR 3/2) to black (7.5YR 2.5/1) color and is the same for all of the sherds. All of the sherds contain bone and coarse quartz sand temper with some limestone inclusions. For the most part, the bone temper consists of small white bone fragments within the paste, as well as on both the exterior and interior surfaces, although there are a few larger angular bone fragments present (Figure 6.11). The sherds appear to have been fired in a reducing (i.e., without oxygen) atmosphere at relatively low temperatures. The ceramic sherds appear technologically similar to the Leon Plain pottery tradition which dates from A.D. 1300 to 1700 and is associated with the Late Prehistoric Toyah Phase (Boyd 2012; Perttula et al. 1995; Texas Beyond History 2018). A Perdiz point (Lot 579-10) was recovered within the same elevation in a nearby unit (i.e., N1001.5 E1004), which is considered to be contemporaneous (Turner et al. 2011). 


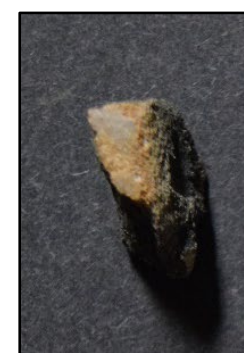

a.

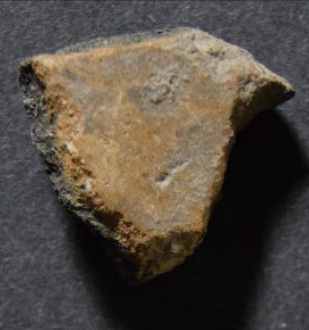

b.
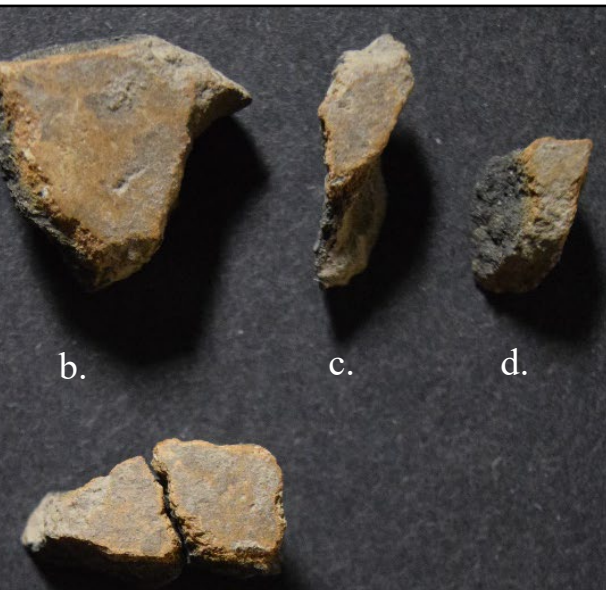

e.

f.

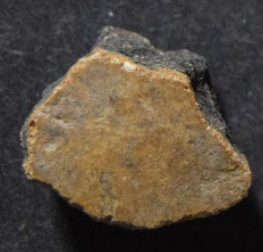

g.

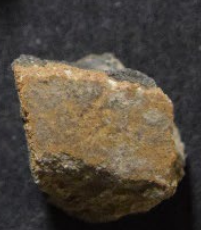

h.

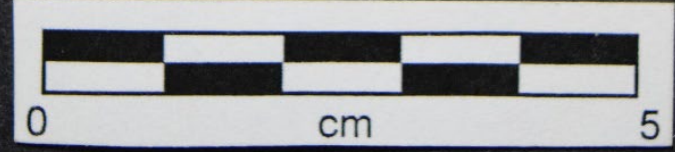

Figure 6.10. Prehistoric ceramics: a-f) Lot 462-22, g) Lot 463-4, and h) Lot 464-5.

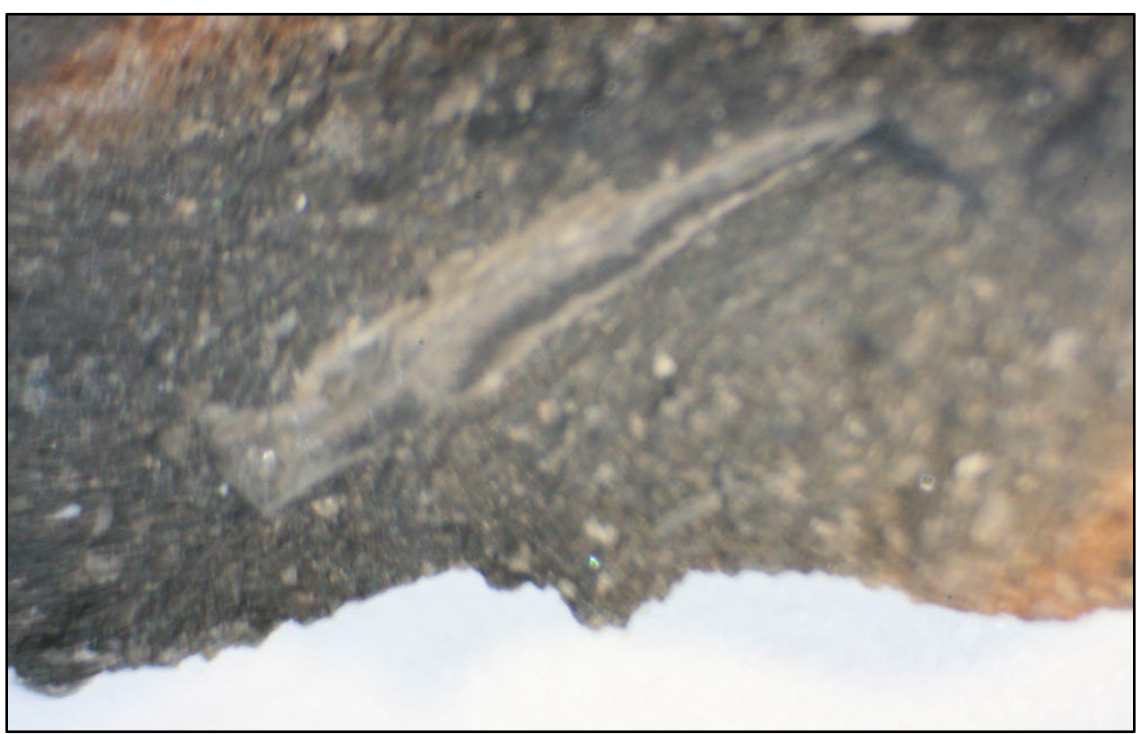

Figure 6.11. Microscopic image of prehistoric ceramic sherd temper with thermally altered angular bone fragment from Unit N1000 E1003, Level 3 (Lot 462-22). 


\section{Historic/Modern Materials}

SWCA recovered 10 historic or modern artifacts, most of which are mid- to late-twentieth-century objects (Appendix B). These items include a colorless glass fragment, a bullet casing, three miscellaneous metal fragments, four sherds of undiagnostic whiteware, and a single Mayer China (1923-1968) ceramic fragment (Figure 6.12). The assemblage was recovered from across the site in the upper levels of the excavation units (99.7-99.1 m). Excavation Blocks C and D recovered a majority of the assemblage, approximately 70 percent.

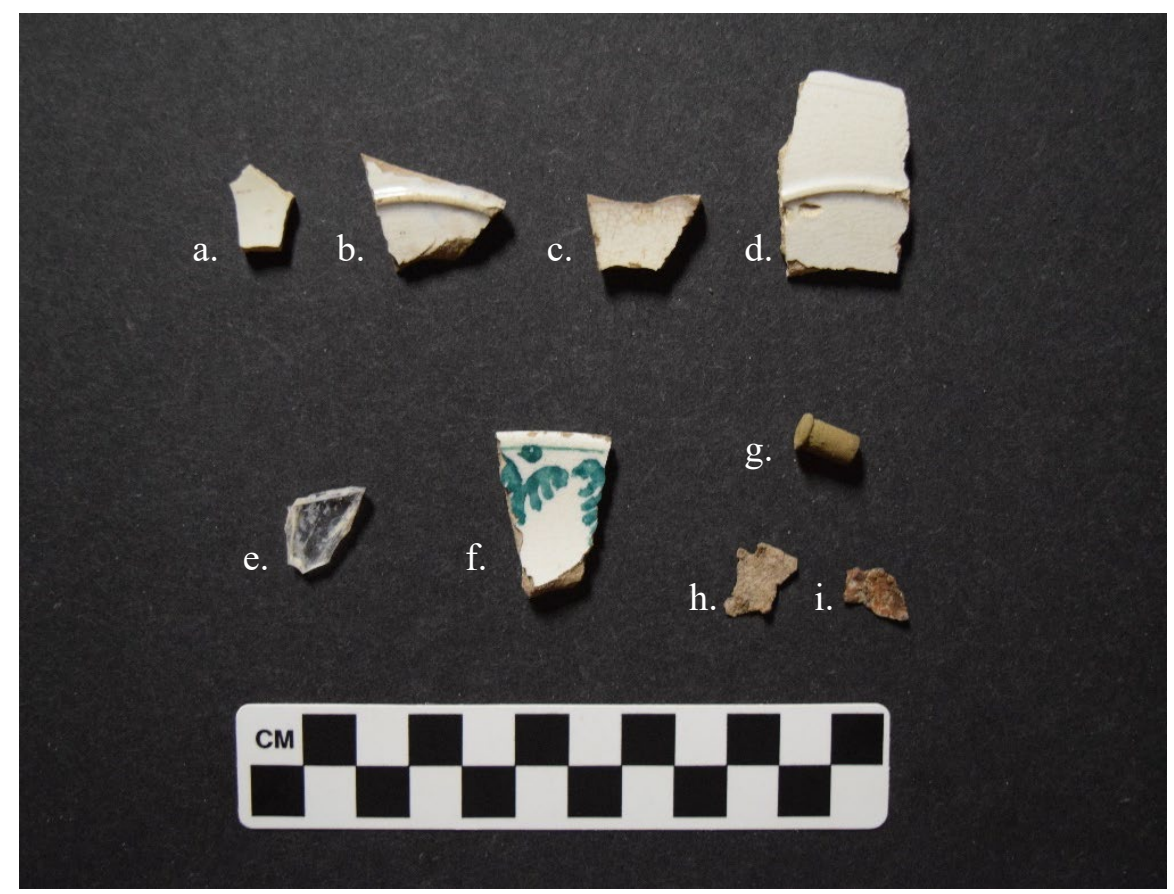

Figure 6.12. Historic Assemblage: Non-diagnostic whiteware sherds: a) Lot 367-5, b) 488-4, c) 497-3, and d) 557-2; Colorless glass: e) Lot 673-5;

Mayer China: f) Lot 578-6; Bullet casing: g) Lot 687-2; and Miscellaneous metal fragments: h) Lot 471-13, and i) Lot 540-6.

All four whiteware sherds (Lots 367-5, 488-4, 497-3, and 557-2) are medial fragments and cannot be identified (see Figure 6.12). The colorless glass fragment (Lot 673-5) is irregular in shape with no diagnostic features; it is thin $(3 \mathrm{~mm})$ and has patina on both faces (see Figure 6.12). The metal bullet casing (Lot 687-2) is a .22 caliber short cartridge casing (see Figure 6.12). These types of cartridges were introduced in 1857. Without the bullet, no further identification could be completed. The three miscellaneous metal fragments (Lots 471-13, 534-7, and 540-6) are small, together only weighing 1.67 grams (Appendix B). The metal fragments are heavily rusted and no identification of their original form or function can be discerned (see Figure 6.12). One piece of miscellaneous metal (Lot 534-7) was encountered in unit N1001 E1003, Level 3 (99.4-99.3 m), which encompasses the western edge and top layer of Feature 4. Feature 4 is discussed in detail later in this chapter.

The single diagnostic ceramic (Lot 578-6) was recovered from unit N1001.5 E1004, Level 2 at an elevation of 99.6 to $99.5 \mathrm{~m}$ (see Figure 4.2). Lot 578-6 is an earthenware rim fragment with a clear glaze and monochromatic green plant-like design (see Figure 6.12), and a very thin green annular ring along the $\mathrm{rim}$. The sherd is thin, $4 \mathrm{~mm}$ at its thickest point, and very slightly curved. Due to the fragmented nature of the specimen, a specific pattern and manufacturing date could not be obtained. Analysis identified the 
most probable manufacturer to be Mayer China (1923-1968), with the closest matching pattern to be the Princess pattern (Snedden 2018).

\section{Burned Clay}

SWCA recovered 41 pieces of burned clay during excavations conducted at the Snakeskin Bluff site (41GU177). All of the pieces of burned clay exhibited amorphous shapes, with sizes ranging from less than $3 \mathrm{~cm}(\mathrm{n}=31)$ to greater than $5 \mathrm{~cm}(\mathrm{n}=1)$, with several in between $(\mathrm{n}=9)$. A representative sampling of the pieces of burned clay is depicted in Figure 6.13. Most of the burned clay was recovered from in and around Feature 3 (discussed below), a single or limited-use hot rock cooking feature radiocarbon dated to 1179-985 cal B.P. (Austin Phase component).

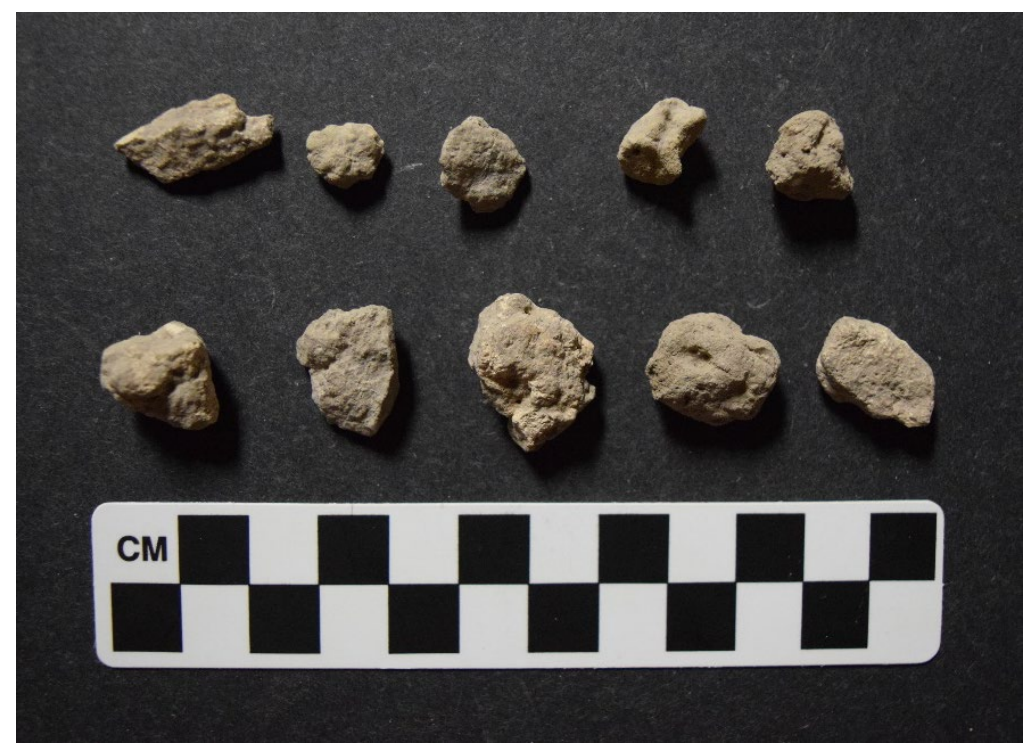

Figure 6.13. Representative sample of pieces of burned clay. Lot $405-9$.

In Feature 3 (unit N996 E1013), the pieces of burned clay were associated with lithic debitage, charcoal flecks, burned bone, snail and mussel shell fragments, and abundant fire-cracked rock (FCR). To the west, in unit N996 E1012, pieces of burned clay were associated with abundant burned bone fragments and charcoal flecking. To the east, in unit N996 E1014, pieces of burned clay were associated with abundant lithic debitage, snail shells, and FCR.

All of the pieces of burned clay contained areas of fire darkening. Fire darkening suggests that these pieces of burned clay continued to be heated or exposed to fire after they were initially baked (Simms et al. 2013:14). On most pieces, this darkening was subtle; however, one piece of burned clay exhibited more extreme darkening. This piece of burned clay was bisected and coronal heat alterations were observed (Figure 6.14). This color gradation, darker at the core and lighter at the surface, reflects differential heating from the core to the surface and suggests that the clay nodule was in its present shape prior to heating (Turpin 2011:287).

Given their association with a hot rock cooking feature, the pieces of burned clay were likely used as heating elements for earth ovens. Pieces of burned clay have been associated with earth ovens archaeologically at sites across North and Central America (Hays et al. 2016; Simms et al. 2013). In experimental recreations, amorphous clay balls have been found to retain heat longer than fire embers and serve to replenish stored heat through conduction (Hunter 1975:67; Hudgins 1993:51). Alternatively, the 
pieces of burned clay could result from natural causes, such as burning brush or tree roots in clay or sandy soils (Hudgins 1993). At the Loeve-Fox site (41WM230) Prewitt (1982) identified a concentration of burned clay that was interpreted as a burned tree root. The pieces of burned clay depicted in the site report appear similar to those recovered from this site (Prewitt 1982:Figure 14a).

At this time, the function of the burned clay pieces remains unclear, although their small size suggests they may be a result of natural causes. Recent studies have shown that microbotanical residues from plants and other materials cooked in earth ovens become embedded in cook stones used as heating elements within the oven (Thoms et al. 2015). Additional research examining these pieces of burned clay for microbotanical residues could confirm whether they were used for cooking or simply a byproduct of brush burning.

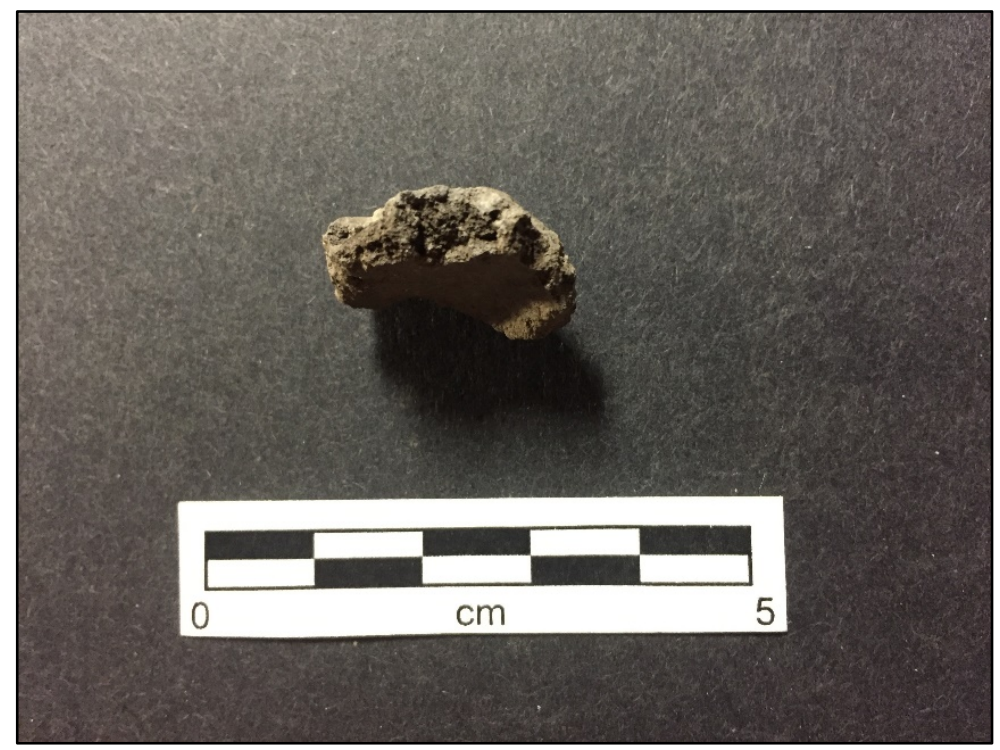

Figure 6.14. Piece of burned clay with coronal heat alteration. Lot 538-6.

\section{FAUNAL REMAINS}

\section{Bone}

SWCA recovered and analyzed 650 vertebrate faunal remains from the excavations (Appendix D). An additional 16 specimens were recovered from the flotation heavy fraction; however, these specimens were not analyzed due to their small size. Each element within the assemblage was thoroughly analyzed by Chris Shelton, M.A., both macroscopically, and with the aid of a 10x microscope combined with an oblique angle light source. SWCA attempted to identify each specimen within the assemblage to skeletal element, as well as to the lowest level of taxonomic classification.

As a result of the high degree of fragmentation, poor preservation environment, and other taphonomic factors, few of the fragments could be attributed to specific elements and/or specific taxa (Appendix D). From the vertebrate assemblage, four specimens could be attributed to biological class Aves, 155 specimens could be attributed to class Mammalia, and 34 specimens of the biological order Testudines (turtle/tortoise). Only 11 specimens could be accurately attributed to the species level, all of which are Odocoileus virginianus (white-tailed deer). A total of 14 specimens could be attributed to large mammals (defined as weighing more than 250 pounds). The large mammal specimens are suspected to be Bison; however, species identification of these elements could not be made with certainty. 
The most prevalent taphonomic processes identified during the analysis are general weathering, geogenic acid etching, and burning (Appendix D). Some form of weathering and/or geogenic acid etching is present on every specimen within the assemblage, and has distorted or obliterated most, if not all, of the cortical surface of the vast majority of the specimens. The poor preservation environment, and high degree of taphonomic processes may have biased the assemblage towards large cortical fragments and larger species. This is evidenced by the complete absence of microfaunal remains and the fact that only 5.6 percent of the assemblage consist of trabecular bone fragments.

Anthropogenic taphonomy is biased against in the assemblage due to the poor preservation of cortical surfaces; however, a total of three specimens were found to exhibit butchering and processing taphonomy (Figure 6.15; Appendix D). One deer metapodial fragment (Lot 576-1) had a distinct percussion notch with a spiral fracture indicative of marrow processing. This specimen also exhibited well-defined pot polish. One other long bone fragment (Lot 323-1) exhibited a percussion notch. This fragment was attributed to a deer-size mammal but could not be definitively attributed to a specific species. Finally, one small fragment (Lot 539-1) exhibited a total of three cut-marks. Non-anthropogenic surface modifications are also biased against in the assemblage, with only a single specimen exhibiting a distinct carnivore tooth pit, and four specimens exhibiting evidence of rodent gnawing.

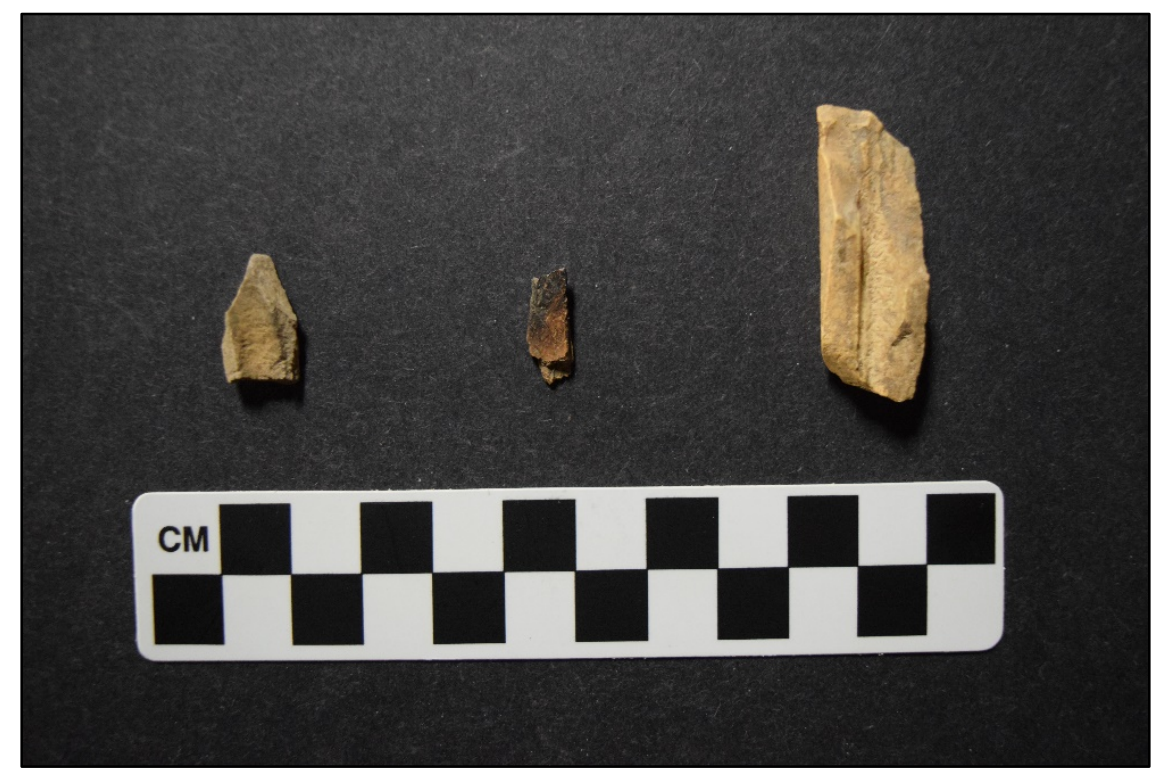

Figure 6.15. Bone exhibiting butchering and processing taphonomy; Percussion notch: a) Lot 323-1; cutmarks: b) Lot 539-1; percussion notch and spiral fracture: c) Lot 576-1.

Although anthropogenic surface modification is biased against in the assemblage, anthropogenic processes can be inferred through the high degree of fragmentation and burning. A total of 98.3 percent of the assemblage consists of bone fragments. Of the fracture margins that can be observed, a high degree showed evidence of green (para-mortem) fracturing. The prevalence of fragmentation, as well as green fracturing, is suggestive of butchering and/or marrow processing. Evidence of burning is present on 13.1 percent of the assemblage and ranges from completely calcined to slight discoloration. The heatrelated discoloration can indicate roasting and cooking, while the calcined and near-calcined elements can indicate the discard of specimens into a campfire. Although fracturing and burning can occur naturally, human processes can be more securely inferred through the presence and proximity of artifacts and thermal features within the site. 


\section{Shell}

Other faunal remains encountered during excavations included 131 mussel shell umbos and numerous observed fragments (Figure 6.16). The mussel shells were collected from a number of contexts, and SWCA Biologist Stephen Van Kampen-Lewis conducted a brief analysis of the shell assemblage, focusing on identifying the various species of mussel shell commonly found in the Guadalupe River. A total of eight species were identified from 46 mussel shell within the $41 \mathrm{GU} 177$ assemblage; the remaining shells were too fragmented for a definitive identification (Table 6.6).

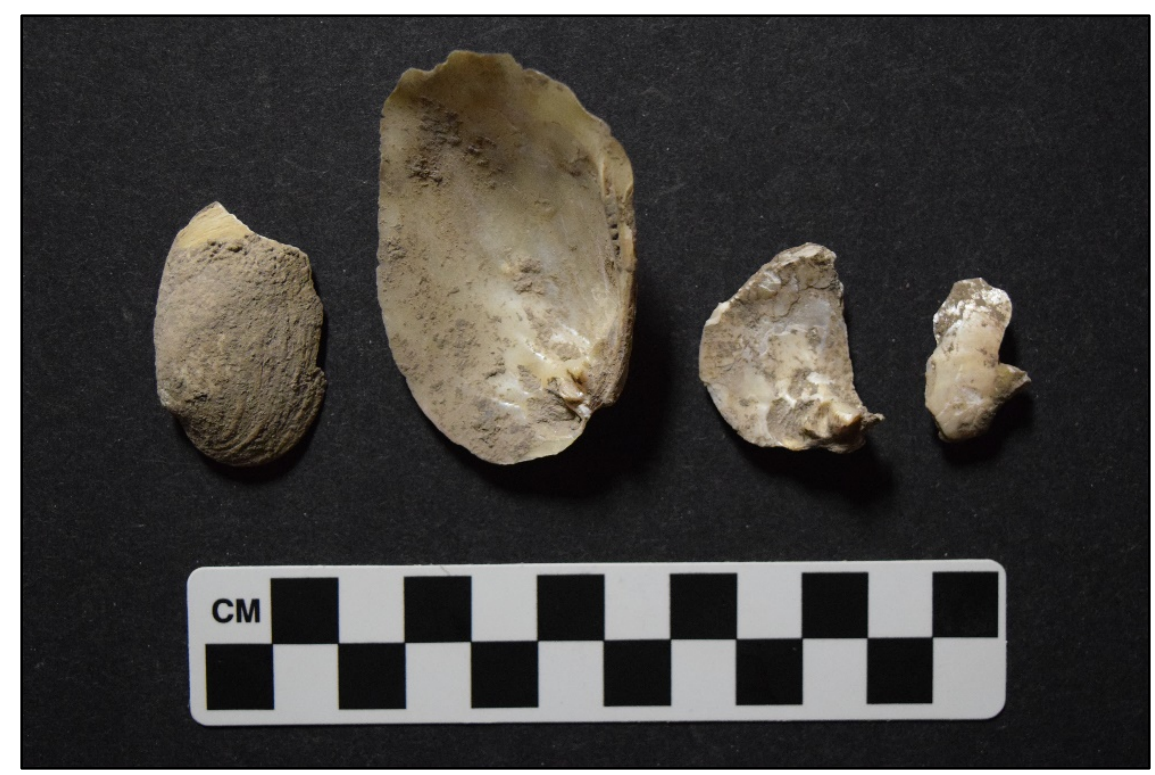

Figure 6.16. Representative sample of mussel shell: a) Lot 589-1 (two on left) and b) Lot 605-6 (two on right).

Table 6.6. Mussel Shell Species Identified within the 41GU177 Assemblage

\begin{tabular}{cccc}
\hline Latin Name & Common Name & Notes & Identified Shells \\
\hline Cyclonaias necki & Guadalupe orb & 33 \\
\hline Cyrtonaias tampicoensis & Tampico pearlymussel & & 3 \\
\hline Fusconaia mitchelli & False spike & P-tooth angled less steeply, W-shaped sculpturing & 1 \\
\hline Megalonaias nervosa & Washboard & P-teeth small, sculptured beak & 1 \\
\hline Toxolasma texasiense & Texas liliput & Lower Guad & 2 \\
\hline Tritogonia verrucosa & Pistolgrip & & 3 \\
\hline Uniomerus declivis & Tapered pondhorn & Lateral teeth more horizontal & 1 \\
\hline Uniomerus tetralasmus & Pondhorn & & 1 \\
\hline
\end{tabular}

Texas' freshwater mussels exhibit phenotypic plasticity depending on inhabited drainage and/or specific environmental conditions that can change the shell shape (e.g., elongate forms occurring in swiftwater and rounded forms occurring in slackwater). Some species are also sexually dimorphic, further complicating identification. As such, identifying individuals is often difficult, even when assessed by experts (Hess et al. 2018). However, knowing a mussel's home drainage greatly reduces the potential list of species and increases the potential for correct identification. SWCA assumed all shells are from the Guadalupe River and the species list was assembled using the North American Freshwater Mussel Identification Guide (application undergoing Beta testing from Science Apps) and Howells (2014). 
Overall, the shells and shell fragments represent a mix of currently common and uncommon species known to inhabit the Guadalupe River drainage. Most identified species are from areas with stable substrate in moving water such as creeks or rivers. However, the pondhorn (Uniomerus tetralasmus) and paper pondshell (Utterbackia imbecillis) are usually found in slow-moving backwaters or impoundments with soft, muddy substrate. Interestingly, the most commonly found species is the Guadalupe orb (Cyclonaias necki), which was taxonomically split by Burlakova et al. (2018) from the Texas pimpleback (Cyclonaias petrina). The Texas pimpleback is rare enough to be considered a candidate species for protection under the Endangered Species Act and the Guadalupe orb may also qualify for such protection. The Guadalupe orb shell color can be a vibrant orange or yellow (Figure 6.17). In addition to a food source, these shells might have been selected for use as pendants or other adornments due to their bright color.

Vertical mussel migration within the substrate during stressful conditions is well documented throughout the United States, with many species burying themselves deeper during winter (Amyot and Downing 1997; Schwalb and Pusch 2007; Block et al. 2013). The Texas Parks and Wildlife Department (2018) prefers mussel relocations from April through November or when water temperatures are above $60^{\circ} \mathrm{F}$, based on relocation protocol from Carlson et al. (2008) and Mackie et al (2008). However, very little research has been conducted on seasonal mussel assemblage movements in Central Texas. As such, it is unclear if any mussel species burrow deeper during winter than during other seasons. It is also unclear if stream dwelling species are physically capable of burrowing deeper, considering the generally rocky nature of Central Texas river substrate. The lack of clarity regarding seasonal vertical migration specifically in Central Texas makes it difficult to determine mussel availability to human gatherers throughout the year.

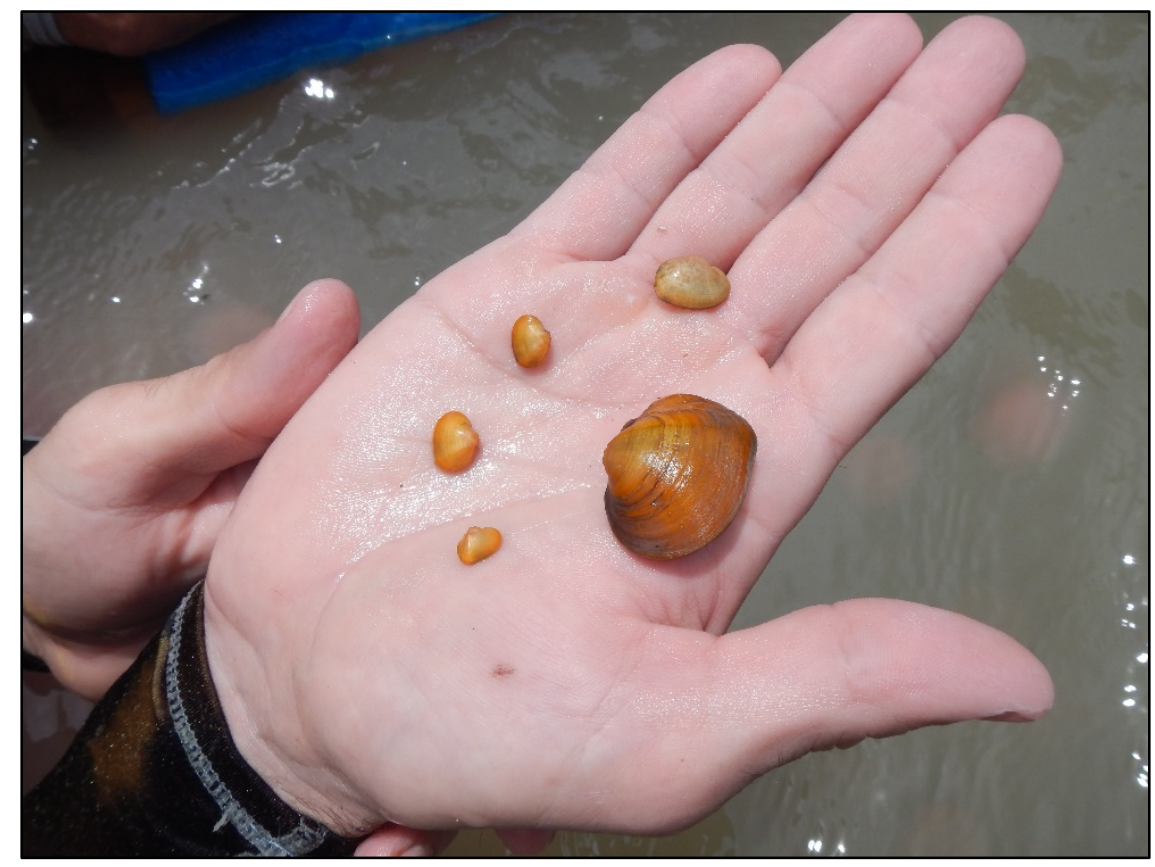

Figure 6.17. Modern Guadalupe orb shell from Palmetto State Park in Gonzales County, Texas. Note the vibrant orange color of the shell.

\section{SPECIAL SAMPLES}

The data recovery excavations at $41 \mathrm{GU} 177$ recovered 305 special samples from appropriate contexts across the site. These included materials for radiometric dating, matrix samples for flotation, flora and 
wood identification samples, and geoarchaeological samples, which included bulk matrix and MS samples.

SWCA recovered 11 charcoal samples from various contexts throughout the site, with the majority procured directly from feature contexts. Of these, five samples were submitted for radiocarbon dating, including three from feature contexts. These results are provided in Appendix E . One additional sample was initially submitted for radiocarbon dating, but ended up being too small of a sample to be processed.

The five feature matrix samples were subjected to flotation. The heavy fraction of the flotation samples was sorted for debitage, bone, mussel shell, and burned rock. The light fraction of the flotation samples, as well as selected flora and wood identification samples recovered from the excavations, were subjected to macrobotanical analysis. The results of the macrobotanical analysis from each of the features, including the flora and wood identification samples, are discussed in the feature section below and are also presented in Appendix F.

SWCA collected 51 bulk matrix samples for particle size analysis and 237 geoarchaeological samples were collected for MS analysis. The bulk matrix and geoarchaeological samples were collected from feature contexts, when feasible, and from one profile in each excavation block. As mentioned in Chapter 5 , particle size and texture analysis was conducted on samples from the southern and northern areas of the excavation area (Blocks A and E) and MS analysis was conducted on six vertical sample columns (Blocks $\mathrm{A}$ and $\mathrm{C}-\mathrm{E}$ ), and one horizontal column centered on Feature 4 (Block C). In addition, five feature matrix samples were collected from the site, with one sample from Feature 2 and two samples each from Features 3 and 4.

\section{FEATURES}

Three cultural features (Features 2-4) were documented over the course of the data recovery investigation across the site; all three features were documented within the Austin Phase component and yielded dates that are consistent with the prevailing chronological placement of the Austin Phase. Flotation samples were collected from each feature and the remaining feature matrix (if present) was screened out in the field and artifacts collected and bagged. It should be noted that an additional feature (Feature 1) was documented during the testing phase of the project and was reported on in the $41 \mathrm{GU} 177$ testing interim report in Appendix A (Rodriguez et al. 2017).

\section{Feature 2}

Feature 2, the smallest observed on the site, was a discrete, irregularly shaped, burned rock concentration associated with the end of the Austin Phase (Table 6.7). It was initially encountered in the southeast corner of excavation unit N1004 E1018 between elevations 99.8 and $99.7 \mathrm{~m}$. Three additional $1 \times 1-\mathrm{m}$ units (N1003 E1018, N1003 E1019, and N1004 E1019) were opened to explore the features extent. The burned rock concentration encompassed portions of the four excavation units centered just southwest of the N1004 and E1019 grid intersection from an elevation of 99.77 to $98.50 \mathrm{~m}$ (Figure 6.18). Feature 2 measured $53 \mathrm{~cm}$ north/south by $30 \mathrm{~cm}$ in size once fully exposed. Bioturbation, namely from roots, was noted within the feature.

The feature consisted of thermally altered limestone $(n=11)$ of various shapes and sizes (see Table 6.7). Most of the altered limestone was 5 to $10 \mathrm{~cm}$ in diameter $(\mathrm{n}=7)$. Three of the limestone rocks exhibited pitting on their surfaces. One large $(15 \mathrm{~cm}$ diameter) limestone cobble with multiple fractures was located at the center of the feature. Five additional rocks were located at the center of the feature with three observed overlapping each other beneath the large central cobble (Figure 6.19). The remaining five burned rocks were encountered scattered to the east in unit N1003 E1019. 
Table 6.7. Feature 2

\begin{tabular}{ll}
\hline Type & Diffuse burned rock cluster \\
\hline Geomorphic Stratum & III and IV \\
\hline Cultural Component & Austin Phase/ Toyah Transition \\
\hline Excavation Block & E \\
\hline Units & N1003-1004 E1018-1019 \\
\hline Center & N1003.94 E1018.90 \\
\hline Top Elev. (m) & 98.77 \\
\hline Bottom Elev. (m) & 98.50 \\
\hline Origination (m) & Unknown \\
\hline Dimensions (cm) & $53 \times 30$ \\
\hline Bulk/Flotation Samples & F-2 and F-3 \\
\hline Special Samples & None \\
\hline Geoarch Samples & S-1 through S-12: Magnetic \\
\hline C-14 Sample & Susceptibility samples \\
\hline $\begin{array}{l}\text { Radiocarbon Age } \\
\text { (Conventional B.P.) }\end{array}$ & $\begin{array}{l}\text { Charred Vetch (Vicia ludoviciana) } \\
\text { from flotation sample (Lot 672-1) }\end{array}$ \\
\hline Associated Diagnostic & Lot 672-1: 670 \pm 30 (Beta \\
Artifacts (Lot No.) & $492582)$ \\
\hline & Fresno Point (Lot 607-6) \\
\hline
\end{tabular}

\begin{tabular}{lrr}
\hline \multicolumn{3}{l}{ Burned Rock Characteristics } \\
\hline Burned Rock Size (cm) & Count & Weight (kg) \\
\hline $\mathbf{0 - 5}$ & 3 & 0.2 \\
\hline $\mathbf{5 - 1 0}$ & 7 & 1 \\
\hline $\mathbf{1 0 - 1 5}$ & 1 & 1.8 \\
\hline $\mathbf{1 5 +}$ & - & - \\
\hline Total & 11 & 3 \\
\hline
\end{tabular}

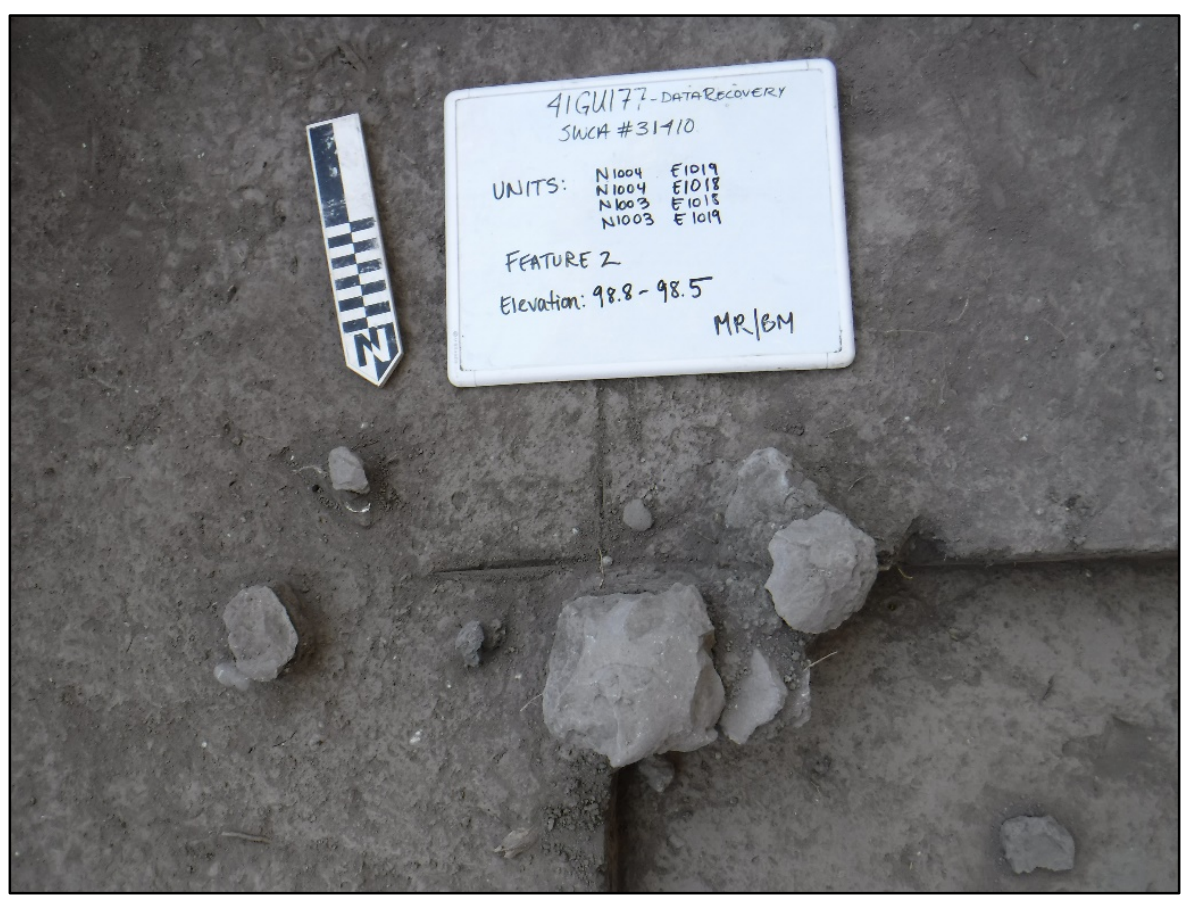

Figure 6.18. Feature 2 plan view. 


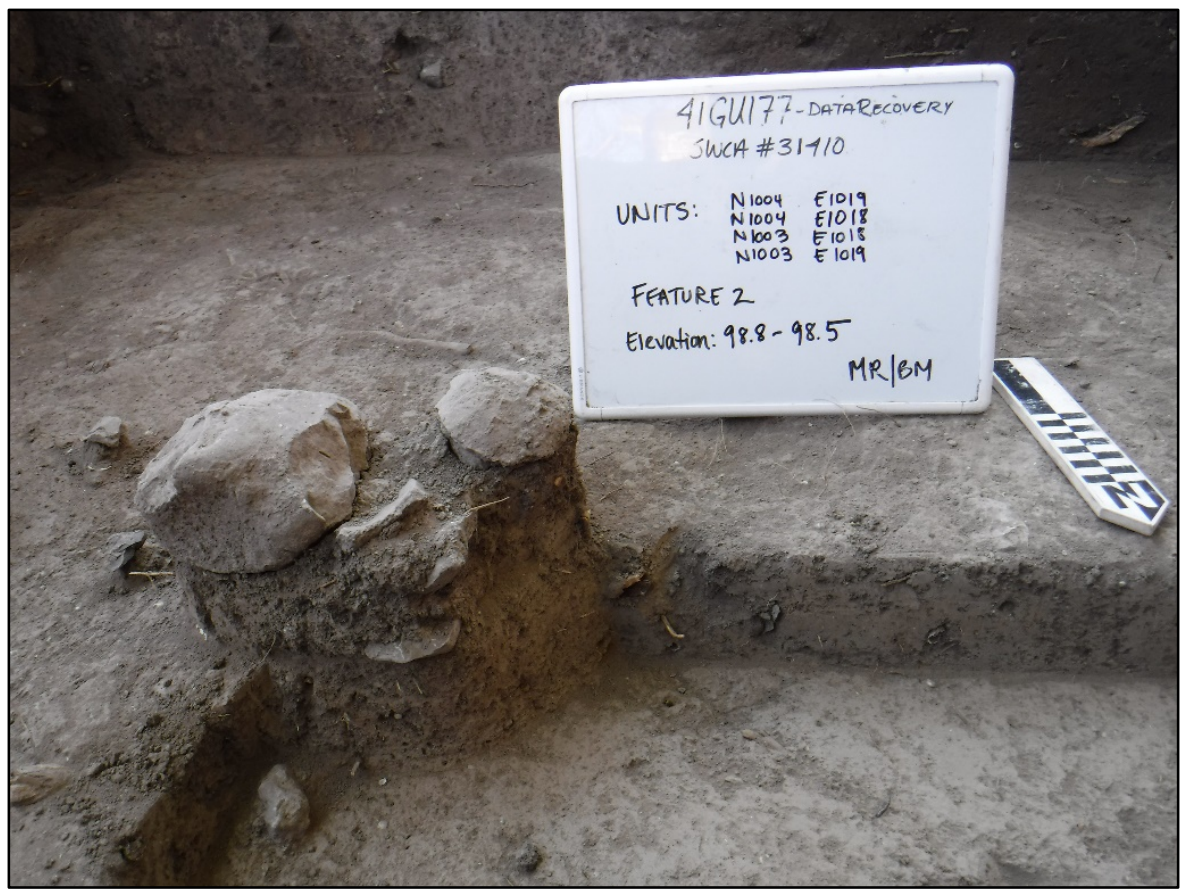

Figure 6.19. Feature 2, northwest profile showing overlapping burned rock, facing south.

Once the feature was exposed in planview, it was bisected, and SWCA collected two gallon-size bags of matrix for flotation and macrobotanical analysis. The remaining matrix was screened in the field; however, no cultural materials were recovered. Additionally, 12 vertical MS samples were collected from the feature bisection profile.

The internal matrix of Feature 2 between 53-80 cmbd (98.77-98.5 m elevation) did not contain any evidence of burning (e.g., charcoal, burned soil, ash) and no evidence of in situ thermal alteration (e.g., reddening) of the Feature 2 soils was observed. However, the macrobotanical analysis of the floated fine fraction from the feature matrix identified several species of charred wood, including elm (Ulmus spp.), mesquite (Prosopis glandulosa), sugarberry (Celtis sp.), acacia/mesquite (Vachellia/Prosopis sp.), hickory/pecan (Carya sp.), grape (Vitis spp.), and an indeterminable hardwood (Appendix F). Charred vetch identified within the flotation sample (Lot 672-1) was submitted for radiocarbon dating and returned a calibrated age range of 676-559 cal B.P. (see Table 6.7). Chronologically, this places Feature 2 within the later part of the Austin Phase or early part of the Toyah Component.

Although no cultural material was encountered from the feature matrix screened in the field, artifacts were recovered from the Feature 2 flotation samples. The assemblage consists of 34 pieces of debitage ( 4 proximal flakes, 3 broken flakes, 19 flaking shatter, and 8 thermal shatter), and one manuport, as well as 28 pieces of small thermally altered stone (FCR). In addition to the artifacts, ecofacts recovered from Feature 2 include one piece of bone and two mussel shell umbos. No temporally diagnostic artifacts were found in direct association with the feature; however, a Late Prehistoric Fresno arrow point was recovered from unit N1003 E1018 between 98.7 and $98.6 \mathrm{~m}$, which is contiguous.

\section{Summary}

Feature 2 is a discrete burned rock cluster located at the transition of Strata III and IV, within the Austin Phase. The lack of a pit or basin and the absence of obvious thermally altered sediment suggests that 
Feature 2 may represent a peripheral clean out area near a cooking locus; however, no feature of this type was identified within excavation Block E.

\section{Feature 3}

Feature 3 consisted of two discrete burned rock and chert clusters (Cluster 1 and 2) with an associated diffuse chert and burned rock scatter within the Austin Phase component (Table 6.8). Burned rock from Cluster 1 was initially encountered in the north wall of excavation unit N995 E1013 between elevations 99.0 and $98.9 \mathrm{~m}$. One additional $1 \times 1-\mathrm{m}$ unit (N996 E1013) was initially opened to the north to explore the feature. A second burned rock cluster (Cluster 2) was encountered in the west wall of this unit, so SWCA subsequently opened two additional $1 \times 1-\mathrm{m}$ units (N996 E1012 and N995 E1012) to further explore the features extent. Cluster 1, in units N995 E1013 and N996 E1013, measured $12 \mathrm{~cm}$ north/south by $22 \mathrm{~cm}$ east/west in size and Cluster 2 in units N996 E 1012 and N996 E1013 measured $35 \mathrm{~cm}$ north/south by $26 \mathrm{~cm}$ east/west (Figure 6.20). Bioturbation from roots and rodents was noted around the feature clusters.

Table 6.8. Feature 3

\begin{tabular}{|c|c|c|}
\hline Type & \multicolumn{2}{|c|}{$\begin{array}{l}\text { Two discrete burned chert clusters with } \\
\text { associated diffuse chert scatter }\end{array}$} \\
\hline Geomorphic Stratum & III & \\
\hline Cultural Component & Austin Phase & \\
\hline Excavation Block & $\mathrm{A}$ & \\
\hline Cluster & Cluster 1 & Cluster 2 \\
\hline Units & N995-996 E1013 & N996 E1012-1013 \\
\hline Center & N996.00 E1013.37 & N996.48 E1013.08 \\
\hline Top Elev. (m) & 98.98 & 99.00 \\
\hline Bottom Elev. (m) & 98.90 & 98.90 \\
\hline Origination (m) & Unknown & Unknown \\
\hline Dimensions (cm) & $35 \times 26$ & $12 \times 22$ \\
\hline Bulk/Flotation Samples & $\mathrm{F}-4$ & $\mathrm{~F}-5$ \\
\hline Special Samples & None & None \\
\hline Geoarch Samples & None & None \\
\hline C-14 Sample & $\begin{array}{l}\text { C-11 (bone); } \\
\text { Bluewood condalia } \\
\text { (Condalia hookeri) } \\
\text { from flotation sample } \\
\text { (Lot 421-1) }\end{array}$ & $\begin{array}{l}\text { None within cluster; } \\
\text { C-12 to C-14 } \\
\text { collected north and } \\
\text { west of cluster }\end{array}$ \\
\hline $\begin{array}{l}\text { Radiocarbon Age } \\
\text { (Conventional B.P.) }\end{array}$ & $\begin{array}{l}\text { Lot 421-1: } 1170 \pm 30 \\
\text { (Beta 492584) }\end{array}$ & None \\
\hline $\begin{array}{l}\text { Associated Diagnostic } \\
\text { Artifacts (Lot No.) }\end{array}$ & None & None \\
\hline
\end{tabular}

Burned Rock Characteristics

\begin{tabular}{crr}
\hline Burned Rock Size (cm) & Count $^{*}$ & Weight (kg) \\
\hline $\mathbf{0 - 5}$ & 2 & 0.2 \\
\hline $\mathbf{5 - 1 0}$ & 21 & 2.4 \\
\hline $\mathbf{1 0 - 1 5}$ & 2 & 0.5 \\
\hline $\mathbf{1 5 +}$ & - & - \\
\hline Total & 25 & 3.1 \\
\hline
\end{tabular}

*burned rock total includes both clusters and associated scatter 


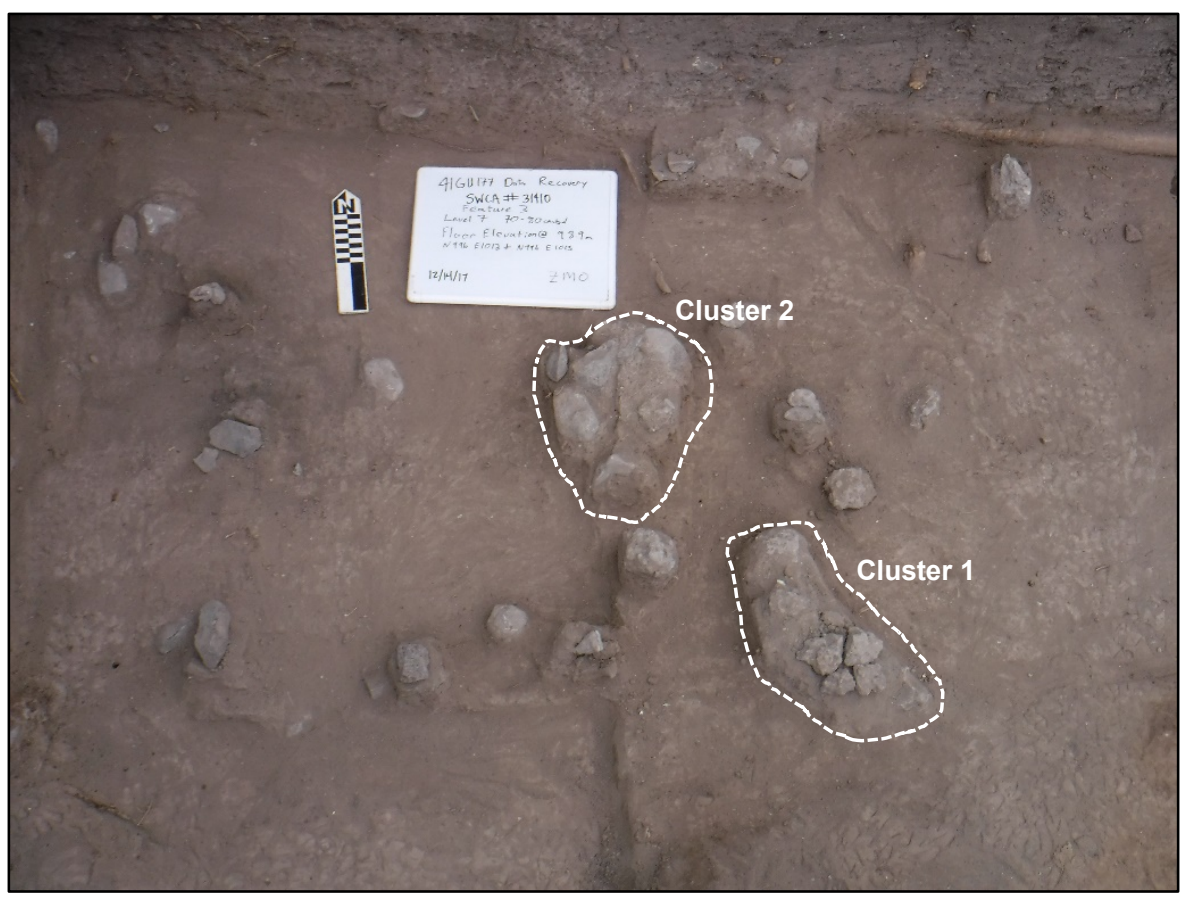

Figure 6.20. Feature 3 plan view showing location of Clusters 1 and 2.

The feature consisted of a single layer of thermally altered limestone $(n=16)$, silicified limestone $(n=1)$, and burned chert $(\mathrm{n}=8)$ of various shapes and sizes. Most of the altered limestone was 5 to $10 \mathrm{~cm}$ in diameter $(\mathrm{n}=21)$ (see Table 6.8). The burned rocks were primarily rounded, while approximately 25 percent were angular. Most of the rocks within Cluster 1 appeared to be fractured in situ, but due to erosion appeared very pitted. Cluster 2 consisted of mostly rounded, unfractured burned chert cobbles with a few adjacent angular limestone. The remaining feature rocks were diffusely scattered throughout units N996 E1012 and N996 E1013 and were a mixture of rounded and pitted limestone, angular limestone, and burned chert, a few of which were also fractured in situ or had refits in proximity. The majority of Feature 3 burned rocks are located within Stratum III between 74 to $77 \mathrm{cmbd}$ (98.96 to 98.97 m elevation), suggesting a consistent occupation surface with no apparent basin morphology.

Once the feature was uncovered, each of the clusters was bisected, and the matrix was collected for flotation. The internal matrix of Feature 3 between 99.9-98.9 contained small flecks of burned bone and charcoal indicating burning. In addition, evidence of in situ thermal alteration was observed and consisted of burned soil around the feature rock, as well as flecks of charcoal below and around the burned rock. One charcoal sample (Sample No. C-11) was collected from Cluster 1; however, analysis determined the sample was burned bone rather than charcoal. No additional radiocarbon samples were taken directly from the feature clusters, as no charcoal large enough for dating was visible during recording and sampling. Three radiocarbon samples (Sample Nos. C-12 through C-14) were collected northeast and west of Cluster 2 and additional charred macrobotanical remains were recovered from within the feature matrix fine fraction (see Table 5.7 and Appendix F).

Charred bluewood condalia from the floatation sample light fraction from Cluster 2 (Lot 421-1) was submitted for radiocarbon dating and produced a calibrated age range of 1179-985 cal B.P.; this date falls within the Austin Phase (see Table 5.7). In addition to the charred bluewood condalia submitted for radiocarbon dating, the macrobotanical analysis of the floated fine fraction from the feature matrix identified several species of charred wood, including red mulberry (Morus rubra), juniper (Juniperus spp.), sugarberry, silktassel (Garrya ovata), and indeterminable hardwood (Appendix F). 
Artifacts recovered from the Cluster 1 flotation sample consisted of 23 pieces of debitage (1 complete flake, 1 proximal flake, 12 flaking shatter, and 9 thermal shatter), and nine pieces of bone. The Cluster 2 flotation sample contained 20 pieces of debitage ( 1 broken flake, 6 flaking shatter, and 13 thermal shatter), and one bone. Additionally, over 150 pieces of debitage were recovered from the surrounding units, of which almost 50 percent consisted of thermal shatter, as well as two cores, one chopper, one edge-modified flake; 11 pieces of burned clay were also recovered from within the feature's extent. No temporally diagnostic artifacts were found in direct association with the feature or within any of the excavation units within Block A.

\section{Summary}

Feature 3 consists of two discrete burned rock and chert clusters and a burned rock and chert scatter located within Stratum III within the Austin Phase component. No pit or basin was observed but the presence of thermally altered sediment and rocks fractured in situ suggests Feature 3 represents a single or limited use hot rock cooking event during the Austin Phase. Subsequent disturbance from rodents likely dispersed portions of the feature rock, resulting in the peripheral scatter observed around the two clusters.

\section{Feature 4}

Feature 4 was a shallow, basin-shaped cooking feature in the Austin Phase component within Block C (Table 6.9). As mentioned in Chapter 4, Feature 4 was first noted as a cluster of burned rocks in the northeast quadrant of unit N1000 E1004, Level 5 in Excavation Block C. Numerous non-traditional, feature-focused units were opened around the initial cluster to further investigate the extent, thickness, and composition of the feature and to identify potential drop zones and/or discard areas (see Figure 4.2). In addition, Level 1 of some of these units was stripped off without screening to just above Feature 4 and were not excavated below Feature 4 after it was documented and removed.

The feature was located primarily within units N1000 E1004, N1001 E1004, and N1001 E1004.5 with the extreme western and northern portions extending into units N1001 E1003 and N1001.5 E1004. The concentration was principally two layers of burned limestone rocks in a slight basin shape with a circular to oval shape.

Layer 1 measured $106 \mathrm{~cm}$ north/south and $96 \mathrm{~cm}$ east/west, and ranged in elevation from $99.42 \mathrm{~m}$ to $99.28 \mathrm{~m}$ and was a mixture of angular and rounded limestone (Figures 6.21 and 6.22; see Table 6.9). Most of the rocks in this layer slanted down slightly towards the center of the burned rock concentration. Another cluster of burned rocks was encountered in Layer 1 just west of the main feature concentration and measured $58 \mathrm{~cm}$ north/south and $68 \mathrm{~cm}$ east/west. These rocks appeared to be in secondary deposition, possibly discarded lid rocks, based on their lack of uniform orientation and lack of in situ thermal alteration in the immediate area (see Figure 6.21).

Layer 2 was primarily located in units N1000 E1004, N1001 E1004, and N1001 E1004.5 and measured $55 \mathrm{~cm} \mathrm{north} /$ south and $68 \mathrm{~cm}$ east/west and ranged in elevation from $99.28 \mathrm{~m}$ to $99.20 \mathrm{~m}$ (Figures 6.23 and 6.24). Most of the rocks in this layer also slanted down slightly towards the center of the burned rock concentration. 
Table 6.9. Feature 4

\begin{tabular}{|c|c|c|}
\hline Type & \multicolumn{2}{|l|}{ Earth Oven } \\
\hline $\begin{array}{l}\text { Geomorphic } \\
\text { Stratum }\end{array}$ & \multicolumn{2}{|l|}{ III } \\
\hline $\begin{array}{l}\text { Cultural } \\
\text { Component }\end{array}$ & \multicolumn{2}{|l|}{ Austin Phase } \\
\hline Excavation Block & \multicolumn{2}{|l|}{$\mathrm{C}$} \\
\hline Layer & Layer 1 & Layer 2 \\
\hline Units & $\begin{array}{l}\text { N1000 E1004; N1001 E1003; } \\
\text { N1001 E1004; } \\
\text { N1001 E1004.5; N1001.5 E1004 }\end{array}$ & $\begin{array}{l}\text { N1000 E1004; } \\
\text { N1001 E1004; } \\
\text { N1001 E1004.5 }\end{array}$ \\
\hline Center & N1001.20 E1004.60 & N1001.15 E1004.70 \\
\hline Top Elev. (m) & 99.42 & 99.28 \\
\hline Bottom Elev. (m) & 99.28 & 99.20 \\
\hline Origination $(\mathrm{m})$ & $\mathrm{N} / \mathrm{A}$ & 99.20 \\
\hline Dimensions (cm) & $\begin{array}{l}106 \times 96 \text { (heating element); } \\
58 \times 68 \text { (possible discard area) }\end{array}$ & $55 \times 68$ \\
\hline $\begin{array}{l}\text { Bulk/Flotation } \\
\text { Samples }\end{array}$ & $F-6$ and $F-7$ & $\mathrm{~F}-8$ and $\mathrm{F}-9$ \\
\hline Special Samples & None & None \\
\hline Geoarch Samples & $M-1$ to $M-5$ & $M-1 A$ to $M 4 A$ \\
\hline C-14 Sample & $\mathrm{C}-15$ to $\mathrm{C}-19$ & $\begin{array}{l}\text { C-20 to C-21; } \\
\text { Camas bulb scale } \\
\text { (Camassia sp.) from } \\
\text { flotation sample (Lot } \\
529-1 \text { ) }\end{array}$ \\
\hline $\begin{array}{l}\text { Radiocarbon Age } \\
\text { (Conventional B.P.) }\end{array}$ & None & $\begin{array}{l}\text { Lot 529-1: } 970 \pm 30 \\
\text { (Beta 492583) }\end{array}$ \\
\hline $\begin{array}{l}\text { Associated } \\
\text { Diagnostic } \\
\text { Artifacts (Lot No.) }\end{array}$ & None & None \\
\hline
\end{tabular}

\section{Burned Rock Characteristics}

\begin{tabular}{lrr}
\hline $\begin{array}{l}\text { Burned Rock } \\
\text { Size }(\mathbf{c m})\end{array}$ & Count $^{*}$ & Weight (kg) \\
\hline $\mathbf{0 - 5}$ & 75 & 0.8 \\
\hline $\mathbf{5 - 1 0}$ & 78 & 12.6 \\
\hline $\mathbf{1 0 - 1 5}$ & 26 & 14.2 \\
\hline $\mathbf{1 5 +}$ & 12 & 15.5 \\
\hline Total & 191 & 43.1 \\
\hline
\end{tabular}

*burned rock total includes both rock layers

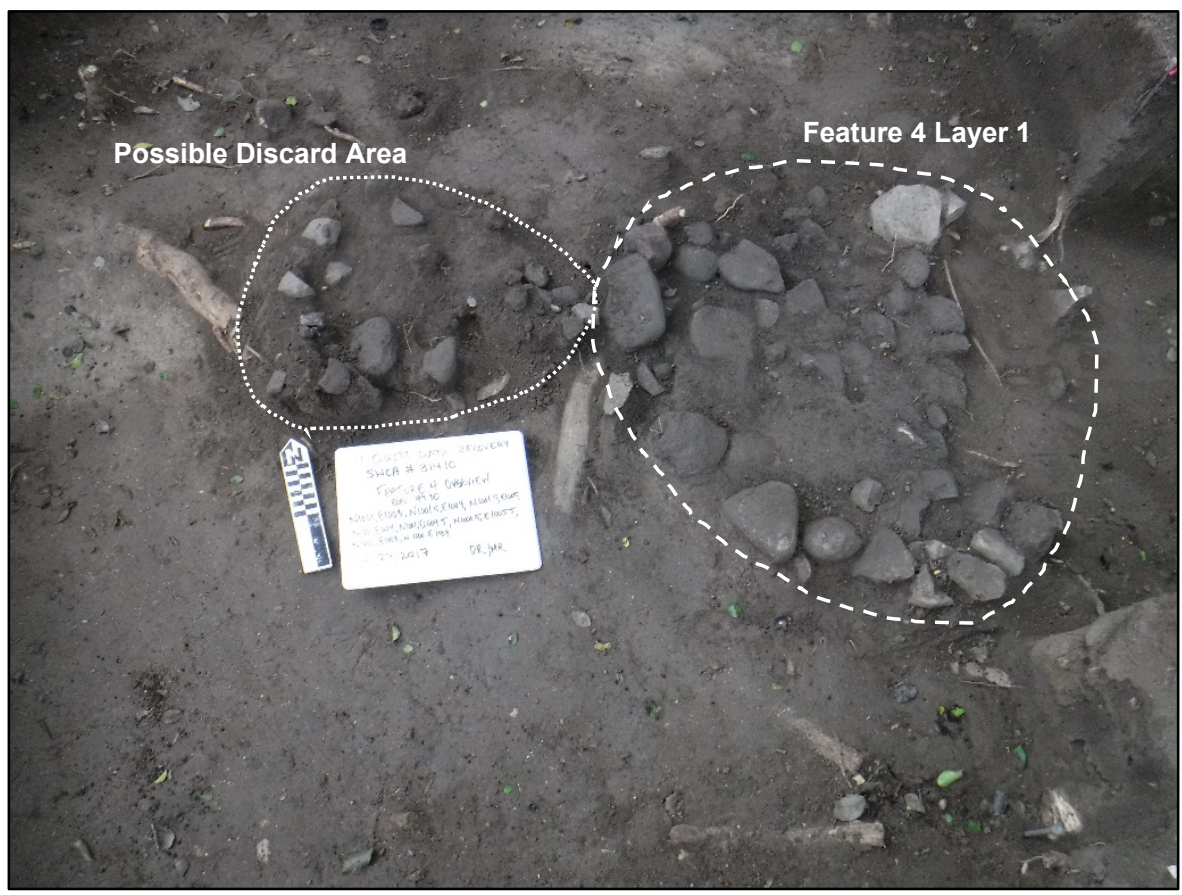

Figure 6.21. Feature 4 Layer 1 plan view, note possible discard area to west. 


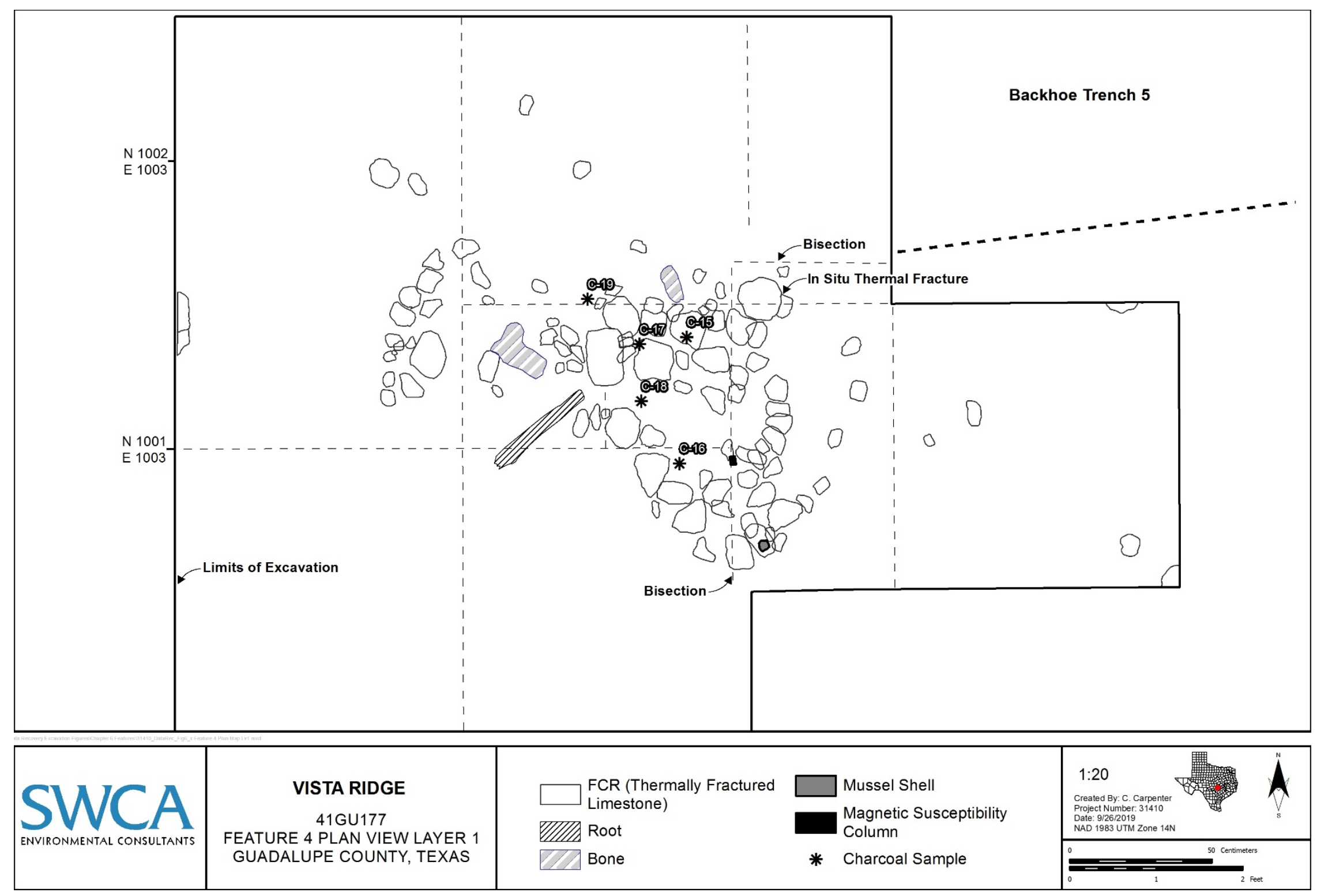

Figure 6.22. Feature 4 Layer 1 Plan map. 


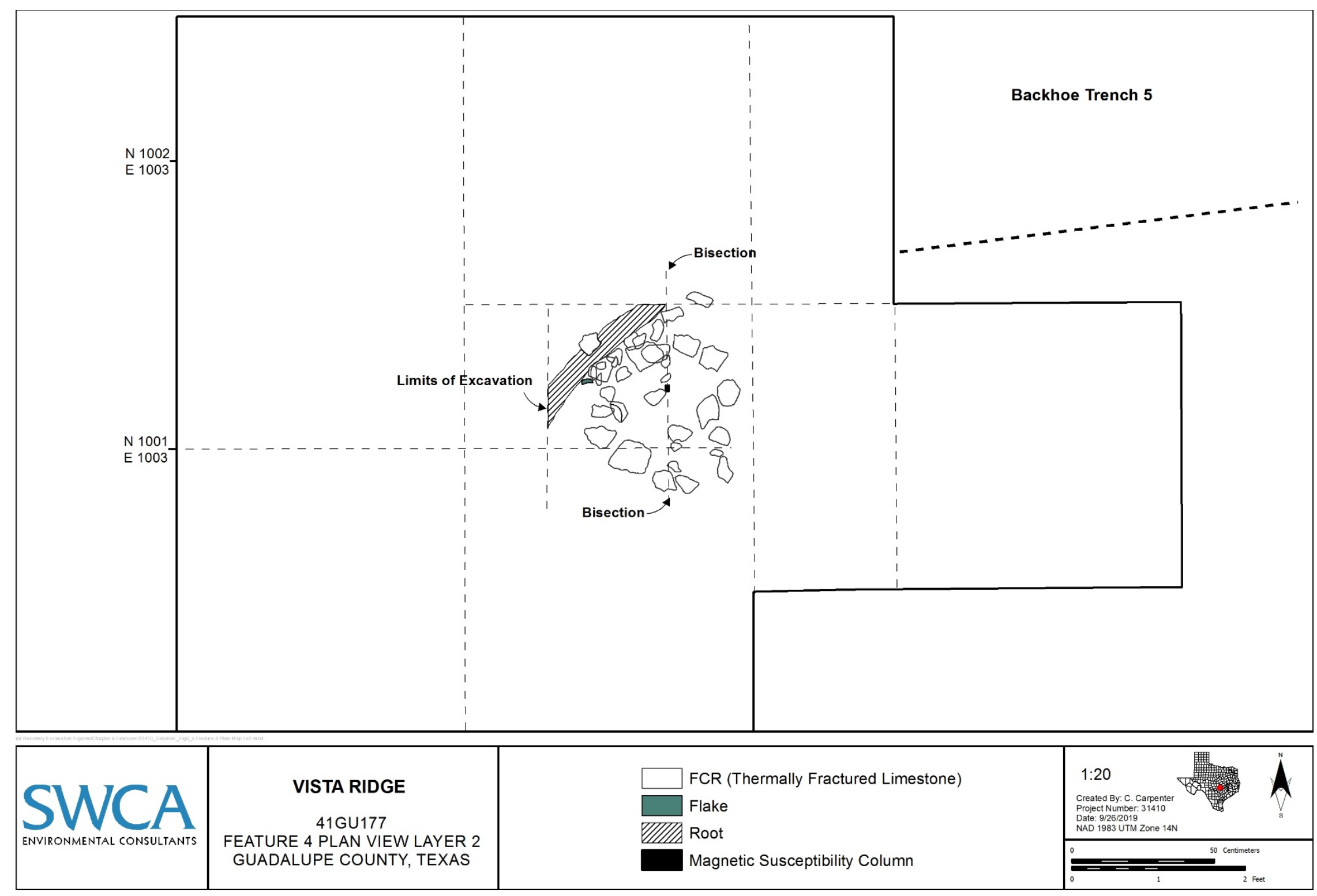

Figure 6.23. Feature 4 Layer 2 Plan map. 


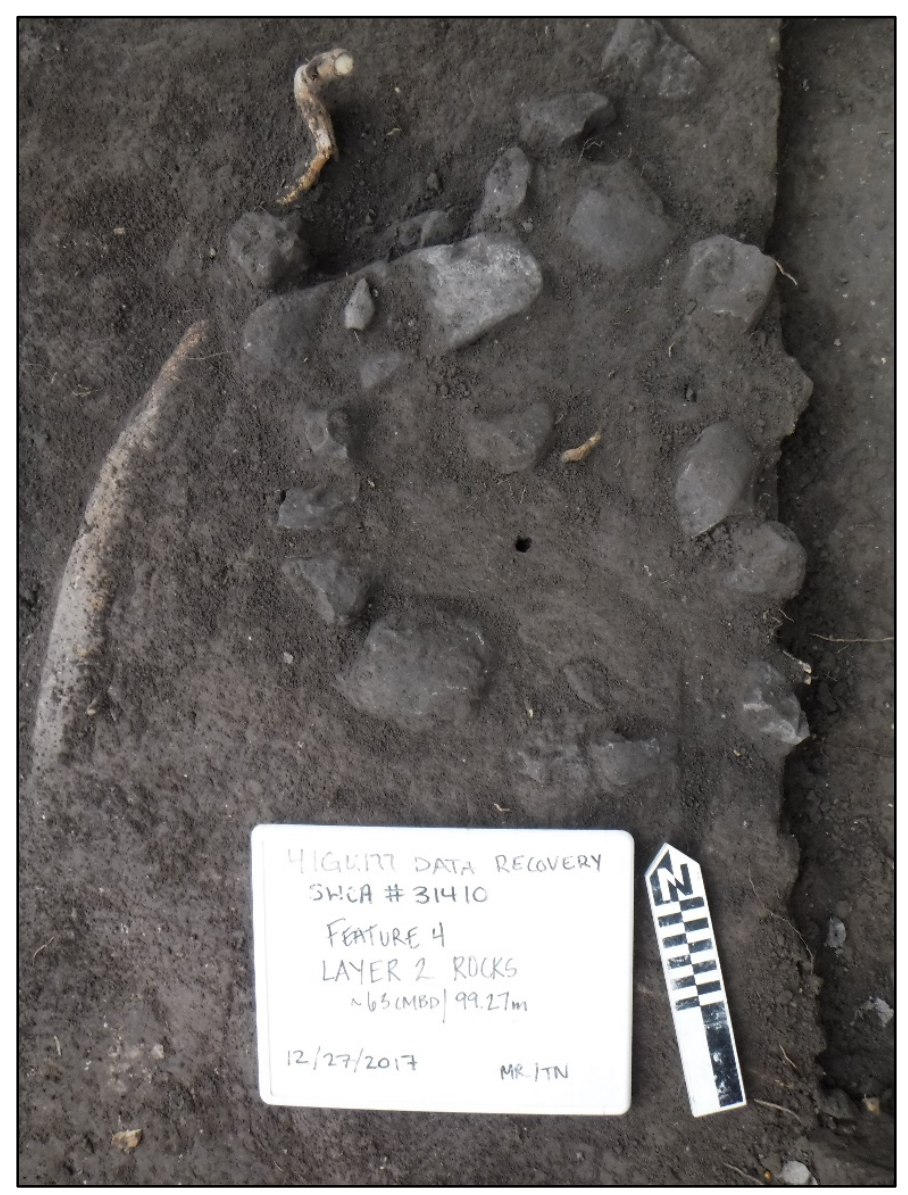

Figure 6.24. Feature 4 Layer 2 plan view.

Charcoal flecking was observed between and around the periphery of the feature. Larger charcoal pieces were recovered from below the rocks in both Layer 1 and 2, and a dark soil stain was observed below the Layer 2 rocks. One radiocarbon sample (Lot 529-1) collected from the feature returned a calibrated age range of 934-796 cal B.P., which falls within the Austin Phase; the sample was a charred camas bulb scale.

In addition to the charred camas bulb submitted for radiocarbon dating, the macrobotanical analysis of the floated fine fraction from the feature matrix identified several species of charred wood including elm, mesquite, red mulberry, juniper, ash (Fraxinus sp.), and indeterminable hardwood, as well as a sugarberry seed and an indeterminable plant part (Appendix F). Artifacts recovered from the Feature include 79 pieces of debitage (over 50 percent of which was thermal shatter), one edge-modified flake, nine pieces of bone, and one burned clay. No temporally diagnostic artifacts were found in direct association with the feature; however, a Toyah Phase Perdiz arrow point was recovered just above the feature in unit N1001.5 E101004 between 99.5 and 99.4 m and Leon Plain ceramics were found in N1000 E1003 from 99.6$99.3 \mathrm{~m}$.

\section{Summary}

Feature 4 comprised burned rocks placed in a shallow basin forming a basal heating element. The presence of a lid in close proximity to the primary feature element further supports this categorization. Overall, Feature 4 is the most substantial burned rock feature identified on the site. The size of the feature 
( $1 \mathrm{~m}$ maximum diameter), quantity of rocks, and basin shape indicate the highest investment of labor of any of the thermal features on the site.

\section{CULTURAL STRATA}

The investigations across the Snakeskin Bluff site (41GU177) encountered cultural components encompassing the Late Archaic to Late Prehistoric periods. A majority of the cultural assemblage at the site was observed intermixed at or near the surface with multiple time periods present. Despite these factors, a cultural stratigraphy could be discerned using the temporally diagnostic artifacts and radiocarbon results at $41 \mathrm{GU} 177$.

As mentioned above, the radiocarbon results at the site indicate that there is an association between Stratum II and the Toyah Phase of the Late Prehistoric, while the underlying Stratum III is associated with the Late Prehistoric. More specifically, the upper portions of Stratum III are associated with the Austin Phase of the Late Prehistoric. Additional stratigraphic resolution is provided when overlaying the temporally diagnostic artifacts to the site stratigraphy. Near the base of Stratum II, a Perdiz arrow point was recovered in Unit N1001 E1004 (Excavation Block C), which correlates with the Toyah Phase. Near the base of Stratum III and top of Stratum IV in Unit N1003 E1018 (Excavation Block E), a Fresno projectile point was recovered. This artifact is commonly associated with the Late Prehistoric. Underlying this projectile point near the base of Stratum IV and top of Stratum V in Unit N1003 E1019 (Excavation Block E), a Zephyr projectile point was encountered. The Zephyr projectile point is commonly identified as a Transitional Archaic artifact. Finally, the one anomalous stratigraphic position for a temporally diagnostic artifact was an Edwards point at the top of Stratum VI in Unit N1004 E1019 (Excavation Block E). This Late Prehistoric Austin Phase artifact appears to be stratigraphically out of context as it is chronologically younger than the projectile points above it. Accordingly, the Edwards projectile point in this unit is interpreted to be vertically displaced (i.e., translocated) due to bioturbation (e.g., burrows or tree roots).

In summary, the cultural strata observed at 41GU177 have some localized areas of distinct stratification and recognizable contextual integrity, but the predominance of the cultural assemblage closer to the ground surface is an intermixed palimpsest. From the surface downward, Stratum I is modern in age and disturbed. Based on temporally diagnostic artifacts (i.e., Perdiz) and radiocarbon data, Stratum II is associated with the Late Prehistoric Toyah Phase and has some areas (particularly the eastern side of the site) that have been disturbed. The underlying Stratum III is definitively associated with the Austin Phase and all of the Late Prehistoric, based on a temporally diagnostic artifact (i.e., Fresno) and radiocarbon data. Based on the Zephyr projectile point, Strata IV-V are associated with the Transitional Archaic. Therefore, the lowermost horizons (Strata VI-VII) predate the Transitional Archaic and are assuredly associated with the Late Archaic or earlier.

Various analyses (e.g., MS, discussed above) corroborate the stratigraphic interpretations during the site excavations. Specifically, the uppermost horizons (Strata I and II) appear to have been affected by earth movement or comparable influences. 


\section{CHAPTER 7. SYNTHESIS AND RECOMMENDATIONS}

\section{SITE SUMMARY}

The Snakeskin Bluff site (41GU177) is a multi-component prehistoric residential camp on the terraces of the Guadalupe River in Guadalupe County, Texas. The uppermost cultural deposits at the site (Stratum I) are a disturbed palimpsest with both modern and prehistoric materials. The Late Prehistoric Toyah Phase component (Stratum II), just below these deposits, exhibited some evidence of disturbance (e.g., bioturbation and heavy equipment); however, these disturbances were primarily observed within the eastern portions of the site. The Austin Phase component (Stratum III) contained intact, well-preserved archaeological deposits that were the primary focus of investigations. Immediately underlying the Austin Phase component, a zone of Transitional Archaic deposits (Strata IV-V) were identified. Below the Transitional Archaic zone, Late Archaic (or possibly earlier) components were also identified (Strata VIVII); however, no temporally diagnostic artifacts were recovered from this earlier component and the dated radiocarbon sample was rejected due to probable translocation, so the actual occupation period for this component is unknown.

Overall, SWCA excavated $34.1 \mathrm{~m}^{3}$ of sediment from the site through both traditional and feature-focused unit excavations. During these excavations, approximately $22 \mathrm{~m}^{2}$ of Late Prehistoric components were exposed, consisting of rock-lined hearths and 6,496 artifacts. The Late Prehistoric artifacts included Edwards points, Perdiz points, a Fresno point, ceramics, bifaces, an end scraper, a shell bead, various informal lithic tools, ground stone, choppers, debitage, and faunal remains. The Late Prehistoric component was approximately $40-80 \mathrm{~cm}$ thick. No cultural features were identified within the underlying Archaic components of the site; however, 3,421 artifacts were recovered including a Zephyr point, bifaces, ground stone, various informal lithic tools, choppers, debitage, and faunal remains. The Transitional Archaic component was approximately 35-60 cm thick; however, the underlying Archaic (and possible older) components were not defined.

The excavations were limited to the right-of-way and the exposure afforded only a partial glimpse of the overall site. Based on the assemblage, the site is interpreted as a logistical base camp, as indicated by both formal and informal tool forms and associated site furniture. Small groups exploited the abundance of lithic raw material and riparian zone resources, making forays into the landscape to hunt and forage. Four radiocarbon dates from the Late Prehistoric components reveal several short-term encampments over the course of several centuries from approximately 600 to 1200 B.P. (A.D. 750 to 1350). The Toyah Phase component has some noted disturbances (especially towards the ground surface), but good stratigraphic integrity from where the radiocarbon sample was collected. The Austin Phase component is vertically and horizontally discrete, contains a substantial amount of archaeological materials, and the site structure and radiocarbon dates suggest multiple, discrete occupations.

\section{REVIEW OF RESEARCH QUESTIONS AND FINDINGS}

The research objectives in the study of the Snakeskin Bluff site were structured around three specific questions that pertain to chronology, lithic technology, and foraging strategies. SWCA designed the specific research questions to address the environmental, technological, chronological, and adaptive changes during the transition from Archaic to Late Prehistoric patterns. The artifact recovery at the site was not as robust as hoped for and disturbances were noted, especially within the upper deposits of the site. Nonetheless, some interpretations on context and site function were possible, despite the relatively small dataset from the site. 


\section{Research Issue 1: Regional Chronology and 41GU177}

The cultural and natural depositional sequence in Central Texas during the shift from the end of the Archaic to Late Prehistory has long been a difficult one to sort out. The lack of well-dated sites with components from this time has been a main contributing factor. We were hopeful that additional radiocarbon data and recovered temporarily diagnostic projectile points from the Snakeskin Bluff site would aid in refining the regional chronology. Unfortunately, a low density of charcoal samples and temporally diagnostic artifacts were recovered within the investigated portions of the site, providing a limited dataset. SWCA submitted five samples from the Snakeskin Bluff site for radiocarbon dating analysis. The samples primarily came from cultural contexts (e.g., feature matrix) and focused on the stratigraphy and cultural zones seemingly affiliated with the Late Prehistoric and older Archaic horizons. Radiocarbon results from the lowest deposits at the site indicate that vertical displacement and/or contamination of archaeological materials (including diagnostics and faunal materials) likely occurred in portions of the site; therefore, one of the radiocarbon dates was rejected.

As mentioned in Chapter 5, the remaining four radiocarbon assays are associated with the Late Prehistoric component in Strata II and III. The faunal sample from Stratum II returned a calibrated age of 552-518 cal B.P. and samples from the three features (i.e., Features 2-4) in Stratum III returned calibrated dates of 1173-1058 cal B.P. (Feature 3), 929-802 cal B.P. (Feature 4), and 670-566 cal B.P. (Feature 2). Based on these data, as well as the recovered temporally diagnostic artifacts, Stratum III appears to date to approximately 1200-600 B.P., while the overlying Stratum II dates from 600 B.P. to sometime before the present. In addition, the radiocarbon data indicate Stratum III may be associated with the Austin Phase, whereas Stratum II appears to be associated with the Toyah Phase (see Collins 2004; Gadus et al. 2006; Johnson 1994; Johnson and Goode 1994; Ricklis and Collins 1995). Recovery of a temporally diagnostic artifact (Zephyr point) at the base of Stratum IV suggests the deposits underlying Stratum III encompass the Transitional Archaic, with possible Late Archaic deposits situated underneath; however, the radiocarbon date from Stratum VI was rejected and no other radiocarbon dates were obtained, so the date range of the underlying components is currently unknown.

In comparing these dates to regional chronologies, the Snakeskin Bluff site supports a growing consensus of chronological breaks at or near 1250 and 650 B.P. All chronologies place the advent of the Scallorn and Edwards stylistic interval at around 1250 B.P. Four chronologies define this as the end of the Archaic and start of the Late Prehistoric, while Johnson and Goode (1994) indicate the Post-Archaic perhaps began earlier with smaller dart points. Snakeskin Bluff dates concur with 1250 B.P. as the earliest extreme of the break, although most of the Austin Phase dates are between 1175 and 670 B.P. (Collins 2004; Prewitt 1985; Black 1989; Johnson 1995; Johnson and Goode 1994; Turner et al. 2011; Carpenter et al. 2013:Figure 9.8). The date for the strata containing Perdiz points on the Snakeskin Bluff site ranges from 518 to 552 B.P., which is consistent with models for the Toyah Phase (Arnn 2012:64-65; Johnson 1994:87; Prewitt 1981:84).

\section{Research Issue 2: Lithic Technology}

The lithic analysis for 41GU177 was focused on determining if lithic reduction strategies and tool production at the site were consistent with other Transitional Archaic and Late Prehistoric sites in the region. The lithic tool assemblage for the individual Late Prehistoric components (i.e., Toyah Phase and Austin Phase) was fairly small and would not be statistically significant, so the following discussion relates to the combined Late Prehistoric components and combined Archaic components.

In broad terms, an assemblage refers to the collective material culture of a community (Deetz 1967:109). Assemblage-based systematics analyze the relative frequency among artifact classes to infer behavioral implications, especially through comparative studies. The basic assumption underlying such studies is 
that "variation in the structure and content of an archaeological assemblage is directly related to the form, nature, and spatial arrangement of human activities" (Binford and Binford 1966:241). The objective in the study of lithic assemblages from the Snakeskin Bluff site is to identify differences in organization of technology and strategies between the Late Prehistoric and Archaic components. The interpretive weight of the analysis of Snakeskin Bluff site assemblages needs to be tempered by a strong word of caution. The components of the site have reasonably good stratigraphic integrity, but the artifacts are more subject to movement. The artifact assemblages for each component, therefore, are undoubtedly mixed to varying degrees, rendering their study a somewhat blunt instrument. These are coarse-grained assemblages, the cumulative debris from repeated occupations. Within this overarching consideration, the patterns are explored here to search for broad trends that can contribute to the overall picture. That said, there are various comparative categories that can be used to assess behavioral change but this section focuses on three specific variables: 1) relative frequency of tool categories; 2) ratio of bifaces and cores; and 3) ratio of debitage to stone tools.

In terms of the first set of variables, a primary consideration is the ratio among cores, bifaces, and flake tools. A more equitable statistical distribution among the forms would be expected at longer-term collector base camps, partly because repeated occupations causes coarse-grained assemblages. Table 7.1 shows the percentages of the various tool categories by component. Several trends are notable:

- Bifaces, as a percentage of each component's assemblage, slightly decrease through time; they compose 25.58 percent of the artifact assemblage in the Late Prehistoric, but only 24.53 percent in the Archaic.

- Cores tend to be underrepresented in the younger component (12.79 percent) and overrepresented in the older component ( 20.75 percent). In other words, cores are 200 percent more represented in the Archaic.

- Modified flakes tend to be overrepresented in the younger component (52.33 percent) and underrepresented in the older component (41.51 percent), which equates to modified flakes being 127 percent more represented in the Late Prehistoric.

Table 7.1. Summary of Lithic Assemblage from Snakeskin Bluff Components

\begin{tabular}{|c|c|c|c|c|c|c|}
\hline \multirow{2}{*}{ Artifact Type } & \multicolumn{2}{|c|}{ Late Prehistoric Component } & \multicolumn{2}{|c|}{ Archaic Component } & \multirow{2}{*}{$\begin{array}{l}\text { Total } \\
\text { Count }\end{array}$} & \multirow{2}{*}{$\begin{array}{c}\begin{array}{c}\text { Relative } \\
\text { Assemblage } \\
\text { Percent* }^{*}\end{array} \\
\text { Percent }^{*}\end{array}$} \\
\hline & Count & Percent* & Count & Percent $^{*}$ & & \\
\hline Projectile Points & 4 & 4.65 & 3 & 5.66 & 7 & 5.04 \\
\hline Scrapers & 1 & 1.16 & 0 & 0.00 & 1 & 0.72 \\
\hline Bifaces & 22 & 25.58 & 13 & 24.53 & 35 & 25.18 \\
\hline Cores & 11 & 12.79 & 11 & 20.75 & 22 & 15.83 \\
\hline Core Tools & 1 & 1.16 & 3 & 5.66 & 4 & 2.88 \\
\hline Ground Stones & 2 & 2.33 & 1 & 1.89 & 3 & 2.16 \\
\hline Modified Flakes & 45 & 52.33 & 22 & 41.51 & 67 & 48.20 \\
\hline Debitage & 5,732 & $\mathrm{~N} / \mathrm{A}$ & 3,122 & $\mathrm{~N} / \mathrm{A}$ & 8,854 & $\mathrm{~N} / \mathrm{A}$ \\
\hline Total $^{\ddagger}$ & 86 & & 53 & & 139 & \\
\hline
\end{tabular}


Some observations are suspect because of low numbers. For example, in terms of assemblage diversity, the component with the highest numbers of tools have the greatest diversity of tools forms, and so the richness index is likely subject to critical thresholds of population size. Additionally, categories such as projectile points, ground stone, core tools, and scrapers, have sample sizes that may be too small to explore trends.

The ratio of bifaces to cores shows a distinct difference in bifacial technology between the components (Table 7.2). The Late Prehistoric component has a much higher ratio of bifaces to cores than the Archaic component, indicating an emphasis on bifacial technology in the Late Prehistoric. Compared to a collector base camp, a forager residential base ought to reveal a decrease in cores relative to bifaces. In general, biface use increases with mobility, although Tomka (2001) identifies mitigating circumstances, and flakecore use increases with longer occupations. While it depends on the site function, if the Snakeskin Bluff site is a residential base camp in both collector and foraging strategies, then the ratio of cores to bifaces should be an indicator of the changing strategies over time.

Table 7.2. Biface to Core Ratio among Snakeskin Bluff Components

\begin{tabular}{lcc}
\hline Artifact Type & Late Prehistoric Component & Archaic Component \\
\hline Bifaces & 22 & 13 \\
\hline Cores & 11 & 11 \\
\hline Ratio & $2: 1$ & $1.2: 1$ \\
\hline
\end{tabular}

Comparing the biface-to-core ratios to other regional data, a two-to-one ratio is within reasonable expectations of quasi-sedentary pattern according to North American data compiled by Parry and Kelly (1987) (Table 7.3). The ratio for the Archaic components suggest a more sedentary pattern, and reliance on bifacial technology appears to have decreased quite a bit during this time.

Table 7.3. Comparative Biface: Core Ratio Data from Parry and Kelly (1987)

\begin{tabular}{lcc}
\hline Archaeological Group & Sedentism/Mobility Pattern & $\begin{array}{c}\text { Biface to Core ratio according to Parry and } \\
\text { Kelly (1987) }\end{array}$ \\
\hline Oaxaca Archaic & Quasi-sedentism & 1.09 \\
Oaxaca Formative & Sedentism & 0.03 \\
\hline Black Mesa Archaic & Mobile hunter-gatherers & 5.75 \\
Black Mesa BMII & Quasi-sedentism & 2.38 \\
Black Mesa PI & Sedentism & 0.45 \\
Black Mesa PII & Sedentism & 0.04 \\
\hline SW Colorado Archaic & Mobile hunter-gatherers & 5.75 \\
SW Colorado BMII & Early quasi-sedentism & 2.83 \\
SW Colorado BMIII & Quasi-sedentism & 0.71 \\
SW Colorado PI & Sedentism & 0.95 \\
SW Colorado PII & Sedentism & 0.7 \\
\hline Chaco Preceramic & Quasi-sedentism & 0.8 \\
Chaco Puebloan & Sedentism & 0.13 \\
\hline Knife River ND Paleo/EA & Mobile hunter-gatherers & 3.52 \\
Knife River ND Archaic & Mobile hunter-gatherers & 2.92 \\
Knife River ND Plains Village & Sedentism & 1.34 \\
\hline
\end{tabular}


The ratio of debitage-to-lithic tools seems to correlate with the biface-to-core ratios; however, there are multiple variables that may affect this ratio. Occupational redundancy, for one, tends to increase the ratio and contributes to a coarser grained assemblage. Raw material availability is likewise a more significant influence, but many of the environmental factors remain fairly constant when looking at a single site, indicating differences throughout the components should reflect technological variation. One important factor is that bifacial reduction yields quite a bit of debitage compared to expedient core-flake production. Along this line, Late Prehistoric yielded the highest ratio of debitage to tools, perhaps correlating with and corroborating the high biface to core ratio in the same component (Table 7.4). Conversely, the Archaic component has a lower debitage-to-tool ratio, and it also has a lower biface-to-core ratio.

Table 7.4. Ratio of Debitage to Tools by Component on the Snakeskin Bluff Site

\begin{tabular}{lccc}
\hline Artifact Type & $\begin{array}{c}\text { Late Prehistoric } \\
\text { Component }\end{array}$ & $\begin{array}{c}\text { Archaic } \\
\text { Component }\end{array}$ & $\begin{array}{c}\text { Relative } \\
\text { Assemblage }\end{array}$ \\
\hline Debitage & 5,732 & 3,122 & 8,854 \\
\hline Total No. of Tools & 86 & 53 & 139 \\
\hline Ratio of Debitage to Tools - No. of Debitage per Tool & 66.65 & 58.91 & 63.70 \\
\hline
\end{tabular}

\section{Research Issue 3: Foraging Strategies}

Foraging strategies pertain to the ways in which the site occupants organized themselves and their technology to interact with their physical setting. The archaeological materials at the Snakeskin Bluff site indicate variation in ecological adaptations through time. The research question on the topic regards the comparison of Snakeskin Bluff site patterns to prevailing models, particularly the transition from Archaic to Late Prehistoric lifeways in the eastern Edwards Plateau cultures. The general approach to the analysis of these strategies at the Snakeskin Bluff site was to look at the relationships among three data sets: 1) environmental data; 2) subsistence-related data; and 3) technological data.

Based on these relationships, the Snakeskin Bluff site data show pronounced diachronic shifts in the longterm subsistence strategies. In the Archaic deposits (approximately 1,320 years ago and older), the site shows minimal occupational evidence. No cultural features were identified and artifact densities were relatively low. Based on the site evidence as well as the regional record, this period is inferred to be a period of generalized foraging with relatively low occupational intensity. Much of the faunal assemblage $(n=98)$ was unidentifiable due to the high level of fragmentation; however, the Archaic assemblage appears to show a relatively low diet breadth, as most of the fragments are suspected to be deer. The high degree of green (para-mortem) fracturing and prevalent fragmentation in the Archaic assemblage suggests butchering and/or marrow processing was occurring at the site during this period. Four fragments of turtle shell were also recovered, along with 80 freshwater mussel shell umbo fragments. As mentioned in Chapter 6, seasonal vertical migration of freshwater mussel shells in Central Texas is not clear, making it difficult to determine if mussels would have been available to human gatherers throughout the year or if they were only available in the warmer months.

This time of low archaeological visibility is followed by a prominent shift in patterns and much higher archaeological visibility in the Austin Phase of the Late Prehistoric, from 1,270 to 670 years ago. The faunal assemblage still shows a low diet breadth, once again focusing on deer. The faunal assemblage indicates groups likely occupied the site on a seasonal basis in the late fall to early winter, intensively harvesting deer. Similar to the Archaic deposits, the faunal assemblage in the Austin Phase deposits also exhibited a high degree of green fracturing and fragmentation, suggesting butchering and/or marrow processing was still occurring at the site during this period. Over a 600 percent increase in exploitation of 
turtles and a 162 percent decrease in the exploitation of freshwater mussels compared to the Archaic was also noted.

Rather than logistical groups moving in for a short duration to exploit deer, the Late Prehistoric Austin phase groups appear to have more substantial occupational debris, notably marked by formal site furniture (e.g., cooking features). SWCA identified three cultural features within the Austin Phase component: Feature 2 is a peripheral clean-out area presumably near a cooking locus (though the cooking locus was not encountered within the project area); Feature 3 is a discrete, burned rock cluster interpreted to be a single- or limited-use hot rock cooking event; and Feature 4 was a large earth oven. The size of Feature 4 ( $1 \mathrm{~m}$ maximum diameter), as well as quantity of rocks and basin shape, indicate a high investment of labor for food processing.

The results of the macrobotanical analysis revealed the archaeological plant remains associated with the cultural features at the Snakeskin Bluff site consist primarily of wood charcoal, interpreted as fuel wood most likely used in earth oven cooking, such as with Feature 4 described above (Appendix F). The most common fuel sources identified from feature contexts at the site include red mulberry, elm, mesquite, juniper, sugarberry, and indeterminable hardwoods. Less-common woods present in smaller numbers and only a single feature context were bluewood, ash, silktassel, pecan/hickory, and grape. However, some findings from the macrobotanical analysis clearly reflect subsistence resources; geophytes and seeds are likely economic resources with traces in the archaeological record. One camas bulb fragment, one sugarberry seed, and two deer pea vetch seeds were identified from direct feature contexts and all were burned (Appendix F).

Overall, the floral subsistence evidence suggests a moderately diverse exploitation of locally available resources but no intensive processing. Caution is warranted, since such remains are the most perishable of all, but the material assemblage partially supports such a view. Classic signatures of plant processing, such as manos, metates, or nutting stones, are not prominent parts of the assemblage, even in relative terms, as only one ground stone specimen (an indeterminate fragment) was recovered from the site that may have been used for plant processing.

The patterns in the Toyah Phase component (670 to 270 years ago) were similar to the Austin Phase; however, bison now appears in the faunal assemblage and cultural features are absent. When the landscape offers a more equitable distribution of critical resources or a higher availability of high-ranking resources (e.g., bison), groups often respond by increasing mobility and exploiting the increased biomass availability, dropping the more intensive processing of low ranked resources. So residential mobility increases, and logistical mobility declines. These economic strategies are the driving force in subsistence selection and the organization of technology (Binford 1980; Kelly 1992; Winterhalder and Smith 1981). As mentioned in the Lithic Technology section above, the biface-to-core ratios increased at the site in the Late Prehistoric, supporting the idea that mobility and biface use increased at the site. Deer is still present in the Toyah Phase assemblage; however, these faunal remains are primarily in mixed contexts and may have come from the overlying historic/modern deposits and/or the underlying Austin Phase component. Based on the site evidence, as well as the regional record, this period is inferred to be a period focused on bison hunting with relatively low occupational intensity and higher mobility. 


\section{SUMMARY AND RECOMMENDATIONS}

The data recovery investigations at the Snakeskin Bluff site sought to address environmental, technological, chronological, and adaptive changes during the transition from Archaic to Late Prehistoric. As mentioned, the overall artifact and feature recovery at the site was low and disturbances and mixing of components was noted, especially within the upper deposits of the site. Disturbances within the Late Prehistoric Toyah Phase were most prevalent in the eastern portions of the site. The Late Prehistoric Austin Phase component contained intact, well-preserved archaeological deposits containing preserved flora and faunal material, cooking features, and diagnostic implements. The Transitional Archaic (and older) occupations were difficult to characterize, due to limited quantities of temporal diagnostic artifacts, lack of cultural features, and low artifact recovery. Despite these limitations, the data recovered from the cultural components show diachronic shifts between the technological and foraging strategies of the Archaic and Late Prehistoric.

The Archaic appears to be a period of relatively low occupational intensity and the faunal assemblages indicate groups had a relatively low diet breadth focused on deer as well as freshwater mussels. Fracturing and fragmentation of the faunal remains suggests butchering and intensive processing (e.g., marrow extraction) was occurring at the site during this period. The Late Prehistoric Austin Phase groups were continuing to exploit mostly deer and local freshwater mussel; however, these groups had more substantial occupational debris, including several cooking features. The Austin Phase groups were exploiting locally available floral resources for both fuel and food sources but intensive processing of plant materials does not appear to have occurred at the site. Bison appear in the faunal assemblage in the Toyah Phase; the lack of cultural features and increase in bifacial technology during this period suggests these groups were becoming more mobile, likely as a response to the availability of high-ranking resources (e.g., bison) and reduced need for intensive processing of lower ranked resources. The suite of radiocarbon and temporally diagnostic artifacts at the site was not robust; however, the Late Prehistoric dates for the Austin and Toyah Phases coincide with accepted regional chronologies for the area.

In concurrence with the 2016-2017 testing recommendations, the Snakeskin Bluff site is considered eligible for designation as an SAL and for the NRHP. Although not all cultural components of the site were stratigraphically discrete, the Late Prehistoric Austin Phase component revealed intact, wellpreserved archaeological deposits that significantly contributed to our understanding of Late Prehistoric patterns. Given the sensitive nature of the cultural deposits at $41 \mathrm{GU} 177$, the main concern following the completion of data recovery excavations was the prevention of significant surface and subsurface impacts to the site during clearing and pipeline construction. As such, SWCA developed a site monitoring protocol; methods and results of the monitoring efforts are provided in Appendix H. Contributing components beyond the impact area will not be affected and will be preserved by avoidance; however, it is important to note that these investigations mitigated the project-specific effects, not the entire site. Any future project that could impact the site's deeper deposits, or those beyond the current right-of-way, warrant further consideration to assess the possibilities for additional contributing components. With these considerations, no further work is recommended. 


\section{CHAPTER 8. LITERATURE CITED}

Acuña, Laura I., Brandon Young, and Rhiana D. Ward

2016 Cultural Resources Investigations of the Vista Ridge Regional Water Supply Project in Burleson, Lee, Bastrop, Caldwell, Guadalupe, Comal and Bexar Counties, Texas. SWCA Cultural Resources Report No. 15-548. Austin, Texas.

Amyot, J., and J. Downing

1997 Seasonal variation in vertical and horizontal movement of the freshwater bivalve Elliptio complanata (Mollusca: Unionidae). Freshwater Biology 37:345-354.

Anderson, S.

1981 Mammals. In Harper \& Row's Complete Field Guide to North American Wildlife: Eastern Edition. by Lester L. Short, S. Anderson, Patricia G. Haneline, Franklin C. Daiber, Harald A. Rehder, and Robert D. Barnes, pp. 221-306. Harper \& Row Publishers, New York, Hagerstown, Philadelphia, San Francisco, Cambridge, London, Mexico City, São Paulo, and Sydney.

Andrefsky, W.

1998 Lithics: Macroscopic Approaches to Analysis. Cambridge University Press, Cambridge.

Arnn, John W.

2012 Land of the Tejas: Native American Identity and Interaction in Texas, A.D. 1300 to 1700. University of Texas Press, Austin.

Ashman, M. R., and G. Puri

2002 Essential Soil Science: A Clear and Concise Introduction to Soil Science. Blackwell Publishing, Malden, MA.

Baker, Frank E.

1979 Soil Survey of Bastrop County, Texas. United States Department of Agriculture, Washington, D.C.

Barnes, V. E.

1983 Geologic Atlas of Texas: San Antonio Sheet. Robert Hamilton Cuyler Memorial Edition, Bureau of Economic Geology, University of Texas, Austin.

Bartlein, Patrick J., Mary E. Edwards, Sarah L. Shafer, and Edward D. Barker, Jr.

1995 Calibration of Radiocarbon Ages and the Interpretation of Paleoenvironmental Records. Quaternary Research 44:417-424.

Bement, L.C.

1994 Hunter-Gatherer Mortuary Practices During the Archaic in Central Texas. Unpublished Ph.D. dissertation, The University of Texas, Austin.

Binford, L. R.

1980 Willow Smoke and Dogs' Tails: Hunter-Gatherer Settlement Systems and Archaeological Site Formation. American Antiquity 45: 4-20. 
Binford, L., and S. Binford

1966 A Preliminary Analysis of Functional Variability in the Mousterian of Levallois Facies. American Anthropologist 68(2):238-295.

Birkeland, Peter W.

1999 Soils and Geomorphology. Third Edition, Oxford University Press, New York and Oxford.

Black, S. L.

1989 Environmental Setting. In From the Gulf to the Rio Grande: Human Adaptation in Central, South, and Lower Pecos Texas, by Thomas R. Hester, Stephen L. Black, D. Gentry Steele, Ben W. Olive, Anne A. Fox, Karl J. Reinhard, and Leland C. Bement, pp. 5-16. Research Series No. 33. Arkansas Archeological Survey, Fayetteville.

Black, S. L., and D. C. Creel

1997 The Central Texas Burned Rock Midden Reconsidered. In Hot Rock Cooking on the Greater Edwards Plateau: Four Burned Rock Midden Sites in West Central Texas, Volume 1, by S. L. Black, L. W. Ellis, D. G. Creel, and G. T. Goode, pp. 269-306. Studies in Archeology 22. Texas Archeological Research Laboratory, The University of Texas at Austin. Archeology Studies Program, Report 2. Environmental Affairs Department, Texas Department of Transportation, Austin.

Black, S. L., and M. Quigg 2018 Unstemmed Point Tradition. Available at: https://www.texasbeyondhistory.net/stplains/prehistory/images/unstemmed.html. Accessed August 2018.

Black, S. L., and A. J. McGraw 1985 The Panther Springs Creek Site: Cultural Change and Continuity within the Upper Salado Creek Watershed, South-Central Texas. Archeological Survey Report No. 100. Center for Archeological Research, The University of Texas at San Antonio.

Black, S. L., L. W. Ellis, D. G. Creel, and G. T. Goode 1997 Hot Rock Cooking on the Greater Edwards Plateau: Four Burned Rock Midden Sites in West Central Texas, Volumes 1 and 2. Studies in Archeology 22. Texas Archeological Research Laboratory, The University of Texas at Austin. Archeology Studies Program, Report 2. Environmental Affairs Department, Texas Department of Transportation, Austin.

Blair, W. F.

1950 The Biotic Provinces of Texas. The Texas Journal of Science 2(1):93-117.

Block, J., G. Gerald, and T. Levine

2013 Temperature effects on burrowing behaviors and performance in a freshwater mussel. Journal of Freshwater Ecology 28(3): 375-384.

Blockley, S. P. E., M. Blaauw, C. Bronk Ramsey, and J. van der Plicht

2007 Building and Testing Age Models for Radiocarbon Dates in Lateglacial and Early Holocene Sediments. Quaternary Science Reviews 26:1915-1926. 
Boyd, Douglas K.

2012 What Is Northern Toyah Phase? The Toyah Phenomenon on the Texas Southern Plains. In The Toyah Phase of Central Texas: Late Prehistoric Economic and Social Processes, edited by Nancy A. Kenmotsu and Douglas K. Boyd, pp. 128-151. Texas A\&M University Press. College Station, Texas.

Bryant, Jr., Vaughn M., and Richard G. Holloway

1985 A Late-Quaternary Paleoenvironmental Record of Texas: An Overview of the Pollen Evidence. In Pollen Records of Late-Quaternary North American Sediments, by Vaughn M. Bryant, Jr. and Richard G. Holloway (editors) pp. 39-70. American Association of Stratigraphic Palynologists Foundation, Austin, Texas.

Bull, J., and J. Farrand, Jr.

1977 The Audubon Society Field Guide to North American Birds: Eastern Region. Fourth Edition. Alfred A. Knopf, Inc. New York.

Burlakova, L., A. Karatayev, E. Froufe, A.E. Bogan, and M. Lopes-Lima

2018 A new freshwater bivalve species of the genus Cyclonaias from Texas (Unionidae: Ampleminae: Quadrulini). The Nautilus 132(2):45-50.

Burt, W. H., and R. P. Grossenheider

1976 Peterson Field Guides: Mammals. Houghton Mifflin Company, Boston and New York.

Callahan, E. C.

1974 A Guide for Flintworking: Stages of Manufacture. Experimental Archeology Papers 3:185192.

1979 The Basics of Biface Knapping in the Eastern Fluted Point Tradition: A Manual for Flintknappers and Lithic Analysts. Archaeology of Eastern North America 7:1-180.

Carlson, S., A. Lawrence, H. Blalock-Herod, K. McCafferty, and S. Abbott

2008 Freshwater mussel survey protocol for the southeastern Atlantic slope and northeastern Gulf drainages in Florida and Georgia. United States Fish and Wildlife Service, Ecological Services and Fisheries Resources Offices. Georgia Department of Transportation, Office of Environment and Location.

Carpenter, S., M. Chavez, K. Miller, and K. Lawrence

2006 The McKinney Roughs Site 41BP627: A Stratified Late Archaic II Site on the Colorado River Terraces Bastrop County, Texas. SWCA Cultural Resources Report No. 02-313, SWCA Environmental Consultants, Austin.

Carpenter, Stephen M., Kevin A. Miller, Mary Jo Galindo, Brett A. Houk, Charles D. Frederick, Mercedes C. Cody, John Lowe, Ken Lawrence, Kevin Hanselka, and Abby Peyton

2013 The Siren Site and the Long Transitional from Archaic to Late Prehistoric Lifeways on the Eastern Edwards Plateau of Central Texas. SWCA Cultural Resources Report No. 12-93, SWCA Environmental Consultants, Austin.

Charlton, R.

2008 Fundamentals of Fluvial Geomorphology. Routledge, London and New York. 
Collins, M. B.

1968 A Note on the Broad Corner-Notched Projectile Points Used In Bison Hunting in Western Texas. The Bull Roarer 3(2) 13-14. The University of Texas Anthropology Society, Department of Anthropology, The University of Texas at Austin.

1974 A Functional Analysis of Lithic Technology among Prehistoric Hunter-Gatherers of Southwestern France and Western Texas. Ph.D. dissertation, University of Arizona, Tuscon.

1995 Forty Years of Archeology in Central Texas. Bulletin of the Texas Archeological Society 66:361-400.

2004 Archeology in Central Texas. In The Prehistory of Texas. Edited by Timothy K. Perttula, pp. 101-126. Texas A\&M University Press, College Station.

Conant, R., and J. T. Collins

1998 Peterson Field Guides: Reptiles and Amphibians Eastern and Central North America. Third Edition. Houghton Mifflin Company, Boston and New York.

Correll, Donovan S., and Marshall C. Johnston

1979 Manual of the Vascular Plants of Texas. University of Texas at Dallas.

Crowther, J., and P. Barker

1995 Magnetic Susceptibility: Distinguishing Anthropogenic Effects from the Natural.

Archaeological Prospection 2:207-215.

Dalan, R. A.

1996 Soil Magnetism, An Approach for Examining Archaeological Landscapes. Geophysical Research Letters 23(2): 185-188.

2006 Magnetic Susceptibility. In Remote Sensing in Archaeology: An Explicitly North American Perspective. J. Johnson, M. Giardano, and K. Kvamme (editors), pp. 161-203. University of Alabama Press, Tuscaloosa, Alabama.

2008 A Review of the Role of Magnetic Susceptibility in Archaeologeophysical Studies in the USA: Recent Developments and Prospects. Archaeological Prospection 15: 1-31.

Dalan, Rinita A., and Subir K. Banerjee

1998 Solving Archaeological Problems Using Techniques of Soil Magnetism. Geoarchaeology: An International Journal 13: 3-36.

Dalan, R. A., and B. W. Bevan

2002 Geophysical Indicators of Culturally Embraced Soils and Sediments. Geoarchaeology: An International Journal 17 (8): 779-810.

Davis, W. B., and D. J Schmidly 1994 The Mammals of Texas. Texas Parks and Wildlife Department, Austin.

Dearing, J.

1999a Magnetic Susceptibility. In Environmental Magnetism: A Practical Guide. J. Walden, F. Oldfield, and J. Smith (editors), pp. 35-62. Technical Guide No. 6. Quaternary Research Association, London. 
1999b Environmental Magnetic Susceptibility Using the Bartington MS2 System. Available at: http://www.gmw.com/magnetic properties/MS2_support.html. Accessed August 2018.

Deetz, J.

1967 Invitation to Archaeology. Ameri-can Museum Science Books, Natu-ral History Press, Garden City, New York.

Dering, $\mathrm{P}$

1999 Earth-Oven Plant Processing in Archaic Period Economies: An Example from a Semi-arid Savannah in South-Central North America. American Antiquity 64(4):659-674.

Dibble, D. S., and D. Lorrain

1968 Bonfire Shelter: A Stratified Bison Kill Site, Val Verde County, Texas. Miscellaneous Papers No. 1. Texas Memorial Museum, The University of Texas at Austin.

Dillehay, T. D.

1974 Late Quaternary Bison Population Changes on the Southern Plains. Plains Anthropologist 19(65):180-196.

Ellwood, B. B., D. E. Peter, W. Balsam, and J. Schieber

1995 Magnetic and Geochemical Variations as Indicators of Palaeoclimate and Archaeological Site Evolution: Examples from 41TR68, Fort Worth, Texas. Journal of Archaeological Science 22: 409-415.

Fields, Ross C., and Eloise F. Gadus (editors)

2012 Archeology of the Nadaco Caddo: The View from the Pine Tree Mound Site (41HS15), Harrison County, Texas. Reports of Investigations 164, Prewitt and Associates, Inc., Austin.

Frederick, Charles D

2010 Geoarchaeological Investigations. In Archaeological Data Recovery on Three Sites Along the San Antonio River, Bexar County, Texas, by Antonio E. Padilla and David L. Nickels, pp. 449-462. Ecological Communications, Corporation, Austin, Texas.

2012 Site Formation Processes and Implications for Archeology in the South Fork of the San Gabriel. In The Siren Site (41WM1126) and the Long Transition from Archaic to Late Prehistoric Lifeways on the Eastern Edwards Plateau, Williamson County, Texas, by Steve Carpenter, Kevin A. Miller, Brett Houk, Mary Jo Galindo, Charles Frederick, John Lowe, Ken Lawrence, Kevin Hanselka, and Abby Peyton, pp. 8-1-8-24. SWCA Environmental Consultants, Austin, Texas.

Gadus, E. Frances, Ross C. Fields, and Karl W. Kibler

2006 Data Recovery Excavations at the J. B. White Site (41MM341), Milam County, Texas. Research Report No. 145. Prewitt \& Associates, Inc. Austin, Texas.

Gale, S. J., and P. G. Hoare

1991 Quaternary Sediments: Petrographic Methods for the Study of Unlithified Rocks. Bellhaven Press and Halsted, Press, New York and Toronto.

Gerstle, A., T. C. Kelly, and C. Assad

1978 The Fort Sam Houston Project: An Archaeological and Historical Assessment. Archaeological Survey Report 40, Center for Archaeological Research. The University of Texas at San Antonio. 
Goode, G. T.

1991 Late Prehistoric Burned Rock Middens in Central Texas. In The Burned Rock Middens of Texas: An Archeological Symposium, edited by Thomas R. Hester, pp. 71-93. Studies in Archeology 13. Texas Archeological Research Laboratory, The University of Texas at Austin.

Gould, F. W.

1969 Texas Plants: A Checklist and Ecological Survey. Texas Agricultural Extension Service, Texas A\&M University, College Station.

Graham, R.W.

1987 Environmental Fluctuations and Evolution of Mammal Faunas during the Last Deglaciation in North America. In North America and Adjacent Oceans during the Last Deglaciation by W. F. Ruddiman and H.E. Wright (editors), pp. 371-402. Geological Society of America. Boulder, Colorado.

Graham, R.W., and E. L. Lundelius

1984 Coevolutionary Disequilibrium and Pleistocene Extinctions. In Quaternary Extinctions: A Prehistoric Revolution, by P.S. Martin and R.G. Klein (editors), pp. 223-249. University of Arizona Press, Tucson.

Hall, Stephen A., and Salvatore Valastro, Jr.

1995 Grassland Vegetation in the Southern Great Plains during the Last Glacial Maximum. Quaternary Research 44:237-245.

Hard, Robert J., Raymond P. Mauldin, and Gerry R. Raymond

1996 Mano Size, Stable Carbon Isotope Ratios, and Macrobotanical Remains as Multiple Lines of Evidence of Maize Dependence in the American Southwest. Journal of Archaeological Method and Theory 3:253-318.

Hays, Christopher T., Richard A. Weinstein, and James B. Stoltman 2016 Poverty Point Objects Reconsidered. Southeastern Archaeology 2016:1-24.

Hess, M.C., K. Inoue, E.T. Tsakiris, M. Hart, J. Morton, and J. Dudding 2018 Misidentification of Sex for Lampsilis teres, Yellow Sandshell, and its Implications for Mussel Conservation and Wildlife Management. PloS one 13(5):e0197107.

Hester, T. R.

1995 The Prehistory of South Texas. Bulletin of the Texas Archeological Society 66:427-459.

2004 The Prehistory of South Texas. In The Prehistory of Texas, edited by T. K. Perttula, pp. 127154. Texas A\&M University Press, College Station.

Houk, B. A., and J. C. Lohse

1993 Archeological Investigations at the Mingo Site, Bandera County, Texas. Bulletin of the Texas Archeological Society 61:193-247.

Houk, B. A., S. Tomka, B. Bousman, C. K. Chandler, B. Moses, M. Renner, and M. Lyons

1997 The Greenbelt Core: A Polyhedral Blade Core from San Antonio, Texas. Current Research in the Pleistocene 14:104-106. 
Howells, R.G.

2014 Field guide to Texas Freshwater Mussels. BioStudies. Kerrville, Texas.

Hua, Quan

2009 Radiocarbon: A Chronological Tool for the Recent Past. Quaternary Geochronology 4:378390 .

Hudgins, Joe D.

1993 Cooking with Clay Balls. In The Cache: Collected Papers on Texas Archeology, by Cathryn A. Hoyt (editor), pp. 47-52. Texas Historical Commission, Austin.

Hudler, D. B.

1997 Determining Clear Fork Tool Function through Use-Wear Analysis: A Discussion of UseWear Methods and Clear Fork Tools. Studies in Archeology 25. Texas Archeological Research Laboratory, The University of Texas at Austin.

Huebner, J. A.

1991 Late Prehistoric Bison Populations in Central and South Texas. Plains Anthropologist 36(137):343-358.

Hunter, Donald G.

1975 Functional Analysis of Poverty Point Clay Objects. Florida Anthropologist 28(1):57-71.

Jelks, E. B.

1962 The Kyle Site: A Stratified Central Texas Aspect Site in Hill County, Texas. Archaeology

Series No. 5. Department of Anthropology, The University of Texas at Austin.

Johnson, L, and G. T. Goode

1994 A New Try at Dating and Characterizing Holocene Climates, as well as Archeological Periods, on the Eastern Edwards Plateau. Bulletin of the Texas Archeological Society 65:151.

Johnson, L, Jr., D. A. Suhm, and C. D. Tunnell

1962 Salvage Archeology of Canyon Reservoir: The Wunderlich, Footbridge, and Oblate Sites. Bulletin No. 5. Texas Memorial Museum, The University of Texas at Austin.

Johnson, L., Jr.

1994 The Life and Times of Toyah-Culture Folk as Seen from the Buckhollow Encampment, Site 31KM16, of Kimble County, Texas. Office of the State Archeologist Report 38. Texas Department of Transportation and Texas Historical Commission, Austin.

1995 Past Cultures and Climates at Jonas Terrace: 41 ME29 of Medina County, Texas. Report No. 40. Office of the State Archeologist, Texas Historical Commission, Austin.

Jones, R. S., and J. J. Leffler

2003 Phase I and II Archaeological Investigations on Camp Mabry, Travis County, Texas, Archaeological Studies Report No. 2. Center for Archaeological Studies, Texas State University-San Marcos, Texas.

Kelly, R. L.

1992 Mobility/Sedentism: Concepts, Archaeological Measures, and Effects. Annual Review of Anthropology 21:43-66. 
Kibler, K. W., and A. M. Scott

2000 Archaic Hunters and Gatherers of the Balcones Canyonlands: Data Recovery Excavations at the Cibolo Crossing Site (41BX377), Camp Bullis Military Reservation, Bexar County, Texas. Reports of Investigations No. 126. Prewitt and Associates, Inc., Austin.

Klein, Richard G., and L. Kathryn Cruz-Uribe

1984 The Analysis of Animal Bones from Archeological Sites. University of Chicago Press.

Kleinbach, K., G. Mehalchick, J. T. Abbott, and J. M. Quigg

1995 Other Analyses. In NRHP Significance Testing of 57 Prehistoric Archeological Sites on Fort Hood, Texas, Volume II, edited by James T. Abbott and W. Nicholas Trierweiler, pp. 765842. Archeological Resource Management Series, Research Report No. 34. United States Army Fort Hood.

Kutac, E. A., and S. C. Caran

1994 Birds and Other Wildlife of South Central Texas. University of Texas Press, Austin.

Lawrence, Ken, and Charles Frederick

2012 Geomorphology-Geoarchaeology: Site Formation and Chronostratigraphy. In Data Recovery Investigations on the Eastern Side of the Siren Site (41WM1126), Williamson County, Texas. A. Peyton, S. Carpenter, J. D. Lowe, and K. Lawrence. SWCA Cultural Resource Report No. 12-236. SWCA Environmental Consultants, Austin.

Lawrence, Ken, J. Kevin Hanselka, John D. Lowe, Christina Nielsen, and Kevin A. Miller 2013 Results of Archaeological Significance Testing at the Turkey Terrace Site (41FR70), Frio County, Texas. SWCA Environmental Consultants, Archaeological Report 12-518 Austin, Texas.

Lowther, A. C., and Werchan, Leroy E.

1978 Soil Survey of Caldwell County, Texas. United States Department of Agriculture, Washington, D.C.

Lyman, R. L.

1994 Vertebrate Taphonomy. Cambridge University Press, Cambridge.

Mackie, G., T. Morris, and D. Ming

2008 Protocol for the Detection and Relocation of Freshwater Mussel Species at Risk in OntarioGreat Lakes Area (OGLA). Canadian Manuscript Report of Fisheries and Aquatic Sciences 2790, Fisheries and Oceans Canada.

Maher, Barbara A., Roy Thompson, and Mark W. Hounslow

1999 Introduction. In Quaternary Climates, Environments, and Magnetism. B.A. Maher and R. Thompson (editors), pp. 1-48. Cambridge University Press. Cambridge.

Mallouf, R. J., B. J. Baskin, and K. L. Killen

1977 A Predictive Assessment of Cultural Resources in Hidalgo and Willacy Counties, Texas. Survey Report 23. Office of the State Archeologist, Texas Historical Commission, Austin.

Mauldin, R. P., and A. L. Figueroa

2006 Data Recovery Excavations at 41PR44, Fort Wolters, Parker County, Texas, Archaeological Report, No. 369, Center for Archaeological Research, University of Texas at San Antonio, San Antonio, Texas. 
McCormac, F. Gerry, and Mike G. Baillie

1993 Radiocarbon to Calendar Date Conversion: Calendrical Band Widths as a Function of Radiocarbon Precision. Radiocarbon 35(2):311-316.

Miksicek, Charles

1987 Formation Processes of the Archaeobotanical Record. In Advances in Archaeological Method and Theory, Vol. 10, edited by M. B. Schiffer, pp. 211-247. Academic Press, New York.

Mock, Cary J., and Patrick J. Bartlein

1995 Spatial Variability of Late-Quaternary Paleoclimates in the Western United States. Quaternary Research 44:425-433.

Mook, W. G., and H. T. Waterbolk

1985 Handbooks for Archaeologists, No. 3 Radiocarbon Dating. European Science Foundation, Strasbourg.

Natural Resources Conservation Service (NRCS)

2018 Web Soil Survey 2.1. National Cooperative Soil Survey. Available at: http://websoilsurvey.nrcs.usda.gov/app/WebSoilSurvey.aspx. Accessed January 2018.

Nelson, D. W., and L. E. Sommers

1982 Total Carbon, Organic Carbon, and Organic Matter. In Methods of Soil Analysis Part 2:

Chemical and Microbiological Properties. Second Edition. A. L. Page, R. H. Miller, and D. R. Keeney (editors), pp. 539-579. American Society of Agronomy, Inc. and Soil Science Society of America, Inc. Publisher, Madison, Wisconsin.

Nickels, D. L.

2010 Stratigraphy, Chronology, and Site Formation Processes. In Archaeological Testing at the San Marcos Springs (41HY160) for the Texas River Center, Hays County, Texas, by David L. Nickels and C. Britt Bousman, pp. 61-82. Archaeological Studies Report No. 13. Center for Archaeological Studies, Texas State University-San Marcos, Texas.

Nordt, Lee C.

1992 Archaeological Geology of the Ft. Hood Military Reservation, Fort Hood, Texas. United States Army Fort Hood, Archeological Resource Management Series, Research Report No. 25. Prewitt \& Associates, Inc. Austin, Texas.

Odell, George H.

2003 Lithic Analysis. Manuals in Archaeological Method, Theory, and Technique Series. Springer, New York.

Olsen, Stanley John

1973 Mammal Remains from Archaeological Sites. Papers of the Peabody Museum of Archaeology and Ethnology Volume 56, Issue 1. Harvard University.

Patterson, Leland W.

1977 An Archeological Complex in Kendall County, Texas. La Tierra 4(2):6-16.

Perttula, Timothy K., M. R. Miller, R. A. Ricklis, D. J. Prikryl, and C. Lintz 1995 Prehistoric and Historic Aboriginal Ceramics in Texas. Bulletin of the Texas Archaeological Society 66:175-235. 
Perttula, Timothy K.

2008 Lake Naconiche Archeology, Nacogdoches County, Texas: Results of the Data Recovery

Excavations at Five Prehistoric Sites Vol. II. Report of Investigations No. 60. Archaeological \& Environmental Consultants, LLC. Austin, Texas.

Perttula, Timothy K., and Bo Nelson

2006 Test Excavations at Three Caddo Sites at Mission Tejas State Park, Houston County, Texas. Report of Investigations No. 76. Archeological \& Environmental Consultants, LLC. Austin, Texas.

Peters, Clare, and Roy Thompson

1999 Supermagnetic Enhancement, Superparamagnetism, and Archaeological Soils.

Geoarchaeology: An International Journal 14 (5): 401-413.

Petrides, G. A.

1988 Peterson Field Guides: A Guide to Eastern Trees. Houghton Mifflin Company, Boston and New York.

Prewitt, E. R.

1981 Cultural Chronology in Central Texas. Bulletin of the Texas Archeological Society 52:65-89.

1982 Archeological Investigations at the Loeve-Fox Site, Williamson County, Texas. Reprints in Archeology No. 1. Prewitt and Associates, Inc. Austin, Texas.

1985 From Circleville to Toyah: Comments on Central Texas Chronology. Bulletin of the Texas Archeological Society 54:201-238.

Ramsey, C. B.

2008 Deposition Models for Chronological Records. Quaternary Science Reviews 27:42-60.

2009 Bayesian Analysis of Radiocarbon Dates. Radiocarbon 51(1):337-360.

Ramsey, R.N., and N. P. Bade

1977 Soil Survey of Guadalupe County, Texas. United States Department of Agriculture, Washington, D.C.

Reitz and Wing

2008 Zooarchaeology. Cambridge Manuals in Archaeology, second edition.

Ricklis, R. A.

1992 The Spread of Late Prehistoric Bison Hunting Complex: Evidence from the South-Central Coastal Prairie of Texas. Plains Anthropologist 37(140):261-273.

Ricklis, Robert A., and Michael B. Collins

1995 Archaic and Late Prehistoric Human Ecology in the Middle Onion Creek Valley, Hays County, Texas. 2 vols. Studies in Archeology No. 19. Austin: Texas Archeological Research Laboratory, University of Texas at Austin.

Rivers, J.M., J.E. Nyquist, Y. Roh, D.O. Terry Jr., and W.E. Doll

2004 Investigation into the Origin of Magnetic Soils on the Oak Ridge Reservation, Tennessee. Soil Science of America Journal 68(5): 1772-1779. 
Rodriguez, Daniel, Ken Lawrence, and Brandon S. Young

2017 Interim Report: Archaeological Investigations at $41 G U 177$ on the Guadalupe River, Guadalupe County, Texas. SWCA Environmental Consultants. Austin, Texas.

Rosendahl, Daniel, Kelsey M. Lowe, Lynley A. Wallis, and Sean Ulm

2014 Integrating Geoarchaeology and Magnetic Susceptibility at Three Shell Mounds: A Pilot Study from Mornington Island, Gulf of Carpentaria, Australia. Journal of Archaeological Science 49:21-32.

Schmidly, D. J.

1983 Texas Mammals East of the Balcones Fault Zone. Texas A\&M Press, College Station.

Schwalb, A., and M. Pusch

2007 Horizontal and Vertical Movements of Unionid Mussels in a Lowland River. Journal of the North American Benthological Society 26(2):261-272.

Shafer, H. J.

2005 There is More to Langtry than Looks: The Uses and Abuses of Texas Point Typology. La Tierra 31(2):9-14.

Short, L.

1981 Birds. In Harper \& Row's Complete Field Guide to North American Wildlife: Eastern Edition. by Lester L. Short, S. Anderson, Patricia G. Haneline, Franklin C. Daiber, Harald A. Rehder, and Robert D. Barnes, pp. 221-306. Harper \& Row Publishers, New York, Hagerstown, Philadelphia, San Francisco, Cambridge, London, Mexico City, São Paulo, and Sydney.

Simms, Stephanie R., Francesco Berna and George J. Bey, III

2013 A Prehispanic Maya Pit Oven Microanalysis of Fired Clay Balls from the Puuc Region, Yucatan, Mexico. Journal of Archaeological Science 40(2):1144-57.

Simpson, B. J.

1988 A Field Guide to Texas Trees. Texas Monthly Field Guide Series. Texas Monthly Press, Austin, Texas.

Snedden, Jeffrey

2018 Legacy of Mayer China Extends Worldwide. The Times online. Available at: https://www.timesonline.com/7b0cf620-aa79-11e6-aefa-673d9e8dd1bd.html. Accessed July 2018.

Sorrow, W. M.

1969 Archeological Investigations at the John Ischy Site: A Burned Rock Midden in Williamson County, Texas. Papers of the Texas Archeological Salvage Project No. 18. The University of Texas at Austin.

Story, D. A.

1985 Adaptive Strategies of Archaic Cultures of the West Gulf Coastal Plain. In Prehistoric Food Production in North America, edited by R. I. Ford, pp. 19-56. Anthropological Papers 75. Museum of Anthropology, University of Michigan, Ann Arbor. 
1990 Cultural History of the Native Americans. In The Archeology and Bioarcheology of the Gulf Coastal Plain, by Dee Ann Story, Janice A. Guy, Barbara A. Burnett, Martha Doty Freeman, Jerome C. Rose, D. Gentry Steele, Ben W. Olive, and Karl J. Reinhard, pp. 163-366.

Research Series No. 38. Arkansas Archeological Survey, Fayetteville.

Struever, Stuart

1968 Flotation Techniques for the Recovery of Small-Scale Archaeological Remains. American Antiquity 33:353-362.

Stuiver, Minze, and Henry A. Polach

1977 Discussion: Reporting of ${ }^{14} \mathrm{C}$ Data. Radiocarbon 19 (3):355-363.

Stuiver, M., and H. S. Suess

1966 On the Relationship Between Radiocarbon Dates and True Sample Ages. Radiocarbon 8:534-540.

Suhm, D. A.

1960 A Review of Central Texas Archeology. Bulletin of the Texas Archeological Society 29:63107.

Suhm, Dee Ann, Alex D. Krieger, and Edward B. Jelks

1954 An Introductory Handbook of Texas Archeology. Bulletin of the Texas Archeological Society, Vol. 25.

Suhm, Dee Ann, and Ed B. Jelks (editors)

1962 Handbook of Texas Archeology: Type Descriptions. Texas Archaeological Society Special Publications No. 1 and Texas Memorial Museum Bulletin No. 4. Austin.

Taylor, R. E.

1997 Radiocarbon Dating. In: Chronometric Dating in Archaeology: Advances in Archaeological and Museum Science Vol. 2. (R. E. Taylor and M. J. Aitken editors) 3:65-96. Plenum Press, New York and London.

2009 Six Decades of Radiocarbon Dating in New World Archaeology. Radiocarbon 51(1):173212.

Taylor, F. B., R. B. Hailey, and D. L. Richmond

1991 Soil Survey of Bexar County, Texas. United States Department of Agriculture, Washington, D.C.

Texas Beyond History

2018 Toyah Culture. Available at:

https://www.texasbeyondhistory.net/plateaus/prehistory/images/toyah.html. Accessed August 2018.

Texas Parks and Wildlife Department

2018 Guidelines for Aquatic Resource Relocation Plan for Fish and Shellfish, Including Freshwater Mussels. Austin, Texas. Available at https://tpwd.texas.gov/publications/pwdpubs/media/pwd_lf_t3200_1958_arrp_guidelines_pac ket.pdf. Accessed August 2018. 
Thompson, R., and F. Oldfield

1986 Environmental Magnetism. Allen \& Unwin Press. London, Boston, and Sydney.

Thoms, Alston, Andrew R. Laurence, Laura Short, and Masahiro Kamiya

2015 Baking Geophytes and Tracking Microfossils: Taphonomic Implications for Earth-Oven and Paleodietary Research. Journal of Archaeological Method and Theory 22(4): 1038-1070.

Tomka, S. A.

2001 The Effect of Processing Require-ments on Reduction Strategies and Tool Form: A New Perspective. In Lithic Debitage: Context, Form, Meaning, edited by W. Andrefsky Jr., pp. 207-224. University of Utah Press, Salt Lake City.

Tomka, S. A., G Mehalchick, K. Kleinbach, and D. K. Boyd

1999 Methods of Investigation. In National Register Testing of 19 Prehistoric Archeological Sites at Fort Hood, Texas: The 1995 Season, by G. Mehalchick, K Kleinbach, D. K. Boyd, S. A. Tomka, and K. W. Kibler, pp. 21-40. Archeological Resource Management Series Research Report No. 37. United States Army, Fort Hood.

Tomka, S. A., H. J. Shafer, and R. P. Mauldin

2003 Chapter 12: Lithic Technology at 41MM340. In Data Recovery Excavations at 41MM340: A Late Archaic Site along Little River in Milam County, Texas, by R. B. Mahoney, S. A. Tomka, R. P. Mauldin, H. J. Shafer, L. C. Nordt, R D. Greaves, and R. R. Galdeano, pp. 133155. Archaeological Survey Report 340. Center for Archaeological Research, The University of Texas at San Antonio. Archeological Studies Program, Report No. 54. Environmental Affairs Division, Texas Department of Transportation, Austin.

Toomey III, Rickard S

1993 Late Pleistocene and Holocene Faunal and Environmental Changes at Hall's Cave, Kerr County, Texas. Vols. 1 and 2 Unpublished Ph.D. dissertation, Department of Geology, The University of Texas at Austin.

Toomey III, Rickard S., Michael D. Blum, and Salvatore Valastro Jr.

1993 Late Quaternary Climates and Environments of the Edwards Plateau, Texas. Global and Planetary Change 7:299-320.

Turner, E. S., and T. R. Hester

1999 A Field Guide to Stone Artifacts of Texas Indians. Third Edition. Texas Monthly Field Guide Series. Gulf Publishing Company, Houston, Texas.

Turner, E. S., T. R. Hester, and R. McReynolds

2011 Stone Artifacts of Texas Indians. Taylor Trade Publishing, New York.

Turpin, Jeff $P$.

2011 Uncommon Cooking Technologies at Two Prehistoric Sites in South-Central Texas. Plains Anthropologist 56(219):285-292.

Van Leusen, P. M., A. Kattenberg, and K. Armstrong 2014 Magnetic Susceptibility Detection of Small Protohistoric Sites in the Raganello Basin, Calabria (Italy). Archaeological Prospection (in press). 
Verosub, K. L., and A. P. Roberts

1995 Environmental Magnetism: Past, Present, and Future. Journal of Geophysical Research 100(B2): 2175-2192.

Waters, Michael R.

1992 Principles of Geoarchaeology: A North American Perspective. University of Arizona Press, Tucson.

Wermund, Edmund G.

2018 "Physiography of Texas," Bureau of Economic Geology. Available at: http://www.beg.utexas.edu/UTopia/images/pagesizemaps/physiography.pdf. Accessed February 2018.

Whittaker, J. C.

1994 Flintknapping: Making and Understanding Stone Tools. University of Texas Press, Austin.

Wiewel, Adam S., and Kenneth L. Kvamme

2014 Magnetic Investigations of Nomadic Group Encampments at Fort Clark State Historic Site, North Dakota. Plains Anthropologist 59(231):261-278.

Winterhalder, B., and E. A. Smith

1981 Hunter-Gatherer Foraging Strategies: Ethnographic and Archeological Analyses. University of Chicago Press, Chicago. 
This page intentionally left blank. 


\section{APPENDIX A}

41GU177 Testing Interim Report 



\section{Interim Report: Archaeological Investigations at 41GU177 on the Guadalupe River, Guadalupe County, Texas}

Texas Antiquities Permit No. 7295

November 2017

SUBMITTED TO:

VRRSP Consultants, LLC.

SUBMITTED BY:

SWCA Environmental Consultants 4407 Monterey Oaks Boulevard

Building 1, Suite 110

Austin, Texas 78749 



\title{
INTERIM REPORT: ARCHAEOLOGICAL INVESTIGATIONS AT 41GU177 ON THE GUADALUPE RIVER, GUADALUPE COUNTY, TEXAS
}

\author{
Prepared for \\ VRRSP CONSULTANTS, LLC. \\ 2000 NW Loop 410 \\ San Antonio, Texas 78213
}

Prepared by

Daniel Rodriguez, Ken Lawrence, and Brandon S. Young

Principal Investigator

Brandon Young, M.A., RPA

\author{
SWCA ENVIRONMENTAL CONSULTANTS \\ 4407 Monterey Oaks Boulevard \\ Building 1, Suite 110 \\ Austin, Texas 78749 \\ www.swca.com
}

Texas Antiquities Permit No. 7295

SWCA Project No. 31410

November 2, 2017 
This page intentionally left blank. 


\section{TABLE OF CONTENTS}

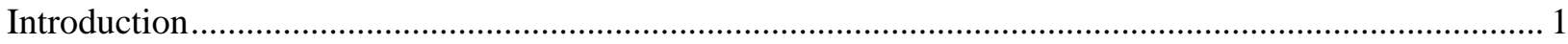

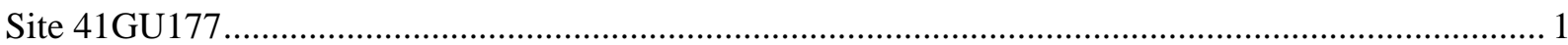

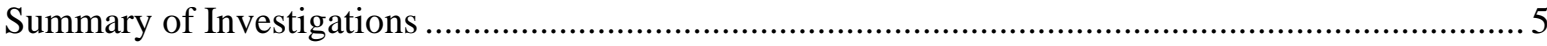

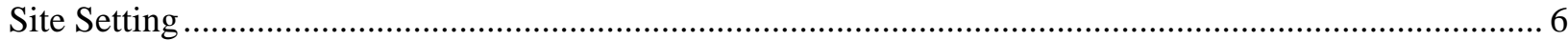

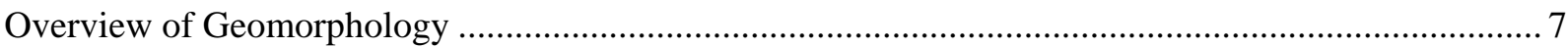

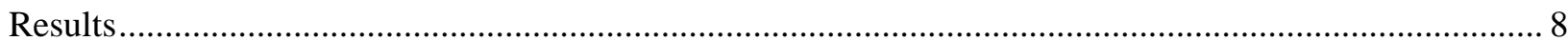

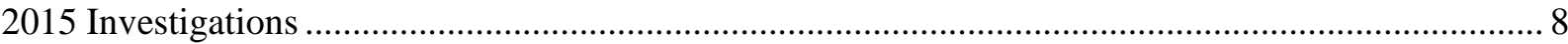

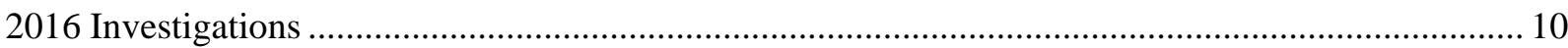

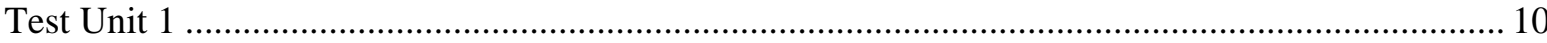

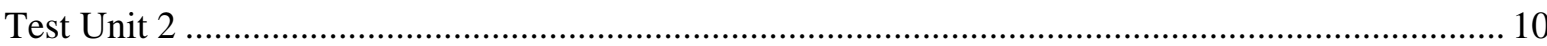

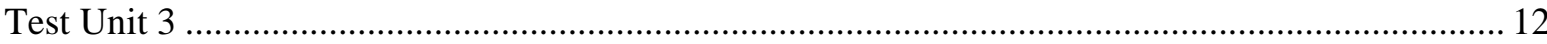

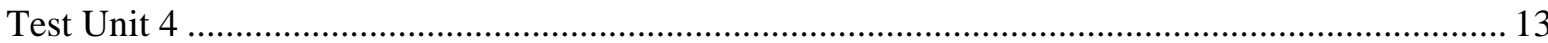

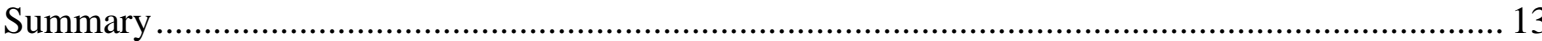

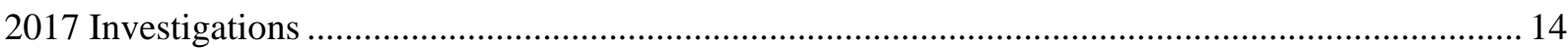

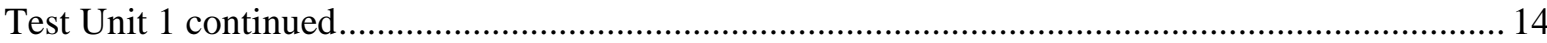

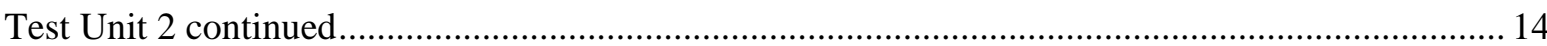

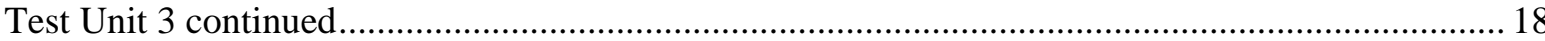

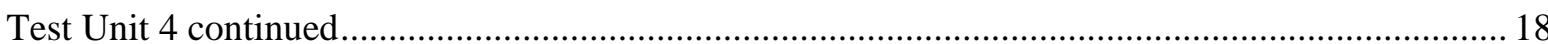

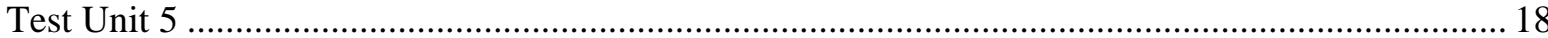

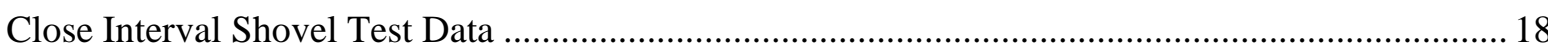

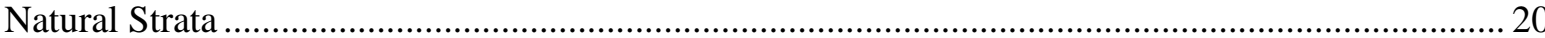

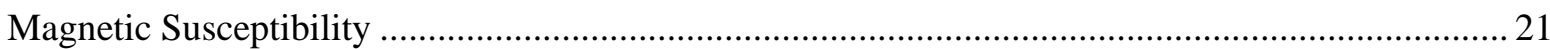

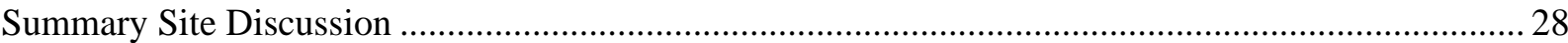

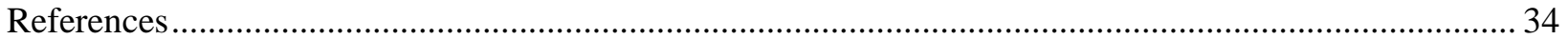




\section{LIST OF FIGURES}

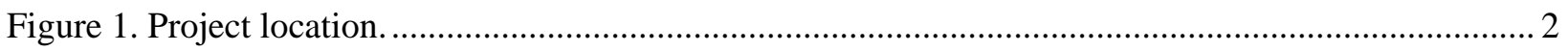

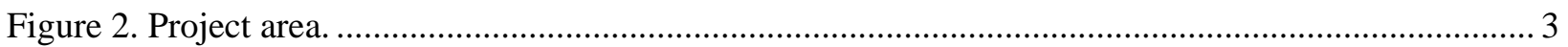

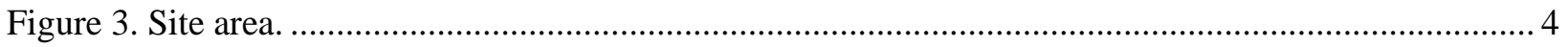

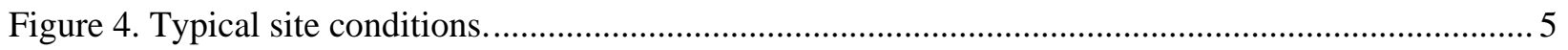

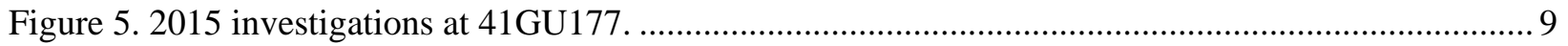

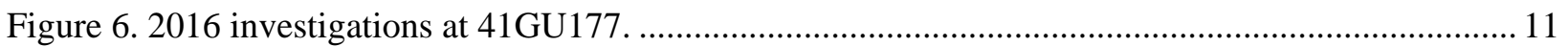

Figure 7. Arrow points and arrow point fragments recovered from 41GU177: a-c. cornernotched, broad stem variant Scallorn arrow points; d. Scallorn preform; e. untyped arrow point fragment; f. untyped arrow point fragment; g. untyped arrow point (possible Sabinal specimen); h. Untyped arrow point preform; i-j. distal tips of an untyped arrow points; k. untyped arrow point fragment; and l. untyped arrow point

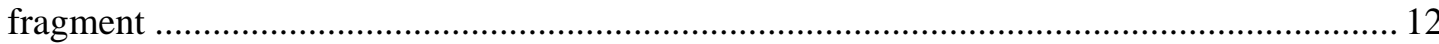

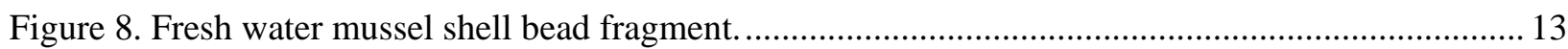

Figure 9. 2017 additional excavations in the proposed bore pit and adjacent work area......................... 15

Figure 10. 2017 close interval shovel test and additional backhoe trench plots. ......................................... 16

Figure 11. Artifact density plot generated from close interval shovel testing data, backhoe trench

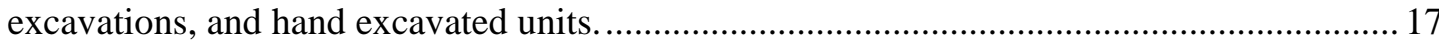

Figure 12. Dart point fragments recovered from 41GU177: a. Montell dart point base; b. Possible Frio or Ensor dart point fragment; and c. untyped dart point distal tip................................... 19

Figure 13. Unifacial axe recovered from TU05 excavations ................................................................. 19

Figure 14. Magnetic susceptibility results from BHT04.................................................................... 23

Figure 15. Magnetic susceptibility results from BHT05 .................................................................... 24

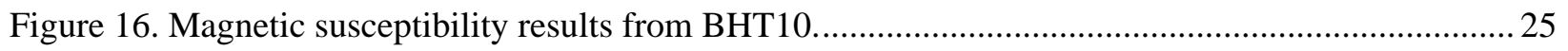

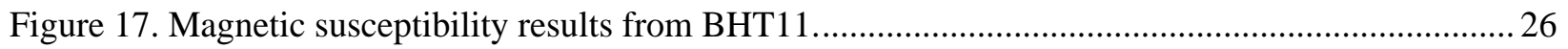

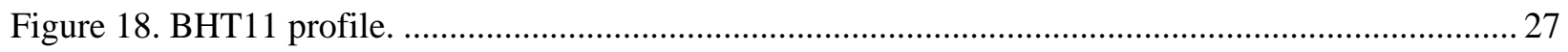

Figure 19. BHT11 overview. The zip-ties in the trench walls are marking artifacts...............................2

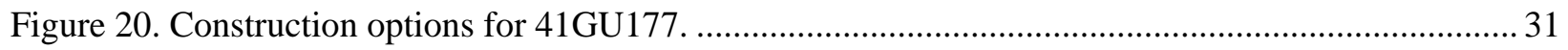

\section{LIST OF APPENDICES}

APPENDIX A Shovel Test Results

APPENDIX B Backhoe Trench Results

APPENDIX C Test Unit Specimen Inventory 


\section{INTRODUCTION}

On behalf of VRRSP Consultants, LLC and the Central Texas Regional Water Supply Corporation (CTRWSC), SWCA Environmental Consultants (SWCA) has prepared this interim report to present the findings of SWCA's ongoing investigations at prehistoric site $41 \mathrm{GU} 177$ on project parcel 50360 (Guadalupe County) and the implications of these investigations regarding potential site preservation and/or data recovery excavations at the site. It is the professional opinion of SWCA that the site warrants State Antiquities Landmark (SAL) designation, based on the density and quality of data recovered from the site to date, as well as the overall integrity of the site's natural and cultural stratigraphy. These investigations are one component of the larger overarching cultural resources investigations for the proposed Vista Ridge Regional Water Supply (Vista Ridge) Project. The project will involve installation of a 140-mile-long, 60inch-diameter water pipeline from north-central San Antonio, Bexar County to Deanville, Burleson County, Texas (Figure 1). The project will also include three pump station locations in Guadalupe, Bastrop, and Burleson Counties. Most of the alignment will follow existing utilities and traverse undeveloped, agricultural parcels in rural settings. The area of potential effects (APE) will consist of the proposed centerline alignment and a 100-foot corridor (50 feet on either side of centerline) for temporary and permanent construction easements. Between June 2015 and the present, the cultural resources inventory has identified 69 cultural resources, including 61 archaeological sites and eight isolated finds. All investigations described in this interim report were conducted under Texas Antiquities Permit No. 7295 issued to Principal Investigator Brandon S. Young. All work has been and will continue to be conducted in accordance with the Antiquities Code of Texas (ACT).

\section{SITE 41GU177}

This report presents the results of a series of investigations at prehistoric site 41GU177 in Guadalupe County (Figure 2). Site 41GU177 is a prehistoric campsite on forested rangeland in northwestern Guadalupe County (Figure 3). The site is on the western high bank of the Guadalupe River with a 30- to 40-foot vertical drop to the river bottom to the east and gently sloping (2-5 percent slope) terrain to the west. Vegetation consists of mixed hardwood trees, large live oak trees, and moderately dense shrub undergrowth (Figure 4). Ground surface visibility ranges from 0 to 20 percent with limestone gravels and cobbles at ground surface. The Guadalupe River is the nearest natural water source, forming the eastern boundary of the site. Soils of the site consist of very dark grayish brown to brown silt loams with 1 to 5 percent inclusions. Inclusions vary with depth, but include limestone cobbles, gravels, Rabdotus snail shell, a variety of other snail shell, and calcium carbonates. Previous impacts to the site area include natural erosion, vegetation clearing, grading, fence lines, and residential house and drive construction.

Certain potential adverse impacts have been proposed (i.e., an approximately $300 \times 8 \times 10$-foot construction trench, a $65 \times 200$-foot temporary workspace, a 20- to 25 -foot-wide haul road for construction activities, vegetation/tree clearing, and a horizontal directional drill [HDD] bore pit) to those portions of the site within the 85-foot-wide pipeline easement (see Figure 3). These impacts will result from work areas needed to tunnel the pipeline beneath the adjacent Guadalupe River and install the 60-inch diameter pipeline. Preliminary survey-level investigations indicated potential for intact, subsurface cultural components. As described in this interim report, SWCA conducted a series of phased survey investigations to fully assess the significance and potential eligibility of site $41 \mathrm{GU} 177$ for listing as a SAL. 


\section{Restricted Information}

\section{Not for Public Disclosure}

Figure 1. Project location. 


\title{
Restricted Information
}

\author{
Not for Public Disclosure
}

Figure 2. Project area. 


\title{
Restricted Information
}

\author{
Not for Public Disclosure
}

Figure 3. Site area. 


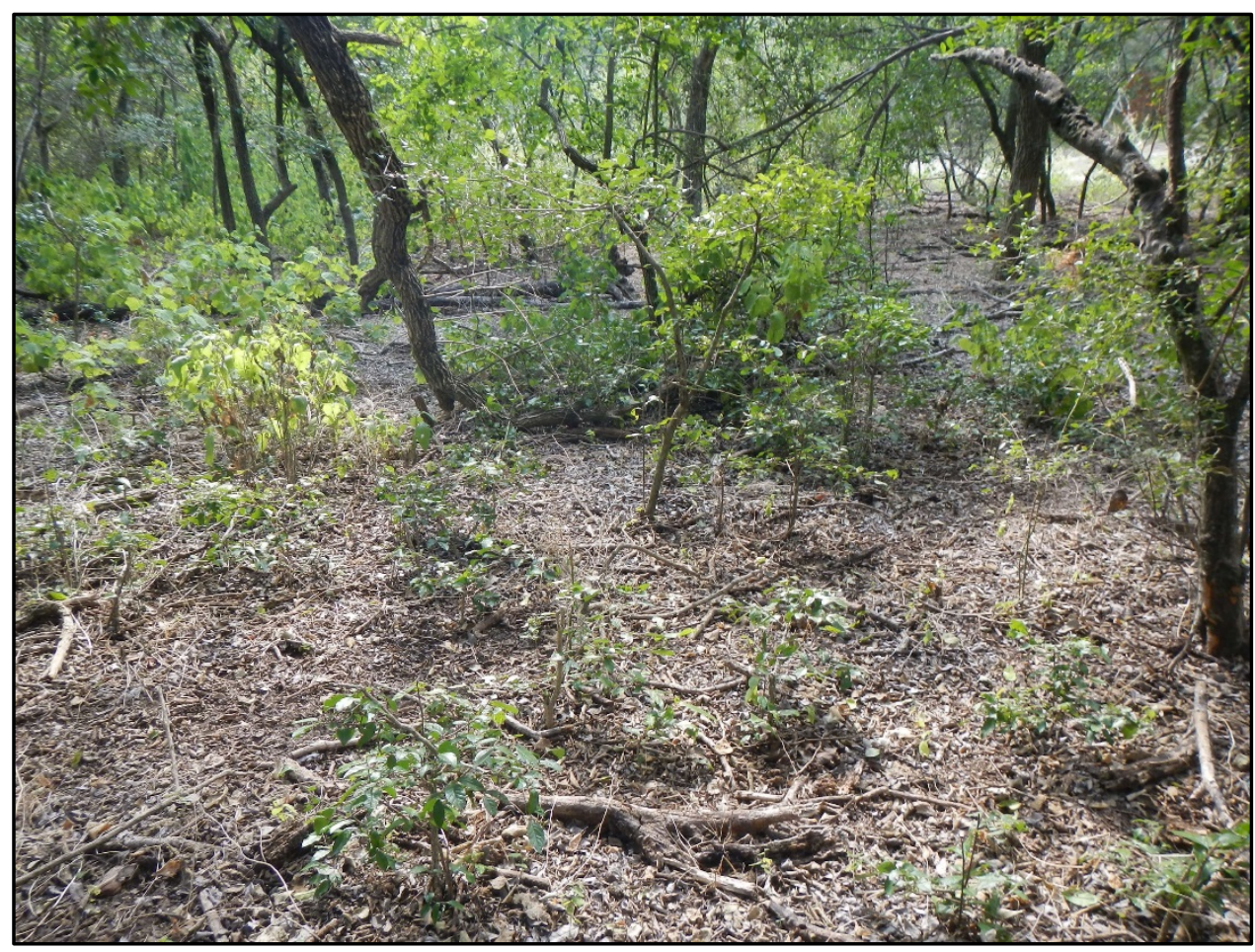

Figure 4. Typical site conditions.

\section{SUMMARY OF INVESTIGATIONS}

Site 41GU177 measures 137 meters (m) northeast/southwest by 30 m northwest/southeast; however, the site likely extends to the northwest and southeast beyond the project boundaries (see Figure 3 ). The site was originally discovered on August 31, 2015, during SWCA's initial Phase I pedestrian survey of the proposed water pipeline alignment. Site boundaries were determined by shovel testing for subsurface cultural deposits within project area boundaries during survey efforts. SWCA initially excavated five shovel tests across the site; however, based upon the nature and depth of the soils identified at the site and the assemblage of artifacts noted during shovel testing, a second phase of survey-level investigations consisting of systematic backhoe trenching occurred at the site on October 16, 2015. Those initial backhoe excavations were limited due to landowner access and clearing restrictions (i.e., no trees could be removed or damaged); as such, the excavation of three backhoe trenches (BHT01-BHT03) occurred only where access was practical. BHT01 and BHT03 contained no cultural materials; however, BHT02, near the northeastern end of the site, contained a partially intact burned rock feature and a moderate density of chipped-stone debitage and faunal remains. Ultimately, the 2015 investigations revealed intact prehistoric subsurface cultural materials and a buried cultural feature in the northeastern/eastern portion of the site.

Given the apparent integrity of the cultural deposit and the depth of observed artifacts, SWCA recommended that a phase additional work was warranted to better explore the cultural deposits through hand excavations and to determine if the cultural deposits retained sufficient integrity to warrant SAL designation. This phase occurred on October 12-14, 2016, until work was halted at the request of the landowner. Investigations involved reopening BHT02 (and extending it for several meters), the excavation of BHT04 that intersected at a right angle with BHT02, and the hand excavation of four, $1 \times 1-\mathrm{m}$ units (Test Units [TUs] 1-4) off BHT02 and BHT04. Due to the recovery of a possible human bone from TU03 on October 13, excavations were halted to contact the Guadalupe County Sheriff's office. Ultimately, the bone was determined to be not human; however, the landowner halted all activity on the site. 
Following negotiations with the landowner, the proposed easement on parcel 50360 was purchased for the project and access was again made available to the site beginning in June 2017. SWCA returned to site 41GU177 on June 28, 2017, to complete the excavation units originally established in 2016, as well as excavate two additional backhoe trenches (BHT05 and BHT06) at the location of a proposed bore pit for a planned HDD that could impact parts of the site. During the excavation of BHT05 and BHT06, in situ cultural materials (including a very large chipped-stone axe and burned rock) were observed in the trench profiles and SWCA excavated a fifth hand excavation unit (TU05) at the intersection of BHT05 and BHT06 to explore the artifact and burned rock feature. Following completion of the limited excavations on July 6, 2017, there was sufficient evidence to recommend that portions of the site warranted SAL designation and potentially a phase of data recovery excavations. However, due to the previous landowner access restrictions, the remaining portions of the site still needed systematic examination to determine the integrity and potential significance of those areas. To accomplish this, in August 2017, SWCA proposed a program of close interval shovel testing and additional backhoe trenching across the remaining site area.

In September and October 2017, SWCA conducted a program of close interval shovel testing followed by additional backhoe trenching at the site to better determine the nature, depth, and extent of its cultural deposits (i.e., was there more than one temporal component represented) and to ultimately refine the proposed data recovery areas. SWCA performed the close interval shovel testing to determine the presence/absence and depth of cultural materials in parts of the site that were not previously subject to intensive examination. SWCA initiated the close interval shovel testing the week of September 10, 2017, and that was completed the following week. The hand excavations involved 53 shovel tests on an approximate $10 \times 10 \mathrm{~m}$ grid. One of the primary goals of the shovel testing was to excavate all shovel tests to a depth of at least 100 centimeters $(\mathrm{cm})$ below ground surface $(\mathrm{cmbs})$, based on the known depth of artifacts determined from previous work on the site. As artifacts were also discovered to extend to depths of $160 \mathrm{cmbs}$ during the previous investigations, every fifth shovel test was extended to a depth of $200 \mathrm{cmbs}$ via a hand auger, to test for more deeply buried cultural materials. The results of the close interval shovel testing and augering indicate that artifacts extend from ground surface to a maximum depth of $140 \mathrm{cmbs}$. All artifacts from the shovel tests were collected and have been processed, washed and analyzed; these artifacts will be curated with the artifacts recovered from the prior investigations, as well as the artifacts that may be collected during any potential future data recovery excavations.

Following completion of the close interval shovel testing, SWCA was scheduled to complete additional mechanical trenching on the site the week of September 24, 2017; due to heavy rains and thunderstorms, those investigations were rescheduled and completed on October 4-5, 2017. The locations of the backhoe trenches were determined by the results of the close interval shovel testing (i.e., areas where shovel testing revealed potentially deeply buried artifacts and possible cultural features). Seven additional trenches (i.e., BHT07- BHT13) were excavated across the site. These deeper excavations revealed similar stratigraphy and artifact densities to those observed during the close interval shovel testing; however, several artifacts were noted at significantly deeper depths (i.e., close to $200 \mathrm{cmbs}$ ) than those in the shovel tests.

Based on the results of the phased investigations at the site, SWCA has refined the location and extent of any proposed data recovery effort, focusing efforts on the more significant and intact parts of the site that may be impacted by construction-related activities. The primary objective for the proposed undertaking is to collect data that complements and expands on what is already known about the site from SWCA's prior investigations between 2015 and 2017. Following is a detailed synopsis of SWCA's phased investigations on 41GU177 between August 2015 and October 2017.

\section{Site SeTTing}

The site is located approximately 4.7 kilometers $(\mathrm{km})$ (2.9 miles) north of McQueeney in Guadalupe Counties, Texas. Site 41GU177 is on the western high bank of the Guadalupe River, with a 30- to 40-foot 
vertical drop to the river bottom to the east and gently sloping (2-5 percent slope) terrain to the west. SWCA's investigations were confined to the portion of the site within the project area; however, SWCA suspects the site continues beyond the limits of the project area to the east and west.

Site 41GU177 is located within forested rangeland with mixed hardwood trees, large live oak trees, and moderately dense shrub undergrowth. Ground surface visibility ranges from 0 to 20 percent, with limestone gravels and cobbles exposed on the ground surface. Vegetation clearing and grading, fence line construction, and natural erosion have impacted site integrity to varying degrees. One property fence and private cemetery borders the northwestern site boundary, and natural erosion from the high bank of the Guadalupe River has impacted the northeastern site boundary.

\section{OVERVIEW OF GEOMORPHOLOGY}

SWCA reviewed the local geology and soils as mapped by the Bureau of Economic Geology and the U.S. Department of Agriculture's Soil Conservation Service, as well as local U.S. Geological Survey (USGS) topographic maps, aerial photographs, and excavation and site profiles, which provided the basis for the general geomorphology of site 41GU177. The site is located on the right bank (west side) of the Guadalupe River, situated roughly 30 to 35 feet above the river.

The surface geology for this area indicates that the project alignment crosses Pleistocene-age terrace deposits (Barnes 1983). These deposits are characterized as gravel, sand, silt, and clay largely derived from the Edwards Plateau situated a few miles upstream (Barnes 1983). These deposits in local areas have calcium carbonate-cemented quartz sand, silt, clay, and gravel intermixed and interbedded in terraces along streams. Low terraces of major rivers are capped by 2 to $4 \mathrm{~m}$ of clayey sand and silt. Sandy gravel on higher terraces varies somewhat in composition from river to river.

The soils at 41GU177 are mapped as Sunev loam (1 to 5 percent slopes) (Natural Resources Conservation Service 2017; Ramsey and Bade 1977). Briefly, the Sunev loams are occasionally identified as Venus loams (Sunev spelled backward) in older soil surveys (Ramsey and Bade 1977; Taylor et al. 1991). These are characterized as occurring on terraces or alluvial fans of rivers and large streams and composed of calcareous brown-dark brown loams and clay loams (Ramsey and Bade 1977; Taylor et al. 1991).

At 41GU177, the Guadalupe River is a competent drainage with an approximately 43-m-wide (142-foot) base and a slow southeast flowing channel. The Guadalupe River, as it winds past the site, has a high sinuosity ratio of 1.4 indicating that it is a sinuous waterway, which is apparent from the New Braunfels East and McQueeney 7.5-minute USGS topographic quadrangle maps (Charlton 2008:138-139). This sinuosity suggests a partially dynamic depositional history where the channel has moved laterally. In this type of setting, the lateral movement can significantly erode the deposits of outside meander bends and, in contrast, deposit significant amounts of sediment upon interior meanders (Charlton 2008). Site 41GU177 is situated on an exterior bend of a meander. Not surprisingly, the right bank of the river, below 41GU177, does not contain broad alluvial landforms.

At this location, the Guadalupe River valley is an unpaired, stair-stepped terrace system consisting of a series of alluvial terraces that bracket the drainage created by various episodes of overbank deposition (Waters 1992:149-151). The left bank (east side) of the Guadalupe River exhibits three terrace landforms that from youngest (closest to the channel) to oldest consist of the $T_{0}, T_{1}$, and $T_{2}$ terraces. In contrast, the right bank, containing 41GU177, has one apparent alluvial terrace landform $\left(\mathrm{T}_{2}\right)$.

On the right bank, the $\mathrm{T}_{2}$ landform abruptly rises about 9.5 to $11 \mathrm{~m}$ (31 to 36 feet) above the Guadalupe River. The $\mathrm{T}_{2}$ landform on both banks is broad (roughly $1 \mathrm{~km}$ ) and gradually grades into the valley margins aligning the drainage valley. Another landform occurs upon the $T_{2}$ terrace near site 41GU177, which could 
be part of the $T_{2}$ landform or remnants of an older alluvial landform (i.e., $T_{3}$ ). This landform parallels the Guadalupe River and is situated at roughly 580 to 585 feet above mean sea level (amsl) and about $1.5 \mathrm{~m}$ (5 feet) above the $T_{2}$ terrace that surrounds it. Site $41 \mathrm{GU} 177$ is primarily situated on the $T_{2}$ terrace between 575 to 580 feet amsl.

\section{RESULTS}

\section{INVESTIGATIONS}

SWCA discovered 41GU177 on August 31, 2015, as part of the intensive pedestrian survey of the Vista Ridge project area. The site was initially identified by a scatter of surface artifacts (Figure 5). SWCA excavated five shovel tests (KS139-KS141 and RW342-RW343), with three (KS139 and RW342-RW343) being positive for cultural materials (Appendix A). Subsurface materials ranged from the ground surface to beyond $80 \mathrm{cmbs}$ and consisted primarily of tertiary flakes with a few secondary flakes and cultural shatter also observed. Materials observed during the ground surface inspection consisted of: two fragmented untyped projectile points (not collected), five to 10 scrapers, two to four choppers, 50 to 80 primary flakes, 100 to 200 secondary flakes, and 100 to 200 pieces of lithic shatter. All lithic materials observed were composed of chert raw material types. SWCA also observed fragments of iridescent water mussel shells during shovel testing. No diagnostic materials or cultural features were observed during these initial shovel test excavations.

Due to the potential for deeply buried cultural deposits, backhoe trench investigations were then conducted at the site on October 16, 2015. A total of three backhoe trenches (BHT01- BHT03) were excavated; BHT01 and BHT02 were excavated within the site boundary and BHT03 was excavated approximately $40 \mathrm{~m}$ southwest of the site (see Figure 5). Due to landowner restrictions (i.e., no damage to oak trees) and the forested terrain, the placement of backhoe trenches was constrained. BHT01 and BHT03 encountered friable brown (10YR 5/3) silt loam overlying light yellowish brown (10YR 6/4) to very pale brown (10YR 7/4) silt loam with increasing gravel and pebble inclusions (Appendix B). In contrast, BHT02 encountered friable very dark grayish brown (10YR 3/2) to brown (10YR 4/3) silt loam horizons containing cultural materials separated by a sterile loose brown (10YR 4/3) silt loam horizon (see Appendix B). Artifacts in BHT02, including numerous lithic flakes and three burned rocks, which were initially observed in the trench wall profile between 0 and 60 cmbs. A second cultural horizon, containing lithic flakes and a blade, was observed from 90 to 120 cmbs.

To investigate the nature of the burned rock and determine if a cultural feature was present, a column sample was excavated off the north wall of BHT02. A total of 10 flakes, two animal bone fragments, one small piece of charcoal, and two burned rocks were recovered from the column sample between 0 and $55 \mathrm{cmbs}$ (Appendix A). In addition, SWCA encountered four small- to medium-sized burned rocks (one 0-5 cm diameter and three 5-10 cm diameter) in a cluster at the base of the column sample. The feature was truncated on the south side by the backhoe; however, a large burned rock appears to continue into the column sample wall, suggesting portions of the feature may remain intact. After documentation was complete, the feature and associated artifacts were covered with soil and the trench was backfilled. 


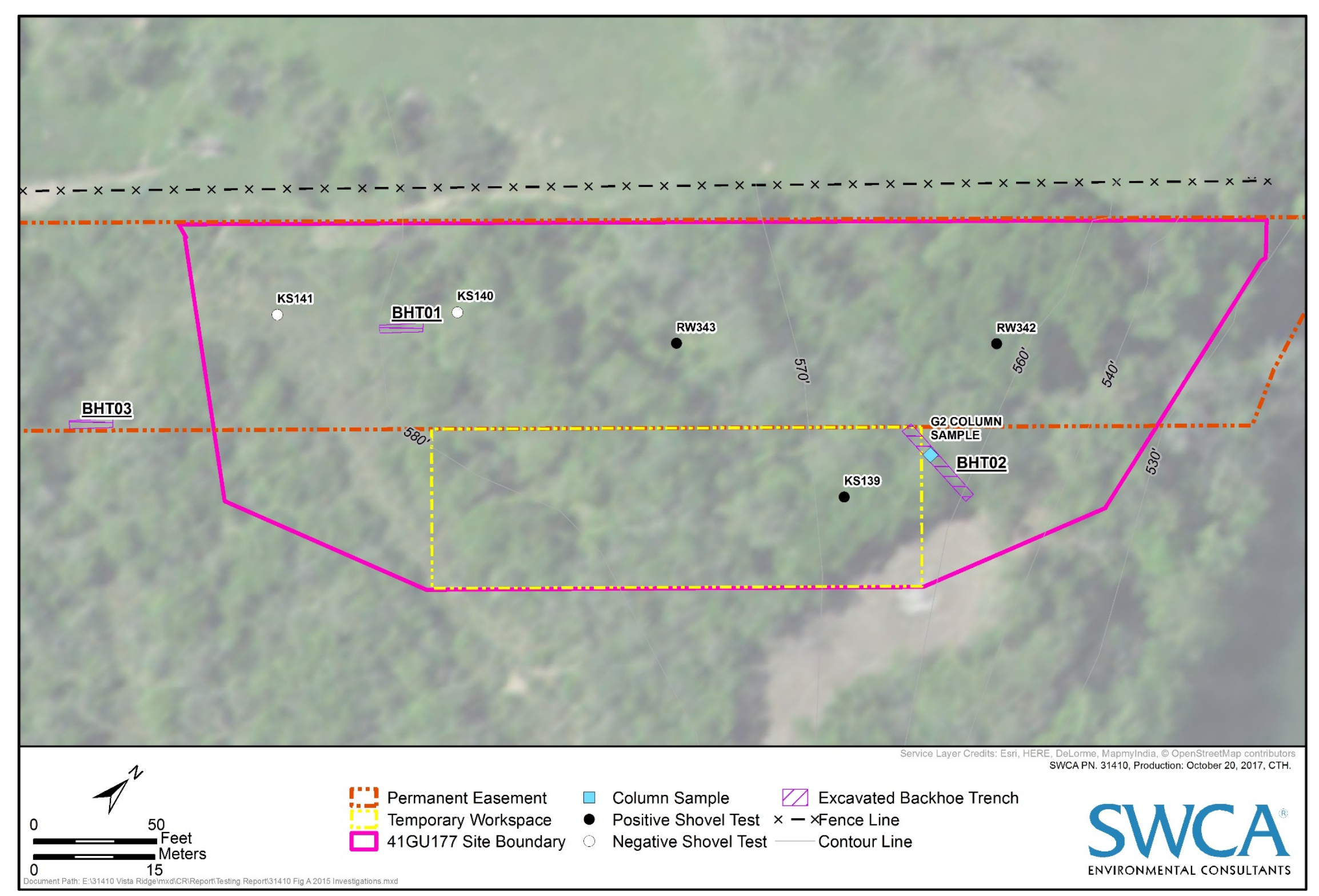

Figure 5. 2015 investigations at 41GU177. 


\section{INVESTIGATIONS}

SWCA revisited site 41GU177 between October 12-14, 2016, to further evaluate the site's significance and potential for deeply buried deposits through further mechanical trenching and hand excavations. SWCA reopened one of the original survey trenches (BHT02) from 2015 and excavated BHT04, which intersected BHT02 (Figure 6). These two trenches bisect each other in a cruciform formation in the eastern-most portion of the site, surrounded by the positive shovel tests and the densest amount of cultural materials observed. The trenches revealed moderately dense cultural materials and one cultural burned rock feature.

BHT02 was originally roughly $5 \mathrm{~m}$ long and excavated to a depth of approximately $180 \mathrm{cmbs}$ during survey investigations (Appendix B). The trench was reopened and extended to the east to a total length of roughly $9 \mathrm{~m}$ long and approximately $122 \mathrm{cmbs}$. Most of the cultural materials were identified along the north wall profile in two cultural zones identified from 0 to $60 \mathrm{cmbs}$ and 90 to $120 \mathrm{cmbs}$ during survey efforts; SWCA observed a similar range and density of artifacts during the subsequent reopening of BHT02. One of the goals of reopening the trench was to locate the previously excavated column sample and truncated partial feature from previous survey investigations and to then establish a $1 \times 1-\mathrm{m}$ excavation unit over it, which was accomplished. BHT04, placed in the southwestern portion of the designated test area, was $8 \mathrm{~m}$ long and excavated to a depth of approximately $120 \mathrm{cmbs}$ (see Figure 6). BHT04 served as an expansion of the trenching area, which allowed for the placement of test units off the east wall. Investigations in October 2016 involved the excavation of four hand excavated $1 \times 1-\mathrm{m}$ test units (i.e., TU01-TU04); SWCA excavated TU01 and TU02 along the north wall of BHT02, and excavated TU03 and TU04 along the east wall of BHT04 (see Figure 6). SWCA excavated the test units to varying depths before the landowner revoked land access on October 14, 2016.

\section{TEST UNIT 1}

TU01 was hand excavated to a depth of $150 \mathrm{cmbs}$ ( $98.5 \mathrm{~cm}$ below datum [cmbd]). TU01 was located on the north wall of BHT02, west of TU02 (see Figure 6). Lithic artifacts include 80 primary lithic flakes, 128 secondary flakes, 574 tertiary flakes, a mano (ground stone artifact) fragment, two utilized flakes, an earlystage biface, a lithic core, a tested cobble, and three projectile points, as well as 75 faunal bones. The projectile points and point fragments consist of two untyped arrow point fragments (Figure 7, Specimens e and k) and one corner-notched, broad stem Scallorn arrow point variant (see Figure 7, Specimen a) (Appendix C). Other artifacts include burned rock between 20 and $90 \mathrm{cmbs}$, a burned nut shell at 70 to 80 cmbs, two fragments of burned seed at 140 to $150 \mathrm{cmbs}$, and freshwater mussel shell throughout.

\section{TEST UNIT 2}

TU02 was hand excavated to a depth of $140 \mathrm{cmbs}$ (98.6 cmbd). TU02 was also located on the north wall of BHT02, east of TU01 (see Figure 6). Lithic artifacts include 129 primary lithic flakes, 217 secondary flakes, 873 tertiary flakes, two blades, one core, two utilized flakes, one hammerstone, one bifacial distal tip, and two projectile points. The projectile points and point fragments include one corner-notched, broad stem Scallorn arrow point variant (see Figure 7, Specimen b), an untyped arrow point preform that may be a Scallorn preform (see Figure 7, Specimen d), and the distal tip of an untyped arrow point (see Figure 7, Specimen i). Other artifacts include 10 animal bone fragments, a probable shell bead fragment (Figure 8), and burned rock at approximately 60 to $70 \mathrm{cmbs}$ (Appendix C) 


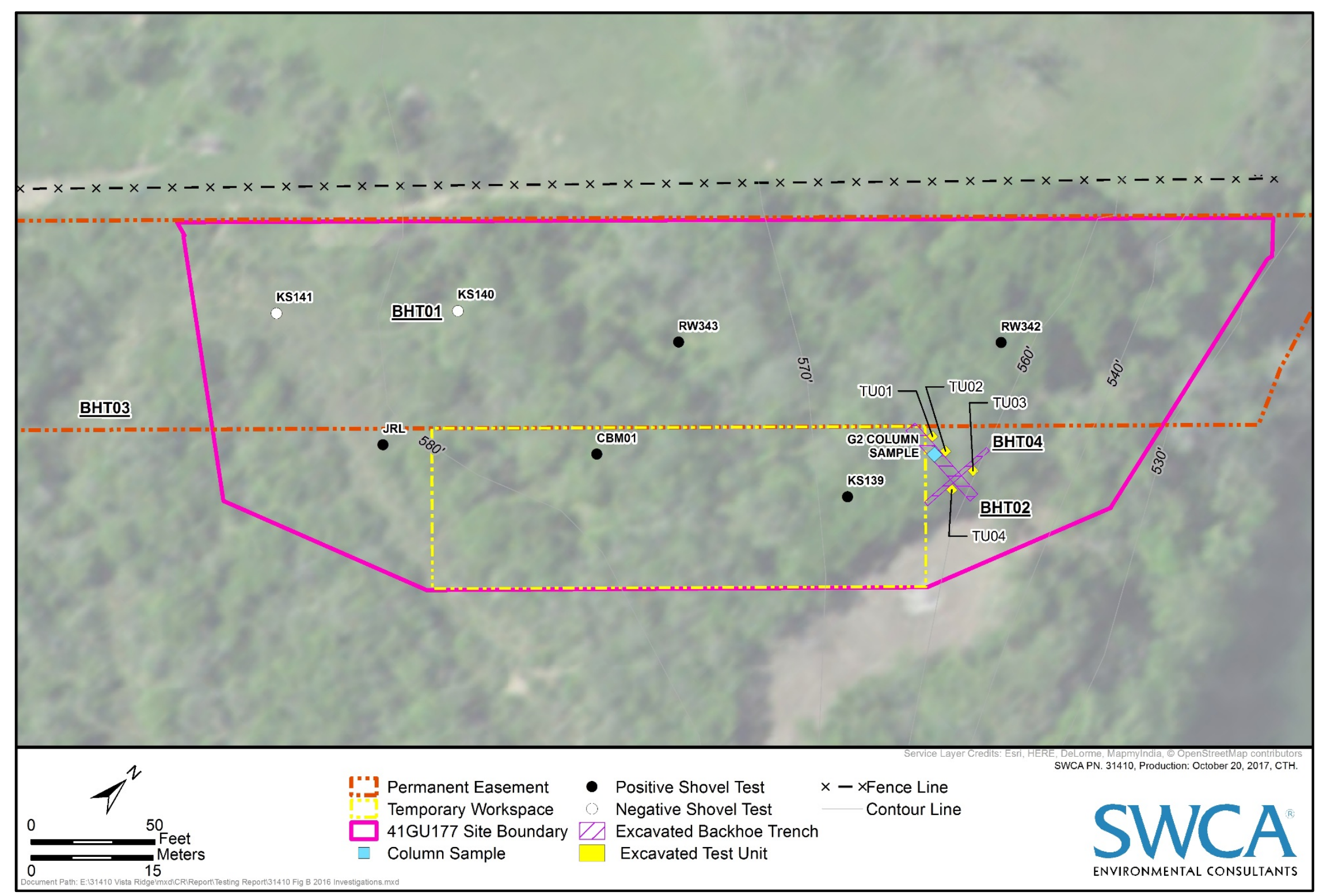

Figure 6. 2016 investigations at 41GU177. 


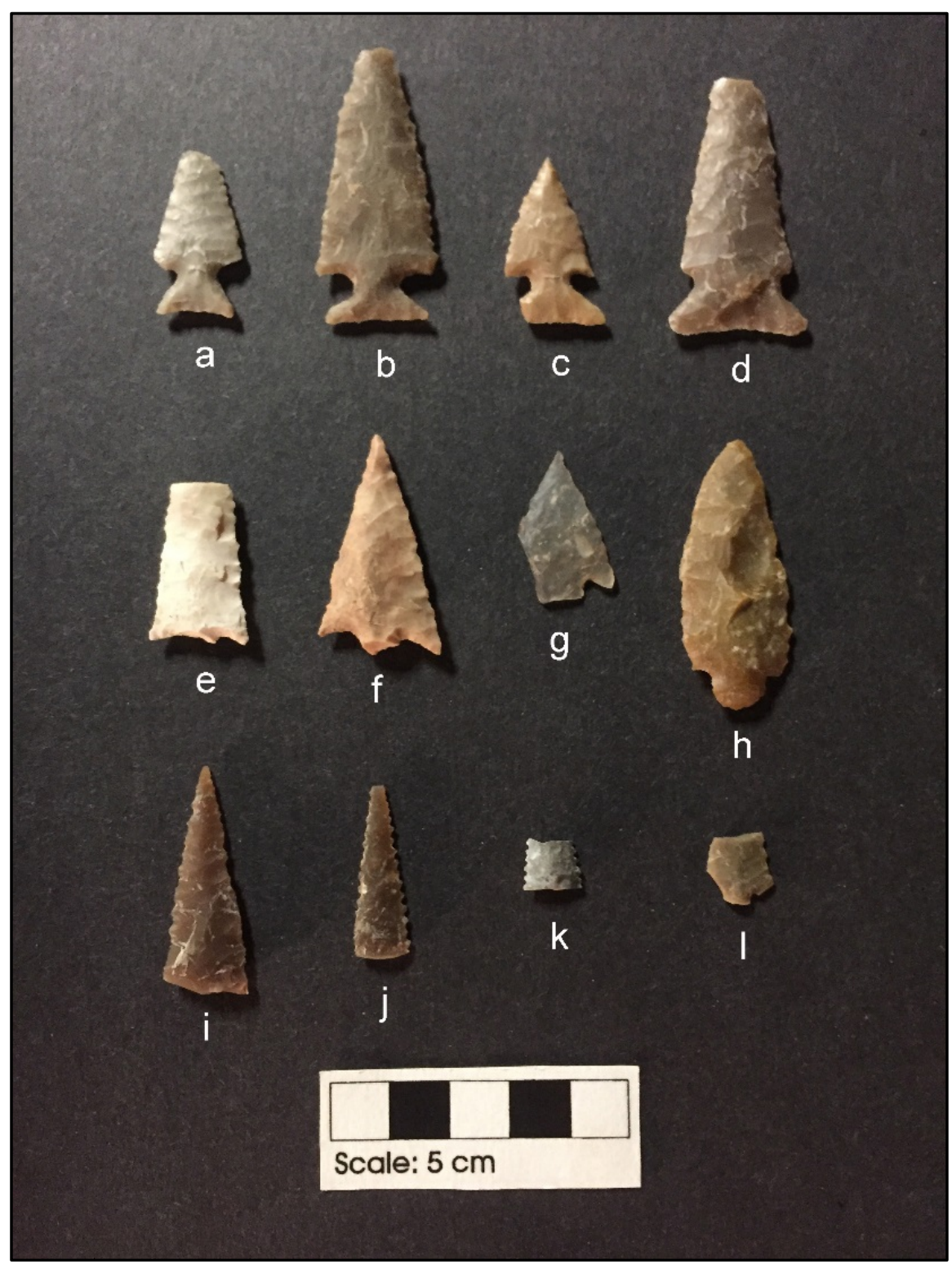

Figure 7. Arrow points and arrow point fragments recovered from 41GU177: a-c. corner-notched, broad stem variant Scallorn arrow points; d. Scallorn preform; e. untyped arrow point fragment; f. untyped arrow point fragment; g. untyped arrow point (possible Sabinal specimen); h. Untyped arrow point preform; i-j. distal tips of an untyped arrow points; k. untyped arrow point fragment; and I. untyped arrow point fragment

\section{TEST UNIT 3}

TU03 was excavated to a depth of $40 \mathrm{cmbs}(99.6 \mathrm{cmbd})$. The unit was located on the east wall of BHT04, just north of the intersection of BHT02 and BHT04 (see Figure 6). Lithic artifacts include nine primary flakes, 21 secondary flakes, 84 tertiary flakes, a utilized flake, several burned rocks, and one large bone initially thought to have the potential to be human (Appendix C). 


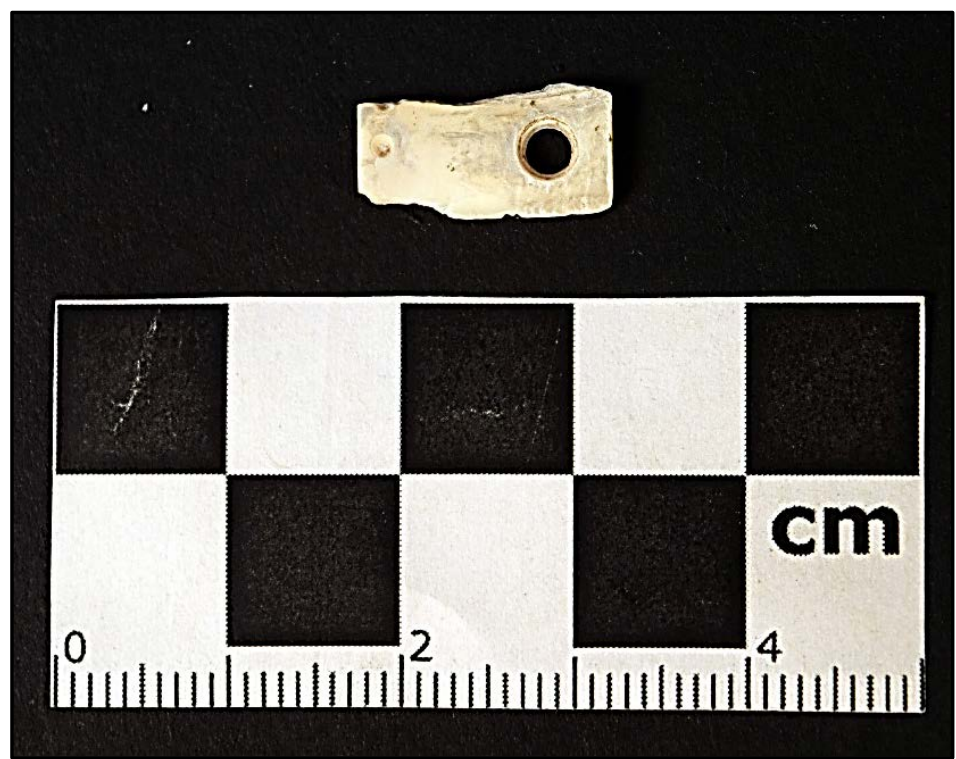

Figure 8. Fresh water mussel shell bead fragment.

Following recovery of the large bone on October 13, 2017, SWCA contacted the Guadalupe County Sheriff's Office and the landowner to inform them of the bone. The Guadalupe County Sheriff's Office assumed custody of the bone to determine if it was human and if the excavation area represented a crime scene. At this point, the landowner revoked access to the site and a 6-week process to definitively identify the bone as human or non-human began. As such, TU03 was only minimally investigated before access to the site was revoked. SWCA's osteologist initially identified the bone as potentially human based on examination of photographs; however, once the Guadalupe County Sheriff's department assumed custody of the bone, they sent photographs to an osteologist at Texas State University, who indicated the bone was not human and probably belonged to a deer. Given that there were two conflicting opinions as to whether the bone was human or not, a third opinion was solicited from osteologist Dr. Michelle D. Hamilton of Texas State University. Following her examination of the bone, Dr. Hamilton indicated that the specimen was non-human and is likely from a bovid/ungulate (i.e., cow, bison, etc.). Given that two of the three osteologists agreed that the specimen was non-human, the bone is not considered a human remain.

\section{TEST UnIT 4}

TU04 was excavated to a depth of $30 \mathrm{cmbs}(99.7 \mathrm{cmbd})$. The unit was located on the east wall of BHT04, just south of the intersection of BHT02 and BHT04 (see Figure 6). Lithic artifacts include 27 primary flakes, 15 secondary flakes, 171 tertiary flakes, and one unifacial scraper (Appendix C). The majority of tertiary flakes came from Level 3, 20 to 30 cmbs, with a total of 145 tertiary flakes recovered. As with TU03, this unit was minimally excavated before access to the site was revoked.

\section{SUMMARY}

The phase of site assessment occurred on October 12-14, 2016, until work was halted at the request of the landowner. Due to the recovery of a possible human bone from TU03 on October 13, excavations were stopped to contact the Guadalupe County Sheriff's office. Ultimately, the bone was determined not to be human; however, the landowner had additional concerns and would not allow any further investigations. 


\section{INVESTIGATIONS}

SWCA returned to site 41GU177 from June 28-July 6, 2017 (following VRRSP's purchase of the easement from the private landowner), to complete the hand excavation units started in 2016 and continue to assess the site and its potential for deeply buried deposits. SWCA cleaned the four excavation units that had been left open from the prior year and continued the excavations to a maximum depth of $180 \mathrm{cmbs}$. In addition to completing the open units, investigators excavated two more trenches (BHT05 and BHT06) in a cruciform pattern to the northwest of the open excavation area, in and adjacent to the proposed bore pit for the HDD. This phase of investigations also excavated a fifth test unit (TU05) based on those results (Figure 9). After hand excavations were complete, SWCAs geoarchaeologist, Ken Lawrence (MA), conducted geoarchaeological analyses of the soil consisting of soil and stratigraphic documentation and magnetic susceptibility testing.

Following completion of hand excavations on July 6, 2017, SWCA determined that additional data from across the site was warranted to further test the site and narrow down the potential data recovery areas. SWCA returned to the site in late September and from October 4-5, 2017, to conduct systematic close interval shovel testing on a $10 \times 10$-m grid across the site to further test the depth, nature, and extent of the cultural deposits (Figure 10). During the late September 2017 mobilization, 53 shovel tests were systematically excavated across the site and, based on the results, SWCA created an artifact density plot map to illustrate where artifacts were recovered (Figure 11; Appendix A). Based on that data, additional backhoe trenches (BHT07-BHT13) were then excavated on October 4-5, 2017, to explore the site further (see Figure 10; Appendix B). SWCA also conducted additional magnetic susceptibility testing during this later phase.

As Figure 11 illustrates, there is an area of high to moderate artifact density in the north-central part of the site. The portion of the site within the high and moderate density areas appears to contain intact archaeological deposits that could be impacted during construction-related activities associated with rightof-way clearing, bore hole excavation, haul road construction and use, and machinery and equipment laydown. Based on the density of cultural materials recovered, and the degree of preservation observed in the high and moderate density areas, it is SWCA's professional opinion that the parts of 41GU177 within those areas may warrant designation as a SAL. The following provides a summary of the completed test unit excavations and additional backhoe trenching results from the 2017 investigations.

\section{TEST UNIT 1 CONTINUED}

TU01 was extended from a depth of $150 \mathrm{cmbs}$ to a maximum depth of $170 \mathrm{cmbs}$ ( $98.3 \mathrm{cmbd})$. These lower levels contained one secondary lithic flake, six tertiary lithic flakes, and five bone fragments (Appendix C). Due to the sparse number of artifacts within the lower levels, it was determined that the unit was below any occupation level and those artifacts within were likely from a secondary context, possibly due to artifact migration and/or bioturbation.

\section{TEST UNIT 2 CONTINUED}

TU02 was extended from a depth of $140 \mathrm{cmbs}$ to a maximum depth of $160 \mathrm{cmbs}$ ( $98.4 \mathrm{cmbd})$. These lower levels contained four secondary lithic flakes and three tertiary lithic flakes (see Appendix C). Like TU01, the lower excavation levels were determined to be below any occupation level, due to the paucity of artifacts, and the unit was terminated. 


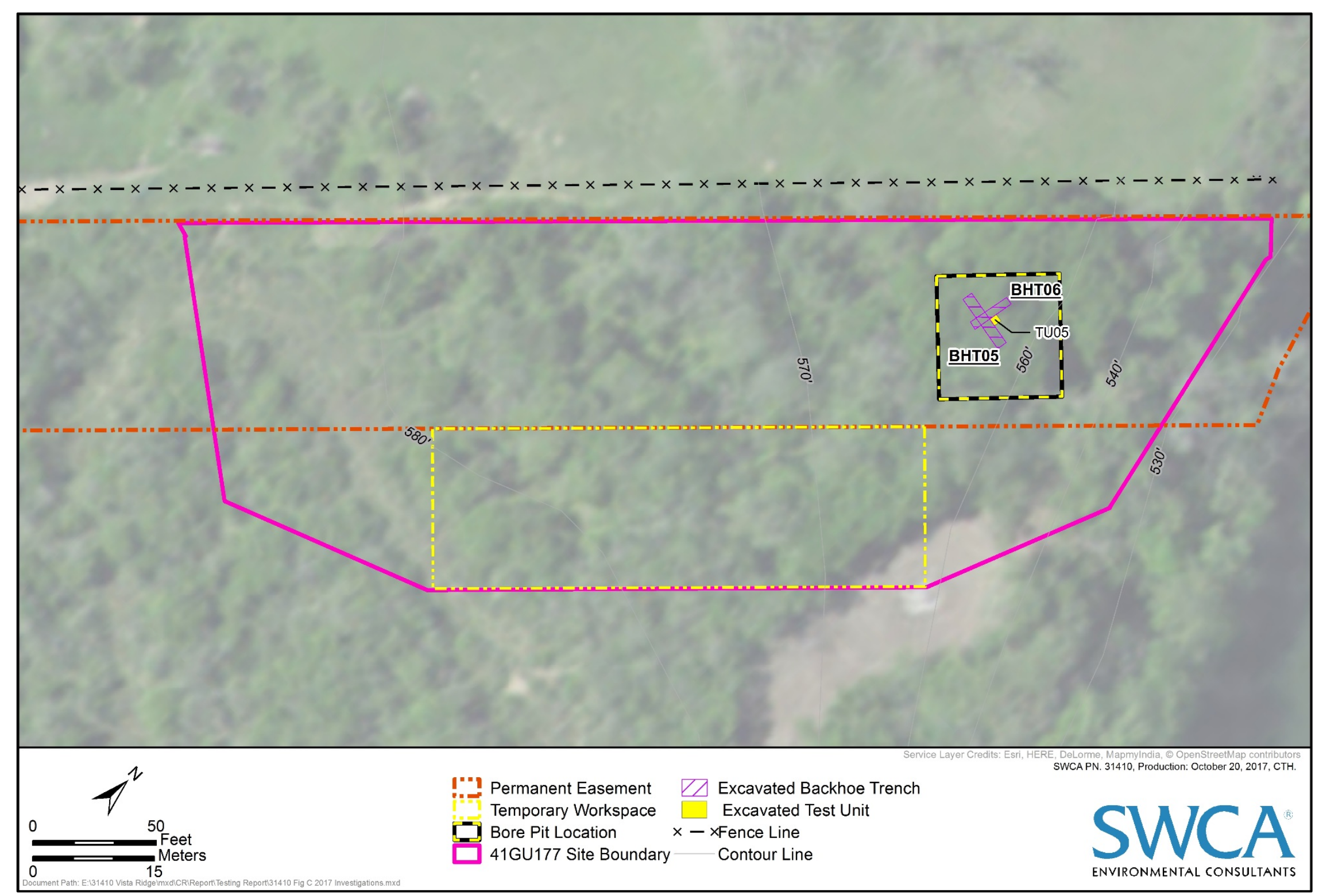

Figure 9. 2017 additional excavations in the proposed bore pit and adjacent work area. 


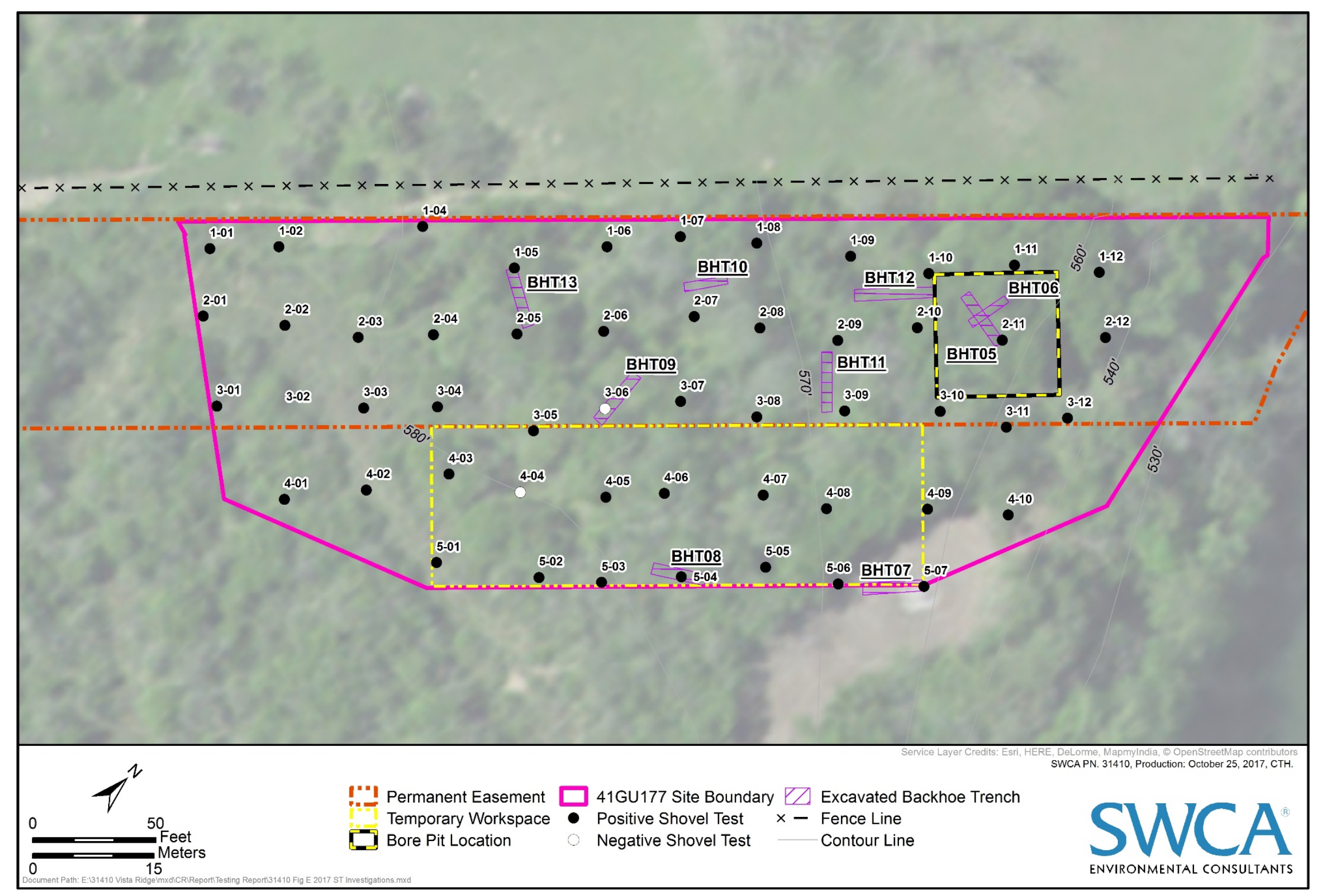

Figure 10. 2017 close interval shovel test and additional backhoe trench plots. 


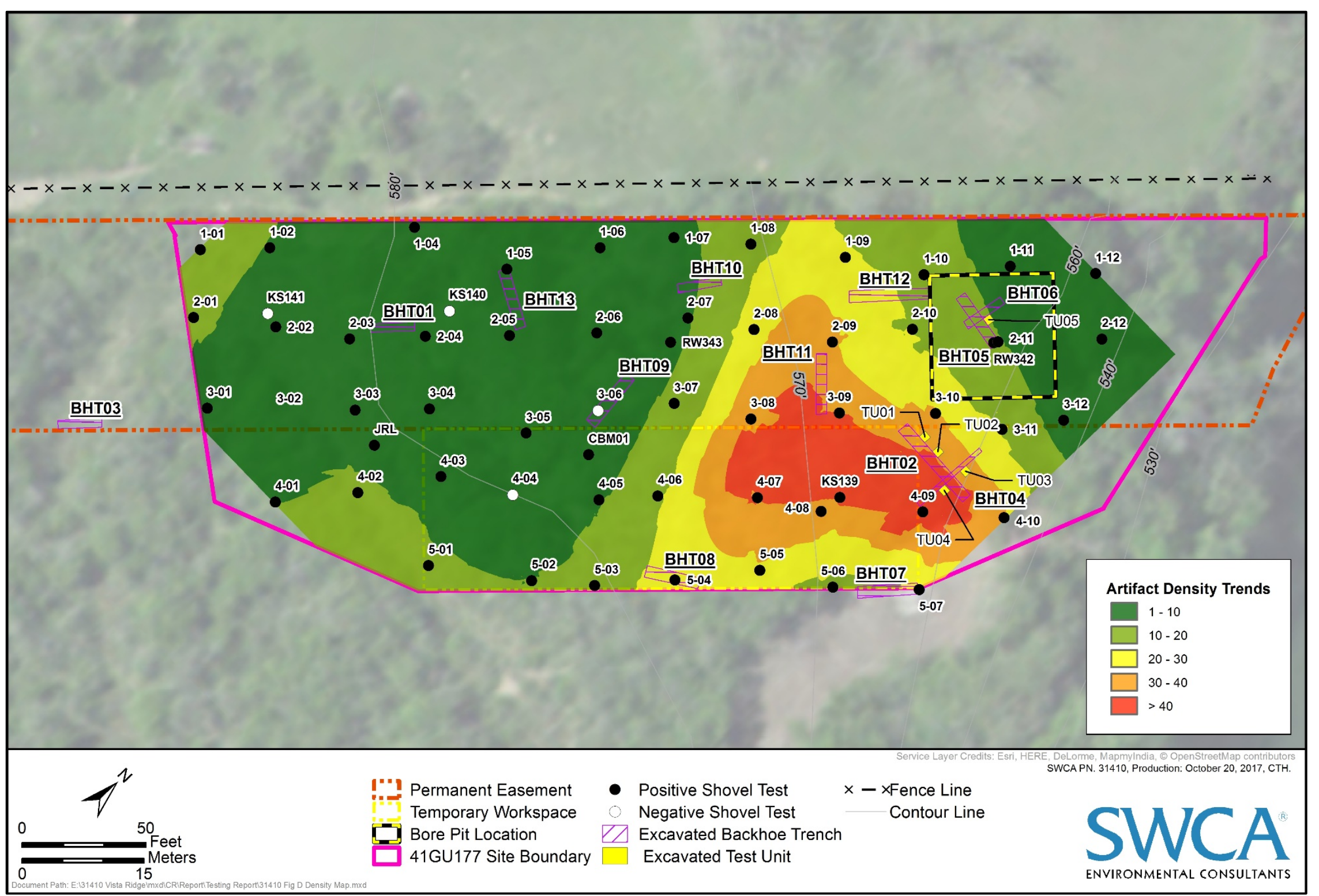

Figure 11. Artifact density plot generated from close interval shovel testing data, backhoe trench excavations, and hand excavated units. 


\section{TEST UNIT 3 CONTINUED}

TU03 was continued from a depth of $40 \mathrm{cmbs}$ to a maximum depth of $180 \mathrm{cmbs}(98.2 \mathrm{cmbd})$. The prehistoric artifact assemblage of the continued levels includes 37 primary lithic flakes, 134 secondary flakes, 553 tertiary flakes, two ground stone fragments, one bifacial tool fragment, two modified flakes, one core, and three projectile points; two untyped arrow point fragments (see Figure 7, Specimens $\mathrm{f}$ and $\mathrm{j}$ ) and an untyped dart point that is possibly a Frio or Ensor specimen (see Figure 12, Specimen b). Additional artifacts include 34 bone fragments and two fresh water mussel shell (Appendix C). The mussel shell was observed between 40 to $50 \mathrm{cmbs}$ and 60 to $70 \mathrm{cmbs}$, while faunal bone fragments were collected throughout the levels. Like TU01 and TU02, the lower excavation levels were determined to be below any occupation level, due to the paucity of recovered artifacts and the unit was terminated.

\section{TEST UNIT 4 CONTINUED}

TU04 was continued from a depth of $30 \mathrm{cmbs}$ to a maximum depth of $160 \mathrm{cmbs}$ (98.4 cmbd). The prehistoric artifact assemblage of the continued levels includes 51 primary lithic flakes, 148 secondary flakes, 466 tertiary flakes, five bifacial fragments, one bifacial blank, six ground stone fragments, one utilized flake, two lithic choppers, and one untyped arrow point preform (see Figure 7, Specimen h and Appendix C). Additional artifacts include 114 bone fragments, 11 freshwater mussel shell fragments, and eight pieces of burned clay (Appendix C). The mussel shell fragments and burned clay were observed above $90 \mathrm{cmbs}$, whereas bone fragments were observed throughout the unit. Most of the unit below $50 \mathrm{cmbs}$ has been heavily bioturbated by rodent burrowing activities. Excavations encountered a hollow rodent den between 50 and $60 \mathrm{cmbs}$, with numerous infilled tunnels above and below the den. Due to the heavy bioturbation and paucity of cultural material in the lower levels, excavations in TU04 was terminated.

\section{TEST UNIT 5}

TU05 was excavated at the northeastern corner of the intersection of BHT05 and BHT06. The unit was placed to investigate a small cluster of burned rocks exposed in the corner of the trenches. TU05 was excavated from surface to a depth of $110 \mathrm{cmbs}(98.9 \mathrm{cmbd})$. The prehistoric artifact assemblage includes 15 primary lithic flakes, 29 secondary flakes, 122 tertiary flakes, one lithic core, four bone fragments, and one fresh water mussel shell fragment (Appendix C). Additionally, during excavation of BHT06, a lithic unifacial axe was uncovered on the E wall near the intersection of BHT05 and BHT06 at a depth of 65 to $70 \mathrm{cmbs}$ (Figure 13). Prehistoric artifacts within the unit become sparse below $70 \mathrm{cmbs}$, and the unit was terminated at the depth of BHT05 (i.e., $150 \mathrm{cmbs}$ ).

\section{Close INTERVAL SHOVEL TEST DATA}

During the close interval shovel testing program conducted on September 15 and 20-22, 2017, SWCA excavated a total of 53 additional shovel tests on a $10 \times 10-\mathrm{m}$ grid to further refine the subsurface data and provide a guide for the backhoe trenching that followed on October 4-5, 2017. Due to variations in the underlying eroded limestone bedrock, the 53 shovel tests ranged in depth from surficial bedrock to as deep as $250 \mathrm{cmbs}$ with the aid of a hand auger. Archaeologists observed cultural material within the shovel tests on surface to a depth of $200 \mathrm{cmbs}$, with most of material located between 20 and $100 \mathrm{cmbs}$. Stratigraphy observed during the shovel testing generally correlated with the strata recorded during the previous phases of investigations (see Natural Strata discussion, below). 


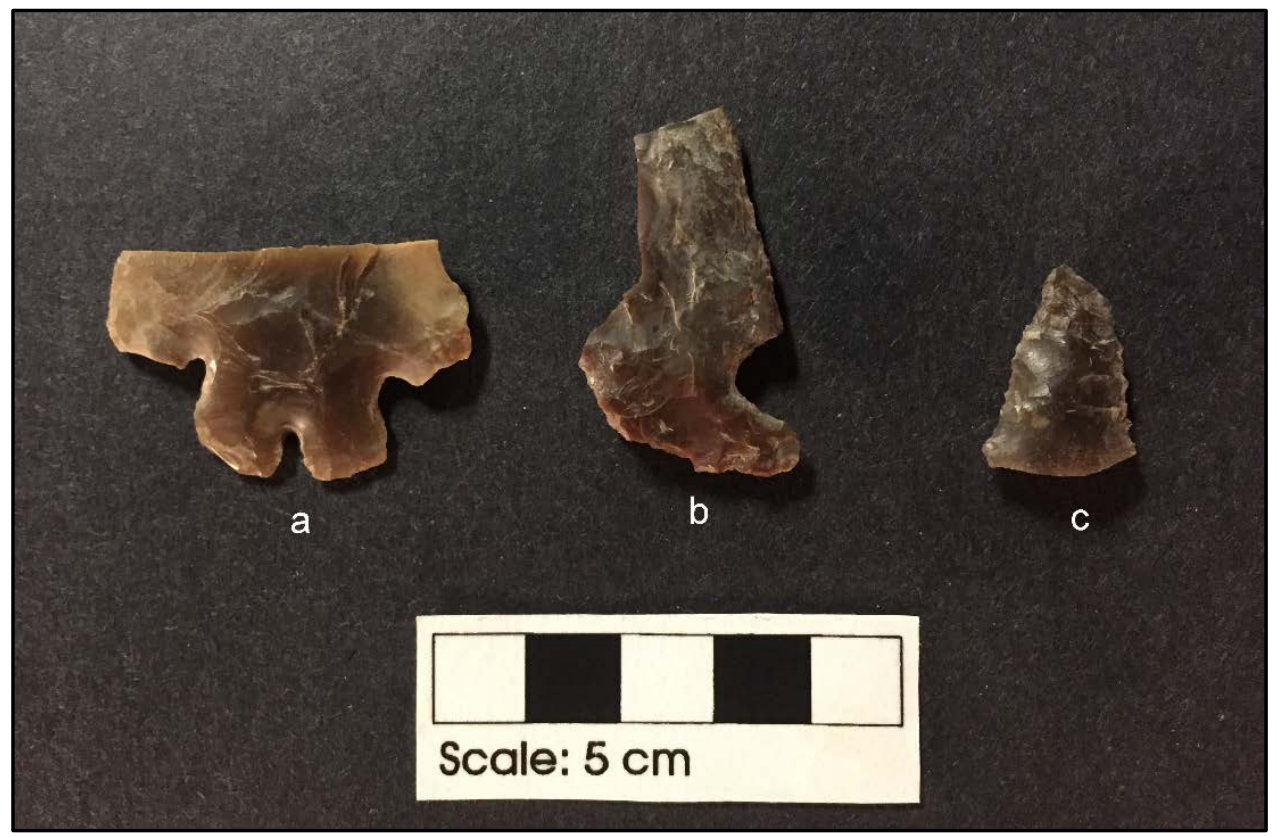

Figure 12. Dart point fragments recovered from 41GU177: a. Montell dart point base; b. Possible Frio or Ensor dart point fragment; and c. untyped dart point distal tip.

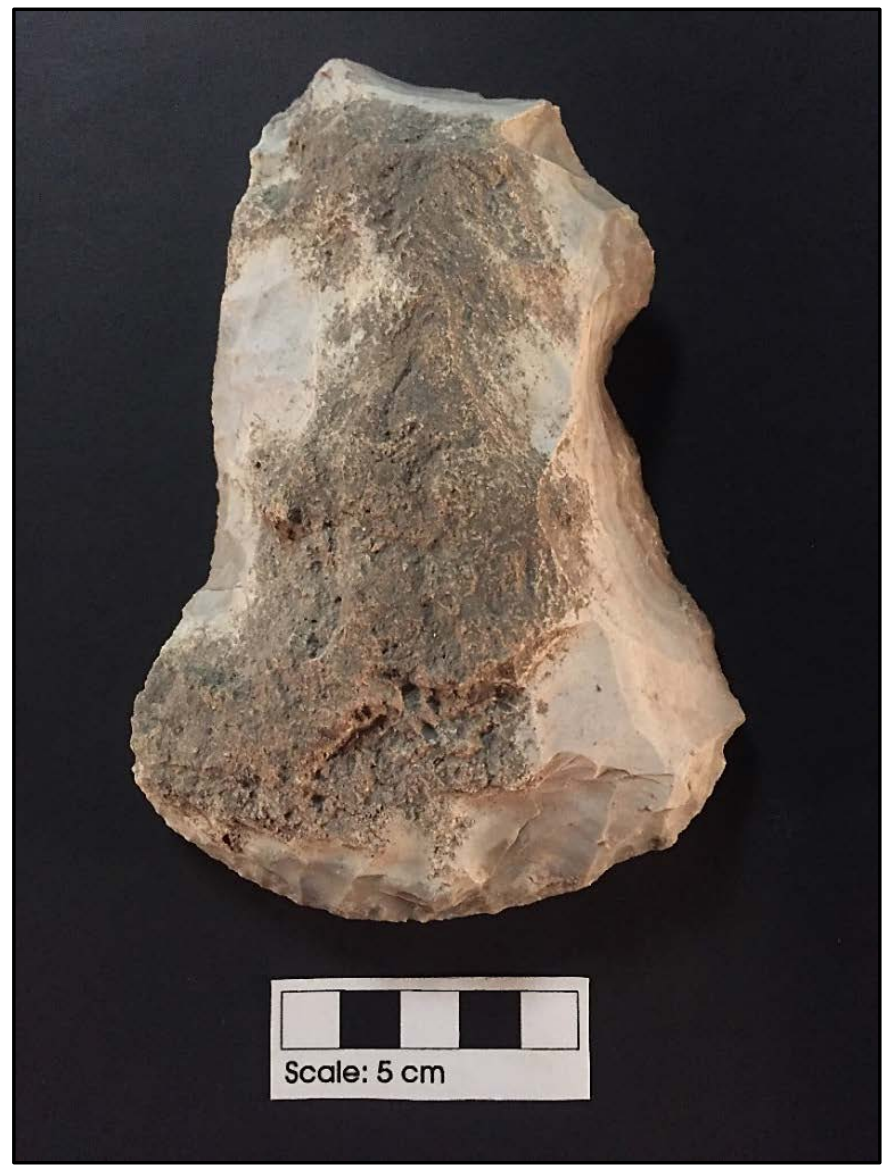

Figure 13. Unifacial axe recovered from TU05 excavations 
Artifactual recovery from the 53 additional shovel tests consist of a total 1,027 pieces of lithic debitage, which included 47 primary flakes, 162 secondary flakes, 758 tertiary flakes, and 60 pieces of lithic shatter. Within the shovel tests of 41GU177, SWCA also recovered five projectile point fragments, one lithic core, one flake toolmodified flake, two ground stone fragments, seven pieces of faunal remains, and 108 pieces of burned rock. The projectile points recovered from these shovel tests include the base of a Montell dart point (see Figure 12, Specimen a); the distal tip of a dart point (see Figure 12, Specimen c); one cornernotched, broad stem Scallorn arrow point variant (see Figure 7, Specimen c); one translucent untyped arrow point that may be a Sabinal specimen (see Figure 7, Specimen g); and one medial section of an untyped arrow point (see Figure 7, Specimen k).

\section{NATURAL STRATA}

As previously indicated, 58 shovel tests, 13 backhoe trenches (BHT01-BHT13) and five test units (TU01TU05) have been excavated at 41GU177 (see Figure 10). The stratigraphy is distinctly different across the site depending on topography and proximity to the Guadalupe River. Specifically, the backhoe trenches on the western end of the site (BHTs 1, 3, 9, and 13) suggest an older deposition than those trenches on the eastern portions of the site (BHTs 2, 4, 5-8, 10-12). The western trenches are situated on a landform roughly 580 to 585 feet amsl and about $1.5 \mathrm{~m}$ (5 feet) above the eastern trenches. These trenches contain relatively shallow $\mathrm{CaCO}_{3}$ filaments and nodules beginning around 30 to $40 \mathrm{cmbs}$, which appear to be Pleistocene terrace deposits. The eastern trenches coincide with the $T_{2}$ terrace landform at 575 to 580 feet amsl (discussed above), whereas the western trenches are situated on the adjacent landform that could be part of the $\mathrm{T}_{2}$ landform or remnants of an older alluvial landform (i.e., $\mathrm{T}_{3}$ ). The stratigraphy in the eastern trenches have deep alluvial deposits that appear younger in age (Holocene) than the western trenches on the adjacent landform. The discussion below summarizes the western and eastern trenches on the two landforms.

\section{EASTERN TRENCHES ( $T_{2}$ TERRACE)}

Overall, SWCA observed six strata in the excavations on the $T_{2}$ terrace (BHTs 2, 4, 5-8, 10-12) (see Figure 10) at $41 \mathrm{GU} 177$ extending to $330 \mathrm{cmbs}$. Below the 6-cm-thick root zone, the upper two horizons (Strata I and II) extending to roughly 52 cmbs are dark gray brown to brown (10YR 4/2-4/3) fine silt loam with a subangular blocky structure, friable consistency, common roots-rootlets (20-30 percent), and Heliodiscus singleyanus snail shell (10 percent). The second horizon (Stratum II) contains some white filaments that are interpreted to be michoryzal fungi (1 percent) and Heliodiscus singleyanus and Rabdotus mooreanus snail shell (10 percent). The lower boundary of Stratum I was clear and slightly wavy, while Stratum II was clear and smooth. Notably, the lower boundary in BHT04 was abrupt and smooth, reflecting the disturbances in the area (e.g., vegetation clearing and earth movement) that likely truncated a portion of Stratum III.

The roughly 35- to 50-cm-thick third horizon (Stratum III) begins at roughly 50 to $75 \mathrm{cmbs}$ and exhibits grayish brown (10YR 5/2) silt loam with a subangular blocky structure, friable consistency; with common roots-rootlets (15 to 20 percent), common insect and worm burrows (10 to 15 percent), and white filaments that are interpreted to be michoryzal fungi ( $<1$ percent), and Heliodiscus singleyanus (1 to 5 percent) and Rabdotus mooreanus (1 to 3 percent) snail shell. SWCA observed rare ( $<1$ percent) subrounded cobbles at the top and bottom of this horizon. The lower boundary of Stratum III was gradual and smooth.

The roughly 50- to 70-cm thick Stratum IV begins at 75 to $85 \mathrm{cmbs}$ and is brown to pale brown (10YR 5/3$6 / 3$ ) silt loam with a friable consistency, a subangular blocky structure; with roots-rootlets (5 to 15 percent), insect and worm burrows (5 to 10 percent), and white filaments that are interpreted to be michoryzal fungi ( $<1$ percent), and Heliodiscus singleyanus (10 percent) and Rabdotus mooreanus (2 percent) snail shell. The lower boundary of Stratum IV was gradual and smooth. 
Stratum V generally begins at $151 \mathrm{cmbs}$ and is a light yellowish brown (10YR 6/4) silt loam with a friableloose consistency and a subangular blocky structure parting to crumb structure; with rootlets (15 percent), insect and worm burrows (5 percent), and Heliodiscus singleyanus (3 percent). This horizon was observed to extend to roughly $230 \mathrm{cmbs}$ and had a gradual and smooth lower boundary.

The last observed stratum (VI) was observed only in BHT06 and BHT07. This horizon typically begins at roughly $230 \mathrm{cmbs}$ and is a light yellowish brown to very pale brown (10YR 6/4-7/4) fine to very fine silt loam. This horizon has a friable consistency, a crumb parting to subangular blocky structure, insect burrows (10 percent), Heliodiscus singleyanus ( $<2$ percent) and Rabdotus mooreanus ( $<5$ percent) snail shell, and calcium carbonate $\left(\mathrm{CaCO}_{3}\right)$ filaments (3-5 percent). The lower boundary of this horizon was unobserved.

\section{WESTERN TRENCHES (T 3 TERRACE?)}

In general, SWCA observed five strata in the western excavations (BHTs 1, 3, 9, and 13) (see Figure 10) at 41GU177 extending to $253 \mathrm{cmbs}$. Notably, the upper three horizons are similar to that observed in the eastern trenches (BHTs 2, 4, 5-8, 10-12), but are thinner and shallower that the stratigraphy observed down slope.

The fourth horizon (Stratum IV) typically begins at roughly 40 to $50 \mathrm{cmbs}$ and exhibits brown to light yellowish brown (10YR 5/4-6/4) silt loam with a crumb to subangular blocky structure, friable consistency; with roots-rootlets (3 to 10 percent), common (5 percent) infilled burrows containing Strat II matrix, small (3 to 4 millimeters) diameter $\mathrm{CaCO}_{3}$ nodules (2 percent), and a gradual and smooth lower boundary.

The fifth horizon (Stratum V) begins around $80 \mathrm{cmbs}$ and contains a light yellowish brown to very pale brown (10YR 6/4-7/6) silt loam with a subangular blocky structure, friable to firm consistency, rootlets (5 percent), $\mathrm{CaCO}_{3}$ nodules ( 2 to $3 \mathrm{~cm}$ ) diameter (3 to 5 percent) that increase in size and prevalence with depth. The lower boundary of this stratum was unobserved, extending to at least $253 \mathrm{cmbs}$. Notably, the horizon appeared to lighten in color toward a very pale brown to yellow (10YR 8/4-8/6) beginning around 140 cmbs.

\section{SUMMARY OF NATURAL STRATA}

In summary, the natural strata (I-VI) observed at 41GU177 suggests some disturbances localized at or near the ground surface. The disturbances are primarily associated with extensive tree root growth, possible earth movement, and erosion. The vertical extent of evident disturbance across the site varied. The excavation areas amongst extensive trees (i.e., BHT05 and BHT06) had moderate, localized disturbance to approximately $75 \mathrm{cmbs}$. In contrast, the excavation areas in proximity to existing roads and previously cleared areas (i.e., BHT02 and BHT04) displayed evidence related to vegetation clearing and earth movement. These disturbances, suggested by the abrupt lower boundary of Stratum II, were shallower (32 cmbs), but more extensive, and may have truncated the upper horizon of Stratum III. The underlying strata (Strata III-VI) across 41GU177 appears generally intact with some disturbance from tree roots and small burrows.

\section{MAGNETIC SUSCEPTIBILITY}

Magnetic susceptibility (MS) analyses of soils have been utilized in archaeological investigations for several decades, and have largely been directed toward surveys (e.g., Crowther and Barker 1995; Dalan 1996, 2008; Rosendahl et al. 2014; Van Leusen et al. 2014; Wiewel and Kvamme 2014). More recently, these investigations have focused on archaeological site formation and associated depositional processes (Dalan 2006, 2008). These analyses have assisted in identifying buried or thermally altered soils associated with cultural activities, as well as identifying the horizontal extent of cultural features (e.g., Dalan and 
Banerjee 1998; Dalan and Bevan 2002; Ellwood et al. 1995; Frederick 2010; Jones and Leffler 2003; Nickels 2010; Peters and Thompson 1999; Mauldin and Figueroa 2006; Rivers et al. 2004).

Extensive reviews of the processes of environmental magnetism and particularly the application of MS have been produced (e.g., Dearing 1999a, 1999b; Gale and Hoare 1991:201-229; Maher et al. 1999; Thompson and Oldfield 1986; Verosub and Roberts 1995). Briefly, magnetic minerals are prevalent in the natural environment and are sensitive to environmental changes (Gale and Hoare 1991:202). The measurement of MS $\chi$ (Chi) is a quantification of the 'magnetisability' of the material (Dalan 2008; Dalan and Banerjee 1998; Dearing 1999b:5; Gale and Hoare 1991:202-204). The mineralogy, the size and shape of the grains, internal stress, and other factors can initially influence the susceptibility value (Dearing 1999a, 1999b; Gale and Hoare 1991:204). However, of relevance to this discussion, factors including organic content, pedogenesis, thermal alteration, and cultural activities (e.g., ash-charcoal and refuse) can subsequently alter (usually increase) the susceptibility values. The implications of using MS for archaeological research is that when examining either vertical or horizontal areas, the susceptibility values can assist in identifying cultural activities areas that may otherwise be blurred at a macro level. Horizontally, the application of MS analysis has been used to define the limits of cultural features and living spaces in excavation blocks (e.g., Mauldin and Figueroa 2006; Wiewel and Kvamme 2014). Vertically, the MS results have been applied to recognizing and delineating cultural horizons (e.g., Frederick 2010, 2012; Lawrence et al. 2013).

Notably, this form of in situ analysis of MS is unable to differentiate between low ( $\chi \mathrm{lf})$ and high ( $\chi$ hf) frequency (470 to $4700 \mathrm{~Hz}$, respectively) and does not examine mass-specific susceptibility. Rather, the in situ analysis examines the volume susceptibility of a specific radius around the probe. Although not as accurate, these analyses are beneficial in that the data can be quickly collected and analyzed in real-time. Although the data derived with this type of analysis (in situ MS) within this setting may not provide definitive identification of cultural versus natural influences, the analysis can be informative regarding integrity of the deposits and the presence of high or low MS values. Specifically, elevated values of MS can suggest an influence by factors including organic content, pedogenesis, thermal alteration, and cultural activities (e.g., ash-charcoal and refuse). Typically, the upper horizons closer to the ground surface will have higher values of MS from the presence of organic matter. Conversely, a stable, intact profile typically has steadily decreasing MS values. Further, "wiggles" are expected in these analyses, but dramatic, sustained changes (increase or decrease) in values may suggest cultural activities or disturbance. This is particularly applicable at recognized horizon boundaries.

MS analysis of four vertical columns were conducted at 41GU177 using either in situ analysis or matrix analysis in the lab. The in situ MS analyses were conducted using a Bartington MS2F probe that collected two readings of data in 2- to 5-cm intervals down profile for each backhoe trench. Briefly, the MS2F probe is $1.5 \mathrm{~cm}$ in diameter and is inserted $2 \mathrm{~cm}$ deep into an exposed profile to collect MS readings. The MS analyses were collected down profile (ground surface to base of exposed profile) at the high sensitivity (0.1) setting at three locations (BHT4 in TU03, BHT5 near TU05, and in BHT10). Due to Occupational Safety and Health Administration (OSHA) safety requirements, these recordings were only able to examine the in situ profile down to a maximum depth of $180 \mathrm{cmbs}$ (6 feet). In contrast, the MS analyses in the fourth column consisted of collecting matrix in 4 to $5 \mathrm{~cm}$ in the western profile of BHT11 ( $\mathrm{n}=33)$.

\section{MS RESULTS}

\section{BHT04 (TU03)}

Regarding the vertical MS results in BHT4/TU03 area, the in situ MS values had several apparent spikes and trends (Figure 14). Examining the MS data down profile, Strata I and II exhibit dynamic fluctuations supporting the interpretation that it is disturbed from recent landform modification. The underlying 
Stratum III exhibits values ranging from 30 to 40 throughout the horizon. The Stratum III results do exhibit two prominent spikes between 70 and $83 \mathrm{cmbs}$ that may coincide with cultural activities. At the transition of Strata III/IV, there is a dramatic drop in values until roughly $103 \mathrm{cmbs}$. At this depth, the values spike until $130 \mathrm{cmbs}$ where the values gradually decrease and transition into Stratum V. The Stratum IV elevated values between 103 and 130 cmbs is likely attributed to cultural activities. Within the underlying Stratum $\mathrm{V}$, a very subtle increase in values occurs around 155 to $170 \mathrm{cmbs}$. Although intriguing, this increase in MS value is inconclusive as to whether this could be attributed to cultural activity or something else with the present data.

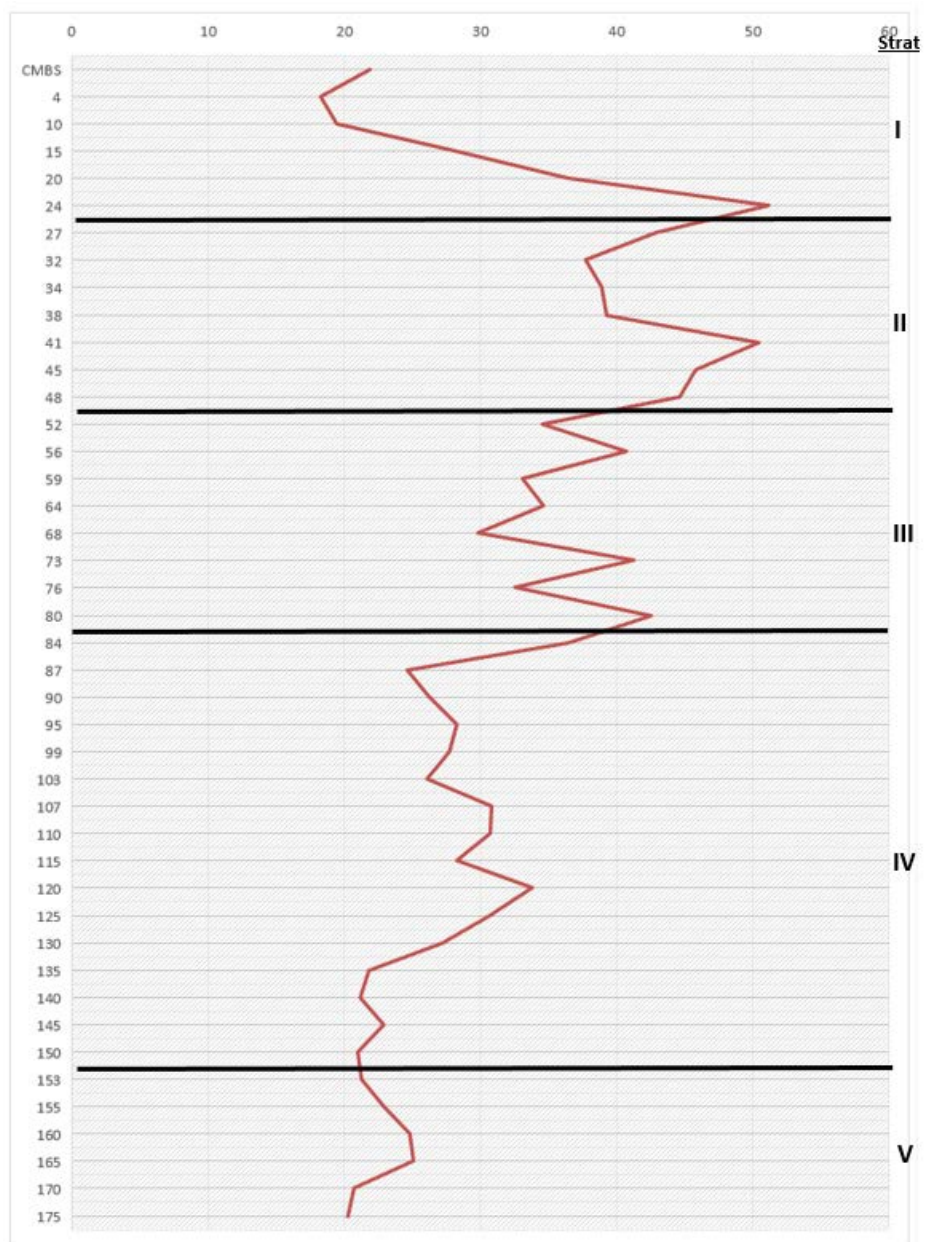

Figure 14. Magnetic susceptibility results from BHT04.

\section{BHT05}

Regarding the vertical MS results in the BHT05 area, the in situ MS values are similar to that exhibited in BHT04 (Figure 15). Interestingly, in Strata I and II (where tree roots and bioturbation were the most extensive) the values are high, but do not have dramatic fluctuations that may indicate a highly organic and homogenous mix. The underlying Stratum III exhibits two prominent spikes. One spike is between 27 and $46 \mathrm{cmbs}$, while the second spike is between 59 and $74 \mathrm{cmbs}$. Since these two "plateaus" are derived from several contiguous readings and separated by consistent decreases, one or both plateaus may indicate distinct cultural zones. Similar to the trends observed in BHT4, the transition between Strata III/IV exhibits a drop-in value from above 48 to below 30 and gradually decreases in values with depth. The underlying Stratum IV does have an increase in values between 81 and $92 \mathrm{cmbs}$. This plateau is like that observed in 
Stratum IV of BHT04 (between 103 and $130 \mathrm{cmbs}$ ), as it has several contiguous readings of higher values and could suggest the presence of cultural activities. Below this slight increase and continuing into Stratum $\mathrm{V}$, no evident values are present to suggest extensive cultural activities in this area. However, the beginnings of an increase in values is evident in Stratum V between 132 and 136 cmbs. This increase in MS value is inconclusive as to whether this could be attributed to cultural activity or something else with the present data.

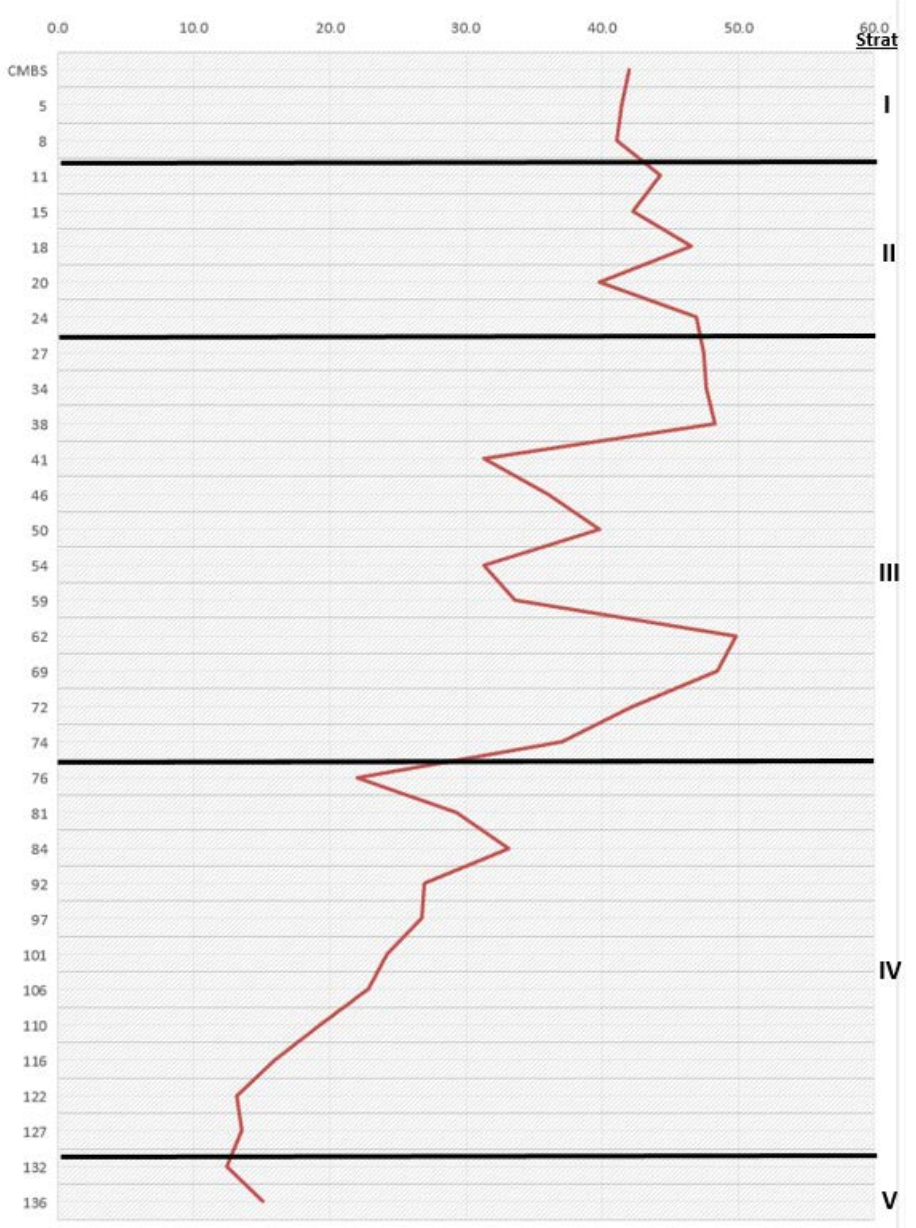

Figure 15. Magnetic susceptibility results from BHT05.

\section{BHT10}

Regarding the vertical MS results in the BHT10 area, the in situ MS values are similar to that exhibited in BHT04 and BHT05 (Figure 16). The upper Strata I and II (where tree roots and bioturbation were again the most extensive) have MS values exhibiting dramatic fluctuations. This likely indicates a highly organic and disturbed mix with cultural activities present. Specifically, a fragment of fire-cracked rock was observed in Stratum II at 39 to $42 \mathrm{cmbs}$ that seems to coincide with the prominent spike in MS values in the horizon (see Figure 16). The underlying Stratum III contains several prominent spikes. One spike is between 65 and $80 \mathrm{cmbs}$, while the second spike is between 95 and 115 cmbs. SWCA observed individual fragments of fire-cracked rock in Stratum III at $76 \mathrm{cmbs}$ and at $103 \mathrm{cmbs}$, respectively, which coincides with these two spikes in MS value. Interestingly, there is a gradual decrease in values between Stratum III and Stratum IV, but then the MS values increase. Stratum IV has one very prominent spike in value between 135 and 145 cmbs (see Figure 16). Not surprisingly, a fragment of fire-cracked rock was observed at 139 cmbs that 
coincides with the prominent spike in MS values for the horizon. Below the spike, the values steadily decrease and transition into Stratum V. This base horizon, Stratum V, contains a very subtle increase in values around 160 to $170 \mathrm{cmbs}$. Although intriguing, this increase in MS value is inconclusive as to whether this could be attributed to cultural activity or something else with the present data.

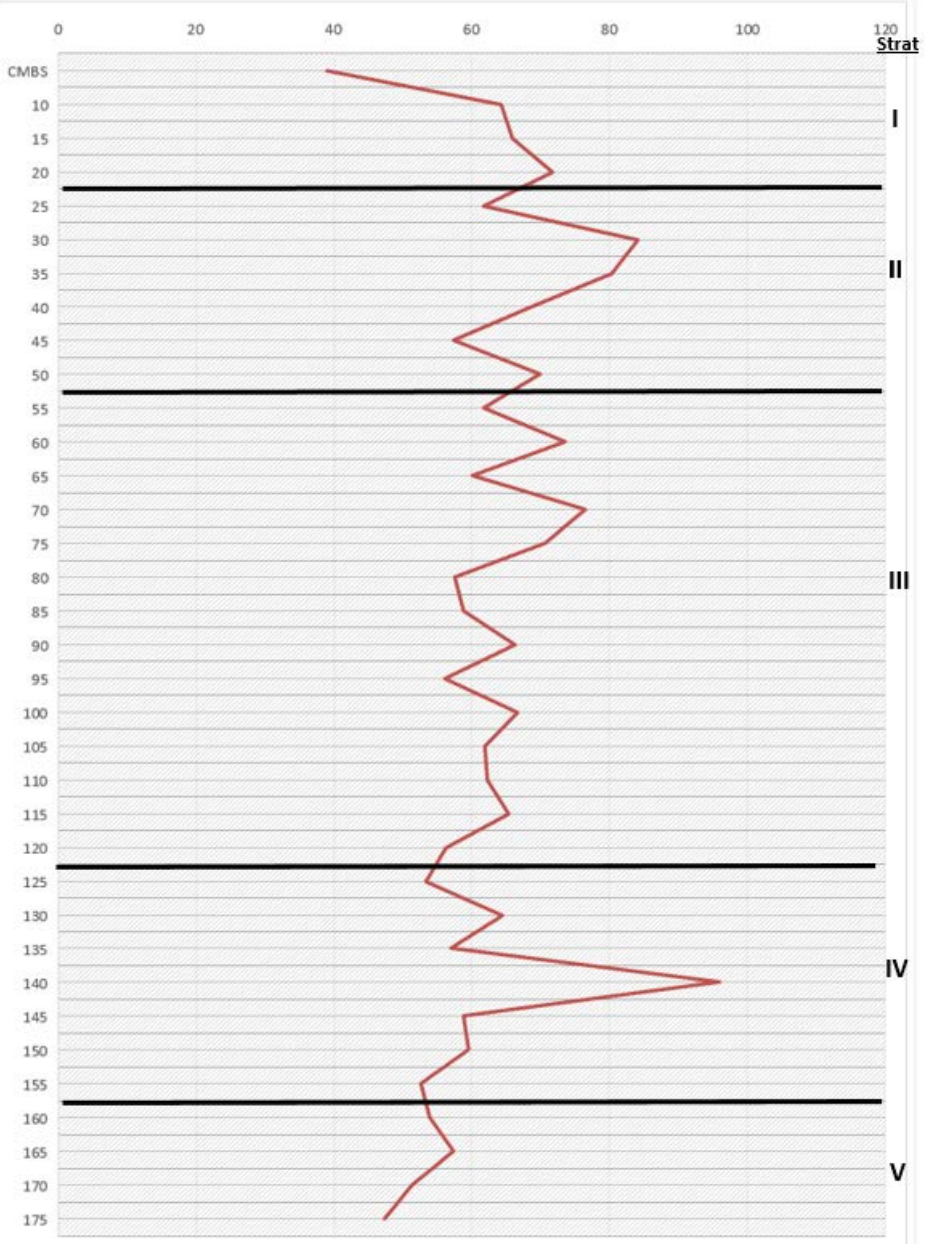

Figure 16. Magnetic susceptibility results from BHT10.

\section{BHT11}

As mentioned above, the MS analysis in BHT11 was conducted differently from the other vertical column analyses that used in situ analysis. The MS analysis in BHT11 utilized collected matrix from the profile that were subsequently examined in the lab. The results of the MS analysis exhibited several notable items (Figure 17). The upper Strata I and II (where tree roots and bioturbation were the most extensive) have MS values that were relatively stable with no dramatic fluctuations. These data suggest a relatively homogenous matrix. The underlying Stratum III exhibited a gradual decrease in values until 45 to $50 \mathrm{cmbs}$, where a slight increase is present. Below that, the gradual decrease in MS values continues into Stratum IV to roughly 55 to $70 \mathrm{cmbs}$, where another spike in value occurs. Below that spike in Stratum IV, the MS values steadily decrease until 120 to 125 cmbs, where a prominent spike in value occurs. At this depth in BHT11, SWCA observed a discontinuous and sloping lens of dark matrix (Figure 18). The lens (also observed in BHT12) is interpreted to be a thin cultural zone, a thin buried soil, or both. Because the lens contained at least one fragment of fire-cracked rock, it is more likely interpreted as a cultural horizon. Below the lens, the MS values decrease until 135 to 160 cmbs, where another prominent spike in MS values is evident. 
Most notable about these MS value spikes is that SWCA observed scattered fragments of fire-cracked rock throughout the trench that coincided with most of these spikes. The one exception is the prominent MS value spike in Stratum V at 135 to $160 \mathrm{cmbs}$ (Figure 19), where no fire-cracked rock fragments were observed in BHT11 at this depth.

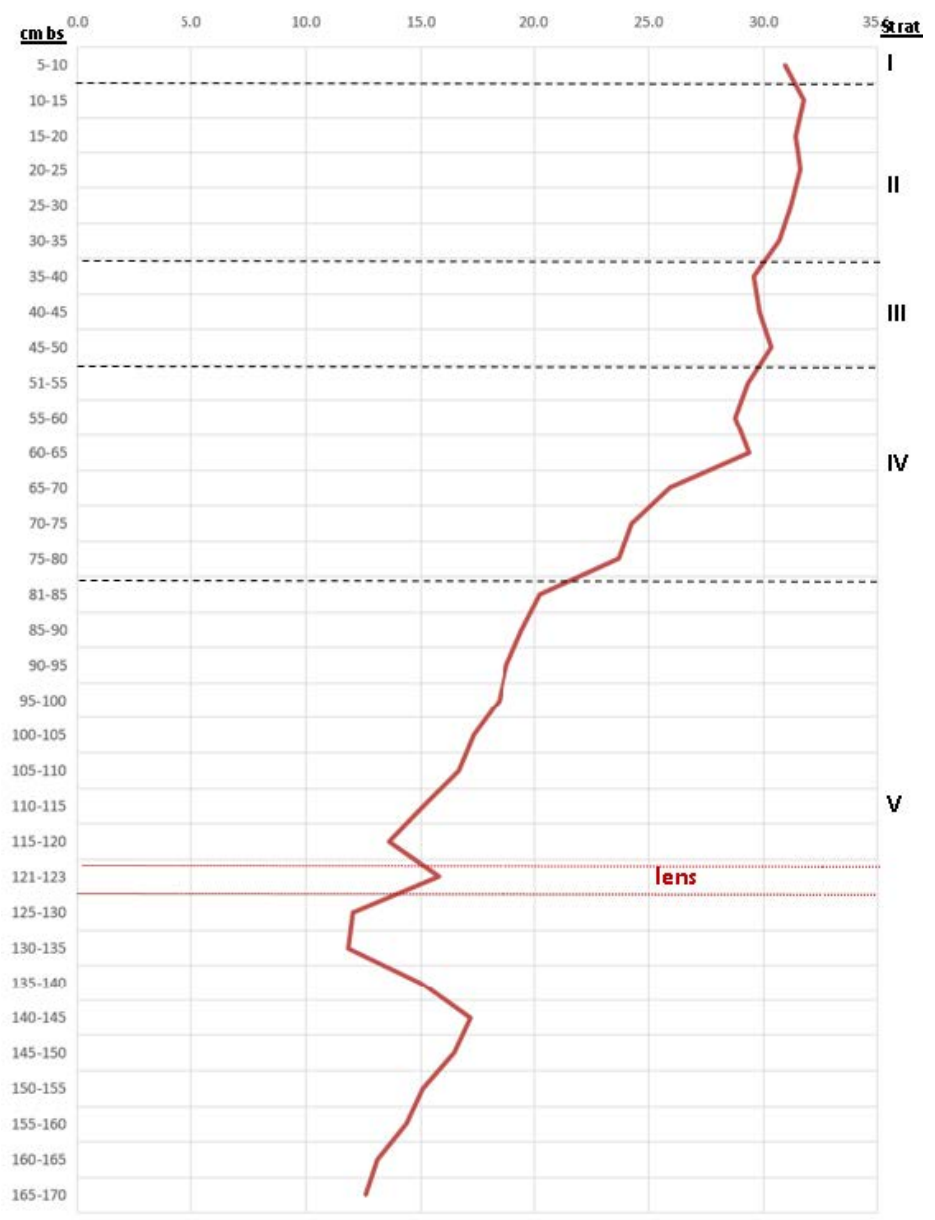

Figure 17. Magnetic susceptibility results from BHT11.

\section{SUMMARY MS RESULTS}

The results of the MS analyses at four different locations across the site provide some interesting data. When the strata and MS results of each vertical column are compared against each other, the data suggests similar factors affecting all the deposits. Specifically, in Stratum II there is an increase in MS values exhibited in BHT04, BHT10, and possibly BHT05 (see Figures 14-16). In Stratum III across all four vertical columns there is at least one plateau exhibited around 70 to $80 \mathrm{cmbs}$; conversely, this occurs at 45 to $50 \mathrm{cmbs}$ in BHT11. Similarly, in Stratum IV, a consistent increase in values is exhibited at roughly 100 to 130 in BHT04, BHT05, and BHT10, while a similar spike is represented from 55 to $70 \mathrm{cmbs}$ in BHT11 (see Figures 14-17). Finally, in Stratum V, an increase in MS is evident in BHT04, BHT10, and BHT11 at roughly 130 to $160 \mathrm{cmbs}$ (see Figures 14-17). Although BHT05 did not extend this deep, there is the beginnings of an increase in MS values between 132 and $136 \mathrm{cmbs}$. Of note, the increase in Stratum V MS values across all trenches was not directly associated with cultural materials. This increase in MS value in Stratum V is inconclusive as to whether this could be attributed to cultural activity or something else with the present data. 


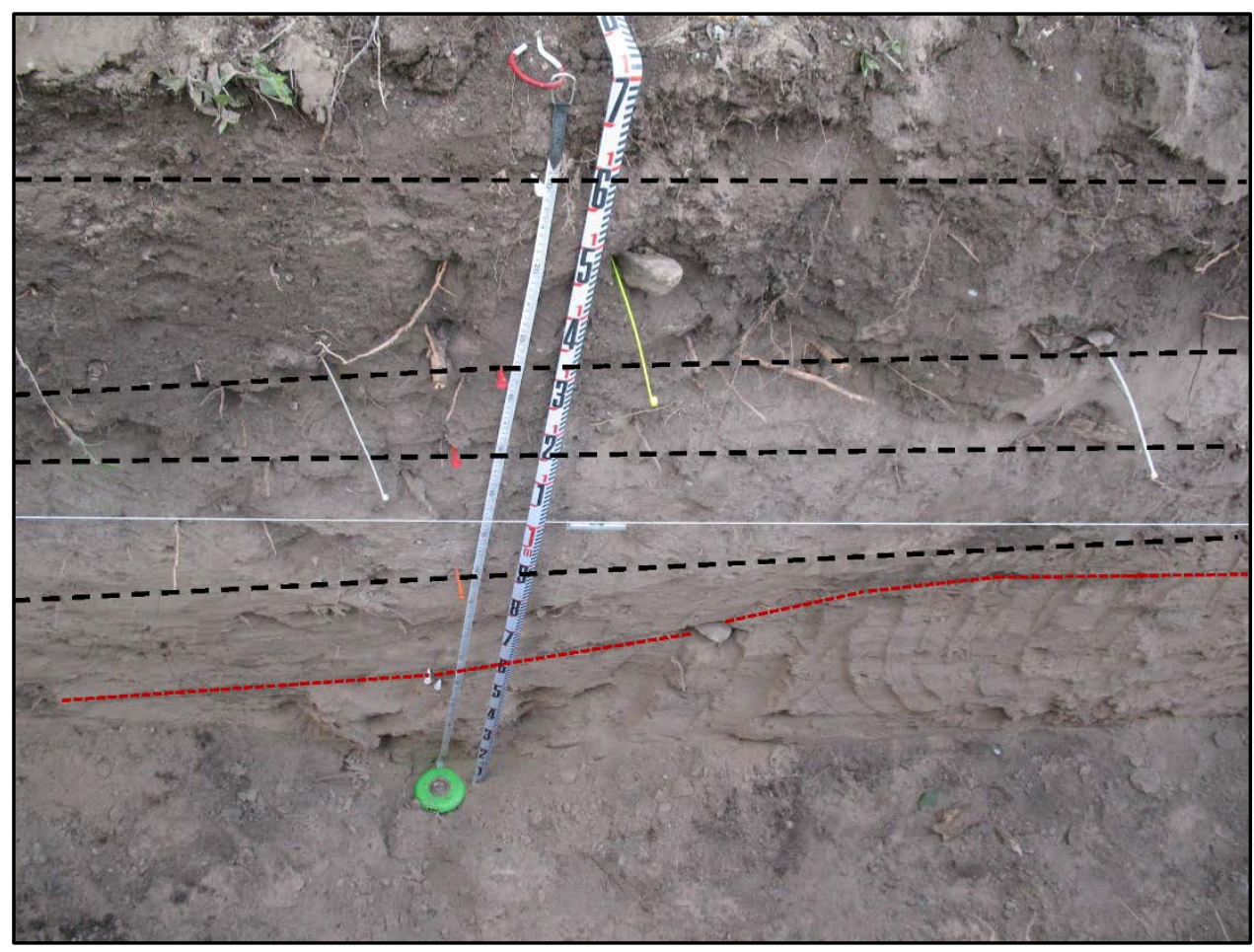

Figure 18. BHT11 profile.

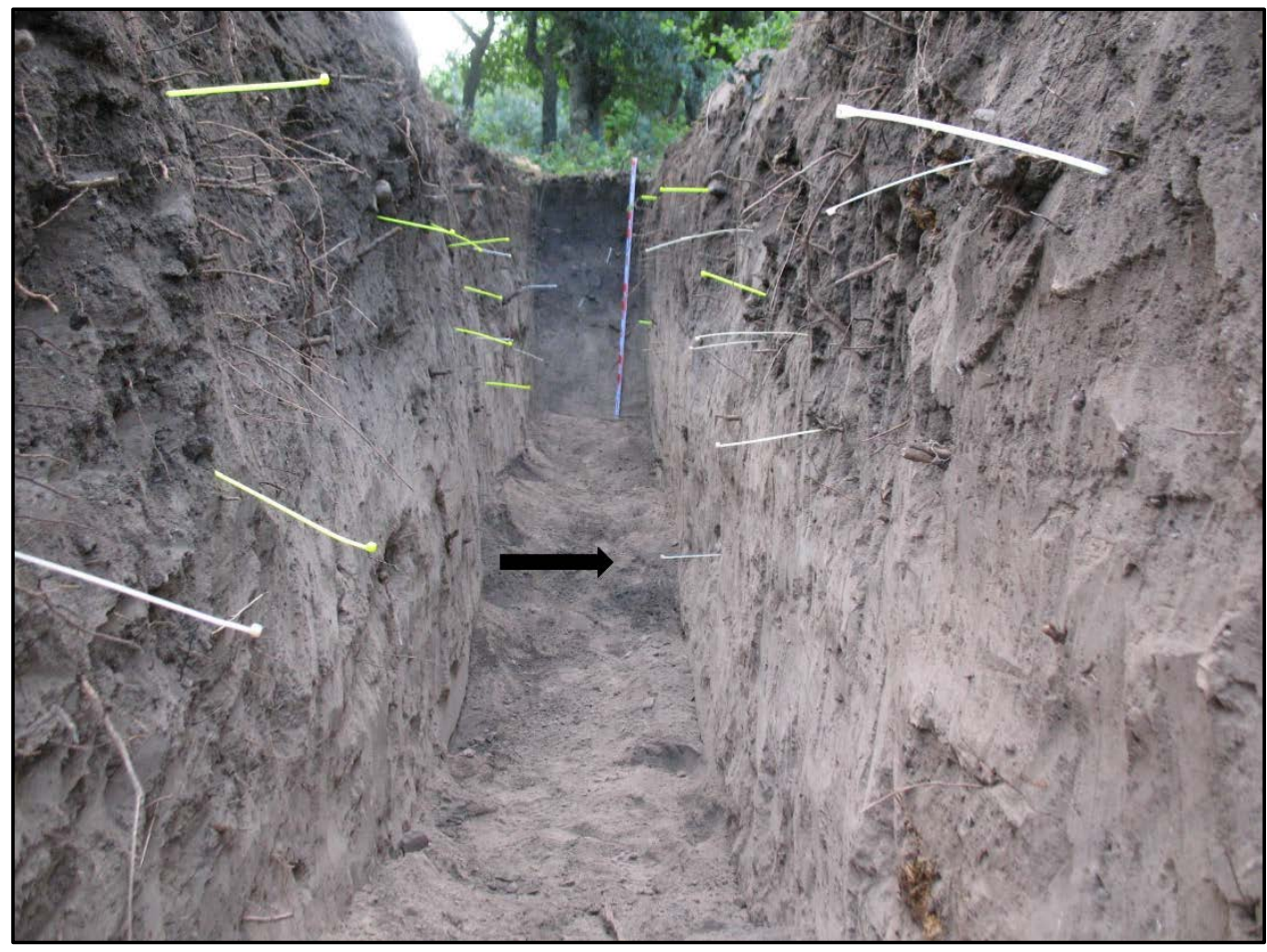

Figure 19. BHT11 overview. The zip-ties in the trench walls are marking artifacts. 
Overall, the data from the MS analyses suggests that although some disturbances from bioturbation and landform modification have disturbed portions of 41GU177, there does appear to be several well-defined, stratified cultural zones present.

\section{SUMMARY Site DISCUSSION}

From the data collected to date, several data realms have been preliminarily addressed, which in turn have implications for more detailed research questions and concerns. The phased investigations have allowed preliminary determinations of the general integrity of the cultural deposits, site chronology, and potential data yield.

Based on the site's natural stratigraphy and tentatively identified cultural zones, the cultural deposits at 41GU177 are relatively intact, with some disturbances from tree roots and krotovina observed. The natural stratigraphy (Strata I-VI) observed at 41GU177 suggests some localized disturbances at or near the ground surface from previous vegetation clearing, extensive tree root growth, possible earth movement, and erosion. The vertical extent of evident disturbance across the site varies. The excavation areas amongst extensive trees (i.e., BHT05 and BHT06) exhibited moderate, localized disturbance to approximately 75 cmbs. In contrast, the excavation areas in proximity to existing roads and previously cleared areas (i.e., BHT02 and BHT04) had evidence that may be related to vegetation clearing and earth movement. These disturbances, suggested by the abrupt lower boundary of Strata II, were shallower (32 cmbs), but more extensive and may have truncated the upper horizon of Strat III. The underlying strata (III-VI) across 41GU177 appear generally intact, with some minor disturbance from tree roots and small burrows.

Investigations to date have yielded a relatively dense assemblage of cultural materials, given that only approximately 7.8 cubic meters have been hand excavated from $1 \times 1$-m test units and 3.9 cubic meters from the close interval shovel testing (for a total of 11.7 cubic meters). The total assemblage encompasses 5,406 artifacts and floral/faunal specimens, including abundant chipped-stone debitage, formal and informal chipped-stone tools (i.e., projectile points, one chipped-stone axe, utilized flakes, bifaces, etc.), ground stone specimens (i.e., mano fragments), and the well-preserved faunal and floral specimens mentioned below (Appendix C). The artifact assemblage is predominately (91 percent) debitage, with a total of 3,897 debitage specimens recovered to date from TU01-TU05 and 1,043 from the shovel testing.

As Appendix C indicates, the majority ( $n=4,469$ [83 percent]) of the debitage assemblage reflects middle to late reduction activities, with secondary and tertiary flakes, and decorticate chips and chunks representing the bulk of the specimens. This suggests that much of the initial reduction of lithic raw materials was occurring off site at raw material source locations, which appear, based on cortex analysis, to include both fluvial gravels (i.e., corticate specimens exhibit smooth water-worn cortex) and in situ outcrops (i.e., corticate specimens exhibiting coarse and/or weathered cortex). Incomplete and complete bifaces (other than projectile points) are poorly represented in the assemblage. Given the preponderance of later-stage reduction debitage (particularly tertiary specimens that represent 67 percent of the debitage assemblage) and the general lack of complete and incomplete bifaces (except occasional broken preforms and the finished and broken projectile points), biface and projectile point manufacturing was a common activity on site, with the completed bifaces (complete projectile points, preforms, and Stage IV bifaces) leaving the site.

In terms of site chronology, temporally diagnostic artifacts recovered from the site to date indicate a general Late Prehistoric use of the site, including a probable Austin Phase (ca. A.D. 600-1200) occupation and hints of an earlier Transitional Archaic (ca. 200 BC to AD 600) occupation. A total of 15 projectile points or point fragments were recovered during the phased investigations. The majority $(n=10)$ are identified as untyped arrow point fragments, whereas three specimens are typed as corner-notched, broad stemmed Scallorn arrow point variants associated with Austin phase occupations. Additional recovered arrow points, 
due to missing bases or reworked bases and stems, are untyped but indicate general Late Prehistoric (ca. A.D. 600-1700) occupations. Traces of earlier site use is potentially evident in the recovery of a dart point fragment identified as belonging to the Montell type that dates to the Late Archaic (ca. 800-400 B.C.), as well as an untyped dart point fragment that may represent a Frio or Ensor type projectile point (Transitional Archaic, ca. 200 B.C. - A.D. 600).

The temporally diagnostic data, when considered in conjunction with the stratigraphic analyses and MS data, suggests site 41GU177 contains evidence for at least two stratified cultural zones with hints of possibly two additional, deeper stratified cultural zones. In the upper reaches of the deposits from roughly 45 to 80 cmbs there are several Scallorn arrow points, suggesting a probable Austin phase occupation that contains some stratigraphic disturbances as previously described. Additionally, a possible trace of an underlying Transitional Archaic occupation is marked by the presence of a possible Frio or Ensor dart point fragment, burned rock, and a spike in the MS data at a depth of approximately 60 to $80 \mathrm{cmbs}$. At this juncture, given the variable depth and thickness of the site stratigraphy and cultural zones due to undulations, data recovery would clarify the actual number of stratified cultural zones, as there is also some evidence of two possible deeper occupations, based on the presence of burned rock and spikes and dips in the MS data from roughly 120 to 150 cmbs.

Within the strata, floral and faunal preservation is good, with a total of 274 recovered faunal remains (262 bones and bone fragments, and 12 mussel shells and shell fragments) recovered from approximately 11.7 cubic meters of hand excavated sediments. TU01 yielded a burned nut shell from 70 to $80 \mathrm{cmbs}$ in the upper possible Austin phase occupation, as well as two burned seed fragments from a depth of 140 to $150 \mathrm{cmbs}$ in the deeper, suggest a possible Transitional Archaic occupation. The recovery of those wellpreserved faunal and floral specimens suggests that additional charred macrobotanical remains are present on site that can be recovered and analyzed to help build a picture of site use and foraging strategies.

A total of 574 burned rock fragments, weighing approximately 27.1 kilograms, were recovered within the five excavation units. All the test units show a rise in burned rock count and weight at approximately 60 cmbs. In addition, TU01 and TU04 display an increase in burned rock counts and weights at approximately 90 to $100 \mathrm{cmbs}$, with TU01 and TU02 indicating a third spike in burned rock weights at 120 to $140 \mathrm{cmbs}$. Although the charred floral remains and the scattered burned rocks and burned rock fragments recovered during excavations suggest that burned rock features (i.e., hearths) were utilized on site, the densities of burned and fire-cracked rock from various excavation contexts has thus far been relatively low and specimens were typically not clustered. This may suggest some degree of disturbance, but the presence of several clustered burned rocks in BHT02 and TU01 also shows that some feature integrity remains and that additional features and/or feature remnants are anticipated. Investigations to date have not identified any evidence for large thermal features, such as a burned rock midden or slab-lined hearths, not uncommon in similar Late Archaic to Transitional Archaic and Late Prehistoric period camp sites.

Following completion of the hand excavations and additional trenching, SWCA proposes that, horizontally, the core of the site/densest cultural materials are concentrated in the area around BHT11, and extending to the east-southeast to BHT02 (TU01 and TU02) and BHT04 (TU03), with TU04 containing compromised data from extensive bioturbation stemming from rodent activities (see Figure 11). The TU05 area exhibits a significantly lower artifact density than the BHT02, BHT04, and TU01-TU03 areas; however, excavations there were halted at $110 \mathrm{cmbs}$, due to time constraints. Similarly, the excavation of closeinterval shovel tests and BHT07-BHT10 to the south and west of BHT11 did not identify a high density of cultural materials. Vertically, cultural materials in the upper 50 to $75 \mathrm{~cm}$ (0 to $75 \mathrm{cmbs}$ ) exhibit some disturbance from tree roots, rodents, and, in some areas, mechanical impacts from prior vegetation clearing. Below $75 \mathrm{cmbs}$, the natural stratigraphy and cultural materials appear relatively intact and have a potential to contain significant intact cultural deposits when considered in conjunction with the stratigraphic, 
artifactual, and MS data collected from the site to date. Based on these data, it is the opinion of SWCA that the site warrants listing as a SAL.

To reiterate, construction impacts to 41GU177 would include the excavation and use of the $50 \times 50$ - $\mathrm{ft}$ bore pit area for tunneling the adjacent Guadalupe River, open cut trenching, a $65 \times 200-\mathrm{ft}$ temporary work space, and a temporary haul road for construction activities. Based on the recovered data, the north-central portions of the site, in SWCA's opinion, represent the core of the potentially significant cultural deposits. In contrast, the parts of the site west-southwest of STs 1-08, 2-08, 3-07, and 4-06 are low artifact density areas with poor preservation and few cultural materials that, in the opinion of SWCA, do not contribute to the site's overall potential SAL eligibility; as a result, SWCA recommends no further investigations are warranted for the southern two-thirds of the site.

Understanding the sensitive nature of the cultural deposits at 41GU177, VRRSP have developed two construction options to minimize construction impacts to the core of the site considered potentially significant by SWCA; Figure 20 was prepared by VRRSP to illustrate those options. As Figure 20 displays, VRRSP has arbitrarily labeled the moderate to high density artifact concentration areas within the existing easement and proposed temporary workspace as Area A. In addition, Area B on the figure denotes portions of the planned construction centerline and associated impact area, while Area $\mathrm{C}$ represents a small area that VRRSP proposes does not need additional work as it would be outside of any surface (i.e., haul road) and subsurface construction impacts, and has been previously investigated. The two construction options (1 and 2) and their respective steps are presented below:

\section{Option 1-Open Cut Trenching Through the Site}

1. Vegetation would be mechanically cleared in Areas A and B using a rubber-tired grinder (fellerbuncher) to not disturb the ground surface and subsurface.

2. Area A would be covered with geotechnical fabric to establish a boundary between the existing ground surface and a 1-foot-thick cap of fill would be placed over the geotechnical fabric to avoid subsurface impacts. An archaeological monitor would be present; the contractor would be responsible for ensuring there would be no impacts to the ground surface and subsurface.

3. Bore pit excavation would proceed, as well as installation of pipe via the open trench in Area B; all areas would then be backfilled upon completion.

4. The protective cap of fill and geotechnical fabric would be carefully removed from Area A with an archaeological monitor present; the contractor would be responsible for ensuring there would be no impacts to the ground surface and subsurface.

\section{Option 2-Tunneling and Limited Open Cut Trenching:}

1. Vegetation would be mechanically cleared in Areas A and B using a rubber-tired grinder (fellerbuncher) to not disturb the ground surface and subsurface.

2. Areas A and B would be covered with geotechnical fabric to establish a boundary between the existing ground surface and a 1-foot-thick cap of fill to be placed over the geotechnical fabric to avoid subsurface impacts.

3. Contractor excavates bore pit, installs tunnel, installs pipe via open trench to the southwest of the delineated sensitive site area, and backfills all areas. The top of the tunnel casing pipe will be at least 6.5 feet below the existing ground surface to avoid disruption to the lower cultural deposits. Potentially significant cultural deposits along the centerline within Area B are therefore avoided.

4. The protective cap of fill and geotechnical fabric would be carefully removed from Area A with an archaeological monitor present; the contractor would be responsible for ensuring there would be no impacts to the ground surface and subsurface. 


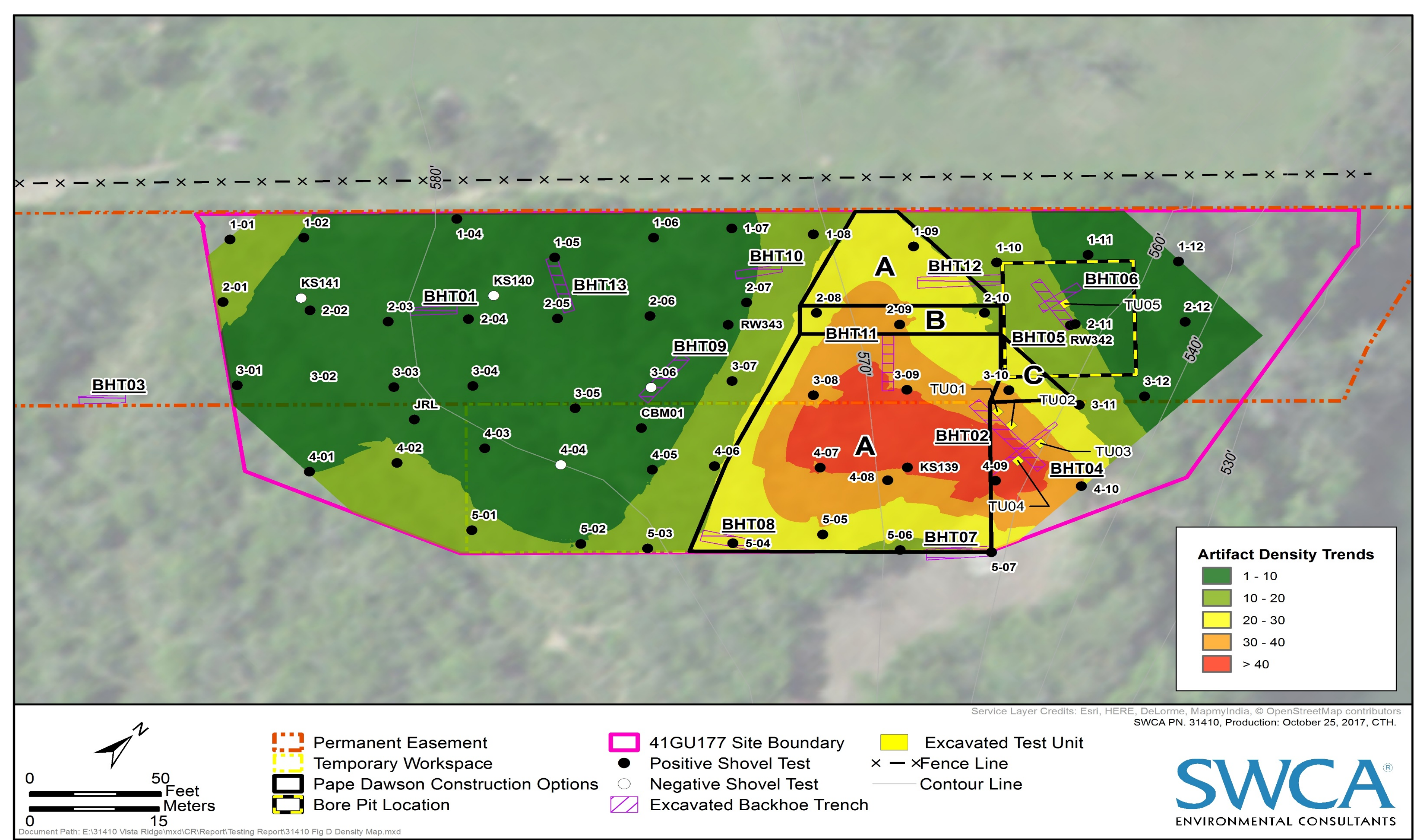

Figure 20. Construction options for 41GU177. 
Given the silty soils on the site, the option to cap the site with fill must consider the potential effects of soil compaction on those cultural materials buried close to the surface. Site capping or burial involves carefully covering an archaeological site with fill to allow project construction or other activities to proceed unimpeded over the site. However, site capping is only an appropriate mitigative measure when it can be demonstrated that important site data will not be lost through soil compaction, accelerated decomposition, horizontal displacement of cultural materials, or changes in soil chemistry. Investigations to date on 41GU177 suggest that approximately the upper 75 centimeters of the site have experienced some localized impacts from bioturbation (i.e., tree roots and burrowing animals) and mechanical impacts during vegetation clearing; however, the extent of the impacts appear moderate and there remain partially intact cultural deposits that should be protected, if the site is capped with fill.

\section{RECOMMENDATIONS}

Given the chronological data currently available from the site, the relative degree of intactness of the natural stratigraphy and cultural deposits, good macrobotanical preservation, and the types and densities of cultural materials recovered during the phased investigations, site 41GU177 appears to have the potential to yield new or important data regarding the Late Prehistoric period from the eastern margins of the Balcones Escarpment. Additionally, the deeper cultural occupation(s) may represent a Transitional Archaic use of the site and may provide valuable information concerning the shift from the Transitional Archaic to the Late Prehistoric period. Based on these data, it is the opinion of SWCA that the site is eligible for listing as a SAL and therefore warrants data recovery in the areas described above, if construction impacts cannot be avoided.

Complete avoidance of the site is currently not possible. The parts of the site west-southwest of STs 1-08, 2-08, 3-07, and 4-06 are low artifact density areas with poor preservation and few cultural materials that, in SWCA's opinion, do not contribute to the site's overall potential SAL eligibility; as a result, no further investigations are recommended for this area. In contrast, the north-central portions of site 41GU177 exhibit a much higher density of cultural materials and good stratigraphic integrity and faunal and floral preservation; this area is associated with tunneling of the Guadalupe River (adjacent to the site) and, in SWCA's opinion, additional investigations are warranted in the bore pit, associated temporary work space, and a proposed haul road.

Although the bore pit area exhibits a relatively lower artifact density, only one controlled hand unit was excavated to a depth of $110 \mathrm{cmbs}$ within the bore pit area; BHTs 5 and 6 were excavated for exploratory purposes. The presence of the unusual unifacial, large chipped-stone axe and potentially associated burned and fire-cracked rocks from TU05 suggests an entirely different activity from the other site activity areas (i.e., tool manufacture and possible mussel shell work areas) may have occurred in the bore pit area. Data recovery here would provide a sample from outside the presumed concentrated core of the site (where multiple living surfaces may be compressed), providing access to potentially more discrete activities and isolable components. Initial MS data collected from BHT 5 also suggests that there is a potential for deeper cultural deposits in this area. As there was a time limitation influencing the work in the bore pit area, we do not feel it has been adequately sampled, considering the potential for the complete destruction of this $50 \times 50$-foot area due to the bore excavation. Including portions of this area for data recovery would allow a further sample of the location; if initial data recovery excavations there determined the location to not be as productive, or the activity areas poorly defined, investigations would then be halted for lack of data.

Based on all the available data collected to date, it is the opinion of SWCA that the cultural deposits at 41GU177 within the identified high and moderate density area in the north-central part of the site (including the bore pit area) is eligible for SAL designation and warrants some level of data recovery investigations and/or measures to minimize site impacts prior to the potential construction impacts. Successful solutions for the treatment of $41 \mathrm{GU} 177$ will require discussions amongst VRRSP, SWCA, and THC to ensure a 
balance between construction needs and schedule, and regulatory compliance. For both construction options, technical discussions will be necessary with THC to address the proposed temporary capping of the site with fill to ensure an appropriate process and suitable materials are used to preserve portions of the site in place, should that be determined a feasible option. 


\section{REFERENCES}

Ashman, M. R. and G. Puri

2002 Essential Soil Science: A Clear and Concise Introduction to Soil Science. Blackwell Publishing, Malden, MA.

Barnes, V. E.

1983 Geologic Atlas of Texas: San Antonio Sheet. Robert Hamilton Cuyler Memorial Edition, Bureau of Economic Geology, University of Texas, Austin.

Charlton, R.

2008 Fundamentals of Fluvial Geomorphology. Routledge, London and New York.

Crowther, J., and P. Barker

1995 Magnetic Susceptibility: Distinguishing Anthropogenic Effects from the Natural. Archaeological Prospection 2:207-215.

Dalan, R. A.

1996 Soil Magnetism, An Approach for Examining Archaeological Landscapes. Geophysical Research Letters 23(2): 185-188.

2006 Magnetic Susceptibility. In Remote Sensing in Archaeology: An Explicitly North American Perspective. J. Johnson, M. Giardano, and K. Kvamme (editors), pp. 161-203. University of Alabama Press, Tuscaloosa, Alabama.

2008 A Review of the Role of Magnetic Susceptibility in Archaeologeophysical Studies in the USA: Recent Developments and Prospects. Archaeological Prospection 15: 1-31.

Dalan, Rinita A. and Subir K. Banerjee

1998 Solving Archaeological Problems Using Techniques of Soil Magnetism. Geoarchaeology: An International Journal 13: 3-36.

Dalan, R. A. and B. W. Bevan

2002 Geophysical Indicators of Culturally Embraced Soils and Sediments. Geoarchaeology: An International Journal 17 (8): 779-810.

Dearing, J.

1999a Magnetic Susceptibility. In Environmental Magnetism: A Practical Guide. J. Walden, F. Oldfield, and J. Smith (editors), pp. 35-62. Technical Guide No. 6. Quaternary Research Association, London.

1999b Environmental Magnetic Susceptibility Using the Bartington MS2 System. Available at: http://www.gmw.com/magnetic_properties/MS2_support.html. Accessed 10/20/17.

Ellwood, B. B., D. E. Peter, W. Balsam, and J. Schieber.

1995 Magnetic and Geochemical Variations as Indicators of Palaeoclimate and Archaeological Site Evolution: Examples from 41TR68, Fort Worth, Texas. Journal of Archaeological Science 22: 409-415.

Frederick, Charles D

2010 Geoarchaeological Investigations. In Archaeological Data Recovery on Three Sites Along the San Antonio River, Bexar County, Texas, by Antonio E. Padilla and David L. Nickels, pp. 449-462. Ecological Communications, Corporation, Austin, Texas. 
2012 Site Formation Processes and Implications for Archeology in the South Fork of the San Gabriel. In The Siren Site (41WM1126) and the Long Transition from Archaic to Late Prehistoric Lifeways on the Eastern Edwards Plateau, Williamson County, Texas, by Steve Carpenter, Kevin A. Miller, Brett Houk, Mary Jo Galindo, Charles Frederick, John Lowe, Ken Lawrence, Kevin Hanselka, and Abby Peyton, pp. 8-1-8-24. SWCA Environmental Consultants, Austin, Texas.

Gale, S. J. and P. G. Hoare

1991 Quaternary Sediments: Petrographic Methods for the Study of Unlithified Rocks. Bellhaven Press and Halsted, Press, New York and Toronto.

Jones, R. S., and J. J. Leffler

2003 Phase I and II Archaeological Investigations on Camp Mabry, Travis County, Texas, Archaeological Studies Report No. 2. Center for Archaeological Studies, Texas State University-San Marcos, Texas.

Lawrence, Ken, J. Kevin Hanselka, John D. Lowe, Christina Nielsen, and Kevin A. Miller

2013 Results of Archaeological Significance Testing at the Turkey Terrace Site (41FR70), Frio County, Texas. SWCA Environmental Consultants, Archaeological Report 12-518 Austin, Texas.

Maher, Barbara A., Roy Thompson, and Mark W. Hounslow

1999 Introduction. In Quaternary Climates, Environments, and Magnetism. B.A. Maher and R. Thompson (editors), pp. 1-48. Cambridge University Press. Cambridge.

Mauldin, R. P., and A. L. Figueroa

2006 Data Recovery Excavations at 41PR44, Fort Wolters, Parker County, Texas, Archaeological Report, No. 369, Center for Archaeological Research, University of Texas at San Antonio, San Antonio, Texas.

Natural Resources Conservation Service (NRCS)

2017 Web Soil Survey 2.1. National Cooperative Soil Survey. Available at: http://websoilsurvey.nrcs.usda.gov/app/WebSoilSurvey.aspx, Accessed July 2017.

Nickels, D. L.

2010 Stratigraphy, Chronology, and Site Formation Processes. In Archaeological Testing at the San Marcos Springs (41HY160) for the Texas River Center, Hays County, Texas, by David L. Nickels and C. Britt Bousman, pp. 61-82. Archaeological Studies Report No. 13. Center for Archaeological Studies, Texas State University-San Marcos, Texas.

Peters, Clare, and Roy Thompson

1999 Supermagnetic Enhancement, Superparamagnetism, and Archaeological Soils. Geoarchaeology: An International Journal 14 (5): 401-413.

Ramsey, R.N. and N. P. Bade

1977 Soil Survey of Guadalupe County, Texas. United States Department of Agriculture, Washington, D.C.

Rivers, J.M., J.E. Nyquist, Y. Roh, D.O. Terry Jr., and W.E. Doll

2004 Investigation into the Origin of Magnetic Soils on the Oak Ridge Reservation, Tennessee. Soil Science of America Journal 68(5): 1772-1779.

Rosendahl, Daniel, Kelsey M. Lowe, Lynley A. Wallis, and Sean Ulm

2014 Integrating Geoarchaeology and Magnetic Susceptibility at Three Shell Mounds: A Pilot Study from Mornington Island, Gulf of Carpentaria, Australia. Journal of Archaeological Science 49:21-32. 
Taylor, F. B., R. B. Hailey, and D. L. Richmond

1991 Soil Survey of Bexar County, Texas. United States Department of Agriculture, Washington, D.C.

Thompson, R. and F. Oldfield

1986 Environmental Magnetism. Allen \& Unwin Press. London, Boston, and Sydney.

Van Leusen, P. M., A. Kattenberg, and K. Armstrong

2014 Magnetic Susceptibility Detection of Small Protohistoric Sites in the Raganello Basin, Calabria (Italy). Archaeological Prospection (in press).

Verosub, K. L. and A. P. Roberts

1995 Environmental Magnetism: Past, Present, and Future. Journal of Geophysical Research 100(B2): 2175-2192.

Waters, Michael R.

1992 Principles of Geoarchaeology: A North American Perspective. University of Arizona Press, Tucson.

Wiewel, Adam S., and Kenneth L. Kvamme

2014 Magnetic Investigations of Nomadic Group Encampments at Fort Clark State Historic Site, North Dakota. Plains Anthropologist 59(231):261-278. 


\section{APPENDIX A}





\begin{tabular}{|c|c|c|c|c|c|c|c|c|c|c|}
\hline ST & Level & $\begin{array}{l}\text { Depth } \\
\text { (cmbs) }\end{array}$ & $\begin{array}{c}\text { Artifact } \\
\text { Type }\end{array}$ & $\begin{array}{c}\text { Artifact } \\
\text { Description }\end{array}$ & Material & $\begin{array}{c}\text { Artifact } \\
\text { No. }\end{array}$ & $\begin{array}{l}\text { Weight } \\
\text { (g) }\end{array}$ & Recorders & Date & Comments \\
\hline $1-1$ & 1 & $0-10$ & Debitage & Tertiary & Lithic & 1 & 1.94 & $\mathrm{JU}$ & $9 / 18 / 2017$ & \\
\hline $1-1$ & 3 & $20-30$ & Debitage & Primary & Lithic & 2 & 3.03 & $\mathrm{JU}$ & 9/18/2017 & \\
\hline $1-1$ & 3 & $20-30$ & Debitage & Secondary & Lithic & 1 & 3.37 & $\mathrm{JU}$ & 9/18/2017 & \\
\hline $1-1$ & 3 & $20-30$ & Debitage & Tertiary & Lithic & 3 & 1.52 & $\mathrm{JU}$ & 9/18/2017 & 3 bifacial thinning flakes \\
\hline $1-1$ & 4 & $30-40$ & Debitage & Secondary & Lithic & 1 & 0.37 & $\mathrm{JU}$ & 9/18/2017 & 1 bifacial thinning flake \\
\hline $1-1$ & 4 & $30-40$ & Debitage & Tertiary & Lithic & 5 & 1.38 & $\mathrm{JU}$ & 9/18/2017 & 4 bifacial thinning flakes \\
\hline $1-1$ & 5 & $40-50$ & Debitage & Secondary & Lithic & 1 & 0.77 & $\mathrm{JU}$ & 9/18/2017 & \\
\hline $1-1$ & 5 & $40-50$ & Debitage & Tertiary & Lithic & 5 & 1.71 & $\mathrm{JU}$ & 9/18/2017 & 4 bifacial thinning flakes \\
\hline $1-1$ & 6 & $50-60$ & Debitage & Tertiary & Lithic & 4 & 0.94 & $\mathrm{JU}$ & $9 / 18 / 2017$ & 4 bifacial thinning flakes \\
\hline $1-2$ & 2 & $10-20$ & Debitage & Secondary & Lithic & 1 & 5.16 & $\mathrm{JU}$ & 9/18/2017 & 1 bifacial thinning flake \\
\hline $1-2$ & 2 & $10-20$ & Debitage & Tertiary & Lithic & 1 & 0.17 & $\mathrm{JU}$ & 9/18/2017 & 1 bifacial thinning flake \\
\hline $1-2$ & 5 & $40-50$ & Debitage & Tertiary & Lithic & 2 & 0.91 & $\mathrm{JU}$ & 9/18/2017 & 1 bifacial thinning flake \\
\hline $1-2$ & 6 & $50-60$ & Debitage & Secondary & Lithic & 1 & 0.24 & $\mathrm{JU}$ & 9/18/2017 & 1 bifacial thinning flake \\
\hline $1-2$ & 6 & $50-60$ & Debitage & Tertiary & Lithic & 1 & 1.78 & $\mathrm{JU}$ & 9/18/2017 & 1 bifacial thinning flake \\
\hline $1-4$ & 1 & $0-10$ & Glass & Colorless & Glass & 1 & 2.61 & $\mathrm{JU}$ & 9/18/2017 & \\
\hline $1-4$ & 1 & $0-10$ & Glass & Milk & Glass & 3 & 4.55 & $\mathrm{JU}$ & 9/18/2017 & \\
\hline $1-4$ & 2 & $10-20$ & Glass & Colorless & Glass & 2 & 1.25 & $\mathrm{JU}$ & 9/18/2017 & \\
\hline $1-4$ & 2 & $10-20$ & Glass & Milk & Glass & 1 & 1.47 & $\mathrm{JU}$ & 9/18/2017 & \\
\hline 1-4 & 3 & $20-30$ & Glass & Colorless & Glass & 2 & 3.84 & $\mathrm{JU}$ & 9/18/2017 & \\
\hline $1-4$ & 3 & $20-30$ & Glass & Milk & Glass & 1 & 0.48 & $\mathrm{JU}$ & $9 / 18 / 2017$ & \\
\hline $1-4$ & 3 & $20-30$ & Debitage & Tertiary & Lithic & 1 & 1.76 & $\mathrm{JU}$ & 9/18/2017 & 1 bifacial thinning flake \\
\hline $1-4$ & 4 & $30-40$ & Glass & Colorless & Glass & 1 & 0.9 & $\mathrm{JU}$ & 9/18/2017 & \\
\hline $1-5$ & 1 & $0-10$ & Debitage & Tertiary & Lithic & 2 & 0.49 & $\mathrm{JU}$ & 9/18/2017 & 1 bifacial thinning flake \\
\hline $1-5$ & 2 & $10-20$ & Debitage & Tertiary & Lithic & 1 & 0.29 & $\mathrm{JU}$ & 9/18/2017 & \\
\hline $1-6$ & 7 & $60-70$ & Debitage & Secondary & Lithic & 1 & 8.32 & $\mathrm{JU}$ & 9/18/2017 & 1 bifacial thinning flake \\
\hline $1-6$ & 8 & $70-80$ & Debitage & Secondary & Lithic & 1 & 1.07 & $\mathrm{JU}$ & 9/18/2017 & \\
\hline $1-6$ & 9 & $80-90$ & $\begin{array}{l}\text { Burned } \\
\text { Rock }\end{array}$ & Burned Rock & Lithic & 2 & 4.33 & JU & 9/18/2017 & \\
\hline $1-7$ & 3 & $20-30$ & Debitage & Tertiary & Lithic & 2 & 0.33 & $\mathrm{JU}$ & 9/18/2017 & \\
\hline $1-8$ & 2 & $10-20$ & Debitage & Tertiary & Lithic & 1 & 0.95 & $\mathrm{JU}$ & 9/18/2017 & 1 bifacial thinning flake \\
\hline $1-8$ & 3 & $20-30$ & Debitage & Tertiary & Lithic & 1 & 0.46 & $\mathrm{JU}$ & $9 / 18 / 2017$ & 1 bifacial thinning flake \\
\hline $1-8$ & 4 & $30-40$ & Debitage & Secondary & Lithic & 1 & 0.86 & $\mathrm{JU}$ & 9/18/2017 & \\
\hline $1-8$ & 4 & $30-40$ & Debitage & Tertiary & Lithic & 2 & 0.57 & $\mathrm{JU}$ & 9/18/2017 & 2 bifacial thinning flakes \\
\hline $1-9$ & 1 & $0-10$ & Debitage & Tertiary & Lithic & 1 & 2.34 & $\mathrm{JU}$ & $9 / 18 / 2017$ & 1 bifacial thinning flake \\
\hline $1-9$ & 5 & $40-50$ & $\begin{array}{l}\text { Faunal } \\
\text { Remains }\end{array}$ & Bone & & 3 & 2.25 & JU & 9/18/2017 & \\
\hline $1-9$ & 6 & $50-60$ & Debitage & Secondary & Lithic & 1 & 22.86 & $\mathrm{JU}$ & 9/18/2017 & \\
\hline $1-9$ & 6 & $50-60$ & Debitage & Tertiary & Lithic & 1 & 0.91 & $\mathrm{JU}$ & 9/18/2017 & 1 bifacial thinning flake \\
\hline $1-9$ & 7 & $60-70$ & Debitage & Primary & Lithic & 1 & 0.23 & $\mathrm{JU}$ & 9/18/2017 & \\
\hline $1-9$ & 7 & $60-70$ & Debitage & Tertiary & Lithic & 1 & 0.24 & $\mathrm{JU}$ & $9 / 18 / 2017$ & 1 bifacial thinning flake \\
\hline $1-9$ & 8 & $70-80$ & Debitage & Tertiary & Lithic & 1 & 0.47 & $\mathrm{JU}$ & 9/18/2017 & \\
\hline $1-9$ & 9 & $80-90$ & Debitage & Tertiary & Lithic & 1 & 0.47 & $\mathrm{JU}$ & 9/18/2017 & 1 bifacial thinning flake \\
\hline 1-10 & 1 & $0-10$ & Debitage & Tertiary & Lithic & 1 & 0.19 & $\mathrm{JU}$ & 9/27/2017 & \\
\hline $1-10$ & 2 & $10-20$ & Debitage & Primary & Lithic & 1 & 15.98 & $\mathrm{JU}$ & 9/27/2017 & \\
\hline $1-10$ & 4 & $40-50$ & $\begin{array}{l}\text { Faunal } \\
\text { Remains }\end{array}$ & Mussel Shell & Shell & 1 & 7.27 & JU & 9/27/2017 & Complete \\
\hline $1-10$ & 4 & $40-50$ & Debitage & Secondary & Lithic & 1 & 11.03 & $\mathrm{JU}$ & 9/27/2017 & 1 bifacial thinning flake \\
\hline $1-10$ & 4 & $40-50$ & Debitage & Tertiary & Lithic & 1 & 0.07 & $\mathrm{JU}$ & 9/27/2017 & \\
\hline $1-10$ & 8 & $70-80$ & Debitage & Secondary & Lithic & 1 & 2.15 & $\mathrm{JU}$ & 9/27/2017 & \\
\hline $1-11$ & 6 & $50-60$ & $\begin{array}{l}\text { Faunal } \\
\text { Remains }\end{array}$ & Mussel Shell & Shell & 2 & 1.29 & JU & 9/27/2017 & 1 umbo \\
\hline $1-12$ & 1 & $0-10$ & Glass & Colorless & Glass & 1 & 1.08 & $\mathrm{JU}$ & 9/27/2017 & \\
\hline $2-1$ & 1 & $0-10$ & Metal & Staple & Metal & 1 & 1 & $\mathrm{JU}$ & 9/18/2017 & Fence Staple \\
\hline $2-1$ & 1 & $0-10$ & Debitage & Tertiary & Lithic & 3 & 0.58 & $\mathrm{JU}$ & 9/18/2017 & 3 bifacial thinning flakes \\
\hline $2-1$ & 2 & $10-20$ & Debitage & Secondary & Lithic & 1 & 2.8 & $\mathrm{JU}$ & 9/18/2017 & 1 bifacial thinning flake \\
\hline $2-1$ & 2 & $10-20$ & Debitage & Tertiary & Lithic & 2 & 1.27 & $\mathrm{JU}$ & 9/18/2017 & 1 bifacial thinning flake \\
\hline $2-1$ & 3 & $20-30$ & Debitage & Secondary & Lithic & 4 & 4.38 & $\mathrm{JU}$ & 9/18/2017 & \\
\hline $2-1$ & 3 & $20-30$ & Debitage & Tertiary & Lithic & 3 & 1.11 & $\mathrm{JU}$ & 9/18/2017 & 3 bifacial thinning flakes \\
\hline $2-1$ & 5 & 46 & $\begin{array}{l}\text { Projectile } \\
\text { Point }\end{array}$ & Montell Point & Lithic & 1 & 4.93 & JU & 9/18/2017 & Proximal point base \\
\hline
\end{tabular}




\begin{tabular}{|c|c|c|c|c|c|c|c|c|c|c|}
\hline ST & Level & $\begin{array}{l}\text { Depth } \\
\text { (cmbs) }\end{array}$ & $\begin{array}{c}\text { Artifact } \\
\text { Type }\end{array}$ & $\begin{array}{c}\text { Artifact } \\
\text { Description }\end{array}$ & Material & $\begin{array}{c}\text { Artifact } \\
\text { No. }\end{array}$ & $\begin{array}{l}\text { Weight } \\
\text { (g) }\end{array}$ & Recorders & Date & Comments \\
\hline $2-1$ & 5 & $40-50$ & Debitage & Tertiary & Lithic & 2 & 0.54 & $\mathrm{JU}$ & $9 / 18 / 2017$ & \\
\hline $2-1$ & 6 & $50-60$ & Debitage & Secondary & Lithic & 1 & 0.23 & $\mathrm{JU}$ & 9/18/2017 & 1 bifacial thinning flake \\
\hline $2-2$ & 1 & $0-10$ & Debitage & Secondary & Lithic & 1 & 0.48 & $\mathrm{JU}$ & 9/18/2017 & 1 bifacial thinning flake \\
\hline $2-2$ & 1 & $0-10$ & Debitage & Tertiary & Lithic & 4 & 3.23 & $\mathrm{JU}$ & 9/18/2017 & 3 bifacial thinning flakes \\
\hline $2-2$ & 2 & $10-20$ & Debitage & Tertiary & Lithic & 1 & 1.12 & $\mathrm{JU}$ & 9/18/2017 & \\
\hline $2-3$ & 1 & $0-10$ & Debitage & Secondary & Lithic & 2 & 0.9 & $\mathrm{JU}$ & 9/18/2017 & \\
\hline $2-3$ & 1 & $0-10$ & Debitage & Tertiary & Lithic & 2 & 0.23 & $\mathrm{JU}$ & 9/18/2017 & 2 bifacial thinning flakes \\
\hline $2-3$ & 3 & $20-30$ & Debitage & Tertiary & Lithic & 2 & 1.54 & $\mathrm{JU}$ & 9/18/2017 & 2 bifacial thinning flakes \\
\hline $2-4$ & 2 & $10-20$ & Debitage & Tertiary & Lithic & 1 & 0.79 & $\mathrm{JU}$ & $9 / 18 / 2017$ & \\
\hline $2-4$ & 3 & $20-30$ & Debitage & Secondary & Lithic & 1 & 0.96 & $\mathrm{JU}$ & 9/18/2017 & 1 bifacial thinning flake \\
\hline $2-4$ & 3 & $20-30$ & Debitage & Tertiary & Lithic & 1 & 0.29 & $\mathrm{JU}$ & 9/18/2017 & 1 bifacial thinning flake \\
\hline $2-5$ & 3 & $20-30$ & Debitage & Secondary & Lithic & 1 & 0.75 & $\mathrm{JU}$ & 9/19/2017 & \\
\hline $2-5$ & 3 & $20-30$ & Debitage & Tertiary & Lithic & 1 & 0.18 & $\mathrm{JU}$ & 9/19/2017 & 1 bifacial thinning flake \\
\hline $2-5$ & 4 & $30-40$ & Debitage & Primary & Lithic & 1 & 0.75 & $\mathrm{JU}$ & 9/19/2017 & \\
\hline $2-5$ & 4 & $30-40$ & Debitage & Tertiary & Lithic & 1 & 0.85 & $\mathrm{JU}$ & 9/19/2017 & \\
\hline $2-6$ & 1 & $0-10$ & $\begin{array}{l}\text { Historic } \\
\text { Ceramic }\end{array}$ & Stoneware & Ceramic & 1 & 3.87 & $\mathrm{JU}$ & 9/18/2017 & Light blue slip \\
\hline $2-6$ & 1 & $0-10$ & Debitage & Secondary & Lithic & 1 & 5.98 & $\mathrm{JU}$ & 9/18/2017 & 1 bifacial thinning flake \\
\hline $2-6$ & 1 & $0-10$ & Debitage & Tertiary & Lithic & 3 & 1.69 & $\mathrm{JU}$ & 9/18/2017 & 3 bifacial thinning flakes \\
\hline $2-6$ & 2 & $10-20$ & Debitage & Primary & Lithic & 2 & 22.62 & $\mathrm{JU}$ & $9 / 18 / 2017$ & \\
\hline $2-6$ & 2 & $10-20$ & Debitage & Secondary & Lithic & 1 & 0.69 & $\mathrm{JU}$ & $9 / 18 / 2017$ & \\
\hline $2-6$ & 2 & $10-20$ & Debitage & Tertiary & Lithic & 7 & 3.2 & $\mathrm{JU}$ & 9/18/2017 & 5 bifacial thinning flakes \\
\hline $2-6$ & 3 & $20-30$ & $\begin{array}{l}\text { Faunal } \\
\text { Remains }\end{array}$ & Bone & & 2 & $<.01$ & JU & 9/18/2017 & 2 burned \\
\hline $2-6$ & 3 & $20-30$ & Debitage & Primary & Lithic & 1 & 0.19 & $\mathrm{JU}$ & 9/18/2017 & \\
\hline $2-6$ & 3 & $20-30$ & Debitage & Secondary & Lithic & 1 & 0.14 & $\mathrm{JU}$ & 9/18/2017 & \\
\hline $2-6$ & 4 & $30-40$ & $\begin{array}{l}\text { Faunal } \\
\text { Remains }\end{array}$ & Bone & & 1 & 0.03 & JU & 9/18/2017 & 1 burned \\
\hline $2-6$ & 4 & $30-40$ & Debitage & Secondary & Lithic & 2 & 13.63 & $\mathrm{JU}$ & 9/18/2017 & \\
\hline $2-6$ & 5 & $40-50$ & Debitage & Tertiary & Lithic & 1 & 0.2 & $\mathrm{JU}$ & 9/18/2017 & 1 bifacial thinning flake \\
\hline $2-6$ & 6 & $50-60$ & $\begin{array}{l}\text { Burned } \\
\text { Rock }\end{array}$ & Burned Rock & Lithic & 1 & 12.98 & JU & 9/18/2017 & \\
\hline $2-6$ & 6 & $50-60$ & Debitage & Secondary & Lithic & 1 & 0.74 & $\mathrm{JU}$ & 9/18/2017 & \\
\hline $2-6$ & 6 & $50-60$ & Debitage & Tertiary & Lithic & 3 & 2.79 & $\mathrm{JU}$ & 9/18/2017 & 3 bifacial thinning flakes \\
\hline $2-7$ & 1 & $0-10$ & Debitage & Tertiary & Lithic & 2 & 1.22 & $\mathrm{JU}$ & 9/18/2017 & 1 bifacial thinning flake \\
\hline $2-7$ & 4 & $30-40$ & Debitage & Secondary & Lithic & 1 & 1.11 & $\mathrm{JU}$ & 9/18/2017 & \\
\hline $2-7$ & 4 & $30-40$ & Debitage & Tertiary & Lithic & 2 & 0.23 & $\mathrm{JU}$ & 9/18/2017 & 2 bifacial thinning flakes \\
\hline $2-7$ & 5 & $40-50$ & Debitage & Tertiary & Lithic & 2 & 0.53 & $\mathrm{JU}$ & 9/18/2017 & 2 bifacial thinning flakes \\
\hline $2-7$ & 6 & $50-60$ & Debitage & Tertiary & Lithic & 1 & 0.11 & $\mathrm{JU}$ & 9/18/2017 & 1 bifacial thinning flake \\
\hline $2-7$ & 8 & $70-80$ & Debitage & Secondary & Lithic & 1 & 2.09 & $\mathrm{JU}$ & 9/18/2017 & \\
\hline $2-8$ & 1 & $0-10$ & Debitage & Tertiary & Lithic & 3 & 1.12 & $\mathrm{JU}$ & 9/19/2017 & 1 bifacial thinning flake \\
\hline $2-8$ & 2 & $10-20$ & Debitage & Tertiary & Lithic & 1 & 0.66 & $\mathrm{JU}$ & 9/19/2017 & 1 bifacial thinning flake \\
\hline $2-8$ & 3 & $20-30$ & Debitage & Tertiary & Lithic & 5 & 4.2 & $\mathrm{JU}$ & 9/19/2017 & \\
\hline $2-8$ & 4 & $30-40$ & Debitage & Primary & Lithic & 1 & 0.54 & $\mathrm{JU}$ & 9/19/2017 & \\
\hline $2-8$ & 4 & $30-40$ & Debitage & Tertiary & Lithic & 2 & 2.55 & $\mathrm{JU}$ & 9/19/2017 & 2 bifacial thinning flakes \\
\hline $2-8$ & 5 & $40-50$ & Debitage & Primary & Lithic & 1 & 0.43 & $\mathrm{JU}$ & 9/19/2017 & \\
\hline $2-8$ & 5 & $40-50$ & Debitage & Tertiary & Lithic & 3 & 1.86 & $\mathrm{JU}$ & 9/19/2017 & 3 bifacial thinning flakes \\
\hline $2-8$ & 6 & $50-60$ & Debitage & Primary & Lithic & 1 & 0.24 & $\mathrm{JU}$ & 9/19/2017 & \\
\hline $2-8$ & 6 & $50-60$ & Debitage & Tertiary & Lithic & 2 & 2.88 & $\mathrm{JU}$ & 9/19/2017 & 2 bifacial thinning flakes \\
\hline $2-8$ & 7 & $60-70$ & Debitage & Tertiary & Lithic & 1 & 0.37 & $\mathrm{JU}$ & 9/19/2017 & Burned \\
\hline $2-8$ & 8 & $70-80$ & Debitage & Tertiary & Lithic & 1 & 0.27 & $\mathrm{JU}$ & 9/19/2017 & 1 bifacial thinning flake \\
\hline $2-8$ & 9 & $80-90$ & Debitage & Tertiary & Lithic & 3 & 0.86 & $\mathrm{JU}$ & 9/19/2017 & 3 bifacial thinning flakes \\
\hline $2-8$ & 10 & $90-100$ & Debitage & Tertiary & Lithic & 5 & 6.45 & $\mathrm{JU}$ & 9/19/2017 & 3 bifacial thinning flakes \\
\hline $2-8$ & 12 & $110-120$ & Debitage & Tertiary & Lithic & 1 & 0.23 & $\mathrm{JU}$ & 9/19/2017 & 1 bifacial thinning flake \\
\hline $2-8$ & 14 & $130-140$ & Debitage & Secondary & Lithic & 1 & 4.28 & $\mathrm{JU}$ & 9/19/2017 & \\
\hline $2-8$ & 14 & $130-140$ & Debitage & Tertiary & Lithic & 1 & 0.25 & $\mathrm{JU}$ & 9/19/2017 & 1 bifacial thinning flake \\
\hline $2-9$ & 3 & $20-30$ & Debitage & Secondary & Lithic & 1 & 0.44 & $\mathrm{JU}$ & 9/19/2017 & \\
\hline $2-9$ & 3 & $20-30$ & Debitage & Tertiary & Lithic & 4 & 0.99 & $\mathrm{JU}$ & 9/19/2017 & 3 bifacial thinning flakes \\
\hline $2-9$ & 4 & $30-40$ & $\begin{array}{l}\text { Faunal } \\
\text { Remains }\end{array}$ & Mussel Shell & Shell & 1 & 1.48 & JU & 9/19/2017 & \\
\hline
\end{tabular}




\begin{tabular}{|c|c|c|c|c|c|c|c|c|c|c|}
\hline ST & Level & $\begin{array}{l}\text { Depth } \\
\text { (cmbs) }\end{array}$ & $\begin{array}{l}\text { Artifact } \\
\text { Type }\end{array}$ & $\begin{array}{c}\text { Artifact } \\
\text { Description }\end{array}$ & Material & $\begin{array}{c}\text { Artifact } \\
\text { No. }\end{array}$ & $\begin{array}{c}\text { Weight } \\
\text { (g) }\end{array}$ & Recorders & Date & Comments \\
\hline $2-9$ & 4 & $30-40$ & Debitage & Tertiary & Lithic & 4 & 2.31 & $\mathrm{JU}$ & $9 / 19 / 2017$ & 3 bifacial thinning flakes \\
\hline $2-9$ & 5 & $40-50$ & Debitage & Secondary & Lithic & 1 & 5.71 & $\mathrm{JU}$ & 9/19/2017 & 1 bifacial thinning flake \\
\hline $2-9$ & 6 & $50-60$ & Debitage & Secondary & Lithic & 1 & 2.06 & $\mathrm{JU}$ & 9/19/2017 & \\
\hline $2-9$ & 6 & $50-60$ & Debitage & Tertiary & Lithic & 6 & 4.2 & $\mathrm{JU}$ & 9/19/2017 & 3 bifacial thinning flakes \\
\hline $2-9$ & 7 & $60-70$ & Debitage & Tertiary & Lithic & 3 & 3.54 & $\mathrm{JU}$ & 9/19/2017 & 2 bifacial thinning flakes \\
\hline $2-9$ & 8 & $70-80$ & Debitage & Secondary & Lithic & 1 & 4.41 & $\mathrm{JU}$ & 9/19/2017 & \\
\hline $2-9$ & 8 & $70-80$ & Debitage & Tertiary & Lithic & 2 & 1.66 & $\mathrm{JU}$ & 9/19/2017 & 2 bifacial thinning flakes \\
\hline $2-10$ & 1 & $0-10$ & Debitage & Secondary & Lithic & 1 & 1.1 & $\mathrm{JU}$ & 9/27/2017 & \\
\hline $2-10$ & 2 & $10-20$ & $\begin{array}{l}\text { Ground } \\
\text { Stone }\end{array}$ & Ground Stone & Lithic & 1 & 35.06 & JU & 9/27/2017 & \\
\hline $2-10$ & 3 & $20-30$ & $\begin{array}{l}\text { Burned } \\
\text { Rock }\end{array}$ & Burned Rock & Lithic & 1 & 42.57 & JU & 9/27/2017 & \\
\hline $2-10$ & 3 & $20-30$ & Debitage & Tertiary & Lithic & 2 & 2.55 & $\mathrm{JU}$ & 9/27/2017 & 1 bifacial thinning flake \\
\hline $2-10$ & 4 & $30-40$ & Debitage & Tertiary & Lithic & 5 & 2.53 & $\mathrm{JU}$ & 9/27/2017 & 3 bifacial thinning flakes \\
\hline $2-10$ & 5 & $40-50$ & Debitage & Secondary & Lithic & 1 & 50.5 & $\mathrm{JU}$ & 9/27/2017 & \\
\hline $2-10$ & 5 & $40-50$ & Debitage & Tertiary & Lithic & 8 & 3.39 & $\mathrm{JU}$ & 9/27/2017 & 5 bifacial thinning flakes \\
\hline $2-10$ & 7 & $60-70$ & Debitage & Tertiary & Lithic & 7 & 11.85 & $\mathrm{JU}$ & 9/27/2017 & 6 bifacial thinning flakes \\
\hline $2-10$ & 7 & $60-70$ & Debitage & Shatter & Lithic & 1 & 1.21 & $\mathrm{JU}$ & 9/27/2017 & \\
\hline $2-10$ & 8 & $70-80$ & Debitage & Secondary & Lithic & 1 & 1.22 & $\mathrm{JU}$ & 9/27/2017 & 1 bifacial thinning flake \\
\hline $2-10$ & 8 & $70-80$ & Debitage & Tertiary & Lithic & 3 & 3.06 & $\mathrm{JU}$ & 9/27/2017 & $\begin{array}{l}1 \text { burned, } 2 \text { bifacial thinning } \\
\text { flakes }\end{array}$ \\
\hline $2-10$ & 9 & $80-90$ & Debitage & Tertiary & Lithic & 1 & 2.36 & $\mathrm{JU}$ & 9/27/2017 & \\
\hline $2-10$ & 10 & $90-100$ & Debitage & Secondary & Lithic & 1 & 1.32 & $\mathrm{JU}$ & 9/27/2017 & \\
\hline $2-10$ & 10 & $90-100$ & Debitage & Shatter & Lithic & 1 & 0.08 & $\mathrm{JU}$ & 9/27/2017 & \\
\hline $2-11$ & 5 & $40-50$ & Debitage & Tertiary & Lithic & 2 & 1.61 & $\mathrm{JU}$ & 9/27/2017 & 2 bifacial thinning flakes \\
\hline $2-11$ & 6 & $50-60$ & Debitage & Primary & Lithic & 1 & 11.82 & $\mathrm{JU}$ & 9/27/2017 & \\
\hline $2-11$ & 6 & $50-60$ & Debitage & Secondary & Lithic & 1 & 1.42 & $\mathrm{JU}$ & 9/27/2017 & 1 bifacial thinning flake \\
\hline $2-11$ & 6 & $50-60$ & Debitage & Tertiary & Lithic & 1 & 0.14 & $\mathrm{JU}$ & 9/27/2017 & 1 bifacial thinning flake \\
\hline $2-11$ & 8 & $70-80$ & Debitage & Secondary & Lithic & 1 & 0.29 & $\mathrm{JU}$ & 9/27/2017 & 1 bifacial thinning flake \\
\hline $2-11$ & 8 & $70-80$ & Debitage & Tertiary & Lithic & 2 & 0.52 & $\mathrm{JU}$ & 9/27/2017 & 2 bifacial thinning flakes \\
\hline $2-12$ & 6 & $50-60$ & Debitage & Secondary & Lithic & 1 & 0.9 & $\mathrm{JU}$ & 9/27/2017 & \\
\hline $3-1$ & 3 & $20-30$ & Debitage & Tertiary & Lithic & 2 & 0.28 & $\mathrm{JU}$ & 9/19/2017 & 1 bifacial thinning flake \\
\hline 3-1 & 5 & $40-50$ & Debitage & Tertiary & Lithic & 2 & 0.32 & $\mathrm{JU}$ & 9/19/2017 & \\
\hline $3-1$ & 6 & $50-60$ & Debitage & Tertiary & Lithic & 1 & 0.08 & $\mathrm{JU}$ & 9/19/2017 & \\
\hline $3-2$ & 1 & $0-10$ & Debitage & Tertiary & Lithic & 1 & 2.48 & $\mathrm{JU}$ & 9/19/2017 & 1 bifacial thinning flake \\
\hline $3-2$ & 2 & $10-20$ & Debitage & Tertiary & Lithic & 1 & 5.81 & $\mathrm{JU}$ & 9/19/2017 & \\
\hline 3-3 & 1 & $0-10$ & Debitage & Primary & Lithic & 1 & 0.98 & $\mathrm{JU}$ & 9/19/2017 & \\
\hline $3-3$ & 1 & $0-10$ & Debitage & Tertiary & Lithic & 1 & 0.36 & $\mathrm{JU}$ & 9/19/2017 & 1 bifacial thinning flake \\
\hline $3-3$ & 2 & $10-20$ & Debitage & Tertiary & Lithic & 3 & 0.73 & $\mathrm{JU}$ & 9/19/2017 & 2 bifacial thinning flakes \\
\hline $3-3$ & 3 & $20-30$ & Debitage & Secondary & Lithic & 2 & 3.05 & $\mathrm{JU}$ & 9/19/2017 & \\
\hline $3-3$ & 3 & $20-30$ & Debitage & Tertiary & Lithic & 3 & 0.74 & $\mathrm{JU}$ & 9/19/2017 & 3 bifacial thinning flakes \\
\hline $3-3$ & 4 & $30-40$ & Debitage & Primary & Lithic & 1 & 4.31 & $\mathrm{JU}$ & 9/19/2017 & \\
\hline $3-3$ & 4 & $30-40$ & Debitage & Tertiary & Lithic & 1 & 0.43 & $\mathrm{JU}$ & 9/19/2017 & \\
\hline $3-4$ & 2 & $10-20$ & Debitage & Secondary & Lithic & 1 & 0.09 & $\mathrm{JU}$ & 9/19/2017 & \\
\hline $3-4$ & 3 & $20-30$ & Debitage & Tertiary & Lithic & 1 & 1.81 & $\mathrm{JU}$ & 9/19/2017 & 1 bifacial thinning flake \\
\hline $3-5$ & 3 & $20-30$ & Debitage & Tertiary & Lithic & 1 & 0.37 & $\mathrm{JU}$ & 9/19/2017 & 1 bifacial thinning flake \\
\hline $3-5$ & 4 & $30-40$ & Debitage & Tertiary & Lithic & 2 & 0.46 & $\mathrm{JU}$ & 9/19/2017 & 1 bifacial thinning flake \\
\hline $3-5$ & 5 & $40-50$ & Debitage & Tertiary & Lithic & 1 & 0.17 & $\mathrm{JU}$ & 9/19/2017 & \\
\hline $3-5$ & 6 & $50-60$ & Debitage & Tertiary & Lithic & 1 & 0.39 & $\mathrm{JU}$ & 9/19/2017 & \\
\hline 3-7 & 3 & $20-30$ & Debitage & Secondary & Lithic & 1 & 0.63 & $\mathrm{JU}$ & 9/19/2017 & \\
\hline $3-7$ & 4 & $30-40$ & Core & Core & Lithic & 1 & 52.67 & $\mathrm{JU}$ & 9/19/2017 & Exhausted \\
\hline $3-7$ & 5 & $40-50$ & Debitage & Tertiary & Lithic & 1 & 3.96 & $\mathrm{JU}$ & 9/19/2017 & 1 bifacial thinning flake \\
\hline $3-7$ & 8 & $70-80$ & Debitage & Secondary & Lithic & 1 & 6.46 & $\mathrm{JU}$ & 9/19/2017 & \\
\hline 3-8 & 2 & $10-20$ & Debitage & Secondary & Lithic & 1 & 0.23 & $\mathrm{JU}$ & 9/19/2017 & \\
\hline $3-8$ & 2 & $10-20$ & Debitage & Tertiary & Lithic & 4 & 3.7 & $\mathrm{JU}$ & 9/19/2017 & 2 bifacial thinning flakes \\
\hline $3-8$ & 3 & $20-30$ & Debitage & Tertiary & Lithic & 4 & 6.47 & $\mathrm{JU}$ & 9/19/2017 & 1 bifacial thinning flake \\
\hline $3-8$ & 4 & $30-40$ & Debitage & Primary & Lithic & 1 & 0.5 & $\mathrm{JU}$ & 9/19/2017 & \\
\hline $3-8$ & 4 & $30-40$ & Debitage & Tertiary & Lithic & 3 & 9.38 & $\mathrm{JU}$ & 9/19/2017 & 2 bifacial thinning flakes \\
\hline $3-8$ & 5 & $40-50$ & Debitage & Secondary & Lithic & 7 & 20.21 & $\mathrm{JU}$ & 9/19/2017 & 2 bifacial thinning flakes \\
\hline $3-8$ & 5 & $40-50$ & Debitage & Tertiary & Lithic & 11 & 7.2 & $\mathrm{JU}$ & 9/19/2017 & 8 bifacial thinning flakes \\
\hline
\end{tabular}




\begin{tabular}{|c|c|c|c|c|c|c|c|c|c|c|}
\hline ST & Level & $\begin{array}{l}\text { Depth } \\
\text { (cmbs) }\end{array}$ & $\begin{array}{l}\text { Artifact } \\
\text { Type }\end{array}$ & $\begin{array}{c}\text { Artifact } \\
\text { Description }\end{array}$ & Material & $\begin{array}{c}\text { Artifact } \\
\text { No. }\end{array}$ & $\begin{array}{l}\text { Weight } \\
\text { (g) }\end{array}$ & Recorders & Date & Comments \\
\hline $3-8$ & 6 & $50-60$ & Debitage & Secondary & Lithic & 2 & 2.29 & $\mathrm{JU}$ & 9/19/2017 & 1 bifacial thinning flake \\
\hline $3-8$ & 6 & $50-60$ & Debitage & Tertiary & Lithic & 9 & 2.71 & $\mathrm{JU}$ & 9/19/2017 & 5 bifacial thinning flakes \\
\hline $3-8$ & 7 & $60-70$ & Debitage & Secondary & Lithic & 1 & 1.27 & $\mathrm{JU}$ & 9/19/2017 & \\
\hline $3-8$ & 7 & $60-70$ & Debitage & Tertiary & Lithic & 5 & 4.49 & $\mathrm{JU}$ & 9/19/2017 & 3 bifacial thinning flakes \\
\hline $3-8$ & 8 & $70-80$ & Debitage & Primary & Lithic & 2 & 1.36 & $\mathrm{JU}$ & 9/19/2017 & \\
\hline $3-8$ & 8 & $70-80$ & Debitage & Secondary & Lithic & 4 & 8.61 & $\mathrm{JU}$ & 9/19/2017 & 1 bifacial thinning flake \\
\hline $3-8$ & 8 & $70-80$ & Debitage & Tertiary & Lithic & 11 & 3.48 & $\mathrm{JU}$ & 9/19/2017 & 7 bifacial thinning flakes \\
\hline $3-8$ & 9 & $80-90$ & Debitage & Primary & Lithic & 1 & 0.85 & $\mathrm{JU}$ & 9/19/2017 & \\
\hline $3-8$ & 9 & $80-90$ & Debitage & Tertiary & Lithic & 12 & 14.98 & $\mathrm{JU}$ & 9/19/2017 & 8 bifacial thinning flakes \\
\hline $3-9$ & 2 & $10-20$ & Debitage & Secondary & Lithic & 3 & 9.23 & $\mathrm{JU}$ & 9/19/2017 & 1 bifacial thinning flake \\
\hline $3-9$ & 2 & $10-20$ & Debitage & Tertiary & Lithic & 1 & 0.29 & $\mathrm{JU}$ & 9/19/2017 & 1 bifacial thinning flake \\
\hline $3-9$ & 3 & $20-30$ & Debitage & Primary & Lithic & 1 & 0.62 & $\mathrm{JU}$ & 9/19/2017 & \\
\hline $3-9$ & 3 & $20-30$ & Debitage & Tertiary & Lithic & 1 & 0.6 & $\mathrm{JU}$ & 9/19/2017 & \\
\hline $3-9$ & 4 & $30-40$ & Debitage & Secondary & Lithic & 2 & 40.47 & $\mathrm{JU}$ & 9/19/2017 & 1 bifacial thinning flake \\
\hline $3-9$ & 4 & $30-40$ & Debitage & Tertiary & Lithic & 7 & 13.24 & $\mathrm{JU}$ & 9/19/2017 & 3 bifacial thinning flakes \\
\hline $3-9$ & 5 & $40-50$ & Core & Core & Lithic & 1 & 331.27 & $\mathrm{JU}$ & 9/19/2017 & \\
\hline $3-9$ & 5 & $40-50$ & Debitage & Tertiary & Lithic & 10 & 7 & $\mathrm{JU}$ & 9/19/2017 & 9 bifacial thinning flakes \\
\hline $3-9$ & 6 & $50-60$ & Debitage & Primary & Lithic & 1 & 0.25 & $\mathrm{JU}$ & 9/19/2017 & \\
\hline $3-9$ & 6 & $50-60$ & Debitage & Secondary & Lithic & 1 & 3.36 & $\mathrm{JU}$ & 9/19/2017 & \\
\hline $3-9$ & 6 & $50-60$ & Debitage & Tertiary & Lithic & 11 & 2.55 & $\mathrm{JU}$ & 9/19/2017 & 7 bifacial thinning flakes \\
\hline $3-9$ & 7 & $60-70$ & Debitage & Secondary & Lithic & 1 & 41.72 & $\mathrm{JU}$ & 9/19/2017 & \\
\hline $3-9$ & 7 & $60-70$ & Debitage & Tertiary & Lithic & 6 & 2.05 & $\mathrm{JU}$ & 9/19/2017 & 6 bifacial thinning flakes \\
\hline $3-9$ & 8 & $70-80$ & Debitage & Secondary & Lithic & 2 & 3.29 & $\mathrm{JU}$ & 9/19/2017 & 1 bifacial thinning flake \\
\hline $3-9$ & 8 & $70-80$ & Debitage & Tertiary & Lithic & 4 & 4.98 & $\mathrm{JU}$ & 9/19/2017 & 3 bifacial thinning flakes \\
\hline $3-9$ & 9 & $80-90$ & Debitage & Primary & Lithic & 1 & 0.38 & $\mathrm{JU}$ & 9/19/2017 & \\
\hline $3-9$ & 9 & $80-90$ & Debitage & Secondary & Lithic & 1 & 18.71 & $\mathrm{JU}$ & 9/19/2017 & 1 bifacial thinning flake \\
\hline $3-9$ & 9 & $80-90$ & Debitage & Tertiary & Lithic & 5 & 7.25 & $\mathrm{JU}$ & 9/19/2017 & 3 bifacial thinning flakes \\
\hline $3-9$ & 10 & $90-100$ & Debitage & Tertiary & Lithic & 2 & 0.84 & $\mathrm{JU}$ & 9/19/2017 & 2 bifacial thinning flakes \\
\hline $3-10$ & 5 & $40-50$ & Debitage & Tertiary & Lithic & 2 & 7.96 & $\mathrm{JU}$ & 9/27/2017 & 1 bifacial thinning flake \\
\hline $3-10$ & 7 & $60-70$ & Debitage & Tertiary & Lithic & 5 & 43.88 & $\mathrm{JU}$ & 9/27/2017 & 3 bifacial thinning flakes \\
\hline $3-10$ & 8 & $70-80$ & Debitage & Tertiary & Lithic & 8 & 3.76 & $\mathrm{JU}$ & 9/27/2017 & 6 bifacial thinning flakes \\
\hline $3-10$ & 9 & $80-90$ & Debitage & Tertiary & Lithic & 4 & 4.93 & $\mathrm{JU}$ & 9/27/2017 & 3 bifacial thinning flakes \\
\hline $3-10$ & 9 & $80-90$ & Debitage & Shatter & Lithic & 1 & 0.4 & $\mathrm{JU}$ & 9/27/2017 & \\
\hline $3-10$ & 9 & $80-90$ & $\begin{array}{l}\text { Faunal } \\
\text { Remains }\end{array}$ & Mussel Shell & Shell & 1 & 0.44 & JU & 9/27/2017 & No umbo \\
\hline 3-10 & 10 & $90-100$ & Debitage & Tertiary & Lithic & 1 & 0.87 & $\mathrm{JU}$ & 9/27/2017 & 1 bifacial thinning flake \\
\hline $3-11$ & 4 & $30-40$ & Debitage & Secondary & Lithic & 2 & 1.41 & $\mathrm{JU}$ & $9 / 27 / 2017$ & \\
\hline $3-11$ & 4 & $30-40$ & Debitage & Tertiary & Lithic & 1 & 0.47 & $\mathrm{JU}$ & 9/27/2017 & 1 bifacial thinning flake \\
\hline 3-11 & 5 & $40-50$ & Debitage & Secondary & Lithic & 2 & 4.09 & $\mathrm{JU}$ & 9/27/2017 & 1 bifacial thinning flake \\
\hline 3-11 & 5 & $40-50$ & Debitage & Tertiary & Lithic & 2 & 4.95 & $\mathrm{JU}$ & 9/27/2017 & 2 bifacial thinning flakes \\
\hline 3-11 & 6 & $50-60$ & Debitage & Tertiary & Lithic & 2 & 0.83 & $\mathrm{JU}$ & 9/27/2017 & 1 bifacial thinning flake \\
\hline $3-11$ & 8 & $70-80$ & Debitage & Secondary & Lithic & 3 & 5.24 & $\mathrm{JU}$ & 9/27/2017 & 1 bifacial thinning flake \\
\hline 3-11 & 8 & $70-80$ & Debitage & Tertiary & Lithic & 15 & 12.38 & $\mathrm{JU}$ & 9/27/2017 & 10 bifacial thinning flakes \\
\hline 3-11 & 8 & $70-80$ & Debitage & Shatter & Lithic & 2 & 3.71 & $\mathrm{JU}$ & 9/27/2017 & \\
\hline 3-11 & 9 & $80-90$ & Debitage & Tertiary & Lithic & 4 & 2.32 & $\mathrm{JU}$ & 9/27/2017 & 4 bifacial thinning flakes \\
\hline $3-11$ & 10 & $90-100$ & Debitage & Secondary & Lithic & 1 & 0.53 & $\mathrm{JU}$ & 9/27/2017 & 1 bifacial thinning flake \\
\hline $3-12$ & 8 & $70-80$ & Debitage & Tertiary & Lithic & 3 & 2.11 & $\mathrm{JU}$ & 9/27/2017 & 1 bifacial thinning flake \\
\hline $3-12$ & 9 & $80-90$ & Debitage & Tertiary & Lithic & 3 & 2.04 & $\mathrm{JU}$ & 9/27/2017 & 2 bifacial thinning flakes \\
\hline 3-12 & 10 & $90-100$ & Debitage & Tertiary & Lithic & 1 & 2.72 & $\mathrm{JU}$ & 9/27/2017 & 1 bifacial thinning flake \\
\hline $3-12$ & 16 & $150-160$ & Debitage & Tertiary & Lithic & 3 & 6.2 & $\mathrm{JU}$ & 9/27/2017 & 1 bifacial thinning flake \\
\hline $4-1$ & 2 & $10-20$ & Debitage & Tertiary & Lithic & 3 & 2.35 & $\mathrm{JU}$ & 9/27/2017 & 3 bifacial thinning flakes \\
\hline $4-1$ & 2 & $10-20$ & Debitage & Shatter & Lithic & 1 & 0.5 & $\mathrm{JU}$ & 9/27/2017 & \\
\hline $4-2$ & 1 & $0-10$ & Debitage & Tertiary & Lithic & 1 & 2.07 & $\mathrm{JU}$ & 9/27/2017 & 1 bifacial thinning flake \\
\hline $4-2$ & 2 & $10-20$ & Debitage & Secondary & Lithic & 1 & 0.56 & $\mathrm{JU}$ & $9 / 27 / 2017$ & \\
\hline $4-2$ & 2 & $10-20$ & Debitage & Tertiary & Lithic & 8 & 3.86 & $\mathrm{JU}$ & 9/27/2017 & 5 bifacial thinning flakes \\
\hline $4-2$ & 2 & $10-20$ & Debitage & Shatter & Lithic & 1 & 0.29 & $\mathrm{JU}$ & 9/27/2017 & \\
\hline $4-2$ & 3 & $20-30$ & Debitage & Secondary & Lithic & 1 & 0.74 & $\mathrm{JU}$ & 9/27/2017 & \\
\hline $4-2$ & 3 & $20-30$ & Debitage & Tertiary & Lithic & 1 & 0.13 & $\mathrm{JU}$ & 9/27/2017 & \\
\hline $4-2$ & 5 & $40-50$ & Debitage & Secondary & Lithic & 1 & 0.83 & $\mathrm{JU}$ & 9/27/2017 & \\
\hline
\end{tabular}




\begin{tabular}{|c|c|c|c|c|c|c|c|c|c|c|}
\hline ST & Level & $\begin{array}{l}\text { Depth } \\
\text { (cmbs) }\end{array}$ & $\begin{array}{l}\text { Artifact } \\
\text { Type }\end{array}$ & $\begin{array}{c}\text { Artifact } \\
\text { Description }\end{array}$ & Material & $\begin{array}{l}\text { Artifact } \\
\text { No. }\end{array}$ & $\begin{array}{c}\text { Weight } \\
\text { (g) }\end{array}$ & Recorders & Date & Comments \\
\hline $4-2$ & 5 & $40-50$ & Debitage & Tertiary & Lithic & 4 & 1.96 & $\mathrm{JU}$ & $9 / 27 / 2017$ & 2 bifacial thinning flakes \\
\hline $4-2$ & 6 & $50-60$ & Debitage & Secondary & Lithic & 2 & 1.4 & $\mathrm{JU}$ & 9/27/2017 & 1 bifacial thinning flake \\
\hline $4-2$ & 6 & $50-60$ & Debitage & Tertiary & Lithic & 3 & 1.04 & $\mathrm{JU}$ & 9/27/2017 & 2 bifacial thinning flakes \\
\hline $4-2$ & 9 & $80-90$ & Debitage & Secondary & Lithic & 1 & 1.09 & $\mathrm{JU}$ & 9/27/2017 & 1 bifacial thinning flake \\
\hline 4-3 & 1 & $0-10$ & Debitage & Tertiary & Lithic & 2 & 0.27 & $\mathrm{JU}$ & 9/27/2017 & 1 bifacial thinning flake \\
\hline $4-3$ & 3 & $20-30$ & Debitage & Tertiary & Lithic & 3 & 2.64 & $\mathrm{JU}$ & 9/27/2017 & 3 bifacial thinning flakes \\
\hline 4-5 & 3 & $20-30$ & Debitage & Tertiary & Lithic & 2 & 0.86 & $\mathrm{JU}$ & 9/27/2017 & 1 bifacial thinning flake \\
\hline $4-6$ & 2 & $10-20$ & Debitage & Tertiary & Lithic & 1 & 15.67 & $\mathrm{JU}$ & 9/27/2017 & 1 bifacial thinning flake \\
\hline $4-6$ & 3 & $20-30$ & Debitage & Secondary & Lithic & 1 & 30.7 & $\mathrm{JU}$ & $9 / 27 / 2017$ & \\
\hline $4-6$ & 5 & $40-50$ & Debitage & Tertiary & Lithic & 1 & 0.35 & $\mathrm{JU}$ & 9/27/2017 & 1 bifacial thinning flake \\
\hline $4-6$ & 5 & $40-50$ & Debitage & Shatter & Lithic & 2 & 1.92 & $\mathrm{JU}$ & $9 / 27 / 2017$ & \\
\hline $4-6$ & 6 & $50-60$ & Debitage & Tertiary & Lithic & 2 & 2.25 & $\mathrm{JU}$ & 9/27/2017 & 2 bifacial thinning flakes \\
\hline 4-7 & 1 & $0-10$ & Debitage & Primary & Lithic & 1 & 1.15 & $\mathrm{JU}$ & 9/27/2017 & \\
\hline 4-7 & 1 & $0-10$ & Debitage & Secondary & Lithic & 2 & 1.83 & $\mathrm{JU}$ & 9/27/2017 & \\
\hline $4-7$ & 1 & $0-10$ & Debitage & Tertiary & Lithic & 14 & 23.12 & $\mathrm{JU}$ & 9/27/2017 & 9 bifacial thinning flakes \\
\hline $4-7$ & 2 & $10-20$ & $\begin{array}{l}\text { Projectile } \\
\text { Point }\end{array}$ & Scallorn Point & Lithic & 1 & 1.55 & JU & 9/27/2017 & $\begin{array}{l}\text { Corner-notched broad stemmed } \\
\text { variant }\end{array}$ \\
\hline 4-7 & 2 & $10-20$ & Debitage & Secondary & Lithic & 4 & 5.64 & $\mathrm{JU}$ & 9/27/2017 & 2 bifacial thinning flakes \\
\hline $4-7$ & 2 & $10-20$ & Debitage & Tertiary & Lithic & 5 & 2.58 & $\mathrm{JU}$ & 9/27/2017 & 3 bifacial thinning flakes \\
\hline $4-7$ & 4 & $30-40$ & Debitage & Secondary & Lithic & 4 & 2.41 & $\mathrm{JU}$ & 9/27/2017 & 1 bifacial thinning flake \\
\hline 4-7 & 4 & $30-40$ & Debitage & Tertiary & Lithic & 9 & 4.71 & $\mathrm{JU}$ & 9/27/2017 & 6 bifacial thinning flakes \\
\hline $4-7$ & 4 & $30-40$ & Debitage & Shatter & Lithic & 2 & 1.73 & $\mathrm{JU}$ & 9/27/2017 & \\
\hline $4-7$ & 5 & $40-50$ & Debitage & Secondary & Lithic & 1 & 5.07 & $\mathrm{JU}$ & 9/27/2017 & \\
\hline $4-7$ & 5 & $40-50$ & Debitage & Tertiary & Lithic & 3 & 3.45 & $\mathrm{JU}$ & 9/27/2017 & 2 bifacial thinning flakes \\
\hline 4-7 & 6 & $50-60$ & Debitage & Tertiary & Lithic & 11 & 17.96 & $\mathrm{JU}$ & 9/27/2017 & 7 bifacial thinning flakes \\
\hline 4-7 & 6 & $50-60$ & Debitage & Shatter & Lithic & 2 & 1.29 & $\mathrm{JU}$ & 9/27/2017 & \\
\hline 4-7 & 7 & $60-70$ & Debitage & Tertiary & Lithic & 2 & 0.68 & $\mathrm{JU}$ & 9/27/2017 & 2 bifacial thinning flakes \\
\hline 4-7 & 8 & $70-80$ & Debitage & Primary & Lithic & 1 & 2.32 & $\mathrm{JU}$ & $9 / 27 / 2017$ & \\
\hline 4-7 & 8 & $70-80$ & Debitage & Secondary & Lithic & 2 & 2.93 & $\mathrm{JU}$ & 9/27/2017 & 1 bifacial thinning flake \\
\hline 4-7 & 8 & $70-80$ & Debitage & Tertiary & Lithic & 4 & 9.66 & $\mathrm{JU}$ & 9/27/2017 & 2 bifacial thinning flakes \\
\hline $4-7$ & 9 & $80-90$ & Debitage & Tertiary & Lithic & 4 & 6.13 & $\mathrm{JU}$ & 9/27/2017 & 2 bifacial thinning flakes \\
\hline 4-7 & 10 & $90-100$ & Debitage & Secondary & Lithic & 2 & 21.51 & $\mathrm{JU}$ & 9/27/2017 & \\
\hline $4-7$ & 10 & $90-100$ & Debitage & Tertiary & Lithic & 3 & 0.59 & $\mathrm{JU}$ & 9/27/2017 & 4 bifacial thinning flakes \\
\hline $4-8$ & 1 & $0-10$ & Debitage & Secondary & Lithic & 2 & 2.74 & $\mathrm{JU}$ & 9/27/2017 & \\
\hline $4-8$ & 1 & $0-10$ & Debitage & Tertiary & Lithic & 18 & 23.21 & $\mathrm{JU}$ & 9/27/2017 & 10 bifacial thinning flakes \\
\hline $4-8$ & 1 & $0-10$ & Debitage & Shatter & Lithic & 2 & 2.42 & $\mathrm{JU}$ & 9/27/2017 & \\
\hline $4-8$ & 1 & $0-10$ & $\begin{array}{l}\text { Burned } \\
\text { Rock }\end{array}$ & Burned Rock & Lithic & 2 & 8 & JU & 9/27/2017 & \\
\hline $4-8$ & 2 & $10-20$ & $\begin{array}{l}\text { Faunal } \\
\text { Remains }\end{array}$ & Bone & & 1 & 0.39 & JU & 9/27/2017 & \\
\hline $4-8$ & 2 & $10-20$ & Debitage & Secondary & Lithic & 1 & 2.82 & $\mathrm{JU}$ & 9/27/2017 & \\
\hline $4-8$ & 2 & $10-20$ & Debitage & Tertiary & Lithic & 9 & 13.88 & $\mathrm{JU}$ & 9/27/2017 & 4 bifacial thinning flakes \\
\hline $4-8$ & 3 & $20-30$ & Debitage & Primary & Lithic & 1 & 0.74 & $\mathrm{JU}$ & 9/28/2017 & \\
\hline $4-8$ & 3 & $20-30$ & Debitage & Secondary & Lithic & 4 & 10.01 & $\mathrm{JU}$ & $9 / 28 / 2017$ & 2 bifacial thinning flakes \\
\hline $4-8$ & 3 & $20-30$ & Debitage & Tertiary & Lithic & 8 & 13.15 & $\mathrm{JU}$ & 9/28/2017 & 5 bifacial thinning flakes \\
\hline $4-8$ & 3 & $20-30$ & Debitage & Shatter & Lithic & 5 & 5.06 & $\mathrm{JU}$ & 9/28/2017 & \\
\hline $4-8$ & 4 & $30-40$ & Debitage & Primary & Lithic & 1 & 1.39 & $\mathrm{JU}$ & 9/28/2017 & \\
\hline $4-8$ & 4 & $30-40$ & Debitage & Tertiary & Lithic & 10 & 6.47 & $\mathrm{JU}$ & 9/28/2017 & 7 bifacial thinning flakes \\
\hline $4-8$ & 4 & $30-40$ & Debitage & Shatter & Lithic & 5 & 3.62 & $\mathrm{JU}$ & 9/28/2017 & \\
\hline $4-8$ & 5 & $40-50$ & Debitage & Secondary & Lithic & 1 & 0.54 & $\mathrm{JU}$ & 9/28/2017 & \\
\hline $4-8$ & 5 & $40-50$ & Debitage & Tertiary & Lithic & 6 & 2.39 & $\mathrm{JU}$ & 9/28/2017 & 3 bifacial thinning flakes \\
\hline $4-8$ & 6 & $50-60$ & Debitage & Primary & Lithic & 2 & 37.56 & $\mathrm{JU}$ & 9/28/2017 & \\
\hline $4-8$ & 6 & $50-60$ & Debitage & Tertiary & Lithic & 1 & 2.3 & $\mathrm{JU}$ & 9/28/2017 & \\
\hline $4-8$ & 7 & $60-70$ & Debitage & Tertiary & Lithic & 4 & 3.05 & $\mathrm{JU}$ & 9/28/2017 & 3 bifacial thinning flakes \\
\hline $4-8$ & 8 & $70-80$ & Debitage & Primary & Lithic & 1 & 0.53 & $\mathrm{JU}$ & 9/28/2017 & \\
\hline $4-8$ & 8 & $70-80$ & Debitage & Secondary & Lithic & 1 & 0.13 & $\mathrm{JU}$ & 9/28/2017 & \\
\hline $4-8$ & 8 & $70-80$ & Debitage & Tertiary & Lithic & 8 & 3.57 & $\mathrm{JU}$ & 9/28/2017 & 5 bifacial thinning flakes \\
\hline 4-8 & 8 & $70-80$ & Debitage & Shatter & Lithic & 3 & 6.33 & $\mathrm{JU}$ & 9/28/2017 & \\
\hline $4-8$ & 9 & $80-90$ & Debitage & Secondary & Lithic & 1 & 0.19 & $\mathrm{JU}$ & 9/28/2017 & \\
\hline $4-8$ & 9 & $80-90$ & Debitage & Tertiary & Lithic & 7 & 4.14 & $\mathrm{JU}$ & 9/28/2017 & 3 bifacial thinning flakes \\
\hline
\end{tabular}




\begin{tabular}{|c|c|c|c|c|c|c|c|c|c|c|}
\hline ST & Level & $\begin{array}{l}\text { Depth } \\
\text { (cmbs) }\end{array}$ & $\begin{array}{c}\text { Artifact } \\
\text { Type }\end{array}$ & $\begin{array}{c}\text { Artifact } \\
\text { Description }\end{array}$ & Material & $\begin{array}{c}\text { Artifact } \\
\text { No. }\end{array}$ & $\begin{array}{c}\text { Weight } \\
\text { (g) }\end{array}$ & Recorders & Date & Comments \\
\hline $4-8$ & 10 & $90-100$ & Debitage & Primary & Lithic & 1 & 1.88 & $\mathrm{JU}$ & 9/28/2017 & \\
\hline $4-8$ & 10 & $90-100$ & Debitage & Secondary & Lithic & 4 & 5.76 & $\mathrm{JU}$ & 9/28/2017 & 2 bifacial thinning flakes \\
\hline $4-8$ & 10 & $90-100$ & Debitage & Tertiary & Lithic & 2 & 1.95 & $\mathrm{JU}$ & 9/28/2017 & 1 bifacial thinning flake \\
\hline $4-9$ & 1 & $0-10$ & Debitage & Secondary & Lithic & 1 & 0.46 & $\mathrm{JU}$ & 9/28/2017 & \\
\hline $4-9$ & 1 & $0-10$ & Debitage & Tertiary & Lithic & 3 & 4.56 & $\mathrm{JU}$ & 9/28/2017 & 1 bifacial thinning flake \\
\hline $4-9$ & 2 & $10-20$ & Debitage & Tertiary & Lithic & 2 & 0.75 & $\mathrm{JU}$ & 9/28/2017 & 2 bifacial thinning flakes \\
\hline $4-9$ & 3 & $20-30$ & Debitage & Tertiary & Lithic & 4 & 1.48 & $\mathrm{JU}$ & 9/28/2017 & 3 bifacial thinning flakes \\
\hline $4-9$ & 4 & $30-40$ & Debitage & Secondary & Lithic & 2 & 1.42 & $\mathrm{JU}$ & 9/28/2017 & 1 bifacial thinning flake \\
\hline $4-9$ & 4 & $30-40$ & Debitage & Tertiary & Lithic & 3 & 0.63 & $\mathrm{JU}$ & 9/28/2017 & 2 bifacial thinning flakes \\
\hline $4-9$ & 5 & $40-50$ & Debitage & Secondary & Lithic & 1 & 8.29 & $\mathrm{JU}$ & 9/28/2017 & 1 bifacial thinning flake \\
\hline $4-9$ & 5 & $40-50$ & Debitage & Tertiary & Lithic & 5 & 2.76 & $\mathrm{JU}$ & 9/28/2017 & 3 bifacial thinning flakes \\
\hline $4-9$ & 6 & $50-60$ & Debitage & Secondary & Lithic & 1 & 12.19 & $\mathrm{JU}$ & 9/28/2017 & 1 bifacial thinning flake \\
\hline $4-9$ & 6 & $50-60$ & Debitage & Tertiary & Lithic & 5 & 2.95 & $\mathrm{JU}$ & 9/28/2017 & 4 bifacial thinning flakes \\
\hline $4-9$ & 8 & $70-80$ & Debitage & Primary & Lithic & 2 & 4.55 & $\mathrm{JU}$ & 9/28/2017 & \\
\hline $4-9$ & 8 & $70-80$ & Debitage & Tertiary & Lithic & 4 & 3.11 & $\mathrm{JU}$ & 9/28/2017 & 3 bifacial thinning flakes \\
\hline $4-9$ & 8 & $70-80$ & Debitage & Shatter & Lithic & 1 & 0.46 & $\mathrm{JU}$ & 9/28/2017 & \\
\hline $4-9$ & 10 & $90-100$ & Debitage & Primary & Lithic & 1 & 1.56 & $\mathrm{JU}$ & 9/28/2017 & \\
\hline $4-9$ & 10 & $90-100$ & Debitage & Secondary & Lithic & 1 & 0.27 & $\mathrm{JU}$ & 9/28/2017 & 1 bifacial thinning flake \\
\hline $4-9$ & 10 & $90-100$ & Debitage & Tertiary & Lithic & 1 & 0.13 & $\mathrm{JU}$ & 9/28/2017 & \\
\hline $4-10$ & 6 & $50-60$ & $\begin{array}{l}\text { Projectile } \\
\text { Point }\end{array}$ & $\begin{array}{l}\text { Untyped Arrow } \\
\text { Point }\end{array}$ & Lithic & 1 & 1 & JU & 9/28/2017 & $\begin{array}{l}\text { Missing proximal end of base; } \\
\text { translucent; probable Sabinal }\end{array}$ \\
\hline $4-10$ & 6 & $50-60$ & Debitage & Primary & Lithic & 1 & 22.75 & $\mathrm{JU}$ & 9/28/2017 & \\
\hline $4-10$ & 6 & $50-60$ & Debitage & Secondary & Lithic & 1 & 0.43 & $\mathrm{JU}$ & 9/28/2017 & \\
\hline $4-10$ & 6 & $50-60$ & Debitage & Tertiary & Lithic & 2 & 0.18 & $\mathrm{JU}$ & 9/28/2017 & 1 bifacial thinning flake \\
\hline $4-10$ & 7 & $60-70$ & Debitage & Secondary & Lithic & 1 & 17.54 & $\mathrm{JU}$ & 9/28/2017 & \\
\hline $4-10$ & 8 & $70-80$ & Debitage & Tertiary & Lithic & 2 & 0.88 & $\mathrm{JU}$ & 9/28/2017 & 2 bifacial thinning flakes \\
\hline $4-10$ & 9 & $80-90$ & Debitage & Secondary & Lithic & 1 & 0.71 & $\mathrm{JU}$ & 9/28/2017 & \\
\hline $4-10$ & 10 & $90-100$ & Debitage & Shatter & Lithic & 1 & 0.09 & $\mathrm{JU}$ & 9/28/2017 & \\
\hline $5-1$ & 1 & $0-10$ & Debitage & Primary & Lithic & 1 & 3.68 & $\mathrm{JU}$ & 9/28/2017 & \\
\hline $5-1$ & 1 & $0-10$ & Debitage & Tertiary & Lithic & 1 & 0.39 & $\mathrm{JU}$ & 9/28/2017 & \\
\hline $5-1$ & 2 & $10-20$ & Debitage & Tertiary & Lithic & 1 & 0.62 & $\mathrm{JU}$ & 9/28/2017 & 1 bifacial thinning flake \\
\hline $5-1$ & 3 & $20-30$ & Debitage & Secondary & Lithic & 1 & 10.3 & $\mathrm{JU}$ & 9/28/2017 & \\
\hline $5-1$ & 3 & $20-30$ & Debitage & Tertiary & Lithic & 2 & 3.38 & $\mathrm{JU}$ & 9/28/2017 & 1 bifacial thinning flake \\
\hline $5-1$ & 4 & $30-40$ & Debitage & Tertiary & Lithic & 3 & 2.16 & $\mathrm{JU}$ & 9/28/2017 & 3 bifacial thinning flakes \\
\hline $5-1$ & 5 & $40-50$ & Debitage & Secondary & Lithic & 1 & 1.04 & $\mathrm{JU}$ & 9/28/2017 & \\
\hline $5-1$ & 5 & $40-50$ & Debitage & Tertiary & Lithic & 2 & 1.62 & $\mathrm{JU}$ & 9/28/2017 & 1 bifacial thinning flake \\
\hline $5-2$ & 2 & $10-20$ & Debitage & Tertiary & Lithic & 2 & 0.69 & $\mathrm{JU}$ & 9/28/2017 & 2 bifacial thinning flakes \\
\hline $5-2$ & 2 & $10-20$ & Debitage & Shatter & Lithic & 1 & 0.86 & $\mathrm{JU}$ & 9/28/2017 & \\
\hline $5-2$ & 3 & $20-30$ & Debitage & Shatter & Lithic & 2 & 0.88 & $\mathrm{JU}$ & 9/28/2017 & \\
\hline $5-2$ & 4 & $30-40$ & Debitage & Tertiary & Lithic & 2 & 2.28 & $\mathrm{JU}$ & 9/28/2017 & 2 bifacial thinning flakes \\
\hline $5-2$ & 5 & $40-50$ & Debitage & Secondary & Lithic & 1 & 2 & $\mathrm{JU}$ & 9/28/2017 & \\
\hline $5-3$ & 1 & $0-10$ & Debitage & Secondary & Lithic & 2 & 1.4 & $\mathrm{JU}$ & 9/28/2017 & \\
\hline $5-3$ & 1 & $0-10$ & Debitage & Tertiary & Lithic & 14 & 5.68 & $\mathrm{JU}$ & 9/28/2017 & 11 bifacial thinning flakes \\
\hline $5-3$ & 1 & $0-10$ & Debitage & Shatter & Lithic & 2 & 0.67 & $\mathrm{JU}$ & 9/28/2017 & \\
\hline $5-3$ & 2 & $10-20$ & $\begin{array}{l}\text { Projectile } \\
\text { Point }\end{array}$ & $\begin{array}{l}\text { Untyped Arrow } \\
\text { Point }\end{array}$ & Lithic & 1 & 0.3 & JU & 9/28/2017 & Medial section \\
\hline $5-3$ & 2 & $10-20$ & Debitage & Secondary & Lithic & 1 & 2.62 & $\mathrm{JU}$ & 9/28/2017 & 1 bifacial thinning flake \\
\hline $5-3$ & 2 & $10-20$ & Debitage & Tertiary & Lithic & 12 & 20.39 & $\mathrm{JU}$ & $9 / 28 / 2017$ & 5 bifacial thinning flakes \\
\hline $5-3$ & 2 & $10-20$ & Debitage & Shatter & Lithic & 2 & 0.72 & $\mathrm{JU}$ & 9/28/2017 & \\
\hline $5-3$ & 4 & $30-40$ & Debitage & Secondary & Lithic & 2 & 6.81 & $\mathrm{JU}$ & 9/28/2017 & \\
\hline $5-3$ & 4 & $30-40$ & Debitage & Tertiary & Lithic & 2 & 1.21 & $\mathrm{JU}$ & 9/28/2017 & 2 bifacial thinning flakes \\
\hline $5-3$ & 6 & $50-60$ & Debitage & Secondary & Lithic & 1 & 0.76 & $\mathrm{JU}$ & 9/28/2017 & \\
\hline $5-3$ & 6 & $50-60$ & Debitage & Tertiary & Lithic & 2 & 2.75 & $\mathrm{JU}$ & 9/28/2017 & 2 bifacial thinning flakes \\
\hline $5-3$ & 7 & $60-70$ & Debitage & Tertiary & Lithic & 2 & 1.17 & $\mathrm{JU}$ & 9/28/2017 & 2 bifacial thinning flakes \\
\hline $5-4$ & 1 & $0-10$ & Debitage & Secondary & Lithic & 4 & 2.23 & $\mathrm{JU}$ & 9/28/2017 & 1 bifacial thinning flake \\
\hline $5-4$ & 1 & $0-10$ & Debitage & Tertiary & Lithic & 15 & 11.7 & $\mathrm{JU}$ & 9/28/2017 & 10 bifacial thinning flakes \\
\hline $5-4$ & 1 & $0-10$ & Debitage & Shatter & Lithic & 4 & 9.92 & $\mathrm{JU}$ & $9 / 28 / 2017$ & \\
\hline $5-4$ & 2 & $10-20$ & Debitage & Primary & Lithic & 1 & 4.97 & $\mathrm{JU}$ & 9/28/2017 & \\
\hline $5-4$ & 2 & $10-20$ & Debitage & Secondary & Lithic & 5 & 11.48 & $\mathrm{JU}$ & $9 / 28 / 2017$ & \\
\hline
\end{tabular}




\begin{tabular}{|c|c|c|c|c|c|c|c|c|c|c|}
\hline ST & Level & $\begin{array}{l}\text { Depth } \\
\text { (cmbs) }\end{array}$ & $\begin{array}{c}\text { Artifact } \\
\text { Type }\end{array}$ & $\begin{array}{c}\text { Artifact } \\
\text { Description }\end{array}$ & Material & $\begin{array}{c}\text { Artifact } \\
\text { No. }\end{array}$ & $\begin{array}{c}\text { Weight } \\
\text { (g) }\end{array}$ & Recorders & Date & Comments \\
\hline $5-4$ & 2 & $10-20$ & Debitage & Tertiary & Lithic & 13 & 14.74 & $\mathrm{JU}$ & 9/28/2017 & 8 bifacial thinning flakes \\
\hline $5-4$ & 2 & $10-20$ & Debitage & Shatter & Lithic & 4 & 1.64 & $\mathrm{JU}$ & 9/28/2017 & \\
\hline $5-4$ & 3 & $20-30$ & Debitage & Primary & Lithic & 2 & 1.03 & $\mathrm{JU}$ & $9 / 28 / 2017$ & \\
\hline $5-4$ & 3 & $20-30$ & Debitage & Secondary & Lithic & 3 & 5.18 & $\mathrm{JU}$ & $9 / 28 / 2017$ & \\
\hline $5-4$ & 3 & $20-30$ & Debitage & Tertiary & Lithic & 6 & 12.22 & $\mathrm{JU}$ & $9 / 28 / 2017$ & 5 bifacial thinning flakes \\
\hline $5-4$ & 3 & $20-30$ & Debitage & Shatter & Lithic & 2 & 3.34 & $\mathrm{JU}$ & 9/28/2017 & \\
\hline $5-4$ & 4 & $30-40$ & Debitage & Tertiary & Lithic & 1 & 0.24 & $\mathrm{JU}$ & 9/28/2017 & 1 bifacial thinning flake \\
\hline $5-4$ & 8 & $70-80$ & Debitage & Tertiary & Lithic & 3 & 8.26 & $\mathrm{JU}$ & 9/28/2017 & 2 bifacial thinning flakes \\
\hline $5-4$ & 9 & $80-90$ & Debitage & Tertiary & Lithic & 4 & 0.62 & $\mathrm{JU}$ & $9 / 28 / 2017$ & 2 bifacial thinning flakes \\
\hline $5-4$ & 10 & $90-100$ & Debitage & Primary & Lithic & 1 & 4.52 & $\mathrm{JU}$ & 9/28/2017 & \\
\hline $5-4$ & 10 & $90-100$ & Debitage & Tertiary & Lithic & 1 & 1.79 & $\mathrm{JU}$ & 9/28/2017 & 1 bifacial thinning flake \\
\hline $5-5$ & 1 & $0-10$ & Debitage & Secondary & Lithic & 1 & 5.25 & $\mathrm{JU}$ & 9/28/2017 & \\
\hline $5-5$ & 1 & $0-10$ & Debitage & Tertiary & Lithic & 10 & 14.78 & $\mathrm{JU}$ & $9 / 28 / 2017$ & 7 bifacial thinning flakes \\
\hline $5-5$ & 2 & $10-20$ & Debitage & Primary & Lithic & 1 & 1.86 & $\mathrm{JU}$ & 9/28/2017 & \\
\hline $5-5$ & 2 & $10-20$ & Debitage & Secondary & Lithic & 1 & 1.36 & $\mathrm{JU}$ & $9 / 28 / 2017$ & \\
\hline $5-5$ & 2 & $10-20$ & Debitage & Tertiary & Lithic & 3 & 1.78 & $\mathrm{JU}$ & $9 / 28 / 2017$ & 2 bifacial thinning flakes \\
\hline $5-5$ & 4 & $30-40$ & Debitage & Primary & Lithic & 1 & 4.41 & $\mathrm{JU}$ & $9 / 28 / 2017$ & \\
\hline $5-5$ & 4 & $30-40$ & Debitage & Tertiary & Lithic & 10 & 22.1 & $\mathrm{JU}$ & $9 / 28 / 2017$ & 7 bifacial thinning flakes \\
\hline $5-5$ & 7 & $60-70$ & Debitage & Primary & Lithic & 1 & 6.27 & $\mathrm{JU}$ & $9 / 28 / 2017$ & \\
\hline $5-5$ & 7 & $60-70$ & Debitage & Secondary & Lithic & 3 & 10.9 & $\mathrm{JU}$ & $9 / 28 / 2017$ & 1 bifacial thinning flake \\
\hline $5-5$ & 7 & $60-70$ & Debitage & Tertiary & Lithic & 9 & 4.12 & $\mathrm{JU}$ & $9 / 28 / 2017$ & 8 bifacial thinning flakes \\
\hline $5-5$ & 8 & $70-80$ & Debitage & Secondary & Lithic & 1 & 0.36 & $\mathrm{JU}$ & 9/28/2017 & \\
\hline $5-5$ & 9 & $80-90$ & Debitage & Secondary & Lithic & 1 & 1.67 & $\mathrm{JU}$ & $9 / 28 / 2017$ & \\
\hline $5-5$ & 9 & $80-90$ & Debitage & Tertiary & Lithic & 1 & 0.43 & $\mathrm{JU}$ & $9 / 28 / 2017$ & 1 bifacial thinning flake \\
\hline $5-6$ & 1 & $0-10$ & Debitage & Secondary & Lithic & 1 & 45.91 & $\mathrm{JU}$ & 9/28/2017 & \\
\hline $5-6$ & 2 & $10-20$ & Debitage & Tertiary & Lithic & 4 & 3.27 & $\mathrm{JU}$ & 9/28/2017 & 4 bifacial thinning flakes \\
\hline $5-6$ & 2 & $10-20$ & Debitage & Shatter & Lithic & 1 & 0.93 & $\mathrm{JU}$ & $9 / 28 / 2017$ & \\
\hline $5-6$ & 3 & $20-30$ & $\begin{array}{l}\text { Modified } \\
\text { Flake }\end{array}$ & Modified Flake & Lithic & 1 & 11.91 & JU & 9/28/2017 & \\
\hline $5-6$ & 4 & $30-40$ & Debitage & Tertiary & Lithic & 7 & 2.76 & $\mathrm{JU}$ & $9 / 28 / 2017$ & 5 bifacial thinning flakes \\
\hline $5-6$ & 4 & $30-40$ & Debitage & Shatter & Lithic & 2 & 0.98 & $\mathrm{JU}$ & 9/28/2017 & \\
\hline $5-6$ & 4 & $30-40$ & $\begin{array}{l}\text { Burned } \\
\text { Rock }\end{array}$ & Burned Rock & Lithic & 1 & 5.13 & $\mathrm{JU}$ & 9/28/2017 & \\
\hline $5-6$ & 5 & $40-50$ & Debitage & Tertiary & Lithic & 4 & 5.64 & $\mathrm{JU}$ & $9 / 28 / 2017$ & 2 bifacial thinning flakes \\
\hline $5-6$ & 6 & $50-60$ & Debitage & Secondary & Lithic & 1 & 16.99 & $\mathrm{JU}$ & $9 / 28 / 2017$ & 1 bifacial thinning flake \\
\hline $5-6$ & 6 & $50-60$ & Debitage & Tertiary & Lithic & 15 & 19.16 & $\mathrm{JU}$ & 9/28/2017 & 12 bifacial thinning flakes \\
\hline $5-6$ & 6 & $50-60$ & $\begin{array}{l}\text { Ground } \\
\text { Stone }\end{array}$ & Ground Stone & Lithic & 1 & 285.57 & JU & 9/28/2017 & \\
\hline $5-6$ & 7 & $60-70$ & $\begin{array}{l}\text { Projectile } \\
\text { Point }\end{array}$ & $\begin{array}{l}\text { Untyped Point } \\
\text { Tip }\end{array}$ & Lithic & 1 & 1.66 & JU & $9 / 28 / 2017$ & Distal end \\
\hline $5-6$ & 7 & $60-70$ & Debitage & Tertiary & Lithic & 6 & 2.97 & $\mathrm{JU}$ & 9/28/2017 & 4 bifacial thinning flakes \\
\hline $5-6$ & 7 & $60-70$ & Debitage & Shatter & Lithic & 1 & 0.63 & $\mathrm{JU}$ & 9/28/2017 & \\
\hline $5-6$ & 8 & $70-80$ & Debitage & Tertiary & Lithic & 4 & 1.49 & $\mathrm{JU}$ & $9 / 28 / 2017$ & 3 bifacial thinning flakes \\
\hline $5-6$ & 9 & $80-90$ & Debitage & Secondary & Lithic & 1 & 5.94 & $\mathrm{JU}$ & 9/28/2017 & \\
\hline $5-6$ & 9 & $80-90$ & Debitage & Tertiary & Lithic & 10 & 15.02 & $\mathrm{JU}$ & $9 / 28 / 2017$ & 7 bifacial thinning flakes \\
\hline $5-6$ & 9 & $80-90$ & $\begin{array}{l}\text { Burned } \\
\text { Rock }\end{array}$ & Burned Rock & Lithic & 1 & 3.33 & JU & 9/28/2017 & \\
\hline $5-6$ & 10 & $90-100$ & Debitage & Tertiary & Lithic & 3 & 0.69 & $\mathrm{JU}$ & 9/28/2017 & 2 bifacial thinning flakes \\
\hline $5-7$ & 1 & $0-10$ & Debitage & Tertiary & Lithic & 3 & 3.54 & $\mathrm{JU}$ & 9/29/2017 & 3 bifacial thinning flakes \\
\hline $5-7$ & 2 & $10-20$ & Debitage & Tertiary & Lithic & 2 & 11.14 & $\mathrm{JU}$ & 9/29/2017 & 2 bifacial thinning flakes \\
\hline $5-7$ & 5 & $40-50$ & Debitage & Tertiary & Lithic & 3 & 1.8 & $\mathrm{JU}$ & 9/29/2017 & 2 bifacial thinning flakes \\
\hline $5-7$ & 5 & $40-50$ & Debitage & Shatter & Lithic & 1 & 0.23 & $\mathrm{JU}$ & 9/29/2017 & \\
\hline $5-7$ & 8 & $70-80$ & Debitage & Tertiary & Lithic & 3 & 1.18 & $\mathrm{JU}$ & 9/29/2017 & 2 bifacial thinning flakes \\
\hline $5-7$ & 14 & $130-140$ & Debitage & Tertiary & Lithic & 1 & 0.13 & $\mathrm{JU}$ & 9/29/2017 & \\
\hline KS139 & 1 & $0-10$ & Debitage & Tertiary & Lithic & 13 & - & DR & $10 / 19 / 2017$ & Not collected \\
\hline KS139 & 2 & $10-20$ & Debitage & Primary & Lithic & 6 & - & DR & $10 / 19 / 2017$ & Not collected \\
\hline KS139 & 3 & $20-30$ & Debitage & Tertiary & Lithic & 9 & - & DR & $10 / 19 / 2017$ & Not collected \\
\hline KS139 & 4 & $30-40$ & Debitage & Tertiary & Lithic & 15 & - & DR & 10/19/2017 & Not collected \\
\hline KS139 & 5 & $40-50$ & Debitage & Tertiary & Lithic & 10 & - & DR & $10 / 19 / 2017$ & Not collected \\
\hline KS139 & 6 & $50-60$ & Debitage & Tertiary & Lithic & 6 & - & $\mathrm{DR}$ & 10/19/2017 & Not collected \\
\hline
\end{tabular}


Interim Report: Archaeological Investigations at 41GU177 on the Guadalupe River

\begin{tabular}{|c|c|c|c|l|c|c|c|c|c|c|}
\hline ST & Level & $\begin{array}{c}\text { Depth } \\
\mathbf{( c m b s )}\end{array}$ & $\begin{array}{c}\text { Artifact } \\
\text { Type }\end{array}$ & $\begin{array}{c}\text { Artifact } \\
\text { Description }\end{array}$ & Material & $\begin{array}{c}\text { Artifact } \\
\text { No. }\end{array}$ & $\begin{array}{c}\text { Weight } \\
\mathbf{( g )}\end{array}$ & Recorders & Date & Comments \\
\hline KS139 & 7 & $70-80$ & Debitage & Tertiary & Lithic & 5 & - & DR & $10 / 19 / 2017$ & Not collected \\
\hline RW342 & $4-8$ & $30-80$ & Debitage & Tertiary & Lithic & 6 & - & DR & $10 / 19 / 2017$ & Not collected \\
\hline RW342 & $4-8$ & $30-80$ & Debitage & Shatter & Lithic & 4 & - & DR & $10 / 19 / 2017$ & Not collected \\
\hline RW343 & $3-4$ & $20-40$ & Debitage & Tertiary & Lithic & 1 & - & DR & $10 / 19 / 2017$ & Not collected \\
\hline
\end{tabular}




\section{APPENDIX B}





\begin{tabular}{|c|c|c|c|c|c|c|c|c|c|c|}
\hline BHT & $\begin{array}{l}\text { Depth } \\
\text { (cmbs) }\end{array}$ & Munsell* & Soil Color* & $\begin{array}{l}\text { Soil } \\
\text { Texture }\end{array}$ & Consistency & $\begin{array}{l}\text { Structure } \\
\text { Type }\end{array}$ & Grade & Inclusions & $\begin{array}{l}\text { Lower } \\
\text { Boundary }\end{array}$ & Comments \\
\hline \multirow{4}{*}{ BHT01 } & $0-58$ & 10YR3/2 & $\begin{array}{l}\text { very dark } \\
\text { grayish } \\
\text { brown }\end{array}$ & silt loam & Friable & $\begin{array}{l}\text { Subangular } \\
\text { Blocky to } \\
\text { Angular Blocky }\end{array}$ & $\begin{array}{l}\text { Weak to } \\
\text { moderate }\end{array}$ & $\begin{array}{l}\text { roots-rootlets } 15-20 \% \text {, few snail shell } \\
\text { (Helio), } 30 \% \text { pin holes, } 5 \% \text { worm } \\
\text { burrows, } 5 \% \text { snail fragments }\end{array}$ & $\begin{array}{l}\text { Gradual and } \\
\text { Smooth }\end{array}$ & Root zone $0-9 \mathrm{cmbs}$ \\
\hline & $58-99$ & 10YR4/3 & brown & Loam & Friable & $\begin{array}{l}\text { Subangular } \\
\text { Blocky }\end{array}$ & $\begin{array}{l}\text { Weak to } \\
\text { moderate }\end{array}$ & $\begin{array}{l}5-10 \% \text { rootlets, } 30 \% \text { pin holes, } 5 \% \\
\text { worm burrows, } 1 \% \text { snail fragments }\end{array}$ & $\begin{array}{l}\text { Gradual and } \\
\text { Smooth }\end{array}$ & Transitional \\
\hline & $99-183$ & 10YR5/4 & $\begin{array}{l}\text { yellowish } \\
\text { brown }\end{array}$ & Silt Loam & Friable & $\begin{array}{l}\text { Subangular } \\
\text { Blocky }\end{array}$ & Moderate & $\begin{array}{l}\text { 3-5\% rootlets, } 30 \% \text { pin holes, } 5 \% \text { worm } \\
\text { burrows, } 10 \% \text { snail - Rabdotus, } 1 \% \\
\text { snail fragments }\end{array}$ & $\begin{array}{l}\text { Clear and } \\
\text { smooth }\end{array}$ & \\
\hline & $183-326$ & $\begin{array}{l}10 Y R 5 / 6 \text { to } \\
10 Y R 6 / 6\end{array}$ & $\begin{array}{l}\text { yellowish } \\
\text { brown to } \\
\text { brownish } \\
\text { yellow }\end{array}$ & $\begin{array}{l}\text { Clay } \\
\text { loam }\end{array}$ & Friable & Angular Blocky & Moderate & $\begin{array}{l}1 \% \text { rootlets, } 5-10 \% \text { pin holes, } 1 \% \text { white } \\
\text { filament }(1 \mathrm{~mm}), 20-30 \% \text { snail - helio } \\
\text { and rabdotus, } 10 \% \text { snail fragments }\end{array}$ & Unobserved & $\begin{array}{l}\mathrm{CaCO}_{3} \text { filaments increase with depth } 3-4 \mathrm{~mm} \\
(5 \%) \sim 280 \mathrm{cmbs} \text {, clay content increases with } \\
\text { depth }\end{array}$ \\
\hline \multirow{4}{*}{ BHT02 } & $0-41$ & 10YR3/2 & $\begin{array}{l}\text { very dark } \\
\text { grayish } \\
\text { brown }\end{array}$ & silt loam & Friable & $\begin{array}{l}\text { Subangular } \\
\text { Blocky to } \\
\text { Angular Blocky }\end{array}$ & $\begin{array}{l}\text { Weak to } \\
\text { moderate }\end{array}$ & $\begin{array}{l}\text { roots-rootlets } 15-20 \% \text {, few snail shell } \\
\text { (Helio), } 30 \% \text { pin holes, } 5 \% \text { worm } \\
\text { burrows, } 5 \% \text { snail fragments }\end{array}$ & $\begin{array}{l}\text { Gradual and } \\
\text { Smooth }\end{array}$ & Root zone $0-9 \mathrm{cmbs}$ \\
\hline & $41-93$ & 10YR4/3 & brown & Loam & Friable & $\begin{array}{l}\text { Subangular } \\
\text { Blocky }\end{array}$ & $\begin{array}{l}\text { Weak to } \\
\text { moderate }\end{array}$ & $\begin{array}{l}5-10 \% \text { rootlets, } 30 \% \text { pin holes, } 5 \% \\
\text { worm burrows, } 1 \% \text { snail fragments }\end{array}$ & $\begin{array}{l}\text { Gradual to } \\
\text { clear and } \\
\text { slightly wavy }\end{array}$ & Transitional \\
\hline & 93-196 & 10YR5/4 & $\begin{array}{l}\text { yellowish } \\
\text { brown }\end{array}$ & Silt Loam & Friable & $\begin{array}{l}\text { Subangular } \\
\text { Blocky }\end{array}$ & Moderate & $\begin{array}{l}3-5 \% \text { rootlets, } 30 \% \text { pin holes, } 5 \% \text { worm } \\
\text { burrows, } 10 \% \text { snail - Rabdotus, } 1 \% \\
\text { snail fragments, pockets of snail - } \\
\text { rabdotus, Insect burrows - } 6 \mathrm{~mm}-1 \% \\
\end{array}$ & $\begin{array}{l}\text { Clear and } \\
\text { smooth }\end{array}$ & \\
\hline & 196-306 & $\begin{array}{l}10 Y R 5 / 6 \text { to } \\
10 Y R 6 / 6\end{array}$ & $\begin{array}{l}\text { yellowish } \\
\text { brown to } \\
\text { brownish } \\
\text { yellow }\end{array}$ & $\begin{array}{l}\text { Clay } \\
\text { loam }\end{array}$ & Friable & Angular Blocky & Moderate & $\begin{array}{l}1 \% \text { rootlets, } 3 \% \text { pin holes, } 1 \% \text { white } \\
\text { filament }(1 \mathrm{~cm}), 20-30 \% \text { snail - helio and } \\
\text { rabdotus, } 10 \% \text { snail fragments, large } \\
\text { insect burrows }\end{array}$ & Unobserved & $\begin{array}{l}\mathrm{CaCO}_{3} \text { filaments increase with depth } 3-4 \mathrm{~mm} \\
(5 \%) \sim 280 \mathrm{cmbs} \text {, clay content increases with } \\
\text { depth }\end{array}$ \\
\hline \multirow{3}{*}{ ВНT03 } & $0-26$ & 10YR 5/3 & brown & $\begin{array}{l}\text { Silty clay } \\
\text { loam }\end{array}$ & $\begin{array}{l}\text { Friable to } \\
\text { Firm }\end{array}$ & $\begin{array}{l}\text { Subangular } \\
\text { Blocky }\end{array}$ & Moderate & $\begin{array}{l}5 \% \text { roots, } 5 \% \text { rootlets, rare gravels, rare } \\
\text { insect casts, } 10 \% \text { white filament }\end{array}$ & $\begin{array}{l}\text { Clear and } \\
\text { irregular }\end{array}$ & No snail shell observed \\
\hline & $26-149$ & 10YR 6/4 & $\begin{array}{l}\text { Light } \\
\text { yellowish } \\
\text { brown }\end{array}$ & Silt loam & Friable & $\begin{array}{l}\text { Subangular } \\
\text { Crumb }\end{array}$ & $\begin{array}{l}\text { Weak to } \\
\text { moderate }\end{array}$ & $\begin{array}{l}1 \% \text { roots, } 1 \% \text { rootlets, } 25 \% \text { limestone } \\
\text { gravels, rare insect casts }\end{array}$ & $\begin{array}{l}\text { Gradual and } \\
\text { smooth }\end{array}$ & $\begin{array}{l}5 \% \mathrm{CaCO}_{3} \text { filaments decrease with depth } \\
\text { starting at approximately } 70 \mathrm{cmbs}\end{array}$ \\
\hline & $149-170$ & 10YR $7 / 4$ & $\begin{array}{l}\text { Very pale } \\
\text { brown }\end{array}$ & Silt loam & Friable & Platy & $\begin{array}{l}\text { Weak to } \\
\text { moderate }\end{array}$ & $\begin{array}{c}>1 \% \text { rootlets, } 20 \% \text { limestone gravels, } \\
1 \% \text { white filament }\end{array}$ & Unobserved & \\
\hline \multirow{5}{*}{ BHT04 } & $0-11$ & 10YR3/2 & $\begin{array}{l}\text { very dark } \\
\text { grayish } \\
\text { brown }\end{array}$ & silt loam & Friable & $\begin{array}{l}\text { Subangular } \\
\text { Blocky to } \\
\text { Angular Blocky }\end{array}$ & $\begin{array}{l}\text { Weak to } \\
\text { moderate }\end{array}$ & $\begin{array}{l}\text { roots-rootlets } 15-20 \% \text {, few snail shell } \\
\text { (Helio), } 30 \% \text { pin holes, } 5 \% \text { worm } \\
\text { burrows, } 5 \% \text { snail fragments }\end{array}$ & $\begin{array}{l}\text { Gradual and } \\
\text { Smooth }\end{array}$ & Root zone $0-9 \mathrm{cmbs}$ \\
\hline & $11-35$ & 10YR4/3 & brown & Loam & Friable & $\begin{array}{l}\text { Subangular } \\
\text { Blocky }\end{array}$ & $\begin{array}{l}\text { Weak to } \\
\text { moderate }\end{array}$ & $\begin{array}{l}5-10 \% \text { rootlets, } 30 \% \text { pin holes, } 5 \% \\
\text { worm burrows, } 1 \% \text { snail fragments }\end{array}$ & $\begin{array}{l}\text { Gradual to } \\
\text { clear and } \\
\text { slightly wavy }\end{array}$ & Transitional \\
\hline & $35-83$ & 10YR5/4 & $\begin{array}{l}\text { yellowish } \\
\text { brown }\end{array}$ & Silt Loam & Friable & $\begin{array}{l}\text { Subangular } \\
\text { Blocky }\end{array}$ & Moderate & $\begin{array}{l}3-5 \% \text { rootlets, } 30 \% \text { pin holes, } 5 \% \text { worm } \\
\text { burrows, } 10 \% \text { snail - Rabdotus, } 1 \% \\
\text { snail fragments, pockets of snail - } \\
\text { rabdotus, Insect burrows - } 6 \mathrm{~mm}-1 \% \\
\end{array}$ & $\begin{array}{l}\text { Clear and } \\
\text { smooth }\end{array}$ & \\
\hline & 83-130 & $\begin{array}{l}10 Y R 5 / 6 \text { to } \\
10 Y R 6 / 6\end{array}$ & $\begin{array}{l}\text { yellowish } \\
\text { brown to } \\
\text { brownish } \\
\text { yellow }\end{array}$ & $\begin{array}{l}\text { Clay } \\
\text { loam }\end{array}$ & Friable & Angular Blocky & Moderate & $\begin{array}{l}1 \% \text { rootlets, } 3 \% \text { pin holes, } 1 \% \text { white } \\
\text { filament }(1 \mathrm{~cm}), 20-30 \% \text { snail - helio and } \\
\text { rabdotus, } 10 \% \text { snail fragments, large } \\
\text { insect burrows }\end{array}$ & Unobserved & $\begin{array}{l}\mathrm{CaCO}_{3} \text { filaments increase with depth } 3-4 \mathrm{~mm} \\
(5 \%) \sim 280 \mathrm{cmbs} \text {, clay content increases with } \\
\text { depth }\end{array}$ \\
\hline & $130-170$ & $\begin{array}{l}10 Y R 6 / 2- \\
6 / 3\end{array}$ & $\begin{array}{l}\text { Light } \\
\text { brownish } \\
\text { gray }\end{array}$ & Silt Loam & Friable & $\begin{array}{l}\text { Subangular, } \\
\text { medium }\end{array}$ & moderate & $\begin{array}{l}\text { rootlets } 5-10 \% \text {, rabdotus }<3 \% \text {, white } \\
\text { filaments } 10 \% \text { - dispersed, limestone } \\
\text { subangular fragments }<19 \%\end{array}$ & Unobserved & \\
\hline
\end{tabular}




\begin{tabular}{|c|c|c|c|c|c|c|c|c|c|c|}
\hline BHT & $\begin{array}{l}\text { Depth } \\
\text { (cmbs) }\end{array}$ & MunselI* & Soil Color* & $\begin{array}{l}\text { Soil } \\
\text { Texture }\end{array}$ & Consistency & $\begin{array}{l}\text { Structure } \\
\text { Type }\end{array}$ & Grade & Inclusions & $\begin{array}{l}\text { Lower } \\
\text { Boundary }\end{array}$ & Comments \\
\hline \multirow{5}{*}{ ВHT05 } & $0-12$ & 10YR3/2 & $\begin{array}{l}\text { very dark } \\
\text { grayish } \\
\text { brown }\end{array}$ & silt loam & Friable & $\begin{array}{l}\text { Subangular } \\
\text { Blocky to } \\
\text { Angular Blocky }\end{array}$ & $\begin{array}{l}\text { Weak to } \\
\text { moderate }\end{array}$ & $\begin{array}{l}\text { roots-rootlets } 15-20 \% \text {, few snail shell } \\
\text { (Helio), } 30 \% \text { pin holes, } 5 \% \text { worm } \\
\text { burrows, } 5 \% \text { snail fragments }\end{array}$ & $\begin{array}{l}\text { Gradual and } \\
\text { Smooth }\end{array}$ & Root zone $0-9 \mathrm{cmbs}$ \\
\hline & $12-40$ & 10YR4/3 & brown & Loam & Friable & $\begin{array}{l}\text { Subangular } \\
\text { Blocky }\end{array}$ & $\begin{array}{l}\text { Weak to } \\
\text { moderate }\end{array}$ & $\begin{array}{l}5-10 \% \text { rootlets, } 30 \% \text { pin holes, } 5 \% \\
\text { worm burrows, } 1 \% \text { snail fragments }\end{array}$ & $\begin{array}{l}\text { Gradual to } \\
\text { clear and } \\
\text { slightly wavy }\end{array}$ & Transitional \\
\hline & $40-76$ & 10YR5/4 & $\begin{array}{l}\text { yellowish } \\
\text { brown }\end{array}$ & Silt Loam & Friable & $\begin{array}{l}\text { Subangular } \\
\text { Blocky }\end{array}$ & Moderate & $\begin{array}{l}\text { 3-5\% rootlets, } 30 \% \text { pin holes, } 5 \% \text { worm } \\
\text { burrows, } 10 \% \text { snail - Rabdotus, } 1 \% \\
\text { snail fragments, pockets of snail - } \\
\text { rabdotus, Insect burrows - } 6 \mathrm{~mm} \mathrm{-1 \%}\end{array}$ & $\begin{array}{l}\text { Clear and } \\
\text { smooth }\end{array}$ & \\
\hline & $76-128$ & $\begin{array}{l}10 \mathrm{YR} 5 / 6 \text { to } \\
10 \mathrm{YR} 6 / 6\end{array}$ & $\begin{array}{l}\text { yellowish } \\
\text { brown to } \\
\text { brownish } \\
\text { yellow }\end{array}$ & $\begin{array}{l}\text { Clay } \\
\text { loam }\end{array}$ & Friable & Angular Blocky & Moderate & $\begin{array}{l}1 \% \text { rootlets, } 3 \% \text { pin holes, } 1 \% \text { white } \\
\text { filament }(1 \mathrm{~cm}), 20-30 \% \text { snail - helio and } \\
\text { rabdotus, } 10 \% \text { snail fragments, large } \\
\text { insect burrows }\end{array}$ & Unobserved & $\begin{array}{l}\mathrm{CaCO}_{3} \text { filaments increase with depth } 3-4 \mathrm{~mm} \\
(5 \%) \sim 280 \mathrm{cmbs} \text {, clay content increases with } \\
\text { depth }\end{array}$ \\
\hline & $128-138$ & $\begin{array}{l}\text { 10YR6/2- } \\
6 / 3\end{array}$ & $\begin{array}{l}\text { Light } \\
\text { brownish } \\
\text { gray }\end{array}$ & Silt Loam & Friable & $\begin{array}{l}\text { Subangular, } \\
\text { medium }\end{array}$ & moderate & $\begin{array}{c}\text { rootlets } 5-10 \% \text {, rabdotus }<3 \% \text {, white } \\
\text { filaments } 10 \% \text { - dispersed, limestone } \\
\text { subangular fragments }<19 \%\end{array}$ & Unobserved & \\
\hline \multirow{5}{*}{ ВНT06 } & $0-10$ & 10YR3/2 & $\begin{array}{l}\text { very dark } \\
\text { grayish } \\
\text { brown }\end{array}$ & silt loam & Friable & $\begin{array}{l}\text { Subangular } \\
\text { Blocky to } \\
\text { Angular Blocky }\end{array}$ & $\begin{array}{l}\text { Weak to } \\
\text { moderate }\end{array}$ & $\begin{array}{l}\text { roots-rootlets } 15-20 \% \text {, few snail shell } \\
\text { (Helio), } 30 \% \text { pin holes, } 5 \% \text { worm } \\
\text { burrows, } 5 \% \text { snail fragments }\end{array}$ & $\begin{array}{l}\text { Gradual and } \\
\text { Smooth }\end{array}$ & Root zone $0-9 \mathrm{cmbs}$ \\
\hline & $10-38$ & 10YR4/3 & brown & Loam & Friable & $\begin{array}{l}\text { Subangular } \\
\text { Blocky }\end{array}$ & $\begin{array}{l}\text { Weak to } \\
\text { moderate }\end{array}$ & $\begin{array}{l}5-10 \% \text { rootlets, } 30 \% \text { pin holes, } 5 \% \\
\text { worm burrows, } 1 \% \text { snail fragments }\end{array}$ & $\begin{array}{l}\text { Gradual to } \\
\text { clear and } \\
\text { slightly wavy }\end{array}$ & Transitional \\
\hline & $38-80$ & 10YR5/4 & $\begin{array}{l}\text { yellowish } \\
\text { brown }\end{array}$ & Silt Loam & Friable & $\begin{array}{l}\text { Subangular } \\
\text { Blocky }\end{array}$ & Moderate & $\begin{array}{l}-5 \% \text { rootlets, } 30 \% \text { pin holes, } 5 \% \text { worm } \\
\text { burrows, } 10 \% \text { snail - Rabdotus, } 1 \% \\
\text { snail fragments, pockets of snail - } \\
\text { rabdotus, Insect burrows- } 6 \mathrm{~mm} \mathrm{-1 \%} \\
\end{array}$ & $\begin{array}{l}\text { Clear and } \\
\text { smooth }\end{array}$ & \\
\hline & 08-132 & $\begin{array}{l}10 \mathrm{YR} 5 / 6 \text { to } \\
10 \mathrm{YR} 6 / 6\end{array}$ & $\begin{array}{l}\text { yellowish } \\
\text { brown to } \\
\text { brownish } \\
\text { yellow }\end{array}$ & $\begin{array}{l}\text { Clay } \\
\text { loam }\end{array}$ & Friable & Angular Blocky & Moderate & $\begin{array}{l}1 \% \text { rootlets, } 3 \% \text { pin holes, } 1 \% \text { white } \\
\text { filament }(1 \mathrm{~cm}), 20-30 \% \text { snail - helio and } \\
\text { rabdotus, } 10 \% \text { snail fragments, large } \\
\text { insect burrows }\end{array}$ & Unobserved & $\begin{array}{l}\mathrm{CaCO}_{3} \text { filaments increase with depth } 3-4 \mathrm{~mm} \\
(5 \%)-280 \mathrm{cmbs} \text {, clay content increases with } \\
\text { depth }\end{array}$ \\
\hline & $132-140$ & $\begin{array}{l}\text { 10YR6/2- } \\
6 / 3\end{array}$ & $\begin{array}{l}\text { Light } \\
\text { brownish } \\
\text { gray }\end{array}$ & Silt Loam & Friable & $\begin{array}{l}\text { Subangular, } \\
\text { medium }\end{array}$ & moderate & $\begin{array}{c}\text { rootlets } 5-10 \% \text {, rabdotus }<3 \% \text {, white } \\
\text { filaments } 10 \% \text { - dispersed, limestone } \\
\text { subangular fragments }<19 \%\end{array}$ & Unobserved & \\
\hline
\end{tabular}




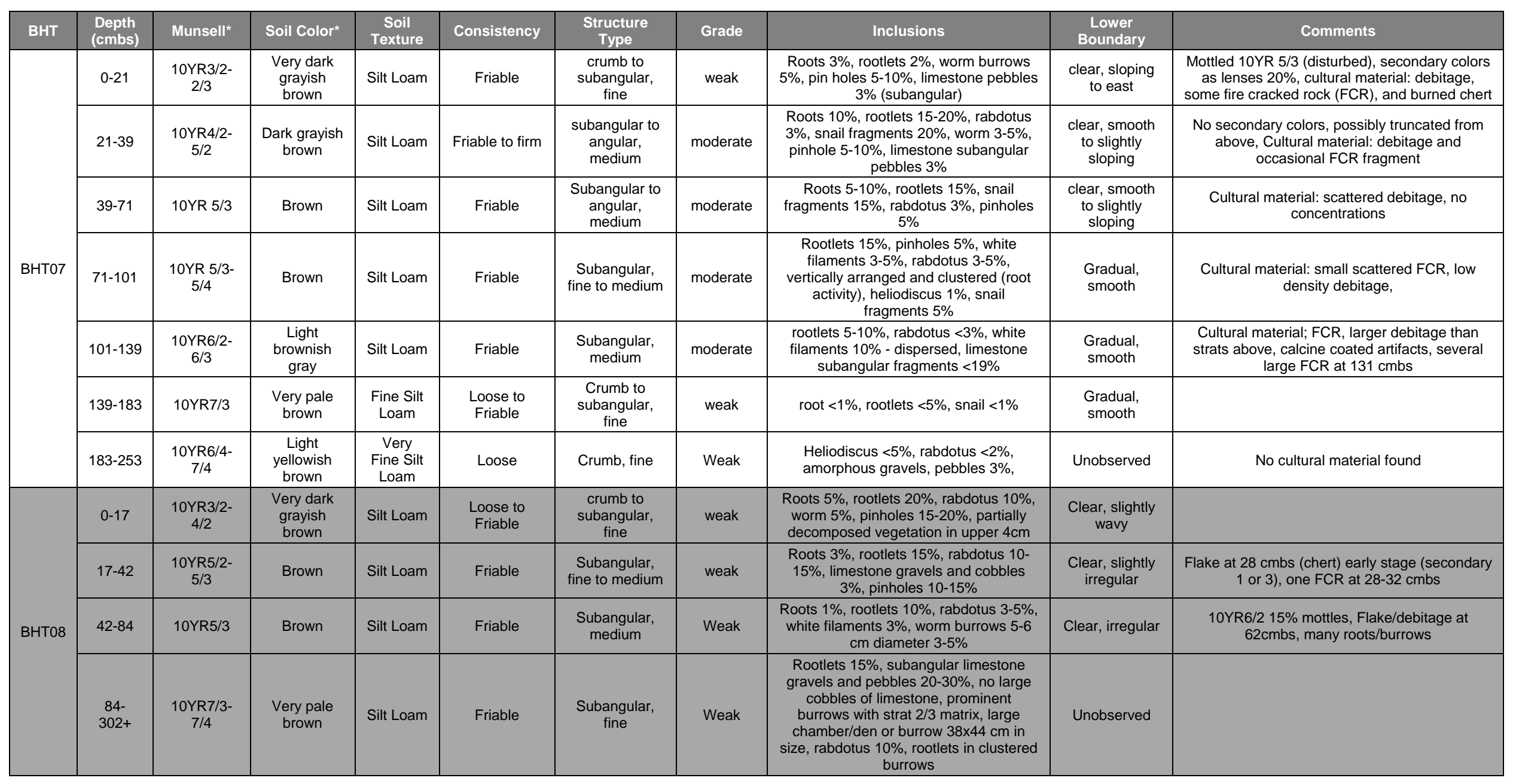




\begin{tabular}{|c|c|c|c|c|c|c|c|c|c|c|}
\hline BHT & $\begin{array}{l}\text { Depth } \\
\text { (cmbs) }\end{array}$ & Munsell* & Soil Color* & $\begin{array}{c}\text { Soil } \\
\text { Texture }\end{array}$ & Consistency & $\begin{array}{c}\text { Structure } \\
\text { Type }\end{array}$ & Grade & Inclusions & $\begin{array}{c}\text { Lower } \\
\text { Boundary }\end{array}$ & Comments \\
\hline \multirow{6}{*}{ ВНT09 } & $0-14$ & 10YR4/2 & $\begin{array}{l}\text { Dark grayish } \\
\text { brown }\end{array}$ & Silt Loam & $\begin{array}{l}\text { Loose to } \\
\text { Friable }\end{array}$ & Crumb, fine & Weak & $\begin{array}{l}\text { Roots } 5 \% \text {, rootlets } 20 \% \text {, rabdotus } 10 \% \text {, } \\
\text { worm burrows } 5 \% \text {, pinholes } 5-10 \%\end{array}$ & $\begin{array}{c}\text { Clear, slightly } \\
\text { wavy }\end{array}$ & $\begin{array}{l}\text { O horizon (decomposing vegetation), Base at } \\
\text { Strat } 1 \text { = Fist size FCR (limestone) }\end{array}$ \\
\hline & $14-37$ & 10YR4/3 & Brown & Silt Loam & Friable & $\begin{array}{c}\text { crumb to } \\
\text { subangular, } \\
\text { fine to medium }\end{array}$ & weak & $\begin{array}{l}\text { Roots } 5 \% \text {, rootlets } 20 \% \text {, rabdotus } 5 \% \text {, } \\
\text { subangular limestone pebbles } 5-10 \% \text {, } \\
\text { worm } 5 \% \text {, pinholes } 10 \%\end{array}$ & $\begin{array}{l}\text { Gradual - } \\
\text { slopes to } \mathrm{N}\end{array}$ & Artifact \\
\hline & $37-56$ & $\begin{array}{l}\text { 10YR5/3- } \\
5 / 4\end{array}$ & Brown & Silt Loam & Friable & $\begin{array}{l}\text { Subangular, } \\
\text { fine to medium }\end{array}$ & $\begin{array}{l}\text { Weak to } \\
\text { moderate }\end{array}$ & $\begin{array}{l}\text { Roots } 5-10 \% \text {, rootlets } 15-10 \% \text {, rabdotus } \\
10 \% \text {, worms } 3-5 \% \text {, pinholes } 3-5 \%, \\
\text { subangular limestone pebbles (5-10\%) }\end{array}$ & $\begin{array}{l}\text { Gradual, } \\
\text { slightly wavy }\end{array}$ & \\
\hline & $56-78$ & 10YR6/3 & Pale brown & Silt Loam & $\begin{array}{l}\text { Loose to } \\
\text { Friable }\end{array}$ & $\begin{array}{l}\text { Crumb to } \\
\text { subangular, } \\
\text { fine }\end{array}$ & Weak & $\begin{array}{c}\text { Roots } 3 \% \text {, rootlets } 10-15 \% \text {, rabdotus } \\
5 \% \text {, heliodiscus } 1-2 \% \text {, pinholes } 5-10 \% \text {, } \\
\text { worm burrows }<3 \% \text {, white filaments } \\
<5 \%\end{array}$ & $\begin{array}{l}\text { Clear, slightly } \\
\text { irregular }\end{array}$ & $\begin{array}{l}\text { Bottom of Strat 4: } 1 \text { debitage specimen in situ } \\
\text { in west wall, this may be a transition strat }\end{array}$ \\
\hline & $78-141$ & $\begin{array}{l}\text { 10YR6/4- } \\
7 / 4\end{array}$ & $\begin{array}{l}\text { Light } \\
\text { yellowish } \\
\text { brown }\end{array}$ & Silt Loam & Friable & $\begin{array}{l}\text { Subangular, } \\
\text { fine to medium }\end{array}$ & $\begin{array}{l}\text { Weak to } \\
\text { moderate }\end{array}$ & $\begin{array}{c}\text { Rootlets } 10 \% \text {, pinholes } 5 \% \text {, roots }<1 \% \text {, } \\
\text { no earth worms, subangular limestone } \\
\text { pebbles (10-15\%), gravels 5-10\%, snail } \\
\text { fragments } 5 \%\end{array}$ & $\begin{array}{l}\text { Gradual, } \\
\text { smooth }\end{array}$ & $\begin{array}{l}\text { No cultural material, small burrows } 2 \% \text { infilled } \\
\text { with strat } 3 \text { or } 4 \text { matrix }\end{array}$ \\
\hline & $\begin{array}{l}141- \\
176+\end{array}$ & 10YR8/4 & $\begin{array}{l}\text { Very pale } \\
\text { brown }\end{array}$ & Silt Loam & $\begin{array}{l}\text { Friable to } \\
\text { Slightly firm }\end{array}$ & $\begin{array}{l}\text { subangular to } \\
\text { angular, } \\
\text { medium }\end{array}$ & moderate & $\begin{array}{c}\text { Rootlets } 5 \% \text {, pinholes } 3 \% \text {, white } \\
\text { filaments } 5 \% \text {, worm burrows } 3 \% \text {, No } \\
\text { pebbles or rabdotus }\end{array}$ & Unobserved & No Cultural material \\
\hline \multirow{5}{*}{ BHT10 } & $0-22$ & 10YR3/2 & $\begin{array}{l}\text { Very dark } \\
\text { grayish } \\
\text { brown }\end{array}$ & Silt Loam & Loose & Crumb, fine & Weak & $\begin{array}{l}\text { Roots } 3 \% \text {, rootlets } 15-20 \% \text {, pinholes } \\
5 \% \text {, worm burrows } 5 \% \text {, rabdotus } 5-10 \%\end{array}$ & $\begin{array}{l}\text { Clear, slightly } \\
\text { wavy }\end{array}$ & Partially decomposed vegetation O horizon \\
\hline & $22-54$ & $\begin{array}{c}\text { 10YR4/2- } \\
4 / 3\end{array}$ & $\begin{array}{l}\text { Dark grayish } \\
\text { brown }\end{array}$ & Silt Loam & $\begin{array}{l}\text { Loose to } \\
\text { Friable }\end{array}$ & $\begin{array}{l}\text { Crumb to } \\
\text { subangular, } \\
\text { fine }\end{array}$ & Weak & $\begin{array}{l}\text { Roots } 10 \% \text {, rootlets } 20 \% \text {, pinholes } 5- \\
10 \% \text {, worm burrows } 5 \% \text {, rabdotus } 3-5 \%\end{array}$ & $\begin{array}{l}\text { Gradual, } \\
\text { smooth }\end{array}$ & Cultural Material: 1 FCR 39-42 \\
\hline & $54-122$ & $\begin{array}{l}\text { 10YR5/3- } \\
5 / 4\end{array}$ & Brown & Silt Loam & Friable & $\begin{array}{l}\text { Subangular, } \\
\text { fine to medium }\end{array}$ & Weak & $\begin{array}{c}\text { Root } 5 \% \text {, Rootlets } 20 \% \text {, worm burrows } \\
10 \% \text {, rabdotus } 5 \% \text {, pinholes } 20 \% \text {, white } \\
\text { filaments } 10-15 \%\end{array}$ & $\begin{array}{l}\text { Gradual, } \\
\text { smooth }\end{array}$ & FCR fragment $103 \mathrm{cmbs}$, one FCR $76 \mathrm{cmbs}$ \\
\hline & $122-159$ & $\begin{array}{l}\text { 10YR5/4- } \\
6 / 4\end{array}$ & $\begin{array}{l}\text { Yellowish } \\
\text { brown }\end{array}$ & Silt Loam & Friable & $\begin{array}{l}\text { subangular to } \\
\text { angular, } \\
\text { medium } \\
\end{array}$ & moderate & $\begin{array}{c}\text { Root }<1 \% \text {, rootlets }<1 \%, \text { Rabdotus } 5 \% \text {, } \\
\text { pinhole }<1 \% \text {, worm burrows } 5 \% \text {, white } \\
\text { filaments }<10 \%\end{array}$ & Clear, smooth & One FCR at $139 \mathrm{cmbs}$ \\
\hline & $159-185$ & 10YR7/3 & $\begin{array}{l}\text { Very pale } \\
\text { brown }\end{array}$ & Silt Loam & $\begin{array}{l}\text { Loose to } \\
\text { Friable }\end{array}$ & $\begin{array}{l}\text { subangular to } \\
\text { angular, } \\
\text { medium }\end{array}$ & moderate & $\begin{array}{c}\text { Limestone pebble subangular }<2 \% \text {, } \\
\text { snail fragments } 5 \% \text {, filaments }<5 \% \text {, } \\
\text { heliodiscus }\end{array}$ & Unobserved & Single FCR at $179 \mathrm{cmbs}$ \\
\hline
\end{tabular}




\begin{tabular}{|c|c|c|c|c|c|c|c|c|c|c|}
\hline BHT & $\begin{array}{l}\text { Depth } \\
\text { (cmbs) }\end{array}$ & Munsell* & Soil Color* & $\begin{array}{l}\text { Soil } \\
\text { Texture }\end{array}$ & Consistency & $\begin{array}{c}\text { Structure } \\
\text { Type }\end{array}$ & Grade & Inclusions & $\begin{array}{l}\text { Lower } \\
\text { Boundary }\end{array}$ & Comments \\
\hline \multirow{6}{*}{ BHT11 } & $0-9$ & $10 Y 3 / 2$ & $\begin{array}{l}\text { Very dark } \\
\text { grayish } \\
\text { brown }\end{array}$ & Loam & Loose & Crumb, fine & Weak & snail rabdotus $5 \%$ & Gradual, wavy & Decomposing vegetation O horizon \\
\hline & 9-35 & $\begin{array}{c}\text { 10YR4/2- } \\
4 / 3\end{array}$ & $\begin{array}{l}\text { Dark grayish } \\
\text { brown }\end{array}$ & Silt Loam & Friable & $\begin{array}{l}\text { Subangular, } \\
\text { fine }\end{array}$ & weak & $\begin{array}{c}\text { Root } 5 \% \text {, Rootlets } 20 \% \text {, rabdotus } 15 \% \text {, } \\
\text { heliodiscus 3\%, pinholes } 15 \% \text {, worm } \\
\text { burrows } 5-10 \% \text {, roots are clustered at } \\
\text { base of strat } \\
\end{array}$ & $\begin{array}{l}\text { Clear, slightly } \\
\text { wavy }\end{array}$ & \\
\hline & $35-51$ & $\begin{array}{l}\text { 10YR4/2- } \\
4 / 3\end{array}$ & $\begin{array}{l}\text { Dark grayish } \\
\text { brown }\end{array}$ & Silt Loam & Friable & $\begin{array}{c}\text { Subangular, } \\
\text { medium }\end{array}$ & $\begin{array}{l}\text { Weak to } \\
\text { moderate }\end{array}$ & $\begin{array}{c}\text { Roots 3-5\%, rootlets } 15 \% \text {, pinholes } \\
15 \% \text {, worm burrows } 5 \% \text {, white filaments } \\
5-10 \% \text { dendritic, rabdotus } 5 \% \text { fragments } \\
15-20 \% \text {, subangular pebbles }<1 \% \\
\text { transitional? }\end{array}$ & $\begin{array}{c}\text { Clear, slightly } \\
\text { wavy }\end{array}$ & \\
\hline & $51-81$ & 10YR5/3 & Brown & Silt Loam & Friable & $\begin{array}{l}\text { subangular to } \\
\text { angular, } \\
\text { medium }\end{array}$ & moderate & $\begin{array}{c}\text { Roots } 3 \% \text {, rootlets } 10 \% \text {, worm burrows } \\
3-5 \% \text {, pinholes } 10-15 \% \text {, white filaments } \\
5-10 \% \text { dendritic, rabdotus } 3-5 \% \text {, snail } \\
\text { fragments } 5 \% \text {, unidentified snail }<1 \% \\
\end{array}$ & $\begin{array}{l}\text { Gradual, } \\
\text { slightly wavy }\end{array}$ & \\
\hline & $81-243$ & 10YR 6/3 & Pale brown & Silt Loam & Friable & $\begin{array}{l}\text { Subangular, } \\
\text { medium }\end{array}$ & moderate & $\begin{array}{l}\text { Roots } 2 \% \text {, rootlets } 5 \% \text {, pinholes } 5-10 \% \text {, } \\
\text { worm burrows }<3 \% \text {, white filaments } \\
<5 \% \text {, rabdotus } 3 \% \text {, snail fragments } 10 \%\end{array}$ & $\begin{array}{l}\text { Gradual, } \\
\text { smooth }\end{array}$ & $\begin{array}{c}\text { Lenses of } 10 Y R \quad 4 / 2 \text { - silt loam, friable, } \\
\text { subangular, fine, weak, rootlets } 3 \% \text {, abrupt } \\
\text { sloping eastward, variable thickness, averaging } \\
3 \mathrm{cmbs} \text {, one burned rock at } 121 \mathrm{cmbs} \text {, ranged } \\
\text { from } 89 \mathrm{cmbs} \text { at } W \text { end to } 144 \text { on east end and } \\
\text { dives to floor stops } 1 / 3 \text { of way to east end of } \\
\text { trench } \\
\end{array}$ \\
\hline & $\begin{array}{l}243- \\
244+\end{array}$ & $\begin{array}{c}\text { 10YR6/4- } \\
7 / 4\end{array}$ & $\begin{array}{l}\text { Light } \\
\text { yellowish } \\
\text { brown }\end{array}$ & Silt Loam & Firm & $\begin{array}{l}\text { Subangular, } \\
\text { coarse }\end{array}$ & moderate & $\begin{array}{c}\text { pinholes } 10 \% \text {, worm burrows }<3 \% \text {, } \\
\text { white filaments (CaCO3) } 5-10 \% \\
\text { increases (1-3mm), rabdotus 3-5\%, } \\
\text { snail fragments } 5 \%\end{array}$ & Unobserved & No cultural material, slightly inundated \\
\hline \multirow{6}{*}{ BHT12 } & $0-11$ & 10YRY3/2 & $\begin{array}{l}\text { Very dark } \\
\text { grayish } \\
\text { brown }\end{array}$ & Loam & Loose & Crumb, fine & Weak & snail rabdotus $5 \%$ & Gradual, wavy & Decomposing vegetation O horizon \\
\hline & 11-39 & $\begin{array}{l}\text { 10YR } 4 / 2- \\
4 / 3\end{array}$ & $\begin{array}{l}\text { Dark grayish } \\
\text { brown }\end{array}$ & Silt Loam & Friable & $\begin{array}{l}\text { Subangular, } \\
\text { fine }\end{array}$ & weak & $\begin{array}{c}\text { Root } 5 \% \text {, Rootlets } 20 \% \text {, rabdotus } 15 \% \text {, } \\
\text { heliodiscus } 3 \% \text {, pinholes } 15 \% \text {, worm } \\
\text { burrows } 5-10 \% \text {, roots are clustered at } \\
\text { base of strat }\end{array}$ & $\begin{array}{l}\text { Clear, slightly } \\
\text { wavy }\end{array}$ & \\
\hline & $39-54$ & $\begin{array}{l}\text { 10YR } 4 / 2- \\
4 / 3\end{array}$ & $\begin{array}{l}\text { Dark grayish } \\
\text { brown }\end{array}$ & Silt Loam & Friable & $\begin{array}{l}\text { Subangular, } \\
\text { medium }\end{array}$ & $\begin{array}{l}\text { Weak to } \\
\text { moderate }\end{array}$ & $\begin{array}{c}\text { Roots 3-5\%, rootlets } 15 \% \text {, pinholes } \\
15 \% \text {, worm burrows } 5 \% \text {, white filaments } \\
5-10 \% \text { dendritic, rabdotus } 5 \% \text { fragments } \\
15-20 \% \text {, subangular pebbles }<1 \% \\
\text { transitional? }\end{array}$ & $\begin{array}{l}\text { Clear, slightly } \\
\text { wavy }\end{array}$ & \\
\hline & $54-73$ & 10YR5/3 & Brown & Silt Loam & Friable & $\begin{array}{l}\text { subangular to } \\
\text { angular, } \\
\text { medium }\end{array}$ & moderate & $\begin{array}{c}\text { Roots } 3 \% \text {, rootlets } 10 \% \text {, worm burrows } \\
3-5 \% \text {, pinholes } 10-15 \% \text {, white filaments } \\
5-10 \% \text { dendritic, rabdotus } 3-5 \% \text {, snail } \\
\text { fragments } 5 \% \text {, unidentified snail }<1 \% \\
\end{array}$ & $\begin{array}{l}\text { Gradual, } \\
\text { slightly wavy }\end{array}$ & insect burrows infilled with strat 5 matrix \\
\hline & $73-126$ & 10YR5/3 & Brown & Silt Loam & Friable & $\begin{array}{l}\text { subangular to } \\
\text { angular, } \\
\text { medium }\end{array}$ & moderate & $\begin{array}{l}\text { pinholes } 3 \% \text {, worm burrows }<3 \% \text {, white } \\
\text { filaments }<3 \% \text {, snail fragments } 5 \%\end{array}$ & Clear, smooth & Lenses prominent \\
\hline & $126-156$ & $\begin{array}{l}\text { 10YR6/3- } \\
6 / 4\end{array}$ & Pale brown & Silt Loam & $\begin{array}{l}\text { Loose to } \\
\text { Friable }\end{array}$ & $\begin{array}{l}\text { crumb to } \\
\text { subangular, } \\
\text { fine to medium }\end{array}$ & Weak & $\begin{array}{c}\text { rootlets } 5 \% \text {, snail rabdotus } 3 \% \text {, root } \\
\text { burrows } 1 \% \text {, white filaments } 1 \% \text { (very } \\
\text { diffuse) }\end{array}$ & Unobserved & $\begin{array}{l}\text { Gritty matrix, became denser and none } \\
\text { compact around } 200 \mathrm{cmbs} \text { very similar to basal } \\
\text { strat BHT11, Lens } 10 Y R 4 / 2-5 / 2 \text { sloping to NE, } \\
\text { discontinuous horizon in all directions }\end{array}$ \\
\hline
\end{tabular}


Interim Report: Archaeological Investigations at 41GU177 on the Guadalupe River

\begin{tabular}{|c|c|c|c|c|c|c|c|c|c|c|}
\hline BHT & $\begin{array}{l}\text { Depth } \\
\text { (cmbs) }\end{array}$ & Munsell* & Soil Color* & $\begin{array}{c}\text { Soil } \\
\text { Texture }\end{array}$ & Consistency & $\begin{array}{c}\text { Structure } \\
\text { Type }\end{array}$ & Grade & Inclusions & $\begin{array}{c}\text { Lower } \\
\text { Boundary }\end{array}$ & Comments \\
\hline \multirow{4}{*}{ BHT13 } & $0-9$ & $\begin{array}{c}\text { 10YR } 3 / 1- \\
3 / 2\end{array}$ & $\begin{array}{l}\text { very dark } \\
\text { grayish } \\
\text { brown }\end{array}$ & Loam & Loose & Crumb, fine & Weak & snail rabdotus $5 \%$ & Gradual, wavy & Decomposing vegetation O horizon \\
\hline & $9-32$ & 10YR $4 / 3$ & $\begin{array}{l}\text { dark grayish } \\
\text { brown }\end{array}$ & $\begin{array}{l}\text { Silty clay } \\
\text { loam }\end{array}$ & $\begin{array}{l}\text { Loose to } \\
\text { Friable }\end{array}$ & $\begin{array}{l}\text { Crumb to } \\
\text { Subangular }\end{array}$ & Weak & $\begin{array}{l}5 \% \text { Roots, } 20 \% \text { Rootlets, } 10 \% \text { Worm } \\
\text { casts, } 10 \% \text { pin holes, insect burrows, } \\
10 \% \text { limestone pebbles (subangular to } \\
\text { sub rounded) }\end{array}$ & $\begin{array}{l}\text { Clear, Slightly } \\
\text { wavy due to } \\
\text { bioturbation }\end{array}$ & Some Rabdotus (3-5\%) \\
\hline & $32-59$ & $\begin{array}{c}10 Y R \text { 6/4- } \\
6 / 6\end{array}$ & Pale brown & Silt loam & Friable & $\begin{array}{l}\text { Crumb to } \\
\text { Subangular }\end{array}$ & Weak & $\begin{array}{c}3 \% \text { roots, } 10 \% \text { rootlets, } 2 \% \text { small } \\
\text { nodules }(3-4 \mathrm{~mm}) \text { of } \mathrm{CaCO}_{3}, 5 \% \text { pin } \\
\text { holes, } 5 \% \text { worm casts, } 5 \% \text { krotovina } \\
\text { infilled with Strat II }\end{array}$ & $\begin{array}{l}\text { Gradual, } \\
\text { smooth }\end{array}$ & Transitional \\
\hline & $59-253$ & $\begin{array}{c}\text { 10YR } 7 / 4- \\
7 / 6\end{array}$ & $\begin{array}{l}\text { Very pale } \\
\text { brown to } \\
\text { yellow } \\
\text { brown }\end{array}$ & Silt loam & $\begin{array}{l}\text { Friable to } \\
\text { Firm }\end{array}$ & $\begin{array}{l}\text { Subangular to } \\
\text { angular }\end{array}$ & Moderate & $\begin{array}{c}5 \% \text { rootlets decreasing with depth, } 3-5 \% \\
\mathrm{CaCO}_{3} \text { nodules }(2-3 \mathrm{~mm}), 5 \% \text { pin holes, } \\
3 \% \text { worm burrows }\end{array}$ & Unobserved & Hue is lighter with depth \\
\hline
\end{tabular}




\section{APPENDIX C}

\section{TEST UNIT SPECIMEN INVENTORY}





\begin{tabular}{|c|c|c|c|c|c|c|c|c|c|c|}
\hline Unit & Level & $\begin{array}{l}\text { Depth } \\
\text { (cmbs) }\end{array}$ & Artifact Type & $\begin{array}{c}\text { Artifact } \\
\text { Description }\end{array}$ & Material & $\begin{array}{c}\text { Artifact } \\
\text { No. }\end{array}$ & $\begin{array}{c}\text { Weight } \\
\text { (g) }\end{array}$ & Recorders & Date & Comments \\
\hline 1 & 1 & $0-10$ & \begin{tabular}{|l|} 
Debitage \\
\end{tabular} & Primary & Lithic & 2 & 7.28 & $\mathrm{JL}$ & $10 / 13 / 2016$ & Shatter \\
\hline 1 & 1 & $0-10$ & Debitage & Tertiary & Lithic & 14 & 14.4 & $\mathrm{JL}$ & 10/13/2016 & Shatter \\
\hline 1 & 1 & $0-10$ & \begin{tabular}{|l|} 
Faunal \\
Remains \\
\end{tabular} & Mussel Shell & Shell & 1 & 0.38 & $\mathrm{JL}$ & $10 / 12 / 2016$ & $\begin{array}{l}\text { Unmodified } \\
\text { fragment }\end{array}$ \\
\hline 1 & 1 & 3 & Groundstone & Mano & Lithic & 1 & 304.81 & $\mathrm{JL}$ & $10 / 12 / 2016$ & $\begin{array}{l}\text { Roughly half } \\
\text { complete }\end{array}$ \\
\hline 1 & 2 & $10-20$ & Biface & $\begin{array}{l}\text { Early-stage } \\
\text { biface }\end{array}$ & Lithic & 1 & 61.97 & $\mathrm{JL}$ & $10 / 13 / 2016$ & Thick and Crude \\
\hline 1 & 2 & $10-20$ & Debitage & Primary & Lithic & 7 & 8.19 & $\mathrm{JL}$ & 10/13/2016 & \\
\hline 1 & 2 & $10-20$ & Debitage & Secondary & Lithic & 15 & 35.9 & $\mathrm{JL}$ & $10 / 13 / 2016$ & $\begin{array}{l}4 \text { Bifacial thinning } \\
\text { flakes }\end{array}$ \\
\hline 1 & 2 & $10-20$ & Debitage & Tertiary & Lithic & 71 & 47.54 & $\mathrm{JL}$ & $10 / 13 / 2016$ & \begin{tabular}{|l|}
56 Bifacial \\
thinning flakes
\end{tabular} \\
\hline 1 & 2 & $10-20$ & $\begin{array}{l}\text { Faunal } \\
\text { Remains } \\
\end{array}$ & Burned Bone & & 1 & 0.17 & $\mathrm{JL}$ & $10 / 13 / 2016$ & \\
\hline 1 & 3 & $20-30$ & Blade & Blade & & 1 & 9.08 & $\mathrm{JL}$ & $10 / 12 / 2016$ & \\
\hline 1 & 3 & $20-30$ & Burned Rock & Burned Rock & Lithic & 4 & 18.26 & $\mathrm{JL}$ & 10/12/2016 & \\
\hline 1 & 3 & $20-30$ & Debitage & Primary & Lithic & 12 & 23.63 & $\mathrm{JL}$ & $10 / 12 / 2016$ & \\
\hline 1 & 3 & $20-30$ & Debitage & Secondary & Lithic & 10 & 14.05 & $\mathrm{JL}$ & $10 / 12 / 2016$ & $\begin{array}{l}7 \text { Bifacial thinning } \\
\text { flakes }\end{array}$ \\
\hline 1 & 3 & $20-30$ & Debitage & Tertiary & Lithic & 38 & 28.86 & $\mathrm{JL}$ & $10 / 12 / 2016$ & \begin{tabular}{|l|}
30 Bifacial \\
thinning flakes \\
\end{tabular} \\
\hline 1 & 4 & $30-40$ & Debitage & Primary & Lithic & 6 & 62.4 & $\mathrm{JL}$ & $10 / 12 / 2016$ & \\
\hline 1 & 4 & $30-40$ & Debitage & Secondary & Lithic & 10 & 26.3 & $\mathrm{JL}$ & $10 / 12 / 2016$ & $\begin{array}{l}7 \text { Bifacial thinning } \\
\text { flakes }\end{array}$ \\
\hline 1 & 4 & $30-40$ & Debitage & Tertiary & Lithic & 53 & 54.92 & $\mathrm{JL}$ & $10 / 12 / 2016$ & \begin{tabular}{|l|}
48 Bifacial \\
thinning flakes
\end{tabular} \\
\hline 1 & 4 & $30-40$ & Burned Rock & Burned Rock & Lithic & 6 & 14.5 & $\mathrm{JL}$ & $10 / 12 / 2016$ & \\
\hline 1 & 4 & $30-40$ & \begin{tabular}{|l} 
Faunal \\
Remains \\
\end{tabular} & Mussel Shell & Shell & 2 & 0.69 & $\mathrm{JL}$ & $10 / 12 / 2016$ & Unmodified \\
\hline 1 & 4 & $30-40$ & \begin{tabular}{|l|} 
Projectile \\
Point \\
\end{tabular} & \begin{tabular}{|l|} 
Untyped Arrow \\
Point
\end{tabular} & Lithic & 1 & 2.15 & $\mathrm{JL}$ & $10 / 12 / 2016$ & $\begin{array}{l}\text { Missing distal tip } \\
\text { and base } \\
\end{array}$ \\
\hline 1 & 5 & 50 & Charcoal & Charcoal & & 4 & 0.15 & $\mathrm{JL}$ & 10/13/2016 & \\
\hline 1 & 5 & $40-50$ & \begin{tabular}{|l|} 
Faunal \\
Remains \\
\end{tabular} & Bone & & 3 & 4.63 & $\mathrm{JL}$ & $10 / 13 / 2016$ & 1 Burned fragment \\
\hline 1 & 5 & $40-50$ & Debitage & Primary & Lithic & 2 & 9.25 & $\mathrm{JL}$ & 10/13/2016 & Shatter \\
\hline 1 & 5 & $40-50$ & Debitage & Secondary & Lithic & 11 & 54.3 & $\mathrm{JL}$ & $10 / 13 / 2016$ & $\begin{array}{l}2 \text { Bifacial thinning } \\
\text { flakes }\end{array}$ \\
\hline 1 & 5 & $40-50$ & Debitage & Tertiary & Lithic & 37 & 37.57 & $\mathrm{JL}$ & $10 / 13 / 2016$ & \begin{tabular}{|l|}
23 Bifacial \\
thinning flakes
\end{tabular} \\
\hline 1 & 5 & $40-50$ & Utilized Flake & Utilized Flake & Lithic & 1 & 1.15 & $\mathrm{JL}$ & $10 / 13 / 2016$ & \\
\hline 1 & 6 & $50-60$ & Biface & \begin{tabular}{|l|} 
Bifacial \\
Fragment \\
\end{tabular} & Lithic & 1 & 53.15 & $\mathrm{JL}$ & $10 / 13 / 2016$ & \\
\hline 1 & 6 & $50-60$ & Core & Core & Lithic & 1 & 133.85 & $\mathrm{JL}$ & $10 / 13 / 2016$ & \\
\hline 1 & 6 & $50-60$ & Debitage & Primary & Lithic & 18 & 29.95 & $\mathrm{JL}$ & $10 / 13 / 2016$ & \\
\hline 1 & 6 & $50-60$ & Debitage & Secondary & Lithic & 24 & 113.78 & $\mathrm{JL}$ & $10 / 13 / 2016$ & $\begin{array}{l}\text { 8 Bifacial thinning } \\
\text { flakes }\end{array}$ \\
\hline 1 & 6 & $50-60$ & Debitage & Tertiary & Lithic & 100 & 84.23 & $\mathrm{JL}$ & $10 / 13 / 2016$ & $\begin{array}{l}76 \text { Bifacial } \\
\text { thinning flakes }\end{array}$ \\
\hline 1 & 6 & $50-60$ & \begin{tabular}{|l|} 
Faunal \\
Remains \\
\end{tabular} & Bone & & 5 & 3.33 & $\mathrm{JL}$ & 10/13/2016 & \\
\hline 1 & 6 & $50-60$ & $\begin{array}{l}\text { Tested } \\
\text { Cobble } \\
\end{array}$ & Tested Cobble & Lithic & 1 & 163.13 & $\mathrm{JL}$ & $10 / 13 / 2016$ & \\
\hline 1 & 7 & $60-70$ & Burned Rock & Burned Rock & Lithic & 1 & 1.3 & $\mathrm{JL}$ & $10 / 12 / 2016$ & \\
\hline 1 & 7 & $60-70$ & Debitage & \begin{tabular}{|l|} 
Primary \\
\end{tabular} & Lithic & 1 & 0.67 & $\mathrm{JL}$ & 10/12/2016 & \\
\hline 1 & 7 & $60-70$ & Debitage & Secondary & Lithic & 11 & 11.22 & $\mathrm{JL}$ & $10 / 12 / 2016$ & $\begin{array}{l}7 \text { Bifacial thinning } \\
\text { flakes }\end{array}$ \\
\hline 1 & 7 & $60-70$ & Debitage & Tertiary & Lithic & 60 & 64.2 & $\mathrm{JL}$ & $10 / 12 / 2016$ & $\begin{array}{l}48 \text { Bifacial } \\
\text { thinning flakes }\end{array}$ \\
\hline 1 & 7 & $60-70$ & \begin{tabular}{|l|} 
Faunal \\
Remains
\end{tabular} & Bone & & 6 & 7.57 & $\mathrm{JL}$ & $10 / 12 / 2016$ & \\
\hline 1 & 7 & $60-70$ & $\begin{array}{l}\text { Projectile } \\
\text { Point }\end{array}$ & Scallorn Point & Lithic & 1 & 1.25 & $\mathrm{JL}$ & $10 / 12 / 2016$ & $\begin{array}{l}\text { Missing distal tip; } \\
\text { corner-notched } \\
\text { broad stemmed } \\
\text { variant }\end{array}$ \\
\hline
\end{tabular}




\begin{tabular}{|c|c|c|c|c|c|c|c|c|c|c|}
\hline Unit & Level & $\begin{array}{l}\text { Depth } \\
\text { (cmbs) }\end{array}$ & Artifact Type & $\begin{array}{c}\text { Artifact } \\
\text { Description }\end{array}$ & Material & $\begin{array}{c}\text { Artifact } \\
\text { No. }\end{array}$ & $\begin{array}{c}\text { Weight } \\
\text { (g) }\end{array}$ & Recorders & Date & Comments \\
\hline 1 & 8 & $70-80$ & Debitage & Primary & Lithic & 8 & 36.06 & $\mathrm{JL}$ & $10 / 14 / 2016$ & $\begin{array}{l}2 \text { Bifacial thinning } \\
\text { flakes }\end{array}$ \\
\hline 1 & 8 & $70-80$ & Debitage & Secondary & Lithic & 13 & 59.03 & $\mathrm{JL}$ & $10 / 14 / 2016$ & $\begin{array}{l}6 \text { Bifacial thinning } \\
\text { flakes }\end{array}$ \\
\hline 1 & 8 & $70-80$ & Debitage & Tertiary & Lithic & 64 & 96.89 & $\mathrm{JL}$ & $10 / 14 / 2016$ & \begin{tabular}{|l|}
49 Bifacial \\
thinning flakes \\
\end{tabular} \\
\hline 1 & 8 & $70-80$ & \begin{tabular}{|l|} 
Faunal \\
Remains \\
\end{tabular} & Bone & & 13 & 6.48 & $\mathrm{JL}$ & $10 / 14 / 2016$ & 1 Burned fragmen \\
\hline 1 & 8 & $70-80$ & \begin{tabular}{|l} 
Faunal \\
Remains \\
\end{tabular} & Mussel Shell & Shell & 2 & 33.36 & $\mathrm{JL}$ & $10 / 14 / 2016$ & $\begin{array}{l}\text { Unmodified } \\
\text { fragments }\end{array}$ \\
\hline 1 & 8 & $70-80$ & $\begin{array}{l}\text { Organic } \\
\text { remains }\end{array}$ & $\begin{array}{l}\text { Burned Nut } \\
\text { Shell } \\
\end{array}$ & & 2 & 0.27 & $\mathrm{JL}$ & $10 / 14 / 2016$ & \\
\hline 1 & 8 & $70-80$ & Utilized Flake & Utilized Flake & Lithic & 1 & 6.52 & $\mathrm{JL}$ & $10 / 14 / 2016$ & \\
\hline 1 & 9 & $80-90$ & Burned Rock & Burned Rock & Lithic & 3 & 28.34 & $\mathrm{JL}$ & $10 / 14 / 2016$ & \\
\hline 1 & 9 & $80-90$ & Core & Core & Lithic & 1 & 68.3 & $\mathrm{JL}$ & $10 / 14 / 2016$ & \begin{tabular}{|l|}
$\begin{array}{l}\text { Broken into } 3 \\
\text { pieces }\end{array}$ \\
\end{tabular} \\
\hline 1 & 9 & $80-90$ & Debitage & Primary & Lithic & 3 & 28.58 & $\mathrm{JL}$ & $10 / 14 / 2016$ & $\begin{array}{l}1 \text { Bifacial thinning } \\
\text { flake }\end{array}$ \\
\hline 1 & 9 & $80-90$ & Debitage & Secondary & Lithic & 4 & 30.54 & $\mathrm{JL}$ & $10 / 14 / 2016$ & $\begin{array}{l}1 \text { Bifacial thinning } \\
\text { flake }\end{array}$ \\
\hline 1 & 9 & $80-90$ & Debitage & Tertiary & Lithic & 24 & 47.94 & $\mathrm{JL}$ & $10 / 14 / 2016$ & \begin{tabular}{|l|}
20 Bifacial \\
thinning flakes
\end{tabular} \\
\hline 1 & 9 & $80-90$ & \begin{tabular}{|l} 
Faunal \\
Remains
\end{tabular} & Bone & & 3 & 0.96 & $\mathrm{JL}$ & $10 / 14 / 2016$ & 1 Burned fragmen \\
\hline 1 & 9 & $80-90$ & \begin{tabular}{|l|} 
Faunal \\
Remains \\
\end{tabular} & Mussel Shell & Shell & 3 & 15.35 & $\mathrm{JL}$ & $10 / 14 / 2016$ & \begin{tabular}{|l|}
$\begin{array}{l}\text { Unmodified } \\
\text { fragments }\end{array}$ \\
\end{tabular} \\
\hline 1 & 10 & 90-100 & Debitage & Primary & Lithic & 7 & 20.05 & $\mathrm{JL}$ & $10 / 12 / 2016$ & $\begin{array}{l}1 \text { Bifacial thinning } \\
\text { flake }\end{array}$ \\
\hline 1 & 10 & 90-100 & Debitage & Secondary & Lithic & 10 & 36.96 & $\mathrm{JL}$ & $10 / 12 / 2016$ & $\begin{array}{l}7 \text { Bifacial thinning } \\
\text { flakes }\end{array}$ \\
\hline 1 & 10 & 90-100 & Debitage & Tertiary & Lithic & 33 & 64 & $\mathrm{JL}$ & $10 / 12 / 2016$ & \begin{tabular}{|l|}
31 Bifacial \\
thinning flakes \\
\end{tabular} \\
\hline 1 & 10 & 90-100 & \begin{tabular}{|l|} 
Faunal \\
Remains
\end{tabular} & Bone & & 5 & 0.07 & $\mathrm{JL}$ & $10 / 12 / 2016$ & \\
\hline 1 & 11 & $\begin{array}{c}100- \\
110\end{array}$ & Debitage & Primary & Lithic & 1 & 0.91 & $\mathrm{JL}$ & $10 / 13 / 2016$ & \\
\hline 1 & 11 & $\begin{array}{c}100- \\
110\end{array}$ & Debitage & Secondary & Lithic & 7 & 20.15 & $\mathrm{JL}$ & $10 / 13 / 2016$ & $\begin{array}{l}\text { 3 Bifacial thinning } \\
\text { flakes }\end{array}$ \\
\hline 1 & 11 & $\begin{array}{c}100- \\
110 \\
\end{array}$ & Debitage & Tertiary & Lithic & 33 & 35.1 & $\mathrm{JL}$ & $10 / 13 / 2016$ & \begin{tabular}{|l|}
25 Bifacial \\
thinning flakes \\
\end{tabular} \\
\hline 1 & 11 & $\begin{array}{c}100- \\
110 \\
\end{array}$ & \begin{tabular}{|l} 
Faunal \\
Remains \\
\end{tabular} & Bone & & 8 & 1.57 & $\mathrm{JL}$ & $10 / 13 / 2016$ & \\
\hline 1 & 11 & $\begin{array}{c}100- \\
110\end{array}$ & \begin{tabular}{|l} 
Faunal \\
Remains
\end{tabular} & Mussel Shell & Shell & 3 & 6.32 & $\mathrm{JL}$ & $10 / 13 / 2016$ & $\begin{array}{l}\text { Unmodified } \\
\text { fragments }\end{array}$ \\
\hline 1 & 12 & $\begin{array}{c}110- \\
120 \\
\end{array}$ & Debitage & Primary & Lithic & 7 & 54.47 & $\mathrm{JL}$ & $10 / 14 / 2016$ & $\begin{array}{l}4 \text { Bifacial thinning } \\
\text { flakes }\end{array}$ \\
\hline 1 & 12 & $\begin{array}{c}110- \\
120\end{array}$ & Debitage & Secondary & Lithic & 6 & 39.54 & $\mathrm{JL}$ & $10 / 14 / 2016$ & $\begin{array}{l}4 \text { Bifacial thinning } \\
\text { flakes }\end{array}$ \\
\hline 1 & 12 & $\begin{array}{c}110- \\
120 \\
\end{array}$ & Debitage & Tertiary & Lithic & 15 & 8.52 & $\mathrm{JL}$ & $10 / 14 / 2016$ & \begin{tabular}{|l|}
13 Bifacial \\
thinning flakes \\
\end{tabular} \\
\hline 1 & 12 & $\begin{array}{c}110- \\
120\end{array}$ & $\begin{array}{l}\text { Faunal } \\
\text { Remains } \\
\end{array}$ & Bone & & 12 & 8.31 & $\mathrm{JL}$ & $10 / 14 / 2016$ & \\
\hline 1 & 13 & $\begin{array}{c}120- \\
130\end{array}$ & Debitage & Primary & Lithic & 2 & 3.12 & $\mathrm{JL}$ & $10 / 13 / 2016$ & \\
\hline 1 & 13 & $\begin{array}{c}120- \\
130\end{array}$ & Debitage & Tertiary & Lithic & 6 & 5.9 & $\mathrm{JL}$ & $10 / 13 / 2016$ & $\begin{array}{l}5 \text { Bifacial thinning } \\
\text { flakes }\end{array}$ \\
\hline 1 & 13 & $\begin{array}{c}120- \\
130\end{array}$ & $\begin{array}{l}\text { Faunal } \\
\text { Remains }\end{array}$ & Mussel Shell & Shell & 1 & 9.52 & $\mathrm{JL}$ & $10 / 13 / 2016$ & $\begin{array}{l}\text { Unmodified } \\
\text { fragments }\end{array}$ \\
\hline 1 & 14 & $\begin{array}{c}130- \\
140 \\
\end{array}$ & Debitage & Primary & Lithic & 4 & 82.57 & $\mathrm{JL}$ & $10 / 13 / 2016$ & \\
\hline 1 & 14 & $\begin{array}{c}130- \\
140\end{array}$ & Debitage & Secondary & Lithic & 5 & 110.35 & $\mathrm{JL}$ & $10 / 13 / 2016$ & $\begin{array}{l}2 \text { Bifacial thinning } \\
\text { flakes }\end{array}$ \\
\hline 1 & 14 & $\begin{array}{c}130- \\
140 \\
\end{array}$ & Debitage & Tertiary & Lithic & 17 & 19.68 & $\mathrm{JL}$ & $10 / 13 / 2016$ & \begin{tabular}{|l|}
16 Bifacial \\
thinning flakes \\
\end{tabular} \\
\hline 1 & 14 & $\begin{array}{c}130- \\
140\end{array}$ & \begin{tabular}{|l|} 
Faunal \\
Remains
\end{tabular} & Bone & & 14 & 5.83 & $\mathrm{JL}$ & $10 / 13 / 2016$ & \\
\hline
\end{tabular}




\begin{tabular}{|c|c|c|c|c|c|c|c|c|c|c|}
\hline Unit & Level & $\begin{array}{l}\text { Depth } \\
\text { (cmbs) }\end{array}$ & Artifact Type & $\begin{array}{c}\text { Artifact } \\
\text { Description }\end{array}$ & Material & \begin{tabular}{|c|}
$\begin{array}{c}\text { Artifact } \\
\text { No. }\end{array}$ \\
\end{tabular} & $\begin{array}{c}\text { Weight } \\
\text { (g) }\end{array}$ & Recorders & Date & Comments \\
\hline 1 & 15 & $\begin{array}{c}140- \\
150 \\
\end{array}$ & Debitage & Secondary & Lithic & 2 & 2.4 & $\mathrm{JL}$ & $10 / 13 / 2016$ & \\
\hline 1 & 15 & $\begin{array}{l}140- \\
150\end{array}$ & Debitage & Tertiary & Lithic & 9 & 9.74 & $\mathrm{JL}$ & $10 / 13 / 2016$ & $\begin{array}{l}8 \text { Bifacial thinning } \\
\text { flakes }\end{array}$ \\
\hline 1 & 15 & $\begin{array}{c}140- \\
150\end{array}$ & \begin{tabular}{|l} 
Faunal \\
Remains \\
\end{tabular} & Bone & & 5 & 3.05 & $\mathrm{JL}$ & $10 / 13 / 2016$ & \\
\hline 1 & 15 & $\begin{array}{c}140- \\
150\end{array}$ & $\begin{array}{l}\text { Organic } \\
\text { remains }\end{array}$ & Burned Seed & & 2 & 0.04 & $\mathrm{JL}$ & $10 / 13 / 2016$ & \\
\hline 1 & 15 & $\begin{array}{l}140- \\
150\end{array}$ & $\begin{array}{l}\text { Projectile } \\
\text { Point }\end{array}$ & $\begin{array}{l}\text { Untyped Arrow } \\
\text { Point }\end{array}$ & Lithic & 1 & 0.25 & $\mathrm{JL}$ & $10 / 13 / 2016$ & $\begin{array}{l}\text { Serrated medial } \\
\text { arrow point } \\
\text { fragment } \\
\end{array}$ \\
\hline 1 & 16 & $\begin{array}{l}150- \\
160\end{array}$ & \begin{tabular}{|l|} 
Faunal \\
Remains \\
\end{tabular} & Bone & & 5 & 15.75 & JU & 8/31/2017 & \\
\hline 1 & 16 & $\begin{array}{l}150- \\
160 \\
\end{array}$ & Debitage & Secondary & Lithic & 1 & 6.28 & $\mathrm{JU}$ & 8/31/2017 & $\begin{array}{l}1 \text { bifacial thinning } \\
\text { flake }\end{array}$ \\
\hline 1 & 16 & $\begin{array}{l}150- \\
160\end{array}$ & Debitage & Tertiary & Lithic & 2 & 2.75 & JU & 8/31/2017 & $\begin{array}{l}1 \text { bifacial thinning } \\
\text { flake }\end{array}$ \\
\hline 1 & 17 & $\begin{array}{l}160- \\
170\end{array}$ & Debitage & Tertiary & Lithic & 4 & 5.98 & JU & 8/31/2017 & $\begin{array}{l}3 \text { bifacial thinning } \\
\text { flakes }\end{array}$ \\
\hline 1 & 6,7 & $56-70$ & Soil Sample & Matrix Sample & & & & & & 3 gal. bags \\
\hline 2 & 1 & $0-10$ & Debitage & Primary & Lithic & 1 & 10.76 & $\mathrm{JL}$ & $10 / 14 / 2016$ & \\
\hline 2 & 1 & 0-10 & Debitage & Tertiary & Lithic & 16 & 11.48 & $\mathrm{JL}$ & $10 / 14 / 2016$ & $\begin{array}{l}14 \text { Bifacial } \\
\text { thinning flakes }\end{array}$ \\
\hline 2 & 2 & $10-20$ & Debitage & Primary & Lithic & 1 & 89.07 & $\mathrm{JL}$ & $10 / 13 / 2016$ & \\
\hline 2 & 2 & 10-20 & Debitage & Secondary & Lithic & 6 & 34.9 & $\mathrm{JL}$ & $10 / 13 / 2016$ & $\begin{array}{l}4 \text { Bifacial thinning } \\
\text { flakes }\end{array}$ \\
\hline 2 & 2 & $10-20$ & Debitage & Tertiary & Lithic & 19 & 23.15 & $\mathrm{JL}$ & $10 / 13 / 2016$ & $\begin{array}{l}18 \text { Bifacial } \\
\text { thinning flakes }\end{array}$ \\
\hline 2 & 3 & $20-30$ & Debitage & Primary & Lithic & 2 & 19.64 & $\mathrm{JL}$ & $10 / 13 / 2016$ & \\
\hline 2 & 3 & $20-30$ & Debitage & Secondary & Lithic & 3 & 4.56 & $\mathrm{JL}$ & $10 / 13 / 2016$ & $\begin{array}{l}1 \text { Bifacial thinning } \\
\text { flake }\end{array}$ \\
\hline 2 & 3 & $20-30$ & Debitage & Tertiary & Lithic & 8 & 3.66 & $\mathrm{JL}$ & $10 / 13 / 2016$ & $\begin{array}{l}4 \text { Bifacial thinning } \\
\text { flakes }\end{array}$ \\
\hline 2 & 4 & $30-40$ & Debitage & Secondary & Lithic & 2 & 1.16 & $\mathrm{JL}$ & $10 / 14 / 2016$ & \\
\hline 2 & 4 & $30-40$ & Debitage & Tertiary & Lithic & 2 & 0.3 & $\mathrm{JL}$ & $10 / 14 / 2016$ & $\begin{array}{l}2 \text { Bifacial thinning } \\
\text { flakes }\end{array}$ \\
\hline 2 & 5 & $40-50$ & Blade & Blade & & 1 & 22.17 & $\mathrm{JL}$ & $10 / 14 / 2016$ & \\
\hline 2 & 5 & $40-50$ & Core & \begin{tabular}{|l|} 
Core \\
\end{tabular} & Lithic & 1 & 44.26 & $\mathrm{JL}$ & $10 / 14 / 2016$ & \\
\hline 2 & 5 & $40-50$ & Debitage & Primary & Lithic & 21 & 154.45 & $\mathrm{JL}$ & $10 / 14 / 2016$ & $\begin{array}{l}1 \text { Bifacial thinning } \\
\text { flake }\end{array}$ \\
\hline 2 & 5 & $40-50$ & Debitage & Secondary & Lithic & 24 & 70.78 & $\mathrm{JL}$ & $10 / 14 / 2016$ & $\begin{array}{l}13 \text { Bifacial } \\
\text { thinning flakes }\end{array}$ \\
\hline 2 & 5 & $40-50$ & Debitage & Tertiary & Lithic & 88 & 76.77 & $\mathrm{JL}$ & $10 / 14 / 2016$ & \begin{tabular}{|l|}
70 Bifacial \\
thinning flakes \\
\end{tabular} \\
\hline 2 & 6 & $50-60$ & Charcoal & Charcoal & & 4 & 0.27 & $\mathrm{JL}$ & $10 / 14 / 2016$ & \\
\hline 2 & 6 & $50-60$ & Debitage & Primary & Lithic & 23 & 115.08 & $\mathrm{JL}$ & $10 / 14 / 2016$ & $\begin{array}{l}5 \text { Bifacial thinning } \\
\text { flakes }\end{array}$ \\
\hline 2 & 6 & $50-60$ & Debitage & Secondary & Lithic & 28 & 211.09 & $\mathrm{JL}$ & $10 / 14 / 2016$ & \begin{tabular}{|l|}
14 Bifacial \\
thinning flakes
\end{tabular} \\
\hline 2 & 6 & $50-60$ & Debitage & Tertiary & Lithic & 149 & 119.02 & $\mathrm{JL}$ & $10 / 14 / 2016$ & $\begin{array}{l}126 \text { Bifacial } \\
\text { thinning flakes }\end{array}$ \\
\hline 2 & 6 & $50-60$ & $\begin{array}{l}\text { Projectile } \\
\text { Point }\end{array}$ & Scallorn Point & Lithic & 1 & 3.79 & $\mathrm{JL}$ & $10 / 14 / 2016$ & $\begin{array}{l}\text { Missing distal tip; } \\
\text { corner-notched } \\
\text { broad stemmed } \\
\text { variant; very large } \\
\text { for arrow point }\end{array}$ \\
\hline 2 & 7 & $60-70$ & Burned Rock & Burned Rock & Lithic & 21 & 270.5 & $\mathrm{JL}$ & $10 / 12 / 2016$ & \\
\hline 2 & 7 & $60-70$ & Charcoal & Charcoal & & 6 & 0.71 & $\mathrm{JL}$ & $10 / 12 / 2016$ & \\
\hline 2 & 7 & $60-70$ & Debitage & Primary & Lithic & 53 & 389.05 & $\mathrm{JL}$ & $10 / 12 / 2016$ & \begin{tabular}{|l|}
11 Bifacial \\
thinning flakes \\
\end{tabular} \\
\hline 2 & 7 & $60-70$ & Debitage & Secondary & Lithic & 86 & 418.54 & $\mathrm{JL}$ & $10 / 12 / 2016$ & $\begin{array}{l}46 \text { Bifacial } \\
\text { thinning flakes }\end{array}$ \\
\hline 2 & 7 & $60-70$ & Debitage & Tertiary & Lithic & 226 & 200.05 & $\mathrm{JL}$ & $10 / 12 / 2016$ & $\begin{array}{l}194 \text { Bifacial } \\
\text { thinning flakes }\end{array}$ \\
\hline 2 & 7 & $60-70$ & $\begin{array}{l}\text { Faunal } \\
\text { Remains }\end{array}$ & Burned Bone & & 8 & 6.93 & $\mathrm{JL}$ & $10 / 12 / 2016$ & $\begin{array}{l}1 \text { Calcified } \\
\text { fragment }\end{array}$ \\
\hline
\end{tabular}




\begin{tabular}{|c|c|c|c|c|c|c|c|c|c|c|}
\hline Unit & Level & $\begin{array}{l}\text { Depth } \\
\text { (cmbs) }\end{array}$ & Artifact Type & $\begin{array}{c}\text { Artifact } \\
\text { Description }\end{array}$ & Material & $\begin{array}{c}\text { Artifact } \\
\text { No. }\end{array}$ & $\begin{array}{c}\text { Weight } \\
\text { (g) }\end{array}$ & Recorders & Date & Comments \\
\hline 2 & 7 & $60-70$ & Shell Artifact & Shell Bead & Shell & 1 & 0.3 & $\mathrm{JL}$ & $10 / 12 / 2016$ & \begin{tabular}{|l} 
Modified shell \\
bead with beveled \\
drill hole and an \\
another decorative \\
incised drill mark
\end{tabular} \\
\hline 2 & 8 & $70-80$ & Debitage & Primary & Lithic & 10 & 20.9 & $\mathrm{JL}$ & $10 / 12 / 2016$ & $\begin{array}{l}\text { 4 Bifacial thinning } \\
\text { flakes }\end{array}$ \\
\hline 2 & 8 & $70-80$ & Debitage & Secondary & Lithic & 19 & 41.23 & $\mathrm{JL}$ & $10 / 12 / 2016$ & $\begin{array}{l}\text { 8 Bifacial thinning } \\
\text { flakes }\end{array}$ \\
\hline 2 & 8 & $70-80$ & Debitage & Tertiary & Lithic & 77 & 50.01 & $\mathrm{JL}$ & $10 / 12 / 2016$ & $\begin{array}{l}66 \text { Bifacial } \\
\text { thinning flakes }\end{array}$ \\
\hline 2 & 8 & $70-80$ & $\begin{array}{l}\text { Projectile } \\
\text { Point }\end{array}$ & $\begin{array}{l}\text { Untyped Arrow } \\
\text { Point Preform }\end{array}$ & Lithic & 1 & 5.4 & $\mathrm{JL}$ & $10 / 12 / 2016$ & \begin{tabular}{|l|} 
Missing distal tip; \\
incomplete \\
manufacturing \\
failure?; unifacially \\
trimmed; likely \\
Scallorn preform \\
\end{tabular} \\
\hline 2 & 8 & $70-80$ & \begin{tabular}{|l|} 
Projectile \\
Point
\end{tabular} & \begin{tabular}{|l|} 
Untyped Arrow \\
Point
\end{tabular} & Lithic & 1 & 1.41 & $\mathrm{JL}$ & $10 / 12 / 2016$ & $\begin{array}{l}\text { Distal arrow point } \\
\text { frag missing base }\end{array}$ \\
\hline 2 & 8 & $70-80$ & Utilized Flake & Utilized Flake & Lithic & 1 & 4.96 & $\mathrm{JL}$ & $10 / 12 / 2016$ & \\
\hline 2 & 9 & $80-90$ & Blade & Blade & & 1 & 5.29 & $\mathrm{JL}$ & 10/13/2016 & \\
\hline 2 & 9 & $80-90$ & \begin{tabular}{|l|} 
Debitage \\
\end{tabular} & Primary & Lithic & 7 & 21.69 & $\mathrm{JL}$ & $10 / 13 / 2016$ & \\
\hline 2 & 9 & $80-90$ & Debitage & Secondary & Lithic & 12 & 23.21 & $\mathrm{JL}$ & $10 / 13 / 2016$ & $\begin{array}{l}\text { 8 Bifacial thinning } \\
\text { flakes }\end{array}$ \\
\hline 2 & 9 & $80-90$ & Debitage & Tertiary & Lithic & 63 & 49.64 & $\mathrm{JL}$ & $10 / 13 / 2016$ & \begin{tabular}{|l|}
60 Bifacial \\
thinning flakes
\end{tabular} \\
\hline 2 & 9 & $80-90$ & Utilized Flake & Utilized Flake & Lithic & 1 & 11.6 & $\mathrm{JL}$ & 10/13/2016 & \\
\hline 2 & 10 & 90-100 & Debitage & Primary & Lithic & 1 & 0.79 & $\mathrm{JL}$ & $10 / 12 / 2016$ & \\
\hline 2 & 10 & 90-100 & Debitage & Secondary & Lithic & 7 & 51.83 & $\mathrm{JL}$ & $10 / 12 / 2016$ & $\begin{array}{l}4 \text { Bifacial thinning } \\
\text { flakes }\end{array}$ \\
\hline 2 & 10 & 90-100 & Debitage & Tertiary & Lithic & 52 & 73.71 & $\mathrm{JL}$ & $10 / 12 / 2016$ & $\begin{array}{l}38 \text { Bifacial } \\
\text { thinning flakes }\end{array}$ \\
\hline 2 & 11 & $\begin{array}{c}100- \\
110\end{array}$ & Charcoal & Charcoal & & 5 & 0.59 & $\mathrm{JL}$ & $10 / 13 / 2016$ & \\
\hline 2 & 11 & $\begin{array}{c}100- \\
110\end{array}$ & Debitage & Primary & Lithic & 4 & 49.89 & $\mathrm{JL}$ & $10 / 13 / 2016$ & \\
\hline 2 & 11 & $\begin{array}{c}100- \\
110\end{array}$ & Debitage & Secondary & Lithic & 8 & 100.74 & $\mathrm{JL}$ & $10 / 13 / 2016$ & $\begin{array}{l}\text { 3 Bifacial thinning } \\
\text { flakes }\end{array}$ \\
\hline 2 & 11 & $\begin{array}{c}100- \\
110\end{array}$ & Debitage & Tertiary & Lithic & 36 & 52.51 & $\mathrm{JL}$ & $10 / 13 / 2016$ & $\begin{array}{l}25 \text { Bifacial } \\
\text { thinning flakes }\end{array}$ \\
\hline 2 & 12 & $\begin{array}{c}110- \\
120\end{array}$ & Debitage & Primary & Lithic & 2 & 0.61 & $\mathrm{JL}$ & $10 / 14 / 2016$ & $\begin{array}{l}2 \text { Bifacial thinning } \\
\text { flakes }\end{array}$ \\
\hline 2 & 12 & $\begin{array}{c}110- \\
120\end{array}$ & Debitage & Secondary & Lithic & 18 & 23.2 & $\mathrm{JL}$ & $10 / 14 / 2016$ & $\begin{array}{l}6 \text { Bifacial thinning } \\
\text { flakes }\end{array}$ \\
\hline 2 & 12 & $\begin{array}{c}110- \\
120\end{array}$ & Debitage & Tertiary & Lithic & 64 & 37.15 & $\mathrm{JL}$ & $10 / 14 / 2016$ & $\begin{array}{l}40 \text { Bifacial } \\
\text { thinning flakes }\end{array}$ \\
\hline 2 & 12 & $\begin{array}{c}110- \\
120\end{array}$ & \begin{tabular}{|l|} 
Faunal \\
Remains
\end{tabular} & Bone & & 2 & 2.18 & $\mathrm{JL}$ & $10 / 14 / 2016$ & \\
\hline 2 & 12 & $\begin{array}{c}110- \\
120\end{array}$ & Hammerstone & Hammerstone & Lithic & 1 & 434.52 & $\mathrm{JL}$ & $10 / 14 / 2016$ & \\
\hline 2 & 13 & $\begin{array}{c}120- \\
130\end{array}$ & Charcoal & Charcoal & & 1 & 0.23 & $\mathrm{JL}$ & $10 / 14 / 2016$ & \\
\hline 2 & 13 & $\begin{array}{c}120- \\
130\end{array}$ & Debitage & Primary & Lithic & 2 & 4.35 & $\mathrm{JL}$ & $10 / 14 / 2016$ & \\
\hline 2 & 13 & $\begin{array}{c}120- \\
130\end{array}$ & Debitage & Secondary & Lithic & 3 & 13.48 & $\mathrm{JL}$ & $10 / 14 / 2016$ & \\
\hline 2 & 13 & $\begin{array}{c}120- \\
130\end{array}$ & Debitage & Tertiary & Lithic & 59 & 46.88 & $\mathrm{JL}$ & $10 / 14 / 2016$ & $\begin{array}{l}36 \text { Bifacial } \\
\text { thinning flakes }\end{array}$ \\
\hline 2 & 14 & $\begin{array}{c}130- \\
140\end{array}$ & Biface & \begin{tabular}{|l|} 
Bifacial \\
Fragment
\end{tabular} & Lithic & 1 & 56.08 & $\mathrm{JL}$ & $10 / 14 / 2016$ & \\
\hline 2 & 14 & $\begin{array}{c}130- \\
140\end{array}$ & Debitage & Primary & Lithic & 2 & 5.78 & $\mathrm{JL}$ & $10 / 14 / 2016$ & \\
\hline 2 & 14 & $\begin{array}{c}130- \\
140\end{array}$ & Debitage & Secondary & Lithic & 1 & 0.72 & $\mathrm{JL}$ & $10 / 14 / 2016$ & \\
\hline 2 & 14 & $\begin{array}{c}130- \\
140\end{array}$ & Debitage & Tertiary & Lithic & 14 & 12 & $\mathrm{JL}$ & $10 / 14 / 2016$ & $\begin{array}{l}10 \text { Bifacial } \\
\text { thinning flakes }\end{array}$ \\
\hline
\end{tabular}




\begin{tabular}{|c|c|c|c|c|c|c|c|c|c|c|}
\hline Unit & Level & $\begin{array}{l}\text { Depth } \\
\text { (cmbs) }\end{array}$ & Artifact Type & $\begin{array}{c}\text { Artifact } \\
\text { Description }\end{array}$ & Material & $\begin{array}{c}\text { Artifact } \\
\text { No. }\end{array}$ & $\begin{array}{c}\text { Weight } \\
\text { (g) }\end{array}$ & Recorders & Date & Comments \\
\hline 2 & 15 & $\begin{array}{c}140- \\
150\end{array}$ & \begin{tabular}{|l|} 
Faunal \\
Remains \\
\end{tabular} & Snail Shell & Shell & 1 & 3.21 & JU & 8/31/2017 & \begin{tabular}{|l|} 
Unknown, \\
complete shell
\end{tabular} \\
\hline 2 & 15 & $\begin{array}{l}140- \\
150 \\
\end{array}$ & Debitage & Secondary & Lithic & 3 & 3.76 & JU & 8/31/2017 & $\begin{array}{l}1 \text { bifacial thinning } \\
\text { flake }\end{array}$ \\
\hline 2 & 15 & $\begin{array}{l}140- \\
150 \\
\end{array}$ & Debitage & Tertiary & Lithic & 3 & 0.88 & JU & 8/31/2017 & $\begin{array}{l}1 \text { bifacial thinning } \\
\text { flake }\end{array}$ \\
\hline 2 & 16 & $\begin{array}{l}150- \\
160 \\
\end{array}$ & Debitage & Secondary & Lithic & 1 & 2.94 & $\mathrm{JU}$ & 8/31/2017 & \\
\hline 3 & 1 & $0-10$ & Debitage & Primary & Lithic & 1 & 50.16 & $\mathrm{JL}$ & $10 / 14 / 2016$ & \\
\hline 3 & 1 & $0-10$ & Debitage & Secondary & Lithic & 1 & 0.35 & $\mathrm{JL}$ & $10 / 14 / 2016$ & \\
\hline 3 & 1 & $0-10$ & Debitage & Tertiary & Lithic & 6 & 2.44 & $\mathrm{JL}$ & $10 / 14 / 2016$ & $\begin{array}{l}5 \text { Bifacial thinning } \\
\text { flakes }\end{array}$ \\
\hline 3 & 1 & $0-10$ & Utilized Flake & Utilized Flake & Lithic & 1 & 2.58 & $\mathrm{JL}$ & 10/14/2016 & \\
\hline 3 & 2 & $10-20$ & Debitage & Secondary & Lithic & 2 & 1.6 & $\mathrm{JL}$ & $10 / 14 / 2016$ & \\
\hline 3 & 2 & $10-20$ & Debitage & Tertiary & Lithic & 6 & 0.98 & $\mathrm{JL}$ & $10 / 14 / 2016$ & $\begin{array}{l}6 \text { Bifacial thinning } \\
\text { flakes }\end{array}$ \\
\hline 3 & 3 & $20-30$ & Debitage & Primary & Lithic & 1 & 0.19 & $\mathrm{JL}$ & $10 / 14 / 2016$ & \\
\hline 3 & 3 & $20-30$ & Debitage & Tertiary & Lithic & 12 & 2.9 & $\mathrm{JL}$ & $10 / 14 / 2016$ & $\begin{array}{l}10 \text { Bifacial } \\
\text { thinning flakes }\end{array}$ \\
\hline 3 & 4 & $30-40$ & Burned Rock & Burned Rock & Lithic & 2 & 18.18 & $\mathrm{JL}$ & $10 / 14 / 2016$ & \\
\hline 3 & 4 & $30-40$ & Debitage & Primary & Lithic & 7 & 48.46 & $\mathrm{JL}$ & $10 / 14 / 2016$ & $\begin{array}{l}1 \text { Bifacial thinning } \\
\text { flake }\end{array}$ \\
\hline 3 & 4 & $30-40$ & Debitage & Secondary & Lithic & 18 & 36.29 & $\mathrm{JL}$ & $10 / 14 / 2016$ & $\begin{array}{l}6 \text { Bifacial thinning } \\
\text { flakes }\end{array}$ \\
\hline 3 & 4 & $30-40$ & Debitage & Tertiary & Lithic & 60 & 31.1 & $\mathrm{JL}$ & $10 / 14 / 2016$ & \begin{tabular}{|l|}
54 Bifacial \\
thinning flakes
\end{tabular} \\
\hline 3 & 5 & $40-50$ & \begin{tabular}{|l|} 
Faunal \\
Remains \\
\end{tabular} & Mussel Shell & Shell & 1 & 2.47 & JU & $8 / 31 / 2017$ & \\
\hline 3 & 5 & $40-50$ & Ground Stone & Ground Stone & & 1 & 85.14 & $\mathrm{JU}$ & $8 / 31 / 2017$ & \\
\hline 3 & 5 & $40-50$ & \begin{tabular}{|l|} 
Modified \\
Flake \\
\end{tabular} & Modified Flake & Lithic & 1 & 10.33 & $\mathrm{JU}$ & 8/31/2017 & \\
\hline 3 & 5 & $40-50$ & Debitage & Primary & Lithic & 6 & 63.93 & $\mathrm{JU}$ & $8 / 31 / 2017$ & \\
\hline 3 & 5 & $40-50$ & Debitage & Secondary & Lithic & 21 & 238.78 & JU & 8/31/2017 & $\begin{array}{l}8 \text { bifacial thinning } \\
\text { flakes }\end{array}$ \\
\hline 3 & 5 & $40-50$ & Debitage & Tertiary & Lithic & 74 & 112.97 & JU & $8 / 31 / 2017$ & $\begin{array}{l}53 \text { bifacial thinning } \\
\text { flakes }\end{array}$ \\
\hline 3 & 6 & $50-60$ & \begin{tabular}{|l|} 
Faunal \\
Remains \\
\end{tabular} & Bone & & 9 & 0.94 & JU & 8/31/2017 & \\
\hline 3 & 6 & $50-60$ & Debitage & Primary & Lithic & 8 & 32.41 & $\mathrm{JU}$ & $8 / 31 / 2017$ & \\
\hline 3 & 6 & $50-60$ & Debitage & Secondary & Lithic & 43 & 122.26 & JU & $8 / 31 / 2017$ & $\begin{array}{l}15 \text { bifacial thinning } \\
\text { flakes }\end{array}$ \\
\hline 3 & 6 & $50-60$ & Debitage & Tertiary & Lithic & 128 & 119.41 & $\mathrm{JU}$ & 8/31/2017 & $\begin{array}{l}85 \text { bifacial thinning } \\
\text { flakes }\end{array}$ \\
\hline 3 & 6 & $50-60$ & \begin{tabular}{|l|} 
Tool \\
\end{tabular} & Biface & Lithic & 1 & 5.13 & $\mathrm{JU}$ & $8 / 31 / 2017$ & thin, proximal \\
\hline 3 & 6 & $50-60$ & \begin{tabular}{|l} 
Projectile \\
Point
\end{tabular} & \begin{tabular}{|l|} 
Untyped Arrow \\
Point Tip
\end{tabular} & Lithic & 1 & 0.63 & JU & $8 / 31 / 2017$ & $\begin{array}{l}\text { Distal tip; serrated } \\
\text { well-thinned }\end{array}$ \\
\hline 3 & 6 & $50-60$ & \begin{tabular}{|l|} 
Projectile \\
Point
\end{tabular} & $\begin{array}{l}\text { Untyped Arrow } \\
\text { Point }\end{array}$ & Lithic & 1 & 3.07 & $\mathrm{JU}$ & 8/31/2017 & Nearly complete \\
\hline 3 & 7 & $60-70$ & $\begin{array}{l}\text { Projectile } \\
\text { Point }\end{array}$ & $\begin{array}{l}\text { Untyped Dart } \\
\text { Point }\end{array}$ & Lithic & 1 & 4.5 & JU & 8/31/2017 & \begin{tabular}{|l|} 
Proximal half of \\
point; heavily \\
burned; probable \\
Frio or Ensor \\
\end{tabular} \\
\hline 3 & 7 & $60-70$ & \begin{tabular}{|l|} 
Faunal \\
Remains \\
\end{tabular} & Mussel Shell & Shell & 1 & 0.21 & JU & 8/31/2017 & \\
\hline 3 & 7 & $60-70$ & Debitage & Primary & Lithic & 3 & 55.44 & JU & 8/31/2017 & $\begin{array}{l}1 \text { bifacial thinning } \\
\text { flake }\end{array}$ \\
\hline 3 & 7 & $60-70$ & Debitage & Secondary & Lithic & 7 & 21.6 & JU & 8/31/2017 & $\begin{array}{l}2 \text { bifacial thinning } \\
\text { flakes }\end{array}$ \\
\hline 3 & 7 & $60-70$ & Debitage & Tertiary & Lithic & 18 & 22.47 & $\mathrm{JU}$ & 8/31/2017 & $\begin{array}{l}13 \text { bifacial thinning } \\
\text { flakes }\end{array}$ \\
\hline 3 & 8 & $70-80$ & Debitage & Primary & Lithic & 3 & 51.34 & $\mathrm{JU}$ & $8 / 31 / 2017$ & \\
\hline 3 & 8 & $70-80$ & Debitage & Secondary & Lithic & 12 & 76.02 & JU & 8/31/2017 & $\begin{array}{l}6 \text { bifacial thinning } \\
\text { flakes } \\
\end{array}$ \\
\hline 3 & 8 & $70-80$ & Debitage & Tertiary & Lithic & 27 & 36.42 & $\mathrm{JU}$ & $8 / 31 / 2017$ & $\begin{array}{l}19 \text { bifacial thinning } \\
\text { flakes }\end{array}$ \\
\hline
\end{tabular}




\begin{tabular}{|c|c|c|c|c|c|c|c|c|c|c|}
\hline Unit & Level & $\begin{array}{l}\text { Depth } \\
\text { (cmbs) }\end{array}$ & Artifact Type & $\begin{array}{c}\text { Artifact } \\
\text { Description }\end{array}$ & Material & \begin{tabular}{|c|}
$\begin{array}{c}\text { Artifact } \\
\text { No. }\end{array}$ \\
\end{tabular} & $\begin{array}{c}\text { Weight } \\
\text { (g) }\end{array}$ & Recorders & Date & Comments \\
\hline 3 & 9 & $80-90$ & \begin{tabular}{|l|} 
Faunal \\
Remains
\end{tabular} & Bone & & 2 & 0.12 & JU & 8/31/2017 & \\
\hline 3 & 9 & $80-90$ & Debitage & Secondary & Lithic & 4 & 45.03 & JU & $8 / 31 / 2017$ & $\begin{array}{l}2 \text { bifacial thinning } \\
\text { flakes }\end{array}$ \\
\hline 3 & 9 & $80-90$ & Debitage & Tertiary & Lithic & 25 & 20.72 & JU & $8 / 31 / 2017$ & $\begin{array}{l}13 \text { bifacial thinning } \\
\text { flakes }\end{array}$ \\
\hline 3 & 10 & $90-100$ & \begin{tabular}{|l|} 
Organic \\
Remains
\end{tabular} & Charcoal & & 1 & 0.71 & JU & $8 / 31 / 2017$ & \\
\hline 3 & 10 & $90-100$ & Ground Stone & Ground Stone & & 3 & 62.89 & $\mathrm{JU}$ & $8 / 31 / 2017$ & \\
\hline 3 & 10 & $90-100$ & Debitage & Secondary & Lithic & 7 & 19.02 & JU & 8/31/2017 & $\begin{array}{l}1 \text { bifacial thinning } \\
\text { flake }\end{array}$ \\
\hline 3 & 10 & $90-100$ & Debitage & Tertiary & Lithic & 25 & 17.51 & JU & 8/31/2017 & $\begin{array}{l}17 \text { bifacial thinning } \\
\text { flakes }\end{array}$ \\
\hline 3 & 10 & $90-100$ & \begin{tabular}{|l} 
Modified \\
Flake
\end{tabular} & Modified Flake & Lithic & 1 & 3.05 & JU & 8/31/2017 & \\
\hline 3 & 11 & $\begin{array}{l}100- \\
110 \\
\end{array}$ & Debitage & Primary & Lithic & 3 & 111.05 & JU & 8/31/2017 & \\
\hline 3 & 11 & $\begin{array}{l}100- \\
110\end{array}$ & Debitage & Secondary & Lithic & 10 & 31.49 & $\mathrm{JU}$ & 8/31/2017 & $\begin{array}{l}3 \text { bifacial thinning } \\
\text { flakes }\end{array}$ \\
\hline 3 & 11 & $\begin{array}{l}100- \\
110\end{array}$ & Debitage & Tertiary & Lithic & 55 & 81.03 & JU & $8 / 31 / 2017$ & $\begin{array}{l}38 \text { bifacial thinning } \\
\text { flakes }\end{array}$ \\
\hline 3 & 12 & $\begin{array}{l}110- \\
120\end{array}$ & \begin{tabular}{|l|} 
Faunal \\
Remains \\
\end{tabular} & Bone & & 5 & 1.04 & JU & 8/31/2017 & 2 burned \\
\hline 3 & 12 & $\begin{array}{l}110- \\
120\end{array}$ & Debitage & Primary & Lithic & 5 & 107.78 & JU & 8/31/2017 & \\
\hline 3 & 12 & $\begin{array}{l}110- \\
120\end{array}$ & Debitage & Secondary & Lithic & 5 & 22.76 & JU & 8/31/2017 & $\begin{array}{l}2 \text { bifacial thinning } \\
\text { flakes }\end{array}$ \\
\hline 3 & 12 & $\begin{array}{l}110- \\
120\end{array}$ & Debitage & Tertiary & Lithic & 62 & 41.93 & JU & 8/31/2017 & $\begin{array}{l}43 \text { bifacial thinning } \\
\text { flakes }\end{array}$ \\
\hline 3 & 12 & $\begin{array}{l}110- \\
120\end{array}$ & Core & Core & Lithic & 1 & 217.1 & JU & 8/31/2017 & \\
\hline 3 & 13 & $\begin{array}{l}120- \\
130\end{array}$ & Debitage & Primary & Lithic & 3 & 12.55 & JU & 8/31/2017 & \\
\hline 3 & 13 & $\begin{array}{l}120- \\
130\end{array}$ & Debitage & Secondary & Lithic & 4 & 3.81 & JU & 8/31/2017 & $\begin{array}{l}2 \text { bifacial thinning } \\
\text { flakes }\end{array}$ \\
\hline 3 & 13 & $\begin{array}{l}120- \\
130\end{array}$ & Debitage & Tertiary & Lithic & 25 & 22.88 & JU & $8 / 31 / 2017$ & $\begin{array}{l}17 \text { bifacial thinning } \\
\text { flakes }\end{array}$ \\
\hline 3 & 14 & $\begin{array}{l}130- \\
140\end{array}$ & \begin{tabular}{|l} 
Faunal \\
Remains
\end{tabular} & Bone & & 9 & 3.16 & JU & 8/31/2017 & \\
\hline 3 & 14 & $\begin{array}{l}130- \\
140\end{array}$ & \begin{tabular}{|l|} 
Faunal \\
Remains
\end{tabular} & Bone & & 1 & 0.12 & JU & 8/31/2017 & \\
\hline 3 & 14 & $\begin{array}{l}130- \\
140 \\
\end{array}$ & Debitage & Secondary & Lithic & 2 & 1.68 & JU & 8/31/2017 & \\
\hline 3 & 14 & $\begin{array}{c}130- \\
140 \\
\end{array}$ & Debitage & Tertiary & Lithic & 23 & 18.76 & JU & 8/31/2017 & $\begin{array}{l}17 \text { bifacial thinning } \\
\text { flakes }\end{array}$ \\
\hline 3 & 15 & $\begin{array}{l}140- \\
150\end{array}$ & \begin{tabular}{|l|} 
Faunal \\
Remains \\
\end{tabular} & Bone & & 3 & 0.85 & JU & $8 / 31 / 2017$ & \\
\hline 3 & 15 & $\begin{array}{l}140- \\
150\end{array}$ & Debitage & Secondary & Lithic & 4 & 4.72 & $\mathrm{JU}$ & 8/31/2017 & $\begin{array}{l}1 \text { bifacial thinning } \\
\text { flake }\end{array}$ \\
\hline 3 & 15 & $\begin{array}{l}140- \\
150 \\
\end{array}$ & Debitage & Tertiary & Lithic & 19 & 17.18 & JU & $8 / 31 / 2017$ & $\begin{array}{l}11 \text { bifacial thinning } \\
\text { flakes }\end{array}$ \\
\hline 3 & 16 & $\begin{array}{l}150- \\
160\end{array}$ & Debitage & Primary & Lithic & 5 & 12.33 & JU & 8/31/2017 & \\
\hline 3 & 16 & $\begin{array}{l}150- \\
160\end{array}$ & Debitage & Secondary & Lithic & 10 & 30.48 & JU & 8/31/2017 & $\begin{array}{l}4 \text { bifacial thinning } \\
\text { flakes }\end{array}$ \\
\hline 3 & 16 & $\begin{array}{l}150- \\
160 \\
\end{array}$ & Debitage & Tertiary & Lithic & 44 & 26.89 & JU & 8/31/2017 & $\begin{array}{l}25 \text { bifacial thinning } \\
\text { flakes }\end{array}$ \\
\hline 3 & 17 & $\begin{array}{l}160- \\
170\end{array}$ & \begin{tabular}{|l} 
Faunal \\
Remains
\end{tabular} & Bone & & 2 & 0.85 & JU & 8/31/2017 & \\
\hline 3 & 17 & $\begin{array}{l}160- \\
170 \\
\end{array}$ & Debitage & Primary & Lithic & 1 & 3.42 & JU & 8/31/2017 & \\
\hline 3 & 17 & $\begin{array}{l}160- \\
170\end{array}$ & Debitage & Secondary & Lithic & 3 & 3.26 & JU & 8/31/2017 & $\begin{array}{l}1 \text { bifacial thinning } \\
\text { flake }\end{array}$ \\
\hline 3 & 17 & $\begin{array}{l}160- \\
170 \\
\end{array}$ & Debitage & Tertiary & Lithic & 15 & 15.66 & $\mathrm{JU}$ & 8/31/2017 & $\begin{array}{l}8 \text { bifacial thinning } \\
\text { flakes }\end{array}$ \\
\hline 3 & 18 & $\begin{array}{l}170- \\
180 \\
\end{array}$ & \begin{tabular}{|l|} 
Faunal \\
Remains \\
\end{tabular} & Bone & & 3 & 2.88 & JU & 8/31/2017 & \\
\hline
\end{tabular}




\begin{tabular}{|c|c|c|c|c|c|c|c|c|c|c|}
\hline Unit & Level & $\begin{array}{l}\text { Depth } \\
\text { (cmbs) }\end{array}$ & Artifact Type & $\begin{array}{c}\text { Artifact } \\
\text { Description }\end{array}$ & Material & $\begin{array}{c}\text { Artifact } \\
\text { No. }\end{array}$ & $\begin{array}{c}\text { Weight } \\
\text { (g) }\end{array}$ & Recorders & Date & Comments \\
\hline 3 & 18 & $\begin{array}{l}170- \\
180 \\
\end{array}$ & Debitage & Secondary & Lithic & 2 & 1.62 & JU & $8 / 31 / 2017$ & $\begin{array}{l}1 \text { bifacial thinning } \\
\text { flake }\end{array}$ \\
\hline 3 & 18 & $\begin{array}{l}170- \\
180 \\
\end{array}$ & Debitage & Tertiary & Lithic & 13 & 24.8 & JU & $8 / 31 / 2017$ & $\begin{array}{l}9 \text { bifacial thinning } \\
\text { flakes }\end{array}$ \\
\hline 4 & 1 & $0-10$ & \begin{tabular}{|l|} 
Debitage \\
\end{tabular} & Primary & Lithic & 1 & 12.19 & $\mathrm{JL}$ & $10 / 14 / 2016$ & \\
\hline 4 & 1 & $0-10$ & Debitage & Tertiary & Lithic & 14 & 7.15 & $\mathrm{JL}$ & $10 / 14 / 2016$ & $\begin{array}{l}12 \text { Bifacial } \\
\text { thinning flakes }\end{array}$ \\
\hline 4 & 2 & $10-20$ & Debitage & Primary & Lithic & 1 & 3.81 & $\mathrm{JL}$ & 10/13/2016 & \\
\hline 4 & 2 & $10-20$ & Debitage & Secondary & Lithic & 1 & 35.43 & $\mathrm{JL}$ & $10 / 13 / 2016$ & \\
\hline 4 & 2 & $10-20$ & Debitage & Tertiary & Lithic & 12 & 10.11 & $\mathrm{JL}$ & $10 / 13 / 2016$ & $\begin{array}{l}10 \text { Bifacial } \\
\text { thinning flakes }\end{array}$ \\
\hline 4 & 3 & $20-30$ & Debitage & Primary & Lithic & 25 & 29.05 & $\mathrm{JL}$ & $10 / 12 / 2016$ & $\begin{array}{l}11 \text { Bifacial } \\
\text { thinning flakes }\end{array}$ \\
\hline 4 & 3 & $20-30$ & Debitage & Secondary & Lithic & 14 & 11.42 & $\mathrm{JL}$ & $10 / 12 / 2016$ & $\begin{array}{l}4 \text { Bifacial thinning } \\
\text { flakes }\end{array}$ \\
\hline 4 & 3 & $20-30$ & Debitage & Tertiary & Lithic & 145 & 68.05 & $\mathrm{JL}$ & $10 / 12 / 2016$ & \begin{tabular}{|l|}
129 Bifacial \\
thinning flakes
\end{tabular} \\
\hline 4 & 3 & $20-30$ & Scraper & \begin{tabular}{|l|} 
Unifacial \\
scraper
\end{tabular} & Lithic & 1 & 4.07 & $\mathrm{JL}$ & $10 / 13 / 2016$ & Unifacially worked \\
\hline 4 & 4 & $30-40$ & Tool & Biface & Lithic & 1 & 3.74 & $\mathrm{JU}$ & $8 / 30 / 2017$ & thin, proximal \\
\hline 4 & 4 & $30-40$ & \begin{tabular}{|l|} 
Faunal \\
Remains \\
\end{tabular} & Mussel Shell & Shell & 2 & 7.76 & JU & $8 / 30 / 2017$ & Shell with hole \\
\hline 4 & 4 & $30-40$ & Ground Stone & Ground Stone & & 4 & 143.67 & $\mathrm{JU}$ & $8 / 30 / 2017$ & \\
\hline 4 & 4 & $30-40$ & \begin{tabular}{|l|} 
Organic \\
Remains \\
\end{tabular} & Charcoal & & 3 & 0.4 & JU & 8/30/2017 & From screen \\
\hline 4 & 4 & $30-40$ & $\begin{array}{l}\text { Special } \\
\text { Sample }\end{array}$ & DAUB & Clay & 5 & 6.45 & JU & 8/30/2017 & \\
\hline 4 & 4 & $30-40$ & \begin{tabular}{|l|} 
Faunal \\
Remains \\
\end{tabular} & Bone & & 7 & 4.96 & JU & 8/30/2017 & 1 burned \\
\hline 4 & 4 & $30-40$ & Debitage & Primary & Lithic & 7 & 72.6 & JU & 8/30/2017 & $\begin{array}{l}1 \text { bifacial thinning } \\
\text { flake }\end{array}$ \\
\hline 4 & 4 & $30-40$ & Debitage & Secondary & Lithic & 27 & 75.38 & JU & $8 / 30 / 2017$ & $\begin{array}{l}9 \text { bifacial thinning } \\
\text { flakes }\end{array}$ \\
\hline 4 & 4 & $30-40$ & Debitage & Tertiary & Lithic & 121 & 151.42 & JU & 8/30/2017 & $\begin{array}{l}70 \text { bifacial thinning } \\
\text { flakes }\end{array}$ \\
\hline 4 & 5 & $40-50$ & \begin{tabular}{|l|} 
Ground Stone \\
\end{tabular} & Ground Stone & & 1 & & $\mathrm{JU}$ & $8 / 30 / 2017$ & \\
\hline 4 & 5 & $40-50$ & \begin{tabular}{|l|} 
Faunal \\
Remains \\
\end{tabular} & Bone & & 1 & 7.14 & JU & 8/30/2017 & \\
\hline 4 & 5 & $40-50$ & Debitage & Primary & Lithic & 8 & 55.47 & JU & $8 / 30 / 2017$ & $\begin{array}{l}2 \text { bifacial thinning } \\
\text { flakes }\end{array}$ \\
\hline 4 & 5 & $40-50$ & Debitage & Secondary & Lithic & 17 & 33.69 & JU & 8/30/2017 & $\begin{array}{l}9 \text { bifacial thinning } \\
\text { flakes }\end{array}$ \\
\hline 4 & 5 & $40-50$ & Debitage & Tertiary & Lithic & 69 & 56.92 & JU & $8 / 30 / 2017$ & $\begin{array}{l}39 \text { bifacial thinning } \\
\text { flakes }\end{array}$ \\
\hline 4 & 6 & $50-60$ & $\begin{array}{l}\text { Organic } \\
\text { Remains } \\
\end{array}$ & Charcoal & & 3 & 0.37 & JU & 8/30/2017 & \\
\hline 4 & 6 & $50-60$ & Tool & Biface & Lithic & 1 & 6.36 & $\mathrm{JU}$ & $8 / 30 / 2017$ & thin, proximal \\
\hline 4 & 6 & $50-60$ & $\begin{array}{l}\text { Special } \\
\text { Sample }\end{array}$ & DAUB & Clay & 3 & 20.61 & JU & 8/30/2017 & \\
\hline 4 & 6 & $50-60$ & \begin{tabular}{|l|} 
Faunal \\
Remains \\
\end{tabular} & Bone & & 12 & 13.34 & JU & 8/30/2017 & 1 burned \\
\hline 4 & 6 & $50-60$ & Debitage & Primary & Lithic & 8 & 10.49 & JU & $8 / 30 / 2017$ & $\begin{array}{l}1 \text { bifacial thinning } \\
\text { flake }\end{array}$ \\
\hline 4 & 6 & $50-60$ & Debitage & Secondary & Lithic & 18 & 114.01 & JU & $8 / 30 / 2017$ & $\begin{array}{l}5 \text { bifacial thinning } \\
\text { flakes }\end{array}$ \\
\hline 4 & 6 & $50-60$ & Debitage & Tertiary & Lithic & 49 & 44.32 & JU & $8 / 30 / 2017$ & $\begin{array}{l}26 \text { bifacial thinning } \\
\text { flakes }\end{array}$ \\
\hline 4 & 7 & $60-70$ & \begin{tabular}{|l|} 
Faunal \\
Remains \\
\end{tabular} & Mussel Shell & Shell & 1 & 2.57 & JU & $8 / 30 / 2017$ & \\
\hline 4 & 7 & $60-70$ & \begin{tabular}{|l} 
Organic \\
Remains \\
\end{tabular} & Charcoal & & 5 & 2.19 & $\mathrm{JU}$ & $8 / 30 / 2017$ & \\
\hline 4 & 7 & $60-70$ & Ground Stone & Ground Stone & & 1 & 46.81 & $\mathrm{JU}$ & $8 / 30 / 2017$ & \\
\hline 4 & 7 & $60-70$ & Debitage & \begin{tabular}{|l|} 
Primary \\
\end{tabular} & Lithic & 3 & 14.9 & $\mathrm{JU}$ & $8 / 30 / 2017$ & \\
\hline 4 & 7 & $60-70$ & Debitage & Secondary & Lithic & 11 & 59.73 & $\mathrm{JU}$ & $8 / 30 / 2017$ & $\begin{array}{l}2 \text { bifacial thinning } \\
\text { flakes }\end{array}$ \\
\hline
\end{tabular}




\begin{tabular}{|c|c|c|c|c|c|c|c|c|c|c|}
\hline Unit & Level & $\begin{array}{l}\text { Depth } \\
\text { (cmbs) }\end{array}$ & Artifact Type & $\begin{array}{c}\text { Artifact } \\
\text { Description }\end{array}$ & Material & \begin{tabular}{|c|}
$\begin{array}{c}\text { Artifact } \\
\text { No. }\end{array}$ \\
\end{tabular} & $\begin{array}{c}\text { Weight } \\
\text { (g) }\end{array}$ & Recorders & Date & Comments \\
\hline 4 & 7 & $60-70$ & Debitage & Tertiary & Lithic & 17 & 42.08 & JU & $8 / 30 / 207$ & $\begin{array}{l}12 \text { bifacial thinning } \\
\text { flakes }\end{array}$ \\
\hline 4 & 8 & $70-80$ & \begin{tabular}{|l} 
Faunal \\
Remains \\
\end{tabular} & Mussel Shell & Shell & 3 & 6.86 & JU & $8 / 30 / 2017$ & \\
\hline 4 & 8 & $70-80$ & \begin{tabular}{|l|} 
Faunal \\
Remains \\
\end{tabular} & Bone & & 3 & 5.3 & $\mathrm{JU}$ & $8 / 30 / 2017$ & \\
\hline 4 & 8 & $70-80$ & \begin{tabular}{|l|} 
Debitage \\
\end{tabular} & Primary & Lithic & 2 & 5.13 & $\mathrm{JU}$ & $8 / 30 / 2017$ & \\
\hline 4 & 8 & $70-80$ & Debitage & Secondary & Lithic & 10 & 60.67 & JU & $8 / 30 / 2017$ & $\begin{array}{l}3 \text { bifacial thinning } \\
\text { flakes } \\
\end{array}$ \\
\hline 4 & 8 & $70-80$ & Debitage & Tertiary & Lithic & 27 & 60.79 & $\mathrm{JU}$ & 8/30/2017 & $\begin{array}{l}15 \text { bifacial thinning } \\
\text { flakes }\end{array}$ \\
\hline 4 & 9 & $80-90$ & \begin{tabular}{|l} 
Faunal \\
Remains \\
\end{tabular} & Mussel Shell & Shell & 4 & 8.46 & $\mathrm{JU}$ & 8/30/2017 & \\
\hline 4 & 9 & $80-90$ & \begin{tabular}{|l|} 
Faunal \\
Remains \\
\end{tabular} & Tooth & Bone & 1 & 2.52 & JU & 8/30/2017 & \\
\hline 4 & 9 & $80-90$ & \begin{tabular}{|l} 
Faunal \\
Remains \\
\end{tabular} & Bone & & 18 & 36.17 & JU & 8/30/2017 & \\
\hline 4 & 9 & $80-90$ & Debitage & Primary & Lithic & 13 & 80.91 & $\mathrm{JU}$ & $8 / 30 / 2017$ & $\begin{array}{l}1 \text { bifacial thinning } \\
\text { flake } \\
\end{array}$ \\
\hline 4 & 9 & $80-90$ & Debitage & Secondary & Lithic & 27 & 176.2 & JU & 8/30/2017 & $\begin{array}{l}7 \text { bifacial thinning } \\
\text { flakes } \\
\end{array}$ \\
\hline 4 & 9 & $80-90$ & Debitage & Tertiary & Lithic & 61 & 110.78 & JU & $8 / 30 / 2017$ & $\begin{array}{l}39 \text { bifacial thinning } \\
\text { flakes }\end{array}$ \\
\hline 4 & 10 & $90-100$ & Tool & Biface & Lithic & 1 & 0.51 & $\mathrm{JU}$ & $8 / 30 / 2017$ & Distal \\
\hline 4 & 10 & $90-100$ & \begin{tabular}{|l|} 
Faunal \\
Remains \\
\end{tabular} & Bone & & 10 & 17.02 & JU & 8/30/2017 & \\
\hline 4 & 10 & $90-100$ & Debitage & Primary & Lithic & 6 & 36.61 & JU & 8/30/2017 & $\begin{array}{l}1 \text { bifacial thinning } \\
\text { flake }\end{array}$ \\
\hline 4 & 10 & $90-100$ & Debitage & Secondary & Lithic & 11 & 62.59 & JU & 8/30/2017 & $\begin{array}{l}4 \text { bifacial thinning } \\
\text { flakes }\end{array}$ \\
\hline 4 & 10 & $90-100$ & Debitage & Tertiary & Lithic & 33 & 62.77 & JU & 8/30/2017 & $\begin{array}{l}25 \text { bifacial thinning } \\
\text { flakes }\end{array}$ \\
\hline 4 & 11 & $\begin{array}{l}100- \\
110\end{array}$ & \begin{tabular}{|l|} 
Faunal \\
Remains \\
\end{tabular} & Bone & & 3 & 2.85 & $\mathrm{JU}$ & 8/30/2017 & \\
\hline 4 & 11 & $\begin{array}{l}100- \\
110 \\
\end{array}$ & Tool & Biface & Lithic & 1 & 4.74 & $\mathrm{JU}$ & 8/30/2017 & thin, distal \\
\hline 4 & 11 & $\begin{array}{l}100- \\
110\end{array}$ & Debitage & Primary & Lithic & 3 & 6.97 & $\mathrm{JU}$ & 8/30/2017 & \\
\hline 4 & 11 & $\begin{array}{l}100- \\
110 \\
\end{array}$ & Debitage & Secondary & Lithic & 8 & 38.54 & $\mathrm{JU}$ & 8/30/2017 & $\begin{array}{l}3 \text { bifacial thinning } \\
\text { flakes }\end{array}$ \\
\hline 4 & 11 & $\begin{array}{l}100- \\
110 \\
\end{array}$ & Debitage & Tertiary & Lithic & 24 & 15.09 & JU & $8 / 30 / 2017$ & $\begin{array}{l}17 \text { bifacial thinning } \\
\text { flakes }\end{array}$ \\
\hline 4 & 11 & $\begin{array}{l}100- \\
110\end{array}$ & Tool & Biface & Lithic & 1 & 116.2 & $\mathrm{JU}$ & 8/30/2017 & $\begin{array}{l}\text { Biface blank, } \\
\text { chunky }\end{array}$ \\
\hline 4 & 12 & $\begin{array}{l}110- \\
120 \\
\end{array}$ & Debitage & Secondary & Lithic & 7 & 31.4 & $\mathrm{JU}$ & 8/30/2017 & $\begin{array}{l}3 \text { bifacial thinning } \\
\text { flakes } \\
\end{array}$ \\
\hline 4 & 12 & $\begin{array}{l}110- \\
120\end{array}$ & Debitage & Tertiary & Lithic & 18 & 20.9 & $\mathrm{JU}$ & 8/30/2017 & $\begin{array}{l}15 \text { bifacial thinning } \\
\text { flakes }\end{array}$ \\
\hline 4 & 13 & $\begin{array}{l}120- \\
130\end{array}$ & \begin{tabular}{|l} 
Projectile \\
Point
\end{tabular} & $\begin{array}{l}\text { Untyped Arrow } \\
\text { Point Preform }\end{array}$ & Lithic & 1 & 4.45 & JU & $8 / 30 / 2017$ & \begin{tabular}{|l|} 
probable \\
unfinished \\
preform-failure to \\
thin; unifacial \\
\end{tabular} \\
\hline 4 & 13 & $\begin{array}{l}120- \\
130\end{array}$ & \begin{tabular}{|l} 
Faunal \\
Remains \\
\end{tabular} & Bone & & 1 & 0.47 & JU & 8/30/2017 & \\
\hline 4 & 13 & $\begin{array}{l}120- \\
130 \\
\end{array}$ & Debitage & Secondary & Lithic & 9 & 52.35 & JU & $8 / 30 / 2017$ & $\begin{array}{l}2 \text { bifacial thinning } \\
\text { flakes }\end{array}$ \\
\hline 4 & 13 & $\begin{array}{l}120- \\
130 \\
\end{array}$ & Debitage & Tertiary & Lithic & 14 & 29.85 & JU & $8 / 30 / 2017$ & $\begin{array}{l}12 \text { bifacial thinning } \\
\text { flakes }\end{array}$ \\
\hline 4 & 13 & $\begin{array}{l}120- \\
130 \\
\end{array}$ & Tool & Biface & Lithic & 1 & 35.09 & JU & $8 / 30 / 2017$ & thin, proximal \\
\hline 4 & 13 & $\begin{array}{l}120- \\
130 \\
\end{array}$ & Tool & Utilized Flake & Lithic & 1 & 23.13 & $\mathrm{JU}$ & 8/30/2017 & \\
\hline 4 & 14 & $\begin{array}{l}130- \\
140\end{array}$ & Debitage & Tertiary & Lithic & 11 & 14.25 & $\mathrm{JU}$ & 8/30/2017 & $\begin{array}{l}8 \text { bifacial thinning } \\
\text { flakes }\end{array}$ \\
\hline 4 & 14 & $\begin{array}{l}130- \\
140 \\
\end{array}$ & \begin{tabular}{|l} 
Faunal \\
Remains \\
\end{tabular} & Bone & & 6 & 1.53 & JU & $8 / 30 / 2017$ & \\
\hline
\end{tabular}




\begin{tabular}{|c|c|c|c|c|c|c|c|c|c|c|}
\hline Unit & Level & $\begin{array}{l}\text { Depth } \\
\text { (cmbs) }\end{array}$ & Artifact Type & $\begin{array}{c}\text { Artifact } \\
\text { Description }\end{array}$ & Material & $\begin{array}{c}\text { Artifact } \\
\text { No. }\end{array}$ & $\begin{array}{c}\text { Weight } \\
\text { (g) }\end{array}$ & Recorders & Date & Comments \\
\hline 4 & 14 & $\begin{array}{c}130- \\
140 \\
\end{array}$ & $\begin{array}{l}\text { Organic } \\
\text { Remains }\end{array}$ & Charcoal & & 4 & 0.28 & JU & 8/30/2017 & \\
\hline 4 & 14 & $\begin{array}{l}130- \\
140 \\
\end{array}$ & Tool & Chopper & Lithic & 1 & 91.32 & JU & 8/30/2017 & \\
\hline 4 & 15 & $\begin{array}{l}140- \\
150 \\
\end{array}$ & \begin{tabular}{|l|} 
Faunal \\
Remains \\
\end{tabular} & Bone & & 9 & 3.64 & JU & $8 / 30 / 2017$ & \\
\hline 4 & 15 & 140 & \begin{tabular}{|l|} 
Faunal \\
Remains \\
\end{tabular} & Bone & & 13 & 10.13 & JU & $8 / 30 / 2017$ & \\
\hline 4 & 15 & 140 & Tool & Chopper & Lithic & 1 & 152.49 & $\mathrm{JU}$ & $8 / 30 / 2017$ & \\
\hline 4 & 15 & $\begin{array}{l}140- \\
150\end{array}$ & \begin{tabular}{|l} 
Faunal \\
Remains \\
\end{tabular} & Tooth & Bone & 1 & 0.86 & JU & 8/30/2017 & \\
\hline 4 & 15 & $\begin{array}{l}140- \\
150 \\
\end{array}$ & \begin{tabular}{|l|} 
Faunal \\
Remains \\
\end{tabular} & Bone & & 29 & 8.94 & JU & 8/30/2017 & \\
\hline 4 & 15 & $\begin{array}{l}140- \\
150\end{array}$ & Debitage & Primary & Lithic & 1 & 5.92 & JU & 8/30/2017 & \\
\hline 4 & 15 & $\begin{array}{l}140- \\
150 \\
\end{array}$ & Debitage & Secondary & Lithic & 2 & 18.65 & $\mathrm{JU}$ & 8/30/2017 & \\
\hline 4 & 15 & $\begin{array}{l}140- \\
150\end{array}$ & Debitage & Tertiary & Lithic & 13 & 12.45 & JU & 8/30/2017 & $\begin{array}{l}9 \text { bifacial thinning } \\
\text { flakes }\end{array}$ \\
\hline 4 & 16 & $\begin{array}{l}150- \\
160 \\
\end{array}$ & \begin{tabular}{|l|} 
Faunal \\
Remains \\
\end{tabular} & Bone & & 2 & 0.48 & $\mathrm{JU}$ & 8/31/2017 & \\
\hline 4 & 16 & $\begin{array}{l}150- \\
160 \\
\end{array}$ & Debitage & Secondary & Lithic & 1 & 75.89 & JU & 8/31/2017 & \\
\hline 4 & 16 & $\begin{array}{l}150- \\
160\end{array}$ & Debitage & Tertiary & Lithic & 9 & 12.33 & JU & 8/31/2017 & $\begin{array}{l}8 \text { bifacial thinning } \\
\text { flakes }\end{array}$ \\
\hline 4 & $5-6$ & - & $\begin{array}{l}\text { Faunal } \\
\text { Remains }\end{array}$ & Mussel Shell & Shell & 1 & 7.13 & JU & 8/30/2017 & $\begin{array}{l}\text { In rodent burrow } \\
\text { between levels } 5 \\
\text { and } 6\end{array}$ \\
\hline 5 & 1 & $0-10$ & Debitage & Secondary & Lithic & 1 & 18.24 & $\mathrm{JU}$ & 9/1/2017 & \\
\hline 5 & 2 & $10-20$ & Debitage & Tertiary & Lithic & 3 & 1.48 & JU & 9/1/2017 & $\begin{array}{l}1 \text { bifacial thinning } \\
\text { flake }\end{array}$ \\
\hline 5 & 2 & $10-20$ & Core & Core & Lithic & 1 & 150.39 & $\mathrm{JU}$ & 9/1/2017 & \\
\hline 5 & 3 & $20-30$ & \begin{tabular}{|l|} 
Faunal \\
Remains \\
\end{tabular} & Bone & & 1 & 0.73 & JU & 9/1/2017 & \\
\hline 5 & 3 & $20-30$ & Debitage & Secondary & Lithic & 1 & 0.35 & $\mathrm{JU}$ & 9/1/2017 & \\
\hline 5 & 3 & $20-30$ & Debitage & Tertiary & Lithic & 3 & 6.1 & JU & 9/1/2017 & $\begin{array}{l}2 \text { bifacial thinning } \\
\text { flakes }\end{array}$ \\
\hline 5 & 4 & $30-40$ & \begin{tabular}{|l|} 
Faunal \\
Remains
\end{tabular} & Mussel Shell & Shell & 1 & 4.32 & $\mathrm{JU}$ & 9/1/2017 & \\
\hline 5 & 4 & $30-40$ & Debitage & Primary & Lithic & 3 & 4.3 & $\mathrm{JU}$ & 9/1/2017 & \\
\hline 5 & 4 & $30-40$ & Debitage & Secondary & Lithic & 5 & 36.14 & JU & 9/1/2017 & $\begin{array}{l}1 \text { bifacial thinning } \\
\text { flake }\end{array}$ \\
\hline 5 & 4 & $30-40$ & Debitage & Tertiary & Lithic & 18 & 33.86 & $\mathrm{JU}$ & $9 / 1 / 2017$ & $\begin{array}{l}11 \text { bifacial thinning } \\
\text { flakes }\end{array}$ \\
\hline 5 & 5 & $40-50$ & Debitage & Primary & Lithic & 2 & 3.25 & JU & 9/1/2017 & $\begin{array}{l}1 \text { bifacial thinning } \\
\text { flake }\end{array}$ \\
\hline 5 & 5 & $40-50$ & Debitage & Secondary & Lithic & 7 & 13.98 & JU & 9/1/2017 & $\begin{array}{l}3 \text { bifacial thinning } \\
\text { flakes }\end{array}$ \\
\hline 5 & 5 & $40-50$ & Debitage & Tertiary & Lithic & 34 & 20.32 & JU & 9/1/2017 & $\begin{array}{l}21 \text { bifacial thinning } \\
\text { flakes }\end{array}$ \\
\hline 5 & 6 & $50-60$ & \begin{tabular}{|l|} 
Faunal \\
Remains \\
\end{tabular} & Bone & & 2 & 0.51 & JU & 9/1/2017 & 1 burned \\
\hline 5 & 6 & $50-60$ & Debitage & Primary & Lithic & 3 & 3.56 & $\mathrm{JU}$ & 9/1/2017 & \\
\hline 5 & 6 & $50-60$ & Debitage & Secondary & Lithic & 4 & 1.35 & JU & 9/1/2017 & $\begin{array}{l}1 \text { bifacial thinning } \\
\text { flake }\end{array}$ \\
\hline 5 & 6 & $50-60$ & Debitage & Tertiary & Lithic & 28 & 29.77 & $\mathrm{JU}$ & 9/1/2017 & $\begin{array}{l}21 \text { bifacial thinning } \\
\text { flakes }\end{array}$ \\
\hline 5 & 7 & $60-70$ & \begin{tabular}{|l} 
Faunal \\
Remains
\end{tabular} & Bone & & 1 & 0.26 & JU & 9/1/2017 & Burned \\
\hline 5 & 7 & $60-70$ & Debitage & Primary & Lithic & 5 & 34.48 & JU & 9/1/2017 & $\begin{array}{l}1 \text { bifacial thinning } \\
\text { flake }\end{array}$ \\
\hline 5 & 7 & $60-70$ & Debitage & Secondary & Lithic & 4 & 20.65 & JU & 9/1/2017 & $\begin{array}{l}2 \text { bifacial thinning } \\
\text { flakes }\end{array}$ \\
\hline 5 & 7 & $60-70$ & Debitage & Tertiary & Lithic & 19 & 22.87 & JU & 9/1/2017 & $\begin{array}{l}8 \text { bifacial thinning } \\
\text { flakes }\end{array}$ \\
\hline 5 & 8 & $70-80$ & Debitage & Secondary & Lithic & 1 & 1.74 & $\mathrm{JU}$ & 9/1/2017 & \\
\hline
\end{tabular}




\begin{tabular}{|c|c|c|c|c|c|c|c|c|c|c|}
\hline Unit & Level & $\begin{array}{l}\text { Depth } \\
\text { (cmbs) }\end{array}$ & Artifact Type & $\begin{array}{c}\text { Artifact } \\
\text { Description }\end{array}$ & Material & \begin{tabular}{|c|}
$\begin{array}{c}\text { Artifact } \\
\text { No. }\end{array}$ \\
\end{tabular} & \begin{tabular}{|c|} 
Weight \\
(g)
\end{tabular} & Recorders & Date & Comments \\
\hline 5 & 8 & $70-80$ & Debitage & Tertiary & Lithic & 5 & 2.59 & JU & 9/1/2017 & $\begin{array}{l}3 \text { bifacial thinning } \\
\text { flakes }\end{array}$ \\
\hline 5 & 9 & $80-90$ & Debitage & Primary & Lithic & 1 & 1.04 & $\mathrm{JU}$ & 9/1/2017 & \\
\hline 5 & 9 & $80-90$ & Debitage & Secondary & Lithic & 2 & 4.8 & $\mathrm{JU}$ & $9 / 1 / 2017$ & \\
\hline 5 & 9 & $80-90$ & Debitage & Tertiary & Lithic & 6 & 11.15 & $\mathrm{JU}$ & 9/1/2017 & $\begin{array}{l}5 \text { bifacial thinning } \\
\text { flakes }\end{array}$ \\
\hline 5 & 10 & $90-100$ & Debitage & Primary & Lithic & 1 & 17.5 & $\mathrm{JU}$ & 9/1/2017 & \\
\hline 5 & 10 & $90-100$ & Debitage & Secondary & Lithic & 2 & 8.49 & $\mathrm{JU}$ & 9/1/2017 & \\
\hline 5 & 10 & $90-100$ & Debitage & Tertiary & Lithic & 3 & 5.45 & $\mathrm{JU}$ & 9/1/2017 & $\begin{array}{l}1 \text { bifacial thinning } \\
\text { flake }\end{array}$ \\
\hline 5 & 11 & $\begin{array}{l}100- \\
110\end{array}$ & Debitage & Secondary & Lithic & 2 & 7.61 & JU & 9/1/2017 & \\
\hline 5 & 11 & $\begin{array}{l}100- \\
110\end{array}$ & Debitage & Tertiary & Lithic & 3 & 8.4 & $\mathrm{JU}$ & 9/1/2017 & $\begin{array}{l}2 \text { bifacial thinning } \\
\text { flakes }\end{array}$ \\
\hline 1 & 2 & $10-20$ & Burned Rock & Burned Rock & Lithic & 7 & 500 & DR & $10 / 19 / 2017$ & Not collected \\
\hline 1 & 3 & $20-30$ & Burned Rock & Burned Rock & Lithic & 1 & $<100$ & DR & $10 / 19 / 2017$ & Not collected \\
\hline 1 & 4 & $30-40$ & Burned Rock & Burned Rock & Lithic & 4 & 200 & DR & 10/19/2017 & Not collected \\
\hline 1 & 5 & $40-50$ & Burned Rock & Burned Rock & Lithic & 8 & 800 & DR & 10/19/2017 & Not collected \\
\hline 1 & 6 & $50-60$ & Burned Rock & Burned Rock & Lithic & 11 & 600 & DR & $10 / 19 / 2017$ & Not collected \\
\hline 1 & 7 & $60-70$ & Burned Rock & Burned Rock & Lithic & 8 & 600 & DR & $10 / 19 / 2017$ & Not collected \\
\hline 1 & 8 & $70-80$ & Burned Rock & Burned Rock & Lithic & 13 & 600 & DR & $10 / 19 / 2017$ & Not collected \\
\hline 1 & 9 & $80-90$ & Burned Rock & Burned Rock & Lithic & 12 & 750 & DR & $10 / 19 / 2017$ & Not collected \\
\hline 1 & 10 & $90-100$ & Burned Rock & Burned Rock & Lithic & 17 & 1250 & DR & $10 / 19 / 2017$ & Not collected \\
\hline 1 & 11 & $\begin{array}{c}100- \\
110 \\
\end{array}$ & Burned Rock & Burned Rock & Lithic & 8 & 600 & DR & $10 / 19 / 2017$ & Not collected \\
\hline 1 & 13 & $\begin{array}{c}120- \\
130 \\
\end{array}$ & Burned Rock & Burned Rock & Lithic & 7 & 205 & DR & $10 / 19 / 2017$ & Not collected \\
\hline 2 & 1 & $0-10$ & Burned Rock & Burned Rock & Lithic & 2 & 100 & DR & $10 / 19 / 2017$ & Not collected \\
\hline 2 & 2 & $10-20$ & Burned Rock & Burned Rock & Lithic & 16 & 300 & DR & $10 / 19 / 2017$ & Not collected \\
\hline 2 & 3 & $20-30$ & Burned Rock & Burned Rock & Lithic & 2 & 110 & DR & $10 / 19 / 2017$ & Not collected \\
\hline 2 & 5 & $40-50$ & Burned Rock & Burned Rock & Lithic & 15 & 300 & DR & $10 / 19 / 2017$ & Not collected \\
\hline 2 & 6 & $50-60$ & Burned Rock & Burned Rock & Lithic & 4 & 250 & DR & $10 / 19 / 2017$ & Not collected \\
\hline 2 & 7 & $60-70$ & Burned Rock & Burned Rock & Lithic & 5 & $>600$ & DR & $10 / 19 / 2017$ & Not collected \\
\hline 2 & 8 & $70-80$ & Burned Rock & Burned Rock & Lithic & 12 & 400 & DR & $10 / 19 / 2017$ & Not collected \\
\hline 2 & 9 & $80-90$ & Burned Rock & Burned Rock & Lithic & 6 & 100 & DR & 10/19/2017 & Not collected \\
\hline 2 & 10 & 90-100 & Burned Rock & Burned Rock & Lithic & 11 & 500 & DR & $10 / 19 / 2017$ & Not collected \\
\hline 2 & 11 & $\begin{array}{c}100- \\
110 \\
\end{array}$ & Burned Rock & Burned Rock & Lithic & 5 & 400 & DR & $10 / 19 / 2017$ & Not collected \\
\hline 2 & 12 & $\begin{array}{c}110- \\
120 \\
\end{array}$ & Burned Rock & Burned Rock & Lithic & 7 & 2700 & DR & $10 / 19 / 2017$ & Not collected \\
\hline 2 & 13 & $\begin{array}{c}120- \\
130\end{array}$ & Burned Rock & Burned Rock & Lithic & 7 & 500 & DR & $10 / 19 / 2017$ & Not collected \\
\hline 3 & 1 & $0-10$ & Burned Rock & Burned Rock & Lithic & 2 & $<100$ & DR & $10 / 19 / 2017$ & Not collected \\
\hline 3 & 5 & $40-50$ & Burned Rock & Burned Rock & Lithic & 5 & 150 & DR & $10 / 19 / 2017$ & Not collected \\
\hline 3 & 6 & $50-60$ & Burned Rock & Burned Rock & Lithic & 32 & 200 & DR & $10 / 19 / 2017$ & Not collected \\
\hline 3 & 9 & $80-90$ & Burned Rock & Burned Rock & Lithic & 5 & 100 & DR & 10/19/2017 & Not collected \\
\hline 3 & 11 & $\begin{array}{l}100- \\
110\end{array}$ & Burned Rock & Burned Rock & Lithic & 5 & 200 & DR & $10 / 19 / 2017$ & Not collected \\
\hline 3 & 12 & $\begin{array}{l}110- \\
120\end{array}$ & Burned Rock & Burned Rock & Lithic & 13 & 600 & DR & $10 / 19 / 2017$ & Not collected \\
\hline 3 & 13 & $\begin{array}{l}120- \\
130 \\
\end{array}$ & Burned Rock & Burned Rock & Lithic & 1 & 100 & DR & $10 / 19 / 2017$ & Not collected \\
\hline 3 & 16 & $\begin{array}{l}150- \\
160\end{array}$ & Burned Rock & Burned Rock & Lithic & 2 & 200 & DR & $10 / 19 / 2017$ & Not collected \\
\hline 4 & 3 & $20-30$ & Burned Rock & Burned Rock & Lithic & 4 & 100 & DR & 10/19/2017 & Not collected \\
\hline 4 & 4 & $30-40$ & Burned Rock & Burned Rock & Lithic & 16 & 600 & DR & $10 / 19 / 2017$ & Not collected \\
\hline 4 & 5 & $40-50$ & Burned Rock & Burned Rock & Lithic & 21 & 1000 & DR & 10/19/2017 & Not collected \\
\hline 4 & 6 & $50-60$ & Burned Rock & Burned Rock & Lithic & 20 & 800 & DR & $10 / 19 / 2017$ & Not collected \\
\hline 4 & 7 & $60-70$ & Burned Rock & Burned Rock & Lithic & 17 & 600 & DR & $10 / 19 / 2017$ & Not collected \\
\hline 4 & 8 & $70-80$ & Burned Rock & Burned Rock & Lithic & 21 & 400 & DR & $10 / 19 / 2017$ & Not collected \\
\hline 4 & 9 & $80-90$ & Burned Rock & Burned Rock & Lithic & 60 & 1100 & DR & 10/19/2017 & Not collected \\
\hline 4 & 10 & $90-100$ & Burned Rock & Burned Rock & Lithic & 20 & 1300 & DR & $10 / 19 / 2017$ & Not collected \\
\hline
\end{tabular}




\begin{tabular}{|c|c|c|c|c|c|c|c|c|c|c|}
\hline Unit & Level & $\begin{array}{l}\text { Depth } \\
\text { (cmbs) }\end{array}$ & Artifact Type & $\begin{array}{c}\text { Artifact } \\
\text { Description }\end{array}$ & Material & $\begin{array}{c}\text { Artifact } \\
\text { No. }\end{array}$ & $\begin{array}{c}\text { Weight } \\
\text { (g) }\end{array}$ & Recorders & Date & Comments \\
\hline 4 & 11 & $\begin{array}{l}100- \\
110\end{array}$ & Burned Rock & Burned Rock & Lithic & 14 & 600 & DR & 10/19/2017 & Not collected \\
\hline 4 & 12 & $\begin{array}{l}110- \\
120 \\
\end{array}$ & Burned Rock & Burned Rock & Lithic & 9 & 200 & DR & 10/19/2017 & Not collected \\
\hline 4 & 13 & $\begin{array}{c}120- \\
130\end{array}$ & Burned Rock & Burned Rock & Lithic & 14 & 400 & DR & 10/19/2017 & Not collected \\
\hline 4 & 14 & $\begin{array}{l}130- \\
140\end{array}$ & Burned Rock & Burned Rock & Lithic & 6 & 100 & DR & 10/19/2017 & Not collected \\
\hline 4 & 15 & $\begin{array}{l}140- \\
150\end{array}$ & Burned Rock & Burned Rock & Lithic & 8 & 100 & DR & 10/19/2017 & Not collected \\
\hline 4 & 16 & $\begin{array}{l}150- \\
160\end{array}$ & Burned Rock & Burned Rock & Lithic & 3 & 200 & DR & 10/19/2017 & Not collected \\
\hline 5 & 4 & $30-40$ & Burned Rock & Burned Rock & Lithic & 3 & 100 & DR & $10 / 19 / 2017$ & Not collected \\
\hline 5 & 5 & $40-50$ & Burned Rock & Burned Rock & Lithic & 17 & 1000 & DR & $10 / 19 / 2017$ & Not collected \\
\hline 5 & 6 & $50-60$ & Burned Rock & Burned Rock & Lithic & 31 & 2200 & DR & 10/19/2017 & Not collected \\
\hline 5 & 7 & $60-70$ & Burned Rock & Burned Rock & Lithic & 17 & 1200 & DR & 10/19/2017 & Not collected \\
\hline 5 & 8 & $70-80$ & Burned Rock & Burned Rock & Lithic & 2 & 100 & DR & $10 / 19 / 2017$ & Not collected \\
\hline 5 & 9 & $80-90$ & Burned Rock & Burned Rock & Lithic & 13 & 100 & DR & $10 / 19 / 2017$ & Not collected \\
\hline 5 & 10 & $90-100$ & Burned Rock & Burned Rock & Lithic & 2 & 100 & DR & $10 / 19 / 2017$ & Not collected \\
\hline 5 & 11 & $\begin{array}{c}100- \\
110\end{array}$ & Burned Rock & Burned Rock & Lithic & 2 & 100 & DR & 10/19/2017 & Not collected \\
\hline Surface & Surface & & Biface & $\begin{array}{l}\text { Late-stage, } \\
\text { thin biface }\end{array}$ & Lithic & 1 & 56.3 & $\mathrm{JL}$ & 10/14/2016 & $\begin{array}{l}\text { Surface collection. } \\
\text { Thin biface. }\end{array}$ \\
\hline
\end{tabular}





\section{APPENDIX B}

41GU177 Data Recovery Specimen Inventory and Burned Rock Tables 



\begin{tabular}{|c|c|c|c|c|c|c|c|c|c|c|c|c|c|c|c|c|c|c|c|c|c|}
\hline Lot No. & \begin{tabular}{|c|}
$\begin{array}{c}\text { Specimen } \\
\text { No. }\end{array}$ \\
\end{tabular} & FS No. & UI No. & FTR No. & $\begin{array}{c}\text { Sample } \\
\text { No. }\end{array}$ & $\begin{array}{c}\text { Excavation } \\
\text { Block }\end{array}$ & Stratum (AU) & Northing & Easting & Level & $\begin{array}{c}\text { Elevation } \\
(\mathrm{m})\end{array}$ & Depth (cmbd) & Point Prov. & Artifact Class & Artifact Type & \begin{tabular}{|c|} 
Artifact \\
Description \\
\end{tabular} & Material & $\begin{array}{c}\text { Number of } \\
\text { artifacts }\end{array}$ & Weight (g) & Comments & Date \\
\hline 314 & 1 & 467 & - & - & - & A & 1 & No995 & E1012 & 1 & $99.7-99.6$ & $0-10$ & - & Detritus & Debitage & Complete Flake & Lithic & 1 & 1.39 & & 12/11/2017 \\
\hline 314 & 2 & 467 & - & - & - & A & 1 & No995 & E1012 & 1 & 99.7-99.6 & $0-10$ & - & Detritus & Debitage & Broken Flake & Lithic & 3 & 6.99 & & 12/11/2017 \\
\hline 314 & 3 & 467 & - & - & - & A & 1 & No995 & E1012 & 1 & $99.7-99.6$ & $0-10$ & - & Detritus & Debitage & Thermal Shatter & Lithic & 2 & 8.18 & & 12/11/2017 \\
\hline 315 & 1 & 475 & - & - & - & A & 1 & No995 & E1012 & 2 & $99.6-99.5$ & $10-20$ & - & Detritus & Debitage & Thermal Shatter & Lithic & 1 & 0.27 & & 12/11/2017 \\
\hline 316 & 1 & 480 & - & - & - & A & "I & No995 & E1012 & 3 & 99.5-99.4 & $20-30$ & - & Detritus & Debitage & Complete Flake & Lithic & 1 & 0.14 & & $\mid 12 / 11 / 2017$ \\
\hline 316 & 2 & 480 & - & - & - & A & "I & No995 & E1012 & 3 & $99.5-99.4$ & $20-30$ & - & Detritus & Debitage & \begin{tabular}{|l|} 
Proximal Flake \\
\end{tabular} & Lithic & 3 & 2.03 & & 12/11/2017 \\
\hline 316 & 3 & 480 & - & - & - & A & "I & No995 & E1012 & 3 & $99.5-99.4$ & $20-30$ & - & Detritus & Debitage & Broken Flake & Lithic & 3 & 0.92 & & 12/11/2017 \\
\hline 316 & 4 & 480 & - & - & - & A & " & No995 & E1012 & 3 & 99.5-99.4 & 20-30 & - & Detritus & Debitage & Thermal Shatter & Lithic & 1 & 0.29 & & $\mid 12 / 11 / 2017$ \\
\hline 316 & 5 & 480 & - & - & - & A & II & No995 & E1012 & 3 & $99.5-99.4$ & $20-30$ & - & Faunal Remains & Shell & Mussel Shell & Shell & 1 & 3.69 & & 12/11/2017 \\
\hline 316 & 7 & 480 & - & - & - & A & "I & N0995 & E1012 & 3 & $99.5-99.4$ & 20-30 & - & Formal Tool & Biface & $\begin{array}{ll}\text { Notched Tool } \\
\end{array}$ & Fine Grain Chert & 1 & 4.72 & & \begin{tabular}{|l|}
$12 / 11 / 2017$ \\
\end{tabular} \\
\hline 317 & 1 & 485 & - & - & - & A & 11 & No995 & E1012 & 4 & 99.4-99.3 & $30-40$ & - & Detritus & Debitage & Complete Flake & Lithic & 5 & 29.72 & & \begin{tabular}{|l|}
$12 / 11 / 2017$ \\
\end{tabular} \\
\hline 317 & 2 & 485 & - & - & - & A & "I & N0995 & E1012 & 4 & $99.4-99.3$ & $30-40$ & - & Detritus & Debitage & Proximal Flake & Lithic & 6 & 4.35 & & \begin{tabular}{|l|}
$12 / 11 / 2017$ \\
\end{tabular} \\
\hline 317 & 3 & 485 & - & - & - & A & "I & N0995 & E1012 & 4 & 99.499 .3 & $30-40$ & - & Detritus & Debitage & Broken Flake & Lithic & 12 & 13.88 & & 12/11/2017 \\
\hline 317 & 4 & 485 & - & - & - & A & "I & N0995 & E1012 & 4 & 99.499 .3 & $30-40$ & - & Detritus & Debitage & Thermal Shatter & Lithic & 4 & 4.58 & & 12/11/2017 \\
\hline 317 & 5 & 485 & - & - & - & A & 11 & No995 & E1012 & 4 & 99.499 .3 & $30-40$ & - & Faunal Remains & Shell & Mussel Shell & Shell & 1 & 0.49 & & 12/11/2017 \\
\hline 318 & 1 & 492 & - & - & - & A & "I & No995 & E1012 & 5 & $99.3-99.2$ & $40-50$ & - & Detritus & Debitage & Complete Flake & Lithic & 9 & 15.27 & & 12/12/2017 \\
\hline 318 & 2 & 492 & - & - & - & A & " & No995 & E1012 & 5 & 99.3-99.2 & $40-50$ & - & Detritus & Debitage & Proximal Flake & Lithic & 8 & 7.1 & & 12/12/2017 \\
\hline 318 & 3 & 492 & - & - & - & A & " & No995 & E1012 & 5 & $99.3-99.2$ & $40-50$ & - & Detritus & Debitage & Broken Flake & Lithic & 22 & 61.31 & & 12/12/2017 \\
\hline 318 & 4 & 492 & - & - & - & A & "I & No995 & E1012 & 5 & $99.3-99.2$ & $40-50$ & - & Detritus & Debitage & Thermal Shatter & Lithic & 10 & 7.51 & & 12/12/2017 \\
\hline 318 & 5 & 492 & - & - & - & A & "I & No995 & E1012 & 5 & $99.3-99.2$ & $40-50$ & - & \begin{tabular}{l|l} 
Informal Tool &
\end{tabular} & \begin{tabular}{|l|} 
Modified Flake \\
\end{tabular} & Edge-modified & Fine Grain Chert & 1 & 23.74 & & 12/12/2017 \\
\hline 318 & 6 & 492 & - & - & - & A & $\|$ & No995 & E1012 & 5 & $99.3-99.2$ & $40-50$ & - & \begin{tabular}{|l|l} 
Informal Tool &
\end{tabular} & Modified Flake & \begin{tabular}{|l|} 
Edge-modified \\
\end{tabular} & Fine Grain Chert & 1 & 1.21 & & 12/12/2/2017 \\
\hline 319 & 1 & 501 & - & - & - & A & IIII & No995 & E1012 & 6 & $99.2-99.1$ & $50-60$ & - & Detritus & Debitage & Complete Flake & Lithic & 6 & 69.41 & & 12/12/2017 \\
\hline 319 & 2 & 501 & - & - & - & A & IIII & N0995 & E1012 & 6 & $99.2-99.1$ & $50-60$ & - & Detritus & Debitage & \begin{tabular}{|l|} 
Proximal Flake \\
\end{tabular} & Lithic & 5 & 8.28 & & 12/12/2017 \\
\hline 319 & 3 & 501 & - & - & - & A & IIII & No995 & E1012 & 6 & $99.2-99.1$ & $50-60$ & - & Detritus & Debitage & Broken Flake & Lithic & 22 & 76.09 & & $\mid$ |12/12/2017 \\
\hline 319 & 4 & 501 & - & - & - & A & IIII & No995 & E1012 & 6 & $99.2-99.1$ & $50-60$ & - & Detritus & Debitage & Thermal Shatter & Lithic & 4 & 2.53 & & 12/12/2017 \\
\hline 319 & 5 & 501 & - & - & - & A & IIII & No995 & E1012 & 6 & 99.2-99.1 & $50-60$ & - & Faunal Remains & Shell & Mussel Shell & Shell & 4 & 0.84 & & 12/12/2017 \\
\hline 320 & 1 & 501 & - & - & - & $\mathrm{A}$ & 11 & No995 & E1012 & 6 & $\begin{array}{l}99.17 \\
\end{array}$ & 53 & \begin{tabular}{|c|} 
N995.50 \\
E1012.34 \\
\end{tabular} & Informal Tool & Modified Flake & Utilized Flake & Fine Grain Chert & 1 & 43.57 & & $\mid$\begin{tabular}{|l|}
$\mid 12 / 12 / 2017$ \\
\end{tabular} \\
\hline 321 & 1 & 506 & - & - & - & A & IIII & No995 & E1012 & 7 & $99.1-99.0$ & $60-70$ & - & Faunal Remains & Bone & Mammal & Bone & 1 & 0.55 & & 12/13/2017 \\
\hline 321 & 2 & 506 & - & - & - & A & IIII & No995 & E1012 & 7 & $99.1-99.0$ & $60-70$ & - & Faunal Remains & Bone & Indeterminate & Bone & 1 & 0.61 & & 12/13/2017 \\
\hline 321 & 3 & 506 & - & - & - & A & IIII & No995 & E1012 & 7 & $99.1-99.0$ & $60-70$ & - & Faunal Remains & Bone & Mammal & Bone & 1 & 0.74 & & 12/13/2017 \\
\hline 321 & 4 & 506 & - & - & - & A & IIII & No995 & E1012 & 7 & $99.1-99.0$ & $60-70$ & - & Faunal Remains & Bone & Indeterminate & Bone & 1 & 0.45 & & $\mid$\begin{tabular}{|l|}
$12 / 13 / 2017$ \\
\end{tabular} \\
\hline 321 & 5 & 506 & - & - & - & A & III & No995 & E1012 & 7 & $99.1-99.0$ & $60-70$ & - & Faunal Remains & Bone & Indeterminate & Bone & 1 & 0.48 & & 12/13/2017 \\
\hline 321 & 6 & 506 & - & - & - & A & III & No995 & E1012 & 7 & $99.1-99.0$ & $60-70$ & - & \begin{tabular}{|l|} 
Faunal Remains \\
\end{tabular} & Bone & Indeterminate & Bone & 1 & 0.4 & & 12/13/2017 \\
\hline 321 & 7 & 506 & - & - & - & A & IIII & No995 & E1012 & 7 & $99.1-99.0$ & $60-70$ & - & Faunal Remains & Bone & Indeterminate & Bone & 1 & 0.29 & & \begin{tabular}{|l|}
$12 / 13 / 2017$ \\
\end{tabular} \\
\hline 321 & 8 & 506 & - & - & - & A & IIII & N0995 & E1012 & 7 & $99.1-99.0$ & $60-70$ & - & Faunal Remains & Bone & Indeterminate & Bone & 1 & 0.57 & & 12/13/2017 \\
\hline 321 & 9 & 506 & - & - & - & A & IIII & No995 & E1012 & 7 & $99.1-99.0$ & $60-70$ & - & Faunal Remains & Bone & Indeterminate & Bone & 1 & 0.28 & & 12/13/2017 \\
\hline 321 & 10 & 506 & - & - & - & A & IIII & No995 & E1012 & 7 & $99.1-99.0$ & $60-70$ & - & Faunal Remains & Bone & Indeterminate & Bone & 1 & 0.35 & & $\mid 12 / 13 / 2017$ \\
\hline 321 & 11 & 506 & - & - & - & A & III & No995 & E1012 & 7 & $99.1-99.0$ & $60-70$ & - & Faunal Remains & Bone & Indeterminate & Bone & 1 & 0.23 & & \begin{tabular}{|l|}
$12 / 13 / 2017$ \\
\end{tabular} \\
\hline 321 & 12 & 506 & - & - & - & A & IIII & N0995 & E1012 & 7 & $99.1-99.0$ & $\begin{array}{ll}60-70 \\
\end{array}$ & - & Faunal Remains & Bone & Mammal & Bone & 1 & 0.56 & & 12/13/2017 \\
\hline 321 & 13 & 506 & - & - & - & A & IIII & No995 & E1012 & 7 & $99.1-99.0$ & $60-70$ & - & Faunal Remains & Bone & Indeterminate & Bone & 1 & 0.46 & & 12/13/2017 \\
\hline 321 & 14 & 506 & - & - & - & A & III & No995 & E1012 & 7 & $99.1-99.0$ & $60-70$ & - & Faunal Remains & Bone & Indeterminate & Bone & 1 & 0.29 & & 12/13/2017 \\
\hline 321 & 15 & 506 & - & - & - & A & IIII & No995 & E1012 & 7 & $99.1-99.0$ & $60-70$ & - & Faunal Remains & Bone & Indeterminate & Bone & 1 & 0.1 & & 12/13/2017 \\
\hline 321 & 16 & 506 & - & - & - & A & IIII & No995 & E1012 & 7 & $99.1-99.0$ & $60-70$ & - & \begin{tabular}{|l|} 
Faunal Remains \\
\end{tabular} & Bone & Indeterminate & Bone & 1 & 0.1 & & 12/13/2017 \\
\hline 321 & 17 & 506 & - & - & - & A & III & No995 & E1012 & 7 & $99.1-99.0$ & $60-70$ & - & Faunal Remains & Bone & Indeterminate & Bone & 1 & 0.17 & & \begin{tabular}{|l|}
$12 / 13 / 2017$ \\
\end{tabular} \\
\hline
\end{tabular}




\begin{tabular}{|c|c|c|c|c|c|c|c|c|c|c|c|c|c|c|c|c|c|c|c|c|c|}
\hline Lot No. & \begin{tabular}{|c|}
$\begin{array}{c}\text { Specimen } \\
\text { No. }\end{array}$ \\
\end{tabular} & FS No. & UI No. & FTR No. & $\begin{array}{c}\text { Sample } \\
\text { No. }\end{array}$ & $\begin{array}{c}\text { Excavation } \\
\text { Block }\end{array}$ & Stratum (AU) & Northing & Easting & Level & $\begin{array}{c}\text { Elevation } \\
(\mathrm{m})\end{array}$ & Depth (cmbd) & \begin{tabular}{|l|} 
Point Prov. \\
\end{tabular} & Artifact Class & Artifact Type & $\begin{array}{c}\text { Artifact } \\
\text { Description }\end{array}$ & Material & $\begin{array}{c}\text { Number of } \\
\text { artifacts }\end{array}$ & Weight (g) & Comments & Date \\
\hline 321 & 18 & 506 & - & - & - & A & III & No995 & E1012 & 7 & $99.1-99.0$ & $60-70$ & - & Faunal Remains & Bone & Indeterminate & Bone & 1 & 0.12 & & 12/13/2017 \\
\hline 321 & 19 & 506 & - & - & - & A & IIII & No995 & E1012 & 7 & $99.1-99.0$ & $60-70$ & - & Faunal Remains & Bone & Indeterminate & Bone & 1 & 0.05 & & $12 / 13 / 2017$ \\
\hline 321 & 20 & 506 & - & - & - & A & IIII & No995 & E1012 & 7 & $99.1-99.0$ & $60-70$ & - & Faunal Remains & Bone & Indeterminate & Bone & 1 & 0.18 & & 12/13/2017 \\
\hline 321 & 21 & 506 & - & - & - & A & IIII & No995 & E1012 & 7 & $99.1-99.0$ & $60-70$ & - & Faunal Remains & Bone & Indeterminate & Bone & 1 & 0.11 & & 12/13/2017 \\
\hline 321 & 22 & 506 & - & - & - & A & III & No995 & E1012 & 7 & $99.1-99.0$ & $60-70$ & - & Faunal Remains & Bone & Indeterminate & Bone & 1 & 0.01 & & 12/13/2017 \\
\hline 321 & 23 & 506 & - & - & - & A & IIII & No995 & E1012 & 7 & $99.1-99.0$ & $60-70$ & - & Faunal Remains & Bone & Indeterminate & Bone & 1 & 0.01 & & 12/13/2017 \\
\hline 321 & 24 & 506 & - & - & - & A & IIII & No995 & E1012 & 7 & $99.1-99.0$ & $60-70$ & - & Detritus & Debitage & Complete Flake & Lithic & 6 & 98.18 & & 12/13/2017 \\
\hline 321 & 25 & 506 & - & - & - & A & III & No995 & E1012 & 7 & $99.1-99.0$ & 60-70 & - & Detritus & Debitage & Proximal Flake & Lithic & 8 & 6 & & $\mid$\begin{tabular}{|l|}
$\mid 12 / 13 / 2017$ \\
\end{tabular} \\
\hline 321 & 26 & 506 & - & - & - & A & III & No995 & E1012 & 7 & $99.1-99.0$ & $60-70$ & - & Detritus & Debitage & Broken Flake & Lithic & 37 & 59.02 & & 12/13/2017 \\
\hline 321 & 27 & 506 & - & - & - & A & IIII & No995 & E1012 & 7 & $99.1-99.0$ & $60-70$ & - & Detritus & Debitage & Thermal Shatter & Lithic & 19 & 27.33 & & 12/13/2017 \\
\hline 321 & 28 & 506 & 25 & - & - & A & IIII & No995 & E1012 & 7 & $99.1-99.0$ & $60-70$ & - & Worked Shell & $\begin{array}{l}\text { Shell } \\
\text { Ornament }\end{array}$ & Shell Bead & Shell & 1 & 0.06 & Rounded rabdotus like shell & 12/13/2017 \\
\hline 322 & 1 & 508 & - & - & - & A & IIII & No995 & E1012 & 7 & 99.07 & 63 & $\begin{array}{l}\begin{array}{l}\text { N999.36 } \\
\text { E91012.90 }\end{array} \\
\end{array}$ & Faunal Remains & Shell & Mussel Shell & Shell & 1 & 24.05 & 1 bivalve shell half & $\mid$\begin{tabular}{|l|}
$12 / 13 / 2017$ \\
\end{tabular} \\
\hline 323 & 1 & 524 & - & - & - & A & III & No995 & E1012 & 8 & 99.0-98.9 & $70-80$ & - & Faunal Remains & Bone & Mammal & Bone & 1 & 0.55 & & 12/13/2017 \\
\hline 323 & 2 & 524 & - & - & - & A & IIII & N0995 & E1012 & 8 & $99.0-98.9$ & $70-80$ & - & Faunal Remains & Bone & Indeterminate & Bone & 1 & 1.37 & & $\mid 12 / 13 / 2017$ \\
\hline 323 & 3 & 524 & - & - & - & A & IIII & No995 & E1012 & 8 & $99.0-98.9$ & $70-80$ & - & Faunal Remains & Bone & Indeterminate & Bone & 1 & 0.63 & & 12/13/2017 \\
\hline 323 & 4 & 524 & - & - & - & A & III & No995 & E1012 & 8 & $99.0-98.9$ & $70-80$ & - & Faunal Remains & Bone & Indeterminate & Bone & 1 & 0.15 & & 12/13/2017 \\
\hline 323 & 5 & 524 & - & - & - & A & IIII & No995 & E1012 & 8 & $99.0-98.9$ & $70-80$ & - & Faunal Remains & Bone & Mammal & Bone & 1 & 0.49 & & 12/13/2017 \\
\hline 323 & 6 & 524 & - & - & - & A & III & No995 & E1012 & 8 & $99.0-98.9$ & $70-80$ & - & Faunal Remains & Bone & Mammal & Bone & 1 & 2.6 & & 12/13/2017 \\
\hline 323 & 7 & 524 & - & - & - & A & IIII & No995 & E1012 & 8 & $99.0-98.9$ & $70-80$ & - & Faunal Remains & Bone & Indeterminate & Bone & 1 & 0.88 & & 12/13/2017 \\
\hline 323 & 8 & 524 & - & - & - & A & IIII & No995 & E1012 & 8 & $99.0-98.9$ & $70-80$ & - & Faunal Remains & Bone & Tortoise/Turtle & Bone & 1 & 0.24 & & 12/13/2017 \\
\hline 323 & 9 & 524 & - & - & - & A & IIII & No995 & E1012 & 8 & 99.098 .9 & $70-80$ & - & Faunal Remains & Bone & Mammal & Bone & 1 & 0.33 & & 12/13/2017 \\
\hline 323 & 10 & 524 & - & - & - & A & IIII & No995 & E1012 & 8 & $99.0-98.9$ & $70-80$ & - & Faunal Remains & Bone & Indeterminate & Bone & 1 & 0.25 & & 12/13/2017 \\
\hline 323 & 11 & 524 & - & - & - & A & IIII & No995 & E1012 & 8 & $99.0-98.9$ & $70-80$ & - & Faunal Remains & Bone & Indeterminate & Bone & 1 & 0.37 & & 12/13/2017 \\
\hline 323 & 12 & 524 & - & - & - & A & IIII & No995 & E1012 & 8 & $99.0-98.9$ & $70-80$ & - & Faunal Remains & Bone & Indeterminate & Bone & 1 & 0.21 & & 12/13/2017 \\
\hline 323 & 13 & 524 & - & - & - & A & IIII & No995 & E1012 & 8 & $99.0-98.9$ & $70-80$ & - & Faunal Remains & Bone & Indeterminate & Bone & 1 & 0.25 & & 12/13/2017 \\
\hline 323 & 14 & 524 & - & - & - & A & IIII & No995 & E1012 & 8 & $99.0-98.9$ & $70-80$ & - & Faunal Remains & Bone & Indeterminate & Bone & 1 & 0.28 & & 12/13/2017 \\
\hline 323 & 15 & 524 & - & - & - & A & IIII & No995 & E1012 & 8 & $99.0-98.9$ & $70-80$ & - & Faunal Remains & Bone & Indeterminate & Bone & 1 & 0.15 & & 12/13/2017 \\
\hline 323 & 16 & 524 & - & - & - & A & IIII & No995 & E1012 & 8 & $99.0-98.9$ & $70-80$ & - & Faunal Remains & Bone & Indeterminate & Bone & 1 & 0.21 & & 12/13/2017 \\
\hline 323 & 17 & 524 & - & - & - & A & IIII & No995 & E1012 & 8 & $99.0-98.9$ & $70-80$ & - & Faunal Remains & Bone & Indeterminate & Bone & 1 & 0.15 & & 12/13/2017 \\
\hline 323 & 18 & 524 & - & - & - & A & IIII & No995 & E1012 & 8 & $99.0-98.9$ & $70-80$ & - & Faunal Remains & Bone & Indeterminate & Bone & 1 & 0.11 & & 12/13/2017 \\
\hline 323 & 19 & 524 & - & - & - & A & IIII & No995 & E1012 & 8 & 99.0-98.9 & $70-80$ & - & Faunal Remains & Bone & Indeterminate & Bone & 1 & 0.08 & & $\mid$\begin{tabular}{|l|}
$12 / 13 / 2017$ \\
\end{tabular} \\
\hline 323 & 20 & 524 & - & - & - & A & III & No995 & E1012 & 8 & $99.0-98.9$ & $70-80$ & - & Faunal Remains & Bone & Indeterminate & Bone & 1 & 0.29 & & 12/13/2017 \\
\hline 323 & 21 & 524 & - & - & - & A & III & No995 & E1012 & 8 & $99.0-98.9$ & $70-80$ & - & Faunal Remains & Bone & Indeterminate & Bone & 1 & 0.14 & & 12/13/2017 \\
\hline 323 & 22 & 524 & - & - & - & A & IIII & No995 & E1012 & 8 & 99.0-98.9 & $70-80$ & - & Faunal Remains & Bone & Indeterminate & Bone & 1 & 0.18 & & \begin{tabular}{|l|}
$12 / 13 / 2017$ \\
\end{tabular} \\
\hline 323 & 23 & 524 & - & - & - & A & IIII & N0995 & E1012 & 8 & $99.0-98.9$ & $\begin{array}{ll}70-80 \\
\end{array}$ & - & Faunal Remains & Bone & Indeterminate & Bone & 1 & 0.06 & & 12/13/2017 \\
\hline 323 & 24 & 524 & - & - & - & A & IIII & No995 & E1012 & 8 & $99.0-98.9$ & $\begin{array}{ll}70-80 \\
\end{array}$ & - & Faunal Remains & Bone & Indeterminate & Bone & 1 & 0.01 & & 12/13/2017 \\
\hline 323 & 25 & 524 & - & - & - & A & III & No995 & E1012 & 8 & $99.0-98.9$ & $70-80$ & - & Detritus & Debitage & Complete Flake & Lithic & 4 & 23.33 & & $\mid 12 / 13 / 2017$ \\
\hline 323 & 26 & 524 & - & - & - & A & IIII & No995 & E1012 & 8 & $99.0-98.9$ & $70-80$ & - & Detritus & Debitage & Proximal Flake & Lithic & 4 & 5.76 & & 12/13/2017 \\
\hline 323 & 27 & 524 & - & - & - & A & IIII & No995 & E1012 & 8 & $99.0-98.9$ & $70-80$ & - & Detritus & Debitage & Broken Flake & Lithic & 57 & 24.02 & & 12/13/2017 \\
\hline 323 & 28 & 524 & - & - & - & A & III & No995 & E1012 & 8 & $99.0-98.9$ & $70-80$ & - & Detritus & Debitage & \begin{tabular}{|l|} 
Flaking Shatter \\
\end{tabular} & Lithic & 2 & 0.67 & & 12/13/2017 \\
\hline 323 & 29 & 524 & - & - & - & A & III & No995 & E1012 & 8 & $99.0-98.9$ & $70-80$ & - & Detritus & Debitage & Thermal Shatter & Lithic & 44 & 48.06 & & \begin{tabular}{|l|}
$12 / 13 / 2017$ \\
\end{tabular} \\
\hline 323 & 30 & 524 & - & - & - & A & IIII & No995 & E1012 & 8 & 99.098 .9 & $70-80$ & - & Informal Tool & \begin{tabular}{|l|} 
Modified Flake \\
\end{tabular} & \begin{tabular}{|l|} 
Edge-modified \\
\end{tabular} & Fine Grain Chert & 1 & 5.38 & & 12/13/2017 \\
\hline 323 & 31 & 524 & - & - & - & $\mathrm{A}$ & III & No995 & E1012 & 8 & $99.0-98.9$ & $70-80$ & - & \begin{tabular}{l|l|} 
Informal Tool \\
\end{tabular} & \begin{tabular}{|l|} 
Modified Flake \\
\end{tabular} & \begin{tabular}{|l|} 
Edge-modified \\
\end{tabular} & \begin{tabular}{|c|}
$\begin{array}{c}\text { Coarse Grain } \\
\text { Chert }\end{array}$ \\
\end{tabular} & 1 & 5.38 & & 12/13/2017 \\
\hline 323 & 32 & 524 & - & - & - & A & III & No995 & E1012 & 8 & $99.0-98.9$ & $70-80$ & - & Informal Tool & Modified Flake & Edge-modified & Fine Grain Chert & 1 & 2.75 & & 12/13/2017 \\
\hline
\end{tabular}




\begin{tabular}{|c|c|c|c|c|c|c|c|c|c|c|c|c|c|c|c|c|c|c|c|c|c|}
\hline Lot No. & \begin{tabular}{|c|}
$\begin{array}{c}\text { Specimen } \\
\text { No. }\end{array}$ \\
\end{tabular} & FS No. & UI No. & FTR No. & $\begin{array}{c}\text { Sample } \\
\text { No. }\end{array}$ & $\begin{array}{c}\text { Excavation } \\
\text { Block }\end{array}$ & Stratum (AU) & Northing & Easting & Level & $\begin{array}{c}\begin{array}{c}\text { Elevation } \\
(\mathrm{m})\end{array} \\
\end{array}$ & Depth (cmbd) & Point Prov. & Artifact Class & Artifact Type & \begin{tabular}{|c|} 
Artifict \\
Description \\
\end{tabular} & Material & $\begin{array}{c}\text { Number of } \\
\text { artifacts }\end{array}$ & Weight (g) & Comments & Date \\
\hline 324 & 1 & 525 & - & - & - & A & III & No995 & E1012 & 8 & 98.9 & 80 & $\begin{array}{r}\text { N995.79 } \\
\text { E1012.13 } \\
\end{array}$ & Other Lithic & $\begin{array}{l}\text { Therrally } \\
\text { Altered Stone } \\
\end{array}$ & FCR & Lithic & 2 & 132.57 & Originally classified as a core & 12/13/2017 \\
\hline 325 & 1 & 578 & - & - & - & A & IV & No995 & E1012 & 9 & $98.9-98.8$ & $80-90$ & - & Faunal Remains & \begin{tabular}{|c|} 
Bone \\
\end{tabular} & \begin{tabular}{|l|} 
Tortoise/Turtle \\
\end{tabular} & Bone & 1 & 0.41 & & $\mid$\begin{tabular}{|l|}
$\mid 12 / 18 / 2017$ \\
\end{tabular} \\
\hline 325 & 2 & 578 & - & - & - & A & IV & No995 & E1012 & 9 & $98.9-98.8$ & $80-90$ & - & Detritus & Debitage & \begin{tabular}{|l|} 
Complete Flake \\
\end{tabular} & Lithic & 4 & 46.2 & & 12/18/2017 \\
\hline 325 & 3 & 578 & - & - & - & A & iv & No995 & E1012 & 9 & $98.9-98.8$ & $80-90$ & - & Detritus & Debitage & \begin{tabular}{|l|} 
Proximal Flake \\
\end{tabular} & Lithic & 1 & 0.26 & & 12/18/2017 \\
\hline 325 & 4 & 578 & - & - & - & A & IV & No995 & E1012 & 9 & $98.9-98.8$ & $80-90$ & - & Detritus & Debitage & Broken Flake & Lithic & 31 & 18.54 & & $\mid 12 / 18 / 2017$ \\
\hline 325 & 5 & 578 & - & - & - & A & IV & No995 & E1012 & 9 & $98.9-98.8$ & $80-90$ & - & Detritus & Debitage & Thermal Shatter & Lithic & 14 & 13.19 & & 12/18/2017 \\
\hline 326 & 1 & 590 & - & - & - & A & iv & No995 & E1012 & 9 & 98.83 & 87 & \begin{tabular}{|c|} 
N996.0 \\
E1012.43
\end{tabular} & \begin{tabular}{|c|} 
Ground/ \\
Battered Stone \\
\end{tabular} & Battered Stone & Tested Cobble & & 1 & 183.52 & Originally classified as a Core & $\mid 12 / 18 / 2017$ \\
\hline 327 & 1 & 589 & - & - & - & A & IV & No995 & E1012 & 10 & $98.8-98.7$ & 90-100 & - & Faunal Remains & Bone & Tortoise/Turtle & Bone & 1 & 0.38 & 1 burned & $\mid$\begin{tabular}{|l|}
$\mid 12 / 18 / 2017$ \\
\end{tabular} \\
\hline 327 & 2 & 589 & - & - & - & A & IV & No995 & E1012 & 10 & 98.8-98.7 & 90-100 & - & Faunal Remains & Bone & Indeterminate & Bone & 1 & 0.52 & 1 burned & 12/18/2017 \\
\hline 327 & 3 & 589 & - & - & - & A & IV & N0995 & E1012 & 10 & $98.8-98.7$ & $90-100$ & - & Faunal Remains & Bone & \begin{tabular}{|l|} 
Indeterminate \\
\end{tabular} & Bone & 1 & 0.28 & 1 burned & \begin{tabular}{|l|}
$22 / 18 / 2017$ \\
\end{tabular} \\
\hline 327 & 4 & 589 & - & - & - & A & iv & No995 & E1012 & 10 & $\begin{array}{l}98.8-98.7 \\
\end{array}$ & $90-100$ & - & \begin{tabular}{|c|} 
Detritus \\
\end{tabular} & Debitage & \begin{tabular}{|c|} 
Complete Flake \\
\end{tabular} & Lithic & 5 & 5.32 & & 12/18/2017 \\
\hline 327 & 5 & 589 & - & - & - & A & IV & No995 & E1012 & 10 & $98.8-98.7$ & $90-100$ & - & Detritus & Debitage & Proximal Flake & Lithic & 2 & 0.68 & & 12/18/2017 \\
\hline 327 & 6 & 589 & - & - & - & A & IV & N0995 & E1012 & 10 & $\begin{array}{ll}98.8-98.7 \\
\end{array}$ & $\begin{array}{l}90-100 \\
\end{array}$ & - & Detritus & Debitage & Broken Flake & Lithic & 27 & 15.89 & & 12/18/2017 \\
\hline 327 & 7 & 589 & - & - & - & A & IV & N0995 & E1012 & 10 & $\begin{array}{ll}98.8-98.7 \\
\end{array}$ & $\begin{array}{l}0-100 \\
\end{array}$ & - & Detritus & Debitage & Thermal Shatter & Lithic & 52 & 71.42 & & 12/18/2017 \\
\hline 327 & 8 & 589 & - & - & - & A & iv & No995 & E1012 & 10 & 98.8-98.7 & $90-100$ & - & Formal Tool & Biface & \begin{tabular}{c|} 
Indeterminate \\
Fragment
\end{tabular} & Fine Grain Chert & 1 & 7.39 & Distal end & 12/18/2017 \\
\hline 327 & 9 & 589 & - & - & - & $\mathrm{A}$ & iv & No995 & E1012 & 10 & 98.8-98.7 & $90-100$ & - & Other Lithic & \begin{tabular}{|l|} 
Thermally \\
Altered Stone \\
\end{tabular} & \begin{tabular}{l|l} 
FCR \\
\end{tabular} & Lithic & 1 & 10.48 & & 12/18/2017 \\
\hline 328 & 1 & 619 & - & - & - & A & IV & No995 & E1012 & 11 & 98.7-98.6 & $100-110$ & - & Detritus & Debitage & Complete Flake & Lithic & 3 & 12.19 & & 12/20/2017 \\
\hline 328 & 2 & 619 & - & - & - & A & iv & No995 & E1012 & 11 & 98.7-98.6 & $100-110$ & - & Detritus & Debitage & Proximal Flake & Lithic & 1 & 0.79 & & 12/20/2017 \\
\hline 328 & 3 & 619 & - & - & - & A & IV & No995 & E1012 & 11 & $\begin{array}{l}98.7-98.6 \\
\end{array}$ & 100-110 & - & Detritus & Debitage & Broken Flake & Lithic & 18 & 2.75 & & 12/20/2017 \\
\hline 328 & 4 & 619 & - & - & - & A & IV & No995 & E1012 & 11 & $\begin{array}{l}98.7-98.6 \\
\end{array}$ & 100-110 & - & Detritus & Debitage & Thermal Shatter & Lithic & 9 & 4.66 & & 12/20/2017 \\
\hline 329 & 1 & 628 & - & - & - & A & IV & No995 & E1012 & 12 & $98.6-98.5$ & $110-120$ & - & Faunal Remains & Bone & Mammal & Tooth & 1 & 0.06 & & 12/20/2017 \\
\hline 329 & 2 & 628 & - & - & - & A & IV & No995 & E1012 & 12 & $98.6-98.5$ & 110-120 & - & Detritus & Debitage & \begin{tabular}{|l|} 
Proximal Flake \\
\end{tabular} & Lithic & 1 & 0.11 & & 12/20/2017 \\
\hline 329 & 3 & 628 & - & - & - & A & IV & No995 & E1012 & 12 & $98.6-98.5$ & $110-120$ & - & Detritus & Debitage & \begin{tabular}{|l|} 
Broken Flake \\
\end{tabular} & Lithic & 8 & 22.95 & & 12/20/2017 \\
\hline 329 & 4 & 628 & - & - & - & A & iv & No995 & E1012 & 12 & $98.6-98.5$ & 110-120 & - & Detritus & Debitage & Thermal Shatter & Lithic & 9 & 11.23 & & 12/20/2017 \\
\hline 330 & 1 & 639 & - & - & - & A & IVIV & No995 & E1012 & 13 & $98.5-98.4$ & $120-130$ & - & Detritus & Debitage & Complete Flake & Lithic & 1 & 11.24 & Complete flake is $95 \%$ complete & 12/20/2017 \\
\hline 330 & 2 & 639 & - & - & - & A & IVN & No995 & E1012 & 13 & $98.5-98.4$ & $120-130$ & - & Detritus & Debitage & \begin{tabular}{|l|} 
Proximal Flake \\
\end{tabular} & Lithic & 2 & 1.52 & & 12/20/2017 \\
\hline 330 & 3 & 639 & - & - & - & A & IVN & No995 & E1012 & 13 & $98.5-98.4$ & $120-130$ & - & Detritus & Debitage & Broken Flake & Lithic & 3 & 1.85 & & 12/20/2017 \\
\hline 331 & 1 & 642 & - & - & - & A & $\mathrm{v}$ & No995 & E1012 & 14 & $98.4-98.3$ & $130-140$ & - & Detritus & Debitage & \begin{tabular}{|l|} 
Complete Flake \\
\end{tabular} & Lithic & 1 & 1.41 & & 12/20/2017 \\
\hline 331 & 2 & 642 & - & - & - & A & $\mathrm{v}$ & No995 & E1012 & 14 & $98.4-98.3$ & $130-140$ & - & Detritus & Debitage & \begin{tabular}{|l|} 
Proximal Flake \\
\end{tabular} & Lithic & 1 & 46.49 & & 12/20/2017 \\
\hline 332 & 1 & 653 & - & - & - & A & $\mathrm{vv}_{1}$ & No995 & E1012 & 15 & $98.3-98.2$ & 140-150 & - & Detritus & Debitage & \begin{tabular}{|c|} 
Complete Flake \\
\end{tabular} & Lithic & 1 & 7.58 & & 12/21/2017 \\
\hline 332 & 2 & 653 & - & - & - & A & vi & No995 & E1012 & 15 & $98.3-98.2$ & $140-150$ & - & Detritus & Debitage & Proximal Flake & Lithic & 4 & 9.64 & & 12/21/2017 \\
\hline 332 & 3 & 653 & - & - & - & A & vi & No995 & E1012 & 15 & $98.3-98.2$ & $140-150$ & - & Detritus & Debitage & Broken Flake & Lithic & 5 & 1.22 & & 12/21/2017 \\
\hline 333 & 1 & 350 & - & - & - & A & 1 & No995 & E1013 & 1 & 99.65-99.6 & $5-10$ & - & Detritus & Debitage & \begin{tabular}{|l|} 
Proximal Flake \\
\end{tabular} & Lithic & 1 & 0.45 & & 11/29/2017 \\
\hline 333 & 2 & 350 & - & - & - & A & 1 & N0995 & E1013 & 1 & \begin{tabular}{|l|l}
$99.65-99.6$ \\
\end{tabular} & $5-10$ & - & Detritus & Debitage & Broken Flake & Lithic & 2 & 3.87 & & \begin{tabular}{|l|}
$11 / 29 / 2017$ \\
\end{tabular} \\
\hline 334 & 1 & 358 & - & - & - & A & 1 & N0995 & E1013 & 2 & $99.6-99.5$ & $10-20$ & - & Detritus & Debitage & \begin{tabular}{|c|} 
Complete Flake \\
\end{tabular} & Lithic & 2 & 0.35 & & 11/29/2017 \\
\hline 334 & 2 & 358 & - & - & - & A & 1 & N0995 & E1013 & 2 & $99.6-99.5$ & $10-20$ & - & Detritus & Debitage & \begin{tabular}{|l|} 
Proximal Flake \\
\end{tabular} & Lithic & 2 & 1.47 & & 11/29/2017 \\
\hline 334 & 3 & 358 & - & - & - & A & 1 & N0995 & E1013 & 2 & $99.6-99.5$ & $10-20$ & - & Detritus & Debitage & Broken Flake & Lithic & 5 & 5.01 & & 11/29/2017 \\
\hline 335 & 1 & 378 & - & - & - & A & 11 & No995 & E1013 & 3 & 99.5-99.4 & $20-30$ & - & Faunal Remains & Bone & Indeterminate & Bone & 1 & 0.42 & & 11/30/2017 \\
\hline 335 & 2 & 378 & - & - & - & A & "I & N0995 & E1013 & 3 & $99.5-99.4$ & $20-30$ & - & Detritus & Debitage & \begin{tabular}{|c|} 
Complete Flake \\
\end{tabular} & Lithic & 2 & 14.26 & & 11/30/2017 \\
\hline 335 & 3 & 378 & - & - & - & A & "I & No995 & E1013 & 3 & $99.5-99.4$ & $20-30$ & - & Detritus & Debitage & \begin{tabular}{|l|} 
Proximal Flake \\
\end{tabular} & Lithic & 2 & 2.99 & & 11/30/2017 \\
\hline 335 & 4 & 378 & - & - & - & A & "I & No995 & E1013 & 3 & $99.5-99.4$ & $20-30$ & - & Detritus & Debitage & $\begin{array}{ll}\text { Broken Flake } \\
\end{array}$ & Lithic & 2 & 1.5 & & 11/30/2017 \\
\hline 336 & 1 & 388 & - & - & - & A & "I & No995 & E1013 & 4 & $99.4-99.3$ & $30-40$ & - & Faunal Remains & Bone & Mammal & Bone & 1 & 6.94 & & 11/30/2017 \\
\hline 336 & 2 & 388 & - & - & - & A & $\|$ & No995 & E1013 & 4 & $99.4-99.3$ & $30-40$ & - & \begin{tabular}{|l|} 
Faunal Remains \\
\end{tabular} & Bone & Indeterminate & Bone & 1 & 0.96 & & 11/30/2017 \\
\hline 336 & 3 & 388 & - & - & - & A & ॥ & No995 & E1013 & 4 & 99.499 .3 & $30-40$ & - & \begin{tabular}{|l|} 
Faunal Remains \\
\end{tabular} & Bone & Indeterminate & Bone & 1 & 0.42 & & 11/30/2017 \\
\hline
\end{tabular}




\begin{tabular}{|c|c|c|c|c|c|c|c|c|c|c|c|c|c|c|c|c|c|c|c|c|c|}
\hline Lot No. & \begin{tabular}{|c|}
$\begin{array}{c}\text { Specimen } \\
\text { No. }\end{array}$ \\
\end{tabular} & FS No. & UI No. & FTR No. & $\begin{array}{c}\text { Sample } \\
\text { No. }\end{array}$ & $\begin{array}{c}\text { Excavation } \\
\text { Block }\end{array}$ & Stratum (AU) & Northing & Easting & Level & $\begin{array}{c}\text { Elevation } \\
(\mathrm{m})\end{array}$ & Depth (cmbd) & \begin{tabular}{|l|} 
Point Prov. \\
\end{tabular} & Artifact Class & Artifact Type & \begin{tabular}{|c|} 
Artifact \\
Description
\end{tabular} & Material & $\begin{array}{c}\text { Number of } \\
\text { artifacts }\end{array}$ & Weight (g) & Comments & Date \\
\hline 336 & \begin{tabular}{|l|} 
\\
\end{tabular} & 388 & - & - & - & A & " & No995 & E1013 & 4 & 99.499 .3 & $30-40$ & - & Faunal Remains & Bone & Indeterminate & Bone & 1 & 0.35 & & 11/30/2017 \\
\hline 336 & 5 & 388 & - & - & - & A & " & No995 & E1013 & 4 & 99.4-99.3 & $30-40$ & - & Detritus & Debitage & Complete Flake & Lithic & 5 & 2.02 & & $\mid$\begin{tabular}{|l|}
$\mid 11 / 30 / 2017$ \\
\end{tabular} \\
\hline 336 & 6 & 388 & - & - & - & A & "I & No995 & E1013 & 4 & 99.499 .3 & $30-40$ & - & Detritus & Debitage & \begin{tabular}{|l|} 
Proximal Flake \\
\end{tabular} & Lithic & 9 & 3.99 & & 11/30/2017 \\
\hline 336 & 7 & 388 & - & - & - & A & "I & No995 & E1013 & 4 & 99.4-99.3 & $30-40$ & - & Detritus & Debitage & Broken Flake & Lithic & 17 & 12.99 & & 11/30/2017 \\
\hline 336 & 8 & 388 & - & - & - & A & "I & No995 & E1013 & 4 & 99.4-99.3 & $30-40$ & - & Detritus & Debitage & Flaking Shatter & Lithic & 1 & 1.6 & & $\mid$\begin{tabular}{|l|}
$\mid 11 / 30 / 2017$ \\
\end{tabular} \\
\hline 336 & 9 & 388 & - & - & - & A & "I & No995 & E1013 & 4 & $99.4-99.3$ & $30-40$ & - & Detritus & Debitage & Thermal Shatter & Lithic & 9 & 6.66 & & 11/30/2017 \\
\hline 336 & 10 & 388 & - & - & - & A & "I & No995 & E1013 & 4 & 99.4-99.3 & $30-40$ & - & Informal Tool & Modified Flake & \begin{tabular}{|l|} 
Edge-modified \\
\end{tabular} & Fine Grain Chert & 1 & 2.07 & & 11/30/2017 \\
\hline 336 & 11 & 388 & - & - & - & A & " & No995 & E1013 & 4 & 99.4-99.3 & $30-40$ & - & Formal Tool & Biface & $\begin{array}{c}\text { Marginal } \\
\text { Fragment }\end{array}$ & Fine Grain Chert & 1 & 6.31 & Medial to proximal end & 11/30/2017 \\
\hline 337 & 1 & 399 & - & - & - & A & "I & No995 & E1013 & 5 & $99.3-99.2$ & $40-50$ & - & Detritus & Debitage & Complete Flake & Lithic & 17 & 14.74 & & 12/1/2017 \\
\hline 337 & 2 & 399 & - & - & - & A & "I & N0995 & E1013 & 5 & $99.3-99.2$ & $40-50$ & - & Detritus & Debitage & \begin{tabular}{|l|} 
Proximal Flake \\
\end{tabular} & Lithic & 17 & 74.62 & & 12/1/2017 \\
\hline 337 & 3 & 399 & - & - & - & A & "I & N0995 & E1013 & 5 & $99.3-99.2$ & $40-50$ & - & Detritus & Debitage & Broken Flake & Lithic & 41 & 40.17 & & 12/1/2017 \\
\hline 337 & 4 & 399 & - & - & - & A & "I & No995 & E1013 & 5 & $99.3-99.2$ & $40-50$ & - & Detritus & Debitage & Flaking Shatter & Lithic & 4 & 3.95 & & 12/1/2017 \\
\hline 337 & 5 & 399 & - & - & - & A & "I & N0995 & E1013 & 5 & $99.3-99.2$ & $40-50$ & - & Detritus & Debitage & Thermal Shatter & Lithic & 21 & 20.23 & & 12/1/2017 \\
\hline 337 & 6 & 399 & - & - & - & A & "I & No995 & E1013 & 5 & $99.3-99.2$ & $40-50$ & - & Informal Tool & \begin{tabular}{|l|} 
Modified Flake \\
\end{tabular} & \begin{tabular}{|l|} 
Edge-modified \\
\end{tabular} & Fine Grain Chert & 1 & 33.73 & & 12/1/2017 \\
\hline 337 & 7 & 399 & - & - & - & A & "I & No995 & E1013 & 5 & $99.3-99.2$ & $40-50$ & - & Informal Tool & \begin{tabular}{|l|} 
Modified Flake \\
\end{tabular} & \begin{tabular}{|l|} 
Edge-modified \\
\end{tabular} & Fine Grain Chert & 1 & 2.01 & & 12/1/2017 \\
\hline 337 & 8 & 399 & - & - & - & $\mathrm{A}$ & 11 & No995 & E1013 & 5 & 99.3-99.2 & $40-50$ & - & Informal Tool & Modified Flake & Utilized Flake & \begin{tabular}{|c|}
$\begin{array}{c}\text { Coarse Grain } \\
\text { Chert }\end{array}$ \\
\end{tabular} & 1 & 60.49 & & 12/1/2017 \\
\hline 338 & 1 & 405 & - & - & - & A & IIII & No995 & E1013 & 6 & 99.2-99.1 & $50-60$ & - & Faunal Remains & Bone & Indeterminate & Bone & 1 & 0.45 & & 12/4/2017 \\
\hline 338 & 2 & 405 & - & - & - & $\mathrm{A}$ & III & No995 & E1013 & 6 & 99.2-99.1 & $50-60$ & - & Faunal Remains & Bone & Indeterminate & Bone & 1 & 0.38 & & 12/1/2017 \\
\hline 338 & 3 & 405 & - & - & - & A & III & No995 & E1013 & 6 & $99.2-99.1$ & 50-60 & - & \begin{tabular}{|l|} 
Faunal Remains \\
\end{tabular} & Bone & Indeterminate & Bone & 1 & 0.17 & & 12/4/2017 \\
\hline 338 & 4 & 405 & - & - & - & A & IIII & No995 & E1013 & 6 & $99.2-99.1$ & 50-60 & - & \begin{tabular}{|l|} 
Faunal Remains \\
\end{tabular} & Bone & Indeterminate & Bone & 1 & 0.34 & & 12/4/2017 \\
\hline 338 & 5 & 405 & - & - & - & A & IIII & No995 & E1013 & 6 & $99.2-99.1$ & $50-60$ & - & Faunal Remains & Bone & Indeterminate & Bone & 1 & 0.17 & & 12/4/2017 \\
\hline 338 & 6 & 405 & - & - & - & A & IIII & No995 & E1013 & 6 & $99.2-99.1$ & $50-60$ & - & Faunal Remains & Shell & Mussel Shell & Shell & 1 & 0.46 & & 12/4/2017 \\
\hline 338 & 7 & 405 & - & - & - & A & IIII & No995 & E1013 & 6 & $99.2-99.1$ & 50-60 & - & Detritus & Debitage & \begin{tabular}{|c|} 
Complete Flake \\
\end{tabular} & Lithic & 14 & 39.25 & & 121/4/2017 \\
\hline 338 & 8 & 405 & - & - & - & A & III & No995 & E1013 & 6 & 99.2-99.1 & 50-60 & - & Detritus & Debitage & \begin{tabular}{|l|} 
Proximal Flake \\
\end{tabular} & Lithic & 13 & 10.05 & & 12/4/2017 \\
\hline 338 & 9 & 405 & - & - & - & A & III & No995 & E1013 & 6 & $99.2-99.1$ & 50-60 & - & Detritus & Debitage & Broken Flake & Lithic & 32 & 12.96 & & 121/4/2017 \\
\hline 338 & 10 & 405 & - & - & - & A & IIII & No995 & E1013 & 6 & $99.2-99.1$ & $50-60$ & - & Detritus & Debitage & Flaking Shatter & Lithic & 2 & 1.71 & & 12/4/2017 \\
\hline 338 & 11 & 405 & - & - & - & A & IIII & No995 & E1013 & 6 & $99.2-99.1$ & $50-60$ & - & Detritus & Debitage & Thermal Shatter & Lithic & 15 & 12.62 & & 12/4/2017 \\
\hline 339 & 1 & 410 & - & - & - & A & IIII & No995 & E1013 & 7 & $99.1-99.0$ & $60-70$ & - & \begin{tabular}{|l|} 
Faunal Remains \\
\end{tabular} & Bone & Avian & Bone & 1 & 0.28 & 2 burned & 12/4/2017 \\
\hline 339 & 2 & 410 & - & - & - & A & IIII & No995 & E1013 & 7 & $99.1-99.0$ & $60-70$ & - & \begin{tabular}{|l|} 
Faunal Remains \\
\end{tabular} & Bone & Indeterminate & Bone & 1 & 0.29 & 2 burned & 121/4/2017 \\
\hline 339 & 3 & 410 & - & - & - & A & IIII & No995 & E1013 & 7 & $99.1-99.0$ & $60-70$ & - & Faunal Remains & Bone & Mammal & Bone & 1 & 0.6 & 2 burned & 12/4/2017 \\
\hline 339 & 4 & 410 & - & - & - & $\mathrm{A}$ & III & No995 & E1013 & 7 & $99.1-99.0$ & 60-70 & - & Formal Tool & Biface & \begin{tabular}{|c|} 
Distal-Medial \\
Fragment \\
\end{tabular} & Fine Grain Chert & 1 & 21.61 & Distal end & 12/4/2017 \\
\hline 339 & 5 & 410 & - & - & - & A & III & No995 & E1013 & 7 & $99.1-99.0$ & $60-70$ & - & Detritus & Debitage & Complete Flake & Lithic & 19 & 37.67 & & 12/4/2017 \\
\hline 339 & 6 & 410 & - & - & - & A & IIII & No995 & E1013 & 7 & $99.1-99.0$ & $60-70$ & - & Detritus & Debitage & \begin{tabular}{|l|} 
Proximal Flake \\
\end{tabular} & Lithic & 17 & 9.68 & & 12/4/2017 \\
\hline 339 & 7 & 410 & - & - & - & A & III & No995 & E1013 & 7 & $99.1-99.0$ & $60-70$ & - & Detritus & Debitage & Broken Flake & Lithic & 41 & 15.89 & & 12/4/2017 \\
\hline 339 & 8 & 410 & - & - & - & A & IIII & N0995 & E1013 & 7 & $99.1-99.0$ & $\begin{array}{ll}60-70 \\
\end{array}$ & - & Detritus & Debitage & \begin{tabular}{|l|} 
Flaking Shatter \\
\end{tabular} & Lithic & 4 & 1.65 & & 12/4/2017 \\
\hline 339 & 9 & 410 & - & - & - & A & IIII & No995 & E1013 & 7 & $99.1-99.0$ & $60-70$ & - & Detritus & Debitage & Thermal Shatter & Lithic & 29 & 20.37 & & 12/4/2017 \\
\hline 339 & 10 & 410 & - & - & - & A & III & N0995 & E1013 & 7 & $99.1-99.0$ & $60-70$ & - & Informal Tool & \begin{tabular}{|l|} 
Modified Flake \\
\end{tabular} & Edge-modified & Fine Grain Chert & 1 & 10.26 & & 12/4/2017 \\
\hline 339 & 11 & 410 & - & - & - & A & III & No995 & E1013 & 7 & $99.1-99.0$ & $60-70$ & - & Detritus & Core & Bifacial & Fine Grain Chert & 1 & 57.01 & Fragment & 12/4/2017 \\
\hline 340 & 1 & 410 & - & - & - & A & III & No995 & E1013 & 7 & 99.05 & 65 & \begin{tabular}{|l|} 
N995.57 \\
E1013.81
\end{tabular} & Faunal Remains & Shell & Mussel Shell & Shell & 4 & 21.57 & & 12/4/2017 \\
\hline 341 & 1 & 421 & - & - & - & $\mathrm{A}$ & III & No995 & E1013 & 8 & 98.93 & 77 & \begin{tabular}{|l|} 
N995.04 \\
E1013.32
\end{tabular} & Formal Tool & Bifiace & Complete & Fine Grain Chert & 1 & 157.9 & Early stage biface & 12/4/2017 \\
\hline 342 & 1 & 422 & - & - & - & A & III & No995 & E1013 & 8 & 99.0-98.9 & $70-80$ & - & Detritus & Debitage & Complete Flake & Lithic & 2 & 39.52 & & 12/4/2017 \\
\hline 342 & 2 & 422 & - & - & - & A & IIII & No995 & E1013 & 8 & $99.0-98.9$ & $70-80$ & - & Detritus & Debitage & \begin{tabular}{|l|} 
Proximal Flake \\
\end{tabular} & Lithic & 4 & 34.46 & & 12/4/2017 \\
\hline 342 & 3 & 422 & - & - & - & A & IIII & No995 & E1013 & 8 & $99.0-98.9$ & $70-80$ & - & Detritus & Debitage & Broken Flake & Lithic & 5 & 5.22 & & 121/4/2017 \\
\hline 342 & 4 & 422 & - & - & - & A & III & No995 & E1013 & 8 & $99.0-98.9$ & $70-80$ & - & Detritus & Debitage & Thermal Shatter & Lithic & 6 & 3.41 & & 12/4/2017 \\
\hline
\end{tabular}




\begin{tabular}{|c|c|c|c|c|c|c|c|c|c|c|c|c|c|c|c|c|c|c|c|c|c|}
\hline Lot No. & \begin{tabular}{|c|}
$\begin{array}{c}\text { Specimen } \\
\text { No. }\end{array}$ \\
\end{tabular} & FS No. & UI No. & FTR No. & $\begin{array}{c}\text { Sample } \\
\text { No. }\end{array}$ & $\begin{array}{c}\text { Excavation } \\
\text { Block }\end{array}$ & Stratum (AU) & Northing & Easting & Level & $\begin{array}{c}\text { Elevation } \\
\text { (m) }\end{array}$ & Depth (cmbd) & Point Prov. & Artifact Class & Artifact Type & \begin{tabular}{|c|} 
Artifact \\
Description
\end{tabular} & Material & $\begin{array}{c}\text { Number of } \\
\text { artifacts }\end{array}$ & Weight (g) & Comments & Date \\
\hline 342 & 5 & 422 & - & - & - & A & III & No995 & E1013 & 8 & $99.0-98.9$ & $70-80$ & - & Informal Tool & Modified Flake & Utilized Flake & Fine Grain Chert & 1 & 86.18 & & 12/4/2017 \\
\hline 343 & 1 & 607 & - & - & - & A & IV & No995 & E1013 & 9 & 98.9-98.8 & $80-90$ & - & Detritus & Debitage & Complete Flake & Lithic & 4 & 2.48 & & 12/20/2017 \\
\hline 343 & 2 & 607 & - & - & - & A & IV & No995 & E1013 & 9 & $98.9-98.8$ & $80-90$ & - & Detritus & Debitage & \begin{tabular}{|l|} 
Proximal Flake \\
\end{tabular} & Lithic & 1 & 8.74 & & 12/20/2017 \\
\hline 343 & 3 & 607 & - & - & - & A & IV & No995 & E1013 & 9 & $98.9-98.8$ & $80-90$ & - & Detritus & Debitage & Broken Flake & Lithic & 13 & 22.8 & & 12/20/2017 \\
\hline 343 & 4 & 607 & - & - & - & A & IV & No995 & E1013 & 9 & 98.9-98.8 & $80-90$ & - & Detritus & Debitage & Thermal Shatter & Lithic & 7 & 4.87 & & 12/20/2017 \\
\hline 344 & 1 & 610 & - & - & - & A & IV & No995 & E1013 & 10 & 98.8-98.7 & 90-100 & - & \begin{tabular}{|l|} 
Faunal Remains \\
\end{tabular} & Bone & Mammal & Bone & 1 & 2.36 & & 12/20/2017 \\
\hline 344 & 2 & 610 & - & - & - & A & IV & No995 & E1013 & 10 & 98.8-98.7 & $90-100$ & - & \begin{tabular}{|l|} 
Faunal Remains \\
\end{tabular} & Bone & Indeterminate & Bone & 2 & 0.44 & & 12/20/2017 \\
\hline 344 & 3 & 610 & - & - & - & A & IV & No995 & E1013 & 10 & 98.8-98.7 & $90-100$ & - & Faunal Remains & Bone & Indeterminate & Bone & 2 & 0.1 & & 12/20/2017 \\
\hline 344 & 4 & 610 & - & - & - & A & IV & No995 & E1013 & 10 & $\begin{array}{l}98.8-98.7 \\
\end{array}$ & 90-100 & - & Faunal Remains & Bone & Indeterminate & Bone & 1 & 0.44 & & $\mid$\begin{tabular}{|l|}
$\mid 12 / 20 / 2017$ \\
\end{tabular} \\
\hline 344 & 5 & 610 & - & - & - & A & IV & N0995 & E1013 & 10 & $\begin{array}{ll}98.8-98.7 \\
\end{array}$ & $\begin{array}{l}0-100 \\
\end{array}$ & - & \begin{tabular}{|l|} 
Faunal Remains \\
\end{tabular} & Bone & Indeterminate & Bone & 1 & 0.13 & & 12/20/2017 \\
\hline 344 & 6 & 610 & - & - & - & A & IV & No995 & E1013 & 10 & 98.8-98.7 & $90-100$ & - & Faunal Remains & Bone & Indeterminate & Bone & 1 & 0.01 & & 12/20/2017 \\
\hline 344 & 7 & 610 & - & - & - & A & IV & N0995 & E1013 & 10 & $98.8-98.7$ & $\begin{array}{l}90-100 \\
\end{array}$ & - & Detritus & Debitage & Complete Flake & Lithic & 5 & 26 & & 12/20/2017 \\
\hline 344 & 8 & 610 & - & - & - & A & IV & No995 & E1013 & 10 & 98.8-98.7 & $90-100$ & - & Detritus & Debitage & Proximal Flake & Lithic & 1 & 0.32 & & 12/20/2017 \\
\hline 344 & 9 & 610 & - & - & - & A & IV & N0995 & E1013 & 10 & 98.8-98.7 & $\begin{array}{l}0-100 \\
\end{array}$ & - & Detritus & Debitage & Broken Flake & Lithic & 8 & 2.62 & & 12/20/2017 \\
\hline 344 & 10 & 610 & - & - & - & A & IV & No995 & E1013 & 10 & 98.8-98.7 & 90-100 & - & Detritus & Debitage & Thermal Shatter & Lithic & 7 & 12.11 & & 12/20/2017 \\
\hline 344 & 11 & 610 & - & - & - & A & iv & No995 & E1013 & 10 & 98.8-98.7 & 90-100 & - & Faunal Remains & Shell & Mussel Shell & Shell & 1 & 4.99 & & 12/20/2017 \\
\hline 345 & 1 & 614 & - & - & - & A & IV & No995 & E1013 & 11 & 98.7-98.6 & $100-110$ & - & Detritus & Debitage & Complete Flake & Lithic & 1 & 0.59 & & 12/20/2017 \\
\hline 345 & 2 & 614 & - & - & - & A & IV & No995 & E1013 & 11 & 98.7-98.6 & $100-110$ & - & Detritus & Debitage & Broken Flake & Lithic & 4 & 5.34 & & 12/20/2017 \\
\hline 345 & 3 & 614 & - & - & - & A & IV & No995 & E1013 & 11 & 98.7-98.6 & $100-110$ & - & Detritus & Debitage & Thermal Shatter & Lithic & 2 & 1.11 & & 12/20/2017 \\
\hline 346 & 1 & 626 & - & - & - & A & IV & No995 & E1013 & 12 & $98.6-98.5$ & $110-120$ & - & Faunal Remains & Bone & Odocoileus sp. & Bone & 1 & 13.06 & & 12/20/2017 \\
\hline 346 & 2 & 626 & - & - & - & A & IV & No995 & E1013 & 12 & $98.6-98.5$ & $110-120$ & - & Faunal Remains & Bone & Mammal & Bone & 1 & 2.79 & & 12/20/2017 \\
\hline 346 & 3 & 626 & - & - & - & A & IV & No995 & E1013 & 12 & $98.6-98.5$ & $110-120$ & - & Faunal Remains & Bone & Mammal & Bone & 1 & 0.4 & & 12/20/2017 \\
\hline 346 & 4 & 626 & - & - & - & A & IV & No995 & E1013 & 12 & $98.6-98.5$ & $110-120$ & - & Detritus & Debitage & \begin{tabular}{|l|} 
Complete Flake \\
\end{tabular} & Lithic & 2 & 24.97 & & 12/20/2017 \\
\hline 346 & 5 & 626 & - & - & - & A & IV & No995 & E1013 & 12 & $98.6-98.5$ & $110-120$ & - & Detritus & Debitage & Broken Flake & Lithic & 1 & 0.87 & & 12/20/2017 \\
\hline 346 & 6 & 626 & - & - & - & A & IV & No995 & E1013 & 12 & $98.6-98.5$ & $110-120$ & - & Detritus & Debitage & Flaking Shatter & Lithic & 1 & 0.15 & & 12/20/2017 \\
\hline 346 & 7 & 626 & - & - & - & A & IV & No995 & E1013 & 12 & $98.6-98.5$ & $110-120$ & - & Detritus & Debitage & Thermal Shatter & Lithic & 8 & 34.88 & & 12/20/2017 \\
\hline 346 & 8 & 626 & - & - & - & A & IV & No995 & E1013 & 12 & $98.6-98.5$ & $110-120$ & - & Detritus & Core & Multidirectional & Fine Grain Chert & 1 & 29.85 & Exhausted Core & 12/20/2017 \\
\hline 347 & 1 & 636 & - & - & - & A & IVN & No995 & E1013 & 13 & $98.5-98.4$ & $120-130$ & - & \begin{tabular}{|l|} 
Faunal Remains \\
\end{tabular} & Bone & Tortoise/Turtle & Bone & 1 & 3.12 & & 12/20/2017 \\
\hline 347 & 2 & 636 & - & - & - & A & IVN & No995 & E1013 & 13 & 98.5-98.4 & $120-130$ & - & Detritus & Debitage & \begin{tabular}{|l|} 
Complete Flake \\
\end{tabular} & Lithic & 1 & 4.17 & & 12/20/2017 \\
\hline 347 & 3 & 636 & - & - & - & A & IVN & No995 & E1013 & 13 & 98.5-98.4 & $120-130$ & - & Detritus & Debitage & \begin{tabular}{|l|} 
Proximal Flake \\
\end{tabular} & Lithic & 1 & 0.16 & & 12/20/2017 \\
\hline 347 & 4 & 636 & - & - & - & A & IVN & No995 & E1013 & 13 & 98.5-98.4 & 120-130 & - & Detritus & Debitage & Broken Flake & Lithic & 2 & 0.36 & & 12/20/2017 \\
\hline 347 & 5 & 636 & - & - & - & A & IVN & No995 & E1013 & 13 & $\begin{array}{l}98.5-98.4 \\
\end{array}$ & $120-130$ & - & Detritus & Debitage & Thermal Shatter & Lithic & 2 & 4.2 & & 12/20/2017 \\
\hline 348 & 1 & 644 & - & - & - & A & $\mathrm{v}$ & No995 & E1013 & 14 & 98.4-98.3 & $130-140$ & - & Faunal Remains & Bone & Odocoileus sp. & Bone & 1 & 8.19 & & 12/21/2017 \\
\hline 348 & 2 & 644 & - & - & - & A & $\mathrm{v}$ & No995 & E1013 & 14 & 98.4-98.3 & $130-140$ & - & Detritus & Debitage & Proximal Flake & Lithic & 1 & 12.38 & & \begin{tabular}{|l|}
$12 / 21 / 2017$ \\
\end{tabular} \\
\hline 348 & 3 & 644 & - & - & - & A & $\mathrm{v}$ & N0995 & E1013 & 14 & $\begin{array}{l}98.4-98.3 \\
\end{array}$ & 130-140 & - & Detritus & Debitage & Broken Flake & Lithic & 1 & 2.3 & & 12/21/2017 \\
\hline 349 & 1 & 670 & - & - & - & A & $\mathrm{vv}_{1}$ & No995 & E1013 & 15 & $98.3-98.2$ & 140-150 & - & Detritus & Debitage & Broken Flake & Lithic & 1 & 2.28 & & 12/28/2017 \\
\hline 349 & 2 & 670 & - & - & - & A & vi & No995 & E1013 & 15 & $98.3-98.2$ & $140-150$ & - & Detritus & Debitage & Thermal Shatter & Lithic & 1 & 0.41 & & $\mid 12 / 28 / 2017$ \\
\hline 350 & 1 & 354 & - & - & - & A & 1 & No995 & E1014 & 1 & 99.59-99.5 & $11-20$ & - & Faunal Remains & Bone & Mammal & Bone & 1 & 0.71 & & 11/29/2017 \\
\hline 350 & 2 & 354 & - & - & - & A & 1 & N0995 & E1014 & 1 & $99.59-99.5$ & $11-20$ & - & Detritus & Debitage & Broken Flake & Lithic & 3 & 1.91 & & 11/29/2017 \\
\hline 351 & 1 & 370 & - & - & - & A & "I & No995 & E1014 & 2 & $99.5-99.4$ & $20-30$ & - & Detritus & Debitage & Broken Flake & Lithic & 3 & 0.75 & & 11/30/2017 \\
\hline 351 & 2 & 370 & - & - & - & A & 11 & No995 & E1014 & 2 & 99.5-99.4 & $20-30$ & - & Informal Tool & Modified Flake & Edge-modified & Fine Grain Chert & 1 & 5.24 & & \begin{tabular}{|l|}
$11 / 30 / 2017$ \\
\end{tabular} \\
\hline 352 & 1 & 382 & - & - & - & A & "I & No995 & E1014 & 3 & $99.4-99.3$ & $30-40$ & - & Detritus & Debitage & \begin{tabular}{|l|} 
Complete Flake \\
\end{tabular} & Lithic & 2 & 1.72 & & 11/30/2017 \\
\hline 352 & 2 & 382 & - & - & - & A & "I & No995 & E1014 & 3 & $99.4-99.3$ & $30-40$ & - & Detritus & Debitage & Broken Flake & Lithic & 5 & 2.31 & & 11/30/2017 \\
\hline 352 & 3 & 382 & - & - & - & A & ॥ & No995 & E1014 & 3 & 99.4-99.3 & $30-40$ & - & Detritus & Debitage & \begin{tabular}{|l|} 
Thermal Shatter \\
\end{tabular} & Lithic & 2 & 2.22 & & 11/30/2017 \\
\hline
\end{tabular}




\begin{tabular}{|c|c|c|c|c|c|c|c|c|c|c|c|c|c|c|c|c|c|c|c|c|c|}
\hline Lot No. & $\begin{array}{c}\text { Specimen } \\
\text { No. }\end{array}$ & FS No. & UI No. & FTR No. & $\begin{array}{c}\text { Sample } \\
\text { No. }\end{array}$ & $\begin{array}{c}\text { Excavation } \\
\text { Block }\end{array}$ & Stratum (AU) & Northing & Easting & Level & $\begin{array}{c}\text { Elevation } \\
(\mathrm{m})\end{array}$ & Depth (cmbd) & \begin{tabular}{|l|} 
Point Prov. \\
\end{tabular} & Artifact Class & Artifact Type & \begin{tabular}{|c|} 
Artifact \\
Description
\end{tabular} & Material & $\begin{array}{c}\text { Number of } \\
\text { artifacts }\end{array}$ & Weight (g) & Comments & Date \\
\hline 353 & 1 & 394 & - & - & - & A & " & No995 & E1014 & 4 & 99.3-99.2 & $40-50$ & - & Faunal Remains & Bone & Mammal & Bone & 1 & 5.72 & & 12/1/2017 \\
\hline 353 & 2 & 394 & - & - & - & A & "I & No995 & E1014 & 4 & 99.3-99.2 & $40-50$ & - & Faunal Remains & Bone & Mammal & Bone & 1 & 3.18 & & 12/1/2017 \\
\hline 353 & 3 & 394 & - & - & - & A & $\|$ & N0995 & E1014 & 4 & $99.3-99.2$ & $40-50$ & - & \begin{tabular}{|c|} 
Detritus \\
\end{tabular} & Debitage & \begin{tabular}{|c|} 
Complete Flake \\
\end{tabular} & Lithic & 11 & 6.63 & & 12/1/2017 \\
\hline 353 & 4 & 394 & - & - & - & $\mathrm{A}$ & 11 & No995 & E1014 & 4 & 99.3-99.2 & $40-50$ & - & Detritus & Debitage & Proximal Flake & Lithic & 18 & 11.63 & & 12/1/2017 \\
\hline 353 & 5 & 394 & - & - & - & A & "I & No995 & E1014 & 4 & $99.3-99.2$ & $40-50$ & - & Detritus & Debitage & Broken Flake & Lithic & 32 & 39.05 & & 12/1/2017 \\
\hline 353 & 6 & 394 & - & - & - & A & "I & No995 & E1014 & 4 & $99.3-99.2$ & $40-50$ & - & Detritus & Debitage & Flaking Shatter & Lithic & 3 & 1.2 & & 12/1/2017 \\
\hline 353 & 7 & 394 & - & - & - & $\mathrm{A}$ & 11 & No995 & E1014 & 4 & 99.3-99.2 & $40-50$ & - & Detritus & Debitage & Thermal Shatter & Lithic & 11 & 15.32 & & 12/1/2017 \\
\hline 354 & 1 & 326 & - & - & - & A & III & No995 & E1014 & 5 & 99.2-99.1 & 50-60 & - & Faunal Remains & Bone & Indeterminate & Bone & 1 & 0.13 & & 12/1/2017 \\
\hline 354 & 2 & 326 & - & - & - & A & III & No995 & E1014 & 5 & 99.2-99.1 & $50-60$ & - & Faunal Remains & Bone & Indeterminate & Bone & 1 & 0.57 & & 12/1/2017 \\
\hline 354 & 3 & 326 & - & - & - & A & IIII & No995 & E1014 & 5 & $99.2-99.1$ & $50-60$ & - & Detritus & Debitage & \begin{tabular}{|l|} 
Complete Flake \\
\end{tabular} & Lithic & 21 & 93.4 & & 12/1/2017 \\
\hline 354 & 4 & 326 & - & - & - & A & IIII & No995 & E1014 & 5 & 99.2-99.1 & $50-60$ & - & Detritus & Debitage & Proximal Flake & Lithic & 15 & 14.3 & & 12/1/2017 \\
\hline 354 & 5 & 326 & - & - & - & A & IIII & No995 & E1014 & 5 & 99.2-99.1 & $50-60$ & - & Detritus & Debitage & Broken Flake & Lithic & 55 & 24.44 & & 12/1/2017 \\
\hline 354 & 6 & 326 & - & - & - & $\mathrm{A}$ & III & No995 & E1014 & 5 & 99.2-99.1 & $50-60$ & - & Detritus & Debitage & Thermal Shatter & Lithic & 20 & 18.25 & & 12/1/2017 \\
\hline 354 & 7 & 326 & - & - & - & A & IIII & No995 & E1014 & 5 & 99.2-99.1 & $50-60$ & - & Informal Tool & \begin{tabular}{|l|} 
Modified Flake \\
\end{tabular} & \begin{tabular}{|l|} 
Edge-modified \\
\end{tabular} & Fine Grain Chert & 1 & 10.97 & & 12/1/2017 \\
\hline 354 & 8 & 326 & - & - & - & A & III & No995 & E1014 & 5 & $99.2-99.1$ & $50-60$ & - & Faunal Remains & Shell & Mussel Shell & Shell & 1 & 3.43 & & 12/1/2017 \\
\hline 355 & 1 & 407 & - & - & - & A & IIII & No995 & E1014 & 6 & $99.1-99.0$ & $60-70$ & - & Faunal Remains & Bone & Mammal & Bone & 1 & 3.42 & & 12/4/2017 \\
\hline 355 & 2 & 407 & - & - & - & A & III & No995 & E1014 & 6 & $99.1-99.0$ & $60-70$ & - & Faunal Remains & Bone & Mammal & Bone & 1 & 1.27 & & 12/4/2017 \\
\hline 355 & 3 & 407 & - & - & - & A & III & No995 & E1014 & 6 & $99.1-99.0$ & $60-70$ & - & Detritus & Debitage & Complete Flake & Lithic & 21 & 43.1 & & 12/4/2017 \\
\hline 355 & 4 & 407 & - & - & - & $\mathrm{A}$ & III & No995 & E1014 & 6 & $99.1-99.0$ & $60-70$ & - & Detritus & Debitage & Proximal Flake & Lithic & 18 & 14.52 & & 12/4/2017 \\
\hline 355 & 5 & 407 & - & - & - & A & IIII & N0995 & E1014 & 6 & $99.1-99.0$ & $\begin{array}{ll}60-70 \\
\end{array}$ & - & Detritus & Debitage & Broken Flake & Lithic & 46 & 45.15 & & 12/4/2017 \\
\hline 355 & 6 & 407 & - & - & - & $\mathrm{A}$ & III & No995 & E1014 & 6 & $99.1-99.0$ & 60-70 & - & Detritus & Debitage & Thermal Shatter & Lithic & 22 & 18.31 & & 12/4/2017 \\
\hline 355 & 7 & 407 & - & - & - & A & IIII & N0995 & E1014 & 6 & $99.1-99.0$ & $60-70$ & - & \begin{tabular}{|l|} 
Faunal Remains \\
\end{tabular} & Shell & Mussel Shell & Shell & 2 & 3.81 & & 12/4/2017 \\
\hline 356 & 1 & 414 & - & - & - & A & III & No995 & E1014 & 7 & 98.96 & 74 & \begin{tabular}{|l|l|} 
N995.97 \\
E1014.38 \\
\end{tabular} & \begin{tabular}{|l|} 
Formal Tool \\
\end{tabular} & Bifface & Complete & Fine Grain Chert & 1 & 108.64 & Early stage biface & 12/4/2017 \\
\hline 357 & 1 & 415 & - & - & - & A & III & No995 & E1014 & 7 & 99.0-98.9 & $70-80$ & - & Faunal Remains & Bone & Indeterminate & Bone & 1 & 0.19 & & 12/4/2017 \\
\hline 357 & 2 & 415 & - & - & - & A & III & No995 & E1014 & 7 & $99.0-98.9$ & $70-80$ & - & Detritus & Debitage & Complete Flake & Lithic & 17 & 73.22 & & 12/4/2017 \\
\hline 357 & 3 & 415 & - & - & - & A & IIII & No995 & E1014 & 7 & $99.0-98.9$ & $70-80$ & - & Detritus & Debitage & \begin{tabular}{|l|} 
Proximal Flake \\
\end{tabular} & Lithic & 17 & 6.64 & & 12/4/2017 \\
\hline 357 & 4 & 415 & - & - & - & $\mathrm{A}$ & III & No995 & E1014 & 7 & 99.0-98.9 & $70-80$ & - & Detritus & Debitage & Broken Flake & Lithic & 37 & 27.46 & & 12/4/2017 \\
\hline 357 & 5 & 415 & - & - & - & $\mathrm{A}$ & III & No995 & E1014 & 7 & 99.0-98.9 & $70-80$ & - & Detritus & Debitage & Thermal Shatter & Lithic & 31 & 22.39 & & 12/4/2017 \\
\hline 358 & 1 & 426 & - & - & - & A & IV & No995 & E1014 & 8 & $98.9-98.8$ & $80-90$ & - & Detritus & Debitage & \begin{tabular}{|l|} 
Complete Flake \\
\end{tabular} & Lithic & 7 & 54.15 & & 12/5/2017 \\
\hline 358 & 2 & 426 & - & - & - & A & IV & No995 & E1014 & 8 & $98.9-98.8$ & $80-90$ & - & Detritus & Debitage & \begin{tabular}{|l|} 
Proximal Flake \\
\end{tabular} & Lithic & 9 & 10.78 & & 12/5/2017 \\
\hline 358 & 3 & 426 & - & - & - & A & IV & No995 & E1014 & 8 & 98.9-98.8 & $80-90$ & - & Detritus & Debitage & Broken Flake & Lithic & 10 & 4.5 & & 12/5/2017 \\
\hline 358 & 4 & 426 & - & - & - & A & IV & No995 & E1014 & 8 & $98.9-98.8$ & $80-90$ & - & Detritus & Debitage & Thermal Shatter & Lithic & 21 & 19.62 & & 12/5/2017 \\
\hline 359 & 1 & 427 & - & - & - & A & IV & No995 & E1014 & 8 & 98.85 & 85 & $\begin{array}{l}\text { N999.53 } \\
\text { E1014.60 }\end{array}$ & Detritus & Core & Multidirectional & Fine Grain Chert & 1 & 240.97 & Core & 12/5/2017 \\
\hline 360 & 1 & 428 & - & - & - & $\mathrm{A}$ & IV & No995 & E1014 & 8 & 98.86 & 84 & \begin{tabular}{|l|} 
N9995.05 \\
E1014.82 \\
\end{tabular} & Formal Tool & Biface & \begin{tabular}{|c|} 
Complete \\
\end{tabular} & Fine Grain Chert & 1 & 181.21 & Bifface-early stage & 12/5/2017 \\
\hline 361 & 1 & 516 & - & - & - & A & IV & No995 & E1014 & 9 & 98.8-98.7 & $90-100$ & - & Detritus & Debitage & Complete Flake & Lithic & 1 & 1.78 & & 12/13/2017 \\
\hline 361 & 2 & 516 & - & - & - & A & IV & No995 & E1014 & 9 & 98.8-98.7 & $90-100$ & - & Detritus & Debitage & Broken Flake & Lithic & 3 & 2.9 & & 12/13/2017 \\
\hline 361 & 3 & 516 & - & - & - & A & IV & No995 & E1014 & 9 & 98.8-98.7 & $90-100$ & - & Detritus & Debitage & Thermal Shatter & Lithic & 1 & 2.93 & & 12/13/2017 \\
\hline 362 & 1 & 532 & - & - & - & A & iv & No995 & E1014 & 10 & $98.7-98.6$ & $100-110$ & - & Detritus & Debitage & Complete Flake & Lithic & 3 & 17.45 & & 12/14/2017 \\
\hline 362 & 2 & 532 & - & - & - & A & iv & No995 & E1014 & 10 & $98.7-98.6$ & $100-110$ & - & Detritus & Debitage & Broken Flake & Lithic & 6 & 2.48 & & 12/14/2017 \\
\hline 362 & 3 & 532 & - & - & - & A & iv & No995 & E1014 & 10 & $98.7-98.6$ & $100-110$ & - & Detritus & Debitage & Thermal Shatter & Lithic & 1 & 0.28 & & 12/14/2017 \\
\hline 363 & 1 & 634 & - & - & - & A & IV & No995 & E1014 & 11 & $98.6-98.5$ & $110-120$ & - & Faunal Remains & Bone & Mammal & Bone & 1 & 1.25 & & 12/20/2017 \\
\hline 363 & 2 & 634 & - & - & - & $\mathrm{A}$ & iv & No995 & E1014 & 11 & 98.6-98.5 & 110-120 & - & Detritus & Debitage & Complete Flake & Lithic & 3 & 7.43 & & 12/20/2017 \\
\hline 363 & 3 & 634 & - & - & - & A & IV & N0995 & E1014 & 11 & $98.6-98.5$ & 110-120 & - & Detritus & Debitage & \begin{tabular}{|l|} 
Broken Flake \\
\end{tabular} & Lithic & 1 & 0.4 & & 12/20/2017 \\
\hline 363 & 4 & 634 & - & - & - & A & IV & No995 & E1014 & 11 & $98.6-98.5$ & $110-120$ & - & Detritus & Debitage & Thermal Shatter & Lithic & 1 & 0.46 & & 12/20/2017 \\
\hline
\end{tabular}




\begin{tabular}{|c|c|c|c|c|c|c|c|c|c|c|c|c|c|c|c|c|c|c|c|c|c|}
\hline Lot No. & \begin{tabular}{|c|}
$\begin{array}{c}\text { Specimen } \\
\text { No. }\end{array}$ \\
\end{tabular} & FS No. & UI No. & FTR No. & $\begin{array}{c}\text { Sample } \\
\text { No. }\end{array}$ & $\begin{array}{c}\text { Excavation } \\
\text { Block }\end{array}$ & Stratum (AU) & Northing & Easting & Level & $\begin{array}{c}\text { Elevation } \\
(\mathrm{m})\end{array}$ & Depth (cmbd) & Point Prov. & Artifact Class & Artifact Type & \begin{tabular}{|c|} 
Artifact \\
Description
\end{tabular} & Material & $\begin{array}{c}\text { Number of } \\
\text { artifacts }\end{array}$ & Weight (g) & Comments & Date \\
\hline 364 & 1 & 647 & - & - & - & A & IVIV & No995 & E1014 & 12 & 98.5-98.4 & 120-130 & - & Detritus & Debitage & \begin{tabular}{|l|} 
Proximal Flake \\
\end{tabular} & Lithic & 2 & 1.63 & & 12/21/2017 \\
\hline 364 & 2 & 647 & - & - & - & A & IVN & No995 & E1014 & 12 & $98.5-98.4$ & $120-130$ & - & Detritus & Debitage & Thermal Shatter & Lithic & 1 & 2.74 & & 12/21/2017 \\
\hline 365 & 1 & 650 & - & - & - & A & $\mathrm{v}$ & No995 & E1014 & 13 & 98.4-98.3 & $130-140$ & - & Detritus & Debitage & \begin{tabular}{|l|} 
Complete Flake \\
\end{tabular} & Lithic & 2 & 36.85 & & 12/21/2017 \\
\hline 365 & 2 & 650 & - & - & - & A & $\mathrm{v}$ & No995 & E1014 & 13 & 98.4-98.3 & $130-140$ & - & Detritus & Debitage & Proximal Flake & Lithic & 1 & 4.73 & & 12/21/2017 \\
\hline 365 & 3 & 650 & - & - & - & A & $\mathrm{v}$ & No995 & E1014 & 13 & 98.4-98.3 & 130-140 & - & Detritus & Debitage & Broken Flake & Lithic & 1 & 0.17 & & 12/21/2017 \\
\hline 366 & 1 & 470 & - & - & - & A & 1 & No996 & E1012 & 1 & $99.7-99.6$ & $0-10$ & - & Faunal Remains & Bone & Mammal & Bone & 1 & 0.07 & & 12/11/2017 \\
\hline 366 & 2 & 470 & - & - & - & A & 1 & No996 & E1012 & 1 & 99.7-99.6 & $0-10$ & - & Detritus & Debitage & Complete Flake & Lithic & 1 & 2.42 & & 12/11/2017 \\
\hline 366 & 3 & 470 & - & - & - & A & 1 & No996 & E1012 & 1 & 99.7-99.6 & $0-10$ & - & Detritus & Debitage & Broken Flake & Lithic & 1 & 1.05 & & $\mid 12 / 11 / 2017$ \\
\hline 366 & 4 & 470 & - & - & - & A & 1 & No996 & E1012 & 1 & 99.7-99.6 & $0-10$ & - & Detritus & Debitage & Thermal Shatter & Lithic & 1 & 12.24 & & 12/11/2017 \\
\hline 367 & 1 & 477 & - & - & - & A & 1 & N0996 & E1012 & 2 & $99.6-99.5$ & $10-20$ & - & Detritus & Debitage & Complete Flake & Lithic & 1 & 4.57 & & \begin{tabular}{|l|}
$12 / 11 / 2017$ \\
\end{tabular} \\
\hline 367 & 2 & 477 & - & - & - & A & 1 & No996 & E1012 & 2 & 99.6-99.5 & $10-20$ & - & Detritus & Debitage & \begin{tabular}{|l|} 
Proximal Flake \\
\end{tabular} & Lithic & 3 & 1.88 & & 12/11/2017 \\
\hline 367 & 3 & 477 & - & - & - & A & 1 & N0996 & E1012 & 2 & $99.6-99.5$ & $10-20$ & - & Detritus & Debitage & Broken Flake & Lithic & 2 & 0.25 & & 12/11/2017 \\
\hline 367 & 4 & 477 & - & - & - & A & 1 & No996 & E1012 & 2 & $99.6-99.5$ & $10-20$ & - & Detritus & Debitage & Thermal Shatter & Lithic & 3 & 0.88 & & 12/11/2017 \\
\hline 367 & 5 & 477 & - & - & - & A & 1 & No996 & E1012 & 2 & $99.6-99.5$ & $10-20$ & - & Historic Artifact & Ceramic & Whiteware & Ceramic & 1 & 0.82 & 1 Whiteware & 12/11/2017 \\
\hline 368 & 1 & 481 & - & - & - & A & 11 & No996 & E1012 & 3 & 99.5-99.4 & $20-30$ & - & Faunal Remains & Bone & Indeterminate & Bone & 1 & 0.22 & & 12/11/2017 \\
\hline 368 & 2 & 481 & - & - & - & A & " & No996 & E1012 & 3 & $99.5-99.4$ & $20-30$ & - & Detritus & Debitage & Complete Flake & Lithic & 1 & 16.39 & & $12 / 11 / 2017$ \\
\hline 368 & 3 & 481 & - & - & - & A & "I & No996 & E1012 & 3 & 99.5-99.4 & $20-30$ & - & Detritus & Debitage & Proximal Flake & Lithic & 2 & 11.95 & & 12/11/2017 \\
\hline 368 & 4 & 481 & - & - & - & A & ॥ & No996 & E1012 & 3 & $99.5-99.4$ & $20-30$ & - & Detritus & Debitage & Broken Flake & Lithic & 5 & 1.9 & & 12/11/2017 \\
\hline 368 & 5 & 481 & - & - & - & A & 11 & No996 & E1012 & 3 & 99.5-99.4 & $20-30$ & - & Detritus & Debitage & Thermal Shatter & Lithic & 7 & 7.68 & & 12/11/2017 \\
\hline 369 & 1 & 487 & - & - & - & A & 11 & No996 & E1012 & 4 & 99.499 .3 & $30-40$ & - & Detritus & Debitage & \begin{tabular}{|l|} 
Complete Flake \\
\end{tabular} & Lithic & 7 & 13.52 & & 12/12/2017 \\
\hline 369 & 2 & 487 & - & - & - & A & 11 & No996 & E1012 & 4 & 99.499 .3 & $30-40$ & - & Detritus & Debitage & \begin{tabular}{|l|} 
Proximal Flake \\
\end{tabular} & Lithic & 10 & 10.19 & & 12/112/2017 \\
\hline 369 & 3 & 487 & - & - & - & A & "I & No996 & E1012 & 4 & $99.4-99.3$ & $30-40$ & - & Detritus & Debitage & Broken Flake & Lithic & 21 & 8.87 & & 12/12/2017 \\
\hline 369 & 4 & 487 & - & - & - & A & "I & No996 & E1012 & 4 & $99.4-99.3$ & $30-40$ & - & Detritus & Debitage & Flaking Shatter & Lithic & 2 & 0.76 & & 12/12/2/2017 \\
\hline 369 & 5 & 487 & - & - & - & A & "I & No996 & E1012 & 4 & $99.4-99.3$ & $30-40$ & - & Detritus & Debitage & Thermal Shatter & Lithic & 12 & 13.22 & & 12/12/2017 \\
\hline 369 & 6 & 487 & - & - & - & A & "I & No996 & E1012 & 4 & $99.4-99.3$ & $30-40$ & - & Faunal Remains & Shell & Mussel Shell & Shell & 1 & 0.17 & & 12/12/2017 \\
\hline 369 & 7 & 487 & - & - & - & A & "I & No996 & E1012 & 4 & 99.499 .3 & $30-40$ & - & Informal Tool & \begin{tabular}{|l|} 
Modified Flake \\
\end{tabular} & \begin{tabular}{|l|} 
Edge-modified \\
\end{tabular} & Fine Grain Chert & 1 & 3.16 & & 12/112/2017 \\
\hline 370 & 1 & 496 & - & - & - & A & "I & No996 & E1012 & 5 & $99.3-99.2$ & $40-50$ & - & Detritus & Debitage & \begin{tabular}{|l|} 
Complete Flake \\
\end{tabular} & Lithic & 11 & 57.41 & & 12/12/2017 \\
\hline 370 & 2 & 496 & - & - & - & A & "I & No996 & E1012 & 5 & $99.3-99.2$ & $40-50$ & - & Detritus & Debitage & \begin{tabular}{|l|} 
Proximal Flake \\
\end{tabular} & Lithic & 14 & 15.37 & & 12/12/2017 \\
\hline 370 & 3 & 496 & - & - & - & A & $\|$ & No996 & E1012 & 5 & $99.3-99.2$ & $40-50$ & - & Detritus & Debitage & Broken Flake & Lithic & 29 & 15.7 & & 12/112/2017 \\
\hline 370 & 4 & 496 & - & - & - & A & "I & No996 & E1012 & 5 & $99.3-99.2$ & $40-50$ & - & Detritus & Debitage & Flaking Shatter & Lithic & 1 & 0.56 & & 12/12/2017 \\
\hline 370 & 5 & 496 & - & - & - & A & " & No996 & E1012 & 5 & 99.3-99.2 & $40-50$ & - & Detritus & Debitage & Thermal Shatter & Lithic & 11 & 26.35 & & $\mid$\begin{tabular}{|l|}
$\mid 12 / 12 / 2017$ \\
\end{tabular} \\
\hline 370 & 6 & 496 & - & - & - & A & II & No996 & E1012 & 5 & $99.3-99.2$ & $40-50$ & - & Faunal Remains & Shell & Mussel Shell & Shell & 1 & 0.95 & & 12/12/2017 \\
\hline 371 & 1 & 497 & - & - & - & A & "I & No996 & E1012 & 5 & 99.26 & 44 & $\begin{array}{l}\begin{array}{l}\text { N996.42 } \\
\text { E91012.23 }\end{array} \\
\end{array}$ & Informal Tool & Modified Flake & \begin{tabular}{|l|} 
Edge-modified \\
\end{tabular} & Fine Grain Chert & 1 & 59.86 & & 12/12/2017 \\
\hline 372 & 1 & 502 & - & - & - & A & IIII & No996 & E1012 & 6 & 99.2-99.1 & $50-60$ & - & Faunal Remains & Bone & Tortoise/Turtle & Bone & 1 & 0.24 & & \begin{tabular}{|l|}
$12 / 12 / 2017$ \\
\end{tabular} \\
\hline 372 & 2 & 502 & - & - & - & A & IIII & No996 & E1012 & 6 & $99.2-99.1$ & 50-60 & - & Detritus & Debitage & \begin{tabular}{|c|} 
Complete Flake \\
\end{tabular} & Lithic & 6 & 32.33 & & 12/12/2017 \\
\hline 372 & 3 & 502 & - & - & - & A & IIII & No996 & E1012 & 6 & $99.2-99.1$ & 50-60 & - & Detritus & Debitage & \begin{tabular}{|l|} 
Proximal Flake \\
\end{tabular} & Lithic & 6 & 8.51 & & 12/12/2017 \\
\hline 372 & 4 & 502 & - & - & - & A & IIII & N0996 & E1012 & 6 & 99.2-99.1 & 50-60 & - & Detritus & Debitage & Broken Flake & Lithic & 23 & 28.37 & & 12/12/2017 \\
\hline 372 & 5 & 502 & - & - & - & A & IIII & No996 & E1012 & 6 & 99.2-99.1 & $50-60$ & - & Detritus & Debitage & Flaking Shatter & Lithic & 1 & 0.53 & & \begin{tabular}{|l|}
$12 / 12 / 2017$ \\
\end{tabular} \\
\hline 372 & 6 & 502 & - & - & - & A & IIII & No996 & E1012 & 6 & 99.2-99.1 & $50-60$ & - & Detritus & Debitage & Thermal Shatter & Lithic & 20 & 54.18 & & 12/12/2017 \\
\hline 373 & 1 & 517 & - & - & - & A & III & No996 & E1012 & 7 & $99.1-99.0$ & $60-70$ & - & Faunal Remains & Bone & Indeterminate & Bone & 1 & 0.26 & & 12/13/2017 \\
\hline 373 & 2 & 517 & - & - & - & A & III & No996 & E1012 & 7 & $99.1-99.0$ & $60-70$ & - & \begin{tabular}{|l|} 
Faunal Remains \\
\end{tabular} & Bone & Indeterminate & Bone & 1 & 3.48 & & \begin{tabular}{|l|}
$12 / 13 / 2017$ \\
\end{tabular} \\
\hline 373 & 3 & 517 & - & - & - & A & IIII & No996 & E1012 & 7 & $99.1-99.0$ & $60-70$ & - & Faunal Remains & Bone & Indeterminate & Bone & 1 & 1.39 & & 12/13/2017 \\
\hline 373 & 4 & 517 & - & - & - & A & IIII & No996 & E1012 & 7 & $99.1-99.0$ & $60-70$ & - & Faunal Remains & Bone & Indeterminate & Bone & 1 & 0.19 & & 12/13/2017 \\
\hline 373 & 5 & 517 & - & - & - & A & III & No996 & E1012 & 7 & $99.1-99.0$ & $60-70$ & - & Faunal Remains & Bone & Indeterminate & Bone & 1 & 0.23 & & \begin{tabular}{|l|}
$12 / 13 / 2017$ \\
\end{tabular} \\
\hline
\end{tabular}




\begin{tabular}{|c|c|c|c|c|c|c|c|c|c|c|c|c|c|c|c|c|c|c|c|c|c|}
\hline Lot No. & \begin{tabular}{|c|}
$\begin{array}{c}\text { Specimen } \\
\text { No. }\end{array}$ \\
\end{tabular} & FS No. & UI No. & FTR No. & $\begin{array}{c}\text { Sample } \\
\text { No. }\end{array}$ & $\begin{array}{c}\text { Excavation } \\
\text { Block }\end{array}$ & Stratum (AU) & Northing & Easting & Level & $\begin{array}{c}\text { Elevation } \\
(\mathrm{m})\end{array}$ & Depth (cmbd) & \begin{tabular}{|l|} 
Point Prov. \\
\end{tabular} & Artifact Class & \begin{tabular}{|l|} 
Artifact Type \\
\end{tabular} & \begin{tabular}{|c|} 
Artifact \\
Description
\end{tabular} & Material & $\begin{array}{c}\text { Number of } \\
\text { artifacts }\end{array}$ & Weight (g) & Comments & Date \\
\hline 373 & 6 & 517 & - & - & - & A & III & No996 & E1012 & 7 & $99.1-99.0$ & $60-70$ & - & Faunal Remains & Bone & Indeterminate & Bone & 1 & 0.58 & & 12/13/2017 \\
\hline 373 & 7 & 517 & - & - & - & A & III & No996 & E1012 & 7 & $99.1-99.0$ & $60-70$ & - & Faunal Remains & Bone & Indeterminate & Bone & 1 & 0.27 & & $12 / 13 / 2017$ \\
\hline 373 & 8 & 517 & - & - & - & A & IIII & No996 & E1012 & 7 & $99.1-99.0$ & $60-70$ & - & \begin{tabular}{|l|} 
Faunal Remains \\
\end{tabular} & Bone & Indeterminate & Bone & 1 & 0.34 & & 12/13/2017 \\
\hline 373 & 9 & 517 & - & - & - & A & IIII & No996 & E1012 & 7 & $99.1-99.0$ & $60-70$ & - & Faunal Remains & Bone & Indeterminate & Bone & 1 & 0.36 & & 12/13/2017 \\
\hline 373 & 10 & 517 & - & - & - & A & IIII & No996 & E1012 & 7 & $99.1-99.0$ & $60-70$ & - & Faunal Remains & Bone & Indeterminate & Bone & 1 & 0.22 & & 12/13/2017 \\
\hline 373 & 11 & 517 & - & - & - & A & IIII & No996 & E1012 & 7 & $99.1-99.0$ & $60-70$ & - & \begin{tabular}{|l|} 
Faunal Remains \\
\end{tabular} & Bone & Indeterminate & Bone & 1 & 0.96 & & 12/13/2017 \\
\hline 373 & 12 & 517 & - & - & - & A & IIII & No996 & E1012 & 7 & $99.1-99.0$ & 60-70 & - & \begin{tabular}{|l|} 
Faunal Remains \\
\end{tabular} & Bone & Indeterminate & Bone & 1 & 0.73 & & 12/13/2017 \\
\hline 373 & 13 & 517 & - & - & - & A & III & No996 & E1012 & 7 & $99.1-99.0$ & 60-70 & - & Detritus & Debitage & Complete Flake & Lithic & 12 & 36.48 & & $\mid$\begin{tabular}{|l|}
$\mid 12 / 13 / 2017$ \\
\end{tabular} \\
\hline 373 & 14 & 517 & - & - & - & A & III & No996 & E1012 & 7 & $99.1-99.0$ & $60-70$ & - & Detritus & Debitage & Proximal Flake & Lithic & 21 & 32.82 & & $\mid$\begin{tabular}{|l|}
$\mid 12 / 13 / 2017$ \\
\end{tabular} \\
\hline 373 & 15 & 517 & - & - & - & A & IIII & N0996 & E1012 & 7 & $99.1-99.0$ & $60-70$ & - & Detritus & Debitage & Broken Flake & Lithic & 59 & 38.36 & & 12/13/2017 \\
\hline 373 & 16 & 517 & - & - & - & A & IIII & No996 & E1012 & 7 & $99.1-99.0$ & $60-70$ & - & Detritus & Debitage & Flaking Shatter & Lithic & 2 & 2.9 & & 12/13/2017 \\
\hline 373 & 17 & 517 & - & - & - & A & IIII & No996 & E1012 & 7 & $99.1-99.0$ & $60-70$ & - & Detritus & Debitage & Thermal Shatter & Lithic & 52 & 51.75 & & 12/13/2017 \\
\hline 373 & 18 & 517 & - & - & - & A & IIII & N0996 & E1012 & 7 & $99.1-99.0$ & $60-70$ & - & \begin{tabular}{|l|} 
Faunal Remains \\
\end{tabular} & Shell & Mussel Shell & Shell & 1 & 2.63 & & 12/13/2017 \\
\hline 373 & 19 & 517 & - & - & - & A & IIII & No996 & E1012 & 7 & $99.1-99.0$ & $60-70$ & - & Other Lithic & \begin{tabular}{l|l} 
Thermally \\
Altered Stone
\end{tabular} & FCR & Lithic & 2 & 85.54 & Originally classified as a Core & 12/13/2017 \\
\hline 373 & 20 & 517 & - & - & - & A & IIII & No996 & E1012 & 7 & $99.1-99.0$ & $60-70$ & - & Detritus & Core & Multidirectional & Fine Grain Chert & 1 & 60.38 & Exhausted Core & 12/13/2017 \\
\hline 373 & 21 & 517 & - & - & - & A & III & No996 & E1012 & 7 & $99.1-99.0$ & $60-70$ & - & Clay & Clay Artifact & Burned Clay & Clay & 5 & 18.47 & & 12/13/2017 \\
\hline 374 & 1 & 549 & - & FTR3 & c-13 & A & IIII & No996 & E1012 & 8 & $99.0-98.9$ & $70-80$ & $\begin{array}{l}\text { N996.53 } \\
\text { E1012.07 }\end{array}$ & $\begin{array}{l}\text { Archaeological } \\
\text { Sample }\end{array}$ & c-14 & not ID'd & & 6 & 0.03 & & 12/13/2017 \\
\hline 375 & 1 & 549 & - & FTR3 & - & A & IIII & No996 & E1012 & 8 & $99.0-98.9$ & $70-80$ & - & Faunal Remains & Bone & Indeterminate & Bone & 1 & 0.26 & & 12/15/2017 \\
\hline 375 & 2 & 549 & - & FTR3 & - & A & IIII & No996 & E1012 & 8 & $99.0-98.9$ & $70-80$ & - & Faunal Remains & Bone & Indeterminate & Bone & 1 & 0.06 & & 12/15/2017 \\
\hline 375 & 3 & 549 & - & FTR3 & - & A & IIII & No996 & E1012 & 8 & $\begin{array}{l}99.0-98.9 \\
\end{array}$ & $70-80$ & - & \begin{tabular}{|l|} 
Faunal Remains \\
\end{tabular} & Bone & Indeterminate & Bone & 1 & 0.29 & & 12/15/2017 \\
\hline 375 & 4 & 549 & - & FTR3 & - & A & IIII & No996 & E1012 & 8 & $99.0-98.9$ & $70-80$ & - & Faunal Remains & Bone & Indeterminate & Bone & 1 & 0.61 & & 12/15/2017 \\
\hline 375 & 5 & 549 & - & FTR3 & - & A & IIII & No996 & E1012 & 8 & $99.0-98.9$ & $70-80$ & - & Faunal Remains & Bone & Indeterminate & Bone & 1 & 0.06 & & 12/15/2017 \\
\hline 375 & 6 & 549 & - & FTR3 & - & A & IIII & No996 & E1012 & 8 & $99.0-98.9$ & $70-80$ & - & Faunal Remains & Bone & Indeterminate & Bone & 1 & 0.46 & & 12/15/2017 \\
\hline 375 & 7 & 549 & - & FTR3 & - & A & IIII & No996 & E1012 & 8 & $99.0-98.9$ & $70-80$ & - & Faunal Remains & Bone & Indeterminate & Bone & 1 & 0.11 & & 12/15/2017 \\
\hline 375 & 8 & 549 & - & FTR3 & - & A & IIII & No996 & E1012 & 8 & $99.0-98.9$ & $70-80$ & - & Faunal Remains & Bone & Indeterminate & Bone & 1 & 0.03 & & 12/15/2017 \\
\hline 375 & 9 & 549 & - & FTR3 & - & A & IIII & No996 & E1012 & 8 & $99.0-98.9$ & $70-80$ & - & \begin{tabular}{|l|} 
Faunal Remains \\
\end{tabular} & Bone & Indeterminate & Bone & 1 & 0.01 & & 12/15/2017 \\
\hline 375 & 10 & 549 & - & FTR3 & - & A & IIII & No996 & E1012 & 8 & $99.0-98.9$ & $70-80$ & - & \begin{tabular}{|l|} 
Faunal Remains \\
\end{tabular} & Bone & Indeterminate & Bone & 1 & 0.01 & & 12/15/2017 \\
\hline 375 & 11 & 549 & - & FTR3 & - & A & IIII & No996 & E1012 & 8 & $99.0-98.9$ & $70-80$ & - & Detritus & Debitage & \begin{tabular}{|l|} 
Complete Flake \\
\end{tabular} & Lithic & 7 & 65.15 & & 12/15/2017 \\
\hline 375 & 12 & 549 & - & FTR3 & - & A & IIII & No996 & E1012 & 8 & $99.0-98.9$ & $70-80$ & - & Detritus & Debitage & \begin{tabular}{|l|} 
Proximal Flake \\
\end{tabular} & Lithic & 5 & 7.52 & & 12/15/2017 \\
\hline 375 & 13 & 549 & - & FTR3 & - & A & IIII & No996 & E1012 & 8 & $99.0-98.9$ & $70-80$ & - & Detritus & Debitage & Broken Flake & Lithic & 23 & 17.78 & & 12/15/2017 \\
\hline 375 & 14 & 549 & - & FTR3 & - & A & IIII & No996 & E1012 & 8 & 99.0-98.9 & $70-80$ & - & Detritus & Debitage & Thermal Shatter & Lithic & 48 & 21.64 & & $\mid 12 / 15 / 2017$ \\
\hline 376 & 1 & 573 & - & - & - & A & iv & No996 & E1012 & 9 & 98.8 & 90 & \begin{tabular}{|l|l}
$\begin{array}{l}\text { N996.66 } \\
\text { E1011.255 }\end{array}$ & \\
\end{tabular} & Faunal Remains & Shell & Mussel Shell & Shell & 1 & 14.85 & & $\mid 12 / 18 / 2017$ \\
\hline 377 & 1 & 573 & - & - & - & A & IV & No996 & E1012 & 9 & $\begin{array}{l}98.9-98.8 \\
\end{array}$ & $80-90$ & - & Faunal Remains & Bone & Indeterminate & Bone & 1 & 0.06 & & 12/18/2017 \\
\hline 377 & 2 & 573 & - & - & - & A & IV & No996 & E1012 & 9 & 98.9-98.8 & $80-90$ & - & Faunal Remains & Bone & Indeterminate & Bone & 1 & 0.17 & & 12/18/2017 \\
\hline 377 & 3 & 573 & - & - & - & A & IV & No996 & E1012 & 9 & $\begin{array}{ll}88.9-98.8 \\
\end{array}$ & \begin{tabular}{ll|l|}
$80-90$ & \\
\end{tabular} & - & Detritus & Debitage & \begin{tabular}{|c|} 
Complete Flake \\
\end{tabular} & Lithic & 4 & 6.51 & & 12/18/2017 \\
\hline 377 & 4 & 573 & - & - & - & A & IV & No996 & E1012 & 9 & $\begin{array}{ll}88.9-98.8 \\
\end{array}$ & \begin{tabular}{ll|l|}
$80-90$ & \\
\end{tabular} & - & Detritus & Debitage & \begin{tabular}{|l|} 
Proximal Flake \\
\end{tabular} & Lithic & 5 & 12.43 & & 12/18/2017 \\
\hline 377 & 5 & 573 & - & - & - & A & IV & No996 & E1012 & 9 & 98.9-98.8 & $80-90$ & - & Detritus & Debitage & Broken Flake & Lithic & 34 & 10.31 & & $\mid 12 / 18 / 2017$ \\
\hline 377 & 6 & 573 & - & - & - & A & IV & No996 & E1012 & 9 & 98.9-98.8 & $80-90$ & - & Detritus & Debitage & Thermal Shatter & Lithic & 83 & 51.17 & & 12/18/2017 \\
\hline 378 & 1 & 586 & - & - & - & A & IV & No996 & E1012 & 10 & $\begin{array}{ll}98.8-98.7 \\
\end{array}$ & $\begin{array}{l}0-100 \\
\end{array}$ & - & Detritus & Debitage & \begin{tabular}{|l|} 
Complete Flake \\
\end{tabular} & Lithic & 7 & 31.79 & & 12/18/2017 \\
\hline 378 & 2 & 586 & - & - & - & A & IV & No996 & E1012 & 10 & $\begin{array}{ll}88.8-98.7 \\
\end{array}$ & 90-100 & - & Detritus & Debitage & \begin{tabular}{|l|} 
Proximal Flake \\
\end{tabular} & Lithic & 5 & 5.19 & & 12/18/2017 \\
\hline 378 & 3 & 586 & - & - & - & A & IV & No996 & E1012 & 10 & 98.8-98.7 & 90-100 & - & Detritus & Debitage & Broken Flake & Lithic & 9 & 11.83 & & 12/18/2017 \\
\hline 378 & 4 & 586 & - & - & - & A & IV & No996 & E1012 & 10 & $\begin{array}{l}98.898 .7 \\
\end{array}$ & $90-100$ & - & Detritus & Debitage & Thermal Shatter & Lithic & 5 & 1.19 & & 12/18/2017 \\
\hline 378 & 5 & 586 & - & - & - & A & IV & No996 & E1012 & 10 & 98.898 .7 & $90-100$ & - & Formal Tool & Biface & \begin{tabular}{c|} 
Indeterminate \\
Fragment
\end{tabular} & Fine Grain Chert & 1 & 14.5 & & 12/18/2017 \\
\hline 379 & 1 & 621 & - & - & - & A & IV & No996 & E1012 & 11 & 98.7-98.6 & $100-110$ & - & Detritus & Debitage & \begin{tabular}{|c|} 
Complete Flake \\
\end{tabular} & Lithic & 1 & 2.06 & & 12/20/2017 \\
\hline
\end{tabular}




\begin{tabular}{|c|c|c|c|c|c|c|c|c|c|c|c|c|c|c|c|c|c|c|c|c|c|}
\hline Lot No. & \begin{tabular}{|c|}
$\begin{array}{c}\text { Specimen } \\
\text { No. }\end{array}$ \\
\end{tabular} & FS No. & UI No. & FTR No. & $\begin{array}{c}\text { Sample } \\
\text { No. }\end{array}$ & $\begin{array}{c}\text { Excavation } \\
\text { Block }\end{array}$ & Stratum (AU) & Northing & Easting & Level & $\begin{array}{c}\begin{array}{c}\text { Elevation } \\
(\mathrm{m})\end{array} \\
\end{array}$ & Depth (cmbd) & Point Prov. & Artifact Class & Artifact Type & \begin{tabular}{|c|} 
Artifact \\
Description
\end{tabular} & Material & $\begin{array}{c}\text { Number of } \\
\text { artifacts }\end{array}$ & Weight (g) & Comments & Date \\
\hline 379 & 2 & 621 & - & - & - & A & IV & No996 & E1012 & 11 & 98.7-98.6 & $100-110$ & - & Detritus & Debitage & \begin{tabular}{|l|} 
Proximal Flake \\
\end{tabular} & Lithic & 2 & 3.21 & & 12/20/2017 \\
\hline 379 & 3 & 621 & - & - & - & A & IV & No996 & E1012 & 11 & $98.7-98.6$ & $100-110$ & - & Detritus & Debitage & Broken Flake & Lithic & 4 & 8.63 & & 12/20/2017 \\
\hline 379 & 4 & 621 & - & - & - & A & IV & No996 & E1012 & 11 & 98.7-98.6 & $100-110$ & - & Detritus & Debitage & Thermal Shatter & Lithic & 5 & 7.54 & & 12/20/2017 \\
\hline 380 & 1 & 627 & - & - & - & A & IV & No996 & E1012 & 12 & $98.6-98.5$ & $110-120$ & - & Detritus & Debitage & Complete Flake & Lithic & 2 & 7.05 & & 12/20/2017 \\
\hline 380 & 2 & 627 & - & - & - & A & IV & No996 & E1012 & 12 & $98.6-98.5$ & $110-120$ & - & Detritus & Debitage & Proximal Flake & Lithic & 1 & 0.72 & & 12/20/2017 \\
\hline 380 & 3 & 627 & - & - & - & A & IV & No996 & E1012 & 12 & $98.6-98.5$ & $110-120$ & - & Detritus & Debitage & Broken Flake & Lithic & 4 & 7.21 & & 12/20/2017 \\
\hline 380 & 4 & 627 & - & - & - & A & IV & No996 & E1012 & 12 & $98.6-98.5$ & $110-120$ & - & Detritus & Debitage & Thermal Shatter & Lithic & 5 & 16.55 & & 12/20/2017 \\
\hline 380 & 5 & 627 & - & - & - & A & IV & No996 & E1012 & 12 & $98.6-98.5$ & $110-120$ & - & Informal Tool & Modified Flake & Edge-modified & Fine Grain Chert & 1 & 4.55 & & 12/20/2017 \\
\hline 381 & 1 & 635 & - & - & - & A & IVN & No996 & E1012 & 13 & $98.5-98.4$ & 120-130 & - & Faunal Remains & Bone & Indeterminate & Bone & 1 & 0.03 & & 12/20/2017 \\
\hline 381 & 2 & 635 & - & - & - & A & IVN & N0996 & E1012 & 13 & $98.5-98.4$ & 120-130 & - & Faunal Remains & Bone & Indeterminate & Bone & 1 & 0.13 & & 12/20/2017 \\
\hline 381 & 3 & 635 & - & - & - & A & IVN & No996 & E1012 & 13 & 98.5-98.4 & $120-130$ & - & Detritus & Debitage & Broken Flake & Lithic & 1 & 6.22 & & 12/20/2017 \\
\hline 381 & 4 & 635 & - & - & - & A & IVN & N0996 & E1012 & 13 & $98.5-98.4$ & 120-130 & - & Detritus & Debitage & Thermal Shatter & Lithic & 4 & 12.85 & & 12/20/2017 \\
\hline 382 & 1 & 652 & - & - & - & A & vi & No996 & E1012 & 15 & 98.3-98.2 & $140-150$ & - & Faunal Remains & Bone & Mammal & Bone & 1 & 0.65 & & 12/21/2017 \\
\hline 382 & 2 & 652 & - & - & - & A & $\mathrm{vv}_{1}$ & No996 & E1012 & 15 & $98.3-98.2$ & 140-150 & - & Faunal Remains & Bone & Mammal & Bone & 1 & 0.09 & & 12/21/2017 \\
\hline 382 & 3 & 652 & - & - & - & A & vi & No996 & E1012 & 15 & 98.3-98.2 & $140-150$ & - & Detritus & Debitage & Proximal Flake & Lithic & 2 & 3.51 & & 12/21/2017 \\
\hline 382 & 4 & 652 & - & - & - & A & $\mathrm{vi}$ & No996 & E1012 & 15 & $98.3-98.2$ & 140-150 & - & Detritus & Debitage & Thermal Shatter & Lithic & 2 & 0.35 & & 12/21/2017 \\
\hline 383 & 1 & 433 & - & - & - & A & 1 & No996 & E1013 & 1 & 99.59-99.5 & $11-20$ & - & Detritus & Debitage & Proximal Flake & Lithic & 1 & 0.17 & & 12/5/2017 \\
\hline 383 & 2 & 433 & - & - & - & A & 1 & No996 & E1013 & 1 & 99.59-99.5 & $11-20$ & - & Detritus & Debitage & Broken Flake & Lithic & 4 & 3.23 & & 12/5/2017 \\
\hline 383 & 3 & 433 & - & - & - & A & 1 & No996 & E1013 & 1 & 99.59-99.5 & $11-20$ & - & Detritus & Debitage & Thermal Shatter & Lithic & 1 & 0.19 & & 12/5/2017 \\
\hline 384 & 1 & 439 & - & - & - & A & "I & No996 & E1013 & 2 & 99.5-99.4 & $20-30$ & - & Detritus & Debitage & \begin{tabular}{|l|} 
Complete Flake \\
\end{tabular} & Lithic & 1 & 3.07 & & 12/5/2017 \\
\hline 384 & 2 & 439 & - & - & - & A & "I & No996 & E1013 & 2 & 99.5-99.4 & $20-30$ & - & Detritus & Debitage & Broken Flake & Lithic & 3 & 0.74 & & 12/5/2017 \\
\hline 384 & 3 & 439 & - & - & - & A & " & No996 & E1013 & 2 & 99.5-99.4 & $20-30$ & - & Detritus & Debitage & Thermal Shatter & Lithic & 1 & 0.77 & & 12/5/2017 \\
\hline 385 & 1 & 444 & - & - & - & $\mathrm{A}$ & 11 & No996 & E1013 & 3 & 99.32 & 38 & \begin{tabular}{|l|l|} 
N996.45 \\
E1013.37 \\
\end{tabular} & \begin{tabular}{l|l|} 
Detritus \\
\end{tabular} & Core & \begin{tabular}{|l|} 
Multidirectional \\
\end{tabular} & \begin{tabular}{|c|}
$\begin{array}{c}\text { Coarse Grain } \\
\text { Chert }\end{array}$ \\
\end{tabular} & 1 & 103.99 & Exhausted Core & 12/5/2017 \\
\hline 386 & 1 & 445 & - & - & - & A & "I & No996 & E1013 & 3 & $99.4-99.3$ & $30-40$ & - & Faunal Remains & Bone & Indeterminate & Bone & 1 & 1.35 & & 12/5/2017 \\
\hline 386 & 2 & 445 & - & - & - & A & "I & No996 & E1013 & 3 & 99.499 .3 & $30-40$ & - & Faunal Remains & Bone & Indeterminate & Bone & 1 & 0.52 & & 12/5/2017 \\
\hline 386 & 3 & 445 & - & - & - & A & "I & No996 & E1013 & 3 & 99.499 .3 & $30-40$ & - & Detritus & Debitage & \begin{tabular}{|l|} 
Complete Flake \\
\end{tabular} & Lithic & 7 & 40.35 & & 12/5/2017 \\
\hline 386 & 4 & 445 & - & - & - & A & "I & No996 & E1013 & 3 & $99.4-99.3$ & $30-40$ & - & Detritus & Debitage & \begin{tabular}{|l|} 
Proximal Flake \\
\end{tabular} & Lithic & 7 & 2.76 & & 12/5/2017 \\
\hline 386 & 5 & 445 & - & - & - & A & "I & No996 & E1013 & 3 & 99.499 .3 & $30-40$ & - & Detritus & Debitage & Broken Flake & Lithic & 8 & 3.81 & & 12/5/2017 \\
\hline 386 & 6 & 445 & - & - & - & A & "I & No996 & E1013 & 3 & 99.499 .3 & $30-40$ & - & Detritus & Debitage & Thermal Shatter & Lithic & 10 & 18.95 & & 1215/2017 \\
\hline 386 & 7 & 445 & - & - & - & A & "I & No996 & E1013 & 3 & $99.4-99.3$ & $30-40$ & - & Informal Tool & \begin{tabular}{|l|} 
Modified Flake \\
\end{tabular} & \begin{tabular}{|l|} 
Edge-modified \\
\end{tabular} & Fine Grain Chert & 1 & 33.3 & & 12/5/2017 \\
\hline 387 & 1 & 452 & - & - & - & A & " & No996 & E1013 & 3 & 99.3 & 40 & \begin{tabular}{|l|l|} 
N996.99 \\
E1013.88 \\
\end{tabular} & Formal Tool & Biface & \begin{tabular}{|c|}
$\begin{array}{c}\text { Distal-Medial } \\
\text { Fragment }\end{array}$ \\
\end{tabular} & Fine Grain Chert & 1 & 37.78 & Distal - Medial & 12/8/20017 \\
\hline 388 & 1 & 362 & - & - & - & A & "1 & No996 & E1013 & 4 & 99.3-99.2 & $40-50$ & - & Detritus & Debitage & Complete Flake & Lithic & 10 & 25.34 & & 12/5/2017 \\
\hline 388 & 1 & 362 & - & - & - & A & "I & No996 & E1013 & 4 & $99.3-99.2$ & $40-50$ & - & Faunal Remains & Bone & Mammal & Bone & 1 & 2.38 & & 12/5/2017 \\
\hline 388 & 2 & 362 & - & - & - & A & "1 & No996 & E1013 & 4 & 99.3-99.2 & $40-50$ & - & Detritus & Debitage & Proximal Flake & Lithic & 8 & 13.89 & & 12/5/2017 \\
\hline 388 & 3 & 362 & - & - & - & A & "I & No996 & E1013 & 4 & $99.3-99.2$ & $40-50$ & - & Detritus & Debitage & Broken Flake & Lithic & 33 & 31.74 & & 12/5/2017 \\
\hline 388 & 4 & 362 & - & - & - & A & "1 & No996 & E1013 & 4 & 99.3-99.2 & $40-50$ & - & Detritus & Debitage & Flaking Shatter & Lithic & 2 & 0.7 & & 12/5/2017 \\
\hline 388 & 5 & 362 & - & - & - & A & "I & No996 & E1013 & 4 & $99.3-99.2$ & $40-50$ & - & Detritus & Debitage & Thermal Shatter & Lithic & 17 & 17.91 & & 12/5/2017 \\
\hline 388 & 6 & 362 & - & - & - & A & "1 & No996 & E1013 & 4 & 99.3-99.2 & $40-50$ & - & \begin{tabular}{l|l} 
Informal Tool &
\end{tabular} & Modified Flake & \begin{tabular}{|l|} 
Edge-modified \\
\end{tabular} & Fine Grain Chert & 1 & 42.64 & & 12/5/2017 \\
\hline 389 & 1 & 451 & - & - & - & A & IIII & No996 & E1013 & 5 & $99.2-99.1$ & 50-60 & - & Faunal Remains & Bone & Odocoileus sp. & Tooth & 1 & 2.3 & & 12/8/2017 \\
\hline 389 & 2 & 451 & - & - & - & A & IIII & No996 & E1013 & 5 & 99.2-99.1 & $50-60$ & - & Detritus & Debitage & Complete Flake & Lithic & 7 & 65.01 & & 12/8/2017 \\
\hline 389 & 3 & 451 & - & - & - & A & III & No996 & E1013 & 5 & 99.2-99.1 & $50-60$ & - & Detritus & Debitage & Proximal Flake & Lithic & 9 & 33.63 & & 12/8/2017 \\
\hline 389 & 4 & 451 & - & - & - & A & IIII & No996 & E1013 & 5 & $99.2-99.1$ & $50-60$ & - & Detritus & Debitage & Broken Flake & Lithic & 28 & 25.75 & & 12/8/2017 \\
\hline 389 & 5 & 451 & - & - & - & A & IIII & N0996 & E1013 & 5 & $99.2-99.1$ & 50-60 & - & Detritus & Debitage & \begin{tabular}{|l|} 
Flaking Shatter \\
\end{tabular} & Lithic & 1 & 0.49 & & 12/8/2017 \\
\hline 389 & 6 & 451 & - & - & - & A & III & No996 & E1013 & 5 & 99.2-99.1 & $50-60$ & - & Detritus & Debitage & \begin{tabular}{|l|} 
Thermal Shatter \\
\end{tabular} & Lithic & 9 & 6.03 & & 12/8/2017 \\
\hline
\end{tabular}




\begin{tabular}{|c|c|c|c|c|c|c|c|c|c|c|c|c|c|c|c|c|c|c|c|c|c|}
\hline Lot No. & \begin{tabular}{|c|}
$\begin{array}{c}\text { Specimen } \\
\text { No. }\end{array}$ \\
\end{tabular} & FS No. & UI No. & FTR No. & \begin{tabular}{c|}
$\begin{array}{c}\text { Sample } \\
\text { No. }\end{array}$ \\
\end{tabular} & $\begin{array}{c}\text { Excavation } \\
\text { Block }\end{array}$ & Stratum (AU) & Northing & Easting & Level & $\begin{array}{c}\text { Elevation } \\
\text { (m) }\end{array}$ & Depth (cmbd) & Point Prov. & Artifact Class & Artifact Type & \begin{tabular}{|c|} 
Artifact \\
Description
\end{tabular} & Material & $\begin{array}{c}\text { Number of } \\
\text { artifacts }\end{array}$ & Weight (g) & Comments & Date \\
\hline 389 & 7 & 451 & - & - & - & A & III & No996 & E1013 & 5 & $99.2-99.1$ & $50-60$ & - & Informal Tool & Modified Flake & Edge-modified & Fine Grain Chert & 1 & 1.54 & & 12/8/2017 \\
\hline 389 & 8 & 451 & - & - & - & A & III & No996 & E1013 & 5 & 99.2-99.1 & $50-60$ & - & \begin{tabular}{|l|} 
Faunal Remains \\
\end{tabular} & Shell & Mussel Shell & Shell & 1 & 1.64 & & 12/8/2017 \\
\hline 390 & 1 & 458 & - & - & - & A & IIII & No996 & E1013 & 6 & $99.1-99.0$ & $60-70$ & - & Detritus & Debitage & \begin{tabular}{|l|} 
Complete Flake \\
\end{tabular} & Lithic & 5 & 15.32 & & 12/8/2017 \\
\hline 390 & 2 & 458 & - & - & - & A & IIII & No996 & E1013 & 6 & $99.1-99.0$ & $60-70$ & - & Detritus & Debitage & Proximal Flake & Lithic & 14 & 5.55 & & 12/8/2017 \\
\hline 390 & 3 & 458 & - & - & - & A & III & No996 & E1013 & 6 & $99.1-99.0$ & $60-70$ & - & Detritus & Debitage & Broken Flake & Lithic & 23 & 15.02 & & 12/8/2017 \\
\hline 390 & 4 & 458 & - & - & - & A & IIII & No996 & E1013 & 6 & $99.1-99.0$ & $60-70$ & - & Detritus & Debitage & \begin{tabular}{|l|} 
Flaking Shatter \\
\end{tabular} & Lithic & 2 & 16.53 & & 12/8/2017 \\
\hline 390 & 5 & 458 & - & - & - & A & IIII & No996 & E1013 & 6 & $99.1-99.0$ & $60-70$ & - & Detritus & Debitage & Thermal Shatter & Lithic & 30 & 23.48 & & 12/8/2017 \\
\hline 390 & 6 & 458 & - & - & - & A & III & No996 & E1013 & 6 & $99.1-99.0$ & 60-70 & - & Formal Tool & Scraper & $\begin{array}{l}\text { End and Side } \\
\text { Scraper }\end{array}$ & Fine Grain Chert & 1 & 20.12 & & 12/8/2017 \\
\hline 391 & 1 & 465 & - & FTR3 & - & A & III & No996 & E1013 & 7 & 99.0-98.9 & $70-80$ & - & Faunal Remains & Bone & Tortoise/Turtle & Bone & 1 & 0.46 & & 12/11/2017 \\
\hline 391 & 2 & 465 & - & FTR3 & - & A & IIII & N0996 & E1013 & 7 & $99.0-98.9$ & $\begin{array}{ll}70-80 \\
\end{array}$ & - & Detritus & Debitage & Complete Flake & Lithic & 8 & 4.49 & & 12/11/2017 \\
\hline 391 & 3 & 465 & - & FTR3 & - & A & IIII & No996 & E1013 & 7 & $99.0-98.9$ & $70-80$ & - & Detritus & Debitage & \begin{tabular}{|l|} 
Proximal Flake \\
\end{tabular} & Lithic & 11 & 7.75 & & 12/11/2017 \\
\hline 391 & 4 & 465 & - & FTR3 & - & A & IIII & N0996 & E1013 & 7 & $99.0-98.9$ & $\begin{array}{ll}70-80 \\
\end{array}$ & - & Detritus & Debitage & Broken Flake & Lithic & 23 & 15.71 & & 12/11/2017 \\
\hline 391 & 5 & 465 & - & FTR3 & - & A & III & No996 & E1013 & 7 & $99.0-98.9$ & $\begin{array}{ll}70-80 \\
\end{array}$ & - & Detritus & Debitage & \begin{tabular}{|l|} 
Flaking Shatter \\
\end{tabular} & Lithic & 1 & 1.24 & & 12/11/2017 \\
\hline 391 & 6 & 465 & - & FTR3 & - & A & III & N0996 & E1013 & 7 & $99.0-98.9$ & $70-80$ & - & Detritus & Debitage & Thermal Shatter & Lithic & 21 & 19.96 & & 12/11/2017 \\
\hline 391 & 7 & 465 & - & FTR3 & - & A & IIII & No996 & E1013 & 7 & $99.0-98.9$ & $70-80$ & - & Clay & Clay Artifact & Burned Clay & Clay & 11 & 147.96 & & 12/8/2017 \\
\hline 392 & 1 & 608 & - & - & - & A & IV & No996 & E1013 & 8 & 98.9-98.8 & $80-90$ & - & Detritus & Debitage & Complete Flake & Lithic & 4 & 55.68 & & 12/2012017 \\
\hline 392 & 2 & 608 & - & - & - & A & IV & No996 & E1013 & 8 & 98.9-98.8 & $80-90$ & - & Detritus & Debitage & Proximal Flake & Lithic & 3 & 5.22 & & 12/20/2017 \\
\hline 392 & 3 & 608 & - & - & - & A & IV & No996 & E1013 & 8 & 98.9-98.8 & $80-90$ & - & Detritus & Debitage & Broken Flake & Lithic & 5 & 8.69 & & 12/2012017 \\
\hline 392 & 4 & 608 & - & - & - & A & IV & No996 & E1013 & 8 & $98.9-98.8$ & $80-90$ & - & Detritus & Debitage & Flaking Shatter & Lithic & 2 & 13.99 & & 121/20/2017 \\
\hline 392 & 5 & 608 & - & - & - & A & IV & No996 & E1013 & 8 & $98.9-98.8$ & $80-90$ & - & Detritus & Debitage & Thermal Shatter & Lithic & 4 & 1.77 & & 121/20/2017 \\
\hline 393 & 1 & 608 & - & FTR3 & c-14 & A & IV & No996 & E1013 & 8 & 98.86 & 84 & \begin{tabular}{|l|} 
N996.86 \\
E1013.50 \\
\end{tabular} & \begin{tabular}{|c|} 
Archaeological \\
Sample
\end{tabular} & C-14 & \begin{tabular}{l|} 
Red mulberry \\
(Morus rubra)
\end{tabular} & & 13 & 0.21 & & 12/14/2017 \\
\hline 393 & 2 & 608 & - & FTR3 & C-14 & A & IV & No996 & E1013 & 8 & 98.86 & 84 & $\begin{array}{l}\text { N996.86 } \\
\text { E1013.50 }\end{array}$ & \begin{tabular}{|c|}
$\begin{array}{c}\text { Archaeological } \\
\text { Sample }\end{array}$ \\
\end{tabular} & \begin{tabular}{|c|} 
Examined \\
Residue $<2 \mathrm{~mm}$
\end{tabular} & & & & 0.03 & & 12/14/2017 \\
\hline 394 & 1 & 612 & - & - & - & A & IV & No996 & E1013 & 9 & 98.8-98.7 & $90-100$ & - & Detritus & Debitage & Complete Flake & Lithic & 2 & 23.71 & & 12/201/2017 \\
\hline 394 & 2 & 612 & - & - & - & A & IV & No996 & E1013 & 9 & 98.8-98.7 & $90-100$ & - & Detritus & Debitage & Broken Flake & Lithic & 2 & 12.27 & & 12/20/2017 \\
\hline 394 & 3 & 612 & - & - & - & A & IV & No996 & E1013 & 9 & 98.8-98.7 & $90-100$ & - & Detritus & Debitage & Thermal Shatter & Lithic & 4 & 6.56 & & 12/20/2017 \\
\hline 395 & 1 & 617 & - & - & - & A & IV & No996 & E1013 & 10 & 98.7-98.6 & $100-110$ & - & Faunal Remains & Bone & Indeterminate & Bone & 1 & 1.14 & & 12/200/2017 \\
\hline 395 & 2 & 617 & - & - & - & A & IV & No996 & E1013 & 10 & 98.7-98.6 & 100-110 & - & \begin{tabular}{|l|} 
Faunal Remains \\
\end{tabular} & Bone & Indeterminate & Bone & 1 & 0.17 & & 121/20/2017 \\
\hline 395 & 3 & 617 & - & - & - & A & IV & No996 & E1013 & 10 & 98.7-98.6 & $100-110$ & - & Faunal Remains & Bone & Indeterminate & Bone & 1 & 0.46 & & 12/2012017 \\
\hline 395 & 4 & 617 & - & - & - & A & IV & No996 & E1013 & 10 & 98.7-98.6 & $100-110$ & - & Faunal Remains & Bone & Indeterminate & Bone & 1 & 0.14 & & 12/200/2017 \\
\hline 395 & 5 & 617 & - & - & - & A & IV & No996 & E1013 & 10 & 98.7-98.6 & $100-110$ & - & Faunal Remains & Bone & Indeterminate & Bone & 1 & 0.19 & & 12/2012017 \\
\hline 395 & 6 & 617 & - & - & - & A & IV & No996 & E1013 & 10 & 98.7-98.6 & $100-110$ & - & Detritus & Debitage & Proximal Flake & Lithic & 2 & 0.42 & & 121/20/2017 \\
\hline 395 & 7 & 617 & - & - & - & A & IV & No996 & E1013 & 10 & 98.7-98.6 & $100-110$ & - & Detritus & Debitage & Broken Flake & Lithic & 2 & 2.12 & & 12/20/2017 \\
\hline 396 & 1 & 624 & - & - & - & A & IV & No996 & E1013 & 11 & $98.6-98.5$ & $110-120$ & - & Detritus & Debitage & Broken Flake & Lithic & 1 & 0.22 & & 121/20/2017 \\
\hline 397 & 1 & 632 & - & - & - & A & IVN & No996 & E1013 & 12 & $\begin{array}{ll}88.5-98.4 \\
\end{array}$ & $120-130$ & - & Detritus & Debitage & Complete Flake & Lithic & 1 & 0.16 & & 12/20/2017 \\
\hline 397 & 2 & 632 & - & - & - & A & IVN & No996 & E1013 & 12 & $98.5-98.4$ & $120-130$ & - & Detritus & Debitage & Broken Flake & Lithic & 2 & 0.88 & & 12/2012017 \\
\hline 398 & 1 & 645 & - & - & - & A & $\mathrm{v}$ & No996 & E1013 & 13 & 98.498 .3 & $130-140$ & - & Faunal Remains & Bone & Indeterminate & Bone & 1 & 0.25 & & 121/21/2017 \\
\hline 398 & 2 & 645 & - & - & - & A & $\mathrm{v}$ & No996 & E1013 & 13 & 98.498 .3 & $130-140$ & - & Faunal Remains & Bone & Indeterminate & Bone & 1 & 0.13 & & 12/21/2017 \\
\hline 398 & 3 & 645 & - & - & - & A & $\mathrm{v}$ & No996 & E1013 & 13 & 98.4-98.3 & $130-140$ & - & Detritus & Debitage & \begin{tabular}{|l|} 
Proximal Flake \\
\end{tabular} & Lithic & 1 & 0.12 & & 121/21/2017 \\
\hline 399 & 1 & 667 & - & - & - & A & vi & No996 & E1013 & 14 & 98.3-98.2 & $140-150$ & - & Faunal Remains & Bone & Indeterminate & Bone & 1 & 0.81 & & 12/27/2017 \\
\hline 399 & 2 & 667 & - & - & - & A & vi & No996 & E1013 & 14 & 98.3-98.2 & $140-150$ & - & Detritus & Debitage & \begin{tabular}{|l|} 
Proximal Flake \\
\end{tabular} & Lithic & 1 & 0.62 & & 121/27/2017 \\
\hline 399 & 3 & 667 & - & - & - & A & vi & No996 & E1013 & 14 & $98.3-98.2$ & $140-150$ & - & Detritus & Debitage & Broken Flake & Lithic & 1 & 2.45 & & 121/27/2017 \\
\hline 400 & 1 & 483 & - & - & - & A & " & No996 & E1014 & 2 & 99.5-99.4 & $20-30$ & - & Faunal Remains & Bone & Indeterminate & Bone & 1 & 0.67 & & 12/11/2017 \\
\hline 400 & 2 & 483 & - & - & - & A & "I & No996 & E1014 & 2 & $99.5-99.4$ & $20-30$ & - & Detritus & Debitage & \begin{tabular}{|l|} 
Complete Flake \\
\end{tabular} & Lithic & 1 & 0.67 & & 12/11/2017 \\
\hline 400 & 3 & 483 & - & - & - & A & "I & No996 & E1014 & 2 & $99.5-99.4$ & $20-30$ & - & Detritus & Debitage & \begin{tabular}{|l|} 
Proximal Flake \\
\end{tabular} & Lithic & 1 & 0.75 & & 12/11/2017 \\
\hline
\end{tabular}




\begin{tabular}{|c|c|c|c|c|c|c|c|c|c|c|c|c|c|c|c|c|c|c|c|c|c|}
\hline Lot No. & \begin{tabular}{|c|}
$\begin{array}{c}\text { Specimen } \\
\text { No. }\end{array}$ \\
\end{tabular} & FS No. & UI No. & FTR No. & $\begin{array}{c}\text { Sample } \\
\text { No. }\end{array}$ & $\begin{array}{c}\text { Excavation } \\
\text { Block }\end{array}$ & Stratum (AU) & Northing & Easting & Level & $\begin{array}{c}\text { Elevation } \\
(\mathrm{m})\end{array}$ & Depth (cmbd) & Point Prov. & Artifact Class & Artifact Type & \begin{tabular}{|c|} 
Artifact \\
Description
\end{tabular} & Material & $\begin{array}{c}\text { Number of } \\
\text { artifacts }\end{array}$ & Weight (g) & Comments & Date \\
\hline 400 & \begin{tabular}{|l|} 
\\
\end{tabular} & 483 & - & - & - & A & " & No996 & E1014 & 2 & 99.5-99.4 & $20-30$ & - & Detritus & Debitage & Broken Flake & Lithic & 2 & 1 & & 12/11/2017 \\
\hline 400 & 5 & 483 & - & - & - & A & " & No996 & E1014 & 2 & 99.5-99.4 & $20-30$ & - & Detritus & Debitage & Thermal Shatter & Lithic & 1 & 0.6 & & 12/11/2017 \\
\hline 401 & 1 & 488 & - & - & - & A & "I & No996 & E1014 & 3 & 99.499 .3 & $30-40$ & - & Detritus & Debitage & \begin{tabular}{|l|} 
Complete Flake \\
\end{tabular} & Lithic & 1 & 9.02 & & 12/12/2017 \\
\hline 401 & 2 & 488 & - & - & - & A & "I & No996 & E1014 & 3 & 99.4-99.3 & $30-40$ & - & Detritus & Debitage & Proximal Flake & Lithic & 3 & 2.45 & & 12/12/2017 \\
\hline 401 & 3 & 488 & - & - & - & A & "I & No996 & E1014 & 3 & 99.4-99.3 & $30-40$ & - & Detritus & Debitage & Broken Flake & Lithic & 10 & 6.65 & & $\mid$\begin{tabular}{|l|}
$\mid 12 / 12 / 2017$ \\
\end{tabular} \\
\hline 401 & 4 & 488 & - & - & - & A & "I & No996 & E1014 & 3 & $99.4-99.3$ & $30-40$ & - & Detritus & Debitage & Thermal Shatter & Lithic & 3 & 0.61 & & 12/12/2017 \\
\hline 401 & 5 & 488 & - & - & - & A & "I & No996 & E1014 & 3 & 99.4-99.3 & $30-40$ & - & Detritus & Core & Multidirectional & Fine Grain Chert & 1 & 46.94 & & 12/12/2017 \\
\hline 401 & 6 & 488 & - & - & - & A & " & No996 & E1014 & 3 & 99.4-99.3 & $30-40$ & - & Informal Tool & Modified Flake & Edge-modified & Fine Grain Chert & 1 & 4.7 & & $\mid$\begin{tabular}{|l|}
$\mid 12 / 12 / 2017$ \\
\end{tabular} \\
\hline 402 & 1 & 490 & - & - & - & A & II & No996 & E1014 & 4 & $99.3-99.2$ & $40-50$ & - & Faunal Remains & Bone & Indeterminate & Bone & 1 & 1.92 & & 12/12/2017 \\
\hline 402 & 2 & 490 & - & - & - & A & "I & No996 & E1014 & 4 & $99.3-99.2$ & $40-50$ & - & Faunal Remains & Bone & Indeterminate & Bone & 1 & 0.38 & & $\mid 12 / 12 / 2017$ \\
\hline 402 & 3 & 490 & - & - & - & A & "I & No996 & E1014 & 4 & 99.3-99.2 & $40-50$ & - & Faunal Remains & Bone & Indeterminate & Bone & 1 & 0.26 & & 12/12/2017 \\
\hline 402 & 4 & 490 & - & - & - & A & "I & N0996 & E1014 & 4 & $99.3-99.2$ & $40-50$ & - & \begin{tabular}{|l|} 
Faunal Remains \\
\end{tabular} & Bone & Indeterminate & Bone & 1 & 0.74 & & \begin{tabular}{|l|}
$12 / 12 / 2017$ \\
\end{tabular} \\
\hline 402 & 5 & 490 & - & - & - & A & "1 & No996 & E1014 & 4 & 99.3-99.2 & $40-50$ & - & Detritus & Debitage & Complete Flake & Lithic & 4 & 25.19 & & 12/12/2017 \\
\hline 402 & 6 & 490 & - & - & - & A & "I & N0996 & E1014 & 4 & $99.3-99.2$ & $40-50$ & - & Detritus & Debitage & Proximal Flake & Lithic & 8 & 4.09 & & $\mid 12 / 12 / 2017$ \\
\hline 402 & 7 & 490 & - & - & - & A & 11 & No996 & E1014 & 4 & 99.3-99.2 & $40-50$ & - & Detritus & Debitage & Broken Flake & Lithic & 23 & 38.05 & & 12/12/2017 \\
\hline 402 & 8 & 490 & - & - & - & A & "1 & No996 & E1014 & 4 & 99.3-99.2 & $40-50$ & - & Detritus & Debitage & Thermal Shatter & Lithic & 7 & 3.01 & & 12/12/2017 \\
\hline 403 & 1 & 495 & - & - & - & A & IIII & No996 & E1014 & 5 & 99.2-99.1 & $50-60$ & - & Faunal Remains & Bone & Indeterminate & Bone & 1 & 0.34 & & 12/12/2017 \\
\hline 403 & 2 & 495 & - & - & - & A & IIII & No996 & E1014 & 5 & $99.2-99.1$ & $50-60$ & - & Faunal Remains & Bone & Indeterminate & Bone & 1 & 0.13 & & 12/11/2/2017 \\
\hline 403 & 3 & 495 & - & - & - & A & IIII & No996 & E1014 & 5 & $99.2-99.1$ & $50-60$ & - & Detritus & Debitage & Complete Flake & Lithic & 16 & 106.41 & & 12/12/2017 \\
\hline 403 & 4 & 495 & - & - & - & A & IIII & No996 & E1014 & 5 & $99.2-99.1$ & $50-60$ & - & Detritus & Debitage & \begin{tabular}{|l|} 
Proximal Flake \\
\end{tabular} & Lithic & 26 & 88.75 & & 12/12/2017 \\
\hline 403 & 5 & 495 & - & - & - & A & IIII & No996 & E1014 & 5 & $99.2-99.1$ & $50-60$ & - & Detritus & Debitage & Broken Flake & Lithic & 62 & 47.28 & & 12/112/2017 \\
\hline 403 & 6 & 495 & - & - & - & A & IIII & No996 & E1014 & 5 & $99.2-99.1$ & $50-60$ & - & Detritus & Debitage & Flaking Shatter & Lithic & 4 & 11.87 & & 12/12/2017 \\
\hline 403 & 7 & 495 & - & - & - & A & IIII & No996 & E1014 & 5 & $99.2-99.1$ & $50-60$ & - & Detritus & Debitage & Thermal Shatter & Lithic & 24 & 50.33 & & 12/12/2017 \\
\hline 403 & 9 & 495 & 23 & - & - & A & IIII & No996 & E1014 & 5 & 99.2-99.1 & $50-60$ & - & Formal Tool & Biface & Complete & Fine Grain Chert & 1 & 53.3 & & $\mid$\begin{tabular}{|l|}
$\mid 12 / 12 / 2017$ \\
\end{tabular} \\
\hline 404 & 1 & 494 & 24 & - & - & $\mathrm{A}$ & III & No996 & E1014 & 6 & 99.0 & 70 & \begin{tabular}{|c|} 
N996.71 \\
E1014.16
\end{tabular} & Formal Tool & Biface & Complete & Fine Grain Chert & 1 & 12.5 & & 12/12/2017 \\
\hline 405 & 1 & 500 & - & - & - & A & IIII & No996 & E1014 & 6 & $99.1-99.0$ & $60-70$ & - & Faunal Remains & Bone & Indeterminate & Bone & 1 & 0.31 & & 12/12/2017 \\
\hline 405 & 2 & 500 & - & - & - & A & IIII & No996 & E1014 & 6 & $99.1-99.0$ & $60-70$ & - & Faunal Remains & Bone & Indeterminate & Bone & 1 & 0.04 & & 12/12/2017 \\
\hline 405 & 3 & 500 & - & - & - & A & IIII & No996 & E1014 & 6 & $99.1-99.0$ & $60-70$ & - & Faunal Remains & Bone & Indeterminate & Bone & 1 & 0.13 & & 12/12/2017 \\
\hline 405 & 4 & 500 & - & - & - & A & IIII & No996 & E1014 & 6 & $99.1-99.0$ & $60-70$ & - & Detritus & Debitage & \begin{tabular}{|l|} 
Complete Flake \\
\end{tabular} & Lithic & 6 & 10.71 & & 12/12/2/2017 \\
\hline 405 & 5 & 500 & - & - & - & A & IIII & No996 & E1014 & 6 & $99.1-99.0$ & $60-70$ & - & Detritus & Debitage & \begin{tabular}{|l|} 
Proximal Flake \\
\end{tabular} & Lithic & 18 & 10.17 & & 12/12/2017 \\
\hline 405 & 6 & 500 & - & - & - & A & IIII & No996 & E1014 & 6 & $99.1-99.0$ & 60-70 & - & Detritus & Debitage & Broken Flake & Lithic & 49 & 49.58 & & $\mid$\begin{tabular}{|l|}
$\mid 12 / 12 / 2017$ \\
\end{tabular} \\
\hline 405 & 7 & 500 & - & - & - & A & III & No996 & E1014 & 6 & $99.1-99.0$ & $60-70$ & - & Detritus & Debitage & Thermal Shatter & Lithic & 21 & 9.39 & & 12/12/2017 \\
\hline 405 & 8 & 500 & - & - & - & A & IIII & No996 & E1014 & 6 & $99.1-99.0$ & $60-70$ & - & \begin{tabular}{|l|} 
Faunal Remains \\
\end{tabular} & Shell & Mussel Shell & Shell & 1 & 0.94 & & 12/12/2017 \\
\hline 405 & 9 & 500 & - & - & - & A & III & No996 & E1014 & 6 & $99.1-99.0$ & $60-70$ & - & Clay & Clay Artifact & Burned Clay & Clay & 10 & 15.79 & & \begin{tabular}{|l|}
$12 / 12 / 2017$ \\
\end{tabular} \\
\hline 406 & 1 & 503 & - & - & - & A & IIII & N0996 & E1014 & 7 & $99.0-98.9$ & $\begin{array}{ll}70-80 \\
\end{array}$ & - & Faunal Remains & Bone & Odocoileus sp. & Tooth & 1 & 1.79 & 1 Cervid tooth & 12/12/2017 \\
\hline 406 & 2 & 503 & - & - & - & A & IIII & No996 & E1014 & 7 & $99.0-98.9$ & $70-80$ & - & Detritus & Debitage & Complete Flake & Lithic & 7 & 8.87 & & 12/12/2017 \\
\hline 406 & 3 & 503 & - & - & - & A & IIII & N0996 & E1014 & 7 & $99.0-98.9$ & $70-80$ & - & Detritus & Debitage & Proximal Flake & Lithic & 13 & 12.27 & & 12/12/2017 \\
\hline 406 & 4 & 503 & - & - & - & A & III & No996 & E1014 & 7 & 99.0-98.9 & $70-80$ & - & Detritus & Debitage & Broken Flake & Lithic & 49 & 38.21 & & 12/12/2017 \\
\hline 406 & 5 & 503 & - & - & - & A & IIII & No996 & E1014 & 7 & $99.0-98.9$ & $\begin{array}{ll}70-80 \\
\end{array}$ & - & Detritus & Debitage & \begin{tabular}{|l|} 
Flaking Shatter \\
\end{tabular} & Lithic & 3 & 2.53 & & 12/12/2017 \\
\hline 406 & 6 & 503 & - & - & - & A & IIII & No996 & E1014 & 7 & 99.0-98.9 & $70-80$ & - & Detritus & Debitage & Thermal Shatter & Lithic & 42 & 48.23 & & 12/12/2017 \\
\hline 406 & 7 & 503 & - & - & - & A & III & No996 & E1014 & 7 & 99.0-98.9 & $70-80$ & - & Informal Tool & Modified Flake & Utilized Flake & $\begin{array}{l}\text { Coarse Grain } \\
\text { Chert }\end{array}$ & 1 & 47.3 & & \begin{tabular}{|l|}
$12 / 12 / 2017$ \\
\end{tabular} \\
\hline 406 & 8 & 503 & - & - & - & A & IIII & No996 & E1014 & 7 & $99.0-98.9$ & $70-80$ & - & \begin{tabular}{l|l} 
Informal Tool &
\end{tabular} & \begin{tabular}{|l|} 
Modified Flake \\
\end{tabular} & \begin{tabular}{|l|} 
Edge-modified \\
\end{tabular} & Fine Grain Chert & 1 & 4.4 & & 12/12/2017 \\
\hline 406 & 9 & 503 & - & - & - & A & IIII & N0996 & E1014 & 7 & $99.0-98.9$ & $70-80$ & - & \begin{tabular}{|l|l} 
Informal Tool &
\end{tabular} & Modified Flake & \begin{tabular}{|l|} 
Edge-modified \\
\end{tabular} & Fine Grain Chert & 1 & 9.55 & & 12/12/2/2017 \\
\hline 406 & 11 & 503 & - & - & - & A & III & No996 & E1014 & 7 & 99.0-98.9 & $70-80$ & - & Formal Tool & Biface & \begin{tabular}{l|} 
Distal-Medial \\
Fragment
\end{tabular} & Fine Grain Chert & 1 & 0.81 & & 12/12/2017 \\
\hline
\end{tabular}




\begin{tabular}{|c|c|c|c|c|c|c|c|c|c|c|c|c|c|c|c|c|c|c|c|c|c|}
\hline Lot No. & \begin{tabular}{|c|}
$\begin{array}{c}\text { Specimen } \\
\text { No. }\end{array}$ \\
\end{tabular} & FS No. & UI No. & FTR No. & $\begin{array}{c}\text { Sample } \\
\text { No. }\end{array}$ & $\begin{array}{c}\text { Excavation } \\
\text { Block }\end{array}$ & Stratum (AU) & Northing & Easting & Level & $\begin{array}{c}\text { Elevation } \\
(\mathrm{m})\end{array}$ & Depth (cmbd) & Point Prov. & Artifiact Class & \begin{tabular}{|l|} 
Artifact Type \\
\end{tabular} & \begin{tabular}{|c|} 
Artifact \\
Description
\end{tabular} & Material & $\begin{array}{c}\text { Number of } \\
\text { artifacts }\end{array}$ & Weight (g) & Comments & Date \\
\hline 407 & 1 & 507 & - & - & - & A & IV & No996 & E1014 & 8 & 98.9-98.8 & $80-90$ & - & Faunal Remains & Bone & Mammal & Bone & 1 & 1.33 & & 12/13/2017 \\
\hline 407 & 2 & 507 & - & - & - & A & IV & No996 & E1014 & 8 & 98.9-98.8 & $80-90$ & - & Detritus & Debitage & Complete Flake & Lithic & 11 & 24.16 & & $12 / 13 / 2017$ \\
\hline 407 & 3 & 507 & - & - & - & A & IV & No996 & E1014 & 8 & $98.9-98.8$ & $80-90$ & - & Detritus & Debitage & \begin{tabular}{|l|} 
Proximal Flake \\
\end{tabular} & Lithic & 7 & 5.78 & & 12/13/2017 \\
\hline 407 & 4 & 507 & - & - & - & A & IV & No996 & E1014 & 8 & $98.9-98.8$ & $80-90$ & - & Detritus & Debitage & Broken Flake & Lithic & 19 & 9.25 & & 12/13/2017 \\
\hline 407 & 5 & 507 & - & - & - & A & IV & No996 & E1014 & 8 & 98.9-98.8 & $80-90$ & - & Detritus & Debitage & Flaking Shatter & Lithic & 2 & 9.72 & & $\mid 12 / 13 / 2017$ \\
\hline 407 & 6 & 507 & - & - & - & A & IV & No996 & E1014 & 8 & $98.9-98.8$ & $80-90$ & - & Detritus & Debitage & Thermal Shatter & Lithic & 12 & 29.31 & & 12/13/2017 \\
\hline 407 & 7 & 507 & - & - & - & A & IV & No996 & E1014 & 8 & 98.9-98.8 & $80-90$ & - & Informal Tool & \begin{tabular}{|l|} 
Modified Flake \\
\end{tabular} & \begin{tabular}{|l|} 
Edge-modified \\
\end{tabular} & Fine Grain Chert & 1 & 45.94 & & 12/13/2017 \\
\hline 407 & 8 & 507 & - & - & - & A & IV & No996 & E1014 & 8 & 98.9-98.8 & $80-90$ & - & Clay & Clay Artifiact & Burned Clay & Clay & 8 & 33.27 & & $\mid$\begin{tabular}{|l|}
$\mid 12 / 13 / 2017$ \\
\end{tabular} \\
\hline 408 & 1 & 507 & - & - & - & A & IV & No996 & E1014 & 8 & 98.87 & 83 & 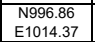 & Detritus & Core & Multidirectional & Fine Grain Chert & 1 & 96.23 & & $12 / 13 / 2017$ \\
\hline 409 & 1 & 514 & - & - & - & A & IV & N0996 & E1014 & 9 & $98.8-98.7$ & $\begin{array}{l}90-100 \\
\end{array}$ & - & Detritus & Debitage & \begin{tabular}{|c|} 
Complete Flake \\
\end{tabular} & Lithic & 6 & 56.27 & & 12/13/2017 \\
\hline 409 & 2 & 514 & - & - & - & A & IV & No996 & E1014 & 9 & 98.8-98.7 & 90-100 & - & Detritus & Debitage & \begin{tabular}{|l|} 
Proximal Flake \\
\end{tabular} & Lithic & 6 & 17.44 & & 12/13/2017 \\
\hline 409 & 3 & 514 & - & - & - & A & IV & N0996 & E1014 & 9 & $98.8-98.7$ & $\begin{array}{l}0-100 \\
\end{array}$ & - & Detritus & Debitage & Broken Flake & Lithic & 12 & 14.51 & & 12/13/2017 \\
\hline 409 & 4 & 514 & - & - & - & A & IV & No996 & E1014 & 9 & $\begin{array}{ll}98.8-98.7 \\
\end{array}$ & $\begin{array}{l}90-100 \\
\end{array}$ & - & Detritus & Debitage & \begin{tabular}{|l|} 
Flaking Shatter \\
\end{tabular} & Lithic & 2 & 1.48 & & 12/13/2017 \\
\hline 409 & 5 & 514 & - & - & - & A & IV & N0996 & E1014 & 9 & $98.8-98.7$ & $90-100$ & - & Detritus & Debitage & Thermal Shatter & Lithic & 27 & 70.74 & & $\mid 12 / 13 / 2017$ \\
\hline 409 & 6 & 514 & - & - & - & A & IV & No996 & E1014 & 9 & 98.8-98.7 & 90-100 & - & Clay & Clay Artifiact & Burned Clay & Clay & 2 & 3.87 & & 12/13/2017 \\
\hline 410 & 1 & 528 & - & - & - & A & iv & No996 & E1014 & 10 & 98.7-98.6 & $100-110$ & - & Detritus & Debitage & Complete Flake & Lithic & 2 & 11.76 & & 12/13/2017 \\
\hline 410 & 2 & 528 & - & - & - & A & iv & No996 & E1014 & 10 & 98.7-98.6 & $100-110$ & - & Detritus & Debitage & \begin{tabular}{|l|} 
Proximal Flake \\
\end{tabular} & Lithic & 14 & 43.22 & & 12/13/2017 \\
\hline 410 & 3 & 528 & - & - & - & A & IV & No996 & E1014 & 10 & 98.7-98.6 & $100-110$ & - & Detritus & Debitage & Broken Flake & Lithic & 23 & 22.02 & & 12/13/2017 \\
\hline 410 & 4 & 528 & - & - & - & A & IV & No996 & E1014 & 10 & $\begin{array}{l}98.7-98.6 \\
\end{array}$ & 100-110 & - & Detritus & Debitage & \begin{tabular}{|l|} 
Flaking Shatter \\
\end{tabular} & Lithic & 1 & 7.53 & & 12/13/2017 \\
\hline 410 & 5 & 528 & - & - & - & $\mathrm{A}$ & iv & No996 & E1014 & 10 & 98.7-98.6 & $100-110$ & - & Detritus & Debitage & Thermal Shatter & Lithic & 30 & 37.36 & & 12/13/2017 \\
\hline 410 & 6 & 528 & - & - & - & A & Iv & No996 & E1014 & 10 & $\begin{array}{l}98.7-98.6 \\
\end{array}$ & 100-110 & - & Clay & Clay Artifact & Burned Clay & Clay & 2 & 15.88 & & 12/13/2017 \\
\hline 411 & 1 & 537 & - & - & - & A & IV & No996 & E1014 & 11 & $98.6-98.5$ & $110-120$ & - & Faunal Remains & Bone & Indeterminate & Bone & 1 & 0.18 & & 12/14/2017 \\
\hline 411 & 2 & 537 & - & - & - & A & IV & No996 & E1014 & 11 & $98.6-98.5$ & 110-120 & - & Faunal Remains & Bone & Indeterminate & Bone & 1 & 0.24 & & 12/14/2017 \\
\hline 411 & 3 & 537 & - & - & - & A & IV & No996 & E1014 & 11 & $98.6-98.5$ & $110-120$ & - & Detritus & Debitage & Complete Flake & Lithic & 5 & 14.17 & & $\mid 12 / 14 / 2017$ \\
\hline 411 & 4 & 537 & - & - & - & A & IV & No996 & E1014 & 11 & $98.6-98.5$ & $110-120$ & - & Detritus & Debitage & Proximal Flake & Lithic & 6 & 7.54 & & 12/14/2017 \\
\hline 411 & 5 & 537 & - & - & - & A & IV & No996 & E1014 & 11 & $98.6-98.5$ & $110-120$ & - & Detritus & Debitage & Broken Flake & Lithic & 4 & 6.44 & & 12/14/2017 \\
\hline 411 & 6 & 537 & - & - & - & A & IV & No996 & E1014 & 11 & $98.6-98.5$ & $110-120$ & - & Detritus & Debitage & \begin{tabular}{|l|} 
Flaking Shatter \\
\end{tabular} & Lithic & 1 & 0.33 & & 12/14/2017 \\
\hline 411 & 7 & 537 & - & - & - & A & IV & No996 & E1014 & 11 & $98.6-98.5$ & $110-120$ & - & Detritus & Debitage & Thermal Shatter & Lithic & 24 & 27.53 & & 12/14/2017 \\
\hline 412 & 1 & 567 & - & - & - & A & IVN & No996 & E1014 & 12 & 98.5-98.4 & $120-130$ & - & Faunal Remains & Bone & Indeterminate & Bone & 1 & 0.11 & & 12/14/2017 \\
\hline 412 & 2 & 567 & - & - & - & A & IVN & No996 & E1014 & 12 & 98.5-98.4 & $120-130$ & - & Detritus & Debitage & \begin{tabular}{|l|} 
Complete Flake \\
\end{tabular} & Lithic & 1 & 68.47 & & 12/14/2017 \\
\hline 412 & 3 & 567 & - & - & - & A & IVN & No996 & E1014 & 12 & 98.5-98.4 & 120-130 & - & Detritus & Debitage & Proximal Flake & Lithic & 2 & 3.76 & & $\mid$\begin{tabular}{|l|}
$\mid 12 / 14 / 2017$ \\
\end{tabular} \\
\hline 412 & 4 & 567 & - & - & - & A & IVN & No996 & E1014 & 12 & $\begin{array}{l}98.5-98.4 \\
\end{array}$ & $120-130$ & - & Detritus & Debitage & Broken Flake & Lithic & 3 & 0.75 & & 12/14/2017 \\
\hline 412 & 5 & 567 & - & - & - & A & IVN & No996 & E1014 & 12 & $98.5-98.4$ & $120-130$ & - & Detritus & Debitage & Thermal Shatter & Lithic & 8 & 2.28 & & 12/14/2017 \\
\hline 413 & 1 & 540 & - & - & - & A & $\mathrm{v}$ & No996 & E1014 & 13 & $98.4-98.3$ & $130-140$ & - & Faunal Remains & Bone & Indeterminate & Bone & 1 & 1.38 & & \begin{tabular}{|l|}
$12 / 14 / 2017$ \\
\end{tabular} \\
\hline 413 & 2 & 540 & - & - & - & A & $\mathrm{v}$ & N0996 & E1014 & 13 & $98.4-98.3$ & $130-140$ & - & Detritus & Debitage & \begin{tabular}{|l|} 
Complete Flake \\
\end{tabular} & Lithic & 2 & 11.29 & & 12/14/2017 \\
\hline 413 & 3 & 540 & - & - & - & A & $\mathrm{v}$ & No996 & E1014 & 13 & $98.4-98.3$ & 130-140 & - & Detritus & Debitage & \begin{tabular}{|l|} 
Proximal Flake \\
\end{tabular} & Lithic & 2 & 0.42 & & 12/14/2017 \\
\hline 413 & 4 & 540 & - & - & - & A & $\mathrm{v}$ & No996 & E1014 & 13 & $98.4-98.3$ & $130-140$ & - & Detritus & Debitage & Broken Flake & Lithic & 2 & 2 & & 12/14/2017 \\
\hline 413 & 5 & 540 & - & - & - & A & $\mathrm{v}$ & No996 & E1014 & 13 & $98.4-98.3$ & $130-140$ & - & Detritus & Debitage & Thermal Shatter & Lithic & 6 & 2.42 & & \begin{tabular}{|l|}
$12 / 14 / 2017$ \\
\end{tabular} \\
\hline 414 & 1 & 543 & - & - & - & A & $\mathrm{vv}_{1}$ & No996 & E1014 & 14 & $98.3-98.2$ & 140-150 & - & Faunal Remains & Bone & Indeterminate & Bone & 1 & 1.09 & & 12/15/2017 \\
\hline 414 & 2 & 543 & - & - & - & A & $\mathrm{vv}_{1}$ & No996 & E1014 & 14 & $98.3-98.2$ & 140-150 & - & Faunal Remains & Bone & Indeterminate & Bone & 1 & 0.26 & & 12/15/2017 \\
\hline 414 & 3 & 543 & - & - & - & A & vi & No996 & E1014 & 14 & $98.3-98.2$ & $140-150$ & - & \begin{tabular}{|l|} 
Faunal Remains \\
\end{tabular} & Bone & Indeterminate & Bone & 1 & 0.41 & & 12/15/2017 \\
\hline 414 & 4 & 543 & - & - & - & A & vI & No996 & E1014 & 14 & 98.3-98.2 & $140-150$ & - & Faunal Remains & Bone & Indeterminate & Bone & 1 & 0.7 & & 12/15/2017 \\
\hline 414 & 5 & 543 & - & - & - & A & vi & No996 & E1014 & 14 & $98.3-98.2$ & $140-150$ & - & Faunal Remains & Bone & Indeterminate & Bone & 1 & 0.18 & & 12/15/2017 \\
\hline 414 & 6 & 543 & - & - & - & A & vi & No996 & E1014 & 14 & $98.3-98.2$ & $140-150$ & - & \begin{tabular}{|c|} 
Detritus \\
\end{tabular} & Debitage & Broken Flake & Lithic & 2 & 0.66 & & 12/15/2017 \\
\hline
\end{tabular}




\begin{tabular}{|c|c|c|c|c|c|c|c|c|c|c|c|c|c|c|c|c|c|c|c|c|c|}
\hline Lot No. & \begin{tabular}{|c|}
$\begin{array}{c}\text { Specimen } \\
\text { No. }\end{array}$ \\
\end{tabular} & FS No. & UI No. & FTR No. & $\begin{array}{c}\text { Sample } \\
\text { No. }\end{array}$ & $\begin{array}{c}\text { Excavation } \\
\text { Block }\end{array}$ & Stratum (AU) & Northing & Easting & Level & $\begin{array}{c}\text { Elevation } \\
(\mathrm{m})\end{array}$ & Depth (cmbd) & Point Prov. & Artifiact Class & \begin{tabular}{|l|} 
Artifact Type \\
\end{tabular} & \begin{tabular}{|c|} 
Artifact \\
Description
\end{tabular} & Material & $\begin{array}{c}\text { Number of } \\
\text { artifacts }\end{array}$ & Weight (g) & Comments & Date \\
\hline 415 & 1 & 547 & - & - & - & A & vi & No996 & E1014 & 15 & 98.2-98.1 & $150-160$ & - & Detritus & Debitage & Broken Flake & Lithic & 1 & 0.06 & & 12/15/2017 \\
\hline 416 & 1 & 553 & 29 & - & - & A & $\mathrm{vl}_{1}$ & No996 & E1014 & 16 & 98.055 & 164.5 & \begin{tabular}{|l|} 
N996.93 \\
E1014.16 \\
\end{tabular} & \begin{tabular}{|c|} 
Ground// \\
Battered Stone \\
\end{tabular} & Groundstone & \begin{tabular}{|l|} 
Grooved Stone \\
\end{tabular} & Limestone & 1 & 185.48 & & $12 / 15 / 2017$ \\
\hline 417 & 1 & 561 & - & - & - & A & $\mathrm{vv}_{1}$ & No996 & E1014 & 17 & $98.0-97.9$ & $\begin{array}{ll}170-180 \\
\end{array}$ & - & \begin{tabular}{|l|} 
Faunal Remains \\
\end{tabular} & Bone & Mammal & Bone & 1 & 4.43 & & 12/15/2017 \\
\hline 417 & 2 & 561 & - & - & - & A & vi & No996 & E1014 & 17 & $98.0-97.9$ & $170-180$ & - & Faunal Remains & Bone & Indeterminate & Bone & 1 & 1.47 & & 12/15/2017 \\
\hline 417 & 3 & 561 & - & - & - & A & $\mathrm{vv}_{1}$ & No996 & E1014 & 17 & 98.0-97.9 & 170-180 & - & Detritus & Debitage & Proximal Flake & Lithic & 2 & 0.29 & & 12/15/2017 \\
\hline 417 & 4 & 561 & - & - & - & A & vi & No996 & E1014 & 17 & $98.0-97.9$ & $170-180$ & - & Detritus & Debitage & Broken Flake & Lithic & 2 & 2.31 & & 12/15/2017 \\
\hline 417 & 5 & 561 & - & - & - & A & vi & No996 & E1014 & 17 & $98.0-97.9$ & 170-180 & - & Formal Tool & Biface & \begin{tabular}{c|} 
Indeterminate \\
Fragment
\end{tabular} & Fine Grain Chert & 1 & 6.44 & & $\mid$\begin{tabular}{|l|}
$\mid 12 / 15 / 2017$ \\
\end{tabular} \\
\hline 418 & 1 & 673 & - & - & - & A & vi & No996 & E1014 & 19 & 97.8-97.7 & $190-200$ & - & Detritus & Debitage & Thermal Shatter & Lithic & 1 & 1.44 & & 12/28/2017 \\
\hline 419 & 1 & 555.1 & - & $\begin{array}{l}\text { FTR3 } \\
\text { Cluster } 1 \\
\end{array}$ & $\mathrm{C}-11$ & A & 'II' & No996 & E1012-1013 & 7 & 98.95 & 75 & $\begin{array}{l}\text { N996.00 } \\
\text { E1013.37 } \\
\end{array}$ & Faunal Remains & Bone & & & 1 & 0.16 & $\begin{array}{c}\text { From Cluster 1; collected as charcoal } \\
\text { sample C-11 but actually bone; not } \\
\text { analyzzed }\end{array}$ & 12/15/2017 \\
\hline 420 & 1 & 555.1 & - & $\begin{array}{l}\text { FTR3 } \\
\text { Cluster } 1\end{array}$ & $F-4$ & A & III & N0995-0996 & E1012-1013 & $7-8$ & 98.97-98.9 & $73-80$ & \begin{tabular}{|c|} 
N995.92- \\
996.07 \\
E1013.28- \\
1013.50 \\
\end{tabular} & \begin{tabular}{|c|}
$\begin{array}{c}\text { Macrobotanical } \\
\text { Remains }\end{array}$ \\
\end{tabular} & Charcoal & $\begin{array}{l}\text { Red muberry } \\
\text { (Morus rubra) }\end{array}$ & & 16 & 1.45 & & 12/15/2017 \\
\hline 420 & 2 & 555.1 & - & $\begin{array}{l}\text { FTR3 } \\
\text { Cluster } 1\end{array}$ & F-4 & A & III & N0995-0996 & E1012-1013 & $7-8$ & 98.97-98.9 & $73-80$ & 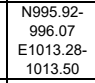 & \begin{tabular}{c|c|} 
Macrobotanical \\
Remains
\end{tabular} & Charcoal & $\mid \begin{array}{c}\text { Juniper } \\
\text { (Juniperus spp.) }\end{array}$ & & 2 & 0.01 & & 12/15/2017 \\
\hline 420 & 3 & 555.1 & - & $\begin{array}{l}\text { FTR3 } \\
\text { Cluster } 1\end{array}$ & $F-4$ & A & III & \begin{tabular}{|l|l} 
No995-0996 \\
\end{tabular} & E1012-1013 & $7-8$ & 98.97-98.9 & $73-80$ & 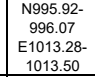 & $\begin{array}{c}\text { Macrobotanical } \\
\text { Remains }\end{array}$ & Charcoal & 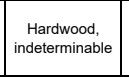 & & 1 & 0.01 & & 12/15/2017 \\
\hline 420 & 4 & 555.1 & - & $\begin{array}{l}\text { FTR3 } \\
\text { Cluster } 1\end{array}$ & F-4 & A & III & N0995-0996 & E1012-1013 & $7-8$ & 98.97-98.9 & $73-80$ & 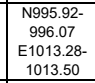 & $\begin{array}{c}\text { Macrobotanical } \\
\text { Remains }\end{array}$ & 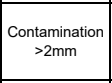 & & & & 5.2 & & 12/15/2017 \\
\hline 420 & 5 & 555.1 & - & $\begin{array}{c}\text { FTR3 } \\
\text { Cluster } 1\end{array}$ & $F-4$ & A & III & No995-0996 & E1012-1013 & $7-8$ & 98.97-98.9 & $73-80$ & \begin{tabular}{|c|} 
N999.92- \\
996.07 \\
E1013.28- \\
1013.50
\end{tabular} & \begin{tabular}{c|} 
Macrobotanical \\
Remains
\end{tabular} & \begin{tabular}{|l|}
$\mid$\begin{tabular}{|l} 
Examined \\
Residue $<2 \mathrm{~mm}$
\end{tabular} \\
\end{tabular} & & & & 2.14 & & 12/15/2017 \\
\hline 420 & 6 & 555.1 & - & $\begin{array}{l}\text { FTR3 } \\
\text { Cluster } 1\end{array}$ & $F-4$ & A & III & No995-0996 & E1012-1013 & $7-8$ & 98.97-98.9 & $73-80$ & 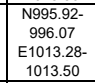 & Other Lithic & \begin{tabular}{|l|} 
Thermally \\
Attered Stone \\
\end{tabular} & $F C R$ & & 7 & 2.5 & & 12/15/2017 \\
\hline 420 & 7 & 555.1 & - & $\begin{array}{l}\text { FTR3 } \\
\text { Cluster } 1\end{array}$ & $F-4$ & A & III & N0995-0996 & E1012-1013 & $7-8$ & 98.97-98.9 & $73-80$ & 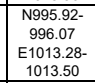 & $\begin{array}{c}\text { Macrobotanical } \\
\text { Remains }\end{array}$ & \begin{tabular}{|l|} 
residual from \\
heavy fraction
\end{tabular} & & & & 36.5 & & 12/15/2017 \\
\hline 420 & 8 & 555.1 & - & $\begin{array}{l}\text { FTR3 } \\
\text { Cluster 1 }\end{array}$ & F-4 & A & III & No995-0996 & E1012-1013 & $7-8$ & 98.97-98.9 & $73-80$ & $\begin{array}{c}\text { N995.92- } \\
996.07 \\
\text { E1013.28- } \\
1013.50 \\
\end{array}$ & Faunal Remains & Bone & & & 9 & 0.5 & $\begin{array}{c}\text { From flotation heavy fraction, not } \\
\text { analyzed }\end{array}$ & 12/15/2017 \\
\hline 420 & 9 & 555.1 & - & $\begin{array}{c}\text { FTR3 } \\
\text { Cluster } 1\end{array}$ & $F-4$ & A & III & \begin{tabular}{|l|l} 
No995-0996 \\
\end{tabular} & E1012-1013 & $7-8$ & 98.97-98.9 & $73-80$ & 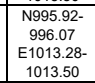 & Detritus & Debitage & Complete Flake & & 1 & 0.2 & & 12/15/2017 \\
\hline 420 & 10 & 555.1 & - & $\begin{array}{l}\text { FTR3 } \\
\text { Cluster } 1\end{array}$ & $F-4$ & A & III & \begin{tabular}{|l|l} 
No995-0996 \\
\end{tabular} & E1012-1013 & $7-8$ & 98.97-98.9 & $73-80$ & 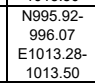 & Detritus & Debitage & Proximal Flake & & 1 & 0.05 & & 12/15/2017 \\
\hline 420 & 11 & 555.1 & - & $\begin{array}{l}\text { FTR3 } \\
\text { Cluster } 1\end{array}$ & $F-4$ & A & III & No995-0996 & E1012-1013 & $7-8$ & 98.97-98.9 & $73-80$ & \begin{tabular}{|c|c|}
$9995.92-$ \\
996.07 \\
E1013.28- \\
1013.50 \\
1
\end{tabular} & Detritus & Debitage & Flaking Shatter & & 12 & 3.01 & & 12115/2017 \\
\hline 420 & 12 & 555.1 & - & $\begin{array}{c}\text { FTR3 } \\
\text { Cluster } 1\end{array}$ & $F-4$ & A & III & No995-0996 & E1012-1013 & $7-8$ & 98.97-98.9 & $73-80$ & 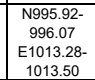 & Detritus & Debitage & Thermal Shatter & & 9 & 1.43 & & 12/15/2017 \\
\hline 420 & N/A & 555.1 & - & $\begin{array}{l}\text { FTR3 } \\
\text { Cluster } 1\end{array}$ & $F-4$ & A & III & No995-0996 & E1012-1013 & $7-8$ & 98.97-98.9 & $73-80$ & 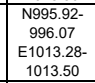 & $\begin{array}{c}\text { Archaeological } \\
\text { Sample }\end{array}$ & Soil & Feature Matrix & & $4.0 \mathrm{~L}$ & 3700 & $\begin{array}{l}\text { ALL FLOATED-NO MATRIX } \\
\text { REMAINING }\end{array}$ & 12115/2017 \\
\hline 421 & \begin{tabular}{|c|}
1 \\
"radiocarbo \\
n dated
\end{tabular} & 555.2 & - & $\begin{array}{l}\text { FTR3 } \\
\text { Cluster 2 }\end{array}$ & $F-5$ & A & III & No995-0996 & E1012-1013 & $7-8$ & 99.0-98.94 & $70-76$ & $\begin{array}{l}\text { N996.32- } \\
\text { 996.59 } \\
\text { E1012.5- } \\
1013.19\end{array}$ & \begin{tabular}{|c|} 
Macrobotanical \\
Remains
\end{tabular} & Charcoal & $\begin{array}{l}\text { Bluewood } \\
\text { condalia } \\
\text { (Condalia } \\
\text { hookeri) }\end{array}$ & & 6 & 0.04 & "sent for radiocarbon dating & 12/15/2017 \\
\hline 421 & 2 & 555.2 & - & $\begin{array}{l}\text { FTR3 } \\
\text { Cluster 2 }\end{array}$ & $F-5$ & A & III & No995-0996 & E1012-1013 & $7-8$ & 99.0-98.94 & $70-76$ & 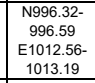 & \begin{tabular}{c|c|} 
Macrobotanical \\
Remains
\end{tabular} & Charcoal & $\begin{array}{l}\text { Sugarbery } \\
\text { (Celtis sp.) }\end{array}$ & & 2 & 0.01 & & $12 / 15 / 2017$ \\
\hline 421 & 3 & 555.2 & - & $\begin{array}{l}\text { FTR3 } \\
\text { Cluster } 2\end{array}$ & $F-5$ & A & III & No995-0996 & E1012-1013 & $7-8$ & 99.0-98.94 & $70-76$ & 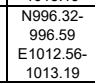 & \begin{tabular}{c|c|} 
Macrobotanical \\
Remains
\end{tabular} & Charcoal & $\left.\begin{array}{c}\text { Silk tassel } \\
\text { (Garrya ovata) }\end{array}\right)$ & & 1 & 0.01 & & 12/15/2017 \\
\hline 421 & 4 & 555.2 & - & $\begin{array}{l}\text { FTR3 } \\
\text { Cluster } 2\end{array}$ & $F-5$ & A & III & N0995-0996 & E1012-1013 & $7-8$ & 99.0-98.94 & $70-76$ & 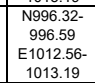 & \begin{tabular}{c|} 
Macrobotanical \\
Remains
\end{tabular} & Charcoal & not id'd & & & 0.01 & & 12/15/2017 \\
\hline
\end{tabular}




\begin{tabular}{|c|c|c|c|c|c|c|c|c|c|c|c|c|c|c|c|c|c|c|c|c|c|}
\hline Lot No. & \begin{tabular}{|c|}
$\begin{array}{c}\text { Specimen } \\
\text { No. }\end{array}$ \\
\end{tabular} & FS No. & UI No. & FTR No. & $\begin{array}{c}\text { Sample } \\
\text { No. }\end{array}$ & $\begin{array}{c}\begin{array}{c}\text { Excavation } \\
\text { Block }\end{array} \\
\end{array}$ & Stratum (AU) & Northing & Easting & Level & \begin{tabular}{|c|} 
Elevation \\
$(\mathrm{m})$
\end{tabular} & Depth (cmbd) & Point Prov. & Artifact Class & Artifact Type & \begin{tabular}{|c|} 
Artifact \\
Description \\
\end{tabular} & Material & $\begin{array}{c}\text { Number of } \\
\text { artifacts }\end{array}$ & Weight $(g)$ & Comments & Date \\
\hline 421 & 5 & 555.2 & - & $\begin{array}{l}\text { FTR3 } \\
\text { Cluster 2 }\end{array}$ & $F-5$ & A & III & N0995-0996 & E1012-1013 & $7-8$ & 99.0-98.94 & $70-76$ & $\begin{array}{c}\text { N996.32- } \\
9966.59 \\
\text { E1012.56- } \\
1013.19\end{array}$ & $\begin{array}{c}\text { Macrobotanical } \\
\text { Remains }\end{array}$ & $\begin{array}{c}\text { Contamination } \\
>2 \mathrm{~mm}\end{array}$ & & & & 31.72 & & 12/15/2017 \\
\hline 421 & 6 & 555.2 & - & $\begin{array}{l}\text { FTR3 } \\
\text { Cluster 2 }\end{array}$ & $F-5$ & A & III & N0995-0996 & E1012-1013 & $7-8$ & 99.0-98.94 & $70-76$ & 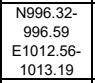 & $\begin{array}{c}\text { Macrobotanical } \\
\text { Remains }\end{array}$ & $\left|\begin{array}{c}\text { Examined } \\
\text { Residue }<2 \mathrm{~mm}\end{array}\right|$ & & & & 5.81 & & 12/15/2017 \\
\hline 421 & 7 & 555.2 & - & $\begin{array}{l}\text { FTR3 } \\
\text { Cluster } 2\end{array}$ & $F-5$ & A & III & N0995-0996 & E1012-1013 & $7-8$ & 99.0-98.94 & $70-76$ & 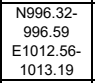 & $\begin{array}{l}\text { Macrobotanical } \\
\text { Remains }\end{array}$ & $\mid \begin{array}{c}\text { residual from } \\
\text { heavy fraction }\end{array}$ & & & & 17 & & 12/15/2017 \\
\hline 421 & 8 & 555.2 & - & $\begin{array}{l}\text { FTR3 } \\
\text { Cluster } 2\end{array}$ & $F-5$ & A & III & N0995-0996 & E1012-1013 & $7-8$ & 99.0-98.94 & $70-76$ & $\begin{array}{c}\text { N996.32- } \\
996.59 \\
\text { E1012.56- } \\
1013.19 \\
\end{array}$ & Faunal Remains & Bone & & & 1 & 0.1 & $\begin{array}{l}\text { From flotation heavy fraction, not } \\
\text { analyzed }\end{array}$ & 12/15/2017 \\
\hline 421 & 9 & 555.2 & - & $\begin{array}{l}\text { FTR3 } \\
\text { Cluster } 2\end{array}$ & $F-5$ & A & III & N0995-0996 & E1012-1013 & $7-8$ & 99.0-98.94 & $70-76$ & 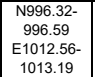 & Detritus & Debitage & Broken Flake & & 1 & 0.25 & & 12/15/2017 \\
\hline 421 & 10 & 555.2 & - & $\begin{array}{l}\text { FTR3 } \\
\text { Cluster } 2\end{array}$ & $F-5$ & A & III & N0995-0996 & E1012-1013 & $7-8$ & 99.0-98.94 & $70-76$ & 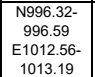 & Detritus & Debitage & Flaking Shatter & & 6 & 0.05 & & 12/15/2017 \\
\hline 421 & 11 & 555.2 & - & $\begin{array}{l}\text { FTR3 } \\
\text { Cluster 2 }\end{array}$ & $F-5$ & A & III & N0995-0996 & E1012-1013 & $7-8$ & 99.0-98.94 & $70-76$ & 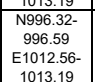 & Detritus & Debitage & Thermal Shatter & & 13 & 1.22 & & 12/15/2017 \\
\hline 421 & N/A & 555.2 & - & $\begin{array}{l}\text { FTR3 } \\
\text { Cluster } 2\end{array}$ & $F-5$ & A & III & No995-0996 & E1012-1013 & $7-8$ & 99.0-98.94 & $70-76$ & 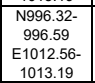 & $\begin{array}{c}\text { Archaeological } \\
\text { Sample }\end{array}$ & Soil & Feature Matrix & & $3.5 \mathrm{~L}$ & 3600 & $\begin{array}{l}\text { ALL FLOATED-NO MATRIX } \\
\text { REMAINING }\end{array}$ & 12/15/2017 \\
\hline 422 & 1 & 555.2 & - & FTR3 & $\mathrm{C}-12$ & A & IIII & No996 & E1013 & 7 & 98.91 & 79 & $\begin{array}{l}\begin{array}{l}\text { N996.90 } \\
\text { E1013.35 }\end{array} \\
\end{array}$ & 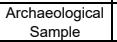 & c-14 & $\begin{array}{l}\begin{array}{l}\text { Red mulbery } \\
\text { (Morus rubra) }\end{array} \\
\end{array}$ & & 15 & 1.44 & $\begin{array}{l}\text { From below Artifict \#1; northeast of } \\
\text { Cluster } 2\end{array}$ & 12/15/2017 \\
\hline 422 & 2 & 555.2 & - & FTR3 & C-12 & A & III & No996 & E1013 & 7 & 98.91 & 79 & \begin{tabular}{|l|l} 
N996.90 \\
E1013.35
\end{tabular} & $\begin{array}{c}\text { Archaeological } \\
\text { Sampleal }\end{array}$ & \begin{tabular}{|c|} 
Examined \\
Residue $<2 \mathrm{~mm}$
\end{tabular} & & & & 0.12 & $\begin{array}{l}\text { From below Atifiact } 1 \text { 1; northeast of } \\
\text { Cluster } 2\end{array}$ & 12/15/2017 \\
\hline 424 & 1 & 595 & - & FTR3 & - & A & III & No996 & E1013 & 7 & 98.92 & 78 & $\begin{array}{l}\text { N996.90 } \\
\text { E1013.37 }\end{array}$ & Detritus & Debitage & \begin{tabular}{|l|} 
Proximal Flake \\
\end{tabular} & Lithic & 1 & 32.73 & Lithic 1: flake & 12/15/2017 \\
\hline 425 & 1 & 596 & - & FTR3 & - & A & IIII & N0996 & E1013 & 7 & 98.95 & 75 & $\begin{array}{l}\text { N996.91 } \\
\text { E1013.09 }\end{array}$ & Detritus & Debitage & \begin{tabular}{|l|} 
Complete Flake \\
\end{tabular} & Lithic & 1 & 29.63 & Lithic 2: flake & 12/15/2017 \\
\hline 426 & 1 & 601 & - & FTR3 & - & $\mathrm{A}$ & III & No996 & E1013 & 7 & 98.98 & 72 & $\begin{array}{l}\text { N999.53 } \\
\text { E1013.09 } \\
\end{array}$ & Detritus & Debitage & \begin{tabular}{|l|} 
Proximal Flake \\
\end{tabular} & Lithic & 1 & 20.74 & Lithic 7: Flake & 12/15/2017 \\
\hline 427 & 1 & 602 & - & FTR3 & - & A & IIII & N0996 & E1013 & 7 & 98.94 & 76 & $\begin{array}{l}\text { N996.53 } \\
\text { E1013.14 }\end{array}$ & Informal Tool & Core Tool & \begin{tabular}{l|} 
Chopper \\
\end{tabular} & Fine Grain Chert & 1 & 355.4 & Lithic 8: Core & $12 / 15 / 2017$ \\
\hline 428 & 1 & 604 & - & FTR3 & - & A & IIII & N0996 & E1013 & 7 & 98.98 & 72 & \begin{tabular}{l|l|} 
N996.57 \\
E1013.07
\end{tabular} & Other Lithic & $\begin{array}{l}\text { Thermally } \\
\text { Altered Stone }\end{array}$ & FCR & Lithic & 1 & 14.1 & Lithic 10: Core & 12/15/2017 \\
\hline 429 & 1 & 597 & - & FTR3 & - & A & IIII & N0996 & E1012 & 8 & 98.91 & 79 & \begin{tabular}{|l|l|} 
No96.37 \\
E110138
\end{tabular} & Informal Tool & \begin{tabular}{|l|} 
Modified Flake \\
\end{tabular} & \begin{tabular}{|l|} 
Edge-modified \\
\end{tabular} & $\begin{array}{l}\text { Coarse Grain } \\
\text { Chert }\end{array}$ & 1 & 180.45 & Lithic 3:flake/ core refitit burned & 12/15/2017 \\
\hline 430 & 1 & 598 & - & FTR3 & - & A & III & No996 & E1012 & 8 & 98.94 & 76 & $\begin{array}{l}\text { No96..11 } \\
\text { E1012.36 } \\
\end{array}$ & Other Lithic & \begin{tabular}{|c|} 
Thermally \\
Altered Stone \\
\end{tabular} & FCC & Lithic & 2 & 335.35 & Lithic 4: Burned Chert Cobble & 12/15/2017 \\
\hline 431 & 1 & 599 & - & FTR3 & - & A & IIII & No996 & E1012 & 8 & 98.97 & 73 & $\begin{array}{l}\begin{array}{l}\text { N999.05 } \\
\text { E1012.67 }\end{array} \\
\end{array}$ & Other Lithic & \begin{tabular}{|l|} 
Thermally \\
Attered Stone \\
\end{tabular} & FCR & Lithic & 1 & 144.9 & Lithic 5: Abrader & 12/15/2017 \\
\hline 432 & 1 & 600 & - & FTR3 & - & A & IV & No996 & E1013 & 8 & 98.83 & 87 & $\begin{array}{r}\text { N996.66 } \\
\text { E1013.31 } \\
\end{array}$ & Informal Tool & Core Tool & Chopper & $\begin{array}{c}\text { Coarse Grain } \\
\text { Chert }\end{array}$ & 1 & 493.32 & Lithic 6: Chopper & 12/15/2017 \\
\hline 433 & 1 & 603 & - & FTR3 & - & A & III & No996 & E1012 & 8 & 98.95 & 75 & \begin{tabular}{|c|} 
N996.51 \\
E1012.98 \\
\end{tabular} & Detritus & Core & Multidirectional & \begin{tabular}{|l|} 
Fine Grain Chert \\
\end{tabular} & 1 & 69.74 & Lithic 9: Core & 12/15/2017 \\
\hline 434 & 1 & 408 & - & - & - & B & 1/11 & No999 & E1013 & 2 & $99.5-99.4$ & $10-20$ & - & Detritus & Debitage & Proximal Flake & Lithic & 1 & 0.26 & & 1214/2017 \\
\hline 434 & 2 & 408 & - & - & - & B & 1/11 & No999 & E1013 & 2 & $99.5-99.4$ & 10-20 & - & Detritus & Debitage & Broken Flake & Lithic & 3 & 22.49 & & 121/4/2017 \\
\hline 435 & 1 & 412 & - & - & - & B & IIII & No999 & E1013 & 3 & $99.4-99.3$ & $20-30$ & - & Detritus & Debitage & Complete Flake & Lithic & 1 & 1.12 & & 121/4/2017 \\
\hline 435 & 2 & 412 & - & - & - & B & IIII & $\begin{array}{ll}\text { N0999 } \\
\end{array}$ & E1013 & 3 & $99.4-99.3$ & $20-30$ & - & Detritus & Debitage & Broken Flake & Lithic & 2 & 1.55 & & 12/4/2017 \\
\hline 436 & 1 & 423 & - & - & - & B & III & No999 & E1013 & 4 & $99.3-99.2$ & $30-40$ & - & Detritus & Debitage & Complete Flake & Lithic & 1 & 0.57 & & 12/5/2017 \\
\hline 436 & 2 & 423 & - & - & - & B & III & No999 & E1013 & 4 & 99.3-99.2 & $30-40$ & - & Detritus & Debitage & \begin{tabular}{|l|} 
Proximal Flake \\
\end{tabular} & Lithic & 3 & 27.51 & & 12/5/2017 \\
\hline 436 & 3 & 423 & - & - & - & B & III & No999 & E1013 & 4 & $99.3-99.2$ & $30-40$ & - & Detritus & Debitage & Broken Flake & Lithic & 3 & 1.08 & & 12/5/2017 \\
\hline 436 & 4 & 423 & - & - & - & B & IIII & No999 & E1013 & 4 & $99.3-99.2$ & $30-40$ & - & Detritus & Debitage & Thermal Shatter & Lithic & 3 & 2.39 & & 121/5/2017 \\
\hline $\begin{array}{lll}437 & \\
\end{array}$ & 1 & 429 & - & - & - & B & IV & No999 & E1013 & 5 & $99.2-99.1$ & $40-50$ & - & Detritus & Debitage & \begin{tabular}{|c|} 
Complete Flake \\
\end{tabular} & Lithic & 10 & 40.32 & & 1215/2017 \\
\hline 437 & 2 & 429 & - & - & - & B & iv & $\begin{array}{ll}\text { No999 } \\
\end{array}$ & E1013 & 5 & $99.2-99.1$ & $40-50$ & - & Detritus & Debitage & \begin{tabular}{|l|} 
Proximal Flake \\
\end{tabular} & Lithic & 3 & 5.65 & & 122/5/2017 \\
\hline $\begin{array}{lll}437 & \\
\end{array}$ & 3 & 429 & - & - & - & B & IV & No999 & E1013 & 5 & $99.2-99.1$ & $40-50$ & - & Detritus & Debitage & Broken Flake & Lithic & 28 & 26.73 & & 121/5/2017 \\
\hline $\begin{array}{lll}437 & \\
\end{array}$ & 4 & 429 & - & - & - & B & IV & No999 & E1013 & 5 & $99.2-99.1$ & $40-50$ & - & Detritus & Debitage & Thermal Shatter & Lithic & 7 & 8.33 & & 12/5/2017 \\
\hline 438 & 1 & 437 & - & - & - & B & IV & No999 & E1013 & 6 & $99.1-99.0$ & 50-60 & - & Detritus & Debitage & Complete Flake & Lithic & 6 & 44.45 & & 1215/2017 \\
\hline 438 & 2 & 437 & - & - & - & B & IV & No999 & E1013 & 6 & $99.1-99.0$ & $50-60$ & - & Detritus & Debitage & \begin{tabular}{|l|} 
Proximal Flake \\
\end{tabular} & Lithic & 1 & 0.56 & & 12/5/2017 \\
\hline 438 & 3 & 437 & - & - & - & B & IV & No999 & E1013 & 6 & $99.1-99.0$ & $50-60$ & - & Detritus & Debitage & Broken Flake & Lithic & 17 & 5.72 & & 1215/2017 \\
\hline 438 & 4 & 437 & - & - & - & $B$ & IV & No999 & E1013 & 6 & $99.1-99.0$ & $50-60$ & - & Detritus & Debitage & Thermal Shatter & Lithic & 23 & 22.37 & & 12/5/2017 \\
\hline
\end{tabular}




\begin{tabular}{|c|c|c|c|c|c|c|c|c|c|c|c|c|c|c|c|c|c|c|c|c|c|}
\hline Lot No. & \begin{tabular}{|c|}
$\begin{array}{c}\text { Specimen } \\
\text { No. }\end{array}$ \\
\end{tabular} & FS No. & UI No. & FTR No. & $\begin{array}{c}\text { Sample } \\
\text { No. }\end{array}$ & $\begin{array}{c}\text { Excavation } \\
\text { Block }\end{array}$ & Stratum (AU) & Northing & Easting & Level & $\begin{array}{c}\text { Elevation } \\
(\mathrm{m})\end{array}$ & Depth (cmbd) & Point Prov. & Artifact Class & Artifact Type & \begin{tabular}{|c|} 
Artifact \\
Description
\end{tabular} & Material & $\begin{array}{c}\text { Number of } \\
\text { artifacts }\end{array}$ & Weight (g) & Comments & Date \\
\hline 439 & 1 & 442 & - & - & - & B & IV & No999 & E1013 & 7 & $99.0-98.9$ & $60-70$ & - & Faunal Remains & Bone & Mammal & Bone & 1 & 1.9 & & 12/5/2017 \\
\hline 439 & 2 & 442 & - & - & - & B & IV & No999 & E1013 & 7 & 99.0-98.9 & $60-70$ & - & Detritus & Debitage & Complete Flake & Lithic & 5 & 2 & & 12/5/2017 \\
\hline 439 & 3 & 442 & - & - & - & B & IV & No999 & E1013 & 7 & $99.0-98.9$ & $60-70$ & - & Detritus & Debitage & \begin{tabular}{|l|} 
Proximal Flake \\
\end{tabular} & Lithic & 4 & 5.49 & & 12/5/2017 \\
\hline 439 & 4 & 442 & - & - & - & B & IV & No999 & E1013 & 7 & $99.0-98.9$ & $60-70$ & - & Detritus & Debitage & Broken Flake & Lithic & 19 & 8.66 & & 12/5/2017 \\
\hline 439 & 5 & 442 & - & - & - & B & IV & No999 & E1013 & 7 & 99.0-98.9 & $60-70$ & - & Detritus & Debitage & Flaking Shatter & Lithic & 3 & 1.43 & & 12/5/2017 \\
\hline 439 & 6 & 442 & - & - & - & B & IV & No999 & E1013 & 7 & $99.0-98.9$ & $60-70$ & - & Detritus & Debitage & \begin{tabular}{|l|} 
Thermal Shatter \\
\end{tabular} & Lithic & 48 & 51.06 & & 12/5/2017 \\
\hline 439 & 7 & 442 & - & - & - & B & IV & No999 & E1013 & 7 & $99.0-98.9$ & $60-70$ & - & Informal Tool & Modified Flake & \begin{tabular}{|l|} 
Edge-modified \\
\end{tabular} & Fine Grain Chert & 1 & 0.54 & & 12/5/2017 \\
\hline 440 & 1 & 448 & - & - & - & B & v & No999 & E1013 & 8 & 98.9-98.8 & $70-80$ & - & Detritus & Debitage & Complete Flake & Lithic & 2 & 10.57 & & 12/5/2017 \\
\hline 440 & 2 & 448 & - & - & - & B & $\mathrm{v}$ & No999 & E1013 & 8 & $\begin{array}{ll}88.9-98.8 \\
\end{array}$ & $70-80$ & - & Detritus & Debitage & \begin{tabular}{|l|} 
Proximal Flake \\
\end{tabular} & Lithic & 5 & 21.5 & & 12/5/2017 \\
\hline 440 & 3 & 448 & - & - & - & B & $\mathrm{v}$ & No999 & E1013 & 8 & $\begin{array}{ll}88.9-98.8 \\
\end{array}$ & $\begin{array}{ll}70-80 \\
\end{array}$ & - & Detritus & Debitage & Broken Flake & Lithic & 19 & 27.54 & & 12/5/2017 \\
\hline 440 & 4 & 448 & - & - & - & B & $\mathrm{v}$ & No999 & E1013 & 8 & 98.9-98.8 & $70-80$ & - & Detritus & Debitage & Thermal Shatter & Lithic & 21 & 28.39 & & 12/5/2017 \\
\hline 440 & 5 & 448 & - & - & - & B & $\mathrm{v}$ & No999 & E1013 & 8 & $98.9-98.8$ & $\begin{array}{ll}70-80 \\
\end{array}$ & - & Informal Tool & \begin{tabular}{|l|} 
Modified Flake \\
\end{tabular} & Edge-modified & Fine Grain Chert & 1 & 76.99 & & 12/5/2017 \\
\hline 441 & 1 & 453 & - & - & - & B & $\mathrm{v}$ & No999 & E1013 & 9 & $98.8-98.7$ & $\begin{array}{ll}80-90 \\
\end{array}$ & - & Detritus & Debitage & \begin{tabular}{l|l} 
Broken Flake \\
\end{tabular} & Lithic & 2 & 1.47 & & 12/8/2017 \\
\hline 441 & 2 & 453 & - & - & - & B & $\mathrm{v}$ & No999 & E1013 & 9 & $\begin{array}{ll}98.8-98.7 \\
\end{array}$ & $\begin{array}{ll}80-90 \\
\end{array}$ & - & Detritus & Debitage & Thermal Shatter & Lithic & 5 & 8.15 & & 12/8/2017 \\
\hline 442 & 1 & 460 & - & - & - & B & vi & No999 & E1013 & 10 & 98.7-98.6 & $90-100$ & - & Detritus & Debitage & Proximal Flake & Lithic & 2 & 4.03 & & 12/8/2017 \\
\hline 442 & 2 & 460 & - & - & - & B & vi & No999 & E1013 & 10 & 98.7-98.6 & 90-100 & - & Detritus & Debitage & Broken Flake & Lithic & 2 & 2.42 & & 12/8/2017 \\
\hline 442 & 3 & 460 & - & - & - & B & vi & No999 & E1013 & 10 & 98.7-98.6 & $90-100$ & - & Detritus & Debitage & Flaking Shatter & Lithic & 1 & 1.94 & & 12/8/2017 \\
\hline 443 & 1 & 476 & - & - & - & B & vi & No999 & E1013 & 12 & 98.5-98.4 & $110-120$ & - & Faunal Remains & Bone & Mammal & Bone & 1 & 0.51 & & 12/11/2017 \\
\hline 443 & 2 & 476 & - & - & - & B & vi & No999 & E1013 & 12 & 98.5-98.4 & $110-120$ & - & Detritus & Debitage & \begin{tabular}{|l|} 
Proximal Flake \\
\end{tabular} & Lithic & 3 & 13.71 & & 12/11/2017 \\
\hline 443 & 3 & 476 & - & - & - & B & vi & No999 & E1013 & 12 & 98.5-98.4 & $110-120$ & - & Detritus & Debitage & Broken Flake & Lithic & 6 & 5.62 & & 12/11/2017 \\
\hline 443 & 4 & 476 & - & - & - & B & $\mathrm{vI}$ & No999 & E1013 & 12 & $\begin{array}{ll}98.5-98.4 \\
\end{array}$ & 110-120 & - & Detritus & Debitage & Thermal Shatter & Lithic & 3 & 5.96 & & 12/11/2017 \\
\hline 444 & 1 & 482 & - & - & - & B & VII & No999 & E1013 & 13 & $98.4-98.3$ & $120-130$ & - & Detritus & Debitage & Proximal Flake & Lithic & 1 & 0.65 & & 12/11/2017 \\
\hline 444 & 2 & 482 & - & - & - & B & VII & No999 & E1013 & 13 & $98.4-98.3$ & $120-130$ & - & Detritus & Debitage & Broken Flake & Lithic & 1 & 0.57 & & 12/11/2017 \\
\hline 444 & 3 & 482 & - & - & - & B & VII & No999 & E1013 & 13 & $98.4-98.3$ & $120-130$ & - & Detritus & Debitage & Thermal Shatter & Lithic & 2 & 3.79 & & 12/11/2017 \\
\hline 444 & 4 & 482 & - & - & - & B & VII & No999 & E1013 & 13 & 98.4-98.3 & $120-130$ & - & Formal Tool & Biface & $\begin{array}{l}\text { Indeterminate } \\
\text { Fragment }\end{array}$ & Fine Grain Chert & 1 & 15.46 & & 12/11/2017 \\
\hline 445 & 1 & 489 & - & - & - & B & VII & No999 & E1013 & 14 & $98.3-98.2$ & $130-140$ & - & Detritus & Debitage & Broken Flake & Lithic & 1 & 1.63 & & 12/112/2017 \\
\hline 445 & 2 & 489 & - & - & - & B & VII & No999 & E1013 & 14 & $98.3-98.2$ & $130-140$ & - & Detritus & Debitage & Thermal Shatter & Lithic & 2 & 1.13 & & 12/12/2017 \\
\hline 446 & 1 & 498 & - & - & - & B & VII & No999 & E1013 & 15 & $98.2-98.1$ & $140-150$ & - & \begin{tabular}{|l|} 
Faunal Remains \\
\end{tabular} & Bone & Indeterminate & Bone & 1 & 0.11 & & 12/12/2017 \\
\hline 446 & 2 & 498 & - & - & - & B & VII & No999 & E1013 & 15 & 98.2-98.1 & $140-150$ & - & Detritus & Debitage & \begin{tabular}{|l|} 
Complete Flake \\
\end{tabular} & Lithic & 1 & 0.2 & & 12/12/2017 \\
\hline 446 & 3 & 498 & - & - & - & B & VII & No999 & E1013 & 15 & $98.2-98.1$ & $140-150$ & - & Detritus & Debitage & \begin{tabular}{|l|} 
Flaking Shatter \\
\end{tabular} & Lithic & 1 & 0.14 & & 12/112/2017 \\
\hline 447 & 1 & 406 & - & - & - & B & 1/11 & No999 & E1014 & 2 & 99.5-99.4 & $10-20$ & - & Detritus & Debitage & Broken Flake & Lithic & 1 & 3.58 & & 12/4/2017 \\
\hline 447 & 2 & 406 & - & - & - & B & 1/11 & No999 & E1014 & 2 & $99.5-99.4$ & $10-20$ & - & Faunal Remains & Shell & Mussel Shell & Shell & 2 & 9.86 & & 12/4/2017 \\
\hline 448 & 1 & 417 & - & - & - & B & III & No999 & E1014 & 3 & 99.4-99.3 & $20-30$ & - & Detritus & Debitage & Thermal Shatter & Lithic & 1 & 0.33 & & 12/4/2017 \\
\hline 449 & 1 & 419 & - & - & - & B & IIII & No999 & E1014 & 4 & 99.3-99.2 & $30-40$ & - & Detritus & Debitage & Proximal Flake & Lithic & 2 & 1.35 & & 12/4/2017 \\
\hline 450 & 1 & 431 & - & - & - & B & IV & No999 & E1014 & 5 & $99.2-99.1$ & $40-50$ & - & Detritus & Debitage & \begin{tabular}{|c|} 
Complete Flake \\
\end{tabular} & Lithic & 7 & 9.15 & & 12/5/2017 \\
\hline 450 & 2 & 431 & - & - & - & B & IV & No999 & E1014 & 5 & $99.2-99.1$ & $40-50$ & - & Detritus & Debitage & \begin{tabular}{|l|} 
Proximal Flake \\
\end{tabular} & Lithic & 3 & 7.62 & & 12/5/2017 \\
\hline 450 & 3 & 431 & - & - & - & B & IV & No999 & E1014 & 5 & 99.2-99.1 & $40-50$ & - & Detritus & Debitage & Broken Flake & Lithic & 27 & 18.68 & & 12/5/2017 \\
\hline 450 & 4 & 431 & - & - & - & B & IV & No999 & E1014 & 5 & 99.2-99.1 & $40-50$ & - & Detritus & Debitage & Flaking Shatter & Lithic & 2 & 11.21 & & 12/5/2017 \\
\hline 450 & 5 & 431 & - & - & - & B & IV & No999 & E1014 & 5 & $99.2-99.1$ & $40-50$ & - & Detritus & Debitage & Thermal Shatter & Lithic & 20 & 10.22 & & 12/5/2017 \\
\hline 451 & 1 & 434 & - & - & - & B & IV & No999 & E1014 & 6 & $99.1-99.0$ & 50-60 & - & Detritus & Debitage & Complete Flake & Lithic & 13 & 36.02 & & 12/5/2017 \\
\hline 451 & 2 & 434 & - & - & - & B & IV & No999 & E1014 & 6 & $99.1-99.0$ & $50-60$ & - & Detritus & Debitage & Proximal Flake & Lithic & 6 & 6.92 & & 12/5/2017 \\
\hline 451 & 3 & 434 & - & - & - & B & iv & No999 & E1014 & 6 & $99.1-99.0$ & 50-60 & - & Detritus & Debitage & Broken Flake & Lithic & 13 & 14.11 & & 12/5/2017 \\
\hline 451 & 4 & 434 & - & - & - & B & IV & No999 & E1014 & 6 & $99.1-99.0$ & $50-60$ & - & Detritus & Debitage & \begin{tabular}{|l|} 
Flaking Shatter \\
\end{tabular} & Lithic & 1 & 14.37 & & 12/5/2017 \\
\hline 451 & 5 & 434 & - & - & - & B & IV & No999 & E1014 & 6 & $99.1-99.0$ & $50-60$ & - & Detritus & Debitage & Thermal Shatter & Lithic & 4 & 21.31 & & 12/5/2017 \\
\hline
\end{tabular}




\begin{tabular}{|c|c|c|c|c|c|c|c|c|c|c|c|c|c|c|c|c|c|c|c|c|c|}
\hline Lot No. & \begin{tabular}{|c|}
$\begin{array}{c}\text { Specimen } \\
\text { No. }\end{array}$ \\
\end{tabular} & FS No. & UI No. & FTR No. & $\begin{array}{c}\text { Sample } \\
\text { No. }\end{array}$ & $\begin{array}{c}\text { Excavation } \\
\text { Block }\end{array}$ & Stratum (AU) & Northing & Easting & Level & $\begin{array}{c}\text { Elevation } \\
\text { (m) }\end{array}$ & Depth (cmbd) & Point Prov. & Artifact Class & \begin{tabular}{|l|} 
Artifact Type \\
\end{tabular} & \begin{tabular}{|c|} 
Artifact \\
Description
\end{tabular} & Material & $\begin{array}{c}\text { Number of } \\
\text { artifacts }\end{array}$ & Weight (g) & Comments & Date \\
\hline 452 & 1 & 440 & - & - & - & B & IV & No999 & E1014 & 7 & $99.0-98.9$ & $60-70$ & - & Detritus & Debitage & Complete Flake & Lithic & 1 & 2.16 & & 12/5/2017 \\
\hline 452 & 2 & 440 & - & - & - & B & IV & No999 & E1014 & 7 & 99.0-98.9 & $60-70$ & - & Detritus & Debitage & Proximal Flake & Lithic & 1 & 0.33 & & 12/5/2017 \\
\hline 452 & 3 & 440 & - & - & - & B & IV & No999 & E1014 & 7 & $99.0-98.9$ & $60-70$ & - & Detritus & Debitage & Broken Flake & Lithic & 2 & 3.2 & & 12/5/2017 \\
\hline 453 & 1 & 449 & - & - & - & B & $\mathrm{v}$ & No999 & E1014 & 8 & $98.9-98.8$ & $70-80$ & - & Detritus & Debitage & Complete Flake & Lithic & 2 & 95.96 & & 12/8/2017 \\
\hline 453 & 2 & 449 & - & - & - & B & $\mathrm{v}$ & No999 & E1014 & 8 & 98.9-98.8 & $70-80$ & - & Detritus & Debitage & Proximal Flake & Lithic & 5 & 17.48 & & 12/8/2017 \\
\hline 453 & 3 & 449 & - & - & - & B & $\mathrm{v}$ & No999 & E1014 & 8 & $98.9-98.8$ & $70-80$ & - & Detritus & Debitage & Broken Flake & Lithic & 42 & 18.2 & & 12/8/2017 \\
\hline 453 & 4 & 449 & - & - & - & B & v & No999 & E1014 & 8 & 98.9-98.8 & $70-80$ & - & Detritus & Debitage & Flaking Shatter & Lithic & 2 & 2.73 & & 12/8/2017 \\
\hline 453 & 5 & 449 & - & - & - & B & v & No999 & E1014 & 8 & 98.9-98.8 & $70-80$ & - & Detritus & Debitage & Thermal Shatter & Lithic & 28 & 39.5 & & 12/8/2017 \\
\hline 454 & 1 & 457 & - & - & - & B & $\mathrm{v}$ & No999 & E1014 & 9 & $\begin{array}{l}98.8-98.7 \\
\end{array}$ & $80-90$ & - & Detritus & Debitage & Complete Flake & Lithic & 3 & 21.37 & & 12/8/2017 \\
\hline 454 & 2 & 457 & - & - & - & B & $\mathrm{v}$ & No999 & E1014 & 9 & $\begin{array}{ll}98.8-98.7 \\
\end{array}$ & $\begin{array}{ll}80-90 \\
\end{array}$ & - & Detritus & Debitage & Broken Flake & Lithic & 4 & 1.13 & & 12/8/2017 \\
\hline 454 & 3 & 457 & - & - & - & B & v & No999 & E1014 & 9 & 98.8-98.7 & $80-90$ & - & Detritus & Debitage & Flaking Shatter & Lithic & 2 & 44.8 & & 12/8/2017 \\
\hline 454 & 4 & 457 & - & - & - & B & $\mathrm{v}$ & No999 & E1014 & 9 & $98.8-98.7$ & $\begin{array}{lll}80-90 \\
\end{array}$ & - & Detritus & Debitage & Thermal Shatter & Lithic & 5 & 6.45 & & 12/8/2017 \\
\hline 455 & 1 & 462 & - & - & - & B & vi & No999 & E1014 & 10 & 98.7-98.6 & $90-100$ & - & Detritus & Debitage & \begin{tabular}{|l|} 
Proximal Flake \\
\end{tabular} & Lithic & 2 & 22.04 & & 12/8/2017 \\
\hline 455 & 2 & 462 & - & - & - & B & $\mathrm{vv}_{1}$ & No999 & E1014 & 10 & $98.7-98.6$ & $\begin{array}{l}0-100 \\
\end{array}$ & - & Detritus & Debitage & Broken Flake & Lithic & 3 & 6.06 & & 12/8/2017 \\
\hline 455 & 3 & 462 & - & - & - & B & vi & No999 & E1014 & 10 & 98.7-98.6 & $90-100$ & - & Detritus & Debitage & Flaking Shatter & Lithic & 1 & 0.63 & & 12/8/2017 \\
\hline 455 & 4 & 462 & - & - & - & B & vi & No999 & E1014 & 10 & 98.7-98.6 & 90-100 & - & Detritus & Debitage & Thermal Shatter & Lithic & 4 & 9.06 & & 12/8/2017 \\
\hline 456 & 1 & 474 & - & - & - & B & vi & No999 & E1014 & 12 & 98.5-98.4 & $110-120$ & - & Detritus & Debitage & Complete Flake & Lithic & 1 & 3.01 & & 12/11/2017 \\
\hline 456 & 2 & 474 & - & - & - & B & vi & No999 & E1014 & 12 & $98.5-98.4$ & $110-120$ & - & Detritus & Debitage & Proximal Flake & Lithic & 1 & 0.23 & & 12/11/2017 \\
\hline 456 & 3 & 474 & - & - & - & B & vi & No999 & E1014 & 12 & 98.5-98.4 & $110-120$ & - & Detritus & Debitage & Broken Flake & Lithic & 2 & 1.16 & & 12/11/2017 \\
\hline 456 & 4 & 474 & - & - & - & B & vi & No999 & E1014 & 12 & $98.5-98.4$ & $110-120$ & - & Detritus & Debitage & Flaking Shatter & Lithic & 1 & 35.72 & & 12/11/2017 \\
\hline 456 & 5 & 474 & - & - & - & B & vi & No999 & E1014 & 12 & $98.5-98.4$ & $110-120$ & - & Detritus & Debitage & \begin{tabular}{|l|} 
Thermal Shatter \\
\end{tabular} & Lithic & 1 & 0.46 & & 12/11/2017 \\
\hline 457 & 1 & 484 & - & - & - & $B$ & VIII & No999 & E1014 & 13 & $98.4-98.3$ & $120-130$ & - & Detritus & Debitage & Broken Flake & Lithic & 2 & 0.62 & & 12/11/2017 \\
\hline 457 & 2 & 484 & - & - & - & B & VII & No999 & E1014 & 13 & $98.4-98.3$ & $120-130$ & - & Detritus & Debitage & \begin{tabular}{|l|} 
Thermal Shatter \\
\end{tabular} & Lithic & 3 & 1.79 & & 12/11/2017 \\
\hline 458 & 1 & 491 & - & - & - & B & VII & No999 & E1014 & 14 & $98.3-98.2$ & $130-140$ & - & Detritus & Debitage & \begin{tabular}{|l|} 
Thermal Shatter \\
\end{tabular} & Lithic & 1 & 0.35 & & 12/12/2017 \\
\hline 459 & 1 & 505 & - & - & - & c & 1 & N1000 & E1003 & 1 & 99.83-99.7 & 7-20 & - & Detritus & Debitage & Broken Flake & Lithic & 2 & 1.26 & & 12/13/2017 \\
\hline 460 & 1 & 511 & - & - & - & c & "I & N1000 & E1003 & 2 & $99.7-99.6$ & $20-30$ & - & Detritus & Debitage & Broken Flake & Lithic & 5 & 1.56 & & 12/13/2017 \\
\hline 460 & 2 & 511 & - & - & - & c & "I & N1000 & E1003 & 2 & $99.7-99.6$ & $20-30$ & - & Detritus & Debitage & Thermal Shatter & Lithic & 1 & 1.56 & & 12/13/2017 \\
\hline 461 & 1 & 512 & - & - & - & $\mathrm{c}$ & 11 & N1000 & E1003 & 2 & 99.65 & 25 & \begin{tabular}{|l|}
$\begin{array}{l}\mathrm{N} 1000.65 \\
\text { E1003.81 }\end{array}$ \\
\end{tabular} & Other Lithic & 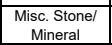 & Manuport & Limestone & 1 & 92.83 & & 12/13/2017 \\
\hline 462 & 1 & 526 & - & - & - & c & "I & N1000 & E1003 & 3 & $99.6-99.5$ & $30-40$ & - & Faunal Remains & Bone & Mammal & Bone & 1 & 3.87 & & 12/13/2017 \\
\hline 462 & 2 & 526 & - & - & - & c & "I & N1000 & E1003 & 3 & $99.6-99.5$ & $30-40$ & - & Faunal Remains & Bone & Mammal & Bone & 1 & 4.19 & & 12/13/2017 \\
\hline 462 & 3 & 526 & - & - & - & c & " & N1000 & E1003 & 3 & 99.6-99.5 & $30-40$ & - & Faunal Remains & Bone & Mammal & Bone & 1 & 3.12 & & 12/13/2017 \\
\hline 462 & 4 & 526 & - & - & - & c & II & N1000 & E1003 & 3 & $99.6-99.5$ & $30-40$ & - & Faunal Remains & Bone & Indeterminate & Bone & 1 & 0.29 & & 12/13/2017 \\
\hline 462 & 5 & 526 & - & - & - & c & "I & N1000 & E1003 & 3 & $99.6-99.5$ & $30-40$ & - & Faunal Remains & Bone & Indeterminate & Bone & 1 & 0.68 & & 12/13/2017 \\
\hline 462 & 6 & 526 & - & - & - & $\mathrm{c}$ & "I & N1000 & E1003 & 3 & $99.6-99.5$ & $30-40$ & - & Faunal Remains & Bone & Indeterminate & Bone & 1 & 0.34 & & 12/13/2017 \\
\hline 462 & 7 & 526 & - & - & - & c & "I & N1000 & E1003 & 3 & $99.6-99.5$ & $30-40$ & - & Faunal Remains & Bone & Indeterminate & Bone & 1 & 0.14 & & 12/13/2017 \\
\hline 462 & 8 & 526 & - & - & - & $\mathrm{c}$ & "I & N1000 & E1003 & 3 & $99.6-99.5$ & $30-40$ & - & \begin{tabular}{|l|} 
Faunal Remains \\
\end{tabular} & Bone & Indeterminate & Bone & 1 & 0.34 & & 12/13/2017 \\
\hline 462 & 9 & 526 & - & - & - & c & "I & N1000 & E1003 & 3 & $99.6-99.5$ & $30-40$ & - & Faunal Remains & Bone & Indeterminate & Bone & 1 & 0.27 & & 12/13/2017 \\
\hline 462 & 10 & 526 & - & - & - & $\mathrm{c}$ & "I & N1000 & E1003 & 3 & $99.6-99.5$ & $30-40$ & - & Faunal Remains & Bone & Indeterminate & Bone & 1 & 1.4 & & 12/13/2017 \\
\hline 462 & 11 & 526 & - & - & - & c & "I & N1000 & E1003 & 3 & $99.6-99.5$ & $30-40$ & - & Faunal Remains & Bone & Indeterminate & Bone & 1 & 0.27 & & 12/13/2017 \\
\hline 462 & 12 & 526 & - & - & - & c & "I & N1000 & E1003 & 3 & $99.6-99.5$ & $30-40$ & - & Faunal Remains & Bone & Indeterminate & Bone & 1 & 0.28 & & 12/13/2017 \\
\hline 462 & 13 & 526 & - & - & - & c & 11 & N1000 & E1003 & 3 & $99.6-99.5$ & $30-40$ & - & Faunal Remains & Bone & Indeterminate & Bone & 1 & 1.64 & & 12/13/2017 \\
\hline 462 & 14 & 526 & - & - & - & c & "I & N1000 & E1003 & 3 & $99.6-99.5$ & $30-40$ & - & Faunal Remains & Bone & Mammal & Bone & 1 & 12.44 & & 12/13/2017 \\
\hline 462 & 15 & 526 & - & - & - & c & "I & N1000 & E1003 & 3 & $99.6-99.5$ & $30-40$ & - & Faunal Remains & Bone & Mammal & Bone & 1 & 8.48 & & 12/13/2017 \\
\hline 462 & 16 & 526 & - & - & - & c & ॥ & N1000 & E1003 & 3 & $99.6-99.5$ & $30-40$ & - & Faunal Remains & Bone & Indeterminate & Bone & 1 & 1.9 & & 12/13/2017 \\
\hline
\end{tabular}




\begin{tabular}{|c|c|c|c|c|c|c|c|c|c|c|c|c|c|c|c|c|c|c|c|c|c|}
\hline Lot No. & \begin{tabular}{|c|}
$\begin{array}{c}\text { Specimen } \\
\text { No. }\end{array}$ \\
\end{tabular} & FS No. & UI No. & FTR No. & $\begin{array}{c}\text { Sample } \\
\text { No. }\end{array}$ & $\begin{array}{c}\text { Excavation } \\
\text { Block }\end{array}$ & Stratum (AU) & Northing & Easting & Level & $\begin{array}{c}\text { Elevation } \\
(\mathrm{m})\end{array}$ & Depth (cmbd) & Point Prov. & Artifact Class & \begin{tabular}{|l|} 
Artifact Type \\
\end{tabular} & \begin{tabular}{|c|} 
Artifact \\
Description
\end{tabular} & Material & $\begin{array}{c}\text { Number of } \\
\text { artifacts }\end{array}$ & Weight (g) & Comments & Date \\
\hline 462 & \begin{tabular}{|l|}
17 \\
\end{tabular} & 526 & - & - & - & $\mathrm{c}$ & " & N1000 & E1003 & 3 & $99.6-99.5$ & $30-40$ & - & Faunal Remains & Bone & Indeterminate & Bone & 1 & 12.2 & & 12/13/2017 \\
\hline 462 & 18 & 526 & - & - & - & c & "I & N1000 & E1003 & 3 & $99.6-99.5$ & $30-40$ & - & Detritus & Debitage & Proximal Flake & Lithic & 6 & 29.14 & & $\mid$\begin{tabular}{|l|}
$\mid 12 / 13 / 2017$ \\
\end{tabular} \\
\hline 462 & 19 & 526 & - & - & - & c & "I & N1000 & E1003 & 3 & $99.6-99.5$ & $30-40$ & - & Detritus & Debitage & Broken Flake & Lithic & 11 & 11.29 & & 12/13/2017 \\
\hline 462 & 20 & 526 & - & - & - & c & "I & N1000 & E1003 & 3 & $99.6-99.5$ & $30-40$ & - & Detritus & Debitage & Thermal Shatter & Lithic & 16 & 17.89 & & 12/13/2017 \\
\hline 462 & 21 & 526 & - & - & - & c & " & N1000 & E1003 & 3 & 99.6-99.5 & $30-40$ & - & Formal Tool & Biface & \begin{tabular}{c|c} 
Medial \\
Fragment
\end{tabular} & Fine Grain Chert & 1 & 0.16 & & $\mid$\begin{tabular}{|l|}
$\mid 12 / 13 / 2017$ \\
\end{tabular} \\
\hline 462 & 22 & 526 & - & - & - & c & $" 1$ & N1000 & E1003 & 3 & 99.6-99.5 & $30-40$ & - & $\begin{array}{c}\text { Prehistoric } \\
\text { Ceramic }\end{array}$ & \begin{tabular}{|l|}
$\begin{array}{l}\text { Undecorated } \\
\text { Body Sherd }\end{array}$ \\
\end{tabular} & $\begin{array}{l}\text { Bone and } \\
\text { coarse sand } \\
\text { temper }\end{array}$ & Ceramic & 6 & 8.2 & 2 sherds reft & 12/13/2017 \\
\hline 463 & 1 & 529 & - & - & - & c & 11/111 & N1000 & E1003 & 4 & 99.5-99.4 & $40-50$ & - & Detritus & Debitage & Complete Flake & Lithic & 6 & 16.23 & & 12/13/2017 \\
\hline 463 & 2 & 529 & - & - & - & c & 11/III & N1000 & E1003 & 4 & $99.5-99.4$ & $40-50$ & - & Detritus & Debitage & \begin{tabular}{|l|} 
Broken Flake \\
\end{tabular} & Lithic & 13 & 5.34 & & 12/13/2017 \\
\hline 463 & 3 & 529 & - & - & - & $\mathrm{c}$ & $11 / 111$ & N1000 & E1003 & 4 & 99.5-99.4 & $40-50$ & - & Detritus & Debitage & Thermal Shatter & Lithic & 24 & 16.89 & & $\mid 12 / 13 / 2017$ \\
\hline 463 & 4 & 529 & - & - & - & c & 11/III & N1000 & E1003 & 4 & 99.5-99.4 & $40-50$ & - & $\begin{array}{c}\text { Prehistoric } \\
\text { Ceramic }\end{array}$ & \begin{tabular}{|l|}
$\begin{array}{l}\text { Undecorated } \\
\text { Body Sherd }\end{array}$ \\
\end{tabular} & \begin{tabular}{|c|}
$\begin{array}{c}\text { Bone and } \\
\text { coarse sand } \\
\text { temper }\end{array}$ \\
\end{tabular} & Ceramic & 1 & 2.46 & & 12/13/2017 \\
\hline 464 & 1 & 536 & - & - & - & c & III & N1000 & E1003 & 5 & 99.4-99.3 & $50-60$ & - & Detritus & Debitage & Complete Flake & Lithic & 3 & 3.15 & & 12/14/2017 \\
\hline 464 & 2 & 536 & - & - & - & c & III & N1000 & E1003 & 5 & 99.4-99.3 & $50-60$ & - & Detritus & Debitage & \begin{tabular}{|l|} 
Proximal Flake \\
\end{tabular} & Lithic & 2 & 2.53 & & 12/14/2017 \\
\hline 464 & 3 & 536 & - & - & - & $\mathrm{c}$ & IIII & N1000 & E1003 & 5 & $99.4-99.3$ & 50-60 & - & Detritus & Debitage & Broken Flake & Lithic & 4 & 2.14 & & $\mid 12 / 14 / 2017$ \\
\hline 464 & 4 & 536 & - & - & - & c & IIII & N1000 & E1003 & 5 & 99.4-99.3 & $50-60$ & - & Detritus & Debitage & Thermal Shatter & Lithic & 22 & 20.93 & & 12/14/2017 \\
\hline 464 & 5 & 536 & - & - & - & c & III & N1000 & E1003 & 5 & 99.4-99.3 & $50-60$ & - & $\begin{array}{l}\text { Prehistoric } \\
\text { Ceramic }\end{array}$ & $\begin{array}{l}\text { Undecorated } \\
\text { Body Sherd }\end{array}$ & \begin{tabular}{|c|}
$\begin{array}{c}\text { Bone and } \\
\text { coarse sand } \\
\text { temper }\end{array}$ \\
\end{tabular} & Ceramic & 1 & 1.73 & & 12/14/2017 \\
\hline 465 & 1 & 661 & - & - & - & $\mathrm{c}$ & III & N1000 & E1003 & 6 & 99.3-99.2 & $60-70$ & - & Detritus & Debitage & \begin{tabular}{|l|} 
Complete Flake \\
\end{tabular} & Lithic & 5 & 110.68 & & 12/27/2017 \\
\hline 465 & 2 & 661 & - & - & - & c & III & N1000 & E1003 & 6 & 99.3-99.2 & $60-70$ & - & Detritus & Debitage & Proximal Flake & Lithic & 2 & 0.99 & & 12/27/2017 \\
\hline 465 & 3 & 661 & - & - & - & $\mathrm{c}$ & IIII & N1000 & E1003 & 6 & $99.3-99.2$ & $60-70$ & - & Detritus & Debitage & Broken Flake & Lithic & 14 & 11.25 & & 12/27/2017 \\
\hline 465 & 4 & 661 & - & - & - & c & IIII & N1000 & E1003 & 6 & $99.3-99.2$ & $60-70$ & - & Detritus & Debitage & \begin{tabular}{|l|} 
Thermal Shatter \\
\end{tabular} & Lithic & 4 & 16.23 & & 12/27/2017 \\
\hline 466 & 1 & 661 & - & - & - & c & III & N1000 & E1003 & 6 & 99.18 & 72 & $\begin{array}{l}\begin{array}{l}\mathrm{N} 1000.40 \\
\text { E1003.64 }\end{array} \\
\end{array}$ & Detritus & Core & Multidirectional & Fine Grain Chert & 1 & 188.54 & & 12/27/2017 \\
\hline 467 & 1 & 663 & - & - & - & c & III & N1000 & E1003 & 7 & 99.2-99.1 & $70-80$ & - & Faunal Remains & Bone & Indeterminate & Bone & 1 & 0.43 & & 12/27/2017 \\
\hline 467 & 2 & 663 & - & - & - & $\mathrm{c}$ & IIII & N1000 & E1003 & 7 & $99.2-99.1$ & $\begin{array}{ll}70-80 \\
\end{array}$ & - & \begin{tabular}{|l|} 
Faunal Remains \\
\end{tabular} & Bone & Indeterminate & Bone & 1 & 0.09 & & 12/27/2017 \\
\hline 467 & 3 & 663 & - & - & - & c & IIII & N1000 & E1003 & 7 & $99.2-99.1$ & $70-80$ & - & Faunal Remains & Bone & Indeterminate & Bone & 1 & 0.27 & & 12/27/2017 \\
\hline 467 & 4 & 663 & - & - & - & $\mathrm{c}$ & IIII & N1000 & E1003 & 7 & $99.2-99.1$ & $\begin{array}{ll}70-80 \\
\end{array}$ & - & Detritus & Debitage & Complete Flake & Lithic & 6 & 17.46 & & 12/27/2017 \\
\hline 467 & 5 & 663 & - & - & - & c & III & N1000 & E1003 & 7 & 99.2-99.1 & $70-80$ & - & Detritus & Debitage & Proximal Flake & Lithic & 4 & 1.75 & & 12/27/2017 \\
\hline 467 & 6 & 663 & - & - & - & $\mathrm{c}$ & IIII & N1000 & E1003 & 7 & $99.2-99.1$ & $\begin{array}{ll}70-80 \\
\end{array}$ & - & Detritus & Debitage & Broken Flake & Lithic & 16 & 7.72 & & 12/27/2017 \\
\hline 467 & 7 & 663 & - & - & - & c & IIII & N1000 & E1003 & 7 & 99.2-99.1 & $70-80$ & - & Detritus & Debitage & Thermal Shatter & Lithic & 14 & 6.85 & & 12/27/2017 \\
\hline 467 & 8 & 663 & - & - & - & c & III & N1000 & E1003 & 7 & $99.2-99.1$ & $70-80$ & - & Clay & Pigment & Red Ochre & Ochre & 2 & 0.69 & & 12/27/2017 \\
\hline 468 & 1 & 665 & - & - & - & c & IIIIV & N1000 & E1003 & 8 & $99.1-99.0$ & $80-90$ & - & Detritus & Debitage & Complete Flake & Lithic & 5 & 20.62 & & 12/27/2017 \\
\hline 468 & 2 & 665 & - & - & - & $\mathrm{c}$ & $\frac{11 \mathrm{IIV}}{\mathrm{N}}$ & N1000 & E1003 & 8 & $99.1-99.0$ & $80-90$ & - & Detritus & Debitage & \begin{tabular}{|l|} 
Proximal Flake \\
\end{tabular} & Lithic & 3 & 5.34 & & 12/27/2017 \\
\hline 468 & 3 & 665 & - & - & - & c & IIIIV & N1000 & E1003 & 8 & $99.1-99.0$ & $80-90$ & - & Detritus & Debitage & Broken Flake & Lithic & 7 & 8.09 & & $\mid$\begin{tabular}{|l|}
$\mid 12 / 27 / 2017$ \\
\end{tabular} \\
\hline 468 & 4 & 665 & - & - & - & $\mathrm{c}$ & III/IV & N1000 & E1003 & 8 & $99.1-99.0$ & $80-90$ & - & Detritus & Debitage & \begin{tabular}{|l|} 
Thermal Shatter \\
\end{tabular} & Lithic & 2 & 1.46 & & 12/27/2017 \\
\hline 469 & 1 & 668 & - & - & - & c & IV & N1000 & E1003 & 9 & $99.0-98.9$ & $90-100$ & - & Faunal Remains & Bone & Indeterminate & Bone & 1 & 0.19 & & 12/28/2017 \\
\hline 469 & 2 & 668 & - & - & - & $\mathrm{c}$ & IV & N1000 & E1003 & 9 & $99.0-98.9$ & $\begin{array}{l}90-100 \\
\end{array}$ & - & Detritus & Debitage & Complete Flake & Lithic & 3 & 12.74 & & $\mid 12 / 28 / 2017$ \\
\hline 469 & 3 & 668 & - & - & - & c & IV & N1000 & E1003 & 9 & $99.0-98.9$ & $90-100$ & - & Detritus & Debitage & \begin{tabular}{|l|} 
Proximal Flake \\
\end{tabular} & Lithic & 2 & 0.61 & & 12/28/2017 \\
\hline 469 & 4 & 668 & - & - & - & c & IV & N1000 & E1003 & 9 & $99.0-98.9$ & 90-100 & - & Detritus & Debitage & Broken Flake & Lithic & 10 & 7.82 & & 12/28/2017 \\
\hline 469 & 5 & 668 & - & - & - & c & IV & N1000 & E1003 & 9 & $99.0-98.9$ & $90-100$ & - & Detritus & Debitage & \begin{tabular}{|l|} 
Thermal Shatter \\
\end{tabular} & Lithic & 5 & 6.39 & & 12/28/2017 \\
\hline 469 & 6 & 668 & - & - & - & $\mathrm{c}$ & IV & N1000 & E1003 & 9 & $99.0-98.9$ & $90-100$ & - & Faunal Remains & Shell & Untyped Shell & Shell & 2 & 6.36 & Burned Oyster? & $\mid$\begin{tabular}{|l|}
$\mid 12 / 28 / 2017$ \\
\end{tabular} \\
\hline 470 & 1 & 509 & - & - & - & c & 1 & N1000 & E1004 & 1 & 99.77-99.7 & $13-20$ & - & Detritus & Debitage & Broken Flake & Lithic & 1 & 0.18 & & 12/13/2017 \\
\hline 471 & 1 & 515 & - & - & - & c & "I & N1000 & E1004 & 2 & $99.7-99.6$ & $20-30$ & - & \begin{tabular}{|l|} 
Faunal Remains \\
\end{tabular} & Bone & Mammal & Bone & 1 & 4.16 & & 12/13/2017 \\
\hline 471 & 2 & 515 & - & - & - & c & " & N1000 & E1004 & 2 & 99.7-99.6 & $20-30$ & - & Faunal Remains & Bone & Mammal & Bone & 1 & 4.39 & & $\mid$\begin{tabular}{|l|}
$\mid 12 / 13 / 2017$ \\
\end{tabular} \\
\hline 471 & 3 & 515 & - & - & - & $\mathrm{c}$ & "I & N1000 & E1004 & 2 & $99.7-99.6$ & $20-30$ & - & \begin{tabular}{|l|} 
Faunal Remains \\
\end{tabular} & Bone & Indeterminate & Bone & 1 & 2.07 & & 12/13/2017 \\
\hline 471 & 4 & 515 & - & - & - & c & "I & N1000 & E1004 & 2 & $99.7-99.6$ & $20-30$ & - & \begin{tabular}{|l|} 
Faunal Remains \\
\end{tabular} & Bone & Indeterminate & Bone & 1 & 1.29 & & 12/13/2017 \\
\hline
\end{tabular}




\begin{tabular}{|c|c|c|c|c|c|c|c|c|c|c|c|c|c|c|c|c|c|c|c|c|c|}
\hline Lot No. & \begin{tabular}{|c|}
$\begin{array}{c}\text { Specimen } \\
\text { No. }\end{array}$ \\
\end{tabular} & FS No. & UI No. & FTR No. & $\begin{array}{c}\text { Sample } \\
\text { No. }\end{array}$ & $\begin{array}{c}\text { Excavation } \\
\text { Block }\end{array}$ & Stratum (AU) & Northing & Easting & Level & $\begin{array}{c}\text { Elevation } \\
\text { (m) }\end{array}$ & Depth (cmbd) & Point Prov. & Artifiact Class & \begin{tabular}{|l|} 
Artifact Type \\
\end{tabular} & $\begin{array}{c}\text { Artifact } \\
\text { Description }\end{array}$ & Material & $\begin{array}{c}\text { Number of } \\
\text { artifacts }\end{array}$ & Weight (g) & Comments & Date \\
\hline 471 & 5 & 515 & - & - & - & $\mathrm{c}$ & " & N1000 & E1004 & 2 & $99.7-99.6$ & $20-30$ & - & Faunal Remains & Bone & Indeterminate & Bone & 1 & 0.32 & & 12/13/2017 \\
\hline 471 & 6 & 515 & - & - & - & $\mathrm{c}$ & "I & N1000 & E1004 & 2 & 99.7-99.6 & $20-30$ & - & Faunal Remains & Bone & Indeterminate & Bone & 1 & 0.62 & & $12 / 13 / 2017$ \\
\hline 471 & 7 & 515 & - & - & - & c & "I & N1000 & E1004 & 2 & $99.7-99.6$ & $20-30$ & - & Faunal Remains & Bone & Indeterminate & Bone & 1 & 0.68 & & 12/13/2017 \\
\hline 471 & 8 & 515 & - & - & - & c & "I & N1000 & E1004 & 2 & $99.7-99.6$ & $20-30$ & - & Faunal Remains & Bone & Indeterminate & Bone & 1 & 0.22 & & 12/13/2017 \\
\hline 471 & 9 & 515 & - & - & - & c & "I & N1000 & E1004 & 2 & 99.7-99.6 & $20-30$ & - & Faunal Remains & Bone & Mammal & Bone & 1 & 0.95 & & $\mid 12 / 13 / 2017$ \\
\hline 471 & 10 & 515 & - & - & - & c & "I & N1000 & E1004 & 2 & $99.7-99.6$ & $20-30$ & - & Detritus & Debitage & \begin{tabular}{|l|} 
Proximal Flake \\
\end{tabular} & Lithic & 3 & 0.95 & & 12/13/2017 \\
\hline 471 & 11 & 515 & - & - & - & c & "I & N1000 & E1004 & 2 & 99.7-99.6 & 20-30 & - & Detritus & Debitage & Broken Flake & Lithic & 4 & 5.61 & & 12/13/2017 \\
\hline 471 & 12 & 515 & - & - & - & c & " & N1000 & E1004 & 2 & 99.7-99.6 & 20-30 & - & Detritus & Debitage & Thermal Shatter & Lithic & 5 & 3.57 & & $\mid$\begin{tabular}{|l|}
$\mid 12 / 13 / 2017$ \\
\end{tabular} \\
\hline 471 & 13 & 515 & - & - & - & c & "I & N1000 & E1004 & 2 & $99.7-99.6$ & $20-30$ & - & Historic Artifiact & Metal & $\begin{array}{l}\text { Miscellaneous } \\
\text { Metal }\end{array}$ & Metal & 1 & 0.22 & & $12 / 13 / 2017$ \\
\hline 472 & 1 & 520 & - & - & - & $\mathrm{c}$ & "I & N1000 & E1004 & 3 & $99.6-99.5$ & $30-40$ & - & Faunal Remains & Bone & Mammal & Bone & 1 & 21.43 & & \begin{tabular}{|l|}
$12 / 13 / 2017$ \\
\end{tabular} \\
\hline 472 & 2 & 520 & - & - & - & $\mathrm{c}$ & "I & N1000 & E1004 & 3 & $99.6-99.5$ & $30-40$ & - & Faunal Remains & Bone & Mammal & Bone & 1 & 19.95 & & \begin{tabular}{|l|}
$12 / 13 / 2017$ \\
\end{tabular} \\
\hline 472 & 3 & 520 & - & - & - & $\mathrm{c}$ & "I & N1000 & E1004 & 3 & $99.6-99.5$ & $30-40$ & - & Faunal Remains & Bone & Mammal & Bone & 1 & 0.75 & & \begin{tabular}{|l|}
$12 / 13 / 2017$ \\
\end{tabular} \\
\hline 472 & 4 & 520 & - & - & - & $\mathrm{c}$ & "I & N1000 & E1004 & 3 & $99.6-99.5$ & $30-40$ & - & Faunal Remains & Bone & Mammal & Bone & 1 & 2.11 & & 12/13/2017 \\
\hline 472 & 5 & 520 & - & - & - & $\mathrm{c}$ & "I & N1000 & E1004 & 3 & $99.6-99.5$ & $30-40$ & - & Faunal Remains & Bone & Mammal & Bone & 1 & 8.58 & & $\mid 12 / 13 / 2017$ \\
\hline 472 & 6 & 520 & - & - & - & c & 11 & N1000 & E1004 & 3 & $99.6-99.5$ & $30-40$ & - & Faunal Remains & Bone & Mammal & Bone & 1 & 13.23 & & \begin{tabular}{|l|}
$12 / 13 / 2017$ \\
\end{tabular} \\
\hline 472 & 7 & 520 & - & - & - & c & "I & N1000 & E1004 & 3 & $99.6-99.5$ & $30-40$ & - & Faunal Remains & Bone & Mammal & Bone & 1 & 5.7 & & \begin{tabular}{|l|}
$12 / 13 / 2017$ \\
\end{tabular} \\
\hline 472 & 8 & 520 & - & - & - & c & "I & N1000 & E1004 & 3 & $99.6-99.5$ & $30-40$ & - & Faunal Remains & Bone & Mammal & Bone & 1 & 1.92 & & 12/13/2017 \\
\hline 472 & 9 & 520 & - & - & - & c & ॥ & N1000 & E1004 & 3 & $99.6-99.5$ & $30-40$ & - & Faunal Remains & Bone & Mammal & Bone & 1 & 4.18 & & 12/13/2017 \\
\hline 472 & 10 & 520 & - & - & - & c & "I & N1000 & E1004 & 3 & $99.6-99.5$ & $30-40$ & - & Faunal Remains & Bone & Mammal & Bone & 1 & 0.71 & & 12/13/2017 \\
\hline 472 & 11 & 520 & - & - & - & c & "I & N1000 & E1004 & 3 & $99.6-99.5$ & $30-40$ & - & Faunal Remains & Bone & Mammal & Bone & 1 & 0.66 & & 12/13/2017 \\
\hline 472 & 12 & 520 & - & - & - & c & "I & N1000 & E1004 & 3 & $99.6-99.5$ & $30-40$ & - & Faunal Remains & Bone & Mammal & Bone & 1 & 3.77 & & 12/13/2017 \\
\hline 472 & 13 & 520 & - & - & - & $\mathrm{c}$ & " & N1000 & E1004 & 3 & $99.6-99.5$ & $30-40$ & - & Faunal Remains & Bone & Mammal & Bone & 1 & 3.77 & & 12/13/2017 \\
\hline 472 & 14 & 520 & - & - & - & $\mathrm{c}$ & $\|$ & N1000 & E1004 & 3 & $99.6-99.5$ & $30-40$ & - & Faunal Remains & Bone & Mammal & Bone & 1 & 3.9 & & 12/13/2017 \\
\hline 472 & 15 & 520 & - & - & - & c & "I & N1000 & E1004 & 3 & $99.6-99.5$ & $30-40$ & - & Faunal Remains & Bone & Mammal & Bone & 1 & 1.13 & & $\mid$\begin{tabular}{|l|}
$\mid 12 / 13 / 2017$ \\
\end{tabular} \\
\hline 472 & 16 & 520 & - & - & - & c & 11 & N1000 & E1004 & 3 & $99.6-99.5$ & $30-40$ & - & Faunal Remains & Bone & Mammal & Bone & 1 & 5.52 & & \begin{tabular}{|l|}
$12 / 13 / 2017$ \\
\end{tabular} \\
\hline 472 & 17 & 520 & - & - & - & c & "I & N1000 & E1004 & 3 & $99.6-99.5$ & $30-40$ & - & Faunal Remains & Bone & Mammal & Bone & 1 & 1.19 & & 12/13/2017 \\
\hline 472 & 18 & 520 & - & - & - & c & "I & N1000 & E1004 & 3 & $99.6-99.5$ & $30-40$ & - & Faunal Remains & Bone & Indeterminate & Bone & 1 & 0.99 & & 12/13/2017 \\
\hline 472 & 19 & 520 & - & - & - & c & "I & N1000 & E1004 & 3 & $99.6-99.5$ & $30-40$ & - & Faunal Remains & Bone & Indeterminate & Bone & 1 & 0.43 & & 12/13/2017 \\
\hline 472 & 20 & 520 & - & - & - & c & "I & N1000 & E1004 & 3 & $99.6-99.5$ & $30-40$ & - & Faunal Remains & Bone & Indeterminate & Bone & 1 & 0.71 & & 12/13/2017 \\
\hline 472 & 21 & 520 & - & - & - & c & "I & N1000 & E1004 & 3 & $99.6-99.5$ & $30-40$ & - & Faunal Remains & Bone & Indeterminate & Bone & 1 & 0.25 & & 12/13/2017 \\
\hline 472 & 22 & 520 & - & - & - & c & " & N1000 & E1004 & 3 & 99.6-99.5 & $30-40$ & - & Faunal Remains & Bone & Indeterminate & Bone & 1 & 0.31 & & $\mid$\begin{tabular}{|l|}
$12 / 13 / 2017$ \\
\end{tabular} \\
\hline 472 & 23 & 520 & - & - & - & c & II & N1000 & E1004 & 3 & $99.6-99.5$ & $30-40$ & - & Faunal Remains & Bone & Indeterminate & Bone & 1 & 0.72 & & 12/13/2017 \\
\hline 472 & 24 & 520 & - & - & - & c & "I & N1000 & E1004 & 3 & $99.6-99.5$ & $30-40$ & - & Faunal Remains & Bone & Mammal & Bone & 1 & 2.53 & & 12/13/2017 \\
\hline 472 & 25 & 520 & - & - & - & $\mathrm{c}$ & "I & N1000 & E1004 & 3 & $99.6-99.5$ & $30-40$ & - & Faunal Remains & Bone & Mammal & Bone & 1 & 2.13 & & \begin{tabular}{|l|}
$12 / 13 / 2017$ \\
\end{tabular} \\
\hline 472 & 26 & 520 & - & - & - & $\mathrm{c}$ & "I & N1000 & E1004 & 3 & $99.6-99.5$ & $30-40$ & - & \begin{tabular}{|l|} 
Faunal Remains \\
\end{tabular} & Bone & Indeterminate & Bone & 1 & 0.75 & & 12/13/2017 \\
\hline 472 & 27 & 520 & - & - & - & $\mathrm{c}$ & "I & N1000 & E1004 & 3 & $99.6-99.5$ & $30-40$ & - & Faunal Remains & Bone & Indeterminate & Bone & 1 & 1.28 & & 12/13/2017 \\
\hline 472 & 28 & 520 & - & - & - & c & "I & N1000 & E1004 & 3 & $99.6-99.5$ & $30-40$ & - & \begin{tabular}{|l|} 
Faunal Remains \\
\end{tabular} & Bone & Indeterminate & Bone & 1 & 0.72 & & 12/13/2017 \\
\hline 472 & 29 & 520 & - & - & - & $\mathrm{c}$ & "I & N1000 & E1004 & 3 & $99.6-99.5$ & $30-40$ & - & Faunal Remains & Bone & Indeterminate & Bone & 1 & 0.36 & & \begin{tabular}{|l|}
$12 / 13 / 2017$ \\
\end{tabular} \\
\hline 472 & 30 & 520 & - & - & - & $\mathrm{c}$ & "I & N1000 & E1004 & 3 & $99.6-99.5$ & $30-40$ & - & Faunal Remains & Bone & Mammal & Bone & 1 & 1.21 & & 12/13/2017 \\
\hline 472 & 31 & 520 & - & - & - & c & "I & N1000 & E1004 & 3 & $99.6-99.5$ & $30-40$ & - & Faunal Remains & Bone & Mammal & Bone & 1 & 0.42 & & 12/13/2017 \\
\hline 472 & 32 & 520 & - & - & - & c & 11 & N1000 & E1004 & 3 & $99.6-99.5$ & $30-40$ & - & Faunal Remains & Bone & Indeterminate & Bone & 1 & 0.4 & & \begin{tabular}{|l|}
$12 / 13 / 2017$ \\
\end{tabular} \\
\hline 472 & 33 & 520 & - & - & - & c & "I & N1000 & E1004 & 3 & $99.6-99.5$ & $30-40$ & - & Faunal Remains & Bone & Mammal & Bone & 1 & 0.68 & & 12/13/2017 \\
\hline 472 & 34 & 520 & - & - & - & c & "I & N1000 & E1004 & 3 & $99.6-99.5$ & $30-40$ & - & \begin{tabular}{|l|} 
Faunal Remains \\
\end{tabular} & Bone & Indeterminate & Bone & 1 & 0.92 & & 12/13/2017 \\
\hline 472 & 35 & 520 & - & - & - & c & ॥ & N1000 & E1004 & 3 & $99.6-99.5$ & $30-40$ & - & Faunal Remains & Bone & Indeterminate & Bone & 1 & 0.7 & & \begin{tabular}{|l|}
$12 / 13 / 2017$ \\
\end{tabular} \\
\hline
\end{tabular}




\begin{tabular}{|c|c|c|c|c|c|c|c|c|c|c|c|c|c|c|c|c|c|c|c|c|c|}
\hline Lot No. & \begin{tabular}{|c|}
$\begin{array}{c}\text { Specimen } \\
\text { No. }\end{array}$ \\
\end{tabular} & FS No. & UI No. & FTR No. & $\begin{array}{c}\text { Sample } \\
\text { No. }\end{array}$ & $\begin{array}{c}\text { Excavation } \\
\text { Block }\end{array}$ & Stratum (AU) & Northing & Easting & Level & $\begin{array}{c}\text { Elevation } \\
\text { (m) }\end{array}$ & Depth (cmbd) & Point Prov. & Artifact Class & \begin{tabular}{|l|} 
Artifact Type \\
\end{tabular} & $\begin{array}{c}\text { Artifact } \\
\text { Description }\end{array}$ & Material & $\begin{array}{c}\text { Number of } \\
\text { artifacts }\end{array}$ & Weight (g) & Comments & Date \\
\hline 472 & 36 & 520 & - & - & - & $\mathrm{c}$ & " & N1000 & E1004 & 3 & $99.6-99.5$ & $30-40$ & - & Faunal Remains & Bone & Mammal & Bone & 1 & 6.84 & & 12/13/2017 \\
\hline 472 & 37 & 520 & - & - & - & c & " & N1000 & E1004 & 3 & 99.6-99.5 & $30-40$ & - & Faunal Remains & Bone & Mammal & Bone & 1 & 2.78 & & $12 / 13 / 2017$ \\
\hline 472 & 38 & 520 & - & - & - & c & "I & N1000 & E1004 & 3 & $99.6-99.5$ & $30-40$ & - & Faunal Remains & Bone & Indeterminate & Bone & 1 & 0.58 & & 12/13/2017 \\
\hline 472 & 39 & 520 & - & - & - & c & "I & N1000 & E1004 & 3 & 99.6-99.5 & $30-40$ & - & Faunal Remains & Bone & Indeterminate & Bone & 1 & 0.21 & & 12/13/2017 \\
\hline 472 & 40 & 520 & - & - & - & c & " & N1000 & E1004 & 3 & 99.6-99.5 & $30-40$ & - & Faunal Remains & Bone & Indeterminate & Bone & 1 & 0.44 & & $\mid 12 / 13 / 2017$ \\
\hline 472 & 41 & 520 & - & - & - & c & "I & N1000 & E1004 & 3 & $99.6-99.5$ & $30-40$ & - & Faunal Remains & Bone & Indeterminate & Bone & 1 & 0.39 & & 12/13/2017 \\
\hline 472 & 42 & 520 & - & - & - & c & "I & N1000 & E1004 & 3 & 99.6-99.5 & $30-40$ & - & Faunal Remains & Bone & Mammal & Bone & 1 & 1.48 & & 12/13/2017 \\
\hline 472 & 43 & 520 & - & - & - & c & " & N1000 & E1004 & 3 & 99.6-99.5 & $30-40$ & - & Faunal Remains & Bone & Indeterminate & Bone & 1 & 0.61 & & $\mid$\begin{tabular}{|l|}
$\mid 12 / 13 / 2017$ \\
\end{tabular} \\
\hline 472 & 44 & 520 & - & - & - & c & II & N1000 & E1004 & 3 & $99.6-99.5$ & $30-40$ & - & Faunal Remains & Bone & Mammal & Bone & 1 & 1.15 & & $\mid$\begin{tabular}{|l|}
$\mid 12 / 13 / 2017$ \\
\end{tabular} \\
\hline 472 & 45 & 520 & - & - & - & $\mathrm{c}$ & "I & N1000 & E1004 & 3 & $99.6-99.5$ & $30-40$ & - & Faunal Remains & Bone & Mammal & Bone & 1 & 0.43 & & $\mid 12 / 13 / 2017$ \\
\hline 472 & 46 & 520 & - & - & - & c & ॥ & N1000 & E1004 & 3 & $99.6-99.5$ & $30-40$ & - & Faunal Remains & Bone & Mammal & Bone & 1 & 0.98 & & \begin{tabular}{|l|}
$12 / 13 / 2017$ \\
\end{tabular} \\
\hline 472 & 47 & 520 & - & - & - & $\mathrm{c}$ & "I & N1000 & E1004 & 3 & $99.6-99.5$ & $30-40$ & - & Faunal Remains & Bone & Mammal & Bone & 1 & 1.07 & & \begin{tabular}{|l|}
$12 / 13 / 2017$ \\
\end{tabular} \\
\hline 472 & 48 & 520 & - & - & - & c & 11 & N1000 & E1004 & 3 & $99.6-99.5$ & $30-40$ & - & Faunal Remains & Bone & Mammal & Bone & 1 & 0.97 & & 12/13/2017 \\
\hline 472 & 49 & 520 & - & - & - & $\mathrm{c}$ & "I & N1000 & E1004 & 3 & 99.6-99.5 & $30-40$ & - & Faunal Remains & Bone & Indeterminate & Bone & 1 & 0.19 & & $\mid 12 / 13 / 2017$ \\
\hline 472 & 50 & 520 & - & - & - & c & 11 & N1000 & E1004 & 3 & $99.6-99.5$ & $30-40$ & - & Faunal Remains & Bone & Indeterminate & Bone & 1 & 0.81 & & \begin{tabular}{|l|}
$12 / 13 / 2017$ \\
\end{tabular} \\
\hline 472 & 51 & 520 & - & - & - & c & "I & N1000 & E1004 & 3 & $99.6-99.5$ & $30-40$ & - & Faunal Remains & Bone & Indeterminate & Bone & 1 & 0.6 & & \begin{tabular}{|l|}
$12 / 13 / 2017$ \\
\end{tabular} \\
\hline 472 & 53 & 520 & - & - & - & c & "I & N1000 & E1004 & 3 & $99.6-99.5$ & $30-40$ & - & Faunal Remains & Bone & Indeterminate & Bone & 1 & 0.61 & & 12/13/2017 \\
\hline 472 & 54 & 520 & - & - & - & c & " & N1000 & E1004 & 3 & $99.6-99.5$ & $30-40$ & - & Faunal Remains & Bone & Indeterminate & Bone & 1 & 0.23 & & 12/13/2017 \\
\hline 472 & 55 & 520 & - & - & - & c & "I & N1000 & E1004 & 3 & $99.6-99.5$ & $30-40$ & - & Faunal Remains & Bone & Indeterminate & Bone & 1 & 0.47 & & 12/13/2017 \\
\hline 472 & 56 & 520 & - & - & - & c & "I & N1000 & E1004 & 3 & $99.6-99.5$ & $30-40$ & - & Faunal Remains & Bone & Indeterminate & Bone & 1 & 0.56 & & 12/13/2017 \\
\hline 472 & 57 & 520 & - & - & - & c & "I & N1000 & E1004 & 3 & $99.6-99.5$ & $30-40$ & - & Faunal Remains & Bone & Indeterminate & Bone & 1 & 0.31 & & 12/13/2017 \\
\hline 472 & 58 & 520 & - & - & - & c & " & N1000 & E1004 & 3 & $99.6-99.5$ & $30-40$ & - & Faunal Remains & Bone & Indeterminate & Bone & 1 & 0.35 & & 12/13/2017 \\
\hline 472 & 59 & 520 & - & - & - & c & "I & N1000 & E1004 & 3 & $99.6-99.5$ & $30-40$ & - & Faunal Remains & Bone & Indeterminate & Bone & 1 & 0.77 & & 12/13/2017 \\
\hline 472 & 60 & 520 & - & - & - & c & "I & N1000 & E1004 & 3 & $99.6-99.5$ & $30-40$ & - & Faunal Remains & Bone & Indeterminate & Bone & 1 & 0.12 & & $\mid$\begin{tabular}{|l|}
$\mid 12 / 13 / 2017$ \\
\end{tabular} \\
\hline 472 & 61 & 520 & - & - & - & c & 11 & N1000 & E1004 & 3 & $99.6-99.5$ & $30-40$ & - & Faunal Remains & Bone & Indeterminate & Bone & 1 & 0.32 & & \begin{tabular}{|l|}
$12 / 13 / 2017$ \\
\end{tabular} \\
\hline 472 & 62 & 520 & - & - & - & c & "I & N1000 & E1004 & 3 & $99.6-99.5$ & $30-40$ & - & Faunal Remains & Bone & Indeterminate & Bone & 1 & 0.87 & & 12/13/2017 \\
\hline 472 & 63 & 520 & - & - & - & c & "I & N1000 & E1004 & 3 & $99.6-99.5$ & $30-40$ & - & Faunal Remains & Bone & Indeterminate & Bone & 1 & 0.6 & & 12/13/2017 \\
\hline 472 & 64 & 520 & - & - & - & c & "I & N1000 & E1004 & 3 & $99.6-99.5$ & $30-40$ & - & Faunal Remains & Bone & Indeterminate & Bone & 1 & 0.12 & & 12/13/2017 \\
\hline 472 & 65 & 520 & - & - & - & c & $\|$ & N1000 & E1004 & 3 & $99.6-99.5$ & $30-40$ & - & Faunal Remains & Bone & Indeterminate & Bone & 1 & 0.16 & & 12/13/2017 \\
\hline 472 & 66 & 520 & - & - & - & c & "I & N1000 & E1004 & 3 & $99.6-99.5$ & $30-40$ & - & Faunal Remains & Bone & Indeterminate & Bone & 1 & 0.15 & & 12/13/2017 \\
\hline 472 & 67 & 520 & - & - & - & c & " & N1000 & E1004 & 3 & 99.6-99.5 & $30-40$ & - & Faunal Remains & Bone & Indeterminate & Bone & 1 & 0.29 & & $\mid$\begin{tabular}{|l|}
$12 / 13 / 2017$ \\
\end{tabular} \\
\hline 472 & 68 & 520 & - & - & - & c & II & N1000 & E1004 & 3 & $99.6-99.5$ & $30-40$ & - & Faunal Remains & Bone & Mammal & Bone & 1 & 0.61 & & 12/13/2017 \\
\hline 472 & 69 & 520 & - & - & - & c & "I & N1000 & E1004 & 3 & $99.6-99.5$ & $30-40$ & - & Faunal Remains & Bone & Indeterminate & Bone & 1 & 0.18 & & 12/13/2017 \\
\hline 472 & 70 & 520 & - & - & - & c & "1 & N1000 & E1004 & 3 & $99.6-99.5$ & $30-40$ & - & Faunal Remains & Bone & Indeterminate & Bone & 1 & 0.18 & & \begin{tabular}{|l|}
$12 / 13 / 2017$ \\
\end{tabular} \\
\hline 472 & 71 & 520 & - & - & - & $\mathrm{c}$ & "I & N1000 & E1004 & 3 & $99.6-99.5$ & $30-40$ & - & Faunal Remains & Bone & Indeterminate & Bone & 1 & 0.16 & & 12/13/2017 \\
\hline 472 & 72 & 520 & - & - & - & $\mathrm{c}$ & "I & N1000 & E1004 & 3 & $99.6-99.5$ & $30-40$ & - & Faunal Remains & Bone & Indeterminate & Bone & 1 & 0.23 & & 12/13/2017 \\
\hline 472 & 73 & 520 & - & - & - & $\mathrm{c}$ & "I & N1000 & E1004 & 3 & $99.6-99.5$ & $30-40$ & - & Faunal Remains & Bone & Indeterminate & Bone & 1 & 0.29 & & $\mid 12 / 13 / 2017$ \\
\hline 472 & 74 & 520 & - & - & - & $\mathrm{c}$ & 11 & N1000 & E1004 & 3 & $99.6-99.5$ & $30-40$ & - & Faunal Remains & Bone & Indeterminate & Bone & 1 & 0.25 & & \begin{tabular}{|l|}
$12 / 13 / 2017$ \\
\end{tabular} \\
\hline 472 & 75 & 520 & - & - & - & $\mathrm{c}$ & "I & N1000 & E1004 & 3 & $99.6-99.5$ & $30-40$ & - & Faunal Remains & Bone & Indeterminate & Bone & 1 & 0.16 & & 12/13/2017 \\
\hline 472 & 76 & 520 & - & - & - & c & "I & N1000 & E1004 & 3 & $99.6-99.5$ & $30-40$ & - & Faunal Remains & Bone & Indeterminate & Bone & 1 & 0.24 & & 12/13/2017 \\
\hline 472 & 77 & 520 & - & - & - & c & 11 & N1000 & E1004 & 3 & $99.6-99.5$ & $30-40$ & - & Faunal Remains & Bone & Mammal & Bone & 1 & 0.44 & & \begin{tabular}{|l|}
$12 / 13 / 2017$ \\
\end{tabular} \\
\hline 472 & 78 & 520 & - & - & - & c & "I & N1000 & E1004 & 3 & $99.6-99.5$ & $30-40$ & - & Faunal Remains & Bone & Mammal & Bone & 1 & 0.53 & & 12/13/2017 \\
\hline 472 & 79 & 520 & - & - & - & $\mathrm{c}$ & $\|$ & N1000 & E1004 & 3 & $99.6-99.5$ & $30-40$ & - & Faunal Remains & Bone & Indeterminate & Bone & 1 & 0.36 & & 12/13/2017 \\
\hline 472 & 80 & 520 & - & - & - & c & ॥ & N1000 & E1004 & 3 & $99.6-99.5$ & $30-40$ & - & Faunal Remains & Bone & Indeterminate & Bone & 1 & 0.11 & & \begin{tabular}{|l|}
$12 / 13 / 2017$ \\
\end{tabular} \\
\hline
\end{tabular}




\begin{tabular}{|c|c|c|c|c|c|c|c|c|c|c|c|c|c|c|c|c|c|c|c|c|c|}
\hline Lot No. & \begin{tabular}{|c|}
$\begin{array}{c}\text { Specimen } \\
\text { No. }\end{array}$ \\
\end{tabular} & FS No. & UI No. & FTR No. & $\begin{array}{c}\text { Sample } \\
\text { No. }\end{array}$ & $\begin{array}{c}\text { Excavation } \\
\text { Block }\end{array}$ & Stratum (AU) & Northing & Easting & Level & $\begin{array}{c}\text { Elevation } \\
(\mathrm{m})\end{array}$ & Depth (cmbd) & \begin{tabular}{|l|} 
Point Prov. \\
\end{tabular} & Artifact Class & \begin{tabular}{|l|} 
Artifact Type \\
\end{tabular} & $\begin{array}{c}\text { Artifact } \\
\text { Description }\end{array}$ & Material & $\begin{array}{c}\text { Number of } \\
\text { artifacts }\end{array}$ & Weight (g) & Comments & Date \\
\hline 472 & \begin{tabular}{|l|}
81 \\
\end{tabular} & 520 & - & - & - & c & " & N1000 & E1004 & 3 & $99.6-99.5$ & $30-40$ & - & Faunal Remains & Bone & Indeterminate & Bone & 1 & 0.23 & & 12/13/2017 \\
\hline 472 & 82 & 520 & - & - & - & c & " & N1000 & E1004 & 3 & 99.6-99.5 & $30-40$ & - & Faunal Remains & Bone & Indeterminate & Bone & 1 & 0.27 & & 12/13/2017 \\
\hline 472 & 83 & 520 & - & - & - & c & "I & N1000 & E1004 & 3 & $99.6-99.5$ & $30-40$ & - & Faunal Remains & Bone & Mammal & Bone & 1 & 0.24 & & 12/13/2017 \\
\hline 472 & 84 & 520 & - & - & - & c & "I & N1000 & E1004 & 3 & 99.6-99.5 & $30-40$ & - & Faunal Remains & Bone & Mammal & Bone & 1 & 0.73 & & 12/13/2017 \\
\hline 472 & 85 & 520 & - & - & - & c & " & N1000 & E1004 & 3 & 99.6-99.5 & $30-40$ & - & Faunal Remains & Bone & Mammal & Bone & 1 & 0.46 & & 12/13/2017 \\
\hline 472 & 86 & 520 & - & - & - & c & "I & N1000 & E1004 & 3 & $99.6-99.5$ & $30-40$ & - & Faunal Remains & Bone & Indeterminate & Bone & 1 & 0.55 & & 12/13/2017 \\
\hline 472 & 87 & 520 & - & - & - & c & "I & N1000 & E1004 & 3 & 99.6-99.5 & $30-40$ & - & Faunal Remains & Bone & Indeterminate & Bone & 1 & 0.12 & & 12/13/2017 \\
\hline 472 & 88 & 520 & - & - & - & c & " & N1000 & E1004 & 3 & 99.6-99.5 & $30-40$ & - & Faunal Remains & Bone & Mammal & Bone & 1 & 0.2 & & 12/13/2017 \\
\hline 472 & 89 & 520 & - & - & - & c & II & N1000 & E1004 & 3 & $99.6-99.5$ & $30-40$ & - & Faunal Remains & Bone & Indeterminate & Bone & 1 & 0.12 & & 12/13/2017 \\
\hline 472 & 90 & 520 & - & - & - & $\mathrm{c}$ & "I & N1000 & E1004 & 3 & $99.6-99.5$ & $30-40$ & - & Faunal Remains & Bone & Indeterminate & Bone & 1 & 0.17 & & 12/13/2017 \\
\hline 472 & 91 & 520 & - & - & - & c & 11 & N1000 & E1004 & 3 & 99.6-99.5 & $30-40$ & - & Faunal Remains & Bone & Indeterminate & Bone & 1 & 0.12 & & 12/13/2017 \\
\hline 472 & 92 & 520 & - & - & - & $\mathrm{c}$ & "I & N1000 & E1004 & 3 & $99.6-99.5$ & $30-40$ & - & Faunal Remains & Bone & Indeterminate & Bone & 1 & 0.57 & & 12/13/2017 \\
\hline 472 & 93 & 520 & - & - & - & c & "1 & N1000 & E1004 & 3 & $99.6-99.5$ & $30-40$ & - & Faunal Remains & Bone & Indeterminate & Bone & 1 & 0.14 & & 12/13/2017 \\
\hline 472 & 94 & 520 & - & - & - & $\mathrm{c}$ & "I & N1000 & E1004 & 3 & 99.6-99.5 & $30-40$ & - & Faunal Remains & Bone & Indeterminate & Bone & 1 & 0.51 & & 12/13/2017 \\
\hline 472 & 95 & 520 & - & - & - & c & ॥ & N1000 & E1004 & 3 & $99.6-99.5$ & $30-40$ & - & Faunal Remains & Bone & Mammal & Bone & 1 & 0.78 & & 12/13/2017 \\
\hline 472 & 96 & 520 & - & - & - & c & "I & N1000 & E1004 & 3 & $99.6-99.5$ & $30-40$ & - & Faunal Remains & Bone & Indeterminate & Bone & 1 & 0.09 & & 12/13/2017 \\
\hline 472 & 97 & 520 & - & - & - & c & "I & N1000 & E1004 & 3 & $99.6-99.5$ & $30-40$ & - & Faunal Remains & Bone & Indeterminate & Bone & 1 & 0.51 & & 12/13/2017 \\
\hline 472 & 98 & 520 & - & - & - & c & " & N1000 & E1004 & 3 & $99.6-99.5$ & $30-40$ & - & Faunal Remains & Bone & Indeterminate & Bone & 1 & 0.16 & & 12/13/2017 \\
\hline 472 & 99 & 520 & - & - & - & c & "I & N1000 & E1004 & 3 & $99.6-99.5$ & $30-40$ & - & Faunal Remains & Bone & Indeterminate & Bone & 1 & 0.09 & & 12/13/2017 \\
\hline 472 & 100 & 520 & - & - & - & c & "I & N1000 & E1004 & 3 & $99.6-99.5$ & $30-40$ & - & Faunal Remains & Bone & Indeterminate & Bone & 1 & 0.07 & & 12/13/2017 \\
\hline 472 & 101 & 520 & - & - & - & c & "I & N1000 & E1004 & 3 & $99.6-99.5$ & $30-40$ & - & Faunal Remains & Bone & Indeterminate & Bone & 1 & 0.23 & & 12/13/2017 \\
\hline 472 & 102 & 520 & - & - & - & c & "I & N1000 & E1004 & 3 & $99.6-99.5$ & $30-40$ & - & Faunal Remains & Bone & Indeterminate & Bone & 1 & 0.18 & & 12/13/2017 \\
\hline 472 & 103 & 520 & - & - & - & c & "I & N1000 & E1004 & 3 & $99.6-99.5$ & $30-40$ & - & Faunal Remains & Bone & Indeterminate & Bone & 1 & 0.23 & & 12/13/2017 \\
\hline 472 & 104 & 520 & - & - & - & c & "I & N1000 & E1004 & 3 & $99.6-99.5$ & $30-40$ & - & Faunal Remains & Bone & Indeterminate & Bone & 1 & 0.34 & & 12/13/2017 \\
\hline 472 & 105 & 520 & - & - & - & c & "I & N1000 & E1004 & 3 & $99.6-99.5$ & $30-40$ & - & Faunal Remains & Bone & Indeterminate & Bone & 1 & 0.51 & & 12/13/2017 \\
\hline 472 & 106 & 520 & - & - & - & c & "I & N1000 & E1004 & 3 & $99.6-99.5$ & $30-40$ & - & Faunal Remains & Bone & Indeterminate & Bone & 1 & 0.11 & & 12/13/2017 \\
\hline 472 & 107 & 520 & - & - & - & c & "I & N1000 & E1004 & 3 & $99.6-99.5$ & $30-40$ & - & Faunal Remains & Bone & Indeterminate & Bone & 1 & 0.06 & & 12/13/2017 \\
\hline 472 & 108 & 520 & - & - & - & c & "I & N1000 & E1004 & 3 & $99.6-99.5$ & $30-40$ & - & Faunal Remains & Bone & Indeterminate & Bone & 1 & 0.11 & & 12/13/2017 \\
\hline 472 & 109 & 520 & - & - & - & c & $\|$ & N1000 & E1004 & 3 & $99.6-99.5$ & $30-40$ & - & Faunal Remains & Bone & Indeterminate & Bone & 1 & 0.07 & & 12/13/2017 \\
\hline 472 & 110 & 520 & - & - & - & c & "I & N1000 & E1004 & 3 & $99.6-99.5$ & $30-40$ & - & Faunal Remains & Bone & Indeterminate & Bone & 1 & 0.17 & & 12/13/2017 \\
\hline 472 & 111 & 520 & - & - & - & c & " & N1000 & E1004 & 3 & 99.6-99.5 & $30-40$ & - & Faunal Remains & Bone & Indeterminate & Bone & 1 & 0.07 & & 12/13/2017 \\
\hline 472 & 112 & 520 & - & - & - & c & II & N1000 & E1004 & 3 & $99.6-99.5$ & $30-40$ & - & Faunal Remains & Bone & Indeterminate & Bone & 1 & 0.12 & & 12/13/2017 \\
\hline 472 & 113 & 520 & - & - & - & c & "I & N1000 & E1004 & 3 & $99.6-99.5$ & $30-40$ & - & Faunal Remains & Bone & Indeterminate & Bone & 1 & 0.16 & & 12/13/2017 \\
\hline 472 & 114 & 520 & - & - & - & c & "1 & N1000 & E1004 & 3 & 99.6-99.5 & $30-40$ & - & Faunal Remains & Bone & Indeterminate & Bone & 1 & 0.09 & & 12/13/2017 \\
\hline 472 & 115 & 520 & - & - & - & $\mathrm{c}$ & "I & N1000 & E1004 & 3 & $99.6-99.5$ & $30-40$ & - & Faunal Remains & Bone & Indeterminate & Bone & 1 & 0.03 & & 12/13/2017 \\
\hline 472 & 116 & 520 & - & - & - & c & " & N1000 & E1004 & 3 & $99.6-99.5$ & $30-40$ & - & Faunal Remains & Bone & Indeterminate & Bone & 1 & 0.10 & & 12/13/2017 \\
\hline 472 & 117 & 520 & - & - & - & $\mathrm{c}$ & "I & N1000 & E1004 & 3 & $99.6-99.5$ & $30-40$ & - & Faunal Remains & Bone & Indeterminate & Bone & 1 & 0.06 & & 12/13/2017 \\
\hline 472 & 118 & 520 & - & - & - & c & 11 & N1000 & E1004 & 3 & 99.6-99.5 & $30-40$ & - & Faunal Remains & Bone & Indeterminate & Bone & 1 & 0.07 & & 12/13/2017 \\
\hline 472 & 119 & 520 & - & - & - & $\mathrm{c}$ & "I & N1000 & E1004 & 3 & $99.6-99.5$ & $30-40$ & - & Detritus & Debitage & \begin{tabular}{|l|} 
Complete Flake \\
\end{tabular} & Lithic & 3 & 22.76 & & 12/13/2017 \\
\hline 472 & 120 & 520 & - & - & - & c & "I & N1000 & E1004 & 3 & $99.6-99.5$ & $30-40$ & - & Detritus & Debitage & \begin{tabular}{|l|} 
Proximal Flake \\
\end{tabular} & Lithic & 1 & 0.51 & & 12/13/2017 \\
\hline 472 & 121 & 520 & - & - & - & c & "I & N1000 & E1004 & 3 & 99.6-99.5 & $30-40$ & - & Detritus & Debitage & Broken Flake & Lithic & 10 & 6.31 & & 12/13/2017 \\
\hline 472 & 122 & 520 & - & - & - & c & $\|$ & N1000 & E1004 & 3 & $99.6-99.5$ & $30-40$ & - & Detritus & Debitage & Thermal Shatter & Lithic & 11 & 19.17 & & 12/13/2017 \\
\hline 473 & 1 & 521 & - & - & - & $\mathrm{c}$ & $\|$ & N1000 & E1004 & 3 & 99.57 & 33 & \begin{tabular}{|l|} 
N1000.17 \\
E1004.07
\end{tabular} & Faunal Remains & Bone & \begin{tabular}{l|} 
Mammal \\
\end{tabular} & Bone & 1 & 12.47 & Bone \#1 & 12/13/2017 \\
\hline 474 & 1 & 522 & - & - & - & c & "I & N1000 & E1004 & 3 & 99.53 & 37 & \begin{tabular}{l|}
$\begin{array}{l}N 11000.94 \\
\text { E1000.42 }\end{array}$ \\
\end{tabular} & Faunal Remains & Bone & Mammal & Bone & 1 & 36.4 & Bone \#2 & 12/13/2017 \\
\hline
\end{tabular}




\begin{tabular}{|c|c|c|c|c|c|c|c|c|c|c|c|c|c|c|c|c|c|c|c|c|c|}
\hline Lot No. & $\begin{array}{c}\text { Specimen } \\
\text { No. } \\
\text { No. }\end{array}$ & FS No. & UI No. & FTR No. & $\begin{array}{l}\text { Sample } \\
\text { No. }\end{array}$ & $\begin{array}{c}\text { Excavation } \\
\text { Block }\end{array}$ & Stratum (AU) & Northing & Easting & Level & $\begin{array}{c}\text { Elevation } \\
(\mathrm{m})\end{array}$ & Depth (cmbd) & Point Prov. & Artifact Class & \begin{tabular}{|l|} 
Artifiact Type \\
\end{tabular} & \begin{tabular}{|c|c} 
Artifact \\
Description
\end{tabular} & Material & $\begin{array}{c}\text { Number of } \\
\text { artifacts }\end{array}$ & Weight (g) & Comments & Date \\
\hline 474 & 2 & 522 & - & - & - & c & $" 1$ & N1000 & E1004 & 3 & 99.53 & 37 & \begin{tabular}{|l|}
$\mathrm{N} 1000.94$ \\
$\mathrm{E} 1004.42$
\end{tabular} & Faunal Remains & Bone & Mammal & Bone & 1 & 10.39 & Bone \#2 & 12/13/2017 \\
\hline 474 & 3 & 522 & - & - & - & c & "I & N1000 & E1004 & 3 & 99.53 & 37 & $\begin{array}{l}\mathrm{N} 110000.94 \\
\mathrm{E} 1004.42\end{array}$ & Faunal Remains & Bone & Mammal & Bone & 1 & 5.55 & Bone \#2 & 12/13/2017 \\
\hline 474 & 4 & 522 & - & - & - & $\mathrm{c}$ & $\|$ & N1000 & E1004 & 3 & 99.53 & 37 & \begin{tabular}{|l|} 
N1000.94 \\
E11004.42
\end{tabular} & Faunal Remains & Bone & Indeterminate & Bone & 1 & 0.13 & Bone \#2 & $12 / 13 / 2017$ \\
\hline 474 & 5 & 522 & - & - & - & c & "I & N1000 & E1004 & 3 & 99.53 & 37 & $\begin{array}{l}\mathrm{N} 10000.94 \\
\mathrm{E} 1004.42\end{array}$ & Faunal Remains & Bone & Indeterminate & Bone & 1 & 0.35 & Bone \#2 & 12/13/2017 \\
\hline 474 & 6 & 522 & - & - & - & $\mathrm{c}$ & $\|$ & N1000 & E1004 & 3 & 99.53 & 37 & \begin{tabular}{|l|} 
N1000.94 \\
E11004.42
\end{tabular} & Faunal Remains & Bone & Indeterminate & Bone & 1 & 0.18 & Bone \#2 & 12/13/2017 \\
\hline 474 & 7 & 522 & - & - & - & c & "I & N1000 & E1004 & 3 & 99.53 & 37 & $\begin{array}{l}\mathrm{N} 110000.94 \\
\mathrm{E} 1004.42\end{array}$ & Faunal Remains & Bone & Indeterminate & Bone & 1 & 0.01 & Bone \#2 & 12/13/2017 \\
\hline 474 & 8 & 522 & - & - & - & $\mathrm{c}$ & $\|$ & N1000 & E1004 & 3 & 99.53 & 37 & \begin{tabular}{|l|} 
N1000.94 \\
E11004.42
\end{tabular} & Faunal Remains & Bone & Indeterminate & Bone & 1 & 0.13 & Bone \#2 & $12 / 13 / 2017$ \\
\hline 474 & 9 & 522 & - & - & - & c & $\|$ & N1000 & E1004 & 3 & 99.53 & 37 & $\begin{array}{l}\text { N1000.94 } \\
\text { E1004.42 }\end{array}$ & Faunal Remains & Bone & Indeterminate & Bone & 1 & 0.01 & Bone \#2 & 12/13/2017 \\
\hline 474 & 10 & 522 & - & - & - & $\mathrm{c}$ & "I & N1000 & E1004 & 3 & 99.53 & 37 & $\begin{array}{l}\text { N1000.94 } \\
\text { E1004.42 }\end{array}$ & Faunal Remains & Bone & Indeterminate & Bone & 1 & 1.82 & Bone \#2 & 12/13/2017 \\
\hline 474 & 11 & 522 & - & - & - & c & "I & N1000 & E1004 & 3 & 99.53 & 37 & \begin{tabular}{|l|l|}
$N 1000.94$ \\
E1004.42
\end{tabular} & Faunal Remains & Bone & Indeterminate & Bone & 1 & 1.23 & Bone \#2 & $12 / 13 / 2017$ \\
\hline 474 & 12 & 522 & - & - & - & $\mathrm{c}$ & $\|$ & N1000 & E1004 & 3 & 99.53 & 37 & $\begin{array}{l}\text { N1000.94 } \\
\text { E100442 }\end{array}$ & \begin{tabular}{|l|} 
Faunal Remains \\
\end{tabular} & Bone & Indeterminate & Bone & 1 & 0.01 & Bone \#2 & 12/13/2017 \\
\hline 474 & 13 & 522 & - & - & - & $\mathrm{c}$ & "I & N1000 & E1004 & 3 & 99.53 & 37 & $\begin{array}{l}\mathrm{N} 11000.94 \\
\mathrm{E} 1004.42\end{array}$ & Faunal Remains & Bone & Indeterminate & Bone & 1 & 1.6 & Bone \#2 & 12/13/2017 \\
\hline 474 & 14 & 522 & - & - & - & $\mathrm{c}$ & 11 & N1000 & E1004 & 3 & 99.53 & 37 & \begin{tabular}{|l|}
$\mathrm{N} 1000.94$ \\
$\mathrm{E} 1004.42$ \\
\end{tabular} & Faunal Remains & Bone & Indeterminate & Bone & 1 & 0.25 & Bone \#2 & 12/13/2017 \\
\hline 474 & 15 & 522 & - & - & - & c & "I & N1000 & E1004 & 3 & 99.53 & 37 & \begin{tabular}{|l|l|}
$N 1000.94$ \\
E1004.42
\end{tabular} & Faunal Remains & Bone & Indeterminate & Bone & 1 & 0.42 & Bone \#2 & $12 / 13 / 2017$ \\
\hline 474 & 16 & 522 & - & - & - & $\mathrm{c}$ & $\|$ & N1000 & E1004 & 3 & 99.53 & 37 & $\begin{array}{l}\text { N1000.94 } \\
\text { E100442 }\end{array}$ & \begin{tabular}{|l|} 
Faunal Remains \\
\end{tabular} & Bone & Indeterminate & Bone & 1 & 1.04 & Bone \#2 & 12/13/2017 \\
\hline 474 & 17 & 522 & - & - & - & c & 11 & N1000 & E1004 & 3 & 99.53 & 37 & $\begin{array}{l}\text { N1000.94 } \\
\text { E1004.42 }\end{array}$ & Faunal Remains & Bone & Indeterminate & Bone & 1 & 0.01 & Bone \#2 & 12/13/2017 \\
\hline 475 & 1 & 534 & - & - & - & c & 11/III & N1000 & E1004 & 4 & $99.5-99.4$ & $40-50$ & - & Faunal Remains & Bone & Indeterminate & Bone & 1 & 0.56 & & 12/14/2017 \\
\hline 475 & 2 & 534 & - & - & - & $\mathrm{c}$ & "1/III & N1000 & E1004 & 4 & 99.5-99.4 & $40-50$ & - & \begin{tabular}{|l|} 
Faunal Remains \\
\end{tabular} & Bone & Indeterminate & Bone & 1 & 0.2 & & 12/14/2017 \\
\hline 475 & 3 & 534 & - & - & - & $\mathrm{c}$ & II/III & N1000 & E1004 & 4 & $99.5-99.4$ & $40-50$ & - & Faunal Remains & Bone & Indeterminate & Bone & 1 & 0.3 & & 12/14/2017 \\
\hline 475 & 4 & 534 & - & - & - & $\mathrm{c}$ & 11/III & N1000 & E1004 & 4 & $99.5-99.4$ & $40-50$ & - & Detritus & Debitage & \begin{tabular}{|c|} 
Complete Flake \\
\end{tabular} & Lithic & 3 & 9.61 & & $12 / 14 / 2017$ \\
\hline 475 & 5 & 534 & - & - & - & $\mathrm{c}$ & $11 / 111$ & N1000 & E1004 & 4 & $99.5-99.4$ & $40-50$ & - & Detritus & Debitage & \begin{tabular}{|l|} 
Proximal Flake \\
\end{tabular} & Lithic & 1 & 0.35 & & $12 / 14 / 2017$ \\
\hline 475 & 6 & 534 & - & - & - & $\mathrm{c}$ & 11/III & N1000 & E1004 & 4 & $99.5-99.4$ & $40-50$ & - & Detritus & Debitage & Broken Flake & Lithic & 13 & 8.98 & & 12/14/2017 \\
\hline 475 & 7 & 534 & - & - & - & $\mathrm{c}$ & $11 / 111$ & N1000 & E1004 & 4 & $99.5-99.4$ & $40-50$ & - & Detritus & Debitage & Thermal Shatter & Lithic & 13 & 7.61 & & 12/14/2017 \\
\hline 475 & 8 & 534 & - & - & - & $\mathrm{c}$ & 11/III & N1000 & E1004 & 4 & $99.5-99.4$ & $40-50$ & - & Formal Tool & Biface & \begin{tabular}{|c|} 
Indeterminate \\
Fragment
\end{tabular} & Fine Grain Chert & 1 & 0.86 & & 12/14/2017 \\
\hline 476 & 1 & 538 & - & - & - & c & III & N1000 & E1004 & 5 & 99.4-99.3 & $50-60$ & - & Faunal Remains & Bone & Indeterminate & Bone & 1 & 0.51 & & 12/14/2017 \\
\hline 476 & 2 & 538 & - & - & - & $\mathrm{c}$ & IIII & N1000 & E1004 & 5 & $\begin{array}{l}99.4-99.3 \\
\end{array}$ & 50-60 & - & Detritus & Debitage & Complete Flake & Lithic & 6 & 108.43 & & 12/14/2017 \\
\hline 476 & 3 & 538 & - & - & - & c & IIII & N1000 & E1004 & 5 & 99.499 .3 & $50-60$ & - & Detritus & Debitage & \begin{tabular}{|l|} 
Proximal Flake \\
\end{tabular} & Lithic & 1 & 1.2 & & 12/14/2017 \\
\hline 476 & 4 & 538 & - & - & - & $\mathrm{c}$ & IIII & N1000 & E1004 & 5 & 99.499 .3 & 50-60 & - & Detritus & Debitage & Broken Flake & Lithic & 16 & 19.07 & & 12/14/2017 \\
\hline 476 & 5 & 538 & - & - & - & $\mathrm{c}$ & III & N1000 & E1004 & 5 & $\begin{array}{l}99.4-99.3 \\
\end{array}$ & $50-60$ & - & Detritus & Debitage & Thermal Shatter & Lithic & 17 & 43.28 & & 12/14/2017 \\
\hline 477 & 1 & 669 & - & - & - & $\mathrm{c}$ & IIII & N1000 & E1004 & 6 & $99.3-99.2$ & $60-70$ & - & Detritus & Debitage & Complete Flake & Lithic & 1 & 3.24 & & 12/28/2017 \\
\hline 477 & 2 & 669 & - & - & - & c & IIII & N1000 & E1004 & 6 & $99.3-99.2$ & $60-70$ & - & Detritus & Debitage & Proximal Flake & Lithic & 4 & 7 & & $\mid$\begin{tabular}{|l|l|}
$12 / 28 / 2017$ \\
\end{tabular} \\
\hline 477 & 3 & 669 & - & - & - & $\mathrm{c}$ & III & N1000 & E1004 & 6 & $99.3-99.2$ & $60-70$ & - & Detritus & Debitage & Broken Flake & Lithic & 7 & 2.15 & & 12/28/2017 \\
\hline 477 & 4 & 669 & - & - & - & $\mathrm{c}$ & III & N1000 & E1004 & 6 & $99.3-99.2$ & $60-70$ & - & Detritus & Debitage & Thermal Shatter & Lithic & 5 & 2.39 & & 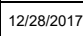 \\
\hline 478 & 1 & 671 & - & - & - & $\mathrm{c}$ & IIII & N1000 & E1004 & 7 & $99.2-99.1$ & $70-80$ & - & \begin{tabular}{|l|} 
Faunal Remains \\
\end{tabular} & Bone & \begin{tabular}{|l|} 
Tortoise//Turtle \\
\end{tabular} & Bone & 1 & 0.32 & & 12/28/2017 \\
\hline 478 & 2 & 671 & - & - & - & $\mathrm{c}$ & IIII & N1000 & E1004 & 7 & 99.2-99.1 & $70-80$ & - & Detritus & Debitage & \begin{tabular}{|l|} 
Complete Flake \\
\end{tabular} & Lithic & 7 & 32.07 & & 12/28/2017 \\
\hline 478 & 3 & 671 & - & - & - & $\mathrm{c}$ & IIII & N1000 & E1004 & 7 & $99.2-99.1$ & $70-80$ & - & Detritus & Debitage & \begin{tabular}{|l|} 
Proximal Flake \\
\end{tabular} & Lithic & 2 & 2.15 & & 12/28/2017 \\
\hline 478 & 4 & 671 & - & - & - & $\mathrm{c}$ & IIII & N1000 & E1004 & 7 & $99.2-99.1$ & $70-80$ & - & Detritus & Debitage & Broken Flake & Lithic & 15 & 5.24 & & 12/28/2017 \\
\hline 478 & 5 & 671 & - & - & - & $\mathrm{c}$ & IIII & N1000 & E1004 & 7 & 99.2-99.1 & $70-80$ & - & Detritus & Debitiage & Thermal Shatter & Lithic & 14 & 10.95 & & $\mid$\begin{tabular}{|l|l|}
$12 / 28 / 2017$ \\
\end{tabular} \\
\hline 479 & 1 & 672 & - & - & - & c & III/V & N1000 & E1004 & 8 & $99.1-99.0$ & $80-90$ & - & Detritus & Debitage & \begin{tabular}{|l|} 
Complete Flake \\
\end{tabular} & Lithic & 3 & 6.44 & & 12/28/2017 \\
\hline 479 & 2 & 672 & - & - & - & $\mathrm{c}$ & III/V & N1000 & E1004 & 8 & $99.1-99.0$ & $80-90$ & - & Detritus & Debitage & \begin{tabular}{|l|} 
Proximal Flake \\
\end{tabular} & Lithic & 5 & 1.93 & & \begin{tabular}{|l|l|}
$12 / 28 / 2017$ \\
\end{tabular} \\
\hline 479 & 3 & 672 & - & - & - & c & III/V & N1000 & E1004 & 8 & $99.1-99.0$ & $80-90$ & - & Detritus & Debitage & Broken Flake & Lithic & 7 & 2.25 & & 12/28/2017 \\
\hline 479 & 4 & 672 & - & - & - & $\mathrm{c}$ & IIIIV & N1000 & E1004 & 8 & $99.1-99.0$ & $80-90$ & - & Detritus & Debitage & Thermal Shatter & Lithic & 10 & 4.07 & & 12/28/2017 \\
\hline 479 & 5 & 672 & - & - & - & c & III/V & N1000 & E1004 & 8 & $99.1-99.0$ & $80-90$ & - & Faunal Remains & Shell & Mussel Shell & Shell & 4 & 3.49 & 1 umbo & \begin{tabular}{|l|l|}
$12 / 28 / 2017$ \\
\end{tabular} \\
\hline
\end{tabular}




\begin{tabular}{|c|c|c|c|c|c|c|c|c|c|c|c|c|c|c|c|c|c|c|c|c|c|}
\hline Lot No. & $\begin{array}{c}\text { Specimen } \\
\text { No. }\end{array}$ & FS No. & UI No. & FTR No. & $\begin{array}{c}\text { Sample } \\
\text { No. }\end{array}$ & $\begin{array}{c}\text { Excavation } \\
\text { Block }\end{array}$ & Stratum (AU) & Northing & Easting & Level & $\begin{array}{c}\text { Elevation } \\
(\mathrm{m})\end{array}$ & Depth (cmbd) & \begin{tabular}{|l} 
Point Prov. \\
\end{tabular} & Artifact Class & \begin{tabular}{|l|} 
Artifiact Type \\
\end{tabular} & $\begin{array}{c}\text { Artifact } \\
\text { Description }\end{array}$ & Material & $\begin{array}{c}\text { Number of } \\
\text { artifacts }\end{array}$ & Weight (g) & Comments & Date \\
\hline 480 & 1 & 674 & - & - & - & c & Iv & N1000 & E1004 & 9 & $99.0-98.9$ & 90-100 & - & Faunal Remains & Bone & Indeterminate & Bone & 1 & 0.12 & & 12/28/2017 \\
\hline 480 & 2 & 674 & - & - & - & c & iv & N1000 & E1004 & 9 & 99.0-98.9 & 90-100 & - & Faunal Remains & Bone & Indeterminate & Bone & 1 & 0.35 & & 12/28/2017 \\
\hline 480 & 3 & 674 & - & - & - & c & IV & N1000 & E1004 & 9 & 99.0-98.9 & $90-100$ & - & Faunal Remains & Bone & Indeterminate & Bone & 1 & 0.16 & & 12/28/2017 \\
\hline 480 & 4 & 674 & - & - & - & c & IV & N1000 & E1004 & 9 & $99.0-98.9$ & 90-100 & - & Faunal Remains & Bone & Indeterminate & Bone & 1 & 0.39 & & 12/28/2017 \\
\hline 480 & 5 & 674 & - & - & - & c & IV & N1000 & E1004 & 9 & $99.0-98.9$ & 90-100 & - & Faunal Remains & Bone & Indeterminate & Bone & 1 & 0.76 & & 12/28/2017 \\
\hline 480 & 6 & 674 & - & - & - & c & IV & N1000 & E1004 & 9 & $99.0-98.9$ & $\begin{array}{l}90-100 \\
\end{array}$ & - & \begin{tabular}{|l|} 
Faunal Remains \\
\end{tabular} & Bone & Indeterminate & Bone & 1 & 0.15 & & 12/28/2017 \\
\hline 480 & 7 & 674 & - & - & - & c & IV & N1000 & E1004 & 9 & 99.0-98.9 & $90-100$ & - & Faunal Remains & Bone & Indeterminate & Bone & 1 & 0.4 & & \begin{tabular}{|l|}
$12 / 28 / 2017$ \\
\end{tabular} \\
\hline 480 & 8 & 674 & - & - & - & c & IV & N1000 & E1004 & 9 & $99.0-98.9$ & 90-100 & - & \begin{tabular}{|l|} 
Faunal Remains \\
\end{tabular} & Bone & Indeterminate & Bone & 1 & 1.09 & & 12/28/2017 \\
\hline 480 & 9 & 674 & - & - & - & $\mathrm{c}$ & IV & N1000 & E1004 & 9 & $99.0-98.9$ & 90-100 & - & \begin{tabular}{|l|} 
Faunal Remains \\
\end{tabular} & Bone & Indeterminate & Bone & 1 & 0.56 & & 12/28/2017 \\
\hline 480 & 10 & 674 & - & - & - & c & IV & N1000 & E1004 & 9 & 99.0-98.9 & $90-100$ & - & Faunal Remains & Bone & Indeterminate & Bone & 1 & 0.6 & & 12/28/2017 \\
\hline 480 & 11 & 674 & - & - & - & $\mathrm{c}$ & IV & N1000 & E1004 & 9 & $99.0-98.9$ & $\begin{array}{l}90-100 \\
\end{array}$ & - & Detritus & Debitage & \begin{tabular}{|c|} 
Complete Flake \\
\end{tabular} & Lithic & 5 & 11.7 & & 12/28/2017 \\
\hline 480 & 12 & 674 & - & - & - & c & IV & N1000 & E1004 & 9 & $99.0-98.9$ & $\begin{array}{l}90-100 \\
\end{array}$ & - & Detritus & Debitage & \begin{tabular}{|l|} 
Proximal Flake \\
\end{tabular} & Lithic & 2 & 1.34 & & 12/28/2017 \\
\hline 480 & 13 & 674 & - & - & - & c & iv & N1000 & E1004 & 9 & $99.0-98.9$ & 90-100 & - & Detritus & Debitage & Broken Flake & Lithic & 14 & 16.28 & & 12/28/2017 \\
\hline 480 & 14 & 674 & - & - & - & c & iv & N1000 & E1004 & 9 & 99.0-98.9 & 90-100 & - & Detritus & Debitage & Thermal Shatter & Lithic & 17 & 3.92 & & 12/28/2017 \\
\hline 481 & 1 & 675 & - & - & - & $\mathrm{c}$ & $\mathrm{v}$ & N1000 & E1004 & 10 & $98.9-98.8$ & 100-110 & - & \begin{tabular}{|l|} 
Faunal Remains \\
\end{tabular} & Bone & Indeterminate & Bone & 1 & 3.61 & & 12/28/2017 \\
\hline 481 & 2 & 675 & - & - & - & c & $\mathrm{v}$ & N1000 & E1004 & 10 & $98.9-98.8$ & $100-110$ & - & Faunal Remains & Bone & Indeterminate & Bone & 1 & 0.66 & & 12/28/2017 \\
\hline 481 & 3 & 675 & - & - & - & c & $\mathrm{v}$ & N1000 & E1004 & 10 & 98.9-98.8 & $100-110$ & - & Faunal Remains & Bone & Indeterminate & Bone & 1 & 0.33 & & 12/28/2017 \\
\hline 481 & 4 & 675 & - & - & - & c & $\mathrm{v}$ & N1000 & E1004 & 10 & $98.9-98.8$ & $100-110$ & - & Faunal Remains & Bone & Indeterminate & Bone & 1 & 0.01 & & 12/28/2017 \\
\hline 481 & 5 & 675 & - & - & - & $\mathrm{c}$ & $\mathrm{v}$ & N1000 & E1004 & 10 & $98.9-98.8$ & 100-110 & - & Detritus & Debitage & \begin{tabular}{|c|} 
Complete Flake \\
\end{tabular} & Lithic & 3 & 2.63 & & 12/28/2017 \\
\hline 481 & 6 & 675 & - & - & - & c & $\mathrm{v}$ & N1000 & E1004 & 10 & 98.9-98.8 & $100-110$ & - & Detritus & Debitage & \begin{tabular}{|l|} 
Proximal Flake \\
\end{tabular} & Lithic & 1 & 0.35 & & 12/28/2017 \\
\hline 481 & 7 & 675 & - & - & - & c & $\mathrm{v}$ & N1000 & E1004 & 10 & 98.9-98.8 & $100-110$ & - & Detritus & Debitage & Broken Flake & Lithic & 4 & 1.9 & & 12/28/2017 \\
\hline 481 & 8 & 675 & - & - & - & c & $\mathrm{v}$ & N1000 & E1004 & 10 & $98.9-98.8$ & $100-110$ & - & Detritus & Debitage & Thermal Shatter & Lithic & 7 & 2.23 & & 12/28/2017 \\
\hline 482 & 1 & 676 & - & - & - & $\mathrm{c}$ & $\mathrm{vv}_{1}$ & N1000 & E1004 & 11 & $98.8-98.7$ & 110-120 & - & Detritus & Debitage & \begin{tabular}{|c|} 
Complete Flake \\
\end{tabular} & Lithic & 1 & 0.12 & & 12/28/2017 \\
\hline 482 & 2 & 676 & - & - & - & c & vi & N1000 & E1004 & 11 & $98.8-98.7$ & 110-120 & - & Detritus & Debitage & Broken Flake & Lithic & 4 & 6.04 & & 12/28/2017 \\
\hline 482 & 3 & 676 & - & - & - & $\mathrm{c}$ & $\mathrm{vv}_{1}$ & N1000 & E1004 & 11 & $98.8-98.7$ & $110-120$ & - & Detritus & Debitage & Thermal Shatter & Lithic & 3 & 5.11 & & 12/28/2017 \\
\hline 483 & 1 & 677 & - & - & - & c & vi & N1000 & E1004 & 12 & $98.7-98.6$ & $120-130$ & - & Detritus & Debitage & Broken Flake & Lithic & 3 & 3.06 & & 12/28/2017 \\
\hline 484 & 1 & 678 & - & - & - & $\mathrm{c}$ & $\mathrm{vv}_{1}$ & N1000 & E1004 & 13 & $98.6-98.5$ & 130-140 & - & Faunal Remains & Bone & Mammal & Bone & 1 & 1.17 & & \begin{tabular}{|l|}
$12 / 28 / 2017$ \\
\end{tabular} \\
\hline 484 & 2 & 678 & - & - & - & c & vi & N1000 & E1004 & 13 & $98.6-98.5$ & $130-140$ & - & Detritus & Debitage & Broken Flake & Lithic & 2 & 4.41 & & 12/28/2017 \\
\hline 485 & 1 & 679 & - & - & - & c & vi & N1000 & E1004 & 14 & $98.5-98.4$ & 140-150 & - & \begin{tabular}{|l|} 
Faunal Remains \\
\end{tabular} & Bone & Mammal & Bone & 1 & 1.94 & & 12/28/2017 \\
\hline 485 & 2 & 679 & - & - & - & c & vi & N1000 & E1004 & 14 & $98.5-98.4$ & $140-150$ & - & Faunal Remains & Bone & Mammal & Bone & 1 & 1.41 & & 12/28/2017 \\
\hline 485 & 3 & 679 & - & - & - & $\mathrm{c}$ & $\mathrm{vv}_{1}$ & N1000 & E1004 & 14 & $98.5-98.4$ & 140-150 & - & \begin{tabular}{|l|} 
Faunal Remains \\
\end{tabular} & Bone & Indeterminate & Bone & 1 & 0.05 & & 12/28/2017 \\
\hline 486 & 1 & 315 & - & - & - & $\mathrm{D}$ & $1 / 111$ & N1000 & E1009 & 1 & \begin{tabular}{|l|}
$99.67-99.59$ \\
\end{tabular} & $2-10$ & - & Detritus & $\begin{array}{l}\text { Debitage } \\
\end{array}$ & $\begin{array}{l}\text { Broken Flake } \\
\end{array}$ & Lithic & 1 & 0.46 & & 11/27/2017 \\
\hline 486 & 2 & 315 & 18 & - & - & D & 1/111 & N1000 & E1009 & 1 & 99.67-99.59 & $2-10$ & - & Formal Tool & Biface & \begin{tabular}{|c|} 
Distal-Medial \\
Fragment
\end{tabular} & Fine Grain Chert & 1 & 6 & & 11/27/2017 \\
\hline 487 & 1 & 317 & - & - & - & $\mathrm{D}$ & $1 / 11$ & N1000 & E1009 & 2 & \begin{tabular}{|l|}
$99.59-99.49$ \\
\end{tabular} & $10-20$ & - & Detritus & Debitage & Complete Flake & Lithic & 1 & 1.01 & & 11/27/2017 \\
\hline 488 & 1 & 319 & - & - & - & $\mathrm{D}$ & 1/111 & N1000 & E1009 & 3 & \begin{tabular}{|l|}
$99.49-99.39$ \\
\end{tabular} & $20-30$ & - & Detritus & Debitage & \begin{tabular}{|c|} 
Complete Flake \\
\end{tabular} & Lithic & 1 & 1.14 & & 11/27/2017 \\
\hline 488 & 2 & 319 & - & - & - & $\mathrm{D}$ & $1 / 11$ & N1000 & E1009 & 3 & \begin{tabular}{|l|}
$99.49-99.39$ \\
\end{tabular} & $20-30$ & - & Detritus & Debitage & Broken Flake & Lithic & 6 & 3.23 & & 11/27/2017 \\
\hline 488 & 3 & 319 & - & - & - & D & 1/11 & N1000 & E1009 & 3 & \begin{tabular}{|l|}
$99.49-99.39$ \\
\end{tabular} & $20-30$ & - & Detritus & Debitage & Thermal Shatter & Lithic & 2 & 1.21 & & 11/27/2017 \\
\hline 488 & 4 & 319 & - & - & - & D & 1/111 & N1000 & E1009 & 3 & \begin{tabular}{|l|l|}
$99.49-99.39$ \\
\end{tabular} & $20-30$ & - & Historic Artifiact & Ceramic & Whiteware & Ceramic & 1 & 2.24 & & 11/27/2017 \\
\hline 489 & 1 & 322 & - & - & - & $\mathrm{D}$ & 1/111 & N1000 & E1009 & 4 & \begin{tabular}{|l|}
$99.39-99.29$ \\
\end{tabular} & $30-40$ & - & Detritus & Debitage & Complete Flake & Lithic & 3 & 7.68 & & 11/27/2018 \\
\hline 489 & 2 & 322 & - & - & - & D & $1 / 11$ & $\mathrm{~N} 1000$ & E1009 & 4 & \begin{tabular}{|l|}
$99.39-99.29$ \\
\end{tabular} & $30-40$ & - & Detritus & Debitage & Proximal Flake & Lithic & 2 & 2.37 & & 11/27/2018 \\
\hline 489 & 3 & 322 & - & - & - & D & $1 / 11$ & N1000 & E1009 & 4 & \begin{tabular}{|l|}
$99.39-99.29$ \\
\end{tabular} & $30-40$ & - & Detritus & Debitage & Broken Flake & Lithic & 13 & 11.14 & & 11/27/2018 \\
\hline 489 & 4 & 322 & - & - & - & $\mathrm{D}$ & 1/111 & N1000 & E1009 & 4 & \begin{tabular}{|l|}
$99.39-99.29$ \\
\end{tabular} & $30-40$ & - & Detritus & Debitage & Thermal Shatter & Lithic & 7 & 7.26 & & 11/27/2018 \\
\hline
\end{tabular}




\begin{tabular}{|c|c|c|c|c|c|c|c|c|c|c|c|c|c|c|c|c|c|c|c|c|c|}
\hline Lot No. & \begin{tabular}{|c|}
$\begin{array}{c}\text { Specimen } \\
\text { No. }\end{array}$ \\
\end{tabular} & FS No. & UI No. & FTR No. & $\begin{array}{c}\text { Sample } \\
\text { No. }\end{array}$ & $\begin{array}{c}\text { Excavation } \\
\text { Block }\end{array}$ & Stratum (AU) & Northing & Easting & Level & $\begin{array}{c}\begin{array}{c}\text { Elevation } \\
(\mathrm{m})\end{array} \\
\end{array}$ & Depth (cmbd) & Point Prov. & Artifiact Class & \begin{tabular}{|l|} 
Artifact Type \\
\end{tabular} & $\begin{array}{c}\text { Artifact } \\
\text { Description }\end{array}$ & Material & $\begin{array}{c}\text { Number of } \\
\text { artifacts }\end{array}$ & Weight (g) & Comments & Date \\
\hline 490 & 1 & 324 & - & - & - & D & $1 / 11$ & N1000 & E1009 & 5 & \begin{tabular}{|l|}
$99.29-99.19$ \\
\end{tabular} & $40-50$ & - & Faunal Remains & Bone & Indeterminate & Bone & 1 & 0.14 & & 11/28/2017 \\
\hline 490 & 2 & 324 & - & - & - & D & $1 / 11$ & N1000 & E1009 & 5 & |99.29-99.19 & $40-50$ & - & Faunal Remains & Bone & Indeterminate & Bone & 1 & 0.17 & & 11/28/2017 \\
\hline 490 & 3 & 324 & - & - & - & D & 1/11 & N1000 & E1009 & 5 & 99.29-99.19 & $40-50$ & - & Detritus & Debitage & Complete Flake & Lithic & 6 & 14.02 & & 11/28/2017 \\
\hline 490 & 4 & 324 & - & - & - & D & 1/11 & N1000 & E1009 & 5 & \begin{tabular}{|l|}
$99.29-99.19$ \\
\end{tabular} & $40-50$ & - & Detritus & Debitage & Proximal Flake & Lithic & 6 & 11.48 & & 11/28/2017 \\
\hline 490 & 5 & 324 & - & - & - & D & $1 / 1 / 1$ & N1000 & E1009 & 5 & 99.29-99.19 & $40-50$ & - & Detritus & Debitage & Broken Flake & Lithic & 35 & 18.96 & & 11/28/2017 \\
\hline 490 & 6 & 324 & - & - & - & D & 1/11 & N1000 & E1009 & 5 & 99.29-99.19 & $40-50$ & - & Detritus & Debitage & Thermal Shatter & Lithic & 26 & 37.44 & & 11/28/2017 \\
\hline 491 & 1 & 329 & - & - & - & D & 1/11 & N1000 & E1009 & 6 & |99.19-99.09 & 50-60 & - & Detritus & Debitage & Complete Flake & Lithic & 6 & 11.77 & & 11/28/2017 \\
\hline 491 & 2 & 329 & - & - & - & D & 1111 & N1000 & E1009 & 6 & 99.19-99.09 & 50-60 & - & Detritus & Debitage & Proximal Flake & Lithic & 8 & 13.07 & & $\mid$\begin{tabular}{|l|}
$\mid 11 / 28 / 2017$ \\
\end{tabular} \\
\hline 491 & 3 & 329 & - & - & - & D & I/II & N1000 & E1009 & 6 & \begin{tabular}{|l|l|}
$99.19-99.09$ \\
\end{tabular} & $50-60$ & - & Detritus & Debitage & Broken Flake & Lithic & 7 & 12.99 & & $\mid$\begin{tabular}{|l|}
$\mid 11 / 28 / 2017$ \\
\end{tabular} \\
\hline 491 & 4 & 329 & - & - & - & $\mathrm{D}$ & 1/11 & N1000 & E1009 & 6 & \begin{tabular}{|l|}
$99.19-99.09$ \\
\end{tabular} & $50-60$ & - & Detritus & Debitage & Thermal Shatter & Lithic & 4 & 5.25 & & 11/28/2017 \\
\hline 492 & 1 & 332 & - & - & - & D & 1/111 & N1000 & E1009 & 7 & \begin{tabular}{|l|l|}
$99.09-98.99$ \\
\end{tabular} & $60-70$ & - & Faunal Remains & Bone & Mammal & Bone & 1 & 1.22 & & 11/28/2017 \\
\hline 492 & 2 & 332 & - & - & - & $\mathrm{D}$ & 1/111 & N1000 & E1009 & 7 & \begin{tabular}{|l|l|}
$99.09-98.99$ \\
\end{tabular} & $60-70$ & - & Detritus & Debitage & Complete Flake & Lithic & 16 & 70.88 & & 11/28/2017 \\
\hline 492 & 3 & 332 & - & - & - & D & 1/11 & N1000 & E1009 & 7 & \begin{tabular}{|l|l|}
$99.09-98.99$ \\
\end{tabular} & $60-70$ & - & Detritus & Debitage & \begin{tabular}{|l|} 
Proximal Flake \\
\end{tabular} & Lithic & 9 & 29.54 & & 11/28/2017 \\
\hline 492 & 4 & 332 & - & - & - & $\mathrm{D}$ & 1/11 & N1000 & E1009 & 7 & |99.09-98.99 & $60-70$ & - & Detritus & Debitage & Broken Flake & Lithic & 40 & 33.78 & & $\mid$\begin{tabular}{|l|}
$\mid 11 / 28 / 2017$ \\
\end{tabular} \\
\hline 492 & 5 & 332 & - & - & - & D & 1/11 & N1000 & E1009 & 7 & \begin{tabular}{|l|l|}
$99.09-98.99$ \\
\end{tabular} & $60-70$ & - & Detritus & Debitage & Thermal Shatter & Lithic & 31 & 21.89 & & 11/28/2017 \\
\hline 492 & 6 & 332 & - & - & - & D & 1/11 & N1000 & E1009 & 7 & \begin{tabular}{|l|l|}
$99.09-98.99$ \\
\end{tabular} & $60-70$ & - & \begin{tabular}{c|} 
Groundl/ \\
Battered Stone
\end{tabular} & \begin{tabular}{|l|} 
Groundstone \\
\end{tabular} & Mano & Limestone & 1 & 214.62 & & \begin{tabular}{|l|}
$11 / 28 / 2017$ \\
\end{tabular} \\
\hline 493 & 1 & 335 & - & - & - & D & |1/111111 & N1000 & E1009 & 8 & \begin{tabular}{|l|}
$88.99-98.89$ \\
\end{tabular} & $70-80$ & - & Detritus & Debitage & Complete Flake & Lithic & 4 & 44.57 & & $\mid$\begin{tabular}{|l|}
$\mid 11 / 28 / 2017$ \\
\end{tabular} \\
\hline 493 & 2 & 335 & - & - & - & D & |11/111 & N1000 & E1009 & 8 & 98.99-98.89 & $70-80$ & - & Detritus & Debitage & Proximal Flake & Lithic & 8 & 25.42 & & 11/28/2017 \\
\hline 493 & 3 & 335 & - & - & - & D & |11/1IIII & N1000 & E1009 & 8 & \begin{tabular}{|l|}
$98.99-98.89$ \\
\end{tabular} & $70-80$ & - & Detritus & Debitage & Broken Flake & Lithic & 8 & 5.26 & & 11/28/2017 \\
\hline 493 & 4 & 335 & - & - & - & $\mathrm{D}$ & I/I/IIIII & N1000 & E1009 & 8 & \begin{tabular}{|l|}
$98.99-98.89$ \\
\end{tabular} & $70-80$ & - & Detritus & Debitage & Thermal Shatter & Lithic & 3 & 12.61 & & 11/28/2017 \\
\hline 494 & 1 & 340 & - & - & - & D & III & N1000 & E1009 & 9 & \begin{tabular}{|l|}
$98.89-98.79$ \\
\end{tabular} & $80-90$ & - & Detritus & Debitage & \begin{tabular}{|l|} 
Complete Flake \\
\end{tabular} & Lithic & 3 & 9.43 & & \begin{tabular}{|l|}
$11 / 29 / 2017$ \\
\end{tabular} \\
\hline 494 & 2 & 340 & - & - & - & D & IIII & N1000 & E1009 & 9 & \begin{tabular}{|l|}
$98.89-98.79$ \\
\end{tabular} & $80-90$ & - & Detritus & Debitage & Proximal Flake & Lithic & 7 & 6.91 & & 11/29/2017 \\
\hline 494 & 3 & 340 & - & - & - & D & IIII & N1000 & E1009 & 9 & \begin{tabular}{|l|l|}
$98.89-98.79$ \\
\end{tabular} & $80-90$ & - & Detritus & Debitage & Broken Flake & Lithic & 16 & 22.52 & & 11/29/2017 \\
\hline 494 & 4 & 340 & - & - & - & D & IIII & N1000 & E1009 & 9 & \begin{tabular}{|l|l|}
$98.89-98.79$ \\
\end{tabular} & $80-90$ & - & Detritus & Debitage & Thermal Shatter & Lithic & 9 & 7.79 & & 11/29/2017 \\
\hline 495 & 1 & 368 & - & - & - & D & IIII & N1000 & E1009 & 10 & \begin{tabular}{|l|l|}
$98.79-98.69$ \\
\end{tabular} & $90-100$ & - & Faunal Remains & Bone & Indeterminate & Bone & 1 & 0.16 & & 11/30/2017 \\
\hline 495 & 2 & 368 & - & - & - & D & IIII & N1000 & E1009 & 10 & \begin{tabular}{|l|}
$98.79-98.69$ \\
\end{tabular} & 90-100 & - & Detritus & Debitage & \begin{tabular}{|l|} 
Complete Flake \\
\end{tabular} & Lithic & 6 & 25.83 & & 11/30/2017 \\
\hline 495 & 3 & 368 & - & - & - & D & IIII & N1000 & E1009 & 10 & \begin{tabular}{|l|}
$98.79-98.69$ \\
\end{tabular} & 90-100 & - & Detritus & Debitage & Proximal Flake & Lithic & 6 & 7 & & 11/30/2017 \\
\hline 495 & 4 & 368 & - & - & - & D & IIII & N1000 & E1009 & 10 & \begin{tabular}{|l|}
$98.79-98.69$ \\
\end{tabular} & 90-100 & - & Detritus & Debitage & Broken Flake & Lithic & 8 & 12.89 & & $\mid 11 / 30 / 2017$ \\
\hline 495 & 5 & 368 & - & - & - & D & IIII & N1000 & E1009 & 10 & \begin{tabular}{|l|l|}
$98.79-98.69$ \\
\end{tabular} & 90-100 & - & Detritus & Debitage & Thermal Shatter & Lithic & 3 & 4.76 & & 11/30/2017 \\
\hline 496 & 1 & 404 & - & - & - & D & IIII & N1000 & E1009 & 11 & \begin{tabular}{|l|l|}
$98.69-98.59$ \\
\end{tabular} & $100-110$ & - & Detritus & Debitage & \begin{tabular}{|l|} 
Complete Flake \\
\end{tabular} & Lithic & 5 & 18.4 & & 1214/2017 \\
\hline 496 & 2 & 404 & - & - & - & D & IIII & N1000 & E1009 & 11 & 98.69-98.59 & $100-110$ & - & Detritus & Debitage & Proximal Flake & Lithic & 5 & 4.11 & & 12/4/2017 \\
\hline 496 & 3 & 404 & - & - & - & D & III & N1000 & E1009 & 11 & \begin{tabular}{|l|l|}
$98.69-98.59$ \\
\end{tabular} & $100-110$ & - & Detritus & Debitage & Broken Flake & Lithic & 19 & 18.57 & & 12/4/2017 \\
\hline 496 & 4 & 404 & - & - & - & D & III & N1000 & E1009 & 11 & \begin{tabular}{|l|l|}
$98.69-98.59$ \\
\end{tabular} & $100-110$ & - & Detritus & Debitage & Thermal Shatter & Lithic & 4 & 2.04 & & 12/4/2017 \\
\hline 497 & 1 & 318 & - & - & - & $\mathrm{D}$ & 1/11 & N1000 & E1010 & 2 & \begin{tabular}{|l|l|}
$99.59-99.49$ \\
\end{tabular} & $10-20$ & - & Detritus & Debitage & Complete Flake & Lithic & 1 & 0.2 & & \begin{tabular}{|l|l|}
$11 / 27 / 2017$ \\
\end{tabular} \\
\hline 497 & 2 & 318 & - & - & - & $\mathrm{D}$ & 1/11 & N1000 & E1010 & 2 & \begin{tabular}{|l|l|}
$99.59-99.49$ \\
\end{tabular} & $10-20$ & - & Detritus & Debitage & Proximal Flake & Lithic & 2 & 1.13 & & 11/27/2017 \\
\hline 497 & 3 & 318 & - & - & - & $\mathrm{D}$ & $1 / 11$ & N1000 & E1010 & 2 & \begin{tabular}{|l|l|}
$99.59-99.49$ \\
\end{tabular} & $10-20$ & - & Historic Artifiact & Ceramic & Whiteware & Ceramic & 1 & 1.59 & & 11/27/2017 \\
\hline 498 & 1 & 320 & - & - & - & $\mathrm{D}$ & $1 / 11$ & N1000 & E1010 & 3 & \begin{tabular}{|l|l|}
$99.49-99.39$ \\
\end{tabular} & $20-30$ & - & Detritus & Debitage & \begin{tabular}{|l|} 
Proximal Flake \\
\end{tabular} & Lithic & 1 & 0.43 & & 11/27/2017 \\
\hline 498 & 2 & 320 & - & - & - & $\mathrm{D}$ & 1/11 & N1000 & E1010 & 3 & \begin{tabular}{|l|l|}
$99.49-99.39$ \\
\end{tabular} & $20-30$ & - & Detritus & Debitage & Broken Flake & Lithic & 3 & 4.57 & & \begin{tabular}{|l|l|}
$11 / 27 / 2017$ \\
\end{tabular} \\
\hline 499 & 1 & 321 & - & - & - & $\mathrm{D}$ & 1/11 & N1000 & E1010 & 4 & \begin{tabular}{|l|l|}
$99.39-99.29$ \\
\end{tabular} & $30-40$ & - & Detritus & Debitage & \begin{tabular}{|l|} 
Complete Flake \\
\end{tabular} & Lithic & 2 & 3.53 & & 11/27/2017 \\
\hline 499 & 2 & 321 & - & - & - & D & $1 / 11$ & N1000 & E1010 & 4 & \begin{tabular}{|l|l|}
$99.39-99.29$ \\
\end{tabular} & $30-40$ & - & Detritus & Debitage & \begin{tabular}{|l|} 
Proximal Flake \\
\end{tabular} & Lithic & 3 & 0.93 & & 11/27/2017 \\
\hline 499 & 3 & 321 & - & - & - & D & 1/11 & N1000 & E1010 & 4 & \begin{tabular}{|l|l|}
$99.39-99.29$ \\
\end{tabular} & $30-40$ & - & Detritus & Debitage & Broken Flake & Lithic & 3 & 2.01 & & 11/27/2017 \\
\hline 499 & 4 & 321 & - & - & - & $\mathrm{D}$ & 1/111 & N1000 & E1010 & 4 & \begin{tabular}{|l|}
$99.39-99.29$ \\
\end{tabular} & $30-40$ & - & Detritus & Debitage & Thermal Shatter & Lithic & 8 & 3.03 & & 11/27/2017 \\
\hline 500 & 1 & 323 & - & - & - & $\mathrm{D}$ & 1/111 & N1000 & E1010 & 5 & \begin{tabular}{|l|}
$99.29-99.19$ \\
\end{tabular} & $40-50$ & - & Detritus & Debitage & \begin{tabular}{|l|} 
Complete Flake \\
\end{tabular} & Lithic & 13 & 37.57 & & 11/27/2017 \\
\hline 500 & 2 & 323 & - & - & - & D & 1/11 & N1000 & E1010 & 5 & \begin{tabular}{|l|}
$99.29-99.19$ \\
\end{tabular} & $40-50$ & - & Detritus & Debitage & \begin{tabular}{|l|} 
Proximal Flake \\
\end{tabular} & Lithic & 9 & 6.52 & & \begin{tabular}{|l|}
$11 / 27 / 2017$ \\
\end{tabular} \\
\hline
\end{tabular}




\begin{tabular}{|c|c|c|c|c|c|c|c|c|c|c|c|c|c|c|c|c|c|c|c|c|c|}
\hline Lot No. & \begin{tabular}{|c|}
$\begin{array}{c}\text { Specimen } \\
\text { No. }\end{array}$ \\
\end{tabular} & FS No. & UI No. & FTR No. & $\begin{array}{c}\text { Sample } \\
\text { No. }\end{array}$ & $\begin{array}{c}\text { Excavation } \\
\text { Block }\end{array}$ & Stratum (AU) & Northing & Easting & Level & $\begin{array}{c}\begin{array}{c}\text { Elevation } \\
(\mathrm{m})\end{array} \\
\end{array}$ & Depth (cmbd) & Point Prov. & Artifact Class & \begin{tabular}{|l|} 
Artifact Type \\
\end{tabular} & \begin{tabular}{|c|} 
Artifact \\
Description
\end{tabular} & Material & $\begin{array}{c}\text { Number of } \\
\text { artifacts }\end{array}$ & Weight (g) & Comments & Date \\
\hline 500 & 3 & 323 & - & - & - & $D$ & $1 / 11$ & N1000 & E1010 & 5 & \begin{tabular}{|l|}
$99.29-99.19$ \\
\end{tabular} & $40-50$ & - & Detritus & Debitage & Broken Flake & Lithic & 13 & 6.36 & & 11/27/2017 \\
\hline 500 & 4 & 323 & - & - & - & D & 1/111 & N1000 & E1010 & 5 & 99.29-99.19 & $40-50$ & - & Detritus & Debitage & Thermal Shatter & Lithic & 22 & 49.77 & & 11/27/2017 \\
\hline 500 & 5 & 323 & - & - & - & D & 1/11 & N1000 & E1010 & 5 & 99.29-99.19 & $40-50$ & - & Detritus & Core & Bifacial & Fine Grain Chert & 1 & 116.04 & Fragment & 11/27/2017 \\
\hline 501 & 1 & 328 & - & - & - & D & 1/11 & N1000 & E1010 & 6 & 99.19-99.09 & $50-60$ & - & Faunal Remains & Bone & Mammal & Bone & 1 & 15.84 & & 11/28/2017 \\
\hline 501 & 2 & 328 & - & - & - & D & $1 / 11$ & N1000 & E1010 & 6 & 99.19-99.09 & $50-60$ & - & Faunal Remains & Bone & Mammal & Bone & 1 & 6.08 & & 11/28/2017 \\
\hline 501 & 3 & 328 & - & - & - & D & $1 / 11$ & N1000 & E1010 & 6 & 99.19-99.09 & $50-60$ & - & Faunal Remains & Bone & Mammal & Bone & 1 & 3.06 & & 11/28/2017 \\
\hline 501 & 4 & 328 & - & - & - & D & 1/11 & N1000 & E1010 & 6 & |99.19-99.09 & 50-60 & - & Faunal Remains & Bone & Mammal & Bone & 1 & 1.32 & & 11/28/2017 \\
\hline 501 & 5 & 328 & - & - & - & D & 1111 & N1000 & E1010 & 6 & 99.19-99.09 & 50-60 & - & Faunal Remains & Bone & Mammal & Bone & 1 & 0.73 & & $\mid$\begin{tabular}{|l|}
$\mid 11 / 28 / 2017$ \\
\end{tabular} \\
\hline 501 & 6 & 328 & - & - & - & D & I/II & N1000 & E1010 & 6 & 99.19-99.09 & $50-60$ & - & Detritus & Debitage & \begin{tabular}{|c|} 
Complete Flake \\
\end{tabular} & Lithic & 11 & 37.91 & & $\mid$\begin{tabular}{|l|}
$\mid 11 / 28 / 2017$ \\
\end{tabular} \\
\hline 501 & 7 & 328 & - & - & - & $\mathrm{D}$ & 1/11 & N1000 & E1010 & 6 & \begin{tabular}{|l|}
$99.19-99.09$ \\
\end{tabular} & 50-60 & - & Detritus & Debitage & Proximal Flake & Lithic & 7 & 15.26 & & \begin{tabular}{|l|l|}
$11 / 28 / 2017$ \\
\end{tabular} \\
\hline 501 & 8 & 328 & - & - & - & $\mathrm{D}$ & 1/11 & N1000 & E1010 & 6 & \begin{tabular}{|l|l|}
$99.19-99.09$ \\
\end{tabular} & $50-60$ & - & Detritus & Debitage & Broken Flake & Lithic & 24 & 10.4 & & \begin{tabular}{|l|}
$11 / 28 / 2017$ \\
\end{tabular} \\
\hline 501 & 9 & 328 & - & - & - & $\mathrm{D}$ & 1/111 & N1000 & E1010 & 6 & \begin{tabular}{|l|l|}
$99.19-99.09$ \\
\end{tabular} & 50-60 & - & Detritus & Debitage & Flaking Shatter & Lithic & 1 & 23.26 & & 11/28/2017 \\
\hline 501 & 10 & 328 & - & - & - & $\mathrm{D}$ & 1/11 & N1000 & E1010 & 6 & \begin{tabular}{|l|l|}
$99.19-99.09$ \\
\end{tabular} & 50-60 & - & Detritus & Debitage & Thermal Shatter & Lithic & 22 & 60.81 & & 11/28/2017 \\
\hline 502 & 1 & 331 & - & - & - & $\mathrm{D}$ & 1/11 & N1000 & E1010 & 7 & |99.09-98.99 & $60-70$ & - & Detritus & Debitage & Complete Flake & Lithic & 12 & 98.38 & & $\mid$\begin{tabular}{|l|}
$\mid 11 / 28 / 2017$ \\
\end{tabular} \\
\hline 502 & 1 & 331 & - & - & - & D & 1/11 & N1000 & E1010 & 7 & \begin{tabular}{|l|l|}
$99.09-98.99$ \\
\end{tabular} & $60-70$ & - & Faunal Remains & Bone & Indeterminate & Bone & 1 & 0.31 & & 11/28/2017 \\
\hline 502 & 2 & 331 & - & - & - & D & 1/11 & N1000 & E1010 & 7 & \begin{tabular}{|l|l|}
$99.09-98.99$ \\
\end{tabular} & $60-70$ & - & Detritus & Debitage & \begin{tabular}{|l|} 
Proximal Flake \\
\end{tabular} & Lithic & 12 & 21.58 & & 11/28/2017 \\
\hline 502 & 2 & 331 & - & - & - & D & 1/11 & N1000 & E1010 & 7 & \begin{tabular}{|l|l|}
$99.09-98.99$ \\
\end{tabular} & $60-70$ & - & Faunal Remains & Bone & Indeterminate & Bone & 1 & 0.21 & & 11/28/2017 \\
\hline 502 & 3 & 331 & - & - & - & D & 1/11 & N1000 & E1010 & 7 & \begin{tabular}{|l|l|}
$99.09-98.99$ \\
\end{tabular} & $60-70$ & - & Detritus & Debitage & Broken Flake & Lithic & 18 & 8.32 & & 11/28/2017 \\
\hline 502 & 4 & 331 & - & - & - & D & 1/11 & N1000 & E1010 & 7 & \begin{tabular}{|l|}
$99.09-98.99$ \\
\end{tabular} & $60-70$ & - & Detritus & Debitage & Flaking Shatter & Lithic & 1 & 7.12 & & 11/28/2017 \\
\hline 502 & 5 & 331 & - & - & - & $\mathrm{D}$ & 1/111 & N1000 & E1010 & 7 & \begin{tabular}{|l|}
$99.099-98.99$ \\
\end{tabular} & $60-70$ & - & Detritus & Debitage & Thermal Shatter & Lithic & 20 & 32.18 & & 11/28/2017 \\
\hline 503 & 1 & 334 & - & - & - & D & VIIIIIII & N1000 & E1010 & 8 & \begin{tabular}{|l|}
$98.99-98.89$ \\
\end{tabular} & $70-80$ & - & Faunal Remains & Bone & Mammal & Bone & 1 & 0.38 & & \begin{tabular}{|l|}
$11 / 28 / 2017$ \\
\end{tabular} \\
\hline 503 & 2 & 334 & - & - & - & D & 111/11111 & N1000 & E1010 & 8 & \begin{tabular}{|l|}
$98.99-98.89$ \\
\end{tabular} & $70-80$ & - & Detritus & Debitage & Complete Flake & Lithic & 15 & 32.27 & & 11/28/2017 \\
\hline 503 & 3 & 334 & - & - & - & D & 111/1111 & N1000 & E1010 & 8 & \begin{tabular}{|l|}
$98.99-98.89$ \\
\end{tabular} & $70-80$ & - & Detritus & Debitage & \begin{tabular}{|l|} 
Proximal Flake \\
\end{tabular} & Lithic & 15 & 12.16 & & 11/28/2017 \\
\hline 503 & 4 & 334 & - & - & - & D & 1111/111 & N1000 & E1010 & 8 & \begin{tabular}{|l|l|}
$98.99-98.89$ \\
\end{tabular} & $70-80$ & - & Detritus & Debitage & Broken Flake & Lithic & 25 & 19.77 & & |11/28/2017 \\
\hline 503 & 5 & 334 & - & - & - & D & |I/IIIII & N1000 & E1010 & 8 & \begin{tabular}{|l|}
$98.99-98.89$ \\
\end{tabular} & $70-80$ & - & Detritus & Debitage & Thermal Shatter & Lithic & 24 & 14.31 & & \begin{tabular}{|l|}
$11 / 28 / 2017$ \\
\end{tabular} \\
\hline 504 & 1 & 336 & - & - & - & D & IIII & N1000 & E1010 & 9 & \begin{tabular}{|l|l|}
$98.89-98.79$ \\
\end{tabular} & $80-90$ & - & \begin{tabular}{|l|} 
Faunal Remains \\
\end{tabular} & Bone & \begin{tabular}{|l|} 
Tortoise/Turtle \\
\end{tabular} & Bone & 1 & 0.15 & & 11/28/2017 \\
\hline 504 & 2 & 336 & - & - & - & D & IIII & N1000 & E1010 & 9 & \begin{tabular}{|l|l|}
$98.89-98.79$ \\
\end{tabular} & $80-90$ & - & Faunal Remains & Bone & Indeterminate & Bone & 1 & 0.25 & & 11/28/2017 \\
\hline 504 & 3 & 336 & - & - & - & D & IIII & N1000 & E1010 & 9 & \begin{tabular}{|l|}
$98.89-98.79$ \\
\end{tabular} & $80-90$ & - & Faunal Remains & Bone & Indeterminate & Bone & 1 & 0.46 & & 11/28/2017 \\
\hline 504 & 4 & 336 & - & - & - & D & IIII & N1000 & E1010 & 9 & \begin{tabular}{|l|l|}
$98.89-98.79$ \\
\end{tabular} & $80-90$ & - & Faunal Remains & Bone & Indeterminate & Bone & 1 & 0.32 & & 11/28/2017 \\
\hline 504 & 5 & 336 & - & - & - & D & IIII & N1000 & E1010 & 9 & \begin{tabular}{|l|l|}
$98.89-98.79$ \\
\end{tabular} & $80-90$ & - & Faunal Remains & Bone & Indeterminate & Bone & 1 & 0.10 & & 11/28/2017 \\
\hline 504 & 6 & 336 & - & - & - & D & IIII & N1000 & E1010 & 9 & 98.89-98.79 & 80-90 & - & Faunal Remains & Bone & Indeterminate & Bone & 1 & 0.16 & & $\mid$\begin{tabular}{|l|}
$\mid 11 / 28 / 2017$ \\
\end{tabular} \\
\hline 504 & 7 & 336 & - & - & - & D & III & N1000 & E1010 & 9 & \begin{tabular}{|l|l|}
$98.89-98.79$ \\
\end{tabular} & $80-90$ & - & Faunal Remains & Bone & Indeterminate & Bone & 1 & 0.13 & & 11/28/2017 \\
\hline 504 & 8 & 336 & - & - & - & D & IIII & N1000 & E1010 & 9 & \begin{tabular}{|l|l|}
$98.89-98.79$ \\
\end{tabular} & $80-90$ & - & Faunal Remains & Bone & Indeterminate & Bone & 1 & 0.31 & & 11/28/2017 \\
\hline 504 & 9 & 336 & - & - & - & $\mathrm{D}$ & IIII & N1000 & E1010 & 9 & \begin{tabular}{|l|l|}
$98.89-98.79$ \\
\end{tabular} & $80-90$ & - & Faunal Remains & Bone & Indeterminate & Bone & 1 & 0.04 & & \begin{tabular}{|l|}
$11 / 28 / 2017$ \\
\end{tabular} \\
\hline 504 & 10 & 336 & - & - & - & $\mathrm{D}$ & IIII & N1000 & E1010 & 9 & \begin{tabular}{|l|l|}
$98.89-98.79$ \\
\end{tabular} & $80-90$ & - & Faunal Remains & Bone & Indeterminate & Bone & 1 & 0.61 & & 11/28/2017 \\
\hline 504 & 11 & 336 & - & - & - & $\mathrm{D}$ & IIII & N1000 & E1010 & 9 & \begin{tabular}{|l|}
$98.89-98.79$ \\
\end{tabular} & \begin{tabular}{ll|l|}
$80-90$ & \\
\end{tabular} & - & Detritus & Debitage & \begin{tabular}{|c|} 
Complete Flake \\
\end{tabular} & Lithic & 16 & 81.69 & & 11/28/2017 \\
\hline 504 & 12 & 336 & - & - & - & $\mathrm{D}$ & IIII & N1000 & E1010 & 9 & \begin{tabular}{|l|}
$98.89-98.79$ \\
\end{tabular} & $80-90$ & - & Detritus & Debitage & Proximal Flake & Lithic & 18 & 15.41 & & 11/28/2017 \\
\hline 504 & 13 & 336 & - & - & - & $\mathrm{D}$ & IIII & N1000 & E1010 & 9 & \begin{tabular}{|l|l|}
$98.89-98.79$ \\
\end{tabular} & $80-90$ & - & Detritus & Debitage & Broken Flake & Lithic & 35 & 17.2 & & \begin{tabular}{|l|}
$11 / 28 / 2017$ \\
\end{tabular} \\
\hline 504 & 14 & 336 & - & - & - & D & IIII & N1000 & E1010 & 9 & \begin{tabular}{|l|l|}
$98.89-98.79$ \\
\end{tabular} & $80-90$ & - & Detritus & Debitage & Thermal Shatter & Lithic & 25 & 15.28 & & 11/28/2017 \\
\hline 505 & 1 & 342 & - & - & - & D & III & N1000 & E1010 & 10 & \begin{tabular}{|l|}
$98.79-98.69$ \\
\end{tabular} & 90-100 & - & Faunal Remains & Bone & Indeterminate & Bone & 1 & 0.23 & & 11/29/2017 \\
\hline 505 & 2 & 342 & - & - & - & D & III & N1000 & E1010 & 10 & \begin{tabular}{|l|}
$98.79-98.69$ \\
\end{tabular} & 90-100 & - & Faunal Remains & Bone & Indeterminate & Bone & 1 & 0.16 & & \begin{tabular}{|l|}
$11 / 29 / 2017$ \\
\end{tabular} \\
\hline 505 & 3 & 342 & - & - & - & D & IIII & N1000 & E1010 & 10 & \begin{tabular}{|l|l|}
$98.79-98.69$ \\
\end{tabular} & $90-100$ & - & Faunal Remains & Bone & Indeterminate & Bone & 1 & 0.08 & & 11/29/2017 \\
\hline 505 & 4 & 342 & - & - & - & D & IIII & N1000 & E1010 & 10 & \begin{tabular}{|l|l|}
$98.79-98.69$ \\
\end{tabular} & $90-100$ & - & Detritus & Debitage & Complete Flake & Lithic & 15 & 79.87 & & 11/29/2017 \\
\hline 505 & 5 & 342 & - & - & - & D & III & N1000 & E1010 & 10 & \begin{tabular}{|l|}
$98.79-98.69$ \\
\end{tabular} & $90-100$ & - & Detritus & Debitage & \begin{tabular}{|l|} 
Proximal Flake \\
\end{tabular} & Lithic & 22 & 18.19 & & \begin{tabular}{|l|}
$11 / 29 / 2017$ \\
\end{tabular} \\
\hline
\end{tabular}




\begin{tabular}{|c|c|c|c|c|c|c|c|c|c|c|c|c|c|c|c|c|c|c|c|c|c|}
\hline Lot No. & \begin{tabular}{|c|}
$\begin{array}{c}\text { Specimen } \\
\text { No. }\end{array}$ \\
\end{tabular} & FS No. & UI No. & FTR No. & $\begin{array}{c}\text { Sample } \\
\text { No. }\end{array}$ & $\begin{array}{c}\text { Excavation } \\
\text { Block }\end{array}$ & Stratum (AU) & Northing & Easting & Level & $\begin{array}{c}\begin{array}{c}\text { Elevation } \\
(\mathrm{m})\end{array} \\
\end{array}$ & Depth (cmbd) & Point Prov. & Artifact Class & Artifact Type & \begin{tabular}{|c|} 
Artifact \\
Description
\end{tabular} & Material & $\begin{array}{c}\text { Number of } \\
\text { artifacts }\end{array}$ & Weight $(g)$ & Comments & Date \\
\hline 505 & 6 & 342 & - & - & - & $D$ & III & N1000 & E1010 & 10 & \begin{tabular}{|l|l|}
$98.79-98.69$ \\
\end{tabular} & $90-100$ & - & Detritus & Debitage & Broken Flake & Lithic & 18 & 14.82 & & 11/29/2017 \\
\hline 505 & 7 & 342 & - & - & - & D & III & N1000 & E1010 & 10 & 98.79-98.69 & $90-100$ & - & Detritus & Debitage & Thermal Shatter & Lithic & 19 & 18.3 & & 11/29/2017 \\
\hline 505 & 8 & 342 & - & - & - & D & IIII & N1000 & E1010 & 10 & 98.79-98.69 & 90-100 & - & Faunal Remains & Shell & Mussel Shell & Shell & 1 & 4.26 & & 11/29/2017 \\
\hline 506 & 1 & 339 & - & - & - & D & IIII & N1000 & E1010 & 11 & \begin{tabular}{|l|l|}
$98.69-98.59$ \\
\end{tabular} & $100-110$ & - & Faunal Remains & Bone & Tortoise/Turtle & Bone & 1 & 0.67 & & 12/1/2017 \\
\hline 506 & 2 & 339 & - & - & - & D & IIII & N1000 & E1010 & 11 & 98.69-98.59 & $100-110$ & - & Faunal Remains & Bone & Tortoise/Turtle & Bone & 1 & 0.62 & & 12/1/2017 \\
\hline 506 & 3 & 339 & - & - & - & D & IIII & N1000 & E1010 & 11 & 98.69-98.59 & $100-110$ & - & Faunal Remains & Bone & \begin{tabular}{|l|} 
Tortoise/Turtle \\
\end{tabular} & Bone & 1 & 0.29 & & 12/1/2017 \\
\hline 506 & 4 & 339 & - & - & - & D & IIII & N1000 & E1010 & 11 & 98.69-98.59 & $100-110$ & - & Faunal Remains & Bone & Tortoise/Turtle & Bone & 1 & 0.27 & & 12/1/2017 \\
\hline 506 & 5 & 339 & - & - & - & D & III & N1000 & E1010 & 11 & 98.69-98.59 & $100-110$ & - & Faunal Remains & Bone & Tortoise/Turtle & Bone & 1 & 0.24 & & 12/1/2017 \\
\hline 506 & 6 & 339 & - & - & - & D & III & N1000 & E1010 & 11 & \begin{tabular}{|l|l|}
$98.69-98.59$ \\
\end{tabular} & $100-110$ & - & Faunal Remains & Bone & Tortoise/Turtle & Bone & 1 & 0.16 & & 12/1/2017 \\
\hline 506 & 7 & 339 & - & - & - & $\mathrm{D}$ & IIII & N1000 & E1010 & 11 & \begin{tabular}{|l|}
$98.69-98.59$ \\
\end{tabular} & $100-110$ & - & Faunal Remains & Bone & Tortoise/Turtle & Bone & 1 & 0.29 & & 12/1/2017 \\
\hline 506 & 8 & 339 & - & - & - & $\mathrm{D}$ & IIII & N1000 & E1010 & 11 & \begin{tabular}{|l|l|}
$98.69-98.59$ \\
\end{tabular} & $100-110$ & - & Faunal Remains & Bone & Tortoise/Turtle & Bone & 1 & 0.33 & & 12/1/2017 \\
\hline 506 & 9 & 339 & - & - & - & $\mathrm{D}$ & IIII & N1000 & E1010 & 11 & \begin{tabular}{|l|l|}
$98.69-98.59$ \\
\end{tabular} & $100-110$ & - & Faunal Remains & Bone & Tortoise/Turtle & Bone & 1 & 0.13 & & 12/1/2017 \\
\hline 506 & 10 & 339 & - & - & - & $\mathrm{D}$ & IIII & N1000 & E1010 & 11 & \begin{tabular}{|l|l|}
$98.69-98.59$ \\
\end{tabular} & $100-110$ & - & Faunal Remains & Bone & \begin{tabular}{|l|} 
Tortoise/Turtle \\
\end{tabular} & Bone & 1 & 0.23 & & 12/1/2017 \\
\hline 506 & 11 & 339 & - & - & - & $\mathrm{D}$ & IIII & N1000 & E1010 & 11 & |98.69-98.59 & $100-110$ & - & Faunal Remains & Bone & Tortoise/Turtle & Bone & 1 & 0.24 & & 12/1/2017 \\
\hline 506 & 12 & 339 & - & - & - & D & IIII & N1000 & E1010 & 11 & 98.69-98.59 & $100-110$ & - & Faunal Remains & Bone & Tortoise/Turtle & Bone & 1 & 0.18 & & 12/1/2017 \\
\hline 506 & 13 & 339 & - & - & - & D & IIII & N1000 & E1010 & 11 & \begin{tabular}{|l|l|}
$98.69-98.59$ \\
\end{tabular} & $100-110$ & - & Faunal Remains & Bone & Indeterminate & Bone & 1 & 0.17 & & 12/1/2017 \\
\hline 506 & 14 & 339 & - & - & - & D & IIII & N1000 & E1010 & 11 & 98.69-98.59 & $100-110$ & - & Faunal Remains & Bone & Tortoise/Turtle & Bone & 1 & 0.09 & & 12/1/2017 \\
\hline 506 & 15 & 339 & - & - & - & D & III & N1000 & E1010 & 11 & \begin{tabular}{|l|l|}
$98.69-98.59$ \\
\end{tabular} & $100-110$ & - & Faunal Remains & Bone & Indeterminate & Bone & 1 & 0.37 & & 12/1/2017 \\
\hline 506 & 16 & 339 & - & - & - & D & IIII & N1000 & E1010 & 11 & \begin{tabular}{|l|l|}
$98.69-98.59$ \\
\end{tabular} & $100-110$ & - & Faunal Remains & Bone & Indeterminate & Bone & 1 & 0.34 & & 12/1/2017 \\
\hline 506 & 17 & 339 & - & - & - & $\mathrm{D}$ & IIII & N1000 & E1010 & 11 & \begin{tabular}{|l|}
$98.69-98.59$ \\
\end{tabular} & 100-110 & - & Faunal Remains & Bone & Indeterminate & Bone & 1 & 0.18 & & 12/1/2017 \\
\hline 506 & 18 & 339 & - & - & - & D & IIII & N1000 & E1010 & 11 & \begin{tabular}{|l|l|}
$98.69-98.59$ \\
\end{tabular} & $100-110$ & - & Faunal Remains & Bone & Indeterminate & Bone & 1 & 0.29 & & 12/1/2017 \\
\hline 506 & 19 & 339 & - & - & - & D & III & N1000 & E1010 & 11 & \begin{tabular}{|l|}
$98.69-98.59$ \\
\end{tabular} & $100-110$ & - & Faunal Remains & Bone & Indeterminate & Bone & 1 & 0.1 & & 12/1/2017 \\
\hline 506 & 20 & 339 & - & - & - & D & IIII & N1000 & E1010 & 11 & \begin{tabular}{|l|l|}
$98.69-98.59$ \\
\end{tabular} & $100-110$ & - & Faunal Remains & Bone & Indeterminate & Bone & 1 & 0.01 & & 12/1/2017 \\
\hline 506 & 21 & 339 & - & - & - & D & IIII & N1000 & E1010 & 11 & \begin{tabular}{|l|l|}
$98.69-98.59$ \\
\end{tabular} & $100-110$ & - & Faunal Remains & Bone & Tortoise/Turle & Bone & 1 & 0.03 & & 12/1/2017 \\
\hline 506 & 22 & 339 & - & - & - & D & III & N1000 & E1010 & 11 & \begin{tabular}{|l|}
$98.69-98.59$ \\
\end{tabular} & $100-110$ & - & Faunal Remains & Bone & Indeterminate & Bone & 1 & 0.16 & & 12/1/2017 \\
\hline 506 & 23 & 339 & - & - & - & D & IIII & N1000 & E1010 & 11 & \begin{tabular}{|l|l|}
$98.69-98.59$ \\
\end{tabular} & $100-110$ & - & Faunal Remains & Bone & Indeterminate & Bone & 1 & 0.07 & & 12/1/2017 \\
\hline 506 & 24 & 339 & - & - & - & D & IIII & N1000 & E1010 & 11 & \begin{tabular}{|l|l|}
$98.69-98.59$ \\
\end{tabular} & $100-110$ & - & Faunal Remains & Bone & Indeterminate & Bone & 1 & 0.01 & & 12/1/2017 \\
\hline 506 & 25 & 339 & - & - & - & D & IIII & N1000 & E1010 & 11 & 98.69-98.59 & $100-110$ & - & Faunal Remains & Bone & Indeterminate & Bone & 1 & 0.01 & & 12/1/2017 \\
\hline 506 & 26 & 339 & - & - & - & D & IIII & N1000 & E1010 & 11 & 98.69-98.59 & $100-110$ & - & Faunal Remains & Bone & Indeterminate & Bone & 1 & 0.03 & & 12/1/2017 \\
\hline 506 & 27 & 339 & - & - & - & D & IIII & N1000 & E1010 & 11 & \begin{tabular}{|l|l|}
$98.69-98.59$ \\
\end{tabular} & $100-110$ & - & Faunal Remains & Bone & Indeterminate & Bone & 1 & 0.01 & & 12/1/2017 \\
\hline 506 & 28 & 339 & - & - & - & D & IIII & N1000 & E1010 & 11 & 98.69-98.59 & $100-110$ & - & Faunal Remains & Bone & Indeterminate & Bone & 1 & 0.01 & & 12/1/2017 \\
\hline 506 & 29 & 339 & - & - & - & D & III & N1000 & E1010 & 11 & \begin{tabular}{|l|l|}
$98.69-98.59$ \\
\end{tabular} & $100-110$ & - & Detritus & Debitage & Complete Flake & Lithic & 25 & 37.98 & & 12/1/2017 \\
\hline 506 & 30 & 339 & - & - & - & D & III & N1000 & E1010 & 11 & \begin{tabular}{|l|l|}
$98.69-98.59$ \\
\end{tabular} & $100-110$ & - & Detritus & Debitage & \begin{tabular}{|l|} 
Proximal Flake \\
\end{tabular} & Lithic & 33 & 38.36 & & 12/1/2017 \\
\hline 506 & 31 & 339 & - & - & - & $\mathrm{D}$ & IIII & N1000 & E1010 & 11 & \begin{tabular}{|l|l|}
$98.69-98.59$ \\
\end{tabular} & $100-110$ & - & Detritus & Debitage & Broken Flake & Lithic & 44 & 31.26 & & 12/1/2017 \\
\hline 506 & 32 & 339 & - & - & - & $\mathrm{D}$ & IIII & N1000 & E1010 & 11 & \begin{tabular}{|l|l|}
$98.69-98.59$ \\
\end{tabular} & $100-110$ & - & Detritus & Debitage & \begin{tabular}{|l|} 
Flaking Shatter \\
\end{tabular} & Lithic & 4 & 15.25 & & 12/1/2017 \\
\hline 506 & 33 & 339 & - & - & - & $\mathrm{D}$ & IIII & N1000 & E1010 & 11 & \begin{tabular}{|l|l|}
$98.69-98.59$ \\
\end{tabular} & $100-110$ & - & Detritus & Debitage & Thermal Shatter & Lithic & 21 & 20.73 & & 12/1/2017 \\
\hline 506 & 34 & 339 & - & - & - & $\mathrm{D}$ & III & N1000 & E1010 & 11 & \begin{tabular}{|l|l|}
$98.69-98.59$ \\
\end{tabular} & $100-110$ & - & \begin{tabular}{l|l} 
Informal Tool &
\end{tabular} & \begin{tabular}{|l|} 
Modified Flake \\
\end{tabular} & \begin{tabular}{|l|} 
Utilized Flake \\
\end{tabular} & Fine Grain Chert & 1 & 42.88 & & 12/1/2017 \\
\hline 507 & 1 & 361 & - & - & - & $\mathrm{D}$ & III & N1000 & E1010 & 11 & 98.57 & 113 & \begin{tabular}{|l|}
$\begin{array}{l}N 1000.25 \\
\text { E1010.26 }\end{array}$ \\
\end{tabular} & \begin{tabular}{|c|} 
Informal Tool \\
\end{tabular} & Modified Flake & Edge-modified & Fine Grain Chert & 1 & 235.17 & & 12/1/2017 \\
\hline 508 & 1 & 411 & - & - & - & $\mathrm{D}$ & IIII & N1000 & E1010 & 12 & \begin{tabular}{|l|}
$98.59-98.49$ \\
\end{tabular} & 110-120 & - & Faunal Remains & Bone & Indeterminate & Bone & 1 & 0.23 & & 12/4/2017 \\
\hline 508 & 2 & 411 & - & - & - & D & III & N1000 & E1010 & 12 & \begin{tabular}{|l|l|}
$98.59-98.49$ \\
\end{tabular} & 110-120 & - & Faunal Remains & Bone & Indeterminate & Bone & 1 & 0.17 & & 12/4/2017 \\
\hline 508 & 3 & 411 & - & - & - & D & III & N1000 & E1010 & 12 & \begin{tabular}{|l|l|}
$98.59-98.49$ \\
\end{tabular} & $110-120$ & - & Detritus & Debitage & Complete Flake & Lithic & 13 & 48.47 & & 12/4/2017 \\
\hline 508 & 4 & 411 & - & - & - & $\mathrm{D}$ & IIII & N1000 & E1010 & 12 & \begin{tabular}{|l|}
$98.59-98.49$ \\
\end{tabular} & 110-120 & - & Detritus & Debitage & \begin{tabular}{|l|} 
Proximal Flake \\
\end{tabular} & Lithic & 29 & 25.05 & & 12/4/2017 \\
\hline 508 & 5 & 411 & - & - & - & $\mathrm{D}$ & IIII & N1000 & E1010 & 12 & \begin{tabular}{|l|}
$98.59-98.49$ \\
\end{tabular} & 110-120 & - & Detritus & Debitage & Broken Flake & Lithic & 50 & 64.53 & & 12/4/2017 \\
\hline 508 & 6 & 411 & - & - & - & D & III & N1000 & E1010 & 12 & \begin{tabular}{|l|}
$98.59-98.49$ \\
\end{tabular} & 110-120 & - & Detritus & Debitage & Flaking Shatter & Lithic & 4 & 2.53 & & 12/4/2017 \\
\hline
\end{tabular}




\begin{tabular}{|c|c|c|c|c|c|c|c|c|c|c|c|c|c|c|c|c|c|c|c|c|c|}
\hline Lot No. & \begin{tabular}{|c|}
$\begin{array}{c}\text { Specimen } \\
\text { No. }\end{array}$ \\
\end{tabular} & FS No. & UI No. & FTR No. & $\begin{array}{c}\text { Sample } \\
\text { No. }\end{array}$ & $\begin{array}{c}\text { Excavation } \\
\text { Block }\end{array}$ & Stratum (AU) & Northing & Easting & Level & $\begin{array}{c}\begin{array}{c}\text { Elevation } \\
(\mathrm{m})\end{array} \\
\end{array}$ & Depth (cmbd) & Point Prov. & Artifact Class & Artifact Type & \begin{tabular}{|c|} 
Artifact \\
Description
\end{tabular} & Material & $\begin{array}{c}\begin{array}{c}\text { Number of } \\
\text { artifacts }\end{array} \\
\end{array}$ & Weight $(g)$ & Comments & Date \\
\hline 508 & 7 & 411 & - & - & - & $D$ & III & N1000 & E1010 & 12 & 98.59-98.49 & $110-120$ & - & Detritus & Debitage & Thermal Shatter & Lithic & 11 & 11.84 & & 12/4/2017 \\
\hline 509 & 1 & 420 & - & - & - & D & IV & N1000 & E1010 & 13 & 98.49-98.39 & 120-130 & - & Faunal Remains & Bone & Indeterminate & Bone & 1 & 1.43 & & 12/4/2017 \\
\hline 509 & 2 & 420 & - & - & - & D & iv & N1000 & E1010 & 13 & 98.49-98.39 & $120-130$ & - & Detritus & Debitage & \begin{tabular}{|l|} 
Complete Flake \\
\end{tabular} & Lithic & 12 & 20.99 & & 12/4/2017 \\
\hline 509 & 3 & 420 & - & - & - & D & iv & N1000 & E1010 & 13 & \begin{tabular}{|l|l|}
$98.49-98.39$ \\
\end{tabular} & $120-130$ & - & Detritus & Debitage & Proximal Flake & Lithic & 15 & 26.14 & & 12/4/2017 \\
\hline 509 & 4 & 420 & - & - & - & D & iv & N1000 & E1010 & 13 & 98.49-98.39 & $120-130$ & - & Detritus & Debitage & Broken Flake & Lithic & 18 & 22.06 & & 12/4/2017 \\
\hline 509 & 5 & 420 & - & - & - & D & IV & N1000 & E1010 & 13 & \begin{tabular}{|l|l|}
$98.49-98.39$ \\
\end{tabular} & $120-130$ & - & Detritus & Debitage & Thermal Shatter & Lithic & 5 & 2.06 & & 12/4/2017 \\
\hline 510 & 1 & 430 & - & - & - & D & iv & N1000 & E1010 & 14 & 98.39-98.29 & 130-140 & - & Faunal Remains & Bone & Indeterminate & Bone & 1 & 0.24 & & 12/5/2017 \\
\hline 510 & 2 & 430 & - & - & - & D & iv & N1000 & E1010 & 14 & 98.39-98.29 & 130-140 & - & Detritus & Debitage & Complete Flake & Lithic & 16 & 34.82 & & 12/5/2017 \\
\hline 510 & 3 & 430 & - & - & - & D & iv & N1000 & E1010 & 14 & \begin{tabular}{|l|}
$98.39-98.29$ \\
\end{tabular} & $130-140$ & - & Detritus & Debitage & Proximal Flake & Lithic & 21 & 32.35 & & 12/5/2017 \\
\hline 510 & 4 & 430 & - & - & - & $\mathrm{D}$ & iv & N1000 & E1010 & 14 & \begin{tabular}{|l|l|}
$98.39-98.29$ \\
\end{tabular} & 130-140 & - & Detritus & Debitage & Broken Flake & Lithic & 17 & 19.98 & & 12/5/2017 \\
\hline 510 & 5 & 430 & - & - & - & D & iv & N1000 & E1010 & 14 & \begin{tabular}{|l|}
$98.39-98.29$ \\
\end{tabular} & $130-140$ & - & Detritus & Debitage & Thermal Shatter & Lithic & 7 & 2.89 & & 1225/2017 \\
\hline 511 & 1 & 438 & - & - & - & $\mathrm{D}$ & iv & N1000 & E1010 & 15 & \begin{tabular}{|l|}
$98.29-98.19$ \\
\end{tabular} & $\begin{array}{ll}140-150 \\
\end{array}$ & - & Faunal Remains & Bone & Tortoise/Turtle & Bone & 1 & 0.24 & & 12/5/2017 \\
\hline 511 & 2 & 438 & - & - & - & D & iv & N1000 & E1010 & 15 & \begin{tabular}{|l|l|}
$98.29-98.19$ \\
\end{tabular} & $140-150$ & - & Detritus & Debitage & Complete Flake & Lithic & 5 & 70.19 & & 12/5/2017 \\
\hline 511 & 3 & 438 & - & - & - & $\mathrm{D}$ & iv & N1000 & E1010 & 15 & \begin{tabular}{|l|}
$98.29-98.19$ \\
\end{tabular} & 140-150 & - & Detritus & Debitage & Proximal Flake & Lithic & 10 & 12.94 & & 122/5/2017 \\
\hline 511 & 4 & 438 & - & - & - & D & iv & N1000 & E1010 & 15 & \begin{tabular}{|l|}
$98.29-98.19$ \\
\end{tabular} & $140-150$ & - & Detritus & Debitage & Broken Flake & Lithic & 23 & 28.91 & & 1225/2017 \\
\hline 511 & 5 & 438 & - & - & - & $\mathrm{D}$ & iv & N1000 & E1010 & 15 & \begin{tabular}{|l|l|}
$98.29-98.19$ \\
\end{tabular} & 140-150 & - & Detritus & Debitage & Thermal Shatter & Lithic & 6 & 14.16 & & 12/5/2017 \\
\hline 511 & 6 & 438 & - & - & - & D & iv & N1000 & E1010 & 15 & \begin{tabular}{|l|}
$98.29-98.19$ \\
\end{tabular} & $140-150$ & - & \begin{tabular}{l|l} 
Informal Tool &
\end{tabular} & Modified Flake & Edge-modified & Fine Grain Chert & 1 & 13.23 & & 1225/2017 \\
\hline 512 & 1 & 447 & - & - & - & D & iv & N1000 & E1010 & 16 & \begin{tabular}{|l|l|}
$98.19-98.09$ \\
\end{tabular} & $150-160$ & - & Faunal Remains & Bone & Indeterminate & Bone & 1 & 1.03 & & 12/5/2017 \\
\hline 512 & 2 & 447 & - & - & - & D & IV & N1000 & E1010 & 16 & \begin{tabular}{|l|}
$98.19-98.09$ \\
\end{tabular} & $150-160$ & - & Detritus & Debitage & Complete Flake & Lithic & 3 & 19.85 & & 1215/2017 \\
\hline 512 & 3 & 447 & - & - & - & D & iv & N1000 & E1010 & 16 & \begin{tabular}{|l|}
$98.19-98.09$ \\
\end{tabular} & $150-160$ & - & Detritus & Debitage & \begin{tabular}{|l|} 
Proximal Flake \\
\end{tabular} & Lithic & 1 & 0.17 & & 1215/2017 \\
\hline 512 & 4 & 447 & - & - & - & D & IV & N1000 & E1010 & 16 & \begin{tabular}{|l|}
$98.19-98.09$ \\
\end{tabular} & $150-160$ & - & Detritus & Debitage & Broken Flake & Lithic & 9 & 15.99 & & 1215/2017 \\
\hline 512 & 5 & 447 & - & - & - & D & IV & N1000 & E1010 & 16 & \begin{tabular}{|l|}
$98.19-98.09$ \\
\end{tabular} & $150-160$ & - & Detritus & Debitage & Thermal Shatter & Lithic & 3 & 11.04 & & 1215/2017 \\
\hline 512 & 6 & 447 & - & - & - & $\mathrm{D}$ & iv & N1000 & E1010 & 16 & \begin{tabular}{|l|}
$98.19-98.09$ \\
\end{tabular} & $150-160$ & - & Formal Tool & Biface & \begin{tabular}{|c|} 
Marginal-Distal \\
Fragment \\
\end{tabular} & Fine Grain Chert & 1 & 0.86 & & $12 / 5 / 2017$ \\
\hline 512 & 7 & 447 & - & - & - & D & iv & N1000 & E1010 & 16 & \begin{tabular}{|l|l|}
$98.19-98.09$ \\
\end{tabular} & $150-160$ & - & Faunal Remains & Shell & Mussel Shell & Shell & 1 & 1.6 & & 12/5/2017 \\
\hline 513 & 1 & 552 & - & - & - & D & $V_{N 1}$ & N1000 & E1010 & 17 & \begin{tabular}{|l|l|} 
98.09-97.99 \\
\end{tabular} & $160-170$ & - & \begin{tabular}{|l|} 
Faunal Remains \\
\end{tabular} & Bone & Indeterminate & Bone & 1 & 0.01 & & 12/15/2017 \\
\hline 513 & 2 & 552 & - & - & - & D & $V N \mid$ & N1000 & E1010 & 17 & 98.09-97.99 & $160-170$ & - & Faunal Remains & Bone & Indeterminate & Bone & 1 & 0.15 & & 12/15/2017 \\
\hline 513 & 3 & 552 & - & - & - & D & $V N 1$ & N1000 & E1010 & 17 & 98.09-97.99 & $160-170$ & - & Faunal Remains & Bone & Indeterminate & Bone & 1 & 0.1 & & 12/15/2017 \\
\hline 513 & 4 & 552 & - & - & - & D & $V N \mid$ & N1000 & E1010 & 17 & 98.09-97.99 & $160-170$ & - & Detritus & Debitage & Complete Flake & Lithic & 1 & 0.38 & & 12/15/2017 \\
\hline 513 & 5 & 552 & - & - & - & D & $V_{N 1}$ & N1000 & E1010 & 17 & \begin{tabular}{|l|l|}
$98.09-97.99$ \\
\end{tabular} & $160-170$ & - & Detritus & Debitage & \begin{tabular}{l|} 
Proximal Flake \\
\end{tabular} & Lithic & 1 & 9.28 & & 12/15/2017 \\
\hline 513 & 6 & 552 & - & - & - & D & $V N \mid$ & N1000 & E1010 & 17 & 98.09-97.99 & $160-170$ & - & Detritus & Debitage & Broken Flake & Lithic & 1 & 0.37 & & 12/15/2017 \\
\hline 513 & 7 & 552 & - & - & - & D & $V_{N 1}$ & N1000 & E1010 & 17 & |98.09-97.99 & $160-170$ & - & Detritus & Debitage & Thermal Shatter & Lithic & 1 & 1.06 & & 12/15/2017 \\
\hline 513 & 8 & 552 & - & - & c-22 & $\mathrm{D}$ & $\mathrm{VNN}$ & N1000 & E1010 & 17 & \begin{tabular}{|l|l|}
$98.09-97.99$ \\
\end{tabular} & $160-170$ & - & $\begin{array}{l}\begin{array}{c}\text { Archaeological } \\
\text { Sample }\end{array} \\
\text { Sale }\end{array}$ & c-14 & not ID'd & & 1 & 0.01 & "not sent for ID-too small & 12/15/2017 \\
\hline 514 & 1 & 554 & - & - & - & D & $\mathrm{V} N \mathrm{NI}$ & N1000 & E1010 & 18 & 97.99-97.89 & $170-180$ & - & Detritus & Debitage & Complete Flake & Lithic & 3 & 27.25 & & 12/15/2017 \\
\hline 514 & 2 & 554 & - & - & - & D & $V_{N 1}$ & N1000 & E1010 & 18 & 97.99-97.89 & 170-180 & - & Detritus & Debitage & Broken Flake & Lithic & 1 & 0.43 & & 12/15/2017 \\
\hline 514 & 3 & 554 & - & - & - & $\mathrm{D}$ & $\frac{\mathrm{V} N 1}{\mathrm{~V}}$ & N1000 & E1010 & 18 & \begin{tabular}{|l|}
$97.99-97.89$ \\
\end{tabular} & $170-180$ & - & Detritus & Debitage & Thermal Shatter & Lithic & 4 & 16.75 & & 12/15/2017 \\
\hline 515 & 1 & 558 & - & - & - & D & $\mathrm{V} N \mathrm{~N}$ & N1000 & E1010 & 19 & \begin{tabular}{|l|l|}
$97.89-97.79$ \\
\end{tabular} & 180-190 & - & Detritus & Debitage & Complete Flake & Lithic & 1 & 2.92 & & 12/15/2017 \\
\hline 515 & 2 & 558 & - & - & - & $\mathrm{D}$ & 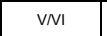 & N1000 & E1010 & 19 & \begin{tabular}{|l|}
$97.89-97.79$ \\
\end{tabular} & $\begin{array}{l}180-190 \\
\end{array}$ & - & Detritus & Debitage & Proximal Flake & Lithic & 2 & 13.54 & & 12/15/2017 \\
\hline 516 & 1 & 623 & - & - & - & D & VII & N1000 & E1010 & 21 & 97.69-97.59 & $200-210$ & - & Detritus & Debitage & Complete Flake & Lithic & 2 & 1.89 & & 121/20/2017 \\
\hline 516 & 2 & 623 & - & - & - & $\mathrm{D}$ & VIII & N1000 & E1010 & 21 & \begin{tabular}{|l|}
$97.69-97.59$ \\
\end{tabular} & $200-210$ & - & Detritus & Debitage & \begin{tabular}{|l|} 
Proximal Flake \\
\end{tabular} & Lithic & 2 & 11.72 & & 121/20/2017 \\
\hline 517 & 1 & 633 & - & - & - & c & "I & N1000.5 & E1005.5 & 2 & 99.6-99.5 & $30-40$ & - & Detritus & Debitage & Complete Flake & Lithic & 1 & 5.28 & & 12/2012017 \\
\hline 517 & 2 & 633 & - & - & - & c & " & N1000.5 & $E 1005.5$ & 2 & 99.6-99.5 & $30-40$ & - & Other Lithic & $\begin{array}{l}\text { Misc. Stone/ } \\
\text { Minaral }\end{array}$ & Silt Stone & Lithic & 1 & 29.75 & & 12/200/2017 \\
\hline 518 & 1 & 640 & - & - & - & c & $\|/\| 11$ & N1000.5 & E1005.5 & 3 & 99.5-99.4 & $40-50$ & - & Faunal Remains & Bone & Indeterminate & Bone & 1 & 0.13 & & 121/20/2017 \\
\hline 518 & 2 & 640 & - & - & - & $\mathrm{c}$ & $\| / 1111$ & N1000.5 & E1005.5 & 3 & $99.5-99.4$ & $40-50$ & - & Faunal Remains & Bone & Indeterminate & Bone & 1 & 0.19 & & 12/20/2017 \\
\hline 518 & 3 & 640 & - & - & - & $\mathrm{c}$ & II/III & $\begin{array}{ll}\text { N1000.5 } \\
\end{array}$ & E1005.5 & 3 & $99.5-99.4$ & $40-50$ & - & \begin{tabular}{|l|} 
Detritus \\
\end{tabular} & Debitage & \begin{tabular}{|l|} 
Complete Flake \\
\end{tabular} & Lithic & 5 & 54.74 & & 12/20/2017 \\
\hline
\end{tabular}




\begin{tabular}{|c|c|c|c|c|c|c|c|c|c|c|c|c|c|c|c|c|c|c|c|c|c|}
\hline Lot No. & $\begin{array}{c}\text { Specimen } \\
\text { No. }\end{array}$ & FS No. & UI No. & FTR No. & $\begin{array}{c}\begin{array}{c}\text { Sample } \\
\text { No. }\end{array} \\
\end{array}$ & $\begin{array}{c}\text { Excavation } \\
\text { Block }\end{array}$ & Stratum (AU) & Northing & Easting & Level & $\begin{array}{l}\text { Elevation } \\
(\mathrm{m})\end{array}$ & Depth (cmbd) & Point Prov. & Artifact Class & Artifact Type & \begin{tabular}{|c|} 
Artifact \\
Description
\end{tabular} & Material & $\begin{array}{c}\text { Number of } \\
\text { artifacts }\end{array}$ & Weight (g) & Comments & Date \\
\hline 518 & 4 & 640 & - & - & - & c & $\| / 111$ & N1000.5 & E1005.5 & 3 & 99.5-99.4 & $40-50$ & - & Detritus & Debitage & Proximal Flake & Lithic & 4 & 1.96 & & 12/20/2017 \\
\hline 518 & 5 & 640 & - & - & - & c & 11/III & $\mathrm{N} 1000.5$ & E1005.5 & 3 & $99.5-99.4$ & $40-50$ & - & Detritus & Debitage & Broken Flake & Lithic & 9 & 4.76 & & $\mid$\begin{tabular}{|l|}
$12 / 20 / 2017$ \\
\end{tabular} \\
\hline 518 & 6 & 640 & - & - & - & c & II/III & $\mathrm{N} 1000.5$ & $E 1005.5$ & 3 & 99.5-99.4 & $40-50$ & - & Detritus & Debitage & Thermal Shatter & Lithic & 11 & 22.29 & & 12/20/2017 \\
\hline 518 & 7 & 640 & - & - & - & $\mathrm{c}$ & $11 / 111$ & N1000.5 & E1005.5 & 3 & 99.5-99.4 & $40-50$ & - & Informal Tool & Modified Flake & \begin{tabular}{|l|} 
Utilized Flake \\
\end{tabular} & Fine Grain Chert & 1 & 14.56 & & 12/20/2017 \\
\hline 518 & 9 & 640 & - & - & - & $\mathrm{c}$ & 11/1111 & N1000.5 & E1005.5 & 3 & 99.5-99.4 & $40-50$ & - & Informal Tool & Modified Flake & Utilized Flake & \begin{tabular}{|c|}
$\begin{array}{c}\text { Coarse Grain } \\
\text { Chert }\end{array}$ \\
\end{tabular} & 1 & 44.44 & & 12/20/2017 \\
\hline 518 & 10 & 640 & - & - & - & $\mathrm{c}$ & $11 / 111$ & N1000.5 & E1005.5 & 3 & $99.5-99.4$ & $40-50$ & - & Other Lithic & \begin{tabular}{|c|}
$\begin{array}{c}\text { Misc. Stone/ } \\
\text { Mineral }\end{array}$ \\
\end{tabular} & Manuport & Limestone & 1 & 58.39 & & 12/20/2017 \\
\hline 519 & 1 & 646 & - & - & - & $\mathrm{c}$ & IIII & N1000.5 & E1005.5 & 4 & $\begin{array}{ll}99.4-99.3 \\
\end{array}$ & 50-60 & - & Faunal Remains & \begin{tabular}{|l|l|} 
Bone \\
5
\end{tabular} & \begin{tabular}{|l|} 
Tortoise/Turtle \\
\end{tabular} & Bone & 1 & 0.18 & & $\mid$\begin{tabular}{|l|}
$12 / 21 / 2017$ \\
\end{tabular} \\
\hline 519 & 2 & 646 & - & - & - & c & III & $\mathrm{N} 1000.5$ & E1005.5 & 4 & 99.4-99.3 & $50-60$ & - & Faunal Remains & Bone & Mammal & Bone & 1 & 2.02 & & 12/21/2017 \\
\hline 519 & 3 & 646 & - & - & - & c & III & N1000.5 & E1005.5 & 4 & 99.4-99.3 & $50-60$ & - & Faunal Remains & Bone & Mammal & Bone & 1 & 0.29 & & 12/21/2017 \\
\hline 519 & 4 & 646 & - & - & - & c & IIII & $\mathrm{N} 1000.5$ & $E 1005.5$ & 4 & 99.499 .3 & $50-60$ & - & Faunal Remains & Bone & Mammal & Bone & 1 & 0.33 & & 12/21/2017 \\
\hline 519 & 5 & 646 & - & - & - & c & III & $\mathrm{N} 1000.5$ & E1005.5 & 4 & 99.4-99.3 & $50-60$ & - & Faunal Remains & Bone & Indeterminate & Bone & 1 & 0.13 & & 12/21/2017 \\
\hline 519 & 6 & 646 & - & - & - & c & IIII & $\mathrm{N} 1000.5$ & $E 1005.5$ & 4 & $99.4-99.3$ & $50-60$ & - & Detritus & Debitage & Complete Flake & Lithic & 7 & 8.79 & & 12/21/2017 \\
\hline 519 & 7 & 646 & - & - & - & $\mathrm{c}$ & III & N1000.5 & E1005.5 & 4 & 99.4-99.3 & $50-60$ & - & Detritus & Debitage & Proximal Flake & Lithic & 8 & 5.34 & & \begin{tabular}{|l|l|}
$12 / 21 / 2017$ \\
\end{tabular} \\
\hline 519 & 8 & 646 & - & - & - & c & IIII & $\mathrm{N} 1000.5$ & $E 1005.5$ & 4 & 99.499 .3 & $50-60$ & - & Detritus & Debitage & Broken Flake & Lithic & 9 & 3.87 & & 12/21/2017 \\
\hline 519 & 9 & 646 & - & - & - & c & III & N1000.5 & E1005.5 & 4 & 99.4-99.3 & $50-60$ & - & Detritus & Debitage & Thermal Shatter & Lithic & 14 & 11.91 & & 12/21/2017 \\
\hline 519 & 10 & 646 & - & - & - & c & III & N1000.5 & E1005.5 & 4 & $99.4-99.3$ & $50-60$ & - & Informal Tool & Modified Flake & Edge-modified & Fine Grain Chert & 1 & 44.49 & & 12/21/2017 \\
\hline 519 & 11 & 646 & - & - & - & c & III & N1000.5 & E1005.5 & 4 & $\begin{array}{l}99.4-99.3 \\
\end{array}$ & $50-60$ & - & Faunal Remains & Shell & \begin{tabular}{|l|} 
Mussel Shell \\
\end{tabular} & Shell & 1 & 4.71 & & 12/21/2017 \\
\hline 520 & 1 & 656 & - & \begin{tabular}{|c|} 
FTR4 Layer \\
1
\end{tabular} & - & c & III & N1000-1001 & E1003-1005 & Layer 1 & 99.42 & 48 & $\begin{array}{l}\mathrm{N} 1001.57 \\
\mathrm{E} 1004.72 \\
\end{array}$ & Faunal Remains & Bone & Mammal & Bone & 1 & 1.53 & 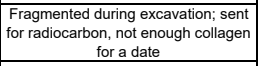 & 12/21/2017 \\
\hline 521 & 1 & 658 & - & \begin{tabular}{|l} 
FTR4 Layer \\
1
\end{tabular} & - & c & III & N1000-1001 & E1003-1005 & Layer 1 & 99.42-99.28 & $48-62$ & - & Detritus & Debitage & \begin{tabular}{|l|} 
Complete Flake \\
\end{tabular} & Lithic & 3 & 1.07 & & 12/27/2017 \\
\hline 521 & 2 & 658 & - & $\begin{array}{c}\text { FTR4 Layer } \\
1\end{array}$ & - & $\mathrm{c}$ & III & N1000-1001 & E1003-1005 & Layer 1 & $99.42-99.28$ & $48-62$ & - & Detritus & Debitage & \begin{tabular}{|l|} 
Proximal Flake \\
\end{tabular} & Lithic & 2 & 0.41 & & 12/27/2017 \\
\hline 521 & 3 & 658 & - & $\begin{array}{c}\text { FTR4 Layer } \\
1\end{array}$ & - & $\mathrm{c}$ & III & N1000-1001 & E1003-1005 & Layer 1 & \begin{tabular}{|l|}
$99.42-99.28$ \\
\end{tabular} & $48-62$ & - & Detritus & Debitage & \begin{tabular}{|l|} 
Broken Flake \\
\end{tabular} & Lithic & 2 & 0.73 & & 12/27/2017 \\
\hline 521 & 4 & 658 & - & $\begin{array}{c}\text { FTR4 Layer } \\
1\end{array}$ & - & $\mathrm{c}$ & III & N1000-1001 & E1003-1005 & Layer 1 & $99.42-99.28$ & $48-62$ & - & Detritus & Debitage & Thermal Shatter & Lithic & 1 & 0.81 & & 12/27/2017 \\
\hline 521 & 5 & 658 & - & $\begin{array}{c}\text { FTR4 Layer } \\
1\end{array}$ & - & $\mathrm{c}$ & III & N1000-1001 & E1003-1005 & Layer 1 & 99.42-99.28 & $48-62$ & - & Faunal Remains & Shell & Untyped Shell & Shell & 2 & 25.24 & Oyster? & 12/27/2017 \\
\hline 522 & 1 & 658 & - & $\begin{array}{c}\text { FTR4 Layer } \\
1\end{array}$ & $F-6 / F-7$ & c & III & N1000-1001 & E1003-1005 & Layer 1 & 99.45-99.3 & $45-60$ & \begin{tabular}{|l|}
$11000.58-$ \\
1000.73 \\
E1003.32- \\
1005.41 \\
\end{tabular} & $\begin{array}{c}\text { Macrobotanical } \\
\text { Remains }\end{array}$ & $\begin{array}{c}\text { Other Plant } \\
\text { Parts }\end{array}$ & $\left|\begin{array}{c}\text { Sugarberry yeed } \\
\text { (Certis } \\
\text { laevigatata) }\end{array}\right|$ & & 1 & 0.01 & $\begin{array}{l}\text { "sent for radiocarbon dating, but } \\
\text { too small too date }\end{array}$ & 12/27/2017 \\
\hline 522 & 2 & 658 & - & FTR4 Layer & $F-6 / F-7$ & c & III & N1000-1001 & E1003-1005 & Layer 1 & 99.45-99.3 & $45-60$ & \begin{tabular}{|c|} 
N1000.58- \\
1000.73 \\
E1003.32- \\
1005.41 \\
\end{tabular} & $\begin{array}{c}\text { Macrobotanical } \\
\text { Remains }\end{array}$ & Charcoal & $\begin{array}{l}\text { Red mulberry } \\
\text { (Morus rubra) }\end{array}$ & & 4 & 0.01 & & 12/27/2017 \\
\hline 522 & 3 & 658 & - & FTR4 Layer & $F-6 / F-7$ & c & III & N1000-1001 & E1003-1005 & Layer 1 & 99.45-99.3 & $45-60$ & \begin{tabular}{|c|} 
N1000.58- \\
1000.73 \\
E1003.382- \\
1005.41 \\
\end{tabular} & $\begin{array}{c}\text { Macrobotanical } \\
\text { Remains }\end{array}$ & Charcoal & $\underset{\text { spp.) }}{\operatorname{Elm}(U \min }$ & & 20 & 2.62 & & 12/27/2017 \\
\hline 522 & 4 & 658 & - & $\begin{array}{c}\text { FTR4 Layer } \\
1\end{array}$ & $F-6 / F-7$ & c & III & N1000-1001 & E1003-1005 & Layer 1 & 99.45-99.3 & $45-60$ & 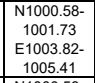 & $\begin{array}{c}\text { Macrobotanical } \\
\text { Remains }\end{array}$ & Charcoal & $\begin{array}{c}\text { Mesquite } \\
\text { (Prosopis } \\
\text { glandulosa) }\end{array}$ & & 13 & 0.53 & & 12/27/2017 \\
\hline 522 & 5 & 658 & - & $\begin{array}{c}\text { FTR4 Layer } \\
1\end{array}$ & $F-6 / F-7$ & c & III & N1000-1001 & E1003-1005 & Layer 1 & 99.45-99.3 & $45-60$ & \begin{tabular}{|c|} 
N1000.58- \\
1000.73 \\
E1003.32-2 \\
1005.41 \\
\end{tabular} & $\begin{array}{c}\text { Macrobotanical } \\
\text { Remains }\end{array}$ & Charcoal & $\begin{array}{c}\text { Juniper } \\
\text { (Juniperus spp.) }\end{array}$ & & 6 & 0.18 & & 12/27/2017 \\
\hline 522 & 6 & 658 & - & FTR4 Layer & $F-6 / F-7$ & c & III & N1000-1001 & E1003-1005 & Layer 1 & 99.45-99.3 & $45-60$ & 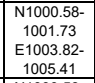 & \begin{tabular}{c|c|} 
Macrobotanical \\
Remains
\end{tabular} & Charcoal & $\begin{array}{l}\text { Ash (Fraxinus } \\
\text { sp.) }\end{array}$ & & 2 & 0.01 & & 12/27/2017 \\
\hline 522 & 7 & 658 & - & $\begin{array}{c}\text { FTR4 Layer } \\
1\end{array}$ & $F-6 / F-7$ & c & III & N1000-1001 & E1003-1005 & Layer 1 & 99.45-99.3 & $45-60$ & \begin{tabular}{|c|} 
N1000.58- \\
1000.73 \\
E1003.32- \\
1005.41 \\
\end{tabular} & $\begin{array}{c}\text { Macrobotanical } \\
\text { Remains }\end{array}$ & $\begin{array}{c}\text { Other Plant } \\
\text { Parts }\end{array}$ & Indeterminable & & 1 & 0.01 & & 12/27/2017 \\
\hline 522 & 8 & 658 & - & FTR4 Layer & $F-6 / F-7$ & c & III & N1000-1001 & E1003-1005 & Layer 1 & 99.45-99.3 & $45-60$ & \begin{tabular}{|l|}
$11000.58-$ \\
1000.73 \\
E1003.32- \\
1005.41 \\
\end{tabular} & \begin{tabular}{c|c|} 
Macrobotanical \\
Remains
\end{tabular} & $\underset{>2 \mathrm{~mm}}{\text { Contamination }}$ & & & & 30.52 & & 12/27/2017 \\
\hline 522 & 9 & 658 & - & $\begin{array}{c}\text { FTR4 Layer } \\
1\end{array}$ & $F-6 / F-7$ & c & III & N1000-1001 & E1003-1005 & Layer 1 & 99.45-99.3 & $45-60$ & \begin{tabular}{|l|} 
N1000.58- \\
1001.73 \\
E1003.32- \\
1005.41 \\
\end{tabular} & \begin{tabular}{c|c|} 
Macrobotanical \\
Remains
\end{tabular} & $\left|\begin{array}{c}\text { Examined } \\
\text { Residue }<2 \mathrm{~mm}\end{array}\right|$ & & & & 7.52 & & 12/27/2017 \\
\hline 522 & 10 & 658 & - & FTR4 Layer & $F-6 / F-7$ & c & III & N1000-1001 & E1003-1005 & Layer 1 & 99.45-99.3 & $45-60$ & \begin{tabular}{|c|} 
N1000.58- \\
1000.73 \\
E1003.32- \\
1005.41 \\
\end{tabular} & Other Lithic & \begin{tabular}{|c|} 
Thermally \\
Attered Stone \\
\end{tabular} & $F C R$ & & 5 & 0.01 & & 12/27/2017 \\
\hline
\end{tabular}




\begin{tabular}{|c|c|c|c|c|c|c|c|c|c|c|c|c|c|c|c|c|c|c|c|c|c|}
\hline Lot No. & $\begin{array}{c}\text { Specimen } \\
\text { No. }\end{array}$ & FS No. & UI No. & FTR No. & \begin{tabular}{c|c}
$\begin{array}{c}\text { Sample } \\
\text { No. }\end{array}$ \\
\end{tabular} & $\begin{array}{c}\text { Excavation } \\
\text { Block }\end{array}$ & Stratum (AU) & Northing & Easting & Level & $\begin{array}{c}\begin{array}{c}\text { Elevation } \\
(\mathrm{m})\end{array} \\
\end{array}$ & Depth (cmbd) & Point Prov. & Artifact Class & Artifact Type & $\begin{array}{c}\text { Artifict } \\
\text { Description }\end{array}$ & Material & $\begin{array}{c}\text { Number of } \\
\text { artifiacts }\end{array}$ & Weight (g) & Comments & Date \\
\hline 522 & 11 & 658 & - & $\left|\begin{array}{c}\text { FTR4 Layer } \\
1\end{array}\right|$ & $F-6 / F-7$ & c & III & N1000-1001 & E1003-1005 & Layer 1 & 99.45-99.3 & $45-60$ & $\begin{array}{c}\text { N1000.58- } \\
1001.73 \\
\text { E1003.82- } \\
1005.41 \\
\end{array}$ & $\begin{array}{c}\begin{array}{c}\text { Macrobotanical } \\
\text { Remains }\end{array} \\
\end{array}$ & \begin{tabular}{|l|}
$\begin{array}{r}\text { residual from } \\
\text { heavy fraction }\end{array}$ \\
|
\end{tabular} & & & & 8.5 & & 12/27/2017 \\
\hline 522 & 12 & 658 & - & $\begin{array}{c}\text { FTR4 Layer } \\
1\end{array}$ & $F-6 / F-7$ & c & III & N1000-1001 & E1003-1005 & Layer 1 & 99.45-99.3 & $45-60$ & $\begin{array}{c}1000.58- \\
1001.73 \\
\text { E1003.82- } \\
1005.41 \\
\end{array}$ & Detritus & Debitage & Thermal Shatter & & 3 & 0.01 & & 12/27/2017 \\
\hline 522 & n/a & 658 & - & $\left|\begin{array}{c}\text { FTR4 Layer } \\
1\end{array}\right|$ & $F-6 / F-7$ & c & III & N1000-1001 & E1003-1005 & Layer 1 & 99.45-99.3 & $45-60$ & \begin{tabular}{|c|} 
N1000.58- \\
1001.73 \\
E1003.82- \\
1005.41 \\
\end{tabular} & $\begin{array}{c}\text { Archaeological } \\
\text { Sample }\end{array}$ & Soil & Feature Matrix & & $3.0 \mathrm{~L}$ & 3000 & all matrix floated & 12/27/2017 \\
\hline 523 & 1 & 658 & - & $\begin{array}{c}\text { FTR4 Layer } \\
1\end{array}$ & $c-15$ & c & III & N1000-1001 & E1003-1005 & Layer 1 & 99.3 & 57 & \begin{tabular}{l|} 
N1001.39 \\
E1004.78 \\
\end{tabular} & \begin{tabular}{|c|} 
Archaeological \\
Sample
\end{tabular} & C-14 & $\begin{array}{c}\text { Junipiper } \\
\text { (Juniperus sp.) }\end{array}$ & & 5 & 0.17 & & 12/27/2017 \\
\hline 524 & 1 & 658 & - & $\begin{array}{c}\text { FTR4 Layer } \\
1\end{array}$ & $c-16$ & c & III & N1000-1001 & E1003-1005 & Layer 1 & 99.3 & 60 & $\begin{array}{l}\text { N1000.96 } \\
\text { E1004.76 } \\
\end{array}$ & \begin{tabular}{|c|} 
Archaeological \\
Sample
\end{tabular} & C-14 & $\begin{array}{c}\text { mesquite } \\
\text { (Prosopis sp.) }\end{array}$ & & 4 & 0.31 & & 12/27/2017 \\
\hline 524 & 2 & 658 & - & \begin{tabular}{|c|} 
FTR4 Layer \\
1
\end{tabular} & $c-16$ & c & III & N1000-1001 & E1003-1005 & Layer 1 & 99.3 & 60 & $\begin{array}{l}\mathrm{N} 1000.96 \\
\mathrm{E} 1004.76\end{array}$ & \begin{tabular}{|c|} 
Archaeological \\
Sample
\end{tabular} & \begin{tabular}{c|} 
Examined \\
Residue $<2 \mathrm{~mm}$
\end{tabular} & & & & 0.14 & & 12/27/2017 \\
\hline 525 & 1 & 658 & - & \begin{tabular}{|c|} 
FTR4 Layer \\
1
\end{tabular} & c-17 & c & III & N1000-1001 & E1003-1005 & Layer 1 & 99.33 & 57 & $\begin{array}{l}\begin{array}{l}\text { N1001.36 } \\
\text { E1004.62 }\end{array} \\
\end{array}$ & \begin{tabular}{|c|} 
Archaeological \\
Sample
\end{tabular} & C-14 & elm (Ulmus sp.) & & 14 & 0.35 & & 12/27/2017 \\
\hline 525 & 2 & 658 & - & \begin{tabular}{|c|} 
FTR4 Layer \\
1
\end{tabular} & $C-17$ & c & "II & N1000-1001 & E1003-1005 & Layer 1 & 99.33 & 57 & $\begin{array}{l}\mathrm{N} 1001.36 \\
\mathrm{E} 1004.62 \\
\end{array}$ & \begin{tabular}{|c|}
$\begin{array}{c}\text { Archaeological } \\
\text { Sample }\end{array}$ \\
\end{tabular} & \begin{tabular}{|c|} 
Examined \\
Residue $<2 \mathrm{~mm}$ \\
\end{tabular} & & & & 0.23 & & 12/27/2017 \\
\hline 526 & 1 & 658 & - & $\begin{array}{c}\text { FTR4 Layer } \\
1\end{array}$ & C-18 & c & III & N1000-1001 & E1003-1005 & Layer 1 & 99.28 & 62 & \begin{tabular}{|l|} 
N1001.16 \\
E1004.62
\end{tabular} & \begin{tabular}{|c|} 
Archaeological \\
Sample
\end{tabular} & C-14 & $\begin{array}{c}\text { Mesquite } \\
\text { (Prosopis sp.) }\end{array}$ & & 8 & 0.21 & & 12/27/2017 \\
\hline 526 & 2 & 658 & - & \begin{tabular}{|c|} 
FTR4 Layer \\
1
\end{tabular} & C-18 & c & III & N1000-1001 & E1003-1005 & Layer 1 & 99.28 & 62 & $\begin{array}{l}\text { N1001.16 } \\
\text { E1004.62 }\end{array}$ & \begin{tabular}{|c|} 
Archaeological \\
Sample
\end{tabular} & \begin{tabular}{|c|} 
Examined \\
Residue $<2 \mathrm{~mm}$ \\
\end{tabular} & & & & 0.22 & & 12/27/2017 \\
\hline 527 & 1 & 658 & - & $\begin{array}{c}\mid \begin{array}{c}|c| R 4 \\
1\end{array} \\
1\end{array}$ & C-19 & $\mathrm{c}$ & III & N1000-1001 & E1003-1005 & Layer 1 & 99.3 & 60 & $\begin{array}{l}\text { N1001.52 } \\
\text { E1004.44 }\end{array}$ & $\begin{array}{c}\text { Archaeological } \\
\text { Sample }\end{array}$ & C-14 & Elm (ulmus sp.) & & 5 & 2.26 & & 12/27/2017 \\
\hline 527 & 2 & 658 & - & \begin{tabular}{|c|} 
FTR4 Layer \\
1
\end{tabular} & C-19 & c & III & N1000-1001 & E1003-1005 & Layer 1 & 99.3 & 60 & $\begin{array}{l}\text { N1001.52 } \\
\text { E1004.44 }\end{array}$ & \begin{tabular}{|c|} 
Archaeological \\
Sample
\end{tabular} & \begin{tabular}{|c|} 
Examined \\
Residue $<2 \mathrm{~mm}$
\end{tabular} & & & & 1.67 & & 12/27/2017 \\
\hline 528 & 1 & 666 & - & 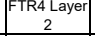 & - & $\mathrm{c}$ & III & N1000-1001 & E1004 & Layer 2 & \begin{tabular}{|l|}
$99.28-99.21$ \\
\end{tabular} & $62-70$ & - & Faunal Remains & Bone & Indeterminate & Bone & 1 & 0.45 & & 12/28/2017 \\
\hline 528 & 2 & 666 & - & \begin{tabular}{|l|l|} 
FTR4 Layer \\
2
\end{tabular} & - & c & IIII & N1000-1001 & E1004 & Layer 2 & \begin{tabular}{|l|l|}
$99.28-99.21$ \\
\end{tabular} & $62-70$ & - & Faunal Remains & Bone & Indeterminate & Bone & 1 & 0.33 & & 12/28/2017 \\
\hline 528 & 3 & 666 & - & $\begin{array}{c}\text { FTR4 Layer } \\
2\end{array}$ & - & $\mathrm{c}$ & III & N1000-1001 & E1004 & Layer 2 & 99.28-99.21 & $62-70$ & - & Faunal Remains & Bone & Indeterminate & Bone & 1 & 0.25 & & 12/28/2017 \\
\hline 528 & 4 & 666 & - & $\begin{array}{l}\text { FTR4 Layer } \\
2\end{array}$ & - & $\mathrm{c}$ & III & N1000-1001 & E1004 & Layer 2 & 99.28-99.21 & $62-70$ & - & Faunal Remains & Bone & Indeterminate & Bone & 1 & 0.06 & & 12/28/2017 \\
\hline 528 & 5 & 666 & - & $\mid \begin{array}{l}\mid \text { TTR4 Layer } \\
2\end{array}$ & - & $\mathrm{c}$ & III & N1000-1001 & E1004 & Layer 2 & 99.28-99.21 & $62-70$ & - & Faunal Remains & Bone & Indeterminate & Bone & 1 & 0.01 & & 12/28/2017 \\
\hline 528 & 6 & 666 & - & $\begin{array}{c}\mid \begin{array}{c}\text { FTR4 Layer } \\
2\end{array} \\
\end{array}$ & - & $\mathrm{c}$ & III & N1000-1001 & E1004 & Layer 2 & 99.28-99.21 & $62-70$ & - & Detritus & Debitage & Complete Flake & Lithic & 4 & 4.94 & & 12/28/2017 \\
\hline 528 & 7 & 666 & - & $\begin{array}{c}\mid \text { FTR4 Layer } \\
2\end{array}$ & - & $\mathrm{c}$ & III & $\begin{array}{ll}\text { N1000-1001 } \\
\end{array}$ & E1004 & Layer 2 & 99.28-99.21 & $62-70$ & - & Detritus & Debitage & Broken Flake & Lithic & 18 & 13.73 & & 12/28/2017 \\
\hline 528 & 8 & 666 & - & FTR4 Layer & - & c & III & N1000-1001 & E1004 & Layer 2 & 99.28-99.21 & $62-70$ & - & Detritus & Debitage & Thermal Shatter & Lithic & 30 & 24.52 & & 12/28/2017 \\
\hline 528 & 9 & 666 & - & $\begin{array}{c}\text { FTR4 Layer } \\
2 \\
\end{array}$ & - & $\mathrm{c}$ & III & $\begin{array}{ll}\text { N1000-1001 } \\
\end{array}$ & E1004 & Layer 2 & 99.28-99.21 & $62-70$ & - & Informal Tool & Modified Flake & Edge-modified & Fine Grain Chert & 1 & 1.2 & & 12/28/2017 \\
\hline 529 & 1 & 666 & - & $\begin{array}{c}\text { FTR4 Layer } \\
2\end{array} \mid$ & $F-8 / F-9$ & c & III & N1000-1001 & E1004 & Layer 2 & |99.28-99.21 & $62-70$ & \begin{tabular}{|c|} 
N1000.86- \\
1001.54 \\
E1004.41- \\
1004.96 \\
\end{tabular} & \begin{tabular}{|c|}
$\begin{array}{c}\text { Macrobotanical } \\
\text { Remains }\end{array}$ \\
\end{tabular} & $\begin{array}{c}\text { Other Plant } \\
\text { Parts }\end{array}$ & \begin{tabular}{|c|}
$\begin{array}{c}\text { Camas bulb } \\
\text { scale (Camassia } \\
\text { sp.) }\end{array}$ \\
\end{tabular} & & 1 & 0.01 & 'sent for radiocarbon dating & 12/27/2017 \\
\hline 529 & 2 & 666 & - & 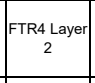 & $F-8 / F-9$ & c & III & N1000-1001 & E1004 & Layer 2 & |99.28-99.21 & $62-70$ & 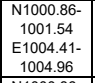 & $\begin{array}{c}\begin{array}{c}\text { Macrobotanical } \\
\text { Remains }\end{array} \\
\end{array}$ & Charcoal & $\begin{array}{l}\text { Red mulberry } \\
\text { (Morus rubra) }\end{array}$ & & 5 & 0.02 & & 12/27/2017 \\
\hline 529 & 3 & 666 & - & $\left|\begin{array}{c}\text { FTR4 Layer } \\
2\end{array}\right|$ & $F-8 / F-9$ & c & III & N1000-1001 & E1004 & Layer 2 & |99.28-99.21 & $62-70$ & $\begin{array}{c}\text { N10000.86- } \\
1001.54 \\
\text { E1004.41- } \\
1004.96 \\
\end{array}$ & \begin{tabular}{|c|}
$\begin{array}{c}\text { Macrobotanical } \\
\text { Remains }\end{array}$ \\
\end{tabular} & Charcoal & $\underset{\text { spp.) }}{\lim _{\text {(UIIus }}}$ & & 11 & 0.5 & & 12/27/2017 \\
\hline 529 & 4 & 666 & - & $\underset{2}{\text { FTR4 Layer }}$ & $F-8 / F-9$ & c & III & N1000-1001 & E1004 & Layer 2 & |99.28-99.21 & $62-70$ & \begin{tabular}{|c|}
$\mathbf{N} 1000.86-1.5$ \\
1001.54 \\
E1004.41- \\
1004.96 \\
\end{tabular} & \begin{tabular}{|c|}
$\begin{array}{c}\text { Macrobotanical } \\
\text { Remains }\end{array}$ \\
\end{tabular} & Charcoal & $\begin{array}{c}\begin{array}{c}\text { Mesquite } \\
\text { (Prosopis } \\
\text { glandulosa) }\end{array} \\
\end{array}$ & & 1 & 0.01 & & 12/27/2017 \\
\hline 529 & 5 & 666 & - & $\mid \begin{array}{c}\text { FTR4 Layer } \\
2\end{array}$ & $F-8 / F-9$ & c & III & N1000-1001 & E1004 & Layer 2 & 99.28-99.21 & $62-70$ & $\begin{array}{c}\text { N1000.86- } \\
1001.54 \\
\text { E1004.41- } \\
1004.96 \\
\end{array}$ & $\begin{array}{c}\text { Macrobotanical } \\
\text { Remains }\end{array}$ & Charcoal & $\begin{array}{c}\text { Juniper } \\
\text { (Juniperus sp..) }\end{array}$ & & 2 & 0.01 & & 12/27/2017 \\
\hline 529 & 6 & 666 & - & $\mid \begin{array}{c}\text { FTR 4 Layer } \\
2\end{array}$ & $F-8 / F-9$ & c & III & N1000-1001 & E1004 & Layer 2 & 99.28-99.21 & $62-70$ & \begin{tabular}{|c|}
$\mathbf{N} 1000.06-16-$ \\
1001.54 \\
E1004.41- \\
1004.96 \\
\end{tabular} & $\begin{array}{c}\text { Macrobotanical } \\
\text { Remains }\end{array}$ & Charcoal & $\begin{array}{c}\text { Hardwood, } \\
\text { indeterminable }\end{array}$ & & 3 & 0.01 & & 12/27/2017 \\
\hline 529 & 7 & 666 & - & $\begin{array}{c}\text { FTR4 Layer } \\
2\end{array}$ & $F-8 / F-9$ & c & III & N1000-1001 & E1004 & Layer 2 & |99.28-99.21 & $62-70$ & $\begin{array}{c}\text { N1000.86- } \\
\text { 1001.54 } \\
\text { E1004.41- } \\
1004.96 \\
1\end{array}$ & \begin{tabular}{|c|}
$\begin{array}{c}\text { Macrobotanical } \\
\text { Remains }\end{array}$ \\
\end{tabular} & $\begin{array}{c}\text { Contamination } \\
>2 \mathrm{~mm}\end{array}$ & & & & 15.33 & & 12/27/2017 \\
\hline 529 & 8 & 666 & - & $\mid \begin{array}{c}\text { FTR 4 Layer } \\
2\end{array}$ & $F-8 / F-9$ & c & III & N1000-1001 & E1004 & Layer 2 & |99.28-99.21 & $62-70$ & \begin{tabular}{|c|}
$\mathbf{N 1} 1000.86-1$ \\
1001.54 \\
E1004.41- \\
1004.96 \\
\end{tabular} & \begin{tabular}{c|}
$\begin{array}{c}\text { Macrobotanical } \\
\text { Remains }\end{array}$ \\
\end{tabular} & $\mid \begin{array}{c}\text { Examined } \\
\text { Residue }<2 \mathrm{~mm}\end{array}$ & & & & 3.35 & & 12/27/2017 \\
\hline 529 & 9 & 666 & - & $\begin{array}{c}\text { FTR4 Layer } \\
2\end{array}$ & $F-8 / F-9$ & c & III & N1000-1001 & E1004 & Layer 2 & 99.28-99.21 & $62-70$ & $\begin{array}{c}\text { N10000.86- } \\
\text { 1001.54 } \\
\text { E1004.41- } \\
1004.96 \\
\end{array}$ & $\begin{array}{c}\text { Macrobotanical } \\
\text { Remains }\end{array}$ & \begin{tabular}{|l|} 
residual from \\
heavy fraction
\end{tabular} & & & & 29 & & 12/27/2017 \\
\hline
\end{tabular}




\begin{tabular}{|c|c|c|c|c|c|c|c|c|c|c|c|c|c|c|c|c|c|c|c|c|c|}
\hline Lot No. & \begin{tabular}{|c|}
$\begin{array}{c}\text { Specimen } \\
\text { No. }\end{array}$ \\
\end{tabular} & FS No. & UI No. & FTR No. & \begin{tabular}{c|} 
Sample \\
No.
\end{tabular} & $\begin{array}{c}\text { Excavation } \\
\text { Block }\end{array}$ & Stratum (AU) & Northing & Easting & Level & $\begin{array}{l}\text { Elevation } \\
\text { (m) }\end{array}$ & Depth (cmbd) & Point Prov. & Artifact Class & \begin{tabular}{|l|} 
Artiffact Type \\
\end{tabular} & \begin{tabular}{|c} 
Artifact \\
Description
\end{tabular} & Material & $\begin{array}{c}\begin{array}{c}\text { Number of } \\
\text { artifacts }\end{array} \\
\end{array}$ & Weight (g) & Comments & Date \\
\hline 529 & 10 & 666 & - & $\underset{2}{\text { FTR4 Layer }}$ & $F-8 / F-9$ & c & III & N1000-1001 & E1004 & Layer 2 & 99.28-99.21 & $62-70$ & $\begin{array}{c}\text { N1000.86- } \\
1001.54 \\
\text { E1000.41- } \\
1004.96 \\
\end{array}$ & Other Lithic & $\begin{array}{c}\text { Thermally } \\
\text { Altered Stone }\end{array}$ & FCR & & 8 & 6.5 & & 12/27/2017 \\
\hline 529 & 11 & 666 & - & $\left|\begin{array}{c}\text { FTR4 Layer } \\
2\end{array}\right|$ & $F-8 / F-9$ & c & III & N1000-1001 & E1004 & Layer 2 & |99.28-99.21 & $62-70$ & \begin{tabular}{|c|}
$\mathbf{N 1} 1000.86-$ \\
1001.54 \\
E1004.41- \\
1004.96
\end{tabular} & Clay & Clay Artifiact & Burned Clay & & 1 & 1.5 & & 12/27/2017 \\
\hline 529 & 12 & 666 & - & $\begin{array}{c}\text { FTR4 Layer } \\
2\end{array}$ & $F-8 / F-9$ & c & III & N1000-1001 & E1004 & Layer 2 & 99.28-99.21 & $62-70$ & \begin{tabular}{|c|}
$\mathbf{N 1} 1000.86-$ \\
1001.54 \\
E1004.41- \\
1004.96 \\
\end{tabular} & Faunal Remains & Bone & & & 3 & 0.5 & $\begin{array}{l}\text { From flotation heavy fraction, not } \\
\text { analyzed }\end{array}$ & 12/27/2017 \\
\hline 529 & 13 & 666 & - & $\begin{array}{c}\text { FTR4 Layer } \\
2\end{array}$ & $F-8 / F-9$ & c & III & N1000-1001 & E1004 & Layer 2 & |99.28-99.21 & $62-70$ & \begin{tabular}{|c|}
$1000.86-$ \\
1001.54 \\
E1004.41- \\
1004.96 \\
\end{tabular} & Detritus & Debitage & Flaking Shatter & & 4 & 0.1 & & 12/27/2017 \\
\hline 529 & 14 & 666 & - & $\begin{array}{c}\text { FTR4 Layer } \\
2\end{array}$ & $F-8 / F-9$ & c & III & N1000-1001 & E1004 & Layer 2 & |99.28-99.21 & $62-70$ & \begin{tabular}{|l|}
$\mathrm{N} 1000.86-$ \\
1001.54 \\
$\mathrm{E} 1004.41-$ \\
1004.96
\end{tabular} & Detritus & Debitage & Thermal Shatter & & 12 & 0.01 & & 12/27/2017 \\
\hline 529 & $\mathrm{n} / \mathrm{a}$ & 666 & - & $\begin{array}{c}\text { FTR4 Layer } \\
2\end{array}$ & $F-8 / F-9$ & c & III & N1000-1001 & E1004 & Layer 2 & |99.28-99.21 & $62-70$ & \begin{tabular}{|c|} 
N1000.86- \\
1001.54 \\
E1000.41- \\
1004.96 \\
\end{tabular} & \begin{tabular}{c|c|}
$\begin{array}{c}\text { Archaeological } \\
\text { Sample }\end{array}$ \\
\end{tabular} & Soil & Feature Matrix & & $2.5 \mathrm{~L}$ & 2400 & all matrix floated & 12/27/2017 \\
\hline 530 & 1 & 666 & - & $\begin{array}{c}\text { FTR4 Layer } \\
2\end{array}$ & $c-20$ & c & IIII & N1000-1001 & E1004 & Layer 2 & 99.26 & 64 & \begin{tabular}{|l|l|} 
N11001.1.13 \\
E1000.47
\end{tabular} & \begin{tabular}{|c|}
$\begin{array}{c}\text { Archaealogical } \\
\text { Sample }\end{array}$ \\
\end{tabular} & C-14 & Elm (ulmus sp.) & & 6 & 0.25 & & 12/27/2017 \\
\hline 531 & 1 & 666 & - & \begin{tabular}{|c|} 
FTR4 Layer \\
2
\end{tabular} & $c-21$ & c & III & N1000-1001 & E1004 & Layer 2 & 99.27 & 63 & \begin{tabular}{l|} 
N1001.18 \\
E1004.45
\end{tabular} & \begin{tabular}{|c|} 
Archaeological \\
Sample
\end{tabular} & C-14 & Elm (ulmus sp.) & & 4 & 0.24 & & 12/27/2017 \\
\hline 532 & 1 & 631 & - & - & - & $\mathrm{c}$ & $\|$ & N1001 & E1003 & 2 & $99.6-99.5$ & $30-40$ & - & Faunal Remains & Bone & Mammal & Bone & 1 & 1.74 & & 12/20/2017 \\
\hline 532 & 2 & 631 & - & - & - & c & $\|$ & N1001 & E1003 & 2 & $99.6-99.5$ & $30-40$ & - & \begin{tabular}{|l|} 
Faunal Remains \\
\end{tabular} & Bone & Mammal & Bone & 1 & 1.67 & & 12/20/2017 \\
\hline 532 & 3 & 631 & - & - & - & $\mathrm{c}$ & $\|$ & N1001 & E1003 & 2 & $99.6-99.5$ & $30-40$ & - & Faunal Remains & Bone & Mammal & Bone & 1 & 1.93 & & 12/20/2017 \\
\hline 532 & 4 & 631 & - & - & - & $\mathrm{c}$ & $"$ & N1001 & E1003 & 2 & $99.6-99.5$ & $30-40$ & - & \begin{tabular}{|l|} 
Faunal Remains \\
\end{tabular} & Bone & Mammal & Bone & 1 & 3.2 & & 12/20/2017 \\
\hline 532 & 5 & 631 & - & - & - & $\mathrm{c}$ & "I & N1001 & E1003 & 2 & $99.6-99.5$ & $30-40$ & - & Faunal Remains & Bone & Indeterminate & Bone & 1 & 0.44 & & 12/20/2017 \\
\hline 532 & 6 & 631 & - & - & - & $\mathrm{c}$ & "I & N1001 & E1003 & 2 & $99.6-99.5$ & $30-40$ & - & Detritus & Debitage & \begin{tabular}{|l|} 
Complete Flake \\
\end{tabular} & Lithic & 2 & 0.77 & & 12/20/2017 \\
\hline 533 & 1 & 641 & - & - & - & $\mathrm{c}$ & $\| / 1111$ & N1001 & E1003 & 3 & $99.5-99.4$ & $40-50$ & - & \begin{tabular}{|l|} 
Faunal Remains \\
\end{tabular} & Bone & Mammal & Bone & 1 & 6.98 & & 12/20/2017 \\
\hline 533 & 2 & 641 & - & - & - & $\mathrm{c}$ & $11 / 111$ & N1001 & E1003 & 3 & $99.5-99.4$ & $40-50$ & - & Faunal Remains & Bone & Indeterminate & Bone & 1 & 0.53 & & 12/20/2017 \\
\hline 533 & 3 & 641 & - & - & - & c & 11/III & N1001 & E1003 & 3 & $99.5-99.4$ & $40-50$ & - & Detritus & Debitage & \begin{tabular}{|l|} 
Proximal Flake \\
\end{tabular} & Lithic & 9 & 32.41 & & 121/20/2017 \\
\hline 533 & 4 & 641 & - & - & - & $\mathrm{c}$ & $\|/\| 1 \|$ & N1001 & E1003 & 3 & $99.5-99.4$ & $40-50$ & - & Detritus & Debitage & Broken Flake & Lithic & 4 & 2.33 & & 12/20/2017 \\
\hline 533 & 5 & 641 & - & - & - & c & 11/III & N1001 & E1003 & 3 & $99.5-99.4$ & $40-50$ & - & Detritus & Debitage & Therrmal Shatter & Lithic & 23 & 33.15 & & 12/20/2017 \\
\hline 533 & 7 & 641 & - & - & - & c & II/III & N1001 & E1003 & 3 & $99.5-99.4$ & $40-50$ & - & Clay & Pigment & Red Ochre & Ochre & 1 & 0.34 & & 12/20/2017 \\
\hline 533 & 8 & 641 & - & - & - & $\mathrm{c}$ & 11/III & N1001 & E1003 & 3 & $99.5-99.4$ & $40-50$ & - & Formal Tool & Biface & Basal Fragment & Fine Grain Chert & 1 & 1.51 & & 12/20/2017 \\
\hline 534 & 1 & 649 & - & - & - & $\mathrm{c}$ & IIII & N1001 & E1003 & 4 & 99.499 .3 & 50-60 & - & Detritus & Debitage & \begin{tabular}{|c|} 
Complete Flake \\
\end{tabular} & Lithic & 1 & 3.86 & & 12/21/2017 \\
\hline 534 & 2 & 649 & - & - & - & $\mathrm{c}$ & IIII & N1001 & E1003 & 4 & $\begin{array}{l}99.4-99.3 \\
\end{array}$ & $50-60$ & - & Detritus & Debitage & \begin{tabular}{|l|} 
Proximal Flake \\
\end{tabular} & Lithic & 5 & 3.41 & & 121/21/2017 \\
\hline 534 & 3 & 649 & - & - & - & $\mathrm{c}$ & III & N1001 & E1003 & 4 & $99.4-99.3$ & $50-60$ & - & Detritus & Debitage & Broken Flake & Lithic & 7 & 2.05 & & 12/21/2017 \\
\hline 534 & 4 & 649 & - & - & - & c & III & N1001 & E1003 & 4 & 99.499 .3 & 50-60 & - & Detritus & Debitage & Flaking Shatter & Lithic & 1 & 1.36 & & 12/21/2017 \\
\hline 534 & 5 & 649 & - & - & - & $\mathrm{c}$ & IIII & N1001 & E1003 & 4 & 99.499 .3 & 50-60 & - & Detritus & Debitage & Thermal Shatter & Lithic & 4 & 5.04 & & 12/21/2017 \\
\hline 534 & 7 & 649 & - & - & - & $\mathrm{c}$ & IIII & N1001 & E1003 & 4 & $99.4-99.3$ & $50-60$ & - & \begin{tabular}{|l|l|} 
Historic Artifiact & \\
\end{tabular} & Metal & \begin{tabular}{|c|} 
Miscellaneous \\
Metal
\end{tabular} & Metal & 1 & 1.03 & & 12/21/2017 \\
\hline 535 & 1 & 657 & - & - & - & c & III & N1001 & E1003 & 4 & $99.4-99.36$ & 50-54 & \begin{tabular}{l|}
$\begin{array}{l}\text { N1001.4 } \\
\text { E1003.9 }\end{array}$ \\
\end{tabular} & Faunal Remains & Bone & Indeterminate & Bone & 1 & 0.13 & & 12/21/2017 \\
\hline 535 & 2 & 657 & - & - & - & $\mathrm{c}$ & III & N1001 & E1003 & 4 & 99.4-99.36 & 50-54 & \begin{tabular}{|l|} 
N11001.4 \\
E1003.9 \\
\end{tabular} & Faunal Remains & Bone & Indeterminate & Bone & 1 & 0.12 & & 12/21/2017 \\
\hline 535 & 3 & 657 & - & - & - & $\mathrm{c}$ & III & N1001 & E1003 & 4 & $99.4-99.36$ & 50-54 & \begin{tabular}{|l|} 
N1001.4 \\
E1003.9
\end{tabular} & Faunal Remains & Bone & Indeterminate & Bone & 1 & 0.09 & & 12/21/2017 \\
\hline 535 & 4 & 657 & - & - & - & $\mathrm{c}$ & III & N1001 & E1003 & 4 & $999.4-99.36$ & 50-54 & \begin{tabular}{|l|} 
N1001.4 \\
E10039.9
\end{tabular} & \begin{tabular}{|l|} 
Faunal Remains \\
\end{tabular} & Bone & Indeterminate & Bone & 1 & 0.11 & & 12/21/2017 \\
\hline 535 & 5 & 657 & - & - & - & c & III & N1001 & E1003 & 4 & $99.4-99.36$ & $50-54$ & $\begin{array}{l}\text { N1001.4 } \\
\text { E10039 }\end{array}$ & \begin{tabular}{|l|} 
Faunal Remains \\
\end{tabular} & Bone & Indeterminate & Bone & 1 & 0.05 & & 12/21/2017 \\
\hline 535 & 6 & 657 & - & - & - & $\mathrm{c}$ & III & N1001 & E1003 & 4 & 99.4-99.36 & 50-54 & \begin{tabular}{l|l|} 
N11001.4 \\
E1003.9 \\
\end{tabular} & Faunal Remains & Bone & Indeterminate & Bone & 1 & 0.04 & & 12/21/2017 \\
\hline 535 & 7 & 657 & - & - & - & $\mathrm{c}$ & III & N1001 & E1003 & 4 & $99.4-99.36$ & 50-54 & \begin{tabular}{|l|} 
N1001.4 \\
E11003.9
\end{tabular} & Faunal Remains & Bone & Indeterminate & Bone & 1 & 0.03 & & 12/21/2017 \\
\hline 535 & 8 & 657 & - & - & - & $\mathrm{c}$ & IIII & N1001 & E1003 & 4 & $99.4-99.36$ & 50-54 & \begin{tabular}{|l|l|} 
N1001.4 \\
E11003.9
\end{tabular} & Faunal Remains & Bone & Indeterminate & Bone & 1 & 0.05 & & 12/21/2017 \\
\hline 535 & 9 & 657 & - & - & - & $\mathrm{c}$ & IIII & N1001 & E1003 & 4 & 99.4-99.36 & $50-54$ & 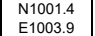 & Faunal Remains & Bone & Indeterminate & Bone & 1 & 0.03 & & 12/21/2017 \\
\hline 535 & 10 & 657 & - & - & - & $\mathrm{c}$ & III & N1001 & E1003 & 4 & $99.4-99.36$ & 50-54 & 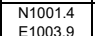 & Faunal Remains & Bone & Indeterminate & Bone & 1 & 0.04 & & 12/21/2017 \\
\hline 535 & 11 & 657 & - & - & - & $\mathrm{c}$ & III & N1001 & E1003 & 4 & $99.4-99.36$ & $50-54$ & $\begin{array}{l}\text { N1001.4 } \\
\text { E10039 }\end{array}$ & \begin{tabular}{|l|} 
Faunal Remains \\
\end{tabular} & Bone & \begin{tabular}{l|} 
Indeterminate \\
\end{tabular} & Bone & 1 & 0.10 & & 12/21/2017 \\
\hline
\end{tabular}




\begin{tabular}{|c|c|c|c|c|c|c|c|c|c|c|c|c|c|c|c|c|c|c|c|c|c|}
\hline Lot No. & \begin{tabular}{|c|}
$\begin{array}{c}\text { Specimen } \\
\text { No. }\end{array}$ \\
\end{tabular} & FS No. & UI No. & FTR No. & $\begin{array}{c}\text { Sample } \\
\text { No. }\end{array}$ & $\begin{array}{c}\text { Excavation } \\
\text { Block }\end{array}$ & Stratum (AU) & Northing & Easting & Level & $\begin{array}{l}\text { Elevation } \\
(\mathrm{m})\end{array}$ & Depth (cmbd) & Point Prov. & Artifact Class & \begin{tabular}{|l|} 
Artifact Type \\
\end{tabular} & $\begin{array}{c}\text { Artifact } \\
\text { Description }\end{array}$ & Material & $\begin{array}{c}\text { Number of } \\
\text { artifacts }\end{array}$ & Weight (g) & Comments & Date \\
\hline 535 & 12 & 657 & - & - & - & c & III & N1001 & E1003 & 4 & $99.4-99.36$ & $50-54$ & $\begin{array}{l}\text { N1001.4 } \\
\text { E1003.9 }\end{array}$ & Faunal Remains & Bone & Indeterminate & Bone & 1 & 0.12 & & 12/21/2017 \\
\hline 535 & 13 & 657 & - & - & - & c & IIII & N1001 & E1003 & 4 & $99.4-99.36$ & 50-54 & $\begin{array}{l}\text { N10101.4. } \\
\text { E1003.9 }\end{array}$ & Faunal Remains & Bone & Indeterminate & Bone & 1 & 0.07 & & 12/21/2017 \\
\hline 535 & 14 & 657 & - & - & - & $\mathrm{c}$ & III & N1001 & E1003 & 4 & 99.4-99.36 & 50-54 & $\begin{array}{l}\text { N1001.4 } \\
\text { E1003.9 } \\
E \text { 1100.9 }\end{array}$ & \begin{tabular}{|l|} 
Faunal Remains \\
\end{tabular} & Bone & \begin{tabular}{ll|} 
Indeterminate \\
\end{tabular} & Bone & 1 & 0.06 & & \begin{tabular}{|l|}
$12 / 21 / 2017$ \\
\end{tabular} \\
\hline 535 & 15 & 657 & - & - & - & $\mathrm{c}$ & III & N1001 & E1003 & 4 & $99.4-99.36$ & $50-54$ & $\begin{array}{l}\text { N1001.4 } \\
\text { E1003.9 }\end{array}$ & Faunal Remains & Bone & Indeterminate & Bone & 1 & 0.04 & & 12/21/2017 \\
\hline 535 & 16 & 657 & - & - & - & $\mathrm{c}$ & III & N1001 & E1003 & 4 & 99.4-99.36 & 50-54 & $\begin{array}{l}\begin{array}{l}\text { N1001.4 } \\
\text { E1003.9 }\end{array}\end{array}$ & Faunal Remains & Bone & Indeterminate & Bone & 1 & 0.05 & & $\mid 12 / 21 / 2017$ \\
\hline 535 & 17 & 657 & - & - & - & $\mathrm{c}$ & III & N1001 & E1003 & 4 & $99.4-99.36$ & $50-54$ & $\begin{array}{l}\text { N10101.4. } \\
\text { E1003.9 }\end{array}$ & Faunal Remains & Bone & Indeterminate & Bone & 1 & 0.03 & & 12/21/2017 \\
\hline 535 & 18 & 657 & - & - & - & $\mathrm{c}$ & IIII & N1001 & E1003 & 4 & $99.4-99.36$ & 50-54 & 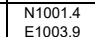 & \begin{tabular}{|l|} 
Faunal Remains \\
\end{tabular} & Bone & Indeterminate & Bone & 1 & 0.03 & & 12/21/2017 \\
\hline 535 & 19 & 657 & - & - & - & $\mathrm{c}$ & III & N1001 & E1003 & 4 & 99.4-99.36 & $50-54$ & $\begin{array}{l}\text { N1001.4 } \\
\text { E1003.9 }\end{array}$ & Faunal Remains & Bone & Indeterminate & Bone & 1 & 0.03 & & $\mid$\begin{tabular}{|l|}
$\mid 12 / 21 / 2017$ \\
\end{tabular} \\
\hline 535 & 20 & 657 & - & - & - & c & III & N1001 & E1003 & 4 & $99.4-99.36$ & $50-54$ & $\begin{array}{l}\text { N1001.4 } \\
\text { E1003.9 }\end{array}$ & Faunal Remains & Bone & Indeterminate & Bone & 1 & 0.03 & & 12/21/2017 \\
\hline 535 & 21 & 657 & - & - & - & $\mathrm{c}$ & IIII & N1001 & E1003 & 4 & 99.4-99.36 & $50-54$ & 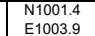 & Faunal Remains & Bone & Indeterminate & Bone & 1 & 0.03 & & 12/21/2017 \\
\hline 535 & 22 & 657 & - & - & - & c & III & N1001 & E1003 & 4 & $99.4-99.36$ & $50-54$ & $\begin{array}{l}\text { N1001.4 } \\
\text { E1003.9 }\end{array}$ & Faunal Remains & Bone & Indeterminate & Bone & 1 & 0.01 & & \begin{tabular}{|l|l|}
$12 / 21 / 2017$ \\
\end{tabular} \\
\hline 535 & 23 & 657 & - & - & - & $\mathrm{c}$ & III & N1001 & E1003 & 4 & $99.4-99.36$ & $50-54$ & $\begin{array}{l}\text { N1001.4 } \\
\text { E1003.9 }\end{array}$ & Faunal Remains & Bone & Indeterminate & Bone & 1 & 0.01 & & 12/21/2017 \\
\hline 535 & 24 & 657 & - & - & - & $\mathrm{c}$ & III & N1001 & E1003 & 4 & 99.4-99.36 & 50-54 & $\begin{array}{l}\text { N1001.4 } \\
\text { E1003.9 }\end{array}$ & Faunal Remains & Bone & Indeterminate & Bone & 1 & 0.01 & & 12/21/2017 \\
\hline 535 & 25 & 657 & - & - & - & $\mathrm{c}$ & IIII & N1001 & E1003 & 4 & 99.4-99.36 & $50-54$ & 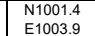 & Faunal Remains & Bone & Indeterminate & Bone & 1 & 0.01 & & 12/21/2017 \\
\hline 535 & 26 & 657 & - & - & - & c & III & N1001 & E1003 & 4 & $99.4-99.36$ & $50-54$ & $\begin{array}{l}\text { N1001.4 } \\
\text { E1003.9 }\end{array}$ & Faunal Remains & Bone & Indeterminate & Bone & 1 & 0.01 & & 12/21/2017 \\
\hline 535 & 27 & 657 & - & - & - & $\mathrm{c}$ & III & N1001 & E1003 & 4 & 99.4-99.36 & 50-54 & $\begin{array}{l}\text { N1001.4 } \\
\text { E1003.9 }\end{array}$ & Faunal Remains & Bone & Indeterminate & Bone & 1 & 0.01 & & 12/21/2017 \\
\hline 535 & 28 & 657 & - & - & - & $\mathrm{c}$ & III & N1001 & E1003 & 4 & $99.4-99.36$ & $50-54$ & $\begin{array}{l}\text { N1001.4 } \\
\text { E1003.9 }\end{array}$ & Faunal Remains & Bone & Indeterminate & Bone & 1 & 0.01 & & 121/21/2017 \\
\hline 535 & 29 & 657 & - & - & - & c & III & N1001 & E1003 & 4 & $99.4-99.36$ & $50-54$ & 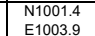 & Faunal Remains & Bone & Indeterminate & Bone & 1 & 0.01 & & 12/21/2017 \\
\hline 535 & 30 & 657 & - & - & - & $\mathrm{c}$ & III & N1001 & E1003 & 4 & $99.4-99.36$ & $50-54$ & $\begin{array}{l}\text { N1001.4 } \\
\text { E1003.9 }\end{array}$ & Faunal Remains & Bone & Indeterminate & Bone & 1 & 0.01 & & \begin{tabular}{|l|l|}
$12 / 21 / 2017$ \\
\end{tabular} \\
\hline 535 & 31 & 657 & - & - & - & $\mathrm{c}$ & III & N1001 & E1003 & 4 & $99.4-99.36$ & 50-54 & $\begin{array}{l}\text { N1001.4 } \\
\text { E1003.9 }\end{array}$ & Faunal Remains & Bone & Indeterminate & Bone & 1 & 0.01 & & 12/21/2017 \\
\hline 535 & 32 & 657 & - & - & - & $\mathrm{c}$ & III & N1001 & E1003 & 4 & $99.4-99.36$ & 50-54 & $\begin{array}{l}\begin{array}{l}\text { N1001.4 } \\
\text { E1003.9 }\end{array} \\
\text { E }\end{array}$ & Faunal Remains & Bone & Indeterminate & Bone & 1 & 0.01 & & 12/21/2017 \\
\hline 535 & 33 & 657 & - & - & - & $\mathrm{c}$ & IIII & N1001 & E1003 & 4 & $999.4-99.36$ & $50-54$ & 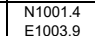 & \begin{tabular}{|l|} 
Faunal Remains \\
\end{tabular} & Bone & Indeterminate & Bone & 1 & 0.01 & & 12/21/2017 \\
\hline 535 & 34 & 657 & - & - & - & $\mathrm{c}$ & III & N1001 & E1003 & 4 & 99.4-99.36 & 50-54 & $\begin{array}{l}\text { N1001.4 } \\
\text { E1003.9 }\end{array}$ & \begin{tabular}{|l|} 
Faunal Remains \\
\end{tabular} & Bone & Indeterminate & Bone & 1 & 0.01 & & 12/21/2017 \\
\hline 535 & 35 & 657 & - & - & - & $\mathrm{c}$ & III & N1001 & E1003 & 4 & 99.4-99.36 & $50-54$ & $\begin{array}{l}\text { N10101.4 } \\
\text { E1003.9 }\end{array}$ & Faunal Remains & Bone & Indeterminate & Bone & 1 & 0.01 & & 12/21/2017 \\
\hline 535 & 36 & 657 & - & - & - & $\mathrm{c}$ & III & N1001 & E1003 & 4 & $99.4-99.36$ & $50-54$ & $\begin{array}{l}\text { N1001.4 } \\
\text { E1003.9 }\end{array}$ & Faunal Remains & Bone & Indeterminate & Bone & 1 & 0.01 & & 12/21/2017 \\
\hline 535 & 37 & 657 & - & - & - & $\mathrm{c}$ & III & N1001 & E1003 & 4 & $99.4-99.36$ & $50-54$ & $\begin{array}{l}\text { N1001.4 } \\
\text { E1003.9 }\end{array}$ & Faunal Remains & Bone & Indeterminate & Bone & 1 & 0.01 & & \begin{tabular}{|l|l|}
$12 / 21 / 2017$ \\
\end{tabular} \\
\hline 535 & 38 & 657 & - & - & - & $\mathrm{c}$ & III & N1001 & E1003 & 4 & 99.4-99.36 & 50-54 & $\begin{array}{l}\text { N1001.4 } \\
\text { E1003.9 }\end{array}$ & Faunal Remains & Bone & Indeterminate & Bone & 1 & 0.01 & & 12/21/2017 \\
\hline 535 & 39 & 657 & - & - & - & $\mathrm{c}$ & III & N1001 & E1003 & 4 & $99.4-99.36$ & 50-54 & $\begin{array}{l}\text { N1001.4 } \\
\text { E1003.9. }\end{array}$ & Faunal Remains & Bone & Indeterminate & Bone & 1 & 0.01 & & 12/21/2017 \\
\hline 535 & 40 & 657 & - & - & - & $\mathrm{c}$ & III & N1001 & E1003 & 4 & $99.4-99.36$ & $50-54$ & $\begin{array}{l}\text { N1001.4 } \\
\text { E1003.9 }\end{array}$ & Faunal Remains & Bone & Indeterminate & Bone & 1 & 0.01 & & 12/21/2017 \\
\hline 535 & 41 & 657 & - & - & - & $\mathrm{c}$ & III & N1001 & E1003 & 4 & 99.4-99.36 & 50-54 & $\begin{array}{l}\begin{array}{l}\text { N1001.4 } \\
\text { E1003.9 }\end{array} \\
\text { L }\end{array}$ & \begin{tabular}{|l|} 
Faunal Remains \\
\end{tabular} & Bone & Indeterminate & Bone & 1 & 0.01 & & \begin{tabular}{|l|l|}
$12 / 21 / 2017$ \\
\end{tabular} \\
\hline 536 & 1 & 572 & - & - & - & c & "I & N1001 & E1004 & 2 & $99.7-99.6$ & $20-30$ & - & Detritus & Debitage & Thermal Shatter & Lithic & 1 & 0.45 & & 12/18/2017 \\
\hline 537 & 1 & 576 & - & - & - & $\mathrm{c}$ & "I & N1001 & E1004 & 3 & 99.6-99.5 & $30-40$ & - & Faunal Remains & Bone & \begin{tabular}{|l|l} 
Indeterminate \\
\end{tabular} & Bone & 1 & 2.12 & & 12/18/2017 \\
\hline 537 & 2 & 576 & - & - & - & c & "I & N1001 & E1004 & 3 & $99.6-99.5$ & $30-40$ & - & Detritus & Debitage & Broken Flake & Lithic & 1 & 2.23 & & 12/18/2017 \\
\hline 537 & 3 & 576 & - & - & - & $\mathrm{c}$ & 11 & N1001 & E1004 & 3 & 99.6-99.5 & $30-40$ & - & Detritus & Debitage & Thermal Shatter & Lithic & 1 & 0.48 & & 12/18/2017 \\
\hline 538 & 1 & 579 & - & - & - & $\mathrm{c}$ & II/III & N1001 & E1004 & 4 & $99.5-99.4$ & $40-50$ & - & \begin{tabular}{|l|} 
Faunal Remains \\
\end{tabular} & Bone & \begin{tabular}{|l|} 
Tortoise/Turtle \\
\end{tabular} & Bone & 1 & 0.13 & & 12/18/2017 \\
\hline 538 & 2 & 579 & - & - & - & $\mathrm{c}$ & 11/111 & N1001 & E1004 & 4 & 99.5-99.4 & $40-50$ & - & Faunal Remains & Bone & Indeterminate & Bone & 1 & 0.59 & & 12/18/2017 \\
\hline 538 & 3 & 579 & - & - & - & $\mathrm{c}$ & 11/III & N1001 & E1004 & 4 & $99.5-99.4$ & $40-50$ & - & Detritus & Debitage & Complete Flake & Lithic & 2 & 0.86 & & $\mid 12 / 18 / 2017$ \\
\hline 538 & 4 & 579 & - & - & - & $\mathrm{c}$ & IIIIII & N1001 & E1004 & 4 & 99.5-99.4 & $40-50$ & - & Detritus & Debitage & \begin{tabular}{|l|l|} 
Broken Flake \\
\end{tabular} & Lithic & 3 & 0.45 & & 12/18/2017 \\
\hline 538 & 5 & 579 & - & - & - & $\mathrm{c}$ & II/III & N1001 & E1004 & 4 & $99.5-99.4$ & $40-50$ & - & Detritus & Debitage & \begin{tabular}{|l|} 
Thermal Shatter \\
\end{tabular} & Lithic & 8 & 6.2 & & 12/18/2017 \\
\hline 538 & 6 & 579 & - & - & - & $\mathrm{c}$ & 11/111 & N1001 & E1004 & 4 & 99.5-99.4 & $40-50$ & - & Clay & Clay Artifiact & Burned Clay & Clay & 2 & 2.83 & 2 fragments refit & 12/18/2017 \\
\hline 539 & 1 & 592 & - & - & - & $\mathrm{c}$ & IIII & N1001 & E1004 & 5 & $99.4-99.3$ & $50-60$ & - & Faunal Remains & Bone & Indeterminate & Bone & 1 & 0.77 & & 12/18/2017 \\
\hline 539 & 2 & 592 & - & - & - & $\mathrm{c}$ & III & N1001 & E1004 & 5 & $\begin{array}{l}99.4-99.3 \\
\end{array}$ & $50-60$ & - & Faunal Remains & Bone & Indeterminate & Bone & 1 & 0.79 & & 12/18/2017 \\
\hline 539 & 3 & 592 & - & - & - & $\mathrm{c}$ & IIII & N1001 & E1004 & 5 & $99.4-99.3$ & 50-60 & - & \begin{tabular}{|l|} 
Faunal Remains \\
\end{tabular} & Bone & Indeterminate & Bone & 1 & 0.05 & & 12/18/2017 \\
\hline 539 & 4 & 592 & - & - & - & $\mathrm{c}$ & III & N1001 & E1004 & 5 & $99.4-99.3$ & $50-60$ & - & \begin{tabular}{|l|} 
Faunal Remains \\
\end{tabular} & Bone & Indeterminate & Bone & 1 & 0.08 & & $\mid 12 / 18 / 2017$ \\
\hline
\end{tabular}




\begin{tabular}{|c|c|c|c|c|c|c|c|c|c|c|c|c|c|c|c|c|c|c|c|c|c|}
\hline Lot No. & \begin{tabular}{|c|}
$\begin{array}{c}\text { Specimen } \\
\text { No. }\end{array}$ \\
\end{tabular} & FS No. & UI No. & FTR No. & $\begin{array}{c}\text { Sample } \\
\text { No. }\end{array}$ & $\begin{array}{c}\text { Excavation } \\
\text { Block }\end{array}$ & Stratum (AU) & Northing & Easting & Level & $\begin{array}{c}\text { Elevation } \\
(\mathrm{m})\end{array}$ & Depth (cmbd) & Point Prov. & Artifact Class & Artifact Type & \begin{tabular}{|c|} 
Artifact \\
Description
\end{tabular} & Material & $\begin{array}{c}\text { Number of } \\
\text { artifacts }\end{array}$ & Weight (g) & Comments & Date \\
\hline 539 & 5 & 592 & - & - & - & $\mathrm{c}$ & III & N1001 & E1004 & 5 & 99.499 .3 & 50-60 & - & Faunal Remains & Bone & Indeterminate & Bone & 1 & 0.16 & & 12/18/2017 \\
\hline 539 & 6 & 592 & - & - & - & c & III & N1001 & E1004 & 5 & 99.4-99.3 & $50-60$ & - & Faunal Remains & Bone & Indeterminate & Bone & 1 & 0.06 & & 12/18/2017 \\
\hline 539 & 7 & 592 & - & - & - & c & IIII & N1001 & E1004 & 5 & 99.499 .3 & $50-60$ & - & \begin{tabular}{|l|} 
Faunal Remains \\
\end{tabular} & Bone & Indeterminate & Bone & 1 & 0.03 & & 12/18/2017 \\
\hline 539 & 8 & 592 & - & - & - & c & IIII & N1001 & E1004 & 5 & 99.4-99.3 & $50-60$ & - & Faunal Remains & Bone & Indeterminate & Bone & 1 & 0.09 & & $\mid$\begin{tabular}{|l|}
$\mid 12 / 18 / 2017$ \\
\end{tabular} \\
\hline 539 & 9 & 592 & - & - & - & c & IIII & N1001 & E1004 & 5 & 99.4-99.3 & $50-60$ & - & Faunal Remains & Bone & Indeterminate & Bone & 1 & 0.03 & & $\mid$\begin{tabular}{|l|}
$\mid 12 / 18 / 2017$ \\
\end{tabular} \\
\hline 539 & 10 & 592 & - & - & - & c & IIII & N1001 & E1004 & 5 & $99.4-99.3$ & $50-60$ & - & \begin{tabular}{|l|} 
Faunal Remains \\
\end{tabular} & Bone & Indeterminate & Bone & 1 & 0.01 & & 12/18/2017 \\
\hline 539 & 11 & 592 & - & - & - & c & IIII & N1001 & E1004 & 5 & 99.4-99.3 & 50-60 & - & \begin{tabular}{|l|} 
Faunal Remains \\
\end{tabular} & Bone & Indeterminate & Bone & 1 & 0.01 & & 12/18/2017 \\
\hline 539 & 12 & 592 & - & - & - & c & III & N1001 & E1004 & 5 & 99.4-99.3 & 50-60 & - & Faunal Remains & Bone & Indeterminate & Bone & 1 & 0.01 & & $\mid$\begin{tabular}{|l|}
$\mid 12 / 18 / 2017$ \\
\end{tabular} \\
\hline 539 & 13 & 592 & - & - & - & c & IIII & N1001 & E1004 & 5 & 99.499 .3 & $50-60$ & - & \begin{tabular}{|l|} 
Faunal Remains \\
\end{tabular} & Bone & Indeterminate & Bone & 1 & 0.01 & & $\mid$\begin{tabular}{|l|}
$\mid 12 / 18 / 2017$ \\
\end{tabular} \\
\hline 539 & 14 & 592 & - & - & - & $\mathrm{c}$ & IIII & N1001 & E1004 & 5 & 99.499 .3 & 50-60 & - & \begin{tabular}{|l|} 
Faunal Remains \\
\end{tabular} & Bone & Indeterminate & Bone & 1 & 0.06 & & $\mid 12 / 18 / 2017$ \\
\hline 539 & 15 & 592 & - & - & - & c & IIII & N1001 & E1004 & 5 & 99.499 .3 & $50-60$ & - & Faunal Remains & Bone & Indeterminate & Bone & 1 & 0.07 & & 12/18/2017 \\
\hline 539 & 16 & 592 & - & - & - & $\mathrm{c}$ & IIII & N1001 & E1004 & 5 & $\begin{array}{l}99.4-99.3 \\
\end{array}$ & 50-60 & - & Detritus & Debitage & Broken Flake & Lithic & 2 & 0.46 & & 12/18/2017 \\
\hline 539 & 17 & 592 & - & - & - & c & IIII & N1001 & E1004 & 5 & $\begin{array}{l}99.499 .3 \\
\end{array}$ & 50-60 & - & Detritus & Debitage & Thermal Shatter & Lithic & 12 & 16.39 & & \begin{tabular}{|l|}
$22 / 18 / 2017$ \\
\end{tabular} \\
\hline 539 & 18 & 592 & - & - & - & $\mathrm{c}$ & IIII & N1001 & E1004 & 5 & 99.4-99.3 & $50-60$ & - & Informal Tool & Modified Flake & Edge-modified & Fine Grain Chert & 1 & 1.06 & & $\mid 12 / 18 / 2017$ \\
\hline 539 & 19 & 592 & - & - & - & c & IIII & N1001 & E1004 & 5 & 99.499 .3 & $50-60$ & - & Faunal Remains & Shell & Mussel Shell & Shell & 1 & 2.2 & & 12/18/2017 \\
\hline 540 & 1 & 542 & - & - & - & c & "I & N1001 & E1004.5 & 2 & $99.7-99.6$ & $20-30$ & - & Faunal Remains & Bone & Indeterminate & Bone & 1 & 0.01 & & 12/14/2017 \\
\hline 540 & 2 & 542 & - & - & - & c & "I & N1001 & E1004.5 & 2 & 99.7-99.6 & $20-30$ & - & Detritus & Debitage & Proximal Flake & Lithic & 1 & 0.21 & & \begin{tabular}{|l|}
$12 / 14 / 2017$ \\
\end{tabular} \\
\hline 540 & 3 & 542 & - & - & - & c & " & N1001 & E1004.5 & 2 & $99.7-99.6$ & $20-30$ & - & Detritus & Debitage & Broken Flake & Lithic & 1 & 0.21 & & 12/14/2017 \\
\hline 540 & 4 & 542 & - & - & - & c & 11 & N1001 & E1004.5 & 2 & $99.7-99.6$ & $20-30$ & - & Detritus & Debitage & Flaking Shatter & Lithic & 1 & 4.48 & & 12/14/2017 \\
\hline 540 & 5 & 542 & - & - & - & c & "I & N1001 & E1004.5 & 2 & $99.7-99.6$ & $20-30$ & - & Detritus & Debitage & Thermal Shatter & Lithic & 2 & 0.61 & & 12/14/2017 \\
\hline 540 & 6 & 542 & - & - & - & c & 11 & N1001 & E1004.5 & 2 & $99.7-99.6$ & $20-30$ & - & \begin{tabular}{|l|} 
Historic Artifiact \\
\end{tabular} & Metal & \begin{tabular}{|c|}
$\begin{array}{c}\text { Miscellaneous } \\
\text { Metal }\end{array}$ \\
\end{tabular} & Metal & 1 & 0.15 & & $\mid 12 / 14 / 2017$ \\
\hline 541 & 1 & 544 & - & - & - & c & " & N1001 & E1004.5 & 3 & $99.6-99.5$ & $30-40$ & - & Detritus & Debitage & Complete Flake & Lithic & 1 & 1.59 & & 12/15/2017 \\
\hline 541 & 2 & 544 & - & - & - & c & "I & N1001 & E1004.5 & 3 & $99.6-99.5$ & $30-40$ & - & Detritus & Debitage & Broken Flake & Lithic & 5 & 2.98 & & 12/15/2017 \\
\hline 541 & 3 & 544 & - & - & - & c & "I & N1001 & E1004.5 & 3 & $99.6-99.5$ & $30-40$ & - & Detritus & Debitage & Flaking Shatter & Lithic & 1 & 0.75 & & $\mid 12 / 15 / 2017$ \\
\hline 541 & 4 & 544 & - & - & - & c & 11 & N1001 & E1004.5 & 3 & $99.6-99.5$ & $30-40$ & - & Detritus & Debitage & Thermal Shatter & Lithic & 2 & 4.35 & & 12/15/2017 \\
\hline 542 & 1 & 546 & - & - & - & c & 11/III & N1001 & $E 1004.5$ & 4 & $99.5-99.4$ & $40-50$ & - & \begin{tabular}{|l|} 
Faunal Remains \\
\end{tabular} & Bone & Mammal & Bone & 1 & 0.46 & & 12/15/2017 \\
\hline 542 & 2 & 546 & - & - & - & c & "1/III & N1001 & $E 1004.5$ & 4 & $99.5-99.4$ & $40-50$ & - & \begin{tabular}{|l|} 
Faunal Remains \\
\end{tabular} & Bone & Mammal & Bone & 1 & 0.82 & & 12/15/2017 \\
\hline 542 & 3 & 546 & - & - & - & c & "1/III & N1001 & $E 1004.5$ & 4 & $99.5-99.4$ & $40-50$ & - & \begin{tabular}{|l|} 
Faunal Remains \\
\end{tabular} & Bone & Mammal & Bone & 1 & 2.26 & & 12/15/2017 \\
\hline 542 & 4 & 546 & - & - & - & c & II/III & N1001 & $E 1004.5$ & 4 & $99.5-99.4$ & $40-50$ & - & \begin{tabular}{|l|} 
Faunal Remains \\
\end{tabular} & Bone & Mammal & Bone & 1 & 1.2 & & 12/15/2017 \\
\hline 542 & 5 & 546 & - & - & - & c & 11/III & N1001 & E1004.5 & 4 & $99.5-99.4$ & $40-50$ & - & \begin{tabular}{|l|} 
Faunal Remains \\
\end{tabular} & Bone & Indeterminate & Bone & 1 & 0.32 & & 12/15/2017 \\
\hline 542 & 6 & 546 & - & - & - & c & 11/111 & N1001 & E1004.5 & 4 & 99.5-99.4 & $40-50$ & - & Faunal Remains & Bone & Mammal & Bone & 1 & 0.48 & & $\mid 12 / 15 / 2017$ \\
\hline 542 & 7 & 546 & - & - & - & c & 11/III & N1001 & E1004.5 & 4 & $99.5-99.4$ & $40-50$ & - & Faunal Remains & Bone & Indeterminate & Bone & 1 & 0.29 & & 12/15/2017 \\
\hline 542 & 8 & 546 & - & - & - & c & 11/III & N1001 & E1004.5 & 4 & $99.5-99.4$ & $40-50$ & - & Faunal Remains & Bone & Indeterminate & Bone & 1 & 0.16 & & 12/15/2017 \\
\hline 542 & 9 & 546 & - & - & - & $\mathrm{c}$ & 11/111 & N1001 & E1004.5 & 4 & 99.5-99.4 & $40-50$ & - & Faunal Remains & Bone & Indeterminate & Bone & 1 & 0.66 & & \begin{tabular}{|l|}
$12 / 15 / 2017$ \\
\end{tabular} \\
\hline 542 & 10 & 546 & - & - & - & $\mathrm{c}$ & II/III & N1001 & E1004.5 & 4 & $99.5-99.4$ & $40-50$ & - & \begin{tabular}{|l|} 
Faunal Remains \\
\end{tabular} & Bone & Odocoileus sp. & Bone & 1 & 0.54 & & 12/15/2017 \\
\hline 542 & 11 & 546 & - & - & - & $\mathrm{c}$ & $\|/\| 1 \|$ & N1001 & E1004.5 & 4 & $99.5-99.4$ & $40-50$ & - & Detritus & Debitage & \begin{tabular}{|l|} 
Complete Flake \\
\end{tabular} & Lithic & 4 & 2.71 & & 12/15/2017 \\
\hline 542 & 12 & 546 & - & - & - & c & 11/III & N1001 & E1004.5 & 4 & $99.5-99.4$ & $40-50$ & - & Detritus & Debitage & Proximal Flake & Lithic & 5 & 3.82 & & $12 / 15 / 2017$ \\
\hline 542 & 13 & 546 & - & - & - & $\mathrm{c}$ & 11/111 & N1001 & $E 1004.5$ & 4 & 99.5-99.4 & $40-50$ & - & Detritus & Debitage & Broken Flake & Lithic & 11 & 10.15 & & \begin{tabular}{|l|}
$12 / 15 / 2017$ \\
\end{tabular} \\
\hline 542 & 14 & 546 & - & - & - & $\mathrm{c}$ & "1/\|!" & N1001 & E1004.5 & 4 & $99.5-99.4$ & $40-50$ & - & Detritus & Debitage & Thermal Shatter & Lithic & 23 & 27.54 & & 12/15/2017 \\
\hline 542 & 15 & 546 & - & - & - & c & 11/111 & N1001 & E1004.5 & 4 & $99.5-99.4$ & $40-50$ & - & Other Lithic & $\begin{array}{l}\text { Thermally } \\
\text { Altered Stone }\end{array}$ & FCR & Lithic & 1 & 81.13 & & \begin{tabular}{|l|l|}
$12 / 15 / 2017$ \\
\end{tabular} \\
\hline 542 & 16 & 546 & - & - & - & c & 11/III & N1001 & E1004.5 & 4 & 99.5-99.4 & $40-50$ & - & Faunal Remains & Shell & Mussel Shell & Shell & 1 & 1.58 & & \begin{tabular}{|l|}
$12 / 15 / 2017$ \\
\end{tabular} \\
\hline 543 & 1 & 560 & - & - & - & c & IIII & N1001 & E1004.5 & 5 & 99.499 .3 & $50-60$ & - & Faunal Remains & Bone & Indeterminate & Bone & 1 & 1.75 & & 12/15/2017 \\
\hline 543 & 2 & 560 & - & - & - & $\mathrm{c}$ & IIII & N1001 & E1004.5 & 5 & $\begin{array}{l}99.4-99.3 \\
\end{array}$ & 50-60 & - & \begin{tabular}{|l|} 
Faunal Remains \\
\end{tabular} & Bone & Indeterminate & Bone & 1 & 0.33 & & 12/15/2017 \\
\hline 543 & 3 & 560 & - & - & - & c & III & N1001 & E1004.5 & 5 & 99.4-99.3 & $50-60$ & - & \begin{tabular}{|l|} 
Faunal Remains \\
\end{tabular} & Bone & Indeterminate & Bone & 1 & 0.21 & & 12/15/2017 \\
\hline
\end{tabular}




\begin{tabular}{|c|c|c|c|c|c|c|c|c|c|c|c|c|c|c|c|c|c|c|c|c|c|}
\hline Lot No. & $\begin{array}{c}\text { Specimen } \\
\text { No. }\end{array}$ & FS No. & UI No. & FTR No. & $\begin{array}{l}\text { Sample } \\
\text { No. }\end{array}$ & $\begin{array}{c}\text { Excavation } \\
\text { Block }\end{array}$ & Stratum (AU) & Northing & Easting & Level & $\begin{array}{c}\begin{array}{c}\text { Elevation } \\
(\mathrm{m})\end{array} \\
\end{array}$ & Depth (cmbd) & \begin{tabular}{|l|} 
Point Prov. \\
\end{tabular} & Artifact Class & Artifact Type & \begin{tabular}{|c|}
$\begin{array}{c}\text { Artifact } \\
\text { Description }\end{array}$ \\
\end{tabular} & Material & $\begin{array}{c}\text { Number of } \\
\text { artifacts }\end{array}$ & Weight (g) & Comments & Date \\
\hline 543 & \begin{tabular}{|l|} 
\\
\end{tabular} & 560 & - & - & - & c & III & N1001 & E1004.5 & 5 & $99.4-99.3$ & 50-60 & - & Detritus & Debitage & \begin{tabular}{|l|} 
Proximal Flake \\
\end{tabular} & Lithic & 1 & 0.04 & & 12/15/2017 \\
\hline 543 & 5 & 560 & - & - & - & c & III & N1001 & E1004.5 & 5 & 99.4-99.3 & 50-60 & - & Detritus & Debitage & Broken Flake & Lithic & 6 & 6.56 & & $\mid 12 / 15 / 2017$ \\
\hline 543 & 6 & 560 & - & - & - & c & III & N1001 & $E 1004.5$ & 5 & $99.4-99.3$ & $50-60$ & - & Detritus & Debitage & Thermal Shatter & Lithic & 13 & 44.02 & & 12/15/2017 \\
\hline 544 & 1 & 325 & - & - & - & D & $1 / 11$ & N1001 & E1009 & 1 & 99.60-99.49 & $9-20$ & - & Detritus & Debitage & Thermal Shatter & Lithic & 1 & 0.76 & & $\mid$\begin{tabular}{|l|}
$\mid 11 / 28 / 2017$ \\
\end{tabular} \\
\hline 545 & 1 & 327 & - & - & - & D & $1 / 11$ & N1001 & E1009 & 2 & 99.49-99.39 & $20-30$ & - & Detritus & Debitage & Proximal Flake & Lithic & 1 & 1.99 & & 11/28/2017 \\
\hline 545 & 2 & 327 & - & - & - & D & 1/11 & N1001 & E1009 & 2 & 99.49-99.39 & $20-30$ & - & Detritus & Debitage & Broken Flake & Lithic & 2 & 0.92 & & 11/28/2017 \\
\hline 545 & 3 & 327 & - & - & - & D & 1/11 & N1001 & E1009 & 2 & |99.49-99.39 & $20-30$ & - & Detritus & Debitage & Flaking Shatter & Lithic & 1 & 0.16 & & $\mid$\begin{tabular}{|l|}
$11 / 28 / 2017$ \\
\end{tabular} \\
\hline 546 & 1 & 327 & - & - & - & D & 1111 & N1001 & E1009 & 2 & 99.40 & 21 & $\begin{array}{l}\text { N1001.30 } \\
\text { E1009.98 }\end{array}$ & Detritus & Core & Bifacial & Fine Grain Chert & 1 & 100.34 & possible tested cobble & $\mid$\begin{tabular}{|l|}
$\mid 11 / 28 / 2017$ \\
\end{tabular} \\
\hline 547 & 1 & 330 & - & - & - & D & $1 / 11$ & N1001 & E1009 & 3 & 99.39-99.29 & $30-40$ & - & Detritus & Debitage & Proximal Flake & Lithic & 2 & 7.21 & & 11/28/2017 \\
\hline 547 & 2 & 330 & - & - & - & D & 1/11 & N1001 & E1009 & 3 & \begin{tabular}{|l|l|}
$99.39-99.29$ \\
\end{tabular} & $30-40$ & - & Detritus & Debitage & Broken Flake & Lithic & 5 & 5.07 & & 11/28/2017 \\
\hline 547 & 3 & 330 & - & - & - & $\mathrm{D}$ & 1/11 & N1001 & E1009 & 3 & \begin{tabular}{|l|l|}
$99.39-99.29$ \\
\end{tabular} & $30-40$ & - & Detritus & Debitage & Thermal Shatter & Lithic & 7 & 14.98 & & 11/28/2017 \\
\hline 547 & 4 & 330 & - & - & - & D & $1 / 11$ & N1001 & E1009 & 3 & \begin{tabular}{|l|}
$99.39-99.29$ \\
\end{tabular} & $30-40$ & - & \begin{tabular}{l|l} 
Informal Tool \\
\end{tabular} & Modified Flake & Edge-modified & Fine Grain Chert & 1 & 2.29 & & \begin{tabular}{|l|}
$11 / 28 / 2017$ \\
\end{tabular} \\
\hline 547 & 5 & 330 & - & - & - & $\mathrm{D}$ & $1 / 1 / 1$ & N1001 & E1009 & 3 & \begin{tabular}{|l|l|}
$99.39-99.29$ \\
\end{tabular} & $30-40$ & - & Faunal Remains & Shell & Mussel Shell & Shell & 1 & 4.98 & & 11/28/2017 \\
\hline 548 & 1 & 333 & - & - & - & D & $1 / 11$ & N1001 & E1009 & 4 & |99.29-99.19 & 40-50 & - & Faunal Remains & Bone & Tortoise/Turtle & Bone & 1 & 6.86 & & 11/28/2017 \\
\hline 548 & 2 & 333 & - & - & - & $\mathrm{D}$ & $1 / 11$ & N1001 & E1009 & 4 & \begin{tabular}{|l|}
$99.29-99.19$ \\
\end{tabular} & $40-50$ & - & Faunal Remains & Bone & Tortoise/Turtle & Bone & 1 & 0.97 & & 11/28/2017 \\
\hline 548 & 3 & 333 & - & - & - & D & 1/111 & N1001 & E1009 & 4 & \begin{tabular}{|l|l|}
$99.29-99.19$ \\
\end{tabular} & $40-50$ & - & \begin{tabular}{|l|} 
Faunal Remains \\
\end{tabular} & Bone & \begin{tabular}{|l|} 
Tortoise/Turtle \\
\end{tabular} & Bone & 1 & 0.47 & & 11128/2017 \\
\hline 548 & 4 & 333 & - & - & - & D & 1/11 & N1001 & E1009 & 4 & \begin{tabular}{|l|l|}
$99.29-99.19$ \\
\end{tabular} & $40-50$ & - & Faunal Remains & Bone & \begin{tabular}{|l|} 
Tortoise/Turtle \\
\end{tabular} & Bone & 1 & 0.36 & & 11/28/2017 \\
\hline 548 & 5 & 333 & - & - & - & D & 1/11 & N1001 & E1009 & 4 & 99.29-99.19 & $40-50$ & - & Faunal Remains & Bone & Tortoise/Turtle & Bone & 1 & 0.46 & & 11/28/2017 \\
\hline 548 & 6 & 333 & - & - & - & D & 1/11 & N1001 & E1009 & 4 & \begin{tabular}{|l|}
$99.29-99.19$ \\
\end{tabular} & $40-50$ & - & Faunal Remains & Bone & Tortoise/Turtle & Bone & 1 & 0.22 & & 11/28/2017 \\
\hline 548 & 7 & 333 & - & - & - & D & 1/11 & N1001 & E1009 & 4 & \begin{tabular}{|l|}
$99.29-99.19$ \\
\end{tabular} & $40-50$ & - & Faunal Remains & Bone & Indeterminate & Bone & 1 & 0.46 & & 11/28/2017 \\
\hline 548 & 8 & 333 & - & - & - & D & 1/11 & N1001 & E1009 & 4 & \begin{tabular}{|l|}
$99.29-99.19$ \\
\end{tabular} & $40-50$ & - & Faunal Remains & Bone & Tortoise/Turle & Bone & 1 & 0.15 & & 11/28/2017 \\
\hline 548 & 9 & 333 & - & - & - & D & 1/11 & N1001 & E1009 & 4 & \begin{tabular}{|l|}
$99.29-99.19$ \\
\end{tabular} & $40-50$ & - & Faunal Remains & Bone & Indeterminate & Bone & 1 & 0.12 & & 11/28/2017 \\
\hline 548 & 10 & 333 & - & - & - & D & 1/11 & N1001 & E1009 & 4 & \begin{tabular}{|l|}
$99.29-99.19$ \\
\end{tabular} & $40-50$ & - & Faunal Remains & Bone & Indeterminate & Bone & 1 & 0.21 & & 11/28/2017 \\
\hline 548 & 11 & 333 & - & - & - & D & 1/11 & N1001 & E1009 & 4 & 99.29-99.19 & $40-50$ & - & Faunal Remains & Bone & Indeterminate & Bone & 1 & 0.2 & & 11/28/2017 \\
\hline 548 & 12 & 333 & - & - & - & D & 1/11 & N1001 & E1009 & 4 & \begin{tabular}{|l|}
$99.29-99.19$ \\
\end{tabular} & $40-50$ & - & Detritus & Debitage & Complete Flake & Lithic & 5 & 8.37 & & 11/28/2017 \\
\hline 548 & 13 & 333 & - & - & - & D & 1/11 & N1001 & E1009 & 4 & \begin{tabular}{|l|}
$99.29-99.19$ \\
\end{tabular} & $40-50$ & - & Detritus & Debitage & \begin{tabular}{|l|} 
Proximal Flake \\
\end{tabular} & Lithic & 14 & 8.79 & & 11/28/2017 \\
\hline 548 & 14 & 333 & - & - & - & D & 1/111 & N1001 & E1009 & 4 & 99.29-99.19 & $40-50$ & - & Detritus & Debitage & Broken Flake & Lithic & 8 & 7.35 & & 11/28/2017 \\
\hline 548 & 15 & 333 & - & - & - & D & 1/111 & N1001 & E1009 & 4 & 99.29-99.19 & $40-50$ & - & Detritus & Debitage & Thermal Shatter & Lithic & 8 & 8.61 & & 111/28/2017 \\
\hline 549 & $\begin{array}{c}1 \\
\begin{array}{c}\text { radiocarbo } \\
\text { n dated }\end{array}\end{array}$ & 337 & - & - & - & D & 1/11 & N1001 & E1009 & 5 & 99.15 & 54 & \begin{tabular}{l|} 
N1001.07 \\
E1009.42
\end{tabular} & Faunal Remains & Bone & Mammal & Bone & 1 & 108.9 & "sent for radiocarbon dating & 11/28/2017 \\
\hline 550 & 1 & 338 & - & - & - & D & $1 / 11$ & N1001 & E1009 & 5 & \begin{tabular}{|l|}
$99.19-99.09$ \\
\end{tabular} & $50-60$ & - & Faunal Remains & Bone & Indeterminate & Bone & 1 & 0.17 & & 11/28/2017 \\
\hline 550 & 2 & 338 & - & - & - & D & 1/11 & N1001 & E1009 & 5 & 99.19-99.09 & $50-60$ & - & Faunal Remains & Bone & Mammal & Bone & 1 & 0.92 & & |11/28/2017 \\
\hline 550 & 3 & 338 & - & - & - & D & 1/11 & N1001 & E1009 & 5 & \begin{tabular}{|l|}
$99.19-99.09$ \\
\end{tabular} & $50-60$ & - & Faunal Remains & Bone & Mammal & Bone & 1 & 0.63 & & 11/28/2017 \\
\hline 550 & 4 & 338 & - & - & - & D & 1/11 & N1001 & E1009 & 5 & \begin{tabular}{|l|}
$99.19-99.09$ \\
\end{tabular} & $50-60$ & - & Faunal Remains & Bone & Mammal & Bone & 1 & 0.64 & & 11/28/2017 \\
\hline 550 & 5 & 338 & - & - & - & D & 1/11 & N1001 & E1009 & 5 & \begin{tabular}{|l|l|}
$99.19-99.09$ \\
\end{tabular} & $50-60$ & - & Faunal Remains & Bone & Mammal & Bone & 1 & 1.67 & & 11/28/2017 \\
\hline 550 & 6 & 338 & - & - & - & D & 1/11 & N1001 & E1009 & 5 & \begin{tabular}{|l|}
$99.19-99.09$ \\
\end{tabular} & $50-60$ & - & Faunal Remains & Bone & Mammal & Bone & 1 & 0.89 & & 11/28/2017 \\
\hline 550 & 7 & 338 & - & - & - & D & 1/11 & N1001 & E1009 & 5 & \begin{tabular}{|l|}
$99.19-99.09$ \\
\end{tabular} & $50-60$ & - & Faunal Remains & Bone & Mammal & Bone & 1 & 1.61 & & 11/28/2017 \\
\hline 550 & 8 & 338 & - & - & - & D & $1 / 11$ & N1001 & E1009 & 5 & 99.19-99.09 & $50-60$ & - & \begin{tabular}{|l|} 
Faunal Remains \\
\end{tabular} & Bone & Mammal & Bone & 1 & 0.81 & & 11/28/2017 \\
\hline 550 & 9 & 338 & - & - & - & D & 1/11 & N1001 & E1009 & 5 & |99.19-99.09 & $50-60$ & - & Faunal Remains & Bone & Mammal & Bone & 1 & 0.49 & & $\mid 11 / 28 / 2017$ \\
\hline 550 & 10 & 338 & - & - & - & D & 1/11 & N1001 & E1009 & 5 & \begin{tabular}{|l|l|}
$99.19-99.09$ \\
\end{tabular} & $50-60$ & - & Faunal Remains & Bone & Mammal & Bone & 1 & 0.52 & & 11/28/2017 \\
\hline 550 & 11 & 338 & - & - & - & D & 1/111 & N1001 & E1009 & 5 & 99.19-99.09 & $50-60$ & - & Faunal Remains & Bone & Mammal & Bone & 1 & 0.81 & & 11/28/2017 \\
\hline 550 & 12 & 338 & - & - & - & D & 1/11 & N1001 & E1009 & 5 & 99.19-99.09 & $50-60$ & - & Faunal Remains & Bone & Mammal & Bone & 1 & 0.39 & & $\mid$\begin{tabular}{|l|}
$\mid 11 / 28 / 2017$ \\
\end{tabular} \\
\hline 550 & 13 & 338 & - & - & - & D & 1/11 & N1001 & E1009 & 5 & 99.19-99.09 & $50-60$ & - & Faunal Remains & Bone & Mammal & Bone & 1 & 0.26 & & 11/28/2017 \\
\hline 550 & 14 & 338 & - & - & - & D & 1/11 & N1001 & E1009 & 5 & 99.19-99.09 & $50-60$ & - & Faunal Remains & Bone & Mammal & Bone & 1 & 0.19 & & 11/28/2017 \\
\hline 550 & 15 & 338 & - & - & - & D & $1 / 11$ & N1001 & E1009 & 5 & \begin{tabular}{|l|}
$99.19-99.09$ \\
\end{tabular} & $50-60$ & - & Faunal Remains & Bone & Mammal & Bone & 1 & 0.14 & & 11/28/2017 \\
\hline
\end{tabular}




\begin{tabular}{|c|c|c|c|c|c|c|c|c|c|c|c|c|c|c|c|c|c|c|c|c|c|}
\hline Lot No. & \begin{tabular}{|c|}
$\begin{array}{c}\text { Specimen } \\
\text { No. }\end{array}$ \\
\end{tabular} & FS No. & UI No. & FTR No. & $\begin{array}{c}\text { Sample } \\
\text { No. }\end{array}$ & $\begin{array}{c}\text { Excavation } \\
\text { Block }\end{array}$ & Stratum (AU) & Northing & Easting & Level & $\begin{array}{c}\begin{array}{c}\text { Elevation } \\
(\mathrm{m})\end{array} \\
\end{array}$ & Depth (cmbd) & Point Prov. & Artifiact Class & \begin{tabular}{|l|} 
Artifact Type \\
\end{tabular} & $\begin{array}{c}\text { Artifact } \\
\text { Description }\end{array}$ & Material & $\begin{array}{c}\text { Number of } \\
\text { artifacts }\end{array}$ & Weight (g) & Comments & Date \\
\hline 550 & 16 & 338 & - & - & - & $D$ & $1 / 11$ & N1001 & E1009 & 5 & \begin{tabular}{|l|l|}
$99.19-99.09$ \\
\end{tabular} & 50-60 & - & Faunal Remains & Bone & Mammal & Bone & 1 & 0.35 & & 11/28/2017 \\
\hline 550 & 17 & 338 & - & - & - & D & $1 / 11$ & N1001 & E1009 & 5 & 99.19-99.09 & $50-60$ & - & Faunal Remains & Bone & Mammal & Bone & 1 & 0.31 & & 11/28/2017 \\
\hline 550 & 18 & 338 & - & - & - & D & 1/11 & N1001 & E1009 & 5 & 99.19-99.09 & $50-60$ & - & Faunal Remains & Bone & Mammal & Bone & 1 & 0.15 & & 11/28/2017 \\
\hline 550 & 19 & 338 & - & - & - & D & 1/11 & N1001 & E1009 & 5 & 99.19-99.09 & $50-60$ & - & Faunal Remains & Bone & Mammal & Bone & 1 & 0.12 & & 11/28/2017 \\
\hline 550 & 20 & 338 & - & - & - & D & I/III & N1001 & E1009 & 5 & 99.19-99.09 & $50-60$ & - & Faunal Remains & Bone & Mammal & Bone & 1 & 0.21 & & 11/28/2017 \\
\hline 550 & 21 & 338 & - & - & - & D & $1 / 11$ & N1001 & E1009 & 5 & 99.19-99.09 & $50-60$ & - & Faunal Remains & Bone & Mammal & Bone & 1 & 0.26 & & 11/28/2017 \\
\hline 550 & 22 & 338 & - & - & - & D & 1/11 & N1001 & E1009 & 5 & 99.19-99.09 & 50-60 & - & Faunal Remains & Bone & Mammal & Bone & 1 & 0.42 & & 11/28/2017 \\
\hline 550 & 23 & 338 & - & - & - & D & 1111 & N1001 & E1009 & 5 & 99.19-99.09 & 50-60 & - & Faunal Remains & Bone & Mammal & Bone & 1 & 0.11 & & $\mid$\begin{tabular}{|l|}
$\mid 11 / 28 / 2017$ \\
\end{tabular} \\
\hline 550 & 24 & 338 & - & - & - & D & I/II & N1001 & E1009 & 5 & 99.19-99.09 & $50-60$ & - & Faunal Remains & Bone & Mammal & Bone & 1 & 0.23 & & $\mid$\begin{tabular}{|l|}
$\mid 11 / 28 / 2017$ \\
\end{tabular} \\
\hline 550 & 25 & 338 & - & - & - & $\mathrm{D}$ & 1/11 & N1001 & E1009 & 5 & \begin{tabular}{|l|l|}
$99.19-99.09$ \\
\end{tabular} & 50-60 & - & Faunal Remains & Bone & Mammal & Bone & 1 & 0.25 & & 11/28/2017 \\
\hline 550 & 26 & 338 & - & - & - & $\mathrm{D}$ & 1/11 & N1001 & E1009 & 5 & \begin{tabular}{|l|l|}
$99.19-99.09$ \\
\end{tabular} & $50-60$ & - & Faunal Remains & Bone & Mammal & Bone & 1 & 0.16 & & \begin{tabular}{|l|}
$11 / 28 / 2017$ \\
\end{tabular} \\
\hline 550 & 27 & 338 & - & - & - & $\mathrm{D}$ & 1/11 & N1001 & E1009 & 5 & \begin{tabular}{|l|l|}
$99.19-99.09$ \\
\end{tabular} & 50-60 & - & Faunal Remains & Bone & Mammal & Bone & 1 & 0.11 & & 11/28/2017 \\
\hline 550 & 28 & 338 & - & - & - & $\mathrm{D}$ & $1 / 1 / 1$ & N1001 & E1009 & 5 & \begin{tabular}{|l|l|}
$99.19-99.09$ \\
\end{tabular} & 50-60 & - & Faunal Remains & Bone & Mammal & Bone & 1 & 0.13 & & 11/28/2017 \\
\hline 550 & 29 & 338 & - & - & - & $\mathrm{D}$ & $1 / 111$ & N1001 & E1009 & 5 & |99.19-99.09 & $50-60$ & - & Faunal Remains & Bone & Mammal & Bone & 1 & 0.31 & & $\mid 11 / 28 / 2017$ \\
\hline 550 & 30 & 338 & - & - & - & D & 1/11 & N1001 & E1009 & 5 & \begin{tabular}{|l|l|}
$99.19-99.09$ \\
\end{tabular} & $50-60$ & - & Faunal Remains & Bone & Mammal & Bone & 1 & 0.15 & & \begin{tabular}{|l|}
$11 / 28 / 2017$ \\
\end{tabular} \\
\hline 550 & 31 & 338 & - & - & - & D & 1/11 & N1001 & E1009 & 5 & \begin{tabular}{|l|l|}
$99.19-99.09$ \\
\end{tabular} & 50-60 & - & Faunal Remains & Bone & Mammal & Bone & 1 & 0.11 & & \begin{tabular}{|l|}
$11 / 28 / 2017$ \\
\end{tabular} \\
\hline 550 & 32 & 338 & - & - & - & D & $1 / 11$ & N1001 & E1009 & 5 & \begin{tabular}{|l|l|}
$99.19-99.09$ \\
\end{tabular} & $50-60$ & - & Faunal Remains & Bone & Mammal & Bone & 1 & 0.25 & & 11/28/2017 \\
\hline 550 & 33 & 338 & - & - & - & D & $1 / 1 / 1$ & N1001 & E1009 & 5 & \begin{tabular}{|l|l|}
$99.19-99.09$ \\
\end{tabular} & $50-60$ & - & Faunal Remains & Bone & Mammal & Bone & 1 & 0.13 & & 11/28/2017 \\
\hline 550 & 34 & 338 & - & - & - & D & $1 / 11$ & N1001 & E1009 & 5 & \begin{tabular}{|l|}
$99.19-99.09$ \\
\end{tabular} & $50-60$ & - & Faunal Remains & Bone & Mammal & Bone & 1 & 0.14 & & 11/28/2017 \\
\hline 550 & 35 & 338 & - & - & - & $\mathrm{D}$ & 1/111 & N1001 & E1009 & 5 & \begin{tabular}{|l|}
$99.19-99.09$ \\
\end{tabular} & 50-60 & - & Faunal Remains & Bone & Mammal & Bone & 1 & 0.11 & & 11/28/2017 \\
\hline 550 & 36 & 338 & - & - & - & D & 1/11 & N1001 & E1009 & 5 & \begin{tabular}{|l|}
$99.19-99.09$ \\
\end{tabular} & $50-60$ & - & Faunal Remains & Bone & Mammal & Bone & 1 & 0.13 & & \begin{tabular}{|l|}
$11 / 28 / 2017$ \\
\end{tabular} \\
\hline 550 & 37 & 338 & - & - & - & $\mathrm{D}$ & $1 / 1 / 1$ & N1001 & E1009 & 5 & \begin{tabular}{|l|}
$99.19-99.09$ \\
\end{tabular} & $50-60$ & - & Faunal Remains & Bone & Mammal & Bone & 1 & 0.16 & & 11/28/2017 \\
\hline 550 & 38 & 338 & - & - & - & D & 1/11 & N1001 & E1009 & 5 & \begin{tabular}{|l|}
$99.19-99.09$ \\
\end{tabular} & $50-60$ & - & Faunal Remains & Bone & Mammal & Bone & 1 & 0.19 & & 11/28/2017 \\
\hline 550 & 39 & 338 & - & - & - & D & 1/11 & N1001 & E1009 & 5 & 99.19-99.09 & $50-60$ & - & Faunal Remains & Bone & Indeterminate & Bone & 1 & 0.32 & & |11/28/2017 \\
\hline 550 & 40 & 338 & - & - & - & D & $1 / 11$ & N1001 & E1009 & 5 & \begin{tabular}{|l|}
$99.19-99.09$ \\
\end{tabular} & $50-60$ & - & Faunal Remains & Bone & Indeterminate & Bone & 1 & 0.04 & & \begin{tabular}{|l|}
$11 / 28 / 2017$ \\
\end{tabular} \\
\hline 550 & 41 & 338 & - & - & - & D & 1/11 & N1001 & E1009 & 5 & 99.19-99.09 & $50-60$ & - & Faunal Remains & Bone & Indeterminate & Bone & 1 & 0.12 & & 11/28/2017 \\
\hline 550 & 42 & 338 & - & - & - & D & 1/11 & N1001 & E1009 & 5 & 99.19-99.09 & $50-60$ & - & Faunal Remains & Bone & Indeterminate & Bone & 1 & 0.11 & & 11/28/2017 \\
\hline 550 & 43 & 338 & - & - & - & D & 1/11 & N1001 & E1009 & 5 & 99.19-99.09 & $50-60$ & - & Faunal Remains & Bone & Indeterminate & Bone & 1 & 0.07 & & 11/28/2017 \\
\hline 550 & 44 & 338 & - & - & - & D & 1/11 & N1001 & E1009 & 5 & 99.19-99.09 & $50-60$ & - & Faunal Remains & Bone & Indeterminate & Bone & 1 & 0.06 & & 11/28/2017 \\
\hline 550 & 45 & 338 & - & - & - & D & $1 / 11$ & N1001 & E1009 & 5 & 99.19-99.09 & $50-60$ & - & Faunal Remains & Bone & Indeterminate & Bone & 1 & 0.04 & & 11/28/2017 \\
\hline 550 & 46 & 338 & - & - & - & D & 1/11 & N1001 & E1009 & 5 & 99.19-99.09 & 50-60 & - & Faunal Remains & Bone & Indeterminate & Bone & 1 & 0.10 & & $\mid$\begin{tabular}{|l|}
$\mid 11 / 28 / 2017$ \\
\end{tabular} \\
\hline 550 & 47 & 338 & - & - & - & D & 1/11 & N1001 & E1009 & 5 & \begin{tabular}{|l|l|}
$99.19-99.09$ \\
\end{tabular} & $50-60$ & - & Faunal Remains & Bone & Indeterminate & Bone & 1 & 0.09 & & 11/28/2017 \\
\hline 550 & 48 & 338 & - & - & - & D & 1/11 & N1001 & E1009 & 5 & \begin{tabular}{|l|l|}
$99.19-99.09$ \\
\end{tabular} & $50-60$ & - & Faunal Remains & Bone & Indeterminate & Bone & 1 & 0.06 & & 11/28/2017 \\
\hline 550 & 49 & 338 & - & - & - & $\mathrm{D}$ & 1/11 & N1001 & E1009 & 5 & \begin{tabular}{|l|}
$99.19-99.09$ \\
\end{tabular} & $50-60$ & - & Faunal Remains & Bone & Indeterminate & Bone & 1 & 0.03 & & \begin{tabular}{|l|}
$11 / 28 / 2017$ \\
\end{tabular} \\
\hline 550 & 50 & 338 & - & - & - & $\mathrm{D}$ & 1/11 & N1001 & E1009 & 5 & \begin{tabular}{|l|l|}
$99.19-99.09$ \\
\end{tabular} & 50-60 & - & Faunal Remains & Bone & Indeterminate & Bone & 1 & 0.01 & & 11/28/2017 \\
\hline 550 & 51 & 338 & - & - & - & $\mathrm{D}$ & 1/11 & N1001 & E1009 & 5 & \begin{tabular}{|l|l|}
$99.19-99.09$ \\
\end{tabular} & 50-60 & - & Faunal Remains & Bone & Indeterminate & Bone & 1 & 0.09 & & 11/28/2017 \\
\hline 550 & 52 & 338 & - & - & - & $\mathrm{D}$ & $1 / 11$ & N1001 & E1009 & 5 & \begin{tabular}{|l|l|}
$99.19-99.09$ \\
\end{tabular} & $50-60$ & - & Faunal Remains & Bone & Indeterminate & Bone & 1 & 0.07 & & 11/28/2017 \\
\hline 550 & 53 & 338 & - & - & - & $\mathrm{D}$ & 1/11 & N1001 & E1009 & 5 & \begin{tabular}{|l|}
$99.19-99.09$ \\
\end{tabular} & $50-60$ & - & Faunal Remains & Bone & Indeterminate & Bone & 1 & 0.12 & & \begin{tabular}{|l|}
$11 / 28 / 2017$ \\
\end{tabular} \\
\hline 550 & 54 & 338 & - & - & - & D & 1/11 & N1001 & E1009 & 5 & 99.19-99.09 & $50-60$ & - & \begin{tabular}{|l|} 
Faunal Remains \\
\end{tabular} & Bone & Indeterminate & Bone & 1 & 0.09 & & 11/28/2017 \\
\hline 550 & 55 & 338 & - & - & - & D & $1 / 11$ & N1001 & E1009 & 5 & \begin{tabular}{|l|l|}
$99.19-99.09$ \\
\end{tabular} & 50-60 & - & Faunal Remains & Bone & Indeterminate & Bone & 1 & 0.05 & & 11/28/2017 \\
\hline 550 & 56 & 338 & - & - & - & D & $1 / 11$ & N1001 & E1009 & 5 & \begin{tabular}{|l|l|}
$99.19-99.09$ \\
\end{tabular} & $50-60$ & - & Faunal Remains & Bone & Indeterminate & Bone & 1 & 0.07 & & \begin{tabular}{|l|}
$11 / 28 / 2017$ \\
\end{tabular} \\
\hline 550 & 57 & 338 & - & - & - & D & 1/11 & N1001 & E1009 & 5 & \begin{tabular}{|l|l|}
$99.19-99.09$ \\
\end{tabular} & $50-60$ & - & Faunal Remains & Bone & Indeterminate & Bone & 1 & 0.08 & & 11/28/2017 \\
\hline 550 & 58 & 338 & - & - & - & D & 1/11 & N1001 & E1009 & 5 & \begin{tabular}{|l|}
$99.19-99.09$ \\
\end{tabular} & $50-60$ & - & \begin{tabular}{|l|} 
Faunal Remains \\
\end{tabular} & Bone & Indeterminate & Bone & 1 & 0.06 & & 11/28/2017 \\
\hline 550 & 59 & 338 & - & - & - & D & $1 / / 1$ & N1001 & E1009 & 5 & \begin{tabular}{|l|}
$99.19-99.09$ \\
\end{tabular} & $50-60$ & - & Faunal Remains & Bone & Indeterminate & Bone & 1 & 0.06 & & \begin{tabular}{|l|}
$11 / 28 / 2017$ \\
\end{tabular} \\
\hline
\end{tabular}




\begin{tabular}{|c|c|c|c|c|c|c|c|c|c|c|c|c|c|c|c|c|c|c|c|c|c|}
\hline Lot No. & \begin{tabular}{|c|}
$\begin{array}{c}\text { Specimen } \\
\text { No. }\end{array}$ \\
\end{tabular} & FS No. & UI No. & FTR No. & $\begin{array}{c}\text { Sample } \\
\text { No. }\end{array}$ & $\begin{array}{c}\text { Excavation } \\
\text { Block }\end{array}$ & Stratum (AU) & Northing & Easting & Level & $\begin{array}{c}\begin{array}{c}\text { Elevation } \\
(\mathrm{m})\end{array} \\
\end{array}$ & Depth (cmbd) & Point Prov. & Artifact Class & Artifact Type & \begin{tabular}{|c|} 
Artifact \\
Description
\end{tabular} & Material & $\begin{array}{c}\text { Number of } \\
\text { artifacts }\end{array}$ & Weight (g) & Comments & Date \\
\hline 550 & 60 & 338 & - & - & - & $D$ & $1 / 11$ & N1001 & E1009 & 5 & \begin{tabular}{|l|l|}
$99.19-99.09$ \\
\end{tabular} & 50-60 & - & Faunal Remains & Bone & Indeterminate & Bone & 1 & 0.08 & & 11/28/2017 \\
\hline 550 & 61 & 338 & - & - & - & D & $1 / 11$ & N1001 & E1009 & 5 & 99.19-99.09 & $50-60$ & - & Faunal Remains & Bone & Indeterminate & Bone & 1 & 0.04 & & 11/28/2017 \\
\hline 550 & 62 & 338 & - & - & - & D & 1/11 & N1001 & E1009 & 5 & 99.19-99.09 & $50-60$ & - & \begin{tabular}{|l|} 
Faunal Remains \\
\end{tabular} & Bone & Indeterminate & Bone & 1 & 0.03 & & 11/28/2017 \\
\hline 550 & 63 & 338 & - & - & - & D & 1/11 & N1001 & E1009 & 5 & 99.19-99.09 & $50-60$ & - & \begin{tabular}{|l|} 
Faunal Remains \\
\end{tabular} & Bone & Indeterminate & Bone & 1 & 0.01 & & 11/28/2017 \\
\hline 550 & 64 & 338 & - & - & - & D & I/III & N1001 & E1009 & 5 & 99.19-99.09 & $50-60$ & - & Detritus & Debitage & Complete Flake & Lithic & 11 & 14.97 & & 11/28/2017 \\
\hline 550 & 65 & 338 & - & - & - & D & $1 / 11$ & N1001 & E1009 & 5 & 99.19-99.09 & $50-60$ & - & Detritus & Debitage & \begin{tabular}{|l|} 
Proximal Flake \\
\end{tabular} & Lithic & 11 & 11.23 & & 11/28/2017 \\
\hline 550 & 66 & 338 & - & - & - & D & 1/11 & N1001 & E1009 & 5 & 99.19-99.09 & $50-60$ & - & Detritus & Debitage & Broken Flake & Lithic & 21 & 14.3 & & 11/28/2017 \\
\hline 550 & 67 & 338 & - & - & - & D & 1111 & N1001 & E1009 & 5 & 99.19-99.09 & 50-60 & - & Detritus & Debitage & Flaking Shatter & Lithic & 1 & 0.71 & & 11/28/2017 \\
\hline 550 & 68 & 338 & - & - & - & D & $1 / 1 / 1$ & N1001 & E1009 & 5 & \begin{tabular}{|l|l|}
$99.19-99.09$ \\
\end{tabular} & $50-60$ & - & Detritus & Debitage & Thermal Shatter & Lithic & 14 & 24.23 & & $\mid$\begin{tabular}{|l|}
$\mid 11 / 28 / 2017$ \\
\end{tabular} \\
\hline 550 & 69 & 338 & 19 & - & - & $\mathrm{D}$ & 1/11 & N1001 & E1009 & 5 & \begin{tabular}{|l|l|}
$99.19-99.09$ \\
\end{tabular} & $50-60$ & - & Formal Tool & \begin{tabular}{|l|} 
Arrow Point \\
\end{tabular} & Indeterminate & Fine Grain Chert & 1 & 1.1 & & 11/28/2017 \\
\hline 551 & 1 & 341 & - & - & - & $\mathrm{D}$ & 1/111 & N1001 & E1009 & 6 & \begin{tabular}{|l|}
$99.09-98.99$ \\
\end{tabular} & $60-70$ & - & Faunal Remains & Bone & Mammal & Bone & 1 & 3.36 & & 11/29/2017 \\
\hline 551 & 2 & 341 & - & - & - & $\mathrm{D}$ & 1/111 & N1001 & E1009 & 6 & \begin{tabular}{|l|l|}
$99.09-98.99$ \\
\end{tabular} & $60-70$ & - & \begin{tabular}{|l|} 
Faunal Remains \\
\end{tabular} & Bone & Indeterminate & Bone & 1 & 0.26 & & 11/29/2017 \\
\hline 551 & 3 & 341 & - & - & - & $\mathrm{D}$ & 1/11 & N1001 & E1009 & 6 & \begin{tabular}{|l|l|}
$99.09-98.99$ \\
\end{tabular} & $60-70$ & - & Detritus & Debitage & \begin{tabular}{|c|} 
Complete Flake \\
\end{tabular} & Lithic & 5 & 29.69 & & 11/29/2017 \\
\hline 551 & 4 & 341 & - & - & - & $\mathrm{D}$ & 1/11 & N1001 & E1009 & 6 & \begin{tabular}{|l|l|}
$99.09-98.99$ \\
\end{tabular} & $60-70$ & - & Detritus & Debitage & Proximal Flake & Lithic & 11 & 9.69 & & 11/29/2017 \\
\hline 551 & 5 & 341 & - & - & - & $\mathrm{D}$ & 1/11 & N1001 & E1009 & 6 & \begin{tabular}{|l|l|}
$99.09-98.99$ \\
\end{tabular} & $60-70$ & - & Detritus & Debitage & Broken Flake & Lithic & 34 & 16.7 & & 11/29/2017 \\
\hline 551 & 6 & 341 & - & - & - & D & 1/11 & N1001 & E1009 & 6 & \begin{tabular}{|l|l|}
$99.09-98.99$ \\
\end{tabular} & 60-70 & - & Detritus & Debitage & Flaking Shatter & Lithic & 2 & 0.72 & & 11/29/2017 \\
\hline 551 & 7 & 341 & - & - & - & D & $1 / 11$ & N1001 & E1009 & 6 & \begin{tabular}{|l|l|}
$99.09-98.99$ \\
\end{tabular} & $60-70$ & - & Detritus & Debitage & Thermal Shatter & Lithic & 17 & 16.93 & & 11/29/2017 \\
\hline 552 & 1 & 347 & - & - & - & D & |11/111 & N1001 & E1009 & 7 & 98.99-98.89 & $70-80$ & - & Faunal Remains & Bone & Tortoise/Turtle & Bone & 1 & 0.13 & & 11/29/2017 \\
\hline 552 & 2 & 347 & - & - & - & $\mathrm{D}$ & VIIIIIIII & N1001 & E1009 & 7 & \begin{tabular}{|l|}
$98.99-98.89$ \\
\end{tabular} & $70-80$ & - & \begin{tabular}{|l|} 
Faunal Remains \\
\end{tabular} & Bone & Indeterminate & Bone & 1 & 0.3 & & 11/29/2017 \\
\hline 552 & 3 & 347 & - & - & - & D & |11/1IIII & N1001 & E1009 & 7 & \begin{tabular}{|l|}
$98.99-98.89$ \\
\end{tabular} & $70-80$ & - & Faunal Remains & Bone & Indeterminate & Bone & 1 & 0.71 & & 11/29/2017 \\
\hline 552 & 4 & 347 & - & - & - & D & |1/1/III & N1001 & E1009 & 7 & \begin{tabular}{|l|}
$98.99-98.89$ \\
\end{tabular} & $70-80$ & - & Detritus & Debitage & Complete Flake & Lithic & 10 & 51.98 & & 11/29/2017 \\
\hline 552 & 5 & 347 & - & - & - & D & 111/1111 & N1001 & E1009 & 7 & \begin{tabular}{|l|}
$98.99-98.89$ \\
\end{tabular} & $70-80$ & - & Detritus & Debitage & Proximal Flake & Lithic & 26 & 46.75 & & 11/29/2017 \\
\hline 552 & 6 & 347 & - & - & - & $\mathrm{D}$ & VIIIIIII & N1001 & E1009 & 7 & \begin{tabular}{|l|}
$98.99-98.89$ \\
\end{tabular} & $70-80$ & - & Detritus & Debitage & Broken Flake & Lithic & 10 & 12.44 & & 11/29/2017 \\
\hline 552 & 7 & 347 & - & - & - & D & 1111/111 & N1001 & E1009 & 7 & \begin{tabular}{|l|l|}
$98.99-98.89$ \\
\end{tabular} & $70-80$ & - & Detritus & Debitage & Thermal Shatter & Lithic & 34 & 25.39 & & 11/29/2017 \\
\hline 552 & 8 & 347 & 20 & - & - & D & 111/IIII & N1001 & E1009 & 7 & \begin{tabular}{|l|}
$98.99-98.89$ \\
\end{tabular} & $70-80$ & - & Formal Tool & Arrow Point & Edwards Point & Fine Grain Chert & 1 & 0.32 & Basal Fragment & 11/29/2017 \\
\hline 552 & 9 & 347 & - & - & - & D & VIIIIIIII & N1001 & E1009 & 7 & \begin{tabular}{|l|l|}
$98.99-98.89$ \\
\end{tabular} & $70-80$ & - & Formal Tool & Biface & \begin{tabular}{|l|} 
Gahagan Biface \\
\end{tabular} & Fine Grain Chert & 1 & 19 & Medial-Basal Fragment & 11/29/2017 \\
\hline 552 & 10 & 347 & - & - & - & D & |I/IIIII & N1001 & E1009 & 7 & \begin{tabular}{|l|}
$98.99-98.89$ \\
\end{tabular} & $70-80$ & - & \begin{tabular}{|l|} 
Faunal Remains \\
\end{tabular} & Shell & Mussel Shell & Shell & 1 & 0.83 & & 11/29/2017 \\
\hline 553 & 1 & 392 & - & - & - & D & III & N1001 & E1009 & 8 & \begin{tabular}{|l|}
$98.89-98.79$ \\
\end{tabular} & $80-90$ & - & Detritus & Debitage & \begin{tabular}{|l|} 
Complete Flake \\
\end{tabular} & Lithic & 1 & 0.34 & & 12/1/2017 \\
\hline 553 & 2 & 392 & - & - & - & D & IIII & N1001 & E1009 & 8 & \begin{tabular}{|l|l|}
$98.89-98.79$ \\
\end{tabular} & $80-90$ & - & Detritus & Debitage & Broken Flake & Lithic & 5 & 21.12 & & 12/112017 \\
\hline 553 & 3 & 392 & - & - & - & D & IIII & N1001 & E1009 & 8 & \begin{tabular}{|l|l|}
$98.89-98.79$ \\
\end{tabular} & $80-90$ & - & Detritus & Debitage & Thermal Shatter & Lithic & 3 & 2.78 & & 12/1/2017 \\
\hline 554 & 1 & 395 & - & - & - & D & IIII & N1001 & E1009 & 9 & 98.79-98.69 & $90-100$ & - & Detritus & Debitage & Complete Flake & Lithic & 2 & 2.63 & & 12/1/2017 \\
\hline 554 & 2 & 395 & - & - & - & D & III & N1001 & E1009 & 9 & \begin{tabular}{|l|l|}
$98.79-98.69$ \\
\end{tabular} & 90-100 & - & Detritus & Debitage & \begin{tabular}{|l|} 
Proximal Flake \\
\end{tabular} & Lithic & 3 & 14.69 & & 12/112017 \\
\hline 554 & 3 & 395 & - & - & - & D & III & N1001 & E1009 & 9 & \begin{tabular}{|l|l|}
$98.79-98.69$ \\
\end{tabular} & 90-100 & - & Detritus & Debitage & Broken Flake & Lithic & 4 & 3.15 & & 12/1/2017 \\
\hline 554 & 4 & 395 & - & - & - & $\mathrm{D}$ & IIII & N1001 & E1009 & 9 & \begin{tabular}{|l|}
$98.79-98.69$ \\
\end{tabular} & $90-100$ & - & Detritus & Debitage & Thermal Shatter & Lithic & 5 & 3.16 & & 12/1/2017 \\
\hline 554 & 5 & 395 & - & - & - & $\mathrm{D}$ & IIII & N1001 & E1009 & 9 & \begin{tabular}{|l|}
$98.79-98.69$ \\
\end{tabular} & $\begin{array}{l}90-100 \\
\end{array}$ & - & Informal Tool & \begin{tabular}{|l|} 
Modified Flake \\
\end{tabular} & \begin{tabular}{|l|} 
Utilized Flake \\
\end{tabular} & Fine Grain Chert & 1 & 20.56 & & 12/1/2017 \\
\hline 555 & 1 & 400 & - & - & - & $\mathrm{D}$ & IIII & N1001 & E1009 & 10 & \begin{tabular}{|l|l|}
$98.69-98.59$ \\
\end{tabular} & $100-110$ & - & Detritus & Debitage & \begin{tabular}{|c|} 
Complete Flake \\
\end{tabular} & Lithic & 1 & 9.29 & & 12/1/2017 \\
\hline 555 & 2 & 400 & - & - & - & $\mathrm{D}$ & IIII & N1001 & E1009 & 10 & \begin{tabular}{|l|l|}
$98.69-98.59$ \\
\end{tabular} & $100-110$ & - & Detritus & Debitage & \begin{tabular}{|l|} 
Proximal Flake \\
\end{tabular} & Lithic & 1 & 0.56 & & 12/1/2017 \\
\hline 555 & 3 & 400 & - & - & - & $\mathrm{D}$ & IIII & N1001 & E1009 & 10 & \begin{tabular}{|l|}
$98.69-98.59$ \\
\end{tabular} & $100-110$ & - & Detritus & Debitage & Broken Flake & Lithic & 5 & 4.32 & & 1211/2017 \\
\hline 555 & 4 & 400 & - & - & - & $\mathrm{D}$ & IIII & N1001 & E1009 & 10 & \begin{tabular}{|l|l|}
$98.69-98.59$ \\
\end{tabular} & $100-110$ & - & Detritus & Debitage & Thermal Shatter & Lithic & 6 & 24.16 & & 12/1/2017 \\
\hline 555 & 5 & 400 & - & - & - & D & III & N1001 & E1009 & 10 & 98.69-98.59 & $100-110$ & - & \begin{tabular}{|c|} 
Ground \\
Battered Stone
\end{tabular} & \begin{tabular}{|l|} 
Groundstone \\
\end{tabular} & \begin{tabular}{|l|} 
Indeterminate \\
\end{tabular} & Limestone & 1 & 7.15 & & 12/112017 \\
\hline 556 & 1 & 349 & - & - & - & D & 1/11 & N1001 & E1010 & 1 & \begin{tabular}{|l|l|}
$99.59-99.49$ \\
\end{tabular} & $10-20$ & - & Detritus & Debitage & Complete Flake & Lithic & 1 & 10.43 & & 11/29/2017 \\
\hline 556 & 2 & 349 & - & - & - & $\mathrm{D}$ & $1 / 11$ & N1001 & E1010 & 1 & \begin{tabular}{|l|}
$99.59-99.49$ \\
\end{tabular} & $10-20$ & - & Detritus & Debitage & \begin{tabular}{|l|} 
Proximal Flake \\
\end{tabular} & Lithic & 1 & 0.12 & & 11/29/2017 \\
\hline 556 & 3 & 349 & - & - & - & $\mathrm{D}$ & 1/111 & N1001 & E1010 & 1 & \begin{tabular}{|l|}
$99.59-99.49$ \\
\end{tabular} & $10-20$ & - & Detritus & Debitage & Broken Flake & Lithic & 7 & 6.13 & & 11/29/2017 \\
\hline 556 & 4 & 349 & - & - & - & D & 1/11 & N1001 & E1010 & 1 & \begin{tabular}{|l|}
$99.59-99.49$ \\
\end{tabular} & $10-20$ & - & Detritus & Debitage & \begin{tabular}{|l|} 
Flaking Shatter \\
\end{tabular} & Lithic & 2 & 0.81 & & 11/29/2017 \\
\hline
\end{tabular}




\begin{tabular}{|c|c|c|c|c|c|c|c|c|c|c|c|c|c|c|c|c|c|c|c|c|c|}
\hline Lot No. & \begin{tabular}{|c|}
$\begin{array}{c}\text { Specimen } \\
\text { No. }\end{array}$ \\
\end{tabular} & FS No. & UI No. & FTR No. & $\begin{array}{c}\text { Sample } \\
\text { No. }\end{array}$ & $\begin{array}{c}\text { Excavation } \\
\text { Block }\end{array}$ & Stratum (AU) & Northing & Easting & Level & $\begin{array}{c}\begin{array}{c}\text { Elevation } \\
(\mathrm{m})\end{array} \\
\end{array}$ & Depth (cmbd) & Point Prov. & Artifact Class & Artifact Type & $\begin{array}{c}\text { Artifact } \\
\text { Description }\end{array}$ & Material & $\begin{array}{c}\text { Number of } \\
\text { artifacts }\end{array}$ & Weight (g) & Comments & Date \\
\hline 557 & 1 & 353 & - & - & - & $D$ & $1 / 11$ & N1001 & E1010 & 2 & \begin{tabular}{|l|l|}
$99.49-99.39$ \\
\end{tabular} & $20-30$ & - & Detritus & Debitage & Broken Flake & Lithic & 1 & 0.4 & & 11/29/2017 \\
\hline 557 & 2 & 353 & - & - & - & D & 1/111 & N1001 & E1010 & 2 & 99.49-99.39 & $20-30$ & - & Historic Artifiact & Ceramic & Whiteware & Ceramic & 1 & 5.43 & & 11/29/2017 \\
\hline 558 & 1 & 356 & - & - & - & D & 1/11 & N1001 & E1010 & 3 & 99.39-99.29 & $30-40$ & - & Detritus & Debitage & Complete Flake & Lithic & 1 & 0.08 & & 11/29/2017 \\
\hline 558 & 2 & 356 & - & - & - & D & 1/11 & N1001 & E1010 & 3 & 99.39-99.29 & $30-40$ & - & Detritus & Debitage & Proximal Flake & Lithic & 2 & 0.9 & & 11/29/2017 \\
\hline 558 & 3 & 356 & - & - & - & D & $1 / 11$ & N1001 & E1010 & 3 & 99.39-99.29 & $30-40$ & - & Detritus & Debitage & Broken Flake & Lithic & 7 & 9.79 & & $\mid$\begin{tabular}{|l|}
$\mid 11 / 29 / 2017$ \\
\end{tabular} \\
\hline 559 & 1 & 360 & - & - & - & D & $1 / 11$ & N1001 & E1010 & 4 & 99.29-99.19 & $40-50$ & - & Faunal Remains & Bone & Indeterminate & Bone & 1 & 1.2 & & 11/29/2017 \\
\hline 559 & 2 & 360 & - & - & - & D & 1/11 & N1001 & E1010 & 4 & 99.29-99.19 & $40-50$ & - & Faunal Remains & Bone & Indeterminate & Bone & 1 & 1.21 & & 11/29/2017 \\
\hline 559 & 3 & 360 & - & - & - & D & 1111 & N1001 & E1010 & 4 & 99.29-99.19 & $40-50$ & - & Detritus & Debitage & Complete Flake & Lithic & 3 & 6.7 & & $\mid$\begin{tabular}{|l|}
$\mid 11 / 29 / 2017$ \\
\end{tabular} \\
\hline 559 & 4 & 360 & - & - & - & D & I/II & N1001 & E1010 & 4 & \begin{tabular}{|l|}
$99.29-99.19$ \\
\end{tabular} & $40-50$ & - & Detritus & Debitage & Proximal Flake & Lithic & 9 & 17.43 & & 11/29/2017 \\
\hline 559 & 5 & 360 & - & - & - & $\mathrm{D}$ & 1/11 & N1001 & E1010 & 4 & \begin{tabular}{|l|}
$99.29-99.19$ \\
\end{tabular} & $40-50$ & - & Detritus & Debitage & Broken Flake & Lithic & 15 & 11.15 & & 11/29/2017 \\
\hline 559 & 6 & 360 & - & - & - & $\mathrm{D}$ & 1/11 & N1001 & E1010 & 4 & \begin{tabular}{|l|}
$99.29-99.19$ \\
\end{tabular} & $40-50$ & - & Detritus & Debitage & Thermal Shatter & Lithic & 19 & 26.31 & & \begin{tabular}{|l|}
$11 / 29 / 2017$ \\
\end{tabular} \\
\hline 559 & 7 & 360 & 21 & - & - & $\mathrm{D}$ & 1/11 & N1001 & E1010 & 4 & \begin{tabular}{|l|}
$99.29-99.19$ \\
\end{tabular} & $40-50$ & - & Formal Tool & Arrow Point & \begin{tabular}{l|} 
Perdiz Point \\
\end{tabular} & Fine Grain Chert & 1 & 0.58 & & 11/29/2017 \\
\hline 560 & 1 & 363 & 22 & - & - & $\mathrm{D}$ & $1 / 11$ & N1001 & E1010 & 4 & 99.22 & 47 & \begin{tabular}{|l|}
$\begin{array}{l}\text { N1001.85 } \\
\text { E1010.05 }\end{array}$ \\
\end{tabular} & Formal Tool & Bifiace & \begin{tabular}{c|} 
Distal-Medial \\
Fragnent \\
Frant
\end{tabular} & Fine Grain Chert & 1 & 10.38 & & \begin{tabular}{|l|}
$11 / 29 / 2017$ \\
\end{tabular} \\
\hline 561 & 1 & 367 & - & - & - & $\mathrm{D}$ & $1 / 111$ & N1001 & E1010 & 5 & \begin{tabular}{|l|l|}
$99.19-99.09$ \\
\end{tabular} & $50-60$ & - & Faunal Remains & Bone & Indeterminate & Bone & 1 & 0.22 & & $\mid$\begin{tabular}{|l|}
$11 / 29 / 2017$ \\
\end{tabular} \\
\hline 561 & 2 & 367 & - & - & - & $\mathrm{D}$ & 1/11 & N1001 & E1010 & 5 & \begin{tabular}{|l|l|}
$99.19-99.09$ \\
\end{tabular} & $50-60$ & - & Faunal Remains & Bone & Indeterminate & Bone & 1 & 0.21 & & 11/29/2017 \\
\hline 561 & 3 & 367 & - & - & - & D & 1/111 & N1001 & E1010 & 5 & \begin{tabular}{|l|l|}
$99.19-99.09$ \\
\end{tabular} & 50-60 & - & Detritus & Debitage & Complete Flake & Lithic & 8 & 13.22 & & 11/29/2017 \\
\hline 561 & 4 & 367 & - & - & - & D & $1 / 11$ & N1001 & E1010 & 5 & \begin{tabular}{|l|l|}
$99.19-99.09$ \\
\end{tabular} & 50-60 & - & Detritus & Debitage & \begin{tabular}{|l|} 
Proximal Flake \\
\end{tabular} & Lithic & 9 & 6.74 & & 11/29/2017 \\
\hline 561 & 5 & 367 & - & - & - & D & $1 / 11$ & N1001 & E1010 & 5 & \begin{tabular}{|l|l|}
$99.19-99.09$ \\
\end{tabular} & $50-60$ & - & Detritus & Debitage & Broken Flake & Lithic & 12 & 21.74 & & 11/29/2017 \\
\hline 561 & 6 & 367 & - & - & - & $\mathrm{D}$ & 1/111 & N1001 & E1010 & 5 & \begin{tabular}{|l|}
$99.19-99.09$ \\
\end{tabular} & 50-60 & - & Detritus & Debitage & Thermal Shatter & Lithic & 15 & 32.36 & & 11/29/2017 \\
\hline 561 & 7 & 367 & - & - & - & $\mathrm{D}$ & 1/11 & N1001 & E1010 & 5 & 99.19-99.09 & 50-60 & - & Formal Tool & Biface & \begin{tabular}{c|} 
Indeterminate \\
Fragment
\end{tabular} & Fine Grain Chert & 1 & 0.64 & & 11/29/2017 \\
\hline 562 & 1 & 371 & - & - & - & D & 1/11 & N1001 & E1010 & 6 & 99.03 & 66 & 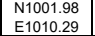 & Detritus & Core & Bifacial & Fine Grain Chert & 1 & 285.11 & & 11/30/2017 \\
\hline 563 & 1 & 372 & - & - & - & $\mathrm{D}$ & 1/11 & N1001 & E1010 & 6 & 99.04 & 65 & \begin{tabular}{|l|} 
N1001.05 \\
E1010.038
\end{tabular} & \begin{tabular}{|l|} 
Informal Tool \\
\end{tabular} & Modified Flake & Edge-modified & Fine Grain Chert & 1 & 8.94 & & \begin{tabular}{|l|}
$11 / 30 / 2017$ \\
\end{tabular} \\
\hline 564 & 1 & 373 & - & - & - & $\mathrm{D}$ & 1/11 & N1001 & E1010 & 6 & 90.1 & 68 & \begin{tabular}{|l|} 
N1001.27 \\
E1010.87
\end{tabular} & \begin{tabular}{|l|} 
Faunal Remains \\
\end{tabular} & Bone & Indeterminate & Bone & 1 & 1.14 & & 11/30/2017 \\
\hline 564 & 2 & 373 & - & - & - & D & 1/111 & N1001 & E1010 & 6 & 90.1 & 68 & \begin{tabular}{|l|}
$N 1001.27$ \\
E101087
\end{tabular} & Faunal Remains & Bone & Indeterminate & Bone & 1 & 1.38 & & 11/30/2017 \\
\hline 564 & 3 & 373 & - & - & - & D & 1/11 & N1001 & E1010 & 6 & 90.1 & 68 & $\begin{array}{l}\text { N1001.27 } \\
\text { E1010.87 }\end{array}$ & \begin{tabular}{|l|} 
Faunal Remains \\
\end{tabular} & Bone & Indeterminate & Bone & 1 & 0.26 & & 11/30/2017 \\
\hline 564 & 4 & 373 & - & - & - & $\mathrm{D}$ & 1/111 & N1001 & E1010 & 6 & 90.1 & 68 & \begin{tabular}{|l|l|}
$N 1001.27$ \\
E1010.87
\end{tabular} & Faunal Remains & Bone & Indeterminate & Bone & 1 & 0.3 & & 11/30/2017 \\
\hline 564 & 5 & 373 & - & - & - & D & 1/11 & N1001 & E1010 & 6 & 90.1 & 68 & $\begin{array}{l}\begin{array}{l}N 1001.127 \\
\text { E1010.87 }\end{array} \\
\end{array}$ & Faunal Remains & Bone & Indeterminate & Bone & 1 & 0.33 & & 11/30/2017 \\
\hline 564 & 6 & 373 & - & - & - & $\mathrm{D}$ & $1 / 111$ & N1001 & E1010 & 6 & 90.1 & 68 & \begin{tabular}{|l|}
$N 1001.27$ \\
E1010.87
\end{tabular} & Faunal Remains & Bone & Indeterminate & Bone & 1 & 0.22 & & 11/30/2017 \\
\hline 564 & 7 & 373 & - & - & - & D & 1/11 & N1001 & E1010 & 6 & 90.1 & 68 & $\begin{array}{l}\begin{array}{l}N 1001.127 \\
\text { E1010.87 }\end{array} \\
\end{array}$ & Faunal Remains & Bone & Indeterminate & Bone & 1 & 0.19 & & 11/30/2017 \\
\hline 564 & 8 & 373 & - & - & - & $\mathrm{D}$ & $1 / 111$ & N1001 & E1010 & 6 & 90.1 & 68 & \begin{tabular}{|l|l|}
$N 1001.27$ \\
E1010.87
\end{tabular} & Faunal Remains & Bone & Indeterminate & Bone & 1 & 0.01 & & \begin{tabular}{|l|}
$1 / 30 / 2017$ \\
\end{tabular} \\
\hline 564 & 9 & 373 & - & - & - & $\mathrm{D}$ & 1/111 & N1001 & E1010 & 6 & 90.1 & 68 & \begin{tabular}{l|} 
N1001.27 \\
E1010.87
\end{tabular} & Faunal Remains & Bone & Indeterminate & Bone & 1 & 0.01 & & $\mid$\begin{tabular}{|l|}
$\mid 11 / 30 / 2017$ \\
\end{tabular} \\
\hline 564 & 10 & 373 & - & - & - & D & $1 / 1 / 1$ & N1001 & E1010 & 6 & 90.1 & 68 & $\begin{array}{l}\text { N11001.127 } \\
\text { E1010.87 }\end{array}$ & Faunal Remains & Bone & Indeterminate & Bone & 1 & 0.01 & & 11/30/2017 \\
\hline 565 & 1 & 374 & - & - & - & D & 1/11 & N1001 & E1010 & 6 & \begin{tabular}{|l|l|}
$99.09-98.99$ \\
\end{tabular} & $60-70$ & - & Detritus & Debitage & Complete Flake & Lithic & 8 & 16.81 & & 11/30/2017 \\
\hline 565 & 2 & 374 & - & - & - & D & 1/11 & N1001 & E1010 & 6 & \begin{tabular}{|l|l|}
$99.09-98.99$ \\
\end{tabular} & $60-70$ & - & Detritus & Debitage & Proximal Flake & Lithic & 3 & 1.32 & & \begin{tabular}{|l|}
$11 / 30 / 2017$ \\
\end{tabular} \\
\hline 565 & 3 & 374 & - & - & - & $\mathrm{D}$ & $1 / 11$ & N1001 & E1010 & 6 & \begin{tabular}{|l|}
$99.09-98.99$ \\
\end{tabular} & $\begin{array}{ll}60-70 \\
\end{array}$ & - & Detritus & Debitage & Broken Flake & Lithic & 21 & 11.47 & & 11/30/2017 \\
\hline 565 & 4 & 374 & - & - & - & $\mathrm{D}$ & $1 / 11$ & N1001 & E1010 & 6 & \begin{tabular}{|l|}
$99.09-98.99$ \\
\end{tabular} & $\begin{array}{ll}60-70 \\
\end{array}$ & - & Detritus & Debitage & Thermal Shatter & Lithic & 10 & 12.64 & & 11/30/2017 \\
\hline 565 & 5 & 374 & - & - & - & $\mathrm{D}$ & $1 / 11$ & N1001 & E1010 & 6 & \begin{tabular}{|l|l|}
$99.09-98.99$ \\
\end{tabular} & $60-70$ & - & Formal Tool & Biface & \begin{tabular}{l|} 
Distal-Medial \\
Fragament
\end{tabular} & Fine Grain Chert & 1 & 20.35 & & 11/30/2017 \\
\hline 566 & 1 & 379 & - & - & - & $\mathrm{D}$ & |I/I/IIII & N1001 & E1010 & 7 & \begin{tabular}{|l|l|}
$98.99-98.89$ \\
\end{tabular} & $70-80$ & - & Detritus & Debitage & \begin{tabular}{|l|} 
Complete Flake \\
\end{tabular} & Lithic & 4 & 2.86 & & \begin{tabular}{|l|}
$11 / 30 / 2017$ \\
\end{tabular} \\
\hline 566 & 2 & 379 & - & - & - & $\mathrm{D}$ & | 1/1/IIII & N1001 & E1010 & 7 & \begin{tabular}{|l|}
$98.99-98.89$ \\
\end{tabular} & $\begin{array}{ll}70-80 \\
\end{array}$ & - & Detritus & Debitage & \begin{tabular}{|l|} 
Proximal Flake \\
\end{tabular} & Lithic & 5 & 2.06 & & 11/30/2017 \\
\hline 566 & 3 & 379 & - & - & - & D & 1/1/IIII & N1001 & E1010 & 7 & \begin{tabular}{|l|}
$98.99-98.89$ \\
\end{tabular} & $70-80$ & - & Detritus & Debitage & Broken Flake & Lithic & 14 & 11.97 & & 11/30/2017 \\
\hline 566 & 4 & 379 & - & - & - & D & |I/I/III & N1001 & E1010 & 7 & \begin{tabular}{|l|}
$98.99-98.89$ \\
\end{tabular} & $70-80$ & - & Detritus & Debitage & Flaking Shatter & Lithic & 3 & 4.78 & & 11/30/2017 \\
\hline 566 & 5 & 379 & - & - & - & D & |IIIIIII & N1001 & E1010 & 7 & \begin{tabular}{|l|}
$98.99-98.89$ \\
\end{tabular} & $70-80$ & - & Detritus & Debitage & Thermal Shatter & Lithic & 12 & 32.31 & & 11/30/2017 \\
\hline 567 & 1 & 384 & - & - & - & D & III & N1001 & E1010 & 8 & \begin{tabular}{|l|}
$98.89-98.79$ \\
\end{tabular} & $80-90$ & - & \begin{tabular}{|l|} 
Faunal Remains \\
\end{tabular} & Bone & Indeterminate & Bone & 1 & 0.08 & & 11/30/2017 \\
\hline 567 & 2 & 384 & - & - & - & D & III & N1001 & E1010 & 8 & \begin{tabular}{|l|}
$98.89-98.79$ \\
\end{tabular} & $80-90$ & - & Faunal Remains & Bone & Indeterminate & Bone & 1 & 0.16 & & \begin{tabular}{|l|}
$11 / 30 / 2017$ \\
\end{tabular} \\
\hline
\end{tabular}




\begin{tabular}{|c|c|c|c|c|c|c|c|c|c|c|c|c|c|c|c|c|c|c|c|c|c|}
\hline Lot No. & \begin{tabular}{|c|}
$\begin{array}{c}\text { Specimen } \\
\text { No. }\end{array}$ \\
\end{tabular} & FS No. & UI No. & FTR No. & $\begin{array}{c}\text { Sample } \\
\text { No. }\end{array}$ & $\begin{array}{c}\text { Excavation } \\
\text { Block }\end{array}$ & Stratum (AU) & Northing & Easting & Level & $\begin{array}{c}\begin{array}{c}\text { Elevation } \\
(\mathrm{m})\end{array} \\
\end{array}$ & Depth (cmbd) & Point Prov. & Artifact Class & Artifact Type & \begin{tabular}{|c|}
$\begin{array}{c}\text { Artifact } \\
\text { Description }\end{array}$ \\
\end{tabular} & Material & $\begin{array}{c}\begin{array}{c}\text { Number of } \\
\text { artifacts }\end{array} \\
\end{array}$ & Weight $(g)$ & Comments & Date \\
\hline 567 & 3 & 384 & - & - & - & $D$ & III & N1001 & E1010 & 8 & \begin{tabular}{|l|l|}
$98.89-98.79$ \\
\end{tabular} & $80-90$ & - & Detritus & Debitage & Complete Flake & Lithic & 4 & 37.75 & & 11/30/2017 \\
\hline 567 & 4 & 384 & - & - & - & D & III & N1001 & E1010 & 8 & |98.89-98.79 & $80-90$ & - & Detritus & Debitage & Proximal Flake & Lithic & 13 & 5.14 & & 11/30/2017 \\
\hline 567 & 5 & 384 & - & - & - & D & IIII & N1001 & E1010 & 8 & \begin{tabular}{|l|l|}
$98.89-98.79$ \\
\end{tabular} & $80-90$ & - & Detritus & Debitage & Broken Flake & Lithic & 12 & 10.53 & & 11/30/2017 \\
\hline 567 & 6 & 384 & - & - & - & D & IIII & N1001 & E1010 & 8 & \begin{tabular}{|l|l|}
$98.89-98.79$ \\
\end{tabular} & $80-90$ & - & Detritus & Debitage & Thermal Shatter & Lithic & 7 & 5.51 & & 11/30/2017 \\
\hline 568 & 1 & 386 & - & - & - & D & IIII & N1001 & E1010 & 9 & |98.79-98.69| & $90-100$ & - & Detritus & Debitage & Proximal Flake & Lithic & 2 & 0.75 & & 11/30/2017 \\
\hline 568 & 2 & 386 & - & - & - & D & IIII & N1001 & E1010 & 9 & 98.79-98.69 & $90-100$ & - & Detritus & Debitage & Broken Flake & Lithic & 2 & 6.38 & & 11/30/2017 \\
\hline 568 & 3 & 386 & - & - & - & D & IIII & N1001 & E1010 & 9 & 98.79-98.69 & $90-100$ & - & Detritus & Debitage & Thermal Shatter & Lithic & 3 & 3.4 & & 11/30/2017 \\
\hline 569 & 1 & 389 & - & - & - & D & III & N1001 & E1010 & 10 & 98.69-98.59 & $100-110$ & - & Detritus & Debitage & Complete Flake & Lithic & 2 & 0.38 & & 11/30/2017 \\
\hline 569 & 2 & 389 & - & - & - & D & IIII & N1001 & E1010 & 10 & \begin{tabular}{|l|l|}
$98.69-98.59$ \\
\end{tabular} & $100-110$ & - & Detritus & Debitage & Proximal Flake & Lithic & 1 & 0.18 & & 11/30/2017 \\
\hline 569 & 3 & 389 & - & - & - & $\mathrm{D}$ & IIII & N1001 & E1010 & 10 & \begin{tabular}{|l|l|}
$98.69-98.59$ \\
\end{tabular} & $100-110$ & - & Detritus & Debitage & Broken Flake & Lithic & 1 & 6.34 & & 11/30/2017 \\
\hline 569 & 4 & 389 & - & - & - & D & IIII & N1001 & E1010 & 10 & 98.69-98.59 & $100-110$ & - & Detritus & Debitage & Thermal Shatter & Lithic & 2 & 0.98 & & 11/30/2017 \\
\hline 569 & 5 & 389 & - & - & - & $\mathrm{D}$ & IIII & N1001 & E1010 & 10 & 98.69-98.59 & $100-110$ & - & Formal Tool & Biface & \begin{tabular}{c|} 
Distal-Medial \\
Fragment
\end{tabular} & Fine Grain Chert & 1 & 0.68 & Originally collected as Projectile Point & 11/30/2017 \\
\hline 570 & 1 & 416 & - & - & - & D & IV & N1001 & E1010 & 12 & 98.49-98.39 & $120-130$ & - & Faunal Remains & Bone & Indeterminate & Bone & 1 & 0.73 & & 12/4/2017 \\
\hline 570 & 2 & 416 & - & - & - & D & IV & N1001 & E1010 & 12 & 98.49-98.39 & $120-130$ & - & Faunal Remains & Bone & Indeterminate & Bone & 1 & 0.74 & & 12/4/2017 \\
\hline 570 & 3 & 416 & - & - & - & $\mathrm{D}$ & IV & N1001 & E1010 & 12 & \begin{tabular}{|c|c|}
$98.49-98.39$ \\
\end{tabular} & 120-130 & - & Detritus & Debitage & Proximal Flake & Lithic & 2 & 29.57 & & 12/42017 \\
\hline 570 & 4 & 416 & - & - & - & D & iv & N1001 & E1010 & 12 & 98.49-98.39 & 120-130 & - & Detritus & Debitage & Broken Flake & Lithic & 1 & 0.24 & & 12/4/2017 \\
\hline 570 & 5 & 416 & - & - & - & $\mathrm{D}$ & iv & N1001 & E1010 & 12 & \begin{tabular}{|l|l|}
$98.49-98.39$ \\
\end{tabular} & 120-130 & - & Detritus & Debitage & Thermal Shatter & Lithic & 1 & 0.16 & & 12/4/2017 \\
\hline 571 & 1 & 425 & - & - & - & D & IV & N1001 & E1010 & 13 & \begin{tabular}{|l|}
$98.39-98.29$ \\
\end{tabular} & $130-140$ & - & Faunal Remains & Bone & Indeterminate & Bone & 1 & 1.25 & & 12/5/2017 \\
\hline 571 & 2 & 425 & - & - & - & D & IV & N1001 & E1010 & 13 & \begin{tabular}{|l|l|}
$98.39-98.29$ \\
\end{tabular} & $130-140$ & - & Faunal Remains & Bone & Indeterminate & Bone & 1 & 1.13 & & 1215/2017 \\
\hline 571 & 3 & 425 & - & - & - & D & iv & N1001 & E1010 & 13 & \begin{tabular}{|l|l|}
$98.39-98.29$ \\
\end{tabular} & $130-140$ & - & Faunal Remains & Bone & Indeterminate & Bone & 1 & 1.13 & & 1215/2017 \\
\hline 571 & 4 & 425 & - & - & - & $\mathrm{D}$ & Iv & N1001 & E1010 & 13 & \begin{tabular}{|l|}
$98.39-98.29$ \\
\end{tabular} & 130-140 & - & Faunal Remains & Bone & Indeterminate & Bone & 1 & 1.36 & & 12/5/2017 \\
\hline 571 & 5 & 425 & - & - & - & $\mathrm{D}$ & Iv & N1001 & E1010 & 13 & \begin{tabular}{|l|}
$98.39-98.29$ \\
\end{tabular} & $130-140$ & - & Faunal Remains & Bone & Indeterminate & Bone & 1 & 0.09 & & 1215/2017 \\
\hline 571 & 6 & 425 & - & - & - & D & IV & N1001 & E1010 & 13 & \begin{tabular}{|l|}
$98.39-98.29$ \\
\end{tabular} & $130-140$ & - & Detritus & Debitage & Thermal Shatter & Lithic & 1 & 0.26 & & 1215/2017 \\
\hline 572 & 1 & 435 & - & - & - & D & iv & N1001 & E1010 & 14 & 98.29-98.19 & 140-150 & - & Detritus & Debitage & Complete Flake & Lithic & 1 & 2.38 & & 1215/2017 \\
\hline 573 & 1 & 443 & - & - & - & D & IV & N1001 & E1010 & 15 & 98.19-98.09 & $150-160$ & - & Detritus & Debitage & \begin{tabular}{|c|} 
Complete Flake \\
\end{tabular} & Lithic & 1 & 0.63 & & 12/5/2017 \\
\hline 573 & 2 & 443 & - & - & - & D & IV & N1001 & E1010 & 15 & 98.19-98.09 & $150-160$ & - & Detritus & Debitage & \begin{tabular}{|l|} 
Proximal Flake \\
\end{tabular} & Lithic & 3 & 54.64 & & 12/5/2017 \\
\hline 573 & 3 & 443 & - & - & - & D & iv & N1001 & E1010 & 15 & 98.19-98.09 & $150-160$ & - & Detritus & Debitage & Broken Flake & Lithic & 2 & 0.39 & & 12/5/2017 \\
\hline 574 & 1 & 606 & - & - & - & D & $V N \mid$ & N1001 & E1010 & 16 & 98.09-97.99 & $160-170$ & - & Faunal Remains & Bone & Indeterminate & Bone & 1 & 0.12 & & 121/20/2017 \\
\hline 574 & 2 & 606 & - & - & - & D & $V_{N 1}$ & N1001 & E1010 & 16 & 98.09-97.99 & $160-170$ & - & Faunal Remains & Bone & Indeterminate & Bone & 1 & 0.05 & & 121/20/2017 \\
\hline 574 & 3 & 606 & - & - & - & D & $V N \mid$ & N1001 & E1010 & 16 & 98.09-97.99 & $160-170$ & - & Detritus & Debitage & Complete Flake & Lithic & 1 & 0.93 & & 12/20/2017 \\
\hline 574 & 4 & 606 & - & - & - & D & $V_{N 1}$ & N1001 & E1010 & 16 & |98.09-97.99 & $160-170$ & - & Detritus & Debitage & Proximal Flake & Lithic & 1 & 0.74 & & 12/20/2017 \\
\hline 574 & 5 & 606 & - & - & - & D & $V N \mid$ & N1001 & E1010 & 16 & \begin{tabular}{|l|l|}
$98.09-97.99$ \\
\end{tabular} & $160-170$ & - & Detritus & Debitage & Broken Flake & Lithic & 1 & 0.85 & & 121/20/2017 \\
\hline 574 & 6 & 606 & - & - & - & D & $V N \mid$ & N1001 & E1010 & 16 & \begin{tabular}{|l|l|}
$98.09-97.99$ \\
\end{tabular} & $160-170$ & - & Detritus & Debitage & Thermal Shatter & Lithic & 1 & 3.17 & & 12/20/2017 \\
\hline 575 & 1 & 611 & - & - & - & $\mathrm{D}$ & $\mathrm{V} N \mathrm{~N}$ & N1001 & E1010 & 18 & \begin{tabular}{|l|}
$97.89-97.79$ \\
\end{tabular} & $\begin{array}{ll}180-190 \\
\end{array}$ & - & Detritus & Debitage & Broken Flake & Lithic & 1 & 0.47 & & 12/20/2017 \\
\hline 576 & 1 & 616 & - & - & - & $\mathrm{D}$ & $\mathrm{V} N 1$ & N1001 & E1010 & 14-16 & 98.09-97.99 & 140-170 & - & Faunal Remains & Bone & Odocoileus sp. & Bone & 1 & 4.65 & $\begin{array}{l}\text { North wall slump; deer metapodiala } \\
\text { green breaks \& pot polish }\end{array}$ & 12/2012017 \\
\hline 577 & 1 & 620 & - & - & - & D & VII & N1001 & E1010 & 20 & 97.69-97.59 & $200-210$ & - & Detritus & Debitage & Complete Flake & Lithic & 2 & 8.41 & & 12/20/2017 \\
\hline 577 & 2 & 620 & - & - & - & $\mathrm{D}$ & VIII & N1001 & E1010 & 20 & \begin{tabular}{|l|l|}
$97.69-97.59$ \\
\end{tabular} & $200-210$ & - & Detritus & Debitage & Broken Flake & Lithic & 1 & 0.04 & & 12/20/2017 \\
\hline 577 & 3 & 620 & - & - & - & D & VII & N1001 & E1010 & 20 & 97.69-97.59 & $200-210$ & - & Detritus & Debitage & Thermal Shatter & Lithic & 1 & 0.12 & & 12/20/2017 \\
\hline 578 & 1 & 637 & - & - & - & $\mathrm{c}$ & "I & $\begin{array}{l}\text { N1001.5 } \\
\end{array}$ & E1004 & 2 & 99.6-99.5 & $30-40$ & - & Faunal Remains & Bone & Indeterminate & Bone & 1 & 1.17 & & 121/20/2017 \\
\hline 578 & 2 & 637 & - & - & - & c & "I & N1001.5 & E1004 & 2 & 99.6-99.5 & $30-40$ & - & Faunal Remains & Bone & Indeterminate & Bone & 1 & 0.61 & & 12/20/2017 \\
\hline 578 & 3 & 637 & - & - & - & $\mathrm{c}$ & "1 & N1001.5 & E1004 & 2 & $99.6-99.5$ & $30-40$ & - & Detritus & Debitage & Proximal Flake & Lithic & 3 & 2.14 & & 12/20/2017 \\
\hline 578 & 4 & 637 & - & - & - & c & " & N1001.5 & E1004 & 2 & $99.6-99.5$ & $30-40$ & - & Detritus & Debitage & Broken Flake & Lithic & 3 & 0.93 & & 121/20/2017 \\
\hline 578 & 5 & 637 & - & - & - & $\mathrm{c}$ & $\|$ & $\begin{array}{ll}\text { N1001.5 } \\
\end{array}$ & E1004 & 2 & $99.6-99.5$ & $30-40$ & - & Detritus & Debitage & Thermal Shatter & Lithic & 1 & 2.01 & & 12/20/2017 \\
\hline 578 & 6 & 637 & - & - & - & $\mathrm{c}$ & ॥ & $\begin{array}{ll}N 1001.5 \\
\end{array}$ & E1004 & 2 & $99.6-99.5$ & $30-40$ & - & Historic Artifact & Ceramic & Whiteware & Ceramic & 1 & 2.73 & Whiteware with green pattern décor & 12/20/2017 \\
\hline
\end{tabular}




\begin{tabular}{|c|c|c|c|c|c|c|c|c|c|c|c|c|c|c|c|c|c|c|c|c|c|}
\hline Lot No. & \begin{tabular}{|c|}
$\begin{array}{c}\text { Specimen } \\
\text { No. }\end{array}$ \\
\end{tabular} & FS No. & UI No. & FTR No. & $\begin{array}{c}\text { Sample } \\
\text { No. }\end{array}$ & $\begin{array}{c}\text { Excavation } \\
\text { Block }\end{array}$ & Stratum (AU) & Northing & Easting & Level & $\begin{array}{c}\text { Elevation } \\
\text { (m) }\end{array}$ & Depth (cmbd) & Point Prov. & Artifiact Class & \begin{tabular}{|l|} 
Artifact Type \\
\end{tabular} & \begin{tabular}{|c|} 
Artifact \\
Description
\end{tabular} & Material & $\begin{array}{c}\text { Number of } \\
\text { artifacts }\end{array}$ & Weight (g) & Comments & Date \\
\hline 579 & 1 & 643 & - & - & - & $\mathrm{c}$ & 11/III & N1001.5 & E1004 & 3 & 99.5-99.4 & $40-50$ & - & Faunal Remains & Bone & Odocoileus sp. & Bone & 1 & 3.63 & & 12/21/2017 \\
\hline 579 & 2 & 643 & - & - & - & c & 11/III & $\mathrm{N} 1001.5$ & E1004 & 3 & 99.5-99.4 & $40-50$ & - & Faunal Remains & Bone & Indeterminate & Bone & 1 & 0.41 & & 12/21/2017 \\
\hline 579 & 3 & 643 & - & - & - & c & II/III & $\mathrm{N} 1001.5$ & E1004 & 3 & $99.5-99.4$ & $40-50$ & - & Faunal Remains & Bone & Indeterminate & Bone & 1 & 0.1 & & 12/21/2017 \\
\hline 579 & 4 & 643 & - & - & - & c & "1/III & $\mathrm{N} 1001.5$ & E1004 & 3 & $99.5-99.4$ & $40-50$ & - & Faunal Remains & Bone & Indeterminate & Bone & 1 & 0.01 & & 12/21/2017 \\
\hline 579 & 5 & 643 & - & - & - & c & $\|/\| 11$ & N1001.5 & E1004 & 3 & 99.5-99.4 & $40-50$ & - & Faunal Remains & Bone & Indeterminate & Bone & 1 & 0.01 & & $\mid$\begin{tabular}{|l|}
$\mid 12 / 21 / 2017$ \\
\end{tabular} \\
\hline 579 & 6 & 643 & - & - & - & c & 11/III & $\mathrm{N} 1001.5$ & E1004 & 3 & $99.5-99.4$ & $40-50$ & - & Detritus & Debitage & \begin{tabular}{|l|} 
Complete Flake \\
\end{tabular} & Lithic & 2 & 0.16 & & 12/21/2017 \\
\hline 579 & 7 & 643 & - & - & - & c & II/III & $\mathrm{N} 1001.5$ & E1004 & 3 & $99.5-99.4$ & $40-50$ & - & Detritus & Debitage & \begin{tabular}{|l|} 
Proximal Flake \\
\end{tabular} & Lithic & 6 & 1.93 & & 12/21/2017 \\
\hline 579 & 8 & 643 & - & - & - & c & $1 / 1 / 11$ & $\mathrm{~N} 1001.5$ & E1004 & 3 & 99.5-99.4 & $40-50$ & - & Detritus & Debitage & Broken Flake & Lithic & 4 & 1.01 & & $\mid$\begin{tabular}{|l|}
$\mid 12 / 21 / 2017$ \\
\end{tabular} \\
\hline 579 & 9 & 643 & - & - & - & c & 11/III & N1001.5 & E1004 & 3 & $99.5-99.4$ & $40-50$ & - & Detritus & Debitage & Thermal Shatter & Lithic & 7 & 3.71 & & 12/21/2017 \\
\hline 579 & 10 & 643 & 32 & - & - & $\mathrm{c}$ & 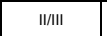 & N1001.5 & E1004 & 3 & $99.5-99.4$ & $40-50$ & - & Formal Tool & Arrow Point & \begin{tabular}{l|} 
Perdiz Point \\
\end{tabular} & Fine Grain Chert & 1 & 1.43 & & 12/21/2017 \\
\hline 580 & 1 & 648 & - & - & - & $\mathrm{c}$ & IIII & N1001.5 & E1004 & 4 & $99.4-99.3$ & $50-60$ & - & Faunal Remains & Bone & Mammal & Bone & 1 & 7.62 & & 12/21/2017 \\
\hline 580 & 2 & 648 & - & - & - & $\mathrm{c}$ & IIII & N1001.5 & E1004 & 4 & $99.4-99.3$ & 50-60 & - & Faunal Remains & Bone & Mammal & Bone & 1 & 4.23 & & 12/21/2017 \\
\hline 580 & 3 & 648 & - & - & - & $\mathrm{c}$ & IIII & N1001.5 & E1004 & 4 & $99.4-99.3$ & 50-60 & - & Faunal Remains & Bone & Indeterminate & Bone & 1 & 0.24 & & 12/21/2017 \\
\hline 580 & 4 & 648 & - & - & - & $\mathrm{c}$ & IIII & N1001.5 & E1004 & 4 & $99.4-99.3$ & 50-60 & - & Faunal Remains & Bone & Tortoise/Turtle & Bone & 1 & 0.09 & & 12/21/2017 \\
\hline 580 & 5 & 648 & - & - & - & c & IIII & $\mathrm{N} 1001.5$ & E1004 & 4 & 99.499 .3 & $50-60$ & - & Faunal Remains & Bone & Indeterminate & Bone & 1 & 0.14 & & 12/21/2017 \\
\hline 580 & 6 & 648 & - & - & - & c & III & N1001.5 & E1004 & 4 & $\begin{array}{l}99.4-99.3 \\
\end{array}$ & 50-60 & - & Faunal Remains & Bone & Indeterminate & Bone & 1 & 0.04 & & 12/21/2017 \\
\hline 580 & 7 & 648 & - & - & - & c & IIII & N1001.5 & E1004 & 4 & 99.4-99.3 & $50-60$ & - & Faunal Remains & Bone & Indeterminate & Bone & 1 & 0.01 & & 12/21/2017 \\
\hline 580 & 8 & 648 & - & - & - & c & IIII & $\mathrm{N} 1001.5$ & E1004 & 4 & 99.499 .3 & $50-60$ & - & Faunal Remains & Bone & Indeterminate & Bone & 1 & 0.01 & & 12/21/2017 \\
\hline 580 & 9 & 648 & - & - & - & c & IIII & $\mathrm{N} 1001.5$ & E1004 & 4 & 99.499 .3 & $50-60$ & - & Faunal Remains & Bone & Indeterminate & Bone & 1 & 0.01 & & 12/21/2017 \\
\hline 580 & 10 & 648 & - & - & - & c & IIII & $\mathrm{N} 1001.5$ & E1004 & 4 & 99.499 .3 & $50-60$ & - & Detritus & Debitage & Complete Flake & Lithic & 2 & 1.62 & & 12/21/2017 \\
\hline 580 & 11 & 648 & - & - & - & $\mathrm{c}$ & IIII & N1001.5 & E1004 & 4 & $\begin{array}{l}99.4-99.3 \\
\end{array}$ & 50-60 & - & Detritus & Debitage & \begin{tabular}{|l|} 
Proximal Flake \\
\end{tabular} & Lithic & 4 & 20.21 & & 12/21/2017 \\
\hline 580 & 12 & 648 & - & - & - & $\mathrm{c}$ & IIII & N1001.5 & E1004 & 4 & $99.4-99.3$ & $50-60$ & - & Detritus & Debitage & Broken Flake & Lithic & 10 & 5.32 & & 12/21/2017 \\
\hline 580 & 13 & 648 & - & - & - & $\mathrm{c}$ & IIII & N1001.5 & E1004 & 4 & $\begin{array}{l}99.4-99.3 \\
\end{array}$ & 50-60 & - & Detritus & Debitage & Thermal Shatter & Lithic & 15 & 15.47 & & 12/21/2017 \\
\hline 580 & 14 & 648 & - & - & - & c & IIII & $\mathrm{N} 1001.5$ & E1004 & 4 & $99.4-99.3$ & $50-60$ & - & Formal Tool & Biface & Complete & Fine Grain Chert & 1 & 90.68 & & 12/21/2017 \\
\hline 581 & 1 & 651 & - & - & - & c & $11 / 111$ & N1001.5 & E1005 & 2 & $99.5-99.4$ & $40-50$ & - & Faunal Remains & Bone & Indeterminate & Bone & 1 & 0.08 & & 12/21/2017 \\
\hline 581 & 2 & 651 & - & - & - & c & 11/III & $\mathrm{N} 1001.5$ & E1005 & 2 & $99.5-99.4$ & $40-50$ & - & Faunal Remains & Bone & Indeterminate & Bone & 1 & 0.26 & & 12/21/2017 \\
\hline 581 & 3 & 651 & - & - & - & c & "1/III & $\mathrm{N} 1001.5$ & E1005 & 2 & $99.5-99.4$ & $40-50$ & - & Detritus & Debitage & \begin{tabular}{|l|} 
Complete Flake \\
\end{tabular} & Lithic & 5 & 15.34 & & 12/21/2017 \\
\hline 581 & 4 & 651 & - & - & - & c & "1/III & N1001.5 & E1005 & 2 & $99.5-99.4$ & $40-50$ & - & Detritus & Debitage & \begin{tabular}{|l|} 
Proximal Flake \\
\end{tabular} & Lithic & 1 & 1.71 & & 12/21/2017 \\
\hline 581 & 5 & 651 & - & - & - & c & II/III & $\mathrm{N} 1001.5$ & E1005 & 2 & $99.5-99.4$ & $40-50$ & - & Detritus & Debitage & Broken Flake & Lithic & 3 & 4.34 & & 12/21/2017 \\
\hline 581 & 6 & 651 & - & - & - & c & $\|/\| 11$ & $\mathrm{~N} 1001.5$ & E1005 & 2 & $99.5-99.4$ & $40-50$ & - & Detritus & Debitage & Thermal Shatter & Lithic & 3 & 0.51 & & 12/21/2017 \\
\hline 582 & 1 & 654 & - & - & - & c & III & $\mathrm{N} 1001.5$ & E1005 & 3 & 99.4-99.3 & 50-60 & - & Faunal Remains & Bone & Indeterminate & Bone & 1 & 0.18 & & $\mid$\begin{tabular}{|l|}
$\mid 12 / 21 / 2017$ \\
\end{tabular} \\
\hline 582 & 2 & 654 & - & - & - & c & III & N1001.5 & E1005 & 3 & $99.4-99.3$ & $50-60$ & - & Detritus & Debitage & \begin{tabular}{|l|} 
Complete Flake \\
\end{tabular} & Lithic & 1 & 0.39 & & 12/21/2017 \\
\hline 582 & 3 & 654 & - & - & - & c & IIII & N1001.5 & E1005 & 3 & 99.4-99.3 & $50-60$ & - & Detritus & Debitage & Broken Flake & Lithic & 9 & 5.78 & & 12/21/2017 \\
\hline 582 & 4 & 654 & - & - & - & $\mathrm{c}$ & IIII & N1001.5 & E1005 & 3 & $\begin{array}{ll}99.4-99.3 \\
\end{array}$ & $50-60$ & - & Detritus & Debitage & Thermal Shatter & Lithic & 11 & 6.94 & & \begin{tabular}{|l|}
$12 / 21 / 2017$ \\
\end{tabular} \\
\hline 583 & 1 & 513 & - & - & - & $\mathrm{E}$ & 1 & N1003 & E1017 & 1 & $99.28-99.2$ & $2-10$ & - & Detritus & Debitage & Thermal Shatter & Lithic & 1 & 2.77 & & 12/13/2017 \\
\hline 584 & 1 & 518 & - & - & - & $\mathrm{E}$ & "I & N1003 & E1017 & 2 & $99.2-99.1$ & $10-20$ & - & Detritus & Debitage & Broken Flake & Lithic & 3 & 0.41 & & 12/13/2017 \\
\hline 585 & 1 & 519 & - & - & - & $\mathrm{E}$ & 11/III & N1003 & E1017 & 3 & $99.1-99.0$ & $20-30$ & - & Detritus & Debitage & \begin{tabular}{|c|} 
Complete Flake \\
\end{tabular} & Lithic & 3 & 2.97 & & 12/13/2017 \\
\hline 585 & 2 & 519 & - & - & - & $\mathrm{E}$ & 11/III & N1003 & E1017 & 3 & $99.1-99.0$ & $20-30$ & - & Detritus & Debitage & Proximal Flake & Lithic & 4 & 3.39 & & \begin{tabular}{|l|}
$12 / 13 / 2017$ \\
\end{tabular} \\
\hline 585 & 3 & 519 & - & - & - & $\mathrm{E}$ & II/III & N1003 & E1017 & 3 & $99.1-99.0$ & $20-30$ & - & Detritus & Debitage & Broken Flake & Lithic & 8 & 26.82 & & 12/13/2017 \\
\hline 585 & 4 & 519 & - & - & - & $\mathrm{E}$ & 11/111 & N1003 & E1017 & 3 & $99.1-99.0$ & $20-30$ & - & Detritus & Debitage & Thermal Shatter & Lithic & 5 & 1.9 & & 12/13/2017 \\
\hline 586 & 1 & 523 & - & - & - & E & III & N1003 & E1017 & 4 & $99.0-98.9$ & $30-40$ & - & Detritus & Debitage & Complete Flake & Lithic & 5 & 3.36 & & \begin{tabular}{|l|}
$12 / 13 / 2017$ \\
\end{tabular} \\
\hline 586 & 2 & 523 & - & - & - & $\mathrm{E}$ & IIII & N1003 & E1017 & 4 & 99.098 .9 & $30-40$ & - & Detritus & Debitage & \begin{tabular}{|l|} 
Proximal Flake \\
\end{tabular} & Lithic & 6 & 33.56 & & 12/13/2017 \\
\hline 586 & 3 & 523 & - & - & - & $\mathrm{E}$ & IIII & N1003 & E1017 & 4 & $\begin{array}{l}99.0-98.9 \\
\end{array}$ & $30-40$ & - & Detritus & Debitage & Broken Flake & Lithic & 21 & 5.64 & & 12/13/2017 \\
\hline 586 & 4 & 523 & - & - & - & E & III & N1003 & E1017 & 4 & $99.0-98.9$ & $30-40$ & - & Detritus & Debitage & Flaking Shatter & Lithic & 2 & 10.9 & & \begin{tabular}{|l|}
$12 / 13 / 2017$ \\
\end{tabular} \\
\hline
\end{tabular}




\begin{tabular}{|c|c|c|c|c|c|c|c|c|c|c|c|c|c|c|c|c|c|c|c|c|c|}
\hline Lot No. & \begin{tabular}{|c|}
$\begin{array}{c}\text { Specimen } \\
\text { No. }\end{array}$ \\
\end{tabular} & FS No. & UI No. & FTR No. & $\begin{array}{c}\text { Sample } \\
\text { No. }\end{array}$ & $\begin{array}{c}\text { Excavation } \\
\text { Block }\end{array}$ & Stratum (AU) & Northing & Easting & Level & $\begin{array}{c}\text { Elevation } \\
(\mathrm{m})\end{array}$ & Depth (cmbd) & Point Prov. & Artifact Class & Artifact Type & \begin{tabular}{|c|} 
Artifact \\
Description
\end{tabular} & Material & $\begin{array}{c}\text { Number of } \\
\text { artifacts }\end{array}$ & Weight (g) & Comments & Date \\
\hline 586 & 5 & 523 & - & - & - & $E$ & III & $\mathrm{N} 1003$ & E1017 & 4 & $99.0-98.9$ & $30-40$ & - & Detritus & Debitage & Thermal Shatter & Lithic & 8 & 2.59 & & 12/13/2017 \\
\hline 587 & 1 & 527 & - & - & - & E & IIII & $\mathrm{N} 1003$ & E1017 & 5 & 98.85 & 45 & $\begin{array}{l}\begin{array}{l}N 11003.08 \\
\text { E1017.80 }\end{array} \\
\end{array}$ & Informal Tool & Modified Flake & \begin{tabular}{|l|} 
Edge-modified \\
\end{tabular} & Fine Grain Chert & 1 & 7.8 & & 12/13/2017 \\
\hline 588 & 1 & 527 & - & - & - & $\mathrm{E}$ & IIII & N1003 & E1017 & 5 & $98.9-98.8$ & $40-50$ & - & \begin{tabular}{|l|} 
Faunal Remains \\
\end{tabular} & Bone & \begin{tabular}{|l|} 
Indeterminate \\
\end{tabular} & Bone & 1 & 0.22 & & 12/13/2017 \\
\hline 588 & 2 & 527 & - & - & - & E & IIII & N1003 & E1017 & 5 & $98.9-98.8$ & $40-50$ & - & Detritus & Debitage & \begin{tabular}{|l|} 
Complete Flake \\
\end{tabular} & Lithic & 7 & 91.11 & & 12/13/2017 \\
\hline 588 & 3 & 527 & - & - & - & E & III & $\mathrm{N} 1003$ & E1017 & 5 & $98.9-98.8$ & $40-50$ & - & Detritus & Debitage & \begin{tabular}{|l|} 
Proximal Flake \\
\end{tabular} & Lithic & 20 & 14.52 & & 12/13/2017 \\
\hline 588 & 4 & 527 & - & - & - & E & IIII & N1003 & E1017 & 5 & $98.9-98.8$ & $40-50$ & - & Detritus & Debitage & Broken Flake & Lithic & 26 & 14.33 & & 12/13/2017 \\
\hline 588 & 5 & 527 & - & - & - & $\mathrm{E}$ & III & N1003 & E1017 & 5 & $98.9-98.8$ & $40-50$ & - & Detritus & Debitage & Thermal Shatter & Lithic & 22 & 21.58 & & 12/13/2017 \\
\hline 589 & 1 & 557 & - & - & - & E & 1111 & N1003 & E1017 & 6 & 98.77 & 53 & $\begin{array}{ll}\mathrm{N} 1003.22 \\
\mathrm{E} 1017.93 \\
\end{array}$ & Faunal Remains & Shell & Mussel Shell & Shell & 2 & 11.7 & & $\mid$\begin{tabular}{|l|}
$\mid 12 / 15 / 2017$ \\
\end{tabular} \\
\hline 590 & 1 & 557 & - & - & - & E & III & $\mathrm{N} 1003$ & E1017 & 6 & 98.8-98.7 & $50-60$ & - & Faunal Remains & Bone & Indeterminate & Bone & 1 & 0.28 & & 12/15/2017 \\
\hline 590 & 2 & 557 & - & - & - & $\mathrm{E}$ & IIII & N1003 & E1017 & 6 & $\begin{array}{ll}98.8-98.7 \\
\end{array}$ & 50-60 & - & Detritus & Debitage & \begin{tabular}{|c|} 
Complete Flake \\
\end{tabular} & Lithic & 11 & 28.69 & & 12/15/2017 \\
\hline 590 & 3 & 557 & - & - & - & E & IIII & $\mathrm{N} 1003$ & E1017 & 6 & 98.8-98.7 & $50-60$ & - & Detritus & Debitage & \begin{tabular}{|l|} 
Proximal Flake \\
\end{tabular} & Lithic & 22 & 51.94 & & 12/15/2017 \\
\hline 590 & 4 & 557 & - & - & - & $\mathrm{E}$ & IIII & N1003 & E1017 & 6 & $\begin{array}{ll}98.8-98.7 \\
\end{array}$ & 50-60 & - & Detritus & Debitage & Broken Flake & Lithic & 19 & 7.03 & & 12/15/2017 \\
\hline 590 & 5 & 557 & - & - & - & E & III & $\mathrm{N} 1003$ & E1017 & 6 & 98.8-98.7 & $50-60$ & - & Detritus & Debitage & Thermal Shatter & Lithic & 34 & 65.88 & & 12/15/2017 \\
\hline 590 & 6 & 557 & - & - & - & $\mathrm{E}$ & IIII & N1003 & E1017 & 6 & $\begin{array}{ll}98.8-98.7 \\
\end{array}$ & 50-60 & - & Informal Tool & Modified Flake & \begin{tabular}{|l|} 
Edge-modified \\
\end{tabular} & Fine Grain Chert & 1 & 3.63 & & 12/15/2017 \\
\hline 590 & 7 & 557 & - & - & - & $\mathrm{E}$ & IIII & $\mathrm{N} 1003$ & E1017 & 6 & 98.8-98.7 & $50-60$ & - & Informal Tool & Modified Flake & Edge-modified & Fine Grain Chert & 1 & 40.92 & & 12/15/2017 \\
\hline 591 & 1 & 563 & - & - & - & $\mathrm{E}$ & iv & N1003 & E1017 & 7 & 98.7-98.6 & $60-70$ & - & Detritus & Debitage & Complete Flake & Lithic & 9 & 36.3 & & 12/15/2017 \\
\hline 591 & 2 & 563 & - & - & - & $\mathrm{E}$ & IV & $\mathrm{N} 1003$ & E1017 & 7 & 98.7-98.6 & $60-70$ & - & Detritus & Debitage & Proximal Flake & Lithic & 28 & 129.27 & & 12/15/2017 \\
\hline 591 & 3 & 563 & - & - & - & $\mathrm{E}$ & iv & N1003 & E1017 & 7 & 98.7-98.6 & $60-70$ & - & Detritus & Debitage & Broken Flake & Lithic & 51 & 58.76 & & 12/15/2017 \\
\hline 591 & 4 & 563 & - & - & - & $\mathrm{E}$ & IV & N1003 & E1017 & 7 & $\begin{array}{l}98.7-98.6 \\
\end{array}$ & $60-70$ & - & Detritus & Debitage & Thermal Shatter & Lithic & 32 & 31.66 & & 12/15/2017 \\
\hline 591 & 5 & 563 & - & - & - & $\mathrm{E}$ & iv & N1003 & E1017 & 7 & $98.7-98.6$ & $60-70$ & - & \begin{tabular}{|l|} 
Faunal Remains \\
\end{tabular} & Shell & Snail Shell & Shell & 1 & & 1 Large snail collected for ID & 12/15/2017 \\
\hline 592 & 1 & 564 & - & - & - & $\mathrm{E}$ & IV & N1003 & E1017 & 7 & 98.64 & 66 & \begin{tabular}{|l|} 
N1003.48 \\
E1017.42
\end{tabular} & Informal Tool & Core Tool & Chopper & $\begin{array}{l}\begin{array}{c}\text { Coarse Grain } \\
\text { Chert }\end{array} \\
\text { (n) }\end{array}$ & 1 & 401.29 & $\begin{array}{l}\text { Core tools are on Biface analysis } \\
\text { sheet }\end{array}$ & $12 / 15 / 2017$ \\
\hline 593 & 1 & 565 & - & - & - & $\mathrm{E}$ & iv & $\mathrm{N} 1003$ & E1017 & 7 & 98.61 & 69 & \begin{tabular}{l|l|} 
N11003.98 \\
E101744
\end{tabular} & Formal Tool & Biface & Complete & Fine Grain Chert & 1 & 52.29 & & 12/15/2017 \\
\hline 594 & 1 & 566 & - & - & - & $\mathrm{E}$ & IV & N1003 & E1017 & 7 & 98.64 & 66 & \begin{tabular}{|l|} 
N1003.65 \\
E1017.62 \\
\end{tabular} & Formal Tool & Biface & Complete & Fine Grain Chert & 1 & 36.81 & & 12/15/2017 \\
\hline 595 & 1 & 570 & - & - & - & E & IV & N1003 & E1017 & 8 & $98.6-98.5$ & $70-80$ & - & Detritus & Debitage & Complete Flake & Lithic & 11 & 43.53 & & 12/18/2017 \\
\hline 595 & 2 & 570 & - & - & - & $\mathrm{E}$ & iv & $\mathrm{N} 1003$ & E1017 & 8 & $98.6-98.5$ & $70-80$ & - & Detritus & Debitage & Proximal Flake & Lithic & 13 & 10.51 & & \begin{tabular}{|l|l|}
$12 / 18 / 2017$ \\
\end{tabular} \\
\hline 595 & 3 & 570 & - & - & - & $\mathrm{E}$ & iv & $\mathrm{N} 1003$ & E1017 & 8 & $98.6-98.5$ & $70-80$ & - & Detritus & Debitage & Broken Flake & Lithic & 40 & 39.39 & & 12/18/2017 \\
\hline 595 & 4 & 570 & - & - & - & $E$ & iv & N1003 & E1017 & 8 & $98.6-98.5$ & $70-80$ & - & Detritus & Debitage & Thermal Shatter & Lithic & 8 & 6.46 & & $12 / 18 / 2017$ \\
\hline 596 & 1 & 574 & - & - & - & $\mathrm{E}$ & $\mathrm{v}$ & N1003 & E1017 & 9 & $98.5-98.4$ & $80-90$ & - & \begin{tabular}{|l|} 
Faunal Remains \\
\end{tabular} & Bone & Indeterminate & Bone & 1 & 0.73 & & 12/18/2017 \\
\hline 596 & 2 & 574 & - & - & - & $\mathrm{E}$ & $\mathrm{v}$ & $\mathrm{N} 1003$ & E1017 & 9 & $98.5-98.4$ & $80-90$ & - & \begin{tabular}{|l|} 
Faunal Remains \\
\end{tabular} & Bone & Indeterminate & Bone & 1 & 0.01 & & 12/18/2017 \\
\hline 596 & 3 & 574 & - & - & - & $\mathrm{E}$ & $\mathrm{v}$ & $\mathrm{N} 1003$ & E1017 & 9 & $98.5-98.4$ & $80-90$ & - & \begin{tabular}{|l|} 
Faunal Remains \\
\end{tabular} & Bone & Indeterminate & Bone & 1 & 0.01 & & 12/18/2017 \\
\hline 596 & 4 & 574 & - & - & - & $E$ & $\mathrm{v}$ & N1003 & E1017 & 9 & 98.5-98.4 & $80-90$ & - & Detritus & Debitage & Complete Flake & Lithic & 1 & 75.04 & & $\mid 12 / 18 / 2017$ \\
\hline 596 & 5 & 574 & - & - & - & $\mathrm{E}$ & $\mathrm{v}$ & $\mathrm{N} 1003$ & E1017 & 9 & $98.5-98.4$ & $80-90$ & - & Detritus & Debitage & \begin{tabular}{|l|} 
Proximal Flake \\
\end{tabular} & Lithic & 3 & 0.58 & & 12/18/2017 \\
\hline 596 & 6 & 574 & - & - & - & $\mathrm{E}$ & $\mathrm{v}$ & $\mathrm{N} 1003$ & E1017 & 9 & $98.5-98.4$ & $80-90$ & - & Detritus & Debitage & Broken Flake & Lithic & 10 & 3.99 & & 12/18/2017 \\
\hline 596 & 7 & 574 & - & - & - & $\mathrm{E}$ & $\mathrm{v}$ & N1003 & E1017 & 9 & $98.5-98.4$ & $80-90$ & - & Detritus & Debitage & Thermal Shatter & Lithic & 8 & 4.92 & & \begin{tabular}{|l|}
$12 / 18 / 2017$ \\
\end{tabular} \\
\hline 597 & 1 & 575 & - & - & - & $\mathrm{E}$ & $\mathrm{V} N \mathrm{~N}$ & N1003 & E1017 & 10 & $98.4-98.3$ & $\begin{array}{l}0-100 \\
\end{array}$ & - & Detritus & Debitage & \begin{tabular}{|c|} 
Complete Flake \\
\end{tabular} & Lithic & 2 & 1.21 & & 12/18/2017 \\
\hline 597 & 2 & 575 & - & - & - & E & $V_{N 1}$ & $\mathrm{~N} 1003$ & E1017 & 10 & 98.4-98.3 & $90-100$ & - & Detritus & Debitage & Proximal Flake & Lithic & 4 & 2.17 & & 12/18/2017 \\
\hline 597 & 3 & 575 & - & - & - & $\mathrm{E}$ & $\mathrm{V} N \mathrm{NI}$ & N1003 & E1017 & 10 & $98.4-98.3$ & $90-100$ & - & Detritus & Debitage & Broken Flake & Lithic & 5 & 2.54 & & $\mid 12 / 18 / 2017$ \\
\hline 597 & 4 & 575 & - & - & - & E & VNI & $\mathrm{N} 1003$ & E1017 & 10 & 98.4-98.3 & $90-100$ & - & Detritus & Debitage & Thermal Shatter & Lithic & 7 & 4.26 & & 12/18/2017 \\
\hline 597 & 5 & 575 & - & - & - & $\mathrm{E}$ & $\mathrm{V} N \mathrm{~N}_{\mathrm{N}}$ & N1003 & E1017 & 10 & 98.4-98.3 & $90-100$ & - & Formal Tool & Biface & \begin{tabular}{c|} 
Indeterminate \\
Fragment \\
\end{tabular} & Fine Grain Chert & 1 & 31.38 & & 12/18/2017 \\
\hline 598 & 1 & 659 & - & - & - & $\mathrm{E}$ & vi & $\mathrm{N} 1003$ & E1017 & 11 & 98.3-98.2 & $100-110$ & - & Detritus & Debitage & Proximal Flake & Lithic & 2 & 4.38 & & 12/27/2017 \\
\hline 598 & 2 & 659 & - & - & - & $\mathrm{E}$ & vi & N1003 & E1017 & 11 & $98.3-98.2$ & 100-110 & - & Detritus & Debitage & Broken Flake & Lithic & 1 & 0.4 & & \begin{tabular}{|l|}
$12 / 27 / 2017$ \\
\end{tabular} \\
\hline 598 & 3 & 659 & - & - & - & $\mathrm{E}$ & $\mathrm{vi}$ & N1003 & E1017 & 11 & $98.3-98.2$ & $100-110$ & - & $\begin{array}{l}\text { Informal Tool } \\
\end{array}$ & \begin{tabular}{|l|} 
Modified Flake \\
\end{tabular} & Utilized Flake & $\begin{array}{c}\begin{array}{c}\text { coarse Grain } \\
\text { Chert }\end{array} \\
\end{array}$ & 1 & 5.79 & & 12/27/2017 \\
\hline 599 & 1 & 660 & - & - & - & $\mathrm{E}$ & vi & $\mathrm{N} 1003$ & E1017 & 12 & 98.2-98.1 & $110-120$ & - & Detritus & Debitage & Broken Flake & Lithic & 1 & 0.08 & & 12/27/2017 \\
\hline 600 & 1 & 662 & - & - & - & $\mathrm{E}$ & vi & N1003 & E1017 & 13 & $98.1-98.0$ & $120-130$ & - & Detritus & Debitage & \begin{tabular}{|l|} 
Proximal Flake \\
\end{tabular} & Lithic & 1 & 0.15 & & 12/27/2017 \\
\hline
\end{tabular}




\begin{tabular}{|c|c|c|c|c|c|c|c|c|c|c|c|c|c|c|c|c|c|c|c|c|c|}
\hline Lot No. & \begin{tabular}{|c|}
$\begin{array}{c}\text { Specimen } \\
\text { No. }\end{array}$ \\
\end{tabular} & FS No. & UI No. & FTR No. & $\begin{array}{c}\text { Sample } \\
\text { No. }\end{array}$ & $\begin{array}{c}\text { Excavation } \\
\text { Block }\end{array}$ & Stratum (AU) & Northing & Easting & Level & $\begin{array}{c}\text { Elevation } \\
(\mathrm{m})\end{array}$ & Depth (cmbd) & Point Prov. & Artifact Class & Artifact Type & \begin{tabular}{c|} 
Artifact \\
Description
\end{tabular} & Material & $\begin{array}{c}\text { Number of } \\
\text { artifacts }\end{array}$ & Weight (g) & Comments & Date \\
\hline 601 & 1 & 664 & - & - & - & $E$ & vi & $\mathrm{N} 1003$ & E1017 & 14 & $98.0-97.9$ & 130-140 & - & Detritus & Debitage & \begin{tabular}{|l|} 
Complete Flake \\
\end{tabular} & Lithic & 1 & 13.86 & & 12/27/2017 \\
\hline 601 & 2 & 664 & - & - & - & E & vi & $\mathrm{N} 1003$ & E1017 & 14 & 98.0-97.9 & 130-140 & - & Detritus & Debitage & Proximal Flake & Lithic & 2 & 6.3 & & $\mid$\begin{tabular}{|l|}
$\mid 12 / 27 / 2017$ \\
\end{tabular} \\
\hline 602 & 1 & 409 & - & - & - & E & "I & N1003 & E1018 & 2 & $99.2-99.1$ & $10-20$ & - & Detritus & Debitage & \begin{tabular}{|l|} 
Proximal Flake \\
\end{tabular} & Lithic & 1 & 2.48 & & 12/4/2017 \\
\hline 602 & 2 & 409 & - & - & - & E & "I & N1003 & E1018 & 2 & 99.2-99.1 & $10-20$ & - & Detritus & Debitage & Broken Flake & Lithic & 2 & 0.25 & & 12/4/2017 \\
\hline 603 & 1 & 424 & - & - & - & E & $\|/\| 11$ & $\mathrm{~N} 1003$ & E1018 & 3 & $99.1-99.0$ & $20-30$ & - & Detritus & Debitage & Proximal Flake & Lithic & 5 & 7.09 & & 12/5/2017 \\
\hline 603 & 2 & 424 & - & - & - & E & 11/III & N1003 & E1018 & 3 & $99.1-99.0$ & $20-30$ & - & Detritus & Debitage & Broken Flake & Lithic & 8 & 4.69 & & 12/5/2017 \\
\hline 603 & 3 & 424 & - & - & - & E & 11/III & N1003 & E1018 & 3 & $99.1-99.0$ & $20-30$ & - & Detritus & Debitage & Thermal Shatter & Lithic & 2 & 0.51 & & 12/5/2017 \\
\hline 604 & 1 & 436 & - & - & - & E & III & N1003 & E1018 & 4 & 99.0-98.9 & $30-40$ & - & Detritus & Debitage & Complete Flake & Lithic & 15 & 24.49 & & 12/5/2017 \\
\hline 604 & 2 & 436 & - & - & - & E & III & N1003 & E1018 & 4 & $99.0-98.9$ & $30-40$ & - & Detritus & Debitage & Proximal Flake & Lithic & 9 & 9.21 & & 12/5/2017 \\
\hline 604 & 3 & 436 & - & - & - & $\mathrm{E}$ & IIII & N1003 & E1018 & 4 & $99.0-98.9$ & $30-40$ & - & Detritus & Debitage & Broken Flake & Lithic & 20 & 8.16 & & 12/5/2017 \\
\hline 604 & 4 & 436 & - & - & - & E & IIII & $\mathrm{N} 1003$ & E1018 & 4 & 99.0-98.9 & $30-40$ & - & Detritus & Debitage & Thermal Shatter & Lithic & 15 & 21.37 & & 12/5/2017 \\
\hline 604 & 5 & 436 & - & - & - & $\mathrm{E}$ & IIII & N1003 & E1018 & 4 & $99.0-98.9$ & $30-40$ & - & Faunal Remains & Shell & Mussel Shell & Shell & 1 & 2.09 & & 12/5/2017 \\
\hline 605 & 1 & 450 & - & - & - & E & III & $\mathrm{N} 1003$ & E1018 & 5 & $98.9-98.8$ & $40-50$ & - & Detritus & Debitage & Complete Flake & Lithic & 10 & 20.52 & & 12/8/2017 \\
\hline 605 & 2 & 450 & - & - & - & $\mathrm{E}$ & IIII & N1003 & E1018 & 5 & $\begin{array}{ll}88.9-98.8 \\
\end{array}$ & $40-50$ & - & Detritus & Debitage & Proximal Flake & Lithic & 10 & 8.61 & & 12/8/2017 \\
\hline 605 & 3 & 450 & - & - & - & $\mathrm{E}$ & IIII & $\mathrm{N} 1003$ & E1018 & 5 & 98.9-98.8 & $40-50$ & - & Detritus & Debitage & Broken Flake & Lithic & 23 & 11.32 & & 12/8/2017 \\
\hline 605 & 4 & 450 & - & - & - & $\mathrm{E}$ & IIII & $\mathrm{N} 1003$ & E1018 & 5 & 98.9-98.8 & $40-50$ & - & Detritus & Debitage & Flaking Shatter & Lithic & 3 & 4.07 & & 12/8/2017 \\
\hline 605 & 5 & 450 & - & - & - & $\mathrm{E}$ & IIII & $\mathrm{N} 1003$ & E1018 & 5 & 98.9-98.8 & $40-50$ & - & Detritus & Debitage & \begin{tabular}{|l|} 
Thermal Shatter \\
\end{tabular} & Lithic & 8 & 12.56 & & 12/8/2017 \\
\hline 605 & 6 & 450 & - & - & - & E & IIII & $\mathrm{N} 1003$ & E1018 & 5 & 98.9-98.8 & $40-50$ & - & Faunal Remains & Shell & Mussel Shell & Shell & 3 & 6.22 & & 12/8/2017 \\
\hline 606 & 1 & 463 & - & - & - & E & IIII & $\mathrm{N} 1003$ & E1018 & 6 & $98.8-98.7$ & $50-60$ & - & Detritus & Debitage & Complete Flake & Lithic & 15 & 104.1 & & 12/8/2017 \\
\hline 606 & 2 & 463 & - & - & - & E & IIII & $\mathrm{N} 1003$ & E1018 & 6 & 98.8-98.7 & $50-60$ & - & Detritus & Debitage & \begin{tabular}{|l|} 
Proximal Flake \\
\end{tabular} & Lithic & 19 & 19.37 & & 12/8/2017 \\
\hline 606 & 3 & 463 & - & - & - & E & IIII & $\mathrm{N} 1003$ & E1018 & 6 & 98.8-98.7 & $50-60$ & - & Detritus & Debitage & Broken Flake & Lithic & 48 & 33.69 & & 12/8/2017 \\
\hline 606 & 4 & 463 & - & - & - & E & IIII & $\mathrm{N} 1003$ & E1018 & 6 & $98.8-98.7$ & $50-60$ & - & Detritus & Debitage & \begin{tabular}{|l|} 
Thermal Shatter \\
\end{tabular} & Lithic & 19 & 32.8 & & 12/8/2017 \\
\hline 606 & 5 & 463 & - & - & - & E & IIII & $\mathrm{N} 1003$ & E1018 & 6 & $98.8-98.7$ & $50-60$ & - & Faunal Remains & Shell & Mussel Shell & Shell & 2 & 4.6 & & 12/8/2017 \\
\hline 607 & 1 & 472 & - & - & - & E & IV & $\mathrm{N} 1003$ & E1018 & 7 & 98.7-98.6 & $60-70$ & - & Detritus & Debitage & Complete Flake & Lithic & 13 & 36.58 & & 12/11/2017 \\
\hline 607 & 2 & 472 & - & - & - & E & IV & N1003 & E1018 & 7 & 98.7-98.6 & $60-70$ & - & Detritus & Debitage & Proximal Flake & Lithic & 12 & 5.74 & & 12/11/2017 \\
\hline 607 & 3 & 472 & - & - & - & E & IV & N1003 & E1018 & 7 & 98.7-98.6 & $60-70$ & - & Detritus & Debitage & Broken Flake & Lithic & 35 & 22.14 & & 12/11/2017 \\
\hline 607 & 4 & 472 & - & - & - & E & IV & $\mathrm{N} 1003$ & E1018 & 7 & 98.7-98.6 & $60-70$ & - & Detritus & Debitage & \begin{tabular}{|l|} 
Flaking Shatter \\
\end{tabular} & Lithic & 2 & 12.68 & & 12/11/2017 \\
\hline 607 & 5 & 472 & - & - & - & E & IV & N1003 & E1018 & 7 & 98.7-98.6 & $60-70$ & - & Detritus & Debitage & Thermal Shatter & Lithic & 18 & 24.22 & & 12/11/2017 \\
\hline 607 & 6 & 472 & - & - & - & E & $1 \mathrm{IIIV}$ & N1003 & E1018 & 7 & 98.7-98.6 & $60-70$ & - & Formal Tool & Arrow Point & \begin{tabular}{l|l|} 
Fresno Point \\
\end{tabular} & Fine Grain Chert & 1 & 2.19 & Proximal end & 12/11/2017 \\
\hline 608 & 1 & 510 & - & - & - & E & IV & N1003 & E1018 & 8 & $98.6-98.5$ & $70-80$ & - & Faunal Remains & Bone & Indeterminate & Bone & 1 & 0.23 & & 12/13/2017 \\
\hline 608 & 2 & 510 & - & - & - & E & IV & N1003 & E1018 & 8 & $98.6-98.5$ & $70-80$ & - & Faunal Remains & Shell & Mussel Shell & Shell & 5 & 17.05 & & 12/13/2017 \\
\hline 608 & 3 & 510 & - & - & - & E & IV & N1003 & E1018 & 8 & $98.6-98.5$ & $70-80$ & - & Informal Tool & Modified Flake & \begin{tabular}{|l|} 
Edge-modified \\
\end{tabular} & Fine Grain Chert & 1 & 6.13 & & 12/13/2017 \\
\hline 608 & 4 & 510 & - & - & - & E & IV & N1003 & E1018 & 8 & $98.6-98.5$ & $70-80$ & - & Informal Tool & Modified Flake & Edge-modified & Fine Grain Chert & 1 & 24.05 & & 12/13/2017 \\
\hline 608 & 5 & 510 & - & - & - & $\mathrm{E}$ & IV & $\mathrm{N} 1003$ & E1018 & 8 & $98.6-98.5$ & $70-80$ & - & Detritus & Debitage & Complete Flake & Lithic & 13 & 16.02 & & 12/13/2017 \\
\hline 608 & 6 & 510 & - & - & - & $\mathrm{E}$ & IV & N1003 & E1018 & 8 & $98.6-98.5$ & $\begin{array}{ll}70-80 \\
\end{array}$ & - & Detritus & Debitage & \begin{tabular}{|l|} 
Proximal Flake \\
\end{tabular} & Lithic & 23 & 25.61 & & 12/13/2017 \\
\hline 608 & 7 & 510 & - & - & - & $\mathrm{E}$ & IV & N1003 & E1018 & 8 & $98.6-98.5$ & \begin{tabular}{ll|l}
$70-80$ \\
\end{tabular} & - & Detritus & Debitage & Broken Flake & Lithic & 41 & 48.34 & & 12/13/2017 \\
\hline 608 & 8 & 510 & - & - & - & $\mathrm{E}$ & IV & N1003 & E1018 & 8 & $98.6-98.5$ & $70-80$ & - & Detritus & Debitage & Thermal Shatter & Lithic & 18 & 14.63 & & 12/13/2017 \\
\hline 609 & 1 & 545 & - & - & - & $\mathrm{E}$ & $\mathrm{v}$ & $\mathrm{N} 1003$ & E1018 & 9 & $98.5-98.4$ & $80-90$ & - & Detritus & Debitage & Complete Flake & Lithic & 9 & 162.77 & & 12/15/2017 \\
\hline 609 & 2 & 545 & - & - & - & $\mathrm{E}$ & $\mathrm{v}$ & $\mathrm{N} 1003$ & E1018 & 9 & 98.5-98.4 & $80-90$ & - & Detritus & Debitage & Proximal Flake & Lithic & 21 & 27.7 & & 12/15/2017 \\
\hline 609 & 3 & 545 & - & - & - & $\mathrm{E}$ & $\mathrm{v}$ & N1003 & E1018 & 9 & 98.5-98.4 & $80-90$ & - & Detritus & Debitage & Broken Flake & Lithic & 32 & 21.25 & & 12/15/2017 \\
\hline 609 & 4 & 545 & - & - & - & E & v & $\mathrm{N} 1003$ & E1018 & 9 & $98.5-98.4$ & $80-90$ & - & Detritus & Debitage & Flaking Shatter & Lithic & 6 & 55.85 & & 12/15/2017 \\
\hline 609 & 5 & 545 & - & - & - & E & $\mathrm{v}$ & $\mathrm{N} 1003$ & E1018 & 9 & 98.5-98.4 & $80-90$ & - & Detritus & Debitage & Thermal Shatter & Lithic & 16 & 28.51 & & 12/15/2017 \\
\hline 610 & 1 & 550 & - & - & - & E & $V N 1$ & $\mathrm{~N} 1003$ & E1018 & 10 & $98.4-98.3$ & 90-100 & - & Detritus & Debitage & \begin{tabular}{|l|} 
Complete Flake \\
\end{tabular} & Lithic & 4 & 5.96 & & 12/15/2017 \\
\hline 610 & 2 & 550 & - & - & - & E & $V_{N I}$ & N1003 & E1018 & 10 & $98.4-98.3$ & $90-100$ & - & Detritus & Debitage & \begin{tabular}{|l|} 
Proximal Flake \\
\end{tabular} & Lithic & 5 & 3.64 & & 12/15/2017 \\
\hline
\end{tabular}




\begin{tabular}{|c|c|c|c|c|c|c|c|c|c|c|c|c|c|c|c|c|c|c|c|c|c|}
\hline Lot No. & \begin{tabular}{|c|}
$\begin{array}{c}\text { Specimen } \\
\text { No. }\end{array}$ \\
\end{tabular} & FS No. & UI No. & FTR No. & $\begin{array}{c}\text { Sample } \\
\text { No. }\end{array}$ & $\begin{array}{c}\text { Excavation } \\
\text { Block }\end{array}$ & Stratum (AU) & Northing & Easting & Level & $\begin{array}{c}\text { Elevation } \\
(\mathrm{m})\end{array}$ & Depth (cmbd) & Point Prov. & Artifact Class & Artifact Type & \begin{tabular}{|c|} 
Artifact \\
Description
\end{tabular} & Material & $\begin{array}{c}\text { Number of } \\
\text { artifacts }\end{array}$ & Weight (g) & Comments & Date \\
\hline 610 & 3 & 550 & - & - & - & $E$ & VNI & $\mathrm{N} 1003$ & E1018 & 10 & 98.498 .3 & $90-100$ & - & Detritus & Debitage & Broken Flake & Lithic & 10 & 15.39 & & 12/15/2017 \\
\hline 610 & 4 & 550 & - & - & - & E & $V N 1$ & $\mathrm{~N} 1003$ & E1018 & 10 & $98.4-98.3$ & $90-100$ & - & Detritus & Debitage & Flaking Shatter & Lithic & 2 & 5.24 & & $12 / 15 / 2017$ \\
\hline 610 & 5 & 550 & - & - & - & E & $V N \mid$ & N1003 & E1018 & 10 & 98.4-98.3 & 90-100 & - & Detritus & Debitage & Thermal Shatter & Lithic & 4 & 9.58 & & 12/15/2017 \\
\hline 611 & 1 & 618 & - & - & - & E & vi & $\mathrm{N} 1003$ & E1018 & 11 & $98.3-98.2$ & $100-110$ & - & Detritus & Debitage & Proximal Flake & Lithic & 4 & 0.51 & & 12/20/2017 \\
\hline 611 & 2 & 618 & - & - & - & E & vi & $\mathrm{N} 1003$ & E1018 & 11 & 98.3-98.2 & $100-110$ & - & Detritus & Debitage & Broken Flake & Lithic & 2 & 2.43 & & 12/20/2017 \\
\hline 611 & 3 & 618 & - & - & - & E & vi & N1003 & E1018 & 11 & $98.3-98.2$ & $100-110$ & - & Detritus & Debitage & \begin{tabular}{|l|} 
Flaking Shatter \\
\end{tabular} & Lithic & 1 & 17.99 & & 12/20/2017 \\
\hline 611 & 4 & 618 & - & - & - & E & vi & N1003 & E1018 & 11 & $98.3-98.2$ & $100-110$ & - & Detritus & Debitage & Thermal Shatter & Lithic & 1 & 1.98 & & 12/20/2017 \\
\hline 612 & 1 & 629 & - & - & - & E & vi & N1003 & E1018 & 13 & $98.1-98.0$ & 120-130 & - & Faunal Remains & Bone & Indeterminate & Bone & 1 & 1.21 & & $\mid$\begin{tabular}{|l|}
$\mid 12 / 20 / 2017$ \\
\end{tabular} \\
\hline 612 & 2 & 629 & - & - & - & E & vi & N1003 & E1018 & 13 & $98.1-98.0$ & $120-130$ & - & Detritus & Debitage & Complete Flake & Lithic & 1 & 5.41 & & 12/20/2017 \\
\hline 613 & 1 & 638 & - & - & - & $\mathrm{E}$ & $\mathrm{vv}_{1}$ & N1003 & E1018 & 15 & $\begin{array}{ll}77.9-97.8 \\
\end{array}$ & 140-150 & - & Detritus & Debitage & \begin{tabular}{|c|} 
Complete Flake \\
\end{tabular} & Lithic & 3 & 34.39 & & 12/20/2017 \\
\hline 613 & 2 & 638 & - & - & - & $\mathrm{E}$ & $\mathrm{vv}_{1}$ & N1003 & E1018 & 15 & $97.9-97.8$ & $140-150$ & - & Detritus & Debitage & Flaking Shatter & Lithic & 2 & 12.97 & & 12/20/2017 \\
\hline 613 & 3 & 638 & - & - & - & $\mathrm{E}$ & $\mathrm{vv}_{1}$ & N1003 & E1018 & 15 & $97.9-97.8$ & 140-150 & - & Detritus & Debitage & Thermal Shatter & Lithic & 1 & 0.31 & & 12/20/2017 \\
\hline 614 & 1 & 413 & - & - & - & $\mathrm{E}$ & "I & N1003 & E1019 & 2 & $99.2-99.1$ & $10-20$ & - & Detritus & Debitage & \begin{tabular}{|l|} 
Proximal Flake \\
\end{tabular} & Lithic & 3 & 1.21 & & $12 / 4 / 2017$ \\
\hline 614 & 2 & 413 & - & - & - & $\mathrm{E}$ & "I & N1003 & E1019 & 2 & $99.2-99.1$ & $10-20$ & - & Detritus & Debitage & Broken Flake & Lithic & 2 & 0.45 & & 12/4/2017 \\
\hline 614 & 3 & 413 & - & - & - & $\mathrm{E}$ & ॥ & $\mathrm{N} 1003$ & E1019 & 2 & 99.2-99.1 & $10-20$ & - & Detritus & Debitage & Thermal Shatter & Lithic & 2 & 0.76 & & 12/4/2017 \\
\hline 615 & 1 & 441 & - & - & - & $\mathrm{E}$ & III & N1003 & E1019 & 4 & $99.0-98.9$ & $30-40$ & - & Detritus & Debitage & Complete Flake & Lithic & 3 & 0.4 & & 12/5/2017 \\
\hline 615 & 2 & 441 & - & - & - & $\mathrm{E}$ & IIII & $\mathrm{N} 1003$ & E1019 & 4 & $99.0-98.9$ & $30-40$ & - & Detritus & Debitage & Proximal Flake & Lithic & 6 & 2.28 & & 12/5/2017 \\
\hline 615 & 3 & 441 & - & - & - & $\mathrm{E}$ & III & $\mathrm{N} 1003$ & E1019 & 4 & 99.0-98.9 & $30-40$ & - & Detritus & Debitage & Broken Flake & Lithic & 11 & 7.77 & & 12/5/2017 \\
\hline 615 & 4 & 441 & - & - & - & $\mathrm{E}$ & III & N1003 & E1019 & 4 & $99.0-98.9$ & $30-40$ & - & Detritus & Debitage & Thermal Shatter & Lithic & 10 & 6.42 & & 12/5/2017 \\
\hline 615 & 5 & 441 & - & - & - & $\mathrm{E}$ & IIII & N1003 & E1019 & 4 & $99.0-98.9$ & $30-40$ & - & \begin{tabular}{l|l} 
Informal Tool &
\end{tabular} & \begin{tabular}{|l|} 
Modified Flake \\
\end{tabular} & \begin{tabular}{|l|} 
Edge-modified \\
\end{tabular} & Fine Grain Chert & 1 & 25.02 & & 1215/2017 \\
\hline 615 & 6 & 441 & - & - & - & $\mathrm{E}$ & IIII & N1003 & E1019 & 4 & 99.098 .9 & $30-40$ & - & Faunal Remains & Shell & Mussel Shell & Shell & 1 & 1.66 & & 12/5/2017 \\
\hline 616 & 1 & 454 & - & - & - & $\mathrm{E}$ & IIII & $\mathrm{N} 1003$ & E1019 & 5 & $98.9-98.8$ & $40-50$ & - & Detritus & Debitage & Complete Flake & Lithic & 5 & 6 & & 12/8/2017 \\
\hline 616 & 2 & 454 & - & - & - & $\mathrm{E}$ & IIII & N1003 & E1019 & 5 & $\begin{array}{ll}98.9-98.8 \\
\end{array}$ & $40-50$ & - & Detritus & Debitage & \begin{tabular}{|l|} 
Proximal Flake \\
\end{tabular} & Lithic & 19 & 11.2 & & 12/8/2017 \\
\hline 616 & 3 & 454 & - & - & - & E & IIII & $\mathrm{N} 1003$ & E1019 & 5 & 98.9-98.8 & $40-50$ & - & Detritus & Debitage & Broken Flake & Lithic & 17 & 12.06 & & 12/8/2017 \\
\hline 616 & 4 & 454 & - & - & - & $\mathrm{E}$ & III & N1003 & E1019 & 5 & $98.9-98.8$ & $40-50$ & - & Detritus & Debitage & Thermal Shatter & Lithic & 15 & 33.83 & & 12/8/2017 \\
\hline 616 & 5 & 454 & - & - & - & E & IIII & N1003 & E1019 & 5 & $98.9-98.8$ & $40-50$ & - & Faunal Remains & Shell & Mussel Shell & Shell & 1 & 2.61 & & 12/8/2017 \\
\hline 617 & 1 & 464 & - & - & - & E & IIII & N1003 & E1019 & 6 & $98.8-98.7$ & $50-60$ & - & Detritus & Debitage & Complete Flake & Lithic & 2 & 0.87 & & 12/11/2017 \\
\hline 617 & 2 & 464 & - & - & - & E & IIII & N1003 & E1019 & 6 & $98.8-98.7$ & $50-60$ & - & Detritus & Debitage & \begin{tabular}{|l|} 
Proximal Flake \\
\end{tabular} & Lithic & 14 & 12.4 & & 12/11/2017 \\
\hline 617 & 3 & 464 & - & - & - & E & IIII & $\mathrm{N} 1003$ & E1019 & 6 & $98.8-98.7$ & $50-60$ & - & Detritus & Debitage & Broken Flake & Lithic & 14 & 16.83 & & 12/11/2017 \\
\hline 617 & 4 & 464 & - & - & - & E & III & N1003 & E1019 & 6 & $98.8-98.7$ & $50-60$ & - & Detritus & Debitage & Thermal Shatter & Lithic & 23 & 21.35 & & 12/11/2017 \\
\hline 617 & 5 & 464 & - & - & - & E & IIII & $\mathrm{N} 1003$ & E1019 & 6 & 98.8-98.7 & 50-60 & - & Faunal Remains & Shell & Mussel Shell & Shell & 2 & 1.86 & & 12/11/2017 \\
\hline 618 & 1 & 479 & - & - & - & E & IV & N1003 & E1019 & 7 & $\begin{array}{l}98.7-98.6 \\
\end{array}$ & $60-70$ & - & Faunal Remains & Bone & Indeterminate & Bone & 1 & 0.07 & & 12/11/2017 \\
\hline 618 & 2 & 479 & - & - & - & E & IV & N1003 & E1019 & 7 & $\begin{array}{l}98.7-98.6 \\
\end{array}$ & $60-70$ & - & Detritus & Debitage & \begin{tabular}{|l|} 
Complete Flake \\
\end{tabular} & Lithic & 14 & 96.06 & & 12/11/2017 \\
\hline 618 & 3 & 479 & - & - & - & $\mathrm{E}$ & IV & N1003 & E1019 & 7 & $98.7-98.6$ & $60-70$ & - & Detritus & Debitage & Proximal Flake & Lithic & 20 & 16.66 & & 12/11/2017 \\
\hline 618 & 4 & 479 & - & - & - & $\mathrm{E}$ & IV & N1003 & E1019 & 7 & $\begin{array}{l}98.7-98.6 \\
\end{array}$ & $\begin{array}{ll}60-70 \\
\end{array}$ & - & Detritus & Debitage & Broken Flake & Lithic & 37 & 29.37 & & 12/11/2017 \\
\hline 618 & 5 & 479 & - & - & - & $\mathrm{E}$ & IV & N1003 & E1019 & 7 & $\begin{array}{l}98.7-98.6 \\
\end{array}$ & $\begin{array}{ll}60-70 \\
\end{array}$ & - & Detritus & Debitage & \begin{tabular}{|l|} 
Flaking Shatter \\
\end{tabular} & Lithic & 4 & 11.69 & & 12/11/2017 \\
\hline 618 & 6 & 479 & - & - & - & $\mathrm{E}$ & IV & N1003 & E1019 & 7 & $\begin{array}{l}98.7-98.6 \\
\end{array}$ & $60-70$ & - & Detritus & Debitage & Thermal Shatter & Lithic & 12 & 7.04 & & 12/11/2017 \\
\hline 618 & 7 & 479 & - & - & - & $\mathrm{E}$ & IV & N1003 & E1019 & 7 & $98.7-98.6$ & $60-70$ & - & \begin{tabular}{l|l} 
Informal Tool &
\end{tabular} & \begin{tabular}{|l|} 
Modified Flake \\
\end{tabular} & \begin{tabular}{|l|} 
Edge-modified \\
\end{tabular} & Fine Grain Chert & 1 & 3.06 & & 12/11/2017 \\
\hline 618 & 8 & 479 & - & - & - & $\mathrm{E}$ & IV & N1003 & E1019 & 7 & $\begin{array}{l}98.7-98.6 \\
\end{array}$ & $\begin{array}{ll}60-70 \\
\end{array}$ & - & \begin{tabular}{l|l} 
Informal Tool &
\end{tabular} & \begin{tabular}{|l|} 
Modified Flake \\
\end{tabular} & \begin{tabular}{|l|} 
Edge-modified \\
\end{tabular} & Fine Grain Chert & 1 & 24.17 & & 12/11/2017 \\
\hline 618 & 9 & 479 & - & - & - & $\mathrm{E}$ & IV & N1003 & E1019 & 7 & 98.7-98.6 & $60-70$ & - & Faunal Remains & Shell & Mussel Shell & Shell & 1 & 2.26 & & 12/11/2017 \\
\hline 619 & 1 & 530 & - & - & - & $\mathrm{E}$ & IV & $\mathrm{N} 1003$ & E1019 & 8 & $98.6-98.5$ & $80-90$ & - & Detritus & Debitage & Complete Flake & Lithic & 7 & 27.92 & & 12/13/2017 \\
\hline 619 & 2 & 530 & - & - & - & $\mathrm{E}$ & IV & N1003 & E1019 & 8 & $98.6-98.5$ & $80-90$ & - & Detritus & Debitage & \begin{tabular}{|l|} 
Proximal Flake \\
\end{tabular} & Lithic & 18 & 17.9 & & 12/13/2017 \\
\hline 619 & 3 & 530 & - & - & - & $\mathrm{E}$ & IV & N1003 & E1019 & 8 & $98.6-98.5$ & $80-90$ & - & Detritus & Debitage & Broken Flake & Lithic & 29 & 29.58 & & 12/13/2017 \\
\hline 619 & 4 & 530 & - & - & - & E & IV & N1003 & E1019 & 8 & $98.6-98.5$ & $80-90$ & - & Detritus & Debitage & Flaking Shatter & Lithic & 3 & 5.01 & & 12/13/2017 \\
\hline
\end{tabular}




\begin{tabular}{|c|c|c|c|c|c|c|c|c|c|c|c|c|c|c|c|c|c|c|c|c|c|}
\hline Lot No. & \begin{tabular}{|c|}
$\begin{array}{c}\text { Specimen } \\
\text { No. }\end{array}$ \\
\end{tabular} & Fs No. & UI No. & FTR No. & $\begin{array}{c}\text { Sample } \\
\text { No. }\end{array}$ & $\begin{array}{c}\text { Excavation } \\
\text { Block }\end{array}$ & Stratum (AU) & Northing & Easting & Level & $\begin{array}{c}\text { Elevation } \\
(\mathrm{m})\end{array}$ & Depth (cmbd) & Point Prov. & Artifact Class & Artifact Type & \begin{tabular}{|c|} 
Artifact \\
Description
\end{tabular} & Material & $\begin{array}{c}\text { Number of } \\
\text { artifacts }\end{array}$ & Weight (g) & Comments & Date \\
\hline 619 & 5 & 530 & - & - & - & $E$ & IV & N1003 & E1019 & 8 & $98.6-98.5$ & $80-90$ & - & Detritus & Debitage & Thermal Shatter & Lithic & 6 & 4.89 & & 12/13/2017 \\
\hline 619 & 6 & 530 & - & - & - & E & IV & N1003 & E1019 & 8 & $98.6-98.5$ & $80-90$ & - & Informal Tool & \begin{tabular}{|l|} 
Modified Flake \\
\end{tabular} & Utilized Flake & Fine Grain Chert & 1 & 7.7 & & 12/13/2017 \\
\hline 619 & 7 & 530 & - & - & - & $\mathrm{E}$ & IV & N1003 & E1019 & 8 & $98.6-98.5$ & $80-90$ & - & Detritus & Core & \begin{tabular}{|l|} 
Multidirectional \\
\end{tabular} & \begin{tabular}{|l|}
$\begin{array}{c}\text { Coarse Grain } \\
\text { Chert }\end{array}$ \\
\end{tabular} & 1 & 100.19 & & 12/13/2017 \\
\hline 619 & 8 & 530 & - & - & - & $\mathrm{E}$ & IV & N1003 & E1019 & 8 & $98.6-98.5$ & $80-90$ & - & \begin{tabular}{|l|} 
Faunal Remains \\
\end{tabular} & Shell & \begin{tabular}{l|} 
Mussel Shell \\
\end{tabular} & Shell & 4 & 16.31 & & 12/13/2017 \\
\hline 620 & 1 & 531 & 26 & - & - & $\mathrm{E}$ & iv & N1003 & E1019 & 8 & 98.53 & 77 & $\begin{array}{l}\text { N1003.75 } \\
\text { E1019.40 } \\
\end{array}$ & Formal Tool & Bifiace & $\begin{array}{c}\text { Distal-Medial } \\
\text { Fragment } \\
\end{array}$ & $\begin{array}{l}\text { Coarse Grain } \\
\text { Chert }\end{array}$ & 1 & 38.43 & & 12/13/2017 \\
\hline 621 & 1 & 539 & - & - & - & E & $\mathrm{v}$ & N1003 & E1019 & 9 & 98.5-98.4 & $80-90$ & - & Detritus & Debitage & \begin{tabular}{|l|} 
Complete Flake \\
\end{tabular} & Lithic & 4 & 97.4 & & 12/14/2017 \\
\hline 621 & 2 & 539 & - & - & - & $\mathrm{E}$ & $\mathrm{v}$ & N1003 & E1019 & 9 & $98.5-98.4$ & $80-90$ & - & Detritus & Debitage & \begin{tabular}{|l|} 
Proximal Flake \\
\end{tabular} & Lithic & 10 & 15.59 & & 12/14/2017 \\
\hline 621 & 3 & 539 & - & - & - & E & $\mathrm{v}$ & $\begin{array}{ll}\text { N1003 } \\
\end{array}$ & E1019 & 9 & 98.5-98.4 & $80-90$ & - & Detritus & Debitage & Broken Flake & Lithic & 30 & 10.55 & & 12/14/2017 \\
\hline 621 & 4 & 539 & - & - & - & $\mathrm{E}$ & $\mathrm{v}$ & N1003 & E1019 & 9 & 98.5-98.4 & $80-90$ & - & Detritus & Debitage & Thermal Shatter & Lithic & 33 & 76.07 & & 12/14/2017 \\
\hline 621 & 5 & 539 & - & - & - & $E$ & $\mathrm{v}$ & N1003 & E1019 & 9 & $98.5-98.4$ & $80-90$ & - & Informal Tool & Modified Flake & Utilized Flake & $\begin{array}{l}\text { Coarse Grain } \\
\text { Chert }\end{array}$ & 1 & 72.36 & & $12 / 14 / 2017$ \\
\hline 621 & 6 & 539 & - & - & - & $E$ & $\mathrm{v}$ & N1003 & E1019 & 9 & $98.5-98.4$ & $\begin{array}{lll}80-90 \\
\end{array}$ & - & \begin{tabular}{|l|} 
Faunal Remains \\
\end{tabular} & Shell & Mussel Shell & Shell & 4 & 9.96 & & 12/14/2017 \\
\hline 621 & 7 & 539 & 27 & - & - & $E$ & $\mathrm{v}$ & N1003 & E1019 & 9 & 98.5-98.4 & $80-90$ & - & Formal Tool & Dart Point & Zephyr Point & Fine Grain Chert & 1 & 3.86 & & $12 / 14 / 2017$ \\
\hline 622 & 1 & 541 & - & - & - & $\mathrm{E}$ & $\begin{array}{lll}V N 1 & \end{array}$ & N1003 & E1019 & 10 & $98.4-98.3$ & $\begin{array}{l}90-100 \\
\end{array}$ & - & Detritus & Debitage & \begin{tabular}{|c|} 
Complete Flake \\
\end{tabular} & Lithic & 9 & 34.79 & & 12/14/2017 \\
\hline 622 & 2 & 541 & - & - & - & $\mathrm{E}$ & $\mathrm{V} N \mathrm{~N}$ & N1003 & E1019 & 10 & $98.4-98.3$ & $\begin{array}{l}90-100 \\
\end{array}$ & - & Detritus & Debitage & \begin{tabular}{|l|} 
Proximal Flake \\
\end{tabular} & Lithic & 16 & 11.66 & & 12/14/2017 \\
\hline 622 & 3 & 541 & - & - & - & $E$ & $V / N 1$ & N1003 & E1019 & 10 & $98.4-98.3$ & $90-100$ & - & Detritus & Debitage & Broken Flake & Lithic & 35 & 37.16 & & 12/14/2017 \\
\hline 622 & 4 & 541 & - & - & - & $\mathrm{E}$ & $\mathrm{VNNI}$ & N1003 & E1019 & 10 & $98.4-98.3$ & 90-100 & - & Detritus & Debitage & Thermal Shatter & Lithic & 11 & 9.61 & & 12/14/2017 \\
\hline 623 & 1 & 581 & - & - & - & $\mathrm{E}$ & vi & N1003 & E1019 & 11 & 98.3-98.2 & 100-110 & - & Detritus & Debitage & Complete Flake & Lithic & 5 & 12.49 & & 12/18/2017 \\
\hline 623 & 2 & 581 & - & - & - & $\mathrm{E}$ & $\mathrm{vI}$ & N1003 & E1019 & 11 & $98.3-98.2$ & 100-110 & - & Detritus & Debitage & Proximal Flake & Lithic & 2 & 3.41 & & 12/18/2017 \\
\hline 623 & 3 & 581 & - & - & - & $\mathrm{E}$ & $\mathrm{vI}$ & N1003 & E1019 & 11 & $98.3-98.2$ & 100-110 & - & Detritus & Debitage & Broken Flake & Lithic & 10 & 14.14 & & 12/18/2017 \\
\hline 623 & 4 & 581 & - & - & - & $\mathrm{E}$ & $\mathrm{vI}$ & N1003 & E1019 & 11 & $98.3-98.2$ & 100-110 & - & Detritus & Debitage & \begin{tabular}{|l|} 
Flaking Shatter \\
\end{tabular} & Lithic & 1 & 58.17 & & 12/18/2017 \\
\hline 623 & 5 & 581 & - & - & - & $E$ & $\mathrm{vl}$ & N1003 & E1019 & 11 & $98.3-98.2$ & $100-110$ & - & Detritus & Debitage & Thermal Shatter & Lithic & 7 & 8.78 & & $12 / 18 / 2017$ \\
\hline 623 & 6 & 581 & - & - & - & $\mathrm{E}$ & $\mathrm{vI}$ & N1003 & E1019 & 11 & $98.3-98.2$ & $100-110$ & - & \begin{tabular}{|l|l} 
Informal Tool &
\end{tabular} & Modified Flake & Utilized Flake & Fine Grain Chert & 1 & 158.32 & & 12/18/2017 \\
\hline 623 & 7 & 581 & - & - & - & $\mathrm{E}$ & vi & N1003 & E1019 & 11 & 98.3-98.2 & $100-110$ & - & \begin{tabular}{c|} 
Informal Tool \\
\end{tabular} & \begin{tabular}{|l|} 
Modified Flake \\
\end{tabular} & \begin{tabular}{|l|} 
Edge-modified \\
\end{tabular} & \begin{tabular}{|c|}
$\begin{array}{c}\text { Coarse Grain } \\
\text { Chert }\end{array}$ \\
\end{tabular} & 1 & 11.21 & & 12/18/2017 \\
\hline 623 & 8 & 581 & - & - & - & $\mathrm{E}$ & vi & N1003 & E1019 & 11 & $98.3-98.2$ & $100-110$ & - & Faunal Remains & Shell & Mussel Shell & Shell & 2 & 4.2 & & 12/18/2017 \\
\hline 623 & 9 & 581 & 30 & - & - & $\mathrm{E}$ & vi & N1003 & E1019 & 11 & $98.3-98.2$ & $100-110$ & - & Formal Tool & Biface & \begin{tabular}{c|} 
Medial-Basal \\
Fragment
\end{tabular} & Fine Grain Chert & 1 & 12.13 & Originally collected as Projectile Point & 12/18/2017 \\
\hline 624 & 1 & 593 & - & - & - & $\mathrm{E}$ & $\mathrm{vi}$ & N1003 & E1019 & 11 & 98.2 & 110 & $\begin{array}{l}\text { N1003.96 } \\
\text { E1019.44 }\end{array}$ & Informal Tool & Core Tool & Chopper & \begin{tabular}{c|}
$\begin{array}{c}\text { Coarse Grain } \\
\text { Chert }\end{array}$
\end{tabular} & 1 & 235.17 & & \begin{tabular}{|l|}
$12 / 18 / 2017$ \\
\end{tabular} \\
\hline 625 & 1 & 587 & - & - & - & E & vi & N1003 & E1019 & 12 & $98.2-98.1$ & $110-120$ & - & Detritus & Debitage & Complete Flake & Lithic & 4 & 10.96 & & 12/17/2017 \\
\hline 625 & 2 & 587 & - & - & - & $\mathrm{E}$ & $\mathrm{vI}$ & N1003 & E1019 & 12 & $98.2-98.1$ & $110-120$ & - & Detritus & 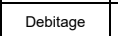 & Proximal Flake & Lithic & 4 & 19.74 & & $12 / 17 / 2017$ \\
\hline 625 & 3 & 587 & - & - & - & $\mathrm{E}$ & $\mathrm{vI}$ & N1003 & E1019 & 12 & $98.2-98.1$ & $110-120$ & - & Detritus & Debitage & Broken Flake & Lithic & 6 & 4.75 & & 12/17/2017 \\
\hline 625 & 4 & 587 & - & - & - & $\mathrm{E}$ & $\mathrm{vI}$ & N1003 & E1019 & 12 & $98.2-98.1$ & $110-120$ & - & Detritus & Debitage & Thermal Shatter & Lithic & 2 & 7.01 & & $12 / 17 / 2017$ \\
\hline 626 & 1 & 591 & - & - & - & $\mathrm{E}$ & $\mathrm{vI}$ & N1003 & E1019 & 13 & $98.1-98.0$ & $120-130$ & - & Faunal Remains & Bone & Odocoileus sp. & Bone & 1 & 2.48 & possible bison tooth & 12/18/2017 \\
\hline 626 & 2 & 591 & - & - & - & $\mathrm{E}$ & $\mathrm{vi}$ & N1003 & E1019 & 13 & $98.1-98.0$ & $120-130$ & - & Faunal Remains & Bone & Odocoileus sp. & Bone & 1 & 0.59 & possible bison tooth & 12/18/2017 \\
\hline 626 & 3 & 591 & - & - & - & E & vi & $\begin{array}{ll}\text { N1003 } \\
\end{array}$ & E1019 & 13 & $98.1-98.0$ & 120-130 & - & Faunal Remains & Bone & Indeterminate & Bone & 1 & 0.81 & possible bison tooth & 12/18/2017 \\
\hline 626 & 4 & 591 & - & - & - & $E$ & $\mathrm{vI}_{\mathrm{N}}$ & N1003 & E1019 & 13 & $98.1-98.0$ & $120-130$ & - & Faunal Remains & Bone & Indeterminate & Bone & 1 & 0.28 & possible bison tooth & 12/18/2017 \\
\hline 626 & 5 & 591 & - & - & - & $E$ & $\mathrm{vl}$ & N1003 & E1019 & 13 & $98.1-98.0$ & $120-130$ & - & Faunal Remains & Bone & Indeterminate & Bone & 1 & 0.42 & possible bison tooth & $12 / 18 / 2017$ \\
\hline 626 & 6 & 591 & - & - & - & $\mathrm{E}$ & vi & N1003 & E1019 & 13 & $98.1-98.0$ & $120-130$ & - & Detritus & Debitage & Complete Flake & Lithic & 3 & 28.66 & & 12/18/2017 \\
\hline 626 & 7 & 591 & - & - & - & $E$ & $\mathrm{vI}$ & N1003 & E1019 & 13 & $98.1-98.0$ & 120-130 & - & Detritus & Debitage & Broken Flake & Lithic & 4 & 3.82 & & 12/18/2017 \\
\hline 626 & 8 & 591 & - & - & - & $E$ & vi & N1003 & E1019 & 13 & $98.1-98.0$ & $120-130$ & - & Detritus & Debitage & Thermal Shatter & Lithic & 2 & 1 & & 12/18/2017 \\
\hline 626 & 9 & 591 & - & - & - & $\mathrm{E}$ & $\mathrm{vI}$ & N1003 & E1019 & 13 & $988.1-98.0$ & 120-130 & - & Faunal Remains & Shell & Mussel Shell & Shell & 1 & 8.11 & & 12/18/2017 \\
\hline 626 & 10 & 591 & - & - & - & $\mathrm{E}$ & vi & N1003 & E1019 & 13 & $98.1-98.0$ & 120-130 & - & \begin{tabular}{|c|}
$\begin{array}{c}\text { Macrobotanical } \\
\text { Remains }\end{array}$ \\
\end{tabular} & \begin{tabular}{|c|}
$\begin{array}{c}\text { Seed } \\
\text { Concentration }\end{array}$ \\
\end{tabular} & \begin{tabular}{c|} 
Seed \\
$\begin{array}{c}\text { Concentration } \\
\text { and matrix }\end{array}$ \\
\end{tabular} & Seed & 1 & 21.41 & Not ID'd & 12/18/2017 \\
\hline 627 & 1 & 613 & - & - & - & $\mathrm{E}$ & vi & N1003 & E1019 & 15 & 97.9-97.8 & $140-150$ & - & Detritus & Debitage & Complete Flake & Lithic & 1 & 9.74 & & 12/20/2017 \\
\hline 627 & 2 & 613 & - & - & - & $E$ & vi & N1003 & E1019 & 15 & 97.9-97.8 & $140-150$ & - & Detritus & Debitage & \begin{tabular}{|l|} 
Proximal Flake \\
\end{tabular} & Lithic & 1 & 22.52 & & 12/20/2017 \\
\hline 628 & 1 & 346 & - & - & - & $E$ & " & N1004 & E1017 & 2 & $99.2-99.1$ & 10-20 & - & Detritus & Debitage & Proximal Flake & Lithic & 3 & 1.24 & & 11/29/2017 \\
\hline 628 & 2 & 346 & - & - & - & $E$ & $\|$ & N1004 & E1017 & 2 & $99.2-99.1$ & $10-20$ & - & Detritus & Debitage & Flaking Shatter & Lithic & 1 & 0.35 & & 11/29/2017 \\
\hline
\end{tabular}




\begin{tabular}{|c|c|c|c|c|c|c|c|c|c|c|c|c|c|c|c|c|c|c|c|c|c|}
\hline Lot No. & \begin{tabular}{|c|}
$\begin{array}{c}\text { Specimen } \\
\text { No. }\end{array}$ \\
\end{tabular} & FS No. & UI No. & FTR No. & $\begin{array}{c}\text { Sample } \\
\text { No. }\end{array}$ & $\begin{array}{c}\text { Excavation } \\
\text { Block }\end{array}$ & Stratum (AU) & Northing & Easting & Level & $\begin{array}{c}\text { Elevation } \\
(\mathrm{m})\end{array}$ & Depth (cmbd) & Point Prov. & Artifact Class & Artifact Type & \begin{tabular}{|c|} 
Artifact \\
Description
\end{tabular} & Material & $\begin{array}{c}\text { Number of } \\
\text { artifacts }\end{array}$ & Weight (g) & Comments & Date \\
\hline 628 & 3 & 346 & - & - & - & $E$ & " & N1004 & E1017 & 2 & $99.2-99.1$ & $10-20$ & - & Detritus & Debitage & \begin{tabular}{|l|} 
Thermal Shatter \\
\end{tabular} & Lithic & 1 & 1.39 & & 11/29/2017 \\
\hline 629 & 1 & 355 & - & - & - & $E$ & 11/III & N1004 & E1017 & 3 & $99.1-99.0$ & $20-30$ & - & Detritus & Debitage & Complete Flake & Lithic & 1 & 3.2 & & 11/29/2017 \\
\hline 629 & 2 & 355 & - & - & - & E & II/III & N1004 & E1017 & 3 & $99.1-99.0$ & $20-30$ & - & Detritus & Debitage & \begin{tabular}{|l|} 
Proximal Flake \\
\end{tabular} & Lithic & 5 & 3.4 & & 11/29/2017 \\
\hline 629 & 3 & 355 & - & - & - & E & "1/III & N1004 & E1017 & 3 & $99.1-99.0$ & $20-30$ & - & Detritus & Debitage & Broken Flake & Lithic & 3 & 1.12 & & 11/29/2017 \\
\hline 629 & 4 & 355 & - & - & - & E & II/II & N1004 & E1017 & 3 & $99.1-99.0$ & $20-30$ & - & Informal Tool & Modified Flake & Edge-modified & Fine Grain Chert & 1 & 1.8 & & 11/29/2017 \\
\hline 630 & 1 & 365 & - & - & - & E & III & N1004 & E1017 & 4 & $99.0-98.9$ & $30-40$ & - & Detritus & Debitage & \begin{tabular}{|l|} 
Complete Flake \\
\end{tabular} & Lithic & 4 & 3.08 & & 11/29/2017 \\
\hline 630 & 2 & 365 & - & - & - & E & IIII & N1004 & E1017 & 4 & $99.0-98.9$ & $30-40$ & - & Detritus & Debitage & \begin{tabular}{|l|} 
Proximal Flake \\
\end{tabular} & Lithic & 11 & 24.91 & & 11/29/2017 \\
\hline 630 & 3 & 365 & - & - & - & E & III & N1004 & E1017 & 4 & 99.0-98.9 & $30-40$ & - & Detritus & Debitage & Broken Flake & Lithic & 10 & 8.77 & & $\mid$\begin{tabular}{|l|}
$\mid 11 / 29 / 2017$ \\
\end{tabular} \\
\hline 630 & 4 & 365 & - & - & - & E & III & N1004 & E1017 & 4 & $99.0-98.9$ & $30-40$ & - & Detritus & Debitage & Thermal Shatter & Lithic & 7 & 5.36 & & $\mid$\begin{tabular}{|l|}
$\mid 11 / 29 / 2017$ \\
\end{tabular} \\
\hline 631 & 1 & 376 & - & - & - & $\mathrm{E}$ & IIII & N1004 & E1017 & 5 & $\begin{array}{ll}98.9-98.8 \\
\end{array}$ & $40-50$ & - & Faunal Remains & Bone & Indeterminate & Bone & 1 & 1.26 & & 11/30/2017 \\
\hline 631 & 2 & 376 & - & - & - & E & IIII & N1004 & E1017 & 5 & 98.9-98.8 & $40-50$ & - & Detritus & Debitage & Complete Flake & Lithic & 2 & 2.3 & & \begin{tabular}{|l|}
$11 / 30 / 2017$ \\
\end{tabular} \\
\hline 631 & 3 & 376 & - & - & - & $\mathrm{E}$ & IIII & N1004 & E1017 & 5 & $98.9-98.8$ & $40-50$ & - & Detritus & Debitage & Proximal Flake & Lithic & 14 & 6.37 & & \begin{tabular}{|l|}
$11 / 30 / 2017$ \\
\end{tabular} \\
\hline 631 & 4 & 376 & - & - & - & $\mathrm{E}$ & III & N1004 & E1017 & 5 & $98.9-98.8$ & $40-50$ & - & Detritus & Debitage & Broken Flake & Lithic & 16 & 11.36 & & 11/30/2017 \\
\hline 631 & 5 & 376 & - & - & - & $\mathrm{E}$ & IIII & N1004 & E1017 & 5 & $\begin{array}{ll}88.9-98.8 \\
\end{array}$ & $40-50$ & - & Detritus & Debitage & Thermal Shatter & Lithic & 10 & 2.67 & & $\mid 11 / 30 / 2017$ \\
\hline 632 & 1 & 385 & - & - & - & $\mathrm{E}$ & IIII & N1004 & E1017 & 6 & 98.8-98.7 & $50-60$ & - & Faunal Remains & Bone & Odocoileus sp. & Tooth & 1 & 1.18 & & 11/30/2017 \\
\hline 632 & 2 & 385 & - & - & - & $\mathrm{E}$ & IIII & N1004 & E1017 & 6 & 98.8-98.7 & 50-60 & - & Detritus & Debitage & Complete Flake & Lithic & 9 & 12.83 & & 11/30/2017 \\
\hline 632 & 3 & 385 & - & - & - & $\mathrm{E}$ & III & N1004 & E1017 & 6 & 98.8-98.7 & $50-60$ & - & Detritus & Debitage & Proximal Flake & Lithic & 10 & 6.47 & & 11/30/2017 \\
\hline 632 & 4 & 385 & - & - & - & E & IIII & N1004 & E1017 & 6 & $98.8-98.7$ & $50-60$ & - & Detritus & Debitage & Broken Flake & Lithic & 23 & 15.4 & & 11/30/2017 \\
\hline 632 & 5 & 385 & - & - & - & E & IIII & N1004 & E1017 & 6 & $98.8-98.7$ & $50-60$ & - & Detritus & Debitage & Flaking Shatter & Lithic & 2 & 0.64 & & 11/30/2017 \\
\hline 632 & 6 & 385 & - & - & - & E & IIII & N1004 & E1017 & 6 & 98.8-98.7 & $50-60$ & - & Detritus & Debitage & Thermal Shatter & Lithic & 11 & 10.23 & & 11/30/2017 \\
\hline 632 & 7 & 385 & - & - & - & $\mathrm{E}$ & IIII & N1004 & E1017 & 6 & $\begin{array}{ll}98.8-98.7 \\
\end{array}$ & $50-60$ & - & Informal Tool & Modified Flake & \begin{tabular}{|l|} 
Edge-modified \\
\end{tabular} & Fine Grain Chert & 1 & 10.54 & & 11/30/2017 \\
\hline 632 & 8 & 385 & - & - & - & $\mathrm{E}$ & IIII & N1004 & E1017 & 6 & $98.8-98.7$ & $50-60$ & - & Detritus & Core & Multidirectional & Fine Grain Chert & 1 & 301.02 & & 11/30/2017 \\
\hline 632 & 9 & 385 & - & - & - & $\mathrm{E}$ & III & N1004 & E1017 & 6 & 98.8-98.7 & $50-60$ & - & Formal Tool & Biface & \begin{tabular}{|c|} 
Marginal \\
Fragment \\
\end{tabular} & Fine Grain Chert & 1 & 0.43 & & 11/30/2017 \\
\hline 633 & 1 & 396 & - & - & - & E & IV & N1004 & E1017 & 7 & 98.7-98.6 & $60-70$ & - & Detritus & Debitage & \begin{tabular}{|c|} 
Complete Flake \\
\end{tabular} & Lithic & 8 & 35.29 & & 12/112017 \\
\hline 633 & 2 & 396 & - & - & - & $\mathrm{E}$ & IV & N1004 & E1017 & 7 & 98.7-98.6 & $60-70$ & - & Detritus & Debitage & \begin{tabular}{|l|} 
Proximal Flake \\
\end{tabular} & Lithic & 9 & 4.13 & & 1211/2017 \\
\hline 633 & 3 & 396 & - & - & - & E & IV & N1004 & E1017 & 7 & 98.7-98.6 & $60-70$ & - & Detritus & Debitage & Broken Flake & Lithic & 30 & 21.12 & & 12/1/2017 \\
\hline 633 & 4 & 396 & - & - & - & E & IV & N1004 & E1017 & 7 & 98.7-98.6 & $60-70$ & - & Detritus & Debitage & Thermal Shatter & Lithic & 16 & 14.31 & & 12/1/2017 \\
\hline 633 & 5 & 396 & - & - & - & E & IV & N1004 & E1017 & 7 & $98.7-98.6$ & $60-70$ & - & Faunal Remains & Shell & Mussel Shell & Shell & 2 & 4.72 & & 12/1/2017 \\
\hline 634 & 1 & 397 & - & - & - & $\mathrm{E}$ & IV & N1004 & E1017 & 7 & 98.61 & 69 & \begin{tabular}{|l|} 
N1004.30 \\
E1017.56
\end{tabular} & Formal Tool & Biface & $\begin{array}{c}\text { Distal-Medial } \\
\text { Fragment }\end{array}$ & Fine Grain Chert & 1 & 12.14 & & $12 / 112017$ \\
\hline 635 & 1 & 455 & - & - & - & E & IV & N1004 & E1017 & 8 & $98.6-98.5$ & $70-80$ & - & Detritus & Debitage & \begin{tabular}{|l|} 
Complete Flake \\
\end{tabular} & Lithic & 8 & 56.49 & & 12/8/2017 \\
\hline 635 & 2 & 455 & - & - & - & E & IV & N1004 & E1017 & 8 & $98.6-98.5$ & $70-80$ & - & Detritus & Debitage & Proximal Flake & Lithic & 6 & 22.92 & & 12/8/2017 \\
\hline 635 & 3 & 455 & - & - & - & E & IV & N1004 & E1017 & 8 & $98.6-98.5$ & $70-80$ & - & Detritus & Debitage & Broken Flake & Lithic & 16 & 11.51 & & 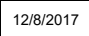 \\
\hline 635 & 4 & 455 & - & - & - & $\mathrm{E}$ & iv & N1004 & E1017 & 8 & $98.6-98.5$ & $70-80$ & - & Detritus & Debitage & \begin{tabular}{|l|} 
Thermal Shatter \\
\end{tabular} & Lithic & 6 & 3.75 & & 12/8/2017 \\
\hline 635 & 5 & 455 & - & - & - & $\mathrm{E}$ & IV & N1004 & E1017 & 8 & $98.6-98.5$ & $70-80$ & - & Faunal Remains & Shell & Mussel Shell & Shell & 1 & 0.53 & & 12/8/2017 \\
\hline 636 & 1 & 456 & - & - & - & $\mathrm{E}$ & IV & N1004 & E1017 & 8 & 98.5 & 80 & \begin{tabular}{|l|} 
N1004.85 \\
E1017.72
\end{tabular} & Detritus & Core & Multidirectional & Fine Grain Chert & 1 & 150.7 & & 12/8/2017 \\
\hline 637 & 1 & 461 & - & - & - & E & v & N1004 & E1017 & 9 & 98.5-98.4 & $80-90$ & - & Detritus & Debitage & Complete Flake & Lithic & 2 & 51.36 & & 12/18/2017 \\
\hline 637 & 2 & 461 & - & - & - & $E$ & $\mathrm{v}$ & N1004 & E1017 & 9 & $98.5-98.4$ & $80-90$ & - & Detritus & Debitage & Proximal Flake & Lithic & 7 & 2.39 & & 12/18/2017 \\
\hline 637 & 3 & 461 & - & - & - & E & v & N1004 & E1017 & 9 & 98.5-98.4 & $80-90$ & - & Detritus & Debitage & Broken Flake & Lithic & 9 & 8.16 & & 12/8/2017 \\
\hline 637 & 4 & 461 & - & - & - & $\mathrm{E}$ & $\mathrm{v}$ & N1004 & E1017 & 9 & $98.5-98.4$ & $\begin{array}{ll}80-90 \\
\end{array}$ & - & Detritus & Debitage & Thermal Shatter & Lithic & 11 & 85.8 & & 12/8/2017 \\
\hline 638 & 1 & 466 & - & - & - & $\mathrm{E}$ & VNII & N1004 & E1017 & 10 & 98.4-98.3 & 90-100 & - & Detritus & Debitage & Complete Flake & Lithic & 2 & 8.56 & & 12/11/2017 \\
\hline 638 & 2 & 466 & - & - & - & E & $V_{N 1}$ & N1004 & E1017 & 10 & $98.4-98.3$ & 90-100 & - & Detritus & Debitage & Proximal Flake & Lithic & 3 & 3.63 & & 12/11/2017 \\
\hline 638 & 3 & 466 & - & - & - & E & $V N 1$ & N1004 & E1017 & 10 & $98.4-98.3$ & $90-100$ & - & Detritus & Debitage & Broken Flake & Lithic & 2 & 0.61 & & 12/11/2017 \\
\hline 638 & 4 & 466 & - & - & - & $\mathrm{E}$ & $\mathrm{V} N \mathrm{~N}$ & $\begin{array}{ll}\text { N1004 } \\
\end{array}$ & E1017 & 10 & $98.4-98.3$ & $90-100$ & - & Detritus & Debitage & Thermal Shatter & Lithic & 1 & 0.44 & & 12/11/2017 \\
\hline 638 & 5 & 466 & - & - & - & E & $V_{N I I}$ & N1004 & E1017 & 10 & $98.4-98.3$ & 90-100 & - & Informal Tool & Modified Flake & \begin{tabular}{|l|} 
Edge-modified \\
\end{tabular} & \begin{tabular}{|l|} 
Fine Grain Chert \\
\end{tabular} & 1 & 5 & & 12/11/2017 \\
\hline
\end{tabular}




\begin{tabular}{|c|c|c|c|c|c|c|c|c|c|c|c|c|c|c|c|c|c|c|c|c|c|}
\hline Lot No. & \begin{tabular}{|c|}
$\begin{array}{c}\text { Specimen } \\
\text { No. }\end{array}$ \\
\end{tabular} & FS No. & UI No. & FTR No. & $\begin{array}{c}\text { Sample } \\
\text { No. }\end{array}$ & $\begin{array}{c}\text { Excavation } \\
\text { Block }\end{array}$ & Stratum (AU) & Northing & Easting & Level & $\begin{array}{c}\text { Elevation } \\
(\mathrm{m})\end{array}$ & Depth (cmbd) & Point Prov. & Artifact Class & \begin{tabular}{|l|l} 
Artifact Type \\
\end{tabular} & \begin{tabular}{|c|}
$\begin{array}{c}\text { Artifact } \\
\text { Description }\end{array}$ \\
\end{tabular} & Material & $\begin{array}{c}\begin{array}{c}\text { Number of } \\
\text { artifacts }\end{array} \\
\end{array}$ & Weight $(g)$ & Comments & Date \\
\hline 639 & 1 & 468 & - & - & - & $E$ & vi & N1004 & E1017 & 11 & $98.3-98.2$ & 100-110 & - & Detritus & Debitage & Complete Flake & Lithic & 1 & 0.12 & & 12/11/2017 \\
\hline 639 & 2 & 468 & - & - & - & E & vi & N1004 & E1017 & 11 & 98.3.98.2 & 100-110 & - & Detritus & Debitage & Proximal Flake & Lithic & 3 & 0.84 & & 12/11/2017 \\
\hline 639 & 3 & 468 & - & - & - & E & vi & N1004 & E1017 & 11 & $98.3-98.2$ & $100-110$ & - & Detritus & Debitage & Thermal Shatter & Lithic & 1 & 1.55 & & 12/11/2017 \\
\hline 640 & 1 & 471 & - & - & - & E & vi & N1004 & E1017 & 12 & $98.2-98.1$ & $110-120$ & - & Detritus & Debitage & Proximal Flake & Lithic & 3 & 8.24 & & 12/11/2017 \\
\hline 640 & 2 & 471 & - & - & - & E & vi & N1004 & E1017 & 12 & 98.2-98.1 & $110-120$ & - & Detritus & Debitage & Broken Flake & Lithic & 1 & 0.08 & & 12/11/2017 \\
\hline 640 & 3 & 471 & - & - & - & E & $\mathrm{vI}$ & N1004 & E1017 & 12 & 98.2-98.1 & $110-120$ & - & Detritus & Debitage & Thermal Shatter & Lithic & 1 & 118.93 & & 12/11/2017 \\
\hline 641 & 1 & 473 & - & - & - & E & vi & N1004 & E1017 & 13 & $98.1-98.0$ & 120-130 & - & Detritus & Debitage & Proximal Flake & Lithic & 2 & 1.95 & & 12/11/2017 \\
\hline 641 & 2 & 473 & - & - & - & E & vi & N1004 & E1017 & 13 & $98.1-98.0$ & 120-130 & - & Detritus & Debitage & Broken Flake & Lithic & 1 & 0.42 & & 12/11/2017 \\
\hline 641 & 3 & 473 & - & - & - & E & vi & N1004 & E1017 & 13 & $98.1-98.0$ & 120-130 & - & Detritus & Debitage & Thermal Shatter & Lithic & 1 & 0.17 & & 12/11/2017 \\
\hline 642 & 1 & 478 & - & - & - & $\mathrm{E}$ & $\mathrm{vi}$ & N1004 & E1017 & 15 & $\begin{array}{ll}77.9-97.8 \\
\end{array}$ & 140-150 & - & Detritus & Debitage & Broken Flake & Lithic & 2 & 1.38 & & 12/11/2017 \\
\hline 643 & 1 & 345 & - & - & - & $\mathrm{E}$ & 1 & N1004 & E1018 & 1 & $99.22-99.2$ & $8-10$ & - & Detritus & Debitage & Proximal Flake & Lithic & 1 & 0.58 & & 11/29/2017 \\
\hline 644 & 1 & 351 & - & - & - & $\mathrm{E}$ & "I & N1004 & E1018 & 2 & $99.2-99.1$ & $10-20$ & - & Detritus & Debitage & Broken Flake & Lithic & 1 & 0.66 & & 11/29/2017 \\
\hline 645 & 1 & 359 & - & - & - & $\mathrm{E}$ & 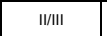 & N1004 & E1018 & 3 & $99.1-99.0$ & 20-30 & - & Faunal Remains & Bone & Indeterminate & Bone & 1 & 0.21 & & 11/29/2017 \\
\hline 645 & 2 & 359 & - & - & - & $E$ & 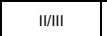 & N1004 & E1018 & 3 & $99.1-99.0$ & 20-30 & - & Detritus & Debitage & Proximal Flake & Lithic & 1 & 0.75 & & 11/29/2017 \\
\hline 645 & 3 & 359 & - & - & - & $\mathrm{E}$ & 11/111 & N1004 & E1018 & 3 & $99.1-99.0$ & $20-30$ & - & Detritus & Debitage & Broken Flake & Lithic & 1 & 0.95 & & 11/29/2017 \\
\hline 646 & 1 & 369 & - & - & - & $\mathrm{E}$ & IIII & N1004 & E1018 & 4 & $99.0-98.9$ & $30-40$ & - & Detritus & Debitage & Complete Flake & Lithic & 4 & 2.96 & & 11/30/2017 \\
\hline 646 & 2 & 369 & - & - & - & $\mathrm{E}$ & IIII & N1004 & E1018 & 4 & $99.0-98.9$ & 30-40 & - & Detritus & Debitage & Proximal Flake & Lithic & 6 & 27.35 & & 11/30/2017 \\
\hline 646 & 3 & 369 & - & - & - & E & IIII & N1004 & E1018 & 4 & 99.0-98.9 & $30-40$ & - & Detritus & Debitage & Broken Flake & Lithic & 6 & 1.97 & & 11/30/2017 \\
\hline 646 & 4 & 369 & - & - & - & $\mathrm{E}$ & IIII & N1004 & E1018 & 4 & 99.0-98.9 & $30-40$ & - & Detritus & Debitage & Thermal Shatter & Lithic & 1 & 1.23 & & 11/30/2017 \\
\hline 646 & 5 & 369 & - & - & - & E & IIII & N1004 & E1018 & 4 & $99.0-98.9$ & $30-40$ & - & Formal Tool & Biface & Basal Fragment & Fine Grain Chert & 1 & 5.3 & & 11/30/2017 \\
\hline 646 & 6 & 369 & - & - & - & E & IIII & N1004 & E1018 & 4 & $99.0-98.9$ & $30-40$ & - & \begin{tabular}{l|l} 
Informal Tool &
\end{tabular} & \begin{tabular}{|l|} 
Modified Flake \\
\end{tabular} & \begin{tabular}{|l|} 
Edge-modified \\
\end{tabular} & Fine Grain Chert & 1 & 7.28 & & 11/30/2017 \\
\hline 647 & 1 & 381 & - & - & - & $\mathrm{E}$ & IIII & N1004 & E1018 & 5 & $98.9-98.8$ & $40-50$ & - & Detritus & Debitage & Complete Flake & Lithic & 5 & 20.33 & & 11/30/2017 \\
\hline 647 & 2 & 381 & - & - & - & E & IIII & N1004 & E1018 & 5 & 98.9-98.8 & $40-50$ & - & Detritus & Debitage & \begin{tabular}{|l|} 
Proximal Flake \\
\end{tabular} & Lithic & 13 & 40.15 & & 11/301/2017 \\
\hline 647 & 3 & 381 & - & - & - & E & IIII & N1004 & E1018 & 5 & 98.9-98.8 & $40-50$ & - & Detritus & Debitage & Broken Flake & Lithic & 9 & 3.81 & & 11/30/2017 \\
\hline 647 & 4 & 381 & - & - & - & E & IIII & N1004 & E1018 & 5 & 98.9-98.8 & $40-50$ & - & Detritus & Debitage & Thermal Shatter & Lithic & 17 & 19.45 & & 11/30/2017 \\
\hline 647 & 5 & 381 & - & - & - & E & IIII & N1004 & E1018 & 5 & $98.9-98.8$ & $40-50$ & - & Informal Tool & \begin{tabular}{|l|} 
Modified Flake \\
\end{tabular} & \begin{tabular}{|l|} 
Edge-modified \\
\end{tabular} & Fine Grain Chert & 1 & 0.63 & & 11/30/2017 \\
\hline 647 & 6 & 381 & - & - & - & E & IIII & N1004 & E1018 & 5 & 98.9-98.8 & $40-50$ & - & Formal Tool & Biface & Distal Fragment & Fine Grain Chert & 1 & 0.45 & & 11/30/2017 \\
\hline 648 & 1 & 390 & - & - & - & E & IIII & N1004 & E1018 & 6 & $98.8-98.7$ & $50-60$ & - & Detritus & Debitage & Complete Flake & Lithic & 9 & 8.71 & & 11/30/2017 \\
\hline 648 & 2 & 390 & - & - & - & $\mathrm{E}$ & IIII & N1004 & E1018 & 6 & $98.8-98.7$ & $50-60$ & - & Detritus & Debitage & \begin{tabular}{|l|} 
Proximal Flake \\
\end{tabular} & Lithic & 12 & 38.48 & & 11/30/2017 \\
\hline 648 & 3 & 390 & - & - & - & E & IIII & N1004 & E1018 & 6 & 98.8-98.7 & $50-60$ & - & Detritus & Debitage & Broken Flake & Lithic & 37 & 15.85 & & 11/30/2017 \\
\hline 648 & 4 & 390 & - & - & - & E & IIII & N1004 & E1018 & 6 & 98.8-98.7 & 50-60 & - & Detritus & Debitage & Flaking Shatter & Lithic & 1 & 1.75 & & 11/30/2017 \\
\hline 648 & 5 & 390 & - & - & - & E & III & N1004 & E1018 & 6 & $\begin{array}{l}98.8-98.7 \\
\end{array}$ & $50-60$ & - & Detritus & Debitage & Thermal Shatter & Lithic & 26 & 23.06 & & 11/30/2017 \\
\hline 648 & 6 & 390 & - & - & - & E & IIII & N1004 & E1018 & 6 & 98.8-98.7 & $50-60$ & - & Faunal Remains & Shell & Mussel Shell & Shell & 2 & 3.74 & & 11/30/2017 \\
\hline 649 & 1 & 402 & - & - & - & $\mathrm{E}$ & IV & N1004 & E1018 & 7 & 98.7-98.6 & $60-70$ & - & Detritus & Debitage & Complete Flake & Lithic & 7 & 5.64 & & 12/112017 \\
\hline 649 & 2 & 402 & - & - & - & $\mathrm{E}$ & iv & N1004 & E1018 & 7 & $\begin{array}{l}98.7-98.6 \\
\end{array}$ & $\begin{array}{ll}60-70 \\
\end{array}$ & - & Detritus & Debitage & Proximal Flake & Lithic & 14 & 8.29 & & 12/1/2017 \\
\hline 649 & 3 & 402 & - & - & - & $\mathrm{E}$ & IV & N1004 & E1018 & 7 & $\begin{array}{l}98.7-98.6 \\
\end{array}$ & $\begin{array}{ll}60-70 \\
\end{array}$ & - & Detritus & Debitage & Broken Flake & Lithic & 19 & 8.31 & & 12/1/2017 \\
\hline 649 & 4 & 402 & - & - & - & $E$ & iv & N1004 & E1018 & 7 & $98.7-98.6$ & 60-70 & - & Detritus & Debitage & Flaking Shatter & Lithic & 1 & 1.16 & & 12/1/2017 \\
\hline 649 & 5 & 402 & - & - & - & $\mathrm{E}$ & iv & N1004 & E1018 & 7 & 98.7-98.6 & $60-70$ & - & Detritus & Debitage & Thermal Shatter & Lithic & 20 & 29.03 & & 12112017 \\
\hline 649 & 6 & 402 & - & - & - & $\mathrm{E}$ & iv & N1004 & E1018 & 7 & $\begin{array}{l}98.7-98.6 \\
\end{array}$ & $\begin{array}{ll}60-70 \\
\end{array}$ & - & Faunal Remains & Shell & Mussel Shell & Shell & 2 & 2.45 & & 12/1/2017 \\
\hline 650 & 1 & 486 & - & - & - & $\mathrm{E}$ & IV & N1004 & E1018 & 8 & 98.6-98.5 & $70-80$ & - & Detritus & Debitage & Complete Flake & Lithic & 9 & 44.31 & & 12/12/2017 \\
\hline 650 & 2 & 486 & - & - & - & $E$ & IV & N1004 & E1018 & 8 & 98.6-98.5 & $70-80$ & - & Detritus & Debitage & Proximal Flake & Lithic & 11 & 4.81 & & 12/12/2017 \\
\hline 650 & 3 & 486 & - & - & - & $\mathrm{E}$ & IV & N1004 & E1018 & 8 & $98.6-98.5$ & $70-80$ & - & Detritus & Debitage & Broken Flake & Lithic & 21 & 6.28 & & 12/12/2017 \\
\hline 650 & 4 & 486 & - & - & - & $\mathrm{E}$ & IV & N1004 & E1018 & 8 & $98.6-98.5$ & $70-80$ & - & Detritus & Debitage & Thermal Shatter & Lithic & 13 & 28.38 & & 12/12/2017 \\
\hline 650 & 5 & 486 & - & - & - & E & IV & N1004 & E1018 & 8 & $98.6-98.5$ & $70-80$ & - & Detritus & Core & Unifacial & 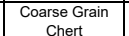 & 1 & 124.26 & Fragment & 12/1/2/2017 \\
\hline
\end{tabular}




\begin{tabular}{|c|c|c|c|c|c|c|c|c|c|c|c|c|c|c|c|c|c|c|c|c|c|}
\hline Lot No. & \begin{tabular}{|c|}
$\begin{array}{c}\text { Specimen } \\
\text { No. }\end{array}$ \\
\end{tabular} & FS No. & UI No. & FTR No. & $\begin{array}{c}\text { Sample } \\
\text { No. }\end{array}$ & $\begin{array}{c}\text { Excavation } \\
\text { Block }\end{array}$ & Stratum (AU) & Northing & Easting & Level & $\begin{array}{c}\text { Elevation } \\
\text { (m) }\end{array}$ & Depth (cmbd) & Point Prov. & Artifact Class & Artifact Type & \begin{tabular}{|c|} 
Artifact \\
Description
\end{tabular} & Material & $\begin{array}{c}\text { Number of } \\
\text { artifacts }\end{array}$ & Weight (g) & Comments & Date \\
\hline 651 & 1 & 486 & - & - & - & $E$ & IV & N1004 & E1018 & 8 & $98.6-98.5$ & $70-80$ & - & Informal Tool & Modified Flake & \begin{tabular}{|l|} 
Edge-modified \\
\end{tabular} & Fine Grain Chert & 1 & 0.6 & & 12/12/2017 \\
\hline 651 & 2 & 486 & - & - & - & E & IV & N1004 & E1018 & 8 & $98.6-98.5$ & $70-80$ & - & \begin{tabular}{|l|} 
Faunal Remains \\
\end{tabular} & Shell & Mussel Shell & Shell & 8 & 16.82 & & 12/12/2017 \\
\hline 652 & 1 & 493 & - & - & - & E & $\mathrm{v}$ & N1004 & E1018 & 9 & 98.5-98.4 & $80-90$ & - & Detritus & Debitage & \begin{tabular}{|l|} 
Complete Flake \\
\end{tabular} & Lithic & 6 & 28.47 & & 12/12/2017 \\
\hline 652 & 2 & 493 & - & - & - & E & $\mathrm{v}$ & N1004 & E1018 & 9 & 98.5-98.4 & $80-90$ & - & Detritus & Debitage & Proximal Flake & Lithic & 8 & 8.68 & & 12/12/2017 \\
\hline 652 & 3 & 493 & - & - & - & E & $\mathrm{v}$ & N1004 & E1018 & 9 & $98.5-98.4$ & $80-90$ & - & Detritus & Debitage & Broken Flake & Lithic & 13 & 8.4 & & $\mid 12 / 12 / 2017$ \\
\hline 652 & 4 & 493 & - & - & - & E & $\mathrm{v}$ & N1004 & E1018 & 9 & 98.5-98.4 & $80-90$ & - & Detritus & Debitage & \begin{tabular}{|l|} 
Thermal Shatter \\
\end{tabular} & Lithic & 4 & 4.15 & & 12/12/2017 \\
\hline 652 & 5 & 493 & - & - & - & E & $\mathrm{v}$ & N1004 & E1018 & 9 & 98.5-98.4 & $80-90$ & - & \begin{tabular}{|l|} 
Faunal Remains \\
\end{tabular} & Shell & Mussel Shell & Shell & 1 & 0.7 & & 12/12/2017 \\
\hline 653 & 1 & 504 & - & - & - & E & $V_{N 1}$ & N1004 & E1018 & 10 & 98.4-98.3 & $90-100$ & - & Detritus & Debitage & Complete Flake & Lithic & 4 & 10.73 & & $\mid$\begin{tabular}{|l|}
$\mid 12 / 13 / 2017$ \\
\end{tabular} \\
\hline 653 & 2 & 504 & - & - & - & E & VNII & N1004 & E1018 & 10 & 98.4-98.3 & 90-100 & - & Detritus & Debitage & Proximal Flake & Lithic & 7 & 19.1 & & 12/13/2017 \\
\hline 653 & 3 & 504 & - & - & - & $\mathrm{E}$ & $\mathrm{V} N \mathrm{~N}$ & N1004 & E1018 & 10 & $98.4-98.3$ & $\begin{array}{l}0-100 \\
\end{array}$ & - & Detritus & Debitage & Broken Flake & Lithic & 15 & 13.29 & & 12/13/2017 \\
\hline 653 & 4 & 504 & - & - & - & $\mathrm{E}$ & $\mathrm{V} N \mathrm{~N}$ & N1004 & E1018 & 10 & $98.4-98.3$ & 90-100 & - & Detritus & Debitage & Thermal Shatter & Lithic & 5 & 1.55 & & 12/13/2017 \\
\hline 654 & 1 & 622 & - & - & - & $\mathrm{E}$ & $\mathrm{vv}_{1}$ & N1004 & E1018 & 11 & $98.3-98.2$ & $100-110$ & - & Detritus & Debitage & Proximal Flake & Lithic & 2 & 2.92 & & 12/20/2017 \\
\hline 654 & 2 & 622 & - & - & - & $\mathrm{E}$ & $\mathrm{vI}$ & N1004 & E1018 & 11 & $98.3-98.2$ & $100-110$ & - & Detritus & Debitage & Broken Flake & Lithic & 3 & 1.27 & & 12/20/2017 \\
\hline 654 & 3 & 622 & - & - & - & $\mathrm{E}$ & $\mathrm{vv}_{1}$ & N1004 & E1018 & 11 & $98.3-98.2$ & $100-110$ & - & Detritus & Debitage & Thermal Shatter & Lithic & 1 & 3.21 & & $\mid 12 / 20 / 2017$ \\
\hline 655 & 1 & 625 & - & - & - & $\mathrm{E}$ & $\mathrm{vv}_{1}$ & N1004 & E1018 & 12 & 98.2-98.1 & $110-120$ & - & Detritus & Debitage & Proximal Flake & Lithic & 2 & 4.62 & & 12/20/2017 \\
\hline 655 & 2 & 625 & - & - & - & $\mathrm{E}$ & vi & N1004 & E1018 & 12 & 98.2-98.1 & 110-120 & - & Detritus & Debitage & Broken Flake & Lithic & 1 & 0.19 & & 12/20/2017 \\
\hline 655 & 3 & 625 & - & - & - & $\mathrm{E}$ & vi & N1004 & E1018 & 12 & 98.2-98.1 & $110-120$ & - & Detritus & Debitage & Thermal Shatter & Lithic & 1 & 0.2 & & 12/20/2017 \\
\hline 655 & 4 & 625 & - & - & - & $\mathrm{E}$ & vi & N1004 & E1018 & 12 & 98.2-98.1 & $110-120$ & - & Faunal Remains & Shell & Mussel Shell & Shell & 1 & 4.95 & & 12/20/2017 \\
\hline 656 & 1 & 630 & - & - & - & $\mathrm{E}$ & vi & N1004 & E1018 & 13 & $98.1-98.0$ & $120-130$ & - & Detritus & Debitage & Complete Flake & Lithic & 2 & 6.1 & & 12/20/2017 \\
\hline 657 & 1 & 418 & - & - & - & E & II/III & N1004 & E1019 & 3 & $99.1-99.0$ & $20-30$ & - & Detritus & Debitage & Thermal Shatter & Lithic & 1 & 1.04 & & 12/4/2017 \\
\hline 658 & 1 & 432 & - & - & - & $\mathrm{E}$ & IIII & N1004 & E1019 & 4 & 99.098 .9 & $30-40$ & - & Detritus & Debitage & Broken Flake & Lithic & 2 & 0.83 & & 12/5/2017 \\
\hline 659 & 1 & 446 & - & - & - & $\mathrm{E}$ & IIII & N1004 & E1019 & 5 & $98.9-98.8$ & $40-50$ & - & Detritus & Debitage & Complete Flake & Lithic & 8 & 13.64 & & $1215 / 2017$ \\
\hline 659 & 2 & 446 & - & - & - & $\mathrm{E}$ & IIII & N1004 & E1019 & 5 & $\begin{array}{ll}98.9-98.8 \\
\end{array}$ & $40-50$ & - & Detritus & Debitage & \begin{tabular}{|l|} 
Proximal Flake \\
\end{tabular} & Lithic & 4 & 2.23 & & 12/5/2017 \\
\hline 659 & 3 & 446 & - & - & - & E & IIII & N1004 & E1019 & 5 & 98.9-98.8 & $40-50$ & - & Detritus & Debitage & Broken Flake & Lithic & 8 & 5.22 & & 1215/2017 \\
\hline 659 & 4 & 446 & - & - & - & $\mathrm{E}$ & III & N1004 & E1019 & 5 & $98.9-98.8$ & $40-50$ & - & Detritus & Debitage & Thermal Shatter & Lithic & 7 & 2.67 & & $1215 / 2017$ \\
\hline 660 & 1 & 459 & - & - & - & E & IIII & N1004 & E1019 & 6 & $98.8-98.7$ & $50-60$ & - & Detritus & Debitage & \begin{tabular}{|l|} 
Complete Flake \\
\end{tabular} & Lithic & 7 & 73.09 & & 12/8/2017 \\
\hline 660 & 2 & 459 & - & - & - & E & IIII & N1004 & E1019 & 6 & $98.8-98.7$ & $50-60$ & - & Detritus & Debitage & \begin{tabular}{|l|} 
Proximal Flake \\
\end{tabular} & Lithic & 12 & 15.17 & & 12/8/2017 \\
\hline 660 & 3 & 459 & - & - & - & E & IIII & N1004 & E1019 & 6 & $98.8-98.7$ & $50-60$ & - & Detritus & Debitage & Broken Flake & Lithic & 14 & 3.2 & & 12/8/2017 \\
\hline 660 & 4 & 459 & - & - & - & E & IIII & N1004 & E1019 & 6 & $98.8-98.7$ & $50-60$ & - & Detritus & Debitage & Thermal Shatter & Lithic & 17 & 24.71 & & 12/8/2017 \\
\hline 660 & 5 & 459 & - & - & - & E & IIII & N1004 & E1019 & 6 & $98.8-98.7$ & $50-60$ & - & Informal Tool & Modified Flake & \begin{tabular}{|l|} 
Edge-modified \\
\end{tabular} & Fine Grain Chert & 1 & 6.24 & & 12/8/2017 \\
\hline 660 & 6 & 459 & - & - & - & E & IIII & N1004 & E1019 & 6 & 98.8-98.7 & 50-60 & - & Informal Tool & Modified Flake & Edge-modified & Fine Grain Chert & 1 & 29.09 & & 12/8/2017 \\
\hline 660 & 7 & 459 & - & - & - & E & III & N1004 & E1019 & 6 & $\begin{array}{l}98.8-98.7 \\
\end{array}$ & $50-60$ & - & Detritus & Core & Multidirectional & Fine Grain Chert & 1 & 55.09 & & 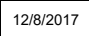 \\
\hline 660 & 8 & 459 & - & - & - & E & III & N1004 & E1019 & 6 & $\begin{array}{l}98.8-98.7 \\
\end{array}$ & $50-60$ & - & Faunal Remains & Shell & Mussel Shell & Shell & 1 & 13.11 & & 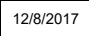 \\
\hline 661 & 1 & 469 & - & - & - & $\mathrm{E}$ & IV & N1004 & E1019 & 7 & $\begin{array}{l}98.7-98.6 \\
\end{array}$ & $60-70$ & - & Detritus & Debitage & Complete Flake & Lithic & 14 & 9.84 & & 12/11/2017 \\
\hline 661 & 2 & 469 & - & - & - & $E$ & IV & N1004 & E1019 & 7 & 98.7-98.6 & $60-70$ & - & Detritus & Debitage & \begin{tabular}{|l|} 
Proximal Flake \\
\end{tabular} & Lithic & 21 & 10.79 & & 12/11/2017 \\
\hline 661 & 3 & 469 & - & - & - & $\mathrm{E}$ & IV & N1004 & E1019 & 7 & $\begin{array}{l}98.7-98.6 \\
\end{array}$ & $\begin{array}{ll}60-70 \\
\end{array}$ & - & Detritus & Debitage & Broken Flake & Lithic & 20 & 7.41 & & 12/11/2017 \\
\hline 661 & 4 & 469 & - & - & - & $\mathrm{E}$ & IV & N1004 & E1019 & 7 & $98.7-98.6$ & $60-70$ & - & Detritus & Debitage & Thermal Shatter & Lithic & 19 & 8.31 & & 12/11/2017 \\
\hline 661 & 5 & 469 & - & - & - & $\mathrm{E}$ & IV & N1004 & E1019 & 7 & $98.7-98.6$ & $60-70$ & - & Informal Tool & Modified Flake & Edge-modified & Fine Grain Chert & 1 & 1.41 & & 12/11/2017 \\
\hline 661 & 6 & 469 & - & - & - & $\mathrm{E}$ & IV & N1004 & E1019 & 7 & $\begin{array}{l}98.7-98.6 \\
\end{array}$ & $\begin{array}{ll}60-70 \\
\end{array}$ & - & Informal Tool & Modified Flake & Edge-modified & Fine Grain Chert & 1 & 1.2 & & 12/11/2017 \\
\hline 661 & 7 & 469 & - & - & - & $\mathrm{E}$ & IV & N1004 & E1019 & 7 & 98.7-98.6 & $60-70$ & - & Formal Tool & Biface & Complete & Fine Grain Chert & 1 & 8.76 & & 12/11/2017 \\
\hline 661 & 8 & 469 & - & - & - & $\mathrm{E}$ & IV & N1004 & E1019 & 7 & 98.7-98.6 & $60-70$ & - & Faunal Remains & Shell & Mussel Shell & Shell & 4 & 7.91 & & 12/11/2017 \\
\hline 662 & 1 & 533 & - & - & - & $\mathrm{E}$ & IV & N1004 & E1019 & 8 & $98.6-98.5$ & $70-80$ & - & \begin{tabular}{|l|} 
Faunal Remains \\
\end{tabular} & Bone & Mammal & Bone & 1 & 1.21 & & 12/14/2017 \\
\hline 662 & 2 & 533 & - & - & - & $\mathrm{E}$ & IV & N1004 & E1019 & 8 & $\begin{array}{l}98.6-98.5 \\
\end{array}$ & $70-80$ & - & Detritus & Debitage & \begin{tabular}{|l|} 
Complete Flake \\
\end{tabular} & Lithic & 4 & 18.47 & & 12/14/2017 \\
\hline 662 & 3 & 533 & - & - & - & $\mathrm{E}$ & IV & N1004 & E1019 & 8 & $98.6-98.5$ & $70-80$ & - & Detritus & Debitage & \begin{tabular}{|l|} 
Proximal Flake \\
\end{tabular} & Lithic & 10 & 7.36 & & 12/14/2017 \\
\hline
\end{tabular}




\begin{tabular}{|c|c|c|c|c|c|c|c|c|c|c|c|c|c|c|c|c|c|c|c|c|c|}
\hline Lot No. & $\begin{array}{c}\text { Specimen } \\
\text { No. }\end{array}$ & FS No. & UI No. & FTR No. & $\begin{array}{c}\text { Sample } \\
\text { No. }\end{array}$ & $\begin{array}{c}\text { Excavation } \\
\text { Block }\end{array}$ & Stratum (AU) & Northing & Easting & Level & $\begin{array}{c}\text { Elevation } \\
(\mathrm{m})\end{array}$ & Depth (cmbd) & \begin{tabular}{|l|} 
Point Prov. \\
\end{tabular} & Artifact Class & Artifact Type & \begin{tabular}{|c|} 
Artifact \\
Description
\end{tabular} & Material & $\begin{array}{c}\text { Number of } \\
\text { artifacts }\end{array}$ & Weight (g) & Comments & Date \\
\hline 662 & 4 & 533 & - & - & - & $\mathrm{E}$ & IV & N1004 & E1019 & 8 & $98.6-98.5$ & $70-80$ & - & Detritus & Debitage & Broken Flake & Lithic & 16 & 27.88 & & 12/14/2017 \\
\hline 662 & 5 & 533 & - & - & - & E & IV & N1004 & E1019 & 8 & $98.6-98.5$ & $70-80$ & - & Detritus & Debitage & Thermal Shatter & Lithic & 5 & 4.64 & & $\mid$\begin{tabular}{|l|}
$12 / 14 / 2017$ \\
\end{tabular} \\
\hline 662 & 6 & 533 & - & - & - & $\mathrm{E}$ & IV & N1004 & E1019 & 8 & $98.6-98.5$ & $70-80$ & - & \begin{tabular}{|l|} 
Faunal Remains \\
\end{tabular} & Shell & \begin{tabular}{|l|} 
Mussel Shell \\
\end{tabular} & Shell & 13 & 22.45 & & 12/14/2017 \\
\hline 663 & 1 & 535 & - & - & - & $E$ & $\mathrm{v}$ & N1004 & E1019 & 9 & $98.5-98.4$ & $80-90$ & - & \begin{tabular}{|l|} 
Detritus \\
\end{tabular} & Debitage & Complete Flake & Lithic & 12 & 91.91 & & 12/14/2017 \\
\hline 663 & 2 & 535 & - & - & - & E & $\mathrm{v}$ & N1004 & E1019 & 9 & $98.5-98.4$ & $80-90$ & - & Detritus & Debitage & Proximal Flake & Lithic & 6 & 3.54 & & $\mid 12 / 14 / 2017$ \\
\hline 663 & 3 & 535 & - & - & - & E & $\mathrm{v}$ & N1004 & E1019 & 9 & 98.5-98.4 & $80-90$ & - & Detritus & Debitage & Broken Flake & Lithic & 14 & 5.44 & & 12/14/2017 \\
\hline 663 & 4 & 535 & - & - & - & $E$ & $\mathrm{v}$ & N1004 & E1019 & 9 & 98.5-98.4 & $80-90$ & - & Detritus & Debitage & Thermal Shatter & Lithic & 11 & 7.26 & & 12/14/2017 \\
\hline 663 & 5 & 535 & - & - & - & $E$ & $\mathrm{v}$ & N1004 & E1019 & 9 & 98.5-98.4 & $80-90$ & - & \begin{tabular}{|l|} 
Faunal Remains \\
\end{tabular} & Shell & Mussel Shell & Shell & 12 & 33.97 & & $\mid 12 / 14 / 2017$ \\
\hline 663 & 6 & 535 & - & - & - & $\mathrm{E}$ & v & N1004 & E1019 & 9 & 98.5-98.4 & $80-90$ & - & Faunal Remains & Shell & Untyped Shell & Shell & 2 & 33.46 & & 12/14/2017 \\
\hline 664 & 1 & 569 & - & - & - & $E$ & $\mathrm{v}$ & N1004 & E1019 & 9 & 98.44 & 86 & \begin{tabular}{|l|} 
N1004.50 \\
E1019.49 \\
\end{tabular} & \begin{tabular}{|l|} 
Detritus \\
\end{tabular} & Core & Multidirectional & \begin{tabular}{|c|} 
Coarse Grain \\
Chert
\end{tabular} & 1 & 109.69 & & \begin{tabular}{|l|}
$12 / 14 / 2017$ \\
\end{tabular} \\
\hline 665 & 1 & 568 & - & - & - & $\mathrm{E}$ & $\begin{array}{l}V I \\
\end{array}$ & N1004 & E1019 & 10 & $98.4-98.3$ & $\begin{array}{l}90-100 \\
\end{array}$ & - & Detritus & Debitage & \begin{tabular}{|l|} 
Complete Flake \\
\end{tabular} & Lithic & 4 & 1.73 & & 12/14/2017 \\
\hline 665 & 2 & 568 & - & - & - & $E$ & $\mathrm{~V} N \mathrm{NI}$ & N1004 & E1019 & 10 & 98.4-98.3 & $90-100$ & - & Detritus & Debitage & Proximal Flake & Lithic & 14 & 8.98 & & 12/14/2017 \\
\hline 665 & 3 & 568 & - & - & - & $\mathrm{E}$ & $\mathrm{V} N \mathrm{~N}$ & N1004 & E1019 & 10 & 98.4-98.3 & $90-100$ & - & Detritus & Debitage & Broken Flake & Lithic & 24 & 9.82 & & $12 / 14 / 2017$ \\
\hline 665 & 4 & 568 & - & - & - & E & $V N 1$ & N1004 & E1019 & 10 & $98.4-98.3$ & 90-100 & - & Detritus & Debitage & Thermal Shatter & Lithic & 10 & 3.81 & & 12/14/2017 \\
\hline 665 & 5 & 568 & - & - & - & $\mathrm{E}$ & $\mathrm{V} N \mathrm{~N}$ & N1004 & E1019 & 10 & 98.4-98.3 & $90-100$ & - & \begin{tabular}{l|l} 
Informal Tool \\
\end{tabular} & Modified Flake & Utilized Flake & Fine Grain Chert & 1 & 121.86 & & 12/14/2017 \\
\hline 665 & 6 & 568 & - & - & - & $\mathrm{E}$ & $V_{N I}$ & N1004 & E1019 & 10 & 98.4-98.3 & $90-100$ & - & \begin{tabular}{|l|} 
Detritus \\
\end{tabular} & \begin{tabular}{|l|l} 
Core \\
\end{tabular} & Multidirectional & Fine Grain Chert & 1 & 71.55 & & \begin{tabular}{|l|}
$12 / 14 / 2017$ \\
\end{tabular} \\
\hline 665 & 7 & 568 & - & - & - & $\mathrm{E}$ & $\mathrm{V} N \mathrm{~N}$ & N1004 & E1019 & 10 & 98.4-98.3 & $90-100$ & - & \begin{tabular}{|c|} 
Detritus \\
\end{tabular} & Core & Multidirectional & \begin{tabular}{|c|}
$\begin{array}{c}\text { Coarse Grain } \\
\text { Chert }\end{array}$ \\
\end{tabular} & 1 & 38.13 & Exhausted Core & 12/14/2017 \\
\hline 665 & 8 & 568 & - & - & - & $\mathrm{E}$ & $\mathrm{VNI}$ & N1004 & E1019 & 10 & $98.4-98.3$ & 90-100 & - & Faunal Remains & Shell & Mussel Shell & Shell & 3 & 7.19 & & 12/14/2017 \\
\hline 666 & 1 & 584 & - & - & - & $E$ & $\mathrm{Vl}$ & N1004 & E1019 & 11 & $98.3-98.2$ & $100-110$ & - & Detritus & Debitage & Complete Flake & Lithic & 5 & 16.06 & & 12/18/2017 \\
\hline 666 & 2 & 584 & - & - & - & $\mathrm{E}$ & $\mathrm{vv}_{1}$ & N1004 & E1019 & 11 & $98.3-98.2$ & 100-110 & - & Detritus & Debitage & \begin{tabular}{|l|} 
Proximal Flake \\
\end{tabular} & Lithic & 4 & 4.08 & & 12/18/2017 \\
\hline 666 & 3 & 584 & - & - & - & E & vi & N1004 & E1019 & 11 & 98.3-98.2 & $100-110$ & - & Detritus & Debitage & Broken Flake & Lithic & 8 & 6.69 & & 12/18/2017 \\
\hline 666 & 4 & 584 & - & - & - & $\mathrm{E}$ & vi & N1004 & E1019 & 11 & $98.3-98.2$ & 100-110 & - & Detritus & Debitage & Thermal Shatter & Lithic & 2 & 10.14 & & 12/18/2017 \\
\hline 666 & 5 & 584 & - & - & - & $E$ & vi & N1004 & E1019 & 11 & 98.3-98.2 & $100-110$ & - & \begin{tabular}{|l|} 
Informal Tool \\
\end{tabular} & Modified Flake & Edge-modified & Fine Grain Chert & 1 & 2.32 & & 12/18/2017 \\
\hline 666 & 6 & 584 & - & - & - & $\mathrm{E}$ & vi & N1004 & E1019 & 11 & 98.3-98.2 & $100-110$ & - & Informal Tool & Modified Flake & Edge-modified & \begin{tabular}{|c|}
$\begin{array}{c}\text { Coarse Grain } \\
\text { Chert }\end{array}$ \\
\end{tabular} & 1 & 43.85 & & 12/18/2017 \\
\hline 666 & 7 & 584 & - & - & - & $\mathrm{E}$ & vi & N1004 & E1019 & 11 & 98.3-98.2 & $100-110$ & - & Faunal Remains & Shell & Mussel Shell & Shell & 2 & 5.58 & & 12/18/2017 \\
\hline 667 & 1 & 594 & - & - & - & $E$ & $\mathrm{vi}$ & $\mathrm{N} 1004$ & E1019 & 11 & 98.2 & 110 & \begin{tabular}{|l|} 
N1004.02 \\
E1019.24 \\
\end{tabular} & Detritus & Core & Bifacial & Fine Grain Chert & 1 & 312.57 & & 12/18/2017 \\
\hline 668 & 1 & 588 & - & - & - & $E$ & $\mathrm{vi}$ & $\mathrm{N} 1004$ & E1019 & 12 & 98.2-98.1 & $110-120$ & - & Faunal Remains & Bone & Mammal & Bone & 1 & 3.93 & & 12/118/2017 \\
\hline 668 & 2 & 588 & - & - & - & $E$ & $\mathrm{vi}$ & N1004 & E1019 & 12 & 98.2-98.1 & $110-120$ & - & Detritus & Debitage & Complete Flake & Lithic & 2 & 9.64 & & 12/18/2017 \\
\hline 668 & 3 & 588 & - & - & - & $E$ & $\mathrm{vi}$ & N1004 & E1019 & 12 & 98.2-98.1 & 110-120 & - & Detritus & Debitage & \begin{tabular}{|l|} 
Proximal Flake \\
\end{tabular} & Lithic & 2 & 16.2 & & 12/18/2017 \\
\hline 668 & 4 & 588 & - & - & - & $\mathrm{E}$ & $\mathrm{vv}_{1}$ & N1004 & E1019 & 12 & $98.2-98.1$ & 110-120 & - & Detritus & Debitage & Thermal Shatter & Lithic & 2 & 0.86 & & 12/18/2017 \\
\hline 668 & 5 & 588 & - & - & - & $E$ & $\mathrm{vi}$ & N1004 & E1019 & 12 & 98.2-98.1 & $110-120$ & - & Faunal Remains & Shell & Mussel Shell & Shell & 1 & 7.68 & & 12/188/2017 \\
\hline 668 & 6 & 588 & 31 & - & - & $E$ & vi & N1004 & E1019 & 12 & $98.2-98.1$ & $110-120$ & - & Formal Tool & Arrow Point & Edwards Point & Fine Grain Chert & 1 & 1.82 & & 12/18/2017 \\
\hline 669 & 1 & 605 & - & - & - & E & vi & N1004 & E1019 & 13 & $98.1-98.0$ & $120-130$ & - & Detritus & Debitage & Complete Flake & Lithic & 3 & 19.6 & & 12/20/2017 \\
\hline 669 & 2 & 605 & - & - & - & $E$ & $\mathrm{vi}$ & N1004 & E1019 & 13 & $98.1-98.0$ & $120-130$ & - & Detritus & Debitage & Proximal Flake & Lithic & 1 & 1.02 & & 12/20/2017 \\
\hline 669 & 3 & 605 & - & - & - & E & vi & N1004 & E1019 & 13 & $98.1-98.0$ & $120-130$ & - & Detritus & Debitage & Broken Flake & Lithic & 5 & 1.38 & & 12/20/2017 \\
\hline 669 & 4 & 605 & - & - & - & $E$ & $\mathrm{vi}$ & N1004 & E1019 & 13 & $98.1-98.0$ & $120-130$ & - & Detritus & Debitage & Thermal Shatter & Lithic & 1 & 0.11 & & 12/20/2017 \\
\hline 670 & 1 & 609 & - & - & - & E & vi & N1004 & E1019 & 14 & $98.0-97.9$ & 130-140 & - & Detritus & Debitage & \begin{tabular}{|l|} 
Complete Flake \\
\end{tabular} & Lithic & 3 & 24.43 & & $\mid 12 / 20 / 2017$ \\
\hline 671 & 1 & 615 & - & - & - & $E$ & vi & N1004 & E1019 & 15 & 97.9-97.8 & 140-150 & - & Detritus & Debitage & Proximal Flake & Lithic & 1 & 8.97 & & 12/20/2017 \\
\hline 672 & 1 & 499 & - & FTR2 & $F-2 / F-3$ & $\mathrm{E}$ & IIIIV & N1003-1004 & E1018-1019 & $6-8$ & 98.77-98.5 & 53-80 & \begin{tabular}{|l|} 
N1003.75- \\
1004.06 \\
E1018.76- \\
1019.28 \\
\end{tabular} & \begin{tabular}{c|}
$\begin{array}{c}\text { Macrobotanical } \\
\text { Remains }\end{array}$ \\
\end{tabular} & \begin{tabular}{|c} 
Other Plant \\
Parts
\end{tabular} & $\begin{array}{l}\text { Vetch (Vicia } \\
\text { ludovicianaa) }\end{array}$ & & 2 & 0.01 & 'sent for radiocarbon dating & 12/12/2017 \\
\hline 672 & 2 & 499 & - & FTR2 & $F-2 / F-3$ & $\mathrm{E}$ & IIIIV & N1003-1004 & E1018-1019 & $6-8$ & 98.77-98.5 & 53-80 & \begin{tabular}{|l|} 
N11003.75- \\
1004.06 \\
E1018.76- \\
1019.28 \\
\end{tabular} & $\begin{array}{c}\text { Macrobotanical } \\
\text { Remains }\end{array}$ & Charcoal & $\underset{\text { spp.) }}{\operatorname{Elm}(\text { UImus }}$ & & 1 & 0.01 & & 12/12/2017 \\
\hline 672 & 3 & 499 & - & FTR2 & $F-2 / F-3$ & $\mathrm{E}$ & IIIIV & N1003-1004 & E1018-1019 & $6-8$ & 98.77-98.5 & $53-80$ & \begin{tabular}{|c|}
$\mathrm{N} 1003.75-$ \\
1004.06 \\
$\mathrm{E} 1018.76-$ \\
1019.28 \\
\end{tabular} & \begin{tabular}{|c|}
$\begin{array}{c}\text { Macrobotanical } \\
\text { Remains }\end{array}$ \\
\end{tabular} & Charcoal & $\begin{array}{c}\text { Mesquite } \\
\text { (Prosopis } \\
\text { glandulosa) }\end{array}$ & & 3 & 0.02 & & 12/12/2017 \\
\hline
\end{tabular}




\begin{tabular}{|c|c|c|c|c|c|c|c|c|c|c|c|c|c|c|c|c|c|c|c|c|c|}
\hline Lot No. & $\begin{array}{c}\begin{array}{c}\text { Specimen } \\
\text { No. }\end{array} \\
\text { Non }\end{array}$ & FS No. & UI No. & FTR No. & \begin{tabular}{c|c} 
Sample \\
No.
\end{tabular} & $\begin{array}{c}\text { Excavation } \\
\text { Block }\end{array}$ & Stratum (AU) & Northing & Easting & Level & $\begin{array}{c}\text { Elevation } \\
(\mathrm{m})\end{array}$ & Depth (cmbd) & Point Prov. & Artifact Class & \begin{tabular}{|l|} 
Artifact Type \\
\end{tabular} & \begin{tabular}{|c|} 
Artifact \\
Description \\
\end{tabular} & Material & $\begin{array}{c}\begin{array}{c}\text { Number of } \\
\text { artifacts }\end{array} \\
\end{array}$ & Weight $(g)$ & Comments & Date \\
\hline 672 & 4 & 499 & - & FTR2 & $F-2 / F=3$ & E & IIIIV & N1003-1004 & E1018-1019 & $6-8$ & 98.77-98.5 & $53-80$ & \begin{tabular}{|c|} 
N11003.75- \\
1004.06 \\
E1018.76- \\
1019.28 \\
\end{tabular} & $\begin{array}{c}\text { Macrobotanical } \\
\text { Remains }\end{array}$ & Charcoal & $\begin{array}{l}\text { Sugarbery } \\
\text { (Celtis sp.) }\end{array}$ & & 6 & 0.01 & & 12/12/2017 \\
\hline 672 & 5 & 499 & - & FTR2 & $F-2 / F-3$ & E & III/V & N1003-1004 & E1018-1019 & $6-8$ & 98.77-98.5 & 53-80 & \begin{tabular}{|c|}
$\mathbf{N} 1000.75-75-$ \\
1004.06 \\
E1019.76- \\
1019.28
\end{tabular} & \begin{tabular}{c|}
$\begin{array}{c}\text { Macrobotanical } \\
\text { Remains }\end{array}$ \\
\end{tabular} & Charcoal & $\begin{array}{c}\text { Hardwood, } \\
\text { indeterminable }\end{array}$ & & 3 & 0.01 & & 12/12/2017 \\
\hline 672 & 6 & 499 & - & FTR2 & $F-2 / F-3$ & E & IIIIV & N1003-1004 & E1018-1019 & $6-8$ & 98.77-98.5 & 53-80 & $\begin{array}{c}\text { N1003.75- } \\
1004.06 \\
\text { E1018.76- } \\
1019.28 \\
\end{array}$ & \begin{tabular}{|c|}
$\begin{array}{c}\text { Macrobotanical } \\
\text { Remains }\end{array}$ \\
\end{tabular} & Charcoal & \begin{tabular}{|c|}
$\begin{array}{c}\text { Acacialmesquite } \\
\text { (Vachellia/Pros } \\
\text { opis sp.) }\end{array}$ \\
\end{tabular} & & 1 & 0.01 & & 12/1/2/2017 \\
\hline 672 & 7 & 499 & - & FTR2 & $F-2 / F-3$ & E & IIIIV & N1003-1004 & E1018-1019 & $6-8$ & 98.77-98.5 & $53-80$ & \begin{tabular}{|l|}
$\mathbf{N} 10003.75-$ \\
1004.06 \\
E1018.76- \\
1019.28
\end{tabular} & \begin{tabular}{|c|} 
Macrobotanical \\
Remains
\end{tabular} & Charcoal & $\begin{array}{c}\text { Hickory/Pecan } \\
\text { (Carya sp.) }\end{array}$ & & 1 & 0.01 & & 12/11/2017 \\
\hline 672 & 8 & 499 & - & FTR2 & $F-2 / F-3$ & E & IIIIV & N1003-1004 & E1018-1019 & $6-8$ & 98.77-98.5 & 53-80 & \begin{tabular}{|c|} 
N1003.75- \\
1004.06 \\
E1018.76- \\
1019.28 \\
\end{tabular} & \begin{tabular}{|c|}
$\begin{array}{c}\text { Macrobotanical } \\
\text { Remains }\end{array}$ \\
\end{tabular} & Charcoal & $\begin{array}{l}\text { Grape (Vitis } \\
\text { spp.) }\end{array}$ & & 1 & 0.01 & & 12/12/2017 \\
\hline 672 & 9 & 499 & - & FTR2 & $F-2 / F-3$ & $\mathrm{E}$ & III/V & N1003-1004 & E1018-1019 & $6-8$ & 98.77-98.5 & $53-80$ & \begin{tabular}{|l|}
$\mathbf{N 1} 1003.75-$ \\
1004.06 \\
E1018.76- \\
1019.28 \\
\end{tabular} & \begin{tabular}{|c|}
$\begin{array}{c}\text { Macrobotanical } \\
\text { Remains }\end{array}$ \\
\end{tabular} & $\underset{>2 \mathrm{~mm}}{\text { Contamination }}$ & & & & 10.97 & & 12/12/2017 \\
\hline 672 & 10 & 499 & - & FTR2 & $F-2 / F-3$ & $\mathrm{E}$ & III/V & N1003-1004 & E1018-1019 & $6-8$ & 98.77-98.5 & $53-80$ & \begin{tabular}{|l|} 
N1003.75- \\
104.06 \\
E1017.76- \\
1019.28
\end{tabular} & \begin{tabular}{c|}
$\begin{array}{c}\text { Macrobotanical } \\
\text { Remains }\end{array}$ \\
\end{tabular} & $\left|\begin{array}{c}\text { Examined } \\
\text { Residue }<2 m m\end{array}\right|$ & & & & 2.01 & & 12/12/2017 \\
\hline 672 & 11 & 499 & - & FTR2 & $F-2 / F-3$ & E & III/V & N1003-1004 & E1018-1019 & $6-8$ & 98.77-98.5 & 53-80 & \begin{tabular}{|l|}
$\mathbf{N 1} 1003.75-$ \\
1004.06 \\
E1019.76- \\
1019.28 \\
\end{tabular} & \begin{tabular}{|c|}
$\begin{array}{c}\text { Macrobotanical } \\
\text { Remains }\end{array}$ \\
\end{tabular} & $\left|\begin{array}{c}\text { residual from } \\
\text { heavy fraction }\end{array}\right|$ & & & & 56 & & 12/12/2017 \\
\hline 672 & 12 & 499 & - & FTR2 & $F-2 / F-3$ & E & III/V & N1003-1004 & E1018-1019 & $6-8$ & 98.77-98.5 & $53-80$ & \begin{tabular}{|l|}
$\mathbf{N 1 0 0 0 3 . 7 5 -}$ \\
1004.06 \\
E1018.76- \\
1019.28
\end{tabular} & \begin{tabular}{c|}
$\begin{array}{c}\text { Macrobotanical } \\
\text { Remains }\end{array}$ \\
\end{tabular} & Charcoal & not $1 D^{\prime} d$ & & 8 & 0.5 & & 12/12/2017 \\
\hline 672 & 13 & 499 & - & FTR2 & $F-2 / F-3$ & E & III/V & N1003-1004 & E1018-1019 & $6-8$ & 98.77-98.5 & $53-80$ & \begin{tabular}{|l|} 
N1003.75- \\
1004.06 \\
E1017.86- \\
1019.28 \\
\end{tabular} & Other Lithic & $\begin{array}{c}\text { Thermally } \\
\text { Altered Stone }\end{array}$ & FCR & & 28 & 8 & & 12/12/2017 \\
\hline 672 & 14 & 499 & - & FTR2 & $F-2 / F-3$ & E & III/V & N1003-1004 & E1018-1019 & $6-8$ & 98.77-98.5 & 53-80 & \begin{tabular}{|l|} 
N1003.75- \\
104.06 \\
E1017.76- \\
1019.28 \\
\end{tabular} & Faunal Remains & Shell & Mussel Shell & & 2 & 9.5 & & 12/12/2017 \\
\hline 672 & 15 & 499 & - & FTR2 & $F-2 / F-3$ & E & III/V & N1003-1004 & E1018-1019 & $6-8$ & 98.77-98.5 & 53-80 & \begin{tabular}{|l|} 
N1003.75- \\
1004.06 \\
E1017.76- \\
1019.28 \\
\end{tabular} & Other Lithic & $\begin{array}{l}\text { Misc. Stone/ } \\
\text { Mineral }\end{array}$ & Manuport & & 1 & 18.5 & & 12/12/2017 \\
\hline 672 & 16 & 499 & - & FTR2 & $F-2 / F-3$ & E & III/V & N1003-1004 & E1018-1019 & $6-8$ & 98.77-98.5 & 53-80 & \begin{tabular}{|l|} 
N1003.75- \\
1004.06 \\
E1018.76- \\
101928
\end{tabular} & Faunal Remains & Bone & & & 1 & 1 & $\begin{array}{l}\text { From flotation heavy fraction, not } \\
\text { analyzed }\end{array}$ & 12/12/2/2017 \\
\hline 672 & 17 & 499 & - & FTR2 & $F-2 / F-3$ & E & III/V & N1003-1004 & E1018-1019 & $6-8$ & 98.77-98.5 & $53-80$ & \begin{tabular}{|c|} 
N11003.75- \\
104.06 \\
E1019.76- \\
1019.28
\end{tabular} & Detritus & Debitage & Proximal Flake & & 4 & 0.67 & & 12/12/2017 \\
\hline 672 & 18 & 499 & - & FTR2 & $F-2 / F=3$ & E & III/V & N1003-1004 & E1018-1019 & $6-8$ & 98.77-98.5 & 53-80 & \begin{tabular}{|c|}
$\mathbf{N} 1000.75-75-$ \\
$1004.06-$ \\
E1018.7.76- \\
10199.28
\end{tabular} & Detritus & Debitage & Broken Flake & & 3 & 0.29 & & 12/11/2017 \\
\hline 672 & 19 & 499 & - & FTR2 & $F-2 / F-3$ & E & III/V & N1003-1004 & E1018-1019 & $6-8$ & 98.77-98.5 & $53-80$ & 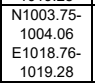 & Detritus & Debitage & Flaking Shatter & & 19 & 0.21 & & 12/12/2017 \\
\hline 672 & 20 & 499 & - & FTR2 & $F-2 / F-3$ & E & III/V & N1003-1004 & E1018-1019 & $6-8$ & 98.77-98.5 & $53-80$ & \begin{tabular}{|c|}
$\mathbf{N 1} 1003.75-$ \\
1004.06 \\
E1019.76- \\
1019.28 \\
\end{tabular} & Detritus & Debitage & Thermal Shatter & & 8 & 0.56 & & 12/112/2017 \\
\hline 672 & n/a & 499 & - & FTR2 & $F-2 / F-3$ & E & III/V & N1003-1004 & E1018-1019 & $6-8$ & 98.77-98.5 & $53-80$ & \begin{tabular}{|c|}
$\mathbf{N} 1000.75-75-$ \\
1004.06 \\
E1018.76- \\
1019.28 \\
\end{tabular} & $\begin{array}{c}\text { Archaeological } \\
\text { Sample }\end{array}$ & Soil & Feature Matrix & & $5.5 \mathrm{~L}$ & 5100 & $\begin{array}{l}\text { Matrix samples floated together; all } \\
\text { floated }\end{array}$ & 12/12/2017 \\
\hline 673 & 1 & 344 & - & - & - & $\mathrm{F}$ & 1 & N1009 & E1011 & 2 & 99.2-99.1 & $10-20$ & - & Faunal Remains & Bone & Indeterminate & Bone & 1 & 0.39 & & 11/29/2017 \\
\hline 673 & 2 & 344 & - & - & - & $\mathrm{F}$ & 1 & N1009 & E1011 & 2 & $99.2-99.1$ & $10-20$ & - & Detritus & Debitage & Broken Flake & Lithic & 1 & 0.19 & & 11/29/2017 \\
\hline 673 & 3 & 344 & - & - & - & $\mathrm{F}$ & 1 & N1009 & E1011 & 2 & $99.2-99.1$ & $10-20$ & - & Detritus & Debitage & Thermal Shatter & Lithic & 1 & 0.83 & & 11/29/2017 \\
\hline 673 & 4 & 344 & - & - & - & $\mathrm{F}$ & 1 & N1009 & E1011 & 2 & 99.2-99.1 & $10-20$ & - & Informal Tool & \begin{tabular}{|l|} 
Modified Flake \\
\end{tabular} & \begin{tabular}{|l|} 
Utilized Flake \\
\end{tabular} & Fine Grain Chert & 1 & 22.47 & & 11/29/2017 \\
\hline 673 & 5 & 344 & - & - & - & $\mathrm{F}$ & 1 & N1009 & E1011 & 2 & $99.2-99.1$ & $10-20$ & - & Historic Artifiact & Glass & \begin{tabular}{|l|} 
Colorless Glass \\
\end{tabular} & Glass & 1 & 0.79 & & 11/29/2017 \\
\hline 674 & 1 & 348 & - & - & - & $\mathrm{F}$ & "I & N1009 & E1011 & 3 & $99.1-99.0$ & $20-30$ & - & Detritus & Debitage & Broken Flake & Lithic & 2 & 2.02 & & 11/29/2017 \\
\hline 674 & 2 & 348 & - & - & - & $\mathrm{F}$ & "1 & N1009 & E1011 & 3 & $99.1-99.0$ & $20-30$ & - & Detritus & Debitage & Thermal Shatter & Lithic & 4 & 3.73 & & 11/29/2017 \\
\hline 675 & 1 & 364 & - & - & - & $\mathrm{F}$ & " & N1009 & E1011 & 4 & $99.0-98.9$ & $30-40$ & - & Detritus & Debitage & Proximal Flake & Lithic & 1 & 0.99 & & 11/29/2017 \\
\hline
\end{tabular}




\begin{tabular}{|c|c|c|c|c|c|c|c|c|c|c|c|c|c|c|c|c|c|c|c|c|c|}
\hline Lot No. & \begin{tabular}{|c|}
$\begin{array}{c}\text { Specimen } \\
\text { No. }\end{array}$ \\
\end{tabular} & FS No. & UI No. & FTR No. & $\begin{array}{c}\text { Sample } \\
\text { No. }\end{array}$ & $\begin{array}{c}\text { Excavation } \\
\text { Block }\end{array}$ & Stratum (AU) & Northing & Easting & Level & $\begin{array}{c}\text { Elevation } \\
(\mathrm{m})\end{array}$ & Depth (cmbd) & Point Prov. & Artifact Class & Artifact Type & \begin{tabular}{|c|} 
Artifact \\
Description
\end{tabular} & Material & $\begin{array}{c}\begin{array}{c}\text { Number of } \\
\text { artifacts }\end{array} \\
\end{array}$ & Weight $(g)$ & Comments & Date \\
\hline 675 & 2 & 364 & - & - & - & $F$ & " & N1009 & E1011 & 4 & 99.0-98.9 & $30-40$ & - & Detritus & Debitage & Broken Flake & Lithic & 2 & 0.63 & & 11/29/2017 \\
\hline 676 & 1 & 366 & - & - & - & $\mathrm{F}$ & III & N1009 & E1011 & 5 & 98.9-98.8 & $40-50$ & - & Faunal Remains & Bone & Indeterminate & Bone & 1 & 1.09 & & 11/29/2017 \\
\hline 676 & 2 & 366 & - & - & - & $\mathrm{F}$ & IIII & N1009 & E1011 & 5 & $98.9-98.8$ & $40-50$ & - & Detritus & Debitage & Complete Flake & Lithic & 3 & 1.03 & & 11/29/2017 \\
\hline 676 & 3 & 366 & - & - & - & $\mathrm{F}$ & IIII & $\begin{array}{ll}\text { N1009 } \\
\end{array}$ & E1011 & 5 & 98.9-98.8 & $40-50$ & - & Detritus & Debitage & Proximal Flake & Lithic & 2 & 0.82 & & 11/29/2017 \\
\hline 676 & 4 & 366 & - & - & - & $\mathrm{F}$ & IIII & N1009 & E1011 & 5 & 98.9-98.8 & $40-50$ & - & Detritus & Debitage & Broken Flake & Lithic & 5 & 4.69 & & 11/29/2017 \\
\hline 676 & 5 & 366 & - & - & - & $\mathrm{F}$ & IIII & N1009 & E1011 & 5 & 98.9-98.8 & $40-50$ & - & Detritus & Debitage & Thermal Shatter & Lithic & 6 & 8.14 & & 11/29/2017 \\
\hline 677 & 1 & 383 & - & - & - & $\mathrm{F}$ & IIII & $\begin{array}{ll}\text { N1009 } \\
\end{array}$ & E1011 & 6 & 98.8-98.7 & $50-60$ & - & Detritus & Debitage & Proximal Flake & Lithic & 4 & 6.93 & & 11/30/2017 \\
\hline 677 & 2 & 383 & - & - & - & $\mathrm{F}$ & III & N1009 & E1011 & 6 & 98.8-98.7 & 50-60 & - & Detritus & Debitage & Broken Flake & Lithic & 1 & 0.42 & & 11/30/2017 \\
\hline 678 & 1 & 387 & - & - & - & $\mathrm{F}$ & III & $\begin{array}{ll}\text { N1009 } \\
\end{array}$ & E1011 & 7 & $98.7-98.6$ & $60-70$ & - & Detritus & Debitage & Broken Flake & Lithic & 1 & 0.58 & & 11/30/2017 \\
\hline 678 & 2 & 387 & - & - & - & $\mathrm{F}$ & IIII & N1009 & E1011 & 7 & $98.7-98.6$ & $\begin{array}{ll}60-70 \\
\end{array}$ & - & Detritus & Debitage & \begin{tabular}{|l|} 
Flaking Shatter \\
\end{tabular} & Lithic & 1 & 10.25 & & 11/30/2017 \\
\hline 678 & 3 & 387 & - & - & - & $\mathrm{F}$ & IIII & N1009 & E1011 & 7 & 98.7-98.6 & $60-70$ & - & Detritus & Debitage & Thermal Shatter & Lithic & 3 & 2.41 & & 11/30/2017 \\
\hline 679 & 1 & 391 & - & - & - & $\mathrm{F}$ & iv & N1009 & E1011 & 8 & $98.6-98.5$ & $\begin{array}{ll}70-80 \\
\end{array}$ & - & Detritus & Debitage & Complete Flake & Lithic & 1 & 5.3 & & 11/30/2017 \\
\hline 679 & 2 & 391 & - & - & - & $\mathrm{F}$ & iv & N1009 & E1011 & 8 & $98.6-98.5$ & $\begin{array}{ll}70-80 \\
\end{array}$ & - & Detritus & Debitage & \begin{tabular}{|l|} 
Proximal Flake \\
\end{tabular} & Lithic & 1 & 26.6 & & 11/30/2017 \\
\hline 679 & 3 & 391 & - & - & - & $\mathrm{F}$ & iv & N1009 & E1011 & 8 & $98.6-98.5$ & $\begin{array}{ll}70-80 \\
\end{array}$ & - & Detritus & Debitage & Broken Flake & Lithic & 2 & 1.63 & & 11/30/2017 \\
\hline 679 & 4 & 391 & - & - & - & $\mathrm{F}$ & iv & N1009 & E1011 & 8 & 98.6-98.5 & $70-80$ & - & Detritus & Debitage & Thermal Shatter & Lithic & 5 & 6.05 & & 11/30/2017 \\
\hline 680 & 1 & 401 & - & - & - & $\mathrm{F}$ & iv & N1009 & E1011 & 9 & $98.5-98.4$ & $80-90$ & - & Detritus & Debitage & Complete Flake & Lithic & 1 & 0.98 & & 12/1/2017 \\
\hline 680 & 2 & 401 & - & - & - & $\mathrm{F}$ & iv & N1009 & E1011 & 9 & 98.5-98.4 & $80-90$ & - & Detritus & Debitage & Thermal Shatter & Lithic & 4 & 4.29 & & 12/1/2017 \\
\hline 681 & 1 & 556 & - & - & - & $\mathrm{F}$ & v & N1009 & E1011 & 11 & 98.3-98.2 & $100-110$ & - & Detritus & Debitage & Broken Flake & Lithic & 1 & 0.4 & & 12/15/2017 \\
\hline 681 & 2 & 556 & - & - & - & $\mathrm{F}$ & $\mathrm{v}$ & N1009 & E1011 & 11 & 98.3-98.2 & $100-110$ & - & Detritus & Debitage & Thermal Shatter & Lithic & 3 & 2.88 & & 12/15/2017 \\
\hline 682 & 1 & 559 & - & - & - & $\mathrm{F}$ & vi & N1009 & E1011 & 12 & 98.2-98.1 & $110-120$ & - & Detritus & Debitage & Thermal Shatter & Lithic & 4 & 2.68 & & 12/15/2017 \\
\hline 683 & 1 & 571 & - & - & - & $\mathrm{F}$ & vi & N1009 & E1011 & 13 & $98.1-98.0$ & $120-130$ & - & Detritus & Debitage & Complete Flake & Lithic & 1 & 8.54 & & 12/18/2017 \\
\hline 683 & 2 & 571 & - & - & - & $\mathrm{F}$ & vi & N1009 & E1011 & 13 & $98.1-98.0$ & $120-130$ & - & Detritus & Debitage & Broken Flake & Lithic & 1 & 0.88 & & 12/18/2017 \\
\hline 683 & 3 & 571 & - & - & - & $\mathrm{F}$ & vi & N1009 & E1011 & 13 & $98.1-98.0$ & $120-130$ & - & Detritus & Debitage & Flaking Shatter & Lithic & 1 & 1.01 & & 12/18/2017 \\
\hline 683 & 4 & 571 & - & - & - & $\mathrm{F}$ & vi & N1009 & E1011 & 13 & $98.1-98.0$ & $120-130$ & - & Detritus & Debitage & Thermal Shatter & Lithic & 2 & 15.93 & & 12/18/2017 \\
\hline 683 & 5 & 571 & - & - & - & $\mathrm{F}$ & vi & N1009 & E1011 & 13 & $98.1-98.0$ & $120-130$ & - & Faunal Remains & Shell & Mussel Shell & Shell & 4 & 4.82 & No umbos but burned & 12/18/2017 \\
\hline 684 & 1 & 577 & - & - & - & $\mathrm{F}$ & vi & N1009 & E1011 & 14 & $98.0-97.9$ & $130-140$ & - & Detritus & Debitage & Broken Flake & Lithic & 2 & 0.42 & & 12/18/2017 \\
\hline 684 & 2 & 577 & - & - & - & $\mathrm{F}$ & $\mathrm{vI}$ & N1009 & E1011 & 14 & $98.0-97.9$ & 130-140 & - & Detritus & Debitage & Thermal Shatter & Lithic & 1 & 0.22 & & 12/18/2017 \\
\hline 685 & 1 & 582 & - & - & - & $\mathrm{F}$ & $\mathrm{vi}$ & N1009 & E1011 & 15 & $97.9-97.8$ & 140-150 & - & Detritus & Core & \begin{tabular}{|l|} 
Multidirectional \\
\end{tabular} & $\begin{array}{l}\text { Coarse Grain } \\
\text { Chert }\end{array}$ & 1 & 18.02 & Exhausted Core & 12/18/2017 \\
\hline 686 & 1 & 585 & - & - & - & $\mathrm{F}$ & $\mathrm{vI}$ & N1009 & E1011 & 16 & $97.8-97.7$ & $150-160$ & - & Detritus & Debitage & Thermal Shatter & Lithic & 1 & 11.86 & & 12/18/2017 \\
\hline 687 & 1 & 343 & - & - & - & $\mathrm{F}$ & 1 & N1009 & E1012 & 1 & 99.21-99.1 & $9-20$ & - & Detritus & Debitage & Broken Flake & Lithic & 1 & 0.32 & & 11/29/2017 \\
\hline 687 & 2 & 343 & - & - & - & $\mathrm{F}$ & 1 & $\begin{array}{ll}\text { N1009 } \\
\end{array}$ & E1012 & 1 & 99.21-99.1 & $9-20$ & - & \begin{tabular}{|l|} 
Historic Artifiact \\
\end{tabular} & Metal & Bullet Casing & Metal & 1 & 0.64 & & 11/29/2017 \\
\hline 688 & 1 & 352 & - & - & - & $\mathrm{F}$ & II & $\begin{array}{ll}\text { N1009 } \\
\end{array}$ & E1012 & 2 & $99.1-99.0$ & $20-30$ & - & Faunal Remains & Bone & Avian & Bone & 1 & 1.09 & & 11/29/2017 \\
\hline 688 & 2 & 352 & - & - & - & $\mathrm{F}$ & "I & N1009 & E1012 & 2 & $99.1-99.0$ & $20-30$ & - & Faunal Remains & Bone & Avian & Bone & 1 & 0.12 & & 11/29/2017 \\
\hline 688 & 3 & 352 & - & - & - & $\mathrm{F}$ & " & N1009 & E1012 & 2 & $99.1-99.0$ & $20-30$ & - & Faunal Remains & Bone & Indeterminate & Bone & 1 & 0.13 & & 11/29/2017 \\
\hline 688 & 4 & 352 & - & - & - & $\mathrm{F}$ & "I & N1009 & E1012 & 2 & $99.1-99.0$ & $20-30$ & - & Faunal Remains & Bone & Avian & Bone & 1 & 0.43 & & 11/29/2017 \\
\hline 688 & 5 & 352 & - & - & - & $\mathrm{F}$ & "I & N1009 & E1012 & 2 & $99.1-99.0$ & $20-30$ & - & Faunal Remains & Bone & Indeterminate & Bone & 1 & 0.17 & & 11/29/2017 \\
\hline 688 & 6 & 352 & - & - & - & $\mathrm{F}$ & "II & N1009 & E1012 & 2 & $99.1-99.0$ & 20-30 & - & \begin{tabular}{|l|} 
Detritus \\
\end{tabular} & Debitage & Proximal Flake & Lithic & 1 & 1.5 & & 11/29/2017 \\
\hline 688 & 7 & 352 & - & - & - & $\mathrm{F}$ & " & N1009 & E1012 & 2 & $99.1-99.0$ & $20-30$ & - & Detritus & Debitage & Broken Flake & Lithic & 1 & 0.35 & & 11/29/2017 \\
\hline 689 & 1 & 357 & - & - & - & $\mathrm{F}$ & "I & N1009 & E1012 & 3 & 99.0-98.9 & $30-40$ & - & \begin{tabular}{|l|} 
Faunal Remains \\
\end{tabular} & Bone & Indeterminate & Bone & 1 & 0.48 & & 11/29/2017 \\
\hline 689 & 2 & 357 & - & - & - & $\mathrm{F}$ & ॥ & N1009 & E1012 & 3 & 99.0-98.9 & $30-40$ & - & Detritus & Debitage & Broken Flake & Lithic & 3 & 7.8 & & 11/29/2017 \\
\hline 689 & 3 & 357 & - & - & - & $\mathrm{F}$ & "I & N1009 & E1012 & 3 & $99.0-98.9$ & $30-40$ & - & Detritus & Debitage & Thermal Shatter & Lithic & 2 & 1.53 & & 11/29/2017 \\
\hline 690 & 1 & 375 & - & - & - & $\mathrm{F}$ & IIII & N1009 & E1012 & 4 & 98.9-98.8 & $40-50$ & - & Detritus & Debitage & Complete Flake & Lithic & 2 & 30.46 & & 11/30/2017 \\
\hline 690 & 2 & 375 & - & - & - & $\mathrm{F}$ & IIII & N1009 & E1012 & 4 & 98.9-98.8 & $40-50$ & - & Detritus & Debitage & \begin{tabular}{|l|} 
Proximal Flake \\
\end{tabular} & Lithic & 3 & 3.11 & & 11/30/2017 \\
\hline 690 & 3 & 375 & - & - & - & $\mathrm{F}$ & III & N1009 & E1012 & 4 & 98.9-98.8 & $40-50$ & - & Detritus & Debitage & Broken Flake & Lithic & 6 & 9.53 & & 11/30/2017 \\
\hline
\end{tabular}




\begin{tabular}{|c|c|c|c|c|c|c|c|c|c|c|c|c|c|c|c|c|c|c|c|c|c|}
\hline Lot No. & $\begin{array}{c}\text { Specimen } \\
\text { No. }\end{array}$ & FS No. & UI No. & FTR No. & $\begin{array}{c}\text { Sample } \\
\text { No. }\end{array}$ & $\begin{array}{c}\text { Excavation } \\
\text { Block }\end{array}$ & Stratum (AU) & Northing & Easting & Level & $\begin{array}{c}\text { Elevation } \\
(\mathrm{m})\end{array}$ & Depth (cmbd) & Point Prov. & Artifact Class & Artifact Type & \begin{tabular}{|c|} 
Artifact \\
Description
\end{tabular} & Material & $\begin{array}{c}\text { Number of } \\
\text { artifacts }\end{array}$ & Weight (g) & Comments & Date \\
\hline 690 & 4 & 375 & - & - & - & $\mathrm{F}$ & III & N1009 & E1012 & 4 & 98.9-98.8 & $40-50$ & - & Detritus & Debitage & Thermal Shatter & Lithic & 3 & 1.57 & & 11/30/2017 \\
\hline 691 & 1 & 377 & - & - & - & $\mathrm{F}$ & III & N1009 & E1012 & 5 & 98.8-98.7 & $50-60$ & - & Detritus & Debitage & Proximal Flake & Lithic & 1 & 0.91 & & 11/30/2017 \\
\hline 691 & 2 & 377 & - & - & - & $\mathrm{F}$ & III & N1009 & E1012 & 5 & 98.8-98.7 & $50-60$ & - & Detritus & Debitage & Broken Flake & Lithic & 4 & 13.16 & & 11/30/2017 \\
\hline 691 & 3 & 377 & - & - & - & $\mathrm{F}$ & III & N1009 & E1012 & 5 & $98.8-98.7$ & $50-60$ & - & Detritus & Debitage & Thermal Shatter & Lithic & 3 & 3.55 & & 11/30/2017 \\
\hline 692 & 1 & 380 & - & - & - & $\mathrm{F}$ & III & N1009 & E1012 & 6 & 98.7-98.6 & $60-70$ & - & Detritus & Debitage & Proximal Flake & Lithic & 2 & 48.61 & & 11/30/2017 \\
\hline 692 & 2 & 380 & - & - & - & $\mathrm{F}$ & III & N1009 & E1012 & 6 & $98.7-98.6$ & $60-70$ & - & Detritus & Debitage & Broken Flake & Lithic & 3 & 0.63 & & 11/30/2017 \\
\hline 692 & 3 & 380 & - & - & - & $\mathrm{F}$ & III & N1009 & E1012 & 6 & $98.7-98.6$ & $60-70$ & - & Detritus & Debitage & Thermal Shatter & Lithic & 5 & 28.86 & & 11/30/2017 \\
\hline 693 & 1 & 393 & - & - & - & $\mathrm{F}$ & IV & N1009 & E1012 & 7 & $98.6-98.5$ & $70-80$ & - & Detritus & Debitage & Proximal Flake & Lithic & 2 & 2.83 & & 12/1/2017 \\
\hline 693 & 2 & 393 & - & - & - & $\mathrm{F}$ & IV & N1009 & E1012 & 7 & $98.6-98.5$ & $70-80$ & - & Detritus & Debitage & Broken Flake & Lithic & 1 & 2.84 & & 12/11/2017 \\
\hline 693 & 3 & 393 & - & - & - & $\mathrm{F}$ & IV & N1009 & E1012 & 7 & $98.6-98.5$ & $\begin{array}{ll}70-80 \\
\end{array}$ & - & Detritus & Debitage & \begin{tabular}{|l|} 
Flaking Shatter \\
\end{tabular} & Lithic & 1 & 5.45 & & 12/1/2017 \\
\hline 693 & 4 & 393 & - & - & - & $\mathrm{F}$ & IV & N1009 & E1012 & 7 & $98.6-98.5$ & $70-80$ & - & Detritus & Debitage & Thermal Shatter & Lithic & 7 & 5.18 & & 12/1/2017 \\
\hline 694 & 1 & 398 & - & - & - & $\mathrm{F}$ & IV & N1009 & E1012 & 8 & $98.5-98.4$ & $\begin{array}{lll}80-90 & \\
\end{array}$ & - & Detritus & Debitage & Complete Flake & Lithic & 1 & 0.84 & & $12 / 1 / 2017$ \\
\hline 694 & 2 & 398 & - & - & - & $\mathrm{F}$ & IV & N1009 & E1012 & 8 & $98.5-98.4$ & $\begin{array}{lll}80-90 & \\
\end{array}$ & - & Detritus & Debitage & \begin{tabular}{|l|} 
Proximal Flake \\
\end{tabular} & Lithic & 1 & 14.17 & & 12/1/2017 \\
\hline 694 & 3 & 398 & - & - & - & $\mathrm{F}$ & IV & N1009 & E1012 & 8 & $98.5-98.4$ & $80-90$ & - & Detritus & Debitage & Thermal Shatter & Lithic & 3 & 5.96 & & 12/1/2017 \\
\hline 694 & 4 & 398 & - & - & - & $\mathrm{F}$ & IV & N1009 & E1012 & 8 & $98.5-98.4$ & $80-90$ & - & Informal Tool & Modified Flake & Edge-modified & Fine Grain Chert & 1 & 18.24 & & 12/1/2017 \\
\hline 695 & 1 & 403 & - & - & - & $\mathrm{F}$ & v & N1009 & E1012 & 9 & 98.4-98.3 & $90-100$ & - & Detritus & Debitage & Complete Flake & Lithic & 1 & 1.3 & & 12/1/2017 \\
\hline 695 & 2 & 403 & - & - & - & $\mathrm{F}$ & v & N1009 & E1012 & 9 & 98.4-98.3 & 90-100 & - & Detritus & Debitage & Broken Flake & Lithic & 1 & 0.8 & & 12/1/2017 \\
\hline 696 & 1 & 548 & - & - & - & $\mathrm{F}$ & vi & N1009 & E1012 & 11 & 98.2-98.1 & $110-120$ & - & Detritus & Debitage & Complete Flake & Lithic & 2 & 10.3 & & 12/15/2017 \\
\hline 696 & 2 & 548 & - & - & - & $\mathrm{F}$ & vi & N1009 & E1012 & 11 & 98.2-98.1 & $110-120$ & - & Detritus & Debitage & Broken Flake & Lithic & 1 & 1.01 & & 12/15/2017 \\
\hline 696 & 3 & 548 & - & - & - & $\mathrm{F}$ & vI & N1009 & E1012 & 11 & 98.2-98.1 & $110-120$ & - & Detritus & Debitage & Thermal Shatter & Lithic & 3 & 5.21 & & 12/15/2017 \\
\hline 696 & 4 & 548 & - & - & - & $\mathrm{F}$ & vi & N1009 & E1012 & 11 & $98.2-98.1$ & $110-120$ & - & Faunal Remains & Shell & Mussel Shell & Shell & 1 & 3.35 & & 12/15/2017 \\
\hline 697 & 1 & 551 & 28 & - & - & $\mathrm{F}$ & 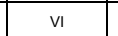 & N1009 & E1012 & 11 & 98.19 & 111 & \begin{tabular}{|l|l|} 
1009.16 \\
E1012.67
\end{tabular} & \begin{tabular}{|l|l|} 
Faunal Remains \\
\end{tabular} & Bone & Odocoileus sp. & Bone & 1 & 17.57 & "portion sent for radiocarbon dating & $12 / 15 / 2017$ \\
\hline 698 & 1 & 562 & - & - & - & $\mathrm{F}$ & vi & N1009 & E1012 & 12 & $98.1-98.0$ & $120-130$ & - & Detritus & Debitage & Complete Flake & Lithic & 1 & 0.94 & & 12/15/2017 \\
\hline 698 & 2 & 562 & - & - & - & $\mathrm{F}$ & vi & N1009 & E1012 & 12 & $98.1-98.0$ & $120-130$ & - & Detritus & Debitage & Broken Flake & Lithic & 1 & 0.66 & & 12/15/2017 \\
\hline 698 & 3 & 562 & - & - & - & $\mathrm{F}$ & vi & N1009 & E1012 & 12 & $98.1-98.0$ & $120-130$ & - & Detritus & Debitage & Thermal Shatter & Lithic & 4 & 5.85 & & 12/15/2017 \\
\hline $\begin{array}{c}\text { not for } \\
\text { curation }\end{array}$ & $\begin{array}{c}\text { not for } \\
\text { curation }\end{array}$ & 680 & - & FTR2 & MS-01 & E & & N1003-1004 & E1018-1019 & $5-8$ & $98.77-98.5$ & $53-80$ & 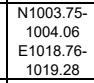 & $\begin{array}{l}\text { Geoarch } \\
\text { Sample }\end{array}$ & Matrix & $\begin{array}{l}\text { Magnetic } \\
\text { Susceptibility }\end{array}$ & & & & & 12/12/2017 \\
\hline $\begin{array}{l}\text { not for } \\
\text { curation }\end{array}$ & $\begin{array}{c}\text { not for } \\
\text { curation }\end{array}$ & 680 & - & FTR2 & MS-02 & $\mathrm{E}$ & & N1003-1004 & E1018-1019 & $5-8$ & $98.77-98.5$ & 53-80 & \begin{tabular}{|c|} 
N1003.75- \\
10004.06 \\
E1018.7- \\
$1019.28-$
\end{tabular} & $\begin{array}{l}\text { Geoarch } \\
\text { Sample }\end{array}$ & Matrix & $\begin{array}{l}\text { Magnetic } \\
\text { Susceptibility }\end{array}$ & & & & & 12/12/2017 \\
\hline $\begin{array}{c}\text { not for } \\
\text { curation }\end{array}$ & $\begin{array}{l}\text { not for } \\
\text { curation }\end{array}$ & 680 & - & FTR2 & MS-03 & E & & N1003-1004 & E1018-1019 & $5-8$ & $98.77-98.5$ & 53-80 & 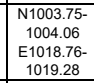 & $\begin{array}{l}\text { Geoarch } \\
\text { Sample }\end{array}$ & Matrix & $\begin{array}{l}\text { Magnetic } \\
\text { Susceptibility }\end{array}$ & & & & & 12/12/2017 \\
\hline $\begin{array}{l}\text { not for } \\
\text { curation }\end{array}$ & $\begin{array}{l}\text { not for } \\
\text { curation }\end{array}$ & 680 & - & FTR2 & MS-04 & E & & N1003-1004 & E1018-1019 & $5-8$ & 98.77-98.5 & $53-80$ & \begin{tabular}{|c|} 
N1003.75- \\
10004.06 \\
E1018.7- \\
$1019.28-$ \\
\end{tabular} & $\begin{array}{l}\text { Geoarch } \\
\text { Sample }\end{array}$ & Matrix & $\begin{array}{l}\text { Magnetic } \\
\text { Susceptibility }\end{array}$ & & & & & $12 / 12 / 2017$ \\
\hline $\begin{array}{l}\text { not for } \\
\text { curation }\end{array}$ & $\begin{array}{l}\text { not for } \\
\text { curation }\end{array}$ & 680 & - & FTR2 & MS- 05 & E & & N1003-1004 & E1018-1019 & $5-8$ & $98.77-98.5$ & 53-80 & \begin{tabular}{|c|}
$1003.75-$ \\
1000.406 \\
E1018.7.6- \\
1019.28 \\
\end{tabular} & $\begin{array}{l}\text { Geoarch } \\
\text { Sample }\end{array}$ & Matrix & $\begin{array}{l}\text { Magnetic } \\
\text { Susceptibility }\end{array}$ & & & & & 12/12/2017 \\
\hline $\begin{array}{l}\text { not for } \\
\text { curation }\end{array}$ & $\begin{array}{l}\text { not for } \\
\text { curation }\end{array}$ & 680 & - & FTR2 & MS-06 & E & & N1003-1004 & E1018-1019 & $5-8$ & $98.77-98.5$ & 53-80 & \begin{tabular}{|c|}
$1003.75-$ \\
$1004.06-$ \\
E11068.7.- \\
1019.28 \\
\end{tabular} & $\begin{array}{l}\text { Geoarch } \\
\text { Sample }\end{array}$ & Matrix & $\begin{array}{l}\text { Magnetic } \\
\text { Susceptibility }\end{array}$ & & & & & 12/12/2017 \\
\hline $\begin{array}{l}\text { not for } \\
\text { curation }\end{array}$ & $\begin{array}{l}\text { not for } \\
\text { curation }\end{array}$ & 680 & - & FTR2 & MS- 07 & E & & N1003-1004 & E1018-1019 & $5-8$ & $98.77-98.5$ & 53-80 & \begin{tabular}{|c|} 
N1003.75- \\
1000.406 \\
E1018.7.6- \\
1019.28
\end{tabular} & $\begin{array}{l}\text { Geoarch } \\
\text { Sample }\end{array}$ & Matrix & $\begin{array}{c}\text { Magnetic } \\
\text { Susceptibility }\end{array}$ & & & & & 12/12/2017 \\
\hline $\begin{array}{c}\text { not for } \\
\text { curation }\end{array}$ & $\begin{array}{l}\text { not for } \\
\text { curation }\end{array}$ & 680 & - & FTR2 & MS- 08 & E & & N1003-1004 & E1018-1019 & $5-8$ & 98.77-98.5 & $53-80$ & \begin{tabular}{|c|}
$10030.7-75-$ \\
1004.06 \\
E11018.76- \\
1019.28 \\
\end{tabular} & $\begin{array}{l}\text { Geoarch } \\
\text { Sample }\end{array}$ & Matrix & $\begin{array}{l}\text { Magnetic } \\
\text { Susceptibility }\end{array}$ & & & & & 12/12/2017 \\
\hline $\begin{array}{l}\text { not for } \\
\text { curation }\end{array}$ & $\begin{array}{l}\text { not for } \\
\text { curation }\end{array}$ & 680 & - & FTR2 & MS-09 & E & & N1003-1004 & E1018-1019 & $5-8$ & $98.77-98.5$ & 53-80 & \begin{tabular}{|c|} 
N1003.75- \\
1000.406 \\
E1018.7. \\
$1019.28-$
\end{tabular} & $\begin{array}{l}\text { Geoarch } \\
\text { Sample }\end{array}$ & Matrix & $\begin{array}{l}\text { Magnetic } \\
\text { Susceptibility }\end{array}$ & & & & & 12/12/2017 \\
\hline
\end{tabular}




\begin{tabular}{|c|c|c|c|c|c|c|c|c|c|c|c|c|c|c|c|c|c|c|c|c|c|}
\hline Lot No. & \begin{tabular}{|c|} 
Specimen \\
No.
\end{tabular} & FS No. & UI No. & FTR No. & $\begin{array}{c}\text { Sample } \\
\text { No. }\end{array}$ & $\begin{array}{c}\text { Excavation } \\
\text { Block }\end{array}$ & Stratum (AU) & Northing & Easting & Level & $\begin{array}{c}\text { Elevation } \\
(\mathrm{m})\end{array}$ & Depth (cmbd) & Point Prov. & Artifact Class & Artifact Type & $\begin{array}{c}\text { Artifact } \\
\text { Description }\end{array}$ & Material & $\begin{array}{c}\begin{array}{c}\text { Number of } \\
\text { artifacts }\end{array} \\
\end{array}$ & Weight (g) & Comments & Date \\
\hline $\begin{array}{l}\text { not for } \\
\text { curation }\end{array}$ & $\begin{array}{l}\text { not for } \\
\text { curation }\end{array}$ & 680 & - & FTR2 & MS-10 & E & & N1003-1004 & E1018-1019 & $5-8$ & \begin{tabular}{|l|}
$98.77-98.5$ \\
\end{tabular} & $53-80$ & $\begin{array}{c}\text { N10003.75- } \\
1004.06 \\
\text { E1018.7.76- } \\
1019.28 \\
\end{array}$ & $\begin{array}{l}\text { Geoarch } \\
\text { Sample }\end{array}$ & Matrix & $\begin{array}{l}\text { Magnetic } \\
\text { Susceptibility }\end{array}$ & & & & & 12/12/2017 \\
\hline $\begin{array}{l}\text { not for } \\
\text { curation }\end{array}$ & $\begin{array}{l}\text { not for } \\
\text { curation }\end{array}$ & 680 & - & FTR2 & MS-11 & E & & N1003-1004 & E1018-1019 & $5-8$ & $98.77-98.5$ & 53-80 & \begin{tabular}{|c|}
$11003.75-5$ \\
1004.06 \\
E1018.8.6- \\
1019.28 \\
\end{tabular} & $\begin{array}{l}\text { Geoarch } \\
\text { Sample }\end{array}$ & Matrix & $\begin{array}{l}\text { Magnetic } \\
\text { Susceptibility }\end{array}$ & & & & & 12/12/2017 \\
\hline $\begin{array}{l}\text { not for } \\
\text { curation }\end{array}$ & $\begin{array}{c}\text { not for } \\
\text { curation }\end{array}$ & 680 & - & FTR2 & MS-12 & E & & N1003-1004 & E1018-1019 & $5-8$ & 98.77-98.5 & $53-80$ & \begin{tabular}{|c|} 
N1003.75- \\
1004.06 \\
E1018.76- \\
1019.28 \\
\end{tabular} & $\begin{array}{l}\text { Geoarch } \\
\text { Sample }\end{array}$ & Matrix & $\begin{array}{l}\text { Magnetic } \\
\text { Susceptibility }\end{array}$ & & & & & 12/12/2017 \\
\hline $\begin{array}{l}\begin{array}{c}\text { not for } \\
\text { curation }\end{array} \\
\end{array}$ & $\begin{array}{l}\begin{array}{c}\text { not for } \\
\text { curation }\end{array} \\
\text { cols }\end{array}$ & 681 & - & - & MS-01 & D & $1 / 11$ & N1000 & E1010 & 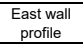 & 99.53 & 16 & - & $\begin{array}{l}\begin{array}{l}\text { Geoarch } \\
\text { Sample }\end{array} \\
\text { Sals }\end{array}$ & Soil & $\begin{array}{l}\text { Magnetic } \\
\text { Susceptibility } \\
\end{array}$ & & & & & 12/18/2017 \\
\hline $\begin{array}{l}\text { not of or } \\
\text { curation }\end{array}$ & $\begin{array}{l}\text { not for } \\
\text { curation }\end{array}$ & 681 & - & - & MS- 02 & D & 1/111 & N1000 & E1010 & 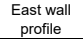 & 99.48 & 21 & - & $\begin{array}{l}\begin{array}{l}\text { Geoarch } \\
\text { Sample }\end{array} \\
\text { Sallo }\end{array}$ & Soil & $\begin{array}{l}\text { Magnetic } \\
\text { Susceptibility }\end{array}$ & & & & & 12/18/2017 \\
\hline \begin{tabular}{l|} 
not for \\
curation
\end{tabular} & $\begin{array}{l}\text { not for } \\
\text { curation }\end{array}$ & 681 & - & - & MS-03 & D & $1 / 11$ & N1000 & E1010 & $\begin{array}{c}\text { East wall } \\
\text { profile }\end{array}$ & 99.43 & 26 & - & $\begin{array}{l}\text { Geoarch } \\
\text { Sample }\end{array}$ & Soil & $\begin{array}{l}\text { Magnetic } \\
\text { Susceptibility }\end{array}$ & & & & & $\mid 12 / 18 / 2017$ \\
\hline $\begin{array}{l}\text { not for } \\
\text { curation }\end{array}$ & $\begin{array}{l}\text { not for } \\
\text { curation }\end{array}$ & 681 & - & - & MS- 04 & D & $1 / 11$ & N1000 & E1010 & $\begin{array}{c}\text { Eastwall } \\
\text { profile } \\
\text { pal }\end{array}$ & 99.38 & 31 & - & $\begin{array}{l}\text { Geourch } \\
\text { Sample }\end{array}$ & Soil & $\begin{array}{l}\text { Magnemici } \\
\text { Susceptibility }\end{array}$ & & & & & $\mid 12 / 18 / 2017$ \\
\hline \begin{tabular}{l|} 
not for \\
curation
\end{tabular} & $\begin{array}{l}\text { not for } \\
\text { curation }\end{array}$ & 681 & - & - & MS-05 & $\mathrm{D}$ & $1 / 11$ & N1000 & E1010 & $\begin{array}{c}\text { East wall } \\
\text { profile } \\
\text { pon }\end{array}$ & 99.33 & 36 & - & $\begin{array}{l}\text { Geoarch } \\
\text { Sample }\end{array}$ & Soil & $\begin{array}{l}\text { Magnetici } \\
\text { Susceptibility }\end{array}$ & & & & & $\mid$\begin{tabular}{|l|}
$\mid 12 / 18 / 2017$ \\
\end{tabular} \\
\hline $\begin{array}{l}\text { not for } \\
\text { curation } \\
\end{array}$ & $\begin{array}{l}\text { not for } \\
\text { curation }\end{array}$ & 681 & - & - & MS-06 & D & $1 / 11$ & N1000 & E1010 & $\begin{array}{c}\text { East wall } \\
\text { profile } \\
\text { res }\end{array}$ & 99.28 & 41 & - & $\begin{array}{l}\text { Geoarch } \\
\text { Sample }\end{array}$ & Soil & $\begin{array}{l}\text { Magneticic } \\
\text { Susceptibility }\end{array}$ & & & & & $\mid$\begin{tabular}{|l|}
$\mid 12 / 18 / 2017$ \\
\end{tabular} \\
\hline $\begin{array}{l}\begin{array}{l}\text { not for } \\
\text { curation }\end{array} \\
\end{array}$ & $\begin{array}{l}\text { not for } \\
\text { curation }\end{array}$ & 681 & - & - & MS-07 & D & 1/11 & N1000 & E1010 & 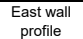 & 99.23 & 46 & - & $\begin{array}{l}\text { Geoarch } \\
\text { Sample }\end{array}$ & Soil & $\begin{array}{l}\text { Magneticic } \\
\text { Susceptibility }\end{array}$ & & & & & $12 / 18 / 2017$ \\
\hline $\begin{array}{l}\text { not for } \\
\text { curation }\end{array}$ & $\begin{array}{l}\text { not for } \\
\text { curation }\end{array}$ & 681 & - & - & MS-08 & D & 1/11 & N1000 & E1010 & $\begin{array}{c}\text { East wall } \\
\text { profile }\end{array}$ & 99.18 & 51 & - & $\begin{array}{l}\text { Geoarch } \\
\text { Sample }\end{array}$ & Soil & $\begin{array}{l}\text { Magnetic } \\
\text { Susceptiility }\end{array}$ & & & & & $\mid 12 / 18 / 2017$ \\
\hline $\begin{array}{c}\text { not tor } \\
\text { curation }\end{array}$ & $\begin{array}{l}\begin{array}{l}\text { not for } \\
\text { curation }\end{array} \\
\end{array}$ & 681 & - & - & MS-09 & $\mathrm{D}$ & 1/11 & N1000 & E1010 & $\begin{array}{c}\text { Eastwall } \\
\text { profile }\end{array}$ & 99.13 & 56 & - & $\begin{array}{l}\text { Gearach } \\
\text { Sample }\end{array}$ & Soil & $\begin{array}{l}\text { Magnetic } \\
\text { Susceptibility }\end{array}$ & & & & & 12/18/2017 \\
\hline $\begin{array}{l}\text { not for } \\
\text { curation }\end{array}$ & $\begin{array}{l}\text { not for } \\
\text { curation }\end{array}$ & 681 & - & - & MS-10 & $\mathrm{D}$ & 1/11 & N1000 & E1010 & $\begin{array}{c}\text { East wall } \\
\text { profile }\end{array}$ & 99.08 & 61 & - & $\begin{array}{l}\text { Geoarch } \\
\text { Sample }\end{array}$ & Soil & $\begin{array}{l}\text { Magnetic } \\
\text { Susceptibility }\end{array}$ & & & & & 12/18/2017 \\
\hline $\begin{array}{l}\text { not tor } \\
\text { curation }\end{array}$ & $\begin{array}{l}\begin{array}{l}\text { not for } \\
\text { curation }\end{array} \\
\end{array}$ & 681 & - & - & MS-11 & $\mathrm{D}$ & 1/11 & N1000 & E1010 & $\begin{array}{c}\text { East wall } \\
\text { profile }\end{array}$ & 99.03 & 66 & - & $\begin{array}{l}\text { Geamprch } \\
\text { Sample }\end{array}$ & Soil & $\begin{array}{l}\text { Mussepunemiti } \\
\text { Susceptibility }\end{array}$ & & & & & 12/18/2017 \\
\hline \begin{tabular}{l|} 
not for \\
curation
\end{tabular} & $\begin{array}{l}\begin{array}{l}\text { not for } \\
\text { curation }\end{array} \\
\end{array}$ & 681 & - & - & MS-12 & D & 1/11 & N1000 & E1010 & $\begin{array}{c}\text { East wall } \\
\text { profile }\end{array}$ & 98.98 & 71 & - & $\begin{array}{l}\text { Geoarch } \\
\text { Sample }\end{array}$ & Soil & $\begin{array}{l}\text { Magnemitic } \\
\text { Susceptibility }\end{array}$ & & & & & 12/18/2017 \\
\hline $\begin{array}{l}\text { not for } \\
\text { curation }\end{array}$ & $\begin{array}{l}\text { not for } \\
\text { curation }\end{array}$ & 681 & - & - & MS-13 & $\mathrm{D}$ & I/II & N1000 & E1010 & $\begin{array}{c}\text { East wall } \\
\text { profile }\end{array}$ & 98.93 & 76 & - & $\begin{array}{l}\text { Geaarch } \\
\text { Sample }\end{array}$ & Soil & $\begin{array}{l}\text { Magnetic } \\
\text { Susceptibility }\end{array}$ & & & & & 12/118/2017 \\
\hline $\begin{array}{l}\text { not for } \\
\text { curation }\end{array}$ & $\begin{array}{l}\text { not for } \\
\text { curation }\end{array}$ & 681 & - & - & MS-14 & D & $1 / 11$ & N1000 & E1010 & $\begin{array}{l}\text { East wall } \\
\text { porfie }\end{array}$ & 98.88 & 81 & - & $\begin{array}{l}\begin{array}{l}\text { Geoarch } \\
\text { Sample }\end{array} \\
\end{array}$ & Soil & $\begin{array}{l}\text { Magnetic } \\
\text { Susceptibility }\end{array}$ & & & & & 12/18/2017 \\
\hline \begin{tabular}{|l|} 
not for \\
curation \\
\end{tabular} & $\begin{array}{l}\begin{array}{l}\text { not for } \\
\text { curation }\end{array} \\
\text { cons }\end{array}$ & 681 & - & - & MS-15 & $\mathrm{D}$ & III & N1000 & E1010 & $\begin{array}{c}\text { East wall } \\
\text { profile } \\
\text { pon }\end{array}$ & 98.83 & 86 & - & $\begin{array}{l}\text { Geaarch } \\
\text { Sample }\end{array}$ & Soil & $\begin{array}{l}\text { Magnentic } \\
\text { Susceptibility }\end{array}$ & & & & & 12/18/2017 \\
\hline $\begin{array}{l}\begin{array}{l}\text { not of or } \\
\text { curation }\end{array} \\
\end{array}$ & $\begin{array}{l}\text { not for } \\
\text { curation }\end{array}$ & 681 & - & - & MS-16 & D & III & N1000 & E1010 & $\begin{array}{c}\begin{array}{c}\text { East wall } \\
\text { profile }\end{array} \\
\text { pats }\end{array}$ & 98.78 & 91 & - & $\begin{array}{l}\begin{array}{l}\text { Geoarch } \\
\text { Sample }\end{array} \\
\text { Sallo }\end{array}$ & Soil & $\begin{array}{l}\text { Magnetic } \\
\text { Susceptibility }\end{array}$ & & & & & 12/18/2017 \\
\hline \begin{tabular}{l|} 
not for \\
curation
\end{tabular} & $\begin{array}{l}\begin{array}{l}\text { not for } \\
\text { curation }\end{array} \\
\end{array}$ & 681 & - & - & MS-17 & $\mathrm{D}$ & III & N1000 & E1010 & $\begin{array}{c}\begin{array}{c}\text { East wall } \\
\text { profile }\end{array} \\
\text { pol }\end{array}$ & 98.73 & 96 & - & $\begin{array}{l}\text { Geoarch } \\
\text { Sample }\end{array}$ & Soil & $\begin{array}{l}\text { Magnetic } \\
\text { Susceptibility }\end{array}$ & & & & & 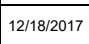 \\
\hline $\begin{array}{c}\text { notat of } \\
\text { curation }\end{array}$ & $\begin{array}{l}\text { nutrotion } \\
\text { curation } \\
\text { curation }\end{array}$ & 681 & - & - & MS-18 & D & III & N1000 & E1010 & 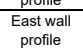 & 98.68 & 101 & - & $\begin{array}{l}\text { Geoarch } \\
\text { Sample }\end{array}$ & Soil & $\begin{array}{c}\text { Magnentic } \\
\text { Susceptibility }\end{array}$ & & & & & 12/18/2017 \\
\hline \begin{tabular}{c|} 
not for \\
curation
\end{tabular} & $\begin{array}{l}\text { not for } \\
\text { curation }\end{array}$ & 681 & - & - & MS-19 & D & III & N1000 & E1010 & $\begin{array}{c}\text { East wall } \\
\text { profile }\end{array}$ & \begin{tabular}{|l|l|}
98.63 \\
\end{tabular} & 106 & - & $\begin{array}{l}\text { Geoarch } \\
\text { Sample }\end{array}$ & Soil & $\begin{array}{l}\text { Magnetici } \\
\text { Susceptibility }\end{array}$ & & & & & 12/18/2017 \\
\hline $\begin{array}{c}\text { not for } \\
\text { curation }\end{array}$ & $\begin{array}{l}\begin{array}{l}\text { not for } \\
\text { curation }\end{array} \\
\text { cons }\end{array}$ & 681 & - & - & MS-20 & D & III & N1000 & E1010 & $\begin{array}{c}\text { East wall } \\
\text { profile }\end{array}$ & 98.56 & 113 & - & $\begin{array}{l}\text { Gearach } \\
\text { Sample }\end{array}$ & Soil & $\begin{array}{l}\text { Magnetic } \\
\text { Susceptibility }\end{array}$ & & & & & 12/18/2017 \\
\hline \begin{tabular}{|c|} 
not for \\
curation
\end{tabular} & $\begin{array}{l}\begin{array}{l}\text { not for } \\
\text { curation }\end{array} \\
\text { cons }\end{array}$ & 681 & - & - & MS-21 & D & IV & N1000 & E1010 & $\begin{array}{c}\text { East wall } \\
\text { profile }\end{array}$ & 98.48 & 121 & - & $\begin{array}{l}\text { Geoarch } \\
\text { Sample }\end{array}$ & Soil & $\begin{array}{l}\text { Magnetic } \\
\text { Susceptibility }\end{array}$ & & & & & 12/18/2017 \\
\hline \begin{tabular}{|c|} 
not for \\
curation
\end{tabular} & $\begin{array}{l}\text { not for } \\
\text { curation }\end{array}$ & 681 & - & - & MS-22 & D & IV & N1000 & E1010 & $\begin{array}{c}\text { East wall } \\
\text { profile }\end{array}$ & 98.43 & 126 & - & $\begin{array}{l}\text { Geoarch } \\
\text { Sample }\end{array}$ & Soil & $\begin{array}{l}\text { Magnetici } \\
\text { Susceptibility }\end{array}$ & & & & & $12 / 18 / 2017$ \\
\hline $\begin{array}{c}\text { not for } \\
\text { curation }\end{array}$ & $\begin{array}{c}\text { not for } \\
\text { curation }\end{array}$ & 681 & - & - & MS-23 & D & iv & N1000 & E1010 & $\begin{array}{c}\text { East wall } \\
\text { profile }\end{array}$ & 98.38 & 131 & - & $\begin{array}{l}\text { Geoarch } \\
\text { Sample }\end{array}$ & Soil & $\begin{array}{l}\text { Magneticic } \\
\text { Susceptibility }\end{array}$ & & & & & 12/18/2017 \\
\hline \begin{tabular}{|c|} 
not for \\
curation
\end{tabular} & $\begin{array}{l}\begin{array}{l}\text { not for } \\
\text { curation }\end{array} \\
\text { cons }\end{array}$ & 681 & - & - & MS-24 & D & IV & N1000 & E1010 & $\begin{array}{c}\text { East wall } \\
\text { profile }\end{array}$ & 98.33 & 136 & - & $\begin{array}{l}\text { Geararch } \\
\text { Sample }\end{array}$ & Soil & $\begin{array}{c}\text { Magnemici } \\
\text { Susceptibility }\end{array}$ & & & & & 12/118/2017 \\
\hline $\begin{array}{l}\begin{array}{l}\text { not of or } \\
\text { curation }\end{array} \\
\end{array}$ & $\begin{array}{l}\text { not for } \\
\text { curation }\end{array}$ & 681 & - & - & MS-25 & D & IV & N1000 & E1010 & $\begin{array}{c}\text { East wall } \\
\text { profile } \\
\end{array}$ & 98.28 & 141 & - & 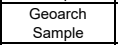 & Soil & $\begin{array}{l}\text { Magnetici } \\
\text { Susceptibility }\end{array}$ & & & & & \begin{tabular}{|l|l|l|l|} 
\\
\end{tabular} \\
\hline \begin{tabular}{|c|} 
not for \\
curation
\end{tabular} & $\begin{array}{l}\text { not for } \\
\text { curation }\end{array}$ & 681 & - & - & MS-26 & D & IV & N1000 & E1010 & $\begin{array}{c}\text { East wall } \\
\text { profile }\end{array}$ & 98.23 & 146 & - & $\begin{array}{l}\text { Geoarch } \\
\text { Sample }\end{array}$ & Soil & $\begin{array}{l}\text { Magnetic } \\
\text { Susceptibility }\end{array}$ & & & & & $12 / 18 / 2017$ \\
\hline \begin{tabular}{|c|} 
not for \\
curation
\end{tabular} & $\begin{array}{c}\text { not for } \\
\text { curation }\end{array}$ & 681 & - & - & MS- 27 & D & iv & N1000 & E1010 & $\begin{array}{c}\text { East wall } \\
\text { profile }\end{array}$ & 98.18 & 151 & - & $\begin{array}{l}\text { Geoarch } \\
\text { Sample }\end{array}$ & Soil & $\begin{array}{l}\text { Magnetici } \\
\text { Susceptibility }\end{array}$ & & & & & $12 / 18 / 2017$ \\
\hline \begin{tabular}{l|} 
not for \\
curation
\end{tabular} & $\begin{array}{l}\text { not for } \\
\text { curation }\end{array}$ & 681 & - & - & MS-28 & D & $V_{N 1}$ & N1000 & E1010 & $\begin{array}{c}\text { East wall } \\
\text { profile }\end{array}$ & 98.13 & 156 & - & $\begin{array}{l}\text { Geoarch } \\
\text { Sample }\end{array}$ & Soil & $\begin{array}{l}\text { Magnetic } \\
\text { Susceptibility }\end{array}$ & & & & & $\mid 12 / 18 / 2017$ \\
\hline \begin{tabular}{|c|} 
not for \\
curation
\end{tabular} & $\begin{array}{l}\text { not for } \\
\text { curation }\end{array}$ & 681 & - & - & MS-29 & $\mathrm{D}$ & $\mathrm{VN}$ & N1000 & E1010 & $\begin{array}{c}\text { East wall } \\
\text { profile }\end{array}$ & 98.08 & 161 & - & $\begin{array}{l}\text { Geoarch } \\
\text { Sample }\end{array}$ & Soil & $\begin{array}{l}\text { Magnetici } \\
\text { Susceptibility }\end{array}$ & & & & & 12/18/2017 \\
\hline \begin{tabular}{c|} 
not for \\
curation
\end{tabular} & $\begin{array}{l}\text { not for } \\
\text { curation }\end{array}$ & 681 & - & - & MS- 30 & D & $\mathrm{VN}$ & N1000 & E1010 & $\begin{array}{c}\text { East wall } \\
\text { profile }\end{array}$ & 98.03 & 166 & - & $\begin{array}{l}\text { Geoarch } \\
\text { Sample }\end{array}$ & Soil & $\begin{array}{l}\text { Magnetici } \\
\text { Susceptibility }\end{array}$ & & & & & $\mid 12 / 18 / 2017$ \\
\hline \begin{tabular}{|c|} 
not for \\
curation
\end{tabular} & $\begin{array}{c}\text { not for } \\
\text { curation }\end{array}$ & 681 & - & - & MS-31 & $\mathrm{D}$ & $\mathrm{V} N 1$ & N1000 & E1010 & $\begin{array}{c}\text { East wall } \\
\text { profile }\end{array}$ & 97.98 & 171 & - & $\begin{array}{l}\text { Geoarch } \\
\text { Sample }\end{array}$ & Soil & $\begin{array}{l}\text { Magnentic } \\
\text { Susceptibility }\end{array}$ & & & & & 12/18/2017 \\
\hline $\begin{array}{c}\text { not tor } \\
\text { curation }\end{array}$ & $\begin{array}{l}\text { not for } \\
\text { curation }\end{array}$ & 681 & - & - & MS-32 & D & VNI & N1000 & E1010 & $\begin{array}{c}\begin{array}{c}\text { East wall } \\
\text { profile }\end{array} \\
\text { pate }\end{array}$ & 97.93 & 176 & - & $\begin{array}{l}\text { Geoarch } \\
\text { Sample }\end{array}$ & Soil & $\begin{array}{l}\text { Magnentic } \\
\text { Suscoptibity }\end{array}$ & & & & & 12/18/2017 \\
\hline $\begin{array}{l}\text { not for } \\
\text { curation }\end{array}$ & $\begin{array}{l}\text { not for } \\
\text { curation }\end{array}$ & 681 & - & - & MS-33 & D & $V_{N 1}$ & N1000 & E1010 & $\begin{array}{c}\text { East wall } \\
\text { profile }\end{array}$ & 97.83 & 186 & - & $\begin{array}{l}\text { Geoarch } \\
\text { Sample }\end{array}$ & Soil & $\begin{array}{l}\text { Magneticic } \\
\text { Susceptibility }\end{array}$ & & & & & 12/18/2017 \\
\hline \begin{tabular}{c|} 
not for \\
curation
\end{tabular} & $\begin{array}{l}\text { not for } \\
\text { curation }\end{array}$ & 681 & - & - & MS- 34 & $\mathrm{D}$ & $\mathrm{VNI}$ & N1000 & E1010 & $\begin{array}{c}\text { East wall } \\
\text { profile }\end{array}$ & 97.79 & 190 & - & $\begin{array}{l}\text { Geoarch } \\
\text { Sample }\end{array}$ & Soil & $\begin{array}{l}\text { Magnetici } \\
\text { Susceptibility }\end{array}$ & & & & & 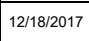 \\
\hline \begin{tabular}{c|} 
not for \\
curation
\end{tabular} & $\begin{array}{l}\text { not for } \\
\text { curation }\end{array}$ & 681 & - & - & BM-01 & $\mathrm{D}$ & $\mathrm{V} N \mathrm{~N}$ & N1000 & E1010 & $\begin{array}{c}\text { East wall } \\
\text { profile }\end{array}$ & \begin{tabular}{|l|}
$97.89-97.79$ \\
\end{tabular} & 180-190 & - & $\begin{array}{l}\text { Geoarch } \\
\text { Sample }\end{array}$ & Soil & Bulk Matrix & & & & & 12/18/2017 \\
\hline \begin{tabular}{|c|} 
not for \\
curation
\end{tabular} & $\begin{array}{l}\text { not for } \\
\text { curation }\end{array}$ & 681 & - & - & BM-02 & $\mathrm{D}$ & IV & N1000 & E1010 & $\begin{array}{c}\text { East wall } \\
\text { profile }\end{array}$ & 98.22-98.12 & 147-157 & - & $\begin{array}{l}\text { Geoarch } \\
\text { Sample }\end{array}$ & Soil & Bulk Matrix & & & & & 12/18/2017 \\
\hline
\end{tabular}




\begin{tabular}{|c|c|c|c|c|c|c|c|c|c|c|c|c|c|c|c|c|c|c|c|c|c|}
\hline Lot No. & \begin{tabular}{|c|}
$\begin{array}{c}\text { Specimen } \\
\text { No. }\end{array}$ \\
\end{tabular} & FS No. & UI No. & FTR No. & $\begin{array}{c}\text { Sample } \\
\text { No. }\end{array}$ & $\begin{array}{c}\text { Excavation } \\
\text { Block }\end{array}$ & Stratum (AU) & Northing & Easting & Level & $\begin{array}{c}\begin{array}{c}\text { Elevation } \\
(\mathrm{m})\end{array} \\
\end{array}$ & Depth (cmbd) & Point Prov. & Artifact Class & \begin{tabular}{|l|} 
Artifact Type \\
\end{tabular} & $\begin{array}{c}\text { Artifact } \\
\text { Description }\end{array}$ & Material & $\begin{array}{c}\text { Number of } \\
\text { artifacts }\end{array}$ & Weight (g) & Comments & Date \\
\hline $\begin{array}{l}\text { not for } \\
\text { curation }\end{array}$ & $\begin{array}{c}\begin{array}{c}\text { not for } \\
\text { curation }\end{array} \\
\end{array}$ & 681 & - & - & ВM-03 & $D$ & IV & N1000 & E1010 & $\begin{array}{c}\text { East wall } \\
\text { profile }\end{array}$ & \begin{tabular}{|l|}
$98.38-98.28$ \\
\end{tabular} & 131-141 & - & $\begin{array}{l}\text { Geoarch } \\
\text { Sample }\end{array}$ & Soil & Bulk Matrix & & & & & 12/18/2017 \\
\hline $\begin{array}{l}\text { not for } \\
\text { curation }\end{array}$ & $\begin{array}{c}\begin{array}{c}\text { not for } \\
\text { curation }\end{array} \\
\end{array}$ & 681 & - & - & BM-04 & D & IIIIV & N1000 & E1010 & $\begin{array}{l}\text { East wall } \\
\text { profie }\end{array}$ & \begin{tabular}{|l|}
$98.53-98.43$ \\
\end{tabular} & $116-126$ & - & $\begin{array}{l}\text { Geoarch } \\
\text { Sample }\end{array}$ & Soil & Bulk Matrix & & & & & \begin{tabular}{|l|}
$12 / 18 / 2017$ \\
\end{tabular} \\
\hline \begin{tabular}{|l|} 
not for \\
curation \\
\end{tabular} & $\begin{array}{l}\begin{array}{l}\text { not for } \\
\text { curation }\end{array} \\
\end{array}$ & 681 & - & - & BM-05 & $\mathrm{D}$ & III & N1000 & E1010 & $\begin{array}{c}\text { East wall } \\
\text { profile } \\
\text { pon }\end{array}$ & \begin{tabular}{|l|}
$98.73-98.63$ \\
\end{tabular} & $96-106$ & - & $\begin{array}{l}\text { Geararch } \\
\text { Sample }\end{array}$ & Soil & Bulk Matrix & & & & & $\mid 12 / 18 / 2017$ \\
\hline 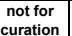 & $\begin{array}{l}\text { not for } \\
\text { curation }\end{array}$ & 681 & - & - & BM-06 & D & III & N1000 & E1010 & 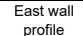 & \begin{tabular}{|l|}
$98.93-98.83$ \\
\end{tabular} & $76-86$ & - & $\begin{array}{l}\text { Geoarch } \\
\text { Sample }\end{array}$ & Soil & Bulk Matrix & & & & & 12/18/2017 \\
\hline $\begin{array}{l}\text { not for } \\
\text { curation }\end{array}$ & $\begin{array}{l}\text { not for } \\
\text { curation }\end{array}$ & 681 & - & - & BM-07 & D & $1 / 11$ & N1000 & E1010 & $\begin{array}{l}\text { East wall } \\
\text { profile } \\
\text { pon }\end{array}$ & \begin{tabular}{|l|}
$99.18-99.08$ \\
\end{tabular} & $51-61$ & - & $\begin{array}{l}\text { Geoarch } \\
\text { Sample }\end{array}$ & Soil & Bulk Matrix & & & & & $\mid$\begin{tabular}{|l|}
$\mid 12 / 18 / 2017$ \\
\end{tabular} \\
\hline $\begin{array}{l}\text { not for } \\
\text { curation }\end{array}$ & $\begin{array}{l}\text { not for } \\
\text { curation }\end{array}$ & 681 & - & - & BM-08 & D & 1/11 & N1000 & E1010 & $\begin{array}{c}\text { East wall } \\
\text { profile }\end{array}$ & \begin{tabular}{|l|l|}
$99.38-99.28$ \\
\end{tabular} & $31-41$ & - & $\begin{array}{l}\text { Geoarch } \\
\text { Sample }\end{array}$ & Soil & Bulk Matrix & & & & & 12/18/2017 \\
\hline \begin{tabular}{l|} 
not for \\
curation
\end{tabular} & $\begin{array}{c}\text { not for } \\
\text { curation }\end{array}$ & 681 & - & - & ВM-09 & $\mathrm{D}$ & 1111 & N1000 & E1010 & $\begin{array}{c}\text { East wall } \\
\text { profile }\end{array}$ & \begin{tabular}{|l|}
$99.58-99.48$ \\
\end{tabular} & $11-21$ & - & $\begin{array}{l}\begin{array}{l}\text { Geoarch } \\
\text { sample }\end{array} \\
\end{array}$ & Soil & Bulk Matrix & & & & & $\mid 12 / 18 / 2017$ \\
\hline $\begin{array}{c}\text { not tor } \\
\text { curation }\end{array}$ & $\begin{array}{l}\text { not for } \\
\text { curation }\end{array}$ & 681 & - & - & BM-10 & D & $\mathrm{V} N \mathrm{~N}_{1}$ & N1000 & E1010 & $\begin{array}{c}\text { East wall } \\
\text { profile }\end{array}$ & \begin{tabular}{|l|}
$98.08-97.98$ \\
\end{tabular} & 161-171 & - & $\begin{array}{l}\text { Geoarch } \\
\text { Sample }\end{array}$ & Soil & Bulk Matrix & & & & & $\mid$\begin{tabular}{|l|}
$\mid 12 / 18 / 2017$ \\
\end{tabular} \\
\hline $\begin{array}{l}\text { not tor } \\
\text { curation }\end{array}$ & $\begin{array}{l}\begin{array}{l}\text { not for } \\
\text { curation }\end{array} \\
\end{array}$ & 682 & - & - & MS-01 & $\mathrm{F}$ & 1 & N1009 & $E 1012$ & $\begin{array}{l}\text { Past wall } \\
\text { profile } \\
\text { pal }\end{array}$ & \begin{tabular}{|l|}
99.15 \\
\end{tabular} & 15 & - & $\begin{array}{l}\text { Geoarch } \\
\text { Sample }\end{array}$ & Soil & $\begin{array}{l}\text { Magnetic } \\
\text { Susceptibility }\end{array}$ & & & & & \begin{tabular}{|l|}
$12 / 21 / 2017$ \\
\end{tabular} \\
\hline $\begin{array}{l}\text { not for } \\
\text { curation }\end{array}$ & $\begin{array}{l}\begin{array}{l}\text { not for } \\
\text { curation }\end{array} \\
\end{array}$ & 682 & - & - & MS-02 & $\mathrm{F}$ & 1 & N1009 & E1012 & $\begin{array}{l}\text { Tast wall } \\
\text { profile }\end{array}$ & 99.12 & 18 & - & $\begin{array}{l}\text { Geoarch } \\
\text { Sample }\end{array}$ & Soil & $\begin{array}{l}\text { Magnetic } \\
\text { Susceptibility }\end{array}$ & & & & & \begin{tabular}{|l|}
$12 / 21 / 2017$ \\
\end{tabular} \\
\hline \begin{tabular}{l|} 
not for \\
curation
\end{tabular} & $\begin{array}{l}\text { not for } \\
\text { curation }\end{array}$ & 682 & - & - & MS-03 & $\mathrm{F}$ & $" 1$ & N1009 & E1012 & $\begin{array}{c}\text { East wall } \\
\text { profile }\end{array}$ & 99.09 & 21 & - & $\begin{array}{l}\text { Geoarch } \\
\text { Sample }\end{array}$ & Soil & $\begin{array}{l}\text { Magnetic } \\
\text { Susceptibility }\end{array}$ & & & & & $\mid$\begin{tabular}{|l|}
$\mid 12 / 21 / 2017$ \\
\end{tabular} \\
\hline \begin{tabular}{l|} 
not for \\
curation \\
\end{tabular} & $\begin{array}{l}\text { not for } \\
\text { curation }\end{array}$ & 682 & - & - & MS-04 & $\mathrm{F}$ & ॥ & N1009 & E1012 & $\begin{array}{l}\text { East wall } \\
\text { profile } \\
\text { pal }\end{array}$ & 99.06 & 24 & - & $\begin{array}{l}\text { Geoarch } \\
\text { Sample }\end{array}$ & Soil & $\begin{array}{l}\text { Magnetic } \\
\text { Susceptibility }\end{array}$ & & & & & $\mid 12 / 21 / 2017$ \\
\hline $\begin{array}{l}\text { not for } \\
\text { curation }\end{array}$ & $\begin{array}{l}\text { not for } \\
\text { curation }\end{array}$ & 682 & - & - & MS-05 & $\mathrm{F}$ & "I & N1009 & E1012 & $\begin{array}{c}\text { East wall } \\
\text { profile }\end{array}$ & 99.04 & 26 & - & $\begin{array}{l}\begin{array}{l}\text { Geoarch } \\
\text { Sample }\end{array} \\
\end{array}$ & Soil & $\begin{array}{l}\text { Magnetic } \\
\text { Susceptibility }\end{array}$ & & & & & \begin{tabular}{|l|}
$12 / 21 / 2017$ \\
\end{tabular} \\
\hline $\begin{array}{l}\text { not for } \\
\text { curation }\end{array}$ & $\begin{array}{l}\text { not for } \\
\text { curation }\end{array}$ & 682 & - & - & MS-06 & $\mathrm{F}$ & " & N1009 & E1012 & $\begin{array}{c}\text { East wall } \\
\text { profile } \\
\end{array}$ & 99.01 & 29 & - & $\begin{array}{l}\begin{array}{l}\text { Geoarch } \\
\text { Sample }\end{array} \\
\end{array}$ & Soil & $\begin{array}{l}\text { Magnetic } \\
\text { Susceptibility }\end{array}$ & & & & & 12/21/2017 \\
\hline $\begin{array}{l}\text { not for } \\
\text { curation }\end{array}$ & $\begin{array}{l}\text { not for } \\
\text { curation }\end{array}$ & 682 & - & - & MS-07 & $\mathrm{F}$ & "I & N1009 & E1012 & $\begin{array}{c}\text { East wall } \\
\text { profile } \\
\text {. }\end{array}$ & 98.97 & 33 & - & $\begin{array}{l}\text { Geoarch } \\
\text { Sample }\end{array}$ & Soil & $\begin{array}{l}\text { Magnetic } \\
\text { Susceptibility } \\
\end{array}$ & & & & & 12/21/2017 \\
\hline \begin{tabular}{c|} 
not for \\
curation
\end{tabular} & $\begin{array}{l}\text { not for } \\
\text { curation }\end{array}$ & 682 & - & - & MS-08 & $\mathrm{F}$ & "I & N1009 & E1012 & $\begin{array}{l}\text { East wall } \\
\text { profile }\end{array}$ & 98.93 & 37 & - & $\begin{array}{l}\text { Geoarch } \\
\text { Sample }\end{array}$ & Soil & $\begin{array}{l}\text { Magnemitic } \\
\text { Suscoptibility }\end{array}$ & & & & & 12/21/2017 \\
\hline $\begin{array}{l}\text { not for } \\
\text { curation }\end{array}$ & $\begin{array}{l}\begin{array}{l}\text { not for } \\
\text { curation }\end{array} \\
\end{array}$ & 682 & - & - & MS-09 & $\mathrm{F}$ & 11 & N1009 & E1012 & $\begin{array}{l}\text { Tastwall } \\
\text { profile }\end{array}$ & 98.9 & 40 & - & $\begin{array}{l}\text { Gearch } \\
\text { Sample }\end{array}$ & Soil & $\begin{array}{l}\text { Magnemitic } \\
\text { Susceptibility }\end{array}$ & & & & & 12/21/2017 \\
\hline \begin{tabular}{l|} 
not for \\
curation
\end{tabular} & $\begin{array}{l}\text { not for } \\
\text { curation }\end{array}$ & 682 & - & - & MS-10 & $\mathrm{F}$ & III & N1009 & E1012 & $\begin{array}{l}\text { East wall } \\
\text { profile }\end{array}$ & 98.87 & 43 & - & $\begin{array}{l}\text { Geoarch } \\
\text { Sample }\end{array}$ & Soil & $\begin{array}{l}\text { Magnetic } \\
\text { Susceptibility }\end{array}$ & & & & & 12/21/2017 \\
\hline \begin{tabular}{l|} 
not for \\
curation
\end{tabular} & \begin{tabular}{|c|}
$\begin{array}{c}\text { not for } \\
\text { curation }\end{array}$ \\
\end{tabular} & 682 & - & - & MS-11 & $\mathrm{F}$ & IIII & N1009 & E1012 & $\begin{array}{l}\text { East wall } \\
\text { profile }\end{array}$ & 98.84 & 46 & - & $\begin{array}{l}\text { Geoarch } \\
\text { Sample }\end{array}$ & Soil & $\begin{array}{l}\text { Magnetic } \\
\text { Susceptibility }\end{array}$ & & & & & 12/21/2017 \\
\hline \begin{tabular}{l|} 
not for \\
curation
\end{tabular} & \begin{tabular}{|c|} 
not for \\
curation
\end{tabular} & 682 & - & - & MS-12 & $\mathrm{F}$ & III & N1009 & E1012 & $\begin{array}{c}\text { East wall } \\
\text { profile }\end{array}$ & 98.81 & 49 & - & $\begin{array}{l}\text { Geoarch } \\
\text { Sample }\end{array}$ & Soil & $\begin{array}{l}\text { Magnetic } \\
\text { Susceptibility }\end{array}$ & & & & & 12/21/2017 \\
\hline $\begin{array}{l}\text { not for } \\
\text { curation }\end{array}$ & $\begin{array}{l}\text { not for } \\
\text { curation }\end{array}$ & 682 & - & - & MS-13 & $\mathrm{F}$ & IIII & N1009 & E1012 & $\begin{array}{l}\text { East wall } \\
\text { profile } \\
\text { pal }\end{array}$ & $\begin{array}{l}98.78 \\
\end{array}$ & 52 & - & $\begin{array}{l}\text { Geararch } \\
\text { Sample }\end{array}$ & Soil & $\begin{array}{l}\text { Magnetic } \\
\text { Susceptibility } \\
\end{array}$ & & & & & 12/21/2017 \\
\hline \begin{tabular}{c|} 
not for \\
curation
\end{tabular} & $\begin{array}{l}\text { not for } \\
\text { curation }\end{array}$ & 682 & - & - & MS-14 & $\mathrm{F}$ & III & N1009 & E1012 & $\begin{array}{c}\text { East wall } \\
\text { profile }\end{array}$ & 98.76 & 54 & - & $\begin{array}{l}\text { Geoarch } \\
\text { Sample }\end{array}$ & Soil & $\begin{array}{l}\text { Magnetici } \\
\text { Susceptibility }\end{array}$ & & & & & 12/21/2017 \\
\hline \begin{tabular}{l|} 
not for \\
curation
\end{tabular} & \begin{tabular}{|c|} 
not for \\
curation
\end{tabular} & 682 & - & - & MS-15 & $\mathrm{F}$ & III & N1009 & E1012 & $\begin{array}{c}\text { Rast wall } \\
\text { profile }\end{array}$ & 98.73 & 57 & - & $\begin{array}{l}\text { Geaarch } \\
\text { Sample }\end{array}$ & Soil & $\begin{array}{l}\text { Magnentic } \\
\text { Susceptibility }\end{array}$ & & & & & 12/21/2017 \\
\hline \begin{tabular}{l|} 
not for \\
curation
\end{tabular} & $\begin{array}{c}\text { not for } \\
\text { curation }\end{array}$ & 682 & - & - & MS-16 & $\mathrm{F}$ & III & N1009 & E1012 & $\begin{array}{c}\text { East wall } \\
\text { profile }\end{array}$ & 98.69 & 61 & - & $\begin{array}{l}\text { Geoarch } \\
\text { Sample }\end{array}$ & Soil & $\begin{array}{l}\text { Magnetic } \\
\text { Susceptibility }\end{array}$ & & & & & 12/21/2017 \\
\hline \begin{tabular}{c|} 
not for \\
curation
\end{tabular} & $\begin{array}{l}\text { not for } \\
\text { curation }\end{array}$ & 682 & - & - & MS-17 & $\mathrm{F}$ & IIII & N1009 & E1012 & $\begin{array}{l}\text { East wall } \\
\text { profile }\end{array}$ & 98.67 & 63 & - & $\begin{array}{l}\text { Geoarch } \\
\text { Sample }\end{array}$ & Soil & $\begin{array}{l}\text { Magnemici } \\
\text { Suscoptibitity }\end{array}$ & & & & & 12/21/2017 \\
\hline $\begin{array}{c}\text { not for } \\
\text { curation }\end{array}$ & 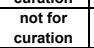 & 682 & - & - & MS-18 & $\mathrm{F}$ & III & N1009 & E1012 & $\begin{array}{c}\text { Past wall } \\
\text { profile }\end{array}$ & 98.64 & 66 & - & $\begin{array}{l}\text { Geoarch } \\
\text { Sample }\end{array}$ & Soil & $\begin{array}{l}\text { Magnetic } \\
\text { Susceptibility }\end{array}$ & & & & & 12/21/2017 \\
\hline \begin{tabular}{|c|} 
not for \\
curation
\end{tabular} & $\begin{array}{l}\begin{array}{l}\text { not for } \\
\text { curation }\end{array} \\
\text { cons }\end{array}$ & 682 & - & - & MS-19 & $\mathrm{F}$ & IIII & N1009 & E1012 & $\begin{array}{c}\text { East wall } \\
\text { profile }\end{array}$ & 98.61 & 69 & - & $\begin{array}{l}\text { Geoarch } \\
\text { Sample }\end{array}$ & Soil & $\begin{array}{l}\text { Magnetic } \\
\text { Susceptibility }\end{array}$ & & & & & 12/21/2017 \\
\hline \begin{tabular}{|c|} 
not for \\
curation
\end{tabular} & $\begin{array}{l}\begin{array}{l}\text { not for } \\
\text { curation }\end{array} \\
\text { cons }\end{array}$ & 682 & - & - & MS-20 & $\mathrm{F}$ & IV & N1009 & E1012 & $\begin{array}{l}\text { East wall } \\
\text { profile }\end{array}$ & 98.58 & 72 & - & $\begin{array}{l}\text { Geoarch } \\
\text { Sample }\end{array}$ & Soil & $\begin{array}{l}\text { Magnetici } \\
\text { Susceptibility }\end{array}$ & & & & & $12 / 21 / 2017$ \\
\hline $\begin{array}{c}\text { not for } \\
\text { curation }\end{array}$ & $\begin{array}{l}\text { not for } \\
\text { curation }\end{array}$ & 682 & - & - & MS-21 & $\mathrm{F}$ & IV & N1009 & E1012 & $\begin{array}{l}\text { East wall } \\
\text { profile }\end{array}$ & 98.55 & 75 & - & $\begin{array}{l}\text { Geoarch } \\
\text { Sample }\end{array}$ & Soil & $\begin{array}{l}\text { Magnentic } \\
\text { Suscoptitily }\end{array}$ & & & & & 12/21/2017 \\
\hline $\begin{array}{l}\text { not for } \\
\text { curation }\end{array}$ & $\begin{array}{c}\text { not for } \\
\text { curation }\end{array}$ & 682 & - & - & MS-22 & $\mathrm{F}$ & IV & N1009 & E1012 & $\begin{array}{c}\text { East wall } \\
\text { profile } \\
\end{array}$ & 98.51 & 79 & - & $\begin{array}{l}\text { Geoarch } \\
\text { Sample }\end{array}$ & Soil & $\begin{array}{l}\text { Magnetici } \\
\text { Susceptibility }\end{array}$ & & & & & 12/21/2017 \\
\hline $\begin{array}{c}\text { not for } \\
\text { curation }\end{array}$ & $\begin{array}{l}\text { not for } \\
\text { curation }\end{array}$ & 682 & - & - & MS-23 & $\mathrm{F}$ & iv & N1009 & E1012 & 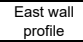 & 98.48 & 82 & - & 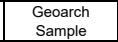 & Soil & $\begin{array}{l}\text { Magnetici } \\
\text { Susceptibility } \\
\text { Sust }\end{array}$ & & & & & $12 / 21 / 2017$ \\
\hline \begin{tabular}{|c|} 
not for \\
curation
\end{tabular} & $\begin{array}{l}\begin{array}{l}\text { not for } \\
\text { curation }\end{array} \\
\text { cons }\end{array}$ & 682 & - & - & MS-24 & $\mathrm{F}$ & IV & N1009 & E1012 & $\begin{array}{l}\text { East wall } \\
\text { profile } \\
\text { pate }\end{array}$ & 98.44 & 86 & - & $\begin{array}{l}\text { Geaarch } \\
\text { Sample }\end{array}$ & Soil & $\begin{array}{l}\text { Magnetic } \\
\text { Susceptibility }\end{array}$ & & & & & 12/21/2017 \\
\hline $\begin{array}{c}\text { not tor } \\
\text { curation }\end{array}$ & $\begin{array}{l}\begin{array}{l}\text { not for } \\
\text { curation }\end{array} \\
\text { cons }\end{array}$ & 682 & - & - & MS- 25 & $\mathrm{~F}$ & IV & N1009 & E1012 & $\begin{array}{l}\text { Past wall } \\
\text { profile }\end{array}$ & 98.41 & 89 & - & $\begin{array}{l}\text { Geoarch } \\
\text { Sample }\end{array}$ & Soil & $\begin{array}{l}\text { Magnetic } \\
\text { Susceptibility }\end{array}$ & & & & & 12/21/2017 \\
\hline $\begin{array}{c}\text { not for } \\
\text { curation }\end{array}$ & 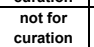 & 682 & - & - & MS-26 & $\mathrm{F}$ & v & N1009 & E1012 & $\begin{array}{l}\text { Tast wall } \\
\text { profile }\end{array}$ & 98.38 & 92 & - & $\begin{array}{l}\text { Geoarch } \\
\text { Sample }\end{array}$ & Soil & $\begin{array}{l}\text { Magnetic } \\
\text { Susceptibility }\end{array}$ & & & & & 12/21/2017 \\
\hline $\begin{array}{l}\text { not for } \\
\text { curation }\end{array}$ & $\begin{array}{l}\begin{array}{l}\text { not for } \\
\text { curation }\end{array} \\
\text { cons }\end{array}$ & 682 & - & - & MS- 27 & $\mathrm{~F}$ & v & N1009 & E1012 & $\begin{array}{c}\text { Rast wall } \\
\text { profile }\end{array}$ & 98.35 & 95 & - & $\begin{array}{l}\text { Geoarch } \\
\text { Sample }\end{array}$ & Soil & $\begin{array}{l}\text { Magnentic } \\
\text { Susceptibility }\end{array}$ & & & & & 12/21/2017 \\
\hline \begin{tabular}{l|} 
not for \\
curation
\end{tabular} & $\begin{array}{l}\begin{array}{l}\text { not for } \\
\text { curation }\end{array} \\
\text { con }\end{array}$ & 682 & - & - & MS-28 & $\mathrm{F}$ & v & N1009 & E1012 & $\begin{array}{c}\text { East wall } \\
\text { profile }\end{array}$ & 98.32 & 98 & - & $\begin{array}{l}\text { Geoarch } \\
\text { Sample }\end{array}$ & Soil & $\begin{array}{l}\text { Magnetici } \\
\text { Susceptibility }\end{array}$ & & & & & $\mid 12 / 21 / 2017$ \\
\hline \begin{tabular}{|c|} 
not for \\
curation
\end{tabular} & $\begin{array}{l}\text { not for } \\
\text { curation }\end{array}$ & 682 & - & - & MS-29 & $\mathrm{F}$ & $v$ & N1009 & E1012 & $\begin{array}{l}\text { East wall } \\
\text { profile } \\
\text { pat }\end{array}$ & 98.30 & 100 & - & $\begin{array}{l}\text { Geoarch } \\
\text { Sample }\end{array}$ & Soil & $\begin{array}{l}\text { Magnetici } \\
\text { Susceptibility }\end{array}$ & & & & & 12/21/2017 \\
\hline \begin{tabular}{|c|} 
not for \\
curation
\end{tabular} & $\begin{array}{l}\text { not for } \\
\text { curation }\end{array}$ & 682 & - & - & MS- 30 & $\mathrm{~F}$ & $v$ & N1009 & E1012 & $\begin{array}{c}\text { East wall } \\
\text { profile }\end{array}$ & \begin{tabular}{|l|l|}
98.27 \\
\end{tabular} & 103 & - & $\begin{array}{l}\text { Geoarch } \\
\text { Sample }\end{array}$ & Soil & $\begin{array}{l}\text { Magnetici } \\
\text { Susceptibility }\end{array}$ & & & & & 12/21/2017 \\
\hline $\begin{array}{l}\text { not for } \\
\text { curation }\end{array}$ & $\begin{array}{l}\text { not for } \\
\text { curation }\end{array}$ & 682 & - & - & MS-31 & $\mathrm{F}$ & $v$ & N1009 & E1012 & $\begin{array}{c}\text { East wall } \\
\text { profile }\end{array}$ & 98.24 & 106 & - & $\begin{array}{l}\text { Geourch } \\
\text { Sample }\end{array}$ & Soil & $\begin{array}{l}\text { Magnetic } \\
\text { Susceptibility }\end{array}$ & & & & & $12 / 21 / 2017$ \\
\hline \begin{tabular}{|c|} 
not for \\
curation
\end{tabular} & $\begin{array}{l}\text { not for } \\
\text { curation }\end{array}$ & 682 & - & - & MS-32 & $\mathrm{F}$ & $\mathrm{v}$ & N1009 & E1012 & 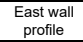 & 98.21 & 109 & - & 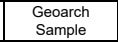 & Soil & $\begin{array}{l}\text { Magnetici } \\
\text { Susceptibility }\end{array}$ & & & & & 12/21/2017 \\
\hline $\begin{array}{c}\text { not tor } \\
\text { curation }\end{array}$ & $\begin{array}{l}\begin{array}{l}\text { not for } \\
\text { curation }\end{array} \\
\text { cons }\end{array}$ & 682 & - & - & MS-33 & $\mathrm{F}$ & vi & N1009 & E1012 & $\begin{array}{c}\text { Pastwo wall } \\
\text { profile }\end{array}$ & 98.18 & 112 & - & $\begin{array}{l}\text { Geoarch } \\
\text { Sample }\end{array}$ & Soil & $\begin{array}{l}\text { Mussagnetic } \\
\text { Susceptibility }\end{array}$ & & & & & 12/21/2017 \\
\hline $\begin{array}{l}\text { not tor } \\
\text { curation }\end{array}$ & $\begin{array}{c}\text { not for } \\
\text { curation }\end{array}$ & 682 & - & - & MS-34 & $\mathrm{F}$ & vi & N1009 & E1012 & $\begin{array}{l}\text { Eastwall } \\
\text { profile }\end{array}$ & 98.15 & 115 & - & $\begin{array}{c}\begin{array}{c}\text { Geoarch } \\
\text { Sample }\end{array} \\
\end{array}$ & Soil & $\begin{array}{l}\text { Magnetic } \\
\text { Susceptibility }\end{array}$ & & & & & 12/21/2017 \\
\hline
\end{tabular}




\begin{tabular}{|c|c|c|c|c|c|c|c|c|c|c|c|c|c|c|c|c|c|c|c|c|c|}
\hline Lot No. & \begin{tabular}{|c|}
$\begin{array}{c}\text { Specimen } \\
\text { No. }\end{array}$ \\
\end{tabular} & FS No. & UI No. & FTR No. & $\begin{array}{c}\text { Sample } \\
\text { No. }\end{array}$ & $\begin{array}{c}\text { Excavation } \\
\text { Block }\end{array}$ & Stratum (AU) & Northing & Easting & Level & $\begin{array}{c}\begin{array}{c}\text { Elevation } \\
(\mathrm{m})\end{array} \\
\end{array}$ & Depth (cmbd) & Point Prov. & Artifact Class & \begin{tabular}{|l|} 
Artifact Type \\
\end{tabular} & $\begin{array}{c}\text { Artifact } \\
\text { Description }\end{array}$ & Material & $\begin{array}{c}\text { Number of } \\
\text { artifacts }\end{array}$ & Weight (g) & Comments & Date \\
\hline $\begin{array}{l}\text { not for } \\
\text { curation }\end{array}$ & $\begin{array}{c}\begin{array}{c}\text { not for } \\
\text { curation }\end{array} \\
\end{array}$ & 682 & - & - & MS-35 & $\mathrm{F}$ & vi & N1009 & E1012 & $\begin{array}{c}\text { East wall } \\
\text { profile }\end{array}$ & 98.12 & 118 & - & $\begin{array}{l}\text { Geoarch } \\
\text { Sample }\end{array}$ & Soil & $\begin{array}{l}\text { Magnetic } \\
\text { Susceptibility }\end{array}$ & & & & & 12/21/2017 \\
\hline \begin{tabular}{c|} 
not for \\
curation
\end{tabular} & $\begin{array}{l}\begin{array}{l}\text { not for } \\
\text { curation }\end{array} \\
\end{array}$ & 682 & - & - & MS-36 & $\mathrm{F}$ & vi & N1009 & E1012 & $\begin{array}{l}\text { East wall } \\
\text { profile }\end{array}$ & 98.09 & 121 & - & $\begin{array}{l}\text { Geoarch } \\
\text { Sample }\end{array}$ & Soil & $\begin{array}{l}\text { Magnetic } \\
\text { Susceptibility }\end{array}$ & & & & & 12/21/2017 \\
\hline \begin{tabular}{|l|} 
not for \\
curation \\
\end{tabular} & $\begin{array}{l}\begin{array}{l}\text { not for } \\
\text { curation }\end{array} \\
\end{array}$ & 682 & - & - & MS-37 & $\mathrm{F}$ & vi & N1009 & E1012 & 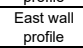 & 98.06 & 124 & - & $\begin{array}{l}\text { Geaarch } \\
\text { Sample }\end{array}$ & Soil & $\begin{array}{l}\text { Magnemici } \\
\text { Susceptibility }\end{array}$ & & & & & $\mid 12 / 21 / 2017$ \\
\hline $\begin{array}{l}\begin{array}{c}\text { not for } \\
\text { curation }\end{array} \\
\end{array}$ & $\begin{array}{l}\text { not for } \\
\text { curation }\end{array}$ & 682 & - & - & MS-38 & $\mathrm{F}$ & vi & N1009 & E1012 & 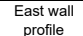 & 98.03 & 127 & - & $\begin{array}{l}\begin{array}{l}\text { Geoarch } \\
\text { Sample }\end{array} \\
\end{array}$ & Soil & $\begin{array}{l}\text { Magnetic } \\
\text { Susceptibility }\end{array}$ & & & & & 12/21/2017 \\
\hline $\begin{array}{l}\text { not for } \\
\text { curation }\end{array}$ & $\begin{array}{l}\text { not for } \\
\text { curation }\end{array}$ & 682 & - & - & MS-39 & $\mathrm{F}$ & vi & N1009 & E1012 & 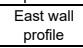 & 98.00 & 130 & - & $\begin{array}{l}\text { Geoarch } \\
\text { Sample }\end{array}$ & Soil & $\begin{array}{l}\text { Magnetic } \\
\text { Susceptibility }\end{array}$ & & & & & $\mid$\begin{tabular}{|l|}
$\mid 12 / 21 / 2017$ \\
\end{tabular} \\
\hline $\begin{array}{l}\text { not for } \\
\text { curation }\end{array}$ & $\begin{array}{l}\text { not for } \\
\text { curation }\end{array}$ & 682 & - & - & MS- 40 & $\mathrm{~F}$ & vi & N1009 & E1012 & $\begin{array}{c}\text { East wall } \\
\text { profile }\end{array}$ & 97.97 & 133 & - & $\begin{array}{l}\text { Geoarch } \\
\text { Sample }\end{array}$ & Soil & $\begin{array}{l}\text { Magnetici } \\
\text { Susceptibility }\end{array}$ & & & & & 12/21/2017 \\
\hline \begin{tabular}{l|} 
not for \\
curation
\end{tabular} & $\begin{array}{l}\text { not for } \\
\text { curation }\end{array}$ & 682 & - & - & MS-41 & $\mathrm{F}$ & vi & N1009 & E1012 & $\begin{array}{l}\text { Eastwall } \\
\text { profile }\end{array}$ & 97.94 & 136 & - & $\begin{array}{l}\text { Geoarch } \\
\text { Sample }\end{array}$ & Soil & $\begin{array}{l}\text { Maganetic } \\
\text { Suscoptibility }\end{array}$ & & & & & $\mid 12 / 21 / 2017$ \\
\hline $\begin{array}{l}\text { not for } \\
\text { curation }\end{array}$ & $\begin{array}{l}\text { not for } \\
\text { curtition }\end{array}$ & 682 & - & - & MS-42 & $\mathrm{F}$ & vi & N1009 & E1012 & $\begin{array}{l}\text { Pastwe wall } \\
\text { profile }\end{array}$ & 97.91 & 139 & - & $\begin{array}{l}\text { Sampie } \\
\text { Geaarch } \\
\text { Sample }\end{array}$ & Soil & $\begin{array}{c}\text { Susceprominy } \\
\text { Muscentic } \\
\text { Suscopibility }\end{array}$ & & & & & $\mid 12 / 21 / 2017$ \\
\hline $\begin{array}{l}\text { not tor } \\
\text { curation }\end{array}$ & $\begin{array}{l}\begin{array}{l}\text { not for } \\
\text { curation }\end{array} \\
\end{array}$ & 682 & - & - & MS-43 & $\mathrm{F}$ & vi & N1009 & $E 1012$ & $\begin{array}{l}\text { Past wall } \\
\text { profile } \\
\text { pal }\end{array}$ & 97.88 & 142 & - & $\begin{array}{l}\text { Geoarch } \\
\text { Sample }\end{array}$ & Soil & $\begin{array}{l}\text { Magnetic } \\
\text { Susceptibility }\end{array}$ & & & & & \begin{tabular}{|l|}
$12 / 21 / 2017$ \\
\end{tabular} \\
\hline $\begin{array}{l}\text { not for } \\
\text { curation }\end{array}$ & $\begin{array}{l}\begin{array}{l}\text { not for } \\
\text { curation }\end{array} \\
\end{array}$ & 682 & - & - & MS-44 & $\mathrm{F}$ & vi & N1009 & E1012 & $\begin{array}{l}\text { Tast wall } \\
\text { profile }\end{array}$ & 97.85 & 145 & - & $\begin{array}{l}\text { Geoarch } \\
\text { Sample }\end{array}$ & Soil & $\begin{array}{l}\text { Magnetic } \\
\text { Susceptibility }\end{array}$ & & & & & $\mid 12 / 21 / 2017$ \\
\hline \begin{tabular}{l|} 
not for \\
curation
\end{tabular} & $\begin{array}{l}\text { not for } \\
\text { curation }\end{array}$ & 682 & - & - & MS-45 & $\mathrm{F}$ & vi & N1009 & E1012 & $\begin{array}{c}\text { East wall } \\
\text { profile }\end{array}$ & 97.82 & 148 & - & $\begin{array}{l}\text { Geoarch } \\
\text { Sample }\end{array}$ & Soil & $\begin{array}{l}\text { Magnetic } \\
\text { Susceptibility }\end{array}$ & & & & & $\mid$\begin{tabular}{|l|}
$\mid 12 / 21 / 2017$ \\
\end{tabular} \\
\hline $\begin{array}{l}\text { not for } \\
\text { curation }\end{array}$ & $\begin{array}{l}\begin{array}{l}\text { not for } \\
\text { curation }\end{array} \\
\end{array}$ & 682 & - & - & MS-46 & $\mathrm{F}$ & vi & N1009 & E1012 & $\begin{array}{l}\text { East wall } \\
\text { profile } \\
\text { pal }\end{array}$ & 97.79 & 151 & - & $\begin{array}{l}\text { Geoarch } \\
\text { Sample }\end{array}$ & Soil & $\begin{array}{l}\text { Magnemic } \\
\text { Susceptibility } \\
\end{array}$ & & & & & $\mid 12 / 21 / 2017$ \\
\hline $\begin{array}{l}\text { not for } \\
\text { curation }\end{array}$ & $\begin{array}{l}\text { not for } \\
\text { curation }\end{array}$ & 682 & - & - & MS-47 & $\mathrm{F}$ & vi & N1009 & E1012 & $\begin{array}{c}\text { East wall } \\
\text { profile }\end{array}$ & 97.75 & 155 & - & $\begin{array}{l}\begin{array}{l}\text { Geoarch } \\
\text { Sample }\end{array} \\
\end{array}$ & Soil & $\begin{array}{l}\text { Magnetic } \\
\text { Susceptibility }\end{array}$ & & & & & 12/21/2017 \\
\hline $\begin{array}{l}\text { not for } \\
\text { curation }\end{array}$ & $\begin{array}{l}\text { not for } \\
\text { curation }\end{array}$ & 682 & - & - & MS- 48 & $\mathrm{~F}$ & vi & N1009 & E1012 & $\begin{array}{c}\text { East wall } \\
\text { profile } \\
\end{array}$ & 97.71 & 159 & - & $\begin{array}{l}\begin{array}{l}\text { Geoarch } \\
\text { Sample }\end{array} \\
\end{array}$ & Soil & $\begin{array}{l}\text { Magnetic } \\
\text { Susceptibility }\end{array}$ & & & & & \begin{tabular}{|l|l|}
$12 / 21 / 2017$ \\
\end{tabular} \\
\hline $\begin{array}{l}\text { not for } \\
\text { curation }\end{array}$ & $\begin{array}{l}\text { not for } \\
\text { curation }\end{array}$ & 682 & - & - & BM-01 & $\mathrm{F}$ & vi & N1009 & E1012 & $\begin{array}{c}\text { East wall } \\
\text { profile } \\
\text {. }\end{array}$ & \begin{tabular}{|l|}
$97.80-97.70$ \\
\end{tabular} & $150-160$ & - & $\begin{array}{l}\text { Geoarch } \\
\text { Sample }\end{array}$ & Soil & Bulk Matrix & & & & & 12/21/2017 \\
\hline \begin{tabular}{c|} 
not for \\
curation
\end{tabular} & $\begin{array}{l}\text { not for } \\
\text { curation }\end{array}$ & 682 & - & - & BM-02 & $\mathrm{F}$ & vi & N1009 & E1012 & $\begin{array}{l}\text { East wall } \\
\text { profile }\end{array}$ & \begin{tabular}{|l|l|}
$98.05-97.95$ \\
\end{tabular} & 125-135 & - & $\begin{array}{l}\text { Geoarch } \\
\text { Sample }\end{array}$ & Soil & Bulk Matrix & & & & & 12/21/2017 \\
\hline $\begin{array}{l}\text { not for } \\
\text { curation }\end{array}$ & $\begin{array}{l}\begin{array}{l}\text { not for } \\
\text { curation }\end{array} \\
\end{array}$ & 682 & - & - & BM-03 & $\mathrm{F}$ & $V_{N 1}$ & N1009 & E1012 & $\begin{array}{l}\text { East wall } \\
\text { profile } \\
\text { pal }\end{array}$ & \begin{tabular}{|l|}
$98.22-98.12$ \\
\end{tabular} & 108-118 & - & $\begin{array}{l}\text { Gemperch } \\
\text { Geample } \\
\text { Sampen }\end{array}$ & Soil & Bulk Matrix & & & & & 12/21/2017 \\
\hline \begin{tabular}{l|} 
not for \\
curation
\end{tabular} & $\begin{array}{l}\text { not for } \\
\text { curation }\end{array}$ & 682 & - & - & BM-04 & $\mathrm{F}$ & $\mathrm{v}$ & N1009 & E1012 & $\begin{array}{l}\text { East wall } \\
\text { profile }\end{array}$ & \begin{tabular}{|l|}
$98.38-98.28$ \\
\end{tabular} & 92-102 & - & $\begin{array}{l}\text { Geoarch } \\
\text { Sample }\end{array}$ & Soil & Bulk Matrix & & & & & 12/21/2017 \\
\hline \begin{tabular}{l|} 
not for \\
curation
\end{tabular} & $\begin{array}{l}\begin{array}{l}\text { not for } \\
\text { curation }\end{array} \\
\end{array}$ & 682 & - & - & BM-05 & $\mathrm{F}$ & IV & N1009 & E1012 & $\begin{array}{l}\text { East wall } \\
\text { profile }\end{array}$ & \begin{tabular}{|l|}
$98.52-98.62$ \\
\end{tabular} & $78-88$ & - & $\begin{array}{l}\text { Geoarch } \\
\text { Sample }\end{array}$ & Soil & Buk Matrix & & & & & 12/21/2017 \\
\hline \begin{tabular}{l|} 
not for \\
curation
\end{tabular} & $\begin{array}{l}\text { not for } \\
\text { curation }\end{array}$ & 682 & - & - & BM-06 & $\mathrm{F}$ & IIIIV & N1009 & E1012 & $\begin{array}{c}\text { East wall } \\
\text { profile }\end{array}$ & \begin{tabular}{|l|}
$98.64-98.54$ \\
\end{tabular} & $66-76$ & - & $\begin{array}{l}\text { Geoarch } \\
\text { Sample }\end{array}$ & Soil & Bulk Matrix & & & & & 12/21/2017 \\
\hline \begin{tabular}{|c|} 
not for \\
curation
\end{tabular} & $\begin{array}{l}\text { not for } \\
\text { curation }\end{array}$ & 682 & - & - & BM-07 & $\mathrm{F}$ & IIII & N1009 & E1012 & $\begin{array}{l}\text { East wall } \\
\text { profile } \\
\text { pate }\end{array}$ & \begin{tabular}{|l|}
$98.80-98.70$ \\
\end{tabular} & 50-60 & - & $\begin{array}{l}\text { Geoarch } \\
\text { Sample }\end{array}$ & Soil & Bulk Matrix & & & & & 12/21/2017 \\
\hline \begin{tabular}{c|} 
not for \\
curation
\end{tabular} & $\begin{array}{l}\begin{array}{l}\text { not for } \\
\text { curation }\end{array} \\
\text { con }\end{array}$ & 682 & - & - & BM-08 & $\mathrm{F}$ & II/III & N1009 & E1012 & $\begin{array}{c}\text { East wall } \\
\text { profile }\end{array}$ & \begin{tabular}{|l|}
$8.92-98.82$ \\
\end{tabular} & $38-48$ & - & $\begin{array}{l}\text { Geoarch } \\
\text { Sample }\end{array}$ & Soil & Bulk Matrix & & & & & 12/21/2017 \\
\hline \begin{tabular}{|c|} 
not for \\
curation
\end{tabular} & $\begin{array}{c}\text { not for } \\
\text { curation }\end{array}$ & 682 & - & - & BM-09 & $\mathrm{F}$ & " & N1009 & E1012 & $\begin{array}{l}\text { East wall } \\
\text { profile } \\
\end{array}$ & \begin{tabular}{|l|}
$99.06-98.96$ \\
\end{tabular} & $24-34$ & - & $\begin{array}{l}\text { Geoarch } \\
\text { Sample }\end{array}$ & Soil & Bulk Matrix & & & & & 12/21/2017 \\
\hline \begin{tabular}{|c|} 
not for \\
curation
\end{tabular} & $\begin{array}{c}\text { not for } \\
\text { curation }\end{array}$ & 682 & - & - & BM-10 & $\mathrm{F}$ & 1 & N1009 & E1012 & $\begin{array}{c}\text { East wall } \\
\text { profile } \\
\text { pat }\end{array}$ & 99.15-99.12 & $15-18$ & - & $\begin{array}{l}\text { Geoarch } \\
\text { Sample }\end{array}$ & Soil & $\begin{array}{l}\text { Magnetici } \\
\text { Susceptibility }\end{array}$ & & & & & 12/21/2017 \\
\hline \begin{tabular}{c|} 
not for \\
curation
\end{tabular} & $\begin{array}{l}\text { not for } \\
\text { curation }\end{array}$ & 683 & - & - & MS-01 & $\mathrm{E}$ & 1 & N1004 & E1017 & $\begin{array}{l}\text { West wall } \\
\text { profile }\end{array}$ & 99.22 & 8 & - & $\begin{array}{l}\text { Geoarch } \\
\text { Sample }\end{array}$ & Soil & $\begin{array}{l}\text { Magnetic } \\
\text { Susceptibitity }\end{array}$ & & & & & 12/26/2017 \\
\hline $\begin{array}{c}\text { not for } \\
\text { curation }\end{array}$ & 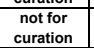 & 683 & - & - & MS-02 & E & " & N1004 & E1017 & $\begin{array}{c}\text { West wall } \\
\text { profile }\end{array}$ & 99.15 & 15 & - & $\begin{array}{l}\text { Geoarch } \\
\text { Sample }\end{array}$ & Soil & $\begin{array}{l}\text { Magnetic } \\
\text { Susceptibility }\end{array}$ & & & & & 12/26/2017 \\
\hline \begin{tabular}{|c|} 
not for \\
curation
\end{tabular} & $\begin{array}{l}\begin{array}{l}\text { not for } \\
\text { curation }\end{array} \\
\text { cons }\end{array}$ & 683 & - & - & MS-03 & $\mathrm{E}$ & "I & N1004 & E1017 & $\begin{array}{c}\text { West wall } \\
\text { profile }\end{array}$ & 99.12 & 18 & - & $\begin{array}{l}\text { Geoarch } \\
\text { Sample }\end{array}$ & Soil & $\begin{array}{l}\text { Magnetic } \\
\text { Susceptibility }\end{array}$ & & & & & 12/26/2017 \\
\hline \begin{tabular}{|c|} 
not for \\
curation
\end{tabular} & $\begin{array}{l}\begin{array}{l}\text { not for } \\
\text { curation }\end{array} \\
\text { cons }\end{array}$ & 683 & - & - & MS-04 & E & "I & N1004 & E1017 & $\begin{array}{c}\text { West wall } \\
\text { profile }\end{array}$ & 99.09 & 21 & - & $\begin{array}{l}\text { Geoarch } \\
\text { Sample }\end{array}$ & Soil & $\begin{array}{l}\text { Magnetici } \\
\text { Susceptibility }\end{array}$ & & & & & $12 / 26 / 2017$ \\
\hline $\begin{array}{c}\text { not for } \\
\text { curation }\end{array}$ & $\begin{array}{l}\text { not for } \\
\text { curation }\end{array}$ & 683 & - & - & MS-05 & E & "I & N1004 & E1017 & $\begin{array}{l}\text { West wall } \\
\text { profile }\end{array}$ & 99.05 & 25 & - & $\begin{array}{l}\text { Geoarch } \\
\text { Sample }\end{array}$ & Soil & $\begin{array}{l}\text { Magnentic } \\
\text { Suscoptitily }\end{array}$ & & & & & 12/26/2017 \\
\hline $\begin{array}{l}\text { not for } \\
\text { curation }\end{array}$ & $\begin{array}{c}\text { not for } \\
\text { curation }\end{array}$ & 683 & - & - & MS-06 & E & III & N1004 & E1017 & $\begin{array}{c}\text { West wall } \\
\text { profile } \\
\end{array}$ & 99.01 & 29 & - & $\begin{array}{l}\text { Geoarch } \\
\text { Sample }\end{array}$ & Soil & $\begin{array}{l}\text { Magnetici } \\
\text { Susceptibility }\end{array}$ & & & & & 121/26/2017 \\
\hline $\begin{array}{c}\text { not for } \\
\text { curation }\end{array}$ & $\begin{array}{l}\text { not for } \\
\text { curation }\end{array}$ & 683 & - & - & MS- 07 & $E$ & III & N1004 & E1017 & $\begin{array}{c}\text { West wall } \\
\text { profile } \\
\end{array}$ & 98.97 & 33 & - & 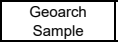 & Soil & $\begin{array}{l}\text { Magnetici } \\
\text { Susceptibility } \\
\text { Sust }\end{array}$ & & & & & $12 / 26 / 2017$ \\
\hline \begin{tabular}{|c|} 
not for \\
curation
\end{tabular} & $\begin{array}{l}\begin{array}{l}\text { not for } \\
\text { curation }\end{array} \\
\text { count }\end{array}$ & 683 & - & - & MS- 08 & $E$ & III & N1004 & E1017 & $\begin{array}{c}\text { West wall } \\
\text { profile } \\
\text { pent }\end{array}$ & 98.94 & 36 & - & $\begin{array}{l}\text { Geaarch } \\
\text { Sample }\end{array}$ & Soil & $\begin{array}{l}\text { Magnetic } \\
\text { Susceptibility }\end{array}$ & & & & & $12 / 26 / 2017$ \\
\hline $\begin{array}{c}\text { not tor } \\
\text { curation }\end{array}$ & $\begin{array}{l}\begin{array}{l}\text { not for } \\
\text { curation }\end{array} \\
\text { cons }\end{array}$ & 683 & - & - & MS-09 & $\mathrm{E}$ & IIII & N1004 & E1017 & $\begin{array}{c}\text { West wall } \\
\text { profile }\end{array}$ & 98.89 & 41 & - & $\begin{array}{l}\text { Geoarch } \\
\text { Sample }\end{array}$ & Soil & $\begin{array}{l}\text { Magnetic } \\
\text { Susceptibility }\end{array}$ & & & & & 12/26/2017 \\
\hline $\begin{array}{c}\text { not for } \\
\text { curation }\end{array}$ & 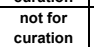 & 683 & - & - & MS-10 & $\mathrm{E}$ & III & N1004 & E1017 & $\begin{array}{c}\text { West wall } \\
\text { profile }\end{array}$ & 98.88 & 42 & - & $\begin{array}{l}\text { Geoarch } \\
\text { Sample }\end{array}$ & Soil & $\begin{array}{l}\text { Magnetic } \\
\text { Susceptibility }\end{array}$ & & & & & $\mid 12 / 26 / 2017$ \\
\hline $\begin{array}{l}\text { not for } \\
\text { curation }\end{array}$ & $\begin{array}{l}\text { not for } \\
\text { curation }\end{array}$ & 683 & - & - & MS-11 & $E$ & III' & N1004 & E1017 & $\begin{array}{c}\text { West wall } \\
\text { profile }\end{array}$ & 98.82 & 48 & - & $\begin{array}{l}\text { Geoarch } \\
\text { Sample }\end{array}$ & Soil & $\begin{array}{l}\text { Magnentic } \\
\text { Susceptibility }\end{array}$ & & & & & 12/26/2017 \\
\hline \begin{tabular}{l|} 
not for \\
curation
\end{tabular} & $\begin{array}{l}\begin{array}{l}\text { not for } \\
\text { curation }\end{array} \\
\text { cons }\end{array}$ & 683 & - & - & MS-12 & E & III & N1004 & E1017 & $\begin{array}{c}\text { West wall } \\
\text { profile }\end{array}$ & 98.77 & 53 & - & $\begin{array}{l}\text { Geoarch } \\
\text { Sample }\end{array}$ & Soil & $\begin{array}{l}\text { Magnetic } \\
\text { Susceptibility }\end{array}$ & & & & & $\mid 12 / 26 / 2017$ \\
\hline \begin{tabular}{|c|} 
not for \\
curation
\end{tabular} & $\begin{array}{l}\text { not for } \\
\text { curation }\end{array}$ & 683 & - & - & MS-13 & $\mathrm{E}$ & III & N1004 & E1017 & $\begin{array}{c}\text { West wall } \\
\text { profile } \\
\text { pent }\end{array}$ & 98.75 & 55 & - & $\begin{array}{l}\text { Geoarch } \\
\text { Sample }\end{array}$ & Soil & $\begin{array}{l}\text { Magnetici } \\
\text { Susceptibility }\end{array}$ & & & & & 12/26/2017 \\
\hline \begin{tabular}{|c|} 
not for \\
curation
\end{tabular} & $\begin{array}{l}\text { not for } \\
\text { curation }\end{array}$ & 683 & - & - & MS-14 & $\mathrm{E}$ & III & N1004 & E1017 & $\begin{array}{c}\text { West wall } \\
\text { profile }\end{array}$ & 98.73 & 57 & - & $\begin{array}{l}\text { Geoarch } \\
\text { Sample }\end{array}$ & Soil & $\begin{array}{l}\text { Magnetici } \\
\text { Susceptibility }\end{array}$ & & & & & $12 / 26 / 2017$ \\
\hline $\begin{array}{l}\text { not for } \\
\text { curation }\end{array}$ & $\begin{array}{c}\text { not for } \\
\text { curation }\end{array}$ & 683 & - & - & MS-15 & E & IV & N1004 & E1017 & $\begin{array}{c}\text { West wall } \\
\text { profile } \\
\text { pats }\end{array}$ & 98.69 & 61 & - & $\begin{array}{l}\begin{array}{l}\text { Geoarch } \\
\text { Sample }\end{array} \\
\text { Sal }\end{array}$ & Soil & $\begin{array}{l}\text { Magnetic } \\
\text { Susceptibility }\end{array}$ & & & & & $12 / 26 / 2017$ \\
\hline \begin{tabular}{|c|} 
not for \\
curation
\end{tabular} & $\begin{array}{l}\text { not for } \\
\text { curation }\end{array}$ & 683 & - & - & MS-16 & $\mathrm{E}$ & iv & N1004 & E1017 & $\begin{array}{c}\text { West wall } \\
\text { profile } \\
\end{array}$ & 98.63 & 67 & - & 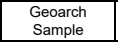 & Soil & $\begin{array}{l}\text { Magnetici } \\
\text { Susceptibility }\end{array}$ & & & & & 12/26/2017 \\
\hline $\begin{array}{c}\text { not tor } \\
\text { curation }\end{array}$ & $\begin{array}{l}\begin{array}{l}\text { not for } \\
\text { curation }\end{array} \\
\text { s. }\end{array}$ & 683 & - & - & MS-17 & $\mathrm{E}$ & IV & N1004 & E1017 & $\begin{array}{c}\text { Wost wall } \\
\text { profile }\end{array}$ & 98.6 & 70 & - & $\begin{array}{l}\text { Geoarch } \\
\text { Sample }\end{array}$ & Soil & $\begin{array}{l}\text { Masseneitic } \\
\text { Susceptibility }\end{array}$ & & & & & 12/26/2017 \\
\hline $\begin{array}{c}\text { not tor } \\
\text { curation }\end{array}$ & $\begin{array}{c}\text { not for } \\
\text { curation }\end{array}$ & 683 & - & - & MS-18 & E & IV & N1004 & E1017 & $\begin{array}{c}\text { West wall } \\
\text { profile }\end{array}$ & 98.57 & 73 & - & $\begin{array}{l}\text { Geoarch } \\
\text { Sample }\end{array}$ & Soil & $\begin{array}{l}\text { Magnetic } \\
\text { Susceptibility }\end{array}$ & & & & & 12/26/2017 \\
\hline
\end{tabular}




\begin{tabular}{|c|c|c|c|c|c|c|c|c|c|c|c|c|c|c|c|c|c|c|c|c|c|}
\hline Lot No. & \begin{tabular}{|c|}
$\begin{array}{c}\text { Specimen } \\
\text { No. }\end{array}$ \\
\end{tabular} & FS No. & UI No. & FTR No. & $\begin{array}{c}\text { Sample } \\
\text { No. }\end{array}$ & $\begin{array}{c}\text { Excavation } \\
\text { Block }\end{array}$ & Stratum (AU) & Northing & Easting & Level & $\begin{array}{c}\begin{array}{c}\text { Elevation } \\
(\mathrm{m})\end{array} \\
\end{array}$ & Depth (cmbd) & Point Prov. & Artifact Class & Artifact Type & $\begin{array}{c}\text { Artifact } \\
\text { Description }\end{array}$ & Material & $\begin{array}{c}\text { Number of } \\
\text { artifacts }\end{array}$ & Weight (g) & Comments & Date \\
\hline $\begin{array}{l}\text { not for } \\
\text { curation }\end{array}$ & $\begin{array}{c}\text { not for } \\
\text { curation }\end{array}$ & 683 & - & - & MS-19 & $\mathrm{E}$ & IV & N1004 & E1017 & $\begin{array}{l}\text { West wall } \\
\text { profile }\end{array}$ & 98.54 & 76 & - & $\begin{array}{l}\text { Geoarch } \\
\text { Sample }\end{array}$ & Soil & $\begin{array}{l}\text { Magnetic } \\
\text { Susceptibility }\end{array}$ & & & & & 12/26/2017 \\
\hline \begin{tabular}{c|} 
not for \\
curation
\end{tabular} & $\begin{array}{l}\begin{array}{l}\text { not for } \\
\text { curation }\end{array} \\
\end{array}$ & 683 & - & - & MS-20 & E & v & N1004 & E1017 & $\begin{array}{c}\text { West wall } \\
\text { profile }\end{array}$ & 98.49 & 81 & - & $\begin{array}{l}\text { Geoarch } \\
\text { Sample }\end{array}$ & Soil & $\begin{array}{l}\text { Magnetic } \\
\text { Susceptibility }\end{array}$ & & & & & 12/26/2017 \\
\hline \begin{tabular}{|l|} 
not for \\
curation \\
\end{tabular} & $\begin{array}{l}\begin{array}{l}\text { not for } \\
\text { curation }\end{array} \\
\end{array}$ & 683 & - & - & MS-21 & $E$ & $\mathrm{v}$ & N1004 & E1017 & $\begin{array}{c}\text { West wall } \\
\text { profile }\end{array}$ & 98.48 & 82 & - & $\begin{array}{l}\text { Geaarch } \\
\text { Sample }\end{array}$ & Soil & $\begin{array}{l}\text { Magnemici } \\
\text { Susceptibility }\end{array}$ & & & & & $\mid 12 / 26 / 2017$ \\
\hline \begin{tabular}{l|} 
not for \\
curation
\end{tabular} & $\begin{array}{l}\begin{array}{l}\text { not for } \\
\text { curation }\end{array} \\
\end{array}$ & 683 & - & - & MS-22 & E & v & N1004 & E1017 & $\begin{array}{c}\text { West wall } \\
\text { profile }\end{array}$ & 98.43 & 87 & - & $\begin{array}{l}\text { Geoarch } \\
\text { Sample }\end{array}$ & Soil & $\begin{array}{l}\text { Magneticic } \\
\text { Susceptibility }\end{array}$ & & & & & 12/26/2017 \\
\hline $\begin{array}{l}\text { not for } \\
\text { curation }\end{array}$ & $\begin{array}{l}\text { not for } \\
\text { curation }\end{array}$ & 683 & - & - & MS-23 & E & v & N1004 & E1017 & $\begin{array}{c}\text { West wall } \\
\text { profile }\end{array}$ & 98.38 & 92 & - & $\begin{array}{l}\text { Geoarch } \\
\text { Sample }\end{array}$ & Soil & $\begin{array}{l}\text { Magnetic } \\
\text { Susceptibility }\end{array}$ & & & & & $\mid$\begin{tabular}{|l|}
$\mid 12 / 26 / 2017$ \\
\end{tabular} \\
\hline $\begin{array}{l}\text { not for } \\
\text { curation }\end{array}$ & $\begin{array}{l}\text { not for } \\
\text { curation }\end{array}$ & 683 & - & - & MS-24 & E & vi & N1004 & E1017 & $\begin{array}{c}\text { West wall } \\
\text { profile }\end{array}$ & 98.33 & 97 & - & $\begin{array}{l}\text { Geoarch } \\
\text { Sample }\end{array}$ & Soil & $\begin{array}{l}\text { Magnetic } \\
\text { Susceptibility }\end{array}$ & & & & & 12/26/2017 \\
\hline \begin{tabular}{l|} 
not for \\
curation
\end{tabular} & $\begin{array}{l}\text { not for } \\
\text { curation }\end{array}$ & 683 & - & - & MS-25 & $\mathrm{E}$ & vi & N1004 & E1017 & $\begin{array}{l}\begin{array}{l}\text { West wall } \\
\text { profile }\end{array} \\
\end{array}$ & 98.31 & 99 & - & $\begin{array}{l}\text { Geoarch } \\
\text { Sample }\end{array}$ & Soil & $\begin{array}{l}\text { Magnentic } \\
\text { Suscoptibility }\end{array}$ & & & & & $\mid$\begin{tabular}{|l|}
$\mid 12 / 26 / 2017$ \\
\end{tabular} \\
\hline $\begin{array}{l}\text { not for } \\
\text { curation }\end{array}$ & $\begin{array}{l}\text { not for } \\
\text { curtition }\end{array}$ & 683 & - & - & MS-26 & $\mathrm{E}$ & vi & N1004 & E1017 & $\begin{array}{c}\text { West wall } \\
\text { profile }\end{array}$ & 98.29 & 101 & - & $\begin{array}{l}\text { Sampie } \\
\text { Geaarch } \\
\text { Sample }\end{array}$ & Soil & $\begin{array}{c}\text { Susceprominy } \\
\text { Muscentic } \\
\text { Suscopibility }\end{array}$ & & & & & $\mid$\begin{tabular}{|l|}
$\mid 12 / 26 / 2017$ \\
\end{tabular} \\
\hline $\begin{array}{l}\text { not tor } \\
\text { curation }\end{array}$ & $\begin{array}{l}\begin{array}{l}\text { not for } \\
\text { curation }\end{array} \\
\end{array}$ & 683 & - & - & MS-27 & $\mathrm{E}$ & vi & N1004 & E1017 & $\begin{array}{c}\text { West wall } \\
\text { profile }\end{array}$ & 98.27 & 103 & - & $\begin{array}{l}\text { Geoarch } \\
\text { Sample }\end{array}$ & Soil & $\begin{array}{l}\text { Magnetic } \\
\text { Susceptibility }\end{array}$ & & & & & \begin{tabular}{|l|}
$12 / 26 / 2017$ \\
\end{tabular} \\
\hline $\begin{array}{l}\text { not for } \\
\text { curation }\end{array}$ & $\begin{array}{l}\begin{array}{l}\text { not for } \\
\text { curation }\end{array} \\
\end{array}$ & 683 & - & - & MS-28 & E & vi & N1004 & E1017 & $\begin{array}{l}\text { West wall } \\
\text { profile }\end{array}$ & 98.25 & 105 & - & $\begin{array}{l}\text { Geoarch } \\
\text { Sample }\end{array}$ & Soil & $\begin{array}{l}\text { Magnetic } \\
\text { Susceptibility }\end{array}$ & & & & & $\mid 12 / 26 / 2017$ \\
\hline \begin{tabular}{l|} 
not for \\
curation
\end{tabular} & $\begin{array}{l}\text { not for } \\
\text { curation }\end{array}$ & 683 & - & - & MS-29 & $\mathrm{E}$ & vi & N1004 & E1017 & $\begin{array}{c}\text { West wall } \\
\text { profile }\end{array}$ & 98.21 & 109 & - & $\begin{array}{l}\text { Geoarch } \\
\text { Sample }\end{array}$ & Soil & $\begin{array}{l}\text { Magnetic } \\
\text { Susceptibility }\end{array}$ & & & & & $\mid$\begin{tabular}{|l|}
$\mid 12 / 26 / 2017$ \\
\end{tabular} \\
\hline \begin{tabular}{l|} 
not for \\
curation
\end{tabular} & $\begin{array}{l}\text { not for } \\
\text { curation }\end{array}$ & 683 & - & - & MS-30 & $E$ & vi & N1004 & E1017 & $\begin{array}{c}\begin{array}{c}\text { West wall } \\
\text { profile }\end{array} \\
\text { pon }\end{array}$ & 98.19 & 111 & - & $\begin{array}{l}\text { Geaarch } \\
\text { Sample }\end{array}$ & Soil & $\begin{array}{l}\text { Magnetici } \\
\text { Susceptibility }\end{array}$ & & & & & $\mid 12 / 26 / 2017$ \\
\hline $\begin{array}{l}\text { not for } \\
\text { curation }\end{array}$ & $\begin{array}{l}\text { not for } \\
\text { curation }\end{array}$ & 683 & - & - & MS-31 & E & vi & N1004 & E1017 & $\begin{array}{c}\text { West wall } \\
\text { profile }\end{array}$ & 98.14 & 116 & - & $\begin{array}{l}\begin{array}{l}\text { Geoarch } \\
\text { Sample }\end{array} \\
\end{array}$ & Soil & $\begin{array}{l}\text { Magnetic } \\
\text { Susceptibility }\end{array}$ & & & & & 12/26/2017 \\
\hline $\begin{array}{l}\text { not for } \\
\text { curation }\end{array}$ & $\begin{array}{l}\text { not for } \\
\text { curation }\end{array}$ & 683 & - & - & MS-32 & E & vi & N1004 & E1017 & $\begin{array}{c}\text { West wall } \\
\text { profile }\end{array}$ & 98.09 & 121 & - & $\begin{array}{l}\begin{array}{l}\text { Geoarch } \\
\text { Sample }\end{array} \\
\end{array}$ & Soil & $\begin{array}{l}\text { Magnetic } \\
\text { Susceptibility }\end{array}$ & & & & & 12/26/2017 \\
\hline $\begin{array}{l}\text { not for } \\
\text { curation }\end{array}$ & $\begin{array}{l}\text { not for } \\
\text { curation }\end{array}$ & 683 & - & - & MS-33 & $\mathrm{E}$ & vi & N1004 & E1017 & $\begin{array}{c}\text { West wall } \\
\text { profile }\end{array}$ & 98.06 & 124 & - & $\begin{array}{l}\text { Geoarch } \\
\text { Sample }\end{array}$ & Soil & $\begin{array}{l}\text { Magnetic } \\
\text { Susceptibility } \\
\end{array}$ & & & & & 12/26/2017 \\
\hline \begin{tabular}{c|} 
not for \\
curation
\end{tabular} & $\begin{array}{l}\text { not for } \\
\text { curation }\end{array}$ & 683 & - & - & MS-34 & E & vi & N1004 & E1017 & $\begin{array}{c}\text { West wall } \\
\text { profile }\end{array}$ & 98.02 & 128 & - & $\begin{array}{l}\text { Geoarch } \\
\text { Sample }\end{array}$ & Soil & $\begin{array}{l}\text { Magnetic } \\
\text { Susceptibility }\end{array}$ & & & & & \begin{tabular}{|l|l|}
$12 / 26 / 2017$ \\
\end{tabular} \\
\hline $\begin{array}{l}\text { not for } \\
\text { curation }\end{array}$ & $\begin{array}{l}\begin{array}{l}\text { not for } \\
\text { curation }\end{array} \\
\text {. }\end{array}$ & 683 & - & - & MS-35 & $\mathrm{E}$ & vi & N1004 & E1017 & $\begin{array}{c}\text { West wall } \\
\text { profile }\end{array}$ & 97.99 & 131 & - & $\begin{array}{l}\text { Geoarch } \\
\text { Sample }\end{array}$ & Soil & $\begin{array}{l}\text { Magnemici } \\
\text { Susceptibility }\end{array}$ & & & & & 12/26/2017 \\
\hline \begin{tabular}{l|} 
not for \\
curation
\end{tabular} & $\begin{array}{l}\text { not for } \\
\text { curation }\end{array}$ & 683 & - & - & MS-36 & E & vi & N1004 & E1017 & $\begin{array}{c}\text { West wall } \\
\text { profile }\end{array}$ & 97.95 & 135 & - & $\begin{array}{l}\text { Geoarch } \\
\text { Sample }\end{array}$ & Soil & $\begin{array}{l}\text { Magnetic } \\
\text { Susceptibility }\end{array}$ & & & & & 12/26/2017 \\
\hline \begin{tabular}{l|} 
not for \\
curation
\end{tabular} & $\begin{array}{l}\begin{array}{l}\text { not for } \\
\text { curation }\end{array} \\
\end{array}$ & 683 & - & - & MS-37 & $\mathrm{E}$ & vi & N1004 & E1017 & $\begin{array}{c}\text { West wall } \\
\text { profile }\end{array}$ & 97.9 & 140 & - & $\begin{array}{l}\text { Geoarch } \\
\text { Sample }\end{array}$ & Soil & $\begin{array}{l}\text { Magnetici } \\
\text { Susceptibility }\end{array}$ & & & & & 12/26/2017 \\
\hline \begin{tabular}{l|} 
not for \\
curation
\end{tabular} & $\begin{array}{l}\begin{array}{l}\text { not for } \\
\text { curation }\end{array} \\
\end{array}$ & 683 & - & - & MS-38 & E & vi & N1004 & E1017 & $\begin{array}{c}\text { West wall } \\
\text { profile }\end{array}$ & 97.86 & 144 & - & $\begin{array}{l}\text { Geoarch } \\
\text { Sample }\end{array}$ & Soil & $\begin{array}{l}\text { Magnetici } \\
\text { Susceptibility }\end{array}$ & & & & & 12/26/2017 \\
\hline \begin{tabular}{|c|} 
not for \\
curation
\end{tabular} & $\begin{array}{l}\text { not for } \\
\text { curation }\end{array}$ & 683 & - & - & MS-39 & $\mathrm{E}$ & vi & N1004 & E1017 & $\begin{array}{c}\text { West wall } \\
\text { profile }\end{array}$ & 97.81 & 149 & - & $\begin{array}{l}\text { Gearach } \\
\text { Sample }\end{array}$ & Soil & $\begin{array}{l}\text { Magnetic } \\
\text { Susceptibity }\end{array}$ & & & & & 12/26/2017 \\
\hline \begin{tabular}{c|} 
not for \\
curation
\end{tabular} & $\begin{array}{l}\begin{array}{l}\text { not for } \\
\text { curation }\end{array} \\
\text { con }\end{array}$ & 683 & - & - & MS -40 & E & vi & N1004 & E1017 & $\begin{array}{c}\text { West wall } \\
\text { profile }\end{array}$ & 97.77 & 153 & - & $\begin{array}{l}\text { Geoarch } \\
\text { Sample }\end{array}$ & Soil & $\begin{array}{l}\text { Magnetic } \\
\text { Susceptibility }\end{array}$ & & & & & 12/26/2017 \\
\hline \begin{tabular}{|c|} 
not for \\
curation
\end{tabular} & $\begin{array}{c}\text { not for } \\
\text { curation }\end{array}$ & 683 & - & - & BM-01 & $\mathrm{E}$ & $\mathrm{vv}_{1}$ & N1004 & E1017 & $\begin{array}{c}\begin{array}{c}\text { West wall } \\
\text { profile }\end{array} \\
\text { pes }\end{array}$ & \begin{tabular}{|l|}
$97.87-97.77$ \\
\end{tabular} & 143-153 & - & 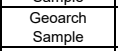 & Soil & Bulk Matrix & & & & & 12/26/2017 \\
\hline \begin{tabular}{|c|} 
not for \\
curation
\end{tabular} & $\begin{array}{c}\text { not for } \\
\text { curation }\end{array}$ & 683 & - & - & BM-02 & E & vi & N1004 & E1017 & $\begin{array}{c}\text { West wall } \\
\text { profile }\end{array}$ & \begin{tabular}{|l|}
$98.05-98.00$ \\
\end{tabular} & 125-130 & - & $\begin{array}{l}\text { Geoarch } \\
\text { Sample }\end{array}$ & Soil & Bulk Matrix & & & & & 12/26/2017 \\
\hline $\begin{array}{c}\text { not tor } \\
\text { curation }\end{array}$ & $\begin{array}{l}\begin{array}{l}\text { not for } \\
\text { curation }\end{array} \\
\text { cons }\end{array}$ & 683 & - & - & BM-03 & E & vi & N1004 & E1017 & $\begin{array}{c}\text { Westivall } \\
\text { profile }\end{array}$ & 98.24-98.17 & $106-113$ & - & $\begin{array}{l}\text { Geoarch } \\
\text { Sample }\end{array}$ & Soil & Bulk Matrix & & & & & 12/26/2017 \\
\hline $\begin{array}{c}\text { not for } \\
\text { curation }\end{array}$ & $\begin{array}{l}\begin{array}{l}\text { not for } \\
\text { curation }\end{array} \\
\text { s. }\end{array}$ & 683 & - & - & BM-04 & $\mathrm{E}$ & $V_{N 1}$ & N1004 & E1017 & $\begin{array}{c}\text { West wall } \\
\text { profile }\end{array}$ & 98.37-98.30 & 93-100 & - & $\begin{array}{l}\text { Geoarch } \\
\text { Sample }\end{array}$ & Soil & Bulk Matrix & & & & & 12/26/2017 \\
\hline \begin{tabular}{c|} 
not for \\
curation
\end{tabular} & $\begin{array}{l}\begin{array}{l}\text { not for } \\
\text { curation }\end{array} \\
\end{array}$ & 683 & - & - & BM-05 & $\mathrm{E}$ & IVN & N1004 & E1017 & $\begin{array}{c}\text { West wall } \\
\text { profile }\end{array}$ & \begin{tabular}{|l|}
$98.52-98.44$ \\
\end{tabular} & $78-86$ & - & $\begin{array}{l}\text { Geoarch } \\
\text { Sample }\end{array}$ & Soil & Bulk Matrix & & & & & 12/26/2017 \\
\hline \begin{tabular}{|c|} 
not for \\
curation
\end{tabular} & $\begin{array}{l}\begin{array}{l}\text { not for } \\
\text { curation }\end{array} \\
\text { cons }\end{array}$ & 683 & - & - & BM-06 & E & IV & N1004 & E1017 & $\begin{array}{c}\text { West wall } \\
\text { profile }\end{array}$ & 98.63-98.53 & $\begin{array}{l}67-77 \\
\end{array}$ & - & $\begin{array}{l}\begin{array}{l}\text { Geoarch } \\
\text { Sample }\end{array} \\
\text { Sals }\end{array}$ & Soil & Bulk Matrix & & & & & $12 / 26 / 2017$ \\
\hline \begin{tabular}{|c|} 
not for \\
curation
\end{tabular} & $\begin{array}{l}\text { not for } \\
\text { curation }\end{array}$ & 683 & - & - & BM-07 & $\mathrm{E}$ & IIII & N1004 & E1017 & $\begin{array}{c}\text { West wall } \\
\text { profile }\end{array}$ & 98.81-98.73 & $49-57$ & - & $\begin{array}{l}\text { Geoarch } \\
\text { Sample }\end{array}$ & Soil & Bulk Matrix & & & & & 12/26/2017 \\
\hline $\begin{array}{l}\text { not for } \\
\text { curation }\end{array}$ & $\begin{array}{c}\text { not for } \\
\text { curation }\end{array}$ & 683 & - & - & BM-08 & E & III & N1004 & E1017 & $\begin{array}{c}\text { West wall } \\
\text { profile }\end{array}$ & \begin{tabular}{|l|}
$98.93-98.87$ \\
\end{tabular} & $37-43$ & - & $\begin{array}{l}\text { Geoarch } \\
\text { Sample }\end{array}$ & Soil & Bulk Matrix & & & & & 121/26/2017 \\
\hline $\begin{array}{c}\text { not for } \\
\text { curation }\end{array}$ & $\begin{array}{l}\text { not for } \\
\text { curation }\end{array}$ & 683 & - & - & BM-09 & $E$ & " & N1004 & E1017 & $\begin{array}{c}\text { West wall } \\
\text { profile }\end{array}$ & 99.14-99.07 & $16-23$ & - & 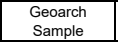 & Soil & Bulk Matrix & & & & & $12 / 26 / 2017$ \\
\hline \begin{tabular}{|c|} 
not for \\
curation
\end{tabular} & $\begin{array}{l}\begin{array}{l}\text { not for } \\
\text { curation }\end{array} \\
\text { count }\end{array}$ & 683 & - & - & BM-10 & E & 1 & N1004 & E1017 & $\begin{array}{c}\begin{array}{c}\text { West wall } \\
\text { profile }\end{array} \\
\text { pes }\end{array}$ & \begin{tabular}{|l|}
$99.30-99.20$ \\
\end{tabular} & $0-10$ & - & $\begin{array}{l}\text { Geaarch } \\
\text { Sample }\end{array}$ & Soil & Bulk Matrix & & & & & 12/26/2017 \\
\hline $\begin{array}{c}\text { not tor } \\
\text { curation }\end{array}$ & $\begin{array}{l}\begin{array}{l}\text { not for } \\
\text { curation }\end{array} \\
\text { cons }\end{array}$ & 683 & - & - & BM-11 & $\mathrm{E}$ & IVIV & N1004 & E1017 & $\begin{array}{c}\text { West wall } \\
\text { profile }\end{array}$ & 98.53-98.42 & $87-98$ & - & $\begin{array}{l}\text { Geoarch } \\
\text { Sample }\end{array}$ & Soil & Bulk Matrix & & & & & 12/26/2017 \\
\hline \begin{tabular}{c|} 
not for \\
curation
\end{tabular} & $\begin{array}{l}\text { not for } \\
\text { curation }\end{array}$ & 683 & - & - & BM-12 & $\mathrm{E}$ & IIIIIV & N1004 & E1017 & $\begin{array}{c}\text { West wall } \\
\text { profile }\end{array}$ & \begin{tabular}{|l|}
$98.77-98.68$ \\
\end{tabular} & 63-72 & - & $\begin{array}{l}\text { Geoarch } \\
\text { Sample }\end{array}$ & Soil & Bulk Matrix & & & & & 12/26/2017 \\
\hline $\begin{array}{l}\text { not for } \\
\text { curation }\end{array}$ & $\begin{array}{l}\text { not for } \\
\text { curation }\end{array}$ & 683 & - & - & BM-13 & $E$ & III' & N1004 & E1017 & $\begin{array}{c}\text { West wall } \\
\text { profile }\end{array}$ & 98.88-98.80 & $42-50$ & - & $\begin{array}{l}\text { Geoarch } \\
\text { Sample }\end{array}$ & Soil & Bulk Matrix & & & & & 12/26/2017 \\
\hline \begin{tabular}{l|} 
not for \\
curation
\end{tabular} & $\begin{array}{l}\begin{array}{l}\text { not for } \\
\text { curation }\end{array} \\
\text { cons }\end{array}$ & 684 & - & - & MS-01 & A & 1 & No996 & E1014 & $\begin{array}{c}\begin{array}{c}\text { North wall } \\
\text { profile }\end{array} \\
\end{array}$ & 99.52 & 18 & - & $\begin{array}{l}\text { Geoarch } \\
\text { Sample }\end{array}$ & Soil & $\begin{array}{l}\text { Magnetici } \\
\text { Susceptibility }\end{array}$ & & & & & $\mid 12 / 27 / 2017$ \\
\hline \begin{tabular}{|c|} 
not for \\
curation
\end{tabular} & $\begin{array}{l}\text { not for } \\
\text { curation }\end{array}$ & 684 & - & - & MS-02 & $\mathrm{A}$ & $"$ & No996 & E1014 & $\begin{array}{c}\text { North wall } \\
\text { profile }\end{array}$ & 99.49 & 21.5 & - & $\begin{array}{l}\text { Geoarch } \\
\text { Sample }\end{array}$ & Soil & $\begin{array}{l}\text { Magneticic } \\
\text { Susceptibility }\end{array}$ & & & & & 12/27/2017 \\
\hline \begin{tabular}{|c|} 
not for \\
curation
\end{tabular} & $\begin{array}{l}\text { not for } \\
\text { curation }\end{array}$ & 684 & - & - & MS-03 & A & " & N0996 & E1014 & $\begin{array}{c}\text { North wall } \\
\text { profile }\end{array}$ & 99.46 & 24 & - & $\begin{array}{l}\text { Geoarch } \\
\text { Sample }\end{array}$ & Soil & $\begin{array}{l}\text { Magnetici } \\
\text { Susceptibility }\end{array}$ & & & & & 12/27/2017 \\
\hline $\begin{array}{l}\text { not for } \\
\text { curation }\end{array}$ & $\begin{array}{c}\text { not for } \\
\text { curation }\end{array}$ & 684 & - & - & MS-04 & A & 11 & No996 & E1014 & $\begin{array}{c}\text { North wall } \\
\text { profile }\end{array}$ & 99.44 & 26 & - & $\begin{array}{l}\text { Geoarch } \\
\text { Sample }\end{array}$ & Soil & $\begin{array}{l}\text { Magneticic } \\
\text { Susceptibility }\end{array}$ & & & & & $12 / 27 / 2017$ \\
\hline \begin{tabular}{|c|} 
not for \\
curation
\end{tabular} & $\begin{array}{l}\text { not for } \\
\text { curation }\end{array}$ & 684 & - & - & MS-05 & A & $"$ & $\begin{array}{l}\text { No996 } \\
\end{array}$ & E1014 & $\begin{array}{c}\text { North wall } \\
\text { profile }\end{array}$ & 99.43 & 27.5 & - & 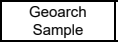 & Soil & $\begin{array}{l}\text { Magnetici } \\
\text { Susceptibility }\end{array}$ & & & & & 12/27/2017 \\
\hline $\begin{array}{l}\text { not for } \\
\text { curation }\end{array}$ & $\begin{array}{l}\text { not for } \\
\text { curation }\end{array}$ & 684 & - & - & MS-06 & $\mathrm{A}$ & 11 & No996 & E1014 & $\begin{array}{c}\begin{array}{c}\text { North wall } \\
\text { profile }\end{array} \\
\end{array}$ & 99.39 & 31 & - & $\begin{array}{l}\text { Geoarch } \\
\text { Sample }\end{array}$ & Soil & $\begin{array}{l}\text { Magneticic } \\
\text { Susceptibity }\end{array}$ & & & & & 12/27/2017 \\
\hline $\begin{array}{l}\text { not tor } \\
\text { curation }\end{array}$ & $\begin{array}{c}\text { not for } \\
\text { curation }\end{array}$ & 684 & - & - & MS-07 & A & " & No996 & E1014 & $\begin{array}{c}\text { North wall } \\
\text { profile }\end{array}$ & 99.36 & 34 & - & $\begin{array}{c}\begin{array}{c}\text { Geoarch } \\
\text { Sample }\end{array} \\
\end{array}$ & Soil & $\begin{array}{l}\text { Magnetic } \\
\text { Susceptibility }\end{array}$ & & & & & 12/27/2017 \\
\hline
\end{tabular}




\begin{tabular}{|c|c|c|c|c|c|c|c|c|c|c|c|c|c|c|c|c|c|c|c|c|c|}
\hline Lot No. & \begin{tabular}{|c|}
$\begin{array}{c}\text { Specimen } \\
\text { No. }\end{array}$ \\
\end{tabular} & FS No. & UI No. & FTR No. & $\begin{array}{c}\text { Sample } \\
\text { No. }\end{array}$ & $\begin{array}{c}\text { Excavation } \\
\text { Block }\end{array}$ & Stratum (AU) & Northing & Easting & Level & $\begin{array}{c}\text { Elevation } \\
\text { (m) }\end{array}$ & Depth (cmbd) & Point Prov. & Artifact Class & Arriffact Type & $\begin{array}{c}\text { Artifact } \\
\text { Description }\end{array}$ & Material & $\begin{array}{c}\text { Number of } \\
\text { artifacts }\end{array}$ & Weight (g) & Comments & Date \\
\hline $\begin{array}{l}\text { not for } \\
\text { curation }\end{array}$ & $\begin{array}{c}\text { not for } \\
\text { curation }\end{array}$ & 684 & - & - & MS-08 & A & " & No996 & E1014 & 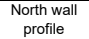 & 99.35 & 35 & - & $\begin{array}{l}\text { Geoarch } \\
\text { Sample }\end{array}$ & Soil & $\begin{array}{l}\text { Magnetic } \\
\text { Susceptibility }\end{array}$ & & & & & 12/27/2017 \\
\hline \begin{tabular}{c|} 
not for \\
curation
\end{tabular} & $\begin{array}{l}\begin{array}{l}\text { not for } \\
\text { curation }\end{array} \\
\end{array}$ & 684 & - & - & MS-09 & A & "I & No996 & E1014 & $\begin{array}{l}\begin{array}{c}\text { North wall } \\
\text { profile }\end{array} \\
\end{array}$ & 99.3 & 40 & - & $\begin{array}{l}\text { Geoarch } \\
\text { Sample }\end{array}$ & Soil & $\begin{array}{l}\text { Magnetic } \\
\text { Susceptibility }\end{array}$ & & & & & 12/27/2017 \\
\hline \begin{tabular}{|l|} 
not for \\
curation \\
\end{tabular} & $\begin{array}{l}\begin{array}{l}\text { not for } \\
\text { curation }\end{array} \\
\end{array}$ & 684 & - & - & MS-10 & A & "I & No996 & E1014 & $\begin{array}{c}\begin{array}{c}\text { North wall } \\
\text { profile }\end{array} \\
\text { pols }\end{array}$ & 99.29 & 41.5 & - & $\begin{array}{l}\text { Geaarch } \\
\text { Sample }\end{array}$ & Soil & $\begin{array}{l}\text { Magnemici } \\
\text { Susceptibility }\end{array}$ & & & & & $\mid 12 / 27 / 2017$ \\
\hline 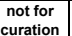 & $\begin{array}{l}\text { not for } \\
\text { curation }\end{array}$ & 684 & - & - & MS-11 & A & ॥ & No996 & E1014 & $\begin{array}{l}\text { North wall } \\
\text { profile }\end{array}$ & 99.25 & 45.5 & - & $\begin{array}{l}\text { Geoarch } \\
\text { Sample }\end{array}$ & Soil & $\begin{array}{l}\text { Magnetic } \\
\text { Susceptitility }\end{array}$ & & & & & 12/27/2017 \\
\hline $\begin{array}{l}\text { not for } \\
\text { curation }\end{array}$ & $\begin{array}{l}\text { not for } \\
\text { curation }\end{array}$ & 684 & - & - & MS-12 & A & " & No996 & E1014 & $\begin{array}{c}\begin{array}{c}\text { North wall } \\
\text { profile }\end{array} \\
\text { pols }\end{array}$ & 99.23 & 47.5 & - & $\begin{array}{l}\text { Geoarch } \\
\text { Sample }\end{array}$ & Soil & $\begin{array}{l}\text { Magnetic } \\
\text { Susceptibility }\end{array}$ & & & & & $\mid$\begin{tabular}{|l|l}
$\mid 12 / 27 / 2017$ \\
\end{tabular} \\
\hline $\begin{array}{l}\text { not for } \\
\text { curation }\end{array}$ & $\begin{array}{l}\text { not for } \\
\text { curation }\end{array}$ & 684 & - & - & MS-13 & A & $" 1$ & No996 & E1014 & $\begin{array}{c}\text { North wall } \\
\text { profile }\end{array}$ & 99.18 & 52 & - & $\begin{array}{l}\text { Geoarch } \\
\text { Sample }\end{array}$ & Soil & $\begin{array}{l}\text { Magnetici } \\
\text { Susceptibility }\end{array}$ & & & & & 12/27/2017 \\
\hline \begin{tabular}{l|} 
not for \\
curation
\end{tabular} & $\begin{array}{l}\text { not for } \\
\text { curation }\end{array}$ & 684 & - & - & MS-14 & A & III & No996 & E1014 & $\begin{array}{l}\text { North wall } \\
\text { profile }\end{array}$ & 99.15 & 55.5 & - & $\begin{array}{l}\text { Geoarch } \\
\text { Sample }\end{array}$ & Soil & $\begin{array}{l}\text { Maganetic } \\
\text { Suscoptibility }\end{array}$ & & & & & $\mid 12 / 27 / 2017$ \\
\hline $\begin{array}{l}\text { not for } \\
\text { curation }\end{array}$ & $\begin{array}{l}\text { not for } \\
\text { curtition }\end{array}$ & 684 & - & - & MS-15 & A & III & No996 & E1014 & $\begin{array}{l}\text { North wall } \\
\text { profile }\end{array}$ & 99.13 & 57 & - & $\begin{array}{l}\text { Sampie } \\
\text { Geaarch } \\
\text { Sample }\end{array}$ & Soil & $\begin{array}{c}\text { Susceprominy } \\
\text { Muscentic } \\
\text { Suscopibility }\end{array}$ & & & & & 12/27/2017 \\
\hline $\begin{array}{l}\text { not tor } \\
\text { curation }\end{array}$ & $\begin{array}{l}\begin{array}{l}\text { not for } \\
\text { curation }\end{array} \\
\end{array}$ & 684 & - & - & MS-16 & A & III & No996 & E1014 & $\begin{array}{c}\begin{array}{c}\text { North wall } \\
\text { profile }\end{array} \\
\text { pols }\end{array}$ & 99.08 & 62.5 & - & $\begin{array}{l}\text { Geoarch } \\
\text { Sample }\end{array}$ & Soil & $\begin{array}{l}\text { Magnetic } \\
\text { Susceptibility }\end{array}$ & & & & & \begin{tabular}{|l|}
$12 / 27 / 2017$ \\
\end{tabular} \\
\hline $\begin{array}{l}\text { not for } \\
\text { curation }\end{array}$ & $\begin{array}{l}\begin{array}{l}\text { not for } \\
\text { curation }\end{array} \\
\end{array}$ & 684 & - & - & MS-17 & A & III & No996 & E1014 & \begin{tabular}{|c|} 
North wall \\
profile
\end{tabular} & 99.06 & 64 & - & $\begin{array}{l}\text { Geoarch } \\
\text { Sample }\end{array}$ & Soil & $\begin{array}{l}\text { Magnetic } \\
\text { Susceptibility }\end{array}$ & & & & & 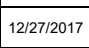 \\
\hline \begin{tabular}{l|} 
not for \\
curation
\end{tabular} & $\begin{array}{l}\text { not for } \\
\text { curation }\end{array}$ & 684 & - & - & MS-18 & A & III & No996 & E1014 & $\begin{array}{c}\text { North wall } \\
\text { profile }\end{array}$ & 99.01 & 69 & - & $\begin{array}{l}\text { Geoarch } \\
\text { Sample }\end{array}$ & Soil & $\begin{array}{l}\text { Magnetic } \\
\text { Susceptibility }\end{array}$ & & & & & $\mid$\begin{tabular}{|l}
$\mid 12 / 27 / 2017$ \\
\end{tabular} \\
\hline $\begin{array}{l}\text { not for } \\
\text { curation }\end{array}$ & $\begin{array}{l}\begin{array}{l}\text { not for } \\
\text { curation }\end{array} \\
\end{array}$ & 684 & - & - & MS-19 & A & III & No996 & E1014 & $\begin{array}{c}\begin{array}{c}\text { North wall } \\
\text { profile }\end{array} \\
\end{array}$ & 99 & 70 & - & $\begin{array}{l}\text { Geoarch } \\
\text { Sample }\end{array}$ & Soil & $\begin{array}{l}\text { Magnemic } \\
\text { Susceptibility } \\
\end{array}$ & & & & & $\mid 12 / 27 / 2017$ \\
\hline $\begin{array}{l}\text { not for } \\
\text { curation }\end{array}$ & $\begin{array}{l}\text { not for } \\
\text { curation }\end{array}$ & 684 & - & - & MS-20 & A & III & No996 & E1014 & $\begin{array}{c}\begin{array}{c}\text { North wall } \\
\text { profile }\end{array} \\
\text { (a) }\end{array}$ & 98.96 & 74 & - & $\begin{array}{l}\begin{array}{l}\text { Geoarch } \\
\text { Sample }\end{array} \\
\end{array}$ & Soil & $\begin{array}{l}\text { Magnetic } \\
\text { Susceptibility }\end{array}$ & & & & & 12/27/2017 \\
\hline $\begin{array}{l}\text { not for } \\
\text { curation }\end{array}$ & $\begin{array}{l}\text { not for } \\
\text { curation }\end{array}$ & 684 & - & - & MS-21 & A & III & No996 & E1014 & $\begin{array}{c}\begin{array}{c}\text { North wall } \\
\text { profile }\end{array} \\
\text { pol }\end{array}$ & 98.93 & 77 & - & $\begin{array}{l}\begin{array}{l}\text { Geoarch } \\
\text { Sample }\end{array} \\
\end{array}$ & Soil & $\begin{array}{l}\text { Magnetic } \\
\text { Susceptibility }\end{array}$ & & & & & 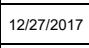 \\
\hline $\begin{array}{l}\text { not for } \\
\text { curation }\end{array}$ & $\begin{array}{l}\text { not for } \\
\text { curation }\end{array}$ & 684 & - & - & MS-22 & A & III & No996 & E1014 & $\begin{array}{c}\text { North wall } \\
\text { profile }\end{array}$ & 98.9 & 80 & - & $\begin{array}{l}\text { Geoarch } \\
\text { Sample }\end{array}$ & Soil & $\begin{array}{l}\text { Magnetic } \\
\text { Susceptibility } \\
\end{array}$ & & & & & 12/27/2017 \\
\hline \begin{tabular}{c|} 
not for \\
curation
\end{tabular} & $\begin{array}{l}\text { not for } \\
\text { curation }\end{array}$ & 684 & - & - & MS-23 & A & IV & No996 & E1014 & $\begin{array}{l}\begin{array}{c}\text { North wall } \\
\text { profile }\end{array} \\
\end{array}$ & $\begin{array}{l}98.87 \\
\end{array}$ & 83 & - & $\begin{array}{l}\text { Geoarch } \\
\text { Sample }\end{array}$ & Soil & $\begin{array}{l}\text { Magnemitic } \\
\text { Suscoptibility }\end{array}$ & & & & & \begin{tabular}{|l|l|l|}
$12 / 27 / 2017$ \\
\end{tabular} \\
\hline $\begin{array}{l}\text { not for } \\
\text { curation }\end{array}$ & $\begin{array}{l}\begin{array}{l}\text { not for } \\
\text { curation }\end{array} \\
\end{array}$ & 684 & - & - & MS-24 & A & iv & No996 & E1014 & $\begin{array}{l}\begin{array}{c}\text { North wall } \\
\text { profile }\end{array} \\
\text { pols }\end{array}$ & 98.84 & 86 & - & $\begin{array}{l}\text { Geoarch } \\
\text { Sample }\end{array}$ & Soil & $\begin{array}{l}\text { Magnemitic } \\
\text { Susceptibility }\end{array}$ & & & & & 12/27/2017 \\
\hline \begin{tabular}{l|} 
not for \\
curation
\end{tabular} & $\begin{array}{l}\text { not for } \\
\text { curation }\end{array}$ & 684 & - & - & MS-25 & A & IV & No996 & E1014 & $\begin{array}{c}\text { North wall } \\
\text { profile }\end{array}$ & 98.81 & 89 & - & $\begin{array}{l}\text { Geoarch } \\
\text { Sample }\end{array}$ & Soil & $\begin{array}{l}\text { Magnetic } \\
\text { Susceptibility }\end{array}$ & & & & & 12/27/2017 \\
\hline \begin{tabular}{l|} 
not for \\
curation
\end{tabular} & $\begin{array}{l}\begin{array}{l}\text { not for } \\
\text { curation }\end{array} \\
\end{array}$ & 684 & - & - & MS-26 & A & IV & No996 & E1014 & 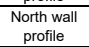 & 98.79 & 91.5 & - & $\begin{array}{l}\text { Geoarch } \\
\text { Sample }\end{array}$ & Soil & $\begin{array}{l}\text { Magneticic } \\
\text { Susceptibility }\end{array}$ & & & & & 121/27/2017 \\
\hline \begin{tabular}{l|} 
not for \\
curation
\end{tabular} & $\begin{array}{l}\text { not for } \\
\text { curation }\end{array}$ & 684 & - & - & MS-27 & A & IV & No996 & E1014 & $\begin{array}{c}\text { North wall } \\
\text { profile }\end{array}$ & 98.75 & 95 & - & $\begin{array}{l}\text { Geoarch } \\
\text { Sample }\end{array}$ & Soil & $\begin{array}{l}\text { Magneticic } \\
\text { Susceptibility }\end{array}$ & & & & & 12/27/2017 \\
\hline \begin{tabular}{l|} 
not for \\
curation
\end{tabular} & $\begin{array}{l}\text { not for } \\
\text { curation }\end{array}$ & 684 & - & - & MS-28 & A & IV & No996 & E1014 & $\begin{array}{c}\text { North wall } \\
\text { profile }\end{array}$ & 98.73 & 97 & - & $\begin{array}{l}\text { Geoarch } \\
\text { Sample }\end{array}$ & Soil & $\begin{array}{l}\text { Magnetic } \\
\text { Susceptibility }\end{array}$ & & & & & 121/27/2017 \\
\hline \begin{tabular}{c|} 
not for \\
curation
\end{tabular} & $\begin{array}{l}\text { not for } \\
\text { curation }\end{array}$ & 684 & - & - & MS-29 & A & IV & No996 & E1014 & $\begin{array}{c}\text { North wall } \\
\text { profile }\end{array}$ & 98.69 & 101 & - & $\begin{array}{l}\text { Geoarch } \\
\text { Sample }\end{array}$ & Soil & $\begin{array}{l}\text { Magnetici } \\
\text { Susceptibility }\end{array}$ & & & & & 121/27/2017 \\
\hline \begin{tabular}{l|} 
not for \\
curation
\end{tabular} & $\begin{array}{c}\text { not for } \\
\text { curation }\end{array}$ & 684 & - & - & MS-30 & A & IV & $\begin{array}{l}\text { No996 } \\
\end{array}$ & E1014 & $\begin{array}{c}\text { North wall } \\
\text { profile }\end{array}$ & 98.67 & 103 & - & $\begin{array}{l}\text { Geoarch } \\
\text { Sample }\end{array}$ & Soil & $\begin{array}{l}\text { Magnentic } \\
\text { Susceptibility }\end{array}$ & & & & & 12/27/2017 \\
\hline \begin{tabular}{l|} 
not for \\
curation
\end{tabular} & $\begin{array}{c}\text { not for } \\
\text { curation }\end{array}$ & 684 & - & - & MS-31 & A & IV & No996 & E1014 & $\begin{array}{c}\text { North wall } \\
\text { profile }\end{array}$ & 98.63 & 107 & - & $\begin{array}{l}\text { Geoarch } \\
\text { Sample }\end{array}$ & Soil & $\begin{array}{l}\text { Magnetic } \\
\text { Susceptibility }\end{array}$ & & & & & 12/27/2017 \\
\hline \begin{tabular}{c|} 
not for \\
curation
\end{tabular} & $\begin{array}{l}\text { not for } \\
\text { curation }\end{array}$ & 684 & - & - & MS-32 & A & IV & No996 & E1014 & $\begin{array}{c}\text { North wall } \\
\text { profile }\end{array}$ & 98.6 & 110 & - & $\begin{array}{l}\text { Geoarch } \\
\text { Sample }\end{array}$ & Soil & $\begin{array}{l}\text { Magnetic } \\
\text { Susceptibility }\end{array}$ & & & & & 12/27/2017 \\
\hline $\begin{array}{l}\text { not for } \\
\text { curation }\end{array}$ & $\begin{array}{l}\begin{array}{l}\text { not for } \\
\text { curation }\end{array} \\
\text { s. }\end{array}$ & 684 & - & - & MS-33 & A & IV & No996 & E1014 & $\begin{array}{c}\text { North wall } \\
\text { profile }\end{array}$ & 98.54 & 116 & - & $\begin{array}{l}\text { Geoarch } \\
\text { Sample }\end{array}$ & Soil & $\begin{array}{l}\text { Magnetic } \\
\text { Susceptibility }\end{array}$ & & & & & 12/27/2017 \\
\hline \begin{tabular}{|c|} 
not for \\
curation
\end{tabular} & $\begin{array}{l}\begin{array}{l}\text { not for } \\
\text { curation }\end{array} \\
\text { cons }\end{array}$ & 684 & - & - & MS-34 & A & IV & $\begin{array}{l}\text { No996 } \\
\end{array}$ & E1014 & $\begin{array}{c}\text { North wall } \\
\text { profile }\end{array}$ & 98.53 & 117.5 & - & $\begin{array}{l}\text { Geoarch } \\
\text { Sample }\end{array}$ & Soil & $\begin{array}{l}\text { Magnetici } \\
\text { Susceptibility }\end{array}$ & & & & & 12/27/2017 \\
\hline \begin{tabular}{|c|} 
not for \\
curation
\end{tabular} & $\begin{array}{l}\begin{array}{l}\text { not for } \\
\text { curation }\end{array} \\
\text { cons }\end{array}$ & 684 & - & - & MS-35 & A & IV & No996 & E1014 & 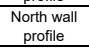 & 98.5 & 120.5 & - & $\begin{array}{l}\text { Geoarch } \\
\text { Sample }\end{array}$ & Soil & $\begin{array}{l}\text { Magnetici } \\
\text { Susceptibility }\end{array}$ & & & & & $12 / 27 / 2017$ \\
\hline $\begin{array}{c}\text { not for } \\
\text { curation }\end{array}$ & $\begin{array}{l}\text { not for } \\
\text { curation }\end{array}$ & 684 & - & - & MS- 36 & A & IVIV & No996 & E1014 & $\begin{array}{l}\text { North wall } \\
\text { profile }\end{array}$ & 98.46 & 124 & - & $\begin{array}{l}\text { Geoarch } \\
\text { Sample }\end{array}$ & Soil & $\begin{array}{l}\text { Magnentic } \\
\text { Suscoptitily }\end{array}$ & & & & & 12/27/2017 \\
\hline $\begin{array}{l}\text { not for } \\
\text { curation }\end{array}$ & $\begin{array}{c}\text { not for } \\
\text { curation }\end{array}$ & 684 & - & - & MS-37 & A & IVIV & No996 & E1014 & $\begin{array}{c}\text { North wall } \\
\text { profile }\end{array}$ & 98.43 & 127 & - & $\begin{array}{l}\text { Geoarch } \\
\text { Sample }\end{array}$ & Soil & $\begin{array}{l}\text { Magnetici } \\
\text { Susceptibility }\end{array}$ & & & & & 12/27/2017 \\
\hline $\begin{array}{c}\text { not for } \\
\text { curation }\end{array}$ & $\begin{array}{l}\text { not for } \\
\text { curation }\end{array}$ & 684 & - & - & MS-38 & A & IVIV & No996 & E1014 & $\begin{array}{c}\text { North wall } \\
\text { profile }\end{array}$ & 98.4 & 130 & - & 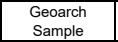 & Soil & $\begin{array}{l}\text { Magnetici } \\
\text { Susceptibility } \\
\text { Sust }\end{array}$ & & & & & $12 / 27 / 2017$ \\
\hline \begin{tabular}{|c|} 
not for \\
curation
\end{tabular} & $\begin{array}{l}\begin{array}{l}\text { not for } \\
\text { curation }\end{array} \\
\text { cons }\end{array}$ & 684 & - & - & MS-39 & A & v & No996 & E1014 & $\begin{array}{c}\text { North wall } \\
\text { profile }\end{array}$ & 98.38 & 132.5 & - & $\begin{array}{l}\text { Geaarch } \\
\text { Sample }\end{array}$ & Soil & $\begin{array}{l}\text { Magnetic } \\
\text { Susceptibility }\end{array}$ & & & & & 12/27/2017 \\
\hline $\begin{array}{c}\text { not tor } \\
\text { curation }\end{array}$ & $\begin{array}{l}\begin{array}{l}\text { not for } \\
\text { curation }\end{array} \\
\text { cons }\end{array}$ & 684 & - & - & MS- 40 & A & v & No996 & E1014 & $\begin{array}{c}\text { North wall } \\
\text { profile }\end{array}$ & 98.35 & 135 & - & $\begin{array}{l}\text { Geoarch } \\
\text { Sample }\end{array}$ & Soil & $\begin{array}{l}\text { Magnetic } \\
\text { Susceptibility }\end{array}$ & & & & & 12/27/2017 \\
\hline $\begin{array}{c}\text { not for } \\
\text { curation }\end{array}$ & $\begin{array}{l}\text { not for } \\
\text { curation }\end{array}$ & 684 & - & - & MS-41 & A & vi & No996 & E1014 & $\begin{array}{c}\text { North wall } \\
\text { profile }\end{array}$ & 98.31 & 139.5 & - & $\begin{array}{l}\text { Geoarch } \\
\text { Sample }\end{array}$ & Soil & $\begin{array}{l}\text { Magnetic } \\
\text { Susceptibility }\end{array}$ & & & & & $\mid 12 / 27 / 2017$ \\
\hline $\begin{array}{l}\text { not for } \\
\text { curation }\end{array}$ & $\begin{array}{l}\text { not for } \\
\text { curation }\end{array}$ & 684 & - & - & MS- 42 & A & vi & No996 & E1014 & $\begin{array}{c}\text { North wall } \\
\text { profile }\end{array}$ & 98.28 & 142 & - & $\begin{array}{l}\text { Geoarch } \\
\text { Sample }\end{array}$ & Soil & $\begin{array}{l}\text { Magnentic } \\
\text { Susceptibility }\end{array}$ & & & & & 12/27/2017 \\
\hline \begin{tabular}{l|} 
not for \\
curation
\end{tabular} & $\begin{array}{l}\begin{array}{l}\text { not for } \\
\text { curation }\end{array} \\
\text { cons }\end{array}$ & 684 & - & - & MS-43 & A & vi & N0996 & E1014 & $\begin{array}{c}\text { North wall } \\
\text { profile }\end{array}$ & 98.25 & 145 & - & 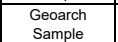 & Soil & $\begin{array}{l}\text { Magneticic } \\
\text { Susceptibility }\end{array}$ & & & & & $\mid 12 / 27 / 2017$ \\
\hline \begin{tabular}{|c|} 
not for \\
curation
\end{tabular} & $\begin{array}{l}\text { not for } \\
\text { curation }\end{array}$ & 684 & - & - & MS-44 & $\mathrm{A}$ & $\mathrm{vi}$ & No996 & E1014 & 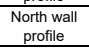 & 98.21 & 149 & - & $\begin{array}{l}\text { Geoarch } \\
\text { Sample }\end{array}$ & Soil & $\begin{array}{l}\text { Magnetici } \\
\text { Susceptibility }\end{array}$ & & & & & 12/27/2017 \\
\hline \begin{tabular}{|c|} 
not for \\
curation
\end{tabular} & $\begin{array}{l}\text { not for } \\
\text { curation }\end{array}$ & 684 & - & - & MS-45 & A & vi & No996 & E1014 & $\begin{array}{c}\text { North wall } \\
\text { profile }\end{array}$ & 98.21 & 149 & - & $\begin{array}{l}\text { Geoarch } \\
\text { Sample }\end{array}$ & Soil & $\begin{array}{l}\text { Magnetici } \\
\text { Susceptibility }\end{array}$ & & & & & 12/27/2017 \\
\hline $\begin{array}{l}\text { not for } \\
\text { curation }\end{array}$ & $\begin{array}{c}\text { not for } \\
\text { curation }\end{array}$ & 684 & - & - & MS-46 & A & vi & No996 & E1014 & $\begin{array}{c}\text { North wall } \\
\text { profile } \\
\end{array}$ & 98.17 & 153.5 & - & $\begin{array}{l}\begin{array}{l}\text { Geoarch } \\
\text { Sample }\end{array} \\
\text { Sal }\end{array}$ & Soil & $\begin{array}{c}\text { Magnentic } \\
\text { Susceptibility }\end{array}$ & & & & & $12 / 27 / 2017$ \\
\hline \begin{tabular}{|c|} 
not for \\
curation
\end{tabular} & $\begin{array}{l}\text { not for } \\
\text { curation }\end{array}$ & 684 & - & - & MS-47 & A & vi & No996 & E1014 & $\begin{array}{c}\text { North wall } \\
\text { profile }\end{array}$ & 98.14 & 156 & - & 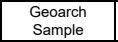 & Soil & $\begin{array}{l}\text { Magnetici } \\
\text { Susceptibility }\end{array}$ & & & & & 12/27/2017 \\
\hline $\begin{array}{l}\text { not for } \\
\text { curation }\end{array}$ & $\begin{array}{l}\text { not for } \\
\text { curation }\end{array}$ & 684 & - & - & MS- 48 & $\mathrm{~A}$ & $\mathrm{vi}$ & No996 & E1014 & $\begin{array}{c}\text { North wall } \\
\text { profile }\end{array}$ & 98.1 & 160 & - & $\begin{array}{l}\text { Geoarch } \\
\text { Sample }\end{array}$ & Soil & $\begin{array}{l}\text { Magneticic } \\
\text { Susceptibity }\end{array}$ & & & & & 12/27/2017 \\
\hline $\begin{array}{l}\text { not tor } \\
\text { curation }\end{array}$ & $\begin{array}{c}\text { not for } \\
\text { curation }\end{array}$ & 684 & - & - & MS-49 & A & vi & N0996 & E1014 & $\begin{array}{c}\text { North wall } \\
\text { profile }\end{array}$ & 98.07 & 163 & - & $\begin{array}{l}\begin{array}{c}\text { Geoarch } \\
\text { Sample }\end{array} \\
\end{array}$ & Soil & $\begin{array}{l}\text { Magnetic } \\
\text { Susceptibility }\end{array}$ & & & & & 12/27/2017 \\
\hline
\end{tabular}




\begin{tabular}{|c|c|c|c|c|c|c|c|c|c|c|c|c|c|c|c|c|c|c|c|c|c|}
\hline Lot No. & \begin{tabular}{|c|}
$\begin{array}{c}\text { Specimen } \\
\text { No. }\end{array}$ \\
\end{tabular} & FS No. & UI No. & FTR No. & $\begin{array}{c}\text { Sample } \\
\text { No. }\end{array}$ & $\begin{array}{c}\text { Excavation } \\
\text { Block }\end{array}$ & Stratum (AU) & Northing & Easting & Level & $\begin{array}{c}\begin{array}{c}\text { Elevation } \\
(\mathrm{m})\end{array} \\
\end{array}$ & Depth (cmbd) & Point Prov. & Artifact Class & Artifact Type & $\begin{array}{c}\text { Artifact } \\
\text { Description }\end{array}$ & Material & $\begin{array}{c}\text { Number of } \\
\text { artifacts }\end{array}$ & Weight (g) & Comments & Date \\
\hline $\begin{array}{l}\text { not for } \\
\text { curation }\end{array}$ & $\begin{array}{c}\text { not for } \\
\text { curation }\end{array}$ & 684 & - & - & MS-50 & A & vi & No996 & E1014 & \begin{tabular}{|c|}
$\begin{array}{c}\text { North wall } \\
\text { profili }\end{array}$ \\
s.
\end{tabular} & 98.03 & 167 & - & $\begin{array}{l}\text { Geoarch } \\
\text { Sample }\end{array}$ & Soil & $\begin{array}{l}\text { Magnetic } \\
\text { Susceptibility }\end{array}$ & & & & & 12/27/2017 \\
\hline \begin{tabular}{c|} 
not for \\
curation
\end{tabular} & $\begin{array}{l}\begin{array}{l}\text { not for } \\
\text { curation }\end{array} \\
\end{array}$ & 684 & - & - & MS-51 & A & vi & No996 & E1014 & $\begin{array}{l}\begin{array}{c}\text { North wall } \\
\text { profile }\end{array} \\
\end{array}$ & 98 & 170 & - & $\begin{array}{l}\text { Geoarch } \\
\text { Sample }\end{array}$ & Soil & $\begin{array}{l}\text { Magnetic } \\
\text { Susceptibility }\end{array}$ & & & & & 12/27/2017 \\
\hline \begin{tabular}{|l|} 
not for \\
curation \\
\end{tabular} & $\begin{array}{l}\begin{array}{l}\text { not for } \\
\text { curation }\end{array} \\
\end{array}$ & 684 & - & - & MS-52 & A & vi & No996 & E1014 & $\begin{array}{c}\text { North wall } \\
\text { profile }\end{array}$ & 97.95 & 175 & - & $\begin{array}{l}\text { Geaarch } \\
\text { Sample }\end{array}$ & Soil & $\begin{array}{l}\text { Magnentic } \\
\text { Susceptibility }\end{array}$ & & & & & 12/27/2017 \\
\hline 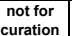 & $\begin{array}{l}\text { not for } \\
\text { curation }\end{array}$ & 684 & - & - & MS-53 & A & vi & No996 & E1014 & $\begin{array}{l}\text { North wall } \\
\text { proflie }\end{array}$ & 97.94 & 176 & - & $\begin{array}{l}\text { Geoarch } \\
\text { Sample }\end{array}$ & Soil & $\begin{array}{l}\text { Magnetic } \\
\text { Suscoptibitit }\end{array}$ & & & & & 12/27/2017 \\
\hline $\begin{array}{l}\text { not for } \\
\text { curation }\end{array}$ & $\begin{array}{l}\text { not for } \\
\text { curation }\end{array}$ & 684 & - & - & MS-54 & A & vi & No996 & E1014 & $\begin{array}{c}\text { North wall } \\
\text { profile }\end{array}$ & 97.9 & 180 & - & $\begin{array}{l}\text { Geaarch } \\
\text { Sample }\end{array}$ & Soil & $\begin{array}{l}\text { Magnetic } \\
\text { Susceptibility }\end{array}$ & & & & & $\mid$\begin{tabular}{|l|}
$\mid 12 / 27 / 2017$ \\
\end{tabular} \\
\hline $\begin{array}{l}\text { not for } \\
\text { curation }\end{array}$ & $\begin{array}{l}\text { not for } \\
\text { curation }\end{array}$ & 684 & - & - & MS- 55 & A & vi & No996 & E1014 & $\begin{array}{c}\begin{array}{c}\text { North wall } \\
\text { profile }\end{array} \\
\end{array}$ & 97.9 & 180 & - & $\begin{array}{l}\text { Geoarch } \\
\text { Sample }\end{array}$ & Soil & $\begin{array}{l}\text { Magnetic } \\
\text { Susceptibility }\end{array}$ & & & & & 12/27/2017 \\
\hline \begin{tabular}{l|} 
not for \\
curation
\end{tabular} & $\begin{array}{l}\text { not for } \\
\text { curation }\end{array}$ & 684 & - & - & MS-56 & A & vi & No996 & E1014 & $\begin{array}{l}\text { North wall } \\
\text { profile }\end{array}$ & 97.86 & 184 & - & $\begin{array}{l}\text { Geoarch } \\
\text { Sample }\end{array}$ & Soil & $\begin{array}{l}\text { Maganetic } \\
\text { Suscoptibility }\end{array}$ & & & & & $\mid$\begin{tabular}{|l|}
$\mid 12 / 27 / 2017$ \\
\end{tabular} \\
\hline $\begin{array}{l}\text { not for } \\
\text { curation }\end{array}$ & $\begin{array}{l}\text { not for } \\
\text { curtition }\end{array}$ & 684 & - & - & MS-57 & A & vi & No996 & E1014 & $\begin{array}{l}\text { North wall } \\
\text { profile }\end{array}$ & 97.84 & 186 & - & $\begin{array}{l}\text { Sampie } \\
\text { Geaarch } \\
\text { Sample }\end{array}$ & Soil & $\begin{array}{c}\text { Susceprominy } \\
\text { Muscentic } \\
\text { Suscopibility }\end{array}$ & & & & & $\mid$\begin{tabular}{|l|}
$\mid 12 / 27 / 2017$ \\
\end{tabular} \\
\hline $\begin{array}{l}\text { not tor } \\
\text { curation }\end{array}$ & $\begin{array}{l}\begin{array}{l}\text { not for } \\
\text { curation }\end{array} \\
\end{array}$ & 684 & - & - & MS-58 & A & vi & No996 & E1014 & $\begin{array}{c}\text { North wall } \\
\text { profile }\end{array}$ & 97.81 & 189 & - & $\begin{array}{l}\text { Geoarch } \\
\text { Sample }\end{array}$ & Soil & $\begin{array}{l}\text { Magnetic } \\
\text { Susceptibility }\end{array}$ & & & & & \begin{tabular}{|l|}
$12 / 27 / 2017$ \\
\end{tabular} \\
\hline $\begin{array}{l}\text { not for } \\
\text { curation }\end{array}$ & $\begin{array}{l}\begin{array}{l}\text { not for } \\
\text { curation }\end{array} \\
\end{array}$ & 684 & - & - & MS-59 & A & vi & No996 & E1014 & $\begin{array}{c}\text { North wall } \\
\text { profile }\end{array}$ & 97.78 & 192 & - & $\begin{array}{l}\text { Geoarch } \\
\text { Sample }\end{array}$ & Soil & $\begin{array}{l}\text { Magnetic } \\
\text { Susceptibility }\end{array}$ & & & & & \begin{tabular}{|l|l|}
$12 / 27 / 2017$ \\
\end{tabular} \\
\hline \begin{tabular}{l|} 
not for \\
curation
\end{tabular} & $\begin{array}{l}\text { not for } \\
\text { curation }\end{array}$ & 684 & - & - & MS-60 & A & vi & No996 & E1014 & $\begin{array}{c}\text { North wall } \\
\text { profile }\end{array}$ & 97.75 & 195 & - & $\begin{array}{l}\text { Geoarch } \\
\text { Sample }\end{array}$ & Soil & $\begin{array}{l}\text { Magnetic } \\
\text { Susceptibility }\end{array}$ & & & & & $\mid$\begin{tabular}{|l|}
$\mid 12 / 27 / 2017$ \\
\end{tabular} \\
\hline $\begin{array}{l}\text { not for } \\
\text { curation }\end{array}$ & $\begin{array}{l}\text { not for } \\
\text { curation }\end{array}$ & 684 & - & - & MS-61 & A & vi & No996 & E1014 & $\begin{array}{c}\begin{array}{c}\text { North wall } \\
\text { profile }\end{array} \\
\end{array}$ & 97.72 & 198 & - & $\begin{array}{l}\text { Geoarch } \\
\text { Sample }\end{array}$ & Soil & $\begin{array}{l}\text { Magnemic } \\
\text { Susceptibility } \\
\end{array}$ & & & & & $\mid$\begin{tabular}{|l|}
$\mid 12 / 27 / 2017$ \\
\end{tabular} \\
\hline $\begin{array}{l}\text { not for } \\
\text { curation }\end{array}$ & $\begin{array}{l}\text { not for } \\
\text { curation }\end{array}$ & 684 & - & - & MS-62 & A & vi & No996 & E1014 & $\begin{array}{c}\begin{array}{c}\text { North wall } \\
\text { profile }\end{array} \\
\end{array}$ & 97.69 & 201 & - & $\begin{array}{l}\begin{array}{l}\text { Geoarch } \\
\text { Sample }\end{array} \\
\end{array}$ & Soil & $\begin{array}{l}\text { Magnetic } \\
\text { Susceptibility }\end{array}$ & & & & & 12/27/2017 \\
\hline $\begin{array}{l}\text { not for } \\
\text { curation }\end{array}$ & $\begin{array}{l}\text { not for } \\
\text { curation }\end{array}$ & 684 & - & - & MS-63 & A & vi & No996 & E1014 & \begin{tabular}{|c|}
$\begin{array}{c}\text { North wall } \\
\text { profile }\end{array}$ \\
\end{tabular} & 97.65 & 205 & - & $\begin{array}{l}\begin{array}{l}\text { Geoarch } \\
\text { Sample }\end{array} \\
\end{array}$ & Soil & $\begin{array}{l}\text { Magnetic } \\
\text { Susceptibility }\end{array}$ & & & & & \begin{tabular}{|l|l|}
$12 / 27 / 2017$ \\
\end{tabular} \\
\hline $\begin{array}{l}\text { not for } \\
\text { curation }\end{array}$ & $\begin{array}{l}\text { not for } \\
\text { curation }\end{array}$ & 684 & - & - & MS-64 & A & vi & No996 & E1014 & $\begin{array}{c}\text { North wall } \\
\text { profile }\end{array}$ & 97.62 & 208 & - & $\begin{array}{l}\text { Geoarch } \\
\text { Sample }\end{array}$ & Soil & $\begin{array}{l}\text { Magnetic } \\
\text { Susceptibility } \\
\end{array}$ & & & & & 12/27/2017 \\
\hline $\begin{array}{l}\text { not for } \\
\text { curation }\end{array}$ & $\begin{array}{l}\text { not for } \\
\text { curation }\end{array}$ & 684 & - & - & BM-01 & A & vi & No996 & E1014 & $\begin{array}{l}\begin{array}{l}\text { North wall } \\
\text { profile }\end{array} \\
\end{array}$ & \begin{tabular}{|l|}
$98.07-97.98$ \\
\end{tabular} & 163-172 & - & $\begin{array}{l}\text { Geoarch } \\
\text { Sample }\end{array}$ & Soil & Bulk Matrix & & & & & 12/27/2017 \\
\hline $\begin{array}{l}\text { not for } \\
\text { curation }\end{array}$ & $\begin{array}{l}\begin{array}{l}\text { not for } \\
\text { curation }\end{array} \\
\end{array}$ & 684 & - & - & BM-02 & A & $\mathrm{vv}_{1}$ & No996 & E1014 & $\begin{array}{c}\begin{array}{c}\text { North wall } \\
\text { profile }\end{array} \\
\end{array}$ & \begin{tabular}{|l|}
$98.08-97.96$ \\
\end{tabular} & $162-174$ & - & $\begin{array}{l}\text { Gampiech } \\
\text { Geaarple } \\
\text { Samper }\end{array}$ & Soil & Bulk Matrix & & & & & 12/27/2017 \\
\hline \begin{tabular}{l|} 
not for \\
curation
\end{tabular} & $\begin{array}{l}\text { not for } \\
\text { curation }\end{array}$ & 684 & - & - & BM-03 & A & $V_{N I}$ & No996 & E1014 & $\begin{array}{l}\begin{array}{c}\text { North wall } \\
\text { profile }\end{array} \\
\end{array}$ & \begin{tabular}{|l|}
$98.36-98.28$ \\
\end{tabular} & 134-142 & - & $\begin{array}{l}\text { Geoarch } \\
\text { Sample }\end{array}$ & Soil & Bulk Matrix & & & & & 12/27/2017 \\
\hline \begin{tabular}{l|} 
not for \\
curation
\end{tabular} & $\begin{array}{l}\begin{array}{l}\text { not for } \\
\text { curation }\end{array} \\
\end{array}$ & 684 & - & - & BM-04 & A & IVN & No996 & E1014 & $\begin{array}{l}\text { North wall } \\
\text { profile }\end{array}$ & 98.48-98.39 & $122-131$ & - & $\begin{array}{l}\text { Geoarch } \\
\text { Sample }\end{array}$ & Soil & Bulk Matrix & & & & & 12/27/2017 \\
\hline \begin{tabular}{l|} 
not for \\
curation
\end{tabular} & $\begin{array}{l}\text { not for } \\
\text { curation }\end{array}$ & 684 & - & - & BM-05 & A & IV & No996 & E1014 & $\begin{array}{c}\text { North wall } \\
\text { profile }\end{array}$ & 98.71-98.61 & 99-109 & - & $\begin{array}{l}\text { Geoarch } \\
\text { Sample }\end{array}$ & Soil & Bulk Matrix & & & & & 12/27/2017 \\
\hline \begin{tabular}{|c|} 
not for \\
curation
\end{tabular} & $\begin{array}{l}\text { not for } \\
\text { curation }\end{array}$ & 684 & - & - & BM-06 & A & IV & No996 & E1014 & $\begin{array}{c}\text { North wall } \\
\text { profile }\end{array}$ & 98.68-98.58 & 102-112 & - & $\begin{array}{l}\text { Geoarch } \\
\text { Sample }\end{array}$ & Soil & Bulk Matrix & & & & & 121/27/2017 \\
\hline \begin{tabular}{c|} 
not for \\
curation
\end{tabular} & $\begin{array}{l}\text { not for } \\
\text { curation }\end{array}$ & 684 & - & - & BM-07 & A & III & No996 & E1014 & $\begin{array}{c}\begin{array}{c}\text { North wall } \\
\text { profile }\end{array} \\
\end{array}$ & 99.07-99.0 & $63-70$ & - & $\begin{array}{l}\text { Geoarch } \\
\text { Sample }\end{array}$ & Soil & Bulk Matrix & & & & & 121/27/2017 \\
\hline \begin{tabular}{|c|} 
not for \\
curation
\end{tabular} & $\begin{array}{c}\text { not for } \\
\text { curation }\end{array}$ & 684 & - & - & BM-08 & A & IIII & $\begin{array}{l}\text { No996 } \\
\end{array}$ & E1014 & $\begin{array}{c}\text { North wall } \\
\text { profile }\end{array}$ & 99.01-98.925 & $69-77.5$ & - & $\begin{array}{l}\text { Geoarch } \\
\text { Sample }\end{array}$ & Soil & Bulk Matrix & & & & & 12/27/2017 \\
\hline \begin{tabular}{|c|} 
not for \\
curation
\end{tabular} & $\begin{array}{c}\text { not for } \\
\text { curation }\end{array}$ & 684 & - & - & BM-09 & A & ॥ & No996 & E1014 & $\begin{array}{c}\begin{array}{c}\text { North wall } \\
\text { profile }\end{array} \\
\end{array}$ & $99.415-99.31$ & $28.5-39$ & - & $\begin{array}{l}\text { Geoarch } \\
\text { Sample }\end{array}$ & Soil & Bulk Matrix & & & & & 12/27/2017 \\
\hline $\begin{array}{c}\text { not tor } \\
\text { curation }\end{array}$ & 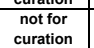 & 684 & - & - & BM-10 & A & "11 & No996 & E1014 & $\begin{array}{c}\text { North wall } \\
\text { profile }\end{array}$ & 99.335-99.26 & $36.5-44$ & - & $\begin{array}{l}\text { Geoarch } \\
\text { Sample }\end{array}$ & Soil & Bulk Matrix & & & & & 12/27/2017 \\
\hline $\begin{array}{l}\text { not for } \\
\text { curation }\end{array}$ & 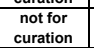 & 684 & - & - & BM-11 & A & 1 & No996 & E1014 & $\begin{array}{c}\text { North wall } \\
\text { profile }\end{array}$ & 99.55-99.52 & $15-18$ & - & $\begin{array}{l}\text { Geoarch } \\
\text { Sample }\end{array}$ & Soil & Bulk Matrix & & & & & 12/27/2017 \\
\hline \begin{tabular}{|c|} 
not for \\
curation
\end{tabular} & $\begin{array}{l}\begin{array}{l}\text { not for } \\
\text { curation }\end{array} \\
\text { cons }\end{array}$ & 684 & - & - & BM-12 & A & $\mathrm{vI}$ & No996 & E1014 & $\begin{array}{c}\begin{array}{c}\text { North wall } \\
\text { profile }\end{array} \\
\end{array}$ & \begin{tabular}{|l|}
$97.72-97.62$ \\
\end{tabular} & $\begin{array}{l}198-208 \\
\end{array}$ & - & $\begin{array}{l}\text { Geoarch } \\
\text { Sample }\end{array}$ & Soil & Bulk Matrix & & & & & 12/27/2017 \\
\hline \begin{tabular}{|c|} 
not for \\
curation
\end{tabular} & $\begin{array}{l}\begin{array}{l}\text { not for } \\
\text { curation }\end{array} \\
\text { cons }\end{array}$ & 685 & - & - & MS-01 & c & 1 & N1000 & E1004 & $\begin{array}{c}\text { South wall } \\
\text { profile }\end{array}$ & 99.74 & 16 & - & $\begin{array}{l}\begin{array}{l}\text { Geoarch } \\
\text { Sample }\end{array} \\
\text { Sals }\end{array}$ & Soil & $\begin{array}{c}\text { Magneticic } \\
\text { Susceptibility }\end{array}$ & & & & & $12 / 29 / 2017$ \\
\hline $\begin{array}{c}\text { not for } \\
\text { curation }\end{array}$ & $\begin{array}{l}\text { not for } \\
\text { curation }\end{array}$ & 685 & - & - & MS-02 & c & 1 & N1000 & E1004 & $\begin{array}{l}\text { South wall } \\
\text { profile }\end{array}$ & 99.72 & 18 & - & $\begin{array}{l}\text { Geoarch } \\
\text { Sample }\end{array}$ & Soil & $\begin{array}{l}\text { Magnetic } \\
\text { Susceptiility }\end{array}$ & & & & & 12/29/2017 \\
\hline $\begin{array}{l}\text { not for } \\
\text { curation }\end{array}$ & $\begin{array}{c}\text { not for } \\
\text { curation }\end{array}$ & 685 & - & - & MS-03 & c & " & N1000 & E1004 & $\begin{array}{c}\text { South wall } \\
\text { profile }\end{array}$ & 99.69 & 21 & - & $\begin{array}{l}\text { Geoarch } \\
\text { Sample }\end{array}$ & Soil & $\begin{array}{l}\text { Magnetic } \\
\text { Susceptibility }\end{array}$ & & & & & 12/299/2017 \\
\hline $\begin{array}{c}\text { not for } \\
\text { curation }\end{array}$ & $\begin{array}{l}\text { not for } \\
\text { curation }\end{array}$ & 685 & - & - & MS- 04 & $\mathrm{c}$ & " & N1000 & E1004 & $\begin{array}{c}\text { South wall } \\
\text { profile }\end{array}$ & 99.63 & 27 & - & 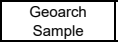 & Soil & $\begin{array}{l}\text { Magnetic } \\
\text { Susceptibility }\end{array}$ & & & & & $12 / 29 / 2017$ \\
\hline \begin{tabular}{|c|} 
not for \\
curation
\end{tabular} & $\begin{array}{l}\begin{array}{l}\text { not for } \\
\text { curation }\end{array} \\
\text { count }\end{array}$ & 685 & - & - & MS-05 & c & "I & N1000 & E1004 & $\begin{array}{c}\text { South wall } \\
\text { profile }\end{array}$ & 99.6 & 30 & - & $\begin{array}{l}\text { Geaarch } \\
\text { Sample }\end{array}$ & Soil & $\begin{array}{l}\text { Magnetic } \\
\text { Susceptibility }\end{array}$ & & & & & $12 / 29 / 2017$ \\
\hline $\begin{array}{c}\text { not tor } \\
\text { curation }\end{array}$ & $\begin{array}{l}\begin{array}{l}\text { not for } \\
\text { curation }\end{array} \\
\text { cons }\end{array}$ & 685 & - & - & MS-06 & c & "I & N1000 & E1004 & $\begin{array}{l}\text { South wall } \\
\text { profile }\end{array}$ & 99.53 & 37 & - & $\begin{array}{l}\text { Geoarch } \\
\text { Sample }\end{array}$ & Soil & $\begin{array}{l}\text { Magnetic } \\
\text { Susceptibility }\end{array}$ & & & & & 12/29/2017 \\
\hline $\begin{array}{c}\text { not for } \\
\text { curation }\end{array}$ & 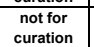 & 685 & - & - & MS-07 & c & "I & N1000 & E1004 & $\begin{array}{c}\text { South wall } \\
\text { profile }\end{array}$ & 99.48 & 42 & - & $\begin{array}{l}\text { Geoarch } \\
\text { Sample }\end{array}$ & Soil & $\begin{array}{l}\text { Magnetic } \\
\text { Susceptibility }\end{array}$ & & & & & 12/29/2017 \\
\hline $\begin{array}{l}\text { not for } \\
\text { curation }\end{array}$ & $\begin{array}{l}\begin{array}{l}\text { not for } \\
\text { curation }\end{array} \\
\text { cons }\end{array}$ & 685 & - & - & MS- 08 & c & III' & N1000 & E1004 & $\begin{array}{c}\text { South wall } \\
\text { profile }\end{array}$ & 99.43 & 47 & - & $\begin{array}{l}\text { Geoarch } \\
\text { Sample }\end{array}$ & Soil & $\begin{array}{l}\text { Magnentic } \\
\text { Susceptibility }\end{array}$ & & & & & 12/29/2017 \\
\hline \begin{tabular}{l|} 
not for \\
curation
\end{tabular} & $\begin{array}{l}\begin{array}{l}\text { not for } \\
\text { curation }\end{array} \\
\text { cons }\end{array}$ & 685 & - & - & MS-09 & c & III & N1000 & E1004 & $\begin{array}{c}\text { South wall } \\
\text { profile }\end{array}$ & 99.37 & 53 & - & $\begin{array}{l}\text { Geoarch } \\
\text { Sample }\end{array}$ & Soil & $\begin{array}{l}\text { Magnetic } \\
\text { Susceptibility }\end{array}$ & & & & & $\mid$\begin{tabular}{|l}
$\mid 12 / 29 / 2017$ \\
\end{tabular} \\
\hline \begin{tabular}{|c|} 
not for \\
curation
\end{tabular} & $\begin{array}{l}\begin{array}{l}\text { not for } \\
\text { curation }\end{array} \\
\text { count }\end{array}$ & 685 & - & - & MS-10 & c & III & N1000 & E1004 & $\begin{array}{c}\text { South wall } \\
\text { profile }\end{array}$ & 99.32 & 58 & - & $\begin{array}{l}\text { Geourch } \\
\text { Sample }\end{array}$ & Soil & $\begin{array}{l}\text { Magnetic } \\
\text { Susceptibitity }\end{array}$ & & & & & 12/29/2017 \\
\hline \begin{tabular}{|c|} 
not for \\
curation
\end{tabular} & $\begin{array}{l}\text { not for } \\
\text { curation }\end{array}$ & 685 & - & - & MS-11 & $\mathrm{c}$ & III & N1000 & E1004 & $\begin{array}{c}\text { South wall } \\
\text { profile }\end{array}$ & $\begin{array}{l}99.3 \\
\end{array}$ & 60 & - & $\begin{array}{l}\text { Geoarch } \\
\text { Sample }\end{array}$ & Soil & $\begin{array}{l}\text { Magnetici } \\
\text { Susceptibility }\end{array}$ & & & & & $12 / 29 / 2017$ \\
\hline $\begin{array}{l}\text { not for } \\
\text { curation }\end{array}$ & $\begin{array}{c}\text { not for } \\
\text { curation }\end{array}$ & 685 & - & - & MS-12 & c & III & N1000 & E1004 & $\begin{array}{c}\text { South wall } \\
\text { profile }\end{array}$ & 99.26 & 64 & - & $\begin{array}{l}\begin{array}{l}\text { Geoarch } \\
\text { Sample }\end{array} \\
\text { Sal }\end{array}$ & Soil & $\begin{array}{l}\text { Magnetic } \\
\text { Susceptibility }\end{array}$ & & & & & $12 / 29 / 2017$ \\
\hline \begin{tabular}{|c|} 
not for \\
curation
\end{tabular} & $\begin{array}{l}\text { not for } \\
\text { curation }\end{array}$ & 685 & - & - & MS-13 & $\mathrm{c}$ & III & N1000 & E1004 & $\begin{array}{c}\text { South wall } \\
\text { profile }\end{array}$ & 99.23 & 67 & - & 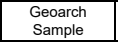 & Soil & $\begin{array}{l}\text { Magnetici } \\
\text { Susceptibility }\end{array}$ & & & & & 12/29/2017 \\
\hline $\begin{array}{l}\text { not for } \\
\text { curation }\end{array}$ & $\begin{array}{l}\text { not for } \\
\text { curation }\end{array}$ & 685 & - & - & MS-14 & $\mathrm{c}$ & III & N1000 & E1004 & $\begin{array}{c}\begin{array}{c}\text { South wall } \\
\text { profile }\end{array} \\
\end{array}$ & 99.18 & 72 & - & $\begin{array}{l}\text { Geoarch } \\
\text { Sample }\end{array}$ & Soil & $\begin{array}{l}\text { Magneticic } \\
\text { Susceptibity }\end{array}$ & & & & & 12/29/2017 \\
\hline $\begin{array}{l}\text { not tor } \\
\text { curation }\end{array}$ & $\begin{array}{c}\text { not for } \\
\text { curation }\end{array}$ & 685 & - & - & MS-15 & c & III & N1000 & E1004 & $\begin{array}{c}\text { South wall } \\
\text { profile }\end{array}$ & 99.13 & 77 & - & $\begin{array}{l}\begin{array}{c}\text { Geoarch } \\
\text { Sample }\end{array} \\
\end{array}$ & Soil & $\begin{array}{l}\text { Magnetic } \\
\text { Susceptibility }\end{array}$ & & & & & 12/29/2017 \\
\hline
\end{tabular}




\begin{tabular}{|c|c|c|c|c|c|c|c|c|c|c|c|c|c|c|c|c|c|c|c|c|c|}
\hline Lot No. & $\begin{array}{c}\text { Specimen } \\
\text { No. }\end{array}$ & FS No. & UI No. & FTR No. & $\begin{array}{c}\begin{array}{c}\text { Sample } \\
\text { No. }\end{array} \\
\end{array}$ & $\begin{array}{c}\text { Excavation } \\
\text { Block }\end{array}$ & Stratum (AU) & Northing & Easting & Level & $\begin{array}{c}\text { Elevation } \\
(\mathrm{m})\end{array}$ & Depth (cmbd) & Point Prov. & Artifact Class & Artifiact Type & $\begin{array}{c}\text { Artifact } \\
\text { Description }\end{array}$ & Material & $\begin{array}{c}\text { Number of } \\
\text { artifacts }\end{array}$ & Weight (g) & Comments & Date \\
\hline $\begin{array}{c}\text { not for } \\
\text { curation }\end{array}$ & $\begin{array}{c}\text { not for } \\
\text { curation }\end{array}$ & 685 & - & - & MS-16 & c & III & N1000 & E1004 & $\begin{array}{c}\text { South wall } \\
\text { profile }\end{array}$ & 99.07 & 83 & - & $\begin{array}{l}\text { Geoarch } \\
\text { Sample }\end{array}$ & Soil & $\begin{array}{l}\text { Magnetic } \\
\text { Susceptibility } \\
\end{array}$ & & & & & 12/29/2017 \\
\hline \begin{tabular}{l|} 
not for \\
curation
\end{tabular} & $\begin{array}{l}\text { not for } \\
\text { curation }\end{array}$ & 685 & - & - & MS-17 & c & IV & N1000 & E1004 & $\begin{array}{c}\begin{array}{c}\text { South wall } \\
\text { profile }\end{array} \\
\text { pol }\end{array}$ & 99.01 & 89 & - & $\begin{array}{l}\begin{array}{l}\text { Geoarch } \\
\text { Sample }\end{array} \\
\text { Sallo }\end{array}$ & Soil & $\begin{array}{l}\text { Magnetic } \\
\text { Susceptibility }\end{array}$ & & & & & 12/29/2017 \\
\hline $\begin{array}{l}\text { not for } \\
\text { curation }\end{array}$ & $\begin{array}{l}\text { not for } \\
\text { curation }\end{array}$ & 685 & - & - & MS-18 & c & IV & N1000 & E1004 & $\begin{array}{c}\text { South wall } \\
\text { profile }\end{array}$ & 98.94 & 96 & - & $\begin{array}{l}\text { Geoarch } \\
\text { Sample }\end{array}$ & Soil & $\begin{array}{l}\text { Magnetic } \\
\text { Susceptibility }\end{array}$ & & & & & 12/29/2017 \\
\hline $\begin{array}{l}\text { not for } \\
\text { curation }\end{array}$ & $\begin{array}{l}\text { not for } \\
\text { curation }\end{array}$ & 685 & - & - & MS-19 & c & iv & N1000 & E1004 & $\begin{array}{c}\begin{array}{c}\text { South wall } \\
\text { profile }\end{array} \\
\text { (a) }\end{array}$ & 98.91 & 99 & - & $\begin{array}{l}\text { Geoarch } \\
\text { Sample }\end{array}$ & Soil & $\begin{array}{l}\text { Magneticic } \\
\text { Susceptibility }\end{array}$ & & & & & \begin{tabular}{|l|}
$12 / 29 / 2017$ \\
\end{tabular} \\
\hline $\begin{array}{l}\text { not for } \\
\text { curation }\end{array}$ & $\begin{array}{l}\text { not for } \\
\text { curation }\end{array}$ & 685 & - & - & MS-20 & c & v & N1000 & E1004 & $\begin{array}{c}\text { South wall } \\
\text { profile }\end{array}$ & 98.83 & 107 & - & $\begin{array}{l}\text { Geoarch } \\
\text { Sample }\end{array}$ & Soil & $\begin{array}{l}\text { Magnetic } \\
\text { Susceptibility }\end{array}$ & & & & & $\mid$\begin{tabular}{|l|}
$\mid 12 / 29 / 2017$ \\
\end{tabular} \\
\hline $\begin{array}{l}\begin{array}{c}\text { not for } \\
\text { curation }\end{array} \\
\end{array}$ & $\begin{array}{l}\text { not for } \\
\text { curation }\end{array}$ & 685 & - & - & MS-21 & c & vi & N1000 & E1004 & $\begin{array}{c}\begin{array}{c}\text { South wall } \\
\text { profile }\end{array} \\
\end{array}$ & 98.78 & 112 & - & $\begin{array}{l}\text { Geoarch } \\
\text { Sample }\end{array}$ & Soil & $\begin{array}{l}\text { Magnetic } \\
\text { Susceptibility } \\
\end{array}$ & & & & & 12/29/2017 \\
\hline $\begin{array}{l}\text { not for } \\
\text { curation }\end{array}$ & $\begin{array}{l}\text { not for } \\
\text { curation }\end{array}$ & 685 & - & - & MS-22 & c & vi & N1000 & E1004 & \begin{tabular}{|c|}
$\begin{array}{c}\text { South wall } \\
\text { profile }\end{array}$ \\
\end{tabular} & 98.71 & 119 & - & 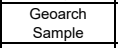 & Soil & $\begin{array}{l}\text { Magnetic } \\
\text { Susceptibility } \\
\text { Sulate }\end{array}$ & & & & & 12/29/2017 \\
\hline $\begin{array}{c}\text { not tor } \\
\text { curation }\end{array}$ & $\begin{array}{l}\text { not for } \\
\text { curation }\end{array}$ & 685 & - & - & MS-23 & c & vi & N1000 & E1004 & $\begin{array}{l}\text { South wall } \\
\text { profile }\end{array}$ & 98.66 & 124 & - & $\begin{array}{l}\text { Geoarch } \\
\text { Sample }\end{array}$ & Soil & $\begin{array}{l}\text { Magnentic } \\
\text { Susceptibility }\end{array}$ & & & & & 12/29/2017 \\
\hline $\begin{array}{l}\text { not for } \\
\text { curation }\end{array}$ & $\begin{array}{l}\text { not for } \\
\text { curation }\end{array}$ & 685 & - & - & MS-24 & c & vi & N1000 & E1004 & $\begin{array}{c}\text { South wall } \\
\text { profile }\end{array}$ & 98.6 & 130 & - & $\begin{array}{l}\text { Geoarch } \\
\text { Sample }\end{array}$ & Soil & $\begin{array}{l}\text { Magnemici } \\
\text { Susceptibility }\end{array}$ & & & & & $\mid$\begin{tabular}{|l|}
$\mid 12 / 29 / 2017$ \\
\end{tabular} \\
\hline $\begin{array}{l}\text { not for } \\
\text { curation }\end{array}$ & $\begin{array}{l}\text { not for } \\
\text { curation }\end{array}$ & 685 & - & - & MS-25 & c & vi & N1000 & E1004 & $\begin{array}{c}\text { South wall } \\
\text { profile }\end{array}$ & 98.55 & 135 & - & $\begin{array}{l}\text { Geoarch } \\
\text { Sample }\end{array}$ & Soil & $\begin{array}{l}\text { Magnemici } \\
\text { Susceptibility }\end{array}$ & & & & & 12/29/2017 \\
\hline $\begin{array}{l}\text { not for } \\
\text { curation }\end{array}$ & $\begin{array}{l}\text { not for } \\
\text { curation }\end{array}$ & 685 & - & - & MS-26 & c & vi & N1000 & E1004 & $\begin{array}{c}\text { South wall } \\
\text { profile }\end{array}$ & 98.49 & 141 & - & $\begin{array}{l}\text { Geoarch } \\
\text { Sample }\end{array}$ & Soil & $\begin{array}{l}\text { Magnetic } \\
\text { Susceptibility }\end{array}$ & & & & & $\mid$\begin{tabular}{|l|}
$\mid 12 / 29 / 2017$ \\
\end{tabular} \\
\hline $\begin{array}{l}\text { not for } \\
\text { curation }\end{array}$ & $\begin{array}{l}\text { not for } \\
\text { curation }\end{array}$ & 685 & - & - & MS-27 & c & vi & N1000 & E1004 & $\begin{array}{c}\text { South wall } \\
\text { profile }\end{array}$ & 98.45 & 145 & - & $\begin{array}{l}\text { Geoarch } \\
\text { Sample }\end{array}$ & Soil & $\begin{array}{l}\text { Magneticic } \\
\text { Susceptibility }\end{array}$ & & & & & 12/29/2017 \\
\hline $\begin{array}{l}\text { not for } \\
\text { curation }\end{array}$ & $\begin{array}{l}\text { not for } \\
\text { curation }\end{array}$ & 685 & - & - & MS-28 & c & vi & N1000 & E1004 & $\begin{array}{c}\begin{array}{c}\text { South wall } \\
\text { profile }\end{array} \\
\end{array}$ & 98.42 & 148 & - & 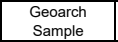 & Soil & $\begin{array}{l}\text { Magnetic } \\
\text { Susceptibility } \\
\end{array}$ & & & & & $\mid$\begin{tabular}{|l|}
$\mid 12 / 29 / 2017$ \\
\end{tabular} \\
\hline $\begin{array}{l}\text { not for } \\
\text { curation }\end{array}$ & $\begin{array}{l}\text { not for } \\
\text { curation }\end{array}$ & 685 & - & - & MS-29 & c & vi & N1000 & E1004 & \begin{tabular}{|c|} 
South wall \\
profile
\end{tabular} & 98.36 & 154 & - & $\begin{array}{l}\begin{array}{l}\text { Geoarch } \\
\text { Sample }\end{array} \\
\end{array}$ & Soil & $\begin{array}{l}\text { Magnetic } \\
\text { Susceptibility }\end{array}$ & & & & & 12/29/2017 \\
\hline $\begin{array}{l}\text { not for } \\
\text { curation }\end{array}$ & $\begin{array}{l}\text { not for } \\
\text { curation }\end{array}$ & 685 & - & - & BM-01 & c & vi & N1000 & E1004 & $\begin{array}{c}\text { South wall } \\
\text { profile }\end{array}$ & \begin{tabular}{|l|}
$98.45-98.37$ \\
\end{tabular} & 145-153 & - & $\begin{array}{l}\text { Geoarch } \\
\text { Sample }\end{array}$ & Soil & Bulk Matrix & & & & & 12/29/2017 \\
\hline $\begin{array}{l}\text { not for } \\
\text { curation }\end{array}$ & $\begin{array}{l}\text { not for } \\
\text { curation }\end{array}$ & 685 & - & - & BM-02 & c & vi & N1000 & E1004 & \begin{tabular}{|c|}
$\begin{array}{c}\text { South wall } \\
\text { profile }\end{array}$ \\
\end{tabular} & \begin{tabular}{|l|}
$98.72-98.63$ \\
\end{tabular} & $118-127$ & - & $\begin{array}{l}\begin{array}{l}\text { Geoarch } \\
\text { Sample }\end{array} \\
\text { Same }\end{array}$ & Soil & Bulk Matrix & & & & & 12/29/2017 \\
\hline \begin{tabular}{l|} 
not for \\
curation
\end{tabular} & $\begin{array}{l}\text { not for } \\
\text { curation }\end{array}$ & 685 & - & - & BM-03 & c & IV & N1000 & E1004 & \begin{tabular}{c|} 
South wall \\
profile
\end{tabular} & \begin{tabular}{|l|}
$98.97-98.89$ \\
\end{tabular} & 93-101 & - & 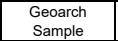 & Soil & Bulk Matrix & & & & & |12/29/2017 \\
\hline \begin{tabular}{l|} 
not for \\
curation
\end{tabular} & $\begin{array}{l}\text { not for } \\
\text { curation }\end{array}$ & 685 & - & - & BM-04 & c & III & N1000 & E1004 & $\begin{array}{c}\text { South wall } \\
\text { profile }\end{array}$ & \begin{tabular}{|l|}
$99.15-99.04$ \\
\end{tabular} & $75-86$ & - & $\begin{array}{l}\text { Geoarch } \\
\text { Sample }\end{array}$ & Soil & Bulk Matrix & & & & & 12/29/2017 \\
\hline $\begin{array}{l}\text { not for } \\
\text { curation }\end{array}$ & $\begin{array}{l}\text { not for } \\
\text { curation }\end{array}$ & 685 & - & - & BM-05 & c & III & N1000 & E1004 & $\begin{array}{c}\text { South wall } \\
\text { profile }\end{array}$ & \begin{tabular}{|l|}
$99.37-99.26$ \\
\end{tabular} & 53-64 & - & $\begin{array}{l}\text { Geoarch } \\
\text { Sample }\end{array}$ & Soil & Bulk Matrix & & & & & $\mid 12 / 29 / 2017$ \\
\hline $\begin{array}{l}\begin{array}{c}\text { not for } \\
\text { curation }\end{array} \\
\end{array}$ & $\begin{array}{l}\text { not for } \\
\text { curation }\end{array}$ & 685 & - & - & BM-06 & c & $" 1$ & N1000 & E1004 & $\begin{array}{c}\text { South wall } \\
\text { profile }\end{array}$ & \begin{tabular}{|l|}
$99.61-99.54$ \\
\end{tabular} & $29 \cdot 36$ & - & $\begin{array}{l}\begin{array}{l}\text { Geoarch } \\
\text { Sample }\end{array} \\
\text { Sam }\end{array}$ & Soil & Bulk Matrix & & & & & 12/29/2017 \\
\hline $\begin{array}{l}\text { not for } \\
\text { curation }\end{array}$ & $\begin{array}{l}\text { not for } \\
\text { curation }\end{array}$ & 685 & - & - & BM-07 & c & 1 & N1000 & E1004 & $\begin{array}{c}\begin{array}{c}\text { South wall } \\
\text { profile }\end{array} \\
\end{array}$ & \begin{tabular}{|l|}
$99.78-99.73$ \\
\end{tabular} & $12-17$ & - & $\begin{array}{l}\begin{array}{l}\text { Geoarch } \\
\text { Sample }\end{array} \\
\end{array}$ & Soil & Bulk Matrix & & & & & \begin{tabular}{|l|}
$12 / 29 / 2017$ \\
\end{tabular} \\
\hline $\begin{array}{c}\text { not for } \\
\text { curation }\end{array}$ & $\begin{array}{c}\text { not for } \\
\text { curation }\end{array}$ & 686 & & \begin{tabular}{|c|} 
FRR4 Layer \\
1 and 2 \\
\end{tabular} & MS-1 & c & III & N1000.96 & E1004.94 & \begin{tabular}{|c} 
Layer 1 and \\
2
\end{tabular} & 99.22 & 68 & $\begin{array}{l}\text { N1000.96 } \\
\text { E1004.94 }\end{array}$ & $\begin{array}{l}\text { Geoarch } \\
\text { Sample }\end{array}$ & Soil & $\begin{array}{l}\text { Magnetic } \\
\text { Susceptibility }\end{array}$ & & & & Cross-section 1 & 12/18/2017 \\
\hline \begin{tabular}{|c|} 
not for \\
curation
\end{tabular} & $\begin{array}{c}\text { not for } \\
\text { curation }\end{array}$ & 686 & & \begin{tabular}{|c|} 
FTR4 Layer \\
1 and 2 \\
\end{tabular} & MS-2 & c & III & $\begin{array}{l}\text { N1000.50- } \\
1001.50\end{array}$ & E1004.94 & \begin{tabular}{|c|} 
Layer 1 and \\
2
\end{tabular} & 99.245 & 65.5 & $\begin{array}{l}\mathrm{N} 1000.96 \\
\text { E1004.94 }\end{array}$ & $\begin{array}{l}\text { Geoarch } \\
\text { Sample }\end{array}$ & Soil & $\begin{array}{c}\text { Magnetic } \\
\text { Suscoptibility }\end{array}$ & & & & Cross-section 1 & $12 / 18 / 2017$ \\
\hline $\begin{array}{c}\text { not for } \\
\text { curation }\end{array}$ & $\begin{array}{c}\text { not for } \\
\text { curation }\end{array}$ & 686 & & \begin{tabular}{|c|} 
FRR4 Layer \\
1 and 2 \\
\end{tabular} & MS-3 & c & III & 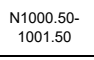 & E1004.94 & \begin{tabular}{|c|} 
Layer 1 and \\
2
\end{tabular} & 99.267 & 63.3 & $\begin{array}{l}\text { N1000.96 } \\
\text { E1004.94 }\end{array}$ & $\begin{array}{l}\text { Geoarch } \\
\text { Sample }\end{array}$ & Soil & $\begin{array}{l}\text { Magnetic } \\
\text { Susceptibility }\end{array}$ & & & & Cross-section 1 & 12/18/2017 \\
\hline $\begin{array}{c}\text { not for } \\
\text { curation }\end{array}$ & $\begin{array}{l}\text { not for } \\
\text { curation }\end{array}$ & 686 & & \begin{tabular}{|c|} 
FTR4 Layer \\
1 and 2
\end{tabular} & MS-4 & c & III & $\begin{array}{l}\text { N1000.50- } \\
1001.50\end{array}$ & E1004.94 & \begin{tabular}{|c|} 
Layer 1 and \\
2
\end{tabular} & 99.29 & 61 & $\begin{array}{l}\text { N1000.96 } \\
\text { E1004.94 }\end{array}$ & $\begin{array}{l}\text { Geoarch } \\
\text { Sample }\end{array}$ & Soil & $\begin{array}{c}\text { Magnetic } \\
\text { Susceptibility }\end{array}$ & & & & Cross-section 1 & 12/18/2017 \\
\hline \begin{tabular}{c|} 
not for \\
curation
\end{tabular} & $\begin{array}{l}\text { not for } \\
\text { curation }\end{array}$ & 686 & & \begin{tabular}{|c|} 
FTR4 Layer \\
1 and 2 \\
\end{tabular} & MS-5 & c & III & $\begin{array}{c}\text { N1000.50- } \\
1001.50 \\
\end{array}$ & E1004.94 & \begin{tabular}{|c|} 
Layer 1 and \\
2
\end{tabular} & 99.315 & 58.5 & $\begin{array}{l}\text { N1000.96 } \\
\text { E1004.94 } \\
\end{array}$ & $\begin{array}{l}\text { Geoarch } \\
\text { Sample }\end{array}$ & Soil & $\begin{array}{c}\text { Magnetic } \\
\text { Susceptibility }\end{array}$ & & & & Cross-section 1 & 12/18/2017 \\
\hline $\begin{array}{l}\begin{array}{c}\text { not for } \\
\text { curation }\end{array} \\
\end{array}$ & $\begin{array}{l}\text { not for } \\
\text { curation }\end{array}$ & 687 & & $\begin{array}{c}\text { FTR4 Layer } \\
2\end{array}$ & MS-1A & $\mathrm{c}$ & III & $\begin{array}{c}\text { N1000.80- } \\
1001.57 \\
\end{array}$ & E1004.70 & Layer 2 & 99.2 & 70 & \begin{tabular}{|l|} 
N1001.21 \\
E1004.70 \\
\end{tabular} & $\begin{array}{l}\text { Geoarch } \\
\text { Sample }\end{array}$ & Soil & $\begin{array}{l}\text { Magnetic } \\
\text { Susceptibility }\end{array}$ & & & & Cross-section 2 & $12 / 18 / 2017$ \\
\hline $\begin{array}{l}\text { not for } \\
\text { curation }\end{array}$ & $\begin{array}{l}\text { not for } \\
\text { curation }\end{array}$ & 687 & & $\begin{array}{c}\text { FTR4 Layer } \\
2\end{array}$ & MS-2A & c & III & $\begin{array}{c}\text { N1000.80- } \\
1001.57 \\
\end{array}$ & E1004.70 & Layer 2 & 99.23 & 67 & $\begin{array}{l}\text { N1001.21 } \\
\text { E1004.70 } \\
\end{array}$ & $\begin{array}{l}\text { Geoarch } \\
\text { Sample }\end{array}$ & Soil & $\begin{array}{l}\text { Magnetic } \\
\text { Susceptibility } \\
\end{array}$ & & & & Cross-section 2 & |12/18/2017 \\
\hline $\begin{array}{l}\begin{array}{c}\text { not for } \\
\text { curation }\end{array} \\
\end{array}$ & $\begin{array}{l}\text { not for } \\
\text { curation }\end{array}$ & 687 & & $\begin{array}{c}\text { FTR4 Layer } \\
2 \\
\end{array}$ & MS-3A & $\mathrm{c}$ & III & $\begin{array}{l}\text { N1000.80- } \\
1001.57 \\
\end{array}$ & E1004.70 & Layer 2 & 99.26 & 64 & $\begin{array}{l}\begin{array}{l}N 1001.21 \\
\text { E1004.70 }\end{array} \\
\end{array}$ & $\begin{array}{l}\begin{array}{l}\text { Geoarch } \\
\text { Sample }\end{array} \\
\end{array}$ & Soil & $\begin{array}{l}\text { Magnetic } \\
\text { Susceptibility } \\
\end{array}$ & & & & Cross-section 2 & 12/18/2017 \\
\hline $\begin{array}{l}\begin{array}{c}\text { not for } \\
\text { curation }\end{array} \\
\end{array}$ & $\begin{array}{l}\text { not for } \\
\text { curation }\end{array}$ & 687 & & $\begin{array}{c}\text { FTR4 Layer } \\
2\end{array}$ & MS-4A & c & III' & $\begin{array}{c}\text { N1000.80- } \\
1001.57 \\
\end{array}$ & E1004.70 & Layer 2 & 99.29 & 61 & $\begin{array}{l}\begin{array}{l}\text { N1001.21 } \\
\text { E1004.70 }\end{array} \\
\end{array}$ & $\begin{array}{l}\text { Geoarch } \\
\text { Sample }\end{array}$ & Soil & $\begin{array}{l}\text { Magnetic } \\
\text { Susceptibility }\end{array}$ & & & & Cross-section 2 & $\mid$\begin{tabular}{|l|}
$\mid 12 / 18 / 2017$ \\
\end{tabular} \\
\hline
\end{tabular}




\begin{tabular}{|c|c|c|c|c|c|c|c|c|c|c|c|c|c|c|}
\hline \multicolumn{5}{|c|}{ Provenience } & \multicolumn{8}{|c|}{ Burned Rock Data } & \multicolumn{2}{|c|}{ Totals } \\
\hline $\begin{array}{c}\text { Excavation } \\
\text { Block }\end{array}$ & Northing & Easting & Level & Elev. (m) & $0-5 \mathrm{Ct}$ & $\begin{array}{c}0-5 \mathrm{Wt} \\
(\mathrm{kg})\end{array}$ & $5-10 \mathrm{Ct}$ & $\begin{array}{c}5-10 \mathrm{Wt} \\
(\mathrm{kg})\end{array}$ & $10-15 \mathrm{Ct}$ & \begin{tabular}{|c|}
$10-15 \mathrm{Wt}$ \\
$(\mathrm{kg})$
\end{tabular} & $15+C t$ & $\begin{array}{c}15+\mathrm{Wt} \\
(\mathrm{kg})\end{array}$ & Total Ct & $\begin{array}{c}\text { Total Wt } \\
(\mathrm{kg})\end{array}$ \\
\hline $\mathrm{A}$ & Ftr 3 & Ftr 3 & & $99.90-98.90$ & 2 & 0.2 & 21 & 2.4 & 2 & 0.5 & & & 25 & 3.1 \\
\hline $\mathrm{A}$ & 995 & 1012 & 1 & $99.70-99.60$ & 1 & 0.10 & 1 & 0.10 & & & & & 2 & 0.20 \\
\hline$A$ & 995 & 1012 & 3 & 99.50-99.40 & & & 1 & 0.20 & & & & & 1 & 0.20 \\
\hline $\mathrm{A}$ & 995 & 1012 & 4 & 99.40-99.30 & 31 & 0.20 & 7 & 0.40 & & & & & 38 & 0.60 \\
\hline $\mathrm{A}$ & 995 & 1012 & 5 & $99.30-99.20$ & 16 & 0.10 & 5 & 0.40 & & & & & 21 & 0.50 \\
\hline$A$ & 995 & 1012 & 6 & $99.20-99.10$ & 16 & 0.10 & 7 & 0.60 & & & & & 23 & 0.70 \\
\hline $\mathrm{A}$ & 995 & 1012 & 7 & $99.10-99.00$ & 24 & 0.20 & 4 & 0.20 & 1 & 0.20 & & & 29 & 0.60 \\
\hline $\mathrm{A}$ & 995 & 1012 & 8 & $99.00-98.90$ & 25 & 0.10 & 15 & 1.30 & & & & & 40 & 1.40 \\
\hline $\mathrm{A}$ & 995 & 1012 & 9 & 98.90-98.80 & 25 & 0.10 & 5 & 1.70 & & & & & 30 & 1.80 \\
\hline$A$ & 995 & 1012 & 10 & \begin{tabular}{|c|}
$98.80-98.70$ \\
\end{tabular} & 50 & 0.10 & 1 & 0.20 & & & & & 51 & 0.30 \\
\hline $\mathrm{A}$ & 995 & 1012 & 11 & $98.70-98.60$ & 11 & 0.10 & 4 & 0.30 & & & & & 15 & 0.40 \\
\hline A & 995 & 1012 & 12 & $98.60-98.50$ & 10 & 0.10 & 1 & 0.10 & & & & & 11 & 0.20 \\
\hline $\mathrm{A}$ & 995 & 1012 & 13 & $98.50-98.40$ & 9 & 0.10 & & & & & & & 9 & 0.10 \\
\hline $\mathrm{A}$ & 995 & 1012 & 14 & $98.40-98.30$ & 5 & 0.10 & 1 & 0.10 & & & & & 6 & 0.20 \\
\hline $\mathrm{A}$ & 995 & 1012 & 15 & $98.30-98.20$ & 11 & 0.10 & & & & & & & 11 & 0.10 \\
\hline $\mathrm{A}$ & 995 & 1013 & 2 & $99.60-99.50$ & & & 3 & 0.40 & & & & & 3 & 0.40 \\
\hline $\mathrm{A}$ & 995 & 1013 & 3 & $99.50-99.40$ & 3 & 0.10 & & & & & & & 3 & 0.10 \\
\hline$A$ & 995 & 1013 & 4 & $99.40-99.30$ & 14 & 0.20 & & & & & & & 14 & 0.20 \\
\hline $\mathrm{A}$ & 995 & 1013 & 5 & $99.30-99.20$ & 9 & 0.10 & 4 & 0.20 & & & & & 13 & 0.30 \\
\hline $\mathrm{A}$ & 995 & 1013 & 6 & $99.20-99.10$ & 11 & 0.10 & 3 & 0.40 & & & & & 14 & 0.50 \\
\hline $\mathrm{A}$ & 995 & 1013 & 7 & $99.10-99.00$ & 27 & 0.30 & 2 & 0.30 & & & & & 29 & 0.60 \\
\hline $\mathrm{A}$ & 995 & 1013 & 8 & $99.00-98.90$ & 12 & 0.10 & 7 & 0.60 & & & & & 19 & 0.70 \\
\hline $\mathrm{A}$ & 995 & 1013 & 9 & $98.90-98.80$ & 3 & 0.10 & & & & & & & 3 & 0.10 \\
\hline$A$ & 995 & 1013 & 10 & $98.80-98.70$ & 7 & 0.10 & & & & & & & 7 & 0.10 \\
\hline $\bar{A}$ & 995 & 1013 & 11 & $98.70-98.60$ & 2 & 0.10 & & & & & & & 2 & 0.10 \\
\hline$A$ & 995 & 1013 & 12 & 98.60-98.50 & 5 & 0.10 & 2 & 0.10 & & & & & 7 & 0.20 \\
\hline $\mathrm{A}$ & 995 & 1013 & 13 & \begin{tabular}{|c|}
$98.50-98.40$ \\
\end{tabular} & 3 & 0.10 & 1 & 0.10 & & & & & 4 & 0.20 \\
\hline$A$ & 995 & 1013 & 14 & $98.40-98.30$ & 3 & 0.10 & & & & & & & 3 & 0.10 \\
\hline $\mathrm{A}$ & 995 & 1013 & 15 & $98.30-98.20$ & 7 & 0.10 & & & & & & & 7 & 0.10 \\
\hline$A$ & 995 & 1014 & 1 & $99.59-99.50$ & 1 & 0.10 & 3 & 0.20 & & & & & 4 & 0.30 \\
\hline $\mathrm{A}$ & 995 & 1014 & 2 & $99.50-99.40$ & 1 & 0.10 & 1 & 0.20 & & & & & 2 & 0.30 \\
\hline$A$ & 995 & 1014 & 4 & $99.30-99.20$ & 18 & 0.30 & 6 & 0.40 & & & & & 24 & 0.70 \\
\hline$A$ & 995 & 1014 & 5 & $99.20-99.10$ & 23 & 0.20 & 10 & 0.60 & & & & & 33 & 0.80 \\
\hline $\mathrm{A}$ & 995 & 1014 & 6 & $99.10-99.00$ & 22 & 0.20 & 3 & 0.30 & & & & & 25 & 0.50 \\
\hline $\bar{A}$ & 995 & 1014 & 7 & $99.00-98.90$ & 30 & 0.20 & 10 & 1.30 & & & & & 40 & 1.50 \\
\hline$A$ & 995 & 1014 & 8 & $98.90-98.80$ & 5 & 0.05 & 5 & 0.60 & & & & & 10 & 0.65 \\
\hline $\mathrm{A}$ & 995 & 1014 & 9 & \begin{tabular}{|c|}
$98.80-98.70$ \\
\end{tabular} & 8 & 0.05 & 1 & 0.05 & & & & & 9 & 0.10 \\
\hline $\bar{A}$ & 995 & 1014 & 10 & \begin{tabular}{|l|}
$98.70-98.60$ \\
\end{tabular} & 13 & 0.05 & & & & & & & 13 & 0.05 \\
\hline$A$ & 995 & 1014 & 11 & $98.60-98.50$ & 7 & 0.10 & 2 & 0.10 & & & & & 9 & 0.20 \\
\hline $\mathrm{A}$ & 995 & 1014 & 12 & $98.50-98.40$ & 2 & 0.10 & & & & & & & 2 & 0.10 \\
\hline$A$ & 995 & 1014 & 13 & $98.40-98.30$ & 2 & 0.10 & & & & & & & 2 & 0.10 \\
\hline$A$ & 996 & 1012 & 2 & $99.60-99.50$ & 2 & 0.10 & 1 & 0.10 & 1 & 0.30 & & & 4 & 0.50 \\
\hline $\mathrm{A}$ & 996 & 1012 & 3 & $99.50-99.40$ & 5 & 0.10 & & & & & & & 5 & 0.10 \\
\hline $\mathrm{A}$ & 996 & 1012 & 4 & \begin{tabular}{|l|}
$99.40-99.30$ \\
\end{tabular} & 10 & 0.10 & 2 & 0.10 & & & & & 12 & 0.20 \\
\hline$A$ & 996 & 1012 & 5 & $99.30-99.20$ & 15 & 0.10 & 2 & 0.20 & & & & & 17 & 0.30 \\
\hline$A$ & 996 & 1012 & 6 & $99.20-99.10$ & 1 & 0.10 & 7 & 0.90 & & & & & 8 & 1.00 \\
\hline$A$ & 996 & 1012 & 7 & \begin{tabular}{|c|}
$99.10-99.00$ \\
\end{tabular} & 43 & 0.40 & 11 & 0.90 & & & & & 54 & 1.30 \\
\hline A & 996 & 1012 & 8 & 99.00-98.90 & 129 & 0.30 & 4 & 0.20 & & & & & 133 & 0.50 \\
\hline A & 996 & 1012 & 9 & 98.90-98.80 & 100 & 1.10 & 27 & 3.30 & & & & & 127 & 4.40 \\
\hline$A$ & 996 & 1012 & 10 & \begin{tabular}{|c|}
$98.80-98.70$ \\
\end{tabular} & 100 & 0.50 & 9 & 0.40 & & & & & 109 & 0.90 \\
\hline $\mathrm{A}$ & 996 & 1012 & 11 & $98.70-98.60$ & 16 & 0.10 & & & & & & & 16 & 0.10 \\
\hline $\mathrm{A}$ & 996 & 1012 & 12 & 98.60-98.50 & 22 & 0.10 & & & & & & & 22 & 0.10 \\
\hline $\mathrm{A}$ & 996 & 1012 & 13 & \begin{tabular}{|c|}
$98.50-98.40$ \\
\end{tabular} & 5 & 0.10 & & & & & & & 5 & 0.10 \\
\hline A & 996 & 1012 & 15 & 98.30-98.20 & 3 & 0.10 & & & & & & & 3 & 0.10 \\
\hline $\mathrm{A}$ & 996 & 1012 & 3 & $99.40-99.30$ & 17 & 0.10 & 1 & 0.10 & & & & & 18 & 0.20 \\
\hline $\mathrm{A}$ & 996 & 1012 & 4 & $99.30-99.20$ & 7 & 0.20 & & & & & & & 7 & 0.20 \\
\hline$A$ & 996 & 1012 & 5 & $99.20-99.10$ & 22 & 0.20 & 1 & 0.20 & & & & & 23 & 0.40 \\
\hline$A$ & 996 & 1012 & 6 & \begin{tabular}{|l|}
$99.10-99.00$ \\
\end{tabular} & 17 & 0.20 & 5 & 0.80 & & & & & 22 & 1.00 \\
\hline $\mathrm{A}$ & 996 & 1012 & 7 & \begin{tabular}{|c|}
$99.00-98.90$ \\
\end{tabular} & 77 & 0.20 & & & & & & & 77 & 0.20 \\
\hline$A$ & 996 & 1012 & 8 & $98.90-98.80$ & 19 & 0.10 & 1 & 0.10 & & & & & 20 & 0.20 \\
\hline$A$ & 996 & 1012 & 9 & \begin{tabular}{|c|}
$98.80-98.70$ \\
\end{tabular} & 1 & 0.10 & 2 & 0.20 & & & & & 3 & 0.30 \\
\hline $\mathrm{A}$ & 996 & 1012 & 10 & $98.70-98.60$ & 6 & 0.10 & & & & & & & 6 & 0.10 \\
\hline$A$ & 996 & 1012 & 11 & 98.60-98.50 & 6 & 0.10 & 1 & 0.10 & & & & & 7 & 0.20 \\
\hline$A$ & 996 & 1012 & 12 & $98.50-98.40$ & 3 & 0.10 & & & & & & & 3 & 0.10 \\
\hline $\bar{A}$ & 996 & 1012 & 14 & $98.30-98.20$ & 2 & 0.10 & & & & & & & 2 & 0.10 \\
\hline $\mathrm{A}$ & 996 & 1013 & 3 & $99.40-99.30$ & 17 & 0.10 & 1 & 0.10 & & & & & 18 & 0.20 \\
\hline $\mathrm{A}$ & 996 & 1013 & 4 & $99.30-99.20$ & 7 & 0.20 & & & & & & & 7 & 0.20 \\
\hline$A$ & 996 & 1013 & 5 & $99.20-99.10$ & 22 & 0.20 & 1 & 0.20 & & & & & 23 & 0.40 \\
\hline $\mathrm{A}$ & 996 & 1013 & 6 & $99.10-99.00$ & 17 & 0.20 & 5 & 0.80 & & & & & 22 & 1.00 \\
\hline $\mathrm{A}$ & 996 & 1013 & 7 & \begin{tabular}{|c|}
$99.00-98.90$ \\
\end{tabular} & 77 & 0.20 & & & & & & & 77 & 0.20 \\
\hline $\mathrm{A}$ & 996 & 1013 & 8 & $98.90-98.80$ & 19 & 0.10 & 1 & 0.10 & & & & & 20 & 0.20 \\
\hline
\end{tabular}




\begin{tabular}{|c|c|c|c|c|c|c|c|c|c|c|c|c|c|c|}
\hline \multicolumn{5}{|c|}{ Provenience } & \multicolumn{8}{|c|}{ Burned Rock Data } & \multicolumn{2}{|c|}{ Totals } \\
\hline $\begin{array}{c}\text { Excavation } \\
\text { Block }\end{array}$ & Northing & Easting & Level & Elev. (m) & $0-5 \mathrm{Ct}$ & \begin{tabular}{|c|}
$0-5 \mathrm{Wt}$ \\
$(\mathrm{kg})$
\end{tabular} & $5-10 \mathrm{Ct}$ & $\begin{array}{c}5-10 \mathrm{Wt} \\
(\mathrm{kg})\end{array}$ & $10-15 \mathrm{Ct}$ & \begin{tabular}{|c|}
$10-15 \mathrm{Wt}$ \\
$(\mathrm{kg})$
\end{tabular} & $15+C t$ & $\begin{array}{c}15+\mathrm{Wt} \\
(\mathrm{kg})\end{array}$ & Total Ct & $\begin{array}{c}\text { Total Wt } \\
(\mathrm{kg})\end{array}$ \\
\hline $\mathrm{A}$ & 996 & 1013 & 9 & $98.80-98.70$ & 1 & 0.10 & 2 & 0.20 & & & & & 3 & 0.30 \\
\hline $\bar{A}$ & 996 & 1013 & 10 & $98.70-98.60$ & 6 & 0.10 & & & & & & & 6 & 0.10 \\
\hline $\mathrm{A}$ & 996 & 1013 & 11 & $98.60-98.50$ & 6 & 0.10 & 1 & 0.10 & & & & & 7 & 0.20 \\
\hline $\mathrm{A}$ & 996 & 1013 & 12 & 98.50-98.40 & 3 & 0.10 & & & & & & & 3 & 0.10 \\
\hline $\mathrm{A}$ & 996 & 1013 & 14 & $98.30-98.20$ & 2 & 0.10 & & & & & & & 2 & 0.10 \\
\hline $\mathrm{A}$ & 996 & 1014 & 2 & $99.50-99.40$ & 1 & 0.50 & & & & & & & 1 & 0.50 \\
\hline $\bar{A}$ & 996 & 1014 & 3 & 99.40-99.30 & 7 & 0.10 & & & & & & & 7 & 0.10 \\
\hline $\mathrm{A}$ & 996 & 1014 & 4 & 99.30-99.20 & 14 & 1.00 & & & & & & & 14 & 1.00 \\
\hline $\mathrm{A}$ & 996 & 1014 & 5 & 99.20-99.10 & 10 & 0.10 & & & & & & & 10 & 0.10 \\
\hline $\bar{A}$ & 996 & 1014 & 6 & 99.10-99.00 & 35 & 0.10 & 4 & 3.50 & & & & & 39 & 3.60 \\
\hline $\bar{A}$ & 996 & 1014 & 7 & 99.00-98.90 & 18 & 0.10 & 5 & 3.20 & & & & & 23 & 3.30 \\
\hline $\bar{A}$ & 996 & 1014 & 8 & 98.90-98.80 & 44 & 0.05 & 4 & 0.25 & & & & & 48 & 0.30 \\
\hline $\bar{A}$ & 996 & 1014 & 9 & $98.80-98.70$ & 39 & 0.10 & & & & & & & 39 & 0.10 \\
\hline $\mathrm{A}$ & 996 & 1014 & 10 & $98.70-98.60$ & 30 & 0.05 & 5 & 0.30 & & & & & 35 & 0.35 \\
\hline $\mathrm{A}$ & 996 & 1014 & 11 & $98.60-98.50$ & 33 & 0.10 & 6 & 1.00 & & & & & 39 & 1.10 \\
\hline $\mathrm{A}$ & 996 & 1014 & 12 & $98.50-98.40$ & 16 & 0.05 & & & & & & & 16 & 0.05 \\
\hline $\mathrm{A}$ & 996 & 1014 & 13 & $98.40-98.30$ & 11 & 0.10 & & & & & & & 11 & 0.10 \\
\hline $\bar{A}$ & 996 & 1014 & 14 & $98.30-98.20$ & 5 & 0.05 & & & & & & & 5 & 0.05 \\
\hline $\mathrm{A}$ & 996 & 1014 & 15 & $98.20-98.10$ & 2 & 0.05 & & & & & & & 2 & 0.05 \\
\hline $\mathrm{A}$ & 996 & 1014 & 16 & $98.10-98.00$ & 3 & 0.10 & & & & & & & 3 & 0.10 \\
\hline$A$ & 996 & 1014 & 17 & $98.00-97.90$ & 2 & 0.05 & & & & & & & 2 & 0.05 \\
\hline $\mathrm{B}$ & 999 & 1013 & 2 & $99.50-99.40$ & 1 & 0.10 & & & & & & & 1 & 0.10 \\
\hline$B$ & 999 & 1013 & 5 & $99.20-99.10$ & 1 & 0.20 & & & & & & & 1 & 0.20 \\
\hline$B$ & 999 & 1013 & 6 & $99.10-99.00$ & 4 & 0.20 & 1 & 0.60 & & & & & 5 & 0.80 \\
\hline $\mathrm{B}$ & 999 & 1013 & 7 & $99.00-98.90$ & 8 & 0.60 & 1 & 0.50 & & & & & 9 & 1.10 \\
\hline $\mathrm{B}$ & 999 & 1013 & 8 & $98.90-98.80$ & 7 & 0.60 & 6 & 0.50 & & & & & 13 & 1.10 \\
\hline$B$ & 999 & 1013 & 9 & $98.80-98.70$ & 2 & 0.70 & & & & & & & 2 & 0.70 \\
\hline $\mathrm{B}$ & 999 & 1013 & 11 & $98.60-98.50$ & 8 & 0.10 & & & & & & & 8 & 0.10 \\
\hline$B$ & 999 & 1013 & 12 & $98.50-98.40$ & 2 & 0.10 & & & & & & & 2 & 0.10 \\
\hline B & 999 & 1014 & 2 & $99.50-99.40$ & 1 & 0.10 & & & & & & & 1 & 0.10 \\
\hline $\mathrm{B}$ & 999 & 1014 & 5 & $99.20-99.10$ & 4 & 0.60 & & & & & & & 4 & 0.60 \\
\hline $\mathrm{B}$ & 999 & 1014 & 6 & $99.10-99.00$ & 3 & 0.60 & & & & & & & 3 & 0.60 \\
\hline$B$ & 999 & 1014 & 7 & $99.00-98.90$ & 3 & 0.60 & & & & & & & 3 & 0.60 \\
\hline $\mathrm{B}$ & 999 & 1014 & 8 & $98.90-98.80$ & 9 & 1.30 & & & & & & & 9 & 1.30 \\
\hline B & 999 & 1014 & 9 & $98.80-98.70$ & 13 & 0.80 & & & & & & & 13 & 0.80 \\
\hline$B$ & 999 & 1014 & 11 & $98.60-98.50$ & 2 & 0.01 & & & & & & & 2 & 0.01 \\
\hline$B$ & 999 & 1014 & 12 & $98.50-98.40$ & 4 & 0.10 & & & & & & & 4 & 0.10 \\
\hline $\mathrm{C}$ & Ftr 4 & Ftr 4 & & $99.42-99.20$ & 75 & 0.80 & 78 & 12.60 & 26 & 14.20 & 12.00 & 15.50 & 191 & 43.10 \\
\hline $\mathrm{C}$ & 1000 & 1003 & 1 & \begin{tabular}{|l|}
$99.83-99.70$ \\
\end{tabular} & 2 & 0.10 & 1 & 0.20 & & & & & 3 & 0.30 \\
\hline$C$ & 1000 & 1003 & 2 & $99.70-99.60$ & 4 & 0.10 & & & & & & & 4 & 0.10 \\
\hline C & 1000 & 1003 & 3 & $99.60-99.50$ & 11 & 0.05 & 1 & 0.05 & & & & & 12 & 0.10 \\
\hline $\mathrm{C}$ & 1000 & 1003 & 4 & $99.50-99.40$ & 13 & 0.10 & 3 & 0.30 & & & & & 16 & 0.40 \\
\hline $\mathrm{C}$ & 1000 & 1003 & 5 & $99.40-99.30$ & 4 & 0.10 & 1 & 0.10 & & & & & 5 & 0.20 \\
\hline $\mathrm{C}$ & 1000 & 1003 & 6 & $99.30-99.20$ & 12 & 0.10 & 3 & 0.30 & & & & & 15 & 0.40 \\
\hline $\mathrm{C}$ & 1000 & 1003 & 7 & $99.20-99.10$ & 33 & 0.30 & 1 & 0.30 & & & & & 34 & 0.60 \\
\hline C & 1000 & 1003 & 8 & $99.10-99.00$ & 16 & 0.10 & & & & & & & 16 & 0.10 \\
\hline $\mathrm{C}$ & 1000 & 1003 & 9 & $99.00-98.90$ & 22 & 0.20 & & & & & & & 22 & 0.20 \\
\hline C & 1000 & 1004 & 1 & $99.77-99.70$ & 1 & 0.10 & & & & & & & 1 & 0.10 \\
\hline $\mathrm{C}$ & 1000 & 1004 & 2 & $99.70-99.60$ & 1 & 0.10 & & & & & & & 1 & 0.10 \\
\hline$C$ & 1000 & 1004 & 3 & $99.60-99.50$ & 7 & 0.10 & & & & & & & 7 & 0.10 \\
\hline $\mathrm{C}$ & 1000 & 1004 & 4 & \begin{tabular}{|c|}
$99.50-99.40$ \\
\end{tabular} & 7 & 0.10 & 4 & 0.50 & & & & & 11 & 0.60 \\
\hline $\mathrm{C}$ & 1000 & 1004 & 5 & \begin{tabular}{|c|}
$99.40-99.30$ \\
\end{tabular} & 8 & 0.10 & 2 & 0.20 & & & & & 10 & 0.30 \\
\hline $\mathrm{C}$ & 1000 & 1004 & 6 & $99.30-99.20$ & 27 & 0.10 & & & & & & & 27 & 0.10 \\
\hline $\mathrm{C}$ & 1000 & 1004 & 7 & $99.20-99.10$ & 7 & 0.20 & & & & & & & 7 & 0.20 \\
\hline $\mathrm{C}$ & 1000 & 1004 & 8 & $99.10-99.00$ & 14 & 0.10 & 2 & 0.10 & & & & & 16 & 0.20 \\
\hline $\mathrm{C}$ & 1000 & 1004 & 9 & $99.00-98.90$ & 23 & 0.10 & 1 & 0.10 & & & & & 24 & 0.20 \\
\hline $\mathrm{C}$ & 1000 & 1004 & 10 & \begin{tabular}{|c|}
$98.90-98.80$ \\
\end{tabular} & 20 & 0.10 & 1 & 0.10 & & & & & 21 & 0.20 \\
\hline C & 1000 & 1004 & 11 & \begin{tabular}{|c|}
$98.80-98.70$ \\
\end{tabular} & 14 & 0.10 & & & & & & & 14 & 0.10 \\
\hline C & 1000 & 1004 & 12 & $98.70-98.60$ & 7 & 0.10 & & & & & & & 7 & 0.10 \\
\hline $\mathrm{C}$ & 1000 & 1004 & 13 & $98.60-98.50$ & 7 & 0.10 & & & & & & & 7 & 0.10 \\
\hline $\mathrm{C}$ & 1000 & 1004 & 14 & $98.50-98.40$ & 5 & 0.10 & & & & & & & 5 & 0.10 \\
\hline $\mathrm{C}$ & 1000 & 1004 & 15 & $98.40-98.30$ & 9 & 0.10 & & & & & & & 9 & 0.10 \\
\hline $\mathrm{C}$ & 1000.5 & 1005.5 & 1 & \begin{tabular}{|c|}
$99.60-99.50$ \\
\end{tabular} & 3 & 0.10 & & & & & & & 3 & 0.10 \\
\hline $\mathrm{C}$ & 1000.5 & 1005.5 & 2 & \begin{tabular}{|l|}
$99.50-99.40$ \\
\end{tabular} & 1 & 0.10 & 14 & 0.20 & & & & & 15 & 0.30 \\
\hline C & 1000.5 & 1005.5 & 3 & $99.40-99.30$ & 33 & 0.10 & 3 & 0.30 & & & & & 36 & 0.40 \\
\hline C & 1001 & 1003 & 1 & $99.60-99.50$ & 10 & 0.20 & & & & & & & 10 & 0.20 \\
\hline $\mathrm{C}$ & 1001 & 1003 & 2 & \begin{tabular}{|l|}
$99.50-99.40$ \\
\end{tabular} & 25 & 0.10 & 4 & 0.20 & & & & & 29 & 0.30 \\
\hline $\mathrm{C}$ & 1001 & 1003 & 4 & \begin{tabular}{|c|}
$99.40-99.30$ \\
\end{tabular} & 14 & 1.10 & 1 & 0.10 & & & & & 15 & 1.20 \\
\hline $\mathrm{C}$ & 1001 & 1004 & 4 & \begin{tabular}{|c|}
$99.50-99.40$ \\
\end{tabular} & 1 & 0.10 & & & & & & & 1 & 0.10 \\
\hline C & 1001 & 1004.5 & 2 & \begin{tabular}{|l|}
$99.70-99.60$ \\
\end{tabular} & 3 & 0.10 & & & & & & & 3 & 0.10 \\
\hline $\mathrm{C}$ & 1001 & 1004.5 & 3 & $99.60-99.50$ & 3 & 0.10 & & & & & & & 3 & 0.10 \\
\hline
\end{tabular}




\begin{tabular}{|c|c|c|c|c|c|c|c|c|c|c|c|c|c|c|}
\hline \multicolumn{5}{|c|}{ Provenience } & \multicolumn{8}{|c|}{ Burned Rock Data } & \multicolumn{2}{|c|}{ Totals } \\
\hline $\begin{array}{c}\text { Excavation } \\
\text { Block }\end{array}$ & Northing & Easting & Level & Elev. (m) & $0-5 \mathrm{Ct}$ & \begin{tabular}{|c|}
$0-5 \mathrm{Wt}$ \\
$(\mathrm{kg})$
\end{tabular} & $5-10 \mathrm{Ct}$ & $\begin{array}{c}5-10 \mathrm{Wt} \\
(\mathrm{kg})\end{array}$ & $10-15 \mathrm{Ct}$ & \begin{tabular}{|c|}
$10-15 \mathrm{Wt}$ \\
$(\mathrm{kg})$
\end{tabular} & $15+C t$ & $\begin{array}{c}15+\mathrm{Wt} \\
(\mathrm{kg})\end{array}$ & Total Ct & $\begin{array}{c}\text { Total Wt } \\
(\mathrm{kg})\end{array}$ \\
\hline C & 1001 & 1004.5 & 4 & $99.50-99.40$ & 16 & 0.10 & & & & & & & 16 & 0.10 \\
\hline $\mathrm{C}$ & 1001 & 1004.5 & 5 & 99.40-99.30 & 13 & 0.10 & & & & & & & 13 & 0.10 \\
\hline $\mathrm{C}$ & 1001.5 & 1004 & 2 & $99.60-99.50$ & 4 & 0.10 & & & & & & & 4 & 0.10 \\
\hline $\mathrm{C}$ & 1001.5 & 1004 & 3 & 99.50-99.40 & 21 & 0.10 & 2 & 0.10 & & & & & 23 & 0.20 \\
\hline $\mathrm{C}$ & 1001.5 & 1004 & 4 & 99.40-99.30 & 5 & 0.20 & 18 & 0.10 & & & & & 23 & 0.30 \\
\hline $\mathrm{C}$ & 1001.5 & 1005 & 2 & $99.50-99.40$ & 10 & 0.10 & 2 & 0.20 & & & & & 12 & 0.30 \\
\hline $\mathrm{C}$ & 1001.5 & 1005 & 3 & 99.40-99.30 & 19 & 0.10 & 1 & 0.10 & & & & & 20 & 0.20 \\
\hline $\bar{D}$ & 1000 & 1009 & 3 & 99.49-99.39 & 1 & 0.10 & & & & & & & 1 & 0.10 \\
\hline $\mathrm{D}$ & 1000 & 1009 & 7 & 99.09-98.99 & 14 & 0.02 & & & & & & & 14 & 0.02 \\
\hline $\mathrm{D}$ & 1000 & 1009 & 8 & 98.99-98.89 & 4 & 0.01 & & & & & & & 4 & 0.01 \\
\hline $\mathrm{D}$ & 1000 & 1009 & 9 & 98.89-98.79 & 18 & 0.03 & & & & & & & 18 & 0.03 \\
\hline $\mathrm{D}$ & 1000 & 1009 & 10 & 98.79-98.69 & 3 & 0.30 & 2 & 0.20 & & & & & 5 & 0.50 \\
\hline $\mathrm{D}$ & 1000 & 1009 & 11 & 98.69-98.59 & 13 & 0.10 & 2 & 0.20 & & & & & 15 & 0.30 \\
\hline $\mathrm{D}$ & 1000 & 1010 & 2 & 99.59-99.49 & 1 & 0.10 & & & & & & & 1 & 0.10 \\
\hline $\mathrm{D}$ & 1000 & 1010 & 4 & $99.39-99.29$ & 1 & 0.10 & & & & & & & 1 & 0.10 \\
\hline $\mathrm{D}$ & 1000 & 1010 & 5 & $99.29-99.19$ & 1 & 0.10 & & & & & & & 1 & 0.10 \\
\hline $\mathrm{D}$ & 1000 & 1010 & 6 & $99.19-99.09$ & 3 & 0.10 & & & & & & & 3 & 0.10 \\
\hline $\mathrm{D}$ & 1000 & 1010 & 7 & 99.09-98.99 & 1 & 0.10 & 1 & 0.10 & & & & & 2 & 0.20 \\
\hline $\mathrm{D}$ & 1000 & 1010 & 8 & $98.99-98.89$ & 11 & 0.10 & & & & & & & 11 & 0.10 \\
\hline $\mathrm{D}$ & 1000 & 1010 & 9 & $98.89-98.79$ & 8 & 0.40 & 3 & 0.60 & & & & & 11 & 1.00 \\
\hline $\mathrm{D}$ & 1000 & 1010 & 10 & $98.79-98.69$ & 9 & 0.10 & & & & & & & 9 & 0.10 \\
\hline $\mathrm{D}$ & 1000 & 1010 & 11 & $98.69-98.59$ & 14 & 0.20 & 3 & 0.40 & & & & & 17 & 0.60 \\
\hline $\mathrm{D}$ & 1000 & 1010 & 12 & $98.59-98.49$ & 23 & 0.20 & 4 & 0.20 & & & & & 27 & 0.40 \\
\hline $\mathrm{D}$ & 1000 & 1010 & 13 & $98.49-98.39$ & 8 & 0.10 & 1 & 0.10 & & & & & 9 & 0.20 \\
\hline $\bar{D}$ & 1000 & 1010 & 14 & $98.39-98.29$ & 17 & 0.10 & 4 & 0.50 & & & & & 21 & 0.60 \\
\hline $\bar{D}$ & 1000 & 1010 & 15 & $98.29-98.19$ & 5 & 0.10 & & & & & & & 5 & 0.10 \\
\hline $\mathrm{D}$ & 1000 & 1010 & 16 & \begin{tabular}{|c|}
$98.19-98.09$ \\
\end{tabular} & 14 & 0.10 & & & & & & & 14 & 0.10 \\
\hline $\mathrm{D}$ & 1000 & 1010 & 17 & $98.09-97.99$ & 4 & 0.10 & & & & & & & 4 & 0.10 \\
\hline $\bar{D}$ & 1000 & 1010 & 18 & $97.99-97.89$ & 1 & 0.10 & 1 & 0.20 & & & & & 2 & 0.30 \\
\hline $\mathrm{D}$ & 1000 & 1010 & 21 & $97.69-97.59$ & 2 & 0.10 & & & & & & & 2 & 0.10 \\
\hline $\mathrm{D}$ & 1001 & 1009 & 1 & $99.60-99.49$ & 3 & 0.10 & 1 & 0.10 & & & & & 4 & 0.20 \\
\hline $\mathrm{D}$ & 1001 & 1009 & 4 & $99.29-99.19$ & 12 & 1.00 & 1 & 0.75 & & & & & 13 & 1.75 \\
\hline $\mathrm{D}$ & 1001 & 1009 & 5 & $99.19-99.09$ & 3 & 1.00 & & & & & & & 3 & 1.00 \\
\hline $\mathrm{D}$ & 1001 & 1009 & 6 & 99.09-98.99 & 11 & 0.20 & & & & & & & 11 & 0.20 \\
\hline $\mathrm{D}$ & 1001 & 1009 & 7 & \begin{tabular}{|l|}
$98.99-98.89$ \\
\end{tabular} & 12 & 1.00 & 2 & 1.30 & & & & & 14 & 2.30 \\
\hline $\mathrm{D}$ & 1001 & 1009 & 8 & $98.89-98.79$ & 4 & 0.10 & 2 & 0.20 & & & & & 6 & 0.30 \\
\hline $\mathrm{D}$ & 1001 & 1009 & 9 & $98.79-98.69$ & 11 & 0.10 & 3 & 0.10 & & & & & 14 & 0.20 \\
\hline $\mathrm{D}$ & 1001 & 1009 & 10 & $98.69-98.59$ & 9 & 0.20 & & & & & & & 9 & 0.20 \\
\hline $\mathrm{D}$ & 1001 & 1009 & 1 & \begin{tabular}{|c|}
$99.59-99.49$ \\
\end{tabular} & 1 & 0.10 & & & & & & & 1 & 0.10 \\
\hline $\mathrm{D}$ & 1001 & 1009 & 3 & \begin{tabular}{|c|}
$99.39-99.29$ \\
\end{tabular} & 1 & 0.10 & & & & & & & 1 & 0.10 \\
\hline $\mathrm{D}$ & 1001 & 1009 & 4 & \begin{tabular}{|l|}
$99.29-99.19$ \\
\end{tabular} & 5 & 0.50 & 1 & 0.50 & & & & & 6 & 1.00 \\
\hline $\mathrm{D}$ & 1001 & 1009 & 5 & 99.19-99.09 & 2 & 0.10 & & & & & & & 2 & 0.10 \\
\hline $\bar{D}$ & 1001 & 1009 & 6 & $99.09-98.99$ & 5 & 0.10 & 3 & 0.30 & & & & & 8 & 0.40 \\
\hline $\mathrm{D}$ & 1001 & 1009 & 7 & \begin{tabular}{|c|}
$98.99-98.89$ \\
\end{tabular} & 6 & 1.00 & 9 & 1.80 & 1 & 0.20 & & & 16 & 3.00 \\
\hline $\mathrm{D}$ & 1001 & 1009 & 8 & \begin{tabular}{|c|}
$98.89-98.79$ \\
\end{tabular} & 2 & 0.10 & & & & & & & 2 & 0.10 \\
\hline $\bar{D}$ & 1001 & 1009 & 9 & \begin{tabular}{|c|}
$98.79-98.69$ \\
\end{tabular} & 5 & 0.10 & & & & & & & 5 & 0.10 \\
\hline $\mathrm{D}$ & 1001 & 1009 & 10 & \begin{tabular}{|c|}
$98.69-98.59$ \\
\end{tabular} & 2 & 0.10 & & & & & & & 2 & 0.10 \\
\hline $\mathrm{D}$ & 1001 & 1009 & 12 & 98.49-98.39 & 3 & 0.20 & & & & & & & 3 & 0.20 \\
\hline $\mathrm{D}$ & 1001 & 1009 & 13 & 98.39-98.29 & 7 & 0.10 & & & & & & & 7 & 0.10 \\
\hline $\mathrm{D}$ & 1001 & 1009 & 14 & \begin{tabular}{|c|}
$98.29-98.19$ \\
\end{tabular} & 4 & 0.10 & & & & & & & 4 & 0.10 \\
\hline $\mathrm{D}$ & 1001 & 1009 & 15 & \begin{tabular}{|c|}
$98.19-98.09$ \\
\end{tabular} & 2 & 0.10 & & & & & & & 2 & 0.10 \\
\hline $\mathrm{D}$ & 1001 & 1009 & 18 & \begin{tabular}{|c|}
$97.89-97.79$ \\
\end{tabular} & 2 & 0.10 & & & & & & & 2 & 0.10 \\
\hline $\bar{D}$ & 1001 & 1009 & 19 & $97.79-97.69$ & 1 & 0.10 & & & & & & & 1 & 0.10 \\
\hline $\mathrm{D}$ & 1001 & 1009 & 20 & $97.69-97.59$ & 4 & 0.10 & & & & & & & 4 & 0.10 \\
\hline $\mathrm{D}$ & 1001 & 1009 & 1 & \begin{tabular}{|l|}
$99.59-99.49$ \\
\end{tabular} & 1 & 0.10 & & & & & & & 1 & 0.10 \\
\hline $\mathrm{D}$ & 1001 & 1009 & 3 & \begin{tabular}{|c|}
$99.39-99.29$ \\
\end{tabular} & 1 & 0.10 & & & & & & & 1 & 0.10 \\
\hline $\mathrm{D}$ & 1001 & 1009 & 4 & 99.29-99.19 & 5 & 0.50 & 1 & 0.50 & & & & & 6 & 1.00 \\
\hline $\mathrm{D}$ & 1001 & 1009 & 5 & \begin{tabular}{|c|}
$99.19-99.09$ \\
\end{tabular} & 2 & 0.10 & & & & & & & 2 & 0.10 \\
\hline $\bar{D}$ & 1001 & 1009 & 6 & $99.09-98.99$ & 5 & 0.10 & 3 & 0.30 & & & & & 8 & 0.40 \\
\hline $\mathrm{D}$ & 1001 & 1009 & 7 & $98.99-98.89$ & 6 & 1.00 & 9 & 1.80 & 1 & 0.20 & & & 16 & 3.00 \\
\hline $\bar{D}$ & 1001 & 1009 & 8 & \begin{tabular}{|c|}
$98.89-98.79$ \\
\end{tabular} & 2 & 0.10 & & & & & & & 2 & 0.10 \\
\hline $\mathrm{D}$ & 1001 & 1009 & 9 & \begin{tabular}{|c|}
$98.79-98.69$ \\
\end{tabular} & 5 & 0.10 & & & & & & & 5 & 0.10 \\
\hline $\mathrm{D}$ & 1001 & 1009 & 10 & \begin{tabular}{|c|}
$98.69-98.59$ \\
\end{tabular} & 2 & 0.10 & & & & & & & 2 & 0.10 \\
\hline $\mathrm{D}$ & 1001 & 1009 & 12 & 98.49-98.39 & 3 & 0.20 & & & & & & & 3 & 0.20 \\
\hline $\mathrm{D}$ & 1001 & 1009 & 13 & $98.39-98.29$ & 7 & 0.10 & & & & & & & 7 & 0.10 \\
\hline $\mathrm{D}$ & 1001 & 1010 & 14 & $98.29-98.19$ & 4 & 0.10 & & & & & & & 4 & 0.10 \\
\hline $\mathrm{D}$ & 1001 & 1010 & 15 & $98.19-98.09$ & 2 & 0.10 & & & & & & & 2 & 0.10 \\
\hline $\mathrm{D}$ & 1001 & 1010 & 18 & \begin{tabular}{|c|}
$97.89-97.79$ \\
\end{tabular} & 2 & 0.10 & & & & & & & 2 & 0.10 \\
\hline $\mathrm{D}$ & 1001 & 1010 & 19 & \begin{tabular}{|c|}
$97.79-97.69$ \\
\end{tabular} & 1 & 0.10 & & & & & & & 1 & 0.10 \\
\hline $\mathrm{D}$ & 1001 & 1010 & 20 & $97.69-97.59$ & 4 & 0.10 & & & & & & & 4 & 0.10 \\
\hline $\bar{E}$ & Ftr 2 & & & $98.77-98.50$ & 3 & 0.20 & 7 & 1.00 & 1 & 1.80 & & & 11 & 3.00 \\
\hline
\end{tabular}




\begin{tabular}{|c|c|c|c|c|c|c|c|c|c|c|c|c|c|c|}
\hline \multicolumn{5}{|c|}{ Provenience } & \multicolumn{8}{|c|}{ Burned Rock Data } & \multicolumn{2}{|c|}{ Totals } \\
\hline $\begin{array}{c}\text { Excavation } \\
\text { Block }\end{array}$ & Northing & Easting & Level & Elev. (m) & $0-5 \mathrm{Ct}$ & \begin{tabular}{|c|}
$0-5 \mathrm{Wt}$ \\
$(\mathrm{kg})$
\end{tabular} & $5-10 \mathrm{Ct}$ & \begin{tabular}{|c|}
$5-10 \mathrm{Wt}$ \\
$(\mathrm{kg})$
\end{tabular} & $10-15 \mathrm{Ct}$ & \begin{tabular}{|c|}
$10-15 \mathrm{Wt}$ \\
$(\mathrm{kg})$
\end{tabular} & $15+C t$ & $\begin{array}{c}15+W t \\
(\mathrm{~kg})\end{array}$ & Total Ct & $\begin{array}{c}\text { Total Wt } \\
(\mathrm{kg})\end{array}$ \\
\hline $\mathrm{E}$ & 1003 & 1017 & 2 & $99.20-99.10$ & 3 & 0.10 & & & & & & & 3 & 0.10 \\
\hline $\mathrm{E}$ & 1003 & 1017 & 4 & $99.00-98.90$ & 7 & 0.10 & & & & & & & 7 & 0.10 \\
\hline $\bar{E}$ & 1003 & 1017 & 5 & $98.90-98.80$ & 10 & 0.10 & & & & & & & 10 & 0.10 \\
\hline$E$ & 1003 & 1017 & 6 & 98.89-98.70 & 12 & 0.10 & 2 & 0.10 & & & & & 14 & 0.20 \\
\hline$E$ & 1003 & 1017 & 7 & $98.70-98.60$ & & & 6 & 0.60 & 15 & 0.10 & & & 21 & 0.70 \\
\hline$E$ & 1003 & 1017 & 8 & $98.60-98.50$ & 11 & 0.10 & 4 & 0.20 & & & & & 15 & 0.30 \\
\hline$E$ & 1003 & 1017 & 9 & $98.50-98.40$ & 4 & 0.10 & 5 & 0.20 & & & & & 9 & 0.30 \\
\hline$E$ & 1003 & 1017 & 10 & $98.40-98.30$ & 2 & 0.10 & & & & & & & 2 & 0.10 \\
\hline$E$ & 1003 & 1017 & 11 & $98.30-98.20$ & 1 & 0.10 & 2 & 0.20 & & & & & 3 & 0.30 \\
\hline$E$ & 1003 & 1017 & 13 & $98.10-98.00$ & 2 & 0.10 & & & & & & & 2 & 0.10 \\
\hline$E$ & 1003 & 1017 & 14 & $98.00-97.90$ & 3 & 0.10 & & & & & & & 3 & 0.10 \\
\hline$E$ & 1003 & 1017 & 15 & $97.90-97.80$ & 3 & 0.10 & & & & & & & 3 & 0.10 \\
\hline $\mathrm{E}$ & 1003 & 1018 & 3 & $99.10-99.00$ & 2 & 0.10 & & & & & & & 2 & 0.10 \\
\hline$E$ & 1003 & 1018 & 4 & $99.00-98.90$ & 3 & 0.10 & & & & & & & 3 & 0.10 \\
\hline$E$ & 1003 & 1018 & 5 & $98.90-98.80$ & 1 & 0.10 & 3 & 0.10 & & & & & 4 & 0.20 \\
\hline$E$ & 1003 & 1018 & 6 & $98.80-98.70$ & 7 & 0.10 & 1 & 0.10 & & & & & 8 & 0.20 \\
\hline$E$ & 1003 & 1018 & 7 & $98.70-98.60$ & 10 & 0.10 & 6 & 0.40 & & & & & 16 & 0.50 \\
\hline$E$ & 1003 & 1018 & 8 & $98.60-98.50$ & 22 & 0.01 & 3 & 0.02 & & & & & 25 & 0.03 \\
\hline$E$ & 1003 & 1018 & 9 & $98.50-98.40$ & 17 & 0.10 & 3 & 0.20 & 1 & 0.40 & & & 21 & 0.70 \\
\hline$E$ & 1003 & 1018 & 10 & $98.40-98.30$ & 11 & 0.10 & 4 & 0.30 & & & & & 15 & 0.40 \\
\hline$E$ & 1003 & 1018 & 11 & $98.30-98.20$ & 3 & 0.10 & 1 & 0.10 & & & & & 4 & 0.20 \\
\hline$E$ & 1003 & 1018 & 12 & $98.10-98.00$ & 2 & 0.10 & & & & & & & 2 & 0.10 \\
\hline$E$ & 1003 & 1018 & 14 & $98.00-97.90$ & 4 & 0.10 & & & & & & & 4 & 0.10 \\
\hline $\mathrm{E}$ & 1003 & 1018 & 15 & $97.90-97.80$ & 6 & 0.10 & & & & & & & 6 & 0.10 \\
\hline$E$ & 1003 & 1019 & 2 & $99.20-99.10$ & 2 & 0.10 & & & & & & & 2 & 0.10 \\
\hline$E$ & 1003 & 1019 & 4 & $99.00-98.90$ & 5 & 0.10 & & & & & & & 5 & 0.10 \\
\hline$E$ & 1003 & 1019 & 5 & $98.90-98.80$ & 10 & 0.10 & 4 & 0.10 & & & & & 14 & 0.20 \\
\hline$E$ & 1003 & 1019 & 6 & $98.80-98.70$ & 14 & 0.10 & 5 & 0.40 & & & & & 19 & 0.50 \\
\hline$E$ & 1003 & 1019 & 7 & $98.70-98.60$ & 24 & 0.20 & 9 & 0.70 & & & & & 33 & 0.90 \\
\hline$E$ & 1003 & 1019 & 8 & $98.60-98.50$ & 16 & 0.20 & 4 & 0.20 & & & & & 20 & 0.40 \\
\hline$E$ & 1003 & 1019 & 9 & $98.50-98.40$ & 10 & 0.10 & 3 & 0.10 & & & & & 13 & 0.20 \\
\hline$E$ & 1003 & 1019 & 10 & $98.40-98.30$ & 11 & 0.10 & 4 & 0.40 & & & & & 15 & 0.50 \\
\hline$E$ & 1003 & 1019 & 11 & $98.30-98.20$ & 10 & 0.10 & 7 & 1.00 & & & & & 17 & 1.10 \\
\hline$E$ & 1003 & 1019 & 12 & $98.20-98.10$ & 2 & 0.10 & & & & & & & 2 & 0.10 \\
\hline$E$ & 1003 & 1019 & 13 & $98.10-98.00$ & 5 & 0.10 & & & & & & & 5 & 0.10 \\
\hline$E$ & 1003 & 1019 & 15 & $97.90-97.80$ & 1 & 0.10 & & & & & & & 1 & 0.10 \\
\hline$E$ & 1004 & 1017 & 2 & \begin{tabular}{|c|}
$99.20-99.10$ \\
\end{tabular} & 3 & 0.10 & & & & & & & 3 & 0.10 \\
\hline$E$ & 1004 & 1017 & 4 & $99.00-98.90$ & 5 & 1.00 & & & & & & & 5 & 1.00 \\
\hline$E$ & 1004 & 1017 & 5 & 98.90-98.80 & 6 & 0.50 & 1 & 0.50 & & & & & 7 & 1.00 \\
\hline $\bar{E}$ & 1004 & 1017 & 6 & $98.80-98.70$ & 8 & 0.10 & 2 & 0.20 & & & & & 10 & 0.30 \\
\hline$E$ & 1004 & 1017 & 7 & $98.70-98.60$ & 4 & 0.10 & 8 & 1.20 & & & & & 12 & 1.30 \\
\hline$E$ & 1004 & 1017 & 8 & $98.60-98.50$ & 14 & 1.00 & & & & & & & 14 & 1.00 \\
\hline$E$ & 1004 & 1017 & 9 & $98.50-98.40$ & 8 & 0.50 & 3 & 1.00 & & & & & 11 & 1.50 \\
\hline$E$ & 1004 & 1017 & 10 & $98.40-98.30$ & 3 & 0.50 & & & & & & & 3 & 0.50 \\
\hline$E$ & 1004 & 1017 & 11 & $98.30-98.20$ & & & 1 & 0.10 & & & & & 1 & 0.10 \\
\hline$E$ & 1004 & 1018 & 4 & $99.00-98.90$ & 1 & 1.00 & & & & & & & 1 & 1.00 \\
\hline$E$ & 1004 & 1018 & 5 & $98.90-98.80$ & 2 & 0.10 & & & & & & & 2 & 0.10 \\
\hline $\bar{E}$ & 1004 & 1018 & 6 & $98.80-98.70$ & 7 & 0.10 & 4 & 0.30 & & & & & 11 & 0.40 \\
\hline$E$ & 1004 & 1018 & 7 & $98.70-98.60$ & 4 & 0.10 & 7 & 1.20 & & & & & 11 & 1.30 \\
\hline$E$ & 1004 & 1018 & 8 & $98.60-98.50$ & 11 & 0.20 & 1 & 0.10 & & & & & 12 & 0.30 \\
\hline$E$ & 1004 & 1018 & 9 & $98.50-98.40$ & 7 & 0.05 & 1 & 0.05 & & & & & 8 & 0.10 \\
\hline$E$ & 1004 & 1018 & 10 & $98.40-98.30$ & 6 & 0.10 & & & & & & & 6 & 0.10 \\
\hline $\mathrm{E}$ & 1004 & 1018 & 11 & $98.30-98.20$ & 2 & 0.10 & & & & & & & 2 & 0.10 \\
\hline $\bar{E}$ & 1004 & 1018 & 12 & $98.20-98.10$ & 3 & 0.10 & 1 & 0.10 & & & & & 4 & 0.20 \\
\hline$E$ & 1004 & 1019 & 5 & $98.90-98.80$ & 3 & 0.10 & & & & & & & 3 & 0.10 \\
\hline$E$ & 1004 & 1019 & 6 & $98.80-98.70$ & 7 & 0.10 & & & & & & & 7 & 0.10 \\
\hline$E$ & 1004 & 1019 & 7 & $98.70-98.60$ & 27 & 0.70 & 5 & 0.20 & & & & & 32 & 0.90 \\
\hline $\mathrm{E}$ & 1004 & 1019 & 8 & $98.60-98.50$ & 5 & 0.10 & 2 & 0.20 & & & & & 7 & 0.30 \\
\hline$E$ & 1004 & 1019 & 9 & $98.50-98.40$ & 11 & 0.10 & 4 & 0.30 & & & & & 15 & 0.40 \\
\hline$E$ & 1004 & 1019 & 10 & $98.40-98.30$ & 13 & 0.10 & 1 & 0.10 & & & & & 14 & 0.20 \\
\hline$E$ & 1004 & 1019 & 11 & $98.30-98.20$ & 6 & 0.10 & & & & & & & 6 & 0.10 \\
\hline$E$ & 1004 & 1019 & 12 & \begin{tabular}{|c|}
$98.20-98.10$ \\
\end{tabular} & 7 & 0.10 & & & & & & & 7 & 0.10 \\
\hline$E$ & 1004 & 1019 & 13 & $98.10-98.00$ & 2 & 0.10 & & & & & & & 2 & 0.10 \\
\hline$E$ & 1004 & 1019 & 14 & $98.00-97.90$ & 12 & 0.20 & & & & & & & 12 & 0.20 \\
\hline$E$ & 1004 & 1019 & 15 & $97.90-97.80$ & 1 & 0.10 & & & & & & & 1 & 0.10 \\
\hline $\mathrm{F}$ & 1009 & 1011 & 5 & $98.90-98.80$ & 3 & 0.3 & & & & & & & 3 & 0.3 \\
\hline $\mathrm{F}$ & 1009 & 1011 & 6 & $98.80-98.70$ & 5 & 0.7 & 1 & 0.6 & & & & & 6 & 1.3 \\
\hline $\mathrm{F}$ & 1009 & 1011 & 7 & $98.70-98.60$ & 15 & 0.7 & 2 & 0.4 & & & & & 17 & 1.1 \\
\hline $\mathrm{F}$ & 1009 & 1011 & 8 & $98.60-98.50$ & 3 & 0.5 & 1 & 0.7 & & & & & 4 & 1.2 \\
\hline $\mathrm{F}$ & 1009 & 1011 & 9 & $98.50-98.40$ & 5 & 0.2 & & & & & & & 5 & 0.2 \\
\hline $\mathrm{F}$ & 1009 & 1011 & 10 & $98.40-98.30$ & 1 & 0.2 & & & & & & & 1 & 0.2 \\
\hline
\end{tabular}




\begin{tabular}{|c|c|c|c|c|c|c|c|c|c|c|c|c|c|c|}
\hline \multicolumn{5}{|c|}{ Provenience } & \multicolumn{8}{|c|}{ Burned Rock Data } & \multicolumn{2}{|c|}{ Totals } \\
\hline $\begin{array}{c}\text { Excavation } \\
\text { Block }\end{array}$ & Northing & Easting & Level & Elev. (m) & $0-5 \mathrm{Ct}$ & \begin{tabular}{|c|}
$0-5 \mathrm{Wt}$ \\
$(\mathrm{kg})$
\end{tabular} & $5-10 \mathrm{Ct}$ & $\begin{array}{c}5-10 \mathrm{Wt} \\
(\mathrm{kg})\end{array}$ & $10-15 \mathrm{Ct}$ & $\begin{array}{c}10-15 \mathrm{Wt} \\
(\mathrm{kg})\end{array}$ & $15+C t$ & \begin{tabular}{|c|}
$15+W t$ \\
$(\mathrm{~kg})$
\end{tabular} & Total Ct & $\begin{array}{c}\text { Total Wt } \\
(\mathrm{kg})\end{array}$ \\
\hline $\mathrm{F}$ & 1009 & 1011 & 11 & $98.30-98.20$ & 5 & 0.1 & & & & & & & 5 & 0.1 \\
\hline $\mathrm{F}$ & 1009 & 1011 & 12 & $98.20-98.10$ & 6 & 0.1 & & & & & & & 6 & 0.1 \\
\hline $\mathrm{F}$ & 1009 & 1011 & 13 & $98.10-98.00$ & 8 & 0.1 & 2 & 0.2 & & & & & 10 & 0.3 \\
\hline $\mathrm{F}$ & 1009 & 1011 & 14 & $98.00-97.90$ & 1 & 0.10 & & & & & & & 1 & 0.1 \\
\hline $\mathrm{F}$ & 1009 & 1012 & 1 & $99.21-99.10$ & 3 & 0.10 & & & & & & & 3 & 0.1 \\
\hline $\mathrm{F}$ & 1009 & 1012 & 3 & $99.00-98.90$ & 2 & 0.10 & & & & & & & 2 & 0.1 \\
\hline $\mathrm{F}$ & 1009 & 1012 & 4 & 98.90-98.80 & 1 & 0.10 & & & & & & & 1 & 0.1 \\
\hline $\mathrm{F}$ & 1009 & 1012 & 5 & $98.80-98.70$ & 3 & 0.7 & & & & & & & 3 & 0.7 \\
\hline $\mathrm{F}$ & 1009 & 1012 & 6 & $98.70-98.60$ & 3 & 0.10 & & & & & & & 3 & 0.1 \\
\hline $\mathrm{F}$ & 1009 & 1012 & 7 & $98.60-98.50$ & 10 & 0.4 & & & & & & & 10 & 0.4 \\
\hline $\mathrm{F}$ & 1009 & 1012 & 8 & $98.50-98.40$ & 3 & 0.2 & & & & & & & 3 & 0.2 \\
\hline $\mathrm{F}$ & 1009 & 1012 & 9 & $98.40-98.30$ & 2 & 0.10 & & & & & & & 2 & 0.1 \\
\hline $\mathrm{F}$ & 1009 & 1012 & 10 & $98.30-98.20$ & 1 & 0.3 & & & & & & & 1 & 0.3 \\
\hline $\mathrm{F}$ & 1009 & 1012 & 11 & $98.20-98.10$ & 6 & 0.2 & & & & & & & 6 & 0.2 \\
\hline $\mathrm{F}$ & 1009 & 1012 & 12 & $98.10-98.00$ & 5 & 0.1 & & & & & & & 5 & 0.1 \\
\hline
\end{tabular}




\section{APPENDIX C}

\section{Artifact Analysis Tables}





\begin{tabular}{|c|c|c|c|c|c|c|c|c|c|c|c|c|c|c|c|c|c|c|c|c|}
\hline $\begin{array}{l}\text { Lot } \\
\text { No. }\end{array}$ & $\begin{array}{l}\text { Spec. } \\
\text { No. }\end{array}$ & $\begin{array}{l}\text { Bag } \\
\text { No. }\end{array}$ & $\begin{array}{l}\text { UI } \\
\text { No. }\end{array}$ & Category & Subcategory & Condition & Raw Material & Munsell & Color & Patination & $\begin{array}{c}\text { Heat } \\
\text { Evidence }\end{array}$ & Breakage & Beveling & Reworking & Shoulders & Basal Margin & $\begin{array}{c}\text { Blade } \\
\text { Observations }\end{array}$ & $\begin{array}{c}\text { Stem } \\
\text { s Observations }\end{array}$ & Notching & General Flaking Patterns \\
\hline \multirow{3}{*}{550} & \multirow{3}{*}{69} & \multirow{3}{*}{338} & \multirow{3}{*}{19} & $\begin{array}{c}\text { Projectile } \\
\text { Point }\end{array}$ & Indet & Blade & $\begin{array}{l}\text { Fine Grain } \\
\text { Chert }\end{array}$ & 10YR5/2 & Grayish Brown & None & None & Use Wear & None & None & $\begin{array}{l}\text { Left: Long and } \\
\text { Right: Short }\end{array}$ & Indet & Straight & Indet & Indet & $\begin{array}{l}\text { Ventral: Oblique Subparallel } \\
\text { and Dorsal: Collateral }\end{array}$ \\
\hline & & & & $\begin{array}{c}\text { Weight } \\
\text { (g) }\end{array}$ & $\begin{array}{l}\text { Maximum } \\
\text { Length }\end{array}$ & $\begin{array}{l}\text { Maximum } \\
\text { Stem } \\
\text { Length }\end{array}$ & $\begin{array}{c}\text { Maximum } \\
\text { Blade Width }\end{array}$ & $\begin{array}{l}\text { Medial Blade } \\
\text { Width }\end{array}$ & $\begin{array}{l}\text { Medial Blade } \\
\text { Thickness }\end{array}$ & $\begin{array}{l}\text { Neck } \\
\text { Width }\end{array}$ & $\begin{array}{l}\text { Stem Base } \\
\text { Width }\end{array}$ & $\begin{array}{c}\text { Medial Stem } \\
\text { Thickness }\end{array}$ & $\begin{array}{c}\text { Basal } \\
\text { Concavity } \\
\text { Width }\end{array}$ & $\begin{array}{l}\text { Base } \\
\text { Depth }\end{array}$ & $\begin{array}{l}\text { Left Notch } \\
\text { Depth }\end{array}$ & $\begin{array}{l}\text { Right Notch } \\
\text { Depth }\end{array}$ & $\begin{array}{l}\text { Left Notch } \\
\text { Width }\end{array}$ & $\begin{array}{l}\text { Right Notch } \\
\text { Width }\end{array}$ & & Comments \\
\hline & & & & 1.1 & Indet & Indet & 15 & Indet & Indet & Indet & Indet & Indet & Indet & Indet & Indet & Indet & Indet & Indet & $\begin{array}{r}\text { Distal tip (ar } \\
\text { broken; }\end{array}$ & $\begin{array}{l}\text { approximately top } 1 / 3 \text { ) and stem } \\
\text {;; Notch appears to be side }\end{array}$ \\
\hline $\begin{array}{l}\text { Lot } \\
\text { No. }\end{array}$ & $\begin{array}{l}\text { Spec. } \\
\text { No. }\end{array}$ & $\begin{array}{l}\text { Bag } \\
\text { No. }\end{array}$ & $\begin{array}{l}\text { UI } \\
\text { No. }\end{array}$ & Category & Subcategory & Condition & Raw Material & Munsell & Color & Patination & $\begin{array}{c}\text { Heat } \\
\text { Evidence }\end{array}$ & Breakage & Beveling & Reworking & Shoulders & Basal Margin & $\begin{array}{c}\text { Blade } \\
\text { Observations } \\
\end{array}$ & $\begin{array}{c}\text { Stem } \\
\text { s Observations }\end{array}$ & Notching & General Flaking Patterns \\
\hline \multirow{3}{*}{552} & \multirow{3}{*}{8} & \multirow{3}{*}{347} & \multirow{3}{*}{20} & $\begin{array}{c}\text { Projectile } \\
\text { Point }\end{array}$ & Edwards & Stem & $\begin{array}{c}\text { Fine Grain } \\
\text { Chert } \\
\end{array}$ & 10YR3/1 & $\begin{array}{c}\text { Very Dark } \\
\text { Gray }\end{array}$ & None & None & Use Wear & Indet & None & Indet & Deep Concave & Indet & Expanding & Side & Random \\
\hline & & & & $\begin{array}{l}\text { Weight } \\
\text { (g) }\end{array}$ & $\begin{array}{c}\text { Maximum } \\
\text { Length }\end{array}$ & $\begin{array}{c}\text { Maximum } \\
\text { Stem } \\
\text { Length }\end{array}$ & $\begin{array}{c}\text { Maximum } \\
\text { Blade Width }\end{array}$ & $\begin{array}{l}\text { Medial Blade } \\
\text { Width }\end{array}$ & $\begin{array}{l}\text { Medial Blade } \\
\text { Thickness }\end{array}$ & $\begin{array}{l}\text { Neck } \\
\text { Width }\end{array}$ & $\begin{array}{l}\text { Stem Base } \\
\text { Width }\end{array}$ & $\begin{array}{l}\text { Medial Stem } \\
\text { Thickness }\end{array}$ & $\begin{array}{c}\text { Basal } \\
\text { Concavity } \\
\text { Width }\end{array}$ & $\begin{array}{l}\text { Base } \\
\text { Depth }\end{array}$ & $\begin{array}{l}\text { Left Notch } \\
\text { Depth }\end{array}$ & $\begin{array}{l}\text { Right Notch } \\
\text { Depth }\end{array}$ & $\begin{array}{l}\text { Left Notch } \\
\text { Width }\end{array}$ & $\begin{array}{l}\text { Right Notch } \\
\text { Width }\end{array}$ & & Comments \\
\hline & & & & 0.32 & Indet & Indet & Indet & Indet & Indet & Indet & 18.04 & 2.76 & 12.99 & 1.53 & Indet & Indet & Indet & Indet & Finely flake & ed edges; Full length unknown \\
\hline $\begin{array}{l}\text { Lot } \\
\text { No. }\end{array}$ & $\begin{array}{l}\text { Spec. } \\
\text { No. }\end{array}$ & $\begin{array}{l}\text { Bag } \\
\text { No. }\end{array}$ & $\begin{array}{l}\text { UI } \\
\text { No. }\end{array}$ & Category & Subcategory & Condition & Raw Material & Munsell & Color & Patination & $\begin{array}{c}\text { Heat } \\
\text { Evidence }\end{array}$ & Breakage & Beveling & Reworking & Shoulders & Basal Margin & $\begin{array}{c}\text { Blade } \\
\text { Observations }\end{array}$ & $\begin{array}{c}\text { Stem } \\
\text { s Observations }\end{array}$ & Notching & General Flaking Patterns \\
\hline \multirow[b]{3}{*}{559} & \multirow[b]{3}{*}{7} & \multirow[b]{3}{*}{360} & \multirow[b]{3}{*}{21} & $\begin{array}{c}\text { Projectile } \\
\text { Point }\end{array}$ & Perdiz & $\begin{array}{c}\text { Basal } \\
\text { Fragment } \\
\end{array}$ & $\begin{array}{c}\text { Fine Grain } \\
\text { Chert } \\
\end{array}$ & $10 \mathrm{G} 5 / 1$ & Greenish Gray & None & None & Use Wear & None & $\begin{array}{c}\text { One } \\
\text { Shoulder }\end{array}$ & $\begin{array}{l}\text { Left: Abrupt and } \\
\text { Right: Long } \\
\end{array}$ & Gentle Convex & Indet & Contracting & Side & Subparallel \\
\hline & & & & $\begin{array}{l}\text { Weight } \\
\text { (g) }\end{array}$ & $\begin{array}{c}\text { Maximum } \\
\text { Length }\end{array}$ & $\begin{array}{c}\text { Maximum } \\
\text { Stem } \\
\text { Length } \\
\end{array}$ & $\begin{array}{c}\text { Maximum } \\
\text { Blade Width }\end{array}$ & $\begin{array}{l}\text { Medial Blade } \\
\text { Width }\end{array}$ & $\begin{array}{l}\text { Medial Blade } \\
\text { Thickness }\end{array}$ & $\begin{array}{l}\text { Neck } \\
\text { Width }\end{array}$ & $\begin{array}{l}\text { Stem Base } \\
\text { Width }\end{array}$ & $\begin{array}{l}\text { Medial Stem } \\
\text { Thickness }\end{array}$ & $\begin{array}{c}\text { Basal } \\
\text { Concavity } \\
\text { Width } \\
\end{array}$ & $\begin{array}{l}\text { Base } \\
\text { Depth }\end{array}$ & $\begin{array}{l}\text { Left Notch } \\
\text { Depth }\end{array}$ & $\begin{array}{l}\text { Right Notch } \\
\text { Depth }\end{array}$ & $\begin{array}{l}\text { Left Notch } \\
\text { Width }\end{array}$ & $\begin{array}{l}\text { Right Notch } \\
\text { Width }\end{array}$ & & Comments \\
\hline & & & & 0.58 & Indet & 9.95 & 14.44 & Indet & Indet & 5.24 & 2.98 & 2.62 & 0 & 0 & 2.64 & 3.84 & 11.86 & 11.74 & $\begin{array}{r}\text { Basal frag } \\
\text { barb is signi } \\
\text { appears } \\
\text { association } \\
\text { from barb to } \\
\text { bar }\end{array}$ & $\begin{array}{l}\text { gment with partial blade; One } \\
\text { ificantly shorter than the other - } \\
\text { s to have been removed in } \\
\text { n with transver flake extending } \\
\text { o opposite lateral margin; Short } \\
\text { rb has been retouched }\end{array}$ \\
\hline $\begin{array}{l}\text { Lot } \\
\text { No. }\end{array}$ & $\begin{array}{l}\text { Spec. } \\
\text { No. }\end{array}$ & $\begin{array}{l}\text { Bag } \\
\text { No. }\end{array}$ & $\begin{array}{l}\mathrm{UI} \\
\text { No. }\end{array}$ & Category & Subcategory & Condition & Raw Material & Munsell & Color & Patination & $\begin{array}{c}\text { Heat } \\
\text { Evidence }\end{array}$ & Breakage & Beveling & Reworking & Shoulders & Basal Margin & $\begin{array}{c}\text { Blade } \\
\text { Observations } \\
\end{array}$ & $\begin{array}{c}\text { Stem } \\
\text { s Observations }\end{array}$ & Notching & General Flaking Patterns \\
\hline \multirow{3}{*}{579} & \multirow{3}{*}{10} & \multirow{3}{*}{643} & \multirow{3}{*}{32} & \begin{tabular}{|c|} 
Projectile \\
Point
\end{tabular} & Perdiz & Complete & $\begin{array}{c}\text { Fine Grain } \\
\text { Chert }\end{array}$ & 10YR5/3 & Brown & None & None & Indet Break & None & None & Long & Gentle Convex & Straight & Contracting & Corner & Oblique Subparallel \\
\hline & & & & $\begin{array}{c}\text { Weight } \\
\text { (g) }\end{array}$ & $\begin{array}{c}\text { Maximum } \\
\text { Length }\end{array}$ & $\begin{array}{c}\text { Maximum } \\
\text { Stem } \\
\text { Length } \\
\end{array}$ & $\begin{array}{c}\text { Maximum } \\
\text { Blade Width }\end{array}$ & $\begin{array}{l}\text { Medial Blade } \\
\text { Width }\end{array}$ & $\begin{array}{l}\text { Medial Blade } \\
\text { Thickness }\end{array}$ & $\begin{array}{l}\text { Neck } \\
\text { Width }\end{array}$ & $\begin{array}{l}\text { Stem Base } \\
\text { Width }\end{array}$ & $\begin{array}{c}\text { Medial Stem } \\
\text { Thickness }\end{array}$ & $\begin{array}{c}\text { Basal } \\
\text { Concavity } \\
\text { Width } \\
\end{array}$ & $\begin{array}{l}\text { Base } \\
\text { Depth }\end{array}$ & $\begin{array}{l}\text { Left Notch } \\
\text { Depth }\end{array}$ & $\begin{array}{l}\text { Right Notch } \\
\text { Depth }\end{array}$ & $\begin{array}{l}\text { Left Notch } \\
\text { Width }\end{array}$ & $\begin{array}{l}\text { Right Notch } \\
\text { Width }\end{array}$ & & Comments \\
\hline & & & & 1.43 & 31.3 & 7.02 & 20.62 & 15.46 & 2.53 & 8.23 & 4.15 & 2.28 & 0 & 0 & 2.65 & 5.03 & 9.49 & 10.01 & Left shoulde & $\begin{array}{l}\text { er tip broken; Mineral impurities } \\
\text { in stem (10YR8/2) }\end{array}$ \\
\hline $\begin{array}{l}\text { Lot } \\
\text { No. }\end{array}$ & $\begin{array}{l}\text { Spec. } \\
\text { No. }\end{array}$ & $\begin{array}{l}\text { Bag } \\
\text { No. }\end{array}$ & $\begin{array}{l}\text { UI } \\
\text { No. }\end{array}$ & Category & Subcategory & Condition & Raw Material & Munsell & Color & Patination & $\begin{array}{c}\text { Heat } \\
\text { Evidence }\end{array}$ & Breakage & Beveling & Reworking & Shoulders & Basal Margin & $\begin{array}{c}\text { Blade } \\
\text { Observations }\end{array}$ & $\begin{array}{c}\text { Stem } \\
\text { s Observations }\end{array}$ & Notching & General Flaking Patterns \\
\hline \multirow{3}{*}{607} & \multirow{3}{*}{6} & \multirow{3}{*}{472} & \multirow{3}{*}{ NA } & $\begin{array}{c}\text { Projectile } \\
\text { Point }\end{array}$ & Fresno & $\begin{array}{c}\text { Basal } \\
\text { Fragment } \\
\end{array}$ & $\begin{array}{c}\text { Fine Grain } \\
\text { Chert }\end{array}$ & 10YR3/1 & $\begin{array}{c}\text { Very Dark } \\
\text { Gray }\end{array}$ & None & None & Use Wear & None & None & Absent & Straight & Convex & Straight & Absent & Oblique Subparallel \\
\hline & & & & \begin{tabular}{|c|}
$\begin{array}{c}\text { Weight } \\
\text { (g) }\end{array}$ \\
\end{tabular} & $\begin{array}{c}\text { Maximum } \\
\text { Length }\end{array}$ & $\begin{array}{l}\text { Maximum } \\
\text { Stem } \\
\text { Length }\end{array}$ & $\begin{array}{c}\text { Maximum } \\
\text { Blade Width } \\
\end{array}$ & $\begin{array}{l}\text { Medial Blade } \\
\text { Width }\end{array}$ & $\begin{array}{c}\text { Medial Blade } \\
\text { Thickness }\end{array}$ & $\begin{array}{l}\text { Neck } \\
\text { Width }\end{array}$ & $\begin{array}{l}\text { Stem Base } \\
\text { Width }\end{array}$ & $\begin{array}{c}\text { Medial Stem } \\
\text { Thickness }\end{array}$ & $\begin{array}{c}\text { Basal } \\
\text { Concavity } \\
\text { Width }\end{array}$ & $\begin{array}{l}\text { Base } \\
\text { Depth }\end{array}$ & $\begin{array}{l}\text { Left Notch } \\
\text { Depth }\end{array}$ & $\begin{array}{l}\text { Right Notch } \\
\text { Depth }\end{array}$ & $\begin{array}{l}\text { Left Notch } \\
\text { Width }\end{array}$ & $\begin{array}{l}\text { Right Notch } \\
\text { Width }\end{array}$ & & Comments \\
\hline & & & & 2.19 & Indet & Indet & 17.88 & 15.38 & 3.69 & Indet & Indet & Indet & Absent & Absent & Absent & Absent & Absent & Absent & \multicolumn{2}{|c|}{$\begin{array}{l}\text { Basal fragment with partial blade; Missing } \\
\text { distal tip; Non-stemmed projectile point }\end{array}$} \\
\hline
\end{tabular}




\begin{tabular}{|c|c|c|c|c|c|c|c|c|c|c|c|c|c|c|c|c|c|c|c|c|}
\hline $\begin{array}{l}\text { Lot } \\
\text { No. }\end{array}$ & $\begin{array}{l}\text { Spec. } \\
\text { No. }\end{array}$ & $\begin{array}{l}\text { Bag } \\
\text { No. }\end{array}$ & $\begin{array}{l}\text { UI } \\
\text { No. }\end{array}$ & Category & Subcategory & Condition & Raw Material & Munsell & Color & Patination & $\begin{array}{c}\text { Heat } \\
\text { Evidence }\end{array}$ & Breakage & Beveling & Reworking & Shoulders & Basal Margin & $\begin{array}{c}\text { Blade } \\
\text { Observations } \\
\end{array}$ & $\begin{array}{c}\text { Stem } \\
\text { s Observations }\end{array}$ & Notching & General Flaking Patterns \\
\hline \multirow{3}{*}{621} & \multirow{3}{*}{7} & \multirow{3}{*}{539} & \multirow{3}{*}{27} & $\begin{array}{c}\text { Projectile } \\
\text { Point }\end{array}$ & Zephyr & Complete & $\begin{array}{l}\text { Fine Grain } \\
\text { Chert }\end{array}$ & 10YR5/3 & Brown & None & None & None & None & $\begin{array}{l}\text { One Lateral } \\
\text { Edge }\end{array}$ & Short & $\begin{array}{l}\text { Straight to } \\
\text { Shallow } \\
\text { Concave }\end{array}$ & $\begin{array}{l}\text { Leff: Convex } \\
\text { and Right: } \\
\text { Straight }\end{array}$ & Expanding & Corner & Random \\
\hline & & & & $\begin{array}{l}\text { Weight } \\
\text { (g) }\end{array}$ & $\begin{array}{c}\text { Maximum } \\
\text { Length }\end{array}$ & $\begin{array}{c}\text { Maximum } \\
\text { Stem } \\
\text { Lenath }\end{array}$ & $\begin{array}{c}\text { Maximum } \\
\text { Blade Width }\end{array}$ & $\begin{array}{l}\text { Medial Blade } \\
\text { Width }\end{array}$ & $\begin{array}{l}\text { Medial Blade } \\
\text { Thickness }\end{array}$ & $\begin{array}{l}\text { Neck } \\
\text { Width }\end{array}$ & $\begin{array}{l}\text { Stem Base } \\
\text { Width }\end{array}$ & $\begin{array}{c}\text { Medial Stem } \\
\text { Thickness }\end{array}$ & $\begin{array}{c}\text { Basal } \\
\text { Concavity } \\
\text { Width }\end{array}$ & $\begin{array}{l}\text { Base } \\
\text { Depth }\end{array}$ & $\begin{array}{l}\text { Left Notch } \\
\text { Depth }\end{array}$ & $\begin{array}{l}\text { Right Notch } \\
\text { Depth }\end{array}$ & $\begin{array}{l}\text { Left Notch } \\
\text { Width }\end{array}$ & $\begin{array}{l}\text { Right Notch } \\
\text { Width }\end{array}$ & & Comments \\
\hline & & & & 3.86 & 37.71 & 10.1 & 19.69 & 14.14 & 4.6 & 14.27 & 20.25 & 5.21 & 4.89 & 0 & 3.3 & 3.1 & 11.08 & 10.5 & $\begin{array}{r}\text { Specklec } \\
\text { predomin }\end{array}$ & $\begin{array}{l}\text { d with } 10 Y R 8 / 2 \text {; Basal margin } \\
\text { lately straight with a very slight } \\
\text { convex curve }\end{array}$ \\
\hline $\begin{array}{l}\text { Lot } \\
\text { No. }\end{array}$ & $\begin{array}{l}\text { Spec. } \\
\text { No. }\end{array}$ & $\begin{array}{l}\text { Bag } \\
\text { No. }\end{array}$ & $\begin{array}{l}\text { UI } \\
\text { No. }\end{array}$ & Category & Subcategory & Condition & Raw Material & Munsell & Color & Patination & $\begin{array}{c}\text { Heat } \\
\text { Evidence }\end{array}$ & Breakage & Beveling & Reworking & Shoulders & Basal Margin & $\begin{array}{c}\text { Blade } \\
\text { Observations } \\
\end{array}$ & $\begin{array}{c}\text { Stem } \\
\text { s Observations } \\
\end{array}$ & Notching & General Flaking Patterns \\
\hline \multirow{3}{*}{668} & \multirow{3}{*}{6} & \multirow{3}{*}{588} & \multirow{3}{*}{31} & $\begin{array}{c}\text { Projectile } \\
\text { Point }\end{array}$ & Edwards & Complete & $\begin{array}{l}\text { Fine Grain } \\
\text { Chert }\end{array}$ & 10YR5/2 & Grayish Brown & None & None & None & None & None & Short & $\begin{array}{l}\text { Shallow } \\
\text { Concave }\end{array}$ & $\begin{array}{l}\text { Straight to } \\
\text { Slightly } \\
\text { Concave }\end{array}$ & Expanding & Corner & Random \\
\hline & & & & $\begin{array}{l}\text { Weight } \\
\text { (g) }\end{array}$ & $\begin{array}{c}\text { Maximum } \\
\text { Length }\end{array}$ & $\begin{array}{c}\text { Maximum } \\
\text { Stem } \\
\text { Length }\end{array}$ & $\begin{array}{c}\text { Maximum } \\
\text { Blade Width }\end{array}$ & $\begin{array}{l}\text { Medial Blade } \\
\text { Width }\end{array}$ & $\begin{array}{l}\text { Medial Blade } \\
\text { Thickness }\end{array}$ & $\begin{array}{l}\text { Neck } \\
\text { Width }\end{array}$ & $\begin{array}{l}\text { Stem Base } \\
\text { Width }\end{array}$ & $\begin{array}{c}\text { Medial Stem } \\
\text { Thickness }\end{array}$ & $\begin{array}{c}\text { Basal } \\
\text { Concavity } \\
\text { Width } \\
\end{array}$ & $\begin{array}{l}\text { Base } \\
\text { Depth }\end{array}$ & $\begin{array}{l}\text { Left Notch } \\
\text { Depth }\end{array}$ & $\begin{array}{l}\text { Right Notch } \\
\text { Depth }\end{array}$ & $\begin{array}{l}\text { Left Notch } \\
\text { Width }\end{array}$ & $\begin{array}{l}\text { Right Notch } \\
\text { Width }\end{array}$ & & Comments \\
\hline & & & & 1.82 & 38.95 & 8.5 & 18.75 & 10.14 & 4.06 & 6.98 & 17.44 & 3.37 & 5.84 & 1.66 & 4.86 & 4.92 & 6.68 & 6.68 & Slight g & $\begin{array}{l}\text { ray banding (10YR6/1); Fine } \\
\text { flaking along edges }\end{array}$ \\
\hline
\end{tabular}




\begin{tabular}{|c|c|c|c|c|c|c|c|c|c|c|c|c|c|c|c|c|c|}
\hline $\begin{array}{l}\text { Lot } \\
\text { No. }\end{array}$ & $\begin{array}{l}\text { Spec. } \\
\text { No. }\end{array}$ & $\begin{array}{l}\text { Bag } \\
\text { No. }\end{array}$ & $\begin{array}{l}\text { UI } \\
\text { No. }\end{array}$ & Category & Subcategory & $\begin{array}{l}\text { Specimen } \\
\text { Condition }\end{array}$ & Breakage & $\begin{array}{c}\text { Raw } \\
\text { Material }\end{array}$ & Munsell & Color & Cortex \% & $\begin{array}{l}\text { Parent } \\
\text { Form }\end{array}$ & $\begin{array}{c}\text { Heat } \\
\text { Evidence }\end{array}$ & $\begin{array}{l}\text { Intentional } \\
\text { Heat } \\
\text { Treatment }\end{array}$ & Patination & $\begin{array}{l}\text { Overall } \\
\text { Shape }\end{array}$ & $\begin{array}{l}\text { Basal } \\
\text { Shape }\end{array}$ \\
\hline \multirow{3}{*}{316} & \multirow{3}{*}{7} & \multirow{3}{*}{480} & \multirow{3}{*}{ NA } & Biface & Notched Tool & Indet Fragment & Manufacture & $\begin{array}{c}\text { Fine Grain } \\
\text { Chert }\end{array}$ & 10YR6/4 & $\begin{array}{l}\text { Light Yellowish } \\
\text { Brown }\end{array}$ & 0 & Indet & Reddened & Yes & None & Indet & Indet \\
\hline & & & & $\begin{array}{c}\begin{array}{c}\text { Cross-section } \\
\text { Shape }\end{array} \\
\end{array}$ & $\begin{array}{l}\text { Flaking } \\
\text { Pattern } \\
\end{array}$ & Beveling & $\begin{array}{c}\begin{array}{c}\text { Reduction } \\
\text { Stage }\end{array} \\
\end{array}$ & Weight (g) & $\begin{array}{c}\text { Max } \\
\text { Length }\end{array}$ & Max Width & $\begin{array}{c}\text { Max } \\
\text { Thickness } \\
\end{array}$ & $\begin{array}{c}\text { Avg Edge } \\
\text { Angle } \\
\end{array}$ & $\begin{array}{c}\begin{array}{c}\text { Callahan's } \\
\text { w/t index }\end{array} \\
\end{array}$ & \multicolumn{4}{|c|}{ Comments } \\
\hline & & & & $\begin{array}{c}\text { Biconvex } \\
\text { Symmetrical }\end{array}$ & Random & None & Indet & 4.72 & Indet & Indet & Indet & Indet & Indet & \multicolumn{4}{|c|}{$\begin{array}{c}\text { Biface broken and reworked into notched tool; } \\
\text { Light 7.5YR5/6 color (possibly heat treatment) } \\
\text { along portion of margins }\end{array}$} \\
\hline
\end{tabular}

\begin{tabular}{|c|c|c|c|c|c|c|c|c|c|c|c|c|c|c|c|c|c|}
\hline $\begin{array}{l}\text { Lot } \\
\text { No. }\end{array}$ & $\begin{array}{c}\text { Spec. } \\
\text { No. }\end{array}$ & $\begin{array}{l}\text { Bag } \\
\text { No. }\end{array}$ & $\begin{array}{l}\text { UI } \\
\text { No. }\end{array}$ & Category & Subcategory & $\begin{array}{l}\text { Specimen } \\
\text { Condition }\end{array}$ & Breakage & $\begin{array}{c}\text { Raw } \\
\text { Material }\end{array}$ & Munsell & Color & Cortex \% & $\begin{array}{l}\text { Parent } \\
\text { Form }\end{array}$ & $\begin{array}{c}\text { Heat } \\
\text { Evidence } \\
\end{array}$ & $\begin{array}{l}\text { Intentional } \\
\text { Heat } \\
\text { Treatment }\end{array}$ & Patination & $\begin{array}{l}\text { Overall } \\
\text { Shape }\end{array}$ & $\begin{array}{l}\text { Basal } \\
\text { Shape }\end{array}$ \\
\hline \multirow{3}{*}{327} & \multirow{3}{*}{8} & \multirow{3}{*}{589} & \multirow{3}{*}{ NA } & Biface & Biface & Indet Fragment & Manufacture & $\begin{array}{c}\text { Fine Grain } \\
\text { Chert }\end{array}$ & $5 Y R 5 / 3$ & Reddish Brown & 0 & Indet & $\begin{array}{l}\text { Reddened } \\
\text { and Potlids }\end{array}$ & No & None & Indet & Indet \\
\hline & & & & $\begin{array}{c}\text { Cross-section } \\
\text { Shape }\end{array}$ & $\begin{array}{l}\text { Flaking } \\
\text { Pattern }\end{array}$ & Beveling & $\begin{array}{c}\text { Reduction } \\
\text { Stage }\end{array}$ & Weight (g) & $\begin{array}{c}\text { Max } \\
\text { Length }\end{array}$ & Max Width & $\begin{array}{c}\text { Max } \\
\text { Thickness }\end{array}$ & $\begin{array}{c}\text { Avg Edge } \\
\text { Angle }\end{array}$ & $\begin{array}{c}\text { Callahan's } \\
\text { w/t index }\end{array}$ & \multicolumn{4}{|c|}{ Comments } \\
\hline & & & & $\begin{array}{c}\text { Biconvex } \\
\text { Symmetrical }\end{array}$ & Random & None & Indet & 7.39 & Indet & Indet & Indet & Indet & Indet & \multicolumn{4}{|c|}{$\begin{array}{l}\text { Fragment appears to be distal end; } 7.5 Y R 4 / 1 \\
\text { coarse grain mineral inclusion on left lateral } \\
\text { margin; Shape is Indeterminate. }\end{array}$} \\
\hline
\end{tabular}

\begin{tabular}{|c|c|c|c|c|c|c|c|c|c|c|c|c|c|c|c|c|c|}
\hline $\begin{array}{l}\text { Lot } \\
\text { No. }\end{array}$ & $\begin{array}{l}\text { Spec. } \\
\text { No. }\end{array}$ & $\begin{array}{l}\text { Bag } \\
\text { No. }\end{array}$ & $\begin{array}{l}\text { UI } \\
\text { No. }\end{array}$ & Category & Subcategory & $\begin{array}{l}\text { Specimen } \\
\text { Condition }\end{array}$ & Breakage & $\begin{array}{c}\text { Raw } \\
\text { Material }\end{array}$ & Munsell & Color & Cortex \% & $\begin{array}{l}\text { Parent } \\
\text { Form }\end{array}$ & $\begin{array}{c}\text { Heat } \\
\text { Evidence }\end{array}$ & $\begin{array}{c}\text { Intentional } \\
\text { Heat } \\
\text { Treatment }\end{array}$ & Patination & $\begin{array}{l}\text { Overall } \\
\text { Shape }\end{array}$ & $\begin{array}{l}\text { Basal } \\
\text { Shape }\end{array}$ \\
\hline \multirow[b]{3}{*}{336} & \multirow[b]{3}{*}{11} & \multirow[b]{3}{*}{388} & \multirow[b]{3}{*}{ NA } & Biface & Biface & $\begin{array}{c}\text { Marginal } \\
\text { Fragment }\end{array}$ & Manufacture & $\begin{array}{c}\text { Fine Grain } \\
\text { Chert } \\
\end{array}$ & 10YR5/3 & Brown & $1-25$ & Indet & $\begin{array}{l}\text { Reddened } \\
\text { and Potlids }\end{array}$ & No & None & Indet & Indet \\
\hline & & & & $\begin{array}{c}\text { Cross-section } \\
\text { Shape }\end{array}$ & $\begin{array}{l}\text { Flaking } \\
\text { Pattern }\end{array}$ & Beveling & $\begin{array}{c}\begin{array}{c}\text { Reduction } \\
\text { Stage }\end{array} \\
\end{array}$ & Weight (g) & $\begin{array}{c}\text { Max } \\
\text { Length }\end{array}$ & Max Width & $\begin{array}{c}\text { Max } \\
\text { Thickness } \\
\end{array}$ & $\begin{array}{c}\text { Avg Edge } \\
\text { Angle }\end{array}$ & $\begin{array}{l}\text { Callahan's } \\
\text { w/t index }\end{array}$ & \multicolumn{4}{|c|}{ Comments } \\
\hline & & & & Indet & Indet & None & Indet & 6.31 & Indet & Indet & Indet & Indet & Indet & \multicolumn{4}{|c|}{$\begin{array}{l}\text { Heavily potlid damage from het treatment and } \\
5 Y R 5 / 6 \text { coloration; Left lateral margin is finely } \\
\text { worked; Very small }(2 \mathrm{~mm}) \text { portion of cortex on } \\
\text { right lateral margin }\end{array}$} \\
\hline
\end{tabular}

\begin{tabular}{|c|c|c|c|c|c|c|c|c|c|c|c|c|c|c|c|c|c|}
\hline $\begin{array}{l}\text { Lot } \\
\text { No. }\end{array}$ & $\begin{array}{c}\text { Spec. } \\
\text { No. }\end{array}$ & $\begin{array}{l}\text { Bag } \\
\text { No. }\end{array}$ & $\begin{array}{l}\text { UI } \\
\text { No. }\end{array}$ & Category & Subcategory & $\begin{array}{l}\text { Specimen } \\
\text { Condition }\end{array}$ & Breakage & $\begin{array}{c}\text { Raw } \\
\text { Material }\end{array}$ & Munsell & Color & Cortex \% & $\begin{array}{l}\text { Parent } \\
\text { Form }\end{array}$ & $\begin{array}{c}\text { Heat } \\
\text { Evidence }\end{array}$ & $\begin{array}{c}\text { Intentional } \\
\text { Heat } \\
\text { Treatment }\end{array}$ & Patination & $\begin{array}{l}\text { Overall } \\
\text { Shape }\end{array}$ & $\begin{array}{l}\text { Basal } \\
\text { Shape }\end{array}$ \\
\hline \multirow{3}{*}{339} & \multirow{3}{*}{4} & \multirow{3}{*}{410} & \multirow{3}{*}{ NA } & Biface & Biface & $\begin{array}{c}\text { Distal-Medial } \\
\text { Fragment }\end{array}$ & Manufacture & $\begin{array}{c}\text { Fine Grain } \\
\text { Chert }\end{array}$ & 10YR5/2 & Grayish Brown & 0 & Indet & $\begin{array}{l}\text { Reddened } \\
\text { and Gloss }\end{array}$ & Yes & None & Indet & Indet \\
\hline & & & & $\begin{array}{c}\text { Cross-section } \\
\text { Shape }\end{array}$ & $\begin{array}{l}\text { Flaking } \\
\text { Pattern }\end{array}$ & Beveling & $\begin{array}{c}\text { Reduction } \\
\text { Stage }\end{array}$ & Weight (g) & $\begin{array}{c}\text { Max } \\
\text { Length }\end{array}$ & Max Width & $\begin{array}{c}\text { Max } \\
\text { Thickness }\end{array}$ & $\begin{array}{c}\text { Avg Edge } \\
\text { Angle }\end{array}$ & $\begin{array}{l}\text { Callahan's } \\
\text { w/t index }\end{array}$ & \multicolumn{4}{|c|}{ Comments } \\
\hline & & & & Crescent & Random & None & Indet & 21.61 & Indet & 41.42 & 7.42 & Indet & Indet & $\begin{array}{l}\text { Appears to } \\
\text { based on a } \\
\text { discolora }\end{array}$ & $\begin{array}{l}\text { be a late sta } \\
\text { vaailable fragr } \\
\text { tion possibly }\end{array}$ & $\begin{array}{l}\text { e (Stage 4 } \\
\text { ent; 10YR } \\
\text { ue to heat }\end{array}$ & $\begin{array}{l}\text { 5) biface } \\
\text { irregular } \\
\text { atment }\end{array}$ \\
\hline
\end{tabular}




\begin{tabular}{|c|c|c|c|c|c|c|c|c|c|c|c|c|c|c|c|c|c|}
\hline $\begin{array}{l}\text { Lot } \\
\text { No. }\end{array}$ & $\begin{array}{l}\text { Spec. } \\
\text { No. }\end{array}$ & $\begin{array}{l}\text { Bag } \\
\text { No. }\end{array}$ & $\begin{array}{l}\text { UI } \\
\text { No. }\end{array}$ & Category & Subcategory & $\begin{array}{l}\text { Specimen } \\
\text { Condition }\end{array}$ & Breakage & $\begin{array}{c}\text { Raw } \\
\text { Material }\end{array}$ & Munsell & Color & Cortex \% & $\begin{array}{l}\text { Parent } \\
\text { Form }\end{array}$ & $\begin{array}{c}\text { Heat } \\
\text { Evidence }\end{array}$ & $\begin{array}{l}\text { Intentional } \\
\text { Heat } \\
\text { Treatment }\end{array}$ & Patination & $\begin{array}{l}\text { Overall } \\
\text { Shape }\end{array}$ & $\begin{array}{l}\text { Basal } \\
\text { Shape }\end{array}$ \\
\hline \multirow{3}{*}{341} & \multirow{3}{*}{1} & \multirow{3}{*}{421} & \multirow{3}{*}{ NA } & Biface & Biface & Complete & None & $\begin{array}{c}\text { Fine Grain } \\
\text { Chert }\end{array}$ & 10YR7/2 & Light Gray & $1-25$ & Indet & None & No & None & Ovate & $\begin{array}{c}\text { Gentle } \\
\text { Convex }\end{array}$ \\
\hline & & & & $\begin{array}{c}\text { Cross-section } \\
\text { Shape }\end{array}$ & $\begin{array}{l}\text { Flaking } \\
\text { Pattern }\end{array}$ & Beveling & $\begin{array}{c}\text { Reduction } \\
\text { Stage }\end{array}$ & Weight (g) & $\begin{array}{c}\text { Max } \\
\text { Length }\end{array}$ & Max Width & $\begin{array}{c}\text { Max } \\
\text { Thickness }\end{array}$ & $\begin{array}{c}\text { Avg Edge } \\
\text { Angle }\end{array}$ & $\begin{array}{l}\text { Callahan's } \\
\text { w/t index }\end{array}$ & \multicolumn{4}{|c|}{ Comments } \\
\hline & & & & $\begin{array}{c}\text { Biconvex } \\
\text { Symmetrical }\end{array}$ & Random & None & Stage 1 & 157.9 & 99.83 & 51.56 & 32.73 & 60 & 1.58 & \multicolumn{4}{|c|}{$\begin{array}{l}\text { Large blank with cortex and only mass thinning; } \\
\text { Platform loss, step and hinge fractures noted; } \\
\text { Numerous thin mineral impurities throughout }\end{array}$} \\
\hline
\end{tabular}

\begin{tabular}{|c|c|c|c|c|c|c|c|c|c|c|c|c|c|c|c|c|c|}
\hline $\begin{array}{l}\text { Lot } \\
\text { No. }\end{array}$ & $\begin{array}{l}\text { Spec. } \\
\text { No. }\end{array}$ & $\begin{array}{l}\text { Bag } \\
\text { No. }\end{array}$ & $\begin{array}{l}\text { UI } \\
\text { No. }\end{array}$ & Category & Subcategory & $\begin{array}{l}\text { Specimen } \\
\text { Condition }\end{array}$ & Breakage & $\begin{array}{c}\text { Raw } \\
\text { Material }\end{array}$ & Munsell & Color & Cortex \% & $\begin{array}{l}\text { Parent } \\
\text { Form }\end{array}$ & $\begin{array}{c}\text { Heat } \\
\text { Evidence }\end{array}$ & $\begin{array}{c}\text { Intentional } \\
\text { Heat } \\
\text { Treatment }\end{array}$ & Patination & $\begin{array}{l}\text { Overall } \\
\text { Shape }\end{array}$ & $\begin{array}{l}\text { Basal } \\
\text { Shape }\end{array}$ \\
\hline \multirow{3}{*}{356} & \multirow{3}{*}{1} & \multirow{3}{*}{414} & \multirow{3}{*}{ NA } & Biface & Biface & Complete & None & $\begin{array}{c}\text { Fine Grain } \\
\text { Chert }\end{array}$ & 10YR6/1 & Gray & 26-50 & Indet & Reddened & Yes & None & Ovate & $\begin{array}{l}\text { Gentle } \\
\text { Convex }\end{array}$ \\
\hline & & & & \begin{tabular}{|c|}
$\begin{array}{c}\text { Cross-section } \\
\text { Shape }\end{array}$ \\
\end{tabular} & $\begin{array}{l}\text { Flaking } \\
\text { Pattern } \\
\end{array}$ & Beveling & $\begin{array}{c}\text { Reduction } \\
\text { Stage }\end{array}$ & Weight (g) & $\begin{array}{c}\text { Max } \\
\text { Length }\end{array}$ & Max Width & $\begin{array}{c}\text { Max } \\
\text { Thickness } \\
\end{array}$ & $\begin{array}{c}\text { Avg Edge } \\
\text { Angle }\end{array}$ & $\begin{array}{c}\text { Callahan's } \\
\text { w/t index }\end{array}$ & \multicolumn{4}{|c|}{ Comments } \\
\hline & & & & $\begin{array}{l}\text { Biconvex } \\
\text { Symmetrical }\end{array}$ & Random & None & Stage 1 & 108.64 & 78.11 & 47.73 & 32.12 & 70 & 1.79 & \multicolumn{4}{|c|}{$\begin{array}{c}\text { Color is mottled with } 10 \text { YR6/2 with } 10 \text { YR6/6 } \\
\text { around cortex; Large blank with cortex with only } \\
\text { mass thinning; Platform loss noted }\end{array}$} \\
\hline
\end{tabular}

\begin{tabular}{|c|c|c|c|c|c|c|c|c|c|c|c|c|c|c|c|c|c|}
\hline $\begin{array}{l}\text { Lot } \\
\text { No. }\end{array}$ & $\begin{array}{l}\text { Spec. } \\
\text { No. }\end{array}$ & $\begin{array}{l}\text { Bag } \\
\text { No. }\end{array}$ & $\begin{array}{l}\text { Ul } \\
\text { No. }\end{array}$ & Category & Subcategory & $\begin{array}{l}\text { Specimen } \\
\text { Condition }\end{array}$ & Breakage & $\begin{array}{c}\text { Raw } \\
\text { Material }\end{array}$ & Munsell & Color & Cortex \% & $\begin{array}{c}\text { Parent } \\
\text { Form }\end{array}$ & $\begin{array}{c}\text { Heat } \\
\text { Evidence }\end{array}$ & $\begin{array}{c}\text { Intentional } \\
\text { Heat } \\
\text { Treatment }\end{array}$ & Patination & $\begin{array}{l}\text { Overall } \\
\text { Shape }\end{array}$ & $\begin{array}{l}\text { Basal } \\
\text { Shape }\end{array}$ \\
\hline \multirow{3}{*}{360} & \multirow{3}{*}{1} & \multirow{3}{*}{428} & \multirow{3}{*}{ NA } & Biface & Biface & Complete & None & $\begin{array}{c}\text { Fine Grain } \\
\text { Chert }\end{array}$ & 10YR4/1 & Dark Gray & $1-25$ & Indet & Reddened & Yes & None & Ovate & $\begin{array}{l}\text { Gentle } \\
\text { Convex }\end{array}$ \\
\hline & & & & $\begin{array}{c}\text { Cross-section } \\
\text { Shape }\end{array}$ & $\begin{array}{l}\text { Flaking } \\
\text { Pattern }\end{array}$ & Beveling & $\begin{array}{c}\text { Reduction } \\
\text { Stage }\end{array}$ & Weight (g) & $\begin{array}{c}\text { Max } \\
\text { Length }\end{array}$ & Max Width & $\begin{array}{c}\text { Max } \\
\text { Thickness }\end{array}$ & $\begin{array}{c}\text { Avg Edge } \\
\text { Angle }\end{array}$ & $\begin{array}{l}\text { Callahan's } \\
\text { w/t index }\end{array}$ & \multicolumn{4}{|c|}{ Comments } \\
\hline & & & & Crescent & Random & None & Stage 2 & 181.21 & 94.69 & 63.08 & 29.98 & 70 & 2.1 & $\begin{array}{r}\text { Speckled } \mathrm{m} \\
10 \mathrm{Y}\end{array}$ & $\begin{array}{l}\text { nineral inclus } \\
\text { YR7/1); Corte }\end{array}$ & $\begin{array}{l}\text { ns through } \\
\text { on distal }\end{array}$ & d $(30 \%-$ \\
\hline
\end{tabular}

\begin{tabular}{|c|c|c|c|c|c|c|c|c|c|c|c|c|c|c|c|c|c|}
\hline $\begin{array}{l}\text { Lot } \\
\text { No. }\end{array}$ & $\begin{array}{l}\text { Spec. } \\
\text { No. }\end{array}$ & $\begin{array}{l}\text { Bag } \\
\text { No. }\end{array}$ & $\begin{array}{l}\text { UI } \\
\text { No. }\end{array}$ & Category & Subcategory & $\begin{array}{l}\text { Specimen } \\
\text { Condition }\end{array}$ & Breakage & $\begin{array}{c}\text { Raw } \\
\text { Material }\end{array}$ & Munsell & Color & Cortex \% & $\begin{array}{l}\text { Parent } \\
\text { Form }\end{array}$ & $\begin{array}{c}\text { Heat } \\
\text { Evidence }\end{array}$ & $\begin{array}{c}\text { Intentional } \\
\text { Heat } \\
\text { Treatment }\end{array}$ & Patination & $\begin{array}{l}\text { Overall } \\
\text { Shape }\end{array}$ & $\begin{array}{l}\text { Basal } \\
\text { Shape }\end{array}$ \\
\hline \multirow[b]{3}{*}{378} & \multirow[b]{3}{*}{5} & \multirow[b]{3}{*}{586} & \multirow{3}{*}{ NA } & Biface & Biface & Indet Fragment & Manufacture & $\begin{array}{l}\text { Fine Grain } \\
\text { Chert }\end{array}$ & 10YR3/1 & $\begin{array}{l}\text { Very Dark } \\
\text { Gray }\end{array}$ & $26-50$ & Indet & None & No & Light & Indet & Indet \\
\hline & & & & $\begin{array}{c}\text { Cross-section } \\
\text { Shape }\end{array}$ & $\begin{array}{l}\text { Flaking } \\
\text { Pattern }\end{array}$ & Beveling & $\begin{array}{c}\text { Reduction } \\
\text { Stage }\end{array}$ & Weight (g) & $\begin{array}{c}\text { Max } \\
\text { Length }\end{array}$ & Max Width & $\begin{array}{c}\text { Max } \\
\text { Thickness }\end{array}$ & $\begin{array}{c}\text { Avg Edge } \\
\text { Angle }\end{array}$ & $\begin{array}{l}\text { Callahan's } \\
\text { w/t index }\end{array}$ & \multicolumn{4}{|c|}{ Comments } \\
\hline & & & & $\begin{array}{l}\text { Crescent to } \\
\text { Biconvex } \\
\text { Symmetrical }\end{array}$ & Random & None & Indet & 14.5 & Indet & Indet & Indet & Indet & Indet & \multicolumn{4}{|c|}{$\begin{array}{l}\text { Approximately } 50 \% \text { of complete specimen; } \\
\text { Possibly a cortical flake; Estimated a stage } 3 \\
\text { based on available fragment; Evidence of } \\
\text { thinning flakes and soft pressure flake removal }\end{array}$} \\
\hline
\end{tabular}




\begin{tabular}{|c|c|c|c|c|c|c|c|c|c|c|c|c|c|c|c|c|c|}
\hline $\begin{array}{l}\text { Lot } \\
\text { No. }\end{array}$ & $\begin{array}{c}\text { Spec. } \\
\text { No. }\end{array}$ & $\begin{array}{l}\text { Bag } \\
\text { No. }\end{array}$ & $\begin{array}{l}\text { Ul } \\
\text { No. }\end{array}$ & Category & Subcategory & $\begin{array}{l}\text { Specimen } \\
\text { Condition }\end{array}$ & Breakage & $\begin{array}{c}\text { Raw } \\
\text { Material }\end{array}$ & Munsell & Color & Cortex \% & $\begin{array}{c}\text { Parent } \\
\text { Form }\end{array}$ & $\begin{array}{c}\text { Heat } \\
\text { Evidence }\end{array}$ & $\begin{array}{c}\text { Intentional } \\
\text { Heat } \\
\text { Treatment }\end{array}$ & Patination & $\begin{array}{l}\text { Overall } \\
\text { Shape }\end{array}$ & $\begin{array}{l}\text { Basal } \\
\text { Shape }\end{array}$ \\
\hline \multirow[b]{3}{*}{387} & \multirow[b]{3}{*}{1} & \multirow[b]{3}{*}{452} & \multirow[b]{3}{*}{ NA } & Biface & Biface & $\begin{array}{l}\text { Distal-Medial } \\
\text { Fragment }\end{array}$ & Manufacture & $\begin{array}{c}\text { Fine Grain } \\
\text { Chert }\end{array}$ & $2.5 \mathrm{YR} 6 / 2$ & Pale Red & $1-25$ & Indet & Reddened & Yes & None & Indet & Indet \\
\hline & & & & $\begin{array}{l}\text { Cross-section } \\
\text { Shape }\end{array}$ & $\begin{array}{l}\text { Flaking } \\
\text { Pattern }\end{array}$ & Beveling & $\begin{array}{l}\text { Reduction } \\
\text { Stage }\end{array}$ & Weight (g) & $\begin{array}{c}\text { Max } \\
\text { Length }\end{array}$ & Max Width & $\begin{array}{c}\text { Max } \\
\text { Thickness }\end{array}$ & $\begin{array}{l}\text { Avg Edge } \\
\text { Angle }\end{array}$ & $\begin{array}{l}\text { Callahan's } \\
\text { w/t index }\end{array}$ & \multicolumn{4}{|c|}{ Comments } \\
\hline & & & & $\begin{array}{l}\text { Biconvex } \\
\text { Symmetrical }\end{array}$ & Random & None & Indet & 37.78 & Indet & Indet & Indet & Indet & Indet & \multicolumn{4}{|c|}{$\begin{array}{c}\text { Heavily banded with } 2.5 Y R 5 / 6 \text { due to heat } \\
\text { treatment; Cortex at proximal end near break; } \\
\text { Estimated a stage } 2 \text { based on available fragment; } \\
\text { Numerous step and hinge fractures along lateral } \\
\text { margin }\end{array}$} \\
\hline $\begin{array}{l}\text { Lot } \\
\text { No. }\end{array}$ & $\begin{array}{c}\text { Spec. } \\
\text { No. }\end{array}$ & $\begin{array}{l}\text { Bag } \\
\text { No. }\end{array}$ & $\begin{array}{l}\text { UI } \\
\text { No. }\end{array}$ & Category & Subcategory & $\begin{array}{l}\text { Specimen } \\
\text { Condition }\end{array}$ & Breakage & $\begin{array}{c}\text { Raw } \\
\text { Material }\end{array}$ & Munsell & Color & Cortex \% & $\begin{array}{c}\text { Parent } \\
\text { Form }\end{array}$ & $\begin{array}{c}\text { Heat } \\
\text { Evidence }\end{array}$ & $\begin{array}{c}\text { Intentional } \\
\text { Heat } \\
\text { Treatment }\end{array}$ & Patination & $\begin{array}{l}\text { Overall } \\
\text { Shape }\end{array}$ & $\begin{array}{l}\text { Basal } \\
\text { Shape }\end{array}$ \\
\hline \multirow[b]{3}{*}{403} & \multirow[b]{3}{*}{9} & \multirow[b]{3}{*}{495} & \multirow[b]{3}{*}{23} & Biface & Biface & Complete & None & $\begin{array}{c}\text { Fine Grain } \\
\text { Chert }\end{array}$ & 10YR6/2 & $\begin{array}{c}\text { Light Brownish } \\
\text { Gray }\end{array}$ & 0 & Indet & None & No & None & $\begin{array}{c}\text { Pointed } \\
\text { Ovate }\end{array}$ & $\begin{array}{l}\text { Gentle } \\
\text { Convex }\end{array}$ \\
\hline & & & & $\begin{array}{l}\text { Cross-section } \\
\text { Shape }\end{array}$ & $\begin{array}{l}\text { Flaking } \\
\text { Pattern }\end{array}$ & Beveling & $\begin{array}{c}\text { Reduction } \\
\text { Stage }\end{array}$ & Weight (g) & $\begin{array}{c}\text { Max } \\
\text { Length }\end{array}$ & Max Width & $\begin{array}{c}\text { Max } \\
\text { Thickness }\end{array}$ & $\begin{array}{l}\text { Avg Edge } \\
\text { Angle }\end{array}$ & $\begin{array}{l}\text { Callahan's } \\
\text { w/t index }\end{array}$ & \multicolumn{4}{|c|}{ Comments } \\
\hline & & & & $\begin{array}{l}\text { Biconvex } \\
\text { Symmetrical }\end{array}$ & Random & None & Stage 5 & 53.3 & 109.29 & 51.19 & 9.13 & 40 & 5.61 & \multicolumn{4}{|c|}{$\begin{array}{l}\text { Finely thinned late stage biface; Use wear and } \\
\text { finely worked left lateral and basal margin; Thick } \\
\text { portion on one face with multiple step fractures } \\
\text { from attempted reduction }\end{array}$} \\
\hline
\end{tabular}

\begin{tabular}{|c|c|c|c|c|c|c|c|c|c|c|c|c|c|c|c|c|c|}
\hline $\begin{array}{l}\text { Lot } \\
\text { No. }\end{array}$ & $\begin{array}{l}\text { Spec. } \\
\text { No. }\end{array}$ & $\begin{array}{l}\text { Bag } \\
\text { No. }\end{array}$ & $\begin{array}{l}\text { Ul } \\
\text { No. }\end{array}$ & Category & Subcategory & $\begin{array}{l}\text { Specimen } \\
\text { Condition }\end{array}$ & Breakage & $\begin{array}{c}\text { Raw } \\
\text { Material }\end{array}$ & Munsell & Color & Cortex \% & $\begin{array}{l}\text { Parent } \\
\text { Form }\end{array}$ & $\begin{array}{c}\text { Heat } \\
\text { Evidence }\end{array}$ & $\begin{array}{c}\text { Intentional } \\
\text { Heat } \\
\text { Treatment }\end{array}$ & Patination & $\begin{array}{l}\text { Overall } \\
\text { Shape }\end{array}$ & $\begin{array}{l}\text { Basal } \\
\text { Shape }\end{array}$ \\
\hline \multirow{3}{*}{404} & \multirow{3}{*}{1} & \multirow{3}{*}{494} & \multirow{3}{*}{24} & Biface & Biface & Complete & None & $\begin{array}{c}\text { Fine Grain } \\
\text { Chert }\end{array}$ & 10YR4/6 & $\begin{array}{c}\text { Dark Yellowish } \\
\text { Brown }\end{array}$ & $1-25$ & Indet & $\begin{array}{l}\text { Reddened } \\
\text { and Gloss }\end{array}$ & Yes & None & Lanceolate & $\begin{array}{l}\begin{array}{l}\text { Shallow } \\
\text { Concave }\end{array} \\
\end{array}$ \\
\hline & & & & $\begin{array}{l}\text { Cross-section } \\
\text { Shape }\end{array}$ & $\begin{array}{l}\text { Flaking } \\
\text { Pattern }\end{array}$ & Beveling & $\begin{array}{c}\text { Reduction } \\
\text { Stage }\end{array}$ & Weight (g) & $\begin{array}{c}\text { Max } \\
\text { Length }\end{array}$ & Max Width & $\begin{array}{c}\text { Max } \\
\text { Thickness }\end{array}$ & $\begin{array}{c}\text { Avg Edge } \\
\text { Angle }\end{array}$ & $\begin{array}{l}\text { Callahan's } \\
\text { w/t index }\end{array}$ & \multicolumn{4}{|c|}{ Comments } \\
\hline & & & & $\begin{array}{l}\text { Biconvex } \\
\text { Symmetrical }\end{array}$ & Random & None & Stage 3 & 12.5 & 53.48 & 28.72 & 8.43 & 35 & 3.41 & $\begin{array}{r}\text { Approxima } \\
\text { basal margin } \\
\text { heat treat }\end{array}$ & $\begin{array}{l}\text { ately } 1 \% \text { corte } \\
\text { n; Prominent } \\
\text { tment; Platfor } \\
\text { mar }\end{array}$ & $\begin{array}{l}\text { ex at distal tip } \\
\text { luster or glos } \\
\text { rm loss on rig } \\
\text { rgin }\end{array}$ & $\begin{array}{l}\text { and right } \\
\text { s likely from } \\
\text { ht lateral }\end{array}$ \\
\hline
\end{tabular}

\begin{tabular}{|c|c|c|c|c|c|c|c|c|c|c|c|c|c|c|c|c|c|}
\hline $\begin{array}{l}\text { Lot } \\
\text { No. }\end{array}$ & $\begin{array}{l}\text { Spec. } \\
\text { No. }\end{array}$ & $\begin{array}{l}\text { Bag } \\
\text { No. }\end{array}$ & $\begin{array}{l}\text { UI } \\
\text { No. }\end{array}$ & Category & Subcategory & $\begin{array}{l}\text { Specimen } \\
\text { Condition }\end{array}$ & Breakage & $\begin{array}{c}\text { Raw } \\
\text { Material }\end{array}$ & Munsell & Color & Cortex \% & $\begin{array}{c}\text { Parent } \\
\text { Form }\end{array}$ & $\begin{array}{c}\text { Heat } \\
\text { Evidence }\end{array}$ & $\begin{array}{c}\text { Intentional } \\
\text { Heat } \\
\text { Treatment }\end{array}$ & Patination & $\begin{array}{l}\text { Overall } \\
\text { Shape }\end{array}$ & $\begin{array}{l}\text { Basal } \\
\text { Shape }\end{array}$ \\
\hline \multirow{3}{*}{406} & \multirow{3}{*}{11} & \multirow{3}{*}{503} & \multirow{3}{*}{ NA } & Biface & Biface & $\begin{array}{c}\text { Distal-Medial } \\
\text { Fragment }\end{array}$ & Manufacture & $\begin{array}{c}\text { Fine Grain } \\
\text { Chert }\end{array}$ & 10YR5/1 & Gray & 76-100 & $\begin{array}{c}\text { Cortical } \\
\text { Flake }\end{array}$ & None & No & None & Indet & Indet \\
\hline & & & & $\begin{array}{l}\text { Cross-section } \\
\text { Shape }\end{array}$ & $\begin{array}{l}\text { Flaking } \\
\text { Pattern }\end{array}$ & Beveling & $\begin{array}{l}\text { Reduction } \\
\text { Stage }\end{array}$ & Weight (g) & $\begin{array}{c}\text { Max } \\
\text { Length }\end{array}$ & Max Width & $\begin{array}{c}\text { Max } \\
\text { Thickness }\end{array}$ & $\begin{array}{c}\text { Avg Edge } \\
\text { Angle }\end{array}$ & $\begin{array}{l}\text { Callahan's } \\
\text { w/t index }\end{array}$ & \multicolumn{4}{|c|}{ Comments } \\
\hline & & & & $\begin{array}{l}\text { Biconvex } \\
\text { Symmetrical }\end{array}$ & Indet & Right Lateral & Stage 5 & 0.81 & Indet & Indet & Indet & Indet & Indet & $\begin{array}{r}\text { Non-diagnos } \\
\text { estime } \\
\text { Pr }\end{array}$ & $\begin{array}{l}\text { stic projectile } \\
\text { late percenta } \\
\text { redominately }\end{array}$ & $\begin{array}{l}\text { ooint fragm } \\
\text { e of compl } \\
\text { laked corte }\end{array}$ & $\begin{array}{l}\text { t; Cannot } \\
\text { on; }\end{array}$ \\
\hline
\end{tabular}




\begin{tabular}{|c|c|c|c|c|c|c|c|c|c|c|c|c|c|c|c|c|c|}
\hline $\begin{array}{l}\text { Lot } \\
\text { No. }\end{array}$ & $\begin{array}{c}\text { Spec. } \\
\text { No. }\end{array}$ & $\begin{array}{l}\text { Bag } \\
\text { No. }\end{array}$ & $\begin{array}{l}\text { UI } \\
\text { No. }\end{array}$ & Category & Subcategory & $\begin{array}{l}\text { Specimen } \\
\text { Condition }\end{array}$ & Breakage & $\begin{array}{c}\text { Raw } \\
\text { Material }\end{array}$ & Munsell & Color & Cortex \% & $\begin{array}{c}\text { Parent } \\
\text { Form }\end{array}$ & $\begin{array}{c}\text { Heat } \\
\text { Evidence }\end{array}$ & $\begin{array}{c}\text { Intentional } \\
\text { Heat } \\
\text { Treatment }\end{array}$ & Patination & $\begin{array}{l}\text { Overall } \\
\text { Shape }\end{array}$ & $\begin{array}{l}\text { Basal } \\
\text { Shape }\end{array}$ \\
\hline \multirow[b]{3}{*}{417} & \multirow[b]{3}{*}{5} & \multirow[b]{3}{*}{561} & \multirow[b]{3}{*}{ NA } & Biface & Biface & Indet Fragment & Manufacture & $\begin{array}{c}\text { Fine Grain } \\
\text { Chert }\end{array}$ & 10YR5/1 & Gray & 1-25 & Indet & None & No & None & Indet & Indet \\
\hline & & & & $\begin{array}{c}\text { Cross-section } \\
\text { Shape }\end{array}$ & $\begin{array}{l}\text { Flaking } \\
\text { Pattern }\end{array}$ & Beveling & $\begin{array}{c}\text { Reduction } \\
\text { Stage }\end{array}$ & Weight (g) & $\begin{array}{c}\text { Max } \\
\text { Length }\end{array}$ & Max Width & $\begin{array}{c}\text { Max } \\
\text { Thickness }\end{array}$ & $\begin{array}{c}\text { Avg Edge } \\
\text { Angle }\end{array}$ & $\begin{array}{l}\text { Callahan's } \\
\text { w/t index }\end{array}$ & \multicolumn{4}{|c|}{ Comments } \\
\hline & & & & Crescent & Collateral & None & Indet & 6.44 & Indet & Indet & Indet & Indet & Indet & \multicolumn{4}{|c|}{$\begin{array}{c}\text { Mineral inclusions (<10\%, 10YR7/1); Indet } \\
\text { fragment that appears to be approximately } 50 \% \\
\text { complete; Very small amount of cortex on distal } \\
\text { tip; Fragment is very thin }(4.6 \mathrm{~mm})\end{array}$} \\
\hline
\end{tabular}

\begin{tabular}{|c|c|c|c|c|c|c|c|c|c|c|c|c|c|c|c|c|c|}
\hline $\begin{array}{l}\text { Lot } \\
\text { No. }\end{array}$ & $\begin{array}{l}\text { Spec. } \\
\text { No. }\end{array}$ & $\begin{array}{l}\text { Bag } \\
\text { No. }\end{array}$ & $\begin{array}{l}\text { UI } \\
\text { No. }\end{array}$ & Category & Subcategory & $\begin{array}{l}\text { Specimen } \\
\text { Condition }\end{array}$ & Breakage & $\begin{array}{c}\text { Raw } \\
\text { Material }\end{array}$ & Munsell & Color & Cortex \% & $\begin{array}{c}\text { Parent } \\
\text { Form }\end{array}$ & $\begin{array}{c}\text { Heat } \\
\text { Evidence }\end{array}$ & $\begin{array}{c}\text { Intentional } \\
\text { Heat } \\
\text { Treatment }\end{array}$ & Patination & $\begin{array}{l}\text { Overall } \\
\text { Shape }\end{array}$ & $\begin{array}{l}\text { Basal } \\
\text { Shape }\end{array}$ \\
\hline \multirow[b]{3}{*}{444} & \multirow[b]{3}{*}{4} & \multirow[b]{3}{*}{482} & \multirow[b]{3}{*}{ NA } & Biface & Biface & Indet Fragment & Manufacture & $\begin{array}{c}\text { Fine Grain } \\
\text { Chert }\end{array}$ & 10YR5/4 & $\begin{array}{c}\text { Yellowish } \\
\text { Brown }\end{array}$ & 0-25 & Indet & Reddened & Yes & None & Indet & Indet \\
\hline & & & & $\begin{array}{l}\text { Cross-section } \\
\text { Shape }\end{array}$ & $\begin{array}{l}\text { Flaking } \\
\text { Pattern }\end{array}$ & Beveling & $\begin{array}{l}\text { Reduction } \\
\text { Stage }\end{array}$ & Weight (g) & $\begin{array}{c}\text { Max } \\
\text { Length }\end{array}$ & Max Width & $\begin{array}{c}\text { Max } \\
\text { Thickness }\end{array}$ & $\begin{array}{c}\text { Avg Edge } \\
\text { Angle }\end{array}$ & $\begin{array}{l}\text { Callahan's } \\
\text { w/t index }\end{array}$ & \multicolumn{4}{|c|}{ Comments } \\
\hline & & & & $\begin{array}{c}\text { Biconvex } \\
\text { Symmetrical }\end{array}$ & Random & None & Indet & 15.46 & Indet & Indet & Indet & Indet & Indet & $\begin{array}{r}\text { Flake is pre } \\
\text { heat treatn } \\
\text { medial po }\end{array}$ & $\begin{array}{l}\text { dominately d } \\
\text { nent; partial } \\
\text { rtion - appea } \\
\text { distal }\end{array}$ & $\begin{array}{l}\mathrm{k} \text { red }(2.5) \\
\text { ace with b } \\
\text { to be the } \\
\text { rtion }\end{array}$ & $\begin{array}{l}3 / 6) \text { due to } \\
\text { ak across } \\
\text { edial and }\end{array}$ \\
\hline
\end{tabular}

\begin{tabular}{|c|c|c|c|c|c|c|c|c|c|c|c|c|c|c|c|c|c|}
\hline $\begin{array}{l}\text { Lot } \\
\text { No. }\end{array}$ & $\begin{array}{l}\text { Spec. } \\
\text { No. }\end{array}$ & $\begin{array}{l}\text { Bag } \\
\text { No. }\end{array}$ & $\begin{array}{l}\text { UI } \\
\text { No. }\end{array}$ & Category & Subcategory & $\begin{array}{l}\text { Specimen } \\
\text { Condition }\end{array}$ & Breakage & $\begin{array}{c}\text { Raw } \\
\text { Material }\end{array}$ & Munsell & Color & Cortex \% & $\begin{array}{c}\text { Parent } \\
\text { Form }\end{array}$ & $\begin{array}{c}\text { Heat } \\
\text { Evidence }\end{array}$ & $\begin{array}{c}\text { Intentional } \\
\text { Heat } \\
\text { Treatment }\end{array}$ & Patination & $\begin{array}{l}\text { Overall } \\
\text { Shape }\end{array}$ & $\begin{array}{l}\text { Basal } \\
\text { Shape }\end{array}$ \\
\hline \multirow{3}{*}{462} & \multirow{3}{*}{21} & \multirow{3}{*}{526} & \multirow{3}{*}{ NA } & Biface & Biface & Medial Fragment & Use & $\begin{array}{l}\text { Fine Grain } \\
\text { Chert }\end{array}$ & N3/ & $\begin{array}{l}\text { Very Dark } \\
\text { Gray }\end{array}$ & 0 & Indet & None & No & None & Indet & Indet \\
\hline & & & & $\begin{array}{c}\text { Cross-section } \\
\text { Shape }\end{array}$ & $\begin{array}{l}\text { Flaking } \\
\text { Pattern }\end{array}$ & Beveling & $\begin{array}{c}\text { Reduction } \\
\text { Stage }\end{array}$ & Weight (g) & $\begin{array}{c}\text { Max } \\
\text { Length }\end{array}$ & Max Width & $\begin{array}{c}\text { Max } \\
\text { Thickness }\end{array}$ & $\begin{array}{c}\text { Avg Edge } \\
\text { Angle }\end{array}$ & $\begin{array}{c}\text { Callahan's } \\
\text { w/t index }\end{array}$ & \multicolumn{4}{|c|}{ Comments } \\
\hline & & & & Indet & $\begin{array}{c}\text { Oblique } \\
\text { Subparallel }\end{array}$ & None & Indet & 0.16 & Indet & Indet & Indet & Indet & Indet & $\begin{array}{r}\text { Small me } \\
\text { point; One } \\
\text { dis }\end{array}$ & $\begin{array}{l}\text { dial fragment } \\
\text { shoulder ma } \\
\text { cern from av }\end{array}$ & $\begin{array}{l}\text { Very likely } \\
\text { y be intact, } \\
\text { ilable fragn }\end{array}$ & $\begin{array}{l}\text { Projectile } \\
t \text { cannot } \\
\text { t }\end{array}$ \\
\hline
\end{tabular}

\begin{tabular}{|c|c|c|c|c|c|c|c|c|c|c|c|c|c|c|c|c|c|}
\hline $\begin{array}{l}\text { Lot } \\
\text { No. }\end{array}$ & $\begin{array}{l}\text { Spec. } \\
\text { No. }\end{array}$ & $\begin{array}{l}\text { Bag } \\
\text { No. }\end{array}$ & $\begin{array}{l}\text { UI } \\
\text { No. }\end{array}$ & Category & Subcategory & $\begin{array}{l}\text { Specimen } \\
\text { Condition }\end{array}$ & Breakage & $\begin{array}{c}\text { Raw } \\
\text { Material }\end{array}$ & Munsell & Color & Cortex \% & $\begin{array}{l}\text { Parent } \\
\text { Form }\end{array}$ & $\begin{array}{c}\text { Heat } \\
\text { Evidence }\end{array}$ & $\begin{array}{l}\text { Intentional } \\
\text { Heat } \\
\text { Treatment }\end{array}$ & Patination & $\begin{array}{l}\text { Overall } \\
\text { Shape }\end{array}$ & $\begin{array}{l}\text { Basal } \\
\text { Shape }\end{array}$ \\
\hline \multirow{3}{*}{475} & \multirow{3}{*}{8} & \multirow{3}{*}{534} & \multirow{3}{*}{ NA } & Biface & Biface & Indet Fragment & Manufacture & $\begin{array}{c}\text { Fine Grain } \\
\text { Chert }\end{array}$ & 10YR6/2 & $\begin{array}{l}\text { Light Grayish } \\
\text { Brown }\end{array}$ & 0 & Indet & None & No & None & Indet & Indet \\
\hline & & & & $\begin{array}{c}\text { Cross-section } \\
\text { Shape } \\
\end{array}$ & $\begin{array}{l}\text { Flaking } \\
\text { Pattern } \\
\end{array}$ & Beveling & $\begin{array}{c}\text { Reduction } \\
\text { Stage }\end{array}$ & Weight (g) & $\begin{array}{c}\text { Max } \\
\text { Length }\end{array}$ & Max Width & $\begin{array}{c}\text { Max } \\
\text { Thickness } \\
\end{array}$ & $\begin{array}{c}\text { Avg Edge } \\
\text { Angle } \\
\end{array}$ & $\begin{array}{c}\text { Callahan's } \\
\text { w/t index }\end{array}$ & \multicolumn{4}{|c|}{ Comments } \\
\hline & & & & Indet & Indet & None & Indet & 0.86 & Indet & Indet & Indet & Indet & Indet & \multicolumn{4}{|c|}{$\begin{array}{c}\text { Small fragment that appears to be a basal } \\
\text { shoulder; Thinning flake removal visible on both } \\
\text { faces }\end{array}$} \\
\hline
\end{tabular}




\begin{tabular}{|c|c|c|c|c|c|c|c|c|c|c|c|c|c|c|c|c|c|}
\hline $\begin{array}{l}\text { Lot } \\
\text { No. }\end{array}$ & $\begin{array}{l}\text { Spec. } \\
\text { No. }\end{array}$ & $\begin{array}{l}\text { Bag } \\
\text { No. }\end{array}$ & $\begin{array}{l}\text { UI } \\
\text { No. }\end{array}$ & Category & Subcategory & $\begin{array}{l}\text { Specimen } \\
\text { Condition }\end{array}$ & Breakage & $\begin{array}{c}\text { Raw } \\
\text { Material }\end{array}$ & Munsell & Color & Cortex \% & $\begin{array}{l}\text { Parent } \\
\text { Form }\end{array}$ & $\begin{array}{c}\text { Heat } \\
\text { Evidence }\end{array}$ & $\begin{array}{l}\text { Intentional } \\
\text { Heat } \\
\text { Treatment }\end{array}$ & Patination & $\begin{array}{l}\text { Overall } \\
\text { Shape }\end{array}$ & $\begin{array}{l}\text { Basal } \\
\text { Shape }\end{array}$ \\
\hline \multirow{3}{*}{486} & \multirow{3}{*}{2} & \multirow{3}{*}{315} & \multirow{3}{*}{18} & Biface & Biface & $\begin{array}{l}\text { Distal-Medial } \\
\text { Fragment }\end{array}$ & Manufacture & $\begin{array}{c}\text { Fine Grain } \\
\text { Chert }\end{array}$ & 10YR5/2 & Grayish Brown & 0 & Indet & None & No & None & Indet & Indet \\
\hline & & & & $\begin{array}{l}\begin{array}{c}\text { Cross-section } \\
\text { Shape }\end{array} \\
\end{array}$ & $\begin{array}{l}\text { Flaking } \\
\text { Pattern }\end{array}$ & Beveling & $\begin{array}{c}\text { Reduction } \\
\text { Stage }\end{array}$ & Weight (g) & $\begin{array}{c}\text { Max } \\
\text { Length }\end{array}$ & Max Width & $\begin{array}{c}\text { Max } \\
\text { Thickness }\end{array}$ & $\begin{array}{l}\begin{array}{c}\text { Avg Edge } \\
\text { Angle }\end{array} \\
\end{array}$ & $\begin{array}{l}\text { Callahan's } \\
\text { w/t index }\end{array}$ & \multicolumn{4}{|c|}{ Comments } \\
\hline & & & & $\begin{array}{l}\text { Biconvex } \\
\text { Symmetrical }\end{array}$ & Collateral & None & Indet & 6 & Indet & Indet & Indet & Indet & Indet & \multicolumn{4}{|c|}{$\begin{array}{l}\text { Very distal tip broken; Large mineral inclusion } \\
\text { throughout both faces focused on distal end; } \\
\text { Incomplete but appears to be a late stage }\end{array}$} \\
\hline
\end{tabular}

\begin{tabular}{|c|c|c|c|c|c|c|c|c|c|c|c|c|c|c|c|c|c|}
\hline $\begin{array}{l}\text { Lot } \\
\text { No. }\end{array}$ & $\begin{array}{l}\text { Spec. } \\
\text { No. }\end{array}$ & $\begin{array}{l}\text { Bag } \\
\text { No. }\end{array}$ & $\begin{array}{l}\text { UI } \\
\text { No. }\end{array}$ & Category & Subcategory & $\begin{array}{l}\text { Specimen } \\
\text { Condition }\end{array}$ & Breakage & $\begin{array}{c}\text { Raw } \\
\text { Material }\end{array}$ & Munsell & Color & Cortex \% & $\begin{array}{l}\text { Parent } \\
\text { Form }\end{array}$ & $\begin{array}{c}\text { Heat } \\
\text { Evidence }\end{array}$ & $\begin{array}{c}\text { Intentional } \\
\text { Heat } \\
\text { Treatment }\end{array}$ & Patination & $\begin{array}{l}\text { Overall } \\
\text { Shape }\end{array}$ & $\begin{array}{l}\text { Basal } \\
\text { Shape }\end{array}$ \\
\hline \multirow{3}{*}{512} & \multirow{3}{*}{6} & \multirow{3}{*}{447} & \multirow{3}{*}{ NA } & Biface & Biface & $\begin{array}{l}\text { Marginal-Distal } \\
\text { Fragment }\end{array}$ & Manufacture & $\begin{array}{l}\text { Fine Grain } \\
\text { Chert }\end{array}$ & $\begin{array}{l}\text { 10YR5/3 to } \\
10 Y R 5 / 4\end{array}$ & $\begin{array}{l}\text { Brown to } \\
\text { Yellowish } \\
\text { Brown }\end{array}$ & 0 & Indet & None & No & None & Indet & Indet \\
\hline & & & & $\begin{array}{c}\text { Cross-section } \\
\text { Shape }\end{array}$ & $\begin{array}{l}\text { Flaking } \\
\text { Pattern }\end{array}$ & Beveling & $\begin{array}{l}\text { Reduction } \\
\text { Stage }\end{array}$ & Weight (g) & $\begin{array}{c}\text { Max } \\
\text { Length }\end{array}$ & Max Width & $\begin{array}{c}\text { Max } \\
\text { Thickness }\end{array}$ & $\begin{array}{c}\text { Avg Edge } \\
\text { Angle }\end{array}$ & $\begin{array}{l}\text { Callahan's } \\
\text { w/t index }\end{array}$ & \multicolumn{4}{|c|}{ Comments } \\
\hline & & & & Indet & Indet & None & Indet & 0.86 & Indet & Indet & Indet & Indet & Indet & $\begin{array}{l}\text { Shoulder fr } \\
\text { margin; Thir }\end{array}$ & $\begin{array}{l}\text { agment with } p \\
\text { nned with fine } \\
\text { of lateral }\end{array}$ & $\begin{array}{l}\text { artial basa } \\
\text { flaking alc } \\
\text { nargin }\end{array}$ & $\begin{array}{l}\text { dd lateral } \\
\text { one face }\end{array}$ \\
\hline
\end{tabular}

\begin{tabular}{|c|c|c|c|c|c|c|c|c|c|c|c|c|c|c|c|c|c|}
\hline $\begin{array}{l}\text { Lot } \\
\text { No. }\end{array}$ & $\begin{array}{l}\text { Spec. } \\
\text { No. }\end{array}$ & $\begin{array}{l}\text { Bag } \\
\text { No. }\end{array}$ & $\begin{array}{l}\text { UI } \\
\text { No. }\end{array}$ & Category & Subcategory & $\begin{array}{l}\text { Specimen } \\
\text { Condition }\end{array}$ & Breakage & $\begin{array}{c}\text { Raw } \\
\text { Material }\end{array}$ & Munsell & Color & Cortex \% & $\begin{array}{c}\text { Parent } \\
\text { Form }\end{array}$ & $\begin{array}{c}\text { Heat } \\
\text { Evidence }\end{array}$ & $\begin{array}{c}\text { Intentional } \\
\text { Heat } \\
\text { Treatment }\end{array}$ & Patination & $\begin{array}{l}\text { Overall } \\
\text { Shape }\end{array}$ & $\begin{array}{l}\text { Basal } \\
\text { Shape }\end{array}$ \\
\hline \multirow{3}{*}{533} & \multirow{3}{*}{8} & \multirow{3}{*}{641} & \multirow[b]{3}{*}{ NA } & Biface & Biface & Basal Fragment & Manufacture & $\begin{array}{l}\text { Fine Grain } \\
\text { Chert }\end{array}$ & 10YR5/1 & Gray & 0 & Indet & None & No & None & Indet & Indet \\
\hline & & & & $\begin{array}{c}\text { Cross-section } \\
\text { Shape }\end{array}$ & $\begin{array}{l}\text { Flaking } \\
\text { Pattern }\end{array}$ & Beveling & $\begin{array}{c}\text { Reduction } \\
\text { Stage }\end{array}$ & Weight (g) & $\begin{array}{c}\text { Max } \\
\text { Length }\end{array}$ & Max Width & $\begin{array}{c}\text { Max } \\
\text { Thickness }\end{array}$ & $\begin{array}{c}\text { Avg Edge } \\
\text { Angle }\end{array}$ & $\begin{array}{c}\text { Callahan's } \\
\text { w/t index }\end{array}$ & \multicolumn{4}{|c|}{ Comments } \\
\hline & & & & Indet & Indet & Indet & Indet & 1.51 & Indet & Indet & Indet & Indet & Indet & \multicolumn{4}{|c|}{$\begin{array}{l}\text { Very small fragment that appears to be one basal } \\
\text { corner/shoulder; Fine (possibly soft pressure) } \\
\text { flake removal with long paralleling thinning flakes } \\
\text { on one face }\end{array}$} \\
\hline
\end{tabular}

\begin{tabular}{|c|c|c|c|c|c|c|c|c|c|c|c|c|c|c|c|c|c|}
\hline $\begin{array}{l}\text { Lot } \\
\text { No. }\end{array}$ & $\begin{array}{l}\text { Spec. } \\
\text { No. }\end{array}$ & $\begin{array}{l}\text { Bag } \\
\text { No. }\end{array}$ & $\begin{array}{l}\text { UI } \\
\text { No. }\end{array}$ & Category & Subcategory & $\begin{array}{l}\text { Specimen } \\
\text { Condition }\end{array}$ & Breakage & $\begin{array}{c}\text { Raw } \\
\text { Material }\end{array}$ & Munsell & Color & Cortex \% & $\begin{array}{c}\text { Parent } \\
\text { Form }\end{array}$ & $\begin{array}{c}\text { Heat } \\
\text { Evidence }\end{array}$ & $\begin{array}{c}\text { Intentional } \\
\text { Heat } \\
\text { Treatment }\end{array}$ & Patination & $\begin{array}{l}\text { Overall } \\
\text { Shape }\end{array}$ & $\begin{array}{l}\text { Basal } \\
\text { Shape }\end{array}$ \\
\hline \multirow{3}{*}{552} & \multirow[b]{3}{*}{70} & \multirow{3}{*}{347} & \multirow[b]{3}{*}{ NA } & Biface & Gahagan Biface & $\begin{array}{c}\text { Medial-Basal } \\
\text { Fragment }\end{array}$ & Use & $\begin{array}{c}\text { Fine Grain } \\
\text { Chert }\end{array}$ & 10YR5/1 & Gray & 0 & Indet & None & No & None & Lanceolate & $\begin{array}{l}\text { Shallow } \\
\text { Concave }\end{array}$ \\
\hline & & & & $\begin{array}{c}\text { Cross-section } \\
\text { Shape }\end{array}$ & $\begin{array}{l}\text { Flaking } \\
\text { Pattern }\end{array}$ & Beveling & $\begin{array}{c}\text { Reduction } \\
\text { Stage }\end{array}$ & Weight (g) & $\begin{array}{c}\text { Max } \\
\text { Length }\end{array}$ & Max Width & $\begin{array}{c}\text { Max } \\
\text { Thickness }\end{array}$ & $\begin{array}{c}\text { Avg Edge } \\
\text { Angle }\end{array}$ & $\begin{array}{l}\text { Callahan's } \\
\text { w/t index }\end{array}$ & \multicolumn{4}{|c|}{ Comments } \\
\hline & & & & $\begin{array}{c}\text { Biconvex } \\
\text { Symmetrical }\end{array}$ & $\begin{array}{c}\text { Oblique } \\
\text { Subparallel }\end{array}$ & None & Indet & 19 & Indet & Indet & Indet & 35 & Indet & $\begin{array}{r}\text { Long late } \\
\text { Slight con } \\
\text { edges; Left } \\
\text { Speckle }\end{array}$ & $\begin{array}{l}\text { stage or com } \\
\text { icave basal } n \\
\text { basal should } \\
\text { es of mineral }\end{array}$ & $\begin{array}{l}\text { plete Gahaga } \\
\text { nargin, recurve } \\
\text { er broken and } \\
\text { inclusion (10) }\end{array}$ & $\begin{array}{l}\text { ed biface; } \\
\text { ed lateral } \\
\text { reworked; } \\
\text { YR6/1) }\end{array}$ \\
\hline
\end{tabular}




\begin{tabular}{|c|c|c|c|c|c|c|c|c|c|c|c|c|c|c|c|c|c|}
\hline $\begin{array}{l}\text { Lot } \\
\text { No. }\end{array}$ & $\begin{array}{l}\text { Spec. } \\
\text { No. }\end{array}$ & $\begin{array}{l}\text { Bag } \\
\text { No. }\end{array}$ & $\begin{array}{l}\text { UI } \\
\text { No. }\end{array}$ & Category & Subcategory & $\begin{array}{l}\text { Specimen } \\
\text { Condition }\end{array}$ & Breakage & $\begin{array}{c}\text { Raw } \\
\text { Material }\end{array}$ & Munsell & Color & Cortex \% & $\begin{array}{c}\text { Parent } \\
\text { Form }\end{array}$ & $\begin{array}{c}\text { Heat } \\
\text { Evidence }\end{array}$ & $\begin{array}{l}\text { Intentional } \\
\text { Heat } \\
\text { Treatment }\end{array}$ & Patination & $\begin{array}{l}\text { Overall } \\
\text { Shape }\end{array}$ & $\begin{array}{l}\text { Basal } \\
\text { Shape }\end{array}$ \\
\hline \multirow{3}{*}{560} & \multirow{3}{*}{1} & \multirow{3}{*}{363} & \multirow{3}{*}{22} & Biface & Biface & $\begin{array}{c}\text { Distal-Medial } \\
\text { Fragment }\end{array}$ & Manufacture & $\begin{array}{c}\text { Fine Grain } \\
\text { Chert }\end{array}$ & 10YR4/2 & $\begin{array}{c}\text { Dark Grayish } \\
\text { Brown }\end{array}$ & 1-25 & Indet & Slight Luster & Yes & None & Indet & Indet \\
\hline & & & & $\begin{array}{c}\text { Cross-section } \\
\text { Shape }\end{array}$ & $\begin{array}{l}\text { Flaking } \\
\text { Pattern }\end{array}$ & Beveling & $\begin{array}{c}\text { Reduction } \\
\text { Stage }\end{array}$ & Weight (g) & $\begin{array}{c}\text { Max } \\
\text { Length }\end{array}$ & Max Width & $\begin{array}{c}\text { Max } \\
\text { Thickness }\end{array}$ & $\begin{array}{c}\text { Avg Edge } \\
\text { Angle }\end{array}$ & $\begin{array}{l}\text { Callahan's } \\
\text { w/t index }\end{array}$ & \multicolumn{4}{|c|}{ Comments } \\
\hline & & & & $\begin{array}{c}\text { Biconvex } \\
\text { Symmetrical }\end{array}$ & Random & None & Indet & 10.38 & Indet & Indet & Indet & 25 & Indet & \multicolumn{4}{|c|}{$\begin{array}{l}\text { Banding on one face (10YR6/1); Thin biface and } \\
\text { appears to be late stage; Break runs from left } \\
\text { basal shoulder to middle of right lateral margin }\end{array}$} \\
\hline
\end{tabular}

\begin{tabular}{|c|c|c|c|c|c|c|c|c|c|c|c|c|c|c|c|c|c|}
\hline $\begin{array}{l}\text { Lot } \\
\text { No. }\end{array}$ & $\begin{array}{l}\text { Spec. } \\
\text { No. }\end{array}$ & $\begin{array}{l}\text { Bag } \\
\text { No. }\end{array}$ & $\begin{array}{l}\text { UI } \\
\text { No. }\end{array}$ & Category & Subcategory & $\begin{array}{l}\text { Specimen } \\
\text { Condition }\end{array}$ & Breakage & $\begin{array}{c}\text { Raw } \\
\text { Material }\end{array}$ & Munsell & Color & Cortex \% & $\begin{array}{c}\text { Parent } \\
\text { Form }\end{array}$ & $\begin{array}{c}\text { Heat } \\
\text { Evidence }\end{array}$ & $\begin{array}{l}\text { Intentional } \\
\text { Heat } \\
\text { Treatment }\end{array}$ & Patination & $\begin{array}{l}\text { Overall } \\
\text { Shape }\end{array}$ & $\begin{array}{l}\text { Basal } \\
\text { Shape }\end{array}$ \\
\hline \multirow{3}{*}{561} & \multirow{3}{*}{7} & \multirow{3}{*}{367} & \multirow{3}{*}{ NA } & Biface & Biface & Indet Fragment & Manufacture & $\begin{array}{c}\text { Fine Grain } \\
\text { Chert }\end{array}$ & 10YR4/2 & $\begin{array}{l}\text { Dark Grayish } \\
\text { Brown }\end{array}$ & 0 & Indet & None & No & None & Indet & Indet \\
\hline & & & & $\begin{array}{l}\text { Cross-section } \\
\text { Shape }\end{array}$ & $\begin{array}{l}\text { Flaking } \\
\text { Pattern }\end{array}$ & Beveling & $\begin{array}{l}\text { Reduction } \\
\text { Stage }\end{array}$ & Weight (g) & $\begin{array}{c}\text { Max } \\
\text { Length }\end{array}$ & Max Width & $\begin{array}{c}\text { Max } \\
\text { Thickness }\end{array}$ & $\begin{array}{l}\text { Avg Edge } \\
\text { Angle }\end{array}$ & $\begin{array}{l}\text { Callahan's } \\
\text { w/t index }\end{array}$ & \multicolumn{4}{|c|}{ Comments } \\
\hline & & & & Indet & Indet & None & Indet & 0.64 & Indet & Indet & Indet & Indet & Indet & \multicolumn{4}{|c|}{$\begin{array}{l}\text { Small fragment that appears to be a basal } \\
\text { shoulder; Step fracture on one face preventing } \\
\text { reduction }\end{array}$} \\
\hline
\end{tabular}

\begin{tabular}{|c|c|c|c|c|c|c|c|c|c|c|c|c|c|c|c|c|c|}
\hline $\begin{array}{l}\text { Lot } \\
\text { No. }\end{array}$ & $\begin{array}{c}\text { Spec. } \\
\text { No. }\end{array}$ & $\begin{array}{l}\text { Bag } \\
\text { No. }\end{array}$ & $\begin{array}{l}\text { UI } \\
\text { No. }\end{array}$ & Category & Subcategory & $\begin{array}{l}\text { Specimen } \\
\text { Condition }\end{array}$ & Breakage & $\begin{array}{c}\text { Raw } \\
\text { Material }\end{array}$ & Munsell & Color & Cortex \% & $\begin{array}{c}\text { Parent } \\
\text { Form }\end{array}$ & $\begin{array}{c}\text { Heat } \\
\text { Evidence }\end{array}$ & $\begin{array}{c}\text { Intentional } \\
\text { Heat } \\
\text { Treatment }\end{array}$ & Patination & $\begin{array}{l}\text { Overall } \\
\text { Shape }\end{array}$ & $\begin{array}{l}\text { Basal } \\
\text { Shape }\end{array}$ \\
\hline \multirow[b]{3}{*}{565} & \multirow[b]{3}{*}{5} & \multirow[b]{3}{*}{374} & \multirow[b]{3}{*}{ NA } & Biface & Biface & $\begin{array}{c}\text { Distal-Medial } \\
\text { Fragment }\end{array}$ & Manufacture & $\begin{array}{l}\text { Fine Grain } \\
\text { Chert }\end{array}$ & 10YR5/2 & Grayish Brown & $1-25$ & $\begin{array}{c}\text { River } \\
\text { Cobble }\end{array}$ & Reddened & Yes & None & Indet & Indet \\
\hline & & & & $\begin{array}{l}\text { Cross-section } \\
\text { Shape }\end{array}$ & $\begin{array}{l}\text { Flaking } \\
\text { Pattern }\end{array}$ & Beveling & $\begin{array}{c}\text { Reduction } \\
\text { Stage }\end{array}$ & Weight (g) & $\begin{array}{c}\text { Max } \\
\text { Length }\end{array}$ & Max Width & $\begin{array}{c}\text { Max } \\
\text { Thickness }\end{array}$ & $\begin{array}{c}\text { Avg Edge } \\
\text { Angle }\end{array}$ & $\begin{array}{l}\text { Callahan's } \\
\text { w/t index }\end{array}$ & \multicolumn{4}{|c|}{ Comments } \\
\hline & & & & $\begin{array}{c}\text { Biconvex } \\
\text { Symmetrical }\end{array}$ & Random & None & Indet & 20.35 & Indet & Indet & Indet & Indet & Indet & \multicolumn{4}{|c|}{$\begin{array}{l}\text { Fragment appears to be the distal-medial due to } \\
\text { lateral margins widening towards break; Cortex } \\
\text { focused on medial portion of one face; Edged } \\
\text { biface with no indication of use or intent; } 20 \% \\
\text { mineral inclusions on one face }\end{array}$} \\
\hline
\end{tabular}

\begin{tabular}{|c|c|c|c|c|c|c|c|c|c|c|c|c|c|c|c|c|c|}
\hline $\begin{array}{l}\text { Lot } \\
\text { No. }\end{array}$ & $\begin{array}{l}\text { Spec. } \\
\text { No. }\end{array}$ & $\begin{array}{l}\text { Bag } \\
\text { No. }\end{array}$ & $\begin{array}{l}\text { UI } \\
\text { No. }\end{array}$ & Category & Subcategory & $\begin{array}{l}\text { Specimen } \\
\text { Condition }\end{array}$ & Breakage & $\begin{array}{c}\text { Raw } \\
\text { Material }\end{array}$ & Munsell & Color & Cortex \% & $\begin{array}{c}\text { Parent } \\
\text { Form }\end{array}$ & $\begin{array}{c}\text { Heat } \\
\text { Evidence }\end{array}$ & $\begin{array}{c}\text { Intentional } \\
\text { Heat } \\
\text { Treatment }\end{array}$ & Patination & $\begin{array}{l}\text { Overall } \\
\text { Shape }\end{array}$ & $\begin{array}{l}\text { Basal } \\
\text { Shape }\end{array}$ \\
\hline \multirow[b]{3}{*}{569} & \multirow[b]{3}{*}{5} & \multirow[b]{3}{*}{389} & \multirow[b]{3}{*}{ NA } & Biface & Biface & $\begin{array}{c}\text { Distal-Medial } \\
\text { Fragment }\end{array}$ & Indet & $\begin{array}{c}\text { Fine Grain } \\
\text { Chert }\end{array}$ & 10YR5/1 & Gray & 0 & Indet & None & No & None & Indet & Indet \\
\hline & & & & $\begin{array}{c}\text { Cross-section } \\
\text { Shape }\end{array}$ & $\begin{array}{l}\text { Flaking } \\
\text { Pattern }\end{array}$ & Beveling & $\begin{array}{c}\text { Reduction } \\
\text { Stage }\end{array}$ & Weight (g) & $\begin{array}{c}\text { Max } \\
\text { Length }\end{array}$ & Max Width & $\begin{array}{c}\text { Max } \\
\text { Thickness }\end{array}$ & $\begin{array}{c}\text { Avg Edge } \\
\text { Angle }\end{array}$ & $\begin{array}{l}\text { Callahan's } \\
\text { w/t index }\end{array}$ & \multicolumn{4}{|c|}{ Comments } \\
\hline & & & & $\begin{array}{l}\text { Biconvex } \\
\text { Symmetrical }\end{array}$ & $\begin{array}{l}\text { Subparallel to } \\
\text { Oblique } \\
\text { Subparallel }\end{array}$ & None & Stage 5 & 0.68 & Indet & Indet & 2.7 & 25 & Indet & $\begin{array}{r}\text { Non-diagnos } \\
\text { is } 10 Y R 7 \\
\text { worked wit }\end{array}$ & $\begin{array}{l}\text { stic projectile } \\
7 / 4 \text { in color; } \\
\text { th possible } n \\
\text { shou }\end{array}$ & $\begin{array}{l}\text { oint fragm } \\
\text { isal margin } \\
\text { tch work be } \\
\text { der }\end{array}$ & $\begin{array}{l}\text { t; Distal tip } \\
\text { slightly } \\
\text { in on left }\end{array}$ \\
\hline
\end{tabular}




\begin{tabular}{|c|c|c|c|c|c|c|c|c|c|c|c|c|c|c|c|c|c|}
\hline $\begin{array}{l}\text { Lot } \\
\text { No. }\end{array}$ & $\begin{array}{l}\text { Spec. } \\
\text { No. }\end{array}$ & $\begin{array}{l}\text { Bag } \\
\text { No. }\end{array}$ & $\begin{array}{l}\text { Ul } \\
\text { No. }\end{array}$ & Category & Subcategory & $\begin{array}{l}\text { Specimen } \\
\text { Condition }\end{array}$ & Breakage & $\begin{array}{c}\text { Raw } \\
\text { Material }\end{array}$ & Munsell & Color & Cortex \% & $\begin{array}{c}\text { Parent } \\
\text { Form }\end{array}$ & $\begin{array}{c}\text { Heat } \\
\text { Evidence }\end{array}$ & $\begin{array}{c}\text { Intentional } \\
\text { Heat } \\
\text { Treatment }\end{array}$ & Patination & $\begin{array}{l}\text { Overall } \\
\text { Shape }\end{array}$ & $\begin{array}{l}\text { Basal } \\
\text { Shape }\end{array}$ \\
\hline \multirow{3}{*}{580} & \multirow{3}{*}{14} & \multirow{3}{*}{648} & \multirow{3}{*}{ NA } & Biface & Biface & Complete & None & $\begin{array}{c}\text { Fine Grain } \\
\text { Chert }\end{array}$ & $\begin{array}{c}10 Y R 3 / 1 \text { to } \\
10 Y R 2 / 1 \\
\end{array}$ & $\begin{array}{c}\text { Very Dark } \\
\text { Gray to Black }\end{array}$ & 0-25 & $\begin{array}{l}\text { Nodular } \\
\text { Cobble }\end{array}$ & None & No & None & Amorphous & Indet \\
\hline & & & & $\begin{array}{c}\text { Cross-section } \\
\text { Shape }\end{array}$ & $\begin{array}{l}\text { Flaking } \\
\text { Pattern } \\
\end{array}$ & Beveling & $\begin{array}{c}\text { Reduction } \\
\text { Stage } \\
\end{array}$ & Weight (g) & $\begin{array}{c}\text { Max } \\
\text { Length } \\
\end{array}$ & Max Width & $\begin{array}{c}\text { Max } \\
\text { Thickness } \\
\end{array}$ & $\begin{array}{c}\text { Avg Edge } \\
\text { Angle }\end{array}$ & $\begin{array}{c}\text { Callahan's } \\
\text { w/t index }\end{array}$ & \multicolumn{4}{|c|}{ Comments } \\
\hline & & & & $\begin{array}{c}\text { Biconvex } \\
\text { Symmetrical }\end{array}$ & Random & None & Stage 2 & 90.68 & 82.04 & 58.92 & 23.08 & 40 & 2.55 & Early stage & $\begin{array}{l}\text { with shape s } \\
\text { hard pressu }\end{array}$ & $\begin{array}{l}\text { started but not } \\
\text { ure reduction }\end{array}$ & clear; Only \\
\hline $\begin{array}{l}\text { Lot } \\
\text { No. }\end{array}$ & $\begin{array}{l}\text { Spec. } \\
\text { No. }\end{array}$ & $\begin{array}{l}\text { Bag } \\
\text { No. }\end{array}$ & $\begin{array}{l}\text { UI } \\
\text { No. }\end{array}$ & Category & Subcategory & $\begin{array}{l}\text { Specimen } \\
\text { Condition }\end{array}$ & Breakage & $\begin{array}{c}\text { Raw } \\
\text { Material }\end{array}$ & Munsell & Color & Cortex \% & $\begin{array}{l}\text { Parent } \\
\text { Form }\end{array}$ & $\begin{array}{c}\text { Heat } \\
\text { Evidence }\end{array}$ & $\begin{array}{c}\text { Intentional } \\
\text { Heat } \\
\text { Treatment }\end{array}$ & Patination & $\begin{array}{l}\text { Overall } \\
\text { Shape }\end{array}$ & $\begin{array}{l}\text { Basal } \\
\text { Shape }\end{array}$ \\
\hline \multirow{3}{*}{593} & \multirow{3}{*}{1} & \multirow{3}{*}{565} & \multirow{3}{*}{ NA } & Biface & Biface & Complete & None & $\begin{array}{c}\text { Fine Grain } \\
\text { Chert }\end{array}$ & 10YR6/3 & Pale Brown & 26-50 & Indet & None & No & None & Teardrop & $\begin{array}{l}\text { Gentle } \\
\text { Convex }\end{array}$ \\
\hline & & & & $\begin{array}{c}\text { Cross-section } \\
\text { Shape }\end{array}$ & $\begin{array}{l}\text { Flaking } \\
\text { Pattern }\end{array}$ & Beveling & $\begin{array}{c}\begin{array}{c}\text { Reduction } \\
\text { Stage }\end{array} \\
\end{array}$ & Weight (g) & $\begin{array}{c}\text { Max } \\
\text { Length }\end{array}$ & Max Width & $\begin{array}{c}\text { Max } \\
\text { Thickness } \\
\end{array}$ & $\begin{array}{c}\text { Avg Edge } \\
\text { Angle }\end{array}$ & $\begin{array}{c}\text { Callahan's } \\
\text { w/t index }\end{array}$ & \multicolumn{4}{|c|}{ Comments } \\
\hline & & & & $\begin{array}{l}\text { Biconvex } \\
\text { Symmetrical }\end{array}$ & Random & None & Stage 3 & 52.29 & 62.64 & 48.08 & 17.75 & 50 & 2.7 & $\begin{array}{r}\text { Platform is } \mathrm{i} \\
\text { encompa } \\
\text { Bar }\end{array}$ & $\begin{array}{l}\text { intact at dista } \\
\text { asses one fac } \\
\text { anding near ce }\end{array}$ & $\begin{array}{l}\text { al end; Cortex c } \\
\text { ce; Mineral incl } \\
\text { cortex (10YR6/4 }\end{array}$ & $\begin{array}{l}\text { completely } \\
\text { lusions; } \\
4 \text { ) } \\
\end{array}$ \\
\hline $\begin{array}{l}\text { Lot } \\
\text { No. }\end{array}$ & $\begin{array}{l}\text { Spec. } \\
\text { No. }\end{array}$ & $\begin{array}{l}\text { Bag } \\
\text { No. }\end{array}$ & $\begin{array}{l}\text { UI } \\
\text { No. }\end{array}$ & Category & Subcategory & $\begin{array}{l}\text { Specimen } \\
\text { Condition }\end{array}$ & Breakage & $\begin{array}{c}\text { Raw } \\
\text { Material }\end{array}$ & Munsell & Color & Cortex \% & $\begin{array}{c}\text { Parent } \\
\text { Form }\end{array}$ & $\begin{array}{c}\text { Heat } \\
\text { Evidence }\end{array}$ & $\begin{array}{c}\text { Intentional } \\
\text { Heat } \\
\text { Treatment }\end{array}$ & Patination & $\begin{array}{l}\text { Overall } \\
\text { Shape }\end{array}$ & $\begin{array}{l}\text { Basal } \\
\text { Shape }\end{array}$ \\
\hline \multirow{3}{*}{594} & \multirow{3}{*}{1} & \multirow{3}{*}{566} & \multirow{3}{*}{ NA } & Biface & Biface & Complete & None & $\begin{array}{c}\text { Fine Grain } \\
\text { Chert }\end{array}$ & 10YR4/4 & $\begin{array}{c}\text { Dark Yellowish } \\
\text { Brown }\end{array}$ & $1-25$ & Indet & None & No & None & $\begin{array}{c}\text { Subtriangula } \\
\mathrm{r}\end{array}$ & $\begin{array}{l}\text { Straight to } \\
\text { Gentle } \\
\text { Convex }\end{array}$ \\
\hline & & & & $\begin{array}{c}\text { Cross-section } \\
\text { Shape }\end{array}$ & $\begin{array}{l}\text { Flaking } \\
\text { Pattern }\end{array}$ & Beveling & $\begin{array}{c}\text { Reduction } \\
\text { Stage } \\
\end{array}$ & Weight (g) & $\begin{array}{c}\text { Max } \\
\text { Length }\end{array}$ & Max Width & $\begin{array}{c}\text { Max } \\
\text { Thickness }\end{array}$ & $\begin{array}{c}\text { Avg Edge } \\
\text { Angle }\end{array}$ & $\begin{array}{c}\text { Callahan's } \\
\text { w/t index }\end{array}$ & \multicolumn{4}{|c|}{ Comments } \\
\hline & & & & Indet & Random & None & Stage 3 & 36.81 & 53.56 & 46.01 & 15.21 & 55 & 3.02 & $\begin{array}{r}\text { Shaped bif } \\
\text { on one face } \\
\text { mar }\end{array}$ & $\begin{array}{l}\text { face with two } \\
\text { e; Edge worki } \\
\text { argin and half }\end{array}$ & $\begin{array}{l}\text { small patches } \\
\text { king begun on o } \\
\text { f of basal margi }\end{array}$ & $\begin{array}{l}\text { of cortex } \\
\text { one lateral } \\
\text { in } \\
\end{array}$ \\
\hline $\begin{array}{l}\text { Lot } \\
\text { No. }\end{array}$ & $\begin{array}{l}\text { Spec. } \\
\text { No. }\end{array}$ & $\begin{array}{l}\text { Bag } \\
\text { No. }\end{array}$ & $\begin{array}{l}\text { UI } \\
\text { No. }\end{array}$ & Category & Subcategory & $\begin{array}{l}\text { Specimen } \\
\text { Condition }\end{array}$ & Breakage & $\begin{array}{c}\text { Raw } \\
\text { Material }\end{array}$ & Munsell & Color & Cortex \% & $\begin{array}{c}\text { Parent } \\
\text { Form }\end{array}$ & $\begin{array}{c}\text { Heat } \\
\text { Evidence }\end{array}$ & $\begin{array}{c}\text { Intentional } \\
\text { Heat } \\
\text { Treatment }\end{array}$ & Patination & $\begin{array}{l}\text { Overall } \\
\text { Shape }\end{array}$ & $\begin{array}{l}\text { Basal } \\
\text { Shape }\end{array}$ \\
\hline \multirow{3}{*}{597} & \multirow{3}{*}{5} & \multirow{3}{*}{575} & \multirow{3}{*}{ NA } & Biface & Biface & Indet Fragment & Manufacture & $\begin{array}{c}\text { Fine Grain } \\
\text { Chert }\end{array}$ & 10YR5/2 & Grayish Brown & 0 & Indet & None & No & None & Indet & Indet \\
\hline & & & & $\begin{array}{l}\text { Cross-section } \\
\text { Shape }\end{array}$ & $\begin{array}{l}\text { Flaking } \\
\text { Pattern }\end{array}$ & Beveling & $\begin{array}{c}\text { Reduction } \\
\text { Stage }\end{array}$ & Weight (g) & $\begin{array}{c}\text { Max } \\
\text { Length }\end{array}$ & Max Width & $\begin{array}{c}\text { Max } \\
\text { Thickness }\end{array}$ & $\begin{array}{c}\text { Avg Edge } \\
\text { Angle }\end{array}$ & $\begin{array}{l}\text { Callahan's } \\
\text { w/t index }\end{array}$ & \multicolumn{4}{|c|}{ Comments } \\
\hline & & & & Crescent & Random & None & Indet & 31.38 & Indet & Indet & Indet & Indet & Indet & $\begin{array}{r}\text { Amorphous } \\
\text { blank stage } \\
\text { discernable } \\
\text { cra }\end{array}$ & $\begin{array}{l}\text { Is fragment w } \\
\text { e due to very } \\
\text { le shape, but } \\
\text { acks and fisst }\end{array}$ & $\begin{array}{l}\text { vith break; Estir } \\
\text { / sinuous edges } \\
\text { it is relatively th } \\
\text { ures throughou }\end{array}$ & $\begin{array}{l}\text { mated as } \\
\text { s, and no } \\
\text { hin; small } \\
\text { ut }\end{array}$ \\
\hline
\end{tabular}




\begin{tabular}{|c|c|c|c|c|c|c|c|c|c|c|c|c|c|c|c|c|c|}
\hline $\begin{array}{l}\text { Lot } \\
\text { No. }\end{array}$ & $\begin{array}{l}\text { Spec. } \\
\text { No. }\end{array}$ & $\begin{array}{l}\text { Bag } \\
\text { No. }\end{array}$ & $\begin{array}{l}\text { UI } \\
\text { No. }\end{array}$ & Category & Subcategory & $\begin{array}{l}\text { Specimen } \\
\text { Condition }\end{array}$ & Breakage & $\begin{array}{c}\text { Raw } \\
\text { Material }\end{array}$ & Munsell & Color & Cortex \% & $\begin{array}{l}\text { Parent } \\
\text { Form }\end{array}$ & $\begin{array}{c}\text { Heat } \\
\text { Evidence }\end{array}$ & $\begin{array}{c}\text { Intentional } \\
\text { Heat } \\
\text { Treatment }\end{array}$ & Patination & $\begin{array}{l}\text { Overall } \\
\text { Shape }\end{array}$ & $\begin{array}{l}\text { Basal } \\
\text { Shape }\end{array}$ \\
\hline \multirow{3}{*}{620} & \multirow{3}{*}{1} & \multirow{3}{*}{531} & \multirow{3}{*}{26} & Biface & Biface & $\begin{array}{l}\text { Distal-Medial } \\
\text { Fragment }\end{array}$ & Manufacture & $\begin{array}{c}\text { Coarse } \\
\text { Grain Chert }\end{array}$ & 10YR5/2 & Grayish Brown & 0 & Indet & None & No & None & Indet & Indet \\
\hline & & & & $\begin{array}{c}\begin{array}{c}\text { Cross-section } \\
\text { Shape }\end{array} \\
\end{array}$ & $\begin{array}{l}\text { Flaking } \\
\text { Pattern }\end{array}$ & Beveling & $\begin{array}{c}\begin{array}{c}\text { Reduction } \\
\text { Stage }\end{array} \\
\end{array}$ & Weight (g) & $\begin{array}{c}\text { Max } \\
\text { Length }\end{array}$ & Max Width & $\begin{array}{c}\text { Max } \\
\text { Thickness }\end{array}$ & $\begin{array}{c}\text { Avg Edge } \\
\text { Angle } \\
\end{array}$ & $\begin{array}{l}\text { Callahan's } \\
\text { w/t index }\end{array}$ & \multicolumn{4}{|c|}{ Comments } \\
\hline & & & & $\begin{array}{c}\text { Biconvex } \\
\text { Symmetrical }\end{array}$ & $\begin{array}{l}\text { Subparallel and } \\
\text { Oblique Parallel }\end{array}$ & None & Stage 5 & 38.43 & Indet & Indet & Indet & 25 & Indet & $\begin{array}{l}\text { Gahagan-l } \\
\text { lateral bas } \\
\text { Mineral incl }\end{array}$ & $\begin{array}{l}\text { like large bla } \\
\text { se to middle } \\
\text { lusions throu }\end{array}$ & $\begin{array}{l}\text { e with brea } \\
\text { f right later } \\
\text { ghout (10Y) }\end{array}$ & $\begin{array}{l}\text { rom left } \\
\text { nargin; } \\
11,20 \%)\end{array}$ \\
\hline
\end{tabular}

\begin{tabular}{|c|c|c|c|c|c|c|c|c|c|c|c|c|c|c|c|c|c|}
\hline $\begin{array}{l}\text { Lot } \\
\text { No. }\end{array}$ & $\begin{array}{c}\text { Spec. } \\
\text { No. }\end{array}$ & $\begin{array}{l}\text { Bag } \\
\text { No. }\end{array}$ & $\begin{array}{l}\text { Ul } \\
\text { No. }\end{array}$ & Category & Subcategory & $\begin{array}{l}\text { Specimen } \\
\text { Condition }\end{array}$ & Breakage & $\begin{array}{c}\text { Raw } \\
\text { Material }\end{array}$ & Munsell & Color & Cortex \% & $\begin{array}{c}\text { Parent } \\
\text { Form }\end{array}$ & $\begin{array}{c}\text { Heat } \\
\text { Evidence }\end{array}$ & $\begin{array}{l}\text { Intentional } \\
\text { Heat } \\
\text { Treatment }\end{array}$ & Patination & $\begin{array}{l}\text { Overall } \\
\text { Shape }\end{array}$ & $\begin{array}{l}\text { Basal } \\
\text { Shape }\end{array}$ \\
\hline \multirow{3}{*}{623} & \multirow{3}{*}{9} & \multirow{3}{*}{581} & \multirow{3}{*}{30} & Biface & Biface & $\begin{array}{c}\text { Medial-Basal } \\
\text { Fragment }\end{array}$ & Manufacture & $\begin{array}{c}\text { Fine Grain } \\
\text { Chert }\end{array}$ & 10YR3/3 & Dark Brown & 0 & Indet & Reddened & Yes & None & Lanceolate & $\begin{array}{l}\text { Shallow } \\
\text { Concave }\end{array}$ \\
\hline & & & & $\begin{array}{c}\text { Cross-section } \\
\text { Shape }\end{array}$ & $\begin{array}{l}\text { Flaking } \\
\text { Pattern }\end{array}$ & Beveling & $\begin{array}{c}\text { Reduction } \\
\text { Stage }\end{array}$ & Weight (g) & $\begin{array}{c}\text { Max } \\
\text { Length }\end{array}$ & Max Width & $\begin{array}{c}\text { Max } \\
\text { Thickness } \\
\end{array}$ & $\begin{array}{c}\text { Avg Edge } \\
\text { Angle }\end{array}$ & $\begin{array}{c}\text { Callahan's } \\
\text { w/t index }\end{array}$ & \multicolumn{4}{|c|}{ Comments } \\
\hline & & & & $\begin{array}{c}\text { Biconvex } \\
\text { Symmetrical }\end{array}$ & Random & None & Stage 3 & 12.13 & Indet & 24.89 & 7.05 & 25 & 3.53 & \multicolumn{4}{|c|}{$\begin{array}{l}\text { Possible projectile point preform due to clear } \\
\text { shaping but still needs thinning; Estimated at } 80 \% \\
\text { complete with only distal tip missing }\end{array}$} \\
\hline
\end{tabular}

\begin{tabular}{|c|c|c|c|c|c|c|c|c|c|c|c|c|c|c|c|c|c|}
\hline $\begin{array}{l}\text { Lot } \\
\text { No. }\end{array}$ & $\begin{array}{c}\text { Spec. } \\
\text { No. }\end{array}$ & $\begin{array}{l}\text { Bag } \\
\text { No. }\end{array}$ & $\begin{array}{l}\text { UI } \\
\text { No. }\end{array}$ & Category & Subcategory & $\begin{array}{l}\text { Specimen } \\
\text { Condition }\end{array}$ & Breakage & $\begin{array}{c}\text { Raw } \\
\text { Material }\end{array}$ & Munsell & Color & Cortex \% & $\begin{array}{c}\text { Parent } \\
\text { Form }\end{array}$ & $\begin{array}{c}\text { Heat } \\
\text { Evidence }\end{array}$ & $\begin{array}{c}\text { Intentional } \\
\text { Heat } \\
\text { Treatment }\end{array}$ & Patination & $\begin{array}{l}\text { Overall } \\
\text { Shape }\end{array}$ & $\begin{array}{l}\text { Basal } \\
\text { Shape }\end{array}$ \\
\hline \multirow[b]{3}{*}{632} & \multirow[b]{3}{*}{9} & \multirow[b]{3}{*}{385} & \multirow[b]{3}{*}{ NA } & Biface & Biface & $\begin{array}{l}\text { Marginal } \\
\text { Fragment }\end{array}$ & Indet & $\begin{array}{c}\text { Fine Grain } \\
\text { Chert }\end{array}$ & 10YR3/3 & Dark Brown & 0 & Indet & None & No & None & Indet & Indet \\
\hline & & & & $\begin{array}{l}\text { Cross-section } \\
\text { Shape }\end{array}$ & $\begin{array}{l}\text { Flaking } \\
\text { Pattern }\end{array}$ & Beveling & $\begin{array}{c}\begin{array}{c}\text { Reduction } \\
\text { Stage }\end{array} \\
\end{array}$ & Weight (g) & $\begin{array}{c}\text { Max } \\
\text { Length }\end{array}$ & Max Width & $\begin{array}{c}\text { Max } \\
\text { Thickness }\end{array}$ & $\begin{array}{c}\text { Avg Edge } \\
\text { Angle }\end{array}$ & $\begin{array}{l}\text { Callahan's } \\
\text { w/t index }\end{array}$ & \multicolumn{4}{|c|}{ Comments } \\
\hline & & & & Indet & Indet & None & Indet & 0.43 & Indet & Indet & Indet & Indet & Indet & $\begin{array}{l}\text { Fragment } m \\
\text { has been ren } \\
\text { or edge coll } \\
\text { soft pressur } \\
\text { complete } p\end{array}$ & $\begin{array}{l}\text { narginal with } \\
\text { noved possit } \\
\text { lapse; Very th } \\
\text { e reduction; } \\
\text { rojectile poin } \\
\text { en }\end{array}$ & $\begin{array}{l}\text { nly one fac } \\
\text { y due to he } \\
n \text { with fine } \\
\text { ppears to } \\
\text {; Indet frac }\end{array}$ & $\begin{array}{l}\text { One face } \\
\text { treatment } \\
\text { king from } \\
\text { portion of } \\
\text { e at one }\end{array}$ \\
\hline
\end{tabular}

\begin{tabular}{|c|c|c|c|c|c|c|c|c|c|c|c|c|c|c|c|c|c|}
\hline $\begin{array}{l}\text { Lot } \\
\text { No. }\end{array}$ & $\begin{array}{l}\text { Spec. } \\
\text { No. }\end{array}$ & $\begin{array}{l}\text { Bag } \\
\text { No. }\end{array}$ & $\begin{array}{l}\text { UI } \\
\text { No. }\end{array}$ & Category & Subcategory & $\begin{array}{l}\text { Specimen } \\
\text { Condition }\end{array}$ & Breakage & $\begin{array}{c}\text { Raw } \\
\text { Material }\end{array}$ & Munsell & Color & Cortex \% & $\begin{array}{l}\text { Parent } \\
\text { Form }\end{array}$ & $\begin{array}{c}\text { Heat } \\
\text { Evidence }\end{array}$ & $\begin{array}{c}\text { Intentional } \\
\text { Heat } \\
\text { Treatment }\end{array}$ & Patination & $\begin{array}{l}\text { Overall } \\
\text { Shape }\end{array}$ & $\begin{array}{l}\text { Basal } \\
\text { Shape }\end{array}$ \\
\hline \multirow{3}{*}{634} & \multirow{3}{*}{1} & \multirow{3}{*}{397} & \multirow{3}{*}{ NA } & Biface & Biface & $\begin{array}{l}\text { Distal-Medial } \\
\text { Fragment }\end{array}$ & Use & $\begin{array}{l}\text { Fine Grain } \\
\text { Chert }\end{array}$ & 10YR4/1 & Dark Gray & 0 & Indet & None & No & None & Indet & Indet \\
\hline & & & & $\begin{array}{l}\text { Cross-section } \\
\text { Shape }\end{array}$ & $\begin{array}{l}\text { Flaking } \\
\text { Pattern }\end{array}$ & Beveling & $\begin{array}{l}\text { Reduction } \\
\text { Stage }\end{array}$ & Weight (g) & $\begin{array}{c}\text { Max } \\
\text { Length }\end{array}$ & Max Width & $\begin{array}{c}\text { Max } \\
\text { Thickness }\end{array}$ & $\begin{array}{c}\text { Avg Edge } \\
\text { Angle }\end{array}$ & $\begin{array}{l}\text { Callahan's } \\
\text { w/t index }\end{array}$ & \multicolumn{4}{|c|}{ Comments } \\
\hline & & & & $\begin{array}{l}\text { Biconvex } \\
\text { Symmetrical }\end{array}$ & $\begin{array}{l}\text { Oblique } \\
\text { Subparallel }\end{array}$ & None & Stage 5 & 12.14 & Indet & Indet & Indet & Indet & Indet & \multicolumn{4}{|c|}{$\begin{array}{c}\text { Very distal tip broken; Mineral inclusions } \\
\text { throughout }(5 \%) ; \text { Estimated at stage } 5 \text { due to } \\
\text { thinness and evidence of fine soft pressure } \\
\text { reduction }\end{array}$} \\
\hline
\end{tabular}




\begin{tabular}{|c|c|c|c|c|c|c|c|c|c|c|c|c|c|c|c|c|c|}
\hline $\begin{array}{l}\text { Lot } \\
\text { No. }\end{array}$ & $\begin{array}{l}\text { Spec. } \\
\text { No. }\end{array}$ & $\begin{array}{l}\text { Bag } \\
\text { No. }\end{array}$ & $\begin{array}{l}\text { Ul } \\
\text { No. }\end{array}$ & Category & Subcategory & $\begin{array}{l}\text { Specimen } \\
\text { Condition }\end{array}$ & Breakage & $\begin{array}{c}\text { Raw } \\
\text { Material }\end{array}$ & Munsell & Color & Cortex \% & $\begin{array}{c}\text { Parent } \\
\text { Form }\end{array}$ & $\begin{array}{c}\text { Heat } \\
\text { Evidence }\end{array}$ & $\begin{array}{c}\text { Intentional } \\
\text { Heat } \\
\text { Treatment }\end{array}$ & Patination & $\begin{array}{l}\text { Overall } \\
\text { Shape }\end{array}$ & $\begin{array}{l}\text { Basal } \\
\text { Shape }\end{array}$ \\
\hline \multirow{3}{*}{646} & \multirow{3}{*}{5} & \multirow{3}{*}{369} & \multirow{3}{*}{ NA } & Biface & Biface & Basal Fragment & Indet & $\begin{array}{c}\text { Fine Grain } \\
\text { Chert }\end{array}$ & 10YR5/4 & $\begin{array}{c}\text { Yellowish } \\
\text { Brown }\end{array}$ & $26-50$ & Indet & None & No & None & Indet & Indet \\
\hline & & & & $\begin{array}{c}\text { Cross-section } \\
\text { Shape }\end{array}$ & $\begin{array}{l}\text { Flaking } \\
\text { Pattern } \\
\end{array}$ & Beveling & $\begin{array}{c}\text { Reduction } \\
\text { Stage } \\
\end{array}$ & Weight (g) & $\begin{array}{c}\text { Max } \\
\text { Length }\end{array}$ & Max Width & $\begin{array}{c}\text { Max } \\
\text { Thickness } \\
\end{array}$ & $\begin{array}{c}\text { Avg Edge } \\
\text { Angle }\end{array}$ & $\begin{array}{c}\text { Callahan's } \\
\text { w/t index }\end{array}$ & \multicolumn{4}{|c|}{ Comments } \\
\hline & & & & Indet & Indet & None & Indet & 5.3 & Indet & Indet & Indet & Indet & Indet & \multicolumn{4}{|c|}{$\begin{array}{l}\text { Small fragment that appears to be a basal } \\
\text { shoulder due to a rounded edge with fine flake } \\
\text { removal }\end{array}$} \\
\hline $\begin{array}{l}\text { Lot } \\
\text { No. }\end{array}$ & $\begin{array}{l}\text { Spec. } \\
\text { No. }\end{array}$ & $\begin{array}{l}\text { Bag } \\
\text { No. }\end{array}$ & $\begin{array}{l}\text { Ul } \\
\text { No. }\end{array}$ & Category & Subcategory & $\begin{array}{l}\text { Specimen } \\
\text { Condition }\end{array}$ & Breakage & $\begin{array}{c}\text { Raw } \\
\text { Material }\end{array}$ & Munsell & Color & Cortex \% & $\begin{array}{c}\text { Parent } \\
\text { Form }\end{array}$ & $\begin{array}{c}\text { Heat } \\
\text { Evidence }\end{array}$ & $\begin{array}{l}\text { Intentional } \\
\text { Heat } \\
\text { Treatment }\end{array}$ & Patination & $\begin{array}{l}\text { Overall } \\
\text { Shape }\end{array}$ & $\begin{array}{l}\text { Basal } \\
\text { Shape }\end{array}$ \\
\hline \multirow{3}{*}{647} & \multirow{3}{*}{6} & \multirow{3}{*}{381} & \multirow{3}{*}{ NA } & Biface & Biface & Distal Fragment & Use & $\begin{array}{c}\text { Fine Grain } \\
\text { Chert }\end{array}$ & 10YR4/1 & Dark Gray & 26-50 & Indet & None & No & None & Indet & Indet \\
\hline & & & & $\begin{array}{c}\begin{array}{c}\text { Cross-section } \\
\text { Shape }\end{array} \\
\end{array}$ & $\begin{array}{l}\text { Flaking } \\
\text { Pattern }\end{array}$ & Beveling & $\begin{array}{c}\text { Reduction } \\
\text { Stage }\end{array}$ & Weight (g) & $\begin{array}{c}\text { Max } \\
\text { Length }\end{array}$ & Max Width & $\begin{array}{c}\text { Max } \\
\text { Thickness }\end{array}$ & $\begin{array}{c}\text { Avg Edge } \\
\text { Angle }\end{array}$ & $\begin{array}{c}\text { Callahan's } \\
\text { w/t index }\end{array}$ & \multicolumn{4}{|c|}{ Comments } \\
\hline & & & & $\begin{array}{l}\text { Biconvex } \\
\text { Symmetrical }\end{array}$ & Subparallel & None & Stage 5 & 0.45 & Indet & Indet & Indet & Indet & Indet & \multicolumn{4}{|c|}{$\begin{array}{l}\text { Distal portion of possible projectile point; Very thin } \\
\text { and small fragment; Cortex is focused at distal tip }\end{array}$} \\
\hline $\begin{array}{l}\text { Lot } \\
\text { No. }\end{array}$ & $\begin{array}{l}\text { Spec. } \\
\text { No. }\end{array}$ & $\begin{array}{l}\text { Bag } \\
\text { No. }\end{array}$ & $\begin{array}{l}\text { UI } \\
\text { No. }\end{array}$ & Category & Subcategory & $\begin{array}{l}\text { Specimen } \\
\text { Condition }\end{array}$ & Breakage & $\begin{array}{c}\text { Raw } \\
\text { Material }\end{array}$ & Munsell & Color & Cortex \% & $\begin{array}{c}\text { Parent } \\
\text { Form }\end{array}$ & $\begin{array}{c}\text { Heat } \\
\text { Evidence }\end{array}$ & $\begin{array}{l}\text { Intentional } \\
\text { Heat } \\
\text { Treatment }\end{array}$ & Patination & $\begin{array}{l}\text { Overall } \\
\text { Shape }\end{array}$ & $\begin{array}{l}\text { Basal } \\
\text { Shape }\end{array}$ \\
\hline \multirow{3}{*}{661} & \multirow{3}{*}{7} & \multirow{3}{*}{469} & \multirow{3}{*}{ NA } & Biface & Biface & Complete & None & $\begin{array}{c}\text { Fine Grain } \\
\text { Chert }\end{array}$ & 10YR4/2 & $\begin{array}{c}\text { Dark Grayish } \\
\text { Brown }\end{array}$ & 0 & Indet & None & No & None & Lanceolate & Irregular \\
\hline & & & & $\begin{array}{c}\text { Cross-section } \\
\text { Shape }\end{array}$ & $\begin{array}{l}\text { Flaking } \\
\text { Pattern }\end{array}$ & Beveling & $\begin{array}{c}\text { Reduction } \\
\text { Stage }\end{array}$ & Weight (g) & $\begin{array}{c}\text { Max } \\
\text { Length }\end{array}$ & Max Width & $\begin{array}{c}\text { Max } \\
\text { Thickness }\end{array}$ & $\begin{array}{c}\text { Avg Edge } \\
\text { Angle }\end{array}$ & $\begin{array}{l}\text { Callahan's } \\
\text { w/t index }\end{array}$ & \multicolumn{4}{|c|}{ Comments } \\
\hline & & & & Crescent & Random & None & Stage 5 & 8.76 & 61.95 & 28.53 & 6.4 & 30 & 4.45 & $\begin{array}{r}\text { Basal marg } \\
\text { Late }\end{array}$ & $\begin{array}{l}\text { in slopes left } \\
\text { e stage with } \mathrm{m}\end{array}$ & $\begin{array}{l}\text { to right at a st } \\
\text { hineral inclusio }\end{array}$ & $\begin{array}{l}\text { teep angle; } \\
\text { ons }\end{array}$ \\
\hline
\end{tabular}




\begin{tabular}{|c|c|c|c|c|c|c|c|c|c|c|c|c|c|c|c|c|}
\hline $\begin{array}{l}\text { Lot } \\
\text { No. }\end{array}$ & $\begin{array}{l}\text { Spec. } \\
\text { No. }\end{array}$ & Bag No. & UI No & Category & Subcategory & $\begin{array}{c}\text { Spec } \\
\text { Condition }\end{array}$ & Breakage & Raw material & Color & $\begin{array}{c}\text { Dorsal } \\
\text { Cortex \% }\end{array}$ & $\begin{array}{c}\text { Heat } \\
\text { Evidence }\end{array}$ & $\begin{array}{c}\text { Intentional } \\
\text { Heat } \\
\text { Treatment }\end{array}$ & Patination & Retouch Detail & $\begin{array}{c}\text { Retouch } \\
\text { Distribution }\end{array}$ & Retouch Location \\
\hline \multirow{3}{*}{390} & \multirow{3}{*}{6} & \multirow{3}{*}{458} & NA & Scraper & $\begin{array}{l}\text { End and Side- } \\
\text { scraper }\end{array}$ & Complete & None & $\begin{array}{c}\text { Fine Grained } \\
\text { Chert }\end{array}$ & $\begin{array}{c}5 Y R 5 / 2 \text { to } \\
5 Y R 4 / 3\end{array}$ & 0 & Reddened & Yes & None & Unifacial-dorsal & Continuous & Distal and Left Lateral Margin \\
\hline & & & & $\begin{array}{l}\text { Weight } \\
\text { (g) }\end{array}$ & $\begin{array}{l}\text { Relative } \\
\text { Length }\end{array}$ & $\begin{array}{l}\text { Relative } \\
\text { Width }\end{array}$ & $\begin{array}{l}\text { Maximum } \\
\text { Thickness }\end{array}$ & $\begin{array}{c}\text { Perimeter } \\
\text { Retouch } \\
\text { Length }\end{array}$ & $\begin{array}{l}\text { Height of } \\
\text { Retouch }\end{array}$ & $\begin{array}{l}\text { Reduction } \\
\text { Index }\end{array}$ & $\begin{array}{c}\text { Average } \\
\text { Edge } \\
\text { Angle } \\
\end{array}$ & $\begin{array}{l}\text { Platform } \\
\text { width }\end{array}$ & $\begin{array}{c}\text { Platform } \\
\text { Thickness }\end{array}$ & \multicolumn{3}{|c|}{ Comments } \\
\hline & & & & 20.12 & 56.94 & 31.74 & 9.16 & 90 & 5.36 & 0.59 & 55 & 14.63 & 6.7 & $\begin{array}{l}\text { Typical Toyah } \\
\text { evidence of } p\end{array}$ & $\begin{array}{r}\text { scraper worke } \\
\text { post deposition } \\
\text { margin near }\end{array}$ & $\begin{array}{l}\text { ed on end and side; possible } \\
\text { nal breakage on right lateral } \\
\text { ar distal end. }\end{array}$ \\
\hline
\end{tabular}




\begin{tabular}{|c|c|c|c|c|c|c|c|c|c|c|c|c|c|c|c|c|c|}
\hline Lot No. & $\begin{array}{l}\text { Spec. } \\
\text { No. }\end{array}$ & $\begin{array}{l}\text { Bag UI No } \\
\text { No. }\end{array}$ & Category & Subcategory & $\begin{array}{c}\text { Retouch } \\
\text { Affiliation }\end{array}$ & $\begin{array}{l}\text { Specimen } \\
\text { Condition }\end{array}$ & Breakage & Raw material & Munsell & Color & $\begin{array}{c}\text { Dorsal } \\
\text { Cortex \% }\end{array}$ & $\begin{array}{c}\text { Heat } \\
\text { Evidence }\end{array}$ & $\begin{array}{c}\text { Intentional } \\
\text { Heat } \\
\text { Treatment }\end{array}$ & Patination & Retouch Detail & $\begin{array}{c}\text { Retouch } \\
\text { Distribution }\end{array}$ & $\begin{array}{l}\text { Retouch } \\
\text { Location }\end{array}$ \\
\hline \multirow{3}{*}{318} & \multirow{3}{*}{5} & \multirow{3}{*}{492 NA } & Modified Flake & Edge-modified & Indet & $\begin{array}{c}\text { Proximal- } \\
\text { Medial }\end{array}$ & Snap & $\begin{array}{c}\text { Fine Grain } \\
\text { Chert }\end{array}$ & $\begin{array}{c}10 \mathrm{YR} 5 / 3 \text { to } \\
10 \mathrm{YR} 5 / 4\end{array}$ & $\begin{array}{c}\text { Brown to } \\
\text { Yellowish Brown }\end{array}$ & $1-25$ & None & NA & None & Unifacial-dorsal & Focused & $\begin{array}{c}\text { Right Lateral } \\
\text { Margin }\end{array}$ \\
\hline & & & Weight (g) & Length & Width & Thickness & $\begin{array}{l}\text { Height of } \\
\text { Retouch }\end{array}$ & $\begin{array}{l}\text { Perimeter } \\
\text { Retouch } \\
\text { Length }\end{array}$ & $\begin{array}{c}\text { Average } \\
\text { Edge } \\
\text { Angle }\end{array}$ & $\begin{array}{c}\text { Reduction } \\
\text { Index }\end{array}$ & $\begin{array}{l}\text { Platform } \\
\text { width }\end{array}$ & $\begin{array}{l}\text { Platform } \\
\text { thickness }\end{array}$ & \multicolumn{5}{|c|}{ Comments } \\
\hline & & & 23.74 & Indet & 67.21 & 10.29 & 1.74 & 19 & 55 & 5.91 & 12.98 & 5.78 & $\begin{array}{l}\text { Irregular in st } \\
\text { focused on }\end{array}$ & $\begin{array}{l}\text { ape with a sic } \\
\text { side struck fla } \\
\text { to }\end{array}$ & $\begin{array}{l}\text { ide struck flake on } \\
\text { ake; Left lateral m } \\
\text { owards distal mar }\end{array}$ & $\begin{array}{l}\text { n right lateral } \mathrm{m} \\
\text { hargin is convex } \\
\text { rgin }\end{array}$ & $\begin{array}{l}\text { nargin; Work is } \\
\text { x and breaks }\end{array}$ \\
\hline
\end{tabular}

\begin{tabular}{|c|c|c|c|c|c|c|c|c|c|c|c|c|c|c|c|c|c|}
\hline Lot No. & $\begin{array}{l}\text { Spec. } \\
\text { No. }\end{array}$ & $\begin{array}{l}\text { Bag UI No } \\
\text { No. }\end{array}$ & Category & Subcategory & $\begin{array}{c}\text { Retouch } \\
\text { Affiliation }\end{array}$ & $\begin{array}{l}\text { Specimen } \\
\text { Condition }\end{array}$ & Breakage & Raw material & Munsell & Color & $\begin{array}{l}\text { Dorsal } \\
\text { Cortex \% }\end{array}$ & $\begin{array}{c}\text { Heat } \\
\text { Evidence }\end{array}$ & $\begin{array}{c}\text { Intentional } \\
\text { Heat } \\
\text { Treatment }\end{array}$ & Patination & Retouch Detail & $\begin{array}{c}\text { Retouch } \\
\text { Distribution }\end{array}$ & $\begin{array}{l}\text { Retouch } \\
\text { Location }\end{array}$ \\
\hline \multirow{3}{*}{318} & \multirow{3}{*}{6} & \multirow{3}{*}{492 NA } & Modified Flake & Edge-modified & Cultural & $\begin{array}{c}\text { Proximal } \\
\text { Missing }\end{array}$ & Snap & $\begin{array}{c}\text { Fine Grain } \\
\text { Chert }\end{array}$ & $\begin{array}{c}\text { 10YR7/4 to } \\
\text { 10YR7/6 }\end{array}$ & $\begin{array}{l}\text { Very Pale } \\
\text { Brown and }\end{array}$ & $0-25$ & Reddened & Yes & None & Unifacial-dorsal & Continuous & $\begin{array}{c}\text { Left Lateral } \\
\text { Margin }\end{array}$ \\
\hline & & & Weight (g) & Length & Width & Thickness & $\begin{array}{l}\text { Height of } \\
\text { Retouch }\end{array}$ & $\begin{array}{l}\text { Perimeter } \\
\text { Retouch } \\
\text { Lenqth }\end{array}$ & $\begin{array}{l}\text { Average } \\
\text { Edge } \\
\text { Anqle }\end{array}$ & $\begin{array}{l}\text { Reduction } \\
\text { Index }\end{array}$ & $\begin{array}{l}\text { Platform } \\
\text { width }\end{array}$ & $\begin{array}{l}\text { Platform } \\
\text { thickness }\end{array}$ & \multicolumn{5}{|c|}{ Comments } \\
\hline & & & 1.21 & Indet & 18.31 & 3.26 & 0.48 & 20.8 & 70 & 6.79 & Indet & Indet & Incomplete & $\begin{array}{r}\text { ke with prox } \\
\text { remo }\end{array}$ & $\begin{array}{l}\text { imal end and plat } \\
\text { val on left lateral }\end{array}$ & $\begin{array}{l}\text { tform missing } \\
\text { margin }\end{array}$ & /ery fine flake \\
\hline
\end{tabular}

\begin{tabular}{|c|c|c|c|c|c|c|c|c|c|c|c|c|c|c|c|c|c|}
\hline Lot No. & $\begin{array}{l}\text { Spec. } \\
\text { No. }\end{array}$ & $\begin{array}{l}\text { Bag UI No } \\
\text { No. }\end{array}$ & Category & Subcategory & $\begin{array}{c}\text { Retouch } \\
\text { Affiliation }\end{array}$ & $\begin{array}{l}\text { Specimen } \\
\text { Condition }\end{array}$ & Breakage & Raw material & Munsell & Color & $\begin{array}{l}\text { Dorsal } \\
\text { Cortex \% }\end{array}$ & $\begin{array}{c}\text { Heat } \\
\text { Evidence }\end{array}$ & $\begin{array}{c}\text { Intentional } \\
\text { Heat } \\
\text { Treatment }\end{array}$ & Patination & Retouch Detail & $\begin{array}{l}\text { Retouch } \\
\text { Distribution }\end{array}$ & $\begin{array}{l}\text { Retouch } \\
\text { Location } \\
\end{array}$ \\
\hline \multirow{3}{*}{320} & \multirow{3}{*}{1} & \multirow{3}{*}{501 NA } & Modified Flake & Utilized Flake & Indet & Complete & None & $\begin{array}{c}\text { Fine Grain } \\
\text { Chert }\end{array}$ & 10YR4/1 & Dark Gray & $26-50$ & None & NA & None & Unifacial-dorsal & Focused & $\begin{array}{c}\text { Left Lateral } \\
\text { Margin }\end{array}$ \\
\hline & & & Weight (g) & Length & Width & Thickness & $\begin{array}{l}\text { Height of } \\
\text { Retouch }\end{array}$ & $\begin{array}{l}\text { Perimeter } \\
\text { Retouch } \\
\text { Length }\end{array}$ & $\begin{array}{l}\text { Average } \\
\text { Edge } \\
\text { Angle }\end{array}$ & $\begin{array}{l}\text { Reduction } \\
\text { Index }\end{array}$ & $\begin{array}{l}\text { Platform } \\
\text { width }\end{array}$ & $\begin{array}{l}\text { Platform } \\
\text { thickness }\end{array}$ & \multicolumn{5}{|c|}{ Comments } \\
\hline & & & 43.57 & 64.5 & 42.48 & 16.49 & 1.55 & 29 & 55 & 10.64 & 12.82 & 7.85 & Nodu & nt roc & Frtical flake with Ir & eguar gral & na mineral \\
\hline
\end{tabular}

\begin{tabular}{|c|c|c|c|c|c|c|c|c|c|c|c|c|c|c|c|c|c|c|}
\hline Lot No. & $\begin{array}{l}\text { Spec. } \\
\text { No. }\end{array}$ & $\begin{array}{l}\text { Bag } \\
\text { No. }\end{array}$ & UI No & Category & Subcategory & $\begin{array}{c}\text { Retouch } \\
\text { Affiliation }\end{array}$ & $\begin{array}{l}\text { Specimen } \\
\text { Condition }\end{array}$ & Breakage & Raw material & Munsell & Color & $\begin{array}{c}\text { Dorsal } \\
\text { Cortex \% }\end{array}$ & $\begin{array}{c}\text { Heat } \\
\text { Evidence }\end{array}$ & $\begin{array}{c}\begin{array}{c}\text { Intentional } \\
\text { Heat } \\
\text { Treatment }\end{array} \\
\end{array}$ & Patination & Retouch Detail & $\begin{array}{l}\text { Retouch } \\
\text { Distribution }\end{array}$ & $\begin{array}{l}\text { Retouch } \\
\text { Location }\end{array}$ \\
\hline \multirow{3}{*}{323} & \multirow{3}{*}{32} & \multirow{3}{*}{524} & \multirow{3}{*}{ NA } & Modified Flake & Edge-modified & Indet & Medial-Distal & Snap & $\begin{array}{c}\text { Fine Grain } \\
\text { Chert }\end{array}$ & 10YR6/2 & $\begin{array}{c}\text { Light Brownish } \\
\text { Gray }\end{array}$ & 0 & None & NA & None & Unifacial-dorsal & Indet & $\begin{array}{l}\text { Left Lateral } \\
\text { Margin }\end{array}$ \\
\hline & & & & Weight (g) & Length & Width & Thickness & $\begin{array}{l}\text { Height of } \\
\text { Retouch }\end{array}$ & $\begin{array}{l}\text { Perimeter } \\
\text { Retouch } \\
\text { Length }\end{array}$ & $\begin{array}{c}\text { Average } \\
\text { Edge } \\
\text { Anqle } \\
\end{array}$ & $\begin{array}{l}\text { Reduction } \\
\text { Index }\end{array}$ & $\begin{array}{l}\text { Platform } \\
\text { width }\end{array}$ & $\begin{array}{c}\text { Platform } \\
\text { thickness }\end{array}$ & \multicolumn{5}{|c|}{ Comments } \\
\hline & & & & 2.75 & Indet & Indet & Indet & Indet & Indet & Indet & Indet & Indet & Indet & \multicolumn{5}{|c|}{$\begin{array}{c}\text { Flake broken in center and the worked edge may have continued; } \\
\text { Modification on left lateral margin with possible use-wear striations on right } \\
\text { lateral margin }\end{array}$} \\
\hline
\end{tabular}

\begin{tabular}{|c|c|c|c|c|c|c|c|c|c|c|c|c|c|c|c|c|c|}
\hline Lot No. & $\begin{array}{l}\text { Spec. } \\
\text { No. }\end{array}$ & $\begin{array}{l}\text { Bag UI No } \\
\text { No. }\end{array}$ & Category & Subcategory & $\begin{array}{c}\text { Retouch } \\
\text { Affiliation }\end{array}$ & $\begin{array}{l}\text { Specimen } \\
\text { Condition }\end{array}$ & Breakage & Raw material & Munsell & Color & $\begin{array}{c}\text { Dorsal } \\
\text { Cortex \% }\end{array}$ & $\begin{array}{c}\text { Heat } \\
\text { Evidence }\end{array}$ & $\begin{array}{c}\text { Intentional } \\
\text { Heat } \\
\text { Treatment }\end{array}$ & Patination & Retouch Detail & $\begin{array}{l}\text { Retouch } \\
\text { Distribution }\end{array}$ & $\begin{array}{l}\text { Retouch } \\
\text { Location }\end{array}$ \\
\hline \multirow{3}{*}{323} & \multirow{3}{*}{30} & \multirow{3}{*}{524} & Modified Flake & Edge-modified & Indet & Medial-Distal & Snap & $\begin{array}{l}\text { Fine Grain } \\
\text { Chert }\end{array}$ & 10YR7/1 & Light Gray & $51-75$ & None & NA & None & Unifacial-dorsal & Focused & $\begin{array}{l}\text { Left Lateral } \\
\text { Margin }\end{array}$ \\
\hline & & & Weight (g) & Length & Width & Thickness & $\begin{array}{l}\text { Height of } \\
\text { Retouch }\end{array}$ & $\begin{array}{l}\text { Perimeter } \\
\text { Retouch } \\
\text { Length }\end{array}$ & $\begin{array}{c}\text { Average } \\
\text { Edge } \\
\text { Angle }\end{array}$ & $\begin{array}{l}\text { Reduction } \\
\text { Index }\end{array}$ & $\begin{array}{l}\text { Platform } \\
\text { width }\end{array}$ & $\begin{array}{l}\text { Platform } \\
\text { thickness }\end{array}$ & \multicolumn{5}{|c|}{ Comments } \\
\hline & & & 5.38 & Indet & Indet & Indet & Indet & Indet & Indet & Indet & Indet & Indet & Platform remo & $\begin{array}{r}\text { ed due to sna } \\
\text { on }\end{array}$ & $\begin{array}{l}\text { nap break through } \\
n \text { dorsal side of bre }\end{array}$ & $\begin{array}{l}\text { assumed cer } \\
\text { reak }\end{array}$ & er of tool; work \\
\hline
\end{tabular}




\begin{tabular}{|c|c|c|c|c|c|c|c|c|c|c|c|c|c|c|c|c|c|c|}
\hline Lot No. & $\begin{array}{l}\text { Spec. } \\
\text { No. }\end{array}$ & $\begin{array}{l}\text { Bag } \\
\text { No. }\end{array}$ & UI No & Category & Subcategory & $\begin{array}{c}\text { Retouch } \\
\text { Affiliation }\end{array}$ & $\begin{array}{l}\text { Specimen } \\
\text { Condition }\end{array}$ & Breakage & Raw material & Munsell & Color & $\begin{array}{c}\text { Dorsal } \\
\text { Cortex \% }\end{array}$ & $\begin{array}{c}\text { Heat } \\
\text { Evidence }\end{array}$ & $\begin{array}{c}\text { Intentional } \\
\text { Heat } \\
\text { Treatment }\end{array}$ & Patination & Retouch Detail & $\begin{array}{c}\text { Retouch } \\
\text { Distribution }\end{array}$ & $\begin{array}{l}\text { Retouch } \\
\text { Location } \\
\end{array}$ \\
\hline \multirow{3}{*}{323} & \multirow{3}{*}{31} & \multirow{3}{*}{524} & \multirow{3}{*}{ NA } & Modified Flake & Edge-modified & Indet & Medial-Distal & Snap & $\begin{array}{c}\text { Coarse Grain } \\
\text { Chert }\end{array}$ & 10YR7/2 & Light Gray & 0 & None & NA & None & Unifacial-dorsal & Focused & $\begin{array}{c}\text { Right Lateral } \\
\text { Margin }\end{array}$ \\
\hline & & & & Weight (g) & Length & Width & Thickness & $\begin{array}{l}\text { Height of } \\
\text { Retouch }\end{array}$ & $\begin{array}{c}\text { Perimeter } \\
\text { Retouch } \\
\text { Length } \\
\end{array}$ & $\begin{array}{l}\text { Average } \\
\text { Edge } \\
\text { Angle } \\
\end{array}$ & $\begin{array}{l}\text { Reduction } \\
\text { Index }\end{array}$ & $\begin{array}{l}\text { Platform } \\
\text { width }\end{array}$ & $\begin{array}{l}\text { Platform } \\
\text { thickness }\end{array}$ & \multicolumn{5}{|c|}{ Comments } \\
\hline & & & & 5.38 & Indet & Indet & Indet & Indet & Indet & Indet & Indet & Indet & Indet & $\begin{array}{l}\text { Incomplete fla } \\
\text { flakes remo }\end{array}$ & $\begin{array}{l}\text { ke missing un } \\
\text { ved from edge }\end{array}$ & $\begin{array}{l}\text { ndetermined amo } \\
\text { e of break along ri }\end{array}$ & $\begin{array}{l}\text { unt; Few (N:7) } \\
\text { right lateral mar } \\
\text { nal }\end{array}$ & $\begin{array}{l}\text { shallow scalar } \\
\text { argin; May not }\end{array}$ \\
\hline
\end{tabular}

\begin{tabular}{|c|c|c|c|c|c|c|c|c|c|c|c|c|c|c|c|c|c|c|}
\hline Lot No. & $\begin{array}{l}\text { Spec. } \\
\text { No. }\end{array}$ & $\begin{array}{l}\text { Bag } \\
\text { No. }\end{array}$ & UI No & Category & Subcategory & $\begin{array}{c}\text { Retouch } \\
\text { Affiliation }\end{array}$ & $\begin{array}{l}\text { Specimen } \\
\text { Condition }\end{array}$ & Breakage & Raw material & Munsell & Color & $\begin{array}{c}\text { Dorsal } \\
\text { Cortex \% } \\
\end{array}$ & $\begin{array}{c}\text { Heat } \\
\text { Evidence }\end{array}$ & $\begin{array}{c}\text { Intentional } \\
\text { Heat } \\
\text { Treatment }\end{array}$ & Patination & Retouch Detail & $\begin{array}{c}\text { Retouch } \\
\text { Distribution }\end{array}$ & $\begin{array}{l}\text { Retouch } \\
\text { Location } \\
\end{array}$ \\
\hline \multirow{3}{*}{336} & \multirow{3}{*}{10} & \multirow{3}{*}{\multicolumn{2}{|c|}{388 NA }} & Modified Flake & Edge-modified & Indet & Complete & None & $\begin{array}{c}\text { Fine Grain } \\
\text { Chert }\end{array}$ & $\begin{array}{c}\text { 10YR7/4 to } \\
\text { 10YR7/6 }\end{array}$ & $\begin{array}{c}\text { Very Pale } \\
\text { Brown to Yellow }\end{array}$ & $0-25$ & None & NA & None & Bifacial & Focused & $\begin{array}{l}\text { Left and Right } \\
\text { Lateral Margin }\end{array}$ \\
\hline & & & & Weight (g) & Length & Width & Thickness & $\begin{array}{l}\text { Height of } \\
\text { Retouch }\end{array}$ & $\begin{array}{l}\text { Perimeter } \\
\text { Retouch } \\
\text { Length } \\
\end{array}$ & $\begin{array}{l}\text { Average } \\
\text { Edge } \\
\text { Angle } \\
\end{array}$ & $\begin{array}{l}\text { Reduction } \\
\text { Index }\end{array}$ & $\begin{array}{c}\text { Platform } \\
\text { width }\end{array}$ & $\begin{array}{l}\text { Platform } \\
\text { thickness }\end{array}$ & \multicolumn{5}{|c|}{ Comments } \\
\hline & & & & 2.07 & 28.95 & 19.66 & 5.02 & 1.27 & 29 & 55 & 3.95 & 6.89 & 4.03 & $\begin{array}{r}\text { Thin flake w } \\
\text { dorsal side ne }\end{array}$ & $\begin{array}{l}\text { th two focuse } \\
\text { ar proximal er }\end{array}$ & $\begin{array}{l}\text { ed locations of retc } \\
\text { nd and right latera } \\
\text { distal end }\end{array}$ & $\begin{array}{l}\text { touch - left late } \\
\text { al margin on ve }\end{array}$ & $\begin{array}{l}\text { eral margin on } \\
\text { entral side near }\end{array}$ \\
\hline
\end{tabular}

\begin{tabular}{|c|c|c|c|c|c|c|c|c|c|c|c|c|c|c|c|c|c|c|}
\hline Lot No. & $\begin{array}{l}\text { Spec. } \\
\text { No. }\end{array}$ & $\begin{array}{l}\text { Bag } \\
\text { No. }\end{array}$ & UI No & Category & Subcategory & $\begin{array}{l}\text { Retouch } \\
\text { Affiliation }\end{array}$ & $\begin{array}{l}\text { Specimen } \\
\text { Condition }\end{array}$ & Breakage & Raw material & Munsell & Color & $\begin{array}{c}\text { Dorsal } \\
\text { Cortex \% }\end{array}$ & $\begin{array}{c}\text { Heat } \\
\text { Evidence }\end{array}$ & $\begin{array}{c}\text { Intentional } \\
\text { Heat } \\
\text { Treatment }\end{array}$ & Patination & Retouch Detail & $\begin{array}{c}\text { Retouch } \\
\text { Distribution }\end{array}$ & $\begin{array}{l}\text { Retouch } \\
\text { Location }\end{array}$ \\
\hline \multirow{3}{*}{337} & \multirow{3}{*}{6} & \multirow{3}{*}{399} & \multirow{3}{*}{ NA } & Modified Flake & Edge-modified & Indet & Complete & None & $\begin{array}{c}\text { Fine Grain } \\
\text { Chert }\end{array}$ & 7.5YR4/2 & Brown & 0 & Reddened & Yes & Yes & Unifacial-dorsal & Continuous & $\begin{array}{c}\text { Left Lateral } \\
\text { margin }\end{array}$ \\
\hline & & & & Weight (g) & Length & Width & Thickness & $\begin{array}{l}\text { Height of } \\
\text { Retouch }\end{array}$ & $\begin{array}{l}\text { Perimeter } \\
\text { Retouch } \\
\text { Length }\end{array}$ & $\begin{array}{c}\text { Average } \\
\text { Edge } \\
\text { Angle }\end{array}$ & $\begin{array}{l}\text { Reduction } \\
\text { Index }\end{array}$ & $\begin{array}{l}\text { Platform } \\
\text { width }\end{array}$ & $\begin{array}{l}\text { Platform } \\
\text { thickness }\end{array}$ & \multicolumn{4}{|c|}{ Comments } & \\
\hline & & & & 33.73 & 54.88 & 37.13 & 18.92 & 2.46 & 45 & 70 & 7.69 & 22.63 & 11.38 & $\begin{array}{r}\text { Mottled with } 5 \\
\text { blocky ea }\end{array}$ & $\begin{array}{l}\text { YR4/2 Dark R } \\
\text { arly stage flake }\end{array}$ & $\begin{array}{l}\text { Reddish Gray band } \\
\text { e; prominent patin }\end{array}$ & $\begin{array}{l}\text { ds from heat tr } \\
\text { na across vent }\end{array}$ & $\begin{array}{l}\text { eatment; Large } \\
\text { al surface }\end{array}$ \\
\hline
\end{tabular}

\begin{tabular}{|c|c|c|c|c|c|c|c|c|c|c|c|c|c|c|c|c|c|}
\hline Lot No. & $\begin{array}{l}\text { Spec. } \\
\text { No. }\end{array}$ & $\begin{array}{l}\text { Bag UI No } \\
\text { No. }\end{array}$ & Category & Subcategory & $\begin{array}{c}\text { Retouch } \\
\text { Affiliation }\end{array}$ & $\begin{array}{l}\text { Specimen } \\
\text { Condition }\end{array}$ & Breakage & Raw material & Munsell & Color & $\begin{array}{c}\text { Dorsal } \\
\text { Cortex \% }\end{array}$ & $\begin{array}{c}\text { Heat } \\
\text { Evidence }\end{array}$ & $\begin{array}{c}\text { Intentional } \\
\text { Heat } \\
\text { Treatment }\end{array}$ & Patination & Retouch Detail & $\begin{array}{c}\text { Retouch } \\
\text { Distribution }\end{array}$ & $\begin{array}{l}\text { Retouch } \\
\text { Location }\end{array}$ \\
\hline \multirow{3}{*}{337} & \multirow{3}{*}{7} & \multirow{3}{*}{399 NA } & Modified Flake & Edge-modified & Cultural & Indet & Snap & $\begin{array}{l}\text { Fine Grain } \\
\text { Chert }\end{array}$ & $\begin{array}{c}\text { 10YR5/1 to } \\
\text { 10YR5/2 }\end{array}$ & $\begin{array}{l}\text { Gray to Grayish } \\
\text { Brown }\end{array}$ & 0 & None & 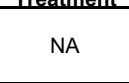 & None & Bifacial & Clustered & $\begin{array}{l}\text { Left and Right } \\
\text { Lateral } \\
\text { Margins }\end{array}$ \\
\hline & & & Weight (g) & Length & Width & Thickness & $\begin{array}{l}\text { Height of } \\
\text { Retouch }\end{array}$ & $\begin{array}{l}\text { Perimeter } \\
\text { Retouch } \\
\text { Length }\end{array}$ & $\begin{array}{l}\text { Average } \\
\text { Edge } \\
\text { Angle } \\
\end{array}$ & $\begin{array}{l}\text { Reduction } \\
\text { Index }\end{array}$ & $\begin{array}{l}\text { Platform } \\
\text { width }\end{array}$ & $\begin{array}{l}\text { Platform } \\
\text { thickness }\end{array}$ & \multicolumn{5}{|c|}{ Comments } \\
\hline & & & 2.01 & Indet & 22.28 & 2.33 & 1.02 & 66 & 45 & 2.29 & Indet & Indet & $\begin{array}{l}\text { Thin flake w } \\
\text { determine o } \\
\mathrm{fla}\end{array}$ & $\begin{array}{l}\text { ith breaks on } \\
\text { ientation; Ret } \\
\text { kes removed }\end{array}$ & $\begin{array}{l}\text { both proximal an } \\
\text { touched on both } \\
\text { from one margin }\end{array}$ & $\begin{array}{l}\text { dd distal margir } \\
\text { lateral margins } \\
\text { on opposite fa }\end{array}$ & $\begin{array}{l}\text { ins - unable to } \\
\text { s with thinning } \\
\text { face }\end{array}$ \\
\hline
\end{tabular}




\begin{tabular}{|c|c|c|c|c|c|c|c|c|c|c|c|c|c|c|c|c|c|}
\hline Lot No. & $\begin{array}{l}\text { Spec. } \\
\text { No. }\end{array}$ & $\begin{array}{l}\text { Bag UI No } \\
\text { No. }\end{array}$ & Category & Subcategory & $\begin{array}{c}\text { Retouch } \\
\text { Affiliation }\end{array}$ & $\begin{array}{l}\text { Specimen } \\
\text { Condition }\end{array}$ & Breakage & Raw material & Munsell & Color & $\begin{array}{c}\text { Dorsal } \\
\text { Cortex \% }\end{array}$ & $\begin{array}{c}\text { Heat } \\
\text { Evidence }\end{array}$ & $\begin{array}{c}\text { Intentional } \\
\text { Heat } \\
\text { Treatment }\end{array}$ & Patination & Retouch Detail & $\begin{array}{l}\text { Retouch } \\
\text { Distribution }\end{array}$ & $\begin{array}{l}\text { Retouch } \\
\text { Location }\end{array}$ \\
\hline \multirow{3}{*}{337} & \multirow{3}{*}{8} & \multirow{3}{*}{399 NA } & Modified Flake & Utilized Flake & Indet & Complete & None & $\begin{array}{c}\text { Coarse Grain } \\
\text { Chert }\end{array}$ & $\begin{array}{c}\text { 10YR5/2 to } \\
\text { 10YR6/2 }\end{array}$ & $\begin{array}{c}\text { Grayish Brown } \\
\text { to Light Grayish } \\
\text { Brown } \\
\end{array}$ & $0-25$ & None & NA & None & Unifacial-dorsal & Focused & $\begin{array}{c}\begin{array}{c}\text { Proximal and } \\
\text { Right Lateral } \\
\text { Margin }\end{array} \\
\end{array}$ \\
\hline & & & Weight (g) & Length & Width & Thickness & $\begin{array}{l}\text { Height of } \\
\text { Retouch }\end{array}$ & $\begin{array}{c}\text { Perimeter } \\
\text { Retouch } \\
\text { Length }\end{array}$ & $\begin{array}{l}\text { Average } \\
\text { Edge } \\
\text { Angle } \\
\end{array}$ & $\begin{array}{l}\text { Reduction } \\
\text { Index }\end{array}$ & $\begin{array}{l}\text { Platform } \\
\text { width }\end{array}$ & $\begin{array}{l}\text { Platform } \\
\text { thickness }\end{array}$ & \multicolumn{5}{|c|}{ Comments } \\
\hline & & & 60.49 & 72.9 & 39.74 & 25.63 & 2.05 & 30 & 80 & 12.5 & 5.78 & 3.97 & Large chunky & $\begin{array}{r}\text { flake with ste } \\
\text { of reto }\end{array}$ & $\begin{array}{l}\text { eeply ground utilize } \\
\text { ouch flaking on an }\end{array}$ & $\begin{array}{l}\text { ed edge; no dis } \\
\text { yy edges }\end{array}$ & listinct evidence \\
\hline Lot No. & $\begin{array}{l}\text { Spec. } \\
\text { No. }\end{array}$ & $\begin{array}{l}\text { Bag UI No } \\
\text { No. }\end{array}$ & Category & Subcategory & $\begin{array}{c}\text { Retouch } \\
\text { Affiliation }\end{array}$ & $\begin{array}{l}\text { Specimen } \\
\text { Condition }\end{array}$ & Breakage & Raw material & Munsell & Color & $\begin{array}{c}\text { Dorsal } \\
\text { Cortex \% }\end{array}$ & $\begin{array}{c}\text { Heat } \\
\text { Evidence }\end{array}$ & $\begin{array}{c}\text { Intentional } \\
\text { Heat } \\
\text { Treatment }\end{array}$ & Patination & Retouch Detail & $\begin{array}{c}\text { Retouch } \\
\text { Distribution }\end{array}$ & $\begin{array}{l}\text { Retouch } \\
\text { Location }\end{array}$ \\
\hline \multirow{3}{*}{339} & \multirow{3}{*}{10} & \multirow{3}{*}{410 NA } & Modified Flake & Edge-modified & Cultural & Indet & Snap & $\begin{array}{c}\text { Fine Grain } \\
\text { Chert }\end{array}$ & $\begin{array}{c}\text { 10YR5/1 to } \\
\text { 10YR5/2 }\end{array}$ & $\begin{array}{l}\text { Gray to Grayish } \\
\text { Brown }\end{array}$ & 0 & None & NA & None & Bifacial & Indet & Indet \\
\hline & & & Weight (g) & Length & Width & Thickness & $\begin{array}{l}\text { Height of } \\
\text { Retouch }\end{array}$ & $\begin{array}{l}\text { Perimeter } \\
\text { Retouch } \\
\text { Lenath }\end{array}$ & $\begin{array}{l}\text { Average } \\
\text { Edge } \\
\text { Angle } \\
\end{array}$ & $\begin{array}{c}\text { Reduction } \\
\text { Index }\end{array}$ & $\begin{array}{c}\text { Platform } \\
\text { width }\end{array}$ & $\begin{array}{l}\text { Platform } \\
\text { thickness }\end{array}$ & \multicolumn{5}{|c|}{ Comments } \\
\hline & & & 10.26 & Indet & Indet & Indet & Indet & Indet & Indet & Indet & Indet & Indet & $\begin{array}{r}\text { Incomplete flak } \\
\text { is slightly c }\end{array}$ & $\begin{array}{l}\text { ke with finely } \\
\text { urved and flak }\end{array}$ & $\begin{array}{l}\text { retouched point ( } \mathrm{r} \text { ke remol is evid }\end{array}$ & $\begin{array}{l}\text { nearly } 90 \text { degr } \\
\text { dent on both si }\end{array}$ & $\begin{array}{l}\text { ree angle); point } \\
\text { sides of point }\end{array}$ \\
\hline Lot No. & $\begin{array}{l}\text { Spec. } \\
\text { No. }\end{array}$ & $\begin{array}{l}\text { Bag UI No } \\
\text { No. }\end{array}$ & Category & Subcategory & $\begin{array}{c}\text { Retouch } \\
\text { Affiliation }\end{array}$ & $\begin{array}{l}\text { Specimen } \\
\text { Condition }\end{array}$ & Breakage & Raw material & Munsell & Color & $\begin{array}{l}\text { Dorsal } \\
\text { Cortex \% }\end{array}$ & $\begin{array}{c}\text { Heat } \\
\text { Evidence }\end{array}$ & $\begin{array}{c}\text { Intentional } \\
\text { Heat } \\
\text { Treatment }\end{array}$ & Patination & Retouch Detail & $\begin{array}{c}\text { Retouch } \\
\text { Distribution }\end{array}$ & $\begin{array}{l}\text { Retouch } \\
\text { Location }\end{array}$ \\
\hline \multirow{3}{*}{342} & \multirow{3}{*}{5} & \multirow{3}{*}{422 NA } & Modified Flake & Utilized Flake & Indet & Complete & None & $\begin{array}{c}\text { Fine Grain } \\
\text { Chert }\end{array}$ & $\begin{array}{c}\text { 10YR5/1 to } \\
\text { 10YR6/1 }\end{array}$ & $\begin{array}{l}\text { Gray to Light } \\
\text { Gray }\end{array}$ & $0-25$ & None & NA & None & Unifacial-dorsal & Continuous & $\begin{array}{c}\text { Left Lateral } \\
\text { Margin and } \\
\text { Distal Margin }\end{array}$ \\
\hline & & & Weight (g) & Length & Width & Thickness & $\begin{array}{l}\text { Height of } \\
\text { Retouch }\end{array}$ & $\begin{array}{l}\text { Perimeter } \\
\text { Retouch } \\
\text { Lenath }\end{array}$ & $\begin{array}{l}\text { Average } \\
\text { Edge } \\
\text { Angle }\end{array}$ & $\begin{array}{l}\text { Reduction } \\
\text { Index }\end{array}$ & $\begin{array}{l}\text { Platform } \\
\text { width }\end{array}$ & $\begin{array}{l}\text { Platform } \\
\text { thickness }\end{array}$ & \multicolumn{5}{|c|}{ Comments } \\
\hline & & & 86.18 & 61.63 & 53.97 & 20.39 & 0.64 & 86 & 80 & 31.86 & 50.75 & 14.14 & $\begin{array}{r}\text { Large flake } \\
\mathrm{pl}\end{array}$ & $\begin{array}{l}\text { with ground I } \\
\text { laced scalar fl }\end{array}$ & $\begin{array}{l}\text { left lateral margin } \\
\text { flakes; Possibly ne }\end{array}$ & $\begin{array}{l}\text { ground with fe } \\
\text { atural processe }\end{array}$ & $\begin{array}{l}\text { ew irregularly } \\
\text { ses }\end{array}$ \\
\hline Lot No. & $\begin{array}{l}\text { Spec. } \\
\text { No. }\end{array}$ & $\begin{array}{l}\text { Bag UI No } \\
\text { No. }\end{array}$ & Category & Subcategory & $\begin{array}{c}\text { Retouch } \\
\text { Affiliation }\end{array}$ & $\begin{array}{l}\text { Specimen } \\
\text { Condition }\end{array}$ & Breakage & Raw material & Munsell & Color & $\begin{array}{c}\text { Dorsal } \\
\text { Cortex \% }\end{array}$ & $\begin{array}{c}\text { Heat } \\
\text { Evidence }\end{array}$ & $\begin{array}{c}\text { Intentional } \\
\text { Heat } \\
\text { Treatment }\end{array}$ & Patination & Retouch Detail & $\begin{array}{c}\text { Retouch } \\
\text { Distribution }\end{array}$ & $\begin{array}{l}\text { Retouch } \\
\text { Location }\end{array}$ \\
\hline \multirow{3}{*}{351} & \multirow{3}{*}{2} & \multirow{3}{*}{370 NA } & Modified Flake & Edge-modified & Indet & Complete & Snap & $\begin{array}{c}\text { Fine Grain } \\
\text { Chert }\end{array}$ & $\begin{array}{c}\text { 10YR5/1 to } \\
\text { 10YR5/2 }\end{array}$ & $\begin{array}{l}\text { Gray to Grayish } \\
\text { Brown }\end{array}$ & 0 & None & NA & None & Unifacial-dorsal & Focused & Distal Margin \\
\hline & & & Weight (g) & Length & Width & Thickness & $\begin{array}{l}\text { Height of } \\
\text { Retouch }\end{array}$ & $\begin{array}{l}\text { Perimeter } \\
\text { Retouch } \\
\text { Lenath }\end{array}$ & $\begin{array}{l}\text { Average } \\
\text { Edge } \\
\text { Angle } \\
\end{array}$ & $\begin{array}{l}\text { Reduction } \\
\text { Index }\end{array}$ & $\begin{array}{l}\text { Platform } \\
\text { width }\end{array}$ & $\begin{array}{l}\text { Platform } \\
\text { thickness }\end{array}$ & \multicolumn{5}{|c|}{ Comments } \\
\hline & & & 5.24 & 28.91 & 30.18 & 6.96 & 2.43 & 15 & 70 & 2.86 & Indet & Indet & \multicolumn{5}{|c|}{$\begin{array}{l}\text { Possible crude flaking on distal end; Undetermined if cultural; Platform has } \\
\text { largely been removed and exhibits step and hinge terminations }\end{array}$} \\
\hline Lot No. & $\begin{array}{l}\text { Spec. } \\
\text { No. }\end{array}$ & $\begin{array}{l}\text { Bag UI No } \\
\text { No. }\end{array}$ & Category & Subcategory & $\begin{array}{c}\text { Retouch } \\
\text { Affiliation }\end{array}$ & $\begin{array}{l}\text { Specimen } \\
\text { Condition }\end{array}$ & Breakage & Raw material & Munsell & Color & $\begin{array}{c}\text { Dorsal } \\
\text { Cortex \% }\end{array}$ & $\begin{array}{c}\text { Heat } \\
\text { Evidence }\end{array}$ & $\begin{array}{c}\text { Intentional } \\
\text { Heat } \\
\text { Treatment }\end{array}$ & Patination & Retouch Detail & $\begin{array}{l}\text { Retouch } \\
\text { Distribution }\end{array}$ & $\begin{array}{l}\text { Retouch } \\
\text { Location }\end{array}$ \\
\hline \multirow{3}{*}{354} & \multirow{3}{*}{7} & \multirow{3}{*}{326 NA } & Modified Flake & Edge-modified & Indet & Complete & None & $\begin{array}{c}\text { Fine Grain } \\
\text { Chert }\end{array}$ & 10YR6/2 & $\begin{array}{c}\text { Light Brownish } \\
\text { Gray }\end{array}$ & 0 & None & NA & None & Unifacial-ventral & Focused & Distal Margin \\
\hline & & & Weight (g) & Length & Width & Thickness & $\begin{array}{l}\text { Height of } \\
\text { Retouch }\end{array}$ & $\begin{array}{l}\text { Perimeter } \\
\text { Retouch } \\
\text { Lenath }\end{array}$ & $\begin{array}{c}\text { Average } \\
\text { Edge } \\
\text { Anqle } \\
\end{array}$ & $\begin{array}{c}\text { Reduction } \\
\text { Index }\end{array}$ & $\begin{array}{l}\text { Platform } \\
\text { width }\end{array}$ & $\begin{array}{c}\text { Platform } \\
\text { thickness }\end{array}$ & \multicolumn{5}{|c|}{ Comments } \\
\hline & & & 10.97 & 39.74 & 48.89 & 5.47 & 1.3 & 26 & 30 & 4.21 & 15.3 & 5.42 & Inclusion & $n$ of $10 Y R 5 / 1$ & gray; Thin flake; $F$ & Retouch may $b$ & be natural \\
\hline
\end{tabular}




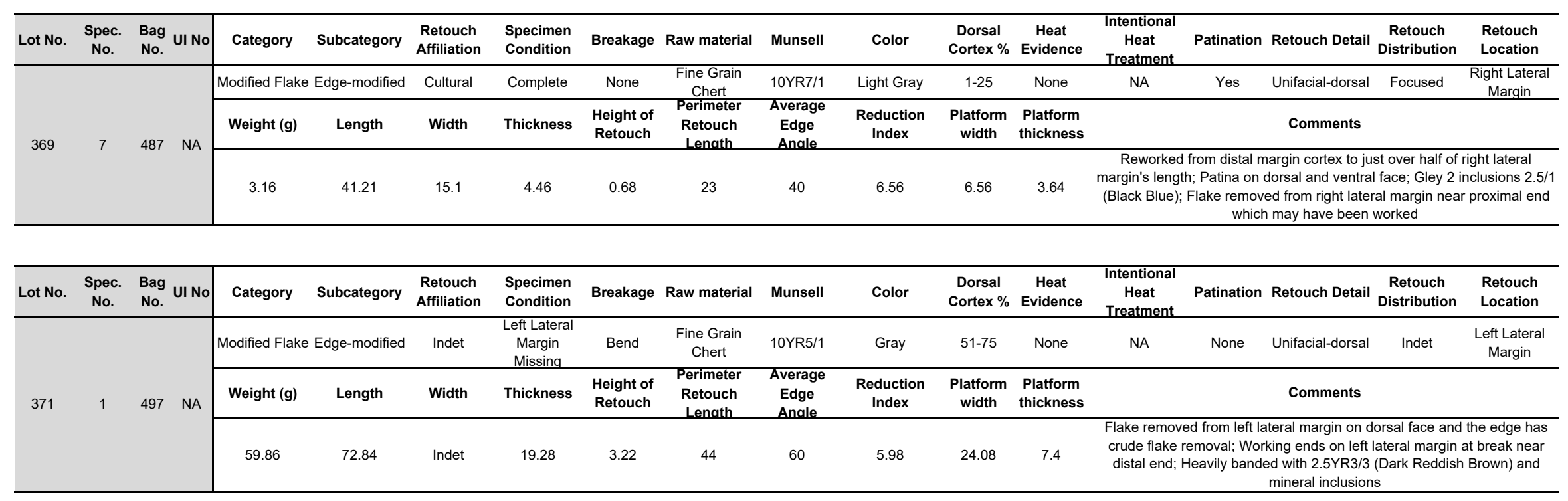

\begin{tabular}{|c|c|c|c|c|c|c|c|c|c|c|c|c|c|c|c|c|c|}
\hline Lot No. & $\begin{array}{l}\text { Spec. } \\
\text { No. }\end{array}$ & $\begin{array}{l}\text { Bag UI No } \\
\text { No. }\end{array}$ & Category & Subcategory & $\begin{array}{l}\text { Retouch } \\
\text { Affiliation }\end{array}$ & $\begin{array}{l}\text { Specimen } \\
\text { Condition }\end{array}$ & Breakage & Raw material & Munsell & Color & $\begin{array}{l}\text { Dorsal } \\
\text { Cortex \% }\end{array}$ & $\begin{array}{c}\text { Heat } \\
\text { Evidence }\end{array}$ & $\begin{array}{c}\text { Intentional } \\
\text { Heat } \\
\text { Treatment }\end{array}$ & Patination & Retouch Detail & $\begin{array}{l}\text { Retouch } \\
\text { Distribution }\end{array}$ & $\begin{array}{l}\text { Retouch } \\
\text { Location }\end{array}$ \\
\hline \multirow{3}{*}{380} & \multirow{3}{*}{5} & \multirow{3}{*}{627 NA } & Modified Flake & Edge-modified & Indet & Complete & None & $\begin{array}{l}\text { Fine Grain } \\
\text { Chert }\end{array}$ & $\begin{array}{l}\text { 10YR4/2 to } \\
\text { 10YR5/2 }\end{array}$ & $\begin{array}{l}\text { Dark Grayish } \\
\text { Brown to } \\
\text { Grayish Brown }\end{array}$ & $0-25$ & None & Ireament & Yes & Unifacial-dorsal & Continuous & $\begin{array}{l}\text { Left and Right } \\
\text { Lateral Margin } \\
\text { and Distal } \\
\text { Marain }\end{array}$ \\
\hline & & & Weight (g) & Length & Width & Thickness & $\begin{array}{l}\text { Height of } \\
\text { Retouch }\end{array}$ & $\begin{array}{c}\text { Perimeter } \\
\text { Retouch } \\
\text { Lenath }\end{array}$ & $\begin{array}{l}\text { Average } \\
\text { Edge } \\
\text { Angle } \\
\end{array}$ & $\begin{array}{l}\text { Reduction } \\
\text { Index }\end{array}$ & $\begin{array}{l}\text { Platform } \\
\text { width }\end{array}$ & $\begin{array}{l}\text { Platform } \\
\text { thickness }\end{array}$ & \multicolumn{5}{|c|}{ Comments } \\
\hline & & & 4.55 & 25.58 & 27.38 & 6.37 & 0.68 & 78 & 40 & 9.37 & 8.91 & 3.13 & \multicolumn{5}{|c|}{$\begin{array}{l}\text { Thin flake with prominent patina on ventral surface; light retouch along all } \\
\text { edges except for platform }\end{array}$} \\
\hline
\end{tabular}

\begin{tabular}{|c|c|c|c|c|c|c|c|c|c|c|c|c|c|c|c|c|c|}
\hline Lot No. & $\begin{array}{l}\text { Spec. } \\
\text { No. }\end{array}$ & $\begin{array}{l}\text { Bag UI No } \\
\text { No. }\end{array}$ & Category & Subcategory & $\begin{array}{c}\text { Retouch } \\
\text { Affiliation }\end{array}$ & $\begin{array}{l}\text { Specimen } \\
\text { Condition }\end{array}$ & Breakage & Raw material & Munsell & Color & $\begin{array}{c}\text { Dorsal } \\
\text { Cortex \% }\end{array}$ & $\begin{array}{c}\text { Heat } \\
\text { Evidence }\end{array}$ & $\begin{array}{c}\text { Intentional } \\
\text { Heat } \\
\text { Treatment }\end{array}$ & Patination & Retouch Detail & $\begin{array}{c}\text { Retouch } \\
\text { Distribution }\end{array}$ & $\begin{array}{l}\text { Retouch } \\
\text { Location }\end{array}$ \\
\hline \multirow{3}{*}{386} & \multirow{3}{*}{7} & \multirow{3}{*}{445 NA } & Modified Flake & Edge-modified & Indet & Complete & None & $\begin{array}{l}\text { Fine Grain } \\
\text { Chert }\end{array}$ & $5 \mathrm{~PB} 5 / 1$ & Bluish Gray & 0 & None & NA & None & Unifacial-dorsal & Focused & Distal Margin \\
\hline & & & Weight (g) & Length & Width & Thickness & $\begin{array}{l}\text { Height of } \\
\text { Retouch }\end{array}$ & $\begin{array}{l}\text { Perimeter } \\
\text { Retouch } \\
\text { Lenqth }\end{array}$ & $\begin{array}{c}\text { Average } \\
\text { Edge } \\
\text { Angle } \\
\end{array}$ & $\begin{array}{l}\text { Reduction } \\
\text { Index }\end{array}$ & $\begin{array}{l}\text { Platform } \\
\text { width }\end{array}$ & $\begin{array}{l}\text { Platform } \\
\text { thickness }\end{array}$ & \multicolumn{5}{|c|}{ Comments } \\
\hline & & & 33.3 & 45.55 & 41.8 & 17.64 & 3.66 & 40 & 65 & 4.82 & 15.29 & 6.98 & $\begin{array}{l}\text { Larger flak } \\
\text { creating a rour }\end{array}$ & $\begin{array}{l}\text { es have been } \\
\text { ded "scraper- }\end{array}$ & $\begin{array}{l}n \text { taken from the } \\
\text { r-like" shape - reto }\end{array}$ & $\begin{array}{l}\text { dorsal side alor } \\
\text { buching is focu }\end{array}$ & $\begin{array}{l}\text { Tumbarmatymin, } \\
\text { ng all edges } \\
\text { used along distal }\end{array}$ \\
\hline
\end{tabular}




\begin{tabular}{|c|c|c|c|c|c|c|c|c|c|c|c|c|c|c|c|c|c|}
\hline Lot No. & $\begin{array}{l}\text { Spec. } \\
\text { No. }\end{array}$ & $\begin{array}{l}\text { Bag } \\
\text { No. }\end{array}$ & UI No & Category & Subcategory & $\begin{array}{l}\text { Retouch } \\
\text { Affiliation }\end{array}$ & $\begin{array}{l}\text { Specimen } \\
\text { Condition }\end{array}$ & Breakage & Raw material & Munsell & Color & $\begin{array}{l}\text { Dorsal } \\
\text { Cortex \% }\end{array}$ & $\begin{array}{c}\text { Heat } \\
\text { Evidence }\end{array}$ & $\begin{array}{l}\text { Intentional } \\
\text { Heat } \\
\text { Treatment }\end{array}$ & Patination & Retouch Detail $\begin{array}{c}\text { Retouch } \\
\text { Distribution }\end{array}$ & $\begin{array}{l}\text { Retouch } \\
\text { Location }\end{array}$ \\
\hline \multirow{3}{*}{388} & \multirow{3}{*}{6} & \multirow{3}{*}{362} & \multirow{3}{*}{ NA } & Modified Flake & Edge-modified & Indet & Distal-Medial & Snap & $\begin{array}{l}\text { Fine Grain } \\
\text { Chert }\end{array}$ & $\begin{array}{l}10 \mathrm{YR} 6 / 1 \\
\text { and } \\
\text { 10YR6/2 }\end{array}$ & $\begin{array}{l}\text { Gray to Light } \\
\text { Brownish Gray }\end{array}$ & $0-25$ & None & NA & None & Unifacial-dorsal & $\begin{array}{l}\text { Right Lateral } \\
\text { Margin }\end{array}$ \\
\hline & & & & Weight (g) & Length & Width & Thickness & $\begin{array}{l}\text { Height of } \\
\text { Retouch }\end{array}$ & $\begin{array}{l}\text { Perimeter } \\
\text { Retouch } \\
\text { Lenath }\end{array}$ & $\begin{array}{l}\text { Average } \\
\text { Edge } \\
\text { Angle }\end{array}$ & $\begin{array}{l}\text { Reduction } \\
\text { Index }\end{array}$ & $\begin{array}{l}\text { Platform } \\
\text { width }\end{array}$ & $\begin{array}{l}\text { Platform } \\
\text { thickness }\end{array}$ & \multicolumn{4}{|c|}{ Comments } \\
\hline & & & & 42.64 & Indet & Indet & Indet & Indet & Indet & Indet & Indet & Indet & Indet & $\begin{array}{r}\text { Large chunky } \\
\text { hammer per } \\
\text { lateral margin }\end{array}$ & $\begin{array}{l}\text { flake with evid } \\
\text { rcussion on le } \\
\text { is irregular ar }\end{array}$ & $\begin{array}{l}\text { dence of crushed edge damage } \\
\text { eft lateral margin; Retouch portio } \\
\text { and includes crushed edges with }\end{array}$ & $\begin{array}{l}\text { likely from hard } \\
\text { on of the right } \\
\text { in step fractures }\end{array}$ \\
\hline Lot No. & $\begin{array}{l}\text { Spec. } \\
\text { No. }\end{array}$ & $\begin{array}{l}\text { Bag } \\
\text { No. }\end{array}$ & UI No & Category & Subcategory & $\begin{array}{l}\text { Retouch } \\
\text { Affiliation }\end{array}$ & $\begin{array}{l}\text { Specimen } \\
\text { Condition }\end{array}$ & Breakage & Raw material & Munsell & Color & $\begin{array}{c}\text { Dorsal } \\
\text { Cortex \% }\end{array}$ & $\begin{array}{c}\text { Heat } \\
\text { Evidence }\end{array}$ & $\begin{array}{l}\text { Intentional } \\
\text { Heat } \\
\text { Treatment }\end{array}$ & Patination & Retouch Detail $\begin{array}{c}\text { Retouch } \\
\text { Distribution }\end{array}$ & $\begin{array}{l}\text { Retouch } \\
\text { Location } \\
\end{array}$ \\
\hline \multirow{3}{*}{389} & \multirow{3}{*}{7} & \multirow{3}{*}{451} & \multirow{3}{*}{ NA } & Modified Flake & Edge-modified & Indet & Complete & None & $\begin{array}{c}\text { Fine Grain } \\
\text { Chert } \\
\end{array}$ & $\begin{array}{l}\text { 10YR5/1 to } \\
\text { 10YR5/3 }\end{array}$ & $\begin{array}{l}\text { Gray to Grayish } \\
\text { Brown }\end{array}$ & 0 & None & NA & None & Focused & $\begin{array}{l}\text { Left and Right } \\
\text { Lateral Margin }\end{array}$ \\
\hline & & & & Weight (g) & Length & Width & Thickness & $\begin{array}{l}\text { Height of } \\
\text { Retouch }\end{array}$ & $\begin{array}{l}\text { Perimeter } \\
\text { Retouch } \\
\text { Length } \\
\end{array}$ & $\begin{array}{l}\text { Average } \\
\text { Edge } \\
\text { Angle } \\
\end{array}$ & $\begin{array}{l}\text { Reduction } \\
\text { Index }\end{array}$ & $\begin{array}{l}\text { Platform } \\
\text { width }\end{array}$ & $\begin{array}{l}\text { Platform } \\
\text { thickness }\end{array}$ & \multicolumn{4}{|c|}{ Comments } \\
\hline & & & & 1.54 & 33.96 & 17.69 & 3.04 & 1.58 & 39 & 55 & 0.52 & 3.86 & 1.4 & Thin flake $n$ & $\begin{array}{l}\text { with hinge brea } \\
\text { retouc }\end{array}$ & $\begin{array}{l}\text { eak on ventral face; Two focused } \\
\text { ch; Both fine scalar flakes }\end{array}$ & locations of \\
\hline Lot No. & $\begin{array}{l}\text { Spec. } \\
\text { No. }\end{array}$ & $\begin{array}{l}\text { Bag } \\
\text { No. }\end{array}$ & UI No & Category & Subcategory & $\begin{array}{c}\text { Retouch } \\
\text { Affiliation }\end{array}$ & $\begin{array}{l}\text { Specimen } \\
\text { Condition }\end{array}$ & Breakage & Raw material & Munsell & Color & $\begin{array}{l}\text { Dorsal } \\
\text { Cortex \% }\end{array}$ & $\begin{array}{c}\text { Heat } \\
\text { Evidence }\end{array}$ & $\begin{array}{l}\text { Intentional } \\
\text { Heat } \\
\text { Treatment }\end{array}$ & Patination & Retouch Detail $\begin{array}{c}\text { Retouch } \\
\text { Distribution }\end{array}$ & $\begin{array}{l}\text { Retouch } \\
\text { Location } \\
\end{array}$ \\
\hline \multirow{3}{*}{401} & \multirow{3}{*}{6} & \multirow{3}{*}{488} & \multirow{3}{*}{ NA } & Modified Flake & Edge-modified & Indet & Complete & None & $\begin{array}{l}\text { Fine Grain } \\
\text { Chert } \\
\end{array}$ & 10YR3/1 & Very Dark Gray & 0 & None & NA & None & Continuous & $\begin{array}{l}\text { Left and Right } \\
\text { Lateral } \\
\text { Margins } \\
\end{array}$ \\
\hline & & & & Weight (g) & Length & Width & Thickness & $\begin{array}{l}\text { Height of } \\
\text { Retouch }\end{array}$ & $\begin{array}{l}\text { Perimeter } \\
\text { Retouch } \\
\text { Lenath }\end{array}$ & $\begin{array}{l}\text { Average } \\
\text { Edge } \\
\text { Angle }\end{array}$ & $\begin{array}{l}\text { Reduction } \\
\text { Index }\end{array}$ & $\begin{array}{l}\text { Platform } \\
\text { width }\end{array}$ & $\begin{array}{c}\text { Platform } \\
\text { thickness }\end{array}$ & \multicolumn{4}{|c|}{ Comments } \\
\hline & & & & 4.7 & 27.7 & 20.96 & 8.67 & 2.6 & 75 & 70 & 0.30 & Indet & Indet & \multicolumn{4}{|c|}{$\begin{array}{l}\text { Small chunky flake with retouch predominately on the dorsal side; steep } \\
\text { angle of breakage with evidence of crushing along edges; retouch } \\
\text { continues around entire flake edge }\end{array}$} \\
\hline Lot No. & $\begin{array}{l}\text { Spec. } \\
\text { No. }\end{array}$ & $\begin{array}{l}\text { Bag } \\
\text { No. }\end{array}$ & UI No & Category & Subcategory & $\begin{array}{c}\text { Retouch } \\
\text { Affiliation }\end{array}$ & $\begin{array}{l}\text { Specimen } \\
\text { Condition }\end{array}$ & Breakage & Raw material & Munsell & Color & $\begin{array}{l}\text { Dorsal } \\
\text { Cortex \% }\end{array}$ & $\begin{array}{c}\text { Heat } \\
\text { Evidence }\end{array}$ & $\begin{array}{l}\text { Intentional } \\
\text { Heat } \\
\text { Treatment }\end{array}$ & Patination & Retouch Detail $\begin{array}{c}\text { Retouch } \\
\text { Distribution }\end{array}$ & $\begin{array}{l}\text { Retouch } \\
\text { Location }\end{array}$ \\
\hline \multirow{3}{*}{406} & \multirow{3}{*}{7} & \multirow{3}{*}{\multicolumn{2}{|c|}{503 NA }} & Modified Flake & Utilized Flake & Indet & Complete & None & $\begin{array}{c}\text { Coarse Grain } \\
\text { Chert }\end{array}$ & 10YR5/1 & Gray & $1-25$ & None & NA & None & Unifacial-ventral Continuous & $\begin{array}{c}\text { Right Lateral } \\
\text { Margin/Distal } \\
\text { Margin } \\
\end{array}$ \\
\hline & & & & Weight (g) & Length & Width & Thickness & $\begin{array}{l}\text { Height of } \\
\text { Retouch }\end{array}$ & $\begin{array}{l}\text { Perimeter } \\
\text { Retouch } \\
\text { Length } \\
\end{array}$ & $\begin{array}{l}\text { Average } \\
\text { Edge } \\
\text { Angle } \\
\end{array}$ & $\begin{array}{l}\text { Reduction } \\
\text { Index }\end{array}$ & $\begin{array}{l}\text { Platform } \\
\text { width }\end{array}$ & $\begin{array}{l}\text { Platform } \\
\text { thickness }\end{array}$ & \multirow{2}{*}{\multicolumn{4}{|c|}{$\begin{array}{l}\text { Prominent coarse mineral inclusions (10YR8/1 - White); Large flake with } \\
\text { light flake removal on dorsal face; Utilization and use-wear noted } \\
\text { completely on ventral face along right margin and distal margin; Light } \\
\text { flaking noticed on medial-proximal area of right lateral margin but unable to } \\
\text { determine if use-wear or actual modification }\end{array}$}} \\
\hline & & & & 47.3 & 62.74 & 46.68 & 14.76 & 1.33 & 89 & 55 & 0.09 & 11.29 & 2.36 & & & & \\
\hline
\end{tabular}




\begin{tabular}{|c|c|c|c|c|c|c|c|c|c|c|c|c|c|c|c|c|}
\hline Lot No. & $\begin{array}{l}\text { Spec. } \\
\text { No. }\end{array}$ & $\begin{array}{l}\text { Bag UI No } \\
\text { No. }\end{array}$ & Category & Subcategory & $\begin{array}{l}\text { Retouch } \\
\text { Affiliation }\end{array}$ & $\begin{array}{l}\text { Specimen } \\
\text { Condition } \\
\end{array}$ & Breakage & Raw material & Munsell & Color & $\begin{array}{l}\text { Dorsal } \\
\text { Cortex \% }\end{array}$ & $\begin{array}{c}\text { Heat } \\
\text { Evidence }\end{array}$ & $\begin{array}{c}\text { Intentional } \\
\text { Heat } \\
\text { Treatment } \\
\end{array}$ & Patination & Retouch Detail $\begin{array}{c}\text { Retouch } \\
\text { Distribution }\end{array}$ & $\begin{array}{l}\text { Retouch } \\
\text { Location } \\
\end{array}$ \\
\hline \multirow{3}{*}{406} & \multirow{3}{*}{8} & \multirow{3}{*}{$503 \mathrm{NA}$} & Modified Flake & Edge-modified & Indet & $\begin{array}{c}\text { Proximal- } \\
\text { Medial }\end{array}$ & Bend & $\begin{array}{c}\text { Fine Grain } \\
\text { Chert } \\
\end{array}$ & $\begin{array}{c}\text { 10YR7/3 to } \\
\text { 10YR7/4 }\end{array}$ & $\begin{array}{l}\text { Very Pale } \\
\text { Brown } \\
\end{array}$ & 0 & Reddened & Yes & None & Unifacial-dorsal & $\begin{array}{l}\text { Right Lateral } \\
\text { Margin }\end{array}$ \\
\hline & & & Weight (g) & Length & Width & Thickness & $\begin{array}{l}\text { Height of } \\
\text { Retouch }\end{array}$ & $\begin{array}{c}\text { Perimeter } \\
\text { Retouch } \\
\text { Length }\end{array}$ & $\begin{array}{c}\text { Average } \\
\text { Edge } \\
\text { Angle } \\
\end{array}$ & $\begin{array}{l}\text { Reduction } \\
\text { Index }\end{array}$ & $\begin{array}{l}\text { Platform } \\
\text { width }\end{array}$ & $\begin{array}{l}\text { Platform } \\
\text { thickness }\end{array}$ & \multicolumn{4}{|c|}{ Comments } \\
\hline & & & 4.4 & Indet & 24.42 & 5.33 & Indet & Indet & Indet & Indet & Indet & Indet & $\begin{array}{l}\text { Thin flake witl } \\
\text { portion; Ple } \\
\text { multiple step }\end{array}$ & $\begin{array}{l}h \text { crude retouc } \\
\text { tform has bee } \\
\text { fractures; Pir }\end{array}$ & $\begin{array}{l}\text { ich on right lateral margin; Break } \\
\text { en removed from the dorsal side } \\
\text { ink coloration along proximal edg } \\
\text { face }\end{array}$ & $\begin{array}{l}\text { k across medial } \\
\text { e resulting in } \\
\text { gge and dorsal }\end{array}$ \\
\hline Lot No. & $\begin{array}{l}\text { Spec. } \\
\text { No. }\end{array}$ & $\begin{array}{l}\text { Bag UI No } \\
\text { No. }\end{array}$ & Category & Subcategory & $\begin{array}{c}\text { Retouch } \\
\text { Affiliation }\end{array}$ & $\begin{array}{l}\text { Specimen } \\
\text { Condition }\end{array}$ & Breakage & Raw material & Munsell & Color & $\begin{array}{l}\text { Dorsal } \\
\text { Cortex \% }\end{array}$ & $\begin{array}{c}\text { Heat } \\
\text { Evidence }\end{array}$ & $\begin{array}{l}\text { Intentional } \\
\text { Heat } \\
\text { Treatment }\end{array}$ & Patination & Retouch Detail $\begin{array}{c}\text { Retouch } \\
\text { Distribution }\end{array}$ & $\begin{array}{l}\text { Retouch } \\
\text { Location }\end{array}$ \\
\hline \multirow{3}{*}{406} & \multirow{3}{*}{9} & \multirow{3}{*}{$503 \mathrm{NA}$} & Modified Flake & Edge-modified & Indet & $\begin{array}{l}\text { Missing Right } \\
\text { Lateral Margin }\end{array}$ & Snap & $\begin{array}{l}\text { Fine Grain } \\
\text { Chert }\end{array}$ & 10YR3/2 & $\begin{array}{l}\text { Very Dark } \\
\text { Grayish Brown }\end{array}$ & 0 & None & NA & None & Unifacial-dorsal & $\begin{array}{l}\text { Left Lateral } \\
\text { Margin }\end{array}$ \\
\hline & & & Weight (g) & Length & Width & Thickness & $\begin{array}{l}\text { Height of } \\
\text { Retouch }\end{array}$ & $\begin{array}{l}\text { Perimeter } \\
\text { Retouch } \\
\text { Lenqth }\end{array}$ & $\begin{array}{l}\text { Average } \\
\text { Edge } \\
\text { Anqle } \\
\end{array}$ & $\begin{array}{l}\text { Reduction } \\
\text { Index }\end{array}$ & $\begin{array}{l}\text { Platform } \\
\text { width }\end{array}$ & $\begin{array}{l}\text { Platform } \\
\text { thickness }\end{array}$ & \multicolumn{4}{|c|}{ Comments } \\
\hline & & & 9.55 & 33.12 & Indet & Indet & 1.04 & (5) & 60 & Indet & Indet & Indet & $\begin{array}{r}\text { Inclusions } \\
\text { missing due } t \\
\text { flak }\end{array}$ & $\begin{array}{l}\text { of } 10 Y R 5 / 3 \mathrm{br} \\
\text { o large break } \\
\text { e removal nea }\end{array}$ & $\begin{array}{l}\text { rown; Thick flake with undeterm } \\
\text { along right lateral margin; Small } \\
\text { ar proximal end of left lateral ma }\end{array}$ & $\begin{array}{l}\text { nined portion } \\
\text { Ill portion of fine } \\
\text { argin }\end{array}$ \\
\hline Lot No. & $\begin{array}{l}\text { Spec. } \\
\text { No. }\end{array}$ & $\begin{array}{l}\text { Bag UI No } \\
\text { No. }\end{array}$ & Category & Subcategory & $\begin{array}{c}\text { Retouch } \\
\text { Affiliation }\end{array}$ & $\begin{array}{l}\text { Specimen } \\
\text { Condition } \\
\end{array}$ & Breakage & Raw material & Munsell & Color & $\begin{array}{c}\text { Dorsal } \\
\text { Cortex \% } \\
\end{array}$ & $\begin{array}{c}\text { Heat } \\
\text { Evidence }\end{array}$ & $\begin{array}{c}\text { Intentional } \\
\text { Heat } \\
\text { Treatment } \\
\end{array}$ & Patination & Retouch Detail $\begin{array}{c}\text { Retouch } \\
\text { Distribution }\end{array}$ & $\begin{array}{l}\text { Retouch } \\
\text { Location }\end{array}$ \\
\hline \multirow{3}{*}{407} & \multirow{3}{*}{7} & \multirow{3}{*}{507 NA } & Modified Flake & Edge-modified & Indet & $\begin{array}{c}\text { Proximal- } \\
\text { Medial }\end{array}$ & Snap & $\begin{array}{c}\text { Fine Grain } \\
\text { Chert } \\
\end{array}$ & 10YR3/2 & $\begin{array}{c}\text { Very Dark } \\
\text { Grayish Brown } \\
\end{array}$ & $26-50$ & None & NA & None & Unifacial-dorsal Continuous & $\begin{array}{l}\text { Left Lateral } \\
\text { Margin } \\
\end{array}$ \\
\hline & & & Weight (g) & Length & Width & Thickness & $\begin{array}{l}\text { Height of } \\
\text { Retouch }\end{array}$ & $\begin{array}{l}\text { Perimeter } \\
\text { Retouch } \\
\text { Lenath }\end{array}$ & $\begin{array}{l}\text { Average } \\
\text { Edge } \\
\text { Anqle } \\
\end{array}$ & $\begin{array}{c}\text { Reduction } \\
\text { Index }\end{array}$ & $\begin{array}{l}\text { Platform } \\
\text { width }\end{array}$ & $\begin{array}{l}\text { Platform } \\
\text { thickness }\end{array}$ & \multicolumn{4}{|c|}{ Comments } \\
\hline & & & 45.94 & 59.59 & 63.02 & 13.19 & 1.49 & 70 & 50 & 0.11 & 19.38 & 5.13 & \multicolumn{4}{|c|}{ Large flake with irregular flake removal and some grinding } \\
\hline Lot No. & $\begin{array}{l}\text { Spec. } \\
\text { No. }\end{array}$ & $\begin{array}{l}\text { Bag UI No } \\
\text { No. }\end{array}$ & Category & Subcategory & $\begin{array}{l}\text { Retouch } \\
\text { Affiliation }\end{array}$ & $\begin{array}{l}\text { Specimen } \\
\text { Condition }\end{array}$ & Breakage & Raw material & Munsell & Color & $\begin{array}{l}\text { Dorsal } \\
\text { Cortex \% }\end{array}$ & $\begin{array}{c}\text { Heat } \\
\text { Evidence }\end{array}$ & $\begin{array}{l}\text { Intentional } \\
\text { Heat } \\
\text { Treatment } \\
\end{array}$ & Patination & Retouch Detail $\begin{array}{c}\text { Retouch } \\
\text { Distribution }\end{array}$ & $\begin{array}{l}\text { Retouch } \\
\text { Location }\end{array}$ \\
\hline \multirow{3}{*}{429} & \multirow{3}{*}{1} & \multirow{3}{*}{597 NA } & Modified Flake & Edge-modified & Indet & $\begin{array}{l}\text { Right Lateral } \\
\text { Margin } \\
\text { Missing }\end{array}$ & Snap & $\begin{array}{l}\text { Coarse Grain } \\
\text { Chert }\end{array}$ & 10YR6/1 & Gray & $51-75$ & None & NA & None & Unifacial-dorsal Continuous & $\begin{array}{l}\text { Proximal/Left } \\
\quad \text { Lateral } \\
\text { Margin/Distal }\end{array}$ \\
\hline & & & Weight (g) & Length & Width & Thickness & $\begin{array}{l}\text { Height of } \\
\text { Retouch }\end{array}$ & $\begin{array}{l}\text { Perimeter } \\
\text { Retouch } \\
\text { Length }\end{array}$ & $\begin{array}{l}\text { Average } \\
\text { Edge } \\
\text { Angle } \\
\end{array}$ & $\begin{array}{l}\text { Reduction } \\
\text { Index }\end{array}$ & $\begin{array}{l}\text { Platform } \\
\text { width }\end{array}$ & $\begin{array}{l}\text { Platform } \\
\text { thickness }\end{array}$ & & & Comments & \\
\hline & & & 180.45 & 92.2 & 54.96 & 29 & 1.85 & 155 & 15.66 & 0.06 & Indet & Indet & \multicolumn{4}{|c|}{$\begin{array}{l}\text { Platform removed; Small portion of right lateral margin missing; Tool is a } \\
\text { large cortical flake with steep flake removal from left lateral margin and } \\
\text { retouch along half of circumference; Specks of mineral inclusions } \\
\text { throughout }\end{array}$} \\
\hline
\end{tabular}




\begin{tabular}{|c|c|c|c|c|c|c|c|c|c|c|c|c|c|c|c|c|c|}
\hline Lot No. & $\begin{array}{l}\text { Spec. } \\
\text { No. }\end{array}$ & $\begin{array}{l}\text { Bag UI No } \\
\text { No. }\end{array}$ & Category & Subcategory & $\begin{array}{c}\text { Retouch } \\
\text { Affiliation }\end{array}$ & $\begin{array}{l}\text { Specimen } \\
\text { Condition }\end{array}$ & Breakage & Raw material & Munsell & Color & $\begin{array}{c}\text { Dorsal } \\
\text { Cortex \% }\end{array}$ & $\begin{array}{c}\text { Heat } \\
\text { Evidence }\end{array}$ & $\begin{array}{c}\text { Intentional } \\
\text { Heat } \\
\text { Treatment }\end{array}$ & Patination & Retouch Detail & $\begin{array}{c}\text { Retouch } \\
\text { Distribution }\end{array}$ & $\begin{array}{l}\text { Retouch } \\
\text { Location }\end{array}$ \\
\hline \multirow{3}{*}{439} & \multirow{3}{*}{7} & \multirow{3}{*}{442 NA } & Modified Flake & Edge-modified & Cultural & Indet & Bend & $\begin{array}{l}\text { Fine Grain } \\
\text { Chert }\end{array}$ & 10YR4/4 & $\begin{array}{l}\text { Dark Yellowish } \\
\text { Brown }\end{array}$ & 0 & None & NA & None & Indet & Focused & Indet \\
\hline & & & Weight (g) & Length & Width & Thickness & $\begin{array}{l}\text { Height of } \\
\text { Retouch }\end{array}$ & $\begin{array}{l}\text { Perimeter } \\
\text { Retouch } \\
\text { Length }\end{array}$ & $\begin{array}{c}\text { Average } \\
\text { Edge } \\
\text { Angle }\end{array}$ & $\begin{array}{c}\text { Reduction } \\
\text { Index }\end{array}$ & $\begin{array}{l}\text { Platform } \\
\text { width }\end{array}$ & $\begin{array}{l}\text { Platform } \\
\text { thickness }\end{array}$ & \multicolumn{5}{|c|}{ Comments } \\
\hline & & & 0.54 & Indet & Indet & Indet & Indet & Indet & Indet & Indet & Indet & Indet & $\begin{array}{l}\text { Incomplete fl } \\
\text { attributes to o } \\
\text { into a point; } \mathrm{O}\end{array}$ & $\begin{array}{l}\text { ake fragment } \\
\text { ient for analy } \\
\text { ee lateral edg } \\
\text { arallel to modi }\end{array}$ & $\begin{array}{l}\text { that is irregular in } \\
\text { ysis; Flake is small } \\
\text { ye of the point has } \\
\text { lified edge restricts }\end{array}$ & $\begin{array}{l}\text { I shape; No dis } \\
\text { II and appears } \\
\text {; been modified } \\
\text { is further analys }\end{array}$ & $\begin{array}{l}\text { ernable flake } \\
\text { have broken } \\
\text { Second break } \\
s\end{array}$ \\
\hline
\end{tabular}

\begin{tabular}{|c|c|c|c|c|c|c|c|c|c|c|c|c|c|c|c|c|c|c|}
\hline Lot No. & $\begin{array}{l}\text { Spec. } \\
\text { No. }\end{array}$ & $\begin{array}{l}\text { Bag } \\
\text { No. }\end{array}$ & UI No & Category & Subcategory & $\begin{array}{c}\text { Retouch } \\
\text { Affiliation }\end{array}$ & $\begin{array}{l}\text { Specimen } \\
\text { Condition }\end{array}$ & Breakage & Raw material & Munsell & Color & $\begin{array}{c}\text { Dorsal } \\
\text { Cortex \% }\end{array}$ & $\begin{array}{c}\text { Heat } \\
\text { Evidence }\end{array}$ & $\begin{array}{c}\text { Intentional } \\
\text { Heat } \\
\text { Treatment }\end{array}$ & Patination & Retouch Detail & $\begin{array}{l}\text { Retouch } \\
\text { Distribution }\end{array}$ & $\begin{array}{l}\text { Retouch } \\
\text { Location }\end{array}$ \\
\hline \multirow{3}{*}{440} & \multirow{3}{*}{5} & \multirow{3}{*}{448} & \multirow{3}{*}{ NA } & Modified Flake & Edge-modified & Indet & Complete & None & $\begin{array}{c}\text { Fine Grain } \\
\text { Chert }\end{array}$ & $\begin{array}{c}10 Y R 6 / 2 \text { to } \\
10 Y R 7 / 2\end{array}$ & $\begin{array}{l}\text { Light Brownish } \\
\text { Gray to Light }\end{array}$ & $26-50$ & None & NA & None & Unifacial-dorsal & Clustered & $\begin{array}{c}\text { Right Lateral } \\
\text { Margin }\end{array}$ \\
\hline & & & & Weight (g) & Length & Width & Thickness & $\begin{array}{l}\text { Height of } \\
\text { Retouch }\end{array}$ & $\begin{array}{l}\text { Perimeter } \\
\text { Retouch } \\
\text { Lenath }\end{array}$ & $\begin{array}{c}\text { Average } \\
\text { Edge } \\
\text { Angle } \\
\end{array}$ & $\begin{array}{c}\text { Reduction } \\
\text { Index }\end{array}$ & $\begin{array}{l}\text { Platform } \\
\text { width }\end{array}$ & $\begin{array}{l}\text { Platform } \\
\text { thickness }\end{array}$ & \multicolumn{5}{|c|}{ Comments } \\
\hline & & & & 76.99 & 75.05 & 71.85 & 17.63 & 1.85 & 35 & 55 & 0.10 & 21.2 & 9.09 & \multicolumn{5}{|c|}{$\begin{array}{c}\text { Large flake with irregular flake removal near proximal end of right lateral } \\
\text { margin }\end{array}$} \\
\hline
\end{tabular}

\begin{tabular}{|c|c|c|c|c|c|c|c|c|c|c|c|c|c|c|c|c|c|c|}
\hline Lot No. & $\begin{array}{l}\text { Spec. } \\
\text { No. }\end{array}$ & $\begin{array}{l}\text { Bag } \\
\text { No. }\end{array}$ & UI No & Category & Subcategory & $\begin{array}{c}\text { Retouch } \\
\text { Affiliation }\end{array}$ & $\begin{array}{l}\text { Specimen } \\
\text { Condition }\end{array}$ & Breakage & Raw material & Munsell & Color & $\begin{array}{c}\text { Dorsal } \\
\text { Cortex \% }\end{array}$ & $\begin{array}{c}\text { Heat } \\
\text { Evidence }\end{array}$ & $\begin{array}{c}\text { Intentional } \\
\text { Heat } \\
\text { Treatment }\end{array}$ & Patination & Retouch Detail & $\begin{array}{c}\text { Retouch } \\
\text { Distribution }\end{array}$ & $\begin{array}{l}\text { Retouch } \\
\text { Location }\end{array}$ \\
\hline \multirow{3}{*}{506} & \multirow{3}{*}{34} & \multirow{3}{*}{339} & \multirow{3}{*}{ NA } & Modified Flake & Utilized Flake & Indet & Complete & None & $\begin{array}{c}\text { Fine Grain } \\
\text { Chert }\end{array}$ & $\begin{array}{c}\text { 10YR5/2 to } \\
\text { 10YR6/2 }\end{array}$ & $\begin{array}{c}\text { Grayish Brown } \\
\text { to Light Grayish } \\
\text { Brown }\end{array}$ & $1-25$ & None & NA & None & Unifacial-ventral & Continuous & $\begin{array}{l}\text { Left and Right } \\
\text { Lateral } \\
\text { Marains }\end{array}$ \\
\hline & & & & Weight (g) & Length & Width & Thickness & $\begin{array}{l}\text { Height of } \\
\text { Retouch }\end{array}$ & $\begin{array}{l}\text { Perimeter } \\
\text { Retouch } \\
\text { Length }\end{array}$ & $\begin{array}{c}\text { Average } \\
\text { Edge } \\
\text { Angle }\end{array}$ & $\begin{array}{c}\text { Reduction } \\
\text { Index }\end{array}$ & $\begin{array}{l}\text { Platform } \\
\text { width }\end{array}$ & $\begin{array}{c}\text { Platform } \\
\text { thickness }\end{array}$ & \multicolumn{5}{|c|}{ Comments } \\
\hline & & & & 42.88 & 57.79 & 57.13 & 14.02 & 0.99 & 111 & 55 & 0.07 & 21.56 & 6.08 & $\begin{array}{r}\text { Large flake wit } \\
\text { be see }\end{array}$ & $\begin{array}{l}\text { th both lateral } \\
\text { en in irregular }\end{array}$ & $\begin{array}{l}\text { margins showing } \\
r \text { patterns - does } n\end{array}$ & $\begin{array}{l}\text { g utilization; } \mathrm{Si} \\
\text { not appear inte }\end{array}$ & $\begin{array}{l}\text { Small flaking can } \\
\text { entional }\end{array}$ \\
\hline
\end{tabular}

\begin{tabular}{|c|c|c|c|c|c|c|c|c|c|c|c|c|c|c|c|c|c|}
\hline Lot No. & $\begin{array}{l}\text { Spec. } \\
\text { No. }\end{array}$ & $\begin{array}{l}\text { Bag UI No } \\
\text { No. }\end{array}$ & Category & Subcategory & $\begin{array}{c}\text { Retouch } \\
\text { Affiliation }\end{array}$ & $\begin{array}{l}\text { Specimen } \\
\text { Condition }\end{array}$ & Breakage & Raw material & Munsell & Color & $\begin{array}{l}\text { Dorsal } \\
\text { Cortex \% }\end{array}$ & $\begin{array}{c}\text { Heat } \\
\text { Evidence }\end{array}$ & $\begin{array}{c}\text { Intentional } \\
\text { Heat } \\
\text { Treatment }\end{array}$ & Patination & Retouch Detail & $\begin{array}{l}\text { Retouch } \\
\text { Distribution }\end{array}$ & $\begin{array}{l}\text { Retouch } \\
\text { Location }\end{array}$ \\
\hline \multirow{3}{*}{507} & \multirow{3}{*}{1} & \multirow{3}{*}{361 NA } & Modified Flak & Edge-modified & Indet & $\begin{array}{l}\text { Proximal } \\
\text { Missing }\end{array}$ & Indet & $\begin{array}{c}\text { Fine Grain } \\
\text { Chert }\end{array}$ & $\begin{array}{c}\text { 10YR7/1 } \\
\text { to10YR7/2 }\end{array}$ & Light Gray & $76-100$ & None & - & None & Bifacial & Focused & $\begin{array}{c}\text { Left Lateral } \\
\text { Margin/Distal } \\
\text { Margin }\end{array}$ \\
\hline & & & Weight (g) & Length & Width & Thickness & $\begin{array}{l}\text { Height of } \\
\text { Retouch }\end{array}$ & $\begin{array}{c}\text { Perimeter } \\
\text { Retouch } \\
\text { Length } \\
\end{array}$ & $\begin{array}{l}\text { Average } \\
\text { Edge } \\
\text { Angle } \\
\end{array}$ & $\begin{array}{l}\text { Reduction } \\
\text { Index }\end{array}$ & $\begin{array}{l}\text { Platform } \\
\text { width }\end{array}$ & $\begin{array}{l}\text { Platform } \\
\text { thickness }\end{array}$ & \multicolumn{5}{|c|}{ Comments } \\
\hline & & & 235.17 & Indet & 79.35 & 21.59 & 2.4 & 145 & 55 & 0.11 & Indet & Indet & $\begin{array}{l}\text { Large cortical } \\
\text { on the vent }\end{array}$ & $\begin{array}{l}\text { flaked worked } \\
\text { ral face; Platfo } \\
\text { 10YR } 4 / 6\end{array}$ & $\begin{array}{l}\text { d predominately } \\
\text { form missing; Colo } \\
\text { (Dark Yellow); Ex }\end{array}$ & $\begin{array}{l}\text { on dorsal face } \\
\text { or change aro } \\
\text { xpedient too }\end{array}$ & $\begin{array}{l}\text { with light flaking } \\
\text { und cortex to }\end{array}$ \\
\hline
\end{tabular}




\begin{tabular}{|c|c|c|c|c|c|c|c|c|c|c|c|c|c|c|c|c|c|}
\hline Lot No. & $\begin{array}{l}\text { Spec. } \\
\text { No. }\end{array}$ & $\begin{array}{l}\text { Bag UI No } \\
\text { No. }\end{array}$ & Category & Subcategory & $\begin{array}{c}\text { Retouch } \\
\text { Affiliation }\end{array}$ & $\begin{array}{l}\text { Specimen } \\
\text { Condition }\end{array}$ & Breakage & Raw material & Munsell & Color & $\begin{array}{c}\text { Dorsal } \\
\text { Cortex \% } \\
\end{array}$ & $\begin{array}{c}\text { Heat } \\
\text { Evidence }\end{array}$ & $\begin{array}{c}\begin{array}{c}\text { Intentional } \\
\text { Heat } \\
\text { Treatment }\end{array} \\
\end{array}$ & Patination & Retouch Detail & $\begin{array}{l}\text { Retouch } \\
\text { Distribution }\end{array}$ & $\begin{array}{l}\text { Retouch } \\
\text { Location } \\
\end{array}$ \\
\hline \multirow{3}{*}{511} & \multirow{3}{*}{6} & \multirow{3}{*}{438} & Modified Flake & Edge-modified & Indet & Complete & None & $\begin{array}{c}\text { Fine Grain } \\
\text { Chert }\end{array}$ & $\begin{array}{c}\text { 10YR5/1 to } \\
\text { 10YR5/2 }\end{array}$ & $\begin{array}{c}\text { Gray to Grayish } \\
\text { Brown }\end{array}$ & $0-25$ & None & NA & None & Unifacial-dorsal & Focused & $\begin{array}{l}\text { Right Lateral } \\
\text { Margin }\end{array}$ \\
\hline & & & Weight (g) & Length & Width & Thickness & $\begin{array}{l}\text { Height of } \\
\text { Retouch }\end{array}$ & $\begin{array}{l}\text { Perimeter } \\
\text { Retouch } \\
\text { Length }\end{array}$ & $\begin{array}{l}\text { Average } \\
\text { Edge } \\
\text { Angle }\end{array}$ & $\begin{array}{l}\text { Reduction } \\
\text { Index }\end{array}$ & $\begin{array}{l}\text { Platform } \\
\text { width }\end{array}$ & $\begin{array}{l}\text { Platform } \\
\text { thickness }\end{array}$ & \multicolumn{5}{|c|}{ Comments } \\
\hline & & & 13.23 & 61.49 & 34.31 & 6.73 & 0.96 & 23 & 45 & 0.14 & 7.38 & 2.84 & \multicolumn{5}{|c|}{$\begin{array}{l}\text { Irregular flake with two localized areas of fine retouch; Shallow scalar } \\
\text { flaking }\end{array}$} \\
\hline Lot No. & $\begin{array}{l}\text { Spec. } \\
\text { No. }\end{array}$ & $\begin{array}{l}\text { Bag UI No } \\
\text { No. }\end{array}$ & Category & Subcategory & $\begin{array}{c}\text { Retouch } \\
\text { Affiliation }\end{array}$ & $\begin{array}{l}\text { Specimen } \\
\text { Condition }\end{array}$ & Breakage & Raw material & Munsell & Color & $\begin{array}{l}\text { Dorsal } \\
\text { Cortex \% }\end{array}$ & $\begin{array}{c}\text { Heat } \\
\text { Evidence }\end{array}$ & $\begin{array}{c}\text { Intentional } \\
\text { Heat } \\
\text { Treatment }\end{array}$ & Patination & Retouch Detail & $\begin{array}{l}\text { Retouch } \\
\text { Distribution }\end{array}$ & $\begin{array}{l}\text { Retouch } \\
\text { Location }\end{array}$ \\
\hline \multirow{3}{*}{518} & \multirow{3}{*}{7} & \multirow{3}{*}{$640 \mathrm{NA}$} & Modified Flake & Utilized Flake & Indet & $\begin{array}{l}\text { Right Lateral } \\
\text { Margin } \\
\text { Missing }\end{array}$ & Snap & $\begin{array}{l}\text { Fine Grain } \\
\text { Chert }\end{array}$ & 10YR6/2 & $\begin{array}{l}\text { Light Brownish } \\
\text { Gray }\end{array}$ & $0-25$ & None & NA & None & Unifacial-dorsal & Focused & $\begin{array}{l}\text { Proximal/Left } \\
\text { Lateral Margin }\end{array}$ \\
\hline & & & Weight (g) & Length & Width & Thickness & $\begin{array}{l}\text { Height of } \\
\text { Retouch }\end{array}$ & $\begin{array}{l}\text { Perimeter } \\
\text { Retouch } \\
\text { Lenqth }\end{array}$ & $\begin{array}{c}\text { Average } \\
\text { Edge } \\
\text { Angle } \\
\end{array}$ & $\begin{array}{l}\text { Reduction } \\
\text { Index }\end{array}$ & $\begin{array}{l}\text { Platform } \\
\text { width }\end{array}$ & $\begin{array}{l}\text { Platform } \\
\text { thickness }\end{array}$ & \multicolumn{5}{|c|}{ Comments } \\
\hline & & & 44.44 & 58.53 & 44.12 & 16.25 & 3.02 & 50 & 35 & 0.19 & 32.41 & 11.63 & \multicolumn{5}{|c|}{$\begin{array}{l}\text { Small portion of right lateral margin (near distal end) is fractured; use-wear } \\
\text { with very small possible removal flakes from middle of proximal margin to } \\
\text { middle of left lateral margin; Prominent banding of 10YR7/2 (Light Gray) } \\
\text { and 10YR6/1 (Gray) }\end{array}$} \\
\hline Lot No. & $\begin{array}{l}\text { Spec. } \\
\text { No. }\end{array}$ & $\begin{array}{l}\text { Bag UI No } \\
\text { No. }\end{array}$ & Category & Subcategory & $\begin{array}{c}\text { Retouch } \\
\text { Affiliation }\end{array}$ & $\begin{array}{l}\text { Specimen } \\
\text { Condition }\end{array}$ & Breakage & Raw material & Munsell & Color & $\begin{array}{c}\text { Dorsal } \\
\text { Cortex \% }\end{array}$ & $\begin{array}{c}\text { Heat } \\
\text { Evidence }\end{array}$ & $\begin{array}{c}\text { Intentional } \\
\text { Heat } \\
\text { Treatment }\end{array}$ & Patination & Retouch Detail & $\begin{array}{c}\text { Retouch } \\
\text { Distribution }\end{array}$ & $\begin{array}{l}\text { Retouch } \\
\text { Location }\end{array}$ \\
\hline \multirow{3}{*}{518} & \multirow{3}{*}{9} & \multirow{3}{*}{640 NA } & Modified Flake & Utilized Flake & Indet & Complete & None & $\begin{array}{c}\text { Coarse Grain } \\
\text { Chert } \\
\end{array}$ & 10YR6/2 & $\begin{array}{c}\text { Light Brownish } \\
\text { Gray }\end{array}$ & $1-25$ & Reddened & Yes & None & Unifacial-ventral & Focused & $\begin{array}{c}\text { Left Lateral } \\
\text { Margin }\end{array}$ \\
\hline & & & Weight (g) & Length & Width & Thickness & $\begin{array}{l}\text { Height of } \\
\text { Retouch }\end{array}$ & $\begin{array}{l}\text { Perimeter } \\
\text { Retouch } \\
\text { Lenath }\end{array}$ & $\begin{array}{c}\text { Average } \\
\text { Edge } \\
\text { Angle }\end{array}$ & $\begin{array}{l}\text { Reduction } \\
\text { Index }\end{array}$ & $\begin{array}{l}\text { Platform } \\
\text { width }\end{array}$ & $\begin{array}{l}\text { Platform } \\
\text { thickness }\end{array}$ & & & Comments & & \\
\hline & & & 14.56 & 46.14 & 44.5 & 10.22 & 1.63 & 50 & 40 & 0.16 & 38 & 8.67 & \multicolumn{5}{|c|}{$\begin{array}{l}\text { Complete flake with modification due to utilization along entire left lateral } \\
\text { margin; Portion of modification near distal end has steeper edge angle and } \\
\text { may be edge modified - unable to determine; Flake is speckled with } \\
\text { mineral inclusions and reddened spots }\end{array}$} \\
\hline Lot No. & $\begin{array}{l}\text { Spec. } \\
\text { No. }\end{array}$ & $\begin{array}{l}\text { Bag UI No } \\
\text { No. }\end{array}$ & Category & Subcategory & $\begin{array}{c}\text { Retouch } \\
\text { Affiliation }\end{array}$ & $\begin{array}{l}\text { Specimen } \\
\text { Condition }\end{array}$ & Breakage & Raw material & Munsell & Color & $\begin{array}{c}\text { Dorsal } \\
\text { Cortex \% }\end{array}$ & $\begin{array}{c}\text { Heat } \\
\text { Evidence }\end{array}$ & $\begin{array}{c}\text { Intentional } \\
\text { Heat } \\
\text { Treatment }\end{array}$ & Patination & Retouch Detail & $\begin{array}{l}\text { Retouch } \\
\text { Distribution }\end{array}$ & $\begin{array}{l}\text { Retouch } \\
\text { Location }\end{array}$ \\
\hline \multirow{3}{*}{519} & \multirow{3}{*}{10} & \multirow{3}{*}{646 NA } & Modified Flake & Edge-modified & Cultural & $\begin{array}{l}\text { Right Lateral } \\
\text { Margin } \\
\text { Missing }\end{array}$ & Bend & $\begin{array}{l}\text { Fine Grain } \\
\text { Chert }\end{array}$ & 10YR3/1 & Very Dark Gray & $51-75$ & None & NA & None & Unifacial-ventral & Continuous & $\begin{array}{l}\text { Left Lateral } \\
\text { Margin }\end{array}$ \\
\hline & & & Weight (g) & Length & Width & Thickness & $\begin{array}{l}\text { Height of } \\
\text { Retouch }\end{array}$ & $\begin{array}{l}\text { Perimeter } \\
\text { Retouch } \\
\text { Lenath }\end{array}$ & $\begin{array}{c}\text { Average } \\
\text { Edge } \\
\text { Anqle } \\
\end{array}$ & $\begin{array}{c}\text { Reduction } \\
\text { Index }\end{array}$ & $\begin{array}{l}\text { Platform } \\
\text { width }\end{array}$ & $\begin{array}{l}\text { Platform } \\
\text { thickness }\end{array}$ & \multicolumn{5}{|c|}{ Comments } \\
\hline & & & 44.49 & 49.06 & Indet & 19.92 & 1.2 & 45 & 55 & 0.06 & 19.33 & 6.35 & Dorsal fa & ce is almost $c$ & completely cortex; & ; Few mineral i & inclusions \\
\hline
\end{tabular}




\begin{tabular}{|c|c|c|c|c|c|c|c|c|c|c|c|c|c|c|c|c|c|}
\hline Lot No. & $\begin{array}{l}\text { Spec. } \\
\text { No. }\end{array}$ & $\begin{array}{l}\text { Bag UI No } \\
\text { No. }\end{array}$ & Category & Subcategory & $\begin{array}{c}\text { Retouch } \\
\text { Affiliation }\end{array}$ & $\begin{array}{l}\text { Specimen } \\
\text { Condition }\end{array}$ & Breakage & Raw material & Munsell & Color & $\begin{array}{c}\text { Dorsal } \\
\text { Cortex \% }\end{array}$ & $\begin{array}{c}\text { Heat } \\
\text { Evidence }\end{array}$ & $\begin{array}{c}\text { Intentional } \\
\text { Heat } \\
\text { Treatment }\end{array}$ & Patination & Retouch Detail & $\begin{array}{l}\text { Retouch } \\
\text { Distribution }\end{array}$ & $\begin{array}{l}\text { Retouch } \\
\text { Location }\end{array}$ \\
\hline \multirow{3}{*}{528} & \multirow{3}{*}{9} & \multirow{3}{*}{666 NA } & Modified Flake & Edge-modified & Cultural & Medial & Snap & $\begin{array}{c}\text { Fine Grain } \\
\text { Chert }\end{array}$ & 10YR7/2 & Light Gray & 0 & None & NA & None & Unifacial-dorsal & Indet & Indet \\
\hline & & & Weight (g) & Length & Width & Thickness & $\begin{array}{l}\text { Height of } \\
\text { Retouch }\end{array}$ & $\begin{array}{l}\text { Perimeter } \\
\text { Retouch } \\
\text { Length }\end{array}$ & $\begin{array}{l}\text { Average } \\
\text { Edge } \\
\text { Angle }\end{array}$ & $\begin{array}{l}\text { Reduction } \\
\text { Index }\end{array}$ & $\begin{array}{l}\text { Platform } \\
\text { width }\end{array}$ & $\begin{array}{l}\text { Platform } \\
\text { thickness }\end{array}$ & & & Comments & & \\
\hline & & & 1.2 & Indet & Indet & Indet & Indet & Indet & Indet & Indet & Indet & Indet & $\begin{array}{c}\text { Medial fragm } \\
\text { margin; Very } \\
\end{array}$ & $\begin{array}{l}\text { ent with only o } \\
\text { small tool fra } \\
\text { inclusions }\end{array}$ & $\begin{array}{l}\text { one lateral margin } \\
\text { agment - cannot a } \\
\text { of } 10 Y R 7 / 1 \text { (Light }\end{array}$ & $\begin{array}{l}\text { in - unable to de } \\
\text { assume propor } \\
\text { ht Gray) color }\end{array}$ & $\begin{array}{l}\text { etermine which } \\
\text { rtions; Mineral }\end{array}$ \\
\hline Lot No. & $\begin{array}{l}\text { Spec. } \\
\text { No. }\end{array}$ & $\begin{array}{l}\text { Bag UI No } \\
\text { No. }\end{array}$ & Category & Subcategory & $\begin{array}{c}\text { Retouch } \\
\text { Affiliation }\end{array}$ & $\begin{array}{l}\text { Specimen } \\
\text { Condition }\end{array}$ & Breakage & Raw material & Munsell & Color & $\begin{array}{l}\text { Dorsal } \\
\text { Cortex \% }\end{array}$ & $\begin{array}{c}\text { Heat } \\
\text { Evidence }\end{array}$ & $\begin{array}{l}\text { Intentional } \\
\text { Heat } \\
\text { Treatment }\end{array}$ & Patination & Retouch Detail & $\begin{array}{c}\text { Retouch } \\
\text { Distribution }\end{array}$ & $\begin{array}{l}\text { Retouch } \\
\text { Location }\end{array}$ \\
\hline \multirow{3}{*}{539} & \multirow{3}{*}{18} & \multirow{3}{*}{592 NA } & Modified Flake & Edge-modified & Cultural & $\begin{array}{c}\text { Proximal- } \\
\text { Medial } \\
\end{array}$ & Snap & $\begin{array}{l}\text { Fine Grain } \\
\text { Chert }\end{array}$ & $\begin{array}{l}\text { 10YR5/4 to } \\
\text { 10YR6/4 } \\
\end{array}$ & $\begin{array}{c}\text { Yellowish Brown } \\
\text { to Light }\end{array}$ & 0 & None & NA & None & Unifacial-dorsal & Indet & $\begin{array}{c}\text { Right Lateral } \\
\text { Margin }\end{array}$ \\
\hline & & & Weight (g) & Length & Width & Thickness & $\begin{array}{l}\text { Height of } \\
\text { Retouch }\end{array}$ & $\begin{array}{c}\text { Perimeter } \\
\text { Retouch } \\
\text { Lenath }\end{array}$ & $\begin{array}{l}\text { Average } \\
\text { Edge } \\
\text { Angle } \\
\end{array}$ & $\begin{array}{l}\text { Reduction } \\
\text { Index }\end{array}$ & $\begin{array}{l}\text { Platform } \\
\text { width }\end{array}$ & $\begin{array}{l}\text { Platform } \\
\text { thickness }\end{array}$ & & & Comments & & \\
\hline & & & 1.06 & Indet & Indet & Indet & Indet & Indet & Indet & Indet & 4.37 & 1.52 & $\begin{array}{r}\text { Thin flake w } \\
\text { margin; B }\end{array}$ & $\begin{array}{l}\text { ith fine overla } \\
\text { reak through } r\end{array}$ & $\begin{array}{l}\text { apping scalar flake } \\
\text { medial portion of } f\end{array}$ & $\begin{array}{l}\text { ke removal alon } \\
f \text { flake prevent } f\end{array}$ & $\begin{array}{l}\text { Ig right lateral } \\
\text { full analysis }\end{array}$ \\
\hline Lot No. & $\begin{array}{l}\text { Spec. } \\
\text { No. }\end{array}$ & $\begin{array}{l}\text { Bag UI No } \\
\text { No. }\end{array}$ & Category & Subcategory & $\begin{array}{c}\text { Retouch } \\
\text { Affiliation }\end{array}$ & $\begin{array}{l}\text { Specimen } \\
\text { Condition }\end{array}$ & Breakage & Raw material & Munsell & Color & $\begin{array}{c}\text { Dorsal } \\
\text { Cortex \% }\end{array}$ & $\begin{array}{c}\text { Heat } \\
\text { Evidence }\end{array}$ & $\begin{array}{c}\text { Intentional } \\
\text { Heat } \\
\text { Treatment }\end{array}$ & Patination & Retouch Detail & $\begin{array}{c}\text { Retouch } \\
\text { Distribution }\end{array}$ & $\begin{array}{l}\text { Retouch } \\
\text { Location }\end{array}$ \\
\hline \multirow{3}{*}{547} & \multirow{3}{*}{4} & \multirow{3}{*}{$330 \quad \mathrm{NA}$} & Modified Flake & Edge-modified & Cultural & Indet & $\begin{array}{c}\text { Snap and } \\
\text { Bend }\end{array}$ & $\begin{array}{c}\text { Fine Grain } \\
\text { Chert } \\
\end{array}$ & $\begin{array}{l}10 Y R 7 / 1 \\
\text { to10YR7/2 } \\
\end{array}$ & Light Gray & 0 & None & NA & None & Unifacial & Indet & Indet \\
\hline & & & Weight (g) & Length & Width & Thickness & $\begin{array}{l}\text { Height of } \\
\text { Retouch }\end{array}$ & $\begin{array}{l}\text { Perimeter } \\
\text { Retouch } \\
\text { Lenath }\end{array}$ & $\begin{array}{l}\text { Average } \\
\text { Edge } \\
\text { Anale } \\
\end{array}$ & $\begin{array}{l}\text { Reduction } \\
\text { Index }\end{array}$ & $\begin{array}{l}\text { Platform } \\
\text { width }\end{array}$ & $\begin{array}{l}\text { Platform } \\
\text { thickness }\end{array}$ & & & Comments & & \\
\hline & & & 2.29 & Indet & Indet & 3.74 & Indet & Indet & Indet & Indet & Indet & Indet & $\begin{array}{l}\text { Incomplete } \\
\text { retouch is } 1.59\end{array}$ & $\begin{array}{l}\text { flake with no } \\
3 \mathrm{~mm} \text {; Worked }\end{array}$ & $\begin{array}{l}\text { discernable platfo } \\
\mathrm{d} \text { edge is incomple }\end{array}$ & $\begin{array}{l}\text { form or orientati } \\
\text { plete due to sma }\end{array}$ & $\begin{array}{l}\text { ion; Available } \\
\text { all snap fracture }\end{array}$ \\
\hline Lot No. & $\begin{array}{l}\text { Spec. } \\
\text { No. }\end{array}$ & $\begin{array}{l}\text { Bag UI No } \\
\text { No. }\end{array}$ & Category & Subcategory & $\begin{array}{c}\text { Retouch } \\
\text { Affiliation }\end{array}$ & $\begin{array}{l}\text { Specimen } \\
\text { Condition }\end{array}$ & Breakage & Raw material & Munsell & Color & $\begin{array}{c}\text { Dorsal } \\
\text { Cortex \% }\end{array}$ & $\begin{array}{c}\text { Heat } \\
\text { Evidence }\end{array}$ & $\begin{array}{c}\text { Intentional } \\
\text { Heat } \\
\text { Treatment }\end{array}$ & Patination & Retouch Detail & $\begin{array}{c}\text { Retouch } \\
\text { Distribution }\end{array}$ & $\begin{array}{l}\text { Retouch } \\
\text { Location }\end{array}$ \\
\hline \multirow{3}{*}{554} & \multirow{3}{*}{5} & \multirow{3}{*}{$395 \quad \mathrm{NA}$} & Modified Flake & Utilized Flake & Indet & Complete & None & $\begin{array}{c}\text { Fine Grain } \\
\text { Chert }\end{array}$ & 10YR6/2 & $\begin{array}{l}\text { Light Brownish } \\
\text { Gray }\end{array}$ & $76-100$ & None & NA & None & Unifacial-dorsal & Focused & $\begin{array}{l}\text { Right Lateral } \\
\text { Margin/Distal } \\
\text { Margin }\end{array}$ \\
\hline & & & Weight (g) & Length & Width & Thickness & $\begin{array}{l}\text { Height of } \\
\text { Retouch }\end{array}$ & $\begin{array}{l}\text { Perimeter } \\
\text { Retouch } \\
\text { Lenath }\end{array}$ & $\begin{array}{l}\text { Average } \\
\text { Edge } \\
\text { Angle }\end{array}$ & $\begin{array}{l}\text { Reduction } \\
\text { Index }\end{array}$ & $\begin{array}{l}\text { Platform } \\
\text { width }\end{array}$ & $\begin{array}{l}\text { Platform } \\
\text { thickness }\end{array}$ & \multicolumn{5}{|c|}{ Comments } \\
\hline & & & 20.56 & 71.47 & 49.2 & 6.5 & 2.32 & 37 & 45 & 0.36 & 13.49 & 1.08 & $\begin{array}{l}\text { Entire dorsa } \\
\text { distal marg }\end{array}$ & $\begin{array}{l}\text { Il face is corte } \\
\text { in to approxim } \\
\text { platform has }\end{array}$ & $\begin{array}{l}\text { ex; Apparent use-v } \\
\text { mately } 1 / 3 \text { of right } \\
\text { as multiple small } s\end{array}$ & $\begin{array}{l}\text {-wear from the } \\
\text { t lateral margin; } \\
\text { step fractures }\end{array}$ & $\begin{array}{l}\text { middle of the } \\
\text {; Dorsal side }\end{array}$ \\
\hline
\end{tabular}




\begin{tabular}{|c|c|c|c|c|c|c|c|c|c|c|c|c|c|c|c|c|c|}
\hline Lot No. & $\begin{array}{l}\text { Spec. } \\
\text { No. }\end{array}$ & $\begin{array}{l}\text { Bag UI No } \\
\text { No. }\end{array}$ & Category & Subcategory & $\begin{array}{c}\text { Retouch } \\
\text { Affiliation }\end{array}$ & $\begin{array}{l}\text { Specimen } \\
\text { Condition }\end{array}$ & Breakage & Raw material & Munsell & Color & $\begin{array}{l}\text { Dorsal } \\
\text { Cortex \% }\end{array}$ & $\begin{array}{c}\text { Heat } \\
\text { Evidence }\end{array}$ & $\begin{array}{l}\text { Intentional } \\
\text { Heat } \\
\text { Treatment }\end{array}$ & Patination & Retouch Detail & $\begin{array}{l}\text { Retouch } \\
\text { Distribution }\end{array}$ & $\begin{array}{l}\text { Retouch } \\
\text { Location }\end{array}$ \\
\hline \multirow{3}{*}{563} & \multirow{3}{*}{1} & \multirow{3}{*}{372} & Modified Flake & Edge-modified & Cultural & Complete & None & $\begin{array}{c}\text { Fine Grain } \\
\text { Chert }\end{array}$ & 10YR4/2 & $\begin{array}{c}\text { Light Brownish } \\
\text { Gray }\end{array}$ & $1-25$ & None & NA & None & Unifacial-ventral & Continuous & $\begin{array}{c}\text { Right Lateral } \\
\text { Margin }\end{array}$ \\
\hline & & & Weight (g) & Length & Width & Thickness & $\begin{array}{l}\text { Height of } \\
\text { Retouch }\end{array}$ & $\begin{array}{l}\text { Perimeter } \\
\text { Retouch } \\
\text { Length } \\
\end{array}$ & $\begin{array}{l}\text { Average } \\
\text { Edge } \\
\text { Angle }\end{array}$ & $\begin{array}{l}\text { Reduction } \\
\text { Index }\end{array}$ & $\begin{array}{l}\text { Platform } \\
\text { width }\end{array}$ & $\begin{array}{l}\text { Platform } \\
\text { thickness }\end{array}$ & \multicolumn{5}{|c|}{ Comments } \\
\hline & & & 8.94 & 40.31 & 23.45 & 13.25 & 2.72 & 30 & 45 & 0.21 & 23.31 & 9.39 & \multicolumn{5}{|c|}{$\begin{array}{c}\text { Cortex at proximal end on dorsal side; Severe step fractures at proximal } \\
\text { end on left lateral margin }\end{array}$} \\
\hline Lot No. & $\begin{array}{l}\text { Spec. } \\
\text { No. }\end{array}$ & $\begin{array}{l}\text { Bag UI No } \\
\text { No. }\end{array}$ & Category & Subcategory & $\begin{array}{c}\text { Retouch } \\
\text { Affiliation }\end{array}$ & $\begin{array}{l}\text { Specimen } \\
\text { Condition }\end{array}$ & Breakage & Raw material & Munsell & Color & $\begin{array}{l}\text { Dorsal } \\
\text { Cortex \% }\end{array}$ & $\begin{array}{c}\text { Heat } \\
\text { Evidence }\end{array}$ & $\begin{array}{l}\text { Intentional } \\
\text { Heat } \\
\text { Treatment }\end{array}$ & Patination & Retouch Detail & $\begin{array}{c}\text { Retouch } \\
\text { Distribution }\end{array}$ & $\begin{array}{l}\text { Retouch } \\
\text { Location }\end{array}$ \\
\hline \multirow{3}{*}{587} & \multirow{3}{*}{1} & \multirow{3}{*}{$527 \quad$ NA } & Modified Flake & Edge-modified & Indet & $\begin{array}{l}\text { Proximal- } \\
\text { Medial }\end{array}$ & Snap & $\begin{array}{l}\text { Fine Grain } \\
\text { Chert }\end{array}$ & 10YR4/1 & Dark Gray & 0 & None & NA & None & Unifacial-dorsal & Continuous & $\begin{array}{l}\text { Left and Right } \\
\text { Lateral } \\
\text { Margins } \\
\end{array}$ \\
\hline & & & Weight (g) & Length & Width & Thickness & $\begin{array}{l}\text { Height of } \\
\text { Retouch }\end{array}$ & $\begin{array}{l}\text { Perimeter } \\
\text { Retouch } \\
\text { Length }\end{array}$ & $\begin{array}{l}\text { Average } \\
\text { Edge } \\
\text { Anqle }\end{array}$ & $\begin{array}{l}\text { Reduction } \\
\text { Index }\end{array}$ & $\begin{array}{l}\text { Platform } \\
\text { width }\end{array}$ & $\begin{array}{l}\text { Platform } \\
\text { thickness }\end{array}$ & \multicolumn{5}{|c|}{ Comments } \\
\hline & & & 7.8 & Indet & Indet & Indet & Indet & Indet & Indet & Indet & 7.98 & 2.13 & \multicolumn{5}{|c|}{$\begin{array}{l}\text { Bands of } 10 Y R 5 / 4 \text { yellowish brown; Breaks on right lateral margin and } \\
\text { distal margin; Thin flake with very fine retouch on all available edges }\end{array}$} \\
\hline Lot No. & $\begin{array}{l}\text { Spec. } \\
\text { No. }\end{array}$ & $\begin{array}{l}\text { Bag UI No } \\
\text { No. }\end{array}$ & Category & Subcategory & $\begin{array}{c}\text { Retouch } \\
\text { Affiliation }\end{array}$ & $\begin{array}{l}\text { Specimen } \\
\text { Condition }\end{array}$ & Breakage & Raw material & Munsell & Color & $\begin{array}{c}\text { Dorsal } \\
\text { Cortex \% }\end{array}$ & $\begin{array}{c}\text { Heat } \\
\text { Evidence }\end{array}$ & $\begin{array}{l}\text { Intentional } \\
\text { Heat } \\
\text { Treatment }\end{array}$ & Patination & Retouch Detail & $\begin{array}{c}\text { Retouch } \\
\text { Distribution }\end{array}$ & $\begin{array}{l}\text { Retouch } \\
\text { Location }\end{array}$ \\
\hline \multirow{3}{*}{590} & \multirow{3}{*}{6} & \multirow{3}{*}{$557 \quad$ NA } & Modified Flake & Edge-modified & Indet & Complete & None & $\begin{array}{l}\text { Fine Grain } \\
\text { Chert }\end{array}$ & 10YR7/1 & Light Gray & $1-25$ & None & NA & None & Unifacial-ventral & Focused & $\begin{array}{l}\text { Left Lateral } \\
\text { Margin }\end{array}$ \\
\hline & & & Weight (g) & Length & Width & Thickness & $\begin{array}{l}\text { Height of } \\
\text { Retouch }\end{array}$ & $\begin{array}{l}\text { Perimeter } \\
\text { Retouch } \\
\text { Lenath }\end{array}$ & $\begin{array}{l}\text { Average } \\
\text { Edge } \\
\text { Anqle }\end{array}$ & $\begin{array}{l}\text { Reduction } \\
\text { Index }\end{array}$ & $\begin{array}{l}\text { Platform } \\
\text { width }\end{array}$ & $\begin{array}{l}\text { Platform } \\
\text { thickness }\end{array}$ & & & Comments & & \\
\hline & & & 40.92 & Indet & 43.92 & 15.92 & 1.63 & 37 & 50 & 0.10 & 27.02 & 14.59 & \multicolumn{5}{|c|}{$\begin{array}{l}\text { Complete except for very distal tip; Distal break has step fractures on a } \\
\text { snap break; Work focused on left lateral margin center and does not } \\
\text { appear to continue to the distal break }\end{array}$} \\
\hline Lot No. & $\begin{array}{l}\text { Spec. } \\
\text { No. }\end{array}$ & $\begin{array}{l}\text { Bag UI No } \\
\text { No. }\end{array}$ & Category & Subcategory & $\begin{array}{c}\text { Retouch } \\
\text { Affiliation }\end{array}$ & $\begin{array}{l}\text { Specimen } \\
\text { Condition }\end{array}$ & Breakage & Raw material & Munsell & Color & $\begin{array}{c}\text { Dorsal } \\
\text { Cortex \% }\end{array}$ & $\begin{array}{c}\text { Heat } \\
\text { Evidence }\end{array}$ & $\begin{array}{l}\text { Intentional } \\
\text { Heat } \\
\text { Treatment }\end{array}$ & Patination & Retouch Detail & $\begin{array}{l}\text { Retouch } \\
\text { Distribution }\end{array}$ & $\begin{array}{l}\text { Retouch } \\
\text { Location }\end{array}$ \\
\hline \multirow{3}{*}{590} & \multirow{3}{*}{7} & \multirow{3}{*}{557 NA } & Modified Flake & Edge-modified & Cultural & Medial & Bend & $\begin{array}{c}\text { Fine Grain } \\
\text { Chert }\end{array}$ & 10YR6/2 & $\begin{array}{c}\text { Light brownish } \\
\text { Gray }\end{array}$ & 0 & None & NA & None & Indet & Indet & Indet \\
\hline & & & Weight (g) & Length & Width & Thickness & $\begin{array}{l}\text { Height of } \\
\text { Retouch }\end{array}$ & $\begin{array}{l}\text { Perimeter } \\
\text { Retouch } \\
\text { Length }\end{array}$ & $\begin{array}{l}\text { Average } \\
\text { Edge } \\
\text { Angle }\end{array}$ & $\begin{array}{l}\text { Reduction } \\
\text { Index }\end{array}$ & $\begin{array}{l}\text { Platform } \\
\text { width }\end{array}$ & $\begin{array}{l}\text { Platform } \\
\text { thickness }\end{array}$ & \multicolumn{5}{|c|}{ 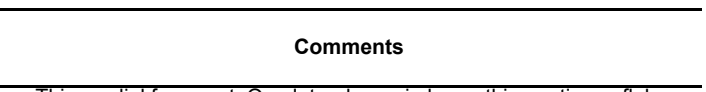 } \\
\hline & & & 3.63 & Indet & 28.21 & 4.07 & Indet & Indet & Indet & Indet & Indet & Indet & \multicolumn{5}{|c|}{$\begin{array}{l}\text { Thin medial fragment; One lateral margin has a thin continues flake } \\
\text { removed creating a sharp edge - no use-wear evident; Parallel margin has } \\
\text { been worked unifacially }\end{array}$} \\
\hline
\end{tabular}




\begin{tabular}{|c|c|c|c|c|c|c|c|c|c|c|c|c|c|c|c|c|c|}
\hline Lot No. & $\begin{array}{l}\text { Spec. } \\
\text { No. }\end{array}$ & $\begin{array}{l}\text { Bag UI No } \\
\text { No. }\end{array}$ & Category & Subcategory & $\begin{array}{c}\text { Retouch } \\
\text { Affiliation }\end{array}$ & $\begin{array}{l}\text { Specimen } \\
\text { Condition }\end{array}$ & Breakage & Raw material & Munsell & Color & $\begin{array}{l}\text { Dorsal } \\
\text { Cortex \% }\end{array}$ & $\begin{array}{c}\text { Heat } \\
\text { Evidence }\end{array}$ & $\begin{array}{c}\text { Intentional } \\
\text { Heat } \\
\text { Treatment }\end{array}$ & Patination & Retouch Detail & $\begin{array}{c}\text { Retouch } \\
\text { Distribution }\end{array}$ & $\begin{array}{l}\text { Retouch } \\
\text { Location } \\
\end{array}$ \\
\hline \multirow{3}{*}{598} & \multirow{3}{*}{3} & \multirow{3}{*}{659} & Modified Flake & Utilized Flake & Indet & $\begin{array}{c}\text { Proximal- } \\
\text { Medial }\end{array}$ & Snap & $\begin{array}{c}\text { Coarse Grain } \\
\text { Chert }\end{array}$ & $\begin{array}{c}\text { 10YR4/1 to } \\
\text { 10YR5/1 }\end{array}$ & $\begin{array}{c}\text { Dark Gray to } \\
\text { Gray }\end{array}$ & 0 & None & NA & Yes & Bifacial & Indet & $\begin{array}{l}\text { Left and Right } \\
\text { Lateral Margin }\end{array}$ \\
\hline & & & Weight (g) & Length & Width & Thickness & $\begin{array}{l}\text { Height of } \\
\text { Retouch }\end{array}$ & $\begin{array}{c}\text { Perimeter } \\
\text { Retouch } \\
\text { Length }\end{array}$ & $\begin{array}{c}\text { Average } \\
\text { Edge } \\
\text { Angle } \\
\end{array}$ & $\begin{array}{c}\text { Reduction } \\
\text { Index }\end{array}$ & $\begin{array}{l}\text { Platform } \\
\text { width }\end{array}$ & $\begin{array}{l}\text { Platform } \\
\text { thickness }\end{array}$ & \multicolumn{5}{|c|}{ Comments } \\
\hline & & & 5.79 & Indet & Indet & Indet & Indet & Indet & Indet & Indet & 8.75 & 2.95 & \multicolumn{5}{|c|}{$\begin{array}{l}\text { Very fine retouching along both lateral margins - difficult to discern; May } \\
\text { have been caused by natural processes }\end{array}$} \\
\hline Lot No. & $\begin{array}{l}\text { Spec. } \\
\text { No. }\end{array}$ & $\begin{array}{l}\text { Bag UI No } \\
\text { No. }\end{array}$ & Category & Subcategory & $\begin{array}{l}\text { Retouch } \\
\text { Affiliation }\end{array}$ & $\begin{array}{l}\text { Specimen } \\
\text { Condition }\end{array}$ & Breakage & Raw material & Munsell & Color & $\begin{array}{c}\text { Dorsal } \\
\text { Cortex \% }\end{array}$ & $\begin{array}{c}\text { Heat } \\
\text { Evidence }\end{array}$ & $\begin{array}{c}\text { Intentional } \\
\text { Heat } \\
\text { Treatment }\end{array}$ & Patination & Retouch Detail & $\begin{array}{c}\text { Retouch } \\
\text { Distribution }\end{array}$ & $\begin{array}{l}\text { Retouch } \\
\text { Location }\end{array}$ \\
\hline \multirow{3}{*}{608} & \multirow{3}{*}{3} & \multirow{3}{*}{510 NA } & Modified Flake & Edge-modified & Indet & $\begin{array}{l}\text { Left Lateral } \\
\text { Margin Distal } \\
\text { Tip Missing }\end{array}$ & Snap & $\begin{array}{l}\text { Fine Grain } \\
\text { Chert }\end{array}$ & $\begin{array}{l}\text { 10YR5/1 to } \\
\text { 10YR5/2 }\end{array}$ & $\begin{array}{l}\text { Gray to Grayish } \\
\text { Brown }\end{array}$ & 0 & None & NA & None & Unifacial-dorsal & Focused & $\begin{array}{l}\text { Distal//Right } \\
\text { Lateral Margin }\end{array}$ \\
\hline & & & Weight (g) & Length & Width & Thickness & $\begin{array}{l}\text { Height of } \\
\text { Retouch }\end{array}$ & $\begin{array}{l}\text { Perimeter } \\
\text { Retouch } \\
\text { Length }\end{array}$ & $\begin{array}{l}\text { Average } \\
\text { Edge } \\
\text { Angle }\end{array}$ & $\begin{array}{l}\text { Reduction } \\
\text { Index }\end{array}$ & $\begin{array}{l}\text { Platform } \\
\text { width }\end{array}$ & $\begin{array}{l}\text { Platform } \\
\text { thickness }\end{array}$ & \multicolumn{5}{|c|}{ Comments } \\
\hline & & & 6.13 & 68.45 & 24.18 & 2.51 & 1.3 & 32 & 30 & 0.52 & Indet & Indet & \multicolumn{5}{|c|}{$\begin{array}{c}\text { Two flakes are in this bag - thin blade flake; Small area with mineral } \\
\text { inclusions; Small break near proximal end on the left lateral margin and at } \\
\text { distal tip; Platform has been removed (step fracture) but the bulb is still } \\
\text { evident; Light use-wear etching on distal end }\end{array}$} \\
\hline
\end{tabular}

\begin{tabular}{|c|c|c|c|c|c|c|c|c|c|c|c|c|c|c|c|c|c|}
\hline Lot No. & $\begin{array}{l}\text { Spec. } \\
\text { No. }\end{array}$ & $\begin{array}{l}\text { Bag UI No } \\
\text { No. }\end{array}$ & Category & Subcategory & $\begin{array}{c}\text { Retouch } \\
\text { Affiliation }\end{array}$ & $\begin{array}{l}\text { Specimen } \\
\text { Condition }\end{array}$ & Breakage & Raw material & Munsell & Color & $\begin{array}{c}\text { Dorsal } \\
\text { Cortex \% }\end{array}$ & $\begin{array}{c}\text { Heat } \\
\text { Evidence }\end{array}$ & $\begin{array}{l}\text { Intentional } \\
\text { Heat } \\
\text { Treatment }\end{array}$ & Patination & Retouch Detail & $\begin{array}{l}\text { Retouch } \\
\text { Distribution }\end{array}$ & $\begin{array}{l}\text { Retouch } \\
\text { Location }\end{array}$ \\
\hline \multirow{3}{*}{608} & \multirow{3}{*}{4} & \multirow{3}{*}{$510 \mathrm{NA}$} & Modified Flake & Edge-modified & Indet & $\begin{array}{l}\text { Left Lateral } \\
\text { Margin } \\
\text { Missing }\end{array}$ & Snap & $\begin{array}{c}\text { Fine Grain } \\
\text { Chert }\end{array}$ & $\begin{array}{l}\text { 10YR5/1 to } \\
10 Y R 5 / 2\end{array}$ & $\begin{array}{l}\text { Gray to Grayish } \\
\text { Brown }\end{array}$ & 0 & None & NA & None & Unifacial-dorsal & Clustered & $\begin{array}{l}\text { Left Lateral } \\
\text { Margin/Distal } \\
\text { Margin }\end{array}$ \\
\hline & & & Weight (g) & Length & Width & Thickness & $\begin{array}{l}\text { Height of } \\
\text { Retouch }\end{array}$ & $\begin{array}{l}\text { Perimeter } \\
\text { Retouch } \\
\text { Lenath }\end{array}$ & $\begin{array}{l}\text { Average } \\
\text { Edge } \\
\text { Angle }\end{array}$ & $\begin{array}{l}\text { Reduction } \\
\text { Index }\end{array}$ & $\begin{array}{l}\text { Platform } \\
\text { width }\end{array}$ & $\begin{array}{l}\text { Platform } \\
\text { thickness }\end{array}$ & \multicolumn{5}{|c|}{ Comments } \\
\hline & & & 24.05 & Indet & 57.32 & 7.75 & $1.75 ; 0.65$ & $64 ; 33$ & $35 ; 70$ & $0.23 ; 0.08$ & 9 & 5 & \multicolumn{5}{|c|}{$\begin{array}{l}\text { Second flake from bag - large flake; Platform is completely cortex; use- } \\
\text { wear striations on distal edge; Two areas of retouch, measurement are } \\
\text { separated for each area }\end{array}$} \\
\hline
\end{tabular}

\begin{tabular}{|c|c|c|c|c|c|c|c|c|c|c|c|c|c|c|c|c|c|}
\hline Lot No. & $\begin{array}{l}\text { Spec. } \\
\text { No. }\end{array}$ & $\begin{array}{l}\text { Bag UI No } \\
\text { No. }\end{array}$ & Category & Subcategory & $\begin{array}{l}\text { Retouch } \\
\text { Affiliation }\end{array}$ & $\begin{array}{l}\text { Specimen } \\
\text { Condition }\end{array}$ & Breakage & Raw material & Munsell & Color & $\begin{array}{c}\text { Dorsal } \\
\text { Cortex \% }\end{array}$ & $\begin{array}{c}\text { Heat } \\
\text { Evidence }\end{array}$ & $\begin{array}{c}\text { Intentional } \\
\text { Heat } \\
\text { Treatment }\end{array}$ & Patination & Retouch Detail & $\begin{array}{c}\text { Retouch } \\
\text { Distribution }\end{array}$ & $\begin{array}{l}\text { Retouch } \\
\text { Location }\end{array}$ \\
\hline \multirow{3}{*}{615} & \multirow[b]{3}{*}{5} & \multirow{3}{*}{$441 \mathrm{NA}$} & Modified Flake & Edge-modified & Indet & Complete & None & $\begin{array}{c}\text { Fine Grain } \\
\text { Chert }\end{array}$ & $\begin{array}{c}10 Y R 5 / 1 \text { to } \\
10 Y R 5 / 2\end{array}$ & $\begin{array}{l}\text { Gray to Grayish } \\
\text { Brown }\end{array}$ & $26-50$ & None & NA & None & Unifacial-ventral & Focused & $\begin{array}{l}\text { Left Lateral } \\
\text { Margin }\end{array}$ \\
\hline & & & Weight (g) & Length & Width & Thickness & $\begin{array}{l}\text { Height of } \\
\text { Retouch }\end{array}$ & $\begin{array}{l}\text { Perimeter } \\
\text { Retouch } \\
\text { Length }\end{array}$ & $\begin{array}{l}\text { Average } \\
\text { Edge } \\
\text { Angle }\end{array}$ & $\begin{array}{c}\text { Reduction } \\
\text { Index }\end{array}$ & $\begin{array}{l}\text { Platform } \\
\text { width }\end{array}$ & $\begin{array}{l}\text { Platform } \\
\text { thickness }\end{array}$ & \multicolumn{5}{|c|}{ Comments } \\
\hline & & & 25.02 & 60.48 & 32.53 & 13.69 & 3.66 & 11 & 60 & 0.27 & Indet & Indet & $\begin{array}{l}\text { Original platf } \\
\text { was then reto } \\
\text { throughout the } \\
\text { lateral margin }\end{array}$ & $\begin{array}{l}\text { rm is gone d } \\
\text { uched to crea } \\
\text { flake; Worke } \\
\text { but larger fla } \\
\text { nargin may ha }\end{array}$ & $\begin{array}{l}\text { lue to break along } \\
\text { ate utilized flake); } \\
\text { ed area is focused } \\
\text { ake removal along } \\
\text { lave been prep for }\end{array}$ & $\begin{array}{l}\text { left lateral mar } \\
\text { Large mineral } \\
\text { near the dista } \\
\text { g medial and pr } \\
r \text { further workin }\end{array}$ & $\begin{array}{l}\text { gin (the break } \\
\text { inclusions are } \\
\text { l end of the left } \\
\text { oximal area of } \\
\text { g }\end{array}$ \\
\hline
\end{tabular}




\begin{tabular}{|c|c|c|c|c|c|c|c|c|c|c|c|c|c|c|c|c|c|}
\hline Lot No. & $\begin{array}{l}\text { Spec. } \\
\text { No. }\end{array}$ & $\begin{array}{l}\text { Bag UI No } \\
\text { No. }\end{array}$ & Category & Subcategory & $\begin{array}{l}\text { Retouch } \\
\text { Affiliation }\end{array}$ & $\begin{array}{l}\text { Specimen } \\
\text { Condition }\end{array}$ & Breakage & Raw material & Munsell & Color & $\begin{array}{l}\text { Dorsal } \\
\text { Cortex } \%\end{array}$ & $\begin{array}{c}\text { Heat } \\
\text { Evidence }\end{array}$ & $\begin{array}{l}\text { Intentional } \\
\text { Heat } \\
\text { Treatment }\end{array}$ & Patination & Retouch Detail & $\begin{array}{c}\text { Retouch } \\
\text { Distribution }\end{array}$ & $\begin{array}{l}\text { Retouch } \\
\text { Location } \\
\end{array}$ \\
\hline \multirow{3}{*}{618} & \multirow{3}{*}{7} & \multirow{3}{*}{479 NA } & Modified Flake & Edge-modified & Indet & Medial & Snap & $\begin{array}{c}\text { Fine Grain } \\
\text { Chert }\end{array}$ & 10YR7/2 & Light Gray & 0 & Reddened & Yes & None & Unifacial-ventral & Indet & $\begin{array}{l}\text { Right Lateral } \\
\text { Margin }\end{array}$ \\
\hline & & & Weight (g) & Length & Width & Thickness & $\begin{array}{l}\text { Height of } \\
\text { Retouch }\end{array}$ & $\begin{array}{l}\text { Perimeter } \\
\text { Retouch } \\
\text { Length }\end{array}$ & $\begin{array}{l}\text { Average } \\
\text { Edge } \\
\text { Angle }\end{array}$ & $\begin{array}{l}\text { Reduction } \\
\text { Index }\end{array}$ & $\begin{array}{l}\text { Platform } \\
\text { width }\end{array}$ & $\begin{array}{l}\text { Platform } \\
\text { thickness }\end{array}$ & \multicolumn{5}{|c|}{ Comments } \\
\hline & & & 24.17 & Indet & Indet & Indet & Indet & Indet & Indet & Indet & Indet & Indet & $\begin{array}{r}\text { Medial fragmer } \\
\text { used for orien }\end{array}$ & $\begin{array}{l}\text { ent missing pro } \\
\text { entation; Heat t }\end{array}$ & $\begin{array}{c}\text { oximal and distal } € \\
\text { treatment evident } \\
\text { Brown) }\end{array}$ & $\begin{array}{l}\text { end; Bulb still } \\
\text { it as } 2.5 Y R 6 / 3 \\
\end{array}$ & $\begin{array}{l}\text { discernable and } \\
\text { (Light Reddish }\end{array}$ \\
\hline Lot No. & $\begin{array}{l}\text { Spec. } \\
\text { No. }\end{array}$ & $\begin{array}{l}\text { Bag UI No } \\
\text { No. }\end{array}$ & Category & Subcategory & $\begin{array}{c}\text { Retouch } \\
\text { Affiliation }\end{array}$ & $\begin{array}{l}\text { Specimen } \\
\text { Condition }\end{array}$ & Breakage & Raw material & Munsell & Color & $\begin{array}{c}\text { Dorsal } \\
\text { Cortex \% }\end{array}$ & $\begin{array}{c}\text { Heat } \\
\text { Evidence }\end{array}$ & $\begin{array}{l}\text { Intentional } \\
\text { Heat } \\
\text { Treatment }\end{array}$ & Patination & Retouch Detail & $\begin{array}{c}\text { Retouch } \\
\text { Distribution }\end{array}$ & $\begin{array}{l}\text { Retouch } \\
\text { Location }\end{array}$ \\
\hline \multirow{3}{*}{618} & \multirow{3}{*}{8} & \multirow{3}{*}{479 NA } & Modified Flake & Edge-modified & Cultural & Indet & Snap & $\begin{array}{c}\text { Fine Grain } \\
\text { Chert }\end{array}$ & 10YR4/1 & Dark Gray & 0 & Reddened & Yes & None & Unifacial-ventral & Focused & Indet \\
\hline & & & Weight (g) & Length & Width & Thickness & $\begin{array}{l}\text { Height of } \\
\text { Retouch }\end{array}$ & $\begin{array}{l}\text { Perimeter } \\
\text { Retouch } \\
\text { Lenqth }\end{array}$ & $\begin{array}{l}\text { Average } \\
\text { Edge } \\
\text { Angle } \\
\end{array}$ & $\begin{array}{l}\text { Reduction } \\
\text { Index }\end{array}$ & $\begin{array}{l}\text { Platform } \\
\text { width }\end{array}$ & $\begin{array}{l}\text { Platform } \\
\text { thickness }\end{array}$ & & & Comments & & \\
\hline & & & 3.06 & Indet & 21.32 & 3 & 2.22 & 13 & 60 & 0.74 & Indet & Indet & \multicolumn{5}{|c|}{$\begin{array}{l}\text { Platform is completely removed but appears to have been at the location } \\
\text { of the modified edge due to a slight bulb of percussion; Modified edge is } \\
\text { small and focused at one end of flake (assumed proximal end); Very light } \\
\text { reddened spots seen throughout material; Snar }\end{array}$} \\
\hline Lot No. & $\begin{array}{l}\text { Spec. } \\
\text { No. }\end{array}$ & $\begin{array}{l}\text { Bag UI No } \\
\text { No. }\end{array}$ & Category & Subcategory & $\begin{array}{c}\text { Retouch } \\
\text { Affiliation }\end{array}$ & $\begin{array}{l}\text { Specimen } \\
\text { Condition }\end{array}$ & Breakage & Raw material & Munsell & Color & $\begin{array}{l}\text { Dorsal } \\
\text { Cortex } \%\end{array}$ & $\begin{array}{c}\text { Heat } \\
\text { Evidence }\end{array}$ & $\begin{array}{l}\text { Intentional } \\
\text { Heat } \\
\text { Treatment }\end{array}$ & Patination & Retouch Detail & $\begin{array}{c}\text { Retouch } \\
\text { Distribution }\end{array}$ & $\begin{array}{l}\text { Retouch } \\
\text { Location }\end{array}$ \\
\hline \multirow{3}{*}{619} & \multirow{3}{*}{6} & \multirow{3}{*}{$530 \mathrm{NA}$} & Modified Flake & Utilized Flake & Indet & $\begin{array}{l}\text { Right Lateral } \\
\text { Margin } \\
\text { Missing }\end{array}$ & Snap & $\begin{array}{l}\text { Fine Grain } \\
\text { Chert }\end{array}$ & 10YR6/2 & $\begin{array}{l}\text { Light Brownish } \\
\text { Gray }\end{array}$ & 0 & None & NA & None & Unifacial-dorsal & Continuous & $\begin{array}{l}\text { Left Lateral } \\
\text { Margin/Distal } \\
\quad \text { Margin }\end{array}$ \\
\hline & & & Weight (g) & Length & Width & Thickness & $\begin{array}{l}\text { Height of } \\
\text { Retouch }\end{array}$ & $\begin{array}{l}\text { Perimeter } \\
\text { Retouch } \\
\text { Length }\end{array}$ & $\begin{array}{l}\text { Average } \\
\text { Edge } \\
\text { Angle } \\
\end{array}$ & $\begin{array}{l}\text { Reduction } \\
\text { Index }\end{array}$ & $\begin{array}{l}\text { Platform } \\
\text { width }\end{array}$ & $\begin{array}{l}\text { Platform } \\
\text { thickness }\end{array}$ & & & Comments & & \\
\hline & & & 7.7 & 57.42 & 36.08 & 4.28 & None & 65 & 20 & Indet & 6.5 & 1.61 & \multicolumn{5}{|c|}{$\begin{array}{l}\text { Very small break on right lateral margin; use almost exclusively on very } \\
\text { edge with very slight flake removal on dorsal side }\end{array}$} \\
\hline Lot No. & $\begin{array}{l}\text { Spec. } \\
\text { No. }\end{array}$ & $\begin{array}{l}\text { Bag UI No } \\
\text { No. }\end{array}$ & Category & Subcategory & $\begin{array}{c}\text { Retouch } \\
\text { Affiliation }\end{array}$ & $\begin{array}{l}\text { Specimen } \\
\text { Condition }\end{array}$ & Breakage & Raw material & Munsell & Color & $\begin{array}{c}\text { Dorsal } \\
\text { Cortex \% }\end{array}$ & $\begin{array}{c}\text { Heat } \\
\text { Evidence }\end{array}$ & $\begin{array}{l}\text { Intentional } \\
\text { Heat } \\
\text { Treatment }\end{array}$ & Patination & Retouch Detail & $\begin{array}{c}\text { Retouch } \\
\text { Distribution }\end{array}$ & $\begin{array}{l}\text { Retouch } \\
\text { Location }\end{array}$ \\
\hline \multirow{3}{*}{621} & \multirow{3}{*}{5} & \multirow{3}{*}{539 NA } & Modified Flake & Utilized Flake & Indet & Complete & None & $\begin{array}{l}\text { Coarse Grain } \\
\text { Chert }\end{array}$ & 10YR6/2 & $\begin{array}{l}\text { Light Brownish } \\
\text { Gray }\end{array}$ & $26-50$ & None & NA & None & Bifacial & Continuous & $\begin{array}{l}\text { Left Lateral } \\
\text { Margin/ Right } \\
\text { Lateral } \\
\text { Margins }\end{array}$ \\
\hline & & & Weight (g) & Length & Width & Thickness & $\begin{array}{l}\text { Height of } \\
\text { Retouch }\end{array}$ & $\begin{array}{l}\text { Perimeter } \\
\text { Retouch } \\
\text { Length } \\
\end{array}$ & $\begin{array}{l}\text { Average } \\
\text { Edge } \\
\text { Angle }\end{array}$ & $\begin{array}{l}\text { Reduction } \\
\text { Index }\end{array}$ & $\begin{array}{l}\text { Platform } \\
\text { width }\end{array}$ & $\begin{array}{l}\text { Platform } \\
\text { thickness }\end{array}$ & \multicolumn{5}{|c|}{ Comments } \\
\hline & & & 72.36 & 100.42 & 46.83 & 20.5 & 1.3 & $93 / 70$ & $60 / 70$ & 0.06 & 17.18 & 7.86 & \multicolumn{5}{|c|}{$\begin{array}{l}\text { Mottled with 10YR7/2 (Light Gray) } 35 \% \text {; Large flake with straight to } \\
\text { concave left lateral margin and convex (pointed) right lateral margin; } \\
\text { Utilized edge bifacial on entire LLM and only on ventral face of RLM }\end{array}$} \\
\hline
\end{tabular}




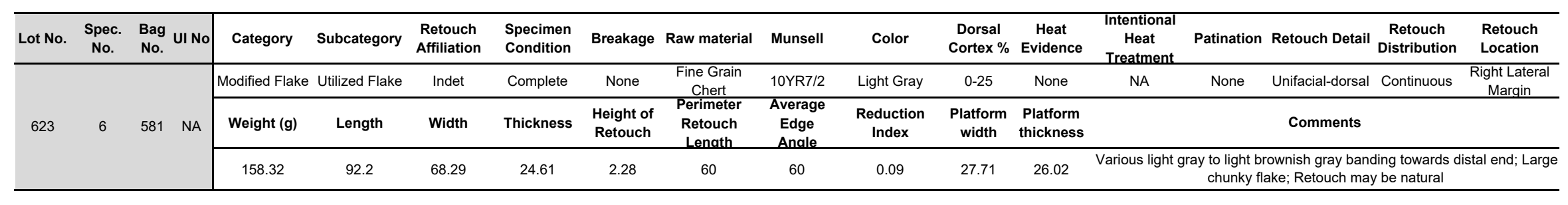

\begin{tabular}{|c|c|c|c|c|c|c|c|c|c|c|c|c|c|c|c|c|c|}
\hline Lot No. & $\begin{array}{l}\text { Spec. } \\
\text { No. }\end{array}$ & $\begin{array}{l}\text { Bag UI No } \\
\text { No. }\end{array}$ & Category & Subcategory & $\begin{array}{c}\text { Retouch } \\
\text { Affiliation }\end{array}$ & $\begin{array}{l}\text { Specimen } \\
\text { Condition }\end{array}$ & Breakage & Raw material & Munsell & Color & $\begin{array}{c}\text { Dorsal } \\
\text { Cortex \% }\end{array}$ & $\begin{array}{c}\text { Heat } \\
\text { Evidence }\end{array}$ & $\begin{array}{c}\text { Intentional } \\
\text { Heat } \\
\text { Treatment }\end{array}$ & Patination & Retouch Detail & $\begin{array}{c}\text { Retouch } \\
\text { Distribution }\end{array}$ & $\begin{array}{l}\text { Retouch } \\
\text { Location } \\
\end{array}$ \\
\hline \multirow{3}{*}{623} & \multirow{3}{*}{7} & \multirow{3}{*}{581 NA } & Modified Flake & Edge-modified & Indet & Medial & Snap & $\begin{array}{c}\text { Coarse Grain } \\
\text { Chert }\end{array}$ & $\begin{array}{c}\text { 10YR5/1 to } \\
\text { 10YR5/2 }\end{array}$ & $\begin{array}{l}\text { Gray to Grayish } \\
\text { Brown }\end{array}$ & 0 & None & NA & None & Bifacial & Indet & $\begin{array}{l}\text { Right and Left } \\
\text { Lateral Margin }\end{array}$ \\
\hline & & & Weight (g) & Length & Width & Thickness & $\begin{array}{l}\text { Height of } \\
\text { Retouch }\end{array}$ & $\begin{array}{c}\text { Perimeter } \\
\text { Retouch } \\
\text { Lenath }\end{array}$ & $\begin{array}{c}\text { Average } \\
\text { Edge } \\
\text { Angle } \\
\end{array}$ & $\begin{array}{l}\text { Reduction } \\
\text { Index }\end{array}$ & $\begin{array}{l}\text { Platform } \\
\text { width }\end{array}$ & $\begin{array}{l}\text { Platform } \\
\text { thickness }\end{array}$ & \multicolumn{5}{|c|}{ Comments } \\
\hline & & & 11.21 & Indet & Indet & Indet & Indet & Indet & Indet & Indet & Indet & Indet & $\begin{array}{l}\text { Working on } \\
\text { inclusions th }\end{array}$ & $\begin{array}{l}\text { both lateral } \mathrm{m} \\
\text { roughout; Med } \\
\text { removal }\end{array}$ & $\begin{array}{l}\text { nargin; Fine fractu } \\
\text { dial portion of tool } \\
\text { l of proximal and d }\end{array}$ & $\begin{array}{l}\text { ures and cluste } \\
\text { I with snap frac } \\
\text { distal ends }\end{array}$ & $\begin{array}{l}\text { ers of mineral } \\
\text { actures causing }\end{array}$ \\
\hline
\end{tabular}

\begin{tabular}{|c|c|c|c|c|c|c|c|c|c|c|c|c|c|c|c|c|c|c|}
\hline Lot No. & $\begin{array}{l}\text { Spec. } \\
\text { No. }\end{array}$ & $\begin{array}{l}\text { Bag } \\
\text { No. }\end{array}$ & UI No & Category & Subcategory & $\begin{array}{c}\text { Retouch } \\
\text { Affiliation }\end{array}$ & $\begin{array}{l}\text { Specimen } \\
\text { Condition }\end{array}$ & Breakage & Raw material & Munsell & Color & $\begin{array}{c}\text { Dorsal } \\
\text { Cortex \% }\end{array}$ & $\begin{array}{c}\text { Heat } \\
\text { Evidence }\end{array}$ & $\begin{array}{c}\text { Intentional } \\
\text { Heat } \\
\text { Treatment } \\
\end{array}$ & Patination & Retouch Detail & $\begin{array}{c}\text { Retouch } \\
\text { Distribution }\end{array}$ & $\begin{array}{l}\text { Retouch } \\
\text { Location } \\
\end{array}$ \\
\hline \multirow{3}{*}{629} & \multirow{3}{*}{4} & \multirow{3}{*}{355} & \multirow{3}{*}{ NA } & Modified Flake & Edge-modified & Cultural & $\begin{array}{l}\text { Proximal- } \\
\text { Medial }\end{array}$ & Snap & $\begin{array}{l}\text { Fine Grain } \\
\text { Chert }\end{array}$ & 10YR4/4 & $\begin{array}{l}\text { Dark Yellowish } \\
\text { Brown }\end{array}$ & 0 & None & - & None & $\begin{array}{l}\text { Bifacial and } \\
\text { Unifacial-dorsal }\end{array}$ & Continuous & $\begin{array}{l}\text { Left Lateral } \\
\text { Margin/Proxim } \\
\text { al/Right Lateral } \\
\text { Marain }\end{array}$ \\
\hline & & & & Weight (g) & Length & Width & Thickness & $\begin{array}{l}\text { Height of } \\
\text { Retouch }\end{array}$ & $\begin{array}{l}\text { Perimeter } \\
\text { Retouch } \\
\text { Length } \\
\end{array}$ & $\begin{array}{c}\text { Average } \\
\text { Edge } \\
\text { Angle } \\
\end{array}$ & $\begin{array}{l}\text { Reduction } \\
\text { Index }\end{array}$ & $\begin{array}{l}\text { Platform } \\
\text { width }\end{array}$ & $\begin{array}{l}\text { Platform } \\
\text { thickness }\end{array}$ & \multicolumn{5}{|c|}{ Comments } \\
\hline & & & & 1.8 & Indet & Indet & Indet & Indet & Indet & Indet & Indet & Indet & Indet & $\begin{array}{l}\text { Flake oriente } \\
\text { margin is unif } \\
\text { bifacially }\end{array}$ & $\begin{array}{l}\text { d by bulb; Ap } \\
\text { acially worked } \\
\text { worked; full }\end{array}$ & $\begin{array}{l}\text { pproximately } 50 \% \\
\text { ed while the proxim } \\
\text { extend of working }\end{array}$ & $\begin{array}{l}\text { of flake missir } \\
\text { nal and right la } \\
g \text { unknown due }\end{array}$ & $\begin{array}{l}\text { ing; Left lateral } \\
\text { ateral margin is } \\
\text { e to break }\end{array}$ \\
\hline
\end{tabular}

\begin{tabular}{|c|c|c|c|c|c|c|c|c|c|c|c|c|c|c|c|c|c|}
\hline Lot No. & $\begin{array}{l}\text { Spec. } \\
\text { No. }\end{array}$ & $\begin{array}{l}\text { Bag UI No } \\
\text { No. }\end{array}$ & Category & Subcategory & $\begin{array}{c}\text { Retouch } \\
\text { Affiliation }\end{array}$ & $\begin{array}{l}\text { Specimen } \\
\text { Condition }\end{array}$ & Breakage & Raw material & Munsell & Color & $\begin{array}{l}\text { Dorsal } \\
\text { Cortex \% }\end{array}$ & $\begin{array}{c}\text { Heat } \\
\text { Evidence }\end{array}$ & $\begin{array}{c}\text { Intentional } \\
\text { Heat } \\
\text { Treatment }\end{array}$ & Patination & Retouch Detail & $\begin{array}{l}\text { Retouch } \\
\text { Distribution }\end{array}$ & $\begin{array}{l}\text { Retouch } \\
\text { Location }\end{array}$ \\
\hline \multirow{3}{*}{632} & \multirow{3}{*}{7} & \multirow{3}{*}{385} & Modified Flake & Edge-modified & Indet & Complete & None & $\begin{array}{l}\text { Fine Grain } \\
\text { Chert }\end{array}$ & $\begin{array}{c}\text { 10YR3/1 to } \\
\text { 10YR3/2 }\end{array}$ & $\begin{array}{l}\text { Very Dark Gray } \\
\text { to Very Dark } \\
\text { Gravish Brow }\end{array}$ & $0-25$ & None & NA & None & Unifacial-ventral & Focused & $\begin{array}{l}\text { Left Lateral } \\
\text { Margin }\end{array}$ \\
\hline & & & Weight (g) & Length & Width & Thickness & $\begin{array}{l}\text { Height of } \\
\text { Retouch }\end{array}$ & $\begin{array}{l}\text { Perimeter } \\
\text { Retouch } \\
\text { Length }\end{array}$ & $\begin{array}{c}\text { Average } \\
\text { Edge } \\
\text { Angle }\end{array}$ & $\begin{array}{l}\text { Reduction } \\
\text { Index }\end{array}$ & $\begin{array}{l}\text { Platform } \\
\text { width }\end{array}$ & $\begin{array}{l}\text { Platform } \\
\text { thickness }\end{array}$ & \multicolumn{5}{|c|}{ Comments } \\
\hline & & & 10.54 & 56.38 & 26.03 & 11.48 & 0.89 & 12 & Indet & 0.08 & 10.57 & 1 & Irregul & ar flake with & one localized area & a of edge mod & fication. \\
\hline
\end{tabular}




\begin{tabular}{|c|c|c|c|c|c|c|c|c|c|c|c|c|c|c|c|c|}
\hline Lot No. & $\begin{array}{l}\text { Spec. } \\
\text { No. }\end{array}$ & $\begin{array}{l}\text { Bag UI No } \\
\text { No. }\end{array}$ & Category & Subcategory & $\begin{array}{c}\text { Retouch } \\
\text { Affiliation }\end{array}$ & $\begin{array}{l}\text { Specimen } \\
\text { Condition }\end{array}$ & Breakage & Raw material & Munsell & Color & $\begin{array}{l}\text { Dorsal } \\
\text { Cortex \% }\end{array}$ & $\begin{array}{c}\text { Heat } \\
\text { Evidence }\end{array}$ & $\begin{array}{l}\text { Intentional } \\
\text { Heat } \\
\text { Treatment }\end{array}$ & Patination & Retouch Detail $\begin{array}{c}\text { Retouch } \\
\text { Distribution }\end{array}$ & $\begin{array}{l}\text { Retouch } \\
\text { Location }\end{array}$ \\
\hline \multirow{3}{*}{638} & \multirow{3}{*}{5} & \multirow{3}{*}{466 NA } & Modified Flake & Edge-modified & Cultural & $\begin{array}{l}\text { Missing Left } \\
\text { Lateral Margin }\end{array}$ & Snap & $\begin{array}{l}\text { Fine Grain } \\
\text { Chert }\end{array}$ & $\begin{array}{l}\text { 10YR5/1 to } \\
10 Y R 5 / 2\end{array}$ & $\begin{array}{l}\text { Gray to Grayish } \\
\text { Brown }\end{array}$ & 0 & None & NA & None & Unifacial-ventral & Distal Margin \\
\hline & & & Weight (g) & Length & Width & Thickness & $\begin{array}{l}\text { Height of } \\
\text { Retouch }\end{array}$ & $\begin{array}{l}\text { Perimeter } \\
\text { Retouch } \\
\text { Lenqth }\end{array}$ & $\begin{array}{l}\text { Average } \\
\text { Edge } \\
\text { Anqle } \\
\end{array}$ & $\begin{array}{l}\text { Reduction } \\
\text { Index }\end{array}$ & $\begin{array}{l}\text { Platform } \\
\text { width }\end{array}$ & $\begin{array}{l}\text { Platform } \\
\text { thickness }\end{array}$ & \multicolumn{4}{|c|}{ Comments } \\
\hline & & & 5 & 44.35 & Indet & 4.18 & 2.61 & Indet & 55 & 0.62 & 12.46 & 3.17 & \multicolumn{4}{|c|}{$\begin{array}{l}\text { Thin flake with removal focused on distal end; Fine flake removal } \\
\text { terminates at break along left lateral margin - incomplete }\end{array}$} \\
\hline Lot No. & $\begin{array}{l}\text { Spec. } \\
\text { No. }\end{array}$ & $\begin{array}{l}\text { Bag UI No } \\
\text { No. }\end{array}$ & Category & Subcategory & $\begin{array}{c}\text { Retouch } \\
\text { Affiliation }\end{array}$ & $\begin{array}{l}\text { Specimen } \\
\text { Condition }\end{array}$ & Breakage & Raw material & Munsell & Color & $\begin{array}{l}\text { Dorsal } \\
\text { Cortex \% }\end{array}$ & $\begin{array}{c}\text { Heat } \\
\text { Evidence }\end{array}$ & $\begin{array}{l}\text { Intentional } \\
\text { Heat } \\
\text { Treatment }\end{array}$ & Patination & Retouch Detail $\begin{array}{c}\text { Retouch } \\
\text { Distribution }\end{array}$ & $\begin{array}{l}\text { Retouch } \\
\text { Location }\end{array}$ \\
\hline \multirow{3}{*}{646} & \multirow{3}{*}{6} & \multirow{3}{*}{369 NA } & Modified Flake & Edge-modified & Cultural & $\begin{array}{l}\text { Right Lateral } \\
\text { Margin } \\
\text { Missing }\end{array}$ & Snap & $\begin{array}{l}\text { Fine Grain } \\
\text { Chert }\end{array}$ & 10YR5/2 & Grayish Brown & $0-25$ & $\begin{array}{c}\text { Potlids and } \\
\text { Reddened }\end{array}$ & Yes & None & Unifacial-dorsal & $\begin{array}{l}\text { Left Lateral } \\
\text { Margin/Distal } \\
\text { Margin }\end{array}$ \\
\hline & & & Weight (g) & Length & Width & Thickness & $\begin{array}{l}\text { Height of } \\
\text { Retouch }\end{array}$ & $\begin{array}{l}\text { Perimeter } \\
\text { Retouch } \\
\text { Lenath }\end{array}$ & $\begin{array}{l}\text { Average } \\
\text { Edge } \\
\text { Angle }\end{array}$ & $\begin{array}{l}\text { Reduction } \\
\text { Index }\end{array}$ & $\begin{array}{l}\text { Platform } \\
\text { width }\end{array}$ & $\begin{array}{c}\text { Platform } \\
\text { thickness }\end{array}$ & \multicolumn{4}{|c|}{ Comments } \\
\hline & & & 7.28 & 44.03 & 25.53 & 7.15 & 2.22 & $40 / 12$ & 65 & 0.31 & 6.68 & 3.73 & \multicolumn{4}{|c|}{$\begin{array}{l}\text { Obvious heat treatment with fractures and potlids under flaking; Working } \\
\text { along both left lateral margin and right distal margin (shoulder); Two areas } \\
\text { of retouch, measurement are separated for each area }\end{array}$} \\
\hline Lot No. & $\begin{array}{l}\text { Spec. } \\
\text { No. }\end{array}$ & $\begin{array}{l}\text { Bag UI No } \\
\text { No. }\end{array}$ & Category & Subcategory & $\begin{array}{c}\text { Retouch } \\
\text { Affiliation }\end{array}$ & $\begin{array}{l}\text { Specimen } \\
\text { Condition }\end{array}$ & Breakage & Raw material & Munsell & Color & $\begin{array}{l}\text { Dorsal } \\
\text { Cortex \% }\end{array}$ & $\begin{array}{c}\text { Heat } \\
\text { Evidence }\end{array}$ & $\begin{array}{l}\text { Intentional } \\
\text { Heat } \\
\text { Treatment }\end{array}$ & Patination & Retouch Detail $\begin{array}{c}\text { Retouch } \\
\text { Distribution }\end{array}$ & $\begin{array}{l}\text { Retouch } \\
\text { Location }\end{array}$ \\
\hline \multirow{3}{*}{647} & \multirow{3}{*}{5} & \multirow{3}{*}{$381 \quad N A$} & Modified Flake & Edge-modified & Cultural & Indet & Snap & $\begin{array}{l}\text { Fine Grain } \\
\text { Chert }\end{array}$ & 10YR4/1 & Dark Gray & 0 & None & NA & None & Bifacial & Indet \\
\hline & & & Weight (g) & Length & Width & Thickness & $\begin{array}{l}\text { Height of } \\
\text { Retouch }\end{array}$ & $\begin{array}{l}\text { Perimeter } \\
\text { Retouch } \\
\text { Length }\end{array}$ & $\begin{array}{l}\text { Average } \\
\text { Edge } \\
\text { Angle }\end{array}$ & $\begin{array}{l}\text { Reduction } \\
\text { Index }\end{array}$ & $\begin{array}{l}\text { Platform } \\
\text { width }\end{array}$ & $\begin{array}{l}\text { Platform } \\
\text { thickness }\end{array}$ & & & Comments & \\
\hline & & & 0.63 & Indet & Indet & Indet & Indet & Indet & Indet & Indet & Indet & Indet & \multicolumn{4}{|c|}{$\begin{array}{c}\begin{array}{c}\text { Small incomplete flake with very fine scalar flake removal on one lateral } \\
\text { margin; Flake consists of single lateral margin - unknown which flake } \\
\text { margin remains }\end{array} \\
\end{array}$} \\
\hline Lot No. & $\begin{array}{l}\text { Spec. } \\
\text { No. }\end{array}$ & $\begin{array}{l}\text { Bag UI No } \\
\text { No. }\end{array}$ & Category & Subcategory & $\begin{array}{l}\text { Retouch } \\
\text { Affiliation }\end{array}$ & $\begin{array}{l}\text { Specimen } \\
\text { Condition }\end{array}$ & Breakage & Raw material & Munsell & Color & $\begin{array}{l}\text { Dorsal } \\
\text { Cortex \% }\end{array}$ & $\begin{array}{c}\text { Heat } \\
\text { Evidence }\end{array}$ & $\begin{array}{c}\text { Intentional } \\
\text { Heat } \\
\text { Treatment }\end{array}$ & Patination & Retouch Detail $\begin{array}{c}\text { Retouch } \\
\text { Distribution }\end{array}$ & $\begin{array}{l}\text { Retouch } \\
\text { Location }\end{array}$ \\
\hline \multirow{3}{*}{651} & \multirow{3}{*}{1} & \multirow{3}{*}{486 NA } & Modified Flake & Edge-modified & Indet & Complete & None & $\begin{array}{l}\text { Fine Grain } \\
\text { Chert }\end{array}$ & 10YR5/2 & Grayish Brown & 0 & None & NA & None & Unifacial-dorsal Continuous & $\begin{array}{l}\text { Left and Right } \\
\text { Lateral } \\
\text { Margins and } \\
\text { Distal Marain }\end{array}$ \\
\hline & & & Weight (g) & Length & Width & Thickness & $\begin{array}{l}\text { Height of } \\
\text { Retouch }\end{array}$ & $\begin{array}{l}\text { Perimeter } \\
\text { Retouch } \\
\text { Lenath }\end{array}$ & $\begin{array}{l}\text { Average } \\
\text { Edge } \\
\text { Angle }\end{array}$ & $\begin{array}{l}\text { Reduction } \\
\text { Index }\end{array}$ & $\begin{array}{l}\text { Platform } \\
\text { width }\end{array}$ & $\begin{array}{l}\text { Platform } \\
\text { thickness }\end{array}$ & \multicolumn{4}{|c|}{ Comments } \\
\hline & & & 0.6 & 26.75 & 9.36 & 2.06 & 0.32 & 59 & 55 & 0.16 & 2.17 & 0.24 & \multicolumn{4}{|c|}{$\begin{array}{l}\text { Very thin flake with very fine retouch flaking on all edges; Flake removal is } \\
\text { steep and fine }\end{array}$} \\
\hline
\end{tabular}




\begin{tabular}{|c|c|c|c|c|c|c|c|c|c|c|c|c|c|c|c|c|c|c|}
\hline Lot No. & $\begin{array}{l}\text { Spec. } \\
\text { No. }\end{array}$ & $\begin{array}{l}\text { Bag } \\
\text { No. }\end{array}$ & UI No & Category & Subcategory & $\begin{array}{c}\text { Retouch } \\
\text { Affiliation }\end{array}$ & $\begin{array}{l}\text { Specimen } \\
\text { Condition }\end{array}$ & Breakage & Raw material & Munsell & Color & $\begin{array}{c}\text { Dorsal } \\
\text { Cortex \% } \\
\end{array}$ & $\begin{array}{c}\text { Heat } \\
\text { Evidence }\end{array}$ & $\begin{array}{c}\text { Intentional } \\
\text { Heat } \\
\text { Treatment } \\
\end{array}$ & Patination & Retouch Detail & $\begin{array}{l}\text { Retouch } \\
\text { Distribution }\end{array}$ & $\begin{array}{l}\text { Retouch } \\
\text { Location } \\
\end{array}$ \\
\hline \multirow{3}{*}{660} & \multirow{3}{*}{5} & \multirow{3}{*}{459} & \multirow{3}{*}{ NA } & Modified Flake & Edge-modified & Indet & Medial-Distal & Snap & $\begin{array}{l}\text { Fine Grain } \\
\text { Chert }\end{array}$ & 10YR6/2 & $\begin{array}{c}\text { Light Brownish } \\
\text { Gray }\end{array}$ & $1-25$ & None & NA & None & Unifacial-ventral & Indet & $\begin{array}{l}\text { Right Lateral } \\
\text { Margin }\end{array}$ \\
\hline & & & & Weight (g) & Length & Width & Thickness & $\begin{array}{l}\text { Height of } \\
\text { Retouch }\end{array}$ & $\begin{array}{l}\text { Perimeter } \\
\text { Retouch } \\
\text { Length }\end{array}$ & $\begin{array}{l}\text { Average } \\
\text { Edge } \\
\text { Angle }\end{array}$ & $\begin{array}{c}\text { Reduction } \\
\text { Index }\end{array}$ & $\begin{array}{c}\text { Platform } \\
\text { width }\end{array}$ & $\begin{array}{l}\text { Platform } \\
\text { thickness }\end{array}$ & \multicolumn{4}{|c|}{ Comments } & \\
\hline & & & & 6.24 & Indet & Indet & Indet & Indet & Indet & Indet & Indet & Indet & Indet & \multicolumn{5}{|c|}{$\begin{array}{c}\text { Flake fragment with two breaks: medial and left lateral margin; Complete } \\
\text { size of flake unknown; Retouching on right lateral margin appears to } \\
\text { continue through break }\end{array}$} \\
\hline Lot No. & $\begin{array}{l}\text { Spec. } \\
\text { No. }\end{array}$ & $\begin{array}{l}\text { Bag } \\
\text { No. }\end{array}$ & UI No & Category & Subcategory & $\begin{array}{c}\text { Retouch } \\
\text { Affiliation }\end{array}$ & $\begin{array}{l}\text { Specimen } \\
\text { Condition }\end{array}$ & Breakage & Raw material & Munsell & Color & $\begin{array}{c}\text { Dorsal } \\
\text { Cortex \% }\end{array}$ & $\begin{array}{c}\text { Heat } \\
\text { Evidence }\end{array}$ & $\begin{array}{c}\text { Intentional } \\
\text { Heat } \\
\text { Treatment }\end{array}$ & Patination & Retouch Detail & $\begin{array}{c}\text { Retouch } \\
\text { Distribution }\end{array}$ & $\begin{array}{l}\text { Retouch } \\
\text { Location }\end{array}$ \\
\hline \multirow{3}{*}{660} & \multirow{3}{*}{6} & \multirow{3}{*}{\multicolumn{2}{|c|}{459 NA }} & Modified Flake & Edge-modified & Indet & Complete & None & $\begin{array}{c}\text { Fine Grain } \\
\text { Chert }\end{array}$ & 10YR5/2 & Grayish Brown & $1-25$ & None & NA & Yes & Unifacial-dorsal & Clustered & $\begin{array}{l}\text { Left Lateral } \\
\text { Margin/Distal } \\
\text { Margin }\end{array}$ \\
\hline & & & & Weight (g) & Length & Width & Thickness & $\begin{array}{l}\text { Height of } \\
\text { Retouch }\end{array}$ & $\begin{array}{l}\text { Perimeter } \\
\text { Retouch } \\
\text { Lenath }\end{array}$ & $\begin{array}{l}\text { Average } \\
\text { Edge } \\
\text { Angle }\end{array}$ & $\begin{array}{l}\text { Reduction } \\
\text { Index }\end{array}$ & $\begin{array}{l}\text { Platform } \\
\text { width }\end{array}$ & $\begin{array}{l}\text { Platform } \\
\text { thickness }\end{array}$ & \multicolumn{5}{|c|}{ Comments } \\
\hline & & & & 29.09 & 88.85 & 32.25 & 14.09 & $3.39 ; 4.24$ & $30 ; 22$ & $65 ; 50$ & $0.24 ; 0.30$ & 15.96 & 6.79 & \multicolumn{5}{|c|}{$\begin{array}{l}\text { Crushing use-wear evident under flaking possibly from preparation for } \\
\text { flake removal; Working is clustered at the distal end of left lateral margin } \\
\text { between cortex and on a spur extending from the right shoulder; Two } \\
\text { areas of retouch, measurement are separated for each area }\end{array}$} \\
\hline Lot No. & $\begin{array}{l}\text { Spec. } \\
\text { No. }\end{array}$ & $\begin{array}{l}\text { Bag } \\
\text { No. }\end{array}$ & UI No & Category & Subcategory & $\begin{array}{c}\text { Retouch } \\
\text { Affiliation }\end{array}$ & $\begin{array}{l}\text { Specimen } \\
\text { Condition }\end{array}$ & Breakage & Raw material & Munsell & Color & $\begin{array}{c}\text { Dorsal } \\
\text { Cortex \% }\end{array}$ & $\begin{array}{c}\text { Heat } \\
\text { Evidence }\end{array}$ & $\begin{array}{l}\text { Intentional } \\
\text { Heat } \\
\text { Treatment }\end{array}$ & Patination & Retouch Detail & $\begin{array}{l}\text { Retouch } \\
\text { Distribution }\end{array}$ & $\begin{array}{l}\text { Retouch } \\
\text { Location }\end{array}$ \\
\hline \multirow{3}{*}{661} & \multirow{3}{*}{5} & \multirow{3}{*}{\multicolumn{2}{|c|}{$469 \mathrm{NA}$}} & Modified Flake & Edge-modified & Indet & Complete & None & $\begin{array}{c}\text { Fine Grain } \\
\text { Chert }\end{array}$ & $\begin{array}{c}\text { 10YR5/1 to } \\
10 Y R 5 / 2\end{array}$ & $\begin{array}{l}\text { Gray to Grayish } \\
\text { Brown }\end{array}$ & 0 & Potlids & No & None & Unifacial-dorsal & Continuous & $\begin{array}{l}\text { Left and Right } \\
\text { Lateral } \\
\text { Margins } \\
\end{array}$ \\
\hline & & & & Weight (g) & Length & Width & Thickness & $\begin{array}{l}\text { Height of } \\
\text { Retouch }\end{array}$ & $\begin{array}{l}\text { Perimeter } \\
\text { Retouch } \\
\text { Lenath }\end{array}$ & $\begin{array}{l}\text { Average } \\
\text { Edge } \\
\text { Angle } \\
\end{array}$ & $\begin{array}{l}\text { Reduction } \\
\text { Index }\end{array}$ & $\begin{array}{l}\text { Platform } \\
\text { width }\end{array}$ & $\begin{array}{l}\text { Platform } \\
\text { thickness }\end{array}$ & \multicolumn{5}{|c|}{ Comments } \\
\hline & & & & 1.41 & 24.88 & 16.36 & 3.48 & 1.14 & 37 & 55 & 0.33 & 6.5 & 1.18 & \multicolumn{5}{|c|}{$\begin{array}{c}\text { Thin flake with removal on dorsal face; Very fine flake removal along both } \\
\text { left and right lateral margin with most prominent work on left margin; Two } \\
\text { areas of shatter that appear to be caused from heat treatment (potlids) on } \\
\text { dorsal face; Inclusions of coarse grain gray mineral spots }\end{array}$} \\
\hline
\end{tabular}

\begin{tabular}{|c|c|c|c|c|c|c|c|c|c|c|c|c|c|c|c|c|c|}
\hline Lot No. & $\begin{array}{l}\text { Spec. } \\
\text { No. }\end{array}$ & $\begin{array}{l}\text { Bag UI No } \\
\text { No. }\end{array}$ & Category & Subcategory & $\begin{array}{c}\text { Retouch } \\
\text { Affiliation }\end{array}$ & $\begin{array}{l}\text { Specimen } \\
\text { Condition }\end{array}$ & Breakage & Raw material & Munsell & Color & $\begin{array}{c}\text { Dorsal } \\
\text { Cortex \% }\end{array}$ & $\begin{array}{c}\text { Heat } \\
\text { Evidence }\end{array}$ & $\begin{array}{c}\text { Intentional } \\
\text { Heat } \\
\text { Treatment }\end{array}$ & Patination & Retouch Detail & $\begin{array}{c}\text { Retouch } \\
\text { Distribution }\end{array}$ & $\begin{array}{l}\text { Retouch } \\
\text { Location }\end{array}$ \\
\hline \multirow{3}{*}{661} & \multirow{3}{*}{6} & \multirow{3}{*}{469 NA } & Modified Flake & Edge-modified & Cultural & $\begin{array}{c}\text { Proximal- } \\
\text { Medial }\end{array}$ & Snap & $\begin{array}{c}\text { Fine Grain } \\
\text { Chert }\end{array}$ & 7.5YR3/2 & Dark Brown & 0 & None & NA & None & Unifacial-ventral & Focused & $\begin{array}{l}\text { Left Lateral } \\
\text { Margin }\end{array}$ \\
\hline & & & Weight (g) & Length & Width & Thickness & $\begin{array}{l}\text { Height of } \\
\text { Retouch }\end{array}$ & $\begin{array}{l}\text { Perimeter } \\
\text { Retouch } \\
\text { Length }\end{array}$ & $\begin{array}{c}\text { Average } \\
\text { Edge } \\
\text { Angle }\end{array}$ & $\begin{array}{l}\text { Reduction } \\
\text { Index }\end{array}$ & $\begin{array}{l}\text { Platform } \\
\text { width }\end{array}$ & $\begin{array}{l}\text { Platform } \\
\text { thickness }\end{array}$ & \multicolumn{5}{|c|}{ Comments } \\
\hline & & & 1.2 & Indet & Indet & Indet & Indet & Indet & Indet & Indet & 9.2 & 1.8 & $\begin{array}{l}\text { Thinned flake } \\
\text { fractures; Visi }\end{array}$ & $\begin{array}{l}\text { (removal on } \\
\text { ible worked } \mathrm{e}\end{array}$ & $\begin{array}{l}\text { dorsal face); Platf } \\
\text { edge has a height } \\
\text { analysis }\end{array}$ & $\begin{array}{l}\text { tform has multi } \\
\text { t of } 2.6 \text { but bre }\end{array}$ & $\begin{array}{l}\text { le small hinge } \\
\mathrm{k} \text { prevents full }\end{array}$ \\
\hline
\end{tabular}




\begin{tabular}{|c|c|c|c|c|c|c|c|c|c|c|c|c|c|c|c|c|c|}
\hline Lot No. & $\begin{array}{l}\text { Spec. } \\
\text { No. }\end{array}$ & $\begin{array}{l}\text { Bag UI No } \\
\text { No. }\end{array}$ & Category & Subcategory & $\begin{array}{c}\text { Retouch } \\
\text { Affiliation }\end{array}$ & $\begin{array}{l}\text { Specimen } \\
\text { Condition }\end{array}$ & Breakage & Raw material & Munsell & Color & $\begin{array}{c}\text { Dorsal } \\
\text { Cortex \% }\end{array}$ & $\begin{array}{c}\text { Heat } \\
\text { Evidence }\end{array}$ & $\begin{array}{l}\text { Intentional } \\
\text { Heat } \\
\text { Treatment }\end{array}$ & Patination & Retouch Detail & $\begin{array}{c}\text { Retouch } \\
\text { Distribution }\end{array}$ & $\begin{array}{l}\text { Retouch } \\
\text { Location }\end{array}$ \\
\hline \multirow{3}{*}{665} & \multirow{3}{*}{5} & \multirow{3}{*}{568 NA } & Modified Flake & Utilized Flake & Indet & Complete & None & $\begin{array}{c}\text { Fine Grain } \\
\text { Chert }\end{array}$ & 10YR2/1 & Black & $26-50$ & None & NA & None & Unifacial-ventral & Focused & $\begin{array}{l}\text { Left Lateral } \\
\text { Margin }\end{array}$ \\
\hline & & & Weight (g) & Length & Width & Thickness & $\begin{array}{l}\text { Height of } \\
\text { Retouch }\end{array}$ & $\begin{array}{l}\text { Perimeter } \\
\text { Retouch } \\
\text { Length }\end{array}$ & $\begin{array}{c}\text { Average } \\
\text { Edge } \\
\text { Angle }\end{array}$ & $\begin{array}{l}\text { Reduction } \\
\text { Index }\end{array}$ & $\begin{array}{l}\text { Platform } \\
\text { width }\end{array}$ & $\begin{array}{l}\text { Platform } \\
\text { thickness }\end{array}$ & \multicolumn{5}{|c|}{ Comments } \\
\hline & & & 121.86 & 80.35 & 69.83 & 17.85 & 0.6 & 73 & 55 & 0.03 & 38.09 & 12.9 & $\begin{array}{l}\text { Prominent b } \\
\text { with cortex }\end{array}$ & $\begin{array}{r}\text { anding } 10 \text { YR5 } \\
\text { on dorsal side } \\
\text { crushed }\end{array}$ & $\begin{array}{l}5 / 1 \text { and } 10 Y R 8 / 1 \text { ( } \\
\text { de; very light and in } \\
\text { ledges on left later }\end{array}$ & $\begin{array}{l}\text { (gray and whit } \\
\text { irregular flake } \\
\text { eral margin }\end{array}$ & $\begin{array}{l}\text { te); Large flake } \\
\text { removal and }\end{array}$ \\
\hline Lot No. & $\begin{array}{l}\text { Spec. } \\
\text { No. }\end{array}$ & $\begin{array}{l}\text { Bag UI No } \\
\text { No. }\end{array}$ & Category & Subcategory & $\begin{array}{c}\text { Retouch } \\
\text { Affiliation }\end{array}$ & $\begin{array}{l}\text { Specimen } \\
\text { Condition }\end{array}$ & Breakage & Raw material & Munsell & Color & $\begin{array}{c}\text { Dorsal } \\
\text { Cortex \% }\end{array}$ & $\begin{array}{c}\text { Heat } \\
\text { Evidence }\end{array}$ & $\begin{array}{c}\text { Intentional } \\
\text { Heat } \\
\text { Treatment }\end{array}$ & Patination & Retouch Detail & $\begin{array}{c}\text { Retouch } \\
\text { Distribution }\end{array}$ & $\begin{array}{l}\text { Retouch } \\
\text { Location }\end{array}$ \\
\hline \multirow{3}{*}{666} & \multirow{3}{*}{5} & \multirow{3}{*}{$584 \quad N A$} & Modified Flake & Edge-modified & Indet & Indet & Snap & $\begin{array}{c}\text { Fine Grain } \\
\text { Chert }\end{array}$ & $\begin{array}{c}10 Y R 5 / 1 \text { to } \\
10 Y R 5 / 2\end{array}$ & $\begin{array}{l}\text { Gray to Grayish } \\
\text { Brown }\end{array}$ & 0 & None & NA & None & Bifacial & Continuous & Indet \\
\hline & & & Weight (g) & Length & Width & Thickness & $\begin{array}{l}\text { Height of } \\
\text { Retouch }\end{array}$ & $\begin{array}{l}\text { Perimeter } \\
\text { Retouch } \\
\text { Length }\end{array}$ & $\begin{array}{l}\text { Average } \\
\text { Edge } \\
\text { Angle } \\
\end{array}$ & $\begin{array}{l}\text { Reduction } \\
\text { Index }\end{array}$ & $\begin{array}{c}\text { Platform } \\
\text { width }\end{array}$ & $\begin{array}{l}\text { Platform } \\
\text { thickness }\end{array}$ & \multicolumn{5}{|c|}{ Comments } \\
\hline & & & 2.32 & Indet & Indet & 5.8 & Indet & Indet & Indet & Indet & Indet & Indet & $\begin{array}{r}\text { Thin incomple } \\
\text { bifacially wor } \\
\text { breaks }\end{array}$ & $\begin{array}{l}\text { te flake with } n \\
\text { ked but work } \\
\text { can be seen }\end{array}$ & $\begin{array}{l}\text { no platform remaii } \\
\text { is predominately } \\
\text { n on both faces ass }\end{array}$ & $\begin{array}{l}\text { aining; single } \mathrm{m} \\
\text { / on one face; } ₫ \\
\text { ssociated with }\end{array}$ & $\begin{array}{l}\text { nargin has been } \\
\text { Step and hinge } \\
\text { thinning }\end{array}$ \\
\hline Lot No. & $\begin{array}{l}\text { Spec. } \\
\text { No. }\end{array}$ & $\begin{array}{l}\text { Bag UI No } \\
\text { No. }\end{array}$ & Category & Subcategory & $\begin{array}{l}\text { Retouch } \\
\text { Affiliation }\end{array}$ & $\begin{array}{l}\text { Specimen } \\
\text { Condition }\end{array}$ & Breakage & Raw material & Munsell & Color & $\begin{array}{c}\text { Dorsal } \\
\text { Cortex \% }\end{array}$ & $\begin{array}{c}\text { Heat } \\
\text { Evidence }\end{array}$ & $\begin{array}{c}\text { Intentional } \\
\text { Heat } \\
\text { Treatment }\end{array}$ & Patination & Retouch Detail & $\begin{array}{c}\text { Retouch } \\
\text { Distribution }\end{array}$ & $\begin{array}{l}\text { Retouch } \\
\text { Location }\end{array}$ \\
\hline \multirow{3}{*}{666} & \multirow{3}{*}{6} & \multirow{3}{*}{584 NA } & Modified Flake & Edge-modified & Indet & Complete & None & $\begin{array}{c}\text { Coarse Grain } \\
\text { Chert }\end{array}$ & 10YR7/2 & Light Gray & $1-25$ & None & NA & None & Bifacial & Continuous & $\begin{array}{c}\text { Right Lateral } \\
\text { Margin/Left } \\
\text { Lateral Margin }\end{array}$ \\
\hline & & & Weight (g) & Length & Width & Thickness & $\begin{array}{l}\text { Height of } \\
\text { Retouch }\end{array}$ & $\begin{array}{l}\text { Perimeter } \\
\text { Retouch } \\
\text { Length }\end{array}$ & $\begin{array}{c}\text { Average } \\
\text { Edge } \\
\text { Angle }\end{array}$ & $\begin{array}{l}\text { Reduction } \\
\text { Index }\end{array}$ & $\begin{array}{l}\text { Platform } \\
\text { width }\end{array}$ & $\begin{array}{l}\text { Platform } \\
\text { thickness }\end{array}$ & \multicolumn{5}{|c|}{ Comments } \\
\hline & & & 43.85 & 72.07 & 39.97 & 14.49 & 0.42 & 110 & 50 & 0.03 & 22.99 & 12.3 & \multicolumn{5}{|c|}{$\begin{array}{l}\text { Cortex at proximal and distal end; Work very fine and on very edge; } \\
\text { Mineral inclusions }\end{array}$} \\
\hline Lot No. & $\begin{array}{l}\text { Spec. } \\
\text { No. }\end{array}$ & $\begin{array}{l}\text { Bag UI No } \\
\text { No. }\end{array}$ & Category & Subcategory & $\begin{array}{c}\text { Retouch } \\
\text { Affiliation }\end{array}$ & $\begin{array}{l}\text { Specimen } \\
\text { Condition }\end{array}$ & Breakage & Raw material & Munsell & Color & $\begin{array}{l}\text { Dorsal } \\
\text { Cortex \% }\end{array}$ & $\begin{array}{c}\text { Heat } \\
\text { Evidence }\end{array}$ & $\begin{array}{c}\text { Intentional } \\
\text { Heat } \\
\text { Treatment }\end{array}$ & Patination & Retouch Detail & $\begin{array}{c}\text { Retouch } \\
\text { Distribution }\end{array}$ & $\begin{array}{l}\text { Retouch } \\
\text { Location }\end{array}$ \\
\hline \multirow{3}{*}{673} & \multirow{3}{*}{4} & \multirow{3}{*}{$344 \mathrm{NA}$} & Modified Flake & Utilized Flake & Indet & $\begin{array}{l}\text { Proximal- } \\
\text { Medial }\end{array}$ & Snap & $\begin{array}{c}\text { Fine Grain } \\
\text { Chert }\end{array}$ & $\begin{array}{c}10 \mathrm{YR} 5 / 2 \text { to } \\
10 \mathrm{YR} 6 / 2 \\
\end{array}$ & $\begin{array}{c}\text { Grayish Brown } \\
\text { to Light Grayish } \\
\text { Brown }\end{array}$ & 0 & Reddened & Yes & None & Bifacial & Continuous & $\begin{array}{l}\text { Left and Right } \\
\text { Lateral } \\
\text { Margins } \\
\end{array}$ \\
\hline & & & Weight (g) & Length & Width & Thickness & $\begin{array}{l}\text { Height of } \\
\text { Retouch }\end{array}$ & $\begin{array}{l}\text { Perimeter } \\
\text { Retouch } \\
\text { Lenath }\end{array}$ & $\begin{array}{c}\text { Average } \\
\text { Edge } \\
\text { Anale }\end{array}$ & $\begin{array}{c}\text { Reduction } \\
\text { Index }\end{array}$ & $\begin{array}{c}\text { Platform } \\
\text { width }\end{array}$ & $\begin{array}{l}\text { Platform } \\
\text { thickness }\end{array}$ & & & Comments & & \\
\hline & & & 22.47 & Indet & Indet & 13.87 & 1.09 & Indet & Indet & Indet & 16.52 & 7.05 & \multicolumn{5}{|c|}{$\begin{array}{l}\text { Large flake with minimal initial removal on both ventral and dorsal faces; } \\
\text { Very light modification along both lateral margins - left lateral ventral and } \\
\text { right lateral dorsal; Modification is very light and irregular appearing to be } \\
\text { utilized and not initial modification }\end{array}$} \\
\hline
\end{tabular}




\begin{tabular}{|c|c|c|c|c|c|c|c|c|c|c|c|c|c|c|c|c|c|c|}
\hline Lot No. & $\begin{array}{l}\text { Spec. } \\
\text { No. }\end{array}$ & $\begin{array}{l}\text { Bag } \\
\text { No. }\end{array}$ & UI No & Category & Subcategory & $\begin{array}{l}\text { Retouch } \\
\text { Affiliation }\end{array}$ & $\begin{array}{l}\text { Specimen } \\
\text { Condition }\end{array}$ & Breakage & Raw material & Munsell & Color & $\begin{array}{c}\text { Dorsal } \\
\text { Cortex \% }\end{array}$ & $\begin{array}{c}\text { Heat } \\
\text { Evidence }\end{array}$ & $\begin{array}{c}\text { Intentional } \\
\text { Heat } \\
\text { Treatment }\end{array}$ & Patination & Retouch Detail & $\begin{array}{l}\text { Retouch } \\
\text { Distribution }\end{array}$ & $\begin{array}{l}\text { Retouch } \\
\text { Location }\end{array}$ \\
\hline \multirow{3}{*}{694} & \multirow{3}{*}{4} & \multirow{3}{*}{398} & \multirow{3}{*}{ NA } & Modified Flak & Edge-modified & Cultural & $\begin{array}{l}\text { Distal Tip } \\
\text { Missing }\end{array}$ & Snap & $\begin{array}{l}\text { Fine Grain } \\
\text { Chert }\end{array}$ & $\begin{array}{c}\text { 10YR5/1 } \\
\text { and } \\
10 Y R 8 / 3\end{array}$ & $\begin{array}{l}\text { Gray and Very } \\
\text { Pale Brown }\end{array}$ & 0 & None & NA & None & Unifacial-ventral & Continuous & $\begin{array}{l}\text { Right Lateral } \\
\text { Margin }\end{array}$ \\
\hline & & & & Weight (g) & Length & Width & Thickness & $\begin{array}{l}\text { Height of } \\
\text { Retouch }\end{array}$ & $\begin{array}{l}\text { Perimeter } \\
\text { Retouch } \\
\text { Length }\end{array}$ & $\begin{array}{c}\text { Average } \\
\text { Edge } \\
\text { Angle }\end{array}$ & $\begin{array}{l}\text { Reduction } \\
\text { Index }\end{array}$ & $\begin{array}{l}\text { Platform } \\
\text { width }\end{array}$ & $\begin{array}{l}\text { Platform } \\
\text { thickness }\end{array}$ & \multicolumn{5}{|c|}{ Comments } \\
\hline & & & & 18.24 & Indet & 43.13 & 9.57 & 1.62 & Indet & 55 & 0.17 & Indet & Indet & \multicolumn{5}{|c|}{$\begin{array}{l}\text { Thinned unifacially worked informal tool; Two major colors with the lighter } \\
\text { 10YR8/3 appearing to be related to previous cortex removal; use-wear } \\
\text { noted on all edges (left lateral margin, proximal and distal ends, and right } \\
\text { lateral margins on ventral and dorsal faces) - all notes focused on modified } \\
\text { edge work on right lateral margin; Current worked edge is } 57 \mathrm{~cm} \text {, but a } \\
\text { break at the distal end prevents full analysis }\end{array}$} \\
\hline
\end{tabular}




\begin{tabular}{|c|c|c|c|c|c|c|c|c|c|c|c|c|c|c|c|c|c|}
\hline $\begin{array}{l}\text { Lot } \\
\text { No. }\end{array}$ & $\begin{array}{l}\text { Spec. } \\
\text { No. }\end{array}$ & $\begin{array}{l}\text { Bag } \\
\text { No. }\end{array}$ & $\begin{array}{l}\text { UI } \\
\text { No. }\end{array}$ & Category & Condition & Subcategory & $\begin{array}{l}\text { Reduction } \\
\text { Technique }\end{array}$ & Breakage & Exhausted & Use Wear & $\begin{array}{l}\text { Use-Wear } \\
\text { Type }\end{array}$ & Raw material & Inclusions & Munsell & Color & $\begin{array}{c}\text { Heat } \\
\text { Evidence }\end{array}$ & $\begin{array}{c}\text { Intentional } \\
\text { Heat } \\
\text { Treatment } \\
\end{array}$ \\
\hline \multirow{3}{*}{339} & \multirow{3}{*}{11} & \multirow{3}{*}{410} & \multirow{3}{*}{ NA } & Core & Core Fragment & Bifacial & Hard & Manufacturing & No & None & None & $\begin{array}{c}\text { Fine Grain } \\
\text { Chert }\end{array}$ & $\begin{array}{c}\text { Irregular Grain and } \\
\text { Minerals } \\
\end{array}$ & 10YR5/2 & Grayish Brown & None & No \\
\hline & & & & Patination & Cortex \% & $\begin{array}{c}\text { Rock } \\
\text { Description }\end{array}$ & Weight (g) & Max Length & Max Width & $\begin{array}{c}\text { Max } \\
\text { Thickness }\end{array}$ & $\begin{array}{l}\text { Max FScar } \\
\text { Length }\end{array}$ & No FScars & \multicolumn{5}{|c|}{ Comments } \\
\hline & & & & None & $26-50$ & Indet & 57.01 & Indet & Indet & Indet & Indet & Indet & Fragment of $\mathrm{c}$ & re with sna & break across app & arent medial & portior \\
\hline
\end{tabular}

\begin{tabular}{|c|c|c|c|c|c|c|c|c|c|c|c|c|c|c|c|c|c|}
\hline $\begin{array}{l}\text { Lot } \\
\text { No. }\end{array}$ & $\begin{array}{l}\text { Spec. } \\
\text { No. }\end{array}$ & $\begin{array}{l}\text { Bag } \\
\text { No. }\end{array}$ & $\begin{array}{l}\text { UI } \\
\text { No. }\end{array}$ & Category & Condition & Subcategory & $\begin{array}{l}\text { Reduction } \\
\text { Technique }\end{array}$ & Breakage & Exhausted & Use Wear & $\begin{array}{l}\text { Use-Wear } \\
\text { Type }\end{array}$ & Raw material & Inclusions & Munsell & Color & $\begin{array}{c}\text { Heat } \\
\text { Evidence }\end{array}$ & $\begin{array}{c}\text { Intentional } \\
\text { Heat } \\
\text { Treatment }\end{array}$ \\
\hline & & & & Core & Complete & Multidirectional & Soft & None & Yes & None & None & $\begin{array}{c}\text { Fine Grain } \\
\text { Chert }\end{array}$ & $\begin{array}{c}\text { Irregular Grain and } \\
\text { Minerals }\end{array}$ & 10YR $4 / 3$ & Brown & None & No \\
\hline 346 & 8 & 626 & NA & Patination & Cortex $\%$ & $\begin{array}{c}\text { Rock } \\
\text { Description } \\
\end{array}$ & Weight (g) & Max Length & Max Width & $\begin{array}{c}\text { Max } \\
\text { Thickness } \\
\end{array}$ & $\begin{array}{l}\text { Max FScar } \\
\text { Length }\end{array}$ & No FScars & \multicolumn{5}{|c|}{ Comments } \\
\hline
\end{tabular}

\begin{tabular}{|c|c|c|c|c|c|c|c|c|c|c|c|c|c|c|c|c|c|}
\hline $\begin{array}{l}\text { Lot } \\
\text { No. }\end{array}$ & $\begin{array}{l}\text { Spec. } \\
\text { No. }\end{array}$ & $\begin{array}{l}\text { Bag } \\
\text { No. }\end{array}$ & $\begin{array}{l}\text { UI } \\
\text { No. }\end{array}$ & Category & Condition & Subcategory & $\begin{array}{l}\text { Reduction } \\
\text { Technique }\end{array}$ & Breakage & Exhausted & Use Wear & $\begin{array}{l}\text { Use-Wear } \\
\text { Type }\end{array}$ & Raw material & Inclusions & Munsell & Color & $\begin{array}{c}\text { Heat } \\
\text { Evidence }\end{array}$ & $\begin{array}{c}\text { Intentional } \\
\text { Heat } \\
\text { Treatment } \\
\end{array}$ \\
\hline \multirow{3}{*}{359} & \multirow{3}{*}{1} & \multirow{3}{*}{427} & \multirow{3}{*}{ NA } & Core & Complete & Multidirectional & Hard & None & No & None & None & $\begin{array}{c}\text { Fine Grain } \\
\text { Chert }\end{array}$ & Fine Fractures & 10YR8/2 & Very Pale Brown & None & No \\
\hline & & & & Patination & Cortex \% & $\begin{array}{c}\text { Rock } \\
\text { Description }\end{array}$ & Weight (g) & Max Length & Max Width & $\begin{array}{c}\text { Max } \\
\text { Thickness } \\
\end{array}$ & $\begin{array}{l}\text { Max FScar } \\
\text { Length }\end{array}$ & No FScars & \multicolumn{5}{|c|}{ Comments } \\
\hline & & & & None & $26-50$ & Nodular & 240.97 & 62 & 75.65 & 46.4 & 53.96 & 15 & Round cobble of & $\begin{array}{l}\text { with fine } \mathrm{fr} \\
\text { Hinge } \mathrm{fr}\end{array}$ & $\begin{array}{l}\text { actures throughout; } \\
\text { actures across face }\end{array}$ & $\begin{array}{l}\text { Platform is } \\
\text { s }\end{array}$ & tact; Step anc \\
\hline
\end{tabular}

\begin{tabular}{|c|c|c|c|c|c|c|c|c|c|c|c|c|c|c|c|c|c|}
\hline $\begin{array}{l}\text { Lot } \\
\text { No. }\end{array}$ & $\begin{array}{l}\text { Spec. } \\
\text { No. }\end{array}$ & $\begin{array}{l}\text { Bag } \\
\text { No. }\end{array}$ & $\begin{array}{l}\text { UI } \\
\text { No. }\end{array}$ & Category & Condition & Subcategory & $\begin{array}{l}\text { Reduction } \\
\text { Technique }\end{array}$ & Breakage & Exhausted & Use Wear & $\begin{array}{c}\text { Use-Wear } \\
\text { Type }\end{array}$ & Raw material & Inclusions & Munsell & Color & $\begin{array}{c}\text { Heat } \\
\text { Evidence }\end{array}$ & $\begin{array}{c}\text { Intentional } \\
\text { Heat } \\
\text { Treatment }\end{array}$ \\
\hline \multirow{3}{*}{373} & \multirow{3}{*}{20} & \multirow{3}{*}{517} & \multirow{3}{*}{ NA } & Core & Complete & Multidirectional & Hard & None & Yes & None & None & $\begin{array}{c}\text { Fine Grain } \\
\text { Chert }\end{array}$ & Fine Fractures & 10YR5/1 & Gray & None & No \\
\hline & & & & Patination & Cortex \% & $\begin{array}{c}\text { Rock } \\
\text { Description } \\
\end{array}$ & Weight (g) & Max Length & Max Width & $\begin{array}{c}\text { Max } \\
\text { Thickness } \\
\end{array}$ & $\begin{array}{l}\text { Max FScar } \\
\text { Length }\end{array}$ & No FScars & \multicolumn{5}{|c|}{ Comments } \\
\hline & & & & None & $1-25$ & Indet & 60.38 & 43.87 & 42.79 & 27.38 & 31.85 & 12 & Exhausted cor & $\begin{array}{l}\text { ith step anc } \\
\text { /R5/6 with }\end{array}$ & $\begin{array}{l}\text { e fracture } \\
\text { ish brow }\end{array}$ & $\begin{array}{l}\text { OSs faces; N } \\
\text { ar cortex }\end{array}$ & Mottled with \\
\hline
\end{tabular}

\begin{tabular}{|c|c|c|c|c|c|c|c|c|c|c|c|c|c|c|c|c|c|}
\hline $\begin{array}{l}\text { Lot } \\
\text { No. }\end{array}$ & $\begin{array}{l}\text { Spec. } \\
\text { No. }\end{array}$ & $\begin{array}{l}\text { Bag } \\
\text { No. }\end{array}$ & $\begin{array}{l}\text { UI } \\
\text { No. }\end{array}$ & Category & Condition & Subcategory & $\begin{array}{l}\text { Reduction } \\
\text { Technique }\end{array}$ & Breakage & Exhausted & Use Wear & $\begin{array}{l}\text { Use-Wear } \\
\text { Type }\end{array}$ & Raw material & Inclusions & Munsell & Color & $\begin{array}{c}\text { Heat } \\
\text { Evidence }\end{array}$ & $\begin{array}{c}\text { Intentional } \\
\text { Heat } \\
\text { Treatment }\end{array}$ \\
\hline \multirow{3}{*}{385} & \multirow{3}{*}{1} & \multirow{3}{*}{444} & \multirow{3}{*}{ NA } & Core & Complete & Multidirectional & Hard and Soft & None & Yes & None & None & $\begin{array}{c}\text { Coarse Grain } \\
\text { Chert }\end{array}$ & $\begin{array}{c}\text { Irregular grain and } \\
\text { Minerals }\end{array}$ & $\begin{array}{c}\text { 10YR3/1 to } \\
\text { 10YR5/1 }\end{array}$ & $\begin{array}{c}\text { Very Dark Gray to } \\
\text { Gray }\end{array}$ & None & No \\
\hline & & & & Patination & Cortex \% & $\begin{array}{c}\text { Rock } \\
\text { Description }\end{array}$ & Weight (g) & Max Length & Max Width & $\begin{array}{c}\text { Max } \\
\text { Thickness } \\
\end{array}$ & $\begin{array}{c}\text { Max FScar } \\
\text { Length }\end{array}$ & No FScars & \multicolumn{5}{|c|}{ Comments } \\
\hline & & & & None & $1-25$ & Nodular & 103.99 & 54.87 & 40.43 & 34.79 & 38.42 & 22 & Speckled with mi & neral inclusio & ons; multiple step an & d hinge frac & tures noted \\
\hline
\end{tabular}




\begin{tabular}{|c|c|c|c|c|c|c|c|c|c|c|c|c|c|c|c|c|c|}
\hline $\begin{array}{l}\text { Lot } \\
\text { No. }\end{array}$ & $\begin{array}{c}\text { Spec. } \\
\text { No. }\end{array}$ & $\begin{array}{l}\text { Bag } \\
\text { No. }\end{array}$ & $\begin{array}{l}\text { UI } \\
\text { No. }\end{array}$ & Category & Condition & Subcategory & $\begin{array}{l}\text { Reduction } \\
\text { Technique }\end{array}$ & Breakage & Exhausted & Use Wear & $\begin{array}{c}\text { Use-Wear } \\
\text { Type }\end{array}$ & Raw material & Inclusions & Munsell & Color & $\begin{array}{c}\text { Heat } \\
\text { Evidence } \\
\end{array}$ & $\begin{array}{c}\text { Intentional } \\
\text { Heat } \\
\text { Treatment } \\
\end{array}$ \\
\hline \multirow{3}{*}{401} & \multirow{3}{*}{5} & \multirow{3}{*}{488} & \multirow{3}{*}{ NA } & Core & Complete & Multidirectional & Hard & None & Yes & None & None & $\begin{array}{c}\text { Fine Grain } \\
\text { Chert }\end{array}$ & Irregular Grain & 10YR6/1 & Gray & None & No \\
\hline & & & & Patination & Cortex $\%$ & $\begin{array}{c}\text { Rock } \\
\text { Description } \\
\end{array}$ & Weight (g) & Max Length & Max Width & $\begin{array}{c}\text { Max } \\
\text { Thickness } \\
\end{array}$ & $\begin{array}{c}\text { Max FScar } \\
\text { Length }\end{array}$ & No FScars & \multicolumn{5}{|c|}{ Comments } \\
\hline & & & & None & $1-25$ & Indet & 46.94 & 46.78 & 33.55 & 31.78 & 36.34 & 14 & Cortex nearly co & $\begin{array}{l}\text { etely remo } \\
\text { edge; Ba }\end{array}$ & $\begin{array}{l}\% \text { rema } \\
\text { (10YR6/ }\end{array}$ & $\begin{array}{l}\text { Slight browr } \\
\text { 10YR5/4) }\end{array}$ & nish red color \\
\hline
\end{tabular}

\begin{tabular}{|c|c|c|c|c|c|c|c|c|c|c|c|c|c|c|c|c|c|}
\hline $\begin{array}{l}\text { Lot } \\
\text { No. }\end{array}$ & $\begin{array}{l}\text { Spec. } \\
\text { No. }\end{array}$ & $\begin{array}{l}\text { Bag } \\
\text { No. }\end{array}$ & $\begin{array}{l}\text { UI } \\
\text { No. }\end{array}$ & Category & Condition & Subcategory & $\begin{array}{l}\text { Reduction } \\
\text { Technique }\end{array}$ & Breakage & Exhausted & Use Wear & $\begin{array}{l}\text { Use-Wear } \\
\text { Type }\end{array}$ & Raw material & Inclusions & Munsell & Color & $\begin{array}{c}\text { Heat } \\
\text { Evidence }\end{array}$ & $\begin{array}{l}\text { Tntentional } \\
\text { Heat } \\
\text { Treatment } \\
\end{array}$ \\
\hline \multirow{3}{*}{408} & \multirow{3}{*}{1} & \multirow{3}{*}{507} & \multirow{3}{*}{ NA } & Core & Complete & Multidirectional & Hard & None & No & None & None & $\begin{array}{c}\text { Fine Grain } \\
\text { Chert }\end{array}$ & Irregular Grain & 5YR4/1 & Dark Gray & None & No \\
\hline & & & & Patination & Cortex \% & $\begin{array}{c}\text { Rock } \\
\text { Description }\end{array}$ & Weight (g) & Max Length & Max Width & $\begin{array}{c}\text { Max } \\
\text { Thickness }\end{array}$ & $\begin{array}{l}\text { Max FScar } \\
\text { Length }\end{array}$ & No FScars & \multirow{2}{*}{\multicolumn{5}{|c|}{ Comments }} \\
\hline & & & & Yes & $1-25$ & Indet & 96.23 & 58.03 & 47.71 & 37.26 & 45.05 & 12 & & & & & \\
\hline
\end{tabular}

\begin{tabular}{|c|c|c|c|c|c|c|c|c|c|c|c|c|c|c|c|c|c|}
\hline $\begin{array}{l}\text { Lot } \\
\text { No. }\end{array}$ & $\begin{array}{l}\text { Spec. } \\
\text { No. }\end{array}$ & $\begin{array}{l}\text { Bag } \\
\text { No. }\end{array}$ & $\begin{array}{l}\text { UI } \\
\text { No. }\end{array}$ & Category & Condition & Subcategory & $\begin{array}{l}\text { Reduction } \\
\text { Technique }\end{array}$ & Breakage & Exhausted & Use Wear & $\begin{array}{l}\text { Use-Wear } \\
\text { Type }\end{array}$ & Raw material & Inclusions & Munsell & Color & $\begin{array}{c}\text { Heat } \\
\text { Evidence }\end{array}$ & $\begin{array}{c}\text { Intentional } \\
\text { Heat } \\
\text { Treatment }\end{array}$ \\
\hline \multirow{3}{*}{433} & \multirow{3}{*}{1} & \multirow{3}{*}{603} & \multirow{3}{*}{ NA } & Core & Complete & Multidirectional & Hard & None & Yes & None & None & $\begin{array}{l}\text { Fine Grain } \\
\text { Chert }\end{array}$ & $\begin{array}{l}\text { Irregular Grain and } \\
\text { Fine Fractures }\end{array}$ & 10YR5/1 & Gray & None & No \\
\hline & & & & Patination & Cortex \% & $\begin{array}{c}\text { Rock } \\
\text { Description }\end{array}$ & Weight (g) & Max Length & Max Width & $\begin{array}{c}\text { Max } \\
\text { Thickness } \\
\end{array}$ & $\begin{array}{l}\text { Max FScar } \\
\text { Length }\end{array}$ & No FScars & \multicolumn{5}{|c|}{ Comments } \\
\hline & & & & None & $1-25$ & Indet & 69.74 & 63.34 & 42.68 & 23.87 & 53.85 & 10 & \multicolumn{5}{|c|}{$\begin{array}{c}\text { Approximately 20\% mineral inclusions; Small step and hinge fractures along } \\
\text { edges }\end{array}$} \\
\hline
\end{tabular}

\begin{tabular}{|c|c|c|c|c|c|c|c|c|c|c|c|c|c|c|c|c|c|}
\hline $\begin{array}{l}\text { Lot } \\
\text { No. }\end{array}$ & $\begin{array}{l}\text { Spec. } \\
\text { No. }\end{array}$ & $\begin{array}{l}\text { Bag } \\
\text { No. }\end{array}$ & $\begin{array}{l}\text { UI } \\
\text { No. }\end{array}$ & Category & Condition & Subcategory & $\begin{array}{l}\text { Reduction } \\
\text { Technique }\end{array}$ & Breakage & Exhausted & Use Wear & $\begin{array}{c}\text { Use-Wear } \\
\text { Type }\end{array}$ & Raw material & Inclusions & Munsell & Color & $\begin{array}{c}\text { Heat } \\
\text { Evidence }\end{array}$ & $\begin{array}{c}\text { Intentional } \\
\text { Heat } \\
\text { Treatment } \\
\end{array}$ \\
\hline \multirow{3}{*}{466} & \multirow{3}{*}{1} & \multirow{3}{*}{661} & \multirow{3}{*}{ NA } & Core & Complete & Multidirectional & Hard & None & Yes & None & None & $\begin{array}{l}\text { Fine Grain } \\
\text { Chert }\end{array}$ & Mineral & $\begin{array}{c}\text { 10YR5/1 to } \\
\text { 10YR5/2 }\end{array}$ & $\begin{array}{c}\text { Gray to Grayish } \\
\text { Brown }\end{array}$ & None & No \\
\hline & & & & Patination & Cortex \% & $\begin{array}{c}\text { Rock } \\
\text { Description } \\
\end{array}$ & Weight (g) & Max Length & Max Width & $\begin{array}{c}\text { Max } \\
\text { Thickness } \\
\end{array}$ & $\begin{array}{c}\text { Max FScar } \\
\text { Length }\end{array}$ & No FScars & \multicolumn{5}{|c|}{ Comments } \\
\hline & & & & None & $26-50$ & Nodular & 188.54 & 64.87 & 52.44 & 47.2 & 45.4 & 16 & & & $\begin{array}{l}\text { Various step and } h \\
\text { platforms }\end{array}$ & ige fra & s on all visible \\
\hline
\end{tabular}

\begin{tabular}{|c|c|c|c|c|c|c|c|c|c|c|c|c|c|c|c|c|c|}
\hline $\begin{array}{l}\text { Lot } \\
\text { No. }\end{array}$ & $\begin{array}{l}\text { Spec. } \\
\text { No. }\end{array}$ & $\begin{array}{l}\text { Bag } \\
\text { No. }\end{array}$ & $\begin{array}{l}\text { UI } \\
\text { No. }\end{array}$ & Category & Condition & Subcategory & $\begin{array}{l}\text { Reduction } \\
\text { Technique }\end{array}$ & Breakage & Exhausted & Use Wear & $\begin{array}{l}\text { Use-Wear } \\
\text { Type }\end{array}$ & Raw material & Inclusions & Munsell & Color & $\begin{array}{c}\text { Heat } \\
\text { Evidence }\end{array}$ & $\begin{array}{c}\text { Intentional } \\
\text { Heat } \\
\text { Treatment }\end{array}$ \\
\hline \multirow{3}{*}{500} & \multirow{3}{*}{5} & \multirow{3}{*}{323} & \multirow{3}{*}{ NA } & Core & Core Fragment & Bifacial & Hard & Manufacturing & No & None & None & Fine Grain & Irregular Grain & 10YR7/2 & Very Pale Brown & None & No \\
\hline & & & & Patination & Cortex \% & $\begin{array}{c}\text { Rock } \\
\text { Description }\end{array}$ & Weight (g) & Max Length & Max Width & $\begin{array}{c}\text { Max } \\
\text { Thickness }\end{array}$ & $\begin{array}{l}\text { Max FScar } \\
\text { Length }\end{array}$ & No FScars & \multicolumn{5}{|c|}{ Comments } \\
\hline & & & & None & $0-25$ & Indet & 116.04 & Indet & 61.09 & 29.07 & Indet & Indet & \multicolumn{5}{|c|}{ Snap fracture at one end, very little $(10 \%)$ cortex on one face } \\
\hline
\end{tabular}




\begin{tabular}{|c|c|c|c|c|c|c|c|c|c|c|c|c|c|c|c|c|c|}
\hline $\begin{array}{l}\text { Lot } \\
\text { No. }\end{array}$ & $\begin{array}{c}\text { Spec. } \\
\text { No. }\end{array}$ & $\begin{array}{l}\text { Bag } \\
\text { No. }\end{array}$ & $\begin{array}{l}\text { UI } \\
\text { No. }\end{array}$ & Category & Condition & Subcategory & $\begin{array}{l}\text { Reduction } \\
\text { Technique }\end{array}$ & Breakage & Exhausted & Use Wear & $\begin{array}{l}\text { Use-Wear } \\
\text { Type }\end{array}$ & Raw material & Inclusions & Munsell & Color & $\begin{array}{c}\text { Heat } \\
\text { Evidence }\end{array}$ & $\begin{array}{c}\text { Intentional } \\
\text { Heat } \\
\text { Treatment }\end{array}$ \\
\hline \multirow{3}{*}{562} & \multirow{3}{*}{1} & \multirow{3}{*}{371} & \multirow{3}{*}{$\mathrm{NA}$} & Core & Complete & Bifacial & Hard & None & No & None & None & $\begin{array}{c}\text { Fine Grain } \\
\text { Chert }\end{array}$ & $\begin{array}{l}\text { Irregular Grain and } \\
\text { Fine Fractures }\end{array}$ & $\begin{array}{c}\text { 10YR4/1 to } \\
10 Y R 5 / 1 \\
\end{array}$ & Dark Gray to Gray & None & No \\
\hline & & & & Patination & Cortex $\%$ & $\begin{array}{c}\text { Rock } \\
\text { Description }\end{array}$ & Weight (g) & Max Length & Max Width & $\begin{array}{c}\text { Max } \\
\text { Thickness } \\
\end{array}$ & $\begin{array}{l}\text { Max FScar } \\
\text { Length }\end{array}$ & No FScars & \multicolumn{5}{|c|}{ Comments } \\
\hline & & & & None & 50 & Nodular & 285.11 & 78.01 & 77 & 35.76 & 55.69 & 10 & \multicolumn{5}{|c|}{ One edge worked with flakes removed in one direction } \\
\hline
\end{tabular}

\begin{tabular}{|c|c|c|c|c|c|c|c|c|c|c|c|c|c|c|c|c|c|}
\hline $\begin{array}{l}\text { Lot } \\
\text { No. }\end{array}$ & $\begin{array}{l}\text { Spec. } \\
\text { No. }\end{array}$ & $\begin{array}{l}\text { Bag } \\
\text { No. }\end{array}$ & $\begin{array}{l}\text { UI } \\
\text { No. }\end{array}$ & Category & Condition & Subcategory & $\begin{array}{l}\text { Reduction } \\
\text { Technique }\end{array}$ & Breakage & Exhausted & Use Wear & $\begin{array}{l}\text { Use-Wear } \\
\text { Type }\end{array}$ & Raw material & Inclusions & Munsell & Color & $\begin{array}{c}\text { Heat } \\
\text { Evidence }\end{array}$ & $\begin{array}{c}\text { Intentional } \\
\text { Heat } \\
\text { Treatment }\end{array}$ \\
\hline \multirow{3}{*}{619} & \multirow{3}{*}{7} & \multirow{3}{*}{530} & \multirow{3}{*}{ NA } & Core & Complete & Multidirectional & Hard & None & No & None & None & $\begin{array}{c}\text { Coarse Grain } \\
\text { Chert }\end{array}$ & Fine Fractures & $\begin{array}{c}10 Y R 7 / 3 \text { to } \\
\text { 10YR7/2 }\end{array}$ & $\begin{array}{l}\text { Very Pale Brown } \\
\text { to Light Gray }\end{array}$ & None & No \\
\hline & & & & Patination & Cortex $\%$ & $\begin{array}{c}\text { Rock } \\
\text { Description }\end{array}$ & Weight (g) & Max Length & Max Width & $\begin{array}{c}\text { Max } \\
\text { Thickness } \\
\end{array}$ & $\begin{array}{c}\text { Max FScar } \\
\text { Length }\end{array}$ & No FScars & \multicolumn{5}{|c|}{ Comments } \\
\hline & & & & None & $1-25$ & Indet & 100.19 & 62.25 & 58.36 & 25.62 & 41.39 & 15 & \multicolumn{5}{|c|}{ Rock appears tabular } \\
\hline
\end{tabular}

\begin{tabular}{|c|c|c|c|c|c|c|c|c|c|c|c|c|c|c|c|c|c|}
\hline $\begin{array}{l}\text { Lot } \\
\text { No. }\end{array}$ & $\begin{array}{l}\text { Spec. } \\
\text { No. }\end{array}$ & $\begin{array}{l}\text { Bag } \\
\text { No. }\end{array}$ & $\begin{array}{l}\text { UI } \\
\text { No. }\end{array}$ & Category & Condition & Subcategory & $\begin{array}{l}\text { Reduction } \\
\text { Technique }\end{array}$ & Breakage & Exhausted & Use Wear & $\begin{array}{l}\text { Use-Wear } \\
\text { Type }\end{array}$ & Raw material & Inclusions & Munsell & Color & $\begin{array}{c}\text { Heat } \\
\text { Evidence }\end{array}$ & $\begin{array}{c}\text { Intentional } \\
\text { Heat } \\
\text { Treatment }\end{array}$ \\
\hline \multirow{3}{*}{632} & \multirow{3}{*}{8} & \multirow{3}{*}{385} & \multirow{3}{*}{ NA } & Core & Complete & Multidirectional & Hard & None & No & None & None & $\begin{array}{l}\text { Fine Grain } \\
\text { Chert }\end{array}$ & None & 10YR7/2 & Light Gray & None & No \\
\hline & & & & Patination & Cortex $\%$ & $\begin{array}{c}\text { Rock } \\
\text { Description } \\
\end{array}$ & Weight (g) & Max Length & Max Width & $\begin{array}{c}\text { Max } \\
\text { Thickness }\end{array}$ & $\begin{array}{c}\text { Max FScar } \\
\text { Length }\end{array}$ & No FScars & \multicolumn{5}{|c|}{ Comments } \\
\hline & & & & Yes & $26-50$ & Nodular & 301.02 & 76.51 & 67.91 & 43.17 & 42.06 & 15 & Banding (10YR7 & Small patch & $\begin{array}{l}\text { patina; Dense } \\
\text { edge }\end{array}$ & ep fractures & along working \\
\hline
\end{tabular}

\begin{tabular}{|c|c|c|c|c|c|c|c|c|c|c|c|c|c|c|c|c|c|}
\hline $\begin{array}{l}\text { Lot } \\
\text { No. }\end{array}$ & $\begin{array}{l}\text { Spec. } \\
\text { No. }\end{array}$ & $\begin{array}{l}\text { Bag } \\
\text { No. }\end{array}$ & $\begin{array}{l}\text { UI } \\
\text { No. }\end{array}$ & Category & Condition & Subcategory & $\begin{array}{l}\text { Reduction } \\
\text { Technique }\end{array}$ & Breakage & Exhausted & Use Wear & $\begin{array}{l}\text { Use-Wear } \\
\text { Type }\end{array}$ & Raw material & Inclusions & Munsell & Color & $\begin{array}{c}\text { Heat } \\
\text { Evidence }\end{array}$ & $\begin{array}{c}\text { Intentional } \\
\text { Heat } \\
\text { Treatment }\end{array}$ \\
\hline \multirow{3}{*}{636} & \multirow{3}{*}{1} & \multirow{3}{*}{456} & \multirow{3}{*}{$\mathrm{NA}$} & Core & Complete & Multidirectional & Hard & None & No & None & None & $\begin{array}{c}\text { Fine Grain } \\
\text { Chert }\end{array}$ & Irregular Grain & 10YR6/2 & $\begin{array}{c}\text { Light Brownish } \\
\text { Gray }\end{array}$ & Reddened & Indet \\
\hline & & & & Patination & Cortex \% & $\begin{array}{c}\text { Rock } \\
\text { Description }\end{array}$ & Weight (g) & Max Length & Max Width & $\begin{array}{c}\text { Max } \\
\text { Thickness }\end{array}$ & $\begin{array}{l}\text { Max FScar } \\
\text { Length }\end{array}$ & No FScars & \multicolumn{5}{|c|}{ Comments } \\
\hline & & & & None & $1-25$ & Indet & 150.7 & 83.1 & 57.6 & 31.78 & 83.1 & 15 & pp & ular; & $\mathrm{ng}$ from heat trea & ment (5YR4) & \\
\hline
\end{tabular}

\begin{tabular}{|c|c|c|c|c|c|c|c|c|c|c|c|c|c|c|c|c|c|}
\hline $\begin{array}{l}\text { Lot } \\
\text { No. }\end{array}$ & $\begin{array}{l}\text { Spec. } \\
\text { No. }\end{array}$ & $\begin{array}{l}\text { Bag } \\
\text { No. }\end{array}$ & $\begin{array}{l}\text { UI } \\
\text { No. }\end{array}$ & Category & Condition & Subcategory & $\begin{array}{l}\text { Reduction } \\
\text { Technique }\end{array}$ & Breakage & Exhausted & Use Wear & $\begin{array}{l}\text { Use-Wear } \\
\text { Type }\end{array}$ & Raw material & Inclusions & Munsell & Color & $\begin{array}{c}\text { Heat } \\
\text { Evidence }\end{array}$ & $\begin{array}{c}\text { Intentional } \\
\text { Heat } \\
\text { Treatment }\end{array}$ \\
\hline \multirow{3}{*}{650} & \multirow{3}{*}{5} & \multirow{3}{*}{486} & \multirow{3}{*}{ NA } & Core & Core Fragment & Unifacial & Hard & Manufacturing & No & None & None & $\begin{array}{c}\text { Coarse Grain } \\
\text { Chert }\end{array}$ & Irregular Grain & 10YR6/2 & $\begin{array}{l}\text { Light Brownish } \\
\text { Gray }\end{array}$ & None & No \\
\hline & & & & Patination & Cortex \% & $\begin{array}{c}\text { Rock } \\
\text { Description }\end{array}$ & Weight (g) & Max Length & Max Width & $\begin{array}{c}\text { Max } \\
\text { Thickness }\end{array}$ & $\begin{array}{l}\text { Max FScar } \\
\text { Length }\end{array}$ & No FScars & \multicolumn{5}{|c|}{ Comments } \\
\hline & & & & None & $26-50$ & Nodular & 124.26 & Indet & Indet & Indet & Indet & iddet & & & & sna & \\
\hline
\end{tabular}




\begin{tabular}{|c|c|c|c|c|c|c|c|c|c|c|c|c|c|c|c|c|c|}
\hline $\begin{array}{l}\text { Lot } \\
\text { No. }\end{array}$ & $\begin{array}{c}\text { Spec. } \\
\text { No. }\end{array}$ & $\begin{array}{l}\text { Bag } \\
\text { No. }\end{array}$ & $\begin{array}{l}\text { UI } \\
\text { No. }\end{array}$ & Category & Condition & Subcategory & $\begin{array}{l}\text { Reduction } \\
\text { Technique }\end{array}$ & Breakage & Exhausted & Use Wear & $\begin{array}{l}\text { Use-Wear } \\
\text { Type }\end{array}$ & Raw material & Inclusions & Munsell & Color & $\begin{array}{c}\text { Heat } \\
\text { Evidence }\end{array}$ & $\begin{array}{c}\text { Intentional } \\
\text { Heat } \\
\text { Treatment }\end{array}$ \\
\hline \multirow{3}{*}{660} & \multirow{3}{*}{7} & \multirow{3}{*}{459} & \multirow{3}{*}{ NA } & Core & Complete & Multidirectional & Hard & None & No & None & None & $\begin{array}{l}\text { Fine Grain } \\
\text { Chert }\end{array}$ & Irregular Grain & 10YR3/1 & Very Dark Gray & None & No \\
\hline & & & & Patination & Cortex \% & $\begin{array}{c}\text { Rock } \\
\text { Description }\end{array}$ & Weight (g) & Max Length & Max Width & $\begin{array}{c}\text { Max } \\
\text { Thickness }\end{array}$ & $\begin{array}{l}\text { Max FScar } \\
\text { Length }\end{array}$ & No FScars & \multicolumn{5}{|c|}{ Comments } \\
\hline & & & & Yes & $26-50$ & Nodular & 55.09 & 59.86 & 46.6 & 25.46 & 59.31 & 11 & \multicolumn{5}{|c|}{ Banding of $10 Y R 4 / 1$} \\
\hline
\end{tabular}

\begin{tabular}{|c|c|c|c|c|c|c|c|c|c|c|c|c|c|c|c|c|c|}
\hline $\begin{array}{l}\text { Lot } \\
\text { No. }\end{array}$ & $\begin{array}{l}\text { Spec. } \\
\text { No. }\end{array}$ & $\begin{array}{l}\text { Bag } \\
\text { No. }\end{array}$ & $\begin{array}{l}\text { UI } \\
\text { No. }\end{array}$ & Category & Condition & Subcategory & $\begin{array}{l}\text { Reduction } \\
\text { Technique }\end{array}$ & Breakage & Exhausted & Use Wear & $\begin{array}{l}\text { Use-Wear } \\
\text { Type }\end{array}$ & Raw material & Inclusions & Munsell & Color & $\begin{array}{l}\text { Heat } \\
\text { Evidence }\end{array}$ & $\begin{array}{c}\begin{array}{c}\text { Intentional } \\
\text { Heat } \\
\text { Treatment }\end{array} \\
\end{array}$ \\
\hline \multirow{3}{*}{664} & \multirow{3}{*}{1} & \multirow{3}{*}{569} & \multirow{3}{*}{ NA } & Core & Complete & Multidirectional & Hard & None & No & None & None & $\begin{array}{c}\text { Coarse Grain } \\
\text { Chert }\end{array}$ & Irregular Grain & $\begin{array}{c}\text { 10YR5/2 to } \\
\text { 10YR5/3 }\end{array}$ & $\begin{array}{l}\text { Grayish Brown to } \\
\text { Brown }\end{array}$ & None & No \\
\hline & & & & Patination & Cortex \% & $\begin{array}{c}\text { Rock } \\
\text { Description }\end{array}$ & Weight (g) & Max Length & Max Width & $\begin{array}{c}\text { Max } \\
\text { Thickness }\end{array}$ & $\begin{array}{l}\text { Max FScar } \\
\text { Length }\end{array}$ & No FScars & \multicolumn{5}{|c|}{ Comments } \\
\hline & & & & Yes & $1-25$ & Indet & 109.69 & 70.82 & 53.71 & 37.97 & 41.39 & 34 & \multicolumn{5}{|c|}{ Banding of $10 \mathrm{YR} 5 / 1$ throughout and $10 \mathrm{YR} 4 / 6$ near cortex } \\
\hline
\end{tabular}

\begin{tabular}{|c|c|c|c|c|c|c|c|c|c|c|c|c|c|c|c|c|c|}
\hline $\begin{array}{l}\text { Lot } \\
\text { No. }\end{array}$ & $\begin{array}{l}\text { Spec. } \\
\text { No. }\end{array}$ & $\begin{array}{l}\text { Bag } \\
\text { No. }\end{array}$ & $\begin{array}{l}\text { UI } \\
\text { No. }\end{array}$ & Category & Condition & Subcategory & $\begin{array}{l}\text { Reduction } \\
\text { Technique }\end{array}$ & Breakage & Exhausted & Use Wear & $\begin{array}{c}\text { Use-Wear } \\
\text { Type }\end{array}$ & Raw material & Inclusions & Munsell & Color & $\begin{array}{c}\text { Heat } \\
\text { Evidence }\end{array}$ & $\begin{array}{c}\text { Intentional } \\
\text { Heat } \\
\text { Treatment }\end{array}$ \\
\hline \multirow{3}{*}{665} & \multirow{3}{*}{6} & \multirow{3}{*}{568} & \multirow{3}{*}{ NA } & Core & Complete & Multidirectional & Hard & None & No & None & None & $\begin{array}{c}\text { Fine Grain } \\
\text { Chert }\end{array}$ & $\begin{array}{c}\text { Irregular Grain and } \\
\text { Carbonaceous } \\
\text { cavities }\end{array}$ & 10YR5/1 & Gray & None & No \\
\hline & & & & Patination & Cortex \% & $\begin{array}{c}\text { Rock } \\
\text { Description } \\
\end{array}$ & Weight (g) & Max Length & Max Width & $\begin{array}{c}\text { Max } \\
\text { Thickness }\end{array}$ & $\begin{array}{c}\text { Max FScar } \\
\text { Length }\end{array}$ & No FScars & \\
\hline & & & & None & $1-25$ & Indet & 71.55 & 51.52 & 55.6 & 35.22 & 55.54 & 10 & \multicolumn{5}{|c|}{ Comments } \\
\hline
\end{tabular}

\begin{tabular}{|c|c|c|c|c|c|c|c|c|c|c|c|c|c|c|c|c|c|}
\hline $\begin{array}{l}\text { Lot } \\
\text { No. }\end{array}$ & $\begin{array}{l}\text { Spec. } \\
\text { No. }\end{array}$ & $\begin{array}{l}\text { Bag } \\
\text { No. }\end{array}$ & $\begin{array}{l}\text { UI } \\
\text { No. }\end{array}$ & Category & Condition & Subcategory & $\begin{array}{l}\text { Reduction } \\
\text { Technique }\end{array}$ & Breakage & Exhausted & Use Wear & $\begin{array}{l}\text { Use-Wear } \\
\text { Type }\end{array}$ & Raw material & Inclusions & Munsell & Color & $\begin{array}{c}\text { Heat } \\
\text { Evidence }\end{array}$ & $\begin{array}{c}\text { Intentional } \\
\text { Heat } \\
\text { Treatment } \\
\end{array}$ \\
\hline \multirow{3}{*}{665} & \multirow{3}{*}{7} & \multirow{3}{*}{568} & \multirow{3}{*}{ NA } & Core & Complete & Multidirectional & Hard & None & Yes & None & None & $\begin{array}{c}\text { Coarse Grain } \\
\text { Chert }\end{array}$ & $\begin{array}{c}\text { Irregular Grain and } \\
\text { Fine Fractures }\end{array}$ & $2.5 Y R 2.5 / 1$ & Reddish Gray & Yes & Indet \\
\hline & & & & Patination & Cortex \% & $\begin{array}{c}\text { Rock } \\
\text { Description }\end{array}$ & Weight (g) & Max Length & Max Width & $\begin{array}{c}\text { Max } \\
\text { Thickness }\end{array}$ & $\begin{array}{l}\text { Max FScar } \\
\text { Length }\end{array}$ & No FScars & \multicolumn{5}{|c|}{ Comments } \\
\hline & & & & None & $1-25$ & Indet & 38.13 & 43.1 & 29.82 & 27.99 & 40.27 & 5 & $\begin{array}{l}\text { Core is black in color } \\
20 \%\end{array}$ & $\begin{array}{l}\text { with crenat } \\
\text { onsists of ve }\end{array}$ & $\begin{array}{l}1 \text { and potlids evid } \\
\text { coarse grain } 2.5\end{array}$ & $\begin{array}{l}\text { nt on faces; } \\
3 / 1 \text { inclusion }\end{array}$ & Approximately \\
\hline
\end{tabular}

\begin{tabular}{|c|c|c|c|c|c|c|c|c|c|c|c|c|c|c|c|c|c|}
\hline $\begin{array}{l}\text { Lot } \\
\text { No. }\end{array}$ & $\begin{array}{l}\text { Spec. } \\
\text { No. }\end{array}$ & $\begin{array}{l}\text { Bag } \\
\text { No. }\end{array}$ & $\begin{array}{l}\text { UI } \\
\text { No. }\end{array}$ & Category & Condition & Subcategory & $\begin{array}{l}\text { Reduction } \\
\text { Technique }\end{array}$ & Breakage & Exhausted & Use Wear & $\begin{array}{l}\text { Use-Wear } \\
\text { Type }\end{array}$ & Raw material & Inclusions & Munsell & Color & $\begin{array}{c}\text { Heat } \\
\text { Evidence }\end{array}$ & $\begin{array}{c}\text { Intentional } \\
\text { Heat } \\
\text { Treatment }\end{array}$ \\
\hline \multirow{3}{*}{667} & \multirow{3}{*}{1} & \multirow{3}{*}{594} & \multirow{3}{*}{ NA } & Core & Complete & Bifacial & Hard & None & No & None & None & $\begin{array}{c}\text { Fine Grain } \\
\text { Chert }\end{array}$ & $\begin{array}{l}\text { Irregular Grain and } \\
\text { Fine Fractures }\end{array}$ & $\begin{array}{c}\text { 10YR7/1 to } \\
10 Y R 7 / 2\end{array}$ & Light Gray & None & No \\
\hline & & & & Patination & Cortex $\%$ & $\begin{array}{c}\text { Rock } \\
\text { Description }\end{array}$ & Weight (g) & Max Length & Max Width & $\begin{array}{c}\text { Max } \\
\text { Thickness } \\
\end{array}$ & $\begin{array}{c}\text { Max FScar } \\
\text { Length }\end{array}$ & No FScars & \multicolumn{5}{|c|}{ Comments } \\
\hline & & & & None & $26-50$ & Nodular & 312.57 & 95.8 & 58.02 & 45.8 & 78.48 & 8 & Large round cobble & with irregula & rain inclusion & Work began & on one edge \\
\hline
\end{tabular}




\begin{tabular}{|c|c|c|c|c|c|c|c|c|c|c|c|c|c|c|c|c|c|}
\hline $\begin{array}{l}\text { Lot } \\
\text { No. }\end{array}$ & $\begin{array}{l}\text { Spec. } \\
\text { No. }\end{array}$ & $\begin{array}{l}\text { Bag } \\
\text { No. }\end{array}$ & $\begin{array}{l}\text { UI } \\
\text { No. }\end{array}$ & Category & Condition & Subcategory & $\begin{array}{l}\text { Reduction } \\
\text { Technique }\end{array}$ & Breakage & Exhausted & Use Wear & $\begin{array}{l}\text { Use-Wear } \\
\text { Type }\end{array}$ & Raw material & Inclusions & Munsell & Color & $\begin{array}{c}\text { Heat } \\
\text { Evidence }\end{array}$ & $\begin{array}{c}\text { Intentional } \\
\text { Heat } \\
\text { Treatment } \\
\end{array}$ \\
\hline \multirow{3}{*}{685} & \multirow{3}{*}{1} & \multirow{3}{*}{582} & \multirow{3}{*}{ NA } & Core & Complete & Multidirectional & Indet & None & Yes & None & None & $\begin{array}{c}\text { Coarse Grain } \\
\text { Chert }\end{array}$ & Irregular Grain & 10YR7/3 & Very Pale Brown & Reddened & Yes \\
\hline & & & & Patination & Cortex \% & $\begin{array}{c}\text { Rock } \\
\text { Description }\end{array}$ & Weight (g) & Max Length & Max Width & $\begin{array}{c}\text { Max } \\
\text { Thickness } \\
\end{array}$ & $\begin{array}{l}\text { Max FScar } \\
\text { Length }\end{array}$ & No FScars & \multicolumn{5}{|c|}{ Comments } \\
\hline & & & & None & 0 & Indet & 18.02 & 26.43 & 24.25 & 20.55 & 25.8 & 12 & \multicolumn{5}{|c|}{$\begin{array}{c}\text { Exhausted core; Very small and weathered smooth; Slightly reddened due to } \\
\text { heat treatment (5YR6/4) }\end{array}$} \\
\hline $\begin{array}{l}\text { Lot } \\
\text { No. }\end{array}$ & $\begin{array}{l}\text { Spec. } \\
\text { No. }\end{array}$ & $\begin{array}{l}\text { Bag } \\
\text { No. }\end{array}$ & $\begin{array}{l}\text { UI } \\
\text { No. }\end{array}$ & Category & Condition & Subcategory & $\begin{array}{l}\text { Reduction } \\
\text { Technique }\end{array}$ & Breakage & Exhausted & Use Wear & $\begin{array}{l}\text { Use-Wear } \\
\text { Type }\end{array}$ & Raw material & Inclusions & Munsell & Color & $\begin{array}{c}\text { Heat } \\
\text { Evidence }\end{array}$ & $\begin{array}{c}\text { Intentional } \\
\text { Heat } \\
\text { Treatment } \\
\end{array}$ \\
\hline \multirow{3}{*}{546} & \multirow{3}{*}{1} & \multirow{3}{*}{327} & \multirow{3}{*}{ NA } & Core & Complete & Bifacial & & & & & & $\begin{array}{c}\text { Fine Grain } \\
\text { Chert }\end{array}$ & Irregular Grain & $\begin{array}{c}\text { 10YR6/2 to } \\
10 Y R 6 / 3\end{array}$ & $\begin{array}{l}\text { Light Brown Gray } \\
\text { to Pale Brown }\end{array}$ & None & No \\
\hline & & & & Patination & Cortex \% & $\begin{array}{c}\text { Rock } \\
\text { Description }\end{array}$ & Weight (g) & Max Length & Max Width & $\begin{array}{c}\text { Max } \\
\text { Thickness } \\
\end{array}$ & $\begin{array}{l}\text { Max FScar } \\
\text { Length }\end{array}$ & No FScars & \multicolumn{5}{|c|}{ Comments } \\
\hline & & & & None & $75-100$ & Tabular & 100.34 & 61.61 & 58.8 & 21.05 & 28.4 & & \multicolumn{5}{|c|}{$\begin{array}{l}\text { Tabular rock with rounded edges that has been broken in half; Break used at } \\
\text { platform with flakes removed from one corner of the rock; very few flakes } \\
\text { removed - tested cobble }\end{array}$} \\
\hline
\end{tabular}




\begin{tabular}{|c|c|c|c|c|c|c|c|c|c|c|c|c|c|c|c|c|c|}
\hline Lot No. & Spec. No. & Bag No. & UI No. & Category & Subcategory & $\begin{array}{l}\text { Specimen } \\
\text { Condition }\end{array}$ & Breakage & Raw Material & Munsell & Color & Cortex \% & Parent Form & $\begin{array}{c}\text { Heat } \\
\text { Evidence }\end{array}$ & $\begin{array}{l}\text { Intentional Heat } \\
\text { Treatment }\end{array}$ & Patination & $\begin{array}{l}\text { Overall } \\
\text { Shape }\end{array}$ & $\begin{array}{l}\text { Basal } \\
\text { Shape }\end{array}$ \\
\hline \multirow{3}{*}{427} & \multirow{3}{*}{1} & \multirow{3}{*}{602} & \multirow{3}{*}{ NA } & Core tool & Chopper & Complete & None & Fine Grain Chert & 10YR7/2 & Light Gray & $51-75$ & Nodular Cobble & None & No & None & Indet & Indet \\
\hline & & & & $\begin{array}{l}\text { Cross-section } \\
\text { Shape }\end{array}$ & $\begin{array}{l}\text { Flaking } \\
\text { Pattern }\end{array}$ & Beveling & $\begin{array}{l}\text { Reduction } \\
\text { Stage }\end{array}$ & Weight (g) & $\begin{array}{c}\text { Max } \\
\text { Length }\end{array}$ & Max Width & $\begin{array}{l}\text { Max } \\
\text { Thickness }\end{array}$ & $\begin{array}{l}\text { Avg Edge } \\
\text { Angle }\end{array}$ & $\begin{array}{l}\text { Callahan's } \\
\text { w/t index }\end{array}$ & \multicolumn{4}{|c|}{ Comments } \\
\hline & & & & Indet & Subparallel & None & Indet & 355.4 & 84.44 & 86.1 & 46.11 & 65 & Indet & \multicolumn{4}{|c|}{ Expedient tool; Banding near cortex (10YR4/1) } \\
\hline Lot No. & Spec. No. & Bag No. & UI No. & Category & Subcategory & $\begin{array}{l}\text { Specimen } \\
\text { Condition }\end{array}$ & Breakage & Raw Material & Munsell & Color & Cortex \% & Parent Form & $\begin{array}{c}\text { Heat } \\
\text { Evidence }\end{array}$ & $\begin{array}{l}\text { Intentional Heat } \\
\text { Treatment }\end{array}$ & Patination & $\begin{array}{l}\text { Overall } \\
\text { Shape }\end{array}$ & $\begin{array}{l}\text { Basal } \\
\text { Shape }\end{array}$ \\
\hline \multirow{3}{*}{432} & \multirow{3}{*}{1} & \multirow{3}{*}{600} & \multirow{3}{*}{ NA } & Core tool & Chopper & Complete & None & Coarse Grain Chert & 10YR7/2 & Light Gray & $51-75$ & Nodular Cobble & None & No & None & Indet & Indet \\
\hline & & & & $\begin{array}{l}\text { Cross-section } \\
\text { Shape }\end{array}$ & $\begin{array}{l}\text { Flaking } \\
\text { Pattern }\end{array}$ & Beveling & $\begin{array}{l}\text { Reduction } \\
\text { Stage }\end{array}$ & Weight (g) & $\begin{array}{c}\text { Max } \\
\text { Length }\end{array}$ & Max Width & $\begin{array}{l}\text { Max } \\
\text { Thickness }\end{array}$ & $\begin{array}{l}\text { Avg Edge } \\
\text { Angle }\end{array}$ & $\begin{array}{l}\text { Callahan's } \\
\text { w/t index }\end{array}$ & \multicolumn{4}{|c|}{ Comments } \\
\hline & & & & Indet & Random & None & Indet & 493.32 & 107.85 & 91.28 & 49.5 & 50 & Indet & \multicolumn{4}{|c|}{ Expedient tool; Banding near cortex (10YR5/1) } \\
\hline Lot No. & Spec. No. & Bag No. & UI No. & Category & Subcategory & $\begin{array}{l}\text { Specimen } \\
\text { Condition }\end{array}$ & Breakage & Raw Material & Munsell & Color & Cortex \% & Parent Form & $\begin{array}{c}\text { Heat } \\
\text { Evidence }\end{array}$ & $\begin{array}{l}\text { Intentional Heat } \\
\text { Treatment }\end{array}$ & Patination & $\begin{array}{l}\text { Overall } \\
\text { Shape }\end{array}$ & $\begin{array}{l}\text { Basal } \\
\text { Shape }\end{array}$ \\
\hline \multirow{3}{*}{592} & \multirow{3}{*}{1} & \multirow{3}{*}{564} & \multirow{3}{*}{ NA } & Core tool & Chopper & Complete & None & Coarse Grain Chert & 10YR7/1 & Light Gray & $51-75$ & Nodular Cobble & None & No & None & Indet & Indet \\
\hline & & & & $\begin{array}{l}\text { Cross-section } \\
\text { Shape }\end{array}$ & $\begin{array}{l}\text { Flaking } \\
\text { Pattern }\end{array}$ & Beveling & $\begin{array}{l}\text { Reduction } \\
\text { Stage }\end{array}$ & Weight (g) & $\begin{array}{c}\text { Max } \\
\text { Length }\end{array}$ & Max Width & $\begin{array}{l}\text { Max } \\
\text { Thickness }\end{array}$ & $\begin{array}{l}\text { Avg Edge } \\
\text { Angle }\end{array}$ & $\begin{array}{l}\text { Callahan's } \\
\text { w/t index }\end{array}$ & \multicolumn{4}{|c|}{ Comments } \\
\hline & & & & Indet & Random & None & Indet & 401.29 & 100.53 & 97.14 & 42.65 & 80 & Indet & \multicolumn{4}{|c|}{$\begin{array}{l}\text { Mottling 10YR62 throughout; Expedient tool with } \\
\text { irregular edges }\end{array}$} \\
\hline Lot No. & Spec. No. & Bag No. & UI No. & Category & Subcategory & $\begin{array}{l}\text { Specimen } \\
\text { Condition }\end{array}$ & Breakage & Raw Material & Munsell & Color & Cortex \% & Parent Form & $\begin{array}{c}\text { Heat } \\
\text { Evidence }\end{array}$ & $\begin{array}{l}\text { Intentional Heat } \\
\text { Treatment }\end{array}$ & Patination & $\begin{array}{l}\text { Overall } \\
\text { Shape }\end{array}$ & $\begin{array}{l}\text { Basal } \\
\text { Shape }\end{array}$ \\
\hline \multirow{3}{*}{624} & \multirow{3}{*}{1} & \multirow{3}{*}{593} & \multirow{3}{*}{ NA } & Core tool & Chopper & Complete & None & Coarse Grain Chert & 10YR7/1 & Light Gray & $26-50$ & Nodular Cobble & None & No & None & Indet & Indet \\
\hline & & & & $\begin{array}{l}\text { Cross-section } \\
\text { Shape }\end{array}$ & $\begin{array}{l}\text { Flaking } \\
\text { Pattern }\end{array}$ & Beveling & $\begin{array}{l}\text { Reduction } \\
\text { Stage }\end{array}$ & Weight (g) & $\begin{array}{c}\text { Max } \\
\text { Length }\end{array}$ & Max Width & $\begin{array}{c}\text { Max } \\
\text { Thickness }\end{array}$ & $\begin{array}{l}\text { Avg Edge } \\
\text { Angle }\end{array}$ & $\begin{array}{l}\text { Callahan's } \\
\text { w/t index }\end{array}$ & \multicolumn{4}{|c|}{ Comments } \\
\hline & & & & Indet & $\begin{array}{c}\text { Oblique } \\
\text { Subparallel }\end{array}$ & None & Indet & 235.17 & 104.74 & 71.32 & 49.99 & 75 & Indet & \multicolumn{4}{|c|}{ Expedient tool; Banding near cortex (10YR3/1) } \\
\hline
\end{tabular}




\begin{tabular}{|c|c|c|c|c|c|c|c|c|c|c|c|c|c|c|c|}
\hline Lot \# & Bag \# & $\begin{array}{c}\text { Complete } \\
\text { Flake } \\
\text { Count }\end{array}$ & $\begin{array}{c}\text { Complete } \\
\text { Flake } \\
\text { Weight (g) }\end{array}$ & $\begin{array}{l}\text { Proximal } \\
\text { Flake } \\
\text { Count }\end{array}$ & $\begin{array}{l}\text { Proximal } \\
\text { Flake } \\
\text { Weight (g) }\end{array}$ & $\begin{array}{c}\text { Broken } \\
\text { Flake } \\
\text { Count }\end{array}$ & $\begin{array}{c}\text { Broken } \\
\text { Flake } \\
\text { Weight (g) }\end{array}$ & $\begin{array}{c}\text { Flaking } \\
\text { Shatter } \\
\text { Count }\end{array}$ & \begin{tabular}{|c|} 
Flaking \\
Shatter \\
Weight (g)
\end{tabular} & $\begin{array}{c}\text { Thermal } \\
\text { Shatter } \\
\text { Count }\end{array}$ & $\begin{array}{c}\text { Thermal } \\
\text { Shatter } \\
\text { Weight (g) }\end{array}$ & Comments & $\begin{array}{c}\text { Total } \\
\text { No. } \\
\text { Flakes }\end{array}$ & $\begin{array}{c}\text { Total } \\
\text { No. } \\
\text { Shatter }\end{array}$ & $\begin{array}{l}\text { Final } \\
\text { Total }\end{array}$ \\
\hline 314 & 467 & 1 & 1.39 & & & 3 & 6.99 & & & \begin{tabular}{|l|}
2 \\
\end{tabular} & \begin{tabular}{|l|}
8.18 \\
\end{tabular} & & 4 & 2 & 6 \\
\hline 315 & 475 & & & & & & & & & 1 & 0.27 & & 0 & 1 & 1 \\
\hline 316 & 480 & 1 & 0.14 & 3 & 2.03 & 3 & 0.92 & & & 1 & 0.29 & & 7 & 1 & 8 \\
\hline 317 & 485 & 5 & 29.72 & 6 & 4.35 & 12 & 13.88 & & & 4 & 4.58 & & 23 & 4 & 27 \\
\hline 318 & 492 & 9 & 15.27 & 8 & 7.1 & 5 & 61.31 & & & 10 & 7.51 & & 22 & 10 & 32 \\
\hline 319 & 501 & 6 & 69.41 & 5 & 8.28 & 22 & 76.09 & & & 4 & 2.53 & & 33 & 4 & 37 \\
\hline 321 & 506 & 6 & 98.18 & 8 & 6 & 37 & 59.02 & & & 19 & 27.33 & & 51 & 19 & 70 \\
\hline 323 & 524 & 4 & 23.33 & 4 & 5.76 & 57 & 24.02 & 2 & 0.67 & 44 & 48.06 & & 65 & 46 & 111 \\
\hline 325 & 578 & 4 & 46.2 & 1 & 0.26 & 31 & 18.54 & & & 14 & 13.19 & & 36 & 14 & 50 \\
\hline 327 & 589 & 5 & 5.32 & 2 & 0.68 & 27 & 15.89 & & & 52 & 71.42 & & 34 & 52 & 86 \\
\hline 328 & 619 & 3 & 12.19 & 1 & 0.79 & 18 & 2.75 & & & 9 & 4.66 & & 22 & 9 & 31 \\
\hline 330 & 639 & 1 & 11.24 & 2 & 1.52 & 3 & 1.85 & & & & & $\begin{array}{l}{ }^{\circ} \text { Complete flake is } \\
95 \% \text { complete }\end{array}$ & 6 & 0 & 6 \\
\hline 331 & 642 & 1 & 1.41 & 1 & 46.49 & & & & & & & & 2 & 0 & 2 \\
\hline 332 & 653 & 1 & 7.58 & 4 & 9.64 & 5 & 1.22 & & & & & & 10 & 0 & 10 \\
\hline 333 & 350 & & & 1 & 0.45 & 2 & 3.87 & & & & & & 3 & 0 & 3 \\
\hline 334 & 358 & 2 & 0.35 & 2 & 1.47 & 5 & 5.01 & & & & & & 9 & 0 & 9 \\
\hline 335 & 378 & 2 & 14.26 & 2 & 2.99 & 2 & 1.5 & & & & & & 6 & 0 & 6 \\
\hline 336 & 388 & 5 & 2.02 & 9 & 3.99 & 17 & 12.99 & 1 & 1.6 & 9 & 6.66 & & 31 & 10 & 41 \\
\hline 337 & 399 & 17 & 14.74 & 17 & 74.62 & 41 & 40.17 & 4 & 3.95 & 21 & 20.23 & & 75 & 25 & 100 \\
\hline 338 & 405 & 14 & 39.25 & 13 & 10.05 & 32 & 12.96 & 2 & 1.71 & 15 & 12.62 & & 59 & 17 & 76 \\
\hline 339 & 410 & 19 & 37.67 & 17 & 9.68 & 41 & 15.89 & 4 & 1.65 & 29 & 20.37 & & 77 & 33 & 110 \\
\hline 342 & 422 & 2 & 39.52 & 4 & 34.46 & 5 & 5.22 & & & 6 & 3.41 & & 11 & 6 & 17 \\
\hline 343 & 607 & 4 & 2.48 & 1 & 8.74 & 13 & 22.8 & & & 7 & 4.87 & & 18 & 7 & 25 \\
\hline 344 & 610 & 5 & 26 & 1 & 0.32 & 8 & 2.62 & & & 7 & 12.11 & & 14 & 7 & 21 \\
\hline 345 & 614 & 1 & 0.59 & & & 4 & 5.34 & & & 2 & 1.11 & & 5 & 2 & 7 \\
\hline 346 & 626 & 2 & 24.97 & & & 1 & 0.87 & 1 & 0.15 & 8 & 34.88 & & 3 & 8 & 11 \\
\hline 347 & 636 & 1 & 4.17 & 1 & 0.16 & 2 & 0.36 & & & 2 & 4.2 & & 4 & 2 & 6 \\
\hline 348 & 644 & & & 1 & 12.38 & 1 & 2.3 & & & & & & 2 & 0 & 2 \\
\hline 349 & 670 & & & & & 1 & 2.28 & & & 1 & 0.41 & & 1 & 1 & 2 \\
\hline 350 & 354 & & & & & 3 & 1.91 & & & & & & 3 & 0 & 3 \\
\hline 351 & 370 & & & & & 3 & 0.75 & & & & & & 3 & 0 & 3 \\
\hline 352 & 382 & 2 & 1.72 & & & 5 & 2.31 & & & 2 & 2.22 & & 7 & 2 & 9 \\
\hline 353 & 394 & 11 & 6.63 & 18 & 11.63 & 32 & 39.05 & 3 & 1.2 & 11 & 15.32 & & 61 & 14 & 75 \\
\hline 354 & 326 & 21 & 93.4 & 15 & 14.3 & 55 & 24.44 & & & 20 & 18.25 & & 91 & 20 & 111 \\
\hline 355 & 407 & 21 & 43.1 & 18 & 14.52 & 46 & 45.15 & & & 22 & 18.31 & & 85 & 22 & 107 \\
\hline 357 & 415 & 17 & 73.22 & 17 & 6.64 & 37 & 27.46 & & & 31 & 22.39 & & 71 & 31 & 102 \\
\hline 358 & 426 & 7 & 54.15 & 9 & 10.78 & 10 & 4.5 & & & 21 & 19.62 & & 26 & 21 & 47 \\
\hline 361 & 516 & 1 & 1.78 & & & 3 & 2.9 & & & 1 & 2.93 & & 4 & 1 & 5 \\
\hline 362 & 532 & 3 & 17.45 & & & 6 & 2.48 & & & 1 & 0.28 & & 9 & 1 & 10 \\
\hline 363 & 634 & 3 & 7.43 & & & 1 & 0.4 & & & 1 & 0.46 & & 4 & 1 & 5 \\
\hline 364 & 647 & & & 2 & 1.63 & & & & & 1 & 2.74 & & 2 & 1 & 3 \\
\hline 365 & 650 & 2 & 36.85 & 1 & 4.73 & 1 & 0.17 & & & & & & 4 & 0 & 4 \\
\hline 366 & 470 & 1 & 2.42 & & & 1 & 1.05 & & & 1 & 12.24 & & 2 & 1 & 3 \\
\hline 367 & 477 & 1 & 4.57 & 3 & 1.88 & 2 & 0.25 & & & 3 & 0.88 & & 6 & 3 & 9 \\
\hline 368 & 481 & 1 & 16.39 & 2 & 11.95 & 5 & 1.9 & & & 7 & 7.68 & & 8 & 7 & 15 \\
\hline 369 & 487 & 7 & 13.52 & 10 & 10.19 & 21 & 8.87 & 2 & 0.76 & 12 & 13.22 & & 38 & 12 & 50 \\
\hline 370 & 496 & 11 & 57.41 & 14 & 15.37 & 29 & 15.7 & 1 & 0.56 & 11 & 26.35 & & 54 & 11 & 65 \\
\hline 372 & 502 & 6 & 32.33 & 6 & 8.51 & 23 & 28.37 & 1 & 0.53 & 20 & 54.18 & & 35 & 21 & 56 \\
\hline
\end{tabular}




\begin{tabular}{|c|c|c|c|c|c|c|c|c|c|c|c|c|c|c|c|}
\hline Lot \# & Bag \# & $\begin{array}{c}\text { Complete } \\
\text { Flake } \\
\text { Count }\end{array}$ & $\begin{array}{c}\text { Complete } \\
\text { Flake } \\
\text { Weight (g) }\end{array}$ & $\begin{array}{l}\text { Proximal } \\
\text { Flake } \\
\text { Count }\end{array}$ & $\begin{array}{l}\text { Proximal } \\
\text { Flake } \\
\text { Weight (g) }\end{array}$ & $\begin{array}{c}\text { Broken } \\
\text { Flake } \\
\text { Count }\end{array}$ & $\begin{array}{c}\text { Broken } \\
\text { Flake } \\
\text { Weight (g) }\end{array}$ & $\begin{array}{c}\text { Flaking } \\
\text { Shatter } \\
\text { Count }\end{array}$ & \begin{tabular}{|c|} 
Flaking \\
Shatter \\
Weight (g)
\end{tabular} & $\begin{array}{c}\text { Thermal } \\
\text { Shatter } \\
\text { Count }\end{array}$ & $\begin{array}{c}\text { Thermal } \\
\text { Shatter } \\
\text { Weight (g) }\end{array}$ & Comments & $\begin{array}{c}\text { Total } \\
\text { No. } \\
\text { Flakes }\end{array}$ & $\begin{array}{c}\text { Total } \\
\text { No. } \\
\text { Shatter }\end{array}$ & $\begin{array}{l}\text { Final } \\
\text { Total }\end{array}$ \\
\hline 373 & 517 & 12 & 36.48 & 21 & 32.82 & 59 & 38.36 & \begin{tabular}{|l|}
2 \\
\end{tabular} & 2.9 & \begin{tabular}{|l|}
52 \\
\end{tabular} & \begin{tabular}{|l|}
51.75 \\
\end{tabular} & & 92 & 54 & 146 \\
\hline 375 & 549 & 7 & 65.15 & 5 & 7.52 & 23 & 17.78 & & & 48 & 21.64 & & 35 & 48 & 83 \\
\hline 377 & 573 & 4 & 6.51 & 5 & 12.43 & 34 & 10.31 & & & 83 & 51.17 & & 43 & 83 & 126 \\
\hline 378 & 586 & 7 & 31.79 & 5 & 5.19 & 9 & 11.83 & & & 5 & 1.19 & & 21 & 5 & 26 \\
\hline 379 & 621 & 1 & 2.06 & 2 & 3.21 & 4 & 8.63 & & & 5 & 7.54 & & 7 & 5 & 12 \\
\hline 380 & 627 & 2 & 7.05 & 1 & 0.72 & 4 & 7.21 & & & 5 & 16.55 & & 7 & 5 & 12 \\
\hline 381 & 635 & & & & & 1 & 6.22 & & & 4 & 12.85 & & 1 & 4 & 5 \\
\hline 382 & 652 & & & 2 & 3.51 & & & & & 2 & 0.35 & & 2 & 2 & 4 \\
\hline 383 & 433 & & & 1 & 0.17 & 4 & 3.23 & & & 1 & 0.19 & & 5 & 1 & 6 \\
\hline 384 & 439 & 1 & 3.07 & & & 3 & 0.74 & & & 1 & 0.77 & & 4 & 1 & 5 \\
\hline 386 & 445 & 7 & 40.35 & 7 & 2.76 & 8 & 3.81 & & & 10 & 18.95 & & 22 & 10 & 32 \\
\hline 388 & 362 & 10 & 25.34 & 8 & 13.89 & 33 & 31.74 & 2 & 0.7 & 17 & 17.91 & & 51 & 17 & 68 \\
\hline 389 & 451 & 7 & 65.01 & 9 & 33.63 & 28 & 25.75 & 1 & 0.49 & 9 & 6.03 & & 44 & 10 & 54 \\
\hline 390 & 458 & 5 & 15.32 & 14 & 5.55 & 23 & 15.02 & 2 & 16.53 & 30 & 23.48 & & 42 & 32 & 74 \\
\hline 391 & 465 & 8 & 4.49 & 11 & 7.75 & 23 & 15.71 & 1 & 1.24 & 21 & 19.96 & & 42 & 22 & 64 \\
\hline 392 & 608 & 4 & 55.68 & 3 & 5.22 & 5 & 8.69 & 2 & 13.99 & 4 & 1.77 & & 12 & 6 & 18 \\
\hline 394 & 612 & 2 & 23.71 & & & 2 & 12.27 & & & 4 & 6.56 & & 4 & 4 & 8 \\
\hline 395 & 617 & & & 2 & 0.42 & 2 & 2.12 & & & & & & 4 & 0 & 4 \\
\hline 396 & 624 & & & & & 1 & 0.22 & & & & & & 1 & $\overline{0}$ & 1 \\
\hline 397 & 632 & 1 & 0.16 & & & 2 & 0.88 & & & & & & 3 & 0 & 3 \\
\hline 398 & 645 & & & 1 & 0.12 & & & & & & & & 1 & 0 & 1 \\
\hline 399 & 667 & & & 1 & 0.62 & 1 & 2.45 & & & & & & 2 & 0 & 2 \\
\hline 400 & 483 & 1 & 0.67 & 1 & 0.75 & 2 & 1 & & & 1 & 0.6 & & 4 & 1 & 5 \\
\hline 401 & 488 & 1 & 9.02 & 3 & 2.45 & 10 & 6.65 & & & 3 & 0.61 & & 14 & 3 & 17 \\
\hline 402 & 490 & 4 & 25.19 & 8 & 4.09 & 23 & 38.05 & & & 7 & 3.01 & & 35 & 7 & 42 \\
\hline 403 & 495 & 16 & 106.41 & 26 & 88.75 & 62 & 47.28 & 4 & 11.87 & 24 & 50.33 & & 104 & 24 & 128 \\
\hline 405 & 500 & 6 & 10.71 & 18 & 10.17 & 49 & 49.58 & & & 21 & 9.39 & & 73 & 21 & 94 \\
\hline 406 & 503 & 7 & 8.87 & 13 & 12.27 & 49 & 38.21 & 3 & 2.53 & 42 & 48.23 & & 69 & 45 & 114 \\
\hline 407 & 507 & 11 & 24.16 & 7 & 5.78 & 19 & 9.25 & 2 & 9.72 & 12 & 29.31 & & 37 & 14 & 51 \\
\hline 409 & 514 & 6 & 56.27 & 6 & 17.44 & 12 & 14.51 & 2 & 1.48 & 27 & 70.74 & & 24 & 29 & 53 \\
\hline 410 & 528 & 2 & 11.76 & 14 & 43.22 & 23 & 22.02 & 1 & 7.53 & 30 & 37.36 & & 39 & 30 & 69 \\
\hline 411 & 537 & 5 & 14.17 & 6 & 7.54 & 4 & 6.44 & 1 & 0.33 & 24 & 27.53 & & 15 & 24 & 39 \\
\hline 412 & 567 & 1 & 68.47 & 2 & 3.76 & 3 & 0.75 & & & 8 & 2.28 & & 6 & 8 & 14 \\
\hline 413 & 540 & 2 & 11.29 & 2 & 0.42 & 2 & 2 & & & 6 & 2.42 & & 6 & 6 & 12 \\
\hline 414 & 543 & & & & & 2 & 0.66 & & & & & & 2 & 0 & 2 \\
\hline 415 & 547 & & & & & 1 & 0.06 & & & & & & 1 & 0 & 1 \\
\hline 417 & 561 & & & 2 & 0.29 & 2 & 2.31 & & & & & & 4 & 0 & 4 \\
\hline 418 & 673 & & & & & & & & & 1 & 1.44 & & 0 & 1 & 1 \\
\hline 424 & 595 & & & 1 & 32.73 & & & & & & & Lithic \#1 & 1 & 0 & 1 \\
\hline 425 & 596 & 1 & 29.63 & & & & & & & & & Lithic \#2 & 1 & 0 & 1 \\
\hline 426 & 601 & & & 1 & 20.74 & & & & & & & Lithic \#7 & 1 & 0 & 1 \\
\hline 434 & 408 & & & 1 & 0.26 & 3 & 22.49 & & & & & & 4 & 0 & 4 \\
\hline 435 & 412 & 1 & 1.12 & & & 2 & 1.55 & & & & & & 3 & 0 & 3 \\
\hline 436 & 423 & 1 & 0.57 & 3 & 27.51 & 3 & 1.08 & & & 3 & 2.39 & & 7 & 3 & 10 \\
\hline 437 & 429 & 10 & 40.32 & 3 & 5.65 & 28 & 26.73 & & & 7 & 8.33 & & 41 & 7 & 48 \\
\hline 438 & 437 & 6 & 44.45 & 1 & 0.56 & 17 & 5.72 & & & 23 & 22.37 & & 24 & 23 & 47 \\
\hline 439 & 442 & 5 & 2 & 4 & 5.49 & 19 & 8.66 & 3 & 1.43 & 48 & 51.06 & & 28 & 48 & 76 \\
\hline 440 & 448 & 2 & 10.57 & 5 & 21.5 & 19 & 27.54 & & & 21 & 28.39 & & 26 & 21 & 47 \\
\hline 441 & 453 & & & & & 2 & 1.47 & & & 5 & 8.15 & & 2 & 5 & 7 \\
\hline
\end{tabular}




\begin{tabular}{|c|c|c|c|c|c|c|c|c|c|c|c|c|c|c|c|}
\hline Lot \# & Bag \# & $\begin{array}{c}\text { Complete } \\
\text { Flake } \\
\text { Count }\end{array}$ & $\begin{array}{c}\text { Complete } \\
\text { Flake } \\
\text { Weight (g) }\end{array}$ & $\begin{array}{l}\text { Proximal } \\
\text { Flake } \\
\text { Count }\end{array}$ & $\begin{array}{l}\text { Proximal } \\
\text { Flake } \\
\text { Weight (g) }\end{array}$ & $\begin{array}{c}\text { Broken } \\
\text { Flake } \\
\text { Count }\end{array}$ & $\begin{array}{c}\text { Broken } \\
\text { Flake } \\
\text { Weight (g) }\end{array}$ & $\begin{array}{c}\text { Flaking } \\
\text { Shatter } \\
\text { Count }\end{array}$ & \begin{tabular}{|c|} 
Flaking \\
Shatter \\
Weight (g)
\end{tabular} & $\begin{array}{c}\text { Thermal } \\
\text { Shatter } \\
\text { Count }\end{array}$ & $\begin{array}{c}\text { Thermal } \\
\text { Shatter } \\
\text { Weight (g) }\end{array}$ & Comments & $\begin{array}{c}\text { Total } \\
\text { No. } \\
\text { Flakes }\end{array}$ & $\begin{array}{c}\text { Total } \\
\text { No. } \\
\text { Shatter }\end{array}$ & $\begin{array}{l}\text { Final } \\
\text { Total }\end{array}$ \\
\hline 442 & 460 & & & 2 & 4.03 & 2 & 2.42 & \begin{tabular}{|l|}
1 \\
\end{tabular} & \begin{tabular}{|l|}
1.94 \\
\end{tabular} & & & & 4 & 1 & 5 \\
\hline 443 & 476 & & & 3 & 13.71 & 6 & 5.62 & & & 3 & 5.96 & & 9 & 3 & 12 \\
\hline 444 & 482 & & & 1 & 0.65 & 1 & 0.57 & & & 2 & 3.79 & & 2 & 2 & 4 \\
\hline 445 & 489 & & & & & 1 & 1.63 & & & 2 & 1.13 & & 1 & 2 & 3 \\
\hline 446 & 498 & 1 & 0.2 & & & & & 1 & 0.14 & & & & 1 & 1 & 2 \\
\hline 447 & 406 & & & & & 1 & 3.58 & & & & & & 1 & 0 & 1 \\
\hline 448 & 417 & & & & & & & & & 1 & 0.33 & & 0 & 1 & 1 \\
\hline 449 & 419 & & & 2 & 1.35 & & & & & & & & 2 & 0 & 2 \\
\hline 450 & 431 & 7 & 9.15 & 3 & 7.62 & 27 & 18.68 & 2 & 11.21 & 20 & 10.22 & & 37 & 22 & 59 \\
\hline 451 & 434 & 13 & 36.02 & 6 & 6.92 & 13 & 14.11 & 1 & 14.37 & 4 & 21.31 & & 32 & 4 & 36 \\
\hline 452 & 440 & 1 & 2.16 & 1 & 0.33 & 2 & 3.2 & & & & & & 4 & 0 & 4 \\
\hline 453 & 449 & 2 & 95.96 & 5 & 17.48 & 42 & 18.2 & 2 & 2.73 & 28 & 39.5 & & 49 & 28 & 77 \\
\hline 454 & 457 & 3 & 21.37 & & & 4 & 1.13 & 2 & 44.8 & 5 & 6.45 & & 7 & 5 & 12 \\
\hline 455 & 462 & & & 2 & 22.04 & 3 & 6.06 & 1 & 0.63 & 4 & 9.06 & & 5 & 4 & 9 \\
\hline 456 & 474 & 1 & 3.01 & 1 & 0.23 & 2 & 1.16 & 1 & 35.72 & 1 & 0.46 & & 4 & 2 & 6 \\
\hline 457 & 484 & & & & & 2 & 0.62 & & & 3 & 1.79 & & 2 & 3 & 5 \\
\hline 458 & 491 & & & & & & & & & 1 & 0.35 & & 0 & 1 & 1 \\
\hline 459 & 505 & & & & & 2 & 1.26 & & & & & & 2 & $\overline{0}$ & 2 \\
\hline 460 & 511 & & & & & 5 & 1.56 & & & 1 & 1.56 & & 5 & 1 & 6 \\
\hline 462 & 526 & & & 6 & 29.14 & 11 & 11.29 & & & 16 & 17.89 & & 17 & 16 & 33 \\
\hline 463 & 529 & 6 & 16.23 & & & 13 & 5.34 & & & 24 & 16.89 & & 19 & 24 & 43 \\
\hline 464 & 536 & 3 & 3.15 & 2 & 2.53 & 4 & 2.14 & & & 22 & 20.93 & & 9 & 22 & 31 \\
\hline 465 & 661 & 5 & 110.68 & 2 & 0.99 & 14 & 11.25 & & & 4 & 16.23 & & 21 & 4 & 25 \\
\hline 467 & 663 & 6 & 17.46 & 4 & 1.75 & 16 & 7.72 & & & 14 & 6.85 & & 26 & 14 & 40 \\
\hline 468 & 665 & 5 & 20.62 & 3 & 5.34 & 7 & 8.09 & & & 2 & 1.46 & & 15 & 2 & 17 \\
\hline 469 & 668 & 3 & 12.74 & 2 & 0.61 & 10 & 7.82 & & & 5 & 6.39 & & 15 & 5 & 20 \\
\hline 470 & 509 & & & & & 1 & 0.18 & & & & & & 1 & 0 & 1 \\
\hline 471 & 515 & 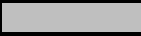 & & 3 & 0.95 & 4 & 5.61 & & & 5 & 3.57 & & 7 & 5 & 12 \\
\hline 472 & 520 & 3 & 22.76 & 1 & 0.51 & 10 & 6.31 & & & 11 & 19.17 & & 14 & 11 & 25 \\
\hline 475 & 534 & 3 & 9.61 & 1 & 0.35 & 13 & 8.98 & & & 13 & 7.61 & & 17 & 13 & 30 \\
\hline 476 & 538 & 6 & 108.43 & 1 & 1.2 & 16 & 19.07 & & & 17 & 43.28 & & 23 & 17 & 40 \\
\hline 477 & 669 & 1 & 3.24 & 4 & 7 & 7 & 2.15 & & & 5 & 2.39 & & 12 & 5 & 17 \\
\hline 478 & 671 & 7 & 32.07 & 2 & 2.15 & 15 & 5.24 & & & 14 & 10.95 & & 24 & 14 & 38 \\
\hline 479 & 672 & 3 & 6.44 & 5 & 1.93 & 7 & 2.25 & & & 10 & 4.07 & & 15 & 10 & 25 \\
\hline 480 & 674 & 5 & 11.7 & 2 & 1.34 & 14 & 16.28 & & & 17 & 3.92 & & 21 & 17 & 38 \\
\hline 481 & 675 & 3 & 2.63 & 1 & 0.35 & 4 & 1.9 & & & 7 & 2.23 & & 8 & 7 & 15 \\
\hline 482 & 676 & 1 & 0.12 & & & 4 & 6.04 & & & 3 & 5.11 & & 5 & 3 & 8 \\
\hline 483 & 677 & & & & & 3 & 3.06 & & & & & & 3 & 0 & 3 \\
\hline 484 & 678 & & & & & 2 & 4.41 & & & & & & 2 & 0 & 2 \\
\hline 486 & 315 & & & & & 1 & 0.46 & & & & & & 1 & 0 & 1 \\
\hline 487 & 317 & 1 & 1.01 & & & & & & & & & & 1 & 0 & 1 \\
\hline 488 & 319 & 1 & 1.14 & & & 6 & 3.23 & & & 2 & 1.21 & & 7 & 2 & 9 \\
\hline 489 & 322 & 3 & 7.68 & 2 & 2.37 & 13 & 11.14 & & & 7 & 7.26 & & 18 & 7 & 25 \\
\hline 490 & 324 & 6 & 14.02 & 6 & 11.48 & 35 & 18.96 & & & 26 & 37.44 & & 47 & 26 & 73 \\
\hline 491 & 329 & 6 & 11.77 & 8 & 13.07 & 7 & 12.99 & & & 4 & 5.25 & & 21 & 4 & 25 \\
\hline 492 & 332 & 16 & 70.88 & 9 & 29.54 & 40 & 33.78 & & & 31 & 21.89 & & 65 & 31 & 96 \\
\hline 493 & 335 & 4 & 44.57 & 8 & 25.42 & 8 & 5.26 & & & 3 & 12.61 & & 20 & 3 & 23 \\
\hline 494 & 340 & 3 & 9.43 & 7 & 6.91 & 16 & 22.52 & & & 9 & 7.79 & & 26 & 9 & 35 \\
\hline 495 & 368 & 6 & 25.83 & 6 & 7 & 8 & 12.89 & & & 3 & 4.76 & & 20 & 3 & 23 \\
\hline
\end{tabular}




\begin{tabular}{|c|c|c|c|c|c|c|c|c|c|c|c|c|c|c|c|}
\hline Lot \# & Bag \# & $\begin{array}{c}\text { Complete } \\
\text { Flake } \\
\text { Count }\end{array}$ & $\begin{array}{l}\text { Complete } \\
\text { Flake } \\
\text { Weight (g) }\end{array}$ & $\begin{array}{l}\text { Proximal } \\
\text { Flake } \\
\text { Count }\end{array}$ & $\begin{array}{l}\text { Proximal } \\
\text { Flake } \\
\text { Weight (g) }\end{array}$ & $\begin{array}{c}\text { Broken } \\
\text { Flake } \\
\text { Count }\end{array}$ & $\begin{array}{c}\text { Broken } \\
\text { Flake } \\
\text { Weight (g) }\end{array}$ & $\begin{array}{c}\text { Flaking } \\
\text { Shatter } \\
\text { Count }\end{array}$ & $\begin{array}{c}\text { Flaking } \\
\text { Shatter } \\
\text { Weight (g) }\end{array}$ & $\begin{array}{c}\text { Thermal } \\
\text { Shatter } \\
\text { Count }\end{array}$ & $\begin{array}{c}\text { Thermal } \\
\text { Shatter } \\
\text { Weight (g) }\end{array}$ & Comments & $\begin{array}{c}\text { Total } \\
\text { No. } \\
\text { Flakes }\end{array}$ & $\begin{array}{c}\text { Total } \\
\text { No. } \\
\text { Shatter }\end{array}$ & $\begin{array}{l}\text { Final } \\
\text { Total }\end{array}$ \\
\hline 496 & 404 & 5 & 18.4 & 5 & 4.11 & 19 & 18.57 & & & 4 & 2.04 & & 29 & 4 & 33 \\
\hline 497 & 318 & 1 & 0.2 & 2 & 1.13 & & & & & & & & 3 & 0 & 3 \\
\hline 498 & 320 & & & 1 & 0.43 & 3 & 4.57 & & & & & & 4 & 0 & 4 \\
\hline 499 & 321 & 2 & 3.53 & 3 & 0.93 & 3 & 2.01 & & & 8 & 3.03 & & 8 & 8 & 16 \\
\hline 500 & 323 & 13 & 37.57 & 9 & 6.52 & 13 & 6.36 & & & 22 & 49.77 & & 35 & 22 & 57 \\
\hline 501 & 328 & 11 & 37.91 & 7 & 15.26 & 24 & 10.4 & 1 & 23.26 & 22 & 60.81 & & 42 & 23 & 65 \\
\hline 502 & 331 & 12 & 98.38 & 12 & 21.58 & 18 & 8.32 & 1 & 7.12 & 20 & 32.18 & & 42 & 21 & 63 \\
\hline 503 & 334 & 15 & 32.27 & 15 & 12.16 & 25 & 19.77 & & & 24 & 14.31 & & 55 & 24 & 79 \\
\hline 504 & 336 & 16 & 81.69 & 18 & 15.41 & 35 & 17.2 & & & 25 & 15.28 & & 69 & 25 & 94 \\
\hline 505 & 342 & 15 & 79.87 & 22 & 18.19 & 18 & 14.82 & & & 19 & 18.3 & & 55 & 19 & 74 \\
\hline 506 & 339 & 25 & 37.98 & 33 & 38.36 & 44 & 31.26 & 4 & 15.25 & 21 & 20.73 & & 102 & 21 & 123 \\
\hline 508 & 411 & 13 & 48.47 & 29 & 25.05 & 50 & 64.53 & 4 & 2.53 & 11 & 11.84 & & 92 & 15 & 107 \\
\hline 509 & 420 & 12 & 20.99 & 15 & 26.14 & 18 & 22.06 & & & 5 & 2.06 & & 45 & 5 & 50 \\
\hline 510 & 430 & 16 & 34.82 & 21 & 32.35 & 17 & 19.98 & & & 7 & 2.89 & & 54 & 7 & 61 \\
\hline 511 & 438 & 5 & 70.19 & 10 & 12.94 & 23 & 28.91 & & & 6 & 14.16 & & 38 & 6 & 44 \\
\hline 512 & 447 & 3 & 19.85 & 1 & 0.17 & 9 & 15.99 & & & 3 & 11.04 & & 13 & 3 & 16 \\
\hline 513 & 552 & 1 & 0.38 & 1 & 9.28 & 1 & 0.37 & & & 1 & 1.06 & & 3 & 1 & 4 \\
\hline 514 & 554 & 3 & 27.25 & & & 1 & 0.43 & & & 4 & 16.75 & & 4 & 4 & 8 \\
\hline 515 & 558 & 1 & 2.92 & 2 & 13.54 & & & & & & & & $\overline{3}$ & 0 & 3 \\
\hline 516 & 623 & 2 & 1.89 & 2 & 11.72 & & & & & & & & 4 & 0 & 4 \\
\hline 517 & 633 & 1 & 5.28 & & & & & & & & & & 1 & 0 & 1 \\
\hline 518 & 640 & 5 & 54.74 & 4 & 1.96 & 9 & 4.76 & & & 11 & 22.29 & & 18 & 11 & 29 \\
\hline 519 & 646 & 7 & 8.79 & 8 & 5.34 & 9 & 3.87 & & & 14 & 11.91 & & 24 & 14 & 38 \\
\hline 521 & 658 & 3 & 1.07 & 2 & 0.41 & 2 & 0.73 & & & 1 & 0.81 & & 7 & 1 & 8 \\
\hline 528 & 666 & 4 & 4.94 & & & 18 & 13.73 & & & 30 & 24.52 & & 22 & 30 & 52 \\
\hline 532 & 631 & 2 & 0.77 & & & & & & & & & & 2 & 0 & 2 \\
\hline 533 & 641 & & & 9 & 32.41 & 4 & 2.33 & & & 23 & 33.15 & & 13 & 23 & 36 \\
\hline 534 & 649 & 1 & 3.86 & 5 & 3.41 & 7 & 2.05 & 1 & 1.36 & 4 & 5.04 & & 13 & 5 & 18 \\
\hline 537 & 576 & & & & & 1 & 2.23 & & & 1 & 0.48 & & 1 & 1 & 2 \\
\hline 536 & 572 & & & & & & & & & 1 & 0.45 & & 0 & 1 & 1 \\
\hline 538 & 579 & 2 & 0.86 & & & 3 & 0.45 & & & 8 & 6.2 & & 5 & 8 & 13 \\
\hline 539 & 592 & & & & & 2 & 0.46 & & & 12 & 16.39 & & 2 & 12 & 14 \\
\hline 540 & 542 & & & 1 & 0.21 & 1 & 0.21 & 1 & 4.48 & 2 & 0.61 & & 2 & 3 & 5 \\
\hline 541 & 544 & 1 & 1.59 & & & 5 & 2.98 & 1 & 0.75 & 2 & 4.35 & & 6 & 3 & 9 \\
\hline 542 & 546 & 4 & 2.71 & 5 & 3.82 & 11 & 10.15 & & & 23 & 27.54 & & 20 & 23 & 43 \\
\hline 543 & 560 & & & 1 & 0.04 & 6 & 6.56 & & & 13 & 44.02 & & 7 & 13 & 20 \\
\hline 544 & 325 & & & & & & & & & 1 & 0.76 & & 0 & 1 & 1 \\
\hline 545 & 327 & & & 1 & 1.99 & 2 & 0.92 & 1 & 0.16 & & & & 3 & 1 & 4 \\
\hline 547 & 330 & & & 2 & 7.21 & 5 & 5.07 & & & 7 & 14.98 & & 7 & 7 & 14 \\
\hline 548 & 333 & 5 & 8.37 & 14 & 8.79 & 8 & 7.35 & & & 8 & 8.61 & & 27 & 8 & 35 \\
\hline 550 & 338 & 11 & 14.97 & 11 & 11.23 & 21 & 14.3 & 1 & 0.71 & 14 & 24.23 & & 43 & 15 & 58 \\
\hline 551 & 341 & 5 & 29.69 & 11 & 9.69 & 34 & 16.7 & 2 & 0.72 & 17 & 16.93 & & 50 & 19 & 69 \\
\hline 552 & 347 & 10 & 51.98 & 26 & 46.75 & 10 & 12.44 & & & 34 & 25.39 & & 46 & 34 & 80 \\
\hline 553 & 392 & 1 & 0.34 & & & 5 & 21.12 & & & 3 & 2.78 & & 6 & 3 & 9 \\
\hline 554 & 395 & 2 & 2.63 & 3 & 14.69 & 4 & 3.15 & & & 5 & 3.16 & & 9 & 5 & 14 \\
\hline 555 & 400 & 1 & 9.29 & 1 & 0.56 & 5 & 4.32 & & & 6 & 24.16 & & 7 & 6 & 13 \\
\hline 556 & 349 & 1 & 10.43 & 1 & 0.12 & 7 & 6.13 & 2 & 0.81 & & & & 9 & 2 & 11 \\
\hline 557 & 353 & & & & & 1 & 0.4 & & & & & & 1 & 0 & 1 \\
\hline 558 & 356 & 1 & 0.08 & 2 & 0.9 & 7 & 9.79 & & & & & & 10 & 0 & 10 \\
\hline
\end{tabular}




\begin{tabular}{|c|c|c|c|c|c|c|c|c|c|c|c|c|c|c|c|}
\hline Lot \# & Bag \# & $\begin{array}{c}\text { Complete } \\
\text { Flake } \\
\text { Count }\end{array}$ & $\begin{array}{c}\text { Complete } \\
\text { Flake } \\
\text { Weight (g) }\end{array}$ & $\begin{array}{l}\text { Proximal } \\
\text { Flake } \\
\text { Count }\end{array}$ & $\begin{array}{l}\text { Proximal } \\
\text { Flake } \\
\text { Weight (g) }\end{array}$ & $\begin{array}{c}\text { Broken } \\
\text { Flake } \\
\text { Count }\end{array}$ & $\begin{array}{c}\text { Broken } \\
\text { Flake } \\
\text { Weight (g) }\end{array}$ & $\begin{array}{c}\text { Flaking } \\
\text { Shatter } \\
\text { Count }\end{array}$ & \begin{tabular}{|c|} 
Flaking \\
Shatter \\
Weight (g)
\end{tabular} & $\begin{array}{c}\text { Thermal } \\
\text { Shatter } \\
\text { Count }\end{array}$ & $\begin{array}{c}\text { Thermal } \\
\text { Shatter } \\
\text { Weight (g) }\end{array}$ & Comments & $\begin{array}{c}\text { Total } \\
\text { No. } \\
\text { Flakes }\end{array}$ & $\begin{array}{c}\text { Total } \\
\text { No. } \\
\text { Shatter }\end{array}$ & $\begin{array}{l}\text { Final } \\
\text { Total }\end{array}$ \\
\hline 559 & 360 & 3 & \begin{tabular}{|l|}
6.7 \\
\end{tabular} & 9 & 17.43 & 15 & 11.15 & & & \begin{tabular}{|l|}
19 \\
\end{tabular} & \begin{tabular}{|l|}
26.31 \\
\end{tabular} & & 27 & 19 & 46 \\
\hline 561 & 367 & 8 & 13.22 & 9 & 6.74 & 12 & 21.74 & & & 15 & 32.36 & & 29 & 15 & 44 \\
\hline 565 & 374 & 8 & 16.81 & 3 & 1.32 & 21 & 11.47 & & & 10 & 12.64 & & 32 & 10 & 42 \\
\hline 566 & 379 & 4 & 2.86 & 5 & 2.06 & 14 & 11.97 & 3 & 4.78 & 12 & 32.31 & & 23 & 15 & 38 \\
\hline 567 & 384 & 4 & 37.75 & 13 & 5.14 & 12 & 10.53 & & & 7 & 5.51 & & 29 & 7 & 36 \\
\hline 568 & 386 & & & 2 & 0.75 & 2 & 6.38 & & & 3 & 3.4 & & 4 & 3 & 7 \\
\hline 569 & 389 & 2 & 0.38 & 1 & 0.18 & 1 & 6.34 & & & 2 & 0.98 & & 4 & 2 & 6 \\
\hline 570 & 416 & & & 2 & 29.57 & 1 & 0.24 & & & 1 & 0.16 & & 3 & 1 & 4 \\
\hline 571 & 425 & & & & & & & & & 1 & 0.26 & & 0 & 1 & 1 \\
\hline 572 & 435 & 1 & 2.38 & & & & & & & & & & 1 & 0 & 1 \\
\hline 573 & 443 & 1 & 0.63 & 3 & 54.64 & 2 & 0.39 & & & & & & 6 & 0 & 6 \\
\hline 574 & 606 & 1 & 0.93 & 1 & 0.74 & 1 & 0.85 & & & 1 & 3.17 & & 3 & 1 & 4 \\
\hline 575 & 611 & & & & & 1 & 0.47 & & & & & & 1 & 0 & 1 \\
\hline 577 & 620 & 2 & 8.41 & & & 1 & 0.04 & & & 1 & 0.12 & & 3 & 1 & 4 \\
\hline 578 & 637 & & & 3 & 2.14 & 3 & 0.93 & & & 1 & 2.01 & & 6 & 1 & 7 \\
\hline 579 & 643 & 2 & 0.16 & 6 & 1.93 & 4 & 1.01 & & & 7 & 3.71 & & 12 & 7 & 19 \\
\hline 580 & 648 & 2 & 1.62 & 4 & 20.21 & 10 & 5.32 & & & 15 & 15.47 & & 16 & 15 & 31 \\
\hline 581 & 651 & 5 & 15.34 & 1 & 1.71 & 3 & 4.34 & & & 3 & 0.51 & & 9 & 3 & 12 \\
\hline 582 & 654 & 1 & 0.39 & & & 9 & 5.78 & & & 11 & 6.94 & & 10 & 11 & 21 \\
\hline 583 & 513 & & & & & & & & & 1 & 2.77 & & 0 & 1 & 1 \\
\hline 584 & 518 & & & & & 3 & 0.41 & & & & & & 3 & 0 & 3 \\
\hline 585 & 519 & 3 & 2.97 & 4 & 3.39 & 8 & 26.82 & & & 5 & 1.9 & & 15 & 5 & 20 \\
\hline 586 & 523 & 5 & 3.36 & 6 & 33.56 & 21 & 5.64 & 2 & 10.9 & 8 & 2.59 & & 32 & 10 & 42 \\
\hline 588 & 527 & 7 & 91.11 & 20 & 14.52 & 26 & 14.33 & & & 22 & 21.58 & & 53 & 22 & 75 \\
\hline 590 & 557 & 11 & 28.69 & 22 & 51.94 & 19 & 7.03 & & & 34 & 65.88 & & 52 & 34 & 86 \\
\hline 591 & 563 & 9 & 36.3 & 28 & 129.27 & 51 & 58.76 & & & 32 & 31.66 & & 88 & 32 & 120 \\
\hline 595 & 570 & 11 & 43.53 & 13 & 10.51 & 40 & 39.39 & & & 8 & 6.46 & & 64 & 8 & 72 \\
\hline 596 & 574 & 1 & 75.04 & 3 & 0.58 & 10 & 3.99 & & & 8 & 4.92 & & 14 & 8 & 22 \\
\hline 597 & 575 & 2 & 1.21 & 4 & 2.17 & 5 & 2.54 & & & 7 & 4.26 & & 11 & 7 & 18 \\
\hline 598 & 659 & & & 2 & 4.38 & 1 & 0.4 & & & & & & 3 & 0 & 3 \\
\hline 599 & 660 & & & & & 1 & 0.08 & & & & & & 1 & 0 & 1 \\
\hline 600 & 662 & & & 1 & 0.15 & & & & & & & & 1 & 0 & 1 \\
\hline 601 & 664 & 1 & 13.86 & 2 & 6.3 & & & & & & & & 3 & 0 & 3 \\
\hline 614 & 413 & & & 3 & 1.21 & 2 & 0.45 & & & 2 & 0.76 & & 5 & 2 & 7 \\
\hline 615 & 441 & 3 & 0.4 & 6 & 2.28 & 11 & 7.77 & & & 10 & 6.42 & & 20 & 10 & 30 \\
\hline 616 & 454 & 5 & 6 & 19 & 11.2 & 17 & 12.06 & & & 15 & 33.83 & & 41 & 15 & 56 \\
\hline 617 & 464 & 2 & 0.87 & 14 & 12.4 & 14 & 16.83 & & & 23 & 21.35 & & 30 & 23 & 53 \\
\hline 618 & 479 & 14 & 96.06 & 20 & 16.66 & 37 & 29.37 & 4 & 11.69 & 12 & 7.04 & & 71 & 16 & 87 \\
\hline 619 & 530 & 7 & 27.92 & 18 & 17.9 & 29 & 29.58 & 3 & 5.01 & 6 & 4.89 & & 54 & 9 & 63 \\
\hline 621 & 539 & 4 & 97.4 & 10 & 15.59 & 30 & 10.55 & & & 33 & 76.07 & & 44 & 33 & 77 \\
\hline 622 & 541 & 9 & 34.79 & 16 & 11.66 & 35 & 37.16 & & & 11 & 9.61 & & 60 & 11 & 71 \\
\hline 623 & 581 & 5 & 12.49 & 2 & 3.41 & 10 & 14.14 & 1 & 58.17 & 7 & 8.78 & & 17 & 8 & 25 \\
\hline 625 & 587 & 4 & 10.96 & 4 & 19.74 & 6 & 4.75 & & & 2 & 7.01 & & 14 & 2 & 16 \\
\hline 626 & 591 & 3 & 28.66 & & & 4 & 3.82 & & & 2 & 1 & & 7 & 2 & 9 \\
\hline 627 & 613 & 1 & 9.74 & 1 & 22.52 & & & & & & & & 2 & 0 & 2 \\
\hline 628 & 346 & & & 3 & 1.24 & & & 1 & 0.35 & 1 & 1.39 & & 3 & 2 & 5 \\
\hline 629 & 355 & 1 & 3.2 & 5 & 3.4 & 3 & 1.12 & & & & & & 9 & 0 & 9 \\
\hline 630 & 365 & 4 & 3.08 & 11 & 24.91 & 10 & 8.77 & & & 7 & 5.36 & & 25 & 7 & 32 \\
\hline 631 & 376 & 2 & 2.3 & 14 & 6.37 & 16 & 11.36 & & & 10 & 2.67 & & 32 & 10 & 42 \\
\hline
\end{tabular}




\begin{tabular}{|c|c|c|c|c|c|c|c|c|c|c|c|c|c|c|c|}
\hline Lot \# & Bag \# & $\begin{array}{c}\text { Complete } \\
\text { Flake } \\
\text { Count }\end{array}$ & $\begin{array}{l}\text { Complete } \\
\text { Flake } \\
\text { Weight (g) }\end{array}$ & $\begin{array}{l}\text { Proximal } \\
\text { Flake } \\
\text { Count }\end{array}$ & $\begin{array}{l}\text { Proximal } \\
\text { Flake } \\
\text { Weight (g) }\end{array}$ & $\begin{array}{c}\text { Broken } \\
\text { Flake } \\
\text { Count }\end{array}$ & $\begin{array}{c}\text { Broken } \\
\text { Flake } \\
\text { Weight (g) }\end{array}$ & $\begin{array}{c}\text { Flaking } \\
\text { Shatter } \\
\text { Count }\end{array}$ & $\begin{array}{c}\text { Flaking } \\
\text { Shatter } \\
\text { Weight (g) }\end{array}$ & $\begin{array}{c}\text { Thermal } \\
\text { Shatter } \\
\text { Count }\end{array}$ & $\begin{array}{c}\text { Thermal } \\
\text { Shatter } \\
\text { Weight (g) }\end{array}$ & Comments & $\begin{array}{c}\text { Total } \\
\text { No. } \\
\text { Flakes }\end{array}$ & $\begin{array}{c}\text { Total } \\
\text { No. } \\
\text { Shatter }\end{array}$ & $\begin{array}{l}\text { Final } \\
\text { Total }\end{array}$ \\
\hline 632 & 385 & 9 & 12.83 & 10 & 6.47 & 23 & 15.4 & 2 & 0.64 & 11 & \begin{tabular}{|l|}
10.23 \\
\end{tabular} & & 42 & 13 & 55 \\
\hline 633 & 396 & 8 & 35.29 & 9 & 4.13 & 30 & 21.12 & & & 16 & 14.31 & & 47 & 16 & 63 \\
\hline 635 & 455 & 8 & 56.49 & 6 & 22.92 & 16 & 11.51 & & & 6 & 3.75 & & 30 & 6 & 36 \\
\hline 637 & 461 & 2 & 51.36 & 7 & 2.39 & 9 & 8.16 & & & 11 & 85.8 & & 18 & 11 & 29 \\
\hline 638 & 466 & 2 & 8.56 & 3 & 3.63 & 2 & 0.61 & & & 1 & 0.44 & & 7 & 1 & 8 \\
\hline 639 & 468 & 1 & 0.12 & 3 & 0.84 & & & & & 1 & 1.55 & & 4 & 1 & 5 \\
\hline 640 & 471 & & & 3 & 8.24 & 1 & 0.08 & & & 1 & 118.93 & & 4 & 1 & 5 \\
\hline 641 & 473 & & & 2 & 1.95 & 1 & 0.42 & & & 1 & 0.17 & & 3 & 1 & 4 \\
\hline 642 & 478 & & & & & 2 & 1.38 & & & & & & 2 & 0 & 2 \\
\hline 644 & 351 & & & & & 1 & 0.66 & & & & & & 1 & 0 & 1 \\
\hline 645 & 359 & & & 1 & 0.75 & 1 & 0.95 & & & & & & 2 & 0 & 2 \\
\hline 646 & 369 & 4 & 2.96 & 6 & 27.35 & 6 & 1.97 & & & 1 & 1.23 & & 16 & 1 & 17 \\
\hline 647 & 381 & 5 & 20.33 & 13 & 40.15 & 9 & 3.81 & & & 17 & 19.45 & & 27 & 17 & 44 \\
\hline 648 & 390 & 9 & 8.71 & 12 & 38.48 & 37 & 15.85 & 1 & 1.75 & 26 & 23.06 & & 58 & 27 & 85 \\
\hline 649 & 402 & 7 & 5.64 & 14 & 8.29 & 19 & 8.31 & 1 & 1.16 & 20 & 29.03 & & 40 & 21 & 61 \\
\hline 650 & 486 & 9 & 44.31 & 11 & 4.81 & 21 & 6.28 & & & 13 & 28.38 & & 41 & 13 & 54 \\
\hline 652 & 493 & 6 & 28.47 & 8 & 8.68 & 13 & 8.4 & & & 4 & 4.15 & & 27 & 4 & 31 \\
\hline 653 & 504 & 4 & 10.73 & 7 & 19.1 & 15 & 13.29 & & & 5 & 1.55 & & 26 & 5 & 31 \\
\hline 654 & 622 & & & 2 & 2.92 & 3 & 1.27 & & & 1 & 3.21 & & 5 & 1 & 6 \\
\hline 655 & 625 & & & 2 & 4.62 & 1 & 0.19 & & & 1 & 0.2 & & 3 & 1 & 4 \\
\hline 656 & 630 & 2 & 6.1 & & & & & & & & & & 2 & 0 & 2 \\
\hline 657 & 418 & & & & & & & & & 1 & 1.04 & & 0 & 1 & 1 \\
\hline 658 & 432 & & & & & 2 & 0.83 & & & & & & 2 & 0 & 2 \\
\hline 659 & 446 & 8 & 13.64 & 4 & 2.23 & 8 & 5.22 & & & 7 & 2.67 & & 20 & 7 & 27 \\
\hline 660 & 459 & 7 & 73.09 & 12 & 15.17 & 14 & 3.2 & & & 17 & 24.71 & & 33 & 17 & 50 \\
\hline 661 & 469 & 14 & 9.84 & 21 & 10.79 & 20 & 7.41 & & & 19 & 8.31 & & 55 & 19 & 74 \\
\hline 662 & 533 & 4 & 18.47 & 10 & 7.36 & 16 & 27.88 & & & 5 & 4.64 & & 30 & 5 & 35 \\
\hline 663 & 535 & 12 & 91.91 & 6 & 3.54 & 14 & 5.44 & & & 11 & 7.26 & & 32 & 11 & 43 \\
\hline 665 & 568 & 4 & 1.73 & 14 & 8.98 & 24 & 9.82 & & & 10 & 3.81 & & 42 & 10 & 52 \\
\hline 666 & 584 & 5 & 16.06 & 4 & 4.08 & 8 & 6.69 & & & 2 & 10.14 & & 17 & 2 & 19 \\
\hline 668 & 588 & 2 & 9.64 & 2 & 16.2 & & & & & 2 & 0.86 & & 4 & 2 & 6 \\
\hline 669 & 605 & 3 & 19.6 & 1 & 1.02 & 5 & 1.38 & & & 1 & 0.11 & & 9 & 1 & 10 \\
\hline 670 & 609 & 3 & 24.43 & & & & & & & & & & 3 & 0 & 3 \\
\hline 671 & 615 & & & 1 & 8.97 & & & & & & & & 1 & 0 & 1 \\
\hline 673 & 344 & & & & & 1 & 0.19 & & & 1 & 0.83 & & 1 & 1 & 2 \\
\hline 674 & 348 & & & & & 2 & 2.02 & & & 4 & 3.73 & & 2 & 4 & 6 \\
\hline 675 & 364 & & & 1 & 0.99 & 2 & 0.63 & & & & & & 3 & 0 & 3 \\
\hline 676 & 366 & 3 & 1.03 & 2 & 0.82 & 5 & 4.69 & & & 6 & 8.14 & & 10 & 6 & 16 \\
\hline 677 & 383 & & & 4 & 6.93 & 1 & 0.42 & & & & & & 5 & 0 & 5 \\
\hline 678 & 387 & & & & & 1 & 0.58 & 1 & 10.25 & 3 & 2.41 & & 1 & 4 & 5 \\
\hline 679 & 391 & 1 & 5.3 & 1 & 26.6 & 2 & 1.63 & & & 5 & 6.05 & & 4 & 5 & 9 \\
\hline 680 & 401 & 1 & 0.98 & & & & & & & 4 & 4.29 & & 1 & 4 & 5 \\
\hline 681 & 556 & & & & & 1 & 0.4 & & & 3 & 2.88 & & 1 & 3 & 4 \\
\hline 682 & 559 & & & & & & & & & 4 & 2.68 & & 0 & 4 & 4 \\
\hline 683 & 571 & 1 & 8.54 & & & 1 & 0.88 & 1 & 1.01 & 2 & 15.93 & & 2 & 3 & 5 \\
\hline 684 & 577 & & & & & 2 & 0.42 & & & 1 & 0.22 & & 2 & 1 & 3 \\
\hline 686 & 585 & & & & & & & & & 1 & 11.86 & & 0 & 1 & 1 \\
\hline 687 & 343 & & & & & 1 & 0.32 & & & & & & 1 & 0 & 1 \\
\hline 688 & 352 & & & 1 & 1.5 & 1 & 0.35 & & & & & & 2 & 0 & 2 \\
\hline
\end{tabular}




\begin{tabular}{|c|c|c|c|c|c|c|c|c|c|c|c|c|c|c|c|}
\hline Lot \# & Bag \# & $\begin{array}{c}\text { Complete } \\
\text { Flake } \\
\text { Count }\end{array}$ & $\begin{array}{c}\text { Complete } \\
\text { Flake } \\
\text { Weight (g) }\end{array}$ & $\begin{array}{l}\text { Proximal } \\
\text { Flake } \\
\text { Count }\end{array}$ & $\begin{array}{l}\text { Proximal } \\
\text { Flake } \\
\text { Weight (g) }\end{array}$ & $\begin{array}{c}\text { Broken } \\
\text { Flake } \\
\text { Count }\end{array}$ & $\begin{array}{c}\text { Broken } \\
\text { Flake } \\
\text { Weight (g) }\end{array}$ & $\begin{array}{c}\text { Flaking } \\
\text { Shatter } \\
\text { Count }\end{array}$ & $\begin{array}{c}\text { Flaking } \\
\text { Shatter } \\
\text { Weight }(\mathrm{g})\end{array}$ & $\begin{array}{c}\text { Thermal } \\
\text { Shatter } \\
\text { Count }\end{array}$ & $\begin{array}{c}\text { Thermal } \\
\text { Shatter } \\
\text { Weight }(\mathrm{g})\end{array}$ & Comments & $\begin{array}{c}\text { Total } \\
\text { No. } \\
\text { Flakes }\end{array}$ & $\begin{array}{c}\text { Total } \\
\text { No. } \\
\text { Shatter }\end{array}$ & $\begin{array}{l}\text { Final } \\
\text { Total }\end{array}$ \\
\hline 689 & 357 & & & & & 3 & 7.8 & & & \begin{tabular}{|l|}
2 \\
\end{tabular} & \begin{tabular}{|l|}
1.53 \\
\end{tabular} & & 3 & 2 & 5 \\
\hline 690 & 375 & 2 & 30.46 & 3 & 3.11 & 6 & 9.53 & & & 3 & 1.57 & & 11 & 3 & 14 \\
\hline 691 & 377 & & & 1 & 0.91 & 4 & 13.16 & & & 3 & 3.55 & & 5 & 3 & 8 \\
\hline 692 & 380 & & & 2 & 48.61 & 3 & 0.63 & & & 5 & 28.86 & & 5 & 5 & 10 \\
\hline 693 & 393 & & & 2 & 2.83 & 1 & 2.84 & 1 & 5.45 & 7 & 5.18 & & 3 & 8 & 11 \\
\hline 694 & 398 & 1 & 0.84 & 1 & 14.17 & & & & & 3 & 5.96 & & 2 & 3 & 5 \\
\hline 695 & 403 & 1 & 1.3 & & & 1 & 0.8 & & & & & & 2 & 0 & 2 \\
\hline 696 & 548 & 2 & 10.3 & & & 1 & 1.01 & & & 3 & 5.21 & & 3 & 3 & 6 \\
\hline 698 & 562 & 1 & 0.94 & & & 1 & 0.66 & & & 4 & 5.85 & & 2 & 4 & 6 \\
\hline 643 & 345 & & & 1 & 0.58 & & & & & & & & 1 & 0 & 1 \\
\hline 329 & 628 & & & 1 & 0.11 & 8 & 22.95 & & & 9 & 11.23 & & 9 & 9 & 18 \\
\hline 602 & 409 & & & 1 & 2.48 & 2 & 0.25 & & & & & & 3 & 0 & 3 \\
\hline 603 & 424 & & & 5 & 7.09 & 8 & 4.69 & & & 2 & 0.51 & & 13 & 2 & 15 \\
\hline 604 & 436 & 15 & 24.49 & 9 & 9.21 & 20 & 8.16 & & & 15 & 21.37 & & 44 & 15 & 59 \\
\hline 605 & 450 & 10 & 20.52 & 10 & 8.61 & 23 & 11.32 & 3 & 4.07 & 8 & $\frac{21.01}{12.56}$ & & 43 & 11 & 54 \\
\hline 606 & 463 & 15 & 104.1 & 19 & 19.37 & 48 & 33.69 & & & 19 & 32.8 & & 82 & 19 & 101 \\
\hline 607 & 472 & 13 & 36.58 & 12 & 5.74 & 35 & 22.14 & 2 & 12.68 & 18 & 24.22 & & 60 & 20 & 80 \\
\hline 608 & 510 & 13 & 16.02 & 23 & 25.61 & 41 & 48.34 & & & 18 & 14.63 & & 77 & 18 & 95 \\
\hline 609 & 545 & 9 & 162.77 & 21 & 27.7 & 32 & 21.25 & 6 & 55.85 & 16 & 28.51 & & 62 & 22 & 84 \\
\hline 610 & 550 & 4 & 5.96 & 5 & 3.64 & 10 & 15.39 & 2 & 5.24 & 4 & 9.58 & & 19 & 6 & 25 \\
\hline 611 & 618 & & & 4 & 0.51 & 2 & 2.43 & $\frac{2}{1}$ & 17.99 & 1 & 1.98 & & 6 & 2 & 8 \\
\hline 612 & 629 & 1 & 5.41 & & & & & & & & & & 1 & 0 & 1 \\
\hline 613 & 638 & 3 & 34.39 & & & & & 2 & 12.97 & 1 & 0.31 & & 3 & 3 & 6 \\
\hline & TOTAL & 1166 & & 1516 & & 3347 & & 89 & & 2598 & & & 6029 & 2687 & 8716 \\
\hline
\end{tabular}




\begin{tabular}{|c|c|c|c|c|c|c|c|c|c|c|c|c|c|c|}
\hline Lot No. & $\begin{array}{c}\text { Spec. } \\
\text { No. }\end{array}$ & Bag No. & UI No. & Category & Subcategory & Condition & Raw material & Overall Form & $\begin{array}{l}\text { Cross-Section } \\
\text { Form } \\
\end{array}$ & $\begin{array}{c}\text { Surface } \\
\text { Modification }\end{array}$ & $\begin{array}{c}\begin{array}{c}\text { Modification } \\
\text { Detail }\end{array} \\
\end{array}$ & $\begin{array}{c}\text { No. of Use } \\
\text { Surfaces }\end{array}$ & $\begin{array}{l}\text { Location of } \\
\text { Modification } \\
\end{array}$ & $\begin{array}{l}\text { Intentional } \\
\text { Modification }\end{array}$ \\
\hline \multirow{3}{*}{416} & \multirow{3}{*}{1} & \multirow{3}{*}{553} & \multirow{3}{*}{ NA } & Groundstone & $\begin{array}{c}\text { Grooved } \\
\text { Stone }\end{array}$ & Marginal Edge & Limestone & Indet & Indet & Grooves & Deep Indention & 1 & $\begin{array}{c}\text { Surface-one } \\
\text { face }\end{array}$ & Tool Shaping \\
\hline & & & & Heat Treatment & Weight (g) & Max Length & Max Width & Max Thickness & \multicolumn{6}{|c|}{ Comments } \\
\hline & & & & None & 185.48 & Indet & Indet & Indet & \multicolumn{6}{|c|}{ Single narrow trough ( $33.99 \mathrm{~mm}$ wide and $10.54 \mathrm{~mm}$ thick); May have extended past the break; Abrader } \\
\hline
\end{tabular}

\begin{tabular}{|c|c|c|c|c|c|c|c|c|c|c|c|c|c|c|}
\hline Lot No. & $\begin{array}{l}\text { Spec. } \\
\text { No. }\end{array}$ & Bag No. & UI No. & Category & Subcategory & Condition & Raw material & Overall Form & $\begin{array}{c}\text { Cross-Section } \\
\text { Form } \\
\end{array}$ & $\begin{array}{c}\text { Surface } \\
\text { Modification }\end{array}$ & $\begin{array}{c}\text { Modification } \\
\text { Detail }\end{array}$ & $\begin{array}{l}\text { No. of Use } \\
\text { Surfaces }\end{array}$ & $\begin{array}{l}\text { Location of } \\
\text { Modification }\end{array}$ & $\begin{array}{c}\text { Intentional } \\
\text { Modification }\end{array}$ \\
\hline \multirow{3}{*}{461} & \multirow{3}{*}{1} & \multirow{3}{*}{512} & \multirow{3}{*}{ NA } & Manuport & Indet & Complete & Limestone & Oval & Planoconvex & None & None & None & NA & NA \\
\hline & & & & Heat Treatment & Weight (g) & Max Length & Max Width & Max Thickness & \multicolumn{6}{|c|}{ Comments } \\
\hline & & & & None & 92.83 & 55.14 & 42.52 & 30.81 & \multicolumn{6}{|c|}{ Non-local stream rolled gravel with one flattened end - no modification } \\
\hline
\end{tabular}

\begin{tabular}{|c|c|c|c|c|c|c|c|c|c|c|c|c|c|c|}
\hline Lot No. & $\begin{array}{l}\text { Spec. } \\
\text { No. }\end{array}$ & Bag No. & UI No. & Category & Subcategory & Condition & Raw material & Overall Form & $\begin{array}{l}\text { Cross-Section } \\
\text { Form }\end{array}$ & $\begin{array}{c}\text { Surface } \\
\text { Modification }\end{array}$ & $\begin{array}{l}\text { Modification } \\
\text { Detail }\end{array}$ & $\begin{array}{c}\text { No. of Use } \\
\text { Surfaces }\end{array}$ & $\begin{array}{l}\text { Location of } \\
\text { Modification }\end{array}$ & $\begin{array}{c}\text { Intentional } \\
\text { Modification }\end{array}$ \\
\hline \multirow{3}{*}{492} & \multirow{3}{*}{6} & \multirow{3}{*}{332} & \multirow{3}{*}{ NA } & Groundstone & Mano & Complete & Limestone & Oval & Biplano & $\begin{array}{c}\text { Grinding and } \\
\text { Residue }\end{array}$ & Smooth & 1 & $\begin{array}{c}\text { Surface-one } \\
\text { face }\end{array}$ & Processing \\
\hline & & & & Heat Treatment & Weight (g) & Max Length & Max Width & Max Thickness & \multicolumn{6}{|c|}{ Comments } \\
\hline & & & & None & 214.62 & 81.5 & 58.12 & 29.52 & \multicolumn{6}{|c|}{$\begin{array}{c}\text { Very small medial fragment with obvious reside across smooth face; Possible heat treatment due to } \\
\text { irregular break and gray color }\end{array}$} \\
\hline
\end{tabular}

\begin{tabular}{|c|c|c|c|c|c|c|c|c|c|c|c|c|c|c|}
\hline Lot No. & $\begin{array}{l}\text { Spec. } \\
\text { No. }\end{array}$ & Bag No. & UI No. & Category & Subcategory & Condition & Raw material & Overall Form & $\begin{array}{l}\text { Cross-Section } \\
\text { Form } \\
\end{array}$ & $\begin{array}{c}\text { Surface } \\
\text { Modification }\end{array}$ & $\begin{array}{c}\begin{array}{c}\text { Modification } \\
\text { Detail }\end{array} \\
\end{array}$ & $\begin{array}{l}\begin{array}{l}\text { No. of Use } \\
\text { Surfaces }\end{array} \\
\end{array}$ & $\begin{array}{l}\text { Location of } \\
\text { Modification } \\
\end{array}$ & $\begin{array}{l}\text { Intentional } \\
\text { Modification } \\
\end{array}$ \\
\hline \multirow{3}{*}{518} & \multirow{3}{*}{10} & \multirow{3}{*}{640} & \multirow{3}{*}{ NA } & Manuport & Indet & Complete & Limestone & Oval & Planoconvex & None & None & None & NA & NA \\
\hline & & & & Heat Treatment & Weight (g) & Max Length & Max Width & Max Thickness & \multicolumn{6}{|c|}{ Comments } \\
\hline & & & & None & 58.39 & 62.18 & 32.04 & 20.84 & \multicolumn{6}{|c|}{ Large rounded limestone gravel } \\
\hline
\end{tabular}

\begin{tabular}{|c|c|c|c|c|c|c|c|c|c|c|c|c|c|c|}
\hline Lot No. & $\begin{array}{l}\text { Spec. } \\
\text { No. }\end{array}$ & Bag No. & UI No. & Category & Subcategory & Condition & Raw material & Overall Form & $\begin{array}{c}\text { Cross-Section } \\
\text { Form } \\
\end{array}$ & $\begin{array}{c}\text { Surface } \\
\text { Modification }\end{array}$ & $\begin{array}{c}\text { Modification } \\
\text { Detail }\end{array}$ & $\begin{array}{l}\text { No. of Use } \\
\text { Surfaces }\end{array}$ & $\begin{array}{l}\text { Location of } \\
\text { Modification }\end{array}$ & $\begin{array}{c}\text { Intentional } \\
\text { Modification }\end{array}$ \\
\hline \multirow{3}{*}{555} & \multirow{3}{*}{5} & \multirow{3}{*}{400} & \multirow{3}{*}{ NA } & Groundstone & Indet & Medial Fragment & Limestone & Indet & Indet & Residue & Smooth & Indet & Indet & Heat Treatment \\
\hline & & & & Heat Treatment & Weight (g) & Max Length & Max Width & Max Thickness & \multicolumn{6}{|c|}{ Comments } \\
\hline & & & & Yes & 7.15 & Indet & Indet & Indet & Very small mec & I fragment wit & $\begin{array}{l}\text { bvious reside ac } \\
\text { irregular break }\end{array}$ & $\begin{array}{l}\text { oss smooth fa } \\
\text { ind gray color }\end{array}$ & Possible heat $\mathrm{t}$ & eatment due to \\
\hline
\end{tabular}





\section{APPENDIX D}

Faunal Analysis 



\begin{tabular}{|c|c|c|c|c|c|c|c|c|c|c|c|c|c|c|c|c|c|c|}
\hline 家 & $\begin{array}{l}\text { 童 } \\
\text { 品 }\end{array}$ & 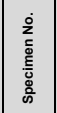 & 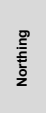 & $\begin{array}{l}\text { 袁 } \\
\text { W }\end{array}$ & $\bar{\Xi}$ & 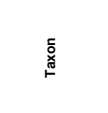 & 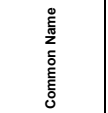 & $\begin{array}{l}\text { बू } \\
\text { 产 } \\
\text { ș }\end{array}$ & 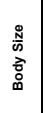 & 总 & 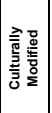 & 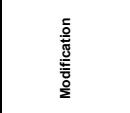 & $\begin{array}{l}\frac{\mathrm{o}}{\mathrm{o}} \\
\frac{\bar{\sigma}}{\mathrm{\omega}}\end{array}$ & $\frac{\circ}{5}$ & 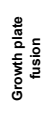 & 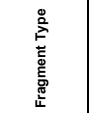 & 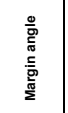 & 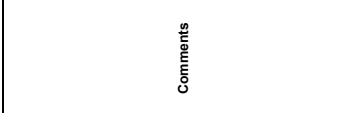 \\
\hline 321 & 506 & s001 & 995 & 1012 & 7 & Mammal & $\mathrm{NA}$ & 0.55 & $\mathrm{NA}$ & Frag & No & None & $\mathrm{NA}$ & $\mathrm{NA}$ & NA & Cortical & Right & Long bone \\
\hline 321 & 506 & s002 & 995 & 1012 & 7 & Indeterminate & NA & 0.61 & $\mathrm{NA}$ & Frag & No & None & NA & $\mathrm{NA}$ & NA & Cortical & Oblique & \\
\hline 321 & 506 & s003 & 995 & 1012 & 7 & Mammal & NA & 0.74 & $\mathrm{NA}$ & Frag & № & None & $\mathrm{NA}$ & $\mathrm{NA}$ & NA & Cortical & Right & \\
\hline 321 & 506 & s004 & 995 & 1012 & 7 & Indeterminate & $\mathrm{NA}$ & 0.45 & $\mathrm{NA}$ & Frag & No & None & $\mathrm{NA}$ & $\mathrm{NA}$ & NA & Cortical & Oblique & \\
\hline 321 & 506 & s005 & 995 & 1012 & 7 & Indeterminate & NA & 0.48 & $\mathrm{NA}$ & Frag & No & None & NA & NA & NA & Cortical & NA & \\
\hline 321 & 506 & soo6 & 995 & 1012 & 7 & Indeterminate & $\mathrm{NA}$ & 0.40 & $\mathrm{NA}$ & Frag & No & None & NA & $\mathrm{NA}$ & NA & Cortical & NA & \\
\hline 321 & 506 & s007 & 995 & 1012 & 7 & Indeterminate & NA & 0.29 & $\mathrm{NA}$ & Frag & No & None & $\mathrm{NA}$ & NA & $\mathrm{NA}$ & Cortical & NA & \\
\hline 321 & 506 & s008 & 995 & 1012 & 7 & Indeterminate & $\mathrm{NA}$ & 0.57 & NA & Frag & № & None & $\mathrm{NA}$ & $\mathrm{NA}$ & NA & Cortical & $\mathrm{NA}$ & \\
\hline 321 & 506 & soog & 995 & 1012 & 7 & Indeterminate & NA & \begin{tabular}{l|l}
0.28 \\
\end{tabular} & $\mathrm{NA}$ & Frag & No & None & NA & $\mathrm{NA}$ & NA & Cortical & NA & \\
\hline 321 & 506 & s010 & 995 & 1012 & 7 & Indeterminate & $\mathrm{NA}$ & 0.35 & $\mathrm{NA}$ & Frag & No & None & $\mathrm{NA}$ & $\mathrm{NA}$ & $\mathrm{NA}$ & Cortical & $\mathrm{NA}$ & \\
\hline 321 & 506 & s011 & 995 & 1012 & 7 & Indeterminate & $\mathrm{NA}$ & 0.23 & $\mathrm{NA}$ & Frag & No & None & NA & $\mathrm{NA}$ & NA & Cortical & $\mathrm{NA}$ & \\
\hline 321 & 506 & s012 & 995 & 1012 & 7 & Mammal & NA & 0.56 & $\mathrm{NA}$ & Frag & No & None & NA & $\mathrm{NA}$ & NA & Cortical & Oblique & \\
\hline 321 & 506 & $\begin{array}{ll}\text { s013 } \\
\end{array}$ & 995 & 1012 & 7 & Indeterminate & $\mathrm{NA}$ & 0.46 & $\mathrm{NA}$ & Frag & No & None & $\mathrm{NA}$ & $\mathrm{NA}$ & $\mathrm{NA}$ & Cortical & $\mathrm{NA}$ & \\
\hline 321 & 506 & s014 & 995 & 1012 & 7 & Indeterminate & NA & \begin{tabular}{l|l|l} 
& \\
\end{tabular} & $\mathrm{NA}$ & Frag & No & None & NA & $\mathrm{NA}$ & NA & Trabecular & $\mathrm{NA}$ & \\
\hline 321 & 506 & s015 & 995 & 1012 & 7 & Indeterminate & NA & 0.10 & NA & Frag & No & None & NA & NA & NA & Cortical & NA & \\
\hline 321 & 506 & s016 & 995 & 1012 & 7 & Indeterminate & NA & 0.10 & $\mathrm{NA}$ & Frag & No & None & $\mathrm{NA}$ & $\mathrm{NA}$ & NA & Cortical & Fresh & \\
\hline 321 & 506 & s017 & 995 & 1012 & 7 & Indeterminate & $\mathrm{NA}$ & 0.17 & $\mathrm{NA}$ & Frag & No & None & $\mathrm{NA}$ & $\mathrm{NA}$ & $\mathrm{NA}$ & Trabecular & $\mathrm{NA}$ & \\
\hline 321 & 506 & s018 & 995 & 1012 & 7 & Indeterminate & $\mathrm{NA}$ & 0.12 & $\mathrm{NA}$ & Frag & No & None & NA & $\mathrm{NA}$ & NA & Trabecular & NA & \\
\hline 321 & 506 & s019 & 995 & 1012 & 7 & Indeterminate & $\mathrm{NA}$ & 0.05 & $\mathrm{NA}$ & Frag & No & None & $\mathrm{NA}$ & $\mathrm{NA}$ & $\mathrm{NA}$ & Cortical & $\mathrm{NA}$ & \\
\hline 321 & 506 & s020 & 995 & 1012 & 7 & Indeterminate & $\mathrm{NA}$ & 0.18 & $\mathrm{NA}$ & Frag & No & None & NA & $\mathrm{NA}$ & NA & Cortical & NA & \\
\hline 321 & 506 & s021 & 995 & 1012 & 7 & Indeterminate & $\mathrm{NA}$ & 0.11 & $\mathrm{NA}$ & Frag & No & None & $\mathrm{NA}$ & $\mathrm{NA}$ & NA & Cortical & NA & \\
\hline 321 & 506 & s022 & 995 & 1012 & 7 & Indeterminate & $\mathrm{NA}$ & $\begin{array}{ll}0.01 \\
\end{array}$ & $\mathrm{NA}$ & Frag & No & None & $\mathrm{NA}$ & NA & NA & Cortical & $\mathrm{NA}$ & \\
\hline 321 & 506 & s023 & 995 & 1012 & 7 & Indeterminate & $\mathrm{NA}$ & 0.01 & $\mathrm{NA}$ & Frag & No & None & $\mathrm{NA}$ & $\mathrm{NA}$ & $\mathrm{NA}$ & $\begin{array}{l}\text { Cortical } \\
\end{array}$ & $\mathrm{NA}$ & \\
\hline 323 & 524 & s001 & 995 & 1012 & 8 & Mammal & $\mathrm{NA}$ & 0.55 & 2 & Frag & Yes & \begin{tabular}{|l} 
Percussion Notch \\
\end{tabular} & $\mathrm{NA}$ & $\mathrm{NA}$ & NA & Cortical & Spiral & Probabaly deer \\
\hline 323 & 524 & s002 & 995 & 1012 & 8 & Indeterminate & $\mathrm{NA}$ & 1.37 & $\mathrm{NA}$ & Frag & No & None & $\mathrm{NA}$ & $\mathrm{NA}$ & $\mathrm{NA}$ & Trabecular & $\mathrm{NA}$ & \\
\hline 323 & 524 & s003 & 995 & 1012 & 8 & Indeterminate & $\mathrm{NA}$ & 0.63 & $\mathrm{NA}$ & Frag & No & None & NA & $\mathrm{NA}$ & NA & Cortical & $\mathrm{NA}$ & \\
\hline 323 & 524 & s004 & 995 & 1012 & 8 & Indeterminate & $\mathrm{NA}$ & 0.15 & $\mathrm{NA}$ & Frag & No & None & $\mathrm{NA}$ & $\mathrm{NA}$ & NA & Cortical & $\mathrm{NA}$ & \\
\hline 323 & 524 & so05 & 995 & 1012 & 8 & Mammal & $\mathrm{NA}$ & 0.49 & $\mathrm{NA}$ & Frag & No & None & $\mathrm{NA}$ & $\mathrm{NA}$ & $\mathrm{NA}$ & Cortical & Multiple & \\
\hline 323 & 524 & s006 & 995 & 1012 & 8 & Mammal & NA & 2.6 & 3-4 & Frag & No & None & $\mathrm{NA}$ & $\mathrm{NA}$ & NA & Cortical & Oblique & \\
\hline 323 & 524 & s007 & 995 & 1012 & 8 & Indeterminate & $\mathrm{NA}$ & 0.88 & $\mathrm{NA}$ & Frag & No & None & $\mathrm{NA}$ & $\mathrm{NA}$ & NA & Trabecular & NA & \\
\hline 323 & 524 & s008 & 995 & 1012 & 8 & Tortoise/Turtle & \begin{tabular}{|l|} 
Tortoise/Turtle \\
\end{tabular} & \begin{tabular}{|l|l|}
0.24 &
\end{tabular} & 1 & Frag & No & None & Carapace & NA & NA & NA & $\mathrm{NA}$ & \\
\hline 323 & 524 & soog & 995 & 1012 & 8 & Mammal & $\mathrm{NA}$ & 0.33 & $\mathrm{NA}$ & Frag & No & None & $\mathrm{NA}$ & $\mathrm{NA}$ & $\mathrm{NA}$ & Cortical & Right & \\
\hline 323 & 524 & s010 & 995 & 1012 & 8 & Indeterminate & $\mathrm{NA}$ & 0.25 & $\mathrm{NA}$ & Frag & No & None & $\mathrm{NA}$ & $\mathrm{NA}$ & $\mathrm{NA}$ & Cortical & $\mathrm{NA}$ & \\
\hline 323 & 524 & s011 & 995 & 1012 & 8 & Indeterminate & $\mathrm{NA}$ & 0.37 & $\mathrm{NA}$ & Frag & No & None & $\mathrm{NA}$ & $\mathrm{NA}$ & NA & Cortical & $\mathrm{NA}$ & \\
\hline 323 & 524 & s012 & 995 & 1012 & 8 & Indeterminate & $\mathrm{NA}$ & 0.21 & $\mathrm{NA}$ & Frag & No & None & NA & $\mathrm{NA}$ & NA & Cortical & NA & \\
\hline 323 & 524 & s013 & 995 & 1012 & 8 & Indeterminate & NA & 0.25 & $\mathrm{NA}$ & Frag & No & None & NA & NA & NA & Cortical & $\mathrm{NA}$ & \\
\hline 323 & 524 & s014 & 995 & 1012 & 8 & Indeterminate & $\mathrm{NA}$ & 0.28 & $\mathrm{NA}$ & Frag & No & None & $\mathrm{NA}$ & $\mathrm{NA}$ & NA & Cortical & $\mathrm{NA}$ & \\
\hline 323 & 524 & s015 & 995 & 1012 & 8 & Indeterminate & NA & 0.15 & $\mathrm{NA}$ & Frag & No & None & NA & NA & NA & Cortical & $\mathrm{NA}$ & \\
\hline 323 & 524 & s016 & 995 & 1012 & 8 & Indeterminate & $\mathrm{NA}$ & 0.21 & $\mathrm{NA}$ & Frag & No & None & NA & $\mathrm{NA}$ & NA & Cortical & $\mathrm{NA}$ & \\
\hline 323 & 524 & s017 & 995 & 1012 & 8 & Indeterminate & $\mathrm{NA}$ & 0.15 & $\mathrm{NA}$ & Frag & No & None & NA & $\mathrm{NA}$ & NA & Cortical & NA & \\
\hline 323 & 524 & s018 & 995 & 1012 & 8 & Indeterminate & $\mathrm{NA}$ & 0.11 & $\mathrm{NA}$ & Frag & No & None & NA & NA & NA & Cortical & $\mathrm{NA}$ & \\
\hline 323 & 524 & s019 & 995 & 1012 & 8 & Indeterminate & $\mathrm{NA}$ & 0.08 & $\mathrm{NA}$ & Frag & No & None & NA & $\mathrm{NA}$ & NA & Cortical & $\mathrm{NA}$ & \\
\hline 323 & 524 & s020 & 995 & 1012 & 8 & Indeterminate & $\mathrm{NA}$ & 0.29 & $\mathrm{NA}$ & Frag & No & None & NA & $\mathrm{NA}$ & NA & Cortical & $\mathrm{NA}$ & \\
\hline 323 & 524 & s021 & 995 & 1012 & 8 & Indeterminate & $\mathrm{NA}$ & 0.14 & $\mathrm{NA}$ & Frag & No & None & $\mathrm{NA}$ & $\mathrm{NA}$ & $\mathrm{NA}$ & $\begin{array}{l}\text { Cortical } \\
\end{array}$ & $\mathrm{NA}$ & \\
\hline 323 & 524 & s022 & 995 & 1012 & 8 & Indeterminate & $\mathrm{NA}$ & 0.18 & $\mathrm{NA}$ & Frag & No & None & $\mathrm{NA}$ & $\mathrm{NA}$ & NA & Cortical & $\mathrm{NA}$ & \\
\hline 323 & 524 & s023 & 995 & 1012 & 8 & Indeterminate & NA & 0.06 & $\mathrm{NA}$ & Frag & No & None & NA & NA & NA & Cortical & NA & \\
\hline 323 & 524 & s024 & 995 & 1012 & 8 & Indeterminate & $\mathrm{NA}$ & 0.01 & $\mathrm{NA}$ & Frag & No & None & NA & $\mathrm{NA}$ & NA & Cortical & $\mathrm{NA}$ & \\
\hline 325 & 578 & s001 & 995 & 1012 & 9 & Tortoise/Turtle & Tortoise/Turtle & \begin{tabular}{|l|l|}
0.41 \\
\end{tabular} & 1 & Frag & No & None & \begin{tabular}{|l|} 
Plastron \\
\end{tabular} & NA & NA & Cortical & Right & Heavily weathered \\
\hline 327 & 589 & s001 & 995 & 1012 & 10 & Tortoise/Turtle & \begin{tabular}{|c|} 
Tortoise/Turtle \\
\end{tabular} & 0.38 & 1 & Frag & No & None & carapace & $\mathrm{NA}$ & $\mathrm{NA}$ & $\mathrm{NA}$ & & Small carapace frag. Possibly burned. \\
\hline 327 & 589 & s002 & 995 & 1012 & 10 & Indeterminate & NA & 0.52 & NA & NA & No & None & NA & NA & NA & NA & & Very weathered and distorted. \\
\hline 327 & 589 & s003 & 995 & 1012 & 10 & Indeterminate & $\mathrm{NA}$ & 0.28 & $\mathrm{NA}$ & NA & No & None & $\mathrm{NA}$ & NA & NA & Cortical & & Heavily weathered \\
\hline 329 & 628 & soo1 & 995 & 1012 & 12 & Mammal & NA & 0.06 & $\mathrm{NA}$ & Frag & No & None & tooth & NA & NA & Enamel & & $\begin{array}{l}\begin{array}{l}\text { Small enamel fragment. Not enough to make a } \\
\text { determination. }\end{array} \\
\end{array}$ \\
\hline 335 & 378 & soo1 & 995 & 1013 & 3 & Indeterminate & NA & 0.42 & NA & Frag & № & None & NA & NA & NA & Cortical & & Long bone fragment, very weathered. \\
\hline 336 & 388 & s001 & 995 & 1013 & 4 & Mammal & $\mathrm{NA}$ & 6.94 & NA & Frag & No & None & NA & $\mathrm{NA}$ & NA & Cortical & & $\begin{array}{l}\text { Large long bone frag. Surface has been obliterated due to to } \\
\text { weathering. }\end{array}$ \\
\hline 336 & 388 & s002 & 995 & 1013 & 4 & Indeterminate & $\mathrm{NA}$ & 0.96 & $\mathrm{NA}$ & Frag & No & None & NA & NA & NA & Cortical & & $\begin{array}{l}\text { Cortical frag. Surface has been oblitierated due to } \\
\text { weathering. }\end{array}$ \\
\hline 336 & 388 & soo3 & 995 & 1013 & 4 & $\begin{array}{l}\text { Indeterminate } \\
\end{array}$ & $\mathrm{NA}$ & 0.42 & $\mathrm{NA}$ & Frag & No & None & $\mathrm{NA}$ & NA & NA & Cortical & & $\begin{array}{l}\text { Cortical frag. Surface has been obliterated due to } \\
\text { weathering. }\end{array}$ \\
\hline
\end{tabular}




\begin{tabular}{|c|c|c|c|c|c|c|c|c|c|c|c|c|c|c|c|c|c|c|}
\hline 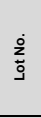 & 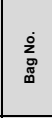 & 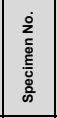 & 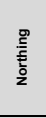 & 喜 & בَّ & $\begin{array}{c}\text { 高 } \\
\text { 恖 }\end{array}$ & 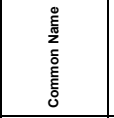 & 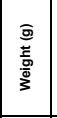 & 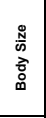 & 总 & 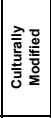 & 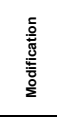 & 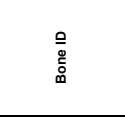 & $\frac{8}{\omega}$ & 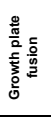 & 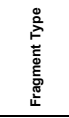 & 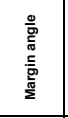 & 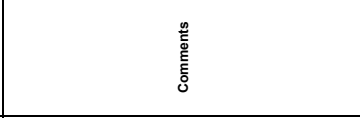 \\
\hline 336 & 388 & s004 & 995 & 1013 & 4 & Indeterminate & $\mathrm{NA}$ & 0.35 & $\mathrm{NA}$ & Frag & № & None & $\mathrm{NA}$ & NA & $\mathrm{NA}$ & Cortical & & \begin{tabular}{|l}
$\begin{array}{l}\text { Cortical frag. Surface has been obliterated due to } \\
\text { weathering. }\end{array}$ \\
\end{tabular} \\
\hline 338 & 405 & s001 & 995 & 1013 & 6 & Indeterminate & $\mathrm{NA}$ & 0.45 & $\mathrm{NA}$ & Frag & No & None & $\mathrm{NA}$ & NA & NA & Cortical & oblique & Long bone frag \\
\hline 338 & 405 & s002 & 995 & 1013 & 6 & Indeterminate & $\mathrm{NA}$ & 0.38 & $\mathrm{NA}$ & Frag & No & None & NA & $\mathrm{NA}$ & NA & Cortical & Right & \\
\hline 338 & 405 & s003 & 995 & 1013 & 6 & Indeterminate & NA & 0.17 & $\mathrm{NA}$ & Frag & No & None & NA & NA & NA & Cortical & Right & \\
\hline 338 & 405 & soo4 & 995 & 1013 & 6 & Indeterminate & $\mathrm{NA}$ & 0.34 & $\mathrm{NA}$ & Frag & № & None & $\mathrm{NA}$ & $\mathrm{NA}$ & $\mathrm{NA}$ & Cortical & Right & \\
\hline 338 & 405 & soos & 995 & 1013 & 6 & Indeterminate & NA & 0.17 & NA & Frag & No & None & NA & NA & NA & Cortical & Right & \\
\hline 339 & 410 & s001 & 995 & 1013 & 7 & Avian & $\mathrm{NA}$ & 0.28 & $\mathrm{NA}$ & Frag & № & None & $\mathrm{NA}$ & NA & $\mathrm{NA}$ & Cortical & & $\begin{array}{l}\text { Long bonen fragment. The esize of the frag and gracile } \\
\text { cortex would indicate a large bird. }\end{array}$ \\
\hline 339 & 410 & s002 & 995 & 1013 & 7 & Indeterminate & NA & 0.29 & NA & Frag & No & None & NA & NA & NA & Cortical & & Too weathered to be identified. \\
\hline 339 & 410 & S003 & 995 & 1013 & 7 & Mammal & $\mathrm{NA}$ & 0.6 & $\mathrm{NA}$ & Frag & No & None & $\mathrm{NA}$ & $\mathrm{NA}$ & $\mathrm{NA}$ & Cortical & & 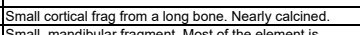 \\
\hline 344 & 610 & s001 & 995 & 1013 & 10 & Mammal & $\mathrm{NA}$ & 2.36 & $\mathrm{NA}$ & Frag & No & None & Mandible & $\mathrm{NA}$ & $\mathrm{NA}$ & Cortical & & $\begin{array}{l}\text { Small, mandibular fragment. Most of the element is } \\
\text { missing, Size class is isther a 1-or a } 2 \text {. }\end{array}$ \\
\hline 344 & 610 & s002 & 995 & 1013 & 10 & Indeterminate & $\mathrm{NA}$ & 0.44 & $\mathrm{NA}$ & Frag & No & None & NA & NA & NA & Cortical & & $\begin{array}{l}\text { Small, fat bone frag. Too small and weathered for } \\
\text { landmarks. }\end{array}$ \\
\hline 344 & 610 & s003 & 995 & 1013 & 10 & Indeterminate & $\mathrm{NA}$ & 0.1 & $\mathrm{NA}$ & Frag & № & None & NA & NA & NA & Cortical & & \begin{tabular}{|l|} 
Small, ortical frag. Too small and weathered for \\
landmarks.
\end{tabular} \\
\hline 344 & 610 & s004 & 995 & 1013 & 10 & Indeterminate & NA & 0.44 & $\mathrm{NA}$ & Frag & No & None & Cranial element & NA & NA & Cortical & & $\begin{array}{l}\begin{array}{l}\text { Small cranial element. Not enough to determine anything } \\
\text { else. }\end{array} \\
\text {. }\end{array}$ \\
\hline 344 & 610 & s005 & 995 & 1013 & 10 & Indeterminate & NA & 0.13 & $\mathrm{NA}$ & Frag & No & None & $\mathrm{NA}$ & NA & $\mathrm{NA}$ & Cortical & & Small frag, \\
\hline 344 & 610 & s006 & 995 & 1013 & 10 & Indeterminate & NA & 0.01 & $\mathrm{NA}$ & Frag & No & None & $\mathrm{NA}$ & NA & NA & Cortical & & Very small frag, mostly trabecular. \\
\hline 346 & 626 & s001 & 995 & 1013 & 12 & Odocoileus sp & Deer & 13.06 & 2 & Whole & № & None & Astragalus & Right & NA & NA & & $\begin{array}{l}\text { Whole deer astragalus. No evidence of butchery. Some } \\
\text { damagag, ubta pappars to be moderm. }\end{array}$ \\
\hline 346 & 626 & s002 & 995 & 1013 & 12 & Mammal & NA & 2.79 & 2 & Frag & No & None & NA & NA & NA & Cortical & & $\begin{array}{l}\text { very weathered large mammal long bone. Best guess } \\
\text { would be a deer metapodial frag, but too weathered to be } \\
\text { definite. }\end{array}$ \\
\hline 346 & 626 & s003 & 995 & 1013 & 12 & Mammal & NA & 0.4 & $\mathrm{NA}$ & Frag & No & None & NA & NA & NA & Cortical & & Small long bone frag. Weathered surface. \\
\hline 347 & 636 & soo1 & 995 & 1013 & 13 & Tortoise/Turle & Tortoise/Turtle & 3.12 & 1 & Frag & No & None & Plastron & NA & NA & NA & & Large piece of plastron \\
\hline 348 & 644 & soo1 & 995 & 1013 & 14 & Odocoileus sp & Deer & 8.19 & 2 & Frag & No & None & Metacarpal & NA & NA & Cortical & & 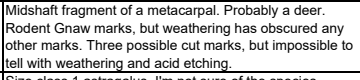 \\
\hline 350 & 354 & s001 & 995 & 1014 & 1 & Mammal & $\mathrm{NA}$ & 0.71 & 1 & Whole & No & None & Astragalus & Right & $\mathrm{NA}$ & NA & & $\begin{array}{l}\text { lize class } 1 \text { astragalus. Im not ture of the species. } \\
\text { Checked several online. Possibly armadillo? }\end{array}$ \\
\hline 353 & 394 & s001 & 995 & 1014 & 4 & Mammal & NA & 5.72 & 2 & Frag & No & None & NA & $\mathrm{NA}$ & NA & Cortical & & $\begin{array}{l}\text { Long bone frag. Heavily weathered. Size class based on } \\
\text { cortical thickness. }\end{array}$ \\
\hline 353 & 394 & s002 & 995 & 1014 & 4 & Mammal & $\mathrm{NA}$ & 3.18 & 2 & Frag & No & None & $\mathrm{NA}$ & $\mathrm{NA}$ & NA & Cortical & & $\begin{array}{l}\text { Long bone frag. Heavily weathered. Size class based on } \\
\text { cortical thickness. }\end{array}$ \\
\hline 354 & 326 & s001 & 995 & 1014 & 5 & Indeterminate & $\mathrm{NA}$ & 0.13 & $\mathrm{NA}$ & Frag & No & None & $\mathrm{NA}$ & NA & NA & Cortical & & Distorted by weathering. \\
\hline 354 & 326 & s002 & 995 & 1014 & 5 & Indeterminate & NA & 0.57 & $\mathrm{NA}$ & Frag & No & None & NA & $\mathrm{NA}$ & $\mathrm{NA}$ & Cortical & & Distorted by weathering. \\
\hline 355 & 407 & s001 & 995 & 1014 & 6 & Mammal & $\mathrm{NA}$ & 3.42 & $\mathrm{NA}$ & Frag & No & None & NA & NA & NA & Cortical & & Long bone fragment, very weathered. \\
\hline 355 & 407 & s002 & 995 & 1014 & 6 & Mammal & NA & 1.27 & $\mathrm{NA}$ & Frag & No & None & NA & NA & NA & Cortical & & Long bone fragment, very weathered. \\
\hline 357 & 415 & s001 & 995 & 1014 & 7 & Indeterminate & $\mathrm{NA}$ & 0.19 & $\mathrm{NA}$ & Frag & No & None & $\mathrm{NA}$ & NA & NA & Cortical & & Too weathered to be identified. \\
\hline 363 & 634 & s001 & 995 & 1014 & 11 & Mammal & NA & 1.25 & $\mathrm{NA}$ & Frag & No & None & NA & NA & NA & Cortical & & Calcined long bone frag. Probably size class 2 \\
\hline 366 & 470 & s001 & 996 & 1012 & 1 & Mammal & NA & 0.07 & $\mathrm{NA}$ & Frag & No & None & tooth & NA & $\mathrm{NA}$ & Enamel & & $\begin{array}{l}\text { Small enamel fragment. Probably cervid, but not enough } \\
\text { to make a determination. }\end{array}$ \\
\hline 368 & 481 & s001 & 996 & 1012 & 3 & Indeterminate & NA & 0.22 & $\mathrm{NA}$ & Frag & No & None & $\mathrm{NA}$ & $\mathrm{NA}$ & NA & Cortical & & Thick cortical frag. \\
\hline 372 & 502 & s001 & 996 & 1012 & 6 & Tortoise/Turle & Tortoise/Turtle & 0.24 & 1 & Frag & No & None & Plastron & NA & NA & NA & NA & \\
\hline 373 & 517 & s001 & 996 & 1012 & 7 & Indeterminate & $\mathrm{NA}$ & 0.26 & $\mathrm{NA}$ & Frag & No & None & NA & $\mathrm{NA}$ & NA & Cortical & NA & \\
\hline 373 & 517 & s002 & 996 & 1012 & 7 & Indeterminate & $\mathrm{NA}$ & 3.48 & $\mathrm{NA}$ & Frag & No & None & NA & $\mathrm{NA}$ & NA & Cortical & $\mathrm{NA}$ & \\
\hline 373 & 517 & s003 & 996 & 1012 & 7 & Indeterminate & $\mathrm{NA}$ & 1.39 & $\mathrm{NA}$ & Frag & No & None & NA & $\mathrm{NA}$ & NA & Cortical & NA & \\
\hline 373 & 517 & s004 & 996 & 1012 & 7 & Indeterminate & $\mathrm{NA}$ & 0.19 & $\mathrm{NA}$ & Frag & No & None & NA & $\mathrm{NA}$ & NA & Cortical & $\mathrm{NA}$ & \\
\hline 373 & 517 & s005 & 996 & 1012 & 7 & Indeterminate & $\mathrm{NA}$ & 0.23 & NA & Frag & No & None & NA & $\mathrm{NA}$ & NA & Cortical & NA & \\
\hline 373 & 517 & s006 & 996 & 1012 & 7 & Indeterminate & $\mathrm{NA}$ & 0.58 & $\mathrm{NA}$ & Frag & No & None & NA & $\mathrm{NA}$ & NA & Cortical & $\mathrm{NA}$ & \\
\hline 373 & 517 & s007 & 996 & 1012 & 7 & Indeterminate & NA & 0.27 & NA & Frag & No & None & $\mathrm{NA}$ & $\mathrm{NA}$ & NA & Cortical & $\mathrm{NA}$ & \\
\hline 373 & 517 & s008 & 996 & 1012 & 7 & Indeterminate & NA & 0.34 & $\mathrm{NA}$ & Frag & No & None & NA & NA & NA & Cortical & NA & \\
\hline 373 & 517 & soog & 996 & 1012 & 7 & Indeterminate & $\mathrm{NA}$ & 0.36 & $\mathrm{NA}$ & Frag & No & None & NA & $\mathrm{NA}$ & NA & NA & $\mathrm{NA}$ & Weathered to just a calcium mass \\
\hline 373 & 517 & s010 & 996 & 1012 & 7 & Indeterminate & NA & 0.22 & $\mathrm{NA}$ & Frag & No & None & NA & $\mathrm{NA}$ & $\mathrm{NA}$ & NA & $\mathrm{NA}$ & Weathered to just a calcium mass \\
\hline 373 & 517 & s011 & 996 & 1012 & 7 & Indeterminate & $\mathrm{NA}$ & 0.96 & $\mathrm{NA}$ & Frag & No & None & NA & $\mathrm{NA}$ & NA & Cortical & Right & |ong bone frag \\
\hline 373 & 517 & s012 & 996 & 1012 & 7 & Indeterminate & $\mathrm{NA}$ & 0.73 & $\mathrm{NA}$ & Frag & No & None & NA & $\mathrm{NA}$ & NA & Cortical & $\mathrm{NA}$ & Roasted \\
\hline 375 & 549 & soo1 & 996 & 1012 & 8 & Indeterminate & NA & 0.26 & $\mathrm{NA}$ & Frag & No & None & NA & NA & NA & Cortical & NA & \\
\hline 375 & 549 & s002 & 996 & 1012 & 8 & Indeterminate & $\mathrm{NA}$ & 0.06 & $\mathrm{NA}$ & Frag & No & None & $\mathrm{NA}$ & $\mathrm{NA}$ & NA & Cortical & $\mathrm{NA}$ & \\
\hline 375 & 549 & s003 & 996 & 1012 & 8 & Indeterminate & $\mathrm{NA}$ & 0.29 & $\mathrm{NA}$ & Frag & No & None & $\mathrm{NA}$ & $\mathrm{NA}$ & NA & Cortical & $\mathrm{NA}$ & \\
\hline 375 & 549 & soo4 & 996 & 1012 & 8 & Indeterminate & $\mathrm{NA}$ & 0.61 & $\mathrm{NA}$ & Frag & No & None & NA & $\mathrm{NA}$ & NA & Cortical & $\mathrm{NA}$ & \\
\hline 375 & 549 & s005 & 996 & 1012 & 8 & Indeterminate & $\mathrm{NA}$ & 0.06 & $\mathrm{NA}$ & Frag & No & None & NA & NA & NA & Cortical & NA & \\
\hline 375 & 549 & s006 & 996 & 1012 & 8 & Indeterminate & $\mathrm{NA}$ & 0.46 & $\mathrm{NA}$ & Frag & No & None & $\mathrm{NA}$ & NA & $\mathrm{NA}$ & Cortical & NA & \\
\hline 375 & 549 & s007 & 996 & 1012 & 8 & Indeterminate & $\mathrm{NA}$ & 0.11 & NA & Frag & No & None & $\mathrm{NA}$ & $\mathrm{NA}$ & NA & Cortical & $\mathrm{NA}$ & \\
\hline 375 & 549 & s008 & 996 & 1012 & 8 & Indeterminate & $\mathrm{NA}$ & 0.03 & $\mathrm{NA}$ & Frag & No & None & $\mathrm{NA}$ & $\mathrm{NA}$ & $\mathrm{NA}$ & Cortical & $\mathrm{NA}$ & \\
\hline 375 & 549 & soog & 996 & 1012 & 8 & Indeterminate & $\mathrm{NA}$ & 0.01 & NA & Frag & No & None & NA & $\mathrm{NA}$ & NA & Cortical & $\mathrm{NA}$ & \\
\hline 375 & 549 & so10 & 996 & 1012 & 8 & Indeterminate & $\mathrm{NA}$ & 0.01 & NA & Frag & No & None & NA & NA & $\mathrm{NA}$ & Cortical & NA & \\
\hline 377 & 573 & s001 & 996 & 1012 & 9 & Indeterminate & $\mathrm{NA}$ & 0.06 & NA & Frag & No & None & $\mathrm{NA}$ & $\mathrm{NA}$ & NA & Cortical & $\mathrm{NA}$ & \\
\hline 377 & 573 & s002 & 996 & 1012 & 9 & Indeterminate & NA & 0.17 & $\mathrm{NA}$ & Frag & No & None & $\mathrm{NA}$ & NA & $\mathrm{NA}$ & Cortical & Right & Calcined \\
\hline
\end{tabular}




\begin{tabular}{|c|c|c|c|c|c|c|c|c|c|c|c|c|c|c|c|c|c|c|}
\hline ْ & 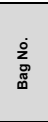 & 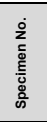 & 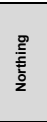 & $\begin{array}{l}\text { 䔍 } \\
\text { 焉 }\end{array}$ & ఏ్త & 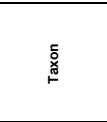 & 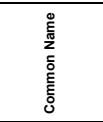 & $\begin{array}{l}\text { 힘 } \\
\text { 흠 } \\
\text { कू } \\
3\end{array}$ & 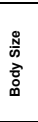 & 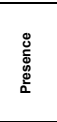 & 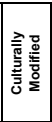 & 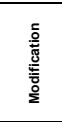 & 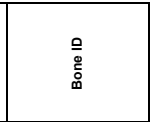 & $\frac{\%}{\omega}$ & 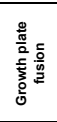 & 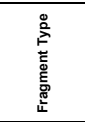 & 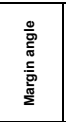 & 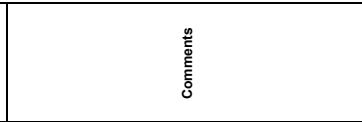 \\
\hline 381 & 635 & so01 & 996 & 1012 & 13 & Indeterminate & $\mathrm{NA}$ & 0.03 & NA & Frag & No & None & $\mathrm{NA}$ & NA & NA & Cortical & Fresh & Burned cortical-calcined \\
\hline 381 & 635 & soo2 & 996 & 1012 & 13 & Indeterminate & NA & 0.13 & NA & Frag & No & None & NA & NA & NA & Cortical & Fresh & Burned cortical-calcined \\
\hline 382 & 652 & so01 & 996 & 1012 & 15 & Mammal & NA & 0.65 & 2 & Frag & No & None & NA & NA & NA & Cortical & & $\begin{array}{l}\text { Unknown long bone mid-shaft frag. Size two, but smaller } \\
\text { than a deer. }\end{array}$ \\
\hline 382 & 652 & s002 & 996 & 1012 & 15 & Mammal & NA & 0.09 & 2 & Frag & No & None & Rib & NA & NA & Cortical & & Small frag, surface obliterated, probable rib frag \\
\hline 386 & 445 & so01 & 996 & 1013 & 3 & Indeterminate & NA & 1.35 & NA & Frag & No & None & NA & NA & NA & Cortical & Right & \\
\hline 386 & 445 & so02 & 996 & 1013 & 3 & Indeterminate & NA & 0.52 & NA & Frag & No & None & $\mathrm{NA}$ & NA & NA & Cortical & Oblique & \\
\hline 388 & 362 & so01 & 996 & 1013 & 4 & Mammal & NA & 2.38 & NA & Frag & No & None & Mandible & NA & NA & Cortical & & $\begin{array}{l}\text { Burned (calcined) Mandible frag. Hardly anything here, } \\
\text { except for some tooth root pits. }\end{array}$ \\
\hline 389 & 451 & so01 & 996 & 1013 & 5 & Odocoileus sp & Deer & 2.3 & 2 & Frag & No & None & tooth (M1 or M2) & Left & NA & NA & & $\begin{array}{l}\text { Cervid, } \mathrm{M} 1 \text { or } \mathrm{M} 2 \text {; left; upper; buchal fragment. Very little } \\
\text { wear (especially for an } \mathrm{M} 1 \text { or } \mathrm{M} 2 \text {. Probably a juvenile. }\end{array}$ \\
\hline 391 & 465 & so01 & 996 & 1013 & 7 & Tortoise/Turtle & Tortoise/Turtle & 0.46 & 1 & Frag & No & None & Plastron (possible rib) & NA & NA & NA & & $\begin{array}{l}\text { Turtle Tortoise Shell. Appears to be plastron with possibly } \\
\text { a bit of rib. Possibly heated }\end{array}$ \\
\hline 395 & 617 & so01 & 996 & 1013 & 10 & Indeterminate & NA & 1.14 & NA & Frag & No & None & NA & NA & NA & Cortical & Spiral & Long bone frag. \\
\hline 395 & 617 & so02 & 996 & 1013 & 10 & Indeterminate & NA & 0.17 & NA & Frag & No & None & NA & NA & NA & Cortical & NA & \\
\hline 395 & 617 & so03 & 996 & 1013 & 10 & Indeterminate & NA & 0.46 & NA & Frag & No & None & NA & NA & NA & Cortical & NA & \\
\hline 395 & 617 & so04 & 996 & 1013 & 10 & Indeterminate & NA & 0.14 & NA & Frag & No & None & NA & NA & NA & Cortical & NA & \\
\hline 395 & 617 & so05 & 996 & 1013 & 10 & Indeterminate & NA & 0.19 & NA & Frag & No & None & NA & NA & NA & Cortical & NA & \\
\hline 398 & 645 & so01 & 996 & 1013 & 13 & Indeterminate & NA & 0.25 & NA & Frag & No & None & NA & NA & NA & Cortical & & Too weathered to be identified. (Refits with S002). \\
\hline 398 & 645 & s002 & 996 & 1013 & 13 & Indeterminate & NA & 0.13 & NA & Frag & No & None & NA & NA & NA & Cortical & & Too weathered to be identified. (Refits with S001). \\
\hline 399 & 667 & so01 & 996 & 1013 & 14 & Indeterminate & NA & 0.81 & NA & Frag & No & None & NA & NA & NA & Cortical & & $\begin{array}{l}\begin{array}{l}\text { Heavily weathered surface. Probably part of a vertebral } \\
\text { body, but not enough to be sure. }\end{array} \\
\end{array}$ \\
\hline 400 & 483 & so01 & 996 & 1014 & 2 & Indeterminate & NA & 0.67 & NA & Frag & No & None & NA & NA & NA & Cortical & Right & \\
\hline 402 & 490 & so01 & 996 & 1014 & 4 & Indeterminate & NA & 1.92 & NA & Frag & No & None & NA & NA & NA & Trabecular & & $\begin{array}{l}\text { Appears to have been a round, articular surface. } \\
\text { However, weathering has destroyed nearly all of the } \\
\text { cortical surface. }\end{array}$ \\
\hline 402 & 490 & so02 & 996 & 1014 & 4 & Indeterminate & NA & 0.38 & NA & Frag & No & None & NA & NA & NA & Cortical & & Not enough present to make a determination \\
\hline 402 & 490 & so03 & 996 & 1014 & 4 & Indeterminate & NA & 0.26 & NA & Frag & No & None & NA & NA & NA & Cortical & & $\begin{array}{l}\begin{array}{l}\text { Might be part of a tortoise shell, but there is not enough } \\
\text { here to make that determination. }\end{array} \\
\end{array}$ \\
\hline 402 & 490 & so04 & 996 & 1014 & 4 & Indeterminate & NA & 0.74 & NA & Frag & No & None & NA & NA & NA & Cortical & & $\begin{array}{l}\begin{array}{l}\text { Appears to be an epiphysis, but is distorted by } \\
\text { weathering. }\end{array} \\
\end{array}$ \\
\hline 403 & 495 & so01 & 996 & 1014 & 5 & Indeterminate & NA & 0.34 & $\mathrm{NA}$ & Frag & No & None & NA & NA & NA & Cortical & & Too small to make a determination. \\
\hline 403 & 495 & so02 & 996 & 1014 & 5 & Indeterminate & NA & 0.13 & NA & Frag & No & None & NA & NA & NA & Cortical & & Too small to make a determination. \\
\hline 405 & 500 & so01 & 996 & 1014 & 6 & Indeterminate & NA & 0.31 & NA & Frag & No & None & NA & NA & NA & Cortical & & Too small to make a determination. \\
\hline 405 & 500 & so02 & 996 & 1014 & 6 & Indeterminate & NA & 0.04 & NA & Frag & No & None & NA & NA & NA & Cortical & & Too small to make a determination. \\
\hline 405 & 500 & so03 & 996 & 1014 & 6 & Indeterminate & NA & 0.13 & NA & Frag & No & None & NA & NA & NA & Cortical & & Too small to make a determination. \\
\hline 406 & 503 & so01 & 996 & 1014 & 7 & Odocoileus sp & Deer & 1.79 & 2 & whole & No & None & Tooth (M1) & Upper left & NA & NA & & $\begin{array}{l}\text { The majority of a cervid M1. Very lightly worn, does not } \\
\text { appear to be fully developed. . Juvenile. }\end{array}$ \\
\hline 407 & 507 & soo1 & 996 & 1014 & 8 & Mammal & NA & 1.33 & 1 & Frag & No & None & Femur & Left & NA & Cortical & & $\begin{array}{l}\text { Incomplete proximal femoral epiphysis. Very heavily } \\
\text { weathered. }\end{array}$ \\
\hline 411 & 537 & soo1 & 996 & 1014 & 11 & Indeterminate & NA & 0.18 & NA & Frag & No & None & NA & NA & NA & Cortical & & Too small and weathered to make a determination. \\
\hline 411 & 537 & so02 & 996 & 1014 & 11 & Indeterminate & NA & 0.24 & NA & Frag & No & None & NA & NA & NA & Trabecular & & No cortical surface. \\
\hline 412 & 567 & so01 & 996 & 1014 & 12 & Indeterminate & NA & 0.11 & NA & Frag & No & None & $\mathrm{NA}$ & NA & NA & Cortical & Fresh & \\
\hline 413 & 540 & soo1 & 996 & 1014 & 13 & Indeterminate & NA & 1.38 & NA & Frag & No & None & NA & NA & NA & NA & Oblique & Calcined \\
\hline 414 & 543 & soo1 & 996 & 1014 & 14 & Indeterminate & NA & 1.09 & NA & Frag & No & None & NA & NA & NA & Cortical & & Completely calcined. \\
\hline 414 & 543 & s002 & 996 & 1014 & 14 & Indeterminate & NA & 0.26 & NA & Frag & No & None & NA & NA & NA & Cortical & & Completely calcined. \\
\hline 414 & 543 & s003 & 996 & 1014 & 14 & Indeterminate & NA & 0.41 & NA & Frag & No & None & $\mathrm{NA}$ & NA & NA & Cortical & & Completely calcined. \\
\hline 414 & 543 & so04 & 996 & 1014 & 14 & Indeterminate & NA & 0.7 & NA & Frag & No & None & $\mathrm{NA}$ & NA & NA & Cortical & & Completely calcined. \\
\hline 414 & 543 & so05 & 996 & 1014 & 14 & Indeterminate & NA & 0.18 & NA & Frag & No & None & $\mathrm{NA}$ & NA & NA & Cortical & & Completely calcined. \\
\hline 417 & 561 & so01 & 996 & 1014 & 17 & Mammal & NA & 4.43 & 1 & Frag & No & None & Humerus & Right & No & Epiphysis & & $\begin{array}{l}\text { Calcined and distorted distal humerus frag. The epiphysia } \\
\text { growth plate is missing, but could be due to the extreme } \\
\text { heat distortion. }\end{array}$ \\
\hline 417 & 561 & s002 & 996 & 1014 & 17 & Indeterminate & NA & 1.47 & NA & Frag & No & None & NA & $\mathrm{NA}$ & NA & Cortical & & $\begin{array}{l}\text { Appears to be an epiphysis, but is heavily distorted by } \\
\text { heat. Completely calcined. }\end{array}$ \\
\hline 439 & 442 & so01 & 999 & 1013 & 7 & Mammal & NA & 1.9 & 2 & Frag & No & None & Vertebra & NA & No & Cortical & & $\begin{array}{l}\text { Most of a vertebral body. Growth plate is not present. } \\
\text { Probably juvenile deer. Burned, not completely calcined. }\end{array}$ \\
\hline 443 & 476 & soo1 & 999 & 1013 & 12 & Mammal & NA & 0.51 & 2 & Frag & No & None & Rib & NA & NA & Cortical & & Small frag. \\
\hline 446 & 498 & so01 & 999 & 1013 & 15 & Indeterminate & NA & 0.11 & NA & Frag & No & None & NA & NA & NA & Cortical & & Very small, burned (calcined) frag. \\
\hline 462 & 526 & soo1 & 1000 & 1003 & 3 & Mammal & NA & 3.87 & 3-4 & Whole & No & None & Sesamoid & NA & $\mathrm{NA}$ & Whole & & \\
\hline 462 & 526 & so02 & 1000 & 1003 & 3 & Mammal & NA & 4.19 & 3-4 & Whole & No & None & Sesamoid & NA & NA & Whole & & \\
\hline 462 & 526 & s003 & 1000 & 1003 & 3 & Mammal & NA & 3.12 & 3-4 & Frag & No & None & Sesamoid & NA & NA & Cortical & & About $50 \%$ remaining \\
\hline 462 & 526 & so04 & 1000 & 1003 & 3 & Indeterminate & NA & 0.29 & NA & Frag & No & None & $\mathrm{NA}$ & NA & NA & Cortical & & \\
\hline 462 & 526 & $\mathbf{s 0 0 5}$ & 1000 & 1003 & 3 & Indeterminate & NA & 0.68 & NA & Frag & No & None & NA & NA & NA & Cortical & & \\
\hline 462 & 526 & soo6 & 1000 & 1003 & 3 & Indeterminate & NA & 0.34 & NA & Frag & No & None & $\mathrm{NA}$ & NA & NA & Cortical & & \\
\hline 462 & 526 & s007 & 1000 & 1003 & 3 & Indeterminate & NA & 0.14 & NA & Frag & No & None & $\mathrm{NA}$ & NA & NA & Cortical & & \\
\hline 462 & 526 & so08 & 1000 & 1003 & 3 & Indeterminate & NA & 0.34 & NA & Frag & No & None & NA & NA & NA & Cortical & & \\
\hline 462 & 526 & soog & 1000 & 1003 & 3 & Indeterminate & NA & 0.27 & NA & Frag & No & None & NA & NA & NA & Cortical & & \\
\hline 462 & 526 & so10 & 1000 & 1003 & 3 & Indeterminate & NA & 1.4 & NA & Frag & No & None & NA & NA & NA & Cortical & & \\
\hline 462 & 526 & s011 & 1000 & 1003 & 3 & Indeterminate & NA & 0.27 & NA & Frag & No & None & $\mathrm{NA}$ & NA & NA & Cortical & & \\
\hline 462 & 526 & so12 & 1000 & 1003 & 3 & Indeterminate & NA & 0.28 & NA & Frag & No & None & $\mathrm{NA}$ & NA & NA & Cortical & & \\
\hline 462 & 526 & s013 & 1000 & 1003 & 3 & Indeterminate & NA & 1.64 & NA & Frag & No & None & NA & NA & NA & Cortical & & Long bone \\
\hline
\end{tabular}




\begin{tabular}{|c|c|c|c|c|c|c|c|c|c|c|c|c|c|c|c|c|c|c|}
\hline ذِ & 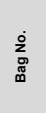 & 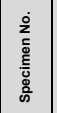 & 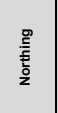 & 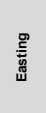 & ఏ్ & 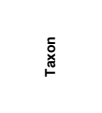 & 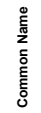 & 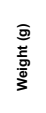 & 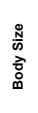 & 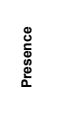 & 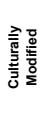 & 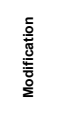 & 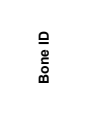 & $\frac{8}{5}$ & 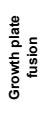 & 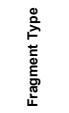 & 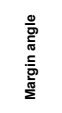 & 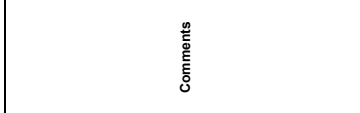 \\
\hline 462 & 526 & s014 & 1000 & 1003 & 3 & Mammal & $\mathrm{NA}$ & 12.44 & NA & Frag & No & None & NA & NA & NA & Cortical & & Large mammal- not sure what size class \\
\hline 462 & 526 & s015 & 1000 & 1003 & 3 & Mammal & NA & 8.48 & NA & Frag & No & None & $\mathrm{NA}$ & NA & NA & Cortical & & Large mammal- not sure what size class \\
\hline 462 & 526 & s016 & 1000 & 1003 & 3 & Indeterminate & $\mathrm{NA}$ & 1.9 & $\mathrm{NA}$ & Frag & No & None & NA & NA & $\mathrm{NA}$ & Cortical & & \\
\hline 462 & 526 & s017 & 1000 & 1003 & 3 & Indeterminate & $\mathrm{NA}$ & 12.2 & NA & Frag & No & None & $\mathrm{NA}$ & NA & $\mathrm{NA}$ & $\mathrm{NA}$ & & Burned clay and bone mixed \\
\hline 467 & 663 & s001 & 1000 & 1003 & 7 & Indeterminate & NA & 0.43 & $\mathrm{NA}$ & Frag & No & None & $\mathrm{NA}$ & $\mathrm{NA}$ & NA & Cortical & $\mathrm{NA}$ & Burned \\
\hline 467 & 663 & s002 & 1000 & 1003 & 7 & Indeterminate & $\mathrm{NA}$ & 0.09 & $\mathrm{NA}$ & Frag & No & None & $\mathrm{NA}$ & NA & NA & Cortical & NA & Burned \\
\hline 467 & 663 & s003 & 1000 & 1003 & 7 & Indeterminate & NA & 0.27 & NA & Frag & No & None & $\mathrm{NA}$ & NA & $\mathrm{NA}$ & Cortical & NA & \\
\hline 469 & 668 & s001 & 1000 & 1003 & 9 & Indeterminate & NA & 0.19 & $\mathrm{NA}$ & Frag & No & None & $\mathrm{NA}$ & $\mathrm{NA}$ & NA & Cortical & $\mathrm{NA}$ & \\
\hline 471 & 515 & soo1 & 1000 & 1004 & 2 & Mammal & NA & 4.16 & NA & Frag & No & None & $\mathrm{NA}$ & NA & NA & Cortical & & Robust cortical, long bone frag. Distorted by weathering \\
\hline 471 & 515 & s002 & 1000 & 1004 & 2 & Mammal & NA & 4.39 & NA & Frag & No & None & NA & $\mathrm{NA}$ & NA & Cortical & & Robust cortical, long bone frag. Distorted by weathering \\
\hline 471 & 515 & s003 & 1000 & 1004 & 2 & Indeterminate & $\mathrm{NA}$ & 2.07 & $\mathrm{NA}$ & Frag & No & None & $\mathrm{NA}$ & NA & NA & Cortical & & Long bone frag. \\
\hline 471 & 515 & s004 & 1000 & 1004 & 2 & Indeterminate & $\mathrm{NA}$ & 1.29 & $\mathrm{NA}$ & Frag & No & None & $\mathrm{NA}$ & NA & $\mathrm{NA}$ & Cortical & & small, but robust cortical frag. Heavily weathered. \\
\hline 471 & 515 & s005 & 1000 & 1004 & 2 & Indeterminate & NA & 0.32 & $\mathrm{NA}$ & Frag & No & None & NA & $\mathrm{NA}$ & NA & Cortical & & small cortical frag. Heavily weathered. \\
\hline 471 & 515 & soo6 & 1000 & 1004 & 2 & Indeterminate & $\mathrm{NA}$ & 0.62 & $\mathrm{NA}$ & Frag & No & None & NA & NA & NA & Cortical & & small cortical frag. Heavily weathered. \\
\hline 471 & 515 & s007 & 1000 & 1004 & 2 & Indeterminate & NA & 0.68 & $\mathrm{NA}$ & Frag & № & None & $\mathrm{NA}$ & $\mathrm{NA}$ & $\mathrm{NA}$ & Cortical & & small cortical frag. Heavily weathered. \\
\hline 471 & 515 & soos & 1000 & 1004 & 2 & Indeterminate & NA & 0.22 & $\mathrm{NA}$ & Frag & No & None & $\mathrm{NA}$ & NA & NA & Cortical & & small cortical frag. \\
\hline 471 & 515 & soog & 1000 & 1004 & 2 & Mammal & NA & 0.95 & 2 & Frag & No & None & Vertebra & $\mathrm{NA}$ & $\mathrm{NA}$ & Cortical & & Articular surface of the transverse process only. \\
\hline 472 & 520 & so01 & 1000 & 1004 & 3 & Mammal & NA & 21.43 & 4 & Whole & No & None & 1st Phalanx & NA & NA & Whole & & $\begin{array}{l}\text { Probably a Bison phalanx. Surface is heavily weathered } \\
\text { and has some recent damage. }\end{array}$ \\
\hline 472 & 520 & soo2 & 1000 & 1004 & 3 & Mammal & $\mathrm{NA}$ & 19.95 & 4 & Frag & No & None & $\mathrm{NA}$ & NA & $\mathrm{NA}$ & Cortical & & $\begin{array}{l}\text { Large, flat cortical frag. Could be a scapula or a inominate } \\
\text { frag, not enough to make a determination. }\end{array}$ \\
\hline 472 & 520 & 5003 & 1000 & 1004 & 3 & Mammal & $\mathrm{NA}$ & 0.75 & NA & Frag & No & None & NA & NA & NA & Cortical & & Small, but thick cortical frag. Evidence of roasting. \\
\hline 472 & 520 & s004 & 1000 & 1004 & 3 & Mammal & $\mathrm{NA}$ & 2.11 & NA & Frag & № & None & $\mathrm{NA}$ & NA & $\mathrm{NA}$ & Cortical & & Small, but thick cortical frag. Evidence of roasting. \\
\hline 472 & 520 & 5005 & 1000 & 1004 & 3 & Mammal & NA & 8.58 & NA & Frag & No & None & NA & NA & NA & Cortical & & Long bone frag. Very heavily weathered. \\
\hline 472 & 520 & s006 & 1000 & 1004 & 3 & Mammal & $\mathrm{NA}$ & 13.23 & $\mathrm{NA}$ & Frag & No & None & $\mathrm{NA}$ & NA & $\mathrm{NA}$ & Cortical & & Long bone frag. Very heavily weathered. \\
\hline 472 & 520 & soor & 1000 & 1004 & 3 & Mammal & NA & 5.7 & $\mathrm{NA}$ & Frag & No & None & NA & NA & $\mathrm{NA}$ & Cortical & & Long bone frag. Very heavily weathered and splintered. \\
\hline 472 & 520 & soos & 1000 & 1004 & 3 & Mammal & NA & 1.92 & $\mathrm{NA}$ & Frag & No & None & NA & NA & NA & Cortical & & Long bone frag. Very heavily weathered.. \\
\hline 472 & 520 & soog & 1000 & 1004 & 3 & Mammal & NA & 4.18 & $\mathrm{NA}$ & Whole & No & None & Sesamoid & NA & $\mathrm{NA}$ & Whole & & Large sesamoid. Heavily weathered. \\
\hline 472 & 520 & s010 & 1000 & 1004 & 3 & Mammal & NA & 0.71 & $\mathrm{NA}$ & Frag & No & None & NA & $\mathrm{NA}$ & NA & Cortical & Oblique & Long bone frag \\
\hline 472 & 520 & so11 & 1000 & 1004 & 3 & Mammal & NA & 0.66 & $\mathrm{NA}$ & Frag & No & None & $\mathrm{NA}$ & NA & $\mathrm{NA}$ & Cortical & Oblique & Long bone frag \\
\hline 472 & 520 & s012 & 1000 & 1004 & 3 & Mammal & $\mathrm{NA}$ & 3.77 & $\mathrm{NA}$ & Frag & No & None & NA & NA & NA & Cortical & Spiral & Long bone frag \\
\hline 472 & 520 & s013 & 1000 & 1004 & 3 & Mammal & NA & 3.77 & $\mathrm{NA}$ & Frag & No & None & $\mathrm{NA}$ & NA & NA & Cortical & Multiple & Long bone frag \\
\hline 472 & 520 & s014 & 1000 & 1004 & 3 & Mammal & $\mathrm{NA}$ & 3.90 & $\mathrm{NA}$ & Frag & No & None & NA & $\mathrm{NA}$ & $\mathrm{NA}$ & Cortical & Spiral & Long bone frag \\
\hline 472 & 520 & s015 & 1000 & 1004 & 3 & Mammal & NA & 1.13 & $\mathrm{NA}$ & Frag & No & None & NA & NA & $\mathrm{NA}$ & Cortical & Right & \\
\hline 472 & 520 & s016 & 1000 & 1004 & 3 & Mammal & NA & 5.52 & $\mathrm{NA}$ & Frag & No & None & $\mathrm{NA}$ & $\mathrm{NA}$ & NA & Cortical & Spiral & $\begin{array}{l}\text { Large mammal long bone frag size } 2-4 \text { (not enough to } \\
\text { determine element. }\end{array}$ \\
\hline 472 & 520 & s017 & 1000 & 1004 & 3 & Mammal & NA & 1.19 & $\mathrm{NA}$ & Frag & No & None & $\mathrm{NA}$ & NA & NA & Cortical & Oblique & \\
\hline 472 & 520 & s018 & 1000 & 1004 & 3 & Indeterminate & NA & 0.99 & $\mathrm{NA}$ & Frag & No & None & NA & NA & NA & Cortical & $\mathrm{NA}$ & Mostly trabecular \\
\hline 472 & 520 & so19 & 1000 & 1004 & 3 & Indeterminate & NA & 0.43 & $\mathrm{NA}$ & Frag & No & None & NA & $\mathrm{NA}$ & $\mathrm{NA}$ & Cortical & Spiral & \\
\hline 472 & 520 & s020 & 1000 & 1004 & 3 & Indeterminate & NA & 0.71 & $\mathrm{NA}$ & Frag & No & None & NA & NA & NA & Cortical & Oblique & \\
\hline 472 & 520 & s021 & 1000 & 1004 & 3 & Indeterminate & NA & 0.25 & $\mathrm{NA}$ & Frag & No & None & NA & NA & NA & Cortical & $\mathrm{NA}$ & \\
\hline 472 & 520 & s022 & 1000 & 1004 & 3 & Indeterminate & NA & 0.31 & $\mathrm{NA}$ & Frag & No & None & NA & NA & NA & Cortical & Spiral & \\
\hline 472 & 520 & s023 & 1000 & 1004 & 3 & Indeterminate & NA & 0.72 & $\mathrm{NA}$ & Frag & No & None & NA & NA & NA & Cortical & Oblique & \\
\hline 472 & 520 & s024 & 1000 & 1004 & 3 & Mammal & NA & 2.53 & $\mathrm{NA}$ & Frag & No & None & NA & $\mathrm{NA}$ & $\mathrm{NA}$ & Cortical & Spiral & Long bone frag \\
\hline 472 & 520 & s025 & 1000 & 1004 & 3 & Mammal & $\mathrm{NA}$ & 2.13 & $\mathrm{NA}$ & Frag & No & None & NA & $\mathrm{NA}$ & $\mathrm{NA}$ & Cortical & Spiral & Long bone frag \\
\hline 472 & 520 & s026 & 1000 & 1004 & 3 & Indeterminate & NA & 0.75 & $\mathrm{NA}$ & Frag & No & None & NA & $\mathrm{NA}$ & $\mathrm{NA}$ & Cortical & Multiple & \\
\hline 472 & 520 & s027 & 1000 & 1004 & 3 & Indeterminate & $\mathrm{NA}$ & 1.28 & $\mathrm{NA}$ & Frag & No & None & $\mathrm{NA}$ & NA & $\mathrm{NA}$ & Cortical & $\mathrm{NA}$ & \\
\hline 472 & 520 & s028 & 1000 & 1004 & 3 & Indeterminate & NA & 0.72 & $\mathrm{NA}$ & Frag & № & None & NA & NA & NA & Cortical & Spiral & Long bone frag \\
\hline 472 & 520 & s029 & 1000 & 1004 & 3 & Indeterminate & NA & 0.36 & $\mathrm{NA}$ & Frag & No & None & NA & $\mathrm{NA}$ & $\mathrm{NA}$ & Cortical & Multiple & \\
\hline 472 & 520 & s030 & 1000 & 1004 & 3 & Mammal & NA & 1.21 & NA & Frag & No & None & NA & NA & $\mathrm{NA}$ & Cortical & Spiral & \\
\hline 472 & 520 & s031 & 1000 & 1004 & 3 & Mammal & NA & 0.42 & $\mathrm{NA}$ & Frag & No & None & NA & $\mathrm{NA}$ & $\mathrm{NA}$ & Cortical & Spiral & \\
\hline 472 & 520 & s032 & 1000 & 1004 & 3 & Indeterminate & $\mathrm{NA}$ & 0.40 & $\mathrm{NA}$ & Frag & No & None & NA & $\mathrm{NA}$ & NA & Cortical & Spiral & \\
\hline 472 & 520 & s033 & 1000 & 1004 & 3 & Mammal & $\mathrm{NA}$ & 0.68 & $\mathrm{NA}$ & Frag & No & None & $\mathrm{NA}$ & NA & NA & Cortical & Spiral & Long bone frag \\
\hline 472 & 520 & $\mathbf{s 0 3 4}$ & 1000 & 1004 & 3 & Indeterminate & NA & 0.92 & NA & Frag & No & None & NA & NA & NA & Cortical & $\mathrm{NA}$ & Mostly trabecular \\
\hline 472 & 520 & s035 & 1000 & 1004 & 3 & Indeterminate & NA & 0.70 & $\mathrm{NA}$ & Frag & No & None & NA & $\mathrm{NA}$ & NA & Cortical & Spiral & \\
\hline 472 & 520 & s036 & 1000 & 1004 & 3 & Mammal & $\mathrm{NA}$ & 6.84 & NA & Frag & No & None & NA & NA & NA & Cortical & Oblique & Metaphysis, not enough to identify element. \\
\hline 472 & 520 & s037 & 1000 & 1004 & 3 & Mammal & NA & 2.78 & $\mathrm{NA}$ & Frag & No & None & NA & $\mathrm{NA}$ & NA & Cortical & Oblique & Metaphysis, not enough to identify element. \\
\hline 472 & 520 & s038 & 1000 & 1004 & 3 & Indeterminate & NA & 0.58 & $\mathrm{NA}$ & Frag & No & None & NA & $\mathrm{NA}$ & $\mathrm{NA}$ & Cortical & Spiral & \\
\hline 472 & 520 & so39 & 1000 & 1004 & 3 & Indeterminate & $\mathrm{NA}$ & 0.21 & $\mathrm{NA}$ & Frag & № & None & NA & NA & NA & Cortical & Spiral & \\
\hline
\end{tabular}




\begin{tabular}{|c|c|c|c|c|c|c|c|c|c|c|c|c|c|c|c|c|c|c|}
\hline 客 & $\begin{array}{l}\text { 童 } \\
\text { 品 }\end{array}$ & 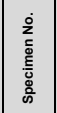 & 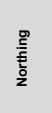 & $\begin{array}{l}\text { 袁 } \\
\text { W }\end{array}$ & $\bar{\Xi}$ & 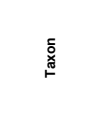 & 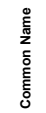 & $\begin{array}{l}\text { बू } \\
\text { 产 } \\
\text { ș }\end{array}$ & 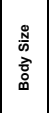 & 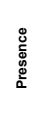 & 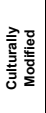 & 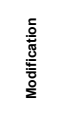 & $\begin{array}{l}\frac{\mathrm{o}}{\mathrm{o}} \\
\frac{\bar{\sigma}}{\mathrm{\omega}}\end{array}$ & $\stackrel{\circ}{\frac{8}{5}}$ & 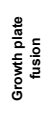 & 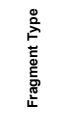 & 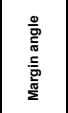 & $\frac{g}{6}$ \\
\hline 472 & 520 & s040 & 1000 & 1004 & 3 & Indeterminate & NA & 0.44 & $\mathrm{NA}$ & Frag & No & None & NA & NA & NA & Cortical & \begin{tabular}{|l|} 
Oblique \\
\end{tabular} & \\
\hline 472 & 520 & s041 & 1000 & 1004 & 3 & Indeterminate & NA & 0.39 & $\mathrm{NA}$ & Frag & No & None & $\mathrm{NA}$ & NA & NA & Cortical & $\mathrm{NA}$ & \\
\hline 472 & 520 & s042 & 1000 & 1004 & 3 & Mammal & NA & 1.48 & $\mathrm{NA}$ & Frag & No & None & $\mathrm{NA}$ & $\mathrm{NA}$ & NA & Cortical & Spiral & Long bone frag \\
\hline 472 & 520 & s043 & 1000 & 1004 & 3 & Indeterminate & NA & 0.61 & $\mathrm{NA}$ & Frag & No & None & NA & $\mathrm{NA}$ & NA & Cortical & \begin{tabular}{|l|l|l|l|l|} 
Mutiple \\
\end{tabular} & \\
\hline 472 & 520 & s044 & 1000 & 1004 & 3 & Mammal & $\mathrm{NA}$ & 1.15 & $\mathrm{NA}$ & Frag & No & None & NA & $\mathrm{NA}$ & NA & Cortical & Spiral & Long bone frag \\
\hline 472 & 520 & s045 & 1000 & 1004 & 3 & Mammal & NA & 0.43 & $\mathrm{NA}$ & Frag & No & None & NA & $\mathrm{NA}$ & NA & Cortical & Oblique & \\
\hline 472 & 520 & s046 & 1000 & 1004 & 3 & Mammal & NA & 0.98 & NA & Frag & No & None & NA & NA & NA & Cortical & Multiple & \\
\hline 472 & 520 & s047 & 1000 & 1004 & 3 & Mammal & NA & 1.07 & $\mathrm{NA}$ & Frag & № & None & $\mathrm{NA}$ & $\mathrm{NA}$ & $\mathrm{NA}$ & Cortical & Multiple & \\
\hline 472 & 520 & s048 & 1000 & 1004 & 3 & Mammal & NA & 0.97 & $\mathrm{NA}$ & Frag & No & None & NA & $\mathrm{NA}$ & NA & Cortical & $\mathrm{NA}$ & Mostly trabecular \\
\hline 472 & 520 & s049 & 1000 & 1004 & 3 & Indeterminate & NA & 0.19 & $\mathrm{NA}$ & Frag & No & None & $\mathrm{NA}$ & $\mathrm{NA}$ & NA & Cortical & Oblique & \\
\hline 472 & 520 & s050 & 1000 & 1004 & 3 & Indeterminate & $\mathrm{NA}$ & 0.81 & $\mathrm{NA}$ & Frag & No & None & NA & $\mathrm{NA}$ & $\mathrm{NA}$ & Cortical & Spiral & Long bone frag \\
\hline 472 & 520 & s051 & 1000 & 1004 & 3 & Indeterminate & NA & 0.60 & $\mathrm{NA}$ & Frag & No & None & NA & $\mathrm{NA}$ & NA & Cortical & Spiral & \\
\hline 472 & 520 & s053 & 1000 & 1004 & 3 & Indeterminate & $\mathrm{NA}$ & 0.61 & $\mathrm{NA}$ & Frag & No & None & $\mathrm{NA}$ & $\mathrm{NA}$ & $\mathrm{NA}$ & Cortical & $\mathrm{NA}$ & \\
\hline 472 & 520 & s054 & 1000 & 1004 & 3 & Indeterminate & NA & 0.23 & $\mathrm{NA}$ & Frag & No & None & NA & $\mathrm{NA}$ & NA & Cortical & $\mathrm{NA}$ & \\
\hline 472 & 520 & so55 & 1000 & 1004 & 3 & Indeterminate & NA & 0.47 & NA & Frag & No & None & NA & NA & NA & Cortical & Right & \\
\hline 472 & 520 & s056 & 1000 & 1004 & 3 & Indeterminate & $\mathrm{NA}$ & 0.56 & $\mathrm{NA}$ & Frag & No & None & $\mathrm{NA}$ & $\mathrm{NA}$ & NA & Cortical & Right & \\
\hline 472 & 520 & s057 & 1000 & 1004 & 3 & Indeterminate & $\mathrm{NA}$ & 0.31 & $\mathrm{NA}$ & Frag & No & None & $\mathrm{NA}$ & $\mathrm{NA}$ & $\mathrm{NA}$ & Cortical & Right & \\
\hline 472 & 520 & s058 & 1000 & 1004 & 3 & Indeterminate & $\mathrm{NA}$ & 0.35 & $\mathrm{NA}$ & Frag & No & None & NA & $\mathrm{NA}$ & NA & Cortical & $\mathrm{NA}$ & \\
\hline 472 & 520 & s059 & 1000 & 1004 & 3 & Indeterminate & $\mathrm{NA}$ & 0.77 & $\mathrm{NA}$ & Frag & No & None & $\mathrm{NA}$ & $\mathrm{NA}$ & $\mathrm{NA}$ & Cortical & Spiral & Long bone \\
\hline 472 & 520 & s060 & 1000 & 1004 & 3 & Indeterminate & NA & 0.12 & $\mathrm{NA}$ & Frag & No & None & NA & $\mathrm{NA}$ & $\mathrm{NA}$ & Cortical & Spiral & \\
\hline 472 & 520 & so61 & 1000 & 1004 & 3 & Indeterminate & $\mathrm{NA}$ & 0.32 & $\mathrm{NA}$ & Frag & No & None & $\mathrm{NA}$ & $\mathrm{NA}$ & NA & Cortical & \begin{tabular}{|l|} 
Multiple \\
\end{tabular} & \\
\hline 472 & 520 & s062 & 1000 & 1004 & 3 & Indeterminate & $\mathrm{NA}$ & 0.87 & $\mathrm{NA}$ & Frag & No & None & NA & $\mathrm{NA}$ & $\mathrm{NA}$ & Cortical & $\mathrm{NA}$ & \\
\hline 472 & 520 & $\begin{array}{ll}\text { s063 } \\
\end{array}$ & 1000 & 1004 & 3 & Indeterminate & $\mathrm{NA}$ & 0.60 & $\mathrm{NA}$ & Frag & No & None & $\mathrm{NA}$ & $\mathrm{NA}$ & NA & Cortical & \begin{tabular}{|l|} 
Oblique \\
\end{tabular} & \\
\hline 472 & 520 & \begin{tabular}{l|l|} 
so64 \\
\end{tabular} & 1000 & 1004 & 3 & Indeterminate & $\mathrm{NA}$ & 0.12 & $\mathrm{NA}$ & Frag & No & None & $\mathrm{NA}$ & NA & NA & Cortical & Spiral & \\
\hline 472 & 520 & s065 & 1000 & 1004 & 3 & Indeterminate & NA & \begin{tabular}{l|l}
0.16 \\
\end{tabular} & $\mathrm{NA}$ & Frag & No & None & $\mathrm{NA}$ & $\mathrm{NA}$ & $\mathrm{NA}$ & Cortical & Spiral & \\
\hline 472 & 520 & s066 & 1000 & 1004 & 3 & Indeterminate & NA & 0.15 & $\mathrm{NA}$ & Frag & No & None & NA & $\mathrm{NA}$ & NA & Cortical & Multiple & \\
\hline 472 & 520 & s067 & 1000 & 1004 & 3 & Indeterminate & $\mathrm{NA}$ & 0.29 & $\mathrm{NA}$ & Frag & No & None & NA & $\mathrm{NA}$ & NA & Cortical & $\begin{array}{l}\text { Spiral } \\
\end{array}$ & \\
\hline 472 & 520 & S068 & 1000 & 1004 & 3 & Mammal & $\mathrm{NA}$ & 0.61 & $\mathrm{NA}$ & Frag & No & None & $\mathrm{NA}$ & NA & NA & Cortical & Spiral & \\
\hline 472 & 520 & so69 & 1000 & 1004 & 3 & Indeterminate & $\mathrm{NA}$ & 0.18 & $\mathrm{NA}$ & Frag & No & None & $\mathrm{NA}$ & NA & NA & Cortical & Oblique & \\
\hline 472 & 520 & s070 & 1000 & 1004 & 3 & Indeterminate & NA & 0.18 & $\mathrm{NA}$ & Frag & No & None & NA & $\mathrm{NA}$ & $\mathrm{NA}$ & Cortical & Spiral & \\
\hline 472 & 520 & s071 & 1000 & 1004 & 3 & Indeterminate & NA & 0.16 & $\mathrm{NA}$ & Frag & No & None & NA & NA & NA & Cortical & Spiral & \\
\hline 472 & 520 & s072 & 1000 & 1004 & 3 & Indeterminate & $\mathrm{NA}$ & 0.23 & $\mathrm{NA}$ & Frag & No & None & $\mathrm{NA}$ & $\mathrm{NA}$ & NA & Cortical & Oblique & \\
\hline 472 & 520 & s073 & 1000 & 1004 & 3 & Indeterminate & $\mathrm{NA}$ & 0.29 & $\mathrm{NA}$ & Frag & No & None & $\mathrm{NA}$ & $\mathrm{NA}$ & NA & Cortical & \begin{tabular}{|l|} 
Spiral \\
\end{tabular} & \\
\hline 472 & 520 & s074 & 1000 & 1004 & 3 & Indeterminate & $\mathrm{NA}$ & 0.25 & $\mathrm{NA}$ & Frag & No & None & NA & $\mathrm{NA}$ & NA & Cortical & Spiral & \\
\hline 472 & 520 & s075 & 1000 & 1004 & 3 & Indeterminate & NA & 0.16 & $\mathrm{NA}$ & Frag & No & None & NA & $\mathrm{NA}$ & NA & Cortical & Fresh & \\
\hline 472 & 520 & s076 & 1000 & 1004 & 3 & Indeterminate & NA & 0.24 & $\mathrm{NA}$ & Frag & No & None & NA & NA & NA & Cortical & NA & \\
\hline 472 & 520 & s077 & 1000 & 1004 & 3 & Mammal & $\mathrm{NA}$ & 0.44 & $\mathrm{NA}$ & Frag & No & None & NA & $\mathrm{NA}$ & NA & Cortical & Right & \\
\hline 472 & 520 & s078 & 1000 & 1004 & 3 & Mammal & $\mathrm{NA}$ & 0.53 & $\mathrm{NA}$ & Frag & No & None & NA & NA & NA & Cortical & $\mathrm{NA}$ & \\
\hline 472 & 520 & s079 & 1000 & 1004 & 3 & Indeterminate & $\mathrm{NA}$ & 0.36 & $\mathrm{NA}$ & Frag & No & None & $\mathrm{NA}$ & $\mathrm{NA}$ & $\mathrm{NA}$ & Cortical & \begin{tabular}{|l|} 
Oblique \\
\end{tabular} & \\
\hline 472 & 520 & s080 & 1000 & 1004 & 3 & Indeterminate & $\mathrm{NA}$ & 0.11 & $\mathrm{NA}$ & Frag & No & None & $\mathrm{NA}$ & NA & NA & Cortical & $\mathrm{NA}$ & \\
\hline 472 & 520 & so81 & 1000 & 1004 & 3 & Indeterminate & NA & 0.23 & $\mathrm{NA}$ & Frag & No & None & NA & $\mathrm{NA}$ & NA & Cortical & \begin{tabular}{|l|l|l|l|l|} 
Mulple & \\
\end{tabular} & \\
\hline 472 & 520 & s082 & 1000 & 1004 & 3 & Indeterminate & $\mathrm{NA}$ & 0.27 & NA & Frag & No & None & NA & $\mathrm{NA}$ & NA & Cortical & Right & \\
\hline 472 & 520 & s083 & 1000 & 1004 & 3 & Mammal & NA & \begin{tabular}{l|l|}
0.24 \\
\end{tabular} & $\mathrm{NA}$ & Frag & No & None & NA & NA & NA & Cortical & $\mathrm{NA}$ & \\
\hline 472 & 520 & s084 & 1000 & 1004 & 3 & Mammal & $\mathrm{NA}$ & 0.73 & $\mathrm{NA}$ & Frag & No & None & $\mathrm{NA}$ & $\mathrm{NA}$ & $\mathrm{NA}$ & Cortical & $\mathrm{NA}$ & \\
\hline 472 & 520 & S085 & 1000 & 1004 & 3 & Mammal & NA & 0.46 & $\mathrm{NA}$ & Frag & No & None & $\mathrm{NA}$ & NA & NA & Cortical & NA & \\
\hline 472 & 520 & s086 & 1000 & 1004 & 3 & Indeterminate & NA & 0.55 & $\mathrm{NA}$ & Frag & No & None & NA & $\mathrm{NA}$ & $\mathrm{NA}$ & Cortical & $\mathrm{NA}$ & \\
\hline 472 & 520 & s087 & 1000 & 1004 & 3 & Indeterminate & $\mathrm{NA}$ & 0.12 & $\mathrm{NA}$ & Frag & No & None & NA & $\mathrm{NA}$ & NA & Cortical & Spiral & \\
\hline 472 & 520 & s088 & 1000 & 1004 & 3 & Mammal & NA & 0.20 & $\mathrm{NA}$ & Frag & No & None & NA & $\mathrm{NA}$ & NA & Cortical & Spiral & \\
\hline 472 & 520 & s089 & 1000 & 1004 & 3 & Indeterminate & $\mathrm{NA}$ & 0.12 & NA & Frag & No & None & $\mathrm{NA}$ & $\mathrm{NA}$ & $\mathrm{NA}$ & Cortical & $\mathrm{NA}$ & \\
\hline 472 & 520 & s090 & 1000 & 1004 & 3 & Indeterminate & $\mathrm{NA}$ & 0.17 & $\mathrm{NA}$ & Frag & No & None & $\mathrm{NA}$ & NA & NA & Cortical & Oblique & \\
\hline 472 & 520 & s091 & 1000 & 1004 & 3 & Indeterminate & $\mathrm{NA}$ & 0.12 & NA & Frag & No & None & NA & NA & NA & Cortical & $\mathrm{NA}$ & \\
\hline 472 & 520 & s092 & 1000 & 1004 & 3 & Indeterminate & NA & 0.57 & NA & Frag & No & None & NA & NA & NA & Cortical & Multiple & \\
\hline 472 & 520 & s093 & 1000 & 1004 & 3 & Indeterminate & NA & 0.14 & NA & Frag & No & None & NA & NA & NA & Cortical & $\mathrm{NA}$ & \\
\hline 472 & 520 & s094 & 1000 & 1004 & 3 & Indeterminate & NA & 0.51 & $\mathrm{NA}$ & Frag & No & None & $\mathrm{NA}$ & $\mathrm{NA}$ & $\mathrm{NA}$ & Cortical & $\mathrm{NA}$ & \\
\hline 472 & 520 & s095 & 1000 & 1004 & 3 & Mammal & NA & 0.78 & $\mathrm{NA}$ & Frag & No & None & NA & NA & NA & Cortical & NA & small, but robust cortical frag. \\
\hline 472 & 520 & s096 & 1000 & 1004 & 3 & Indeterminate & $\mathrm{NA}$ & 0.09 & $\mathrm{NA}$ & Frag & No & None & NA & NA & $\mathrm{NA}$ & Cortical & Spiral & \\
\hline
\end{tabular}




\begin{tabular}{|c|c|c|c|c|c|c|c|c|c|c|c|c|c|c|c|c|c|c|}
\hline ْ & 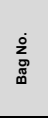 & 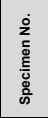 & 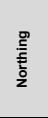 & 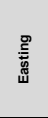 & ळ్ & 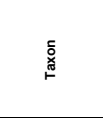 & 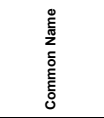 & 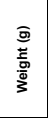 & 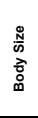 & 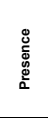 & 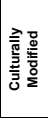 & 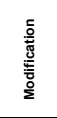 & 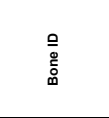 & $\frac{0}{5}$ & 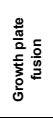 & 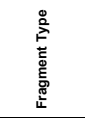 & 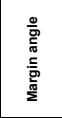 & 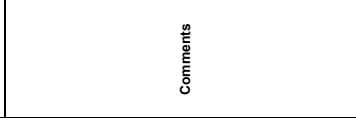 \\
\hline 472 & 520 & S097 & 1000 & 1004 & 3 & Indeterminate & NA & 0.51 & NA & Frag & No & None & NA & NA & NA & Cortical & $\mathrm{NA}$ & \\
\hline 472 & 520 & S098 & 1000 & 1004 & 3 & Indeterminate & NA & 0.16 & NA & Frag & No & None & NA & NA & NA & Cortical & NA & \\
\hline 472 & 520 & S099 & 1000 & 1004 & 3 & Indeterminate & NA & 0.09 & NA & Frag & No & None & NA & NA & NA & Cortical & $\mathrm{NA}$ & \\
\hline 472 & 520 & S100 & 1000 & 1004 & 3 & Indeterminate & NA & 0.07 & NA & Frag & No & None & NA & $\mathrm{NA}$ & NA & Cortical & NA & \\
\hline 472 & 520 & S101 & 1000 & 1004 & 3 & Indeterminate & NA & 0.23 & $\mathrm{NA}$ & Frag & No & None & NA & NA & NA & Cortical & NA & \\
\hline 472 & 520 & S102 & 1000 & 1004 & 3 & Indeterminate & NA & 0.18 & NA & Frag & No & None & NA & NA & NA & Cortical & Spiral & \\
\hline 472 & 520 & S103 & 1000 & 1004 & 3 & Indeterminate & NA & 0.23 & NA & Frag & No & None & $\mathrm{NA}$ & NA & NA & Cortical & $\mathrm{NA}$ & \\
\hline 472 & 520 & S104 & 1000 & 1004 & 3 & Indeterminate & NA & 0.34 & NA & Frag & No & None & NA & NA & NA & Cortical & NA & \\
\hline 472 & 520 & S105 & 1000 & 1004 & 3 & Indeterminate & NA & 0.51 & NA & Frag & No & None & $\mathrm{NA}$ & $\mathrm{NA}$ & NA & Cortical & Spiral & \\
\hline 472 & 520 & S106 & 1000 & 1004 & 3 & Indeterminate & NA & 0.11 & NA & Frag & No & None & NA & NA & NA & Cortical & Multiple & \\
\hline 472 & 520 & S107 & 1000 & 1004 & 3 & Indeterminate & NA & 0.06 & NA & Frag & No & None & NA & NA & NA & Cortical & Fresh & \\
\hline 472 & 520 & S108 & 1000 & 1004 & 3 & Indeterminate & NA & 0.11 & NA & Frag & No & None & NA & NA & NA & Cortical & Fresh & \\
\hline 472 & 520 & s109 & 1000 & 1004 & 3 & Indeterminate & NA & 0.07 & NA & Frag & No & None & NA & NA & NA & Cortical & Multiple & \\
\hline 472 & 520 & s110 & 1000 & 1004 & 3 & Indeterminate & NA & 0.17 & NA & Frag & No & None & NA & NA & NA & Cortical & NA & \\
\hline 472 & 520 & s111 & 1000 & 1004 & 3 & Indeterminate & NA & 0.07 & NA & Frag & No & None & NA & NA & NA & Cortical & NA & \\
\hline 472 & 520 & S112 & 1000 & 1004 & 3 & Indeterminate & NA & 0.12 & $\mathrm{NA}$ & Frag & No & None & NA & NA & NA & Trabecular & $\mathrm{NA}$ & \\
\hline 472 & 520 & s113 & 1000 & 1004 & 3 & Indeterminate & NA & 0.16 & NA & Frag & No & None & NA & NA & NA & Cortical & Oblique & \\
\hline 472 & 520 & S114 & 1000 & 1004 & 3 & Indeterminate & NA & 0.09 & NA & Frag & No & None & NA & NA & NA & Cortical & Right & \\
\hline 472 & 520 & S115 & 1000 & 1004 & 3 & Indeterminate & NA & 0.03 & NA & Frag & No & None & NA & NA & NA & Trabecular & NA & \\
\hline 472 & 520 & S116 & 1000 & 1004 & 3 & Indeterminate & NA & 0.10 & $\mathrm{NA}$ & Frag & No & None & $\mathrm{NA}$ & NA & $\mathrm{NA}$ & Cortical & Spiral & \\
\hline 472 & 520 & S117 & 1000 & 1004 & 3 & Indeterminate & NA & 0.06 & NA & Frag & No & None & $\mathrm{NA}$ & $\mathrm{NA}$ & NA & Cortical & Multiple & \\
\hline 472 & 520 & S118 & 1000 & 1004 & 3 & Indeterminate & NA & 0.07 & $\mathrm{NA}$ & Frag & No & None & NA & NA & NA & Cortical & Multiple & \\
\hline 473 & 521 & S001 & 1000 & 1004 & 3 & Mammal & NA & 12.47 & 4 & Frag & No & None & Metapodial & NA & NA & Epiphysis & & Large, distal metapodial epiphysis frag. Cow size. \\
\hline 474 & 522 & s001 & 1000 & 1004 & 3 & Mammal & NA & 36.4 & 4 & Frag & No & None & Carpal/Tarsal? & NA & NA & Cortical & & Very large carpal or tarsal bone. \\
\hline 474 & 522 & s002 & 1000 & 1004 & 3 & Mammal & NA & 10.39 & 4 & Frag & No & None & $\mathrm{NA}$ & $\mathrm{NA}$ & $\mathrm{NA}$ & Cortical & & $\begin{array}{l}\begin{array}{l}\text { Articular surface, not enough present to determine what } \\
\text { element. }\end{array} \\
\end{array}$ \\
\hline 474 & 522 & s003 & 1000 & 1004 & 3 & Mammal & NA & 5.55 & $\mathrm{NA}$ & Frag & No & None & NA & $\mathrm{NA}$ & $\mathrm{NA}$ & Cortical & & Not enough present to determine what element. \\
\hline 474 & 522 & S004 & 1000 & 1004 & 3 & Indeterminate & NA & 0.13 & NA & Frag & No & None & NA & NA & NA & Trabecular & & Trabecular bone frag, unable to identify. \\
\hline 474 & 522 & S005 & 1000 & 1004 & 3 & Indeterminate & NA & 0.35 & NA & Frag & No & None & NA & NA & NA & Trabecular & & Trabecular bone frag, unable to identify. \\
\hline 474 & 522 & so06 & 1000 & 1004 & 3 & Indeterminate & NA & 0.18 & NA & Frag & No & None & NA & NA & NA & Trabecular & & Trabecular bone frag, unable to identify. \\
\hline 474 & 522 & S007 & 1000 & 1004 & 3 & Indeterminate & NA & 0.01 & NA & Frag & No & None & $\mathrm{NA}$ & NA & NA & Trabecular & & Trabecular bone frag, unable to identify. \\
\hline 474 & 522 & s008 & 1000 & 1004 & 3 & Indeterminate & NA & 0.13 & NA & Frag & No & None & NA & NA & NA & Trabecular & & Trabecular bone frag, unable to identify. \\
\hline 474 & 522 & soog & 1000 & 1004 & 3 & Indeterminate & NA & 0.01 & NA & Frag & No & None & NA & NA & NA & Trabecular & & Trabecular bone frag, unable to identify. \\
\hline 474 & 522 & s010 & 1000 & 1004 & 3 & Indeterminate & NA & 1.82 & NA & Frag & No & None & NA & NA & NA & Cortical & & Distorted by weathering. \\
\hline 474 & 522 & s011 & 1000 & 1004 & 3 & Indeterminate & NA & 1.23 & NA & Frag & No & None & NA & NA & NA & Cortical & & Distorted by weathering. \\
\hline 474 & 522 & S012 & 1000 & 1004 & 3 & Indeterminate & NA & 0.01 & NA & Frag & No & None & NA & NA & NA & Trabecular & & Trabecular bone frag, unable to identify. \\
\hline 474 & 522 & s013 & 1000 & 1004 & 3 & Indeterminate & NA & 1.6 & NA & Frag & No & None & NA & NA & NA & Trabecular & & Trabecular bone frag, unable to identify. \\
\hline 474 & 522 & S014 & 1000 & 1004 & 3 & Indeterminate & NA & 0.25 & NA & Frag & No & None & NA & NA & NA & Trabecular & & Trabecular bone frag, unable to identify. \\
\hline 474 & 522 & s015 & 1000 & 1004 & 3 & Indeterminate & NA & 0.42 & NA & Frag & No & None & NA & NA & NA & Cortical & & Not enough to make a determination \\
\hline 474 & 522 & S016 & 1000 & 1004 & 3 & Indeterminate & NA & 1.04 & NA & Frag & No & None & NA & NA & NA & Cortical & & Possible vert frag, Not enough to make a determination \\
\hline 474 & 522 & s017 & 1000 & 1004 & 3 & Indeterminate & NA & 0.01 & NA & Frag & No & None & NA & NA & NA & Trabecular & & Trabecular bone frag, unable to identify. \\
\hline 475 & 534 & s001 & 1000 & 1004 & 4 & Indeterminate & NA & 0.56 & $\mathrm{NA}$ & Frag & No & None & NA & NA & NA & Cortical & Right & Evidence of roasting \\
\hline 475 & 534 & s002 & 1000 & 1004 & 4 & Indeterminate & NA & 0.2 & NA & Frag & No & None & NA & NA & NA & Cortical & Right & Evidence of roasting \\
\hline 475 & 534 & S003 & 1000 & 1004 & 4 & Indeterminate & NA & 0.3 & NA & Frag & No & None & NA & NA & NA & Cortical & Right & Evidence of roasting \\
\hline 476 & 538 & so01 & 1000 & 1004 & 5 & Indeterminate & NA & 0.51 & NA & Frag & No & None & NA & NA & NA & Cortical & & Weathered piece of cortical bone. \\
\hline 478 & 671 & s001 & 1000 & 1004 & 7 & Tortoise/Turtle & Tortoise/Turtle & 0.32 & 1 & Frag & No & None & Plastron & NA & NA & NA & & Small plastron fragment. \\
\hline 480 & 674 & so01 & 1000 & 1004 & 9 & Indeterminate & NA & 0.12 & NA & Frag & No & None & NA & NA & NA & Cortical & & Small, burned cortical frag. \\
\hline 480 & 674 & s002 & 1000 & 1004 & 9 & Indeterminate & NA & 0.35 & NA & Frag & No & None & NA & NA & NA & Cortical & & Distorted by weathering. Refits with $\mathrm{S} 003$ \\
\hline 480 & 674 & s003 & 1000 & 1004 & 9 & Indeterminate & NA & 0.16 & NA & Frag & No & None & NA & NA & NA & Cortical & & Distorted by weathering. Refits with S002 \\
\hline 480 & 674 & s004 & 1000 & 1004 & 9 & Indeterminate & NA & 0.39 & NA & Frag & No & None & NA & NA & NA & Cortical & & Distorted by weathering. \\
\hline 480 & 674 & s005 & 1000 & 1004 & 9 & Indeterminate & NA & 0.76 & NA & Frag & No & None & NA & NA & NA & Cortical & & Too small to make a determination. Burned \\
\hline 480 & 674 & so06 & 1000 & 1004 & 9 & Indeterminate & NA & 0.15 & NA & Frag & No & None & NA & NA & NA & Cortical & & Too small to make a determination. Burned \\
\hline 480 & 674 & s007 & 1000 & 1004 & 9 & Indeterminate & NA & 0.4 & NA & Frag & No & None & NA & NA & NA & Cortical & & Too small to make a determination. Burned \\
\hline 480 & 674 & s008 & 1000 & 1004 & 9 & Indeterminate & NA & 1.09 & NA & Frag & No & None & $\mathrm{NA}$ & NA & NA & Cortical & & Distorted by weathering. Refits with $\mathrm{S} 009$ \\
\hline 480 & 674 & so09 & 1000 & 1004 & 9 & Indeterminate & NA & 0.56 & NA & Frag & No & None & NA & NA & NA & Cortical & & Distorted by weathering. Refits with $\mathrm{S} 008$ \\
\hline 480 & 674 & s010 & 1000 & 1004 & 9 & Indeterminate & NA & 0.6 & NA & Frag & No & None & $\mathrm{NA}$ & NA & NA & Cortical & & Distorted by weathering. \\
\hline 481 & 675 & s001 & 1000 & 1004 & 10 & Indeterminate & NA & 3.61 & NA & Frag & No & None & Humerus & Left & NA & Cortical & & $\begin{array}{l}\text { Incomplete proximal humerus epiphysis. Weathering has } \\
\text { obliterated the surface, and distorted the shape. Cortical } \\
\text { bone is very gracile, may represent a large bird. }\end{array}$ \\
\hline
\end{tabular}




\begin{tabular}{|c|c|c|c|c|c|c|c|c|c|c|c|c|c|c|c|c|c|c|}
\hline 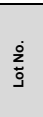 & 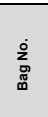 & 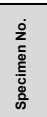 & 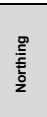 & 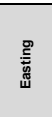 & 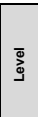 & 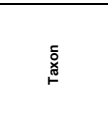 & 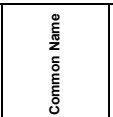 & 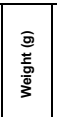 & $\begin{array}{l}\frac{\AA}{10} \\
\text { ते̀ } \\
\text { ते }\end{array}$ & 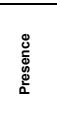 & 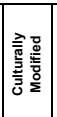 & 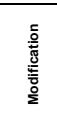 & 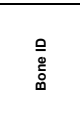 & $\frac{8}{\omega}$ & 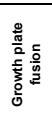 & 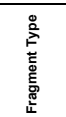 & 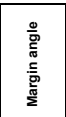 & 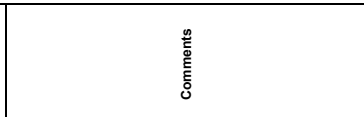 \\
\hline 481 & 675 & so02 & 1000 & 1004 & 10 & Indeterminate & NA & 0.66 & NA & Frag & No & None & NA & NA & NA & Cortical & & Distorted by weathering. \\
\hline 481 & 675 & so03 & 1000 & 1004 & 10 & Indeterminate & NA & 0.33 & NA & Frag & No & None & NA & NA & NA & Cortical & & $\begin{array}{l}\text { Distorted by weathering. Possible epiphyseal growth } \\
\text { plate. }\end{array}$ \\
\hline 481 & 675 & so04 & 1000 & 1004 & 10 & Indeterminate & NA & 0.01 & $\mathrm{NA}$ & Frag & No & None & NA & NA & NA & Cortical & & Too small to make a determination. \\
\hline 484 & 678 & soo1 & 1000 & 1004 & 13 & Mammal & NA & 1.17 & NA & Frag & No & None & NA & NA & NA & Cortical & & Long bone frag. Heavily weathered. \\
\hline 485 & 679 & s001 & 1000 & 1004 & 14 & Mammal & NA & 1.94 & NA & Frag & No & None & NA & NA & NA & Cortical & & Long bone frag with fluvial wear. Refits with S002 \\
\hline 485 & 679 & so02 & 1000 & 1004 & 14 & Mammal & NA & 1.41 & NA & Frag & No & None & NA & NA & NA & Cortical & & Long bone frag with fluvial wear. Refits with S001 \\
\hline 485 & 679 & so03 & 1000 & 1004 & 14 & Indeterminate & NA & 0.05 & NA & Frag & No & None & NA & NA & NA & Cortical & & A laminar piece of cortical bone. \\
\hline 490 & 324 & so01 & 1000 & 1009 & 5 & Indeterminate & NA & 0.14 & NA & Frag & No & None & $\mathrm{NA}$ & NA & NA & Cortical & & Heavily weathered, small frag. \\
\hline 490 & 324 & soo2 & 1000 & 1009 & 5 & Indeterminate & NA & 0.17 & NA & Frag & No & None & $\mathrm{NA}$ & NA & NA & Cortical & & Heavily weathered, small frag. \\
\hline 492 & 332 & soo1 & 1000 & 1009 & 7 & Mammal & NA & 1.22 & NA & Frag & No & None & NA & NA & NA & Cortical & & No landmarks. One tooth pit. \\
\hline 495 & 368 & s001 & 1000 & 1009 & 10 & Indeterminate & NA & 0.16 & NA & Frag & No & None & NA & NA & NA & Cortical & Oblique & Probably turtle shell, but not definitive. \\
\hline 501 & 328 & so01 & 1000 & 1010 & 6 & Mammal & NA & 15.84 & 3 or 4 & Frag & No & None & NA & NA & NA & Cortical & & $\begin{array}{l}\text { Large cortical frag. Surface has been obliterated due to } \\
\text { weathering. The thickness of the cortex suggests a large } \\
\text { mammal size } 3 \text { to } 4 \text { (probably } 4 \text { ). }\end{array}$ \\
\hline 501 & 328 & s002 & 1000 & 1010 & 6 & Mammal & NA & 6.08 & NA & Frag & No & None & NA & NA & NA & Cortical & & $\begin{array}{l}\text { Large cortical frag. Surface has been obliterated due to } \\
\text { weathering. }\end{array}$ \\
\hline 501 & 328 & so03 & 1000 & 1010 & 6 & Mammal & NA & 3.06 & NA & Frag & No & None & NA & NA & NA & Cortical & & $\begin{array}{l}\text { Large cortical frag. Surface has been obliterated due to } \\
\text { weathering. }\end{array}$ \\
\hline 501 & 328 & so04 & 1000 & 1010 & 6 & Mammal & NA & 1.32 & NA & Frag & No & None & NA & NA & NA & Cortical & & $\begin{array}{l}\text { Large cortical frag. Surface has been obliterated due to } \\
\text { weathering. }\end{array}$ \\
\hline 501 & 328 & so05 & 1000 & 1010 & 6 & Mammal & NA & 0.73 & NA & Frag & No & None & NA & NA & NA & Cortical & & $\begin{array}{l}\text { Cortical frag. Surface has been obliterated due to } \\
\text { weathering. }\end{array}$ \\
\hline 502 & 331 & so01 & 1000 & 1010 & 7 & Indeterminate & NA & 0.31 & NA & Frag & No & None & NA & NA & NA & Cortical & & $\begin{array}{l}\text { Heavily burned, not completely calcined. But, distorted by } \\
\text { heat and weathering. }\end{array}$ \\
\hline 502 & 331 & so02 & 1000 & 1010 & 7 & Indeterminate & NA & 0.21 & NA & Frag & No & None & NA & NA & $\mathrm{NA}$ & Cortical & & Hardly any surface on this frag. \\
\hline 503 & 334 & soo1 & 1000 & 1010 & 8 & Mammal & NA & 0.38 & 1 & Frag & No & None & Femur & Left & NA & Cortical & & Incomplete proximal femoral epiphysis. \\
\hline 504 & 336 & so01 & 1000 & 1010 & 9 & Tortoise/Turtle & Tortoise/Turtle & 0.15 & 1 & Frag & No & None & Carapace & NA & NA & NA & NA & \\
\hline 504 & 336 & soo2 & 1000 & 1010 & 9 & Indeterminate & NA & 0.25 & NA & Frag & No & None & NA & NA & NA & Cortical & Fresh & \\
\hline 504 & 336 & s003 & 1000 & 1010 & 9 & Indeterminate & NA & 0.46 & $\mathrm{NA}$ & Frag & No & None & $\mathrm{NA}$ & NA & $\mathrm{NA}$ & Cortical & Multiple & \\
\hline 504 & 336 & s004 & 1000 & 1010 & 9 & Indeterminate & NA & 0.32 & NA & Frag & No & None & NA & NA & $\mathrm{NA}$ & Cortical & Multiple & \\
\hline 504 & 336 & S005 & 1000 & 1010 & 9 & Indeterminate & NA & 0.10 & NA & Frag & No & None & $\mathrm{NA}$ & NA & $\mathrm{NA}$ & Cortical & Fresh & \\
\hline 504 & 336 & S006 & 1000 & 1010 & 9 & Indeterminate & NA & 0.16 & $\mathrm{NA}$ & Frag & No & None & $\mathrm{NA}$ & NA & $\mathrm{NA}$ & Cortical & Right & \\
\hline 504 & 336 & s007 & 1000 & 1010 & 9 & Indeterminate & $\mathrm{NA}$ & 0.13 & NA & Frag & No & None & $\mathrm{NA}$ & $\mathrm{NA}$ & $\mathrm{NA}$ & Cortical & Fresh & \\
\hline 504 & 336 & so08 & 1000 & 1010 & 9 & Indeterminate & NA & 0.31 & NA & Frag & No & None & $\mathrm{NA}$ & NA & $\mathrm{NA}$ & Cortical & Right & \\
\hline 504 & 336 & s009 & 1000 & 1010 & 9 & Indeterminate & $\mathrm{NA}$ & 0.04 & $\mathrm{NA}$ & Frag & No & None & $\mathrm{NA}$ & NA & NA & Cortical & Fresh & \\
\hline 504 & 336 & s010 & 1000 & 1010 & 9 & Indeterminate & NA & 0.61 & $\mathrm{NA}$ & Frag & No & None & NA & $\mathrm{NA}$ & $\mathrm{NA}$ & Cortical & Fresh & \\
\hline 505 & 342 & so01 & 1000 & 1010 & 10 & Indeterminate & $\mathrm{NA}$ & 0.23 & NA & Frag & No & None & $\mathrm{NA}$ & NA & $\mathrm{NA}$ & Cortical & & $\begin{array}{l}\text { Cortical frag. Surface has been obliterated due to } \\
\text { weathering. }\end{array}$ \\
\hline 505 & 342 & s002 & 1000 & 1010 & 10 & Indeterminate & NA & 0.16 & NA & Frag & No & None & NA & NA & $\mathrm{NA}$ & Cortical & & $\begin{array}{l}\text { Cortical frag. Surface has been obliterated due to } \\
\text { weathering. }\end{array}$ \\
\hline 505 & 342 & $\mathbf{s} 003$ & 1000 & 1010 & 10 & Indeterminate & NA & 0.08 & NA & Frag & No & None & $\mathrm{NA}$ & NA & NA & Cortical & & $\begin{array}{l}\text { Cortical frag. Too small to make a determination. Appears } \\
\text { to be slightly burned (roasted). }\end{array}$ \\
\hline 506 & 339 & s001 & 1000 & 1010 & 11 & Tortoise/Turtle & Tortoise/Turtle & 0.67 & 1 & Frag & No & None & carapace & NA & NA & Cortical & NA & \\
\hline 506 & 339 & so02 & 1000 & 1010 & 11 & Tortoise/Turtle & Tortoise/Turtle & 0.62 & 1 & Frag & No & None & carapace & NA & NA & Cortical & NA & \\
\hline 506 & 339 & so03 & 1000 & 1010 & 11 & Tortoise/Turtle & Tortoise/Turtle & 0.29 & 1 & Frag & No & None & carapace & NA & NA & Cortical & NA & \\
\hline 506 & 339 & S004 & 1000 & 1010 & 11 & Tortoise/Turtle & Tortoise/Turtle & 0.27 & 1 & Frag & No & None & carapace & NA & NA & Cortical & NA & \\
\hline 506 & 339 & $\mathbf{s 0 0 5}$ & 1000 & 1010 & 11 & Tortoise/Turtle & Tortoise/Turtle & 0.24 & 1 & Frag & No & None & carapace & NA & $\mathrm{NA}$ & Cortical & NA & \\
\hline 506 & 339 & s006 & 1000 & 1010 & 11 & Tortoise/Turtle & Tortoise/Turtle & \begin{tabular}{|l|}
0.16 \\
\end{tabular} & 1 & Frag & No & None & carapace & NA & $\mathrm{NA}$ & Cortical & $\mathrm{NA}$ & \\
\hline 506 & 339 & S007 & 1000 & 1010 & 11 & Tortoise/Turtle & Tortoise/Turtle & 0.29 & 1 & Frag & No & None & Plastron & NA & NA & Cortical & NA & \\
\hline 506 & 339 & S008 & 1000 & 1010 & 11 & Tortoise/Turtle & Tortoise/Turtle & 0.33 & 1 & Frag & No & None & Plastron & NA & $\mathrm{NA}$ & Cortical & NA & \\
\hline 506 & 339 & soog & 1000 & 1010 & 11 & Tortoise/Turtle & Tortoise/Turtle & \begin{tabular}{|l|}
0.13 \\
\end{tabular} & 1 & Frag & No & None & Plastron & NA & $\mathrm{NA}$ & Cortical & $\mathrm{NA}$ & \\
\hline 506 & 339 & s010 & 1000 & 1010 & 11 & Tortoise/Turtle & Tortoise/Turtle & 0.23 & 1 & Frag & No & None & Plastron & NA & NA & Cortical & NA & \\
\hline 506 & 339 & S011 & 1000 & 1010 & 11 & Tortoise/Turtle & Tortoise/Turtle & 0.24 & 1 & Frag & No & None & Plastron & NA & $\mathrm{NA}$ & Cortical & NA & \\
\hline 506 & 339 & S012 & 1000 & 1010 & 11 & Tortoise/Turtle & Tortoise/Turtle & 0.18 & 1 & Frag & No & None & Plastron & NA & NA & Cortical & $\mathrm{NA}$ & \\
\hline 506 & 339 & S013 & 1000 & 1010 & 11 & Indeterminate & NA & 0.17 & $\mathrm{NA}$ & Frag & No & None & NA & NA & $\mathrm{NA}$ & Cortical & Spiral & \\
\hline 506 & 339 & S014 & 1000 & 1010 & 11 & Tortoise/Turtle & Tortoise/Turtle & 0.09 & 1 & Frag & No & None & Plastron & $\mathrm{NA}$ & NA & Cortical & NA & \\
\hline 506 & 339 & s015 & 1000 & 1010 & 11 & Indeterminate & $\mathrm{NA}$ & 0.37 & NA & Frag & No & None & NA & $\mathrm{NA}$ & $\mathrm{NA}$ & Cortical & Oblique & Refits with S016 \\
\hline 506 & 339 & s016 & 1000 & 1010 & 11 & Indeterminate & $\mathrm{NA}$ & 0.34 & $\mathrm{NA}$ & Frag & No & None & $\mathrm{NA}$ & NA & $\mathrm{NA}$ & Cortical & Oblique & Refits with S015 \\
\hline 506 & 339 & s017 & 1000 & 1010 & 11 & Indeterminate & $\mathrm{NA}$ & 0.18 & $\mathrm{NA}$ & Frag & No & None & $\mathrm{NA}$ & NA & NA & Cortical & Fresh & \\
\hline 506 & 339 & s018 & 1000 & 1010 & 11 & Indeterminate & NA & 0.29 & $\mathrm{NA}$ & Frag & No & None & $\mathrm{NA}$ & NA & $\mathrm{NA}$ & Cortical & Oblique & \\
\hline 506 & 339 & S019 & 1000 & 1010 & 11 & Indeterminate & NA & 0.10 & NA & Frag & No & None & NA & NA & $\mathrm{NA}$ & Cortical & NA & Possibly a skull frag. \\
\hline 506 & 339 & so20 & 1000 & 1010 & 11 & Indeterminate & NA & 0.01 & $\mathrm{NA}$ & Frag & No & None & NA & $\mathrm{NA}$ & NA & Cortical & Fresh & \\
\hline 506 & 339 & s021 & 1000 & 1010 & 11 & Tortoise/Turtle & Tortoise/Turtle & 0.03 & 1 & Frag & No & None & Plastron & $\mathrm{NA}$ & $\mathrm{NA}$ & Cortical & $\mathrm{NA}$ & \\
\hline 506 & 339 & S022 & 1000 & 1010 & 11 & Indeterminate & NA & 0.16 & NA & Frag & No & None & $\mathrm{NA}$ & NA & $\mathrm{NA}$ & Cortical & NA & \\
\hline 506 & 339 & s023 & 1000 & 1010 & 11 & Indeterminate & NA & 0.07 & NA & Frag & No & None & NA & NA & NA & Cortical & Fresh & \\
\hline 506 & 339 & S024 & 1000 & 1010 & 11 & Indeterminate & NA & 0.01 & NA & Frag & No & None & NA & NA & NA & Cortical & Fresh & \\
\hline
\end{tabular}




\begin{tabular}{|c|c|c|c|c|c|c|c|c|c|c|c|c|c|c|c|c|c|c|}
\hline$\frac{0}{9}$ & $\begin{array}{l}\text { i } \\
\text { 总 } \\
\text { m }\end{array}$ & 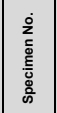 & 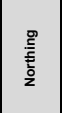 & 墨 & 舀 & 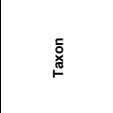 & 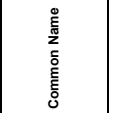 & 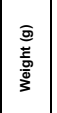 & 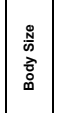 & 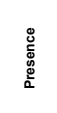 & 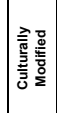 & 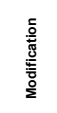 & $\begin{array}{l}\frac{0}{\circ} \\
\stackrel{\circ}{\circ} \\
\text { ஸे }\end{array}$ & $\frac{\circ}{5}$ & 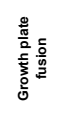 & 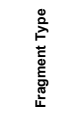 & 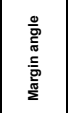 & 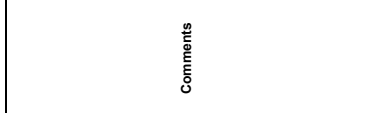 \\
\hline 506 & 339 & s025 & 1000 & 1010 & 11 & Indeterminate & $\mathrm{NA}$ & 0.01 & $\mathrm{NA}$ & Frag & No & None & NA & $\mathrm{NA}$ & NA & Cortical & Fresh & \\
\hline 506 & 339 & s026 & 1000 & 1010 & 11 & Indeterminate & NA & 0.03 & $\mathrm{NA}$ & Frag & No & None & NA & NA & NA & Cortical & NA & \\
\hline 506 & 339 & s027 & 1000 & 1010 & 11 & Indeterminate & NA & 0.01 & $\mathrm{NA}$ & Frag & No & None & $\mathrm{NA}$ & NA & NA & Cortical & $\mathrm{NA}$ & \\
\hline 506 & 339 & s028 & 1000 & 1010 & 11 & Indeterminate & $\mathrm{NA}$ & 0.01 & $\mathrm{NA}$ & Frag & No & None & $\mathrm{NA}$ & NA & NA & Cortical & $\mathrm{NA}$ & \\
\hline 508 & 411 & soo1 & 1000 & 1010 & 12 & Indeterminate & NA & 0.23 & $\mathrm{Na}$ & Frag & No & None & NA & $\mathrm{NA}$ & NA & Cortical & & Possibly a spinal articular facet. \\
\hline 508 & 411 & s002 & 1000 & 1010 & 12 & Indeterminate & $\mathrm{NA}$ & 0.17 & $\mathrm{Na}$ & Frag & No & None & $\mathrm{NA}$ & $\mathrm{NA}$ & NA & Cortical & & Possibly a spinal articular facet. \\
\hline 509 & 420 & s001 & 1000 & 1010 & 13 & Indeterminate & NA & 1.43 & NA & Frag & No & None & $\mathrm{NA}$ & $\mathrm{NA}$ & NA & Cortical & Right & \\
\hline 510 & 430 & soo1 & 1000 & 1010 & 14 & Indeterminate & NA & 0.24 & $\mathrm{NA}$ & Frag & No & None & $\mathrm{NA}$ & $\mathrm{NA}$ & NA & Cortical & & Small long bone frag. \\
\hline 511 & 438 & soo1 & 1000 & 1010 & 15 & Tortoise/Turtle & \begin{tabular}{|l|} 
Tortoise/Turtle \\
\end{tabular} & 0.24 & 1 & Frag & No & None & carapace & NA & NA & NA & & \\
\hline 512 & 447 & soo1 & 1000 & 1010 & 16 & Indeterminate & NA & 1.03 & $\mathrm{NA}$ & Frag & No & None & $\mathrm{NA}$ & $\mathrm{NA}$ & NA & Cortical & $\mathrm{NA}$ & Long bone frag \\
\hline 513 & 552 & s001 & 1000 & 1010 & 17 & Indeterminate & NA & 0.01 & $\mathrm{NA}$ & Frag & No & None & $\mathrm{NA}$ & $\mathrm{NA}$ & NA & Cortical & \begin{tabular}{|l|} 
Oblique \\
\end{tabular} & Burned \\
\hline 513 & 552 & s002 & 1000 & 1010 & 17 & Indeterminate & NA & 0.15 & $\mathrm{NA}$ & Frag & No & None & NA & NA & NA & Cortical & oblique & Burned \\
\hline 513 & 552 & s003 & 1000 & 1010 & 17 & $\begin{array}{l}\text { Indeterminate } \\
\mathrm{C}\end{array}$ & $\mathrm{NA}$ & 0.1 & $\mathrm{NA}$ & Frag & No & None & $\mathrm{NA}$ & $\mathrm{NA}$ & NA & Cortical & Right & Burned \\
\hline 518 & 640 & soo1 & 1000.5 & 1005.5 & 3 & Indeterminate & NA & 0.13 & $\mathrm{NA}$ & Frag & No & None & NA & NA & NA & Cortical & & Small and weathered. Trabecula remnant on one side. \\
\hline 518 & 640 & s002 & 1000.5 & 1005.5 & 3 & Indeterminate & NA & 0.19 & $\mathrm{NA}$ & Frag & No & None & $\mathrm{NA}$ & NA & NA & Cortical & & Small frag, unidentifiable \\
\hline 519 & 646 & soo1 & 1000.5 & 1005.5 & 4 & Tortoise/Turtle & Tortoise/Turtle & 0.18 & 1 & Frag & No & None & Plastron & $\mathrm{NA}$ & NA & NA & & Appears to be a plastron fragment. \\
\hline 519 & 646 & s002 & 1000.5 & 1005.5 & 4 & Mammal & NA & 2.02 & 2 & Frag & No & None & NA & NA & NA & Cortical & & $\begin{array}{l}\text { Mid-shaft long bone frag. No landmarks visible. One } \\
\text { fracture margin is a fresh break, the other is acute and } \\
\text { spiral. }\end{array}$ \\
\hline 519 & 646 & s003 & 1000.5 & 1005.5 & 4 & Mammal & $\mathrm{NA}$ & 0.29 & NA & Frag & No & None & $\mathrm{NA}$ & NA & $\mathrm{NA}$ & Cortical & & $\begin{array}{l}\text { Heavily weathered bone fragment. Fresh break margin, } \\
\text { other margin has been obliterated. }\end{array}$ \\
\hline 519 & 646 & s004 & 1000.5 & 1005.5 & 4 & Mammal & $\mathrm{NA}$ & 0.33 & NA & Frag & No & None & NA & NA & $\mathrm{NA}$ & Cortical & & $\begin{array}{l}\text { Heavily weathered bone fragment. Fresh break margin } \\
\text { rearly lal margins are fresh breaks. One small margin is } \\
\text { right angle. }\end{array}$ \\
\hline $\begin{array}{ll}519 \\
520\end{array}$ & $\begin{array}{ll}646 \\
656\end{array}$ & \begin{tabular}{|l|}
5005 \\
$s 001$ \\
\end{tabular} & \begin{tabular}{|l|l|}
1000.5 \\
101.57 \\
\end{tabular} & $\begin{array}{ll}1005.5 \\
1004.72\end{array}$ & \begin{tabular}{|c|}
4 \\
$4-5$ \\
\end{tabular} & \begin{tabular}{|c|} 
Indeterminate \\
Mammal \\
\end{tabular} & NA & \begin{tabular}{|l|}
0.13 \\
1.53 \\
\end{tabular} & \begin{tabular}{|c|}
$\mathrm{NA}$ \\
4 \\
\end{tabular} & $\begin{array}{l}\text { Frag } \\
\text { Frag }\end{array}$ & No & $\begin{array}{l}\text { None } \\
\text { None }\end{array}$ & $\begin{array}{ll}\mathrm{NA} \\
\text { Femur }\end{array}$ & $\begin{array}{ll}\mathrm{NA} \\
\text { Left }\end{array}$ & $\begin{array}{ll}\text { NA } \\
\text { Fused }\end{array}$ & $\begin{array}{l}\text { Cortical } \\
\text { Epiphysis }\end{array}$ & fon & 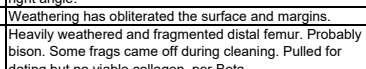 \\
\hline 528 & 666 & soo1 & FTR 4 & FTR4 & Lyr 2 & Indeterminate & $\mathrm{NA}$ & 0.45 & $\mathrm{NA}$ & Frag & No & None & NA & NA & NA & Cortical & $\mathrm{NA}$ & \\
\hline 528 & 666 & s002 & FTR 4 & FTR4 & Lyr 2 & \begin{tabular}{|l|} 
Indeterminate \\
\end{tabular} & NA & 0.33 & $\mathrm{NA}$ & Frag & No & None & $\mathrm{NA}$ & $\mathrm{NA}$ & NA & Cortical & $\mathrm{NA}$ & \\
\hline 528 & 666 & s003 & FTR 4 & FTR4 & Lyr 2 & Indeterminate & NA & 0.25 & $\mathrm{NA}$ & Frag & No & None & NA & NA & $\mathrm{NA}$ & Cortical & $\mathrm{NA}$ & \\
\hline 528 & 666 & soo4 & \begin{tabular}{|l|l|} 
FTR 4 \\
\end{tabular} & FTR4 & Lyr 2 & Indeterminate & NA & 0.06 & $\mathrm{NA}$ & Frag & No & None & NA & NA & NA & Cortical & $\mathrm{NA}$ & \\
\hline 528 & 666 & soos & \begin{tabular}{|l|l|} 
FTR 4 \\
\end{tabular} & FTR4 & Lyr 2 & Indeterminate & $\mathrm{NA}$ & 0.01 & $\mathrm{NA}$ & Frag & No & None & $\mathrm{NA}$ & $\mathrm{NA}$ & $\mathrm{NA}$ & Cortical & Fresh & \\
\hline 532 & 631 & s001 & 1001 & 1003 & 2 & Mammal & $\mathrm{NA}$ & 1.74 & NA & Frag & No & None & NA & NA & $\mathrm{NA}$ & Cortical & Multiple & Large mammal frage-size 2-4 \\
\hline 532 & 631 & s002 & 1001 & 1003 & 2 & Mammal & $\mathrm{NA}$ & 1.67 & 3-4 & Frag & No & None & $\mathrm{NA}$ & NA & $\mathrm{NA}$ & Cortical & Multiple & \\
\hline 532 & 631 & s003 & 1001 & 1003 & 2 & Mammal & NA & 1.93 & $\mathrm{NA}$ & Frag & No & None & NA & NA & NA & Cortical & Multiple & Large mammal frage- size 2-4 \\
\hline 532 & 631 & soo4 & 1001 & 1003 & 2 & Mammal & NA & 3.20 & $\mathrm{NA}$ & Frag & No & None & $\mathrm{NA}$ & $\mathrm{NA}$ & $\mathrm{NA}$ & Cortical & Multiple & Large mammal frage- size 2-4 \\
\hline 532 & 631 & s005 & 1001 & 1003 & 2 & Indeterminate & $\mathrm{NA}$ & 0.44 & NA & Frag & No & None & NA & $\mathrm{NA}$ & $\mathrm{NA}$ & Cortical & Multiple & \\
\hline 533 & 641 & soo1 & 1001 & 1003 & 3 & Mammal & NA & 6.98 & 3 to 4 & Frag & No & None & NA & $\mathrm{NA}$ & $\mathrm{NA}$ & Cortical & & $\begin{array}{l}\text { Very thick long bone frag. Heavily weathered. Not enough } \\
\text { to make a apoceies estimate, but cortical thickness } \\
\text { suggests size } 3-4 .\end{array}$ \\
\hline 533 & 641 & s002 & 1001 & 1003 & 3 & Indeterminate & $\mathrm{NA}$ & 0.53 & $\mathrm{NA}$ & Frag & No & None & $\mathrm{NA}$ & $\mathrm{NA}$ & $\mathrm{NA}$ & Cortical & & cortical frag. \\
\hline 535 & 657 & s001 & 1001 & 1003 & 4 & Indeterminate & NA & 0.13 & NA & Frag & No & None & NA & NA & NA & Cortical & $\mathrm{NA}$ & \\
\hline 535 & 657 & s002 & 1001 & 1003 & 4 & Indeterminate & $\mathrm{NA}$ & 0.12 & $\mathrm{NA}$ & Frag & No & None & NA & NA & NA & Cortical & NA & \\
\hline 535 & 657 & s003 & 1001 & 1003 & 4 & Indeterminate & NA & 0.09 & $\mathrm{NA}$ & Frag & No & None & NA & NA & NA & Cortical & $\mathrm{NA}$ & \\
\hline 535 & 657 & soo4 & 1001 & 1003 & 4 & Indeterminate & $\mathrm{NA}$ & 0.11 & $\mathrm{NA}$ & Frag & No & None & $\mathrm{NA}$ & NA & NA & Cortical & $\mathrm{NA}$ & \\
\hline 535 & 657 & soos & 1001 & 1003 & 4 & Indeterminate & $\mathrm{NA}$ & 0.05 & $\mathrm{NA}$ & Frag & No & None & NA & NA & NA & Cortical & NA & \\
\hline 535 & 657 & soo6 & 1001 & 1003 & 4 & Indeterminate & NA & 0.04 & $\mathrm{NA}$ & Frag & No & None & NA & NA & NA & Cortical & NA & \\
\hline 535 & 657 & s007 & 1001 & 1003 & 4 & Indeterminate & $\mathrm{NA}$ & 0.03 & NA & Frag & No & None & NA & $\mathrm{NA}$ & NA & $\begin{array}{l}\text { Cortical } \\
\end{array}$ & $\mathrm{NA}$ & \\
\hline 535 & 657 & s008 & 1001 & 1003 & 4 & Indeterminate & $\mathrm{NA}$ & \begin{tabular}{l|l|} 
& 0.05 \\
\end{tabular} & $\mathrm{NA}$ & Frag & No & None & NA & $\mathrm{NA}$ & NA & Cortical & $\mathrm{NA}$ & \\
\hline 535 & 657 & soog & 1001 & 1003 & 4 & Indeterminate & $\mathrm{NA}$ & \begin{tabular}{l|l}
0.03 \\
\end{tabular} & NA & Frag & No & None & NA & NA & NA & Cortical & $\mathrm{NA}$ & \\
\hline 535 & 657 & s010 & 1001 & 1003 & 4 & Indeterminate & $\mathrm{NA}$ & 0.04 & $\mathrm{NA}$ & Frag & No & None & NA & NA & NA & Cortical & $\mathrm{NA}$ & \\
\hline 535 & 657 & so11 & 1001 & 1003 & 4 & Indeterminate & NA & 0.10 & $\mathrm{NA}$ & Frag & No & None & NA & NA & NA & Cortical & $\mathrm{NA}$ & \\
\hline 535 & 657 & s012 & 1001 & 1003 & 4 & Indeterminate & $\mathrm{NA}$ & 0.12 & NA & Frag & No & None & $\mathrm{NA}$ & NA & NA & Cortical & $\mathrm{NA}$ & \\
\hline 535 & 657 & s013 & 1001 & 1003 & 4 & Indeterminate & $\mathrm{NA}$ & 0.07 & $\mathrm{NA}$ & Frag & No & None & NA & NA & NA & Cortical & $\mathrm{NA}$ & \\
\hline 535 & 657 & s014 & 1001 & 1003 & 4 & Indeterminate & $\mathrm{NA}$ & 0.06 & NA & Frag & No & None & NA & NA & NA & Cortical & NA & \\
\hline 535 & 657 & s015 & 1001 & 1003 & 4 & Indeterminate & $\mathrm{NA}$ & 0.04 & NA & Frag & No & None & NA & NA & NA & Cortical & $\mathrm{NA}$ & \\
\hline 535 & 657 & s016 & 1001 & 1003 & 4 & Indeterminate & $\mathrm{NA}$ & 0.05 & $\mathrm{NA}$ & Frag & No & None & $\mathrm{NA}$ & $\mathrm{NA}$ & NA & Cortical & $\mathrm{NA}$ & \\
\hline 535 & 657 & s017 & 1001 & 1003 & 4 & Indeterminate & NA & 0.03 & NA & Frag & No & None & $\mathrm{NA}$ & $\mathrm{NA}$ & NA & $\begin{array}{l}\text { Cortical } \\
\end{array}$ & $\mathrm{NA}$ & \\
\hline 535 & 657 & s018 & 1001 & 1003 & 4 & Indeterminate & $\mathrm{NA}$ & \begin{tabular}{|l|l|} 
& 0.03 \\
\end{tabular} & $\mathrm{NA}$ & Frag & No & None & $\mathrm{NA}$ & NA & NA & Cortical & $\mathrm{NA}$ & \\
\hline 535 & 657 & s019 & 1001 & 1003 & 4 & Indeterminate & $\mathrm{NA}$ & 0.03 & NA & Frag & No & None & $\mathrm{NA}$ & $\mathrm{NA}$ & $\mathrm{NA}$ & Cortical & $\mathrm{NA}$ & \\
\hline 535 & 657 & s020 & 1001 & 1003 & 4 & Indeterminate & $\mathrm{NA}$ & 0.03 & $\mathrm{NA}$ & Frag & No & None & NA & NA & NA & Cortical & $\mathrm{NA}$ & \\
\hline 535 & 657 & s021 & 1001 & 1003 & 4 & Indeterminate & $\mathrm{NA}$ & 0.03 & $\mathrm{NA}$ & Frag & No & None & $\mathrm{NA}$ & $\mathrm{NA}$ & $\mathrm{NA}$ & Cortical & $\mathrm{NA}$ & \\
\hline 535 & 657 & s022 & 1001 & 1003 & 4 & Indeterminate & $\mathrm{NA}$ & 0.01 & NA & Frag & No & None & NA & $\mathrm{NA}$ & NA & Cortical & $\mathrm{NA}$ & \\
\hline
\end{tabular}




\begin{tabular}{|c|c|c|c|c|c|c|c|c|c|c|c|c|c|c|c|c|c|c|}
\hline 家 & 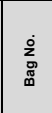 & 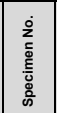 & $\begin{array}{l}\frac{0}{\underline{\underline{z}}} \\
\frac{5}{2} \\
\end{array}$ & $\begin{array}{l}\text { 总 } \\
\text { 覀 }\end{array}$ & $\bar{g}$ & $\begin{array}{l}\text { 离 } \\
\text { 离 }\end{array}$ & 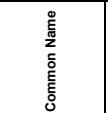 & $\begin{array}{l}\text { बू } \\
\text { 产 } \\
\text { ș }\end{array}$ & 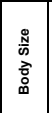 & 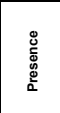 & 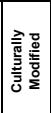 & 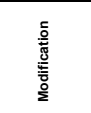 & $\begin{array}{l}\frac{\mathrm{o}}{\mathrm{o}} \\
\frac{\mathrm{\sigma}}{\mathrm{\omega}}\end{array}$ & $\frac{\circ}{5}$ & 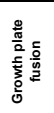 & 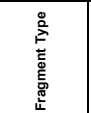 & 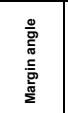 & 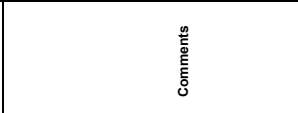 \\
\hline 535 & 657 & s023 & 1001 & 1003 & 4 & Indeterminate & NA & 0.01 & $\mathrm{NA}$ & Frag & No & None & $\mathrm{NA}$ & NA & NA & Cortical & NA & \\
\hline 535 & 657 & s024 & 1001 & 1003 & 4 & Indeterminate & $\mathrm{NA}$ & 0.01 & $\mathrm{NA}$ & Frag & No & None & NA & NA & NA & Cortical & NA & \\
\hline 535 & 657 & s025 & 1001 & 1003 & 4 & Indeterminate & $\mathrm{NA}$ & 0.01 & $\mathrm{NA}$ & Frag & No & None & $\mathrm{NA}$ & $\mathrm{NA}$ & NA & Cortical & $\mathrm{NA}$ & \\
\hline 535 & 657 & s026 & 1001 & 1003 & 4 & Indeterminate & $\mathrm{NA}$ & 0.01 & $\mathrm{NA}$ & Frag & No & None & $\mathrm{NA}$ & $\mathrm{NA}$ & $\mathrm{NA}$ & Cortical & $\mathrm{NA}$ & \\
\hline 535 & 657 & S027 & 1001 & 1003 & 4 & Indeterminate & $\mathrm{NA}$ & 0.01 & $\mathrm{NA}$ & Frag & No & None & $\mathrm{NA}$ & $\mathrm{NA}$ & NA & Cortical & $\mathrm{NA}$ & \\
\hline 535 & 657 & s028 & 1001 & 1003 & 4 & Indeterminate & $\mathrm{NA}$ & 0.01 & $\mathrm{NA}$ & Frag & No & None & $\mathrm{NA}$ & $\mathrm{NA}$ & $\mathrm{NA}$ & Cortical & $\mathrm{NA}$ & \\
\hline 535 & 657 & s029 & 1001 & 1003 & 4 & Indeterminate & $\mathrm{NA}$ & 0.01 & $\mathrm{NA}$ & Frag & No & None & $\mathrm{NA}$ & $\mathrm{NA}$ & NA & Cortical & $\mathrm{NA}$ & \\
\hline 535 & 657 & s030 & 1001 & 1003 & 4 & Indeterminate & NA & 0.01 & NA & Frag & No & None & NA & NA & NA & Cortical & $\mathrm{NA}$ & \\
\hline 535 & 657 & s031 & 1001 & 1003 & 4 & Indeterminate & $\mathrm{NA}$ & 0.01 & $\mathrm{NA}$ & Frag & No & None & NA & NA & NA & Cortical & $\mathrm{NA}$ & \\
\hline 535 & 657 & s032 & 1001 & 1003 & 4 & Indeterminate & NA & 0.01 & $\mathrm{NA}$ & Frag & No & None & NA & $\mathrm{NA}$ & NA & Cortical & NA & \\
\hline 535 & 657 & s033 & 1001 & 1003 & 4 & Indeterminate & $\mathrm{NA}$ & 0.01 & $\mathrm{NA}$ & Frag & No & None & $\mathrm{NA}$ & $\mathrm{NA}$ & NA & Cortical & $\mathrm{NA}$ & \\
\hline 535 & 657 & s034 & 1001 & 1003 & 4 & Indeterminate & $\mathrm{NA}$ & 0.01 & $\mathrm{NA}$ & Frag & No & None & NA & $\mathrm{NA}$ & NA & Cortical & $\mathrm{NA}$ & \\
\hline 535 & 657 & s035 & 1001 & 1003 & 4 & Indeterminate & NA & 0.01 & $\mathrm{NA}$ & Frag & No & None & NA & NA & NA & Cortical & NA & \\
\hline 535 & 657 & s036 & 1001 & 1003 & 4 & Indeterminate & NA & 0.01 & NA & Frag & No & None & NA & NA & NA & Cortical & $\mathrm{NA}$ & \\
\hline 535 & 657 & s037 & 1001 & 1003 & 4 & Indeterminate & NA & \begin{tabular}{|l|l|}
0.01 \\
\end{tabular} & NA & Frag & No & None & NA & NA & NA & Cortical & $\mathrm{NA}$ & \\
\hline 535 & 657 & s038 & 1001 & 1003 & 4 & Indeterminate & $\mathrm{NA}$ & 0.01 & $\mathrm{NA}$ & Frag & No & None & $\mathrm{NA}$ & $\mathrm{NA}$ & $\mathrm{NA}$ & Cortical & $\mathrm{NA}$ & \\
\hline 535 & 657 & s039 & 1001 & 1003 & 4 & Indeterminate & NA & 0.01 & $\mathrm{NA}$ & Frag & No & None & $\mathrm{NA}$ & $\mathrm{NA}$ & NA & Cortical & $\mathrm{NA}$ & \\
\hline 535 & 657 & s040 & 1001 & 1003 & 4 & Indeterminate & $\mathrm{NA}$ & 0.01 & $\mathrm{NA}$ & Frag & No & None & $\mathrm{NA}$ & $\mathrm{NA}$ & $\mathrm{NA}$ & Cortical & $\mathrm{NA}$ & \\
\hline 535 & 657 & s041 & 1001 & 1003 & 4 & Indeterminate & $\mathrm{NA}$ & 0.01 & NA & Frag & No & None & NA & NA & NA & Cortical & $\mathrm{NA}$ & \\
\hline 537 & 576 & soo1 & 1001 & 1004 & 3 & Indeterminate & NA & 2.12 & $\mathrm{NA}$ & Frag & No & None & NA & NA & NA & Cortical & & Too distorted by weathering to identify. \\
\hline 538 & 579 & s001 & 1001 & 1004 & 4 & Tortoise/Turtle & Tortoise/Turtle & 0.13 & 1 & Frag & No & None & Plastron/carapace & $\mathrm{NA}$ & $\mathrm{NA}$ & Cortical & Right & Burned, can't determine which side of the shell. \\
\hline 538 & 579 & s002 & 1001 & 1004 & 4 & Indeterminate & NA & 0.59 & $\mathrm{NA}$ & Frag & No & None & $\mathrm{NA}$ & $\mathrm{NA}$ & NA & Cortical & Right & sightyly burned \\
\hline 539 & 592 & s001 & 1001 & 1004 & 5 & Indeterminate & $\mathrm{NA}$ & 0.77 & $\mathrm{NA}$ & Frag & Yes & Cut marks & $\mathrm{NA}$ & $\mathrm{NA}$ & $\mathrm{NA}$ & Cortical & Right & \\
\hline 539 & 592 & s002 & 1001 & 1004 & 5 & Indeterminate & $\mathrm{NA}$ & 0.79 & $\mathrm{NA}$ & Frag & No & None & $\mathrm{NA}$ & $\mathrm{NA}$ & NA & Trabecular & $\mathrm{NA}$ & Burned (possibly roasted) \\
\hline 539 & 592 & s003 & 1001 & 1004 & 5 & Indeterminate & NA & 0.05 & $\mathrm{NA}$ & Frag & No & None & NA & NA & NA & Trabecular & NA & \\
\hline 539 & 592 & s004 & 1001 & 1004 & 5 & Indeterminate & $\mathrm{NA}$ & 0.08 & $\mathrm{NA}$ & Frag & No & None & NA & $\mathrm{NA}$ & NA & Cortical & $\mathrm{NA}$ & \\
\hline 539 & 592 & s005 & 1001 & 1004 & 5 & Indeterminate & $\mathrm{NA}$ & 0.16 & $\mathrm{NA}$ & Frag & No & None & NA & $\mathrm{NA}$ & NA & Cortical & $\mathrm{NA}$ & \\
\hline 539 & 592 & s006 & 1001 & 1004 & 5 & Indeterminate & $\mathrm{NA}$ & 0.06 & $\mathrm{NA}$ & Frag & No & None & $\mathrm{NA}$ & $\mathrm{NA}$ & $\mathrm{NA}$ & $\begin{array}{l}\text { Cortical } \\
\end{array}$ & $\mathrm{NA}$ & \\
\hline 539 & 592 & s007 & 1001 & 1004 & 5 & Indeterminate & $\mathrm{NA}$ & $\begin{array}{ll}0.03 \\
\end{array}$ & $\mathrm{NA}$ & Frag & No & None & $\mathrm{NA}$ & NA & NA & Cortical & $\mathrm{NA}$ & \\
\hline 539 & 592 & s008 & 1001 & 1004 & 5 & Indeterminate & $\mathrm{NA}$ & 0.09 & $\mathrm{NA}$ & Frag & No & None & $\mathrm{NA}$ & NA & NA & Cortical & $\mathrm{NA}$ & \\
\hline 539 & 592 & soog & 1001 & 1004 & 5 & Indeterminate & $\mathrm{NA}$ & 0.03 & $\mathrm{NA}$ & Frag & No & None & NA & NA & NA & Cortical & $\mathrm{NA}$ & \\
\hline 539 & 592 & s010 & 1001 & 1004 & 5 & Indeterminate & NA & 0.01 & $\mathrm{NA}$ & Frag & No & None & NA & $\mathrm{NA}$ & NA & Cortical & NA & \\
\hline 539 & 592 & s011 & 1001 & 1004 & 5 & Indeterminate & $\mathrm{NA}$ & 0.01 & $\mathrm{NA}$ & Frag & No & None & $\mathrm{NA}$ & $\mathrm{NA}$ & NA & Cortical & $\mathrm{NA}$ & \\
\hline 539 & 592 & s012 & 1001 & 1004 & 5 & Indeterminate & $\mathrm{NA}$ & 0.01 & $\mathrm{NA}$ & Frag & No & None & NA & $\mathrm{NA}$ & NA & Cortical & NA & \\
\hline 539 & 592 & s013 & 1001 & 1004 & 5 & Indeterminate & $\mathrm{NA}$ & 0.01 & $\mathrm{NA}$ & Frag & No & None & NA & $\mathrm{NA}$ & $\mathrm{NA}$ & Cortical & $\mathrm{NA}$ & \\
\hline 539 & 592 & s014 & 1001 & 1004 & 5 & Indeterminate & $\mathrm{NA}$ & 0.06 & $\mathrm{NA}$ & Frag & No & None & NA & $\mathrm{NA}$ & NA & Cortical & Oblique & \\
\hline 539 & 592 & s015 & 1001 & 1004 & 5 & Indeterminate & $\mathrm{NA}$ & 0.07 & $\mathrm{NA}$ & Frag & No & None & NA & $\mathrm{NA}$ & NA & Cortical & Oblique & \\
\hline 540 & 542 & s001 & 1001 & 1004.5 & 2 & Indeterminate & $\mathrm{NA}$ & 0.01 & NA & Frag & No & None & $\mathrm{NA}$ & $\mathrm{NA}$ & $\mathrm{NA}$ & Cortical & Fresh & \\
\hline 542 & 546 & s001 & 1001 & 1004.5 & 4 & Mammal & $\mathrm{NA}$ & 0.46 & $\mathrm{NA}$ & Frag & No & None & NA & NA & NA & Cortical & Spiral & Probably size 2 \\
\hline 542 & 546 & s002 & 1001 & 1004.5 & 4 & Mammal & $\mathrm{NA}$ & 0.82 & $\mathrm{NA}$ & Frag & No & None & $\mathrm{NA}$ & $\mathrm{NA}$ & $\mathrm{NA}$ & Cortical & \begin{tabular}{|l|} 
Oblique \\
\end{tabular} & \\
\hline 542 & 546 & s003 & 1001 & 1004.5 & 4 & Mammal & NA & 2.26 & $\mathrm{NA}$ & Frag & No & None & $\mathrm{NA}$ & NA & NA & Cortical & Oblique & Probably size 2 \\
\hline 542 & 546 & s004 & 1001 & 1004.5 & 4 & Mammal & $\mathrm{NA}$ & 1.20 & $\mathrm{NA}$ & Frag & No & None & NA & $\mathrm{NA}$ & NA & Cortical & Fresh & Probably size 2 \\
\hline 542 & 546 & s005 & 1001 & 1004.5 & 4 & Indeterminate & $\mathrm{NA}$ & 0.32 & $\mathrm{NA}$ & Frag & No & None & $\mathrm{NA}$ & $\mathrm{NA}$ & NA & Cortical & Right & \\
\hline 542 & 546 & s006 & 1001 & 1004.5 & 4 & Mammal & NA & 0.48 & $\mathrm{NA}$ & Frag & No & None & $\mathrm{NA}$ & $\mathrm{NA}$ & NA & Cortical & Oblique & \\
\hline 542 & 546 & s007 & 1001 & 1004.5 & 4 & Indeterminate & $\mathrm{NA}$ & 0.29 & NA & Frag & No & None & $\mathrm{NA}$ & $\mathrm{NA}$ & $\mathrm{NA}$ & Cortical & Oblique & \\
\hline 542 & 546 & s008 & 1001 & 1004.5 & 4 & Indeterminate & NA & 0.16 & $\mathrm{NA}$ & Frag & No & None & $\mathrm{NA}$ & $\mathrm{NA}$ & NA & Cortical & Fresh & \\
\hline 542 & 546 & soog & 1001 & 1004.5 & 4 & Indeterminate & NA & 0.66 & NA & Frag & No & None & NA & NA & NA & Cortical & Right & \\
\hline 542 & 546 & so10 & 1001 & 1004.5 & 4 & Odocoileus $s p$ & Deer & 0.54 & 2 & Frag & No & None & Tooth & $\mathrm{NA}$ & NA & NA & $\mathrm{NA}$ & Not enough to make a determination \\
\hline 543 & 560 & s001 & 1001 & 1004.5 & 5 & $\begin{array}{l}\text { Indeterminate } \\
\end{array}$ & $\mathrm{NA}$ & 1.75 & $\mathrm{NA}$ & Frag & No & None & $\mathrm{NA}$ & $\mathrm{NA}$ & $\mathrm{NA}$ & Cortical & Fresh & \\
\hline$\overline{543}$ & 560 & s002 & 1001 & 1004.5 & 5 & $\begin{array}{l}\text { Indeterminate } \\
\end{array}$ & $\mathrm{NA}$ & 0.33 & $\mathrm{NA}$ & Frag & No & None & $\mathrm{NA}$ & $\mathrm{NA}$ & $\mathrm{NA}$ & Cortical & Right & \\
\hline 543 & 560 & s003 & 1001 & 1004.5 & 5 & Indeterminate & NA & 0.21 & $\mathrm{NA}$ & Frag & No & None & NA & NA & NA & Cortical & NA & \\
\hline 548 & 333 & s001 & 1001 & 1009 & 4 & Tortoise/Turtle & \begin{tabular}{|c|} 
Tortoise/Turtle \\
\end{tabular} & 6.86 & 1 & Frag & No & None & Plastron & $\mathrm{NA}$ & NA & NA & & Large plastron frag. Refits with S002 \\
\hline 548 & 333 & s002 & 1001 & 1009 & 4 & Tortoise/Turtle & \begin{tabular}{|l|l|l|l} 
Tortoise/Turtle \\
\end{tabular} & \begin{tabular}{|l|l|}
0.97 & \\
\end{tabular} & 1 & Frag & No & None & Plastron & NA & NA & NA & & Plastron frag. Refits with S001 \\
\hline 548 & 333 & s003 & 1001 & 1009 & 4 & Tortoise/Turtle & Tortoise/Turtle & 0.47 & 1 & Frag & No & None & Plastron & $\mathrm{NA}$ & $\mathrm{NA}$ & $\mathrm{NA}$ & & Plastron frag. \\
\hline 548 & 333 & s004 & 1001 & 1009 & 4 & Tortoise/ Turtle & Tortoise/Turtle & 0.36 & 1 & Frag & No & None & carapace & $\mathrm{NA}$ & NA & NA & & Carapace frag. \\
\hline 548 & 333 & s005 & 1001 & 1009 & 4 & Tortoise/Turtle & Tortoise/Turtle & \begin{tabular}{|l|l}
0.46 \\
\end{tabular} & 1 & Frag & No & None & Plastron & $\mathrm{NA}$ & NA & $\mathrm{NA}$ & & Plastron frag. \\
\hline
\end{tabular}




\begin{tabular}{|c|c|c|c|c|c|c|c|c|c|c|c|c|c|c|c|c|c|c|}
\hline$\frac{\dot{s}}{2}$ & 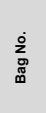 & 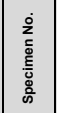 & $\begin{array}{l}\text { 㝘 } \\
\text { 总 }\end{array}$ & 墨 & פ్ & 高 & 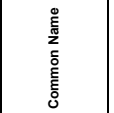 & 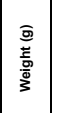 & 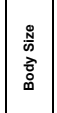 & 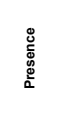 & 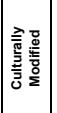 & 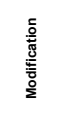 & 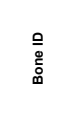 & $\frac{\circ}{\omega}$ & 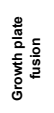 & 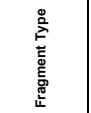 & 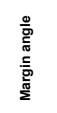 & 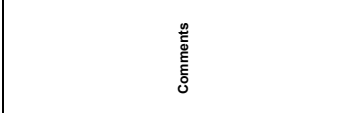 \\
\hline 548 & 333 & soo6 & 1001 & 1009 & 4 & Tortoise/Turtle & Tortoise/Turtle & 0.22 & 1 & Frag & No & None & Plastron & $\mathrm{NA}$ & NA & NA & & Plastron frag. \\
\hline 548 & 333 & s007 & 1001 & 1009 & 4 & Indeterminate & NA & 0.46 & $\mathrm{NA}$ & Frag & No & None & NA & NA & NA & Trabecular & & Trabecular bone frag, unable to identify. \\
\hline 548 & 333 & s008 & 1001 & 1009 & 4 & Tortoise//urtle & Tortoise/Turle & 0.15 & 1 & Frag & No & None & Plastron & NA & NA & NA & & Very small plastron frag (possibly carapace). \\
\hline 548 & 333 & soog & 1001 & 1009 & 4 & Indeterminate & NA & 0.12 & $\mathrm{NA}$ & Frag & No & None & $\mathrm{NA}$ & NA & NA & Cortical & & Cortical bone frag. Too small to identify. \\
\hline 548 & 333 & s010 & 1001 & 1009 & 4 & Indeterminate & $\mathrm{NA}$ & 0.21 & $\mathrm{NA}$ & Frag & No & None & NA & NA & NA & Cortical & & Cortical bone frag. Too weathered to identify. \\
\hline 548 & 333 & s011 & 1001 & 1009 & 4 & Indeterminate & $\mathrm{NA}$ & 0.2 & $\mathrm{NA}$ & Frag & No & None & NA & $\mathrm{NA}$ & NA & Cortical & & Cortical bone frag. Too weathered to identify. \\
\hline 549 & 337 & soo1 & 1001 & 1009 & 5 & Mammal & NA & 108.9 & 3 to 4 & Frag & No & None & Humerus & Right & Yes & Epiphysis & & $\begin{array}{l}\text { Distah humerale pepiphysis. Large, size } 3-4 \text { mammal. } \\
\text { Growth plate is completely fused. Too smal to be con or } \\
\text { bison. Possibiby horse. Pulled for radiocarbon dating. }\end{array}$ \\
\hline 550 & 338 & soo1 & 1001 & 1009 & 5 & Indeterminate & NA & 0.17 & NA & Frag & No & None & $\mathrm{NA}$ & $\mathrm{NA}$ & NA & Cortical & $\mathrm{NA}$ & Possibly tortoise \\
\hline 550 & 338 & s002 & 1001 & 1009 & 5 & Mammal & NA & 0.92 & 2 & Frag & № & None & NA & $\mathrm{NA}$ & NA & Cortical & Fresh & Probably deer \\
\hline 550 & 338 & s003 & 1001 & 1009 & 5 & Mammal & $\mathrm{NA}$ & 0.63 & 2 & Frag & No & None & $\mathrm{NA}$ & NA & NA & Cortical & Fresh & Probably deer \\
\hline 550 & 338 & soo4 & 1001 & 1009 & 5 & Mammal & $\mathrm{NA}$ & 0.64 & 2 & Frag & No & None & NA & $\mathrm{NA}$ & NA & Cortical & Fresh & Probably deer \\
\hline 550 & 338 & s005 & 1001 & 1009 & 5 & Mammal & $\mathrm{NA}$ & 1.67 & 2 & Frag & No & None & $\mathrm{NA}$ & $\mathrm{NA}$ & $\mathrm{NA}$ & Cortical & Fresh & Probably deer \\
\hline 550 & 338 & s006 & 1001 & 1009 & 5 & Mammal & $\mathrm{NA}$ & 0.89 & 2 & Frag & No & None & $\mathrm{NA}$ & $\mathrm{NA}$ & $\mathrm{NA}$ & Cortical & Fresh & Probably deer \\
\hline 550 & 338 & s007 & 1001 & 1009 & 5 & Mammal & $\mathrm{NA}$ & 1.61 & 2 & Frag & No & None & $\mathrm{NA}$ & $\mathrm{NA}$ & $\mathrm{NA}$ & Cortical & Fresh & Probabaly deer \\
\hline 550 & 338 & s008 & 1001 & 1009 & 5 & Mammal & $\mathrm{NA}$ & 0.81 & 2 & Frag & No & None & $\mathrm{NA}$ & $\mathrm{NA}$ & $\mathrm{NA}$ & Cortical & Fresh & Probably deer \\
\hline 550 & 338 & soog & 1001 & 1009 & 5 & Mammal & $\mathrm{NA}$ & 0.49 & 2 & Frag & No & None & $\mathrm{NA}$ & NA & NA & Cortical & Fresh & Probably deer \\
\hline 550 & 338 & s010 & 1001 & 1009 & 5 & Mammal & $\mathrm{NA}$ & 0.52 & 2 & Frag & No & None & $\mathrm{NA}$ & $\mathrm{NA}$ & $\mathrm{NA}$ & Cortical & Fresh & Probably deer \\
\hline 550 & 338 & s011 & 1001 & 1009 & 5 & Mammal & $\mathrm{NA}$ & 0.81 & 2 & Frag & No & None & $\mathrm{NA}$ & $\mathrm{NA}$ & NA & Cortical & Fresh & Probably deer \\
\hline 550 & 338 & s012 & 1001 & 1009 & 5 & Mammal & $\mathrm{NA}$ & 0.39 & 2 & Frag & No & None & $\mathrm{NA}$ & $\mathrm{NA}$ & NA & Cortical & Fresh & Probably deer \\
\hline 550 & 338 & s013 & 1001 & 1009 & 5 & Mammal & $\mathrm{NA}$ & 0.26 & 2 & Frag & No & None & $\mathrm{NA}$ & $\mathrm{NA}$ & $\mathrm{NA}$ & Cortical & Fresh & Probably deer \\
\hline 550 & 338 & s014 & 1001 & 1009 & 5 & Mammal & $\mathrm{NA}$ & 0.19 & 2 & Frag & No & None & NA & $\mathrm{NA}$ & $\mathrm{NA}$ & Cortical & Fresh & Probably deer \\
\hline 550 & 338 & s015 & 1001 & 1009 & 5 & Mammal & $\mathrm{NA}$ & 0.14 & 2 & Frag & No & None & $\mathrm{NA}$ & $\mathrm{NA}$ & $\mathrm{NA}$ & Cortical & Fresh & Probably deer \\
\hline 550 & 338 & s016 & 1001 & 1009 & 5 & Mammal & $\mathrm{NA}$ & 0.35 & 2 & Frag & No & None & NA & $\mathrm{NA}$ & $\mathrm{NA}$ & Cortical & Fresh & Probably deer \\
\hline 550 & 338 & s017 & 1001 & 1009 & 5 & Mammal & $\mathrm{NA}$ & 0.31 & 2 & Frag & No & None & $\mathrm{NA}$ & $\mathrm{NA}$ & $\mathrm{NA}$ & Cortical & Fresh & Probably deer \\
\hline 550 & 338 & s018 & 1001 & 1009 & 5 & Mammal & $\mathrm{NA}$ & 0.15 & 2 & Frag & No & None & $\mathrm{NA}$ & $\mathrm{NA}$ & $\mathrm{NA}$ & Cortical & Fresh & Probably deer \\
\hline 550 & 338 & s019 & 1001 & 1009 & 5 & Mammal & NA & 0.12 & 2 & Frag & No & None & NA & NA & NA & Cortical & Fresh & Probably deer \\
\hline 550 & 338 & s020 & 1001 & 1009 & 5 & Mammal & $\mathrm{NA}$ & 0.21 & 2 & Frag & No & None & $\mathrm{NA}$ & $\mathrm{NA}$ & NA & Cortical & Fresh & Probably deer \\
\hline 550 & 338 & s021 & 1001 & 1009 & 5 & Mammal & $\mathrm{NA}$ & 0.26 & 2 & Frag & No & None & NA & $\mathrm{NA}$ & NA & Cortical & Fresh & Probably deer \\
\hline 550 & 338 & s022 & 1001 & 1009 & 5 & Mammal & NA & 0.42 & 2 & Frag & No & None & NA & NA & NA & Cortical & Fresh & Probably deer \\
\hline 550 & 338 & s023 & 1001 & 1009 & 5 & Mammal & $\mathrm{NA}$ & 0.11 & 2 & Frag & No & None & $\mathrm{NA}$ & $\mathrm{NA}$ & $\mathrm{NA}$ & Cortical & Fresh & Probably deer \\
\hline 550 & 338 & s024 & 1001 & 1009 & 5 & Mammal & $\mathrm{NA}$ & 0.23 & 2 & Frag & No & None & $\mathrm{NA}$ & NA & $\mathrm{NA}$ & Cortical & Fresh & Probably deer \\
\hline 550 & 338 & S025 & 1001 & 1009 & 5 & Mammal & $\mathrm{NA}$ & 0.25 & 2 & Frag & No & None & $\mathrm{NA}$ & $\mathrm{NA}$ & $\mathrm{NA}$ & Cortical & Fresh & Probably deer \\
\hline 550 & 338 & s026 & 1001 & 1009 & 5 & Mammal & $\mathrm{NA}$ & 0.16 & 2 & Frag & No & None & $\mathrm{NA}$ & NA & NA & Cortical & Fresh & Probably deer \\
\hline 550 & 338 & s027 & 1001 & 1009 & 5 & Mammal & $\mathrm{NA}$ & 0.11 & 2 & Frag & No & None & $\mathrm{NA}$ & $\mathrm{NA}$ & $\mathrm{NA}$ & Cortical & Fresh & Probably deer \\
\hline 550 & 338 & s028 & 1001 & 1009 & 5 & Mammal & NA & 0.13 & 2 & Frag & No & None & $\mathrm{NA}$ & $\mathrm{NA}$ & NA & Cortical & Fresh & Probably deer \\
\hline 550 & 338 & s029 & 1001 & 1009 & 5 & Mammal & $\mathrm{NA}$ & 0.31 & 2 & Frag & No & None & $\mathrm{NA}$ & $\mathrm{NA}$ & $\mathrm{NA}$ & Cortical & Fresh & Probably deer \\
\hline 550 & 338 & so30 & 1001 & 1009 & 5 & Mammal & NA & 0.15 & 2 & Frag & No & None & $\mathrm{NA}$ & $\mathrm{NA}$ & $\mathrm{NA}$ & Cortical & Fresh & Probably deer \\
\hline 550 & 338 & s031 & 1001 & 1009 & 5 & Mammal & $\mathrm{NA}$ & 0.11 & 2 & Frag & No & None & $\mathrm{NA}$ & $\mathrm{NA}$ & NA & Cortical & Fresh & Probably deer \\
\hline 550 & 338 & s032 & 1001 & 1009 & 5 & Mammal & NA & 0.25 & 2 & Frag & No & None & NA & $\mathrm{NA}$ & NA & Cortical & Fresh & Probably deer \\
\hline 550 & 338 & s033 & 1001 & 1009 & 5 & Mammal & $\mathrm{NA}$ & 0.13 & 2 & Frag & No & None & $\mathrm{NA}$ & $\mathrm{NA}$ & NA & Cortical & Fresh & Probably deer \\
\hline 550 & 338 & s034 & 1001 & 1009 & 5 & Mammal & $\mathrm{NA}$ & 0.14 & 2 & Frag & No & None & NA & NA & NA & Cortical & Fresh & Probably deer \\
\hline 550 & 338 & s035 & 1001 & 1009 & 5 & Mammal & NA & 0.11 & 2 & Frag & No & None & $\mathrm{NA}$ & $\mathrm{NA}$ & NA & Cortical & Fresh & Probably deer \\
\hline 550 & 338 & s036 & 1001 & 1009 & 5 & Mammal & $\mathrm{NA}$ & 0.13 & 2 & Frag & No & None & NA & $\mathrm{NA}$ & NA & Cortical & Fresh & Probably deer \\
\hline 550 & 338 & s037 & 1001 & 1009 & 5 & Mammal & $\mathrm{NA}$ & 0.16 & 2 & Frag & No & None & NA & $\mathrm{NA}$ & $\mathrm{NA}$ & Cortical & Fresh & Probably deer \\
\hline 550 & 338 & s038 & 1001 & 1009 & 5 & Mammal & $\mathrm{NA}$ & 0.19 & 2 & Frag & No & None & $\mathrm{NA}$ & $\mathrm{NA}$ & NA & Cortical & Fresh & Probably deer \\
\hline 550 & 338 & s039 & 1001 & 1009 & 5 & Indeterminate & NA & 0.32 & $\mathrm{NA}$ & Frag & No & None & $\mathrm{NA}$ & $\mathrm{NA}$ & $\mathrm{NA}$ & Trabecular & $\mathrm{NA}$ & \\
\hline 550 & 338 & s040 & 1001 & 1009 & 5 & Indeterminate & $\mathrm{NA}$ & \begin{tabular}{|l|l|}
0.04 \\
\end{tabular} & $\mathrm{NA}$ & Frag & No & None & $\mathrm{NA}$ & $\mathrm{NA}$ & NA & Trabecular & $\mathrm{NA}$ & \\
\hline 550 & 338 & s041 & 1001 & 1009 & 5 & Indeterminate & NA & 0.12 & $\mathrm{NA}$ & Frag & No & None & NA & $\mathrm{NA}$ & NA & Trabecular & $\mathrm{NA}$ & \\
\hline 550 & 338 & s042 & 1001 & 1009 & 5 & Indeterminate & $\mathrm{NA}$ & 0.11 & NA & Frag & No & None & $\mathrm{NA}$ & $\mathrm{NA}$ & $\mathrm{NA}$ & Trabecular & $\mathrm{NA}$ & \\
\hline 550 & 338 & s043 & 1001 & 1009 & 5 & Indeterminate & $\mathrm{NA}$ & \begin{tabular}{|l|}
0.07 \\
\end{tabular} & NA & Frag & No & None & NA & $\mathrm{NA}$ & NA & Trabecular & $\mathrm{NA}$ & \\
\hline 550 & 338 & s044 & 1001 & 1009 & 5 & Indeterminate & $\mathrm{NA}$ & 0.06 & $\mathrm{NA}$ & Frag & No & None & $\mathrm{NA}$ & $\mathrm{NA}$ & $\mathrm{NA}$ & Trabecular & $\mathrm{NA}$ & \\
\hline 550 & 338 & $\begin{array}{ll}\text { s045 } \\
\end{array}$ & 1001 & 1009 & 5 & Indeterminate & $\mathrm{NA}$ & 0.04 & \begin{tabular}{|l|}
$\mathrm{NA}$ \\
\end{tabular} & Frag & No & None & NA & $\mathrm{NA}$ & $\mathrm{NA}$ & Trabecular & $\mathrm{NA}$ & \\
\hline 550 & 338 & s046 & 1001 & 1009 & 5 & Indeterminate & NA & 0.10 & NA & Frag & No & None & NA & $\mathrm{NA}$ & NA & Trabecular & $\mathrm{NA}$ & \\
\hline 550 & 338 & s047 & 1001 & 1009 & 5 & Indeterminate & $\mathrm{NA}$ & 0.09 & $\mathrm{NA}$ & Frag & No & None & $\mathrm{NA}$ & $\mathrm{NA}$ & $\mathrm{NA}$ & Trabecular & $\mathrm{NA}$ & \\
\hline 550 & 338 & s048 & 1001 & 1009 & 5 & Indeterminate & $\mathrm{NA}$ & 0.06 & $\mathrm{NA}$ & Frag & No & None & NA & $\mathrm{NA}$ & NA & Trabecular & $\mathrm{NA}$ & \\
\hline 550 & 338 & s049 & 1001 & 1009 & 5 & Indeterminate & NA & 0.03 & $\mathrm{NA}$ & Frag & No & None & $\mathrm{NA}$ & $\mathrm{NA}$ & $\mathrm{NA}$ & Trabecular & $\mathrm{NA}$ & \\
\hline
\end{tabular}




\begin{tabular}{|c|c|c|c|c|c|c|c|c|c|c|c|c|c|c|c|c|c|c|}
\hline ò & 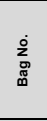 & 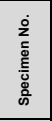 & 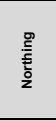 & 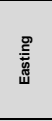 & $\bar{\Xi}$ & 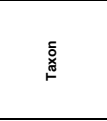 & 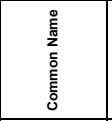 & 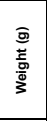 & 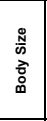 & 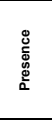 & 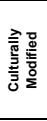 & 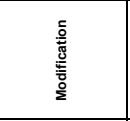 & $\begin{array}{l}\circ \\
\stackrel{0}{\circ} \\
\text { ஸे }\end{array}$ & $\frac{\pi}{\infty}$ & 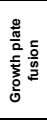 & 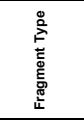 & $\begin{array}{l}\frac{0}{0} \\
\frac{1}{5} \\
\frac{5}{5} \\
\frac{5}{2} \\
\frac{\pi}{2}\end{array}$ & 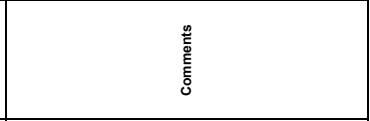 \\
\hline 550 & 338 & so50 & 1001 & 1009 & 5 & Indeterminate & NA & 0.01 & $\mathrm{NA}$ & Frag & No & None & NA & NA & NA & Trabecular & NA & \\
\hline 550 & 338 & s051 & 1001 & 1009 & 5 & Indeterminate & NA & 0.09 & $\mathrm{NA}$ & Frag & No & None & NA & NA & $\mathrm{NA}$ & Cortical & NA & \\
\hline 550 & 338 & S052 & 1001 & 1009 & 5 & Indeterminate & NA & 0.07 & $\mathrm{NA}$ & Frag & No & None & NA & NA & NA & Cortical & NA & \\
\hline 550 & 338 & s053 & 1001 & 1009 & 5 & Indeterminate & NA & 0.12 & $\mathrm{NA}$ & Frag & No & None & NA & NA & NA & Cortical & NA & \\
\hline 550 & 338 & S054 & 1001 & 1009 & 5 & Indeterminate & NA & 0.09 & NA & Frag & No & None & NA & NA & NA & Cortical & NA & \\
\hline 550 & 338 & S055 & 1001 & 1009 & 5 & Indeterminate & NA & 0.05 & NA & Frag & No & None & NA & NA & NA & Cortical & NA & \\
\hline 550 & 338 & s056 & 1001 & 1009 & 5 & Indeterminate & $\mathrm{NA}$ & 0.07 & NA & Frag & No & None & NA & NA & NA & Cortical & NA & \\
\hline 550 & 338 & S057 & 1001 & 1009 & 5 & Indeterminate & NA & 0.08 & $\mathrm{NA}$ & Frag & No & None & $\mathrm{NA}$ & NA & NA & Cortical & NA & \\
\hline 550 & 338 & S058 & 1001 & 1009 & 5 & Indeterminate & NA & 0.06 & NA & Frag & No & None & NA & NA & NA & Cortical & NA & \\
\hline 550 & 338 & s059 & 1001 & 1009 & 5 & Indeterminate & NA & 0.06 & NA & Frag & No & None & NA & NA & NA & Cortical & NA & \\
\hline 550 & 338 & so60 & 1001 & 1009 & 5 & Indeterminate & NA & 0.08 & NA & Frag & No & None & NA & NA & NA & Cortical & NA & \\
\hline 550 & 338 & s061 & 1001 & 1009 & 5 & Indeterminate & NA & 0.04 & $\mathrm{NA}$ & Frag & No & None & NA & NA & NA & Cortical & NA & \\
\hline 550 & 338 & S062 & 1001 & 1009 & 5 & Indeterminate & NA & 0.03 & NA & Frag & No & None & NA & NA & NA & Cortical & NA & \\
\hline 550 & 338 & so63 & 1001 & 1009 & 5 & Indeterminate & NA & 0.01 & NA & Frag & No & None & NA & NA & NA & Cortical & NA & \\
\hline 551 & 341 & soo1 & 1001 & 1009 & 6 & Mammal & NA & 3.36 & 2 & Frag & No & None & NA & NA & NA & Cortical & & Near epiphysis, long bone frag. Heavily weathered. \\
\hline 551 & 341 & s002 & 1001 & 1009 & 6 & Indeterminate & NA & 0.26 & $\mathrm{NA}$ & Frag & No & None & $\mathrm{NA}$ & NA & NA & Cortical & & Small cortical frag \\
\hline 552 & 347 & so01 & 1001 & 1009 & 7 & Tortoise/Turtle & Tortoise/Turtle & 0.13 & 1 & Frag & No & None & carapace & NA & NA & NA & & \\
\hline 552 & 347 & soo2 & 1001 & 1009 & 7 & Indeterminate & NA & 0.3 & NA & Frag & No & None & NA & NA & NA & Cortical & Right & Burned-nearly calcined \\
\hline 552 & 347 & so03 & 1001 & 1009 & 7 & Indeterminate & NA & 0.71 & NA & Frag & No & None & NA & NA & NA & Cortical & Right & Long bone- Possibly bird, not sure \\
\hline 559 & 360 & so01 & 1001 & 1010 & 4 & Indeterminate & NA & 1.2 & NA & Frag & No & None & NA & NA & NA & Cortical & & Large cortical frag, severely weathered. \\
\hline 559 & 360 & so02 & 1001 & 1010 & 4 & Indeterminate & NA & 1.21 & NA & Frag & No & None & NA & NA & NA & Cortical & & Large cortical frag, severely weathered. \\
\hline 561 & 367 & soo1 & 1001 & 1010 & 5 & Indeterminate & NA & 0.22 & $\mathrm{NA}$ & Frag & No & None & NA & NA & $\mathrm{NA}$ & Cortical & Right & \\
\hline 561 & 367 & so02 & 1001 & 1010 & 5 & Indeterminate & NA & 0.21 & $\mathrm{NA}$ & Frag & No & None & NA & NA & NA & Cortical & Right & Possibly turtle/tortoise \\
\hline 564 & 373 & soo1 & 1001 & 1010 & 6 & Indeterminate & NA & 1.14 & NA & Frag & No & None & NA & NA & $\mathrm{NA}$ & Cortical & Fresh & \\
\hline 564 & 373 & s002 & 1001 & 1010 & 6 & Indeterminate & NA & 1.38 & $\mathrm{NA}$ & Frag & No & None & NA & NA & $\mathrm{NA}$ & Cortical & Oblique & \\
\hline 564 & 373 & so03 & 1001 & 1010 & 6 & Indeterminate & NA & 0.26 & $\mathrm{NA}$ & Frag & No & None & NA & NA & NA & Cortical & Oblique & \\
\hline 564 & 373 & so04 & 1001 & 1010 & 6 & Indeterminate & NA & 0.3 & $\mathrm{NA}$ & Frag & No & None & NA & NA & NA & Cortical & $\mathrm{NA}$ & \\
\hline 564 & 373 & so05 & 1001 & 1010 & 6 & Indeterminate & $\mathrm{NA}$ & 0.33 & $\mathrm{NA}$ & Frag & No & None & $\mathrm{NA}$ & NA & $\mathrm{NA}$ & Cortical & NA & \\
\hline 564 & 373 & s006 & 1001 & 1010 & 6 & Indeterminate & $\mathrm{NA}$ & 0.22 & $\mathrm{NA}$ & Frag & No & None & $\mathrm{NA}$ & NA & NA & Cortical & $\mathrm{NA}$ & \\
\hline 564 & 373 & s007 & 1001 & 1010 & 6 & Indeterminate & $\mathrm{NA}$ & 0.19 & NA & Frag & No & None & NA & NA & NA & Cortical & $\mathrm{NA}$ & \\
\hline 564 & 373 & so08 & 1001 & 1010 & 6 & Indeterminate & $\mathrm{NA}$ & 0.01 & $\mathrm{NA}$ & Frag & No & None & NA & NA & $\mathrm{NA}$ & Cortical & NA & \\
\hline 564 & 373 & soog & 1001 & 1010 & 6 & Indeterminate & NA & 0.01 & $\mathrm{NA}$ & Frag & No & None & NA & NA & $\mathrm{NA}$ & Cortical & NA & \\
\hline 564 & 373 & so10 & 1001 & 1010 & 6 & Indeterminate & $\mathrm{NA}$ & 0.01 & $\mathrm{NA}$ & Frag & No & None & $\mathrm{NA}$ & $\mathrm{NA}$ & $\mathrm{NA}$ & Cortical & $\mathrm{NA}$ & \\
\hline 567 & 384 & soo1 & 1001 & 1010 & 8 & Indeterminate & NA & 0.08 & $\mathrm{NA}$ & Frag & No & None & NA & NA & $\mathrm{NA}$ & Cortical & & Small, heavily weathered frag. \\
\hline 567 & 384 & soo2 & 1001 & 1010 & 8 & Indeterminate & NA & 0.16 & NA & Frag & No & None & NA & NA & NA & Cortical & & $\begin{array}{l}\text { well preserved (unlike S001), but too small to make a } \\
\text { determination. }\end{array}$ \\
\hline 570 & 416 & so01 & 1001 & 1010 & 12 & Indeterminate & NA & 0.73 & NA & Frag & No & None & NA & NA & NA & Trabecular & & Trabecular bone frag, unable to identify. \\
\hline 570 & 416 & so02 & 1001 & 1010 & 12 & Indeterminate & NA & 0.74 & NA & Frag & No & None & NA & NA & NA & Cortical & & $\begin{array}{l}\text { Heavily weathered. Weathering has destroyed any } \\
\text { landmarks. }\end{array}$ \\
\hline 571 & 425 & soo1 & 1001 & 1010 & 13 & Indeterminate & NA & 1.25 & $\mathrm{NA}$ & Frag & No & None & NA & NA & NA & Cortical & Right & Possible chop marks \\
\hline 571 & 425 & so02 & 1001 & 1010 & 13 & Indeterminate & $\mathrm{NA}$ & 1.13 & $\mathrm{NA}$ & Frag & No & None & $\mathrm{NA}$ & $\mathrm{NA}$ & NA & Cortical & Fresh & \\
\hline 571 & 425 & s003 & 1001 & 1010 & 13 & Indeterminate & $\mathrm{NA}$ & 1.13 & $\mathrm{NA}$ & Frag & No & None & NA & NA & NA & Cortical & Fresh & \\
\hline 571 & 425 & so04 & 1001 & 1010 & 13 & Indeterminate & NA & 1.36 & $\mathrm{NA}$ & Frag & No & None & NA & NA & NA & Cortical & Fresh & \\
\hline 571 & 425 & $\mathbf{s 0 0 5}$ & 1001 & 1010 & 13 & Indeterminate & NA & 0.09 & $\mathrm{NA}$ & Frag & No & None & NA & NA & NA & Cortical & Fresh & \\
\hline 574 & 606 & soo1 & 1001 & 1010 & 16 & Indeterminate & $\mathrm{NA}$ & 0.12 & $\mathrm{NA}$ & Frag & No & None & NA & NA & $\mathrm{NA}$ & Cortical & Fresh & \\
\hline 574 & 606 & s002 & 1001 & 1010 & 16 & Indeterminate & NA & 0.05 & $\mathrm{NA}$ & Frag & No & None & NA & NA & NA & Cortical & Fresh & \\
\hline 576 & 616 & soo1 & 1001 & 1010 & - & Odocoileus $s p$ & Deer & 4.65 & 2 & Frag & Yes & Percussion Notch & Metapodial & NA & NA & Cortical & & $\begin{array}{l}\text { Mid-shaft metaposial frag with percussion notch and } \\
\text { hertzian cone in margin. Spiral fracture. Marrow } \\
\text { processed. Edges of the margin are polished (possibly pot } \\
\text { polishing). }\end{array}$ \\
\hline 578 & 637 & soo1 & 1001.5 & 1004 & 2 & Indeterminate & NA & 1.17 & NA & Frag & No & None & NA & NA & NA & Cortical & & Distorted by weathering \\
\hline 578 & 637 & s002 & 1001.5 & 1004 & 2 & Indeterminate & NA & 0.61 & NA & Frag & No & None & NA & NA & NA & Cortical & & Distorted by weathering \\
\hline 579 & 643 & so01 & 1001.5 & 1004 & 3 & Odocoileus sp & Deer & 3.63 & 2 & whole & No & None & Tooth (M1) & Right & NA & NA & & $\begin{array}{l}\begin{array}{l}\text { Possibly an M2. Only slightly worn, and appears under- } \\
\text { developed. Juvenile }\end{array} \\
\end{array}$ \\
\hline 579 & 643 & so02 & 1001.5 & 1004 & 3 & Indeterminate & NA & 0.41 & $\mathrm{NA}$ & Frag & No & None & NA & NA & NA & Trabecular & & Trabecular bone frag, unable to identify. \\
\hline 579 & 643 & so03 & 1001.5 & 1004 & 3 & Indeterminate & NA & 0.1 & $\mathrm{NA}$ & Frag & No & None & NA & NA & NA & Cortical & & Too small to identify. \\
\hline 579 & 643 & soo4 & 1001.5 & 1004 & 3 & Indeterminate & NA & 0.01 & NA & Frag & No & None & NA & NA & NA & Cortical & & Too small to identify. \\
\hline 579 & 643 & so05 & 1001.5 & 1004 & 3 & Indeterminate & NA & 0.01 & NA & Frag & No & None & NA & NA & NA & Cortical & & Too small to identify. \\
\hline 580 & 648 & soo1 & 1001.5 & 1004 & 4 & Mammal & NA & 7.62 & NA & Frag & No & None & NA & NA & NA & Cortical & & Very large cortical frag. Heavily weathered surface. \\
\hline 580 & 648 & so02 & 1001.5 & 1004 & 4 & Mammal & NA & 4.23 & NA & Frag & No & None & NA & NA & NA & Cortical & & Very large cortical frag. Heavily weathered surface. \\
\hline 580 & 648 & s003 & 1001.5 & 1004 & 4 & Indeterminate & NA & 0.24 & NA & Frag & No & None & NA & NA & NA & Trabecular & & Trabecular bone frag, unable to identify. \\
\hline
\end{tabular}




\begin{tabular}{|c|c|c|c|c|c|c|c|c|c|c|c|c|c|c|c|c|c|c|}
\hline soj & 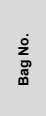 & 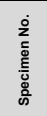 & 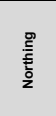 & 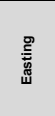 & ฮ্ & 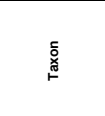 & 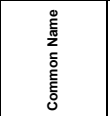 & $\begin{array}{l}\text { 힘 } \\
\text { 흠 } \\
\text { कू } \\
3\end{array}$ & $\begin{array}{l}\frac{N}{n} \\
\text { ते } \\
\text { ֻे }\end{array}$ & 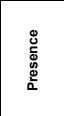 & 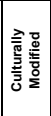 & 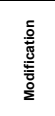 & 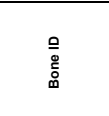 & $\frac{\circ}{0}$ & 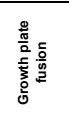 & 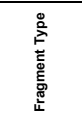 & 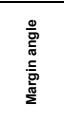 & 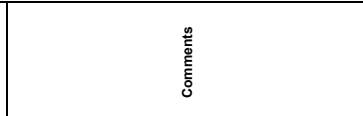 \\
\hline 580 & 648 & S004 & 1001.5 & 1004 & 4 & Tortoise/Turtle & Tortoise/Turtle & 0.09 & 1 & Frag & No & None & Plastron & NA & NA & NA & & Very small plastron frag. \\
\hline 580 & 648 & S005 & 1001.5 & 1004 & 4 & Indeterminate & NA & 0.14 & NA & Frag & No & None & NA & $\mathrm{NA}$ & NA & Cortical & & Very small cortical frag. \\
\hline 580 & 648 & s006 & 1001.5 & 1004 & 4 & Indeterminate & NA & 0.04 & $\mathrm{NA}$ & Frag & No & None & NA & NA & NA & Cortical & & Very small cortical frag. \\
\hline 580 & 648 & S007 & 1001.5 & 1004 & 4 & Indeterminate & NA & 0.01 & NA & Frag & No & None & NA & NA & NA & Cortical & & Very small cortical frag. \\
\hline 580 & 648 & soos & 1001.5 & 1004 & 4 & Indeterminate & NA & 0.01 & $\mathrm{NA}$ & Frag & No & None & $\mathrm{NA}$ & $\mathrm{NA}$ & NA & Cortical & & Very small cortical frag. \\
\hline 580 & 648 & soog & 1001.5 & 1004 & 4 & Indeterminate & NA & 0.01 & NA & Frag & No & None & NA & NA & NA & Cortical & & Very small cortical frag. \\
\hline 581 & 651 & s001 & 1001.5 & 1005 & 2 & Indeterminate & NA & 0.08 & $\mathrm{NA}$ & Frag & No & None & $\mathrm{NA}$ & $\mathrm{NA}$ & NA & Cortical & Oblique & \\
\hline 581 & 651 & S002 & 1001.5 & 1005 & 2 & Indeterminate & NA & 0.26 & $\mathrm{NA}$ & Frag & No & None & $\mathrm{NA}$ & $\mathrm{NA}$ & NA & Cortical & Right & \\
\hline 582 & 654 & s001 & 1001.5 & 1005 & 3 & Indeterminate & NA & 0.18 & NA & Frag & No & None & NA & NA & NA & Cortical & & $\begin{array}{l}\text { Small cortical frag. No landmarks. One fracture margin is } \\
\text { right, the other is acute. }\end{array}$ \\
\hline 588 & 527 & s001 & 1003 & 1017 & 5 & Indeterminate & NA & 0.22 & NA & Frag & No & None & NA & NA & NA & Cortical & NA & Burned-calcined \\
\hline 590 & 557 & soo1 & 1003 & 1017 & 6 & Indeterminate & NA & 0.28 & NA & Frag & No & None & NA & NA & NA & Cortical & Right & Burned \\
\hline 596 & 574 & soo1 & 1003 & 1017 & 9 & Indeterminate & NA & 0.73 & NA & Frag & No & None & NA & NA & NA & Cortical & NA & Possibly turtle/tortoise \\
\hline 596 & 574 & s002 & 1003 & 1017 & 9 & Indeterminate & NA & 0.01 & NA & Frag & No & None & NA & NA & NA & Cortical & NA & \\
\hline 596 & 574 & s003 & 1003 & 1017 & 9 & Indeterminate & NA & 0.01 & NA & Frag & No & None & NA & NA & NA & Cortical & NA & \\
\hline 608 & 510 & s001 & 1003 & 1018 & 8 & Indeterminate & NA & 0.23 & NA & Frag & No & None & NA & NA & NA & Cortical & Fresh & \\
\hline 612 & 629 & s001 & 1003 & 1018 & 13 & Indeterminate & NA & 1.21 & NA & Frag & No & None & NA & NA & NA & Cortical & & Looks like an auditory meatus \\
\hline 618 & 479 & soo1 & 1003 & 1019 & 7 & Indeterminate & NA & 0.07 & NA & Frag & No & None & NA & NA & NA & Cortical & Oblique & \\
\hline 626 & 591 & s001 & 1003 & 1019 & 13 & Odocoileus sp & Deer & 2.48 & 2 & Frag & No & None & Tooth (molar) & NA & NA & NA & & $\begin{array}{l}\text { Not enough remains to determine which molar. Refits with } \\
\text { S002 }\end{array}$ \\
\hline 626 & 591 & so02 & 1003 & 1019 & 13 & Odocoileus sp & Deer & 0.59 & 2 & Frag & No & None & Tooth (molar) & NA & $\mathrm{NA}$ & NA & & $\begin{array}{l}\text { Not enough remains to determine which molar. Refits with } \\
\text { s001 }\end{array}$ \\
\hline 626 & 591 & so03 & 1003 & 1019 & 13 & Indeterminate & NA & 0.81 & NA & NA & No & None & $\mathrm{NA}$ & NA & NA & NA & & $\begin{array}{l}\text { Enamel frag with some dentin. Not enough to determine } \\
\text { size or shape. Probably associate with other teeth in the } \\
\text { level. }\end{array}$ \\
\hline 626 & 591 & S004 & 1003 & 1019 & 13 & Indeterminate & NA & 0.28 & NA & NA & No & None & NA & $\mathrm{NA}$ & NA & NA & & $\begin{array}{l}\text { Enamel frag with some dentin. Not enough to determine } \\
\text { size or shape. Probably associate with other teeth in the } \\
\text { level. }\end{array}$ \\
\hline 626 & 591 & S005 & 1003 & 1019 & 13 & Indeterminate & NA & 0.42 & NA & NA & No & None & NA & NA & $\mathrm{NA}$ & NA & & $\begin{array}{l}\text { Enamel frag with some dentin. Not enough to determine } \\
\text { size or shape. Probably associate with other teeth in the } \\
\text { level. }\end{array}$ \\
\hline 631 & 376 & S001 & 1004 & 1017 & 5 & Indeterminate & NA & 1.26 & NA & NA & No & None & $\mathrm{NA}$ & $\mathrm{NA}$ & NA & Cortical & & $\begin{array}{l}\text { Just an articular surface, not enough to determine from } \\
\text { what element. Possibly a growth plate from an epiphysis. }\end{array}$ \\
\hline 632 & 385 & s001 & 1004 & 1017 & 6 & Odocoileus sp & Deer & 1.18 & 2 & whole & No & None & Tooth (M1) & NA & NA & NA & & $\begin{array}{l}\text { The majority of a cervid M1. Very heavily weathered, nut } \\
\text { does not appear to be worn. Juvenile. }\end{array}$ \\
\hline 645 & 359 & \begin{tabular}{|l}
5001 \\
\end{tabular} & 1004 & 1018 & 3 & Indeterminate & NA & \begin{tabular}{|l|}
0.21 \\
\end{tabular} & $\mathrm{NA}$ & Frag & № & None & NA & $\mathrm{NA}$ & $\mathrm{NA}$ & Cortical & & Distorted by weathering \\
\hline 662 & 533 & soo1 & 1004 & 1019 & 8 & Mammal & NA & 1.21 & 1 & Frag & No & None & Femur & Left & NA & Cortical & & $\begin{array}{l}\begin{array}{l}\text { Near proximal epiphysis, long bone frag. Distorted by } \\
\text { weathering. }\end{array} \\
\text {. }\end{array}$ \\
\hline 668 & 588 & s001 & 1004 & 1019 & 12 & Mammal & NA & 3.93 & NA & Frag & No & None & NA & NA & NA & Cortical & & Long bone frag, not enough to determine element. \\
\hline 673 & 344 & soo1 & 1009 & 1011 & 2 & Indeterminate & NA & 0.39 & NA & Frag & No & None & NA & NA & NA & Cortical & NA & Probably avian, but can't make determination. \\
\hline 676 & 366 & S001 & 1009 & 1011 & 5 & Indeterminate & $\mathrm{NA}$ & 1.09 & $\mathrm{NA}$ & Frag & No & None & $\mathrm{NA}$ & $\mathrm{NA}$ & $\mathrm{NA}$ & Cortical & & Not enough present to distinguish. \\
\hline 688 & 352 & so01 & 1009 & 1012 & 2 & Avian & NA & 1.09 & NA & Frag & No & None & Tibia & Right & No & Epiphysis & & $\begin{array}{l}\text { Distal and midshaft tibia. Refits with S002-the astragalus. } \\
\text { These aren't fused, so possibly a juvenile. Smaller than a } \\
\text { domestic chicken, larger than a dove. }\end{array}$ \\
\hline 688 & 352 & s002 & 1009 & 1012 & 2 & Avian & NA & 0.12 & NA & Whole & No & None & Astragalus & Right & No & Whole & & $\begin{array}{l}\text { Refits with S001- These aren't fused, so possibly a } \\
\text { juvenile. }\end{array}$ \\
\hline 688 & 352 & so03 & 1009 & 1012 & 2 & Indeterminate & NA & 0.13 & NA & whole & No & None & Carpal/Tarsal? & $\mathrm{NA}$ & NA & Whole & & $\begin{array}{l}\text { It looks to be part of the avian skeleton in this level, but I } \\
\text { am unsure of what bone it is. }\end{array}$ \\
\hline 688 & 352 & S004 & 1009 & 1012 & 2 & Avian & NA & 0.43 & NA & Frag & No & None & $\mathrm{NA}$ & $\mathrm{NA}$ & NA & Cortical & & $\begin{array}{l}\begin{array}{l}\text { Appears to be a corocoid frag. Not enough to be } \\
\text { definitive. }\end{array} \\
\end{array}$ \\
\hline 688 & 352 & s005 & 1009 & 1012 & 2 & Indeterminate & NA & 0.17 & NA & Frag & No & None & NA & NA & NA & Cortical & & $\begin{array}{l}\text { It looks to be part of the avian skeleton in this level, but I } \\
\text { am unsure of what bone it is. }\end{array}$ \\
\hline 689 & 357 & S001 & 1009 & 1012 & 3 & Indeterminate & NA & 0.48 & NA & Frag & No & None & NA & NA & NA & Cortical & Right & \\
\hline 697 & 551 & s001 & 1009 & 1012 & 10 & Odocoileus $s p$ & Deer & 17.57 & 2 & Frag & No & None & Humerus & Right & Fused & Epiphysis & & $\begin{array}{l}\text { Distal humerus, weathered surface. Portion pulled for } \\
\text { dating. Broken into several pieces to obtain sample. }\end{array}$ \\
\hline 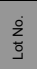 & 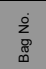 & 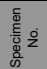 & $\begin{array}{l}\frac{9}{\underline{\underline{E}}} \\
\frac{\mathrm{E}}{\mathrm{c}} \\
\mathrm{z}\end{array}$ & 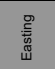 & $\stackrel{\bar{g}}{\ddot{g}}$ & & & & 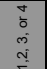 & 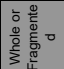 & 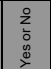 & & & & 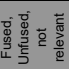 & 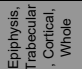 & & \\
\hline
\end{tabular}




\begin{tabular}{|c|c|c|c|c|c|c|c|c|c|c|c|c|c|c|}
\hline $\begin{array}{l}\text { ì } \\
\text { to }\end{array}$ & 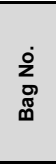 & 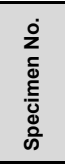 & $\begin{array}{l}\text { 일 } \\
\stackrel{\Xi}{E} \\
\text { 늠 }\end{array}$ & 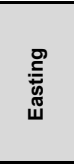 & ग्త & 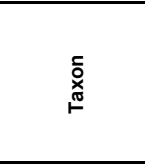 & 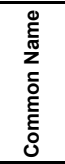 & $\begin{array}{l}\text { 오 } \\
\text { t. } \\
. \frac{0}{0} \\
3\end{array}$ & $\begin{array}{l}\stackrel{N}{N} \\
\text { क) } \\
\text { ते } \\
\text { வ }\end{array}$ & 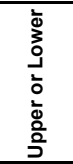 & 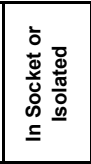 & $\frac{0}{\infty}$ & $\begin{array}{l}\text { 으 } \\
\text { 호 } \\
\stackrel{\circ}{\circ}\end{array}$ & 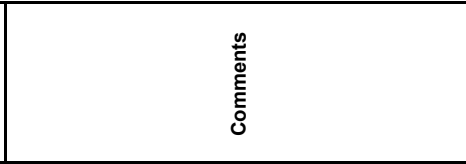 \\
\hline 329 & 628 & S001 & 995 & 1012 & 12 & NA & NA & 0.06 & NA & NA & NA & NA & NA & $\begin{array}{l}\text { Small enamel fragment. Not enough to make a } \\
\text { determination. }\end{array}$ \\
\hline 366 & 470 & S001 & 996 & 1012 & 1 & NA & NA & 0.07 & NA & NA & NA & NA & NA & $\begin{array}{l}\text { Small enamel fragment. Probably cervid, but not } \\
\text { enough to make a determination. }\end{array}$ \\
\hline 389 & 451 & S001 & 996 & 1013 & 5 & Odocoileus sp & Deer & 2.3 & 2 & Upper & Isolated & Left & M1 or M2 & Bucchal frag \\
\hline 406 & 503 & S001 & 996 & 1014 & 7 & Odocoileus sp & Deer & 1.79 & 2 & Upper & Isolated & Left & M1 & $\begin{array}{l}\text { The majority of a cervid M1. Very lightly worn, does } \\
\text { not appear to be fully developed. . Juvenile. }\end{array}$ \\
\hline 542 & 546 & S010 & 1001 & 1004.5 & 4 & Odocoileus sp & Deer & 0.54 & 2 & NA & Isolated & NA & NA & Not enough remains to determine which tooth. \\
\hline 579 & 643 & S001 & 1001.5 & 1004 & 3 & Odocoileus sp & Deer & 3.63 & 2 & NA & Isolated & Right & M1 & $\begin{array}{l}\text { Possibly an M2. Only slightly worn, and appears } \\
\text { under-developed. Juvenile }\end{array}$ \\
\hline 626 & 591 & S001 & 1003 & 1019 & 13 & Odocoileus sp & Deer & 2.48 & 2 & NA & Isolated & NA & NA & $\begin{array}{l}\text { Not enough remains to determine which molar. } \\
\text { Refits with } \mathrm{S} 002\end{array}$ \\
\hline 626 & 591 & S002 & 1003 & 1019 & 13 & Odocoileus sp & Deer & 0.59 & 2 & NA & Isolated & NA & NA & $\begin{array}{l}\text { Not enough remains to determine which molar. } \\
\text { Refits with S001 }\end{array}$ \\
\hline 626 & 591 & S003 & 1003 & 1019 & 13 & NA & NA & 0.81 & NA & NA & Isolated & NA & NA & $\begin{array}{l}\text { Not enough remains to determine size and shape. } \\
\text { Probably associated with other tooth frags in level. }\end{array}$ \\
\hline 626 & 591 & S004 & 1003 & 1019 & 13 & NA & NA & 0.28 & NA & NA & Isolated & NA & NA & $\begin{array}{l}\text { Not enough remains to determine size and shape. } \\
\text { Probably associated with other tooth frags in level. }\end{array}$ \\
\hline 626 & 591 & S005 & 1003 & 1019 & 13 & NA & NA & 0.42 & NA & NA & Isolated & NA & NA & $\begin{array}{l}\text { Not enough remains to determine size and shape. } \\
\text { Probably associated with other tooth frags in level. }\end{array}$ \\
\hline 632 & 385 & S001 & 1004 & 1017 & 6 & Odocoileus $s p$ & Deer & 1.18 & 2 & NA & Isolated & NA & M1 & Very heavily weathered, but not worn. Juvenile \\
\hline
\end{tabular}




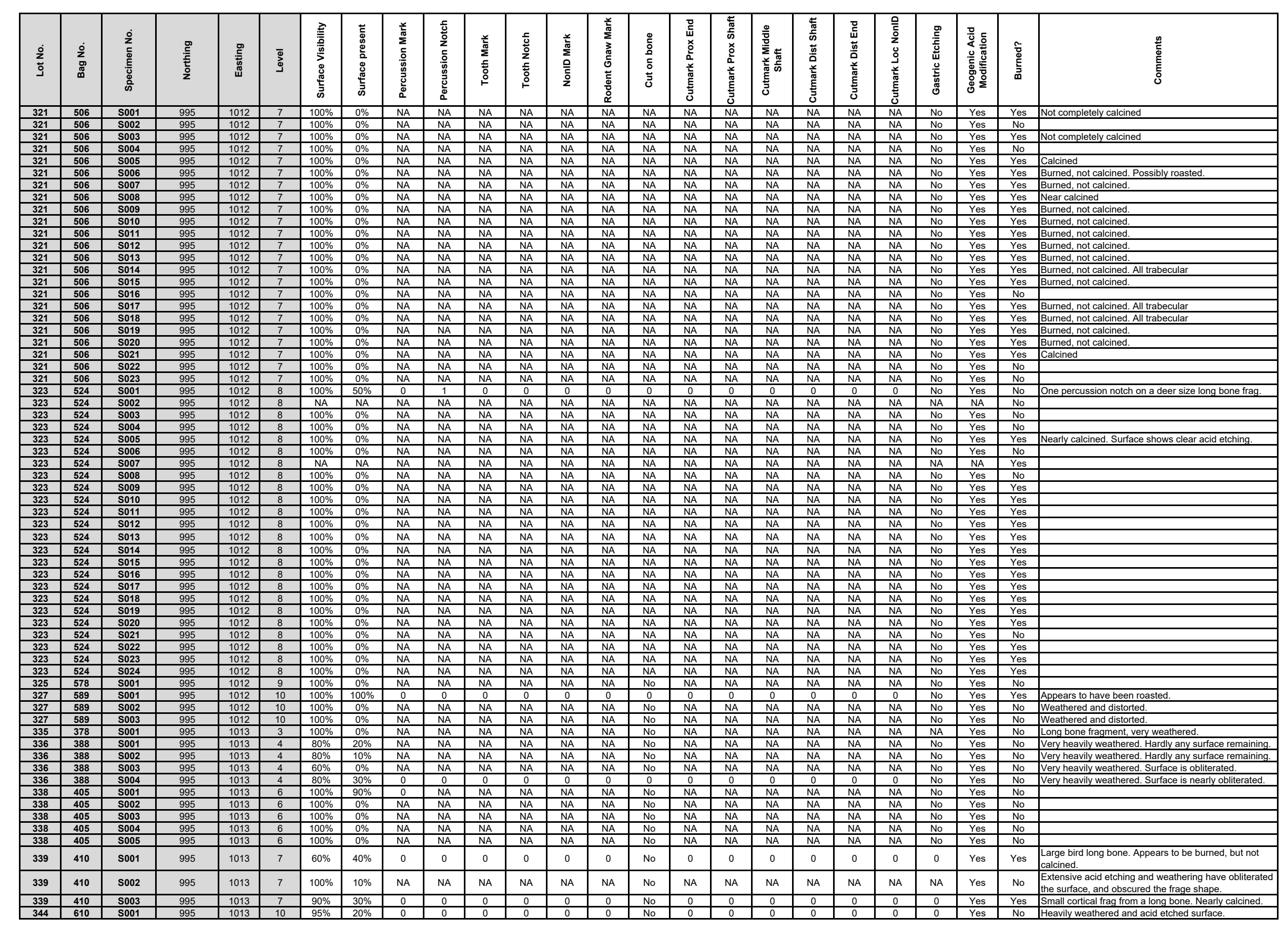




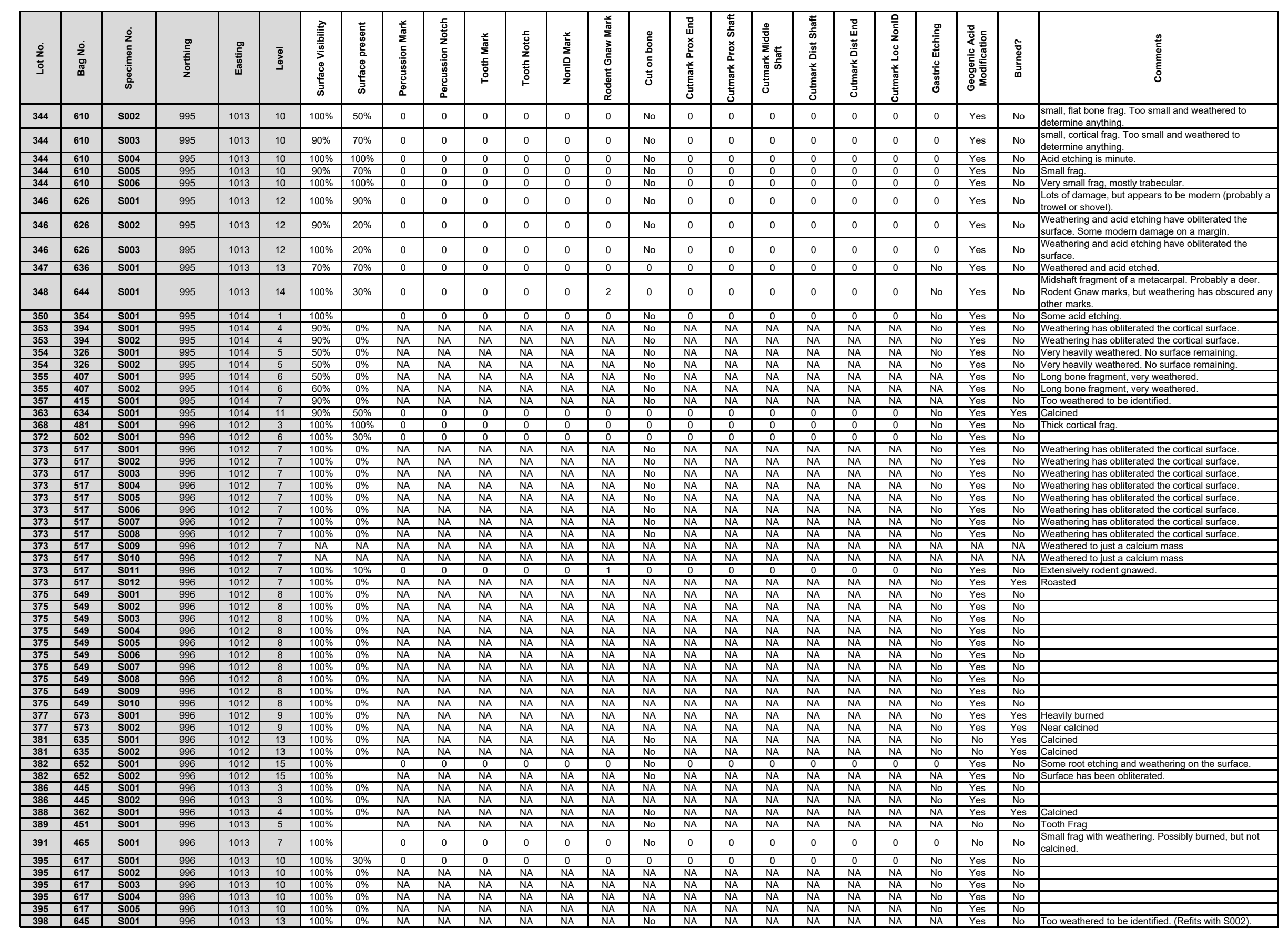




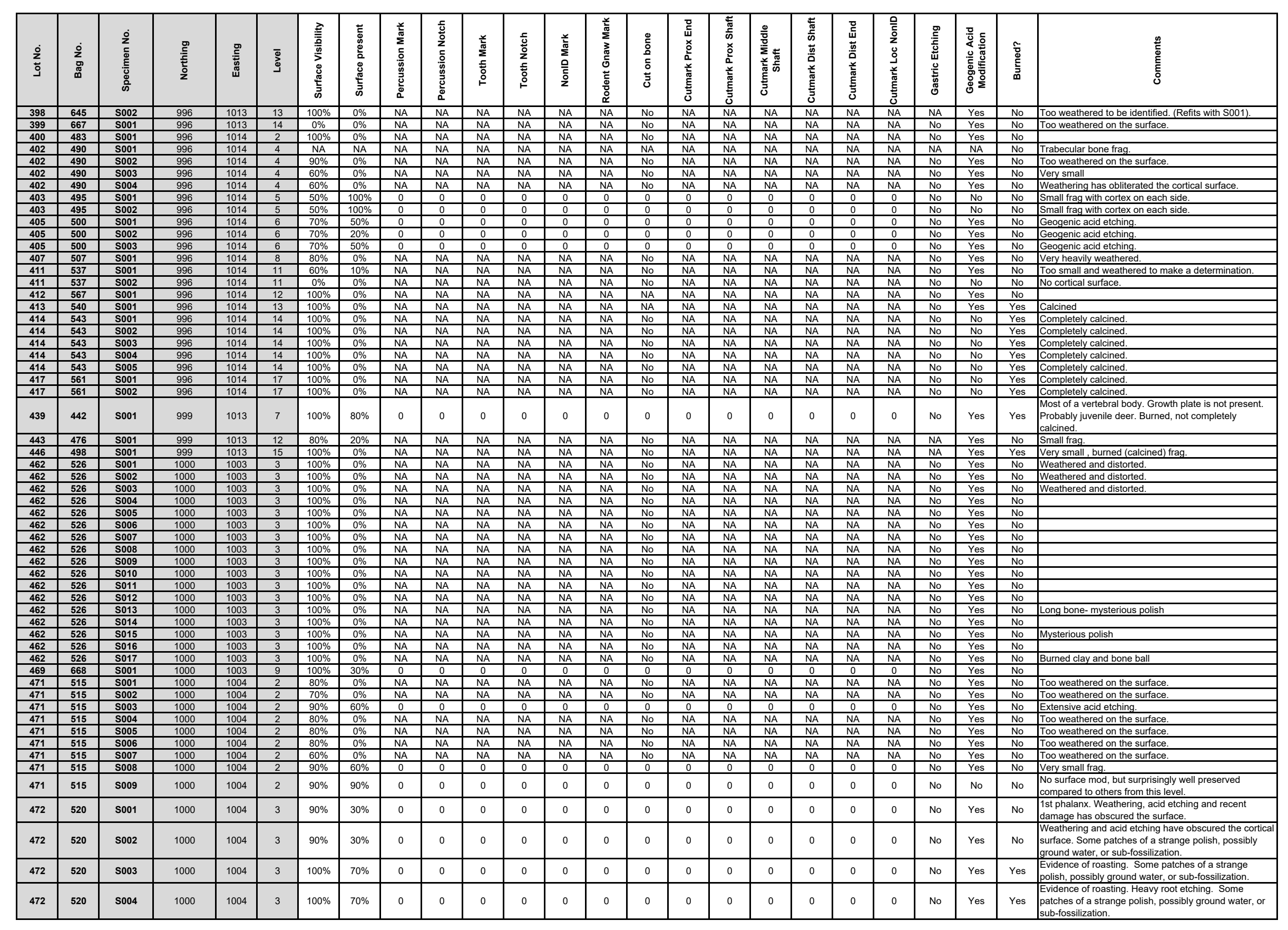




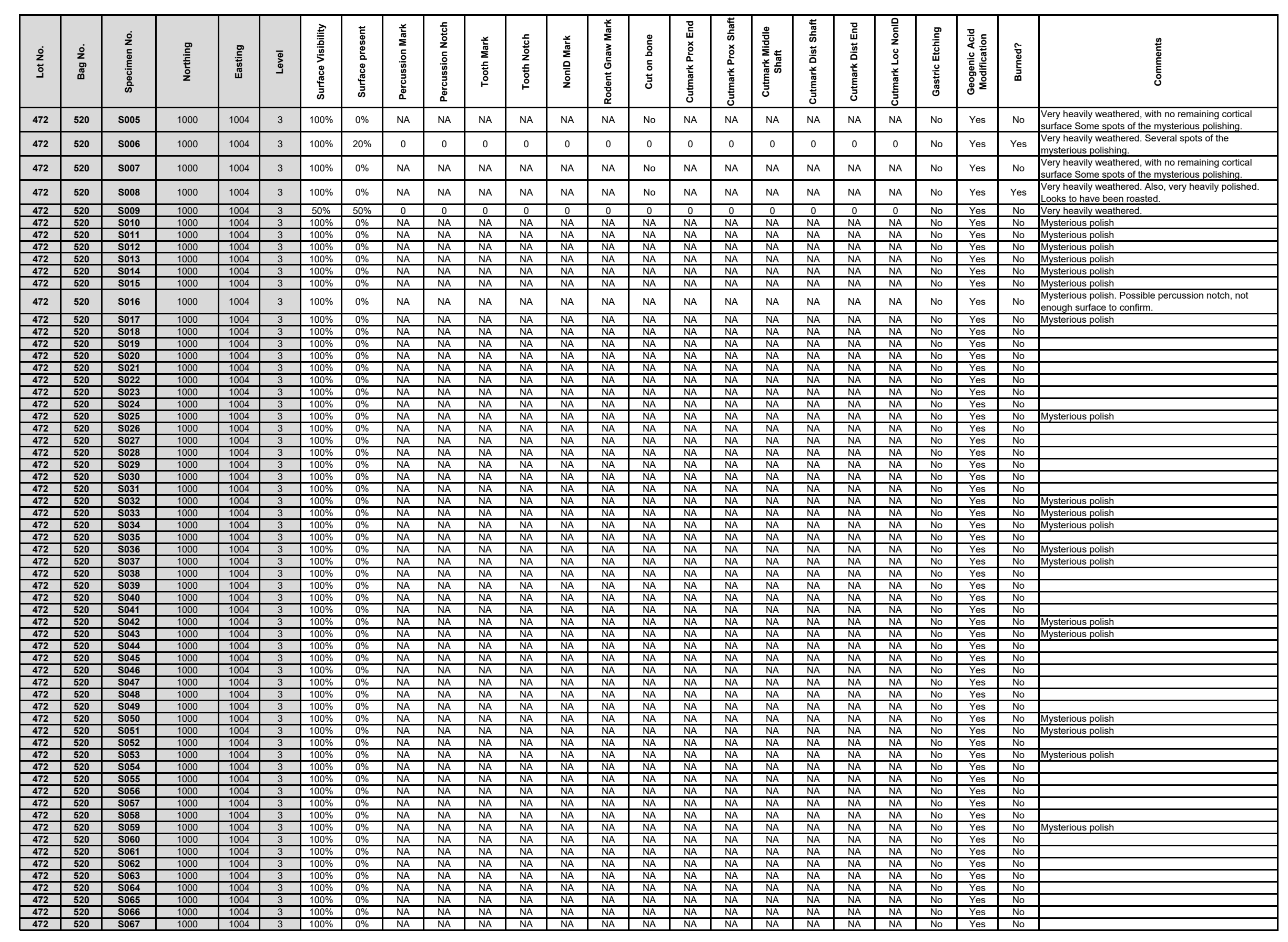




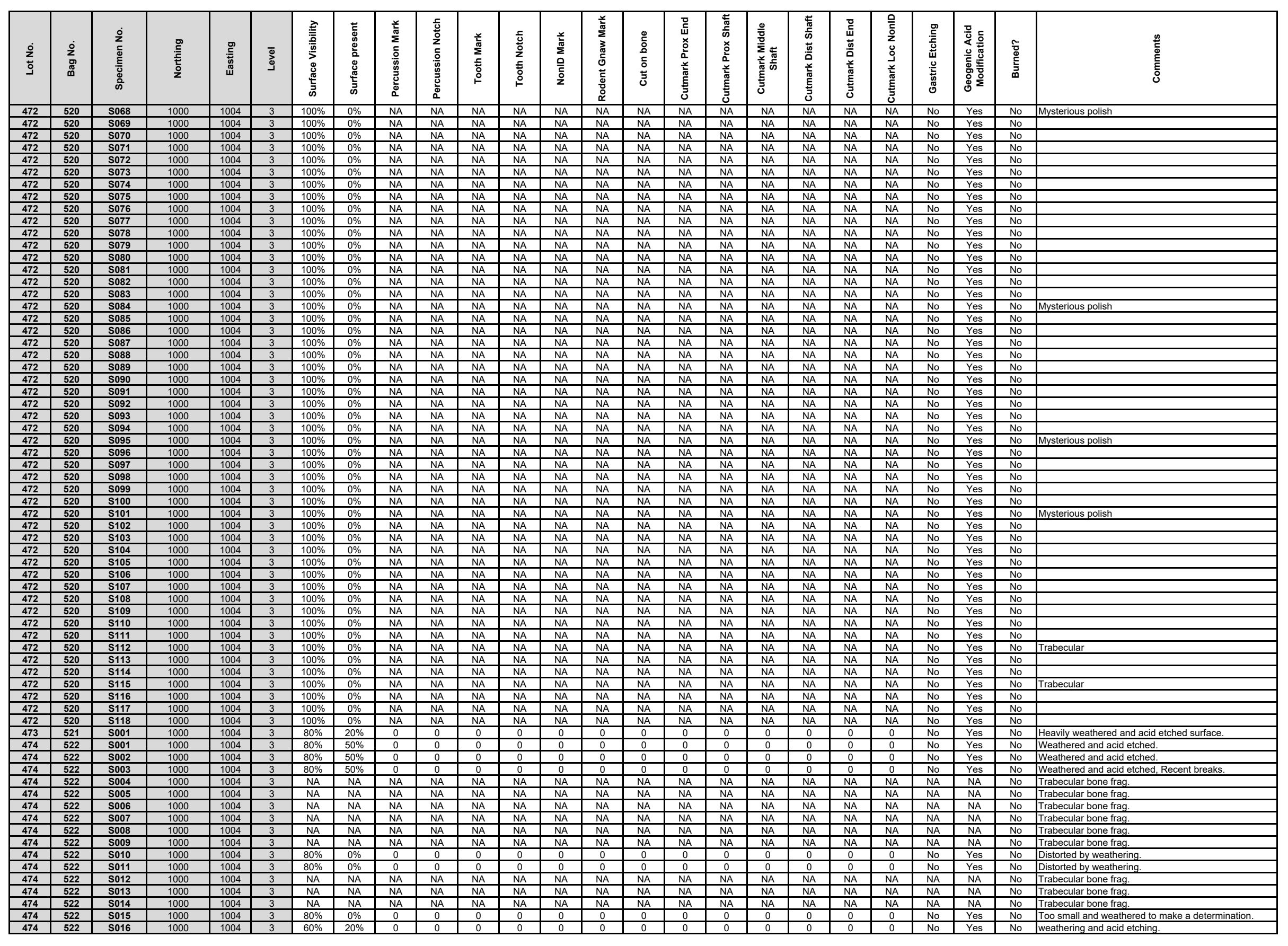




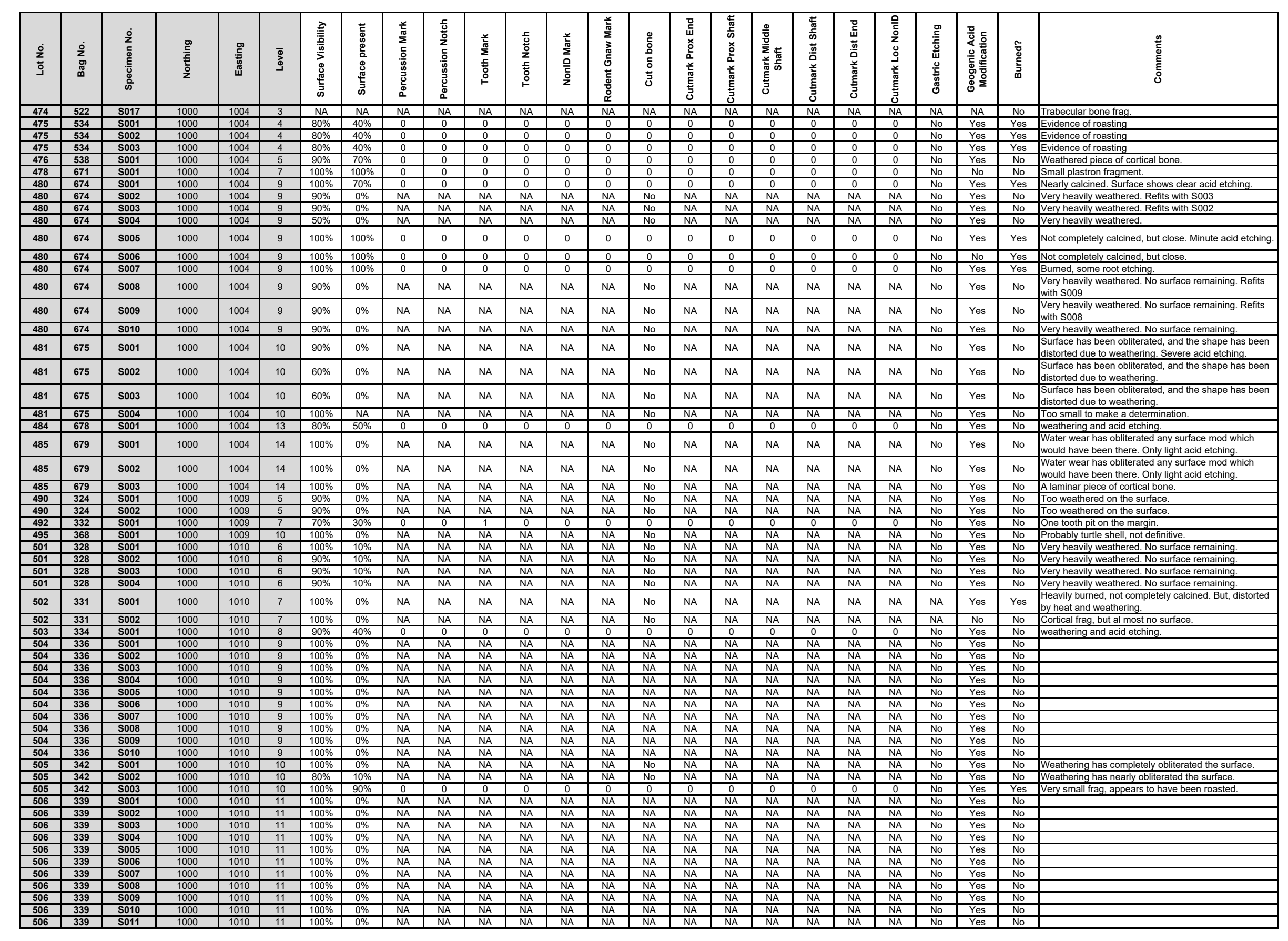




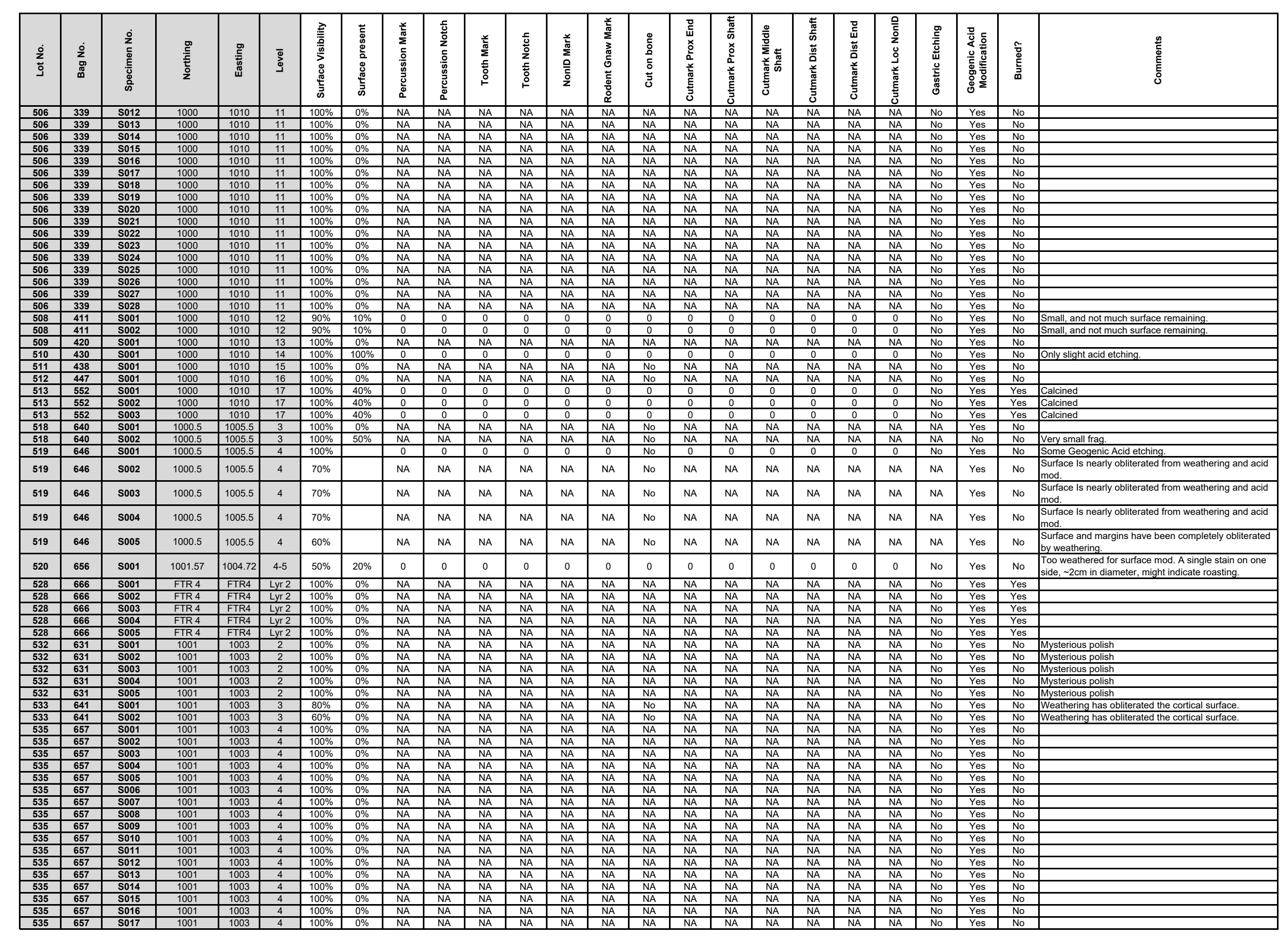




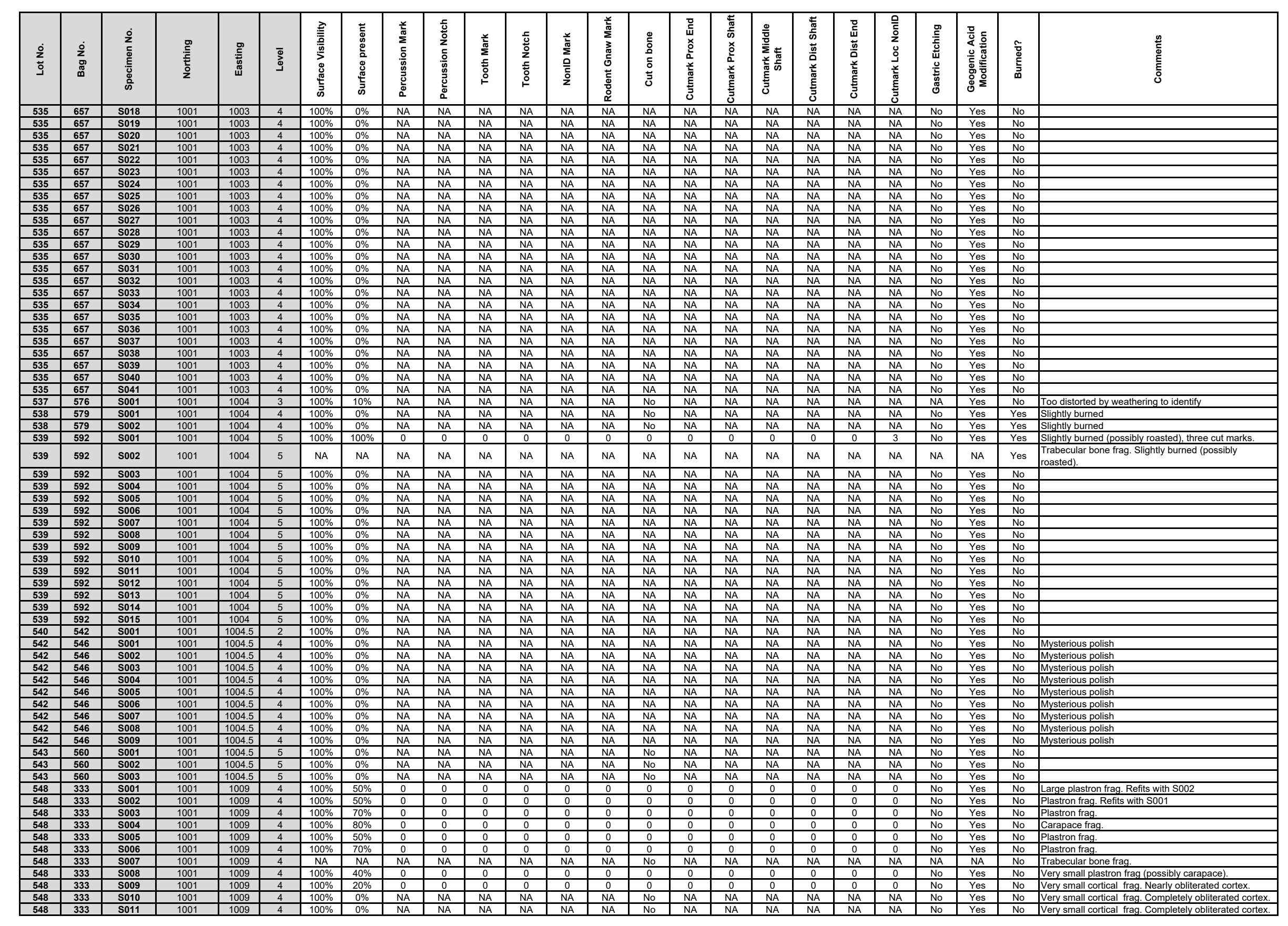




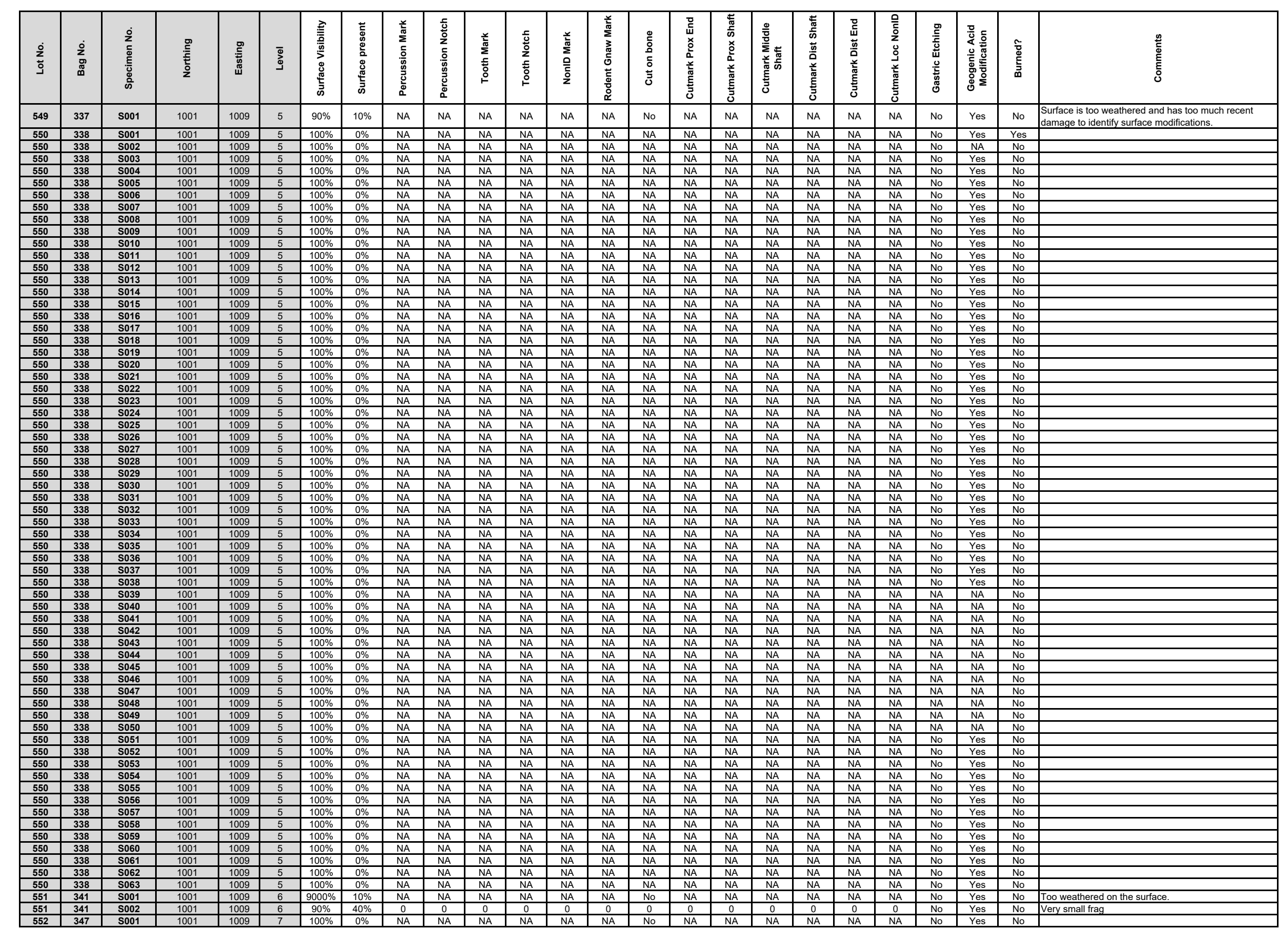




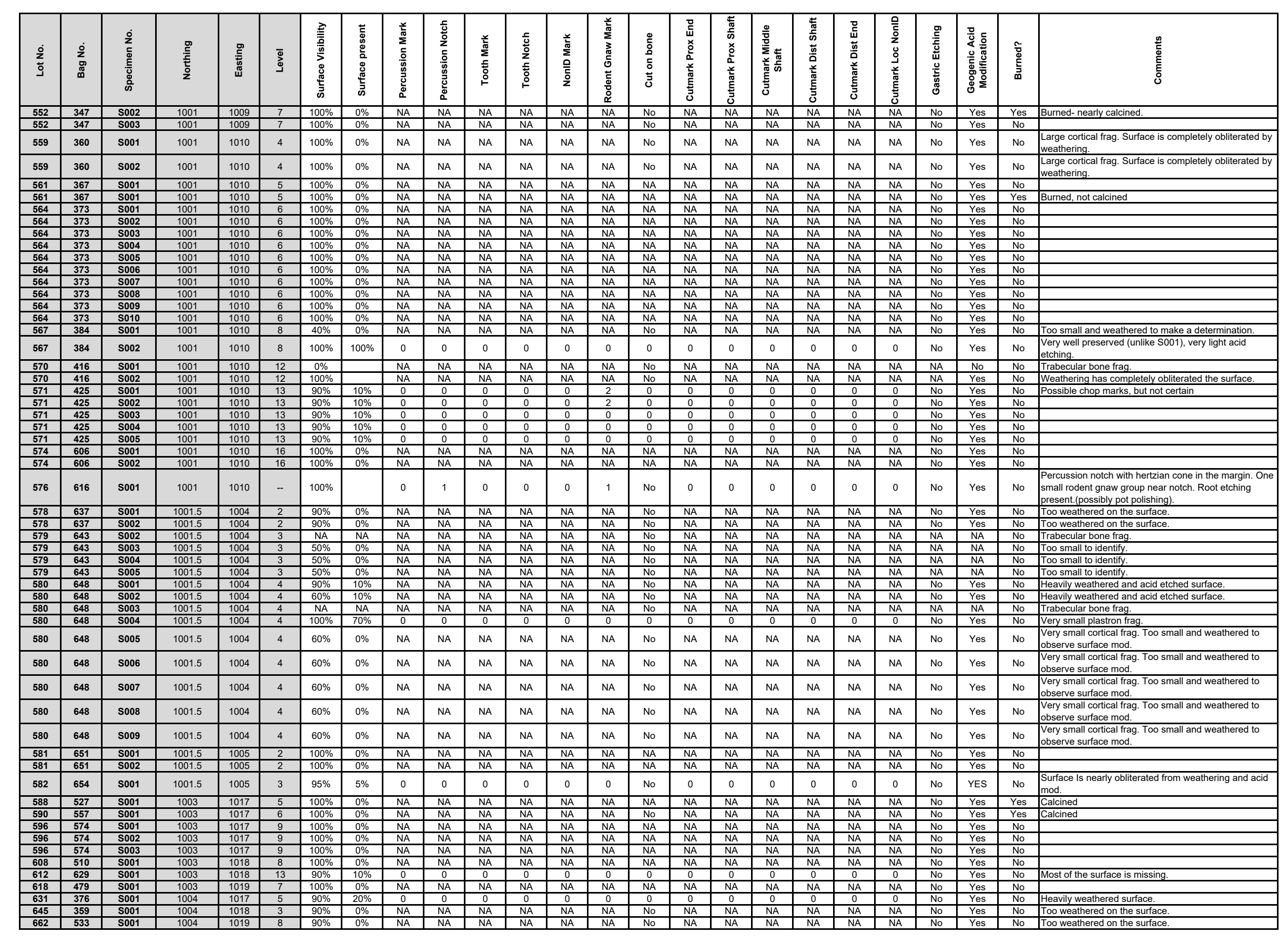




\begin{tabular}{|c|c|c|c|c|c|c|c|c|c|c|c|c|c|c|c|c|c|c|c|c|c|c|c|c|}
\hline 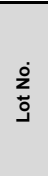 & 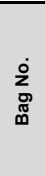 & 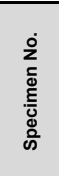 & 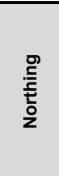 & 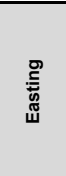 & 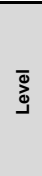 & 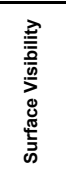 & 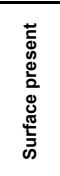 & 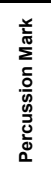 & 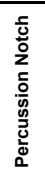 & 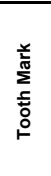 & 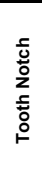 & 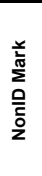 & 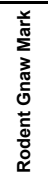 & 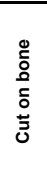 & 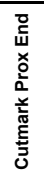 & 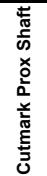 & 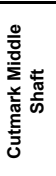 & 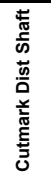 & 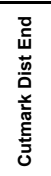 & 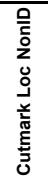 & 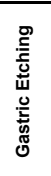 & 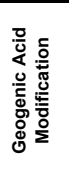 & 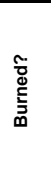 & 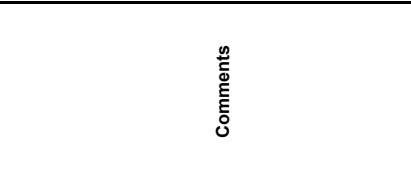 \\
\hline 668 & 588 & S001 & 1004 & 1019 & 12 & $100 \%$ & $20 \%$ & 0 & 0 & 0 & 0 & 0 & 0 & 0 & 0 & $\begin{array}{l}0 \\
0 \\
\end{array}$ & 0 & 0 & 0 & 0 & No & Yes & No & $\begin{array}{l}\text { Nearly obliterated cortical surface from weathering. } \\
\end{array}$ \\
\hline 676 & 366 & S001 & 1009 & 1011 & 5 & $60 \%$ & $0 \%$ & $\mathrm{NA}$ & $\mathrm{NA}$ & $\mathrm{NA}$ & $\mathrm{NA}$ & $\mathrm{NA}$ & NA & No & $\mathrm{NA}$ & NA & $\mathrm{NA}$ & $\mathrm{NA}$ & $\mathrm{NA}$ & $\mathrm{NA}$ & No & Yes & No & Too weathered on the surface. \\
\hline 688 & 352 & $S 001$ & 1009 & 1012 & 2 & $80 \%$ & $70 \%$ & 0 & 0 & 0 & 0 & 0 & 0 & 0 & 0 & 0 & 0 & 0 & 0 & 0 & No & Yes & No & Some acid etching. \\
\hline 688 & 352 & $\frac{5002}{503}$ & 1009 & $\frac{1012}{1012}$ & $\frac{2}{2}$ & $100 \%$ & $100 \%$ & 0 & 0 & 0 & 0 & 0 & 0 & 0 & 0 & 0 & 0 & 0 & 0 & 0 & No & Yes & No & Well preserved, no surface mod. \\
\hline 688 & 352 & S003 & $\frac{1009}{1009}$ & $\frac{1012}{1012}$ & $\frac{2}{2}$ & $100 \%$ & $100 \%$ & 0 & 0 & 0 & 0 & 0 & 0 & 0 & 0 & 0 & 0 & 0 & 0 & 0 & No & Yes & No & Well preserved, no surface mod. \\
\hline 688 & $\frac{352}{352}$ & 5004 & 1009 & $\frac{1012}{1012}$ & $\frac{2}{2}$ & $90 \%$ & $80 \%$ & 0 & 0 & 0 & 0 & & 0 & 0 & 0 & 0 & 0 & 0 & 0 & 0 & No & Yes & No & No anthropogenic surface mod \\
\hline 688 & $\frac{352}{357}$ & $\begin{array}{l}S 005 \\
S 001\end{array}$ & $\frac{1009}{1009}$ & $\frac{1012}{1012}$ & $\frac{2}{3}$ & $90 \%$ & $80 \%$ & $\frac{0}{N A}$ & 0 & 0 & 0 & 0 & 0 & 0 & 0 & 0 & 0 & 0 & 0 & 0 & No & Yes & No & No anthropogenic surface mod \\
\hline 689 & $\frac{357}{551}$ & $\frac{5001}{5001}$ & $\begin{array}{l}1009 \\
1009\end{array}$ & $\frac{1012}{1012}$ & $\frac{3}{10}$ & $\frac{100 \%}{100 \%}$ & $\frac{0 \%}{20 \%}$ & $\frac{\mathrm{NA}}{0}$ & $\frac{N A}{0}$ & $\frac{\mathrm{NA}}{0}$ & $\frac{\mathrm{NA}}{0}$ & $\frac{N A}{0}$ & $\frac{N A}{0}$ & $\frac{\text { No }}{0}$ & $\frac{N A}{0}$ & $\frac{\mathrm{NA}}{0}$ & $\mathrm{NA}$ & $\frac{N A}{0}$ & $\frac{\mathrm{NA}}{0}$ & $\frac{\mathrm{NA}}{0}$ & $\frac{\text { No }}{\text { No }}$ & $\begin{array}{l}\text { Yes } \\
\text { YYes }\end{array}$ & No & Suffac is wathered and hearily acid atched \\
\hline
\end{tabular}




\section{APPENDIX E}

Radiocarbon Assays 

Beta Analytic Inc

4985 SW 74 Court

Miami, Florida 33155

Tel: 305-667-5167

Fax: 305-663-0964

beta@radiocarbon.com
Mr. Darden Hood

President

Mr. Ronald Hatfield

Mr. Christopher Patrick

Deputy Directors

ISO/IEC 17025:2005 Accredited Test Results: Testing results recognized by all Signatories to the ILAC Mutual Recognition Arrangement

May 01, 2018

Mr. Brandon S. Young

Blanton \& Associates

4407 Monterey Oaks Blvd.

Building 1, Suite 110

Austin, TX 78749

USA

RE: Radiocarbon Dating Results

Dear Mr. Young,

Enclosed are the radiocarbon dating results for five samples recently sent to us. As usual, the method of analysis is listed on the report with the results and calibration data is provided where applicable. The Conventional Radiocarbon Ages have all been corrected for total fractionation effects and where applicable, calibration was performed using 2013 calibration databases (cited on the graph pages).

The web directory containing the table of results and PDF download also contains pictures, a cvs spreadsheet download option and a quality assurance report containing expected vs. measured values for 3-5 working standards analyzed simultaneously with your samples.

Reported results are accredited to ISO/IEC 17025:2005 Testing Accreditation PJLA \#59423 standards and all chemistry was performed here in our laboratory and counted in our own accelerators here. Since Beta is not a teaching laboratory, only graduates trained to strict protocols of the ISO/IEC 17025:2005 Testing Accreditation PJLA \#59423 program participated in the analyses.

As always Conventional Radiocarbon Ages and sigmas are rounded to the nearest 10 years per the conventions of the 1977 International Radiocarbon Conference. When counting statistics produce sigmas lower than $+/-30$ years, a conservative $+/-30$ $\mathrm{BP}$ is cited for the result. The reported $\mathrm{d} 13 \mathrm{C}$ values were measured separately in an IRMS (isotope ratio mass spectrometer). They are NOT the AMS d13C which would include fractionation effects from natural, chemistry and AMS induced sources.

When interpreting the results, please consider any communications you may have had with us regarding the samples.

Thank you for prepaying the analyses. As always, if you have any questions or would like to discuss the results, don't hesitate to contact us.

Sincerely ,

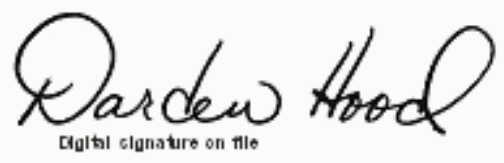


Beta Analytic Inc

4985 SW 74 Court

Miami, Florida 33155

Tel: $305-667-5167$

Fax: 305-663-0964

beta@radiocarbon.com
Mr. Darden Hood

President

Mr. Ronald Hatfield

Mr. Christopher Patrick

Deputy Directors

\section{REPORT OF RADIOCARBON DATING ANALYSES}

Brandon S. Young

Blanton \& Associates

Laboratory Number
Report Date: May 01, 2018

Material Received: April 20, 2018
Conventional Radiocarbon Age (BP) or Percent Modern Carbon (pMC) \& Stable Isotopes

Calendar Calibrated Results: 95.4 \% Probability High Probability Density Range Method (HPD)

Beta - 492582

Sample Code Number

$670+/-30$ BP

IRMS $\delta 13 C:-22.3$ o/oo

$\begin{array}{lll}(53.1 \%) & 1274-1320 \text { cal AD } & (676-630 \text { cal BP }) \\ (42.3 \%) & 1350-1391 \text { cal AD } & (600-559 \text { cal BP })\end{array}$

Submitter Material: Seeds

Pretreatment: (charred material) acid/alkali/acid

Analyzed Material: Charred material

Analysis Service: AMS-Standard delivery

Percent Modern Carbon: $92.00+/-0.34$ pMC

Fraction Modern Carbon: $0.9200+/-0.0034$

D14C: $-80.02+/-3.44$ o/oo

$\Delta 14 \mathrm{C}: \quad-87.56+/-3.44$ o/oo(1950:2,018.00)

Measured Radiocarbon Age: (without d13C correction): $630+/-30 \mathrm{BP}$

Calibration: BetaCal3.21: HPD method: INTCAL13

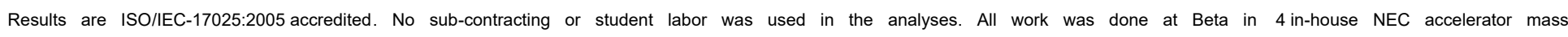

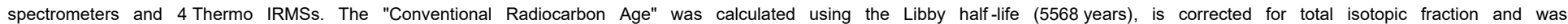

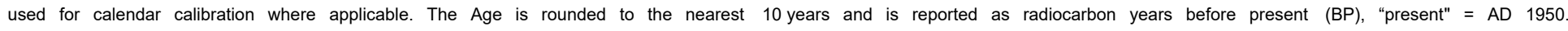

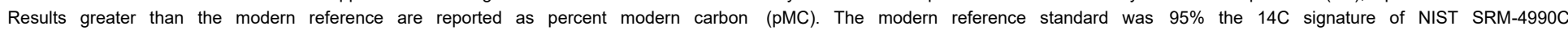

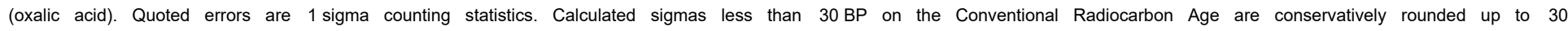

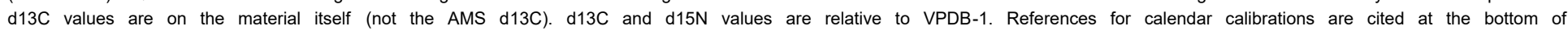
calibration graph pages. 
Beta Analytic Inc

4985 SW 74 Court

Miami, Florida 33155

Tel: $305-667-5167$

Fax: 305-663-0964

beta@radiocarbon.com
Mr. Darden Hood

President

Mr. Ronald Hatfield

Mr. Christopher Patrick

Deputy Directors

\title{
REPORT OF RADIOCARBON DATING ANALYSES
}

Brandon S. Young

Blanton \& Associates

Laboratory Number
Report Date: May 01, 2018

Material Received: April 20, 2018
Conventional Radiocarbon Age (BP) or Percent Modern Carbon (pMC) \& Stable Isotopes

Calendar Calibrated Results: 95.4 \% Probability High Probability Density Range Method (HPD)

Beta -492583

Sample Code Number

$970+/-30$ BP

IRMS $\delta 13 C:-25.5$ o/oo

\author{
(95.4\%) $\quad 1016-1154$ cal AD $\quad(934-796$ cal BP) \\ Submitter Material: Plant \\ Pretreatment: (charred material) acid/alkali/acid \\ Analyzed Material: Charred material \\ Analysis Service: AMS-Standard delivery \\ Percent Modern Carbon: $88.63+/-0.33$ pMC \\ Fraction Modern Carbon: $0.8863+/-0.0033$ \\ D14C: $-113.75+/-3.31$ o/o0 \\ $\Delta 14 \mathrm{C}: \quad-121.01+/-3.31$ o/oo(1950:2,018.00) \\ Measured Radiocarbon Age: (without d13C correction): $980+/-30 \mathrm{BP}$ \\ Calibration: BetaCal3.21: HPD method: INTCAL13
}

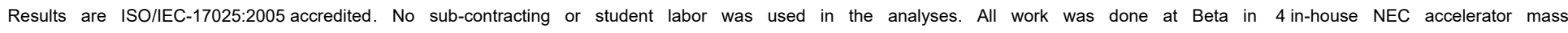

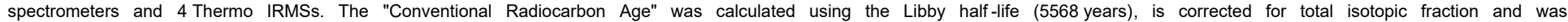

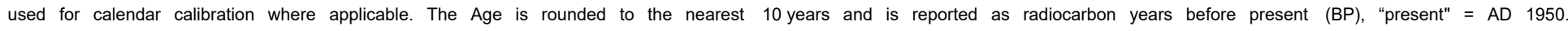

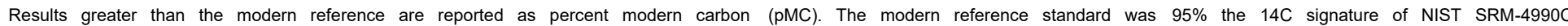

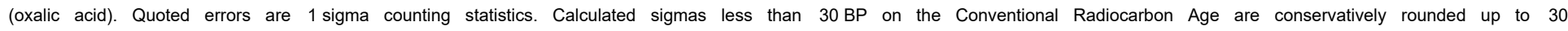

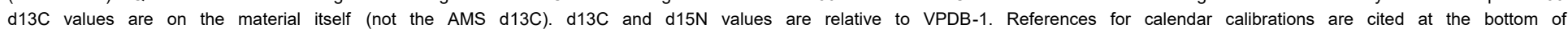
calibration graph pages. 
Beta Analytic Inc

4985 SW 74 Court

Miami, Florida 33155

Tel: $305-667-5167$

Fax: 305-663-0964

beta@radiocarbon.com
Mr. Darden Hood

President

Mr. Ronald Hatfield

Mr. Christopher Patrick

Deputy Directors

\title{
REPORT OF RADIOCARBON DATING ANALYSES
}

Brandon S. Young

Blanton \& Associates

Laboratory Number
Report Date: May 01, 2018

Material Received: April 20, 2018
Conventional Radiocarbon Age (BP) or Percent Modern Carbon (pMC) \& Stable Isotopes

Calendar Calibrated Results: 95.4 \% Probability High Probability Density Range Method (HPD)

Beta - 492584

Sample Code Number

$170+/-30$ BP

IRMS $\delta 13 C:-25.2$ o/oo

\author{
(80.8\%) $771-903 \mathrm{cal}$ AD $\quad(1179-1047 \mathrm{cal}$ BP $)$ \\ (14.6\%) $918-965 \mathrm{cal} A D \quad(1032-985 \mathrm{cal} B P)$ \\ Submitter Material: Charcoal \\ Pretreatment: (charred material) acid/alkali/acid \\ Analyzed Material: Charred material \\ Analysis Service: AMS-Standard delivery \\ Percent Modern Carbon: $86.45+/-0.32$ pMC \\ Fraction Modern Carbon: $0.8645+/-0.0032$ \\ D14C: $-135.54+/-3.23$ o/oo \\ $\Delta 14 \mathrm{C}: \quad-142.62+/-3.23$ o/oo(1950:2,018.00) \\ Measured Radiocarbon Age: (without d13C correction): $1170+/-30$ BP \\ Calibration: BetaCal3.21: HPD method: INTCAL13
}

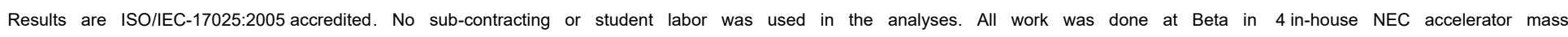

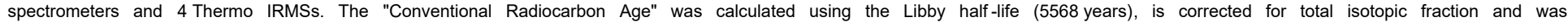

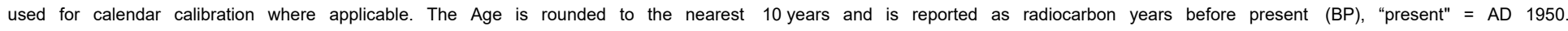

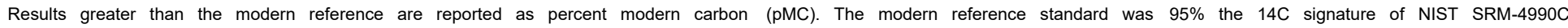

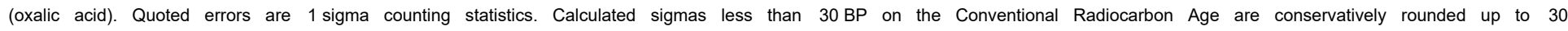

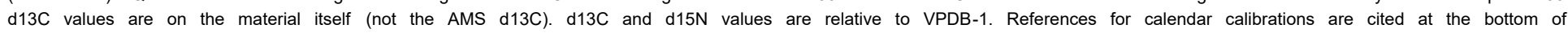
calibration graph pages. 
Beta Analytic Inc

4985 SW 74 Court

Miami, Florida 33155

Tel: $305-667-5167$

Fax: 305-663-0964

beta@radiocarbon.com
Mr. Darden Hood

President

Mr. Ronald Hatfield

Mr. Christopher Patrick

Deputy Directors

\section{REPORT OF RADIOCARBON DATING ANALYSES}

Brandon S. Young

Blanton \& Associates

Laboratory Number
Report Date: May 01, 2018

Material Received: April 20, 2018
Conventional Radiocarbon Age (BP) or Percent Modern Carbon (pMC) \& Stable Isotopes

Calendar Calibrated Results: 95.4 \% Probability High Probability Density Range Method (HPD)

Beta - 492587

Sample Code Number

$530+/-30$ BP

41GU177_FS337_S001

1390 - 1440 cal AD

(560 - 510 cal BP)

(75.9\%)

1320 - 1350 cal AD

(630 - $600 \mathrm{cal}$ BP)

Submitter Material: Bone (Non-heated)

Pretreatment: (bone collagen) collagen extraction; with alkali

Analyzed Material: Bone collagen

Analysis Service: AMS-Standard delivery

Percent Modern Carbon: $93.62+/-0.35$ pMC

Fraction Modern Carbon: $0.9362+/-0.0035$

D14C: $-63.85+/-3.50$ o/oo

$\Delta 14 \mathrm{C}:-71.52+/-3.50$ o/oo(1950:2,018.00)

Measured Radiocarbon Age: (without d13C correction): $300+/-30 \mathrm{BP}$

Calibration: BetaCal3.21: HPD method: INTCAL13

Carbon/Nitrogen: $\mathrm{CN}: 3.3 \quad \% \mathrm{C}: 42.25 \% \mathrm{~N}: 15.04$

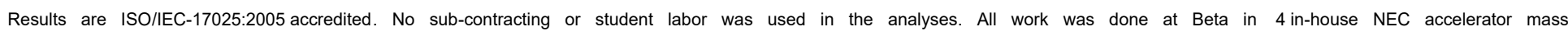

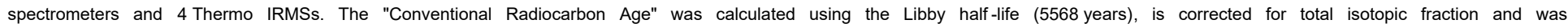

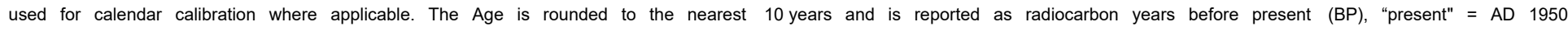

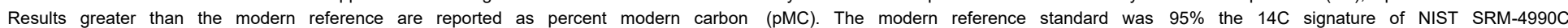

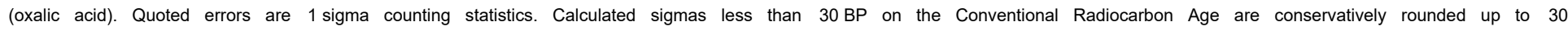

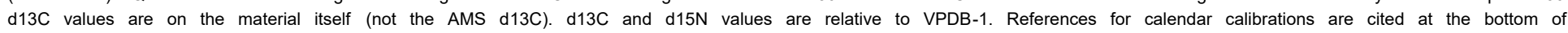
calibration graph pages. 
Beta Analytic Inc

4985 SW 74 Court

Miami, Florida 33155

Tel: $305-667-5167$

Fax: 305-663-0964

beta@radiocarbon.com
Mr. Darden Hood

President

Mr. Ronald Hatfield

Mr. Christopher Patrick

Deputy Directors

\section{REPORT OF RADIOCARBON DATING ANALYSES}

Brandon S. Young

Blanton \& Associates

Laboratory Number
Report Date: May 01, 2018

Material Received: April 20, 2018
Conventional Radiocarbon Age (BP) or Percent Modern Carbon (pMC) \& Stable Isotopes

Calendar Calibrated Results: 95.4 \% Probability High Probability Density Range Method (HPD)
Beta - 492588
Sample Code Number

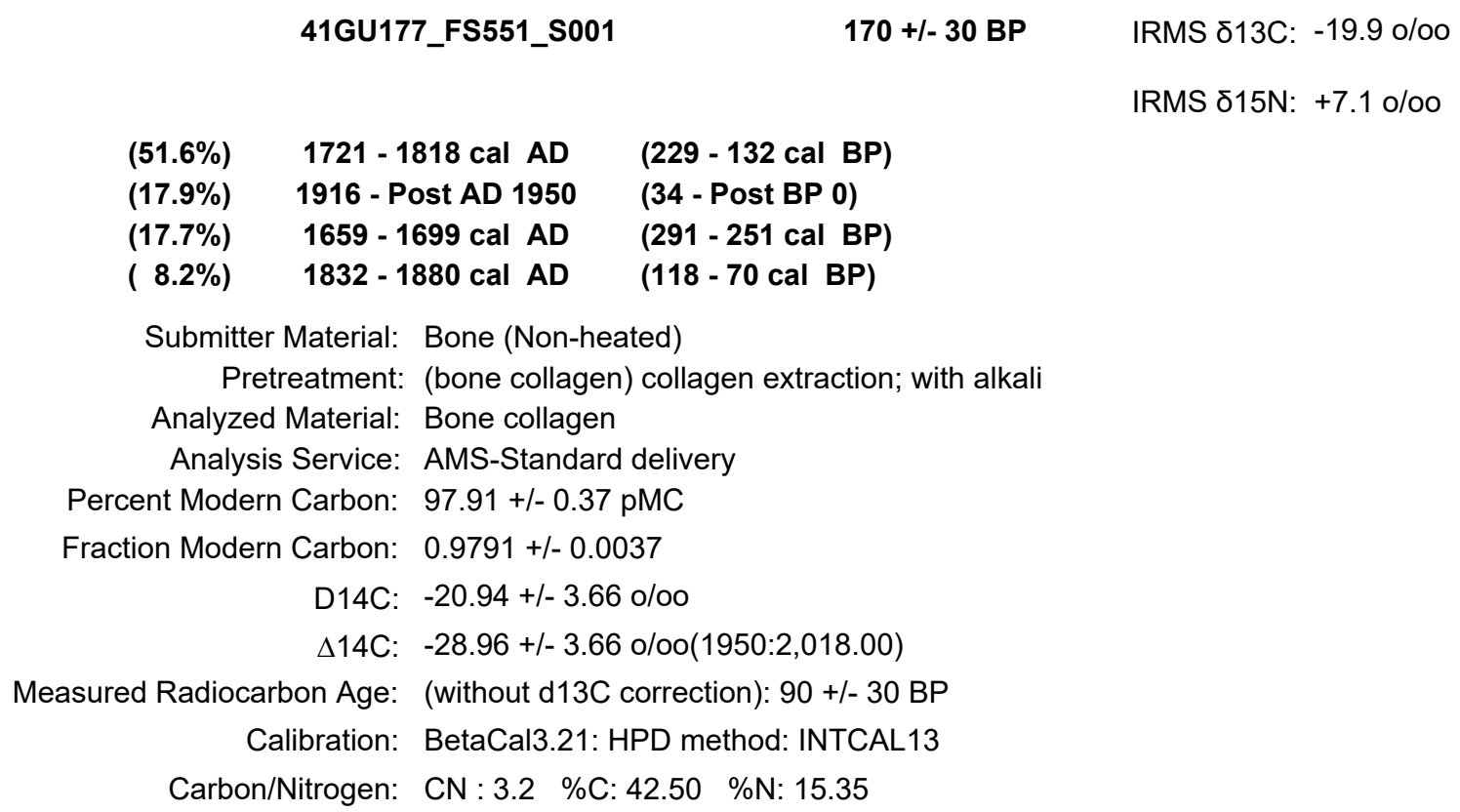

IRMS $\delta 13 C:-19.9$ o/oo

IRMS $\delta 15 \mathrm{~N}:+7.1 \mathrm{o} / 00$
$(51.6 \%)$
$(17.9 \%)$
$(17.7 \%)$
1721 - 1818 cal AD
1916 - Post AD 1950
( $8.2 \%)$
(229 - 132 cal BP)
(34 - Post BP 0)
(291 - 251 cal BP)
(118 - 70 cal BP)

Submitter Material: Bone (Non-heated)

Pretreatment: (bone collagen) collagen extraction; with alkali

Analyzed Material: Bone collagen

Analysis Service: AMS-Standard delivery

Percent Modern Carbon: $97.91+/-0.37$ pMC

Fraction Modern Carbon: $0.9791+/-0.0037$

D14C: $-20.94+/-3.66$ o/oo

$\Delta 14 \mathrm{C}: \quad-28.96+/-3.66$ o/oo(1950:2,018.00)

Measured Radiocarbon Age: (without d13C correction): 90 +/- 30 BP

Calibration: BetaCal3.21: HPD method: INTCAL13

Carbon/Nitrogen: CN: 3.2 \%C: $42.50 \%$ N: 15.35

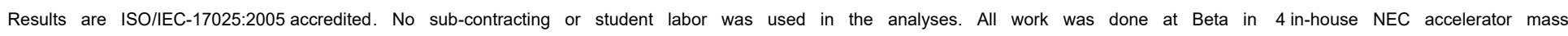

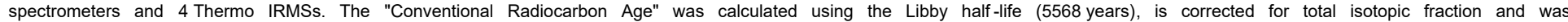

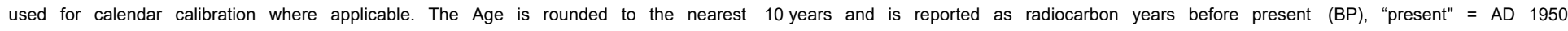

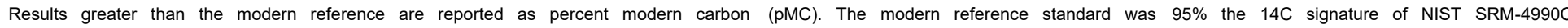

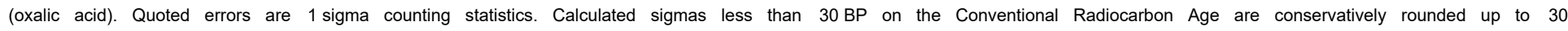

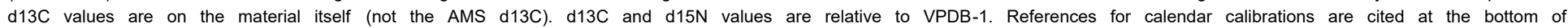
calibration graph pages. 


\title{
Calibration of Radiocarbon Age to Calendar Years
}

(High Probability Density Range Method (HPD): INTCAL13)

\author{
(Variables: $\mathrm{d} 13 \mathrm{C}=-22.3 \mathrm{o} / \mathrm{oo})$ \\ Laboratory number Beta-492582 \\ Conventional radiocarbon age $\quad 670 \pm 30 \mathrm{BP}$
}

$95.4 \%$ probability
(53.1\%) $\quad 1274-1320$ cal AD
$(676-630$ cal BP)
(42.3\%) $1350-1391 \mathrm{cal} A D$
$(600-559$ cal BP)

$68.2 \%$ probability
(38.6\%) $1280-1305$ cal AD
(670 - 645 cal BP)
(29.6\%) $1364-1384 \mathrm{cal} \mathrm{AD}$
(586 - 566 cal BP)

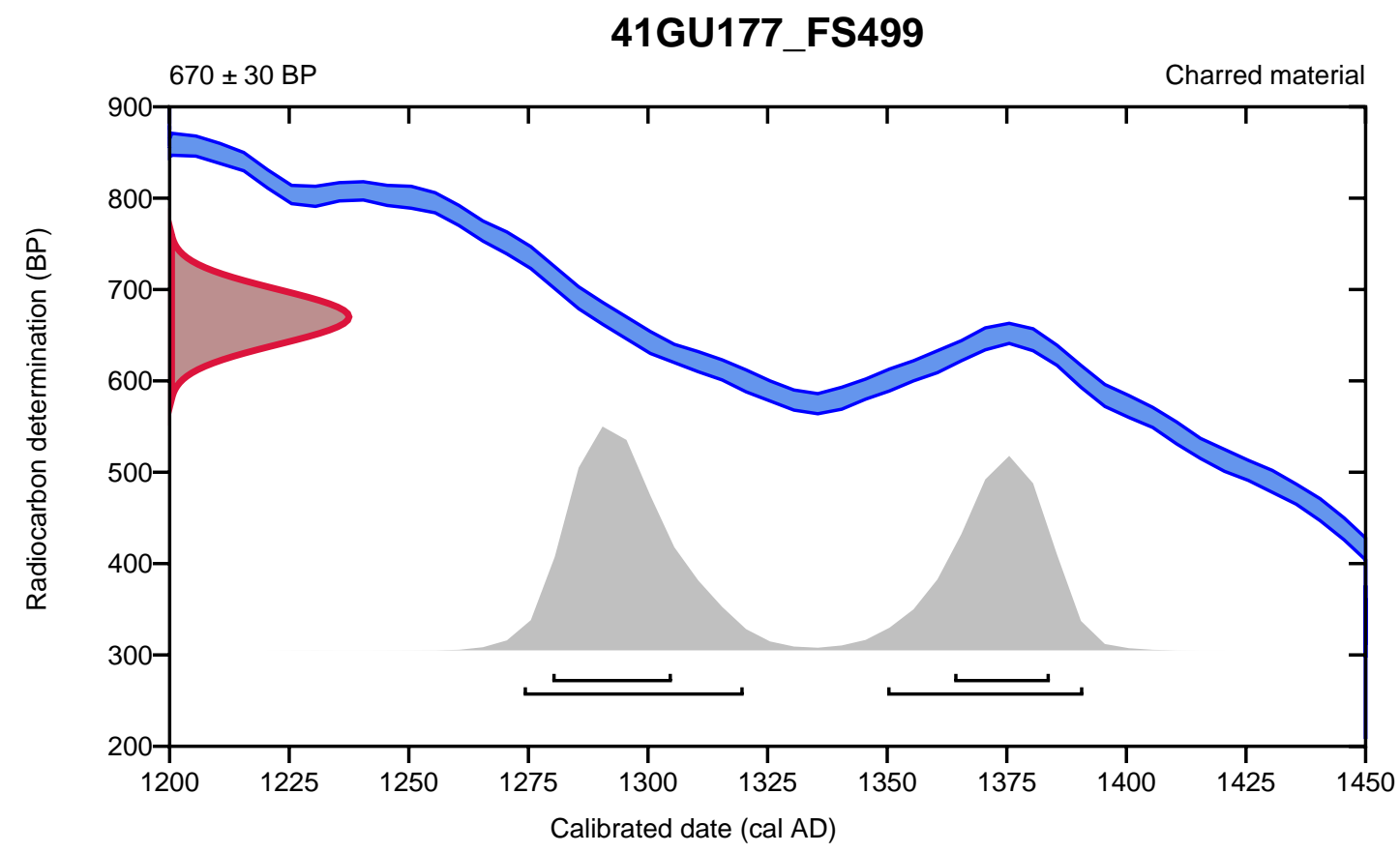

\section{Database used}

INTCAL13

\section{References}

References to Probability Method

Bronk Ramsey, C. (2009). Bayesian analysis of radiocarbon dates. Radiocarbon, 51(1), 337-360.

References to Database INTCAL13

Reimer, et.al., 2013, Radiocarbon55(4).

\section{Beta Analytic Radiocarbon Dating Laboratory}




\section{Calibration of Radiocarbon Age to Calendar Years}

(High Probability Density Range Method (HPD): INTCAL13)

(Variables: $\mathrm{d} 13 \mathrm{C}=-25.5 \mathrm{o} / \mathrm{oo}$ )

Laboratory number Beta-492583

Conventional radiocarbon age $\quad 970 \pm 30 \mathrm{BP}$

$95.4 \%$ probability

(95.4\%) $\quad 1016-1154 \mathrm{cal} \mathrm{AD} \quad(934-796 \mathrm{cal} \mathrm{BP})$

$68.2 \%$ probability

$\begin{array}{lll}(31.4 \%) & 1088-1122 \mathrm{cal} \mathrm{AD} & (862-828 \mathrm{cal} \text { BP }) \\ (28.3 \%) & 1021-1048 \mathrm{cal} \mathrm{AD} & (929-902 \mathrm{cal} \text { BP }) \\ (8.4 \%) & 1138-1148 \mathrm{cal} \mathrm{AD} & (812-802 \mathrm{cal} \text { BP })\end{array}$

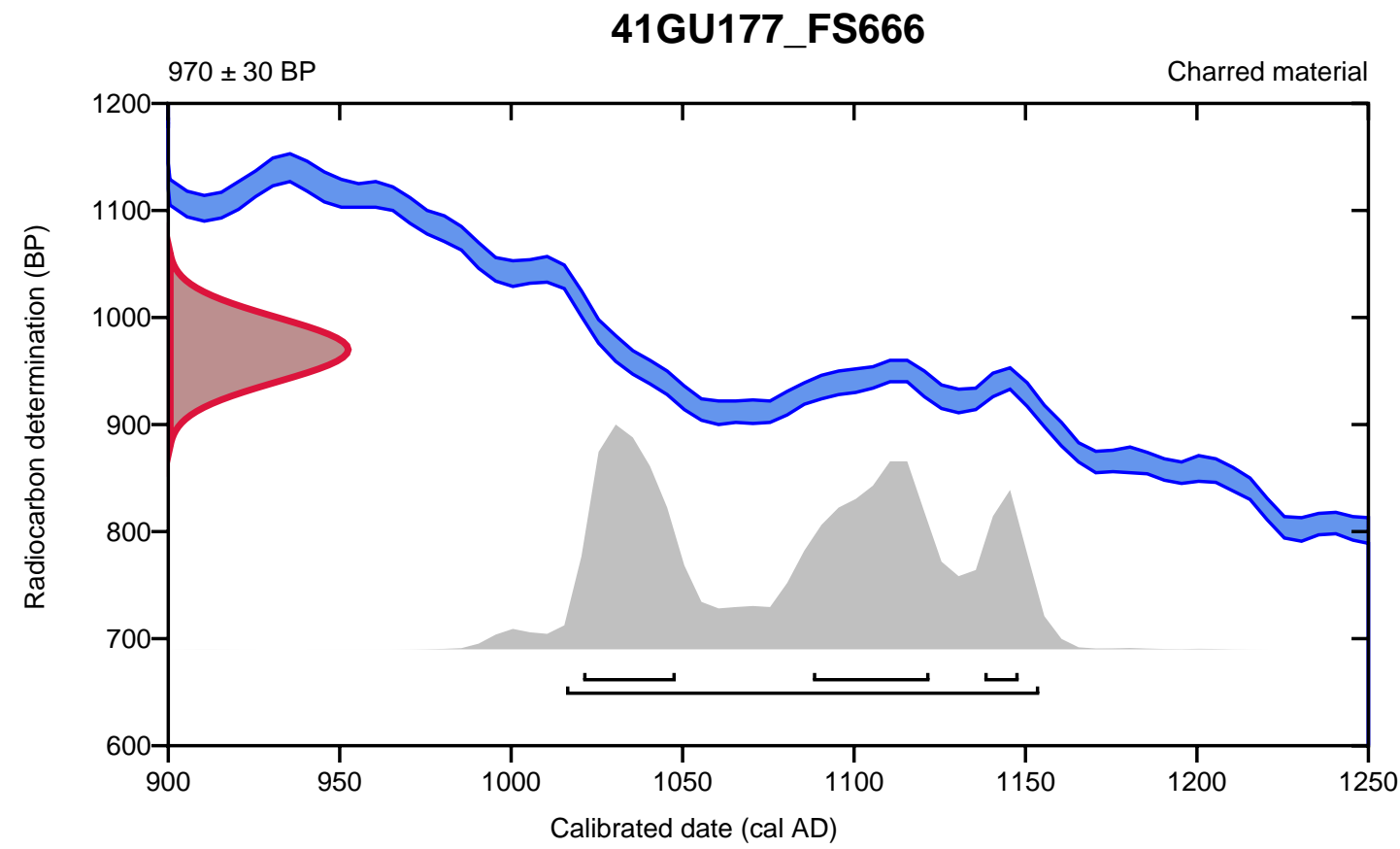

Database used

INTCAL13

\section{References}

References to Probability Method

Bronk Ramsey, C. (2009). Bayesian analysis of radiocarbon dates. Radiocarbon, 51(1), 337-360.

References to Database INTCAL13

Reimer, et.al., 2013, Radiocarbon55(4).

\section{Beta Analytic Radiocarbon Dating Laboratory}




\title{
Calibration of Radiocarbon Age to Calendar Years
}

(High Probability Density Range Method (HPD): INTCAL13)

\author{
(Variables: $\mathrm{d} 13 \mathrm{C}=-25.2 \mathrm{o} / \mathrm{oo}$ ) \\ Laboratory number Beta-492584 \\ Conventional radiocarbon age $\quad 1170 \pm 30 \mathrm{BP}$
}

95.4\% probability
(80.8\%) $771-903$ cal AD
$(1179-1047$ cal BP)
(14.6\%) $918-965 \mathrm{cal} A D$
(1032 - 985 cal BP)

$68.2 \%$ probability
(57.1\%) $801-892$ cal AD
$(1149-1058$ cal BP)
(11.1\%) $777-793$ cal AD
$(1173-1157$ cal BP)

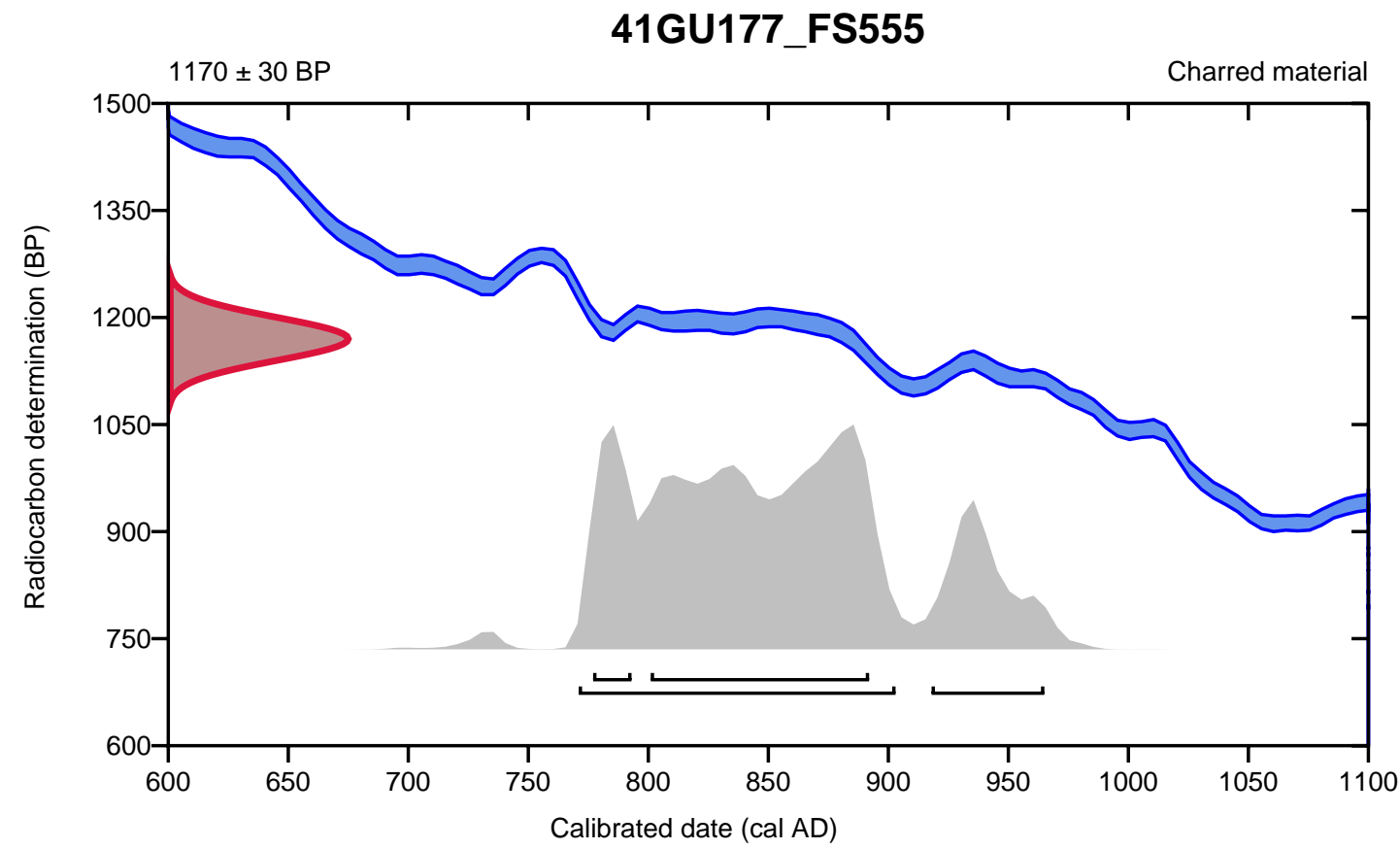

Database used

INTCAL13

\section{References}

References to Probability Method

Bronk Ramsey, C. (2009). Bayesian analysis of radiocarbon dates. Radiocarbon, 51(1), 337-360.

References to Database INTCAL13

Reimer, et.al., 2013, Radiocarbon55(4).

\section{Beta Analytic Radiocarbon Dating Laboratory}


BetaCal 3.9

\section{Calibration of Radiocarbon Age to Calendar Years}

(High Probability Density Range Method (HPD): INTCAL13)

(Variables: $\mathrm{d} 13 \mathrm{C}=-11.1 \mathrm{o} / \mathrm{oo})$

Laboratory number Beta-492587

Conventional radiocarbon age $\quad 530 \pm 30 \mathrm{BP}$

$95.4 \%$ probability

$\begin{array}{lll}(75.9 \%) & 1390-1440 \mathrm{cal} \text { AD } & (560-510 \mathrm{cal} \text { BP }) \\ (19.5 \%) & 1320-1350 \mathrm{cal} \mathrm{AD} & (630-600 \mathrm{cal} \text { BP })\end{array}$

$68.2 \%$ probability

$(68.2 \%) \quad 1398-1432$ cal AD

(552 - 518 cal BP)

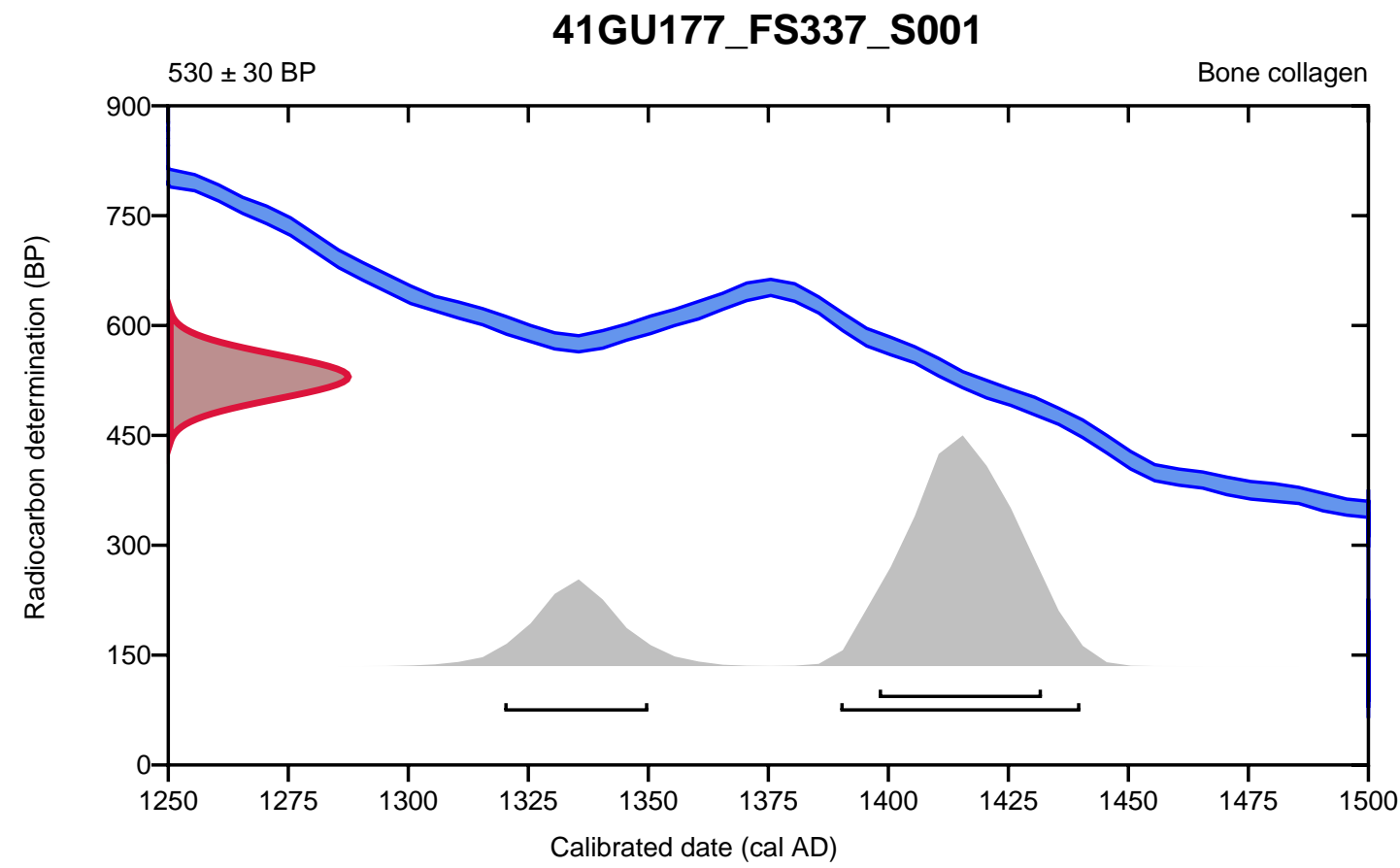

Database used

INTCAL13

\section{References}

References to Probability Method

Bronk Ramsey, C. (2009). Bayesian analysis of radiocarbon dates. Radiocarbon, 51(1), 337-360.

References to Database INTCAL13

Reimer, et.al., 2013, Radiocarbon55(4).

\section{Beta Analytic Radiocarbon Dating Laboratory}




\section{Calibration of Radiocarbon Age to Calendar Years}

(High Probability Density Range Method (HPD): INTCAL13)

(Variables: d13C $=-19.9$ o/oo)

\section{Laboratory number Beta-492588}

Conventional radiocarbon age $\quad 170 \pm 30 \mathrm{BP}$

$95.4 \%$ probability

$\begin{array}{lll}(51.6 \%) & 1721-1818 \mathrm{cal} \mathrm{AD} & (229-132 \mathrm{cal} \mathrm{BP}) \\ (17.9 \%) & 1916-\text { Post cal AD } 1950 & (34-\text { Post cal BP } 0) \\ (17.7 \%) & 1659-1699 \mathrm{cal} \mathrm{AD} & (291-251 \mathrm{cal} \mathrm{BP}) \\ (8.2 \%) & 1832-1880 \mathrm{cal} \mathrm{AD} & (118-70 \text { cal BP })\end{array}$

$68.2 \%$ probability

$\begin{array}{lll}(35.4 \%) & 1732-1782 \mathrm{cal} \mathrm{AD} & (218-168 \mathrm{cal} \mathrm{BP}) \\ (14.2 \%) & 1928-\text { Post cal AD } 1950 & (22-\text { Post cal BP } 0) \\ (11.7 \%) & 1668-1685 \mathrm{cal} \mathrm{AD} & (282-265 \text { cal BP }) \\ (7 \%) & 1797-1808 \mathrm{cal} \mathrm{AD} & (153-142 \mathrm{cal} \mathrm{BP})\end{array}$

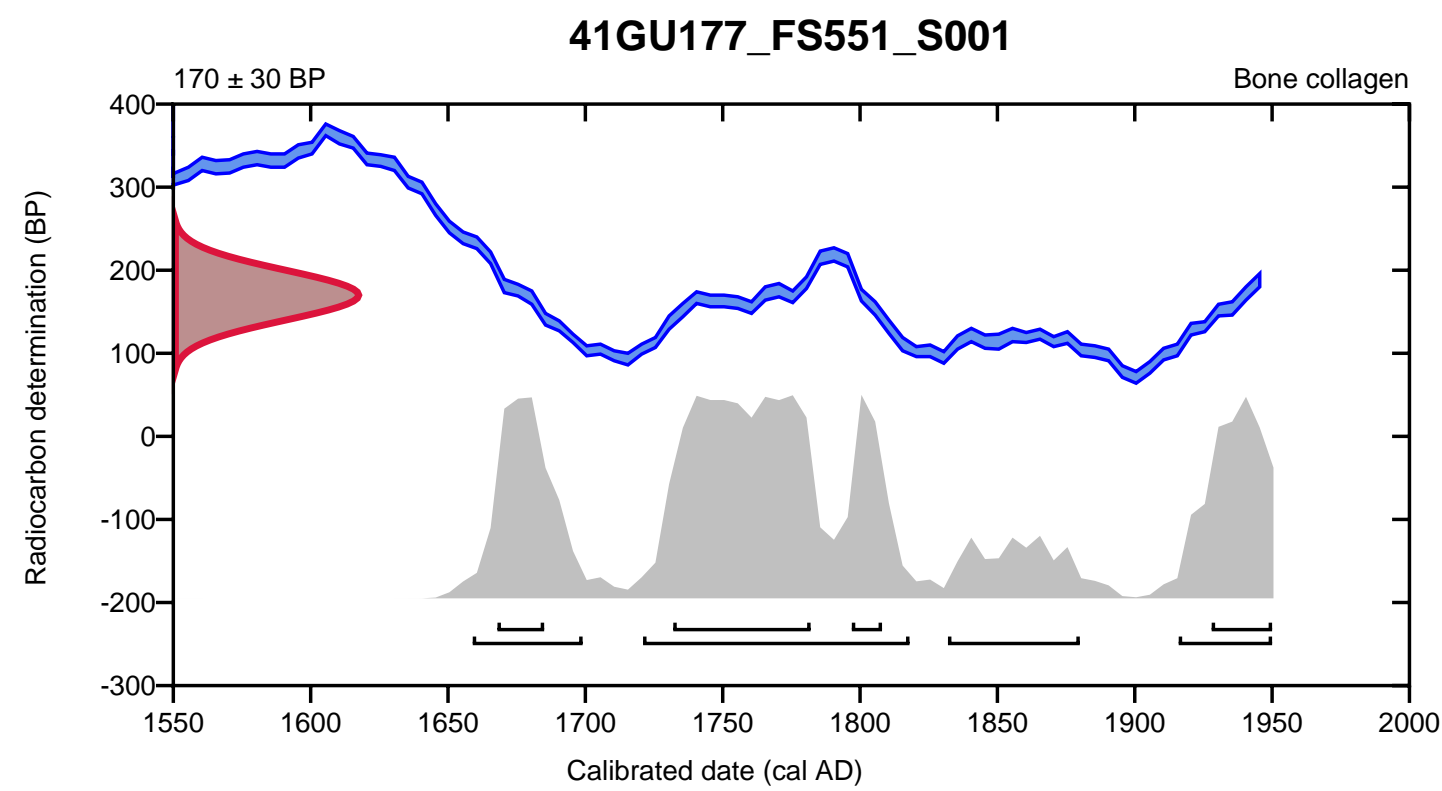

Database used

INTCAL13

\section{References}

References to Probability Method

Bronk Ramsey, C. (2009). Bayesian analysis of radiocarbon dates. Radiocarbon, 51(1), 337-360.

References to Database INTCAL13

Reimer, et.al., 2013, Radiocarbon55(4).

\section{Beta Analytic Radiocarbon Dating Laboratory}





\section{APPENDIX F}

Macrobotanical Analysis 



\title{
FLOTATION SAMPLES AND \\ RADIOCARBON MATERIAL FROM \\ SITE 41GU177, Guadalupe County, TeXas
}

March 27, 2018

\author{
Prepared for: \\ Brandon Young, Archaeologist \\ SWCA Environmental Consultants \\ 4407 Monterey Oaks Blvd. \\ Austin, TX 78749
}


Macrobotanical Analysis, 8/22/18, p. 2

Prepared by:

Leslie L. Bush, Ph.D., R.P.A.

Macrobotanical Analysis

12308 Twin Creeks Rd., B-104

Manchaca, Texas 78652 
Ten carbon samples and five flotation samples from Site $41 \mathrm{GU} 177$ were submitted for sorting, identification, and analysis. The site is situated on a bluff above the Guadalupe River outside modern New Braunfels, near the northwestern border of Guadalupe County. The location is just below the confluence of an unnamed, intermittent tributary or canyon. Ecologically, the area is the southwesternmost extension of the Blackland Prairie vegetation region. Although little of the original prairie ecosystems remain today, vegetation in presettlement times would have been trallgrass prairie with the most common grasses being little bluestem (Schizachyrium scoparium), Indiangrass (Sorghastrum nutans), and big bluestem (Andropogon gerardii). Community types vary in localized areas primarily due to differences in soil (Diggs et al. 1999:40). Wooded areas are present in the prairies in scattered upland areas and near larger rivers and streams such as the Guadalupe River. Wildfires tended to make smaller tributaries treeless in pre-settlement times. Because of its proximity to other major vegetation regions, the Blackland Prairie belt in Guadalupe County shares plant species with the South Texas Plains, Post Oak Savannah, and the Edwards Plateau.

\section{METHODS}

Radiocarbon samples were placed in a No. 10 mesh to separate larger fragments from soil and wood charcoal flecks. Materials were examined under a Leica S9i stereoscopic microscope at 6-55 X magnification. Wood charcoal fragments were snapped to reveal a clean transverse section. When necessary, tangential or radial sections were examined for ray seriation, presence of spiral thickenings, types and sizes of intervessel pitting, and other minute characteristics. After identification, specimens of each taxon were counted, labeled, recorded, and weighed on an Ohaus Scout II $200 \times 0.01 \mathrm{~g}$ electronic balance. To retain suitability for radiometric dating, carbon samples were placed on freshly washed glassware when not in specimen containers, and they were handled only with vinyl gloves and metal forceps. Contact with paper and other plant products was avoided. Material that fell through the No. 10 mesh was examined under the microscope for plant parts other than wood, especially annual plant parts such as seeds. None were found.

Flotation samples were sorted according to standard procedures (Pearsall 2015). Each flotation light fraction was weighed on an Ohaus Scout II $200 \times 0.01 \mathrm{~g}$ electronic balance before being size-sorted through a stack of graduated geologic mesh. All carbonized botanical materials that did not pass through the No. 10 mesh (2 mm square openings) were sorted under a Leica S9i stereozoom microscope at 6-55 X, then counted, weighed, recorded, and labeled. At Site 
41GU177, these larger remains consisted almost exclusively of wood charcoal. Gastropods, small rocks, and uncarbonized botanical material larger than $2 \mathrm{~mm}$ (usually rootlets) were weighed, recorded, and labeled as "contamination". Materials that fell through the $2 \mathrm{~mm}$ mesh ("residue") were examined under a stereoscopic microscope at 6-55 X magnification for carbonized botanical remains that had not been previously identified in the $2 \mathrm{~mm}$ size fraction. Identifiable botanical materials were removed from residue, counted, weighed, recorded, and labeled. Uncarbonized macrobotanical remains other than rootlets (at this site, seeds, leaves, and fruits) were recorded on a presence/absence basis on laboratory forms.

Wood charcoal identification was attempted for up to twenty specimens from each flotation sample. When fewer than twenty wood charcoal fragments were present in the $2 \mathrm{~mm}$ size fraction, as was the case for all flotation samples at Site 41GU177, all such fragments were identified, and identification was attempted for progressively smaller fragments until either twenty fragments were identified or identification became impractical. Wood charcoal fragments were snapped to reveal a transverse section and examined under a stereoscopic microscope at 6$55 \mathrm{X}$ magnification. When necessary, tangential or radial sections were examined for ray seriation, presence of spiral thickenings, types and sizes of intervessel pitting, and other characteristics.

Botanical materials were identified to the lowest possible taxonomic level by comparison to materials in the Macrobotanical Analysis comparative collection and through the use of standard reference works (e.g., Core et al. 1979; Davis 1993; Hoadley 1990; InsideWood 2004; Martin and Barkley 1961; Musil 1963; Panshin and de Zeeuw 1980; Wheeler 2011). Plant nomenclature follows that of the PLANTS Database (USDA, NCRS 2018).

\section{RESULTS}

\section{Radiocarbon material}

Identifications of material for possible radiocarbon dating are given in Table B.1. Four of these radiocarbon samples were removed from flotation samples prior to processing. They are included in the flotation tables as well as the radiocarbon table so that the flotation tables reflect the actual density of charcoal per volume.

Plant material for radiocarbon dating consisted of wood charcoal. Four taxa were identified: red mulberry (Morus rubra), juniper (Juniperus sp.), mesquite (Prosopis sp.), and elm (Ulmus sp.). From the location near the southwestern edge of the Blackland Prairie, the juniper species is likely Juniperus virginiana, sometimes called redcedar, and the mesquite is probably Prosopis glandulosa, called honey 
mesquite. Some elm specimens in C-20 and C-21 show a period of approximately four years of inhibited growth (Figure 1).

\section{Flotation samples}

Archaeological plant materials recovered are given in Tables B.2 and B. 3 by count and weight respectively. As noted above, the tables include charcoal designated for possible radiocarbon dating that is also reported in Table B.1. Uncarbonized plant materials other than rootlets are shown on a presence/absence basis in Table B.4.

Uncarbonized (modern) plant remains. Most uncarbonized plant parts in the samples appear in the form of rootlets that are clearly related to the modern vegetation at the site. Uncarbonized seeds are a common occurrence on most archaeological sites, and they usually represent seeds of modern plants that have made their way into the soil either through their own dispersal mechanisms or by faunalturbation, floralturbation, or argilliturbation (Bryant 1985:51-52; Keepax 1977; Miksicek 1987:231-232). In all except the driest areas of North America, uncarbonized plant material on open-air sites can be assumed to be of modern origin unless compelling evidence suggests otherwise (Lopinot and Brussell 1982; Miksicek 1987:231). The seeds, leaves, and fruits at 41GU177 consist of a weedy annual (sandmat [Chamaesyce spp.]), and parts of woody plants (sugarberry [Celtis laevigata], mesquite [Prosopis glandulosa], Texas persimmon [Diospyros texana], and sumac [Rhus spp.]) that relate to the current vegetation. All uncarbonized plant parts are interpreted here as modern, and further discussion concerns ancient (carbonized) plant parts only.

Carbonized (ancient) plant remains. Camas bulb (Camassia spp.). One camas bulb fragment was recovered from Feature 4, Layer 2. This may represent the Atlantic camas (Camassia scilloides) or the prairie camas (C. angusta). Bulbs of the two species are similar, with the plants distinguished largely by the smaller scape height, fewer floral bracts, and earlier bloom period in Atlantic camas and persistent flower stalks in prairie camas (G. Yatskievych 1999:503-504; K. Yatskievych 2000:297-298). Archaeologists have documented a long history of bulb exploitation in Texas, with camas bulbs at the Wilson-Leonard Site currently the oldest known, dating to approximately 8,000 years before present (Acuña 2006:57; Mehalchick et al. 2004:Table 8.18). The use of earth oven facilities to transform otherwise inedible geophytes into nutritionally optimal starches and sugars seems to be part of a continent-wide trend that began in the early Holocene (Thoms 2008; Wandschnider 1997). West of the Pecos River, geophytes cooked in earth ovens include bulbs such as wild onion and, more commonly, the larger basal rosettes of desert plants such as lechuguilla and sotol (Dering 1999). 
Seeds. A fragment of a sugarberry seed (Celtis laevigata) was recovered from Feature 4, Layer 1. Uncarbonized sugarberry seeds were recovered in all three features. Because of their high mineral content, uncarbonized sugarberry seeds survive well in the soil and frequently appear in geological deposits in North America (Wang et al. 1997). The Feature 4 specimen is the only carbonized sugarberry seed fragment present, however. Because it has been exposed to fire and is in feature context, it is interpreted as ancient. The thin, sweet pulp of sugarberry fruits covers a relatively large seed with high calcium content. The fruits and seeds are edible raw or cooked. Historically, they were often pounded, shaped into cakes or balls with other ingredients such as fat, and dried or baked for future use (Moerman 1998:147).

Two deer pea vetch seeds (Vicia ludoviciana), one whole, one fragmentary, were recovered from Feature 2. Deer pea vetch is a small weedy legume that produces small pods about an inch long. Although no ethnographic records are available for this particular species, seeds of the closely related American vetch (Vicia americana) were used by Native people of western North America for food (Moerman 1998:595-596). The immature pod could also have been used in cooking, analogous to how snow pea pods are used today. Although it is possible that the vetch seeds at Site 41GU177 because carbonized when the crop of a gamebird was discarded in the fire, no other seeds favored by gamebirds such as croton, broomweed, or acorns were recovered (Leif and Smith 1993; Riley et al. 1993). Vetch has been recovered from at least four other archaeological sites in Central Texas, where it is also interpreted as a human food plant (Table B.5).

Table B.5: Sites with deer pea vetch (Vicia ludoviciana) in Texas

\begin{tabular}{|l|r|l|r|l|}
\hline Site & $\begin{array}{r}\text { \# vetch } \\
\text { seeds }\end{array}$ & Affiliation & $\begin{array}{r}\text { Soil } \\
\text { volume } \\
\text { (liters) }\end{array}$ & Reference \\
\hline $\begin{array}{l}\text { Barnhill \#3 } \\
\text { (41CV1646) }\end{array}$ & 80 & $\begin{array}{l}\text { Austin \& Toyah (with } \\
\text { corn) }\end{array}$ & 99 & $\begin{array}{l}\text { Bush } \\
2017 a\end{array}$ \\
\hline 41 FK148 & 24 & Late Caddo & 228 & $\begin{array}{l}\text { Bush } \\
2013\end{array}$ \\
\hline 41 RT113 & 2 & $\begin{array}{l}\text { Late Prehistoric } \\
\text { (Perdiz) }\end{array}$ & 106 & $\begin{array}{l}\text { Bush } \\
2017 b\end{array}$ \\
\hline Baker (41SS192) & 427 & $\begin{array}{l}\text { Toyah (with corn and } \\
\text { Caddo ceramics) }\end{array}$ & 66 & $\begin{array}{l}\text { report in } \\
\text { progress }\end{array}$ \\
\hline
\end{tabular}




\begin{tabular}{|l|r|l|r|l|}
\hline 41GU177 & 2 & Toyah & 18.5 & $\begin{array}{l}\text { this } \\
\text { report }\end{array}$ \\
\hline
\end{tabular}

Wood charcoal. Of the 117 wood charcoal fragments that could be identified to genus or species, mulberry $(n=38)$ and elm $(n=32)$ were the most numerous. Mulberry was more common in Feature 3 and elm in Feature 4. Mesquite $(n=17)$ was found in Features 2 and 4 , and juniper $(n=10)$ in Features 3 and 4. Woods that were present in smaller numbers and only a single context were bluewood (Condalia hookeri, $\mathrm{n}=6$, Feature 3), ash (Fraxinus sp.; $\mathrm{n}=2$, Feature 4), silktassel (Garrya ovata, n=1, Feature 3), and pecan/hickory (Carya sp.) and grape (Vitis sp.) (1 each, Feature 2).

Although they would all have occurred in the site area, none of the woods recovered at Site $41 \mathrm{GU} 177$ are considered outstanding fuelwoods, with the possible exception of mesquite. The absence of oaks (Quercus spp.), a staple of earth oven cooking, is particularly is puzzling. The two common oaks of the area, live oak (Quercus fusiformis) and post oak (Q. stellata), have excellent coaling qualities and specific gravities of 0.88 and 0.67 respectively (Alden 1995:101). In general, the heat value of a wood is directly related to its specific gravity (Marcouiller and Anderson n.d.). Coaling properties, which are especially important in earth oven cooking, relate to the third stage of the burning process. After evaporation of within-cell moisture (first stage), wood is converted to charcoal (second stage, signified by flames). In the third stage, the glowing coals burn slowly, without flame, and can be left for hours without attention (Collier and Turner 1981, Marcouiller and Anderson n.d.). Elm and mulberry, the two most common woods in the 41GU177 samples, are moderately good fuel woods with specific gravities of 0.64 and 0.66 , respectively (Alden 1995:120). Mulberry is said to throw sparks but produce excellent coals, while elm has a reputation for smoke (Marcoullier and Anderson n.d.). Mesquite (specific gravity 0.82 ) is possibly the best fuelwood represented, assuming its distinctive odor is considered desirable (Alden 1995:94). Other woods recovered in the samples would also have been useful in fires. For example, softwoods such as juniper tend to ignite more easily than hardwoods (Collier and Turner 1981).

\section{SUMMARY}

Archaeological plant remains at Site 41GU177 consisted mostly of wood charcoal. The wood charcoal is interpreted as fuel wood, possibly associated with earth 
oven cooking of geophytes and other plant material. A camas bulb fragment was recovered from Feature 4. Other ancient plant parts recovered were a sugarberry seed fragment (Feature 4) and two vetch seeds (Feature 2). These are also interpreted as food items.

\section{REFERENCES CITED}

Acuña, Laura I.

2006 The Economic Contribution of Root and Other Geophytes in Prehistoric Texas. M.A. thesis, Department of Anthropology, Texas State University, San Marcos, Texas.

Alden, Harry A.

1995 Hardwoods of North America. General Technical Report FPL-GTR83. United States Department of Agriculture, Forest Service, Forest Products Laboratory, Madison, Wisconsin.

Bryant, John A.

1985 Seed Physiology. The Institute of Biology's Studies in Biology No. 165. Edward Arnold, Ltd., London.

Bush, Leslie L.

2013 Plant Remains from Eight Features at Site 41FK148, Franklin County, Texas. Manuscript submitted to Steve Carpenter, Principal Investigator, SWCA Environmental Consultants, Austin, Texas. November 12, 2013.

Bush, Leslie L.

2017a Four Additional Flotation Samples from Feature 25, an Earth Oven at Barnhill Rockshelter \#3 (41CV1646), Coryell County, Texas. Manuscript submitted to Carol Macauley-Jameson, Department of Anthropology, Baylor University, Waco, Texas. October 12, 2017.

Bush, Leslie L.

2017b Plant Remains from Three Sites in Robertson County, Texas. Manuscript submitted to Meg Cruse, Cultural Resources Manager, Blanton \& Associates, Inc., Austin, Texas. July 10, 2017.

Collier, Kathy, and Larry Turner 
1981 Obtaining, Seasoning and Burning Wood. AEES-18. University of Kentucky College of Agriculture, Cooperative Extension Service, Lexington, Kentucky. https://www.bae.uky.edu/publications/AEES/AEES18.pdf. Accessed March 27, 2015.

Core, H. A., W. A. Cote and A. C. Day

1979 Wood Structure and Identification. 2nd ed. Syracuse University Press, Syracuse, New York.

Davis, Linda W.

1993 Weed Seeds of the Great Plains: A Handbook for Identification. University Press of Kansas, Lawrence.

Dering, Phil

1999 Earth-Oven Plant Processing in Archaic Period Economies: An Example from a Semi-Arid Savannah in South-Central North America. American Antiquity 64(4): 659-674.

Diggs Jr., George M., Barney L. Lipscomb and Robert J. O'Kennon 1999 Shinners and Mahler's Illustrated Flora of North Central Texas. Second Printing, 2000, with minor corrections. Illustrated Texas Floras Project. Botanical Research Institute of Texas, Fort Worth.

Hoadley, R. Bruce

1990 Identifying Wood: Accurate Results with Simple Tools. The Taunton Press, Newtown, Connecticut.

InsideWood

2004-onwards Published on the Internet. http://insidewood.lib.ncsu.edu/search. Accessed February 27, 2018.

Keepax, Carole

1977 Contamination of archaeological deposits by seeds of modern origin with particular reference to the use of flotation machines. Journal of Archaeological Science 4:221-229.

Leif, Anthony P., and Smith, Loren M.

1993 Winter Diet Quality, Gut Morphology and Condition of Northern Bobwhite and Scaled Quail in West Texas. Journal of Field Ornithology 64(4): 527-538. 
Lopinot, Neal H. and David Eric Brussell

1982 Assessing Uncarbonized Seeds from Open-air Sites in Mesic

Environments: An Example from Southern Illinois. Journal of Archaeological Science 9:95-108.

Marcouiller, Dave, and Steven Anderson

n.d. Firewood: How to Obtain, Measure, Season, and Burn. NREM-9440. Oklahoma Cooperative Extension Service, Division of Agricultural Sciences and Natural Resources, Oklahoma State University, Stillwater, Oklahoma. http://pods.dasnr.okstate.edu/docushare/dsweb/Get/Document2507/NREM-9440web.pdf. Accessed March 27, 2015.

Martin, Alexander C. and William D. Barkley

1961 Seed Identification Manual. University of California Press, Berkeley.

Mehalchick, Gemma, Douglas Boyd, Karl W. Kibler, and Christopher W. Ringstaff 2004 Shifting Sands and Geophytes: Geoarcheological Investigations at Paluxy Sites on Fort Hood, Texas. United States Army Fort Hood Archeological Resource Management Series No. 48. Prewitt and Associates, Inc., Austin, Texas.

Miksicek, Charles H.

1987 Formation Processes of the Archaeobotanical Record. In Advances in Archaeological Method and Theory, Vol. 10, edited by Michael B. Schiffer, pp. 211-247. Academic Press, Inc.

Moerman, Daniel E.

1998 Native American Ethnobotany. Timber Press, Portland, Oregon.

Musil, Albina F.

1963 Identification of Crop and Weed Seeds Agriculture Handbook No. 219. U.S. Department of Agriculture, Washington, D.C.

Panshin, A. J. and Carol de Zeeuw

1980 Textbook of Wood Technology: Structure, Identification, Properties, and Uses of the Commercial Woods of the United States and Canada. Fourth ed. McGraw-Hill Book Company, New York.

Pearsall, Deborah M. 
2015 Paleoethnobotany: A Handbook of Procedures. 3rd ed. Left Coast Press, Walnut Creek, California.

Riley, Terry Z., Davis, Charles A., and Smith, Randall A.

1993 Autumn and Winter Foods of the Lesser Prairie-Chicken (Tympanuchus Pallidicinctus) (Galliformes:Tetraonidae). Great Basin Naturalist 53(2): 186189.

Thoms, Alston V.

2008 Ancient Savannah Roots of the Carbohydrate Revolution in SouthCentral North America. Plains Anthropologist 53: 121-136.

USDA, NRCS (United States Department of Agriculture, Natural Resources Conservation Service)

2018 The PLANTS Database. http://plants.usda.gov. National Plant Data Center, Greensboro, North Carolina. Accessed March 16, 2018.

Wandsnider, LuAnn

1997 The Roasted and the Boiled: Food Composition and Heat Treatment with Special Emphasis on Pit-Hearth Cooking. Journal of Anthropological Archaeology 16: 1-48.

Wang, Yang, A. Hope Jahren, and Ronald Amundson

1997 Potential for 14C Dating of Biogenic Carbonate in Hackberry (Celtis) Endocarps. Quaternary Research 47: 337-343.

Wheeler, Elizabeth A.

2011 InsideWood - A Web Resource for Hardwood Anatomy. IAWA Journa/32(2): 199-211.

Yatskievych, George

1999 Steyermark's Flora of Missouri, Volume 1. The Missouri Department of Conservation and The Missouri Botanical Garden Press, Jefferson City and St. Louis, Missouri.

Yatskievych, Kay

2000 Field Guide to Indiana Wildflowers. Indiana University Press, Bloomington and Indianapolis. 
Figure 1: Transverse section of Ulmus sp. wood charcoal from C-21, FTR 4 Layer 2.

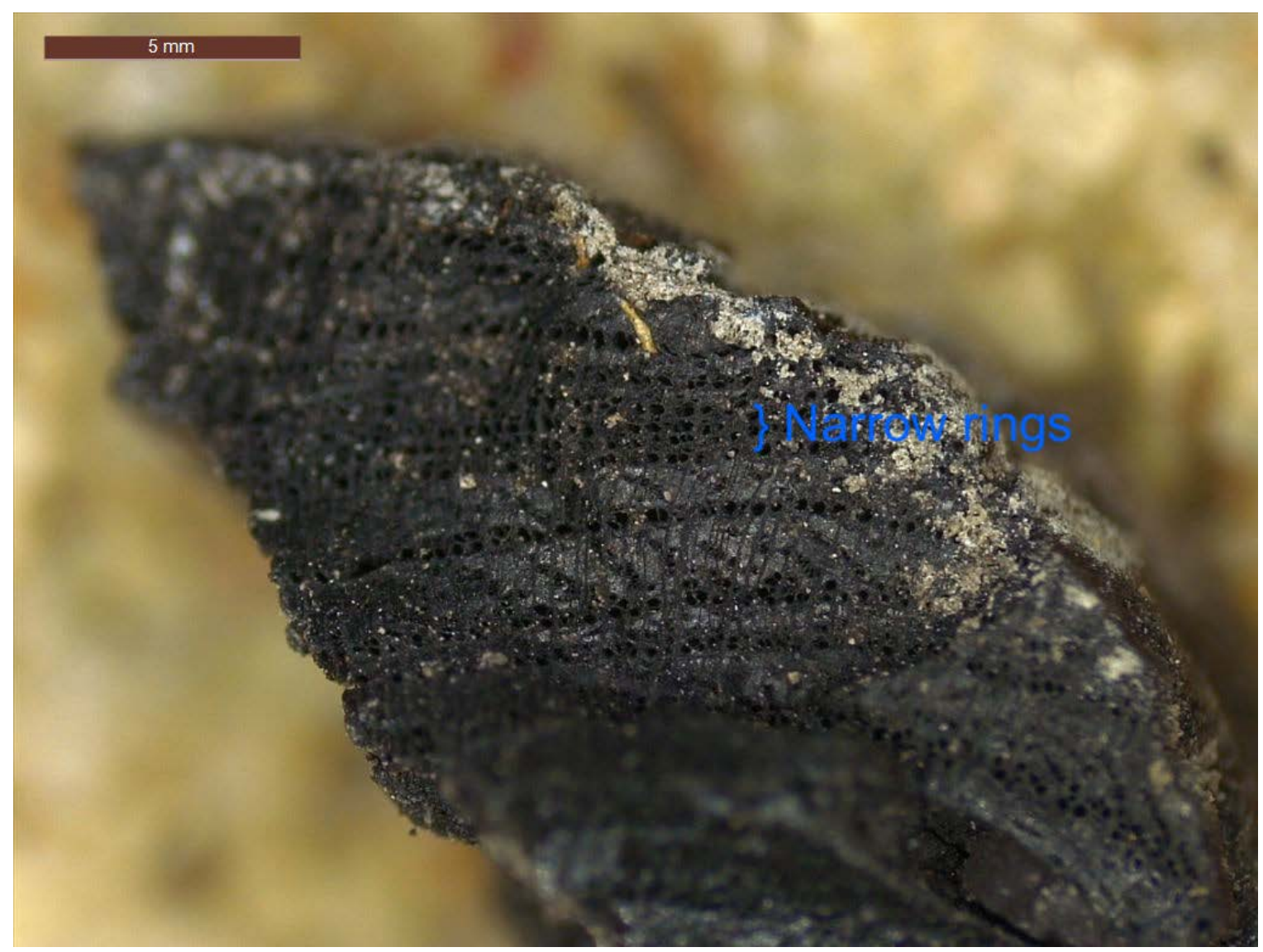


Table B.1: Samples for Radiocarbon Dating, Site 41GU177

\begin{tabular}{|c|c|c|c|c|c|c|c|c|c|}
\hline FS\# & Sample \# & FTR & Level/Layer & $\begin{array}{r}\text { Depth } \\
\text { (cmbd) }\end{array}$ & $\begin{array}{l}\text { Material Type } \\
\text { (MNR=Minimum Number of } \\
\text { Rings) }\end{array}$ & $\begin{array}{l}\text { Botanical } \\
\text { name }\end{array}$ & $\begin{array}{l}\text { Common } \\
\text { name }\end{array}$ & $\begin{array}{r}\text { Number of } \\
\text { Individual } \\
\text { Specimens }\end{array}$ & Weight (g) \\
\hline 555 & C-11 & 3 & Level 7 & 75 & Bone & & & 1 & 0.16 \\
\hline \multirow[t]{2}{*}{555} & $\mathrm{C}-12$ & 3 & Level 7 & 79 & Wood charcoal, MNR $=8$ & Morus rubra & Red mulberry & 15 & 1.44 \\
\hline & & & & & Examined residue $<2 \mathrm{~mm}$ & & & & 0.12 \\
\hline \multirow[t]{2}{*}{608} & C-14 & 3 & Level 8 & 84 & Wood charcoal, MNR=5 & Morus rubra & Red mulberry & 13 & 0.21 \\
\hline & & & & & Examined residue $<2 \mathrm{~mm}$ & & & & 0.03 \\
\hline 658 & C-15 & 4 & Layer 1 & 57 & Wood charcoal, MNR=2 & Juniperus sp. & Juniper & 5 & 0.17 \\
\hline \multirow[t]{2}{*}{658} & C-16 & 4 & Layer 1 & 60 & Wood charcoal, MNR=6 & Prosopis sp. & Mesquite & 4 & 0.31 \\
\hline & & & & & Examined residue $<2 \mathrm{~mm}$ & & & & 0.14 \\
\hline \multirow[t]{2}{*}{658} & C-17 & 4 & Layer 1 & 57 & Wood charcoal, MNR=6 & Ulmus sp. & Elm & 14 & 0.35 \\
\hline & & & & & Examined residue $<2 \mathrm{~mm}$ & & & & 0.23 \\
\hline \multirow[t]{2}{*}{658} & $C-18$ & 4 & Layer 1 & 62 & Wood charcoal, MNR=6 & Prosopis sp. & Mesquite & 8 & 0.21 \\
\hline & & & & & Examined residue $<2 \mathrm{~mm}$ & & & & 0.22 \\
\hline \multirow[t]{2}{*}{658} & C-19 & 4 & Layer 1 & 60 & Wood charcoal, MNR=7 & Ulmus sp. & Elm & 5 & 2.26 \\
\hline & & & & & Examined residue $<2 \mathrm{~mm}$ & & & & 1.67 \\
\hline 666 & $C-20$ & 4 & Level 5-6 & 64 & Wood charcoal, MNR=7 & Ulmus sp. & Elm & 6 & 0.25 \\
\hline 666 & $C-21$ & 4 & Level 5-6 & 63 & Wood charcoal, MNR $=18$ & Ulmus sp. & Elm & 4 & 0.24 \\
\hline
\end{tabular}


Table B.2: Carbonized (ancient) Plant Remains from Site 41GU177

Number of Individual Specimens

\begin{tabular}{|c|c|c|c|c|c|c|c|}
\hline FS\# & 608 & 499 & 555.1 & 555.2 & 658 & 666 & Site \\
\hline Lot\# & 393 & 672 & 420 & 421 & 522 & 529 & Total \\
\hline Feature & 3 & 2 & 3 & 3 & 4 & 4 & \\
\hline Portion & & & Cluster 1 & Cluster 2 & Layer 1 & Layer 2 & \\
\hline Level & 8 & $6-8$ & $7-8$ & $7-8$ & $4-5$ & $5-6$ & \\
\hline Depth (cmbd) & 84 & $53-80$ & $73-80$ & $70-76$ & $45-60$ & $60-69$ & \\
\hline & N/A (carbon & & & & & & \\
\hline Flotation volume (cu. dm.) & sample only) & 5.5 & 4 & 3.5 & 3 & 2.5 & 18.5 \\
\hline \multicolumn{8}{|l|}{ Wood charcoal } \\
\hline Red mulberry (Morus rubra) & 13 & & 16 & & 4 & 5 & 38 \\
\hline Elm (Ulmus spp.) & & 1 & & & 20 & 11 & 32 \\
\hline Mesquite (Prosopis glandulosa) & & 3 & & & 13 & 1 & 17 \\
\hline Juniper (Juniperus spp.) & & & 2 & & 6 & 2 & 10 \\
\hline Sugarberry (Celtis sp.) & & 6 & & 2 & & & 8 \\
\hline Hardwood, indeterminable & & 3 & 1 & & & 3 & 7 \\
\hline Bluewood condalia (Condalia hookeri) & & & & 6 & & & 6 \\
\hline Ash (Fraxinus sp.) & & & & & 2 & & 2 \\
\hline Acacia/Mesquite (Vachellia/Prosopis sp.) & & 1 & & & & & 1 \\
\hline Hickory/Pecan (Carya sp.) & & 1 & & & & & 1 \\
\hline Silktassel (Garrya ovata) & & & & 1 & & & 1 \\
\hline Grape (Vitis spp.) & & 1 & & & & & 1 \\
\hline \multicolumn{8}{|l|}{ Other plant parts } \\
\hline Camas bulb scale (Camassia sp.) & & & & & & 1 & 1 \\
\hline Sugarberry seed (Celtis laevigata) & & & & & 1 & & 1 \\
\hline Vetch seed (Vicia ludoviciana) & & 2 & & & & & 2 \\
\hline Indeterminable & & & & & 1 & & 1 \\
\hline
\end{tabular}


Table B.3: Carbonized (ancient) Plant Remains from Site 41GU177

Weights in grams

\begin{tabular}{|c|c|c|c|c|c|c|c|}
\hline FS\# & 465 & 499 & 555.1 & 555.2 & 658 & 666 & Site \\
\hline Lot\# & 393 & 672 & 420 & 421 & 522 & 529 & Total \\
\hline Feature & 3 & 2 & 3 & 3 & 4 & 4 & \\
\hline Portion & & & Cluster 1 & Cluster 2 & Layer 1 & Layer 2 & \\
\hline Level & 8 & $6-8$ & 7-8 & 7-8 & 4-5 & $5-6$ & \\
\hline Depth (cmbd) & 84 & $53-80$ & $73-80$ & 70-76 & $45-60$ & $60-69$ & \\
\hline Flotation volume (cu. dm.) & $\begin{array}{l}\text { N/A (carbon } \\
\text { sample only) }\end{array}$ & 5.5 & 4 & 3.5 & 3 & 2.5 & 18.5 \\
\hline \multicolumn{8}{|l|}{ Wood charcoal } \\
\hline Red mulberry (Morus rubra) & 0.21 & & 1.45 & & 0.01 & 0.02 & 1.69 \\
\hline Elm (Ulmus spp.) & & 0.01 & & & 2.62 & 0.5 & 3.13 \\
\hline Mesquite (Prosopis glandulosa) & & 0.02 & & & 0.53 & 0.01 & 0.56 \\
\hline Juniper (Juniperus spp.) & & & 0.01 & & 0.18 & 0.01 & 0.2 \\
\hline Sugarberry (Celtis sp.) & & 0.01 & & 0.01 & & & 0.02 \\
\hline Hardwood, indeterminable & & 0.01 & 0.01 & & & 0.01 & 0.03 \\
\hline Bluewood condalia (Condalia hookeri) & & & & 0.04 & & & 0.04 \\
\hline Ash (Fraxinus sp.) & & & & & 0.01 & & 0.01 \\
\hline Acacia/Mesquite (Vachellia/Prosopis sp.) & & 0.01 & & & & & 0.01 \\
\hline Hickory/Pecan (Carya sp.) & & 0.01 & & & & & 0.01 \\
\hline Silktassel (Garrya ovata) & & & & 0.01 & & & 0.01 \\
\hline Grape (Vitis spp.) & & 0.01 & & & & & 0.01 \\
\hline \multicolumn{8}{|l|}{ Other plant parts } \\
\hline Camas bulb scale (Camassia sp.) & & & & & & 0.01 & 0.01 \\
\hline Sugarberry seed (Celtis laevigata) & & & & & 0.01 & & 0.01 \\
\hline Vetch (Vicia ludoviciana) & & 0.01 & & & & & 0.01 \\
\hline Indeterminable & & & & & 0.01 & & 0.01 \\
\hline Contamination $>2 \mathrm{~mm}$ & & 10.97 & 5.2 & 31.72 & 30.52 & 15.33 & 93.74 \\
\hline Examined residue $<2 \mathrm{~mm}$ & 0.03 & 2.01 & 2.14 & 5.81 & 7.52 & 3.35 & 20.86 \\
\hline
\end{tabular}


Table B.4: Uncarbonized (modern) Plant Parts from Site 41GU177

$\mathrm{X}=$ present

\begin{tabular}{|c|c|c|c|c|c|c|c|}
\hline FS\# & 465 & 499 & 555.1 & 555.2 & 658 & 666 & Total \\
\hline Lot\# & 393 & 672 & 420 & 421 & 522 & 529 & Occurrences \\
\hline Feature & 3 & 2 & 3 & 3 & 4 & 4 & \\
\hline Portion & & & Cluster 1 & Cluster 2 & Layer 1 & Layer 2 & \\
\hline Level & 8 & $6-8$ & $7-8$ & 7-8 & 4-5 & 5-6 & \\
\hline Depth (cmbd) & 84 & $53-80$ & $73-80$ & $70-76$ & $45-60$ & $60-69$ & \\
\hline Flotation volume (cu. dm.) & $\begin{array}{r}\text { sample } \\
\text { only) }\end{array}$ & 5.5 & 4 & 3.5 & 3 & 2.5 & 18.5 \\
\hline Sugarberry seed (Celtis laevigata) & & $X$ & $X$ & $X$ & $x$ & $\mathrm{X}$ & 5 \\
\hline Sugarberry leaf (Celtis laevigata) & & & & $\mathrm{X}$ & $\mathrm{X}$ & $\mathrm{X}$ & 3 \\
\hline Sandmat seed (Chamaesyce spp.) & & & & & $\mathrm{X}$ & $\mathrm{X}$ & 2 \\
\hline Mesquite endocarp (Prosopis glandulosa) & & & $\mathrm{X}$ & & & & 1 \\
\hline Texas persimmon fruit, immature (Diospyros texana) & & & & & $\mathrm{x}$ & & 1 \\
\hline Acorn, immature (Quercus spp.) & & & & & & $\mathrm{X}$ & 1 \\
\hline Sumac leaflet (Rhus cf. lanceolata) & & $\mathrm{X}$ & & & & & 1 \\
\hline Total taxa & 0 & 2 & 2 & 2 & 4 & 4 & 14 \\
\hline
\end{tabular}




\section{APPENDIX G}

Geoarchaeological Analysis Raw Data 

Appendix G1. Particle Size Analysis

\begin{tabular}{|c|c|c|c|c|c|c|c|c|c|c|c|c|c|c|c|}
\hline Unit & $\begin{array}{l}\text { Sample } \\
\text { Matrix \# }\end{array}$ & 14 VC Sand & $\begin{array}{c}18 \mathrm{VC}-\mathrm{C} \\
\text { Sand }\end{array}$ & VC Sand & $35 \mathrm{C}$ Sand & C Sand & $60 \mathrm{M}$ Sand & Med Sand & $120 \mathrm{~F}$ Sand & Fine Sand & $\begin{array}{c}230 \mathrm{VF} \\
\text { Sand }\end{array}$ & VF Sand & $<230$ Silt-Clay & Silt-Clay & Total \\
\hline N996 E1014 & 3 & 0 & 0 & 0 & 19.142 & 19.142 & 53.554 & 53.554 & 73.858 & 73.858 & 30.073 & 30.073 & 19.858 & 19.858 & 196.485 \\
\hline N996 E1014 & 4 & 0 & 15.52 & 15.52 & 37.157 & 37.157 & 62.504 & 62.504 & 80.000 & 80.000 & 33.073 & 33.073 & 26.415 & 26.415 & 254.669 \\
\hline N996 E1014 & 5 & 0 & 21.698 & 21.698 & 57.59 & 57.59 & 67.285 & 67.285 & 79.628 & 79.628 & 38.843 & 38.843 & 31.308 & 31.308 & 296.352 \\
\hline N996 E1014 & 7 & 0 & 19.447 & 19.447 & 33.358 & 33.358 & 27.521 & 27.521 & 23.821 & 23.821 & 12.137 & 12.137 & 13.428 & 13.428 & 129.712 \\
\hline N996 E1014 & 9 & 9.196 & 28.927 & 38.123 & 47.339 & 47.339 & 33.099 & 33.099 & 30.524 & 30.524 & 18.237 & 18.237 & 20.311 & 20.311 & 187.633 \\
\hline N996 E1014 & 11 & 12.728 & 21.072 & 33.8 & 38.719 & 38.719 & 42.325 & 42.325 & 47.489 & 47.489 & 25.058 & 25.058 & 31.339 & 31.339 & 218.73 \\
\hline N996 E1014 & 12 & 12.712 & 8.429 & 21.141 & 32.183 & 32.183 & 51.479 & 51.479 & 54.432 & 54.432 & 18.286 & 18.286 & 12.589 & 12.589 & 190.11 \\
\hline N1004 E1017 & 1 & 0 & 4.251 & 4.251 & 30.749 & 30.749 & 52.614 & 52.614 & 59.772 & 59.772 & 21.563 & 21.563 & 12.164 & 12.164 & 181.113 \\
\hline N1004 E1017 & 4 & 0 & 14.275 & 14.275 & 44.557 & 44.557 & 42.991 & 42.991 & 45.883 & 45.883 & 21.271 & 21.271 & 17.699 & 17.699 & 186.676 \\
\hline N1004 E1017 & 5 & 0 & 26.183 & 26.183 & 51.019 & 51.019 & 38.686 & 38.686 & 37.517 & 37.517 & 19.784 & 19.784 & 22.042 & 22.042 & 195.231 \\
\hline N1004 E1017 & 8 & 0 & 29.76 & 29.76 & 47.235 & 47.235 & 26.216 & 26.216 & 20.514 & 20.514 & 11.699 & 11.699 & 16.571 & 16.571 & 151.995 \\
\hline N1004 E1017 & 9 & 0 & 34.829 & 34.829 & 48.151 & 48.151 & 33.106 & 33.106 & 30.835 & 30.835 & 17.046 & 17.046 & 18.978 & 18.978 & 182.945 \\
\hline N1004 E1017 & 10 & 0 & 19.654 & 19.654 & 33.256 & 33.256 & 21.286 & 21.286 & 22.741 & 22.741 & 14.64 & 14.64 & 15.506 & 15.506 & 127.083 \\
\hline
\end{tabular}




\begin{tabular}{|c|c|c|c|c|c|c|c|c|c|}
\hline Unit-Column & Sample & $\begin{array}{l}\text { Elevation } \\
(\mathrm{m})\end{array}$ & LF1 & LF2 & HF1 & HF2 & $\begin{array}{l}\text { Sample + Cube } \\
\text { Mass (g) }\end{array}$ & LF avg. & Hf avg. \\
\hline N996/E1014 & MS01 & 99.52 & 18.7000008 & 18.7000008 & 16.8999996 & 17 & 15.346 & 18.7000008 & 16.9499998 \\
\hline N996/E1014 & $\mathrm{MSO2}$ & 99.49 & 21 & 21.1000004 & 19.3999996 & 19.5 & 14.982 & 21.0500002 & 19.4499998 \\
\hline N996/E1014 & $\mathrm{MS} 03$ & 99.46 & 21.2000008 & 21.1000004 & 19.8999996 & 20 & 14.862 & 21.1500006 & 19.9499998 \\
\hline N996/E1014 & MS04 & 99.44 & 22.7999992 & 22.8999996 & 21.3999996 & 21.7000008 & 15.349 & 22.8499994 & 21.5500002 \\
\hline N996/E1014 & MS05 & 99.43 & 22.3999996 & 22.5 & 19.8999996 & 20 & 15.378 & 22.4499998 & 19.9499998 \\
\hline N996/E1014 & MS06 & 99.39 & 24 & 24 & 22.1000004 & 22.2000008 & 15.533 & 24 & 22.1500006 \\
\hline N996/E1014 & MS07 & 99.36 & 25 & 25 & 23.2999992 & 23.2999992 & 15.273 & 25 & 23.2999992 \\
\hline N996/E1014 & MS08 & 99.35 & 24.7999992 & 24.7999992 & 23.1000004 & 23.1000004 & 15.053 & 24.7999992 & 23.1000004 \\
\hline N996/E1014 & MS09 & 99.3 & 25.7000008 & 25.7000008 & 23.8999996 & 23.8999996 & 15.379 & 25.7000008 & 23.8999996 \\
\hline N996/E1014 & MS10 & 99.29 & 24.7000008 & 24.7000008 & 23 & 23 & 14.863 & 24.7000008 & 23 \\
\hline N996/E1014 & MS11 & 99.25 & 26.3999996 & 26.3999996 & 23.6000004 & 23.5 & 15.09 & 26.3999996 & 23.5500002 \\
\hline N996/E1014 & MS12 & 99.23 & 27.6000004 & 27.6000004 & 24.7999992 & 24.8999996 & 15.253 & 27.6000004 & 24.8499994 \\
\hline N996/E1014 & MS13 & $\begin{array}{l}0.218 \\
99.18\end{array}$ & 27.5 & 27.5 & 24.8999996 & 24.7999992 & 15.169 & 27.5 & 24.8499994 \\
\hline N996/E1014 & MS14 & 99.15 & 27.7000008 & 27.6000004 & 25.2000008 & 25.2000008 & 14.938 & 27.6500006 & 25.2000008 \\
\hline N996/E1014 & MS15 & 99.13 & 29.8999996 & 29.8999996 & 27.1000004 & 27.2000008 & 16.032 & 29.8999996 & 27.1500006 \\
\hline N996/E1014 & MS16 & 99.08 & 26.6000004 & 26.6000004 & 23.7999992 & 23.8999996 & 15.288 & 26.6000004 & 23.8499994 \\
\hline N996/E1014 & MS17 & 99.06 & 26.7999992 & 26.7999992 & 24.1000004 & 24.2000008 & 15.13 & 26.7999992 & 24.1500006 \\
\hline 996/E1014 & MS18 & 99.01 & 25 & 24.8999996 & 22.6000004 & 22.5 & 15.589 & 24.9499998 & 22.5500002 \\
\hline N996/E1014 & MS19 & 99 & 24.7999992 & 24.7999992 & 22.3999996 & 22.5 & 15.545 & 24.7999992 & 22.4499998 \\
\hline N996/E1014 & MS20 & 98.96 & 23.6000004 & 23.7000008 & 21.6000004 & 21.6000004 & 15.194 & 23.6500006 & 21.6000004 \\
\hline N996/E1014 & MS21 & 98.93 & 22.7999992 & 22.7999992 & 20.7000008 & 20.7000008 & 15.416 & 22.7999992 & 20.7000008 \\
\hline N996/E1014 & MS22 & 98.9 & 22.5 & 22.5 & 20.3999996 & 20.5 & 15.073 & 22.5 & 20.4499998 \\
\hline N996/E1014 & MS23 & 98.87 & 22.7999992 & 22.7999992 & 20.7999992 & 20.7999992 & 15.519 & 22.7999992 & 20.7999992 \\
\hline N996/E1014 & MS24 & 98.84 & 22.8999996 & 22.8999996 & 20.8999996 & 20.8999996 & 15.68 & 22.8999996 & 20.8999996 \\
\hline$\frac{\text { N996/E1014 }}{4}$ & MS25 & 98.81 & 22 & 22 & 20.1000004 & 20.1000004 & 15.196 & 22 & 20.1000004 \\
\hline N996/E1014 & MS26 & 98.79 & 23.6000004 & 23.6000004 & 21.2000008 & 21.2000008 & 15.974 & 23.6000004 & 21.2000008 \\
\hline N996/E1014 & MS27 & 98.75 & 23.6000004 & 23.5 & 21.1000004 & 21.1000004 & 15.988 & 23.5500002 & 21.1000004 \\
\hline$\frac{N 966 / E 1014}{\text { N996 }}$ & MS28 & 98.73 & 21.79999992 & 21.7000008 & 19.6000004 & 19.6000004 & 15.534 & 21.75 & 19.6000004 \\
\hline N996/E1014 & MS29 & 98.69 & 22.2999992 & 22.2000008 & 20.1000004 & 20.1000004 & 16.376 & 22.25 & 20.1000004 \\
\hline N996/E1014 & MS30 & 98.67 & 20.6000004 & 20.6000004 & 18.6000004 & 18.7000008 & 16.278 & 20.6000004 & 18.6500006 \\
\hline N996/E1014 & MS31 & 98.63 & 19.3999996 & 19.3999996 & 17.5 & 17.5 & 16.331 & 19.3999996 & 17.5 \\
\hline N996/E1014 & MS32 & 98.6 & 18.7999992 & 18.7999992 & 17 & 17.1000004 & 16.148 & 18.7999992 & 17.0500002 \\
\hline N996/E1014 & MS33 & 98.54 & 17.1000004 & 17.1000004 & 15.6000004 & 15.6999998 & 15.979 & 17.1000004 & 15.6500001 \\
\hline N996/E1014 & MS34 & 98.53 & 19.7999992 & 19.8999996 & 18.2999992 & 18.2000008 & 16.098 & 19.8499994 & 18.25 \\
\hline N996/E1014 & MS35 & 98.5 & 16.2999992 & 16.3999996 & 15 & 15 & 16.262 & 16.3499994 & 15 \\
\hline N996/E1014 & MS36 & 98.46 & 16.1000004 & 16.1000004 & 14.6000004 & 14.6000004 & 16.562 & 16.1000004 & 14.6000004 \\
\hline N996/E1014 & MS37 & 98.43 & 16.2000008 & 16.1000004 & 14.6999998 & 14.6000004 & 16.193 & 16.1500006 & 00001 \\
\hline N996/E1014 & MS38 & 98.4 & 14.3999996 & 14.5 & 13.1000004 & 13.1000004 & 16.194 & 14.4499998 & 13.1000004 \\
\hline N996/E1014 & MS39 & 98.38 & 12.8999996 & 12.8999996 & 11.8999996 & 11.8999996 & 16.064 & 12.8999996 & 11.8999996 \\
\hline & MS40 & 98.35 & 15 & 15 & 13.6999998 & 13.6999998 & & 15 & \\
\hline N996/E1014 & MS41 & 98.31 & 13.1999998 & 13.1999998 & 12.1000004 & 12.1000004 & 16.34 & 13.1999998 & 00004 \\
\hline N996/E1014 & MS42 & 98.28 & 12.5 & 12.5 & 11.6000004 & 11.6000004 & 16.624 & 12.5 & 11.6000004 \\
\hline N996/E1014 & MS43 & 98.25 & 12.8000002 & 12.8000002 & 12 & 12 & & 12.8000002 & 12 \\
\hline N996/E1014 & MS44 & 98.21 & 11.8999996 & 12 & 11.3000002 & 11.3999996 & 16.406 & 11.9499998 & 11.34 \\
\hline N996/E1014 & MS45 & 98.21 & 12.1999998 & 12.1999998 & 11.6000004 & 11.6999998 & 16.145 & 12.1999998 & 11.6500001 \\
\hline 996/E1014 & MS46 & 98.17 & 11.1999998 & 11.3000002 & 10.1000004 & 10.1000004 & & 11.25 & \\
\hline N996/E1014 & MS47 & 98.14 & 11.6999998 & 11.6999998 & 10.6999998 & 10.6000004 & 16.524 & 11.6999998 & 10.6500001 \\
\hline N996/E1014 & MS48 & 98.1 & 10.8999996 & 10.8999996 & 9.89999962 & 10 & 16.627 & 9996 & 9981 \\
\hline & MS49 & 98.07 & 10.8999996 & 10.8999996 & 10 & 10.1000004 & & & \\
\hline N996/E1014 & MS50 & 98.03 & 10.3000002 & 10.3999996 & 9.5 & 9.5 & 16.875 & 10.3499999 & 9.5 \\
\hline N996/E1014 & MS51 & 98 & 9.69999981 & 9.69999981 & 8.80000019 & 8.69999981 & 16.609 & 9.69999981 & 8.75 \\
\hline & MS52 & 97.95 & 9.5 & 9.5 & 8.69999981 & 8.69999981 & & 9.5 & \\
\hline 1996/E1014 & MS53 & 97.94 & 9 & 89999962 & 8.100 & 8.10000038 & 16.333 & 9981 & 038 \\
\hline N996/E1014 & MS54 & 97.9 & 9.5 & 9.60000038 & 8.80000019 & 8.80000019 & 16.895 & 9.55000019 & 0019 \\
\hline N996/E1014 & MS55 & 97.9 & 10 & 10 & 9.300 & 9.30000019 & 17.247 & 10 & 0019 \\
\hline N996/E1014 & 1556 & 97.86 & 9.10000038 & 9.10000038 & 8.30 & 8.30000019 & 17.201 & 0038 & 8.3000 \\
\hline 996/E1014 & MS57 & 97.84 & 9.5 & 9.60000038 & 8.80000019 & 8.69999981 & 16.735 & 0019 & 8.75 \\
\hline N996/E1014 & MS58 & 97.81 & 8.60000038 & 8.69999981 & 8 & & 16.872 & 8.6500001 & 8 \\
\hline N996/E1014 & S59 & 97.78 & 9.5 & 9.5 & 8.80000019 & 8.80000019 & 16.5 & 9.5 & 8.80 \\
\hline N996/E1014 & MS60 & 97.75 & 9.5 & 9.5 & 8.89999962 & 8.89999962 & 16.8 & 9.5 & 9962 \\
\hline N996/E1014 & MS61 & 97.72 & 10.1000004 & 10.1000004 & 9.19999981 & 9.19999981 & 16.551 & 10.1000004 & 99981 \\
\hline 996/E1014 & S62 & 97.69 & 12.8000002 & 12.8000002 & 11.6999998 & 11.6000004 & 17.849 & 12.8000002 & \\
\hline N996/E1014 & MS63 & 97.65 & 11.1000004 & 11.1000004 & 10.1999998 & 10.1000004 & 16.784 & 00004 & 10.1500001 \\
\hline N996/E1014 & MS64 & 97.62 & 13 & 13 & 12 & 12 & 17.14 & 13 & 12 \\
\hline & & & & & & & & & \\
\hline V1000/E1004 & MS01 & 99.74 & & 008 & & & & 23.6 & \\
\hline 04 & $\mathrm{MSO2}$ & 99.72 & 00004 & 5.1000004 & 22.8999996 & 22.8999996 & 14.434 & 00004 & 99996 \\
\hline & MS & 99.69 & $25.8 \mathrm{~s}$ & 996 & & & & & \\
\hline E1004 & MS04 & $\begin{array}{l}99.03 \\
9.63\end{array}$ & & 26.8999996 & 24.3999996 & 24.3999996 & & & \\
\hline N1000/E1004 & MS05 & 99.6 & 25.3999996 & 25.5 & 23.1000004 & 23.1000004 & 15.245 & 25.4499998 & 23.1000004 \\
\hline & MS06 & 99.53 & & 2000008 & & & & & \\
\hline & MS07 & 99.48 & 0.7000008 & 20.7000008 & 19.1000004 & & 5.053 & & \\
\hline 1004 & MS08 & 99.43 & 19.89999966 & 19.8999996 & 18.3999996 & 18.3999996 & 15.01 & 99996 & 99996 \\
\hline & MS09 & 99.3 & 20.3999996 & 20.39999 & 18.7000008 & 18.7000 & & 20.3999 & \\
\hline & MS10 & 99.32 & 20.5 & 20.6000004 & 19 & & 15.574 & & 500002 \\
\hline & MS11 & 99.3 & 19.5 & 19.6000004 & 17.7999992 & 17.8999996 & 15.184 & & 199994 \\
\hline & $\mathrm{MS} 12$ & 99.26 & & 20.2999992 & 18.6000004 & & & 9992 & \\
\hline & $\mathrm{MS} 13$ & 99.23 & 0004 & 20.6000004 & 19 & 19.1000004 & & & 19.0500002 \\
\hline & MS14 & 99. & & 20.5 & 18.5 & & & & 18.5 \\
\hline & MS15 & 99.13 & & 23.1000004 & 20.7000008 & 20.7000008 & & & 20.7000008 \\
\hline /E1004 & MS16 & 99.07 & & 20.79 & 18.5 & 18.5 & 16.077 & 20.7999992 & 18.5 \\
\hline & MS17 & 99.01 & 8.2999992 & 18.2999992 & 16.3999996 & 16.3999996 & 15.18 & 18.2999992 & 16.3999996 \\
\hline & MS18 & 98.94 & 7.6000004 & 17.6000004 & 15.8999996 & 15.8999996 & 15.11 & 000004 & 9996 \\
\hline & 40 & 98.91 & & & 15.60 & & 15.375 & & \\
\hline & MS20 & 98.83 & 96 & 17.79 & 16 & & 16 & & \\
\hline N1000/E1004 & MS21 & 98.78 & 15.1999998 & 15.1999998 & 13.8000002 & 13.8999996 & 15.489 & 15.1999998 & 13.8499999 \\
\hline & MS22 & 98.71 & 15.3000002 & & 14.1000004 & 14.1000004 & 16.148 & & 00004 \\
\hline N1000/E1004 & MS23 & 98.66 & 14.1999998 & 14.1000004 & 13.1999998 & 13.1999998 & 15.632 & 14.1500001 & 13.1999998 \\
\hline
\end{tabular}




\begin{tabular}{|c|c|c|c|c|c|c|c|c|c|c|c|c|}
\hline Unit-Column & Sample & $\begin{array}{l}\text { Elevation } \\
(\mathrm{m})\end{array}$ & LF1 & LF2 & HF1 & HF2 & $\begin{array}{l}\text { Sample + Cube } \\
\text { Mass (g) }\end{array}$ & LF avg. & Hf avg. & $\begin{array}{l}\text { cube wt } \\
\text { (g) }\end{array}$ & $\begin{array}{l}\text { sample Wt } \\
\text { (g) }\end{array}$ & $\begin{array}{c}\text { mass } \\
(\mathbf{k g})\end{array}$ \\
\hline N1000/E1004 & MS24 & 98.6 & 13.3000002 & 13.1999998 & 12.5 & 12.5 & 15.375 & 13.25 & 12.5 & 5.82 & 9.555 & 0.009555 \\
\hline N1000/E1004 & MS25 & 98.55 & 13.6999998 & 13.6000004 & 12.8000002 & 12.8000002 & 15.602 & 13.6500001 & 12.8000002 & 5.82 & 9.782 & 0.009782 \\
\hline N1000/E1004 & MS26 & 98.49 & 14 & 14 & 12.8000002 & 12.6999998 & 15.577 & 14 & 12.75 & 5.82 & 9.757 & 0.009757 \\
\hline N1000/E1004 & MS27 & 98.45 & 13.1999998 & 13.3000002 & 12.1000004 & 12.1999998 & 15.664 & 13.25 & 12.1500001 & 5.82 & 9.844 & 0.009844 \\
\hline N1000/E1004 & MS28 & 98.42 & 13.8999996 & 14 & 12.8999996 & 12.8000002 & 16.061 & 13.9499998 & 12.8499999 & 5.82 & 10.241 & 0.010241 \\
\hline V1000/E1004 & MS29 & 98.36 & 13.1999998 & 13.1999998 & 12.1000004 & 12.1000004 & 16.322 & 13.1999998 & 12.1000004 & 5.82 & 10.502 & 0.010502 \\
\hline V1001/E1010 & MS01 & 99.43 & 23.5 & 23.5 & 21.2999992 & 21.2999992 & 14.117 & 23.5 & 21.2999992 & 5.82 & 8.297 & 0.008297 \\
\hline N1001/E1010 & $\mathrm{MS} 02$ & 99.38 & 24 & 24 & 21.7999992 & 21.7999992 & 14.344 & 24 & 21.7999992 & 5.82 & 8.524 & 0.008524 \\
\hline N1001/E1010 & MS03 & 99.33 & 23.7999992 & 23.7999992 & 21.6000004 & 21.6000004 & 14.176 & 23.7999992 & 21.6000004 & 5.82 & 8.356 & 0.008356 \\
\hline N1001/E1010 & MS04 & 99.28 & 26.2999992 & 26.3999996 & 23.8999996 & 23.8999996 & 15.049 & 26.3499994 & 23.8999996 & 5.82 & 9.229 & 0.009229 \\
\hline N1001/E1010 & MS05 & 99.23 & 25.3999996 & 25.3999996 & 23.1000004 & 23.2000008 & 14.745 & 25.3999996 & 23.1500006 & 5.82 & 8.925 & 0.008925 \\
\hline N1001/E1010 & MS06 & $\begin{array}{l}9.218 \\
99.18\end{array}$ & 25.7999992 & 25.7999992 & 23.1000004 & 23.1000004 & 14.905 & 25.7999992 & 23.1000004 & 5.82 & 9.085 & 0.009085 \\
\hline N1001/E1010 & MS07 & 99.13 & 24.1000004 & 24.1000004 & 21.7999992 & 21.7000008 & 14.692 & 24.1000004 & 21.75 & 5.82 & 8.872 & 0.008872 \\
\hline N1001/E1010 & MS08 & 99.08 & 22.5 & 22.3999996 & 20.2000008 & 20.2999992 & 15.045 & 22.4499998 & 20.25 & 5.82 & 9.225 & 0.009225 \\
\hline N1001/E1010 & MS09 & 99.03 & 21.7000008 & 21.7000008 & 19.6000004 & 19.7000008 & 15.053 & 21.7000008 & 19.6500006 & 5.82 & 9.233 & 0.009233 \\
\hline N1001/E1010 & MS10 & 98.98 & 21.1000004 & 21.1000004 & 19.2000008 & 19.2000008 & 15.037 & 21.1000004 & 19.2000008 & 5.82 & 9.217 & 0.009217 \\
\hline N1001/E1010 & MS11 & 98.93 & 20.7000008 & 20.7000008 & 19 & 18.8999996 & 15.307 & 20.7000008 & 18.9499998 & 5.82 & 9.487 & 0.009487 \\
\hline N1001/E1010 & MS12 & 98.85 & 21.2000008 & 21.2999992 & 19.2000008 & 19.2000008 & $\frac{10.001}{15.449}$ & 21.25 & 19.2000008 & 5.82 & $\begin{array}{l}3.4029 \\
9.629\end{array}$ & 0.009629 \\
\hline N1001/E1010 & MS13 & 98.83 & 20.3999996 & 20.3999996 & 18.3999996 & 18.3999996 & 15.87 & 20.3999996 & 18.3999996 & 5.82 & 10.05 & 0.01005 \\
\hline N1001/E1010 & $\mathrm{MS14}$ & 98.78 & 18.7999992 & 18.7999992 & 16.7999992 & 16.7999992 & 15.173 & 18.7999992 & 16.7999992 & 5.82 & 9.353 & 0.009353 \\
\hline N1001/E1010 & MS15 & 98.73 & 21.2999992 & 21.2999992 & 19.2999992 & 19.2999992 & 16.156 & 21.2999992 & 19.2999992 & 5.82 & 10.336 & 0.010336 \\
\hline N1001/E1010 & MS16 & 98.68 & 21.7999992 & 21.7999992 & 19.7000008 & 19.7999992 & 15.72 & 21.7999992 & 19.75 & 5.82 & 9.9 & 0.0099 \\
\hline N1001/E1010 & MS17 & 98.63 & 22.6000004 & 22.5 & 20.2000008 & 20.2000008 & 15.646 & 22.5500002 & 20.2000008 & 5.82 & 9.826 & 0.009826 \\
\hline N1001/E1010 & MS18 & 98.58 & 23.7000008 & 23.7000008 & 21.5 & 21.6000004 & 15.912 & 23.7000008 & 21.5500002 & 5.82 & 10.092 & 0.010092 \\
\hline N1001/E1010 & MS19 & 98.53 & 19.7000008 & 19.7000008 & 17.8999996 & 17.8999996 & 15.326 & 19.7000008 & 17.8999996 & 5.82 & 9.506 & 0.009506 \\
\hline N1001/E1010 & MS20 & 98.46 & 16.1000004 & 16 & 14.5 & 14.5 & 15.867 & 16.0500002 & 14.5 & 5.82 & 10.047 & 0.010047 \\
\hline N100 & MS21 & 98.38 & 15.1999998 & 15.1999998 & 13.6999998 & 13.8000002 & 16.256 & 15.1999998 & 13.75 & 5.82 & 10.436 & 0.010436 \\
\hline & MS22 & 98.33 & 14.3999996 & 14.3999996 & 13 & 13 & 15.944 & 14.3999996 & 13 & 5.82 & 10.124 & 0.010124 \\
\hline$\overline{\mathrm{N} 1001 / \mathrm{E} 1010}$ & MS23 & 98.28 & 13.8000002 & 13.6999998 & 12.5 & 12.6000004 & 16.629 & 13.75 & 12.5500002 & 5.82 & 10.809 & 0.010809 \\
\hline IE1010 & MS24 & 98.23 & 11.6999998 & 11.6000004 & 10.6999998 & 10.6999998 & 16.235 & 11.6500001 & 10.6999998 & 5.82 & 10.415 & 0.010415 \\
\hline N1001/E1010 & MS25 & 98.18 & 10.6999998 & 10.6999998 & 9.69999981 & 9.69999981 & 15.439 & 10.6999998 & 9.69999981 & 5.82 & 9.619 & 0.009619 \\
\hline N1001/E1010 & MS26 & 98.13 & 11.1999998 & 11.1000004 & 10.1000004 & 10.1000004 & 15.541 & 11.1500001 & 10.1000004 & 5.82 & 9.721 & 0.009721 \\
\hline N1001/E1010 & MS27 & 98.08 & 11.8999996 & 12 & 10.6999998 & 10.6000004 & 15.726 & 11.9499998 & 10.6500001 & 5.82 & 9.906 & 0.009906 \\
\hline N1001/E1010 & MS28 & 98.03 & 11.6000004 & 11.6000004 & 10.3999996 & 10.3999996 & 16.231 & 11.6000004 & 999996 & 5.82 & 10.411 & 0.010411 \\
\hline N1001/E1010 & MS29 & 97.98 & 10.8000002 & 10.8000002 & 9.80000019 & 9.80000019 & 15.647 & 10.8000002 & 9.80000019 & 5.82 & 9.827 & 0.009827 \\
\hline N1001/E1010 & MS30 & 97.93 & 12.1000004 & 12.1000004 & 10.8999996 & 10.8999996 & 17.909 & 12.1000004 & 10.8999996 & $\frac{3.02}{5.82}$ & $\frac{9.021}{12.089}$ & $\begin{array}{l}0.090221 \\
0.012089\end{array}$ \\
\hline N1001/E1010 & MS31 & 97.88 & 10.3000002 & 10.3000002 & 9.60000038 & 9.60000038 & 16.025 & 10.3000002 & 9.60000038 & 5.82 & 10.205 & 0.010205 \\
\hline N1001/E1010 & MS32 & 97.83 & 10.3999996 & 10.3999996 & 9.69999981 & 9.69999981 & 15.885 & 10.3999996 & 9.69999981 & 5.82 & 10.065 & 0.010065 \\
\hline & MS33 & 97.73 & 8.69999981 & 8.69999981 & 8.19999981 & 8.10000038 & 16.973 & 8.69999981 & & 5.82 & 11.153 & 0.011153 \\
\hline N1001/E1010 & MS34 & 97.64 & 9.10000038 & 9.10000038 & 8.69999981 & 8.60000038 & 16.17 & 9.10000038 & 00001 & 5.82 & 10.35 & 0.01035 \\
\hline & $\mathrm{MS} 01$ & 9922 & 9996 & 9992 & 21.5 & & 806 & 8499994 & & 582 & 7986 & \\
\hline & MSO2 & 99.15 & 6.07000008 & 26.7000008 & 27 & & 16.3902 & 26.7000008 & & 5.82 & 10.572 & 0.010572 \\
\hline N1004/E1017 & MSO3 & 99.12 & 26.2000008 & 26.2000008 & 26.7999992 & 26.8999996 & 16.514 & 26.2000008 & 26.8499994 & 5.82 & 10.694 & 0.010694 \\
\hline & MS04 & 99.09 & 21.5 & 21.3999996 & 23.2000008 & 23.2000008 & 14.813 & 21.4499998 & 23.2000008 & 5.82 & 8.993 & \\
\hline N1004/E1017 & MS05 & 99.05 & 22.1000004 & 22 & 23.8999996 & 23.89999966 & 15.327 & 22.0500002 & 23.8999996 & 5.82 & 9.507 & 0.009507 \\
\hline N1004/E1017 & MS06 & 99.01 & 21.6000004 & 21.6000004 & 19.2999992 & 19.39999966 & 15.555 & 21.6000004 & 99994 & 5.82 & 9.735 & 0.009735 \\
\hline & MS07 & & 24.5 & 24.5 & 22.1000004 & 22.2000008 & & 24.5 & & 5.82 & & \\
\hline N1004/E1017 & MS08 & 98.94 & 25.7999992 & 25.8999996 & 23.3999996 & 23.5 & 14.872 & 25.8499994 & 99998 & 5.82 & 9.052 & 0.009052 \\
\hline IE1017 & MS09 & 98.89 & 24.5 & 24.3999996 & 22.3999996 & 22.3999996 & 14.267 & 24.4499998 & 9999996 & 5.82 & 8.447 & 0.008447 \\
\hline & MS10 & 98.88 & & 24.7999992 & 22.7999992 & 22.7000008 & & 7999992 & 22. & 5.82 & & \\
\hline 4/E1017 & MS11 & 98.82 & 24.89 & 24.8999996 & 22.2999992 & 22.399 & 14.648 & 24.8999996 & 99994 & 5.82 & 8.828 & 0.008828 \\
\hline N1004/E1017 & MS12 & 98.77 & 23.8999996 & 99992 & 21.5 & 00004 & 14.499 & 8499994 & & 5.82 & 8.679 & 0.008679 \\
\hline & MS13 & 98.75 & 23.2999992 & 23.2999992 & 21 & 0004 & 14.479 & 23.2999992 & 0002 & 5.82 & 8.659 & 0.008659 \\
\hline & MS14 & 98.73 & 23 & 3.1000004 & 21.1000004 & 0004 & 14.76 & 0500002 & & 5.82 & 8.9 & 0.00894 \\
\hline & MS15 & 98.69 & 23.2999992 & 23.3999996 & 21.2999992 & 21.2999992 & 15.012 & 23.3499994 & 9992 & 5.82 & 9.1 & 0.009192 \\
\hline N100 & MS16 & 98.63 & 22.5 & 22.6000004 & 20.2999992 & 20.2999992 & 14.839 & 22.5500002 & 9992 & 5.82 & 9.019 & 0.009019 \\
\hline & MS17 & 98.6 & 1.6000004 & 21.6000004 & 19.5 & 9996 & & 6000004 & & 5.82 & 9.524 & 0.009524 \\
\hline & MS18 & 98.57 & 20.6000004 & 20.7000008 & 18.6000004 & & & 20.6500006 & & 5.82 & 9.566 & 0.009566 \\
\hline$\overline{\mathrm{N} 10}$ & MS19 & 98.54 & 20.5 & 20.5 & 18.6000004 & 18.6 & 15.113 & 20.5 & 0004 & 5.82 & 9.293 & 0.009293 \\
\hline & $\mathrm{MS} 20$ & 98.49 & 20 & 20 & 000004 & 18.2 & & 20 & & 5.82 & & 0.009563 \\
\hline & MS21 & $\begin{array}{l}98.45 \\
98.48\end{array}$ & 19.2999992 & 19.3999996 & 17.3999996 & 17.2999992 & 15.033 & 19.3499994 & 3499994 & 5.02 & 9.2 & 0.009213 \\
\hline N10 & MS22 & 98.43 & 18.2000008 & 18.2000008 & 16.1000004 & 16.2000008 & 15.621 & 18.2000008 & 16.1500006 & 5.82 & 9.801 & 0.009801 \\
\hline & MS23 & 98.38 & & 17.5 & 15.5 & 15.5 & & & & 5.82 & & 0.009957 \\
\hline & MS24 & 98.33 & 15.5 & 15.5 & 13.8000002 & 13.6999998 & 15.422 & 15.5 & 13. & 5.82 & 9.602 & 0.009602 \\
\hline & MS25 & 98.31 & 15.3999996 & 15.3999996 & 13.8000002 & 13.8000002 & 14.987 & 15.3999996 & 3000002 & 5.82 & 9.167 & 0.009167 \\
\hline & $\mathrm{MS}$ & 98.29 & 14.6000004 & 14.6000004 & 12.8000002 & 12.8999996 & 15.299 & 000004 & 499999 & 5.82 & 9.479 & 0.009479 \\
\hline & MS27 & 98.27 & 16 & 16.1000004 & 14.1999998 & 14.1999998 & 16.045 & 00002 & 99998 & 5.82 & 10.225 & 0.010225 \\
\hline & MS28 & 98.25 & 14.6000004 & 14.6000004 & 12.8000002 & 12.8999996 & 15.647 & 00004 & 12.8499999 & 5.82 & 9.827 & 0.009827 \\
\hline & MS29 & 98.21 & 0004 & 14 & 12.5 & 12.5 & & & & 5.82 & 9.8 & 0.00986 \\
\hline & MS30 & 98.19 & 12.5 & 12.6000004 & 11.5 & 11.5 & 15.071 & 12.5500002 & 11.5 & 5.82 & 9.251 & 0.009251 \\
\hline & MS31 & 98.14 & 13.3000002 & 13.3000002 & 11.8999996 & 11.8999996 & 15.969 & 13.3000002 & 11.8999996 & 5.82 & 10.149 & 0.010149 \\
\hline & MS32 & 98.09 & 12.6000004 & 12.6000004 & 11.3999996 & & & 6000004 & & 5.82 & 9.9 & 0.009921 \\
\hline & MS33 & 98.06 & & 13.5 & 12.3000002 & 12.3000002 & 16.719 & & & 5.82 & & 0.010899 \\
\hline & MS34 & 98.02 & 12.6000004 & 12.6000004 & 11.6000004 & 11.6000004 & 16.368 & 6000004 & & 5.82 & 10.548 & 0.010548 \\
\hline & $M S$ & 97.99 & & 12.6000004 & 11.3999996 & 11.300 & 16.027 & 00002 & & 5.82 & 10.207 & 0.010207 \\
\hline & MS36 & 97.95 & 11.69 & 11.6999998 & 10.6000004 & 10.5 & 15.7 & 11.6999998 & 10.5500002 & 5.82 & & 0.009927 \\
\hline & MS37 & 97.9 & & 12.6999998 & & & & 12.6999998 & & 5.82 & 10. & 0.01017 \\
\hline & MS38 & 97.86 & 12.6999998 & 12.69 & 11.3000002 & & 16.196 & & & 5.82 & 10.376 & 0.010376 \\
\hline & MS39 & 97.81 & & & 11.8999996 & 11.8999996 & & & 11.8999996 & 5.82 & & 0.011226 \\
\hline N1004/E1017 & MS40 & 97.77 & 12.1999998 & 12.3000002 & 10.8999996 & 10.8000002 & 16.021 & 12.25 & 10.8499999 & 5.82 & 10.201 & 0.010201 \\
\hline & MS01 & & 2999992 & .2999992 & & & & & & 5.82 & & 0.008476 \\
\hline & MSO2 & 99.12 & & 21 & & 19.3999996 & & & & 5.82 & 9.321 & \\
\hline 9/E1012 & MSO3 & 99.09 & 22.5 & 22.6000004 & 20.8999996 & 21 & 15.697 & 22.5500002 & 20.9499998 & 5.82 & 9.877 & 0.009877 \\
\hline & MS04 & 99.06 & 23.1000004 & 23.2000008 & 21.5 & 21.5 & & & & 5.82 & & \\
\hline N1009/E1012 & MS05 & 99.04 & 21.8999996 & 22 & 19.7000008 & 19.8999996 & 15.251 & 21.9499998 & 19.8000002 & 5.82 & 9.431 & 0.009431 \\
\hline
\end{tabular}




\begin{tabular}{|c|c|c|c|c|c|c|c|c|c|c|c|c|c|c|}
\hline Unit-Column Sample & $\begin{array}{c}\text { Elevation } \\
(\mathrm{m})\end{array}$ & LF1 & LF2 & HF1 & HF2 & $\begin{array}{c}\text { Sample + Cube } \\
\text { Mass (g) }\end{array}$ & LF avg. & Hf avg. & $\begin{array}{c}\text { cube wt } \\
\text { (g) }\end{array}$ & $\begin{array}{c}\text { sample Wt } \\
\text { (g) }\end{array}$ & $\begin{array}{c}\operatorname{mass} \\
(\mathrm{kg})\end{array}$ & Xhf & XIf & Xfd \\
\hline N1009/E1012 MS06 & 99.01 & 20.6000004 & 20.6000004 & 18.7999992 & 18.7999992 & 15.326 & 20.6000004 & 18.7999992 & 5.82 & 9.506 & 0.009506 & 19.8 & 21.7 & 8.7 \\
\hline N1009/E1012 MS07 & 98.97 & 20.2000008 & 20.2999992 & 18.5 & 18.5 & 15.739 & 20.25 & 18.5 & 5.82 & 9.919 & 0.009919 & 18.7 & 20.4 & 8.6 \\
\hline N1009/E1012 MS08 & 98.93 & 21.3999996 & 21.3999996 & 19.5 & 19.5 & 15.086 & 21.3999996 & 19.5 & 5.82 & 9.266 & 0.009266 & 21.0 & 23.1 & 8.9 \\
\hline N1009/E1012 MS09 & 98.9 & 21.3999996 & 21.3999996 & 19.6000004 & 19.6000004 & 14.97 & 21.3999996 & 19.6000004 & 5.82 & 9.15 & 0.00915 & 21.4 & 23.4 & 8.4 \\
\hline N1009/E1012 MS10 & 98.87 & 21.8999996 & 22 & 20 & 19.8999996 & 15.271 & 21.9499998 & 19.9499998 & 5.82 & 9.451 & 0.009451 & 21.1 & 23.2 & 9.1 \\
\hline N1009/E1012 MS11 & 98.84 & 21.5 & 21.5 & 19.3999996 & 19.5 & 14.797 & 21.5 & 19.4499998 & 5.82 & 8.977 & 0.008977 & 21.7 & 24.0 & 9.5 \\
\hline N1009/E1012 MS12 & 98.81 & 21.7000008 & 21.7999992 & 19.7000008 & 19.7000008 & 14.784 & 21.75 & 19.7000008 & 5.82 & 8.964 & 0.008964 & 22.0 & 24.3 & 9.4 \\
\hline N1009/E1012 MS13 & 98.78 & 22.1000004 & 22.1000004 & 20 & 20.1000004 & 14.518 & 22.1000004 & 20.0500002 & 5.82 & 8.698 & 0.008698 & 23.1 & 25.4 & 9.3 \\
\hline N1009/E1012 MS14 & 98.76 & 23 & 22.8999996 & 21.1000004 & 21.1000004 & 15 & 22.9499998 & 21.1000004 & 5.82 & 9.18 & 0.00918 & 23.0 & 25.0 & 8.1 \\
\hline N1009/E1012 MS15 & 98.73 & 24.1000004 & 24.1000004 & 21.7000008 & 21.7000008 & 15.492 & 24.1000004 & 21.7000008 & 5.82 & 9.672 & 0.009672 & 22.4 & 24.9 & 10.0 \\
\hline N1009/E1012 MS16 & 98.69 & 21.2000008 & 21.2000008 & 19.2000008 & 19.2000008 & 14.538 & 21.2000008 & 19.2000008 & 5.82 & 8.718 & 0.008718 & 22.0 & 24.3 & 9.4 \\
\hline N1009/E1012 MS17 & 98.67 & 22.2999992 & 22.3999996 & 20.2999992 & 20.2999992 & 15.143 & 22.3499994 & 20.2999992 & 5.82 & 9.323 & 0.009323 & 21.8 & 24.0 & 9.2 \\
\hline N1009/E1012 MS18 & 98.64 & 23.2999992 & 23.2999992 & 21.1000004 & 21.2000008 & 14.798 & 23.2999992 & 21.1500006 & 5.82 & 8.978 & 0.008978 & 23.6 & 26.0 & 9.2 \\
\hline N1009/E1012 MS19 & 98.61 & 23.2000008 & 23.2999992 & 21.2000008 & 21.2000008 & 14.982 & 23.25 & 21.2000008 & 5.82 & 9.162 & 0.009162 & 23.1 & 25.4 & 8.8 \\
\hline N1009/E1012 MS20 & 98.58 & 21.7000008 & 21.7000008 & 19.5 & 19.6000004 & 14.881 & 21.7000008 & 19.5500002 & 5.82 & 9.061 & 0.009061 & 21.6 & 23.9 & 9.9 \\
\hline N1009/E1012 MS21 & 98.55 & 21.8999996 & 22 & 19.8999996 & 20 & 15.274 & 21.9499998 & 19.9499998 & 5.82 & 9.454 & 0.009454 & 21.1 & 23.2 & 9.1 \\
\hline N1009/E1012 MS22 & 98.51 & 21.1000004 & 21 & 19.1000004 & 19.1000004 & 15.064 & 21.0500002 & 19.1000004 & 5.82 & 9.244 & 0.009244 & 20.7 & 22.8 & 9.3 \\
\hline N1009/E1012 MS23 & 98.48 & 19.7999992 & 19.8999996 & 18.2000008 & 18.2000008 & 15.281 & 19.8499994 & 18.2000008 & 5.82 & 9.461 & 0.009461 & 19.2 & 21.0 & 8.3 \\
\hline N1009/E1012 MS24 & 98.44 & 19 & 19 & 17.3999996 & 17.3999996 & 15.582 & 19 & 17.3999996 & 5.82 & 9.762 & 0.009762 & 17.8 & 19.5 & 8.4 \\
\hline N1009/E1012 MS25 & 98.41 & 17.6000004 & 17.7000008 & 16.2999992 & 16.2999992 & 15.566 & 17.6500006 & 16.2999992 & 5.82 & 9.746 & 0.009746 & 16.7 & 18.1 & 7.6 \\
\hline N1009/E1012 MS26 & 98.38 & 17.6000004 & 17.7000008 & 15.8999996 & 15.8999996 & 15.438 & 17.6500006 & 15.8999996 & 5.82 & 9.618 & 0.009618 & 16.5 & 18.4 & 9.9 \\
\hline N1009/E1012 MS27 & 98.35 & 15.1000004 & 15 & 13.6000004 & 13.6999998 & 14.617 & 15.0500002 & 13.6500001 & 5.82 & 8.797 & 0.008797 & 15.5 & 17.1 & 9.3 \\
\hline N1009/E1012 MS28 & 98.32 & 15.5 & 15.6000004 & 14.1999998 & 14.1999998 & 15.44 & 15.5500002 & 14.1999998 & 5.82 & 9.62 & 0.00962 & 14.8 & 16.2 & 8.7 \\
\hline N1009/E1012 MS29 & 98.3 & 16.6000004 & 16.5 & 15.1000004 & 15.1000004 & 15.626 & 16.5500002 & 15.1000004 & 5.82 & 9.806 & 0.009806 & 15.4 & 16.9 & 8.8 \\
\hline N1009/E1012 MS30 & 98.27 & 14.8999996 & 14.8000002 & 13.5 & 13.600 & 15.119 & 999 & 0002 & 5.82 & 9.299 & 9299 & 14.6 & 16.0 & 8.8 \\
\hline N1009/E1012 MS31 & 98.24 & 15.1000004 & 15.1000004 & 13.6000004 & 13.6000004 & 15.305 & 15.1000004 & 13.6000004 & 5.82 & 9.485 & 0.009485 & 14.3 & 15.9 & 9.9 \\
\hline N1009/E1012 MS32 & 98.21 & 14.5 & 14.3999996 & 13.6999998 & 13.8000002 & 15.238 & 14.4499998 & 13.75 & 5.82 & 9.418 & 0.009418 & 14.6 & 15.3 & 4.8 \\
\hline N1009/E1012 MS33 & 98.18 & 14.6999998 & 14.8000002 & 14.1000004 & 14.1000004 & 16.326 & 14.75 & 14.1000004 & 5.82 & 10.506 & 0.010506 & 13.4 & 14.0 & 4.4 \\
\hline N1009/E1012 MS34 & 98.15 & 14.1000004 & 14.1000004 & 13.6999998 & 13.6000004 & 15.26 & 14.1000004 & 13.6500001 & 5.82 & 9.44 & 0.00944 & 14.5 & 14.9 & 3.2 \\
\hline N1009/E1012 MS35 & 98.12 & 14.1000004 & 14.1000004 & 13 & 12.8999996 & 15.255 & 14.1000004 & 12.9499998 & 5.82 & 9.435 & 0.009435 & 13.7 & 14.9 & 8.2 \\
\hline N1009/E1012 MS36 & 98.09 & 14.8000002 & 14.8000002 & 13.1999998 & 13.1999998 & 15.8 & 14.8000002 & 13.1999998 & 5.82 & 9.98 & 0.00998 & 13.2 & 14.8 & 10.8 \\
\hline N1009/E1012 MS37 & 98.06 & 14.1999998 & 14.1999998 & 12.6000004 & 12.6000004 & 15.706 & 14.1999998 & 12.6000004 & 5.82 & 9.886 & 0.009886 & 12.7 & 14.4 & 11.3 \\
\hline N1009/E1012 MS38 & 98.03 & 13.8999996 & 13.8999996 & 12.1999998 & 12.1999998 & 15.283 & 13.8999996 & 12.1999998 & 5.82 & 9.463 & 0.009463 & 12.9 & 14.7 & 12.2 \\
\hline N1009/E1012 MS39 & 98 & 13.1999998 & 13.1999998 & 12 & 12.1000004 & 15.357 & 13.1999998 & 12.0500002 & 5.82 & 9.537 & 0.009537 & 12.6 & 13.8 & 8.7 \\
\hline N1009/E1012 MS40 & 97.97 & 13.6000004 & 13.6000004 & 12.1000004 & 12.1000004 & 16.405 & 13.6000004 & 12.1000004 & 5.82 & 10.585 & 0.010585 & 11.4 & 12.8 & 11.0 \\
\hline N1009/E1012 MS41 & 97.94 & 12.5 & 12.3999996 & 11.1999998 & 11.1999998 & 15.926 & 12.4499998 & 11.1999998 & 5.82 & 10.106 & 0.010106 & 11.1 & 12.3 & 10.0 \\
\hline N1009/E1012 MS42 & 97.91 & 12.5 & 12.5 & 11.3999996 & 11.5 & 16.041 & 12.5 & 11.4499998 & 5.82 & 10.221 & 0.010221 & 11.2 & 12.2 & 8.4 \\
\hline N1009/E1012 MS43 & 97.88 & 12.3000002 & 12.3000002 & 11.1999998 & 11.1999998 & 15.747 & 12.3000002 & 11.1999998 & 5.82 & 9.927 & 0.009927 & 11.3 & 12.4 & 8.9 \\
\hline N1009/E1012 MS44 & 97.85 & 12.3000002 & 12.3999996 & 11.3000002 & 11.3000002 & 15.48 & 12.3499999 & 11.3000002 & 5.82 & 9.66 & 0.00966 & 11.7 & 12.8 & 8.5 \\
\hline N1009/E1012 MS45 & 97.82 & 12.1999998 & 12.1000004 & 10.8000002 & 10.8000002 & 15.847 & 12.1500001 & 10.8000002 & 5.82 & 10.027 & 0.010027 & 10.8 & 12.1 & 11.1 \\
\hline N1009/E1012 MS46 & 97.79 & 12.6000004 & 12.6999998 & 11.1000004 & 11.1000004 & 16.181 & 12.6500001 & 11.1000004 & 5.82 & 10.361 & 0.010361 & 10.7 & 12.2 & 12.3 \\
\hline N1009/E1012 MS47 & 97.75 & 11.8000002 & 11.8999996 & 10.5 & 10.5 & 15.563 & 11.8499999 & 10.5 & 5.82 & 9.743 & 0.009743 & 10.8 & 12.2 & 11.4 \\
\hline N1009/E1012 MS48 & 97.71 & 11.8000002 & 11.8000002 & 10.6000004 & 10.6000004 & 15.995 & 11.8000002 & 10.6000004 & 5.82 & 10.175 & 0.010175 & 10.4 & 11.6 & 10.2 \\
\hline
\end{tabular}




\begin{tabular}{|c|c|c|c|c|c|c|c|c|c|c|c|c|c|c|c|c|}
\hline North & East & Sample & $\begin{array}{c}\text { Elevation } \\
\text { (m) }\end{array}$ & LF1 & LF2 & HF1 & HF2 & $\begin{array}{c}\text { Mass of Sample } \\
+ \text { Cube } \\
\text { grams }\end{array}$ & LF avg. & Hf avg. & $\begin{array}{c}\text { cube wt } \\
\text { (g) }\end{array}$ & $\begin{array}{c}\text { sample } \\
\text { Wt }\end{array}$ & $\begin{array}{c}\text { mass } \\
(\mathrm{kg})\end{array}$ & Xhf & XIf & $X f d$ \\
\hline N1001.10 & E1004.70 & $0,-1 \mathrm{~A}$ & 99.34 & 21.7999992 & 21.7999992 & 19.7999992 & 19.8999996 & 15.5 & 21.7999992 & 19.8499994 & 5.82 & 9.68 & 0.0 & 20.5 & 22.5 & 8.9 \\
\hline N1001.10 & E1004.50 & $0,-2 \mathrm{~A}$ & 99.34 & 21.7000008 & 21.7999992 & 19.8999996 & 19.8999996 & 15.668 & 21.75 & 19.8999996 & 5.82 & 9.848 & 0.0 & 20.2 & 22.1 & 8.5 \\
\hline N1001.10 & E1004.30 & $0,-3 \mathrm{~A}$ & 99.34 & 21.8999996 & 21.8999996 & 20.2999992 & 20.3999996 & 15.876 & 21.8999996 & 20.3499994 & 5.82 & 10.056 & 0.0 & 20.2 & 21.8 & 7.1 \\
\hline N1001.10 & E1004.10 & $0,-4 \mathrm{~A}$ & 99.34 & 19.8999996 & 19.8999996 & 19 & 19 & 15.700 & 19.8999996 & 19 & 5.82 & 9.88 & 0.0 & 19.2 & 20.1 & 4.5 \\
\hline N1001.10 & E1003.90 & $0,-5 \mathrm{~A}$ & 99.34 & 18.6000004 & 18.7000008 & 17.7999992 & 17.8999996 & 15.624 & 18.6500006 & 17.8499994 & 5.82 & 9.804 & 0.0 & 18.2 & 19.0 & 4.3 \\
\hline N1001.10 & E1003.70 & $0,-6 \mathrm{~A}$ & 99.34 & 19 & 19 & 17.3999996 & 17.5 & 15.863 & 19 & 17.4499998 & 5.82 & 10.043 & 0.0 & 17.4 & 18.9 & 8.2 \\
\hline N1001.10 & E1003.50 & $0,-7 \mathrm{~A}$ & 99.34 & 18.1000004 & 18.1000004 & 16.7000008 & 16.7999992 & 15.725 & 18.1000004 & 16.75 & 5.82 & 9.905 & 0.0 & 16.9 & 18.3 & 7.5 \\
\hline N1001.10 & E1003.30 & $0,-8 \mathrm{~A}$ & 99.34 & 21.3999996 & 21.3999996 & 19.6000004 & 19.6000004 & 16.150 & 21.3999996 & 19.6000004 & 5.82 & 10.33 & 0.0 & 19.0 & 20.7 & 8.4 \\
\hline N1001.10 & E1003.10 & $0,-9 \mathrm{~A}$ & 99.34 & 21.1000004 & 21.1000004 & 19.6000004 & 19.6000004 & 15.41 & 21.1000004 & 19.6000004 & 5.82 & 9.59 & 0.0 & 20.4 & 22.0 & 7.1 \\
\hline N1000.50 & E1004.90 & $-3,0 \mathrm{~A}$ & 99.34 & 23.3999996 & 23.2999992 & 21.7999992 & 21.7999992 & 16.258 & 23.3499994 & 21.7999992 & 5.82 & 10.438 & 0.0 & 20.9 & 22.4 & 6.6 \\
\hline N1001.10 & E1004.90 & $0,0 \mathrm{~A}$ & 99.34 & 21.2999992 & 21.2000008 & 19.8999996 & 19.8999996 & 15.694 & 21.25 & 19.8999996 & 5.82 & 9.874 & 0.0 & 20.2 & 21.5 & 6.4 \\
\hline N1001.10 & E1005.10 & $0,1 \mathrm{~A}$ & 99.34 & 21.8999996 & 21.8999996 & 19.3999996 & 19.3999996 & 15.739 & 21.8999996 & 19.3999996 & 5.82 & 9.919 & 0.0 & 19.6 & 22.1 & 11.4 \\
\hline N1001.10 & E1005.30 & $0,2 \mathrm{~A}$ & 99.34 & 20.6000004 & 20.7000008 & 18.2999992 & 18.2999992 & 15.252 & 20.6500006 & 18.2999992 & 5.82 & 9.432 & 0.0 & 19.4 & 21.9 & 11.4 \\
\hline N1001.10 & E1005.50 & $0,3 \mathrm{~A}$ & 99.34 & 22.1000004 & 22.1000004 & 19.3999996 & 19.3999996 & 15.567 & 22.1000004 & 19.3999996 & 5.82 & 9.747 & 0.0 & 19.9 & 22.7 & 12.2 \\
\hline N1001.10 & E1005.70 & $0,4 \mathrm{~A}$ & 99.34 & 22.3999996 & 22.3999996 & 19.7999992 & 19.7999992 & 15.909 & 22.3999996 & 19.7999992 & 5.82 & 10.089 & 0.0 & 19.6 & 22.2 & 11.6 \\
\hline N1001.10 & E1005.90 & $0,5 \mathrm{~A}$ & 99.34 & 23.2000008 & 23.2000008 & 20.7000008 & 20.7000008 & 15.938 & 23.2000008 & 20.7000008 & 5.82 & 10.118 & 0.0 & 20.5 & 22.9 & 10.8 \\
\hline N1001.10 & E1006.10 & $0,6 \mathrm{~A}$ & 99.34 & 21.7000008 & 21.7999992 & 19.3999996 & 19.5 & 15.188 & 21.75 & 19.4499998 & 5.82 & 9.368 & 0.0 & 20.8 & 23.2 & 10.6 \\
\hline N1001.10 & E1006.30 & $0,7 \mathrm{~A}$ & 99.34 & 22.1000004 & 22.2000008 & 19.8999996 & 19.8999996 & 15.441 & 22.1500006 & 19.8999996 & 5.82 & 9.621 & 0.0 & 20.7 & 23.0 & 10.2 \\
\hline $\mathrm{N} 1001.10$ & E1006.50 & $0,8 \mathrm{~A}$ & 99.34 & 23 & 22.8999996 & 20.7000008 & 20.7000008 & 15.579 & 22.9499998 & 20.7000008 & 5.82 & 9.759 & 0.0 & 21.2 & 23.5 & 9.8 \\
\hline N1001.30 & E1004.90 & $1,0 \mathrm{~A}$ & 99.34 & 21.3999996 & 21.3999996 & 18.7000008 & 18.7000008 & 15.949 & 21.3999996 & 18.7000008 & 5.82 & 10.129 & 0.0 & 18.5 & 21.1 & 12.6 \\
\hline N1001.50 & E1004.90 & $2,0 \mathrm{~A}$ & 99.34 & 22 & 22 & 18.7999992 & 18.7000008 & 15.769 & 22 & 18.75 & 5.82 & 9.949 & 0.0 & 18.8 & 22.1 & 14.8 \\
\hline N1001.70 & E1004.90 & $3,0 \mathrm{~A}$ & 99.34 & 20.6000004 & 20.6000004 & 17.5 & 17.3999996 & 15.186 & 20.6000004 & 17.4499998 & 5.82 & 9.366 & 0.0 & 18.6 & 22.0 & 15.3 \\
\hline N1001.90 & E1004.90 & $4,0 \mathrm{~A}$ & 99.34 & 21.1000004 & 21 & 18.5 & 18.5 & 15.374 & 21.0500002 & 18.5 & 5.82 & 9.554 & 0.0 & 19.4 & 22.0 & 12.1 \\
\hline N1002.10 & E1004.90 & $5,0 \mathrm{~A}$ & 99.34 & 21.7999992 & 21.7999992 & 19.2999992 & 19.2999992 & 15.174 & 21.7999992 & 19.2999992 & 5.82 & 9.354 & 0.0 & 20.6 & 23.3 & 11.5 \\
\hline N1002.30 & E1004.90 & $6,0 \mathrm{~A}$ & 99.34 & 22.7999992 & 22.7999992 & 20 & 19.8999996 & 15.867 & 22.7999992 & 19.9499998 & 5.82 & 10.047 & 0.0 & 19.9 & 22.7 & 12.5 \\
\hline N1002.50 & E1004.90 & $7,0 \mathrm{~A}$ & 99.34 & 22.7000008 & 22.7000008 & 19.5 & 19.5 & 15.618 & 22.7000008 & 19.5 & 5.82 & 9.798 & 0.0 & 19.9 & 23.2 & 14.1 \\
\hline N1002.70 & E1004.90 & $8,0 \mathrm{~A}$ & 99.34 & 23.1000004 & 23.2000008 & 19.7999992 & 19.7999992 & 15.814 & 23.1500006 & 19.7999992 & 5.82 & 9.994 & 0.0 & 19.8 & 23.2 & 14.5 \\
\hline N1000.50 & 4.90 & $-3,0 \mathrm{~B}$ & 99.30 & 22.8999996 & 22.8999996 & 21.5 & 21.5 & 16.242 & 22.8999996 & 21.5 & 5.82 & 10.422 & 0.0 & 20.6 & 22.0 & 6.1 \\
\hline N1000.30 & E1004.90 & $-4,0 \mathrm{~B}$ & 99.30 & 20.7000008 & 20.6000004 & 19.7000008 & 19.7000008 & 15.443 & 20.6500006 & 19.7000008 & 5.82 & 9.623 & 0.0 & 20.5 & 21.5 & 4.6 \\
\hline N1001.10 & E1004.50 & $0,-2 \mathrm{~B}$ & 99.30 & 21.3999996 & 21.5 & 19.7999992 & 19.8999996 & 16.207 & 21.4499998 & 19.8499994 & 5.82 & 10.387 & 0.0 & 19.1 & 20.7 & 7.5 \\
\hline N1001.10 & E1004.30 & $0,-3 \mathrm{~B}$ & 99.30 & 18.6000004 & 18.6000004 & 17.3999996 & 17.5 & 15.292 & 18.6000004 & 17.4499998 & 5.82 & 9.472 & 0.0 & 18.4 & 19.6 & 6.2 \\
\hline N1001.10 & E1004.10 & $0,-4 \mathrm{~B}$ & 99.30 & 17.7999992 & 17.7999992 & 17 & 17 & 15.293 & 17.7999992 & 17 & 5.82 & 9.473 & 0.0 & 17.9 & 18.8 & 4.5 \\
\hline N1001.10 & E1003.90 & $0,-5 \mathrm{~B}$ & 99.30 & 19.3999996 & 19.3999996 & 17.6000004 & 17.6000004 & 16.106 & 19.3999996 & 17.6000004 & 5.82 & 10.286 & 0.0 & 17.1 & 18.9 & 9.3 \\
\hline N1001.10 & E1003.70 & $0,-6 \mathrm{~B}$ & 99.30 & 17.3999996 & 17.3999996 & 16 & 15.8999996 & 15.816 & 17.3999996 & 15.9499998 & 5.82 & 9.996 & 0.0 & 16.0 & 17.4 & 8.3 \\
\hline N1001.10 & E1003.50 & $0,-7 \mathrm{~B}$ & 99.30 & 21.8999996 & 21.7999992 & 20 & 20 & 16.166 & 21.8499994 & 20 & 5.82 & 10.346 & 0.0 & 19.3 & 21.1 & 8.5 \\
\hline N1001.10 & E1003.30 & $0,-8 \mathrm{~B}$ & 99.30 & 20.3999996 & 20.3999996 & 18.8999996 & 18.8999996 & 15.237 & 20.3999996 & 18.8999996 & 5.82 & 9.417 & 0.0 & 20.1 & 21.7 & 7.4 \\
\hline N1001.70 & E1004.90 & $3,0 \mathrm{~B}$ & 99.30 & 20.2999992 & 20.2999992 & 18.7000008 & 18.7000008 & 15.339 & 20.2999992 & 18.7000008 & 5.82 & 9.519 & 0.0 & 19.6 & 21.3 & 7.9 \\
\hline N1001.90 & E1004.90 & $4,0 \mathrm{~B}$ & 99.30 & 19.7000008 & 19.7000008 & 18.2999992 & 18.2999992 & 15.465 & 19.7000008 & 18.2999992 & 5.82 & 9.645 & 0.0 & 19.0 & 20.4 & 7.1 \\
\hline N1002.10 & E1004.90 & $5,0 \mathrm{~B}$ & 99.30 & 20.2999992 & 20.2999992 & 18.7999992 & 18.8999996 & 15.443 & 20.2999992 & 18.8499994 & 5.82 & 9.623 & 0.0 & 19.6 & 21.1 & 7.1 \\
\hline N1002.30 & E1004.90 & $6,0 \mathrm{~B}$ & 99.30 & 20.7000008 & 20.7000008 & 19.2000008 & 19.2000008 & 15.441 & 20.7000008 & 19.2000008 & 5.82 & 9.621 & 0.0 & 20.0 & 21.5 & 7.2 \\
\hline N1002.50 & E1004.90 & $7,0 \mathrm{~B}$ & 99.30 & 21.5 & 21.3999996 & 20.1000004 & 20.1000004 & 15.832 & 21.4499998 & 20.1000004 & 5.82 & 10.012 & 0.0 & 20.1 & 21.4 & 6.3 \\
\hline N1002.70 & E1004.90 & $8,0 \mathrm{~B}$ & 99.30 & 21.2999992 & 21.2999992 & 20 & 20 & 15.466 & 21.2999992 & 20 & 5.82 & 9.646 & 0.0 & 20.7 & 22.1 & 6.1 \\
\hline N1001.10 & E1005.30 & $0,2 \mathrm{~B}$ & 99.30 & 22.1000004 & 22.1000004 & 20.1000004 & 20.1000004 & 15.976 & 22.1000004 & 20.1000004 & 5.82 & 10.156 & 0.0 & 19.8 & 21.8 & 9.0 \\
\hline N1001.10 & E1005.50 & $0,3 \mathrm{~B}$ & 99.30 & 21.7999992 & 21.8999996 & 19.8999996 & 19.8999996 & 15.702 & 21.8499994 & 19.8999996 & 5.82 & 9.882 & 0.0 & 20.1 & 22.1 & 8.9 \\
\hline N1001.10 & E1005.70 & $0,4 \mathrm{~B}$ & 99.30 & 21.2999992 & 21.2000008 & 19.5 & 19.5 & 15.54 & 21.25 & 19.5 & 5.82 & 9.72 & 0.0 & 20.1 & 21.9 & 8.2 \\
\hline N1001.10 & E1005.90 & $0,5 \mathrm{~B}$ & 99.30 & 22.2999992 & 22.2999992 & 20.8999996 & 20.8999996 & 16.076 & 22.2999992 & 20.8999996 & 5.82 & 10.256 & 0.0 & 20.4 & 21.7 & 6.3 \\
\hline N1001.10 & E1006.10 & $0,6 \mathrm{~B}$ & 99.30 & 20.6000004 & 20.7000008 & 19.3999996 & 19.3999996 & 15.394 & 20.6500006 & 19.3999996 & 5.82 & 9.574 & 0.0 & 20.3 & 21.6 & 6.1 \\
\hline N1001.10 & E1006.30 & $0,7 \mathrm{~B}$ & 99.30 & 19.2000008 & 19.1000004 & 18 & 18 & 14.838 & 19.1500006 & 18 & 5.82 & 9.018 & 0.0 & 20.0 & 21.2 & 6.0 \\
\hline N1001.10 & E1006.50 & $0,8 \mathrm{~B}$ & 99.30 & 19.2999992 & 19.2999992 & 18.3999996 & 18.5 & 14.976 & 19.2999992 & 18.4499998 & 5.82 & 9.156 & 0.0 & 20.2 & 21.1 & 4.4 \\
\hline
\end{tabular}





\section{APPENDIX H}

Construction Monitoring Memo 



\title{
TECHNICAL MEMORANDUM
}

\author{
To: $\quad$ Carissa Shelley \\ VRRSP Consultants, LLC,. \\ c/o Pape-Dawson Engineers, Inc \\ 2000 NW Loop 410 \\ San Antonio, Texas 78213 \\ From: Christina Nielsen, Project Manager \\ Date: $\quad$ November 8, 2019 \\ Re: $\quad$ Monitoring of Construction Activities on Site 41GU177 on Parcel 50360 (Mullins), \\ Guadalupe County, Texas / SWCA Project No. 31410
}

On behalf of the Central Texas Regional Water Supply Corporation (CTRWSC) and VRRSP Consultants, LLC, SWCA Environmental Consultants (SWCA) completed construction monitoring at prehistoric site 41GU177 (Snakeskin Bluff site) in Guadalupe County, Texas. The site was initially recorded by SWCA during Phase I survey investigations that occurred between August 2015 and March 2018 for the proposed Vista Ridge Regional Water Supply (Vista Ridge) Project (Figure 1).

Investigations were conducted in compliance with Section 106 of the National Historic Preservation Act (54 United States Code 306108) and its implementing regulations (36 Code of Federal Regulations 800), in anticipation of a Nationwide Permit 12 from the U.S. Army Corps of Engineers in accordance with Section 404 of the Clean Water Act. In addition, the work is subject to compliance with the Antiquities Code of Texas (ACT), as the Vista Ridge Project ultimately will be owned by CTRWSC, a political subdivision of the State of Texas. Testing and data recovery investigations at the site were conducted between October 2016 and December 2017, and included intensive shovel testing, geomorphological study with mechanical excavations, and subsequent hand excavations. The survey and testing investigations were conducted under ACT Permit No. 7295, and the subsequent data recovery was conducted under Permit No. 8231.

Site 41GU177 is a stratified prehistoric site on the western high bank of the Guadalupe River southeast of New Braunfels, Texas, near the community of McQueeney. The site contains components deposited intermittently from approximately the Late Archaic to Transitional Archaic periods through Late Prehistoric times. The primary components investigated in the excavations span the final Late Archaic period and into the Austin phase of the Late Prehistoric, a timeframe from approximately 2,600 to 900 years ago.

Given the sensitive nature of the cultural deposits at 41GU177, the main concern following the completion of data recovery excavations was the prevention of significant surface and subsurface impacts to the site during vegetation clearing and pipeline construction. Potential impacts included rutting by wheeled equipment and dragging large objects, such as tree trunks, through the sensitive site area. As such, SWCA developed a site monitoring protocol. 


\section{Restricted Information}

\section{Not for Public Disclosure}

Figure 1. Location map for Vista Ridge Project and Site 41GU177. 


\section{CONSTRUCTION MONITORING METHODS}

The cultural resources monitor was required to be on-site during all construction activities associated with vegetation removal and the subsequent placement of a protective cap of fill over the sensitive areas of the site. For vegetation clearing, the monitor ensured that:

1. All trees and brush were cut off at the ground surface to avoid pulling roots out of the ground and disturbing the buried cultural deposits.

2. A wheeled feller-buncher was used for tree and brush removal, provided the ground was not wet and could support the weight of the feller-buncher to avoid rutting.

3. Trees and other vegetation were not dragged across the sensitive site areas, to avoid potential subsurface disturbances to the cultural deposits.

For placement, use, and removal of the protective fill cap, the monitor:

1. Was on site for the placement of geofabric over the ground surface in the sensitive site areas prior to the placement of fill; the geofabric served as a boundary between the original ground surface and the cap of protective fill.

2. Ensured that during the placement of the fill, all material was placed by working it from the southwest side of the protective area and advancing towards the river side. The fill would be placed and pushed out so that heavy equipment was always supported by the protective fill and did not run directly over any geofabric.

3. Ensured that all construction activities related to the sensitive site areas stay in the prescribed work areas that were covered by geofabric and fill placement.

4. Ensured that the fill cap and geofabric were carefully removed to avoid gouging of the ground surface and impacts to buried cultural materials following completion of all construction activities at the site.

\section{CONSTRUCTION MONITORING RESULTS}

Construction monitoring of site 41 GU177 occurred September 3-4, 2019. The monitoring was performed to ensure no cultural resources associated with site $41 \mathrm{GU} 177$ were disturbed during pipeline construction activities. During excavation of the pipeline trench, SWCA monitored the profile and back dirt for the presence of any cultural resources. Soils observed during the trench excavation consisted of a brown (10YR 5/4) to light yellowish brown (10YR 6/4) silt loam with subangular limestone pebble and snail shell inclusions $(5 \%-10 \%)$. The construction crew continued excavating the trench while also setting up metal boxes to provide shoring for the trench walls, and eventually laying the pipe down after enough soil was excavated (Figure 2). Some welding was conducted after the pipe was properly positioned. SWCA monitored the trenching activity until the identified point past the deeper soils and site area was reached. No cultural resources were observed during the monitoring. 


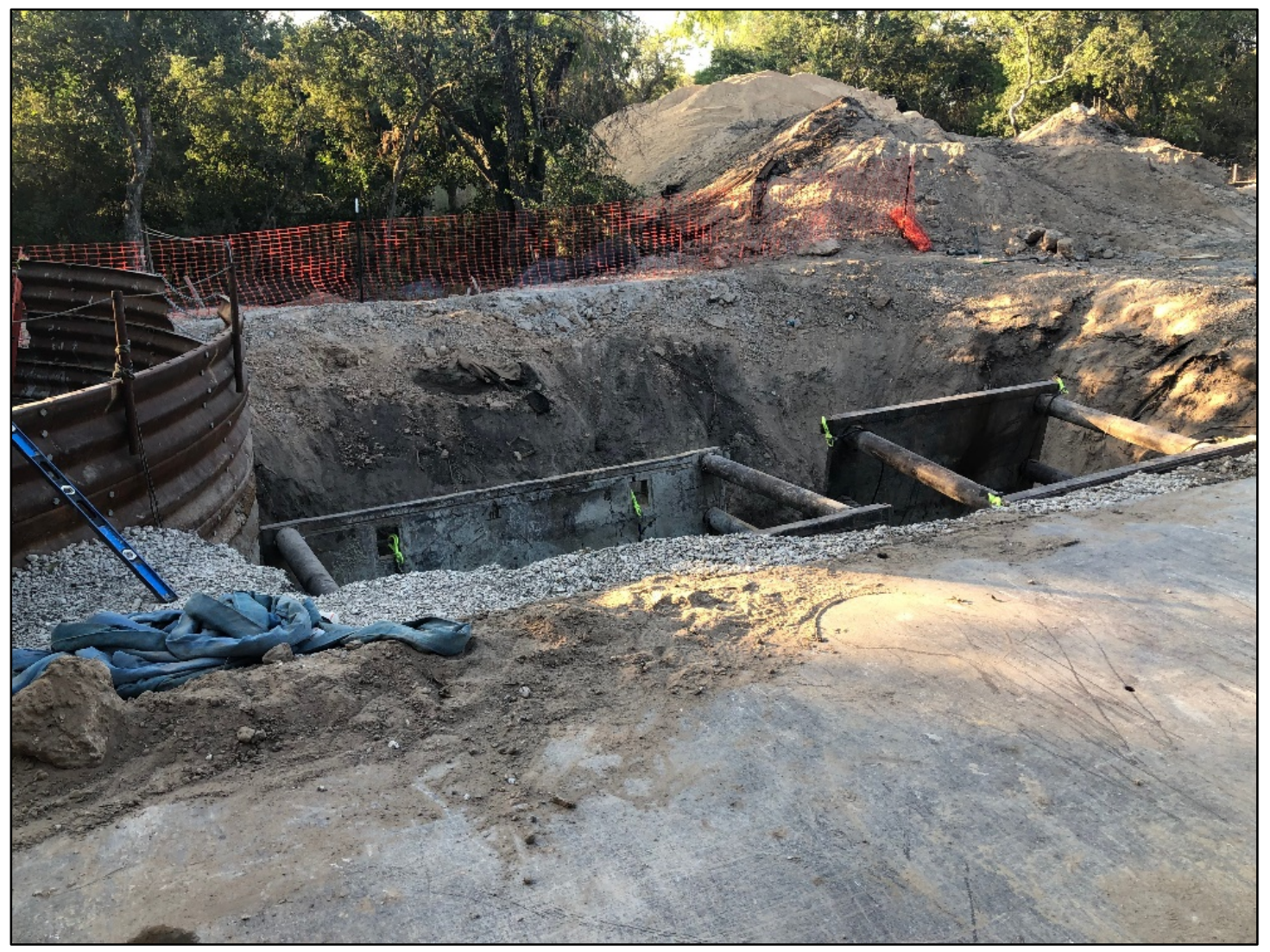

Figure 2. Overview of construction monitoring at site 41GU177 showing shoring placed in trench prior to installation of pipe, facing northeast. Backdirt pile to north was inspected for cultural resources.

An SWCA archaeologist returned to site 41 GU177 on November 5, 2019 to monitor the removal of the protective fill and geofabric. The monitoring was performed to ensure no cultural resources associated with site $41 \mathrm{GU} 177$ were disturbed during the fill removal. The construction crew used an excavator with a flat bar welded across the bucket teeth to slowly remove the protective fill and to pull back the geofabric separating the fill from the original ground surface (Figure 3). SWCA closely monitored the process to ensure the excavator was not digging below the original ground surface and to ensure no new artifacts or features were uncovered. No cultural material was observed during the monitoring (Figure 4). 


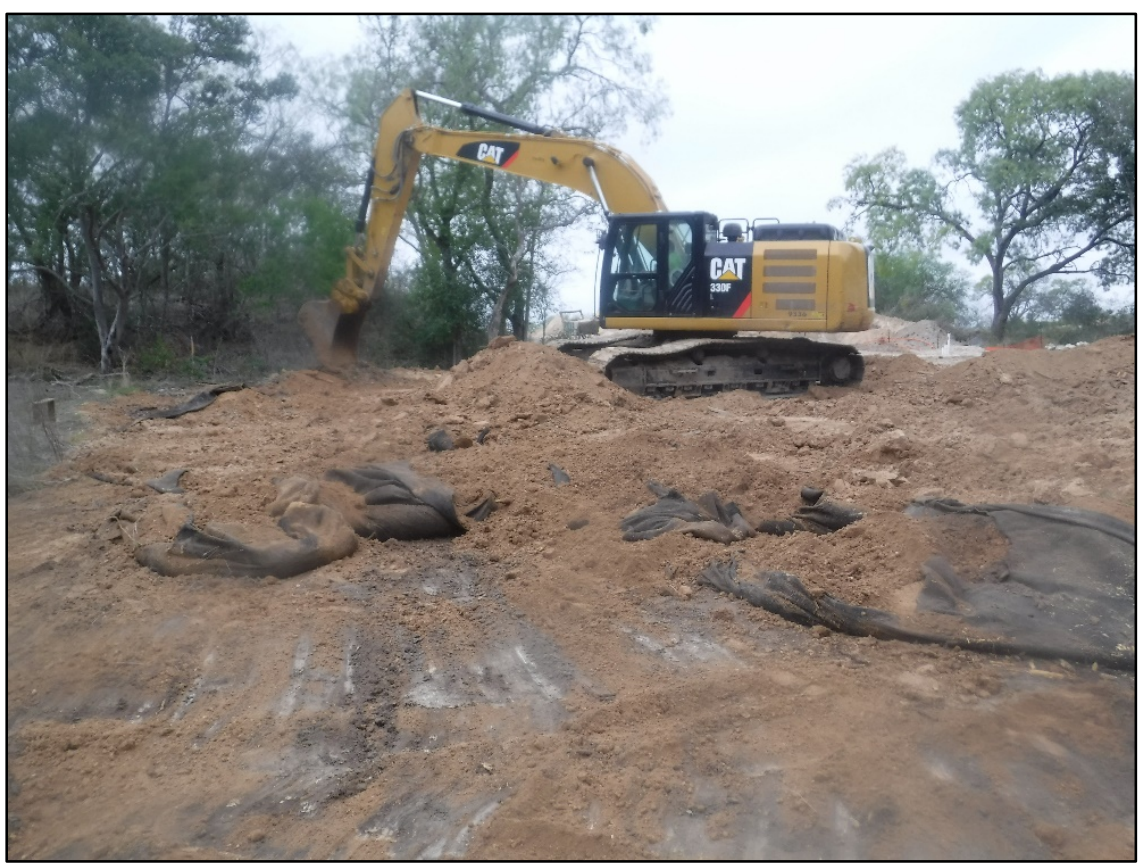

Figure 3. Overview of construction monitoring at site 41GU177 showing the removal of the protective fill layer and geofabric, facing northeast.

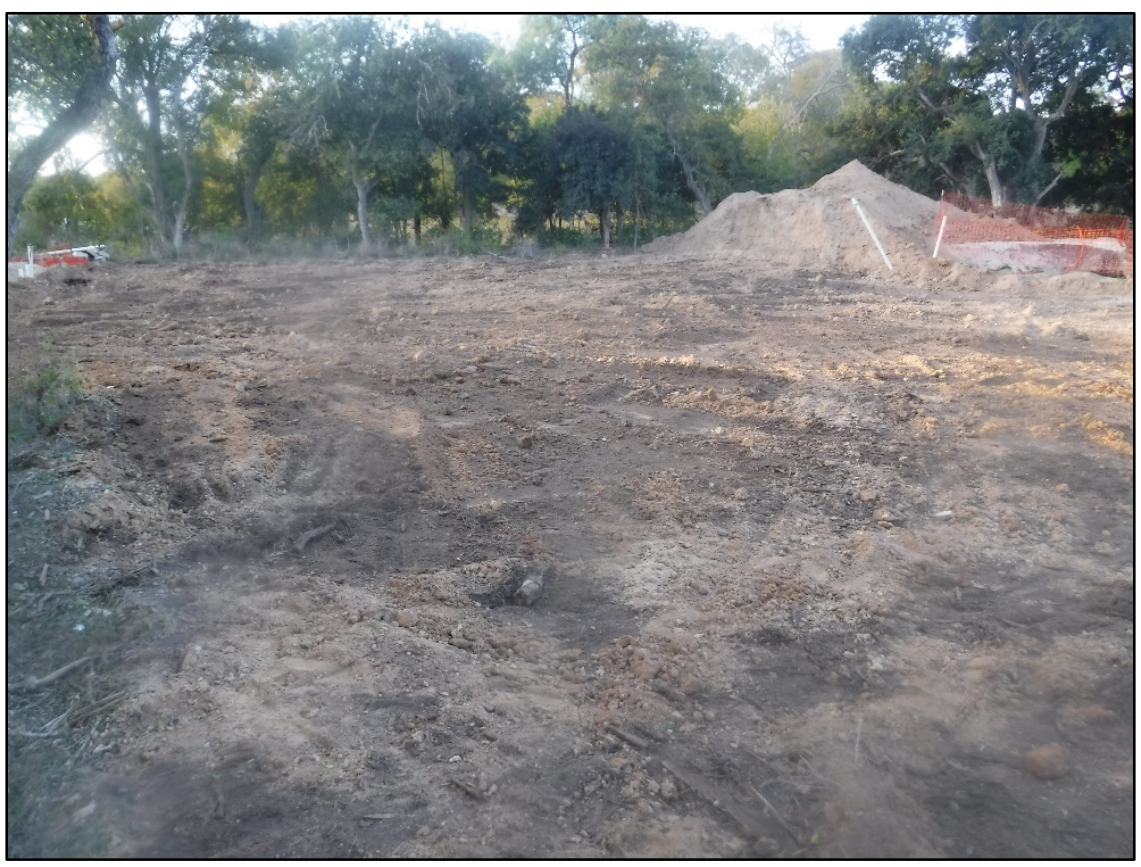

Figure 4. Overview of site 41GU177 after the protective fill and geofabric had been removed, facing northeast. 


\section{RECOMMENDATIONS}

The Snakeskin Bluff site is considered eligible for designation as a State Antiquities Landmark and for the National Register of Historic Places. Although data recovery investigations at the site revealed that some cultural components of the site exhibited signs of disturbance, the Late Prehistoric Austin Phase component revealed intact, well-preserved archaeological deposits that significantly contributed to our understanding of Late Prehistoric patterns. Contributing components beyond the impact area will not be affected and will be preserved by avoidance; however, it is important to note that these investigations mitigated the project-specific effects, not the entire site. Any future project that could impact the site's deeper deposits, or those beyond the current right-of-way, warrant further consideration to assess the possibilities for additional contributing components. With these considerations, no further work is recommended. 